Supporting Information for

\title{
Strongly directing substituents in the radical arylation of substituted
} benzenes

(atom coordinates and absolute energies)

\author{
Josefa Hofmann, ${ }^{\mathrm{a}}$ Timothy Clark, ${ }^{\mathrm{b}}$ Markus R. Heinrich ${ }^{\mathrm{a}, *}$
}

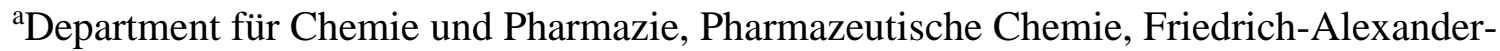

Universität Erlangen-Nürnberg, Schuhstraße 19, 91052 Erlangen, Germany

${ }^{\mathrm{b}}$ Computer-Chemie-Centrum and Interdisciplinary Center for Molecular

Materials, Friedrich-Alexander-Universität Erlangen-Nürnberg, Nägelsbachstrasse 25, 91052

Erlangen, Germany

Markus.Heinrich@fau.de

\section{Content}

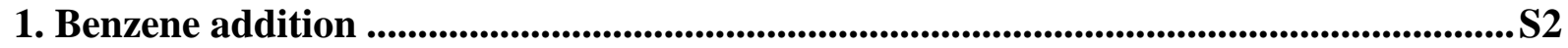

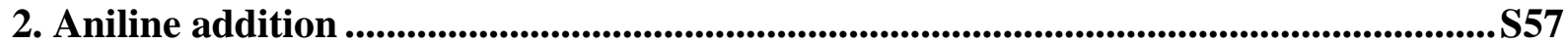

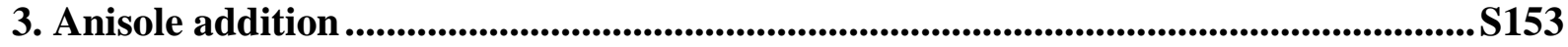

4. Cyanobenzene addition ...........................................................................................................S225

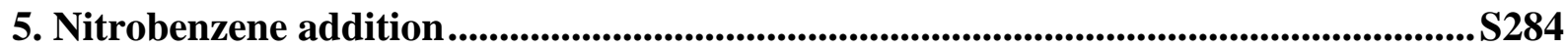

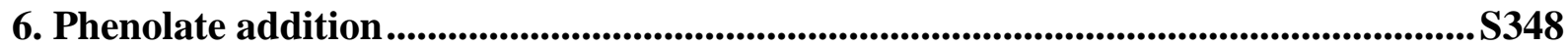

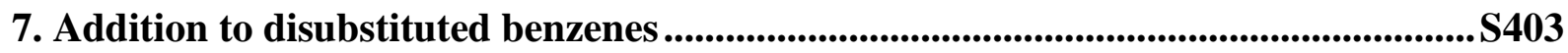




\section{Benzene addition}

Phenyl radical addition to Benzene pre-Reaction Complex

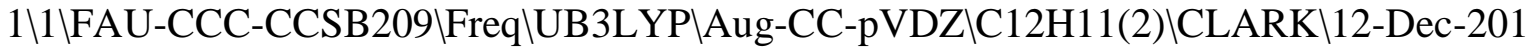
4\0\\\# ub3lyp/aug-cc-pvdz freq name=clark \\Addition precursor product Ph-radical + benzenel|0,2\C,-1.862698,-0.313801,0.218581\C,-2.491272,$0.715634,-0.965256 \backslash \mathrm{C},-3.851441,-0.451641,-1.160814 \backslash \mathrm{C},-4.584023,0.21429$ $9,-0.172048 \backslash \mathrm{C},-3.955942,0.616279,1.01198 \backslash \mathrm{C},-2.595708,0.352261,1.206987$ $\backslash \mathrm{H},-0.801916,-0.520906,0.370545 \backslash \mathrm{H},-1.920448,-1.235509,-1.735728 \backslash \mathrm{H},-4.3$ $41131,-0.765695,-2.083597 \backslash \mathrm{H},-4.527046,1.135043,1.78314 \backslash \mathrm{H},-2.106495,0.6$ $65589,2.13015 \backslash \mathrm{H},-5.644429,0.420001,-0.324121 \backslash \mathrm{C}, 4.519603,0.531579,-0.23$ $8462 \backslash \mathrm{C}, 4.366224,-0.740326,0.3253 \backslash \mathrm{C}, 3.079832,-1.261253,0.557974 \backslash \mathrm{C}, 2.016$ $968,-0.456342,0.2029 \backslash \mathrm{C}, 2.104093,0.802593,-0.355505 \backslash \mathrm{C}, 3.401062,1.301496$ ,-0.577921\H,5.520506,0.926707,-0.414653\Н,5.243462,-1.334587,0.587159 $\backslash \mathrm{H}, 2.945049,-2.250778,0.995944 \backslash \mathrm{H}, 1.223104,1.390354,-0.614746 \backslash \mathrm{H}, 3.52915$ 2,2.29272,-1.016378।IVersion=EM64L-G09RevA.02 $\backslash$ State $=2-A \backslash H F=-463.863035$ $6 \backslash S 2=0.757286 \backslash S 2-1=0 . \backslash S 2 A=0.750031 \backslash \mathrm{RMSD}=9.825 \mathrm{e}-09 \backslash \mathrm{RMSF}=2.273 \mathrm{e}-05 \backslash \mathrm{ZeroP}$ oint $=0.1872051 \backslash$ Thermal $=0.197615 \backslash Z \mathrm{ZPE}=117.4729689 \backslash \mathrm{Dipole}=0.3950675,0.122$ $3643,-0.0561472 \backslash$ DipoleDeriv $=0.1187332,-0.0235418,0.0141872,-0.0729415$, $-0.0981512,0.0260688,0.0503512,0.027022,-0.0556077,-0.0484991,-0.05442$ $49,-0.037074,-0.0446762,-0.0759096,0.0697679,-0.0279592,0.0718592,-0.0$ $007285,-0.0231155,0.0031271,0.0676088,-0.0002904,-0.1019713,0.0677701$, $0.060967,0.0679267,0.0450762,0.0674761,-0.045772,0.0288492,-0.039493,-$ $0.1095897,0.0276141,0.0263743,0.0277147,-0.061231,-0.0158608,-0.056508$ $8,-0.0492814,-0.0506104,-0.0796327,0.0820125,-0.0411704,0.0823892,0.01$ $01099,-0.0584573,-0.0045595,0.0621408,-0.0092828,-0.0974225,0.060293,0$ $.0387562,0.0564708,0.0342582,-0.0272949,0.040701,-0.0270405,0.061923,0$ $.0890756,-0.0207026,-0.0452307,-0.0212657,0.0545601,0.0339573,0.057880$ $2,0.0574506,0.0610415,0.0743567,-0.0759966,0.061475,-0.0758307,-0.0059$ $672,0.0239882,-0.0089792,-0.0699162,-0.0076816,0.1012447,-0.0671296,-0$ $.0777223,-0.0674055,-0.047239,0.0106791,0.0548014,0.056693,0.0614612,0$ $.0771836,-0.0830783,0.059224,-0.0826196,-0.0107989,0.0434528,-0.008597$ $4,-0.0736874,-0.0082514,0.0975168,-0.0629535,-0.0749763,-0.0622545,-0$. $0418811,-0.1019014,0.0448302,-0.029736,0.0473475,0.1106037,-0.0206031$, $-0.0308989,-0.0206276,0.0728054,0.0082356,0.0634822,-0.0282179,0.06352$ $11,-0.1000568,-0.0158499,-0.0282313,-0.0156926,-0.1285769,0.1045608,-0$ $.0228101,0.0096996,0.0173336,0.0323699,-0.0753393,-0.0077999,-0.075623$ $7,-0.104761,-0.1512753,0.0220081,-0.0102416,-0.040925,0.0353452,-0.054$ $5083,0.0178231,-0.0542027,-0.0639174,-0.0642299,-0.0555175,0.0249442,-$ $0.0370081,-0.0241611,-0.0315883,0.0185411,-0.0312916,-0.081467,-0.0703$ $725,-0.078574,0.0350313,-0.0877529,-0.066417,-0.0066129,0.0388157,-0.0$ $064385,-0.0782798,0.1083741,0.0272071,-0.0124505,0.0149777,0.0654614,-$ $0.0875169,-0.0060584,-0.0871941,-0.093934,-0.071031,-0.0518094,0.02318$ $33,-0.0521093,0.0594507,0.036252,0.0228849,0.0361964,0.125155,-0.05846$ $95,0.0755867,-0.0332305,0.056649,0.0279201,0.04483,-0.0246453,0.044910$ $5,0.109497,0.0958242,-0.0145594,0.0064993,-0.008726,-0.0188141,0.07268$ $73,0.0037235,0.0721719,0.1130998,0.028992,0.0623066,-0.0272321,0.09353$ $25,0.0564672,0.0336848,-0.0424546,0.0332938,0.1176964,0.0462337,-0.026$ $2765,0.0118209,-0.0180386,-0.0548694,0.0808987,0.0082114,0.0804915,0.0$ 921316\Polar=178.8780334,-8.544101,125.5169364,3.9537981,-0.0990996,12 $2.595433 \backslash \mathrm{PG}=\mathrm{C} 01[\mathrm{X}(\mathrm{C} 12 \mathrm{H} 11)] \mathrm{NImag}=1 \backslash 0.70204722,-0.12068387,0.25719443$ 
$, 0.06106086,0.19053733,0.56616709,-0.16371870,-0.00139492,-0.05909771$, $0.65099067,-0.02820151,-0.09413074,-0.08111138,-0.12527378,0.27235802$, $-0.11452331,-0.06668343,-0.26451677,0.02247151,0.21893784,0.60308030$,$0.02010099,-0.02644420,-0.07053361,-0.32127435,0.06425966,-0.00686646$, $0.64522458,-0.00712187,0.01995202,0.02179196,0.03777270,-0.08557945,-0$ $.02358987,-0.09541136,0.26294730,-0.03004061,0.01134874,0.01451664,-0$. $06239939,-0.00921448,-0.11738451,0.07983743,0.21096312,0.62057429,-0.0$ $0209456,0.00022190,0.00313329,-0.02892947,0.01877683,0.01875873,-0.178$ $85408,0.07818585,0.10083230,0.70273809,0.00017356,-0.01877537,-0.02439$ $400,0.03822256,0.00352507,0.01034622,0.05149774,-0.12722673,-0.1001588$ $9,-0.11955843,0.25969044,0.00315844,-0.02438736,-0.05616567,0.05932219$ ,-0.00009239,0.04065174,0.04529323,-0.08574975,-0.21961251,0.06070851, $0.18974365,0.56705240,-0.02883158,0.03789152,0.05931895,-0.04671664,-0$ $.00397446,-0.03060605,0.05989250,-0.01859120,-0.01037789,-0.16339079,-$ $0.00233650,-0.05856172,0.65087551,0.01862072,0.00269791,0.00025984,-0$. $00401982,-0.00560471,-0.00034352,0.00094749,-0.00203864,-0.02186514,-0$ $.02891415,-0.09648213,-0.08028787,-0.12458710,0.27374899,0.01886321,0$. $01060757,0.04042705,-0.03073633,-0.00034738,-0.02420295,0.03023455,-0$. $03241659,-0.04250704,-0.11418011,-0.06582147,-0.26567394,0.02185294,0$. $21791937,0.60394906,-0.17920626,0.05266665,0.04484388,0.05979612,0.000$ $26776,0.03032274,-0.05157966,0.02121084,0.01935562,-0.02025178,-0.0263$ $3737,-0.07074701,-0.32123034,0.06419557,-0.00685939,0.64524546,0.07895$ $020,-0.12445431,-0.08655239,-0.01909711,-0.00374590,-0.03148286,0.0210$ $7558,-0.01514427,-0.00660225,-0.00697088,0.02062164,0.02153966,0.03782$ $309,-0.08545024,-0.02357059,-0.09631740,0.26052836,0.10035789,-0.10085$ $749,-0.21846414,-0.00997322,-0.02104714,-0.04271936,0.01923148,-0.0065$ $9125,-0.00968051,-0.03011715,0.01103092,0.01476537,-0.06237519,-0.0092$ $2667,-0.11734775,0.07990953,0.21157904,0.62006663,-0.31852853,0.055370$ $16,-0.03970591,-0.00926966,0.00378552,0.00097663,0.00146169,-0.0000134$ $2,-0.00319218,0.00060717,-0.00027900,0.00021495,0.00100758,0.00278562$, $0.00237802,-0.01116669,0.00143307,-0.00490209,0.33602529,0.05575500,-0$ $.05158692,-0.00007823,-0.00924833,0.00478522,-0.00147818,0.00029739,0$. $00530664,-0.00414539,-0.00026612,-0.00099450,-0.00009191,0.00248444,0$. $00440585,-0.00486678,0.01405300,0.00065669,0.00288857,-0.06104788,0.04$ $411326,-0.03981313,-0.00017496,-0.05941329,-0.02573757,0.00558718,0.00$ $057889,-0.00253217,-0.00430207,-0.00298807,0.00020905,-0.00006544,-0.0$ $0108711,0.00170762,-0.00476572,-0.00156350,0.02194807,-0.00408541,0.00$ $651532,0.04341878,0.00359057,0.05797705,0.01025797,-0.00712839,-0.0106$ $7756,-0.13257361,0.07408063,0.10141870,-0.01020947,0.01435059,0.022603$ $28,-0.00488246,0.00190468,-0.00273172,-0.00068332,-0.00045038,-0.00061$ $184,0.00010131,0.00026417,-0.00376247,0.00057040,-0.00086583,0.0006238$ $1,0.13703980,0.00567827,-0.00102097,-0.00507149,0.07402356,-0.10464549$ ,-0.10207997,0.00146659,0.00055530,-0.00459161,0.00224895,0.00595108,$0.00152375,-0.00043757,-0.00075234,0.00057402,-0.00004397,0.00588435$,$0.00359586,-0.00094294,-0.00278898,0.00185595,-0.08123939,0.10073795,0$ $.01599236,-0.01211044,-0.01350629,0.10144704,-0.10201780,-0.19305507,-$ $0.00434382,0.00244095,0.00592110,-0.00195111,-0.00167752,0.00263249,-0$ $.00065924,0.00055597,-0.00024735,-0.00429665,-0.00341150,-0.00202870,0$ $.00043241,0.00184340,0.00022672,-0.10796409,0.11280921,0.20092529,-0.0$ $0506970,0.00296781,-0.00042332,-0.00807405,-0.00948263,-0.02621952,-0$. $11228861,-0.03210842,-0.10829452,0.01032652,0.00519145,0.01452773,-0.0$ 
$0057803,0.00311167,0.00229181,-0.00077432,0.00027514,0.00062173,-0.000$ $52092,-0.00045723,-0.00133467,0.00071669,-0.00090107,0.00068608,0.1153$ $1754,0.00268657,0.00561113,-0.00183658,0.00333064,0.00495057,0.0049869$ $4,-0.03202365,-0.06449516,-0.07566461,-0.00774426,-0.00023807,-0.01199$ $230,0.00348032,0.00426253,-0.00409992,0.00023899,-0.00086479,0.0003551$ $3,0.00021616,0.00018235,0.00021481,-0.00107076,-0.00287094,0.00171859$, $0.03244549,0.05725595,-0.00114314,-0.00171422,0.00310606,0.00065365,-0$ $.00201458,-0.00047845,-0.10828116,-0.07567129,-0.25364427,-0.01239379$, $-0.00494307,-0.01361607,0.00301662,-0.00429314,-0.00016123,0.00061470$, $0.00037657,0.00009629,0.00009685,-0.00014868,-0.00012871,0.00036568,0$. $00179315,-0.00002272,0.11706433,0.08469887,0.26583860,-0.00491973,0.00$ $189059,-0.00264568,-0.00065479,-0.00038871,-0.00064598,0.00006864,0.00$ $012193,-0.00362983,0.01018308,-0.00698906,-0.01094257,-0.13267151,0.07$ $381424,0.10168353,-0.01022142,0.01443828,0.02261785,-0.00062003,0.0007$ $4315,0.00113278,-0.00001220,-0.00021094,0.00017935,0.00036883,-0.00035$ $131,-0.00062760,0.13718932,0.00223671,0.00590291,-0.00150484,-0.000388$ $79,-0.00062258,0.00050097,-0.00022551,0.00552716,-0.00345128,0.0058999$ $6,-0.00051223,-0.00512295,0.07379141,-0.10459632,-0.10157239,0.0015780$ $0,0.00071841,-0.00475301,0.00005728,-0.00001025,-0.00027272,-0.0002105$ $4,-0.00089623,0.00039988,0.00032363,-0.00005018,-0.00030631,-0.0810963$ $2,0.10028438,-0.00196186,-0.00160750,0.00262928,-0.00063538,0.00048375$ ,-0.00023391,-0.00436158,-0.00325790,-0.00209575,0.01600385,-0.0121802 $5,-0.01318442,0.10163492,-0.10155954,-0.19317910,-0.00421636,0.0022315$ $9,0.00587005,-0.00029045,0.00008443,0.00017342,0.00018118,0.00039715,-$ $0.00022293,0.00077996,-0.00067501,-0.00080138,-0.10810521,0.11248614,0$ $.20098063,0.01040769,0.00488074,0.01449683,-0.00052397,0.00328742,0.00$ $229271,-0.00081380,0.00019460,0.00063475,-0.00502772,0.00305032,-0.000$ $39015,-0.00810702,-0.00954218,-0.02614143,-0.11211658,-0.03174021,-0.1$ $0822704,0.00057118,-0.00101645,0.00025457,0.00036691,0.00027635,0.0007$ $5824,-0.00000040,-0.00026865,0.00006413,0.00072770,-0.00093540,0.00060$ $676,0.11512845,-0.00789589,-0.00081596,-0.01186854,0.00356030,0.004611$ $31,-0.00426392,0.00023563,-0.00099696,0.00042860,0.00267626,0.00567311$ ,-0.00190216,0.00334597,0.00472265,0.00513501,-0.03176058,-0.06386736, $-0.07576322,-0.00091660,-0.00272355,0.00186948,-0.00037624,-0.00012400$ ,-0.00063985,-0.00027459,-0.00087760,0.00040857,-0.00110551,-0.0028576 $0,0.00173360,0.03228816,0.05705682,-0.01217097,-0.00478442,-0.01396494$ $, 0.00285414,-0.00446047,-0.00006089,0.00067654,0.00044100,0.00007761,-$ $0.00114774,-0.00173131,0.00308849,0.00077872,-0.00189002,-0.00040011,-$ $0.10825963,-0.07583867,-0.25394273,0.00045061,0.00184190,0.00018405,-0$ $.00059388,-0.00026275,-0.00081121,0.00005939,0.00040724,-0.00024809,0$. $00029825,0.00180466,-0.00003595,0.11699542,0.08463334,0.26624542,0.000$ $53028,-0.00026908,0.00020403,0.00099044,0.00280650,0.00253806,-0.01151$ $392,0.00141515,-0.00526967,-0.32037915,0.05545458,-0.03985340,-0.00954$ $286,0.00398754,0.00118592,0.00148857,-0.00012171,-0.00329919,-0.000125$ $89,-0.00021429,0.00009523,-0.00069115,0.00009107,-0.00028424,0.0006054$ $8,-0.00094274,0.00057298,0.00059266,-0.00103394,0.00036467,-0.00059288$ $, 0.00023171,0.00005978,0.33864763,-0.00026253,-0.00085290,-0.00011503$, $0.00245065,0.00409006,-0.00483133,0.01432500,0.00120260,0.00294778,0.0$ $5543720,-0.05122807,-0.00012663,-0.00891237,0.00511422,-0.00156002,0.0$ $0024489,0.00508766,-0.00403241,-0.00022365,-0.00087388,0.00045928,0.00$ $076756,0.00001615,0.00007578,-0.00109745,-0.00287162,0.00180436,-0.000$ 
$87671,-0.00288668,0.00186643,-0.00044508,0.00021333,-0.00015388,-0.061$ $40848,0.04299041,0.00020334,-0.00012241,-0.00100126,0.00178346,-0.0046$ $6811,-0.00165832,0.02166306,-0.00409630,0.00682260,-0.03985563,-0.0001$ $3935,-0.05884173,-0.02574458,0.00550111,0.00091067,-0.00255253,-0.0041$ $9944,-0.00314196,0.00010101,0.00046472,-0.00013625,0.00113051,-0.00028$ 426,0.00018570,0.00024787,0.00188549,0.00005717,0.00068666,0.00178660, $0.00009183,-0.00135333,0.00020700,-0.00011823,0.04369038,0.00366466,0$. $05682967,0.00015591,-0.00005096,0.00004926,-0.00005517,0.00000411,0.00$ $004571,0.00000885,0.00000549,-0.00000947,-0.00004120,0.00000745,-0.000$ $00266,-0.00000044,0.00001185,0.00000353,-0.00005207,-0.00003544,-0.000$ $04911,0.00001996,0.00008410,-0.00005709,0.00000971,0.00001140,0.000008$ $14,-0.00002005,-0.00000114,-0.00001062,-0.00002059,0.00000727,0.000005$ $59,0.00000933,0.00000088,-0.00000588,-0.00001173,0.00000160,-0.0000007$ $2,0.72223510,0.00004668,-0.00001574,0.00001656,0.00000495,0.00003896,0$ $.00000133,-0.00000900,-0.00000833,-0.00001430,0.00000825,0.00000933,0$. $00000059,-0.00000284,-0.00000724,0.00001613,0.00000289,0.00003747,-0.0$ $0003511,-0.00005431,-0.00007020,0.00002440,-0.00001297,-0.00002175,0.0$ $0000812,-0.00000326,-0.00000198,0.00000088,-0.00000284,-0.00000259,-0$. $00000026,-0.00000858,-0.00001325,0.00000928,-0.00000073,-0.00000383,0$. $00000089,0.02607158,0.58087861,-0.00001571,-0.00004406,-0.00001439,-0$. $00001317,-0.00000924,0.00000040,-0.00001142,0.00001241,-0.00000088,-0$. $00000143,0.00000047,0.00000849,0.00001174,-0.00001490,-0.00000788,0.00$ $001949,0.00001347,0.00000992,0.00001866,0.00006180,0.00000403,0.000005$ $27,-0.00000233,0.00001139,-0.00000021,0.00000035,-0.00000457,0.0000003$ $3,0.00000164,0.00000009,0.00000381,0.00001031,-0.00000510,0.00000159,0$ $.00000036,-0.00000297,-0.01235959,-0.19680538,0.22343197,-0.00000101,-$ $0.00010730,0.00005724,-0.00000703,-0.00001103,0.00003295,-0.00000440$, $0.00000682,-0.00000706,0.00000717,0.00000238,-0.00000055,-0.00000424,0$ $.00000852,0.00000715,-0.00001571,-0.00003860,-0.00001728,0.00006096,0$. $00014049,-0.00006974,0.00000115,0.00002963,-0.00000249,-0.00000248,0.0$ 0000586,-0.00000113,-0.00000204,0.00000367,-0.00000395,0.00000807,0.00 $001303,-0.00001521,0.00000128,0.00000085,-0.00000032,-0.12330867,0.007$ $86646,-0.00332577,0.68122845,-0.00007791,0.00003546,-0.00004865,0.0000$ 4386,-0.00004081,-0.00001899,0.00000842,0.00000717,0.00002418,0.000004 $46,-0.00000288,-0.00000412,-0.00000057,-0.00000975,-0.00001235,0.00005$ $727,-0.00000931,0.00004195,0.00000058,0.00005833,0.00000932,-0.0000017$ $2,0.00001207,-0.00000563,0.00001491,-0.00000015,0.00000908,0.00001268$, $-0.00000545,-0.00000369,-0.00000098,0.00000419,-0.00000612,0.00000550$, $0.00000040,0.00000148,-0.04759079,-0.28703111,0.09818923,-0.04043573,0$ $.58545710,0.00002255,0.00002879,-0.00000581,-0.00002242,-0.00000656,0$. $00001095,0.00002231,0.00000637,-0.00000559,-0.00000934,-0.00001043,-0$. $00000711,-0.00001609,0.00003217,0.00001553,-0.00004391,-0.00004090,-0$. $00002638,0.00002667,-0.00003443,0.00003111,-0.00000097,0.00000598,-0.0$ $0000365,-0.00000054,-0.00000163,0.00000066,-0.00000669,0.00000235,0.00$ $000136,0.00000644,0.00000249,0.00001193,-0.00000227,0.00000148,0.00000$ $325,0.02120857,0.09817553,-0.10892889,0.01701064,-0.20114258,0.2203810$ $5,0.00002229,-0.00003452,0.00005940,-0.00013055,0.00011273,0.00008715$, $0.00000020,-0.00002893,-0.00004423,-0.00001794,0.00000953,0.00000104,-$ $0.00000803,0.00001867,0.00005040,-0.00014089,0.00000288,-0.00014012,0$. $00018498,0.00004187,-0.00008335,0.00003709,-0.00002968,-0.00001160,-0$. $00002566,0.00000250,-0.00000918,-0.00002971,0.00000903,0.00000123,0.00$ 
$000986,0.00000109,0.00002776,-0.00001129,0.00000203,-0.00000062,0.0153$ $7925,-0.07224183,0.03199926,-0.27553525,-0.03550472,0.01603834,0.59343$ $261,0.00026885,-0.00010048,0.00005798,-0.00015704,0.00005797,0.0000031$ $8,-0.00002479,0.00001033,-0.00003365,-0.00003542,0.00000964,0.00001233$ ,-0.00001289,0.00002599,0.00002635,-0.00013816,0.00001470,-0.00007282, $0.00012374,-0.00001477,0.00001740,0.00006259,-0.00000879,0.00002723,-0$ $.00002650,-0.00000538,-0.00002171,-0.00003809,0.00001391,0.00001028,0$. $00002918,0.00000941,-0.00000210,-0.00001763,0.00000424,-0.00000188,-0$. $02913114,-0.00925991,0.00792224,-0.10371142,-0.13278824,0.03178721,-0$. $01028823,0.63302890,-0.00009173,-0.00004382,-0.00006988,0.00001760,0.0$ $0003461,-0.00001425,-0.00002089,-0.00000301,0.00002788,0.00002108,0.00$ $002028,0.00001415,0.00002421,-0.00005066,-0.00000331,0.00011489,0.0000$ $2659,0.00004849,-0.00006618,0.00006175,0.00004536,0.00000384,-0.000033$ $69,0.00001043,0.00002124,-0.00000184,0.00000194,0.00000488,-0.00000307$ ,-0.00000667,-0.00004911,0.00001697,-0.00002579,0.00000939,-0.00000257 $, 0.00000045,0.01290484,0.00802776,0.00511300,0.04617543,0.03162769,-0$. $07566615,0.00392409,-0.22675927,0.22104370,-0.00142640,0.00014762,-0.0$ $0017132,0.00031299,-0.00010743,-0.00003553,-0.00000028,0.00001895,0.00$ $008952,0.00002710,0.00000372,-0.00001001,0.00002363,-0.00006313,-0.000$ $07855,0.00031451,-0.00000756,0.00017538,-0.00011607,-0.00008929,0.0001$ $0964,-0.00005986,0.00003974,-0.00001840,0.00003913,-0.00000459,0.00001$ $176,0.00004389,-0.00001214,0.00000314,-0.00005086,0.00003487,-0.000039$ $30,0.00001189,0.00000243,-0.00000237,-0.01462547,0.02199654,-0.0097410$ $6,-0.06418400,-0.00554678,0.00257975,-0.23964382,0.12557088,-0.0553205$ $8,0.39336213,-0.00005960,0.00005133,0.00013418,0.00017772,-0.00009988$, $-0.00003201,0.00003147,0.00001990,0.00004205,-0.00000628,-0.00001577,0$ $.00000648,0.00000411,0.00001628,-0.00003661,0.00004130,-0.00005476,0.0$ 0003794,-0.00024906,-0.00005707,-0.00026927,-0.00002989,0.00004789,0.0 $0001984,0.00001022,0.00000521,0.00000881,0.00001914,-0.00000228,0.0000$ $0403,-0.00001226,-0.00000408,-0.00001787,0.00000183,0.00000195,-0.0000$ $0036,0.02187615,-0.05079095,0.01907790,0.04516350,0.05124665,-0.017050$ $07,0.10215021,-0.18150714,0.05778693,-0.10963408,0.52637945,0.00001238$ $, 0.00017905,0.00025772,0.00013603,-0.00008749,-0.00009928,0.00004168,-$ $0.00001305,0.00004127,0.00000585,0.00000284,0.00000528,-0.00004484,0.0$ $0004291,0.00001698,-0.00014995,-0.00000288,-0.00005589,0.00000150,-0.0$ $0024119,-0.00049135,0.00001713,0.00003284,0.00002096,-0.00000949,0.000$ $00920,0.00001079,-0.00000013,0.00000218,0.00000891,-0.00000616,-0.0000$ $0196,0.00006671,-0.00000684,0.00000204,0.00000395,-0.00973162,0.019096$ $76,-0.01625003,-0.01986989,-0.01697974,0.02067539,-0.04497086,0.057778$ $76,-0.07610926,0.04793836,-0.20098307,0.16132297,0.00054468,-0.0000357$ $5,0.00012134,-0.00018873,0.00009892,0.00003102,-0.00003103,0.00001359$, $-0.00009656,-0.00003408,-0.00000874,0.00001110,-0.00004466,0.00008900$, $0.00005673,-0.00022686,0.00002156,-0.00014671,-0.00012010,-0.00014910$, $-0.00001328,0.00004503,-0.00004555,0.00004284,-0.00004161,-0.00000811$, $-0.00000815,-0.00004616,0.00000024,0.00000671,0.00005351,-0.00005697,0$ $.00001711,-0.00001778,-0.00000456,0.00000302,-0.05466282,0.03120090,-0$ $.01375451,-0.02008920,-0.03230392,0.01433762,0.04254432,0.02627524,-0$. $01169129,-0.09845647,0.00439007,-0.00168632,0.65741179,0.00005714,-0.0$ $0023027,0.00011083,-0.00004967,0.00004113,0.00014871,0.00003027,-0.000$ $04304,0.00003121,-0.00001883,0.00006413,-0.00001525,-0.00000944,-0.000$ $05639,0.00003267,0.00001029,-0.00005622,-0.00017975,-0.00028528,0.0001$ 
$5981,-0.00004320,0.00001228,0.00002982,-0.00002173,-0.00000251,0.00001$ $294,-0.00001035,-0.00000862,0.00002025,-0.00000658,0.00000913,0.000019$ $86,0.00002412,-0.00000735,-0.00000097,0.00000252,-0.01166704,0.0494484$ $8,-0.01801572,-0.02875882,-0.03637519,0.01460496,0.05146093,-0.0309387$ $3,0.01557401,-0.01906830,-0.29882054,0.11004879,-0.06543378,0.57985106$ $,-0.00002653,0.00014388,0.00008035,-0.00000112,-0.00009863,-0.00008040$ $, 0.00002879,0.00004359,-0.00000332,0.00000237,-0.00001687,0.00003401,-$ $0.00003199,0.00002343,0.00001645,0.00000897,0.00007839,-0.00001603,0.0$ $0014036,-0.00011961,-0.00002045,-0.00001610,0.00003417,0.00001910,0.00$ $000652,-0.00001383,0.00000335,-0.00000450,-0.00000328,-0.00000098,-0.0$ $0000697,-0.00003231,0.00002802,0.00000580,0.00000420,0.00000432,0.0053$ $0942,-0.01810129,0.01690490,0.01273480,0.01461754,-0.01009301,-0.02283$ $547,0.01557446,-0.00252275,0.00862215,0.11002495,-0.09949550,0.0282504$ $3,-0.20288209,0.21185301,-0.00021267,0.00011252,-0.00007643,0.00009491$ ,-0.00000769,-0.00000698,0.00000714,0.00000025,0.00002728,0.00002857,-$0.00000378,0.00000223,0.00001235,-0.00002783,-0.00001956,0.00010001,0$. $00000223,0.00003166,-0.00004382,-0.00009821,0.00005676,-0.00003035,-0$. $00002506,-0.00001384,0.00002354,0.00000135,0.00001357,0.00002505,-0.00$ $000874,-0.00000715,-0.00002614,0.00000042,0.00001178,0.00001697,-0.000$ $00149,0.00000055,-0.25518176,0.06556880,-0.02887240,0.04118756,0.06095$ $032,-0.02701461,-0.06536979,0.00671082,-0.00291695,0.02258860,-0.08019$ $446,0.03555396,-0.28340781,-0.02850587,0.01279467,0.63411640,0.0000047$ $8,0.00022585,-0.00006172,0.00000267,0.00000527,-0.00006000,-0.00000333$ ,-0.00001129,-0.00001676,0.00002277,-0.00002027,0.00001337,-0.00001499 $, 0.00003561,0.00001428,-0.00005035,0.00001498,0.00001749,0.00014867,-0$ $.00023926,0.00008590,-0.00000851,-0.00001577,0.00000992,0.00000493,-0$. $00000247,0.00001013,0.00000063,-0.00000857,-0.00000025,-0.00002000,-0$. $00001868,0.00002387,0.00000746,0.00000091,0.00000259,0.12090525,-0.177$ $20557,0.04927888,0.01970840,-0.03347362,0.01814664,0.01050894,0.001600$ $20,-0.00214200,-0.02971537,-0.02152968,0.01531181,-0.09673693,-0.12619$ $942,0.02869657,0.00054310,0.62504925,-0.00001481,-0.00002717,0.0000594$ $7,0.00003961,-0.00000136,-0.00001547,0.00001376,-0.00003195,0.00003891$ $,-0.00000393,0.00001684,0.00000131,-0.00001325,0.00001647,-0.00001359$, $-0.00001547,-0.00005652,-0.00001627,-0.00004787,0.00006757,-0.00005403$ $, 0.00001830,0.00003754,-0.00000039,0.00000926,0.00000665,0.00000355,-0$ $.00000520,0.00000310,0.00000368,-0.00000512,0.00000594,0.00001183,-0.0$ $0000547,0.00000241,0.00000362,-0.05336483,0.04927959,-0.08721712,-0.00$ $871265,0.01816631,-0.00039765,-0.00459899,-0.00215677,-0.00234769,0.01$ $313015,0.01548611,0.00628885,0.04300044,0.02848045,-0.07455677,-0.0006$ $6205,-0.21848354,0.22779214,0.00003203,-0.00005515,0.00002590,-0.00001$ $314,0.00001447,0.00001164,0.00000058,-0.00000265,0.00000128,-0.0000055$ $9,0.00000493,-0.00000138,-0.00000001,-0.00000032,0.00000157,-0.0000112$ $3,-0.00000430,-0.00002043,-0.00000290,0.00003624,-0.00001587,0.0000069$ $7,0.00000463,0.00000575,-0.00000490,0.00000009,-0.00000369,-0.00000554$ $, 0.00000274,0.00000173,0.00000525,-0.00000003,-0.00000508,-0.00000506$, $0.00000069,-0.00000048,-0.29421389,-0.09381580,0.04191490,-0.00020742$, $0.00140686,-0.00061792,0.00222116,-0.00033605,0.00015415,0.00046873,0$. $00094174,-0.00042388,-0.00219488,0.00347102,-0.00152313,-0.01749518,-0$ $.01144673,0.00509855,0.31073521,0.00000557,0.00001367,-0.00001666,-0.0$ $0000617,0.00000214,0.00000004,-0.00000010,0.00000233,-0.00000091,-0.00$ $000195,-0.00000204,-0.00000159,0.00000356,-0.00000085,-0.00000314,0.00$ 
$000034,0.00000164,0.00000867,0.00000405,-0.00001734,0.00002172,0.00000$ $013,-0.00000489,0.00000010,-0.00000113,-0.00000036,-0.00000080,0.00000$ $029,-0.00000017,0.00000028,0.00000144,-0.00000162,-0.00000264,-0.00000$ $069,0.00000003,-0.00000050,-0.09381662,-0.09008507,0.02425351,-0.02633$ $013,-0.00538468,0.00396293,-0.00030336,-0.00354291,0.00424874,0.000929$ $05,-0.00133977,0.00030415,0.00347196,0.00015369,0.00259856,0.01627779$, $0.00908137,-0.00249120,0.10024824,0.09102312,0.00000283,-0.00001322,-0$ $.00000783,-0.00000731,0.00000741,0.00001404,-0.00000293,-0.00000133,-0$ $.00000701,0.00000052,-0.00000182,-0.00000518,0.00000295,-0.00000295,-0$ $.00000094,0.00000362,0.00000202,0.00000757,-0.00000320,0.00001736,0.00$ $000964,-0.00000378,-0.00000357,-0.00000737,-0.00000245,0.00000036,-0.0$ $0000065,0.00000190,-0.00000061,-0.00000048,0.00000327,-0.00000114,-0.0$ $0000613,0.00000008,-0.00000048,-0.00000035,0.04192331,0.02421694,-0.04$ $635480,0.01168348,0.00394632,0.00175212,0.00013449,0.00424608,0.004145$ $42,-0.00041880,0.00030953,-0.00074147,-0.00151938,0.00258551,0.0048453$ $9,-0.00719378,-0.00244299,0.00462387,-0.04480092,-0.02941103,0.0379181$ $3,0.00008345,-0.00002311,0.00002564,-0.00004315,0.00001332,0.00001983$, $0.00000082,-0.00000528,-0.00000377,-0.00001401,0.00000404,-0.00000175$, $-0.00000429,0.00000091,0.00000913,-0.00004441,-0.00000855,-0.00003199$, $0.00005000,0.00000905,-0.00002098,0.00001390,0.00000302,0.00000605,-0$. $00000921,0.00000200,-0.00000701,-0.00000984,0.00000643,0.00000187,0.00$ $001146,-0.00000217,-0.00000233,-0.00000592,0.00000156,-0.00000074,0.00$ $555907,-0.00420628,0.00185829,-0.23631590,0.12331299,-0.05428922,-0.01$ $765196,0.01620026,-0.00715125,-0.00558320,-0.00216101,0.00098887,0.000$ $95032,-0.00094874,0.00041216,0.00155452,-0.00086115,0.00039151,0.00080$ $030,0.00017572,-0.00008615,0.24924711,0.00001457,-0.00000590,0.0000049$ $9,-0.00001165,0.00000884,0.00000107,-0.00000175,-0.00000051,-0.0000047$ $9,-0.00000117,0.00000130,0.00000011,-0.00000239,0.00000214,0.00000302$, $-0.00000893,0.00000588,-0.00000626,0.00001612,-0.00001416,0.00000483,0$ $.00000188,-0.00000310,0.00000031,-0.00000207,-0.00000062,-0.00000061,-$ $0.00000152,-0.00000012,-0.00000002,-0.00000022,-0.00000205,0.00000186$, $-0.00000042,-0.00000042,-0.00000019,0.02353591,-0.00934185,0.00571590$, $0.12241825,-0.13584422,0.04399203,-0.01124689,0.00789139,-0.00149545$,$0.00285699,0.00185962,0.00201259,-0.00014771,-0.00063166,-0.00004347,-$ $0.00188550,-0.00321579,0.00414874,-0.00014776,0.00015162,-0.00175587$, $0.13136669,0.13971018,-0.00000807,0.00000396,0.00000584,0.00000718,-0$. $00000628,0.00000229,-0.00000087,0.00000173,-0.00000087,0.00000053,0.00$ $000061,0.00000236,0.00000245,-0.00000515,-0.00000305,0.00000738,0.0000$ $0375,0.00000538,-0.00000848,0.00000529,-0.00000763,-0.00000124,0.00000$ $210,-0.00000189,-0.00000015,-0.00000027,-0.00000038,0.00000146,-0.0000$ $0026,-0.00000082,-0.00000145,-0.00000133,-0.00000279,0.00000023,-0.000$ $00004,-0.00000061,-0.01041176,0.00573561,0.00107356,-0.05391874,0.0440$ $1496,-0.05554913,0.00502753,-0.00153144,0.00515989,0.00128746,0.002018$ $31,0.00549819,0.00006165,-0.00004936,-0.00071952,0.00084062,0.00414977$ $, 0.00431127,0.00005572,-0.00176255,-0.00303687,0.05782115,-0.05090419$, $0.04686715,-0.00007417,-0.00001740,0.00000105,0.00001518,-0.00003827,-$ $0.00000546,-0.00000111,0.00000377,0.00000167,0.00000572,-0.00000525,0$. $00000116,0.00000158,0.00000137,-0.00000836,0.00001849,-0.00001571,0.00$ $004077,0.00000061,0.00006168,-0.00002183,-0.00000477,0.00001268,-0.000$ $01182,0.00000684,0.00000349,0.00000288,0.00000819,-0.00000034,-0.00000$ $380,-0.00000298,0.00001178,-0.00000255,0.00000614,0.00000029,-0.000000$ 
$21,-0.00463457,-0.00149962,0.00067099,0.00081782,-0.02621667,0.0116039$ $9,-0.05615253,-0.03011290,0.01335823,0.00564535,0.02116600,-0.00935991$ $,-0.00327560,0.00382375,-0.00170689,-0.00138099,-0.00013738,0.00007583$ ,-0.00009557,-0.00123729,0.00054958,0.00119780,0.00040408,-0.00018927, $0.05763706,-0.00003286,0.00003432,-0.00002900,0.00001068,0.00000521,-0$ $.00001418,-0.00000529,-0.00001021,0.00000488,-0.00000032,0.00000202,-0$ $.00000822,0.00000189,-0.00001267,-0.00001042,0.00002040,0.00002374,0.0$ $0003152,-0.00003441,0.00000199,0.00002436,-0.00000377,-0.00001202,-0.0$ $0000127,0.00000004,0.00000174,0.00000057,0.00000576,-0.00000202,-0.000$ $00057,0.00000513,-0.00001698,0.00000552,0 .,-0.00000213,-0.00000010,-0$. $00233105,0.00247622,0.00185706,0.00165022,-0.00618064,0.00510006,-0.03$ $458273,-0.28178773,0.10993392,0.00009270,-0.01326322,0.00553027,0.0018$ $5363,0.00080345,0.00195238,0.00039868,0.00027323,-0.00030276,0.0000874$ $2,-0.00040774,0.00016151,0.00009600,0.00005416,-0.00177262,0.03303045$, $0.29850942,0.00003507,-0.00001441,-0.00000416,-0.00002322,0.00000760,0$ $.00004680,-0.00000716,-0.00000198,-0.00002562,-0.00000004,-0.00001046$, $-0.00000414,0.00001354,0.00000556,-0.00001361,-0.00001110,-0.00000381$, $0.00000720,0.00001483,0.00001378,0.00005319,-0.00000603,0.00000868,-0$. $00001787,-0.00000900,0.00000158,-0.00000209,0.00000349,-0.00000206,0.0$ $0000102,0.00001043,-0.00000713,-0.00000808,0.00000015,-0.00000033,-0.0$ $0000305,0.00105144,0.00186705,0.00586605,-0.00070641,0.00513824,0.0030$ $2311,0.01530514,0.10993305,-0.08189816,-0.00005937,0.00549419,-0.00325$ $571,-0.00081259,0.00195512,0.00435617,-0.00017202,-0.00031396,-0.00028$ $053,-0.00003830,0.00016238,-0.00011682,-0.00004721,-0.00176792,-0.0031$ $5426,-0.01465845,-0.12239212,0.07604582,0.00001303,0.00012089,-0.00009$ $308,0.00000966,-0.00005126,-0.00010026,0.00000865,-0.00000111,-0.00000$ $610,0.00001276,-0.00001574,0.00000541,0.00000640,0.00000129,0.00000565$ $, 0.00001411,0.00005640,0.00010864,-0.00007814,-0.00024336,0.00015214,-$ $0.00002964,0.00000045,-0.00000977,0.00001468,0.00000392,0.00001037,0.0$ $0001619,-0.00000476,-0.00000800,-0.00004478,0.00000036,0.00000714,0.00$ $001073,-0.00000020,0.00000050,-0.00337823,-0.00337179,0.00152560,-0.00$ $020846,-0.00054947,0.00023066,0.00143185,-0.00228928,0.00104350,0.0060$ $7577,-0.00010407,0.00000277,-0.23761285,0.12143725,-0.05349320,-0.0173$ $4597,0.01713185,-0.00756767,-0.00091141,0.00080396,-0.00035498,-0.0000$ $7511,0.00003105,-0.00001432,0.00021783,-0.00020939,0.00009414,0.250608$ $53,-0.00022224,0.00026863,-0.00023387,0.00006934,-0.00000831,-0.000057$ $50,-0.00003227,0.00000695,-0.00002217,0.00005401,-0.00004308,-0.000012$ $93,0.00005147,-0.00003368,-0.00000361,0.00008896,0.00006073,0.00017703$ $, 0.00009810,-0.00028236,0.00022179,-0.00005263,-0.00006487,-0.00003001$ $, 0.00001833,0.00000004,0.00001705,0.00002816,-0.00001441,-0.00000770,-$ $0.00003622,0.00001433,-0.00004114,0.00001824,-0.00000108,-0.00000413,-$ $0.00260450,0.00139492,0.00231341,-0.00102680,-0.00066444,0.00012423,-0$ $.00035692,-0.00323839,0.00377319,0.02107593,-0.01339202,0.00565076,0.1$ $2555759,-0.13128701,0.04292265,-0.01068580,0.00940807,-0.00175729,-0.0$ $0052020,0.00029007,-0.00014094,0.00006623,-0.00009432,-0.00026453,0.00$ $079031,-0.00030696,0.00014890,-0.13278481,0.13801480,0.00012861,-0.000$ $26621,-0.00011710,-0.00009423,0.00012238,0.00013528,-0.00007746,0.0000$ $2932,-0.00006452,-0.00002234,-0.00001501,-0.00005758,0.00006676,-0.000$ $04198,-0.00001383,-0.00001059,-0.00000979,0.00003935,-0.00005067,0.000$ 26430,0.00008968,0.00000367,-0.00009907,-0.00002404,-0.00002182,0.0000 $0225,-0.00000918,-0.00000238,0.00000544,0.00000503,0.00005572,0.000026$ 
$44,-0.00007870,-0.00000873,-0.00000588,-0.00000790,0.00115508,0.002321$ $07,0.00557296,0.00045741,0.00011321,-0.00044470,0.00017391,0.00379000$, $0.00361678,-0.00931783,0.00562997,-0.00316887,-0.05537411,0.04296513$,$0.05298937,0.00479241,-0.00180273,0.00620913,0.00022978,-0.00014595,0$. $00003224,-0.00002843,-0.00026664,-0.00057379,-0.00034806,0.00015158,-0$ $.00002869,0.05848663,-0.05103958,0.04504356,0.00008974,-0.00002574,0.0$ $0002694,-0.00003696,0.00002890,0.00000219,-0.00000193,-0.00000266,-0.0$ $0001185,-0.00001334,0.00000695,-0.00000307,-0.00000577,0.00001047,0.00$ $001143,-0.00004034,0.00001485,-0.00003294,0.00003292,-0.00001030,-0.00$ $000877,0.00000991,-0.00001016,0.00000880,-0.00000932,-0.00000215,-0.00$ $000207,-0.00000999,0.00000012,0.00000214,0.00000771,-0.00001189,0.0000$ 0596,-0.00000446,-0.00000223,0.00000117,0.00682948,0.02246278,-0.00993 $465,-0.00342663,0.00241140,-0.00106354,-0.00054619,0.00107800,-0.00046$ $940,-0.00476727,-0.00352277,0.00156134,-0.00109903,-0.02554782,0.01132$ $947,-0.05926114,-0.02999471,0.01323988,0.00089648,-0.00022466,0.000087$ $62,0.00028824,0.00125978,-0.00055904,0.00004369,-0.00005317,0.00002266$ $, 0.00124443,0.00040571,-0.00019333,0.05977976,0.00005969,0.00000190,0$. $00000870,-0.00002234,-0.00000189,0.00000945,0.00000603,0.00001691,-0.0$ $0000434,-0.00001864,-0.00000661,0.00000300,0.00000454,0.00001278,-0.00$ $000714,-0.00002038,-0.00000908,-0.00000727,0.00000746,0.00000309,-0.00$ $000822,0.00000651,-0.00000448,0.00000648,-0.00000674,-0.00000314,-0.00$ $000327,-0.00000521,0.00000065,0.00000158,0.00000376,-0.00000532,-0.000$ $00140,-0.00000251,-0.00000040,-0.00000004,-0.00529434,-0.01042463,0.00$ $618270,0.00341540,0.00098911,0.00231336,0.00029739,0.00052581,-0.00059$ $704,-0.00280679,0.00124888,0.00230443,0.00195840,-0.00596517,0.0045966$ $1,-0.02912185,-0.28375436,0.10959084,0.00010991,0.00006776,-0.00171709$ ,-0.00030856,-0.00053040,0.00036192,-0.00002883,-0.00018315,-0.0002219 $5,0.00003780,-0.00002762,-0.00172824,0.03172931,0.29804936,-0.00002769$ $, 0.00000180,0.00000204,0.00000864,0.00000250,-0.00000104,-0.00000330,-$ $0.00000411,0.00000017,0.00000651,0.00000404,-0.00000062,-0.00000058,-0$ $.00000957,0.00000346,0.00001372,0.00000594,0.00000814,-0.00000426,-0.0$ $0000103,0.00000194,-0.00000171,-0.00000246,-0.00000370,-0.00000023,-0$. $00000146,-0.00000064,0.00000530,-0.00000018,-0.00000292,-0.00000231,0$. $00000381,-0.00000534,0.00000149,-0.00000016,-0.00000060,0.00233846,0.0$ $0615785,0.00078989,-0.00150790,0.00232235,0.00522160,-0.00013530,-0.00$ $060689,-0.00056358,0.00126581,0.00230806,0.00544941,-0.00083711,0.0046$ $6000,0.00235304,0.01283027,0.10956270,-0.08444813,-0.00004988,-0.00172$ $412,-0.00306253,0.00013634,0.00035995,0.00012565,0.00001045,-0.0002223$ $5,-0.00058655,-0.00002577,-0.00173362,-0.00316574,-0.01402094,-0.12108$ $306,0.07788603 \backslash \backslash-0.00002591,-0.00001222,-0.00000676,-0.00001338,0.0000$ $0007,-0.00002443,-0.00002928,0.00001738,0.00000194,0.00000972,-0.00001$ $047,-0.00002426,0.00000466,-0.00000890,0.00002865,0.00004154,-0.000011$ $14,-0.00000030,0.00002205,0.00004406,0.00004650,0.00000045,0.00000130$, $0.00000586,-0.00000941,-0.00000640,0.00000341,0.00000145,-0.00000538$,$0.00000040,0.00000547,0.00000606,-0.00000325,-0.00000313,0.00000350,-0$ $.00000527,0.00000414,-0.00005353,-0.00000137,-0.00000279,0.00003829,0$. $00001259,0.00003340,0.00005747,0.00002089,-0.00006918,-0.00002257,-0.0$ $0001802,0.00005346,-0.00002421,-0.00006243,-0.00001469,-0.00001347,0.0$ $0000164,-0.00000527,0.00000019,0.00000002,-0.00000580,-0.00000212,-0.0$ $0000387,-0.00000084,-0.00000564,-0.00001660,-0.00000101,0.00000183,0.0$ 0001409,0.00000437,0.00000589,0.000031361II@ 
Addition Transition State

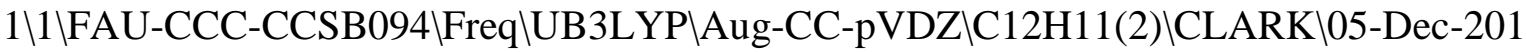
410\\N Geom=AllCheck Guess=TCheck SCRF=Check GenChk UB3LYP/Aug-CC-pVD $\mathrm{Z}$ Freq \\Addition transition state Ph-radical + benzenel\0,2\C,1.199520

2886,-1.1698942646,0.226960418\C,1.7288320607,-0.4337490717,1.32139146 $1 \backslash \mathrm{C}, 2.6260874065,0.606372746,1.1011581265 \backslash \mathrm{C}, 3.0676303008,0.9000703349$, $-0.1993550019 \backslash \mathrm{C}, 2.615591419,0.1254066754,-1.2801479159 \backslash \mathrm{C}, 1.7182891343$, $-0.9171247522,-1.0718223803 \backslash \mathrm{H}, 0.6889060571,-2.1117524653,0.4194440093 \backslash$ $\mathrm{H}, 1.4017812288,-0.6692154177,2.3341082811 \backslash \mathrm{H}, 3.0030155808,1.1849087388$, $1.9455931805 \backslash \mathrm{H}, 2.9843644093,0.3302108677,-2.2860855801 \backslash \mathrm{H}, 1.383087453,-$ $1.5266375697,-1.9109506821 \backslash \mathrm{H}, 3.776020737,1.7117892274,-0.3664242306 \backslash \mathrm{C}$, $-3.4549077953,0.6386554577,-0.1178188246 \backslash \mathrm{C},-3.2598172188,-0.7194166901$ $, 0.1557341506 \backslash \mathrm{C},-1.956837629,-1.2376804349,0.2546926834 \backslash \mathrm{C},-0.895396357$ $1,-0.3662975581,0.0739164408 \backslash \mathrm{C},-1.0500785999,0.982663333,-0.1979792516$ \C,-2.3559115458,1.4890748478,-0.2945232246\H,-4.4673225596,1.03673366 $39,-0.1937758338 \mathrm{UH},-4.1186646246,-1.3793395008,0.292882402 \backslash \mathrm{H},-1.803154$ $2603,-2.297595485,0.4681905698 \backslash \mathrm{H},-0.186711405,1.6356007452,-0.33374507$ 41\H,-2.5137410805,2.5480545719,-0.5078087232llVersion=EM64L-G09RevC.0 $1 \backslash$ State $=2-\mathrm{A} \backslash \mathrm{HF}=-463.852827 \backslash \mathrm{S} 2=0.779854 \backslash \mathrm{S} 2-1=0 . \mathrm{SS} 2 \mathrm{~A}=0.750637 \backslash \mathrm{RMSD}=7.008$ $\mathrm{e}-09 \backslash \mathrm{RMSF}=2.840 \mathrm{e}-06 \backslash \mathrm{ZeroPoint}=0.1871804 \backslash \mathrm{Thermal}=0.1970552 \backslash \mathrm{ZPE}=117.4574$ 821 $\backslash$ Dipole $=0.012431,0.0147195,-0.0030328 \backslash$ DipoleDeriv $=0.2459835,0.03064$ $07,-0.0074422,-0.026582,0.0792661,-0.0244895,0.0039317,-0.0254552,-0.0$ $383763,-0.1479239,0.0794682,-0.0797379,0.0839093,-0.1284342,0.022638,-$ $0.0499553,-0.0557415,0.0500472,-0.0650691,0.078936,0.0342633,0.0958654$ $,-0.0239065,0.0796328,0.0796217,0.0539068,0.061183,-0.1201278,0.114540$ $8,-0.0226568,0.0090163,0.0052271,-0.0126256,-0.0014363,-0.01277,-0.053$ $9264,-0.0667809,0.0587296,-0.0608212,0.0571482,-0.0589841,-0.0291379,-$ $0.1097875,-0.0045977,0.0980845,-0.1470727,0.1053259,0.0439753,0.097356$ $9,-0.0897042,0.0347111,0.0153208,0.1144373,0.0101902,0.0973577,-0.0957$ $089,0.0190292,-0.0807959,-0.0255526,0.0165586,0.0160857,0.0166801,0.05$ $16024,0.0830899,-0.050557,0.0622694,-0.0379026,0.0793964,0.0155308,0.0$ $345976,0.0258197,-0.0616054,0.0769341,-0.0573522,-0.0357495,-0.075391$, $0.0483679,-0.0763305,-0.0551891,-0.0711641,-0.0373214,0.0778405,-0.039$ $0582,0.0539043,-0.0478527,0.0883097,0.0237222,0.0790602,0.0188306,-0.0$ $784751,0.0823707,-0.0717298,-0.0387393,-0.0487201,0.0439866,-0.0592317$ $,-0.0181729,-0.0694113,-0.0249339,0.0240552,-0.1093224,0.0223782,-0.11$ $75302,0.0024752,0.0124849,0.0238944,0.0123064,0.0585647,-0.0160888,-0$. $0558103,0.0103855,-0.0501672,-0.0769274,-0.0134227,0.0100193,-0.012561$ $4,-0.1427509,0.0668602,0.0533096,-0.0118074,-0.1005476,0.0494602,-0.03$ $66801,0.0192836,-0.0377238,-0.1271847,-0.3756043,0.0144416,-0.0012672$, $0.3352297,0.0922568,-0.042143,-0.0663623,-0.0402466,-0.09917,0.6221205$ - $-0.0704263,0.0110224,-0.2506953,-0.0299152,-0.0035111,0.0480637,-0.00$ $35892,-0.0537701,-0.4815569,0.0826479,-0.0151348,0.0654605,-0.0608884$, $-0.0055114,-0.0116572,-0.0060449,-0.0853197,0.1442271,-0.0349377,0.006$ $2645,0.1065366,0.1182421,-0.0498967,-0.0231782,-0.0501092,-0.1156763,-$ $0.095513,0.0578703,-0.0106229,0.0630175,0.0482856,0.0172405,-0.0116865$ $, 0.0174035,0.1318925,-0.1233789,-0.0876389,0.0187829,-0.0680118,-0.001$ $3632,0.0263287,0.0147686,0.0263346,0.1226965,0.0877928,0.0508337,-0.01$ $00859,-0.0187043,-0.0953024,0.0444119,0.0039284,0.0440743,0.1151344,0$. 
$0360583,-0.0833084,0.0171591,-0.054094,0.0375541,0.0138936,0.0111761,0$ $.013862,0.1019791,-0.0055741,0.0291058,-0.0053689,0.0634544,-0.1018496$ $, 0.0458271,-0.0123266,0.0457595,0.1171358 \backslash$ Polar $=205.5345764,13.5302399$ ,137.3372133,-3.1264854,-4.1531502,117.965759\PG=C01 [X(C12H11)]/NImag $=1 \backslash 0.18640752,0.27748043,0.53684111,-0.05418628,0.01329978,0.60629577$ ,-0.07761851,-0.06539482,-0.03718931,0.36529138,-0.03855705,-0.1500911 $2,-0.05402169,0.26075061,0.43605729,-0.03332599,-0.09790429,-0.2307062$ $0,-0.07055675,-0.00641379,0.68818649,-0.01930345,-0.02293359,-0.033574$ $96,-0.17948673,-0.13406279,0.04592495,0.36217309,-0.03217778,-0.021203$ $25,-0.03763669,-0.14157808,-0.21875124,0.04565863,0.26973731,0.4610374$ $9,-0.00107261,-0.01461438,0.04084682,0.00304587,-0.00683685,-0.1288121$ $9,-0.01442964,0.07553737,0.68027405,0.00362258,0.00252069,-0.00076119$, $-0.00839029,-0.01606728,0.02064707,-0.10475763,-0.03783748,0.08111412$, $0.37655528,0.01447880,-0.00631155,-0.00983768,-0.01041178,-0.00023776$, $0.02527169,-0.04534897,-0.10444983,0.05621559,0.28980698,0.48145754,-0$ $.00317662,-0.00978047,-0.05273979,0.05191337,0.06466621,0.00854218,0.0$ $3663145,0.00308710,-0.28806343,-0.05741683,0.02838626,0.62287718,-0.01$ $891450,-0.00782139,0.04019218,-0.01966538,-0.02041333,-0.00623227,0.02$ $908045,0.02354916,-0.01935826,-0.10562734,-0.05704024,-0.01739318,0.36$ $152389,-0.02875764,0.00688671,0.05212318,-0.02007571,-0.03352841,-0.00$ $840492,0.02894010,0.03497531,-0.03491177,-0.06694378,-0.15310498,-0.05$ $922297,0.25452843,0.43915361,0.01379077,0.02897782,0.01233868,-0.01122$ $595,-0.01548595,-0.00549541,0.00801630,-0.00224371,-0.05264087,-0.0613$ $9162,-0.11236409,-0.23852021,-0.08848090,0.02362492,0.70277834,-0.0768$ $5938,-0.04549198,0.05800092,0.02070951,0.01981825,-0.00396810,-0.01944$ $604,-0.01402243,0.01818217,-0.00895339,-0.02985800,-0.04348490,-0.1794$ $2595,-0.13178246,0.05265042,0.36561417,-0.02263349,-0.10777729,0.00239$ $563,0.01976311,0.02910175,-0.00760434,-0.01621076,-0.02071255,0.022013$ $74,-0.02326764,-0.03100437,-0.04773340,-0.14105942,-0.21861448,0.04596$ $464,0.26867714,0.47772194,0.04410530,0.04650036,-0.27377314,-0.0045453$ $1,-0.01989987,-0.03574256,0.01369746,0.01493657,-0.01854265,-0.0123992$ $8,-0.00832382,0.03984478,0.01014077,-0.00652095,-0.12897382,-0.0336559$ $9,0.09244258,0.64617692,-0.08319921,-0.12463701,0.02529521,-0.00833833$ ,-0.01335615,-0.00087997,0.00530684,-0.00130123,-0.00412942,-0.0009828 $0,-0.00065490,0.00012686,0.00536196,0.00040222,0.00423039,-0.00819762$, $-0.01174412,0.00605499,0.10882141,-0.13949851,-0.25976748,0.04176734,-$ $0.00397904,-0.00630158,0.00728083,-0.00244443,0.00003562,-0.00214623,0$ $.00114036,0.00087371,-0.00037401,-0.00187004,0.00108810,0.00008333,0.0$ $0107722,0.00103971,-0.00442235,0.13469426,0.27160207,0.02829602,0.0417$ $0275,-0.06456016,-0.01228303,-0.02179232,0.00785801,-0.00099277,-0.002$ $41475,-0.00384121,-0.00023098,-0.00036349,-0.00086447,0.00177966,0.000$ $33552,-0.00494030,0.01297874,0.02474734,0.00037308,-0.02744336,-0.0444$ $0324,0.06378470,0.00581424,0.00253049,-0.01292563,-0.06883699,-0.02883$ $268,0.08131915,-0.00178401,-0.00363651,0.01928381,0.00200971,-0.006304$ $66,0.00006401,-0.00075715,0.00008608,-0.00033944,0.00251005,-0.0038744$ $0,-0.00121541,-0.00169896,0.00107966,0.00001803,0.06464271,0.00588516$, $0.00663326,-0.01491576,-0.02990194,-0.06121856,0.05387469,-0.00634916$, $-0.00034043,0.02252380,-0.00648446,-0.00034051,-0.00040758,0.00012876$, $-0.00094189,-0.00039287,-0.00401420,0.00095156,-0.00326031,0.00198456$, $-0.00027149,0.00067924,0.03615232,0.05683760,0.00872448,0.00688655,-0$. $01723231,0.07957843,0.05596154,-0.30013868,-0.00043235,-0.00147756,-0$. 
$00108487,0.00068468,0.00033373,0.00216067,-0.00041894,-0.00045238,0.00$ $038365,-0.00244162,-0.00337566,-0.00170834,-0.00025804,0.00002556,0.00$ $087140,-0.08615752,-0.05856774,0.31684725,0.00192273,-0.00650984,0.002$ $58990,-0.00337041,-0.01012245,-0.01657434,-0.07835052,-0.06109887,-0.0$ $7353816,0.00625097,0.00517847,0.00870898,0.00356334,-0.00321844,0.0026$ $0443,-0.00052551,0.00041900,0.00008656,-0.00024059,-0.00059204,-0.0010$ $8476,-0.00208067,0.00269753,-0.00023849,0.07446616,-0.00613210,0.00032$ $449,0.00099442,-0.00669364,-0.00733082,-0.01936594,-0.06162673,-0.1275$ $0703,-0.11523573,0.00220198,0.00650421,0.00590839,-0.00322404,0.003495$ $90,0.00147849,0.00011658,-0.00068066,0.00072260,-0.00042858,-0.0003345$ $9,-0.00067722,0.00246426,-0.00108368,0.00043511,0.07039642,0.12800336$, $0.00166603,0.00090236,0.00159914,0.00332592,0.00376795,0.00655543,-0.0$ $7333866,-0.11572603,-0.22480043,-0.01156439,-0.01697395,-0.01641692,0$. $00313190,0.00207213,-0.00397427,0.00053305,0.00070426,0.00012037,0.000$ $10999,0.00026697,0.00012412,-0.00055239,0.00017584,0.00078493,0.077662$ $39,0.12521982,0.23599438,0.00190837,-0.00700653,0.00014307,-0.00053139$ $, 0.00034740,-0.00023820,0.00352291,-0.00401854,-0.00119572,0.00626341$, $0.00145498,-0.01026383,-0.07691188,-0.02734853,0.08973460,-0.00323068$, $-0.00290073,0.01925872,-0.00023031,-0.00012654,0.00123163,-0.00055651$, $0.00055743,-0.00001313,0.00017560,0.00001649,-0.00047283,0.07292607,-0$ $.00630244,-0.00013712,-0.00019320,-0.00010263,-0.00107120,-0.00022588$, $-0.00422358,0.00111510,-0.00357420,0.00638533,0.00699581,-0.01581474,-$ $0.02791197,-0.05945544,0.04573005,-0.00726882,0.00035680,0.02200808,-0$ $.00043097,-0.00011799,0.00078046,0.00056228,-0.00040937,0.00008058,0.0$ $0019009,0.00028013,-0.00091381,0.03429032,0.05457235,0.00084528,-0.000$ $10702,0.00208052,-0.00052407,-0.00019668,0.00050839,-0.00168924,-0.004$ $17307,-0.00155370,0.00961334,0.00707771,-0.01692187,0.08976221,0.04621$ $299,-0.29428658,-0.00044884,-0.00111762,-0.00127641,0.00006598,-0.0001$ $6315,-0.00010225,-0.00007545,0.00015356,-0.00007022,0.00042074,0.00039$ $126,-0.00095601,-0.09697672,-0.04899241,0.31096532,0.00583507,0.007212$ $87,0.01074962,0.00255309,-0.00308581,0.00256926,-0.00075551,0.00021518$ $, 0.00029144,0.00202941,-0.00583038,0.00238904,-0.00192949,-0.01081671$, $-0.01632574,-0.07011416,-0.05930283,-0.06526793,-0.00170248,0.00099292$ ,-0.00041272,0.00042016,0.00025552,0.00049222,-0.00055697,0.00054940,$0.00014583,-0.00208105,0.00249263,-0.00042268,0.06599519,0.00208175,0$. $00589384,0.00513359,-0.00273968,0.00294002,0.00133233,0.00028607,-0.00$ $043642,0.00073230,-0.00623521,0.00008142,0.00134809,-0.00585747,-0.007$ $95020,-0.01953363,-0.05959243,-0.13630091,-0.12218431,0.00192792,-0.00$ $035678,-0.00017845,0.00004614,0.00017351,0.00034055,0.00051800,-0.0004$ $4624,-0.00000253,0.00258561,-0.00103074,0.00055820,0.06801192,0.137674$ $71,-0.01054406,-0.01668156,-0.01651668,0.00375925,0.00145073,-0.003739$ $48,0.00034339,0.00079397,-0.00012122,0.00188127,0.00059985,0.00172821$, $0.00293787,0.00446258,0.00666369,-0.06325134,-0.12426456,-0.22377086$,$0.00050719,0.00048249,0.00095969,-0.00056461,-0.00081306,-0.00093533,-$ $0.00019780,0.00007247,-0.00003657,-0.00080219,0.00030651,0.00073199,0$. $06703422,0.13324312,0.23465315,-0.00028263,0.00091003,-0.00018723,0.00$ $422023,-0.00199103,0.00252430,-0.00281978,-0.00727703,-0.00068034,-0.1$ $6238850,-0.14785463,0.03032967,-0.00296015,-0.00660606,0.00354514,0.00$ $419184,-0.00284507,-0.00162114,-0.00055728,0.00025897,-0.00005008,-0.0$ $0021306,-0.00042744,-0.00004471,-0.00168744,0.00217207,-0.00024161,-0$. $00168723,0.00210244,-0.00059876,-0.00021960,-0.00038358,0.00020942,0.1$ 
$6427343,0.00067338,-0.00023172,-0.00016422,-0.00254274,0.00366608,0.00$ $086442,-0.00329489,-0.00119756,-0.00167248,-0.14762566,-0.20325728,0.0$ $3008414,-0.01029540,-0.00807649,0.00542248,-0.00313034,0.00191481,-0.0$ $0382304,0.00044705,-0.00031958,0.00008448,-0.00019224,-0.00011297,-0.0$ $0011185,0.00206996,-0.00106539,0.00042505,0.00215220,-0.00094665,0.000$ $36744,-0.00054136,-0.00047547,0.00024450,0.16211981,0.21005494,-0.0001$ 4094,-0.00016873,-0.00099206,0.00194015,0.00033090,-0.00520662,0.01878 $852,0.02173887,0.00061870,0.03027508,0.03008162,-0.06376560,-0.0159783$ $7,-0.01798845,0.00762411,-0.00086787,-0.00328750,-0.00341396,-0.000085$ $28,0.00008651,0.00009193,0.00094292,0.00110805,-0.00016546,-0.00061739$ $, 0.00008715,0.00098627,-0.00021006,0.00070757,0.00086896,-0.00079329,-$ $0.00097595,0.00020130,-0.03322095,-0.03172623,0.06307829,-0.00004283$, $0.00009476,0.00001353,-0.00030602,0.00002567,0.00014344,-0.00000647,0$. $00013813,-0.00001377,0.00005698,-0.00004548,-0.00000373,-0.00000010,0$. $00014067,-0.00004168,-0.00031167,-0.00002976,-0.00014138,-0.00004016,-$ $0.00006192,0.00001411,0.00001834,-0.00001328,-0.00001513,-0.00000198$,$0.00001248,-0.00001266,-0.00000465,-0.00000635,0.00002390,0.00001416$, $0.00000649,0.00002379,-0.00002839,-0.00000392,0.00000655,0.72163518,-0$ $.00012703,-0.00036113,0.00003819,-0.00008163,-0.00003091,-0.00001800,-$ $0.00010369,0.00008160,-0.00004424,0.00011308,0.00022687,-0.00003529,-0$ $.00013259,0.00011184,-0.00002796,-0.00004117,-0.00002458,0.00006575,-0$ $.00023270,0.00029444,-0.00005004,-0.00001689,-0.00005945,-0.00002141,0$ $.00001904,0.00001737,0.00000320,0.00002242,0.00001095,-0.00000228,-0.0$ $0002223,-0.00005925,0.00002293,-0.00000352,-0.00002018,0.00000297,-0.0$ $2862640,0.64548238,0.00002444,0.00003908,-0.00014219,-0.00007755,0.000$ $04768,0.00004867,0.00007219,-0.00009762,-0.00003461,-0.00003086,-0.000$ $05413,0.00000455,-0.00001924,0.00005513,-0.00004034,0.00011591,-0.0000$ $0377,0.00002829,0.00004485,-0.00005110,0.00004775,0.00001524,0.0000331$ $6,-0.00004893,-0.00001814,0.00001248,0.00001494,0.00000814,-0.00001917$ $, 0.00001480,-0.00001230,-0.00003649,-0.00004164,0.00000319,0.00000733$, $0.00001438,0.00319675,-0.10293351,0.15619785,0.00095908,-0.00131644,0$. $00028031,-0.00025141,0.00003994,-0.00004846,-0.00011136,0.00019369,-0$. $00005849,0.00004279,0.00005326,-0.00002219,-0.00009909,0.00020649,0.00$ $000046,-0.00025724,0.00005025,0.00000791,-0.00005237,0.00052077,-0.000$ $10604,-0.00005719,-0.00004341,-0.00006104,0.00003886,-0.00000194,0.000$ $00868,0.00004291,-0.00000614,-0.00001094,-0.00005275,-0.00002237,0.000$ $07887,0.00001451,0.00001894,-0.00000044,-0.12494129,-0.00200948,0.0006$ $5957,0.68117705,-0.00064298,-0.00034705,0.00034364,-0.00028326,0.00040$ $147,-0.00010413,0.00022017,0.00002754,0.00025972,-0.00039035,-0.000512$ $03,0.00010748,0.00022637,-0.00007414,-0.00018825,-0.00049698,0.0003955$ $4,-0.00009820,-0.00022176,-0.00013199,0.00003017,-0.00001664,0.0000028$ $9,-0.00005442,-0.00002048,-0.00001066,-0.00004003,-0.00001482,-0.00000$ $693,0.00003597,-0.00002044,0.00004101,0.00006342,0.00000667,0.00002431$ - $-0.00001115,0.05797344,-0.32076071,0.05148980,0.04044487,0.65829053,0$ $.00013170,0.00034967,0.00127267,0.00061344,-0.00009285,-0.00010047,-0$. $00006446,0.00005058,0.00009912,0.00008185,0.00010411,-0.00002574,-0.00$ $002487,0.00001688,0.00019288,-0.00045726,-0.00012313,-0.00008948,0.000$ $04561,0.00002638,0.00000537,0.00001017,-0.00002366,0.00006752,-0.00001$ $021,0.00002860,-0.00001059,0.00001670,-0.00002819,-0.00001280,-0.00000$ $105,0.00003844,0.00002961,-0.00000153,-0.00000937,-0.00002150,-0.01145$ $107,0.05175344,-0.07507625,-0.01059753,-0.10647082,0.15381810,-0.00460$ 
$946,-0.00220366,0.00046853,-0.00103966,0.00112570,0.00069418,0.0002755$ $9,-0.00021530,0.00039263,-0.00064089,-0.00065962,0.00015690,0.00026493$ - $-0.00035909,-0.00028472,-0.00104476,0.00076315,-0.00105315,0.00048800$ ,-0.00086447,0.00017156,-0.00010107,0.00009685,-0.00027067,-0.00005718 ,-0.00013031,-0.00006555,-0.00005360,-0.00009384,0.00010098,-0.0000941 $1,0.00019492,0.00020171,-0.00008585,0.00004273,-0.00001821,0.01315714$, $0.07889404,-0.01597251,-0.28537335,0.03501190,-0.00608479,0.60632687,0$ $.00148777,0.00194746,-0.00028812,0.00115601,-0.00080380,0.00032400,-0$. $00025173,-0.00022652,-0.00029444,0.00058843,0.00089852,-0.00035229,-0$. $00006146,0.00020698,0.00047416,0.00096116,-0.00105368,-0.00000450,0.00$ $154627,0.00054164,-0.00014801,0.00004447,0.00006471,0.00009999,0.00001$ $997,0.00004764,0.00010040,0.00002823,0.00000207,-0.00011657,0.00003151$ ,-0.00001059,-0.00011962,0.00007648,-0.00000560,0.00000890,0.03255200, $-0.00985574,0.00346224,0.10524213,-0.14131159,0.01546099,0.00262525,0$. $70075309,-0.00027908,-0.00028906,0.00054014,0.00026226,0.00059355,-0.0$ $0020659,-0.00037216,-0.00057584,0.00055297,-0.00012272,-0.00033423,-0$. $00070019,0.00043211,0.00072081,0.00011837,-0.00067424,-0.00022812,0.00$ $005708,-0.00031535,-0.00013816,-0.00013091,0.00003726,0.00002487,-0.00$ $005047,-0.00001174,0.00000218,-0.00001713,0.00000473,-0.00000888,0.000$ $02872,-0.00005299,-0.00005318,0.00000685,-0.00001511,0.00000415,0.0000$ $2106,-0.00659578,0.00328111,0.00748683,-0.02028009,0.01576258,-0.06562$ $313,-0.00266672,-0.11625925,0.14899416,0.07601912,-0.01101707,0.001850$ $28,-0.02498328,-0.01729200,-0.02316295,0.00616884,0.00951573,-0.008145$ $22,-0.00339193,-0.00565452,0.00116284,0.00620942,0.01189982,0.00373698$ $,-0.02469513,-0.00689230,0.02826359,-0.01352967,0.00827793,-0.00160993$ $, 0.00074568,-0.00045814,0.00107101,0.00054809,0.00040606,0.00005587,0$. $00054070,0.00035289,-0.00020390,0.00073151,-0.00083280,-0.00080940,0.0$ $0029155,0.00001676,-0.00000054,-0.01118955,-0.02260865,0.00459905,-0.0$ $5975366,0.00625974,-0.00094887,-0.22821849,-0.13536031,0.02813659,0.34$ $585767,-0.02853176,-0.01006178,0.00075619,0.00219750,0.00697097,0.0061$ $9857,-0.00177435,-0.00266043,0.00200550,0.00098559,0.00183488,0.000250$ $92,-0.00226305,-0.00437320,-0.00138370,0.00512246,0.00432359,-0.008952$ $26,0.01208853,-0.00798196,0.00163345,-0.00014510,0.00020929,-0.0002472$ $9,-0.00001215,-0.00025905,0.00000422,-0.00011074,-0.00021314,0.0001026$ $1,-0.00023368,0.00039839,0.00023439,-0.00016720,0.00005432,-0.00000436$ $,-0.02246067,-0.05832361,0.01041179,-0.04889434,0.05564594,-0.00841282$ $,-0.10238338,-0.20169959,0.03062195,0.11837772,0.59593410,0.00539378,0$ $.00067900,-0.00706966,-0.00803027,-0.00255846,-0.00203996,0.00149848,0$ $.00264740,-0.00287538,-0.00017800,0.00026014,0.00298797,-0.00074566,-0$ $.00209649,-0.00117449,0.00677002,-0.00006708,0.00029856,-0.00238253,0$. $00165250,0.00005183,0.00025024,-0.00019484,0.00035687,0.00024614,-0.00$ $001798,0.00003579,-0.00022947,0.00010930,-0.00001612,-0.00018233,0.000$ $18171,0.00019144,0.00003511,-0.00000055,0.00004859,0.00456202,0.010404$ $31,-0.00949222,0.01018626,-0.00865202,0.01466088,0.02146115,0.03073961$ ,-0.05871711,-0.02505958,-0.10366160,0.10649827,-0.00378141, 0.00459823 $,-0.00090331,-0.00046620,0.00169048,0.00107262,0.00017141,0.00007607,0$ $.00034145,-0.00072513,-0.00127678,0.00024482,0.00018285,-0.00005523,-0$ $.00032635,-0.00048164,0.00113505,-0.00165849,-0.00101759,0.00121254,-0$ $.00024112,-0.00006289,-0.00006933,-0.00023796,-0.00011651,-0.00006606$, $-0.00013201,-0.00011252,-0.00000970,0.00014744,-0.00005842,0.00002323$, $0.00025322,-0.00012647,0.00006369,-0.00000767,-0.05430431,-0.03663292$, 
$0.00767122,-0.02330216,0.03270064,-0.00652272,0.04025780,-0.02240173,0$ $.00436660,-0.09296649,0.00050376,0.00006022,0.66713869,0.00196400,-0.0$ $0125345,0.00015375,-0.00007076,0.00049285,-0.00072044,-0.00072815,-0.0$ $0027358,0.00014279,0.00067016,-0.00043644,-0.00006312,-0.00058304,-0.0$ $0025470,0.00006260,-0.00015011,0.00057607,0.00065209,0.00039473,-0.000$ $13324,0.00004640,0.00008657,-0.00003176,0.00015573,-0.00001051,0.00000$ $812,0.00001226,-0.00002510,0.00003018,0.00000752,0.00007747,-0.0000754$ $2,-0.00010674,-0.00006124,0.00001838,0.00000115,0.00859306,0.05657697$, $-0.00970250,0.03143956,-0.04037880,0.00705086,-0.05365036,-0.03661178$, $0.00840898,0.03266450,-0.32950374,0.05557998,0.05753651,0.65106948,-0$. $00038058,0.00016101,-0.00041284,0.00023090,0.00042948,0.00035820,-0.00$ $023968,0.00001821,0.00025953,-0.00012960,-0.00005691,-0.00075267,0.000$ 49956,0.00018618,0.00022771,-0.00018494,-0.00053902,0.00029203,-0.0000 $7491,0.00003914,0.00008561,-0.00000532,0.00001268,0.00004814,0.0000309$ $9,-0.00004303,0.00006653,-0.00002121,0.00006354,0.00004700,-0.00002397$ $, 0.00004461,0.00009366,0.00001304,0.00000294,0.00003013,-0.00146398,-0$ $.00951314,0.01062920,-0.00626493,0.00704890,-0.00617330,0.01068801,0.0$ $0828220,0.00211121,-0.00643480,0.05570501,-0.06501949,-0.01401715,-0.1$ $0617075,0.14845324,0.00151060,0.00009323,-0.00002401,0.00062165,-0.000$ $34749,-0.00014224,0.00000161,-0.00033174,0.00007184,-0.00010424,0.0002$ $1434,-0.00002453,-0.00001701,-0.00034639,0.00004634,0.00062952,-0.0002$ $6289,0.00027690,0.00016883,-0.00028472,0.00005551,-0.00002090,0.000097$ $02,0.00005649,-0.00002244,-0.00000090,0.00002827,-0.00002275,-0.000011$ $68,-0.00002756,-0.00001915,0.00007411,-0.00009587,0.00002996,-0.000010$ $29,-0.00000512,-0.24957506,-0.07012512,0.01499120,0.04414537,-0.062909$ $62,0.01254900,-0.06440895,-0.00861291,0.00200547,0.01627880,0.08559801$ ,-0.01730634,-0.29469854,0.03116229,-0.00526728,0.64119699,-0.00028386 ,-0.00070132,0.00034220,-0.00002286,-0.00023786,-0.00003506,-0.0000464 $9,0.00024691,-0.00011630,0.00041593,0.00037125,-0.00009056,-0.00003470$ $, 0.00017565,0.00007532,0.00000940,-0.00023678,0.00009308,0.00023255,-0$ $.00023872,0.00005294,0.00003711,-0.00002189,0.00005371,0.00007088,-0.0$ $0001119,0.00003178,0.00007127,-0.00002571,-0.00003550,0.00003866,-0.00$ $000705,-0.00003417,0.00001657,-0.00000117,-0.00000168,-0.12994078,-0.1$ $9770244,0.02733953,-0.01860237,-0.04010754,0.00970618,-0.01120643,-0.0$ $0083875,-0.00065484,0.03088814,-0.01863888,0.00620724,0.10146361,-0.13$ $840855,0.01484691,-0.00659234,0.69854962,0.00005502,0.00033494,0.00086$ $267,-0.00008577,0.00009665,-0.00005608,-0.00000293,0.00024124,0.000054$ $23,-0.00007707,-0.00007241,-0.00000046,0.00001659,-0.00030665,0.000101$ $37,0.00007377,-0.00003136,-0.00004569,-0.00004469,0.00005704,0.0000260$ $0,-0.00000445,-0.00007982,0.00003161,-0.00000814,-0.00000016,-0.000033$ $66,-0.00001940,0.00000433,-0.00001271,-0.00000719,0.00010306,0.0000108$ $5,-0.00000591,-0.00000541,-0.00002559,0.02706663,0.02707478,-0.0708002$ $4,0.00361023,0.00990060,0.00548982,0.00253655,-0.00064366,-0.00421543$, $-0.00627027,0.00595961,0.01163141,-0.01946419,0.01514566,-0.06569994,-$ $0.00092348,-0.11416060,0.15646767,-0.00000725,0.00001702,-0.00000367,-$ $0.00001621,0.00000297,0.00004033,0.00001661,0.00001719,0.00001738,-0.0$ $0002674,0.00001777,-0.00000056,0.00001693,0.00000788,-0.00002134,-0.00$ $001623,-0.00001443,-0.00003750,0.00001379,0.00000405,-0.00000068,0.000$ $00405,0.00000129,-0.00000180,-0.00000373,-0.00000531,-0.00000435,-0.00$ $000289,-0.00000293,0.00000366,0.00000531,0.00000092,-0.00000046,-0.000$ $00013,-0.00000938,0.00000053,-0.29880914,0.09536431,-0.01810814,-0.001$ 
$07413,-0.00149625,0.00032291,0.00208789,0.00066239,-0.00011619,0.00055$ $650,-0.00089056,0.00017053,-0.00203840,-0.00396376,0.00083995,-0.01716$ $597,0.01190134,-0.00231076,0.31584300,-0.00001397,-0.00004721,-0.00004$ $019,0.00002179,0.00000387,0.00000842,-0.00000239,0.00000663,-0.0000016$ $1,0.00001034,0.00001574,-0.00000939,0.00000524,0.00003969,-0.00000251$, $0.00000314,-0.00000264,-0.00000677,-0.00005640,0.00005329,-0.00000938$, $-0.00000477,-0.00000132,-0.00000759,0.00000649,0.00000199,0.00000382,0$ $.00000700,0.00000288,-0.00000590,0.00000079,-0.00000482,0.00000999,0.0$ $0000376,0.00000413,0.00000066,0.09532584,-0.09309552,0.01120133,0.0284$ $9958,-0.00573856,0.00170153,0.00023360,-0.00510139,0.00234585,-0.00086$ $604,-0.00126049,0.00006569,-0.00355843,-0.00111475,0.00151430,-0.01804$ $294,0.00979523,-0.00120533,-0.10211006,0.09526668,0.00000228,-0.000042$ $42,-0.00025301,0.00004121,0.00001214,0.00002040,-0.00000873,-0.0000796$ $5,0.00001229,-0.00000152,-0.00000565,-0.00000481,0.00000869,0.00007166$ ,-0.00001546,-0.00004691,-0.00000565,0.00002357,0.00001061,-0.00000966 $, 0.00000873,-0.00001243,0.00001237,-0.00000298,-0.00000122,-0.00000892$ $,-0.00000107,-0.00000212,0.00000625,-0.00000309,0.00001327,-0.00001122$ ,-0.00000044,-0.00000019,-0.00000038,-0.00000123,-0.01809809,0.0112056 $1,-0.03774443,-0.00574153,0.00183396,0.00295751,-0.00002506,0.00234078$ $, 0.00603730,0.00016829,0.00007076,-0.00095347,0.00075369,0.00151701,0$. $00598837,0.00373927,-0.00134250,0.00367521,0.01934537,-0.01377385,0.02$ $756632,0.00016967,-0.00026055,0.00004805,-0.00022662,0.00010351,-0.000$ $00286,0.00003902,0.00004946,0.00004825,-0.00010997,-0.00017059,0.00003$ $371,0.00003522,0.00002766,-0.00007023,-0.00022709,0.00010039,-0.000031$ $39,0.00001392,-0.00003038,0.00000627,-0.00000819,-0.00000573,-0.000027$ $34,-0.00000691,-0.00001685,-0.00001783,-0.00000860,-0.00000873,0.00002$ $685,-0.00001036,0.00000780,0.00002851,-0.00001097,-0.00000202,0.000001$ $54,0.00602001,0.00487913,-0.00098978,-0.22766915,-0.13280473,0.0276806$ $8,-0.01738766,-0.01859314,0.00385479,-0.00452559,0.00221721,-0.0003929$ $8,0.00018485,0.00106277,-0.00022314,0.00161498,0.00136142,-0.00025894$, $0.00082559,-0.00020590,0.00002052,0.23998591,0.00023001,0.00005520,-0$. $00000903,0.00001356,-0.00005737,-0.00001317,0.00000233,0.00001433,-0.0$ $0002163,0.00002337,0.00004215,0.00000184,-0.00001601,-0.00000240,0.000$ $00677,0.00002724,-0.00002878,0.00003899,0.00002507,0.00001057,0.000004$ $13,0.00000965,0.00000001,0.00001256,0.00000361,0.00000170,0.00000446,0$ $.00000509,0.00000070,-0.00000212,0.00000870,-0.00000376,-0.00000994,0$. $00000589,-0.00000251,0.00000358,-0.02500160,-0.01197575,0.00324134,-0$. $13183257,-0.15718489,0.02505618,0.01101991,0.00866368,-0.00095972,0.00$ $224893,0.00130933,0.00100394,0.00059795,-0.00060129,-0.00004142,0.0022$ $5615,-0.00465494,0.00229948,0.00020356,0.00067486,-0.00094092,0.141958$ $87,0.16392633,-0.00004684,-0.00000632,0.00001784,-0.00004306,-0.000025$ $32,0.00003648,0.00005125,0.00004984,-0.00003614,-0.00000371,0.00001005$ $, 0.00007687,-0.00005011,-0.00007059,-0.00002324,0.00003525,0.00005419$, $0.00001510,-0.00000397,0.00000455,0.00003045,0.00000070,-0.00000321,-0$ $.00000291,-0.00000533,-0.00000130,-0.00000209,0.00000468,0.00000237,0$. $00000026,-0.00000315,0.00000398,-0.00000177,-0.00000236,0.00000062,0.0$ $0000340,0.00504149,0.00310593,0.00286035,0.02748969,0.02506091,-0.0412$ $5120,-0.00213257,-0.00082533,0.00431929,-0.00040725,0.00099930,0.00612$ $493,-0.00012252,-0.00004324,-0.00077631,-0.00043593,0.00230646,0.00631$ $770,-0.00006209,-0.00093777,-0.00378458,-0.02963968,-0.02880647,0.0304$ $2378,0.00065612,-0.00116205,0.00022436,-0.00004649,0.00016707,-0.00004$ 
$061,-0.00006309,0.00006550,0.00003217,0.00006485,0.00005826,-0.0000104$ $5,-0.00006543,0.00004748,-0.00005927,-0.00004327,0.00017288,-0.0000215$ $4,-0.00029983,0.00044229,-0.00008703,-0.00000072,-0.00005717,0.0000074$ $2,0.00003943,0.00000940,0.00001060,0.00003795,0.00000452,-0.00001280,-$ $0.00000187,-0.00005422,0.00001386,0.00001179,0.00001045,-0.00000260,-0$ $.00500863,0.00141593,-0.00022944,0.00002724,0.02881627,-0.00580412,-0$. $05977538,0.03660432,-0.00728540,0.00646121,-0.02263925,0.00454967,-0.0$ $0325017,-0.00383685,0.00081685,-0.00126362,-0.00008389,0.00001521,-0.0$ $0014325,0.00140667,-0.00028263,0.00113941,-0.00043710,0.00006548,0.061$ $17374,0.00097563,-0.00031235,-0.00012959,-0.00025813,-0.00001307,-0.00$ $031295,0.00011749,0.00020857,0.00001821,-0.00013306,-0.00019801,0.0000$ $4332,0.00011743,0.00015955,-0.00015253,-0.00013517,0.00017589,0.000326$ $86,-0.00072096,0.00038331,-0.00004483,-0.00005834,-0.00004815,-0.00003$ 099,0.00001980,0.00002047,-0.00000602,0.00001262,0.00003092,0.00000081 ,-0.00002280,-0.00003654,0.00002336,0.00001978,0.00000243,-0.00000062, $0.00250464,0.00202400,0.00097408,-0.00115772,-0.00785387,0.00249924,0$. $04109275,-0.31143524,0.05575774,0.00024040,-0.01599688,0.00317534,-0.0$ $0238600,-0.00034259,0.00141892,-0.00049797,0.00036858,-0.00024397,-0.0$ $0007583,-0.00049457,0.00012552,0.00002256,0.00053892,-0.00088030,-0.04$ $003436,0.33338265,-0.00020277,-0.00011444,-0.00089863,-0.00025660,-0.0$ $0011695,0.00020308,-0.00003810,-0.00002307,-0.00014829,0.00002983,0.00$ $004460,0.00001949,-0.00001043,-0.00010403,-0.00009171,0.00033349,0.000$ $11766,0.00000798,0.00014616,-0.00005011,0.00015956,-0.00008637,0.00000$ $193,-0.00006442,0.00000953,-0.00003074,0.00001016,-0.00001738,0.000021$ $37,-0.00000265,0.00010316,-0.00000941,-0.00007191,-0.00000289,-0.00000$ $058,-0.00000802,-0.00045801,0.00097320,0.00665921,0.00025149,0.0023696$ $2,0.00402108,-0.00818429,0.05577266,-0.04585587,-0.00008181,0.00327036$ ,-0.00097915,0.00052280,0.00143109,0.00635953,0.00010777,-0.00024055,$0.00076149,0.00001771,0.00011780,0.00010154,-0.00002759,-0.00087823,-0$ $.00363069,0.00792220,-0.06248793,0.03586824,-0.00015623,0.00029869,-0$. $00006234,0.00023695,-0.00004912,0.00018153,0.00009448,-0.00007177,-0.0$ $0025257,-0.00070679,0.00001385,0.00000301,0.00009663,0.00003441,0.0002$ $6038,0.00023416,-0.00011775,-0.00015020,-0.00001708,-0.00000929,0.0000$ $0205,0.00001909,-0.00004231,0.00003039,0.00004059,0.00001003,0.0000376$ $1,0.00004099,-0.00000606,-0.00004075,0.00001851,-0.00005219,-0.0000127$ $8,0.00005844,0.00003101,-0.00000688,-0.00361002,0.00364088,-0.00069093$ ,-0.00009167,0.00061149,-0.00012552,0.00110565,0.00233066,-0.00044882, $0.00684030,0.00051808,-0.00013696,-0.22945439,-0.13027965,0.02718769$,$0.01725417,-0.01893176,0.00392500,-0.00092528,-0.00090856,0.00018802,-$ $0.00005209,-0.00005588,0.00000701,0.00028073,0.00029574,-0.00006012,0$. $24207199,-0.00127428,0.00003484,-0.00014743,0.00015380,0.00007511,0.00$ $032172,0.00013788,0.00002302,0.00004952,0.00008036,0.00044354,0.000018$ $98,0.00007368,0.00010798,-0.00015830,0.00004750,-0.00011113,-0.0002910$ $3,-0.00000822,0.00005550,-0.00001507,-0.00001321,0.00001320,-0.0000492$ $4,-0.00003851,0.00001579,-0.00004573,-0.00001625,0.00001531,0.00002236$ ,-0.00000697,0.00000407,0.00002803,-0.00009259,-0.00002963,0.00000620, $0.00283547,0.00068058,0.00122672,0.00097708,-0.00061547,-0.00002308,0$. $00080274,-0.00473098,0.00224301,-0.02264693,-0.01654880,0.00339175,-0$. $13357145,-0.15239117,0.02430124,0.01065823,0.00970839,-0.00103613,0.00$ $055489,0.00033956,-0.00005547,-0.00006603,-0.00000099,-0.00020423,-0.0$ $0095073,-0.00046371,0.00012838,0.14272853,0.16283148,0.00025444,-0.000$ 
$15747,-0.00070662,0.00023550,0.00014433,-0.00003327,0.00013723,-0.0003$ $6989,-0.00014972,-0.00001092,0.00001637,0.00051619,-0.00018227,0.00025$ $832,-0.00024271,-0.00027846,-0.00011067,0.00015620,0.00000245,-0.00001$ $488,-0.00001676,-0.00000497,0.00004789,-0.00004036,-0.00004461,0.00002$ $852,-0.00003344,0.00005526,-0.00004818,-0.00003071,0.00000879,-0.00006$ $598,-0.00003306,0.00001783,0.00000711,0.00000696,-0.00052612,0.0012284$ $8,0.00660183,-0.00019837,-0.00002358,-0.00071324,-0.00013977,0.0022419$ $5,0.00594753,0.00454436,0.00329338,-0.00091271,0.02785409,0.02428159,-$ $0.04011069,-0.00205834,-0.00090961,0.00497824,-0.00010755,-0.00004864$, $0.00008654,0.00000856,-0.00020499,-0.00097648,0.00019110,0.00012034,0$. $00011639,-0.02981014,-0.02881259,0.02932730,-0.00005026,0.00018801,-0$. $00003804,-0.00005890,0.00010556,0.00005593,0.00003373,0.00000934,0.000$ 05306,-0.00009033,-0.00016616,0.00003215,0.00003785,-0.00001120,-0.000 04896,-0.00005950,0.00007463,-0.00009349,-0.00007048,0.00006043,-0.000 $01110,-0.00000572,0.00001015,-0.00002640,-0.00002491,-0.00000375,-0.00$ $002221,-0.00002485,0.00000537,0.00002133,-0.00000650,0.00001806,0.0000$ $2032,-0.00002427,0.00000224,0.00000033,0.00727847,-0.02358479,0.004747$ $10,-0.00336098,-0.00295322,0.00064193,-0.00107347,-0.00067579,0.000136$ $58,-0.00399561,0.00289736,-0.00053950,-0.00097234,0.02770791,-0.005575$ $49,-0.06162551,0.03923858,-0.00781581,0.00085967,0.00024522,-0.0000708$ $0,0.00021463,-0.00129672,0.00026303,0.00006927,0.00006150,-0.00001781$, $0.00112920,-0.00036499,0.00005192,0.06182078,-0.00032404,-0.00001310,0$ $.00003569,0.00008032,-0.00003571,-0.00001239,-0.00003968,-0.00001330,-$ $0.00002871,0.00004232,0.00010710,-0.00001892,-0.00004492,-0.00002499,0$ $.00003291,0.00012402,-0.00000982,0.00000045,0.00004982,-0.00002257,-0$. $00000010,-0.00000359,-0.00000457,0.00001053,0.00001402,0.00000815,0.00$ $001357,0.00001673,0.00000109,-0.00001812,-0.00000434,-0.00001279,-0.00$ $000869,0.00001692,0.00000281,0.00000054,0.00621498,-0.01323773,0.00335$ $659,-0.00379976,0.00020501,0.00134083,-0.00013706,0.00055948,-0.000265$ $50,0.00248015,0.00084073,0.00115112,-0.00157411,-0.00696080,0.00228899$ $, 0.03822362,-0.31738285,0.05656809,-0.00018708,0.00065672,-0.00091977$, $0.00037386,-0.00072512,0.00020587,0.00003457,-0.00010033,-0.00019262,0$ $.00001098,0.00054352,-0.00090179,-0.04156773,0.33561907,0.00006623,0.0$ $0003285,0.00014903,-0.00012862,-0.00006042,-0.00003982,0.00002246,0.00$ $005809,-0.00001300,-0.00000856,-0.00001537,0.00001806,-0.00000704,-0.0$ $0006113,-0.00000938,0.00008672,0.00005184,-0.00005707,-0.00000839,0.00$ $000024,-0.00002219,0.00000245,0.00001079,-0.00000676,-0.00000933,-0.00$ $000048,-0.00000987,0.00000470,-0.00000175,-0.00000086,0.00000015,-0.00$ $000883,-0.00000150,-0.00000488,-0.00000055,0.00000595,-0.00127082,0.00$ $348594,0.00281218,0.00081657,0.00133584,0.00648314,0.00002580,-0.00025$ $610,-0.00070470,-0.00045437,0.00114402,0.00636321,0.00034531,0.0021573$ $3,0.00389515,-0.00761736,0.05656599,-0.04799550,0.00001674,-0.00092047$ $,-0.00371321,-0.00007599,0.00021438,0.00025897,-0.00001304,-0.00019403$ ,-0.00101343,-0.00002467,-0.00089963,-0.00373742,0.00823594,-0.0626385 $6,0.03733903 \backslash \backslash 0.00000157,0.00000157,-0.00000327,-0.00000019,-0.0000005$ $8,-0.00000225,0.00000017,0.00000333,-0.00000548,-0.00000310,0.00000306$ $, 0.00000033,-0.00000059,0.00000461,0.00000243,0.00000051,0.00000164,0$. $00000413,0.00000598,0.00000310,-0.00000056,0.00000168,0.00000249,-0.00$ $000031,-0.00000094,0.00000398,-0.00000110,-0.00000104,0.00000413,-0.00$ $000046,0.00000171,0.00000164,-0.00000012,-0.00000248,0.00000520,-0.000$ $00099,-0.00000149,-0.00000445,0.00000107,0.00000078,-0.00000470,0.0000$ 
0035,0.00000067,-0.00000912,0.00000173,0.00000065,0.00000531,0.,-0.000 $00066,-0.00000034,-0.00000002,-0.00000035,-0.00000296,0.00000070,-0.00$ $000142,-0.00000600,0.00000122,0.00000223,-0.00000568,0.00000113,0.0000$ $0247,-0.00000300,0.00000093,-0.00000277,0.00000020,-0.00000011,-0.0000$ 0341,-0.00000345,0.00000065।I!@

\section{Addition Product}

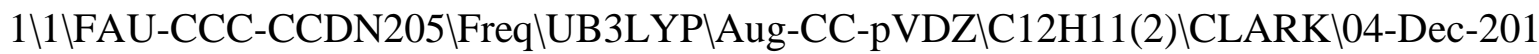
4I0\\\# Geom=AllCheck Guess=TCheck SCRF=Check GenChk UB3LYP/Aug-CC-pVD $\mathrm{Z}$ Freq \\Addition product Ph-radical + benzene $\backslash 0,2 \backslash \mathrm{C},-6.2588211088,1.4$ 320481589,1.6846167634\C,-4.9904673737,0.7114638449,2.077971996lC,-3.7 436413776,1.2111896688,1.8134464464\C,-3.5680176133,2.4545258288,1.147 9809727\C,-4.7111311132,3.2017755003,0.754931487\C,-5.9799442437,2.747 $2946566,0.9953807591 \backslash \mathrm{H},-6.8081492537,0.7845636744,0.9690598497 \backslash \mathrm{H},-5.10$ $34347082,-0.2420320476,2.5956162687 \backslash \mathrm{H},-2.8635646317,0.6439371132,2.122$ $2767298 \mathrm{\backslash H},-4.5729129288,4.1608310332,0.2521476137 \backslash \mathrm{H},-6.8441326913,3.33$ 93226199,0.6911489477\H,-2.5671176481,2.8342899738,0.9473496646\C,-8.9 453393747,1.9283709655,5.0739436718\C,-9.3362816176,1.1553066406,3.977 8372669\C,-8.4720067695,0.9961346504,2.8883369839\C,-7.2104993945,1.60 42767395,2.878402678\C,-6.8267768807,2.3788963402,3.9840213622\C,-7.68 $61185208,2.5402390223,5.0731051307 \backslash \mathrm{H},-9.6161923548,2.0544510029,5.9243$ 64979\H,-10.3152028986,0.6743706932,3.9685873693\Н,-8.784948539,0.3905 $066611,2.0357359232 \backslash \mathrm{H},-5.8470913766,2.8578883266,3.9889080732 \backslash \mathrm{H},-7.373$ 417143,3.1454450076,5.9251140449\\Version=EM64L-G09RevC.01 \State $=2-\mathrm{A} \backslash \mathrm{H}$ $\mathrm{F}=-463.8941122 \backslash \mathrm{S} 2=0.786154 \backslash \mathrm{S} 2-1=0 . \mathrm{IS} 2 \mathrm{~A}=0.750744 \backslash \mathrm{RMSD}=8.788 \mathrm{e}-09 \backslash \mathrm{RMSF}=3$. 530e-06\ZeroPoint $=0.1891002 \backslash$ Thermal $=0.1986841 \mathrm{ZPE}=118.6621896 \backslash$ Dipole $=-$ $0.0730566,-0.0286685,0.0128771 \backslash$ DipoleDeriv $=-0.0227097,0.0262482,0.0505$ $305,-0.0165597,0.0459485,0.142525,-0.0293772,0.1042382,0.2005854,0.079$ $3448,-0.0036436,-0.0310153,0.0089703,-0.0235656,-0.0445348,0.1093388,-$ $0.0872378,-0.2453454,-0.0025542,-0.0828409,0.0928888,-0.0186852,0.0316$ $361,-0.1119607,0.0172646,-0.0888952,-0.0564076,0.0826791,0.0557592,-0$. $0252933,0.061074,-0.0571113,-0.0518387,-0.0164537,-0.0479789,-0.134442$ $4,-0.0216301,-0.0023204,0.009269,-0.0886378,0.0882381,-0.0589427,0.045$ $7068,-0.1004892,-0.0942732,0.0125624,0.0346833,0.0218421,0.111996,-0.0$ $259119,-0.1921179,0.0471778,-0.069843,-0.1751449,-0.0529436,-0.0920428$ $,-0.0598087,-0.0819091,-0.0028368,-0.0641336,-0.0404967,-0.0547553,0.0$ $05224,0.0440699,-0.0149467,-0.0047977,-0.0154824,-0.0420037,0.0836158$, $-0.0089762,0.0963953,0.0784846,-0.0723756,0.0761635,-0.0183293,0.06858$ $25,0.0254354,0.0620707,-0.0601233,0.0538537,0.1112307,0.0462067,-0.013$ $9837,0.0237536,-0.0413387,-0.0625369,0.1216138,-0.0002981,0.0977848,0$. $0810689,-0.0363981,0.0538645,-0.0421814,0.0552086,0.0121782,0.0566078$, $-0.0356509,0.0450964,0.1047987,-0.0831935,-0.0453072,0.0344763,-0.0514$ $553,0.0491783,0.0501295,0.0232038,0.0448131,0.1105574,-0.0712831,0.009$ $9319,-0.0436408,-0.0020435,-0.1113565,0.0524897,-0.0660819,0.0414437,-$ $0.000299,0.0532499,0.089953,-0.0014145,0.0956947,-0.0683741,0.0353993$, $0.0091488,0.0404714,-0.0657413,-0.0745648,0.0482604,0.0515313,0.072443$ $5,-0.0467665,0.0688488,0.0966759,0.0906675,-0.0357145,0.1517141,0.0061$ $159,-0.1709042,-0.0382833,-0.0539309,0.022474,-0.253941,-0.0178079,0.1$ $509856,-0.0708339,0.0361042,0.0474015,0.0593358,-0.0709824,-0.0134921$, $0.0916723,0.0083869,-0.1614399,0.0169579,0.0587462,-0.0204206,0.071235$ 
$7,-0.0425092,0.0930842,0.0031087,0.1041615,0.0673987,0.0046294,-0.0093$ $708,0.0994373,-0.0080859,0.1082051,-0.0383799,0.1016897,-0.037336,-0.0$ $305386,-0.0817657,-0.0897745,0.0231396,-0.091726,0.072683,-0.0208429,0$ $.0194058,-0.0226775,0.0678708,0.0814773,-0.0268209,-0.0154727,-0.04041$ $6,0.0521652,-0.0909944,-0.0406449,-0.1029918,-0.0368658,-0.0360997,-0$. $0655009,0.0045713,-0.0715396,0.0684603,0.0005208,-0.007242,-0.005183,0$ $.0999118,0.0534604,-0.049278,-0.0255627,-0.0383787,0.0537579,-0.102141$ $7,-0.0051072,-0.0921169,-0.0419039 \backslash$ Polar $=175.354193,14.2232728,120.187$ $8852,-28.9486706,-2.169275,136.8113278 \backslash \mathrm{PG}=\mathrm{C} 01[\mathrm{X}(\mathrm{C} 12 \mathrm{H} 11)] \mathrm{NImag}=0 \backslash 10.4$ $7528698,0.02487109,0.48183697,0.03911809,0.00527069,0.44107735,-0.1550$ $7038,0.04971567,-0.03010013,0.64017830,0.04056039,-0.11222625,0.026976$ $08,0.08384894,0.53649964,-0.02639823,0.02638881,-0.08005308,-0.0437458$ $4,-0.22139932,0.24951741,-0.03004302,-0.00389688,0.00164548,-0.3531092$ $4,-0.09959877,0.05286151,0.71772226,0.00710732,0.01259026,-0.00450596$, $-0.05677136,-0.12320984,0.03777198,-0.04199661,0.49423901,-0.00436628$, $-0.00285641,0.01066941,0.02875943,0.03738656,-0.07144042,0.02494324,-0$ $.19981913,0.22781717,0.00155974,0.00361862,-0.00200215,0.00933423,-0.0$ $2869429,0.01574957,-0.12227689,-0.04346178,0.02303315,0.67465468,0.003$ $64186,-0.00708576,-0.00152205,-0.06815373,-0.03425045,0.02355133,0.035$ $87924,-0.20536827,0.08128381,0.04996063,0.46440756,-0.00191322,-0.0014$ $7350,-0.00907131,0.03664095,0.02287249,-0.00336430,-0.01935382,0.08097$ $811,-0.09768231,-0.02710636,-0.19191196,0.20605242,-0.00419413,-0.0151$ $8628,0.00604504,-0.03575317,-0.01229234,0.00657235,0.04575234,0.058294$ $81,-0.03069502,-0.19302950,0.01606064,-0.00826499,0.60779213,-0.025703$ $34,-0.00603270,0.00865846,0.00478024,0.00236131,-0.00248798,0.01991831$ ,-0.02874701,0.01767930,0.09563255,-0.15134655,0.05111583,0.04949888,0 $.58315456,0.01290443,0.00740110,0.00347991,-0.00264415,-0.00248467,-0$. $00040804,-0.01063454,0.01840233,-0.00452899,-0.05019186,0.05103538,-0$. $08099262,-0.02556062,-0.24327020,0.24888074,-0.08742274,-0.01463153,0$. $00682120,0.02320069,0.03123182,-0.01590290,-0.01919605,-0.02631504,0.0$ $1372224,-0.07506238,0.04201735,-0.02220377,-0.33079839,-0.11893560,0.0$ $6172418,0.74337427,-0.00650300,-0.16948431,0.05188728,0.01157268,-0.01$ $761699,0.00838262,-0.00911943,-0.01020964,0.00454851,0.00280989,0.0315$ $6597,-0.01159771,-0.07498493,-0.14042509,0.04672210,-0.00448087,0.4633$ $4431,0.00118694,0.05155359,-0.09050241,-0.00645844,0.01047980,-0.00508$ $777,0.00467478,0.00463040,-0.00437656,-0.00074534,-0.01199664,0.015256$ $17,0.03961161,0.04728540,-0.07658852,-0.00098857,-0.17378901,0.2195790$ $0,-0.09130398,-0.06637021,-0.08167797,-0.02278200,-0.01178595,-0.01633$ $485,0.00026906,0.00425827,-0.00295940,-0.00125326,-0.00115176,0.000528$ $01,0.00224172,-0.00059828,0.00014560,-0.00434918,-0.00936762,0.0005717$ $4,0.11384296,-0.06113235,-0.12013894,-0.08710376,0.00619816,0.01198040$ $, 0.00857124,0.00174970,-0.00089245,0.00027837,-0.00146278,0.00070472,-$ $0.00052576,0.00398250,-0.00385698,0.00078067,-0.01840294,-0.02040115$,$0.01164295,0.07282352,0.13412897,-0.07182814,-0.08231917,-0.13325853,-$ $0.00746913,-0.00304496,-0.00308811,0.00056147,0.00187190,-0.00226297$,$0.00005585,-0.00080920,0.00022341,0.00052812,0.00108161,-0.00127170,0$. $00853388,0.00815669,0.01086160,0.07746328,0.09397718,0.15468989,0.0041$ $9154,0.02271767,-0.01071077,-0.05822420,-0.02412959,0.01288135,0.00100$ $632,-0.02587671,0.01400912,-0.00489993,-0.00110114,0.00066655,-0.00061$ $999,0.00015627,-0.00014782,-0.00501997,0.00138722,-0.00077324,0.000705$ $78,-0.00075545,-0.00018449,0.05992641,-0.00323743,-0.00875367,0.005017$ 
$80,-0.02652528,-0.26533489,0.12714638,0.00193970,-0.00610033,0.0062159$ $5,-0.00220427,0.00330516,0.00269666,0.00010709,0.00015842,-0.00048933$, $0.00288655,0.00079187,0.00072828,0.00057496,0.00012017,0.00051680,0.02$ $759090,0.27680736,0.00235882,0.00628309,-0.00247829,0.01413291,0.12814$ $169,-0.09888600,-0.00126621,0.00594099,0.00227848,0.00100407,0.0024114$ $8,0.00645527,-0.00002423,-0.00038755,-0.00053010,-0.00163734,0.0003335$ $2,0.00152811,0.00067899,-0.00041555,0.00056453,-0.01490519,-0.13977753$ $, 0.09484888,-0.00163746,-0.00315670,0.00190143,-0.02003505,0.01668999$, $-0.00925938,-0.23633556,0.11583136,-0.06294898,0.00568858,-0.00338079$, $0.00175089,0.00150175,-0.00126400,0.00064262,0.00003708,-0.00054768,0$. $00031872,-0.00056777,0.00026759,-0.00015430,0.00106365,-0.00013735,0.0$ $0015372,0.25005900,-0.00196019,0.00180413,0.00430384,-0.01045534,0.009$ $18586,-0.00305853,0.11605201,-0.12847858,0.04951088,0.02305702,-0.0087$ $2467,0.00634762,-0.00190541,-0.00280908,0.00428199,-0.00076291,-0.0003$ $3465,-0.00010290,0.00037801,-0.00034834,0.00003979,0.00006087,-0.00047$ $310,-0.00269440,-0.12460787,0.13017232,0.00170555,0.00389425,0.0074251$ $6,0.00546639,-0.00251319,0.00494306,-0.06362278,0.04953641,-0.06419645$ $,-0.01228561,0.00615160,-0.00045569,0.00100356,0.00441740,0.00281812,0$ $.00040504,-0.00008798,-0.00053562,0.00107580,-0.00089093,0.00032486,-0$ $.00007013,-0.00277006,-0.00397219,0.06794059,-0.05688336,0.05627000,-0$ $.00407564,-0.00006824,0.00032302,-0.00086565,0.00001350,0.00003741,-0$. $00379413,0.00256076,-0.00135248,0.00750252,0.02157570,-0.01137072,-0.0$ $6188412,-0.02958771,0.01564468,-0.00032112,-0.02710757,0.01408781,-0.0$ $0012092,-0.00054625,0.00023995,0.00015731,-0.00000012,0.00000971,0.000$ $18293,0.00117785,-0.00064990,0.06154969,-0.00110809,0.00398489,0.00300$ $170,0.00023342,0.00034643,-0.00048288,0.00324637,0.00117327,0.00209320$ ,-0.00489986,-0.01024812,0.00684881,-0.03019772,-0.26680381,0.12046092 $, 0.00044607,-0.00658363,0.00520299,-0.00007226,0.00036250,-0.00025822$, $-0.00004465,-0.00022916,-0.00027736,-0.00023457,-0.00064426,0.00041128$ $, 0.03297452,0.27965308,0.00084537,0.00357313,0.00767067,-0.00009052,-0$ $.00051786,-0.00029542,-0.00159661,0.00201545,0.00413361,0.00256359,0.0$ $0699452,-0.00074078,0.01560380,0.12008826,-0.10032568,0.00009008,0.005$ $00740,0.00100858,0.00011145,0.00120757,-0.00083329,0.00003197,-0.00034$ $768,-0.00061247,0.00014838,0.00031635,-0.00015318,-0.01733820,-0.13332$ $006,0.09529271,0.00620095,-0.00482489,0.00343972,0.00034576,-0.0015188$ $3,0.00094326,0.00005873,-0.00048683,0.00020783,-0.00319071,-0.00346498$ $, 0.00211471,-0.01794267,0.01803549,-0.00936584,-0.23201177,0.11996778$, $-0.06222772,0.00023159,0.00050322,-0.00041536,0.00048688,-0.00022121,0$ $.00010646,0.00001469,0.00009637,-0.00008848,0.00098333,-0.00015849,-0$. $00003747,0.24498943,0.02104808,-0.01056206,0.00698762,-0.00318467,-0.0$ $0341391,0.00262428,-0.00035529,-0.00043177,-0.00019892,-0.00261900,0.0$ $0176495,0.00312116,-0.01021945,0.00879425,-0.00165458,0.12267720,-0.13$ $316578,0.05373293,-0.00004576,0.00081514,-0.00075222,0.00142674,-0.000$ $55700,0.00049140,0.00008583,-0.00008942,-0.00032972,0.00015008,-0.0002$ $9663,-0.00269312,-0.12861731,0.13622564,-0.00977695,0.00564402,-0.0026$ $7460,0.00146010,0.00285086,0.00037178,0.00028242,-0.00020503,-0.000614$ $02,0.00126117,0.00315380,0.00627056,0.00510005,-0.00176754,0.00635082$, $-0.06287209,0.05303423,-0.05728490,0.00019214,0.00089619,0.00034541,-0$ $.00075979,0.00047068,0.00009651,-0.00002195,-0.00037798,-0.00060814,-0$ $.00005729,-0.00267088,-0.00407562,0.06600332,-0.06057449,0.05036860,0$. $00053782,0.00034377,-0.00007338,0.00168023,0.00003733,0.00000293,0.000$ 
$18653,0.00169093,-0.00089442,-0.29809006,-0.09121386,0.04825533,-0.015$ $92346,-0.01138485,0.00599171,-0.00203156,0.00363764,-0.00192746,0.0000$ $3712,-0.00001538,-0.00002328,-0.00007899,0.00010038,-0.00005793,0.0006$ $8176,-0.00029919,0.00012720,0.00084350,0.00007501,-0.00006336,-0.00100$ $563,-0.00052544,0.00027135,0.31310349,0.00027416,-0.00034979,-0.000430$ $74,0.00057140,-0.00379182,0.00401420,-0.02485513,-0.00365286,0.0036270$ $0,-0.09132255,-0.08510328,0.03178253,0.01519745,0.00907386,-0.00300513$ $, 0.00319321,-0.00091615,0.00251893,-0.00007000,0.00004016,0.00005572,-$ $0.00130722,-0.00040766,0.00028327,0.00022693,0.00028463,-0.00147380,-0$ $.00041360,0.00014478,-0.00141566,0.00087463,0.00031857,-0.00009533,0.0$ $9760228,0.08421484,-0.00021705,-0.00049849,-0.00099658,-0.00018458,0.0$ $0409855,0.00160700,0.01329862,0.00359621,0.00144761,0.04804620,0.03167$ $953,-0.04195340,-0.00811815,-0.00293078,0.00484582,-0.00155450,0.00252$ $021,0.00242715,-0.00012423,0.00000637,0.00016996,0.00067475,0.00028566$ ,-0.00001394,-0.00010931,-0.00146123,-0.00170516,0.00023581,-0.0013979 $4,-0.00173024,-0.00048238,-0.00010791,0.00019131,-0.05158372,-0.035858$ $69,0.03555814,-0.00120370,-0.00019494,-0.00022447,0.00000711,0.0000298$ $1,0.00080226,-0.00008091,0.00016582,0.00004132,0.00013748,0.00012900,0$ $.00007100,0.00001999,-0.00002013,0.00014475,-0.00019942,0.00050848,0.0$ $0050211,-0.00002205,0.00004417,0.00020952,-0.00002472,-0.00001444,-0.0$ $0004071,-0.00001385,-0.00007098,-0.00015484,0.00002652,-0.00009806,-0$. $00011722,-0.00000448,-0.00003976,-0.00005895,-0.00001724,-0.00001652,0$ $.00001846,0.60378404,-0.00010361,-0.00090531,0.00036079,-0.00004568,0$. $00023603,0.00018720,0.00007308,-0.00003651,0.00010086,0.00011500,0.000$ $07370,0.00004881,-0.00003620,-0.00003226,0.00010432,0.00004384,0.00018$ $449,0.00031273,-0.00004258,0.00019712,0.00020904,-0.00000793,-0.000070$ $13,-0.00004426,0.00003662,0.00000827,-0.00007819,0.00001429,-0.0000200$ $2,-0.00011522,-0.00005688,-0.00001898,-0.00000843,-0.00001633,0.000008$ $99,-0.00000108,0.16063570,0.31476262,-0.00003990,0.00045297,-0.0001590$ $4,-0.00001722,0.00034984,-0.00021176,0.00001101,-0.00010150,0.00008768$ $, 0.00008146,0.00005570,0.00000983,-0.00008293,0.00017884,-0.00006205,0$ $.00030570,-0.00034528,0.00010596,0.00003903,0.00012538,0.00030982,-0.0$ $0004096,-0.00007239,0.00002273,0.00008166,-0.00001730,-0.00002414,0.00$ $007869,0.00003445,-0.00002470,-0.00007196,0.00001481,-0.00005751,0.000$ $00568,-0.00000775,0.00000363,-0.12677459,0.19149527,0.61076505,-0.0012$ $1158,-0.00447033,-0.00011505,0.00026552,0.00018374,0.00022231,-0.00000$ $789,-0.00004009,0.00001390,0.00009546,0.00005051,-0.00003790,0.0001366$ $3,-0.00030851,0.00019301,-0.00096534,0.00055262,0.00075086,0.00012482$, $0.00023200,0.00030887,-0.00010748,0.00003137,-0.00005821,0.00004110,0$. $00000005,-0.00001676,-0.00007145,-0.00004999,-0.00010455,-0.00006913,-$ $0.00010145,0.00003635,0.00000995,0.00002721,0.00000528,-0.13092173,-0$. $06609762,-0.06322500,0.63653011,-0.00456859,0.00413324,-0.00282150,-0$. $00085593,-0.00062497,0.00110140,-0.00017113,0.00040998,-0.00024752,-0$. $00006752,-0.00010851,-0.00001226,-0.00010139,0.00027915,-0.00025948,0$. $00104552,-0.00019334,-0.00062072,0.00004722,0.00003088,0.00003523,-0.0$ $0005124,0.00004205,0.00002262,-0.00011203,-0.00009834,-0.00001435,0.00$ $007418,0.00007856,0.00012856,0.00005708,-0.00006415,0.00003065,0.00002$ $930,-0.00000683,0.00001995,-0.03885418,-0.15087958,-0.12753829,0.19833$ $426,0.33001157,-0.00029754,-0.00291806,0.00265213,0.00054008,-0.000022$ $35,-0.00096562,0.00020181,-0.00025719,-0.00002537,-0.00028040,-0.00012$ $577,-0.00005517,0.00016814,-0.00017847,-0.00002419,-0.00060566,0.00001$ 
$279,-0.00018644,-0.00003615,-0.00013184,-0.00022636,0.00005559,0.00010$ $039,-0.00000031,-0.00000631,0.00011539,0.00016573,-0.00009730,0.000008$ $05,0.00009724,0.00006694,0.00001959,0.00006877,0.00001735,0.00002467,-$ $0.00000110,-0.01201521,-0.10263384,-0.24615054,-0.08556757,0.18520271$, $0.56153267,-0.01893777,-0.00792947,0.00684360,0.00014878,-0.00185023,0$ $.00058192,-0.00022499,0.00057561,-0.00044149,-0.00000249,0.00001797,0$. $00006302,0.00054104,-0.00063702,0.00024121,-0.00142510,0.00113081,-0.0$ $0132556,0.00025402,-0.00127790,-0.00281384,-0.00002720,0.00042262,-0.0$ $0006583,-0.00018441,-0.00007479,0.00010272,-0.00024299,0.00000974,0.00$ $016869,0.00016448,-0.00035884,0.00033492,-0.00001509,0.00000158,0.0000$ $1946,0.04951229,0.03656856,0.03054725,-0.19480449,-0.01850485,0.085641$ $99,0.58110059,0.00151624,0.00547421,-0.00030382,0.00084566,-0.00069891$ ,-0.00106732,0.00028825,0.00014812,0.00005471,0.00014003,0.00001806,0. $00003817,-0.00004287,0.00029045,-0.00012462,0.00070738,-0.00024370,-0$. $00067715,0.00150933,0.00024438,-0.00058187,-0.00027077,0.00009878,-0.0$ $0000489,0.00005616,0.00007328,0.00019131,0.00005152,0.00005787,-0.0000$ $2470,0.00009045,0.00014642,0.00000211,-0.00001444,0.00000570,-0.000049$ $11,0.01636763,0.00856287,-0.01328672,0.00919907,-0.08630588,-0.0520698$ $9,0.17591499,0.33228601,0.02462627,0.00834413,-0.00194316,0.00112926,0$ $.00144055,-0.00218659,0.00057491,-0.00072278,0.00062347,0.00021813,0.0$ $0010163,-0.00003162,-0.00009024,0.00100058,-0.00024494,0.00230180,-0.0$ $0116715,-0.00114414,0.00243795,0.00197419,0.00129545,0.00011695,-0.000$ $31478,0.00015575,0.00032120,0.00009467,0.00000151,0.00025559,0.0002187$ $9,0.00005643,-0.00028789,0.00009993,-0.00008144,0.00001398,-0.00004726$ ,-0.00008110,-0.00746360,-0.03177631,-0.04522940,0.13772474,-0.0267408 $6,-0.23928456,-0.07934341,0.21466259,0.60872365,-0.10139245,0.00863791$ $, 0.04630356,-0.03058545,0.00718890,0.01934183,0.00059931,0.00444932,-0$ $.00114181,-0.00105855,-0.00072468,0.00033995,0.00264597,0.00070316,0.0$ $0091490,-0.00901523,-0.01099280,0.01221516,0.00437951,-0.00327985,-0.0$ $0952901,0.00147142,-0.00130428,-0.00041775,-0.00034544,-0.00065714,-0$. $00094138,0.00019460,-0.00004657,-0.00019973,0.00070949,0.00071333,-0.0$ $0140034,0.00012688,0.00001113,0.00002482,-0.03252281,-0.02488463,-0.02$ $286757,-0.03722413,0.00893784,0.05608789,-0.27485876,-0.11237737,-0.01$ $909917,0.56757437,0.00224329,-0.07238288,-0.00925886,0.00868636,-0.000$ $85820,-0.01066540,0.00086371,-0.00101978,0.00028627,-0.00051040,0.0005$ $5007,-0.00013115,0.00189007,-0.00419567,0.00185771,-0.01977627,0.00400$ $192,0.02132500,0.00979574,-0.00419106,-0.01813686,0.00004521,0.0007316$ $9,-0.00022662,0.00015733,0.00045062,0.00050946,-0.00084984,-0.00095749$ ,-0.00093751,-0.00021133,0.00034116,-0.00020662,-0.00003257,0.00013181 $, 0.00007147,-0.02508809,-0.02707774,-0.01607758,-0.01183723,0.02120517$ $, 0.03989696,-0.08330041,-0.12353967,-0.03454771,0.16486045,0.34238780$, $0.03425250,-0.01511693,-0.12826817,0.00567375,-0.00550809,0.01021289$,$0.00300780,0.00093720,-0.00227296,0.00079157,0.00009613,0.00029387,-0$. $00179402,-0.00102849,-0.00116142,0.01225734,0.00955508,-0.01617555,0.0$ $1505703,-0.00619089,-0.02470380,-0.00111833,0.00096415,0.00054097,-0.0$ $0087787,-0.00047742,-0.00029220,-0.00023409,0.00006498,0.00058623,0.00$ $008366,-0.00101955,0.00169568,-0.00007388,0.00002091,0.00022762,-0.023$ $21939,-0.01624487,-0.01565702,0.01702212,0.02090052,0.02947461,0.03555$ $426,-0.00797482,-0.11215385,-0.04882420,0.16375320,0.52764443,0.007400$ $57,0.01177475,0.01722454,0.00161514,0.00027728,0.00124691,-0.00061350$, $0.00004993,0.00018842,-0.00012893,0.00004309,-0.00008105,-0.00045962,-$ 
$0.00047315,0.00003510,0.00106451,0.00132898,0.00035581,-0.00179402,0.0$ $0099757,0.00447199,0.00006068,-0.00008841,0.00004875,-0.00002397,-0.00$ $009815,-0.00012729,-0.00001801,-0.00011576,-0.00008660,0.00005371,-0.0$ $0010364,0.00007485,-0.00003969,-0.00000734,0.00006780,-0.03669484,-0.0$ $1160858,0.01910922,-0.00451495,0.00569793,0.01063626,-0.00225110,-0.01$ $868618,-0.02694997,-0.13148358,-0.06385500,-0.06035739,0.64228733,0.00$ $286947,0.00086734,-0.00485888,-0.00062052,0.00071203,-0.00071384,-0.00$ $039241,-0.00034419,-0.00031091,-0.00012187,-0.00038618,-0.00000700,-0$. $00041588,0.00006320,0.00035970,-0.00009164,-0.00016213,0.00041815,-0.0$ $0009604,0.00096356,-0.00006526,0.00002521,0.00009917,-0.00003061,-0.00$ $003691,0.00007626,-0.00000078,-0.00001904,0.00003209,-0.00004413,-0.00$ $009843,0.00039314,-0.00022470,-0.00002020,0.00004944,0.00001581,0.0083$ $0607,0.02278116,0.02006859,0.00566627,-0.01353508,-0.02199563,-0.03773$ $945,-0.01283499,-0.00224025,-0.03731831,-0.14558377,-0.11515898,0.1933$ $3256,0.32277977,0.00050872,-0.01300405,-0.02283686,-0.00221619,0.00046$ $563,-0.00119482,-0.00011593,-0.00024139,0.00026943,-0.00042426,-0.0001$ $4676,-0.00026898,-0.00032748,-0.00006821,-0.00028622,-0.00059711,-0.00$ $286151,0.00022842,0.00241531,-0.00106817,-0.00316294,0.00032593,-0.000$ $36584,0.00018071,0.00002796,0.00004337,0.00011964,0.00004033,0.0000517$ $1,0.00014902,0.00003297,0.00021547,-0.00010927,0.00005085,-0.00000265$, $0.00002066,0.05653549,0.03828002,0.02832699,0.01058350,-0.02201912,-0$. $05567244,-0.06277232,-0.01966446,0.02714020,-0.01046013,-0.09089652,-0$ $.22752352,-0.10139933,0.17852797,0.56219986,0.00183922,-0.00325939,-0$. $00223712,-0.00030213,-0.00003861,-0.00054018,0.00017537,-0.00014091,-0$ $.00006128,-0.00007461,-0.00008318,-0.00005557,-0.00001070,-0.00012402$, $-0.00017861,-0.00038462,-0.00040181,-0.00006078,0.00029138,-0.00051648$ ,-0.00107307,0.00004515,0.00003834,0.00004064,-0.00004567,0.00007403,0 $.00013041,-0.00008440,0.00002353,0.00010059,0.00005126,0.00012513,0.00$ $009989,-0.00000020,0.00000640,-0.00001300,-0.28473836,-0.08806235,0.03$ $564252,-0.00180035,-0.03936176,-0.06519492,-0.05219472,-0.00942422,0.0$ $2552983,0.04987865,0.03784321,0.03122781,-0.19772100,-0.01829992,0.089$ $30926,0.58395211,-0.00326420,0.00221363,-0.00429672,-0.00057547,0.0000$ $4486,0.00049906,-0.00008491,0.00008355,-0.00009413,0.00002403,0.000024$ $85,-0.00003144,0.00018360,0.00034969,-0.00001635,0.00011615,-0.0003573$ $3,0.00012902,0.00030282,-0.00011078,-0.00082906,0.00008038,-0.00001077$ $, 0.00008001,-0.00003816,-0.00007525,-0.00003636,0.00005047,0.00000519$, $0.00003918,0.00000768,-0.00005505,-0.00011239,-0.00000051,-0.00002287$, $0.00000064,-0.11529094,-0.12497628,-0.00800378,-0.01940121,-0.01279943$ ,-0.02106168,-0.01007800,-0.00631889,0.00653094,0.01718175,0.00818692, $-0.01199547,0.00939053,-0.08624579,-0.05339443,0.17886305,0.33745911,-$ $0.00225999,-0.00429973,0.00005120,-0.00021289,0.00045355,0.00042418,0$. $00028004,0.00016993,0.00026096,0.00015040,0.00004308,0.00003340,0.0001$ $5707,0.00007998,0.00018251,-0.00039274,0.00013845,0.00089440,0.0004678$ $7,-0.00007548,-0.00039663,0.00000221,-0.00011852,-0.00016300,0.0000658$ $0,-0.00002008,-0.00012123,0.00000184,-0.00005951,-0.00016101,-0.000012$ $68,0.00001922,-0.00012047,-0.00002699,0.00000374,-0.00001523,-0.015544$ $11,-0.03290637,-0.11228733,-0.02766885,-0.00281658,0.02784406,0.024286$ $29,0.00593095,-0.01596064,-0.00761298,-0.03087851,-0.04489387,0.141367$ $57,-0.02807516,-0.24445654,-0.07330551,0.21534099,0.60785744,-0.000091$ $25,0.00038190,-0.00014783,-0.00001387,-0.00003357,0.00006886,-0.000007$ $17,0.00000560,-0.00002620,0.00002162,0.00000575,-0.00000260,0.00002287$ 
$, 0.00003900,0.00001041,0.00003837,0.00002184,0.00001626,-0.00001520,0$. $00002845,0.00006967,-0.00000016,-0.00000217,-0.00000461,-0.00000727,-0$ $.00000384,-0.00000255,0.00000519,0.00000462,-0.00000201,0.00000135,-0$. $00000721,-0.00001537,-0.00000714,-0.00000130,-0.00000069,-0.15987785,0$ $.01328643,0.13819031,0.00815352,-0.00196085,-0.00820643,0.00172027,-0$. $00311602,-0.00086912,-0.00054084,-0.00036478,-0.00088613,-0.00309577,-$ $0.00491391,-0.00028784,-0.01144566,0.00487989,0.02268159,0.16526398,0$. $00038704,-0.00064949,0.00036484,0.00007259,0.00007313,-0.00003633,0.00$ $004907,0.00000786,0.00005780,0.00001460,0.00000159,0.00000043,-0.00000$ $858,-0.00004746,-0.00002761,-0.00009042,0.00003300,0.00002804,-0.00001$ $329,0.00003398,0.00006824,-0.00000224,-0.00000299,-0.00001979,0.000022$ $75,0.00000204,-0.00000022,-0.00001188,-0.00000950,-0.00000411,-0.00000$ $162,-0.00000182,0.00000738,0.00000194,-0.00000012,-0.00000036,0.013281$ $49,-0.04696921,-0.03264705,0.01134850,0.00184974,-0.01293414,-0.003561$ $63,0.00289508,-0.00480253,-0.00033057,-0.00094584,-0.00004907,-0.00450$ $374,0.00333526,-0.00220148,-0.00839838,0.00502458,0.01086814,-0.011427$ $08,0.03969965,-0.00013190,0.00037231,-0.00020991,-0.00002273,-0.000005$ $55,0.00002259,-0.00001539,-0.00001618,-0.00001738,0.00000598,0.0000048$ 4,-0.00000037,0.00000246,0.00004632,0.00000998,0.00007557,-0.00001646, $0.00000565,-0.00000766,0.00003059,0.00006813,-0.00000714,0.00000767,0$. $00000087,0.00000094,-0.00000502,-0.00000564,0.00001437,0.00001055,-0.0$ $0000839,0.00000278,-0.00001306,-0.00000281,0.00000844,0.00000430,-0.00$ $000157,0.13817611,-0.03265408,-0.22379487,0.01681879,-0.00076577,-0.01$ $360639,-0.00170120,-0.00520684,-0.00105380,-0.00082334,-0.00001872,-0$. $00004223,0.00048642,-0.00182666,0.00281794,-0.00228401,-0.00127075,0.0$ $0302691,-0.14976017,0.03815119,0.23362430,-0.00033396,-0.00043168,-0.0$ $0045847,-0.00006900,-0.00012235,0.00018461,-0.00001791,0.00007173,-0.0$ $0002909,-0.00003313,0.00000472,0.00000858,0.00003722,-0.00006945,0.000$ $03778,-0.00015211,0.00014692,0.00013113,0.00002730,0.00000103,-0.00003$ $653,-0.00001186,0.00001617,-0.00001762,-0.00003600,-0.00000384,-0.0000$ $0759,-0.00001646,-0.00003317,-0.00001400,0.00001446,-0.00001847,0.0000$ $0238,-0.00000615,-0.00000256,0.00000340,-0.00423449,-0.00235612,0.0026$ $2348,-0.27904903,-0.11729692,0.00146692,-0.01273146,-0.00942424,-0.002$ $96837,0.00082337,-0.00064209,0.00449300,0.00024772,0.00068920,0.000035$ $23,0.00263975,-0.00258884,-0.00068862,0.00007079,0.00148334,-0.0009714$ $9,0.29323349,0.00024803,0.00008764,-0.00014996,-0.00000849,0.00005807$, $0.00005279,-0.00000179,0.00001364,-0.00000311,0.00000454,-0.00001342,0$ $.00000637,0.00001254,0.00000687,0.00002137,-0.00004631,-0.00001006,-0$. $00009111,0.00000637,-0.00000931,0.00000864,0.00001389,-0.00000147,-0.0$ $0000118,0.00000309,-0.00000664,-0.00002076,-0.00000656,0.00000510,0.00$ $001748,-0.00000252,-0.00000810,-0.00000125,-0.00000006,-0.00000322,-0$. $00000120,-0.01560557,-0.00321704,0.00166197,-0.11732382,-0.09768670,-0$ $.00829464,0.00383810,0.00467907,-0.00110225,-0.00098267,0.00517619,-0$. $00263683,0.00070119,-0.00081624,-0.00004882,-0.00222014,0.00323355,-0$. $00544308,0.00168557,-0.00233846,0.00166111,0.12881822,0.09409100,0.000$ $81530,0.00047538,0.00018983,0.00003415,0.00000996,-0.00021824,0.000027$ $68,-0.00003587,0.00004532,0.00002534,0.00001218,-0.00001729,-0.0000259$ $5,0.00005421,-0.00001343,0.00014125,-0.00012527,-0.00005194,-0.0000277$ $0,0.00001242,0.00003205,0.00001083,-0.00001572,0.00000828,0.00002551,0$ $.00001221,0.00002166,0.00002383,0.00001883,-0.00000580,-0.00000071,0.0$ $0002727,-0.00001279,0.00000276,-0.00000069,-0.00000613,-0.02228079,-0$. 
$01044884,0.00421318,0.00141619,-0.00832014,-0.05316460,0.02196987,0.01$ $102501,0.00404062,0.00384808,-0.00295143,-0.00200980,0.00006368,-0.000$ $03570,-0.00125920,0.00000019,-0.00510732,-0.00239530,-0.00058966,0.001$ $84638,-0.00003055,-0.00305408,0.01216509,0.05064825,0.00021502,0.00057$ $332,-0.00283666,-0.00015756,-0.00000957,0.00035542,-0.00002845,0.00000$ $191,0.00001134,0.00004159,0.00003338,0.00000533,-0.00008360,0.00014063$ ,-0.00008814,0.00038928,0.00012456,0.00016300,-0.00002248,0.00044292,0 $.00107740,-0.00005386,-0.00001090,-0.00002708,-0.00000138,-0.00003378$, $-0.00004714,0.00004140,0.00001424,-0.00002810,-0.00001343,0.00001478,-$ $0.00005034,0.00002140,0.00000486,-0.00000490,-0.00090922,-0.00161486,0$ $.00438362,0.00887788,0.01124469,0.01566311,-0.07673550,-0.05102627,-0$. $05814330,-0.00391898,-0.01508614,-0.02170198,-0.00399326,-0.00428615,0$ $.00176839,-0.00103222,0.00035190,0.00048277,0.00031658,0.00050670,0.00$ $086020,0.00012760,0.00139825,-0.00109431,0.07719035,0.00235594,-0.0025$ $4884,0.00073455,0.00041101,0.00051752,-0.00018897,0.00015636,-0.000208$ $13,0.00008585,-0.00000437,0.00001677,-0.00000646,0.00005120,-0.0001340$ $1,0.00011059,-0.00056692,0.00009673,0.00029832,-0.00018594,0.00048542$, $0.00063550,0.00005584,-0.00006632,-0.00001151,0.00005948,0.00002772,-0$ $.00000263,-0.00002271,-0.00003962,-0.00006027,-0.00003831,-0.00009443$, $0.00002237,0.00001186,-0.00000128,0.00000487,-0.00115189,0.00549843,-0$ $.00219810,-0.00216156,0.00104003,-0.00149571,-0.05123977,-0.12752800,-$ $0.12636389,-0.00258206,-0.00422591,-0.01191256,-0.00473220,0.00380212$, $-0.00122194,0.00023753,-0.00064958,0.00088315,-0.00018176,0.00006317$, $0.00012384,0.00159546,-0.00232895,0.00157432,0.05770256,0.12717259,0.0$ $0049949,0.00235947,-0.00010976,-0.00031335,-0.00002286,0.00022812,-0.0$ $0000479,0.00011956,-0.00003350,-0.00003640,-0.00002311,-0.00000441,0.0$ $0001077,0.00011717,-0.00006267,0.00037895,-0.00009203,0.00012500,-0.00$ $011119,0.00005703,0.00044911,-0.00005979,0.00000885,-0.00003539,-0.000$ $00851,-0.00002165,-0.00003908,0.00004085,0.00001092,-0.00000796,-0.000$ $00536,0.00000967,-0.00004645,-0.00000224,0.00000366,0.00000777,0.00527$ $034,-0.00176509,-0.00091792,-0.00954357,-0.01375134,-0.01424766,-0.058$ $55713,-0.12656596,-0.21983706,0.00181043,-0.00048141,0.00081217,0.0009$ $2475,-0.00162804,0.00284041,0.00026342,0.00077479,-0.00001575,-0.00043$ $334,-0.00075281,-0.00080214,-0.00072046,0.00175474,0.00001830,0.060664$ $00,0.13958876,0.23186859,-0.00065086,0.00134843,0.00027182,0.00011135$, $0.00008240,0.00035118,0.00000224,-0.00000447,-0.00002613,0.00006499,-0$ $.00011814,-0.00002557,0.00005656,0.00023057,0.00018101,0.00013415,0.00$ $020010,0.00004978,-0.00004309,-0.00000682,-0.00010679,-0.00003550,0.00$ $001463,-0.00001649,-0.00002304,0.00003244,0.00004482,0.00001688,0.0000$ $3279,0.00000264,-0.00006228,-0.00003512,-0.00011487,-0.00001112,0.0000$ $0447,-0.00000838,0.00084961,-0.00071786,0.00463025,0.00031023,0.000694$ $54,-0.00006486,0.00226188,-0.00253351,-0.00054083,-0.00484027,-0.00323$ $844,0.00130582,-0.27855822,-0.11712002,0.00135570,-0.01221063,-0.00905$ $299,-0.00278542,-0.00069989,-0.00048826,-0.00014656,-0.00022103,0.0003$ $0150,-0.00019524,-0.00020391,-0.00011386,0.00014167,0.29367210,0.00072$ $760,-0.00188288,0.00168515,0.00014614,-0.00003509,-0.00013162,0.000273$ $63,0.00010168,0.00031147,-0.00000827,0.00018759,-0.00013526,0.00008385$ ,-0.00012596,-0.00025686,-0.00018291,0.00018115,0.00024347,0.00001474, $-0.00005198,0.00015458,-0.00000744,-0.00012077,-0.00009619,0.00006631$, $-0.00000183,-0.00000989,-0.00001475,-0.00002025,0.00008905,0.00006400$, $-0.00004065,0.00011901,-0.00001249,-0.00002068,0.00001130,-0.00110972$, 
$0.00530084,-0.00289793,0.00074344,-0.00082715,0.00000978,-0.00215904,0$ $.00298572,-0.00535384,-0.01592332,-0.00382309,0.00125182,-0.11694970,-$ $0.09766815,-0.00859396,0.00422173,0.00488487,-0.00099952,0.00020180,0$. $00018416,-0.00010853,0.00030109,-0.00068637,0.00041165,-0.00082019,-0$. $00021879,-0.00000990,0.12883576,0.09415257,-0.00090492,0.00111962,-0.0$ $0021101,-0.00015152,-0.00009560,0.00010772,0.00019986,-0.00007040,-0.0$ $0023190,0.00017402,-0.00004442,0.00003806,0.00020559,0.00025713,-0.000$ $04172,-0.00004310,-0.00009817,-0.00015423,-0.00006234,0.00017622,0.000$ $24492,-0.00000389,0.00012897,0.00000529,-0.00002102,0.00005480,0.00002$ $068,0.00004353,0.00001132,-0.00000347,0.00001522,-0.00003355,-0.000045$ $54,-0.00003566,-0.00000006,0.00000646,0.00389403,-0.00325836,-0.002297$ $04,0.00002085,0.00005155,-0.00111627,0.00015921,-0.00501316,-0.0026933$ $0,-0.02253753,-0.01034270,0.00441346,0.00167249,-0.00843512,-0.0536198$ $3,0.02217584,0.01113584,0.00402406,0.00115052,0.00052160,0.00007349,-0$ $.00019249,0.00041215,-0.00009869,-0.00118452,-0.00065272,0.00003992,-0$ $.00353477,0.01248528,0.05156839,0.00033708,0.00043743,0.00064653,0.000$ $08059,-0.00006999,-0.00002230,-0.00003645,0.00003044,0.00001258,-0.000$ $00045,-0.00002759,-0.00002875,-0.00004886,-0.00002904,0.00000018,0.000$ $11663,0.00011619,-0.00012859,-0.00013128,0.00012537,0.00022565,-0.0000$ $1128,0.00002315,0.00000515,-0.00001367,0.00000478,0.00001237,-0.000009$ $00,-0.00000110,0.00001182,-0.00000600,-0.00000978,0.00000901,-0.000002$ $36,0.00000050,0.00000627,-0.00287139,-0.01512229,-0.02260975,-0.003797$ $71,-0.00425617,0.00182627,-0.00106729,0.00022916,0.00050530,-0.0006170$ $9,-0.00159277,0.00437621,0.00866080,0.01070189,0.01561094,-0.07682760$, $-0.05105704,-0.05943267,0.00018754,0.00166968,-0.00058050,-0.00030909$, $-0.00083336,-0.00113624,-0.00006724,0.00034412,-0.00020854,0.00007985$, $0.00150774,-0.00103939,0.07635428,-0.00026971,0.00001058,0.00000341,0$. $00011421,0.00004602,-0.00005908,-0.00001710,-0.00006800,0.00000960,0.0$ $0000003,0.00001421,-0.00002448,0.00002844,0.00002481,0.00005063,-0.000$ $08175,-0.00009547,0.00003341,0.00008870,-0.00010641,-0.00001411,-0.000$ $00581,-0.00002642,-0.00000064,0.00001261,-0.00000099,0.00000053,0.0000$ $0541,0.00000873,0.00000335,0.00000493,0.00000048,0.00001334,0.00000485$ $, 0.00000379,-0.00000377,-0.00191293,-0.00341145,-0.01124414,-0.0045772$ $3,0.00404959,-0.00127115,0.00023144,-0.00049599,0.00071162,-0.00127953$ $, 0.00563776,-0.00223000,-0.00237196,0.00162198,-0.00178485,-0.05110178$ $,-0.12988879,-0.12823863,0.00147164,-0.00223835,0.00174257,-0.00013815$ $,-0.00022517,-0.00060393,0.00033314,-0.00067664,0.00030958,0.00165185$, $-0.00245493,0.00168979,0.05780869,0.12827067,-0.00068737,-0.00064621,-$ $0.00044295,-0.00021033,0.00008887,-0.00003457,0.00005505,0.00000496,0$. $00004968,0.00002358,0.00000489,0.00000527,-0.00002493,0.00005873,-0.00$ $002563,0.00004056,-0.00011831,0.00006803,0.00015730,-0.00004810,-0.000$ $37479,0.00000628,-0.00001639,0.00000531,0.00002820,-0.00000104,-0.0000$ $0169,0.00002256,0.00001834,-0.00001649,-0.00002102,0.00001379,-0.00002$ $589,0.00001474,-0.00000116,-0.00000867,0.00222850,0.00083589,0.0030284$ $4,0.00122069,-0.00156526,0.00289180,0.00050636,0.00071051,-0.00011147$, $0.00496834,-0.00194248,-0.00074757,-0.00897293,-0.01373858,-0.01390266$ ,-0.05951634,-0.12828058,-0.22361213,-0.00095307,0.00156147,-0.0000738 $3,0.00017229,0.00003248,0.00006625,-0.00022810,0.00030031,-0.00022963$, $-0.00076879,0.00182094,-0.00002944,0.06193845,0.14090694,0.23352263$ I$0.00000537,0.00000008,0.00000348,0.00000109,-0.00000683,0.00000157,-0$. $00000413,0.00000096,0.00000017,0.00000270,0.00000231,0.00000100,-0.000$ 
$00572,0.00000020,0.00000045,0.00000781,0.00000313,-0.00000623,0.000000$ $44,0.00000053,-0.00000145,0.00000049,0.00000059,0.00000046,0.00000024$, $-0.00000057,-0.00000080,-0.00000096,0 ., 0.00000009,-0.00000122,-0.00000$ $102,0.00000096,-0.00000021,-0.00000161,-0.00000024,0.00000544,0.000006$ $19,0.00000446,0.00000191,-0.00000377,-0.00000687,-0.00001049,-0.000001$ $39,0.00000541,0.00001040,0.00000451,-0.00000011,0.00000145,-0.00000201$ $,-0.00000772,-0.00000628,-0.00000276,0.00000632,0.00000014,-0.00000009$ $,-0.00000039,0.00000027,0.00000068,0.00000115,0.00000118,0.00000024,-0$ $.00000091,-0.00000029,0.00000028,0.00000043,0.00000112,0.00000037,-0.0$ 0000121II@@

Chlorophenyl radical addition to benzene

Pre-reaction Complex

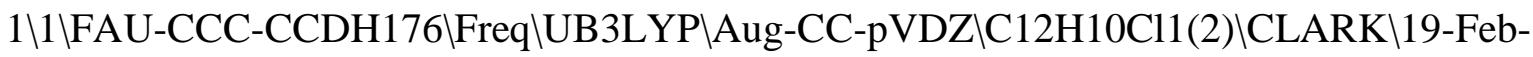
201610॥\#N Geom=AllCheck Guess=TCheck SCRF=Check GenChk UB3LYP/Aug-CCpVDZ Freq $\backslash p$-CHLOROPHENYL RADICAL pre-addition complex with benzene, C 2V\\0,2\C,-3.5809768581,1.7295093951,0.211754923\C,-3.5390665445,0.882 $7625868,1.3253050931 \backslash \mathrm{C},-3.4689170972,-0.5037063558,1.147769028 \backslash \mathrm{C},-3.44$ $08128794,-1.0430627844,-0.1433952121 \backslash \mathrm{C},-3.4817022078,-0.196234304,-1.2$ $569035707 \backslash \mathrm{C},-3.5517686894,1.1901041914,-1.0792693638 \mathrm{H},-3.6350260335,2$ $.8098263695,0.3501505744 \backslash \mathrm{H},-3.5612942792,1.3032816011,2.3312079323 \backslash \mathrm{H},-$ $3.4373112341,-1.1634670892,2.0155133616 \backslash \mathrm{H},-3.4596511922,-0.6165863415$, $-2.2629338752 \downarrow \mathrm{H},-3.5833285498,1.8500460571,-1.9468181175 \backslash \mathrm{H},-3.38886367$ $21,-2.1235968351,-0.2820329288 \backslash \mathrm{C}, 2.4855192419,-0.5886866209,0.01466623$ $21 \backslash \mathrm{C}, 3.2477516144,0.5822158571,0.0206974421 \backslash \mathrm{C}, 2.5890364598,1.823937454$ $7,0.0628565981 \backslash \mathrm{C}, 1.2097922389,1.8018700021,0.0963529368 \backslash \mathrm{C}, 0.4221276973$ $, 0.6692692534,0.0907143239 \backslash \mathrm{C}, 1.0889083943,-0.5681166458,0.0484636098 \backslash \mathrm{C}$ $1,3.3153272811,-2.1465554887,-0.0380876346 \backslash \mathrm{H}, 4.3354670453,0.530502493$, $-0.0067614655 \backslash \mathrm{H}, 3.1615467018,2.7518591756,0.068424882 \backslash \mathrm{H},-0.6669420173$, $0.7078420234,0.1164075822 \backslash \mathrm{H}, 0.5253235698,-1.500015831,0.0418380551 \backslash \mathrm{Ve}$ rsion=ES64L-G09RevD.01 \State $=2-A \backslash H F=-923.4823269 \backslash \mathrm{S} 2=0.757664 \backslash \mathrm{S} 2-1=0 . \mathrm{IS}$ $2 \mathrm{~A}=0.750033 \backslash \mathrm{RMSD}=9.349 \mathrm{e}-09 \backslash \mathrm{RMSF}=1.184 \mathrm{e}-05 \backslash$ ZeroPoint $=0.1779075 \backslash$ Thermal $=$ $0.1903305 \backslash Z P E=111.6386263 \backslash$ Dipole $=-0.3797536,0.3525382,0.0069712 \backslash$ Dipole Deriv $=-0.1545652,-0.0009943,-0.0000848,-0.042437,0.0974668,0.0197755,-$ $0.0039603,0.0197064,-0.0541902,-0.156761,-0.0005258,0.0032938,-0.01941$ $2,-0.0346486,0.0571879,-0.0331431,0.0566855,0.0751763,-0.1558405,-0.01$ $04221,0.0077559,0.0133188,-0.0008957,-0.0760285,-0.0234198,-0.0717359$, $0.0395271,-0.1574725,-0.0151262,-0.0010747,0.0187055,0.0896377,0.01919$ $8,0.0040513,0.0179816,-0.0535276,-0.1519943,-0.0077253,-0.0091281,0.00$ $58395,-0.0345935,0.0577578,0.027536,0.0540669,0.0747994,-0.1524888,0.0$ $006242,-0.0023022,-0.0249878,0.0004407,-0.0777895,0.028325,-0.0765987$, $0.0407676,0.1431649,0.0012183,0.0001228,0.0127228,-0.0867189,-0.019597$ $3,0.0010585,-0.0192177,0.0609618,0.1424612,0.0004053,-0.0022451,0.0060$ $472,0.0401887,-0.0558429,0.0042585,-0.0537877,-0.0691361,0.1423083,0.0$ $105488,-0.0081758,0.0057122,0.0060048,0.0754866,-0.0030628,0.0733239,-$ $0.0354317,0.1412974,0.0070757,0.0080986,0.0041629,0.0396352,-0.0559765$ $, 0.001436,-0.0543266,-0.0692383,0.1414756,0.0000747,0.000955,0.0081347$ $, 0.006952,0.0755039,-0.004817,0.0730856,-0.0350888,0.1432099,0.0197357$ 
$, 0.001726,0.01219,-0.088682,-0.0196549,0.0013153,-0.0190415,0.0603677$, $0.2495069,-0.4869405,-0.0164732,-0.5246666,0.8736917,0.03002,-0.018216$ $8,0.0312269,-0.0419671,-0.0121277,0.055966,-0.0020605,0.0531488,-0.150$ $5507,-0.0017516,-0.0008434,-0.0024867,-0.1018245,-0.0018737,0.0212543$, $-0.0021708,0.0811341,0.0238112,-0.0000384,0.0000021,0.0021177,-0.07985$ $03,-0.0456205,0.0707756,-0.0005001,0.0780942,-0.1188926,-0.0020523,-0$. $0004232,-0.002984,-0.0972004,0.0574563,0.0522498,0.0021893,-0.0375571$, $-0.0305158,0.0008213,-0.0023152,-0.0006035,-0.0504179,-0.0785399,0.079$ $255,-0.0001469,0.1117484,-0.1180359,-0.0025323,0.0012532,-0.0023888,-0$ $.0863894,-0.2648655,0.2818588,0.0096863,0.3129814,-0.6171955,-0.017848$ $4,0.0113083,-0.0182955,-0.0685439,-0.031669,0.005453,0.0038673,0.03104$ $4,0.0765051,-0.0019022,0.004511,-0.0012896,0.1288855,0.0404219,-0.0544$ $212,0.0011546,-0.0559975,-0.011403,-0.0020037,0.0015329,-0.0019932,0.1$ $450931,0.08438,0.0092563,0.0043692,0.0134909,0.0406398,-0.001152,0.002$ $9842,-0.0010316,0.1019728,0.0781362,-0.0395958,0.0011435,-0.0634174,-0$ $.0028416,-0.0015804,0.000629,-0.0024134,0.115255 \backslash$ Polar $=153.3708847,-9$. $6064988,176.7763938,-1.0820236,1.207502,128.1523734 \backslash \mathrm{PG}=\mathrm{C} 01[\mathrm{X}(\mathrm{C} 12 \mathrm{H} 10 \mathrm{Cl}$ 1) $\mathrm{MNImag}=0 \backslash 10.13600603,-0.03021139,0.73381094,-0.00141665,0.00996067$, $0.65918001,-0.06325731,0.00684174,-0.00321295,0.13685700,0.00726374,-0$ $.20203191,0.06987739,-0.02717211,0.66834613,-0.00641542,0.13320524,-0$. $25841634,-0.00210516,0.02808244,0.72499979,0.00729960,0.00251493,-0.00$ $351938,-0.06431392,0.01327206,0.00319347,0.13648447,0.00199924,-0.0384$ $1608,0.06529023,0.01372198,-0.33191592,-0.05816129,-0.02732449,0.68485$ $560,-0.00115441,0.01962018,0.04516245,-0.00020728,0.00499977,-0.128235$ $99,0.00106507,-0.03791569,0.70766304,-0.00670741,-0.00029265,-0.000274$ 63,0.00737790,0.00114733,0.00156758,-0.06298103,0.00460221,0.00549722, $0.13568991,-0.00020652,-0.00249369,0.00838515,0.00106845,-0.01253103,-$ $0.03458971,0.00478506,-0.15566515,-0.10665228,-0.02907032,0.73306853,-$ $0.00035795,0.00841671,-0.06695258,0.00392293,-0.08023377,0.01914713,0$. $00247153,-0.04352875,-0.30444379,-0.00082350,0.00985933,0.65895027,0.0$ $0725390,0.00117190,0.00416816,-0.00622611,0.00257247,-0.00112712,0.007$ $01719,-0.00254961,-0.00188645,-0.06366573,0.00667988,-0.00343127,0.136$ $76922,0.00096969,-0.01242481,-0.08027651,0.00259131,-0.05813278,0.0237$ $7761,-0.00267731,0.06081767,0.03780063,0.00651882,-0.20187302,0.070120$ $51,-0.02648368,0.66833195,0.00182546,-0.03462553,0.01920988,-0.0012526$ $1,0.02383230,-0.01148187,0.00053289,-0.00776655,-0.05434662,-0.0066389$ $7,0.13318135,-0.25844666,-0.00163088,0.02806065,0.72487362,-0.06283991$ $, 0.00458958,0.00257609,0.00679375,-0.00276324,0.00031611,-0.00631378,0$ $.00190321,0.00177546,0.00745315,0.00222148,-0.00323780,-0.06403000,0.0$ $1363219,0.00289087,0.13617487,0.00473813,-0.15601938,-0.04343238,-0.00$ $268376,0.06086558,-0.00780262,0.00190201,-0.04350229,-0.03219031,0.002$ $37657,-0.03843851,0.06525273,0.01343197,-0.33213191,-0.05828916,-0.027$ $61526,0.68546107,0.00593297,-0.10680280,-0.30454287,-0.00185755,0.0378$ $1260,-0.05443940,0.00159600,-0.03219205,-0.02604561,-0.00088162,0.0195$ $8646,0.04524487,-0.00041751,0.00497104,-0.12821847,0.00114077,-0.03787$ $724,0.70794507,-0.03717299,0.01470053,0.00182512,0.00307671,0.00086593$ $, 0.00023146,0.00721324,0.00034135,-0.00018722,-0.00090425,-0.00006950$, $-0.00000926,0.00725229,0.00028334,0.00014260,0.00307305,0.00057980,-0$. $00005214,0.02606757,0.01512014,-0.33263670,-0.03526963,0.00078332,-0.0$ $1386706,-0.00563598,0.00040798,0.00011190,0.00385469,-0.00009723,0.000$ $59642,0.00021687,0.00034336,0.00157142,-0.00250120,0.00044852,-0.00780$ 
$569,0.00177991,-0.01638651,0.35187458,0.00185123,-0.03525597,-0.061978$ $99,-0.00133155,0.02515562,0.00711150,-0.00011003,0.00310952,-0.0036824$ $6,-0.00002942,0.00022012,-0.00109034,0.00010436,-0.00175198,-0.0051410$ $7,0.00150138,-0.02902217,0.00102260,-0.00197004,0.03779495,0.06172343$, $0.00317837,-0.00038164,-0.00091578,-0.03676313,0.00326089,0.00520535,0$ $.00324734,0.00052537,0.00150841,0.00717833,0.00065178,-0.00001599,-0.0$ $0104905,-0.00001505,-0.00001742,0.00740025,0.00044652,-0.00019161,-0.0$ $0388157,-0.00023949,0.00003924,0.02540477,-0.00046251,0.01058696,0.018$ $37104,0.00340754,-0.09896678,-0.09986199,0.00047848,-0.00653500,-0.029$ $28415,0.00068717,-0.00575013,0.00026027,-0.00004316,-0.00080221,0.0005$ $8423,0.00051254,-0.00159061,0.00362099,-0.00022703,0.00080655,-0.00036$ $035,-0.00401603,0.10140121,0.00044447,-0.01239449,-0.01744415,0.005566$ $24,-0.09979289,-0.29607448,-0.00021521,0.00141887,-0.00023123,0.000131$ $94,-0.00045208,0.00217076,-0.00014968,0.00060262,0.00038997,-0.0000196$ 2,0.00431236,-0.00195994,0.00000530,0.00007187,0.00093851,-0.00566692, $0.10696546,0.31266684,0.00718482,0.00062910,0.00013033,0.00326877,0.00$ $082587,-0.00135539,-0.03682788,0.00591976,-0.00644372,0.00313753,-0.00$ $037507,0.00061237,0.00737384,0.00035956,0.00011492,-0.00100941,-0.0000$ $5049,0.00003978,0.00016006,0.00004772,-0.00006021,-0.00390459,-0.00024$ $291,0.00002064,0.02551150,0.00057767,-0.00530292,-0.00221232,0.0008330$ $1,-0.01295494,0.02602525,0.00575762,-0.15980501,0.13503156,-0.00018982$ $, 0.01023746,-0.01112475,0.00024746,0.00031848,-0.00303403,0.00001676,-$ $0.00045436,-0.00079276,0.00005654,-0.00081149,0.00127774,-0.00022916,0$ $.00100527,-0.00018599,-0.00684421,0.16665756,0.00016166,-0.00147791,0$. $00172307,0.00013710,-0.00471446,0.00616406,-0.00620998,0.13506629,-0.2$ $3490577,-0.00108768,0.01958013,-0.01707497,0.00032024,-0.00370917,-0.0$ $0386792,-0.00003361,-0.00081745,0.00001841,0.00001651,-0.00028896,0.00$ $015288,-0.00002450,0.00024922,0.00073061,0.00685486,-0.14475027,0.2470$ $9473,0.00722780,0.00065699,-0.00001924,-0.00105164,-0.00003159,-0.0000$ $4501,0.00735504,0.00042764,-0.00011637,0.00324545,-0.00033496,-0.00095$ $511,-0.03686518,0.00316887,0.00525695,0.00325776,0.00048861,0.00149368$ $, 0.00014683,0.00003087,0.00007104,-0.00113437,-0.00005726,0.00000845,0$ $.00010996,0.00000360,-0.00005890,0.02546030,0.00062865,-0.00576762,0.0$ $0025424,-0.00000137,-0.00080477,0.00057749,0.00038802,-0.00159465,0.00$ $363149,-0.00030003,0.01054320,0.01831624,0.00305678,-0.09885143,-0.099$ $73949,0.00058745,-0.00652511,-0.02929617,0.00004272,-0.00050761,-0.001$ $45032,-0.00005499,0.00002787,-0.00005041,-0.00000623,0.00032804,0.0009$ $4453,-0.00392129,0.10132486,-0.00006270,-0.00047279,0.00217880,0.00005$ $820,0.00059790,0.00039237,-0.00036832,0.00429079,-0.00196459,0.0008484$ $1,-0.01235715,-0.01741320,0.00491547,-0.09977345,-0.29607114,0.0000851$ $0,0.00142965,-0.00026337,-0.00000428,0.00012016,-0.00014724,0.00000087$ ,-0.00004760,-0.00007358,0.00004849,-0.00060395,-0.00098771,-0.0055858 $4,0.10689637,0.31264816,0.00313820,-0.00040774,0.00056466,0.00739156,0$ $.00035480,0.00020176,-0.00100494,-0.00001625,0.00001989,0.00719293,0.0$ $0062571,0.00013446,0.00323003,0.00086919,-0.00133499,-0.03680855,0.005$ $87410,-0.00635099,-0.00387909,-0.00022323,-0.00004918,0.00010381,-0.00$ $001534,0.00000167,-0.00114904,-0.00005806,-0.00000463,-0.00389777,-0.0$ $0022054,-0.00000360,0.02544728,-0.00043080,0.01025072,-0.01116104,0.00$ $043522,0.00032892,-0.00303073,-0.00005776,-0.00045318,-0.00079069,0.00$ $066859,-0.00528771,-0.00221626,0.00067513,-0.01293172,0.02598973,0.006$ $13784,-0.15997600,0.13520167,-0.00021796,0.00074962,0.00030225,-0.0000$ 
$1844,0.00032128,-0.00060821,-0.00005599,-0.00000169,0.00006563,-0.0002$ $5464,0.00099683,-0.00017537,-0.00684730,0.16681253,-0.00078791,0.01961$ $165,-0.01711289,0.00004920,-0.00372439,-0.00386865,0.00013801,-0.00080$ $856,0.00001835,-0.00002293,-0.00149908,0.00171409,0.00038363,-0.004714$ $52,0.00615318,-0.00669592,0.13514374,-0.23493999,-0.00000592,-0.000121$ $41,0.00099566,-0.00002552,0.00094707,-0.00099474,-0.00000929,0.0000658$ $2,-0.00003761,-0.00003705,0.00024202,0.00073685,0.00689138,-0.14484610$ $, 0.24714337,-0.00088908,-0.00008641,-0.00001938,0.00721797,0.00031307$, $0.00016481,0.00304180,0.00060214,-0.00011099,-0.03691156,0.01417570,0$. $00175027,0.00310820,0.00087233,0.00031462,0.00720693,0.00037121,-0.000$ $20842,-0.00117924,-0.00006385,0.00000113,0.00016668,0.00002996,-0.0000$ $0828,-0.00387143,-0.00026626,0.00000657,-0.00389776,-0.00026148,-0.000$ $03079,0.00015717,0.00004817,0.00001921,0.02585751,-0.00004718,0.000589$ $51,0.00022262,0.00020941,0.00157879,-0.00251938,0.00061586,-0.00783122$ $, 0.00177191,0.01401712,-0.33223860,-0.03528825,0.00088040,-0.01389638$, $-0.00564164,0.00028765,0.00010902,0.00387574,-0.00004453,-0.00008469,-$ $0.00001953,0.00003269,-0.00051696,0.00012127,-0.00019954,0.00074991,-0$ $.00013376,-0.00018539,0.00080736,0.00007173,0.00004686,-0.00082137,-0$. $00029604,-0.01569602,0.35152280,-0.00001267,0.00022494,-0.00109025,0.0$ $0011629,-0.00174883,-0.00514100,0.00142600,-0.02898489,0.00102822,0.00$ $168845,-0.03527442,-0.06186108,-0.00119970,0.02507080,0.00710763,-0.00$ $016214,0.00310554,-0.00368739,0.00000197,-0.00002057,0.00007549,0.0000$ $7314,-0.00145124,-0.00014169,0.00000203,0.00031363,0.00098483,-0.00000$ $304,-0.00034679,0.00093561,-0.00007001,0.00127926,0.00016052,-0.001871$ $46,0.03783636,0.06158752,0.00004185,0.00000509,-0.00001142,0.00002752$, $0.00005515,0.00003750,0.00005571,-0.00002310,0.00006058,0.00011304,-0$. $00010505,-0.00002620,0.00004874,-0.00000200,-0.00005134,0.00002188,0.0$ $0007189,-0.00000574,-0.00002060,-0.00002484,-0.00000389,-0.00001801,0$. $00000106,-0.00000733,-0.00004515,-0.00000819,0.00000187,-0.00004180,-0$ $.00000837,-0.00000664,-0.00001476,-0.00000610,0.00000914,-0.00003048,-$ $0.00001155,0.00000358,0.61587600,-0.00006824,-0.00006351,-0.00001262,-$ $0.00008969,-0.00008375,-0.00006759,-0.00012404,0.00006406,-0.00016868$, $-0.00013759,0.00013563,0.00001305,-0.00010402,0.00001251,0.00018007,-0$ $.00009510,-0.00005731,0.00005248,0.00006073,-0.00001191,-0.00000503,0$. $00006362,0.00001132,-0.00000633,0.00007991,0.00000155,-0.00000273,0.00$ $006723,0.00000551,-0.00000380,0.00006594,0.00000639,0.00000815,0.00008$ $305,0.00001346,0.00000977,0.06490655,0.52913588,0.00000772,-0.00000049$ $, 0.00004214,-0.00005111,0.00001190,0.00000740,-0.00000369,0.00002070,-$ $0.00002337,-0.00001963,0.00000112,0.00000849,-0.00001028,-0.00002036,0$ $.00000198,0.00004939,-0.00002713,0.00001092,0.00000005,-0.00000623,0.0$ $0001407,0.00002196,0.00001833,-0.00001571,0.00000519,-0.00000804,0.000$ $01271,0.00000104,0.00000739,0.00000168,-0.00002476,-0.00000074,-0.0000$ $2076,0.00001045,0.00000379,-0.00000713,-0.01049138,0.00684372,0.125553$ $74,-0.00008109,-0.00003536,-0.00000690,-0.00001747,-0.00002808,-0.0000$ $5974,-0.00003809,0.00003887,-0.00005201,-0.00006057,0.00004921,0.00000$ $661,-0.00004258,0.00001996,0.00006248,-0.00001362,-0.00004250,0.000047$ $66,0.00002916,0.00000264,-0.00000004,0.00001058,-0.00000404,0.00000316$ $, 0.00001529,-0.00000627,0.00000223,0.00001234,-0.00000178,-0.00000579$, $0.00001189,0.00000356,-0.00000473,0.00002578,0.00000468,0.00000115,-0$. $18631694,-0.13652800,0.00017308,0.73582206,0.00003320,0.00006296,0.000$ $01858,0.00003558,0.00000205,0.00003099,-0.00001059,-0.00003285,0.00001$ 
$579,0.00001684,-0.00000560,0.00000243,-0.00000951,-0.00001497,-0.00002$ $671,0.00003796,-0.00000097,-0.00003986,-0.00002316,0.00000913,0.000000$ $17,-0.00002057,-0.00000143,0.00000724,0.00000431,0.00000928,-0.0000106$ $9,0.00000183,0.00000244,0.00000943,-0.00002299,0.00000326,-0.00000801$, $0.00000294,0.00001218,0.00000103,-0.07342974,-0.24522327,-0.00198710,-$ $0.00138997,0.63101652,0.00000913,-0.00000456,0.00000540,-0.00000061,0$. $00000157,-0.00001167,0.00002018,-0.00000331,0.00001638,0.00000418,0.00$ $000083,-0.00000439,-0.00000475,-0.00000231,0.00000011,-0.00002516,0.00$ $001507,-0.00001433,-0.00000273,0.00000261,-0.00001400,-0.00000749,-0.0$ $0001518,0.00000424,-0.00000543,0.00000062,-0.00000926,0.00000855,-0.00$ $000073,0.00000255,0.00001982,0.00000859,0.00000343,-0.00000486,-0.0000$ $0239,0.00000417,0.00149688,-0.00049639,-0.06418948,-0.01491843,0.01067$ $400,0.12501438,0.00009275,-0.00000399,0.00001563,-0.00000726,-0.000030$ $16,-0.00000433,-0.00003422,-0.00000449,-0.00005367,-0.00005189,0.00003$ $358,0.00001550,-0.00001524,-0.00000898,0.00003661,0.00000693,-0.000014$ $90,-0.00001676,-0.00003733,0.00001684,0.00000085,-0.00001641,0.0000113$ $6,0.00001752,0.00001965,0.00001178,-0.00001171,0.00001102,-0.00000041$, $0.00001030,-0.00002079,0.00000996,-0.00001337,0.00001831,0.00002233,0$. $00000321,0.06089519,0.02701988,-0.00073206,-0.15464902,0.04387791,0.00$ $324150,0.66152579,0.00002010,-0.00003327,-0.00000441,-0.00000867,-0.00$ $001445,-0.00001788,-0.00000534,-0.00000165,0.00000756,-0.00003183,0.00$ $002040,0.00000764,0.00000040,-0.00001437,-0.00000359,0.00000705,-0.000$ $00806,0.00002134,-0.00004761,0.00002741,0.00000568,-0.00001149,-0.0000$ $0835,0.00000709,-0.00001050,0.00000755,-0.00000907,-0.00000906,0.00000$ $314,0.00000346,-0.00001542,-0.00000260,-0.00001258,0.00000542,0.000033$ $54,0.00000058,-0.02966456,-0.05748710,-0.00061222,0.12036985,-0.271190$ $13,-0.00738772,0.07063240,0.66580825,-0.00003439,0.00000681,0.00001190$ $, 0.00000593,-0.00002379,0.00000014,-0.00004813,0.00000809,-0.00001114$, $-0.00001950,-0.00000832,0.00002441,0.00006567,0.00000112,-0.00001141,0$ $.00003873,0.00001406,0.00000114,0.00001028,-0.00000303,0.00001288,-0.0$ $0000069,0.00001564,-0.00000344,0.00001586,0.00000426,0.00000072,-0.000$ $02939,-0.00000364,-0.00001371,-0.00001899,-0.00001644,0.00000064,0.000$ $00544,0.00000110,-0.00000390,-0.00193660,-0.00201087,0.00631336,0.0048$ $6079,-0.00551506,-0.05919067,-0.01173168,0.00974518,0.12180374,0.00002$ $724,-0.00001385,0.00000437,0.00000733,-0.00006572,-0.00006952,-0.00003$ $494,0.00003048,-0.00004821,-0.00000280,0.00008579,0.00001557,-0.000012$ $92,-0.00000677,0.00006127,0.00000678,-0.00005014,0.00004038,-0.0000309$ $1,0.00000657,0.00000589,-0.00000875,0.00000680,0.00001718,-0.00000707$, $-0.00000038,-0.00000218,-0.00000841,0.00000264,-0.00000174,-0.00000593$ $, 0.00001038,-0.00001494,-0.00000074,0.00000464,0.00000153,-0.05974176$, $-0.03017084,0.00061666,-0.03546980,0.08257133,0.00294352,-0.34864364,-$ $0.02457344,0.00686513,0.59474634,0.00008485,0.00014625,0.00001120,0.00$ $008229,0.00004459,0.00004546,0.00002317,-0.00005634,0.00002301,0.00004$ $677,-0.00012213,-0.00001999,0.00003782,-0.00002767,-0.00003688,0.00005$ $228,0.00007720,-0.00003553,0.00001386,-0.00004072,-0.00001251,-0.00001$ $693,0.00001945,-0.00001425,0.00000050,-0.00001821,-0.00001035,-0.00001$ $322,-0.00001968,0.00000722,-0.00001020,0.00001062,0.00002111,0.0000089$ $3,0.00000060,0.00000762,-0.02985780,-0.01930461,0.00040390,0.02760493$, $0.03113428,-0.00025030,0.00129108,-0.09778310,-0.00098800,0.13475964,0$ $.41392920,0.00005699,-0.00000918,-0.00001688,0.00006801,0.00003930,-0$. $00001252,0.00007180,-0.00002468,0.00005450,0.00003915,0.00001373,-0.00$ 
$001681,-0.00009888,0.00000627,0.00004882,-0.00014731,-0.00002009,-0.00$ $002557,-0.00001898,0.00000838,-0.00003218,-0.00004585,-0.00002728,0.00$ $000423,-0.00002391,-0.00000946,-0.00001082,0.00004250,0.00001040,0.000$ $00888,0.00007901,0.00002245,0.00000622,-0.00001137,-0.00000384,-0.0000$ $0244,0.00062445,0.00041344,-0.00577577,0.00173393,-0.00162989,0.012269$ $24,0.00739190,-0.00040050,-0.05005963,-0.00990591,0.00413801,0.0700807$ $5,0.00014077,0.00031247,0.00004976,-0.00003982,0.00003666,0.00026370,0$ $.00007443,-0.00010650,0.00013972,-0.00005622,-0.00018763,-0.00002513,0$ $.00005593,-0.00006246,-0.00014772,-0.00001366,0.00008276,-0.00023711,-$ $0.00002633,-0.00008253,-0.00001477,-0.00002244,-0.00003311,-0.00004234$ ,-0.00000897,0.00003579,-0.00004817,-0.00000454,0.00002299,0.00005301, $-0.00000668,-0.00004082,0.00003599,0.00002283,0.00005454,0.00000546,-0$ $.02151294,0.02610788,0.00124958,0.00296377,-0.00325495,-0.00017036,-0$. $03083978,-0.03422569,0.00010236,-0.18712503,-0.13373037,0.00039476,0.7$ $3269348,0.00026795,0.00006482,0.00005365,0.00003854,-0.00027550,0.0001$ $0737,-0.00012569,-0.00004370,-0.00022741,-0.00018182,0.00018137,0.0000$ $7490,-0.00002629,-0.00012070,0.00019796,0.00009683,-0.00018332,-0.0001$ $8561,-0.00004099,0.00005853,0.00000367,-0.00002263,0.00002595,0.000035$ $55,-0.00000135,0.00004217,-0.00001674,-0.00000382,0.00003642,0.0000183$ 6,-0.00003086,0.00004351,-0.00003996,0.00003026,0.00003019,0.00000569, $0.08263353,0.02547798,-0.00159806,-0.00644229,-0.06576853,-0.00119564$, $-0.06337985,0.03458125,0.00217781,-0.10717182,-0.25962638,-0.00194182$, $0.02505615,0.59635636,0.00003397,0.00006043,-0.00036346,0.00019220,0.0$ $0012312,0.00014573,0.00020617,-0.00016602,-0.00009385,-0.00007605,0.00$ $004490,-0.00021347,-0.00016951,0.00015424,0.00004087,-0.00015703,-0.00$ $019800,0.00009599,-0.00000744,0.00000111,0.00004034,0.00001231,0.00005$ $089,0.00006800,-0.00001525,-0.00003017,0.00005559,0.00001046,0.0000141$ $0,0.00006077,-0.00003222,-0.00004904,0.00004623,0.00001022,-0.00000075$ $, 0.00001884,0.00244476,-0.00016588,0.00603714,-0.00018190,-0.00128785$, $-0.00082457,-0.00051934,0.00147905,0.00323629,0.00090209,-0.00133061$, $0.05024608,-0.01357389,0.00949003,0.12491247,-0.00016918,-0.00015630,-$ $0.00002223,-0.00005057,0.00003908,-0.00016390,-0.00001392,0.00009101,-$ $0.00003443,0.00009793,0.00006613,0.00000978,-0.00000312,0.00006864,0.0$ $0005271,-0.00006354,-0.00000093,0.00016168,0.00001602,0.00001633,0.000$ $00286,-0.00000561,0.00000502,0.00001153,0.00000766,-0.00001732,0.00002$ $346,0.00000350,-0.00000448,-0.00002124,-0.00000465,0.00000457,-0.00000$ $794,-0.00007179,-0.00001243,-0.00000752,-0.32406420,0.01909056,0.00662$ $229,-0.03121178,-0.06972801,-0.00055480,-0.04895938,0.03174709,0.00183$ $119,0.06138128,0.01732606,-0.00079077,-0.15983149,0.04669518,0.0033278$ $0,0.66279430,-0.00001262,-0.00021436,-0.00000579,-0.00008015,0.0000009$ $8,-0.00004559,0.00004301,0.00004451,0.00010125,0.00006786,0.00004964,0$ $.00001243,0.00001950,0.00006625,-0.00010264,-0.00005776,-0.00007438,0$. $00005175,0.00002442,0.00000351,0.00000571,0.00003765,-0.00001357,-0.00$ $001831,-0.00005787,0.00001934,0.00005588,-0.00002653,0.00002649,-0.000$ 02950,0.00003330,-0.00002444,0.00000900,-0.00013106,-0.00008983,-0.000 $03051,-0.04404898,-0.10745332,0.00019558,-0.02480122,0.03216532,0.0011$ $1587,0.02778341,-0.01408259,-0.00098355,-0.03709221,-0.06577222,-0.000$ $70161,0.12391684,-0.26547347,-0.00734723,0.04789625,0.70453893,-0.0000$ $2010,-0.00000759,0.00003253,0.00004565,0.00002908,-0.00003679,0.000080$ $35,-0.00001904,0.00000159,-0.00001457,-0.00000563,-0.00002283,-0.00007$ $844,0.00000761,-0.00000819,-0.00001060,-0.00002512,-0.00003808,0.00000$ 
$376,0.00000864,-0.00000549,-0.00003764,-0.00001395,0.00002721,-0.00005$ $709,-0.00001342,0.00002570,0.00004553,0.00001414,0.00003057,0.00003285$ ,-0.00000322,0.00003233,0.00001023,0.00001660,-0.00000214,0.00525577,-$0.00131347,-0.06405496,0.00038279,0.00221516,0.00715466,0.00175044,-0$. $00105225,-0.00040350,-0.00193732,-0.00210473,0.01160488,0.00497325,-0$. $00546500,-0.05896163,-0.01172732,0.01118958,0.12422228,-0.00006015,-0$. 00005996,-0.00000719,-0.00004913,-0.00002207,-0.00006241,-0.00003913,0 $.00003971,-0.00004990,-0.00004593,0.00005738,0.00001144,-0.00003698,0$. $00002058,0.00005716,-0.00004329,-0.00003434,0.00005320,0.00003450,-0.0$ $0000592,-0.00000109,0.00002509,0.00000225,-0.00000404,0.00002060,-0.00$ $000018,0.00000296,0.00001874,0.00000186,-0.00000422,0.00002533,0.00000$ $197,0.00000462,0.00002428,-0.00000411,-0.00000099,-0.06324512,0.046340$ $70,0.00193907,0.00506183,-0.00399090,-0.00010046,-0.00069686,-0.000724$ $49,0.00010635,0.00101367,0.00147696,-0.00001086,-0.00388050,-0.0042553$ $3,0.00009745,-0.01717728,0.03737944,0.00131393,0.07737863,0.00009088,0$ $.00008282,0.00000734,0.00007839,0.00003617,0.00008041,0.00005257,-0.00$ $005158,0.00007295,0.00004166,-0.00009467,-0.00001441,0.00005033,-0.000$ $02918,-0.00008076,0.00007626,0.00004938,-0.00006887,-0.00006102,0.0000$ $2186,0.00000443,-0.00004756,-0.00000563,0.00001352,-0.00002260,-0.0000$ $0357,-0.00001528,-0.00002488,-0.00000833,0.00001315,-0.00005019,0.0000$ $0031,-0.00001502,-0.00001002,0.00001481,0.00000295,0.04615966,-0.12576$ $805,-0.00324912,0.02243650,-0.03964161,-0.00147370,-0.00624318,-0.0004$ $8802,0.00005507,0.00138661,-0.00107437,-0.00004418,0.00139977,0.002781$ $14,-0.00005812,0.01106684,-0.01749398,-0.00073637,-0.07727845,0.181677$ $67,0.00000311,0.00000322,0.00000353,0.00001658,0.00000155,0.00001275,-$ $0.00000100,0.00000122,-0.00000281,0.00000407,-0.00000648,-0.00000291,0$ $.00000167,-0.00000054,-0.00000587,-0.00000690,0.00000103,0.00000427,-0$ $.00000211,0.00000103,-0.00000278,-0.00000564,0.00000028,-0.00000233,-0$ $.00000479,0.00000093,0.00000006,0.00000284,-0.00000329,0.00000250,0.00$ $000078,-0.00000012,-0.00000266,-0.00000080,0.00000098,-0.00000585,0.00$ $193783,-0.00325876,-0.02328181,0.00046018,-0.00082623,0.00453305,-0.00$ $001135,-0.00008230,0.00425264,-0.00001675,-0.00004173,-0.00086827,0.00$ $022282,0.00007436,0.00418630,0.00075324,-0.00136607,0.00465132,-0.0032$ $0872,0.00541588,0.01176534,-0.00000040,0.00000037,-0.00000025,-0.00000$ $699,-0.00000450,-0.00000754,-0.00000080,0.00000301,-0.00000796,-0.0000$ $0975,0.00000935,0.00000207,0.00000501,0.00000051,0.00000759,-0.0000082$ $4,-0.00000408,0.00000758,0.00000656,-0.00000218,-0.00000030,0.00000285$ $, 0.00000150,-0.00000160,0.00000420,-0.00000040,-0.00000076,0.00000202$, $-0.00000055,0.00000029,0.00000374,0.00000100,0.00000168,0.00000373,0.0$ $0000106,0.00000050,-0.01263437,0.00532896,0.00050034,-0.34158612,0.009$ $90596,0.00762597,-0.00873061,-0.00247636,0.00026485,-0.00014206,0.0030$ $4970,0.00022547,0.00096235,-0.00012934,-0.00004731,0.00053969,-0.00314$ $689,0.00003776,0.00120393,0.00044596,-0.00008086,0.36017959,0.00000765$ ,-0.00000072,-0.00000031,0.00000861,-0.00000199,0.00000678,0.00000095, $0.00000003,-0.00000722,-0.00001792,-0.00000203,0.00000016,0.00000142,-$ $0.00000433,0.00000684,0.00000905,0.00000153,-0.00000749,-0.00000900,0$. $00000574,0.00000098,-0.00000460,0.00000086,0.00000271,0.00000224,0.000$ 00136,-0.00000177,0.00000146,0.00000104,0.00000117,-0.00000538,0.00000 $097,-0.00000305,0.00000600,0.00000646,0.00000125,-0.02580194,0.0036409$ $6,0.00062327,0.01380107,-0.05434938,-0.00075225,0.02658354,0.00151451$, $-0.00071389,0.00386814,-0.00435822,-0.00031364,-0.00076272,-0.00054927$ 
$, 0.00001782,-0.00219682,-0.00412941,-0.00013013,-0.00070677,0.00095467$ $, 0.00009251,-0.01308310,0.05569195,0.00000074,0.00000104,-0.00000434,-$ $0.00000039,-0.00000326,0.00000111,-0.00000584,-0.00000067,0.00000227,0$ $.00000241,0.00000142,0.00000311,0.00000514,0.00000178,0.00000083,-0.00$ $000139,0.00000185,0.00000114,-0.00000033,0.00000004,0.00000080,0.00000$ $124,-0.00000112,-0.00000063,0.00000229,0.00000220,-0.00000171,-0.00000$ $302,-0.00000159,-0.00000249,-0.00000137,0.00000068,0.00000009,-0.00000$ $075,-0.00000138,0.00000170,-0.00014992,-0.00012214,0.00319356,0.007705$ $18,-0.00066551,-0.03483753,0.00088104,-0.00000941,0.00423473,0.0002341$ $1,-0.00030899,0.00642250,-0.00006671,0.00001715,-0.00088682,0.00005793$ ,-0.00010626,0.00476858,-0.00010519,0.00006455,-0.00257054,-0.00842098 $, 0.00101463,0.02369674,-0.00001100,-0.00002630,-0.00000289,-0.00001473$ ,-0.00000460,-0.00002078,-0.00000328,0.00001107,-0.00000894,-0.0000242 $2,0.00002236,0.00000480,0 ., 0.00000503,0.00001201,-0.00000705,-0.000012$ $75,0.00002019,-0.00000198,0.00000612,0.00000144,0.00000416,-0.00000496$ $, 0.00000015,0.00000176,0.00000113,-0.00000156,0.00000113,-0.00000027,0$ $.00000022,0.00000084,-0.00000207,-0.00000385,0.00000607,0.00000423,-0$. $00000014,0.00012593,0.00266532,0.00021495,0.01011898,0.01404078,0.0001$ $7963,-0.12970551,-0.12946206,-0.00039977,-0.01334015,-0.02438574,-0.00$ $018741,-0.00525998,-0.00013411,0.00024326,-0.00069027,0.00108101,0.000$ $03245,-0.00003253,0.00095178,0.00002054,0.00073155,-0.00038138,-0.0001$ $2207,0.13829675,-0.00000718,0.00001285,-0.00000083,-0.00001302,-0.0000$ $0118,-0.00001219,0.00002844,0.00000644,-0.00000016,-0.00002350,-0.0000$ $0446,0.00000091,0.00002308,0.00000290,0.00000435,-0.00000952,-0.000007$ $34,0.00000619,0.00001106,-0.00000253,-0.00000047,0.00000320,0.00000228$ $, 0.00000012,-0.00000256,-0.00000342,0.00000050,-0.00000258,-0.00000194$ ,-0.00000143,0.00000296,0.00000270,0.00000190,0.00000374,-0.00000176,0 $.00000012,0.00317043,-0.00182073,-0.00024640,-0.01596602,-0.01846420,-$ $0.00012232,-0.12479033,-0.25790910,-0.00169312,-0.00142333,0.00382939$, $0.00013980,0.00180347,0.00171568,-0.00012168,0.00055714,-0.00009679,-0$ $.00001451,-0.00009115,-0.00137322,-0.00002669,-0.00008380,0.00117667,0$ $.00011343,0.13666351,0.27282834,-0.00000398,0.00000067,0.00000173,-0.0$ $0000828,0.00000227,0.00000734,-0.00000575,0.00000575,-0.00000478,0.000$ $00819,-0.00000368,-0.00000237,-0.00000737,-0.00000349,-0.00000378,0.00$ $001923,-0.00000381,0.00000788,0.00000120,-0.00000155,0.00000417,0.0000$ $0802,-0.00000099,-0.00000670,0.00000208,0.00000319,-0.00000133,0.00000$ $075,-0.00000289,-0.00000131,-0.00001047,0.00000520,-0.00000702,-0.0000$ $0125,-0.00000008,0.00000051,0.00021582,-0.00024124,0.00656207,-0.00044$ $194,-0.00084374,0.00555632,-0.00030776,-0.00158438,-0.03410148,0.00029$ $363,0.00069637,-0.00020937,0.00029080,-0.00006841,0.00495227,0.0000189$ $9,-0.00002715,-0.00041678,-0.00000271,-0.00005032,-0.00002769,-0.00011$ $806,0.00012108,-0.00402099,0.00005155,0.00195815,0.02223200,-0.0003428$ $5,0.00029479,0.00008821,-0.00021124,0.00005782,0.00023724,-0.00024791$, $-0.00020952,0.00017662,-0.00015934,-0.00014099,-0.00006268,-0.00018533$ ,-0.00016805,-0.00016117,-0.00023188,0.00013087,-0.00031409,0.00004241 ,-0.00009397,-0.00000856,0.00008069,-0.00002795,-0.00009913,0.00004393 $, 0.00005555,-0.00005834,0.00005696,0.00003594,0.00007479,0.00008738,-0$ $.00004917,0.00008662,0.00004223,0.00006242,0.00000910,0.00140838,0.002$ $26480,0.00015944,0.00052805,-0.00026618,-0.00002321,0.00034148,-0.0016$ $8903,0.00005666,-0.01282796,-0.00165364,0.00027812,-0.33771489,0.01299$ $154,0.00712724,-0.00980261,-0.00195790,0.00034576,-0.00038689,0.000058$ 
$54,0.00000962,-0.00007175,0.00004161,-0.00000647,-0.00022418,0.0001786$ $0,0.00000819,0.35895693,-0.00034000,-0.00022018,-0.00007044,-0.0000877$ $8,0.00040613,-0.00018721,0.00010949,0.00001361,0.00039296,0.00024104,-$ $0.00042814,-0.00014491,0.00006172,0.00017754,-0.00026199,-0.00008028,0$ $.00022701,0.00031890,0.00015958,-0.00008989,-0.00000695,0.00005338,-0$. $00002287,-0.00004864,-0.00003209,-0.00002904,0.00000469,-0.00000190,-0$ $.00002464,-0.00000225,0.00005331,-0.00003964,0.00004883,-0.00006221,-0$ $.00001486,-0.00000176,0.00170533,-0.00314609,-0.00024834,0.00028652,-0$ $.00128027,-0.00002533,-0.00375925,-0.00386409,-0.00008999,-0.02480619$, $0.00366329,0.00063894,0.00811353,-0.05214108,-0.00055250,0.02807739,0$. $00183581,-0.00075843,0.00130814,-0.00083972,-0.00004917,0.00000463,0.0$ $0003976,0.00000860,-0.00096062,0.00019154,0.00003022,-0.00964669,0.054$ $34054,-0.00005988,-0.00005504,0.00042232,-0.00011375,-0.00024762,-0.00$ $025061,-0.00027481,0.00036636,-0.00000925,0.00007648,-0.00005540,0.000$ $25327,0.00021798,-0.00032911,-0.00023833,0.00008531,0.00034883,-0.0001$ $8044,0.00002365,-0.00001322,0.00001083,0.00006712,-0.00001106,-0.00004$ $641,0.00007374,0.00002317,-0.00001745,-0.00006028,-0.00000910,-0.00001$ $850,-0.00003610,0.00001810,-0.00001915,-0.00000573,0.00000639,-0.00000$ $628,0.00015677,-0.00027028,0.00649256,-0.00001661,-0.00001776,-0.00032$ $270,0.00003527,-0.00013968,0.00490527,-0.00021893,0.00011521,0.0002018$ $6,0.00697447,-0.00068007,-0.03651130,0.00097948,-0.00005341,0.00598944$ $, 0.00003838,-0.00001794,-0.00003372,-0.00001092,0.00000977,-0.00042663$ $,-0.00001842,0.00000354,-0.00008672,-0.00780285,0.00088166,0.02394315$, $-0.00005998,-0.00004188,0.00000656,-0.00000922,0.00005719,0.00001736,0$ $.00006504,-0.00002307,0.00011858,0.00009456,-0.00004183,-0.00001243,0$. $00001790,0.00004427,-0.00013307,-0.00001243,0.00001471,-0.00000035,0.0$ $0001590,-0.00001066,-0.00000034,0.00000100,-0.00000935,-0.00001412,-0$. $00003603,0.00002333,0.00001455,-0.00000737,0.00001474,0.00000001,0.000$ $00204,-0.00001530,0.00000990,-0.00004740,-0.00004315,-0.00000475,-0.01$ $092809,-0.02682762,-0.00023831,-0.00511263,0.00161795,0.00026893,-0.00$ $050315,0.00017325,-0.00001008,0.00022026,0.00380906,0.00023517,0.00942$ $971,0.01413621,0.00017201,-0.13272098,-0.12686949,-0.00042743,0.000887$ $21,-0.00055786,-0.00009436,-0.00045412,-0.00136211,-0.00000562,0.00002$ $770,-0.00003264,-0.00001087,0.00081838,-0.00039702,-0.00011036,0.13831$ $171,-0.00001545,0.00001729,-0.00000583,0.00000116,-0.00002729,-0.00000$ $686,-0.00005443,-0.00000920,-0.00008717,-0.00008925,0.00010220,0.00001$ $689,-0.00003735,-0.00004328,0.00007267,0.00000452,-0.00001555,-0.00000$ $069,-0.00001257,0.00002162,0.00000447,0.00000349,0.00000456,0.00001292$ $, 0.00003245,0.00000668,-0.00000826,0.00002003,0.00000258,0.00000789,-0$ $.00000267,0.00001070,-0.00001057,0.00002440,0.00002705,-0.00000051,0.0$ $0428353,0.00191440,-0.00012546,0.00062775,0.00155580,-0.00008703,0.000$ $94149,0.00089084,0.00002055,0.00264168,-0.00465122,-0.00030456,-0.0144$ $5606,-0.01721342,-0.00012783,-0.13063098,-0.26383241,-0.00181793,0.000$ $54466,0.00125309,0.00006839,0.00017920,0.00035884,-0.00000703,-0.00007$ $747,-0.00008211,0.00000884,-0.00026938,0.00124530,0.00012668,0.1363412$ $7,0.27846353,-0.00000139,-0.00000519,-0.00005099,-0.00003710,-0.000077$ $80,-0.00001998,-0.00003932,-0.00000631,0.00005447,0.00002042,0.0000296$ $8,0.00002899,0.00002951,0.00000656,0.00001444,0.00001906,0.00008063,0$. $00000317,-0.00000145,0.00000153,-0.00000860,0.00001737,-0.00000852,-0$. $00000534,0.00005252,0.00001677,-0.00004883,-0.00002855,-0.00000399,-0$. $00004190,-0.00000898,0.00000439,-0.00000749,-0.00001886,-0.00002144,0$. 
$00004412,0.00044550,0.00062515,0.00311715,0.00024884,-0.00011641,0.004$ $85464,0.00000227,0.00003842,-0.00099898,0.00021417,-0.00031523,0.00657$ $449,-0.00042947,-0.00083776,0.00420435,-0.00052278,-0.00191943,-0.0345$ $9070,-0.00006680,0.00009383,-0.00260453,0.00002244,0.00003079,0.000424$ $51,-0.00001535,0.00001079,-0.00043398,-0.00012110,0.00012311,-0.004051$ $22,0.00021905,0.00225043,0.02354220 \backslash 0.00000379,-0.00001932,-0.0000246$ $1,-0.00000136,-0.00000266,-0.00000321,-0.00000222,0.00000384,-0.000008$ $18,0.00000239,0.00000348,0.00001667,0.00000011,-0.00000274,-0.00000190$ $,-0.00000017,0.00000677,0.00000078,0.00000017,0.00001179,0.00000030,-0$ $.00000122,0.00000428,-0.00000210,-0.00000139,0.00000245,0.00000195,0.0$ $0000003,-0.00000462,-0.00000102,0.00000099,-0.00000236,-0.00000274,-0$. $00000055,-0.00000132,0.00000475,-0.00001076,-0.00000081,0.00000076,0.0$ $0000117,0.00000384,0.00000185,-0.00000347,-0.00002084,-0.00005163,0.00$ $000446,0.00002678,0.00006161,0.00000687,-0.00000299,-0.00000025,0.0000$ $0641,-0.00000015,0.00000492,-0.00000064,-0.00000188,0.00000513,-0.0000$ $0223,-0.00000083,0.00000089,-0.00000102,-0.00000031,-0.00000428,-0.000$ 00007,-0.00000072,-0.00000250,-0.00000129,-0.00000170,0.00000281॥I@

\section{Addition Transition State}

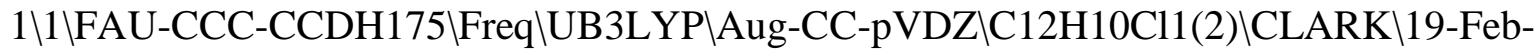
201610\॥N Geom=AllCheck Guess=TCheck SCRF=Check GenChk UB3LYP/Aug-CCpVDZ Freq \p-CHLOROPHENYL RADICAL addition TS to benzenel $10,2 \backslash C, 1.6723$ $914439,-0.5757175624,-0.9215514364 \backslash C, 2.2241385597,-1.2477248683,0.2019$ $299299 \backslash \mathrm{C}, 3.0125232471,-0.556256672,1.1161390228 \backslash \mathrm{C}, 3.3230408681,0.79733$ $74405,0.904966073 \backslash \mathrm{C}, 2.8529000846,1.4499015129,-0.2460098045 \backslash \mathrm{C}, 2.063416$ $2704,0.7685485907,-1.1668895383 \backslash \mathrm{H}, 1.252968854,-1.1633527105,-1.7362715$ $255 \backslash \mathrm{H}, 1.9993873625,-2.3024772344,0.3595448568 \backslash \mathrm{H}, 3.4080968103,-1.073161$ $1274,1.99135642 \backslash \mathrm{H}, 3.1231708938,2.4906595517,-0.4285303915 \backslash \mathrm{H}, 1.71480671$ $71,1.272813771,-2.068109028 \mathrm{lH}, 3.9469604222,1.3330312039,1.6207421086 \mathrm{lC}$ ,-3.1002248039,0.0367655111,0.416438467\C,-2.0865170271,0.4269482402,1 $.2958006479 \backslash \mathrm{C},-0.7488394701,0.2386810809,0.9167352244 \backslash \mathrm{C},-0.4836720157$, $-0.3292323944,-0.3181632904 \backslash \mathrm{C},-1.4780858433,-0.7185256746,-1.200326242$ $3 \backslash \mathrm{C},-2.8197698197,-0.5334965806,-0.8264322482 \backslash \mathrm{Cl},-4.784924186,0.271780$ 9893,0.8926404372\Н,-2.3390333652,0.8692406628,2.2588245907\Н,0.056015 $8339,0.535132669,1.5903442742 \backslash \mathrm{H},-1.2480921557,-1.1636488794,-2.1702234$ 177\H,-3.6317487077,-0.8280946101,-1.4903564186॥Version=ES64L-G09RevD $.01 \backslash \mathrm{State}=2-\mathrm{A} \backslash \mathrm{HF}=-923.4727295 \backslash \mathrm{S} 2=0.779905 \backslash \mathrm{S} 2-1=0 . \backslash \mathrm{S} 2 \mathrm{~A}=0.750645 \backslash \mathrm{RMSD}=6$. 082e-09\RMSF=3.097e-06\ZeroPoint $=0.1777893 \backslash$ Thermal $=0.1888361 \backslash \mathrm{ZPE}=111.5$ 644897\Dipole $=0.8436929,-0.0693548,-0.1883954 \backslash$ DipoleDeriv $=0.1761756,0$. $0397719,0.0270317,-0.0040842,0.0019655,0.0628697,-0.0236239,0.057704,0$ $.045213,-0.143486,0.0849709,0.0446361,0.048813,0.0563158,-0.0023473,0$. $1075685,-0.0727082,-0.1119381,-0.08575,-0.0080643,0.087353,-0.0791222$, $-0.0121421,-0.0562691,0.116168,-0.0848576,0.0446711,-0.1149881,0.05197$ $69,0.0929531,0.0101135,-0.0171753,0.0351776,-0.0009308,0.0303177,0.002$ $9703,-0.0950367,0.0640834,0.0273012,0.1100464,0.1043835,-0.0486686,0.0$ $017392,-0.0244287,-0.0529699,-0.1454382,-0.0116781,0.1239056,0.0615183$ ,-0.0585008,-0.1094849,0.0922306,-0.0419535,-0.0074881,0.124906,-0.041 $6149,-0.0723774,-0.0391721,0.0254462,-0.0443067,-0.0663716,-0.0450128$, $-0.0081172,0.0989733,-0.0615047,-0.0243524,-0.0288201,-0.0657303,0.026$ $7178,-0.0247503,0.0351137,0.0722858,0.0797499,0.0142045,-0.0698914,0.0$ 
$265632,0.0279912,0.074722,-0.0985011,0.0785536,-0.0223073,0.093841,-0$. $04739,-0.0205098,-0.0795368,-0.074304,0.0380917,-0.0173186,0.035483,0$. $0787493,0.0880824,0.0110337,-0.0839989,-0.0006954,0.0364066,0.061692,-$ $0.0547446,0.0603231,-0.0160756,0.0436796,-0.0567089,-0.0871772,-0.0662$ $784,0.0332912,-0.0414847,-0.0948617,-0.037695,0.0118823,1.1746678,-0.1$ $411503,-0.2841463,-0.1785509,-0.018045,0.0550152,-0.3480593,0.0501276$, $0.0742411,-0.1872991,0.0142624,-0.0070537,0.0727651,-0.05955,0.0534641$ $, 0.1419589,0.0559204,0.0315841,-0.4375628,-0.0132269,0.0567624,-0.0219$ $763,-0.0625511,0.0271458,-0.0589436,0.027227,-0.0172844,0.6707629,0.02$ $51356,-0.0385983,-0.0875897,-0.077536,-0.0042781,-0.1900509,-0.01894,-$ $0.0569352,-0.2933565,-0.0388767,-0.0128919,0.1492131,-0.0463702,0.0779$ $6,0.3279003,0.0988948,0.0824381,-0.2197766,0.048283,0.1072524,0.012376$ $7,-0.0926107,0.0240624,-0.0014707,0.0163493,-0.047324,-0.8774292,0.076$ $4774,0.176004,0.1094435,-0.0893558,-0.0304363,0.2225399,-0.0285753,-0$. $1390488,0.0084155,0.0122705,0.0378884,0.023885,0.0998397,-0.0592346,0$. $0485983,-0.0583232,-0.0020953,0.0365652,-0.0214682,-0.074514,-0.020532$ $2,0.089043,-0.026604,-0.0398926,-0.0253052,0.0399045,0.0679241,0.02974$ $81,0.0594398,-0.0021724,0.0854441,-0.0784512,-0.002562,-0.0762594,-0.0$ $513324,-0.0636202,-0.0305351,-0.0650166,-0.0162071,0.1137444,-0.035352$ $9,-0.0366217,-0.0319552,0.0489771 \backslash$ Polar $=237.2508724,9.7621952,127.9564$ $783,7.3343295,8.9346746,145.1636185 \backslash \mathrm{PG}=\mathrm{C} 01[\mathrm{X}(\mathrm{C} 12 \mathrm{H} 10 \mathrm{Cl1})] \mathrm{NImag}=1 \backslash 0.1$ $4107960,0.11187704,0.61397462,0.22112588,-0.00518840,0.57759633,-0.075$ $14435,0.01581956,-0.07871951,0.31485606,0.02117520,-0.16191266,0.09747$ $595,0.13297925,0.68842421,-0.04903538,0.05597944,-0.22215821,0.2150489$ $2,-0.05563535,0.48910251,-0.01708717,0.01459320,-0.03164013,-0.1533927$ $8,-0.09052439,-0.10219635,0.31812014,-0.01786881,0.05055210,-0.0192456$ $3,-0.05615686,-0.17217036,-0.03306899,0.09359852,0.61723072,-0.0300388$ $8,0.00564195,-0.03345307,-0.11990942,-0.08917327,-0.20233582,0.2463483$ $3,-0.10004966,0.56937387,0.00141921,0.00482711,0.00054690,-0.00092132$, $-0.02200883,-0.00247182,-0.09026819,-0.06988618,-0.01068017,0.32284592$ $, 0.01199216,-0.03734703,0.02383824,-0.04718980,-0.03194507,-0.05725388$ ,-0.03379380,-0.29493938,0.06795944,0.14010413,0.61438479,0.01013664,0 $.02454337,-0.01962471,0.01048505,-0.01521160,0.03322965,-0.02891745,0$. $01087616,-0.11237844,0.24291970,-0.03758105,0.54618101,-0.01149522,-0$. $03761823,0.00790566,-0.01777119,-0.00335455,-0.02062419,0.02374614,0.0$ $2949964,0.01222461,-0.10209304,-0.00233082,-0.06593197,0.30880084,-0.0$ $2250056,-0.02364438,-0.01914410,0.00084977,-0.00137032,-0.00014654,0.0$ $0780585,-0.02278803,0.02940545,0.03211856,-0.16285591,0.11262823,0.149$ $32839,0.68286524,-0.01855439,-0.04573377,0.03597536,-0.02136149,-0.007$ $21340,-0.03995399,0.02402528,0.06468967,0.01080740,-0.08627925,0.05496$ $079,-0.23404857,0.20395164,-0.08188823,0.51403135,-0.06477807,-0.05298$ $451,-0.01992597,0.01669697,0.01341202,0.01288103,-0.01485249,-0.019471$ $58,-0.00538493,-0.00853968,0.02200743,-0.03984166,-0.15211231,-0.09549$ $711,-0.09698673,0.31697995,-0.02930093,-0.27745014,0.01519933,0.013577$ $57,-0.01391111,0.04153349,-0.01665466,-0.03616243,-0.01186398,-0.00241$ $107,0.05205064,-0.02960626,-0.06212052,-0.17324186,-0.03242889,0.11473$ $358,0.58195472,-0.00873099,0.06036231,-0.11632545,0.01383329,0.0286114$ $8,0.01119110,-0.00815216,-0.01909744,-0.00796705,-0.02602958,0.0127582$ $5,-0.04350636,-0.11683834,-0.08902861,-0.20142676,0.23582867,-0.094692$ $66,0.59009361,-0.06073081,-0.06065436,-0.08895496,-0.00781846,-0.00574$ $864,-0.01394056,0.00521498,0.00391614,-0.00282817,-0.00097023,-0.00050$ 
$919,-0.00059705,0.00575065,-0.00275495,0.00192959,-0.00571928,-0.01015$ $190,-0.00678830,0.08472441,-0.06882656,-0.13762978,-0.11172617,0.00712$ $334,0.01119114,0.01345746,0.00054398,-0.00117216,0.00290774,0.00061698$ ,-0.00037706,0.00088033,-0.00150797,-0.00472040,0.00197392,-0.01053792 ,-0.00896168,-0.02283313,0.06857153,0.14108522,-0.10090259,-0.11276188 ,-0.21023707,-0.00694345,-0.01545928,-0.01037713,-0.00260667,0.0028014 $7,-0.00238009,0.00076006,0.00100530,0.00029732,-0.00098225,0.00186535$, $0.00042465,0.00446302,0.00765870,0.00805898,0.09736941,0.11901407,0.21$ $855409,0.00471293,0.01354306,-0.00237589,-0.05352176,-0.06174522,-0.00$ $020661,0.00041952,-0.01738471,0.00314871,0.00311699,-0.00235614,-0.005$ $53984,-0.00083821,0.00021865,-0.00000804,0.00285005,-0.00032918,-0.004$ $18667,-0.00188986,0.00014533,0.00102300,0.04743525,-0.00406399,-0.0100$ $6777,-0.00011157,-0.06046749,-0.31969544,0.04164662,-0.00135683,-0.010$ $36983,0.00503641,-0.00294470,0.00116116,-0.00196388,0.00032539,0.00055$ $879,-0.00007297,0.00071389,0.00107228,0.00289963,0.00071405,0.00063384$ $,-0.00025366,0.06685804,0.33724703,0.00652654,0.02364339,0.00045382,-0$ $.00197896,0.04334557,-0.05733509,-0.00429392,-0.02055768,0.00696453,-0$ $.00553991,-0.00116160,-0.00051116,0.00000288,-0.00018708,-0.00104539,-$ $0.00475437,0.00269042,-0.00219379,0.00180229,-0.00079136,0.00029170,0$. $00551760,-0.04658976,0.05385697,0.00328706,-0.00454164,-0.00482779,-0$. $00294375,0.00941545,-0.01407861,-0.08013720,0.04440915,-0.09014640,0.0$ $0540265,-0.00454886,0.00597098,0.00437817,-0.00280710,-0.00211521,-0.0$ $0046111,0.00010263,0.00049299,-0.00022550,0.00068780,-0.00088822,-0.00$ $256101,0.00102661,0.00239713,0.07548411,-0.00357740,-0.00025275,-0.001$ $68448,-0.00647040,0.01062087,-0.01284199,0.04387006,-0.11941369,0.1088$ $7672,0.01198672,-0.00853366,0.02469551,-0.00319067,-0.00484435,0.00081$ $001,-0.00046506,-0.00053528,-0.00081573,-0.00030587,0.00028697,-0.0006$ $4276,0.00117757,0.00050871,-0.00043476,-0.04484677,0.12197400,-0.00487$ $353,-0.00181630,0.00070419,-0.00584082,0.01197613,-0.01174765,-0.09046$ $673,0.10839690,-0.23153205,-0.00204580,0.00017372,-0.00053946,-0.00202$ $171,0.00133792,0.00340081,0.00043686,-0.00076442,0.00003300,-0.0003837$ $0,0.00040191,-0.00045058,0.00210714,-0.00069345,-0.00039315,0.10068691$ , $-0.11823056,0.24143925,0.00303868,-0.00264217,-0.00617147,-0.00063178$ $, 0.00023390,0.00029803,0.00400152,-0.00033289,-0.00429542,0.00548149,0$ $.01064940,-0.00236943,-0.06027505,-0.07149593,0.00382701,-0.00105905,-$ $0.01725296,0.00374854,-0.00008809,-0.00111012,0.00033023,-0.00065129,0$ $.00018129,0.00051823,0.00016331,0.00043498,-0.00012503,0.05430506,-0.0$ $0307080,0.00130993,-0.00151012,0.00032895,0.00044649,-0.00040935,0.000$ $01015,0.00152338,0.00355563,-0.00466926,-0.00967258,-0.00036716,-0.071$ $76989,-0.31017071,0.04753278,-0.00178775,-0.01044895,0.00509110,-0.000$ $19950,-0.00049310,0.00029309,0.00026196,-0.00013224,-0.00016388,-0.000$ $25114,-0.00054833,-0.00008607,0.07876433,0.32734575,-0.00526079,-0.001$ $38226,-0.00046539,-0.00025590,-0.00037149,-0.00096953,-0.00459520,0.00$ $287553,-0.00247518,0.00727739,0.02433988,0.00062915,0.00330920,0.04805$ $985,-0.06097549,-0.00533076,-0.01983710,0.00739883,-0.00030465,-0.0007$ $7019,0.00010911,0.00048401,-0.00013552,-0.00023864,0.00022915,0.001296$ $95,-0.00004221,0.00029965,-0.05252907,0.05747827,0.00499913,-0.0056573$ $9,0.00864738,0.00343705,-0.00287484,-0.00196301,-0.00070781,-0.0001564$ $5,0.00033558,0.00331183,-0.00410501,-0.00428994,-0.00140901,0.00910954$ $,-0.01449243,-0.07038136,0.03733925,-0.08405835,-0.00198826,0.00048009$ $, 0.00078606,0.00041434,-0.00025903,0.00031198,-0.00068009,0.00029621,0$ 
$.00045126,-0.00258816,0.00112277,0.00209208,0.06524608,0.01087112,-0.0$ $0864618,0.02437235,-0.00380792,-0.00454300,0.00091332,-0.00018357,-0.0$ $0073803,-0.00059403,-0.00382160,-0.00006987,-0.00154143,-0.00566043,0$. $01039674,-0.01377155,0.03533076,-0.11567923,0.11197226,0.00095887,0.00$ $083732,-0.00078237,0.00058919,-0.00055990,0.00120940,0.00033563,-0.000$ $03844,-0.00016247,0.00151206,0.00044865,-0.00049442,-0.03585078,0.1181$ $3900,-0.00185182,0.00055844,-0.00109494,-0.00123572,0.00115937,0.00278$ $354,0.00043572,-0.00059590,0.00020992,-0.00474069,-0.00231365,0.000602$ $62,-0.00510192,0.01204791,-0.01209436,-0.08344650,0.11003940,-0.244510$ $03,0.00150858,-0.00002749,0.00004318,-0.00014739,-0.00003873,-0.000127$ $23,0.00039495,-0.00006375,-0.00033349,0.00209175,-0.00076939,-0.000234$ $19,0.09306594,-0.12040463,0.25517070,-0.00044915,0.00059173,0.00069937$ $, 0.00484189,-0.00226077,-0.00117327,-0.00051144,-0.00175142,-0.0051251$ $7,-0.13406492,-0.08457323,-0.11571519,-0.00236337,-0.00651333,-0.00560$ $551,0.00452056,0.00062780,-0.00336810,-0.00061944,0.00006765,0.0002425$ $8,-0.00010114,-0.00007118,-0.00029873,-0.00208383,0.00085195,0.0018893$ $6,-0.00209193,0.00115649,0.00180728,-0.00021477,-0.00037221,-0.0003021$ $9,0.13299768,0.00038789,-0.00060476,0.00047733,-0.00194825,-0.00488200$ $, 0.00273637,-0.01704802,-0.00890293,-0.02079350,-0.08439970,-0.1235108$ $9,-0.08721997,0.00893809,0.01018411,0.01044225,-0.00006421,-0.00006186$ $, 0.00383925,0.00020140,-0.00002454,-0.00016594,-0.00083810,-0.00057082$ ,-0.00101129,0.00109601,0.00077641,-0.00051973,0.00085552,0.00041012,-$0.00093552,0.00043853,0.00035039,0.00059536,0.09231302,0.12677330,0.00$ $053218,0.00049566,-0.00045109,-0.00181356,0.00213320,0.00265316,0.0034$ $1431,0.00430098,0.00617585,-0.11545890,-0.08721791,-0.17270038,-0.0139$ $1235,-0.01462307,-0.01113007,-0.00345543,0.00433124,-0.00166281,0.0003$ $8257,-0.00017122,-0.00015181,0.00016161,0.00029842,0.00023074,0.001708$ $19,-0.00084091,-0.00043666,0.00191451,-0.00060794,-0.00012363,-0.00070$ $211,-0.00069624,-0.00069538,0.12708004,0.09258763,0.17824845,-0.000347$ $68,-0.00012522,0.00011073,0.00007504,0.00011885,-0.00015836,-0.0000897$ $4,0.00020129,-0.00017297,0.00013767,-0.00005540,0.00012678,0.00012564$, $-0.00008941,0.00030973,-0.00007339,-0.00017351,-0.00014010,-0.00023535$ $, 0.00007054,-0.00001539,-0.00007694,-0.00004808,-0.00003208,0.00002705$ ,-0.00002457,0.00011599,-0.00003963,0.00008342,-0.00003794,-0.00005216 ,-0.00000843,0.00003894,0.00002056,0.00009510,0.00003122,0.50729898,-0 $.00000589,-0.00002303,-0.00025086,-0.00006569,-0.00010396,-0.00006831$, $0.00001208,-0.00001449,0.00028001,0.00010349,0.00007900,0.00005658,-0$. $00017319,-0.00002090,-0.00000453,-0.00001164,0.00004239,0.00004026,-0$. $00005847,0.00004940,0.00007000,0.00004170,0.00001683,0.00000570,-0.000$ $03295,0.00007858,-0.00012347,0.00002755,-0.00005256,0.00005253,-0.0000$ $4971,-0.00007449,0.00001059,-0.00005215,-0.00008824,-0.00003436,0.0151$ $3515,0.21418932,-0.00001352,-0.00031122,-0.00029851,-0.00006306,-0.000$ $03950,-0.00001417,-0.00013275,0.00002441,0.00007714,0.00008327,-0.0000$ $3467,0.00015853,-0.00011126,0.00012519,0.00020372,-0.00023082,0.000098$ $50,-0.00007245,-0.00017399,0.00007968,0.00016282,0.00000976,-0.0000078$ $7,-0.00005358,-0.00000613,0.00004390,-0.00003876,0.00000156,-0.0000403$ $9,0.00000123,0.00001938,-0.00009100,0.00000748,-0.00003644,-0.00002027$ ,-0.00004170,0.04137696,0.19324479,0.55095972,0.00135011,0.00052715,-0 $.00004184,0.00047262,-0.00019874,-0.00024569,0.00003857,-0.00021731,0$. $00007197,-0.00009557,0.00026763,0.00000228,-0.00010016,-0.00016673,-0$. $00049618,0.00073205,-0.00020347,0.00000614,0.00024206,-0.00024439,-0.0$ 
$0015935,0.00001303,0.00016877,0.00012843,-0.00008478,0.00007648,-0.000$ $17658,-0.00000658,-0.00010553,0.00009003,-0.00002572,0.00005094,-0.000$ $04932,-0.00004967,-0.00015737,-0.00004785,-0.20495748,-0.03068582,-0.0$ $6998424,0.64195910,-0.00022591,0.00057477,-0.00038642,0.00021351,-0.00$ $001176,-0.00009318,0.00002537,0.00002637,-0.00022076,0.00018568,0.0000$ $9472,0.00021717,0.00009873,0.00015891,0.00020196,0.00002060,-0.0000189$ $5,-0.00007465,0.00006727,-0.00001557,-0.00009532,-0.00001119,0.0000189$ $8,0.00005502,0.00003132,-0.00002694,0.00003520,-0.00000763,-0.00001492$ ,-0.00000465,0.00003787,-0.00001597,-0.00005533,0.00000284,-0.00002121 ,-0.00001531,-0.05717256,-0.09205087,-0.06033389,-0.01412348,0.2323818 $3,0.00003850,-0.00056990,-0.00053776,-0.00018690,0.00003789,-0.0002056$ $6,-0.00006337,0.00029257,0.00020556,0.00025229,0.00018847,0.00030096,-$ $0.00003574,-0.00011872,0.00015068,0.00015311,-0.00033226,-0.00019682,0$ $.00023512,-0.00004207,-0.00020204,0.00006222,-0.00011123,-0.00002338,0$ $.00008621,-0.00002244,0.00003116,0.00003621,0.00003232,-0.00005312,0.0$ $0000396,0.00006826,-0.00003650,0.00002906,0.00002996,-0.00000898,-0.12$ $869327,-0.05954200,-0.19878769,-0.01808506,0.22855089,0.62850149,-0.00$ $456023,0.00149611,0.00405542,-0.00036833,-0.00036944,0.00212444,0.0001$ $5623,-0.00050273,0.00020062,-0.00045766,-0.00058403,-0.00102359,0.0001$ $8793,0.00027487,-0.00008084,-0.00065042,0.00207981,0.00021671,-0.00108$ $874,0.00051501,0.00092421,-0.00009808,0.00017694,-0.00012115,-0.000174$ $26,0.00012617,-0.00010856,-0.00008436,-0.00014921,0.00000908,-0.000070$ $71,-0.00027147,0.00017507,-0.00014700,0.00002122,0.00002315,-0.0529688$ $0,-0.02109179,-0.04754833,-0.30466959,0.04203914,0.08584407,0.65704315$ $, 0.00073983,-0.00066134,0.00025339,0.00001003,0.00025849,-0.00051545,-$ $0.00006846,-0.00000896,-0.00017785,0.00019169,-0.00056260,0.00012975,-$ $0.00065059,0.00003888,-0.00043017,0.00026437,0.00059755,0.00047246,0.0$ $0004120,0.00004101,-0.00008093,0.00001545,0.00004684,0.00001647,-0.000$ $03620,0.00004509,0.00000222,0.00004349,0.00005259,-0.00005456,-0.00000$ $521,0.00008554,-0.00005847,-0.00002562,0.00003887,0.00001718,0.0028294$ $3,0.01645790,0.01979417,0.01062365,-0.07422264,-0.02840622,0.01774857$, $0.22382381,0.00129868,-0.00082371,-0.00029431,-0.00005742,0.00092235,0$ $.00031871,-0.00080820,-0.00065505,-0.00033672,0.00058273,0.00017961,-0$ $.00064383,-0.00028092,-0.00009091,-0.00002711,-0.00032622,0.00008355,0$ $.00055912,0.00044475,-0.00004893,0.00000314,0.00008378,-0.00007312,0.0$ $0005331,-0.00005211,-0.00001265,-0.00002289,-0.00002532,-0.00002310,0$. $00003833,0.00007931,0.00004153,-0.00010982,-0.00007841,-0.00001389,0.0$ $0001760,0.00456087,0.01888465,0.04898066,0.01770846,-0.02750507,-0.121$ $61049,0.05280834,0.20933456,0.58951636,0.07753106,0.00282894,-0.005938$ $35,-0.02616369,0.01225767,-0.02658621,0.00517857,0.01238453,0.00605055$ ,-0.00297325,-0.00412403,-0.00467411,0.00539179,0.00177754,0.01258040, $-0.02168262,-0.03181384,0.00356901,-0.01488584,0.00335340,0.00664369,0$ $.00086973,-0.00119885,-0.00004688,0.00058598,0.00007399,0.00055193,0.0$ $0048873,0.00046273,0.00022891,0.00080554,0.00058977,-0.00107943,0.0003$ $8808,0.00012712,0.00007654,-0.00951505,-0.00819316,-0.01847074,0.00743$ $345,0.01352973,0.02952094,-0.09804948,0.02315255,0.04936933,0.33188343$ $,-0.00776982,-0.00834007,-0.00283960,0.00579985,-0.00007843,0.00418316$ $,-0.00111705,-0.00336578,-0.00135883,0.00012784,0.00190756,-0.00086238$ $, 0.00012314,-0.00012348,0.00150270,-0.00659564,0.00065019,0.00050249,0$ $.00576086,-0.00099228,-0.00271245,-0.00026104,0.00035703,0.00006085,-0$ $.00014324,-0.00001532,-0.00004683,0.00018632,-0.00005728,-0.00013755,0$ 
$.00019515,0.00008802,-0.00017700,-0.00005871,0.00009108,0.00001524,-0$. $00854194,-0.01765189,-0.02301848,0.03664775,0.00883174,-0.00825424,0.0$ $0925376,-0.10375335,-0.10690243,0.03685187,0.17896149,-0.02091759,-0.0$ $0332393,-0.01057522,-0.00167248,-0.00238164,0.00617424,-0.00066484,-0$. $00107234,-0.00111331,0.00072875,-0.00051041,0.00204682,-0.00186499,-0$. $00034817,-0.00457629,0.00556322,0.00852213,0.00090879,0.01001059,-0.00$ 265544,-0.00498056,-0.00008013,0.00012967,0.00006476,0.00013800,-0.000 $09969,-0.00020974,-0.00015687,-0.00014740,-0.00010099,-0.00028467,-0.0$ $0008314,0.00044022,-0.00014361,-0.00001938,0.00003263,-0.01863246,-0.0$ $2314093,-0.05856314,0.08017960,-0.00915813,-0.00402134,0.01838031,-0.1$ $0656255,-0.28770284,0.09054076,0.20562265,0.53720360,-0.00476134,-0.00$ $117283,-0.00242242,-0.00098598,-0.00024663,0.00146548,0.00004693,-0.00$ $047876,-0.00014401,-0.00038621,-0.00051294,-0.00057630,0.00035637,0.00$ $022888,-0.00037245,-0.00085222,0.00141618,0.00034893,0.00047421,-0.000$ $47074,-0.00074317,-0.00016514,0.00036028,-0.00004323,-0.00008501,0.000$ $11486,-0.00024038,-0.00011805,-0.00020705,-0.00003285,-0.00009802,-0.0$ $0015105,0.00018519,-0.00016931,-0.00001072,-0.00004425,0.00532998,0.03$ $673447,0.08073247,-0.06144047,-0.00636533,-0.01542337,0.04556520,-0.02$ $085704,-0.04435640,-0.21087115,-0.04202476,-0.09635451,0.60732706,0.00$ $066982,0.00068248,0.00044935,-0.00000764,-0.00055746,-0.00058006,0.000$ $32469,0.00077319,0.00025245,0.00017071,-0.00021814,0.00072097,-0.00033$ $683,-0.00031788,-0.00064978,0.00079695,0.00008129,0.00008020,0.0004507$ $3,0.00002443,0.00028277,-0.00002717,-0.00005728,-0.00000638,0.00000920$ ,-0.00003183,0.00002503,0.00001378,0.00003183,0.00000587,0.00005972,0. $00010148,-0.00001019,0.00002218,-0.00002445,-0.00001381,0.01272993,0.0$ $0523479,-0.00412069,-0.00527860,-0.00281815,-0.00015807,-0.00705347,-0$ $.00381141,-0.01797266,-0.05630718,-0.08210714,-0.06389150,-0.00572460$, $0.23046083,0.00127547,0.00073404,0.00161554,0.00103603,-0.00023350,-0$. $00056761,-0.00033568,0.00009314,-0.00037518,0.00030074,0.00068534,0.00$ $041293,0.00020109,-0.00028815,0.00063532,0.00067053,-0.00054179,-0.000$ $86891,0.00122642,0.00023255,0.00052848,0.00002758,-0.00008560,0.000037$ $22,0.00004457,-0.00008394,0.00011256,-0.00000282,0.00011567,-0.0000670$ $4,0.00003977,0.00014678,-0.00002736,0.00009483,0.00005929,0.00000304,0$ $.02800621,-0.00325314,0.00064719,-0.01321892,-0.00006893,-0.00363400,-$ $0.01381856,-0.01843775,-0.03632007,-0.12805489,-0.06394075,-0.19684496$ ,-0.00093490,0.22476624,0.62114803,0.00105378,-0.00046288,-0.00116631, $-0.00044408,-0.00010676,0.00002378,0.00003773,0.00005608,0.00026079,-0$ $.00001351,0.00006497,0.00007768,-0.00024695,0.00007923,-0.00006431,-0$. $00021491,0.00008671,0.00011810,0.00002481,0.00021285,0.00044926,0.0000$ $0637,-0.00001785,-0.00003645,0.00004603,-0.00003859,0.00001522,0.00007$ $608,0.00001779,0.00001559,-0.00005658,-0.00009457,0.00007144,0.0000270$ $7,-0.00002118,0.00004335,-0.11066939,-0.00116008,-0.00319255,0.0471678$ $4,-0.00445975,-0.00853216,-0.02737156,0.01371578,0.02884288,-0.0545437$ $6,-0.02278152,-0.05213019,-0.29882391,0.04169269,0.08611780,0.68869777$ ,-0.00040768,0.00066399,-0.00059423,-0.00054993,0.00006011,0.00026132, $-0.00002481,0.00003004,-0.00028721,-0.00026696,-0.00012080,-0.00022719$ $, 0.00014227,0.00019477,-0.00006779,0.00023454,0.00002831,0.00006256,-0$ $.00005108,0.00011768,-0.00000983,-0.00003661,-0.00010618,-0.00005771,0$ $.00012482,-0.00019199,0.00019727,-0.00001098,0.00014241,-0.00006553,0$. $00005780,0.00008622,0.00004270,0.00013447,0.00017128,0.00009863,0.0253$ $4474,-0.10967767,-0.09770727,-0.02248416,-0.00069662,-0.02019281,0.013$ 
$98720,-0.00927211,-0.01337846,0.00005038,0.02052966,0.01560977,0.01063$ $812,-0.07444508,-0.02792592,0.01614739,0.22306570,-0.00039730,-0.00061$ $037,-0.00019962,-0.00024780,0.00019030,0.00031923,0.00020777,-0.000194$ $79,0.00010635,-0.00025396,-0.00018119,-0.00035801,0.00017287,0.0001262$ $2,-0.00017604,-0.00051348,0.00014276,0.00028950,-0.00019921,-0.0000324$ $3,-0.00014119,-0.00004675,0.00009566,-0.00001652,-0.00002470,0.0000225$ $4,-0.00000887,0.00000203,-0.00003402,0.00002241,-0.00003731,-0.0000486$ $4,0.00004353,0.00000218,-0.00000093,0.00002605,0.05545658,-0.09826374$, $-0.27919440,-0.04968103,-0.01963680,-0.03612388,0.03020948,-0.01344593$ ,-0.03129916,-0.00127106,0.01492283,0.04653762,0.01814604,-0.02694844, $-0.12102138,0.04792852,0.21045454,0.59007680,0.00027642,-0.00001324,-0$ $.00011079,-0.00020463,-0.00015265,0.00022229,0.00002806,-0.00009521,0$. $00011032,-0.00013535,-0.00002812,-0.00012294,-0.00002226,0.00012036,-0$ $.00006205,-0.00022429,0.00020114,-0.00002097,0.00017850,-0.00004353,-0$ $.00005396,0.00005373,-0.00002630,0.00000347,0.00000336,-0.00001509,-0$. $00004483,0.00000743,-0.00005310,-0.00001471,0.00005436,0.00002731,-0.0$ $0000912,-0.00000582,-0.00001510,-0.00001719,-0.13948997,0.01413996,0.0$ 2842056,-0.04157755,0.00637131,0.01287615,0.00037185,-0.00272654,-0.00 $599959,-0.00021488,0.00041916,0.00081360,0.00250875,0.00129001,0.00277$ $874,-0.02637921,0.00401132,0.00805776,0.20514756,0.00000467,-0.0001578$ $6,0.00010182,-0.00000999,0.00003558,-0.00003417,-0.00000186,0.00004780$ ,-0.00000194,-0.00001546,-0.00000533,0.00003008,0.00003477,-0.00003757 $,-0.00001655,0.00000564,-0.00001872,-0.00000036,-0.00004726,0.00001794$ $, 0.00001180,-0.00000656,-0.00001001,-0.00001279,0.00000819,-0.00001191$ $, 0.00003052,0.00000088,0.00001850,-0.00001202,-0.00000573,0.00000356,0$ $.00000652,0.00001368,0.00001694,0.00000601,0.01407822,-0.02805845,-0.0$ $0987474,-0.00480731,0.00498558,0.00072391,0.00000353,0.00328280,-0.002$ $37101,0.00021764,-0.00053184,0.00099127,-0.00125212,0.00299538,-0.0033$ $7275,0.01517900,0.00206220,-0.00481401,-0.02373730,0.01929412,-0.00008$ $961,0.00006648,0.00000870,0.00007305,0.00004323,-0.00003665,0.00000494$ $, 0.00001913,-0.00003505,0.00002461,0.00002150,0.00003817,0.00001706,-0$ $.00001336,0.00002946,0.00007293,-0.00005860,0.00000073,-0.00008574,0.0$ $0004386,0.00004471,-0.00001699,-0.00000577,-0.00000194,0.00001602,-0.0$ $0001244,0.00002978,0.00000268,0.00002842,-0.00000197,-0.00001539,-0.00$ $000757,0.00000236,0.00001774,0.00001506,0.00001113,0.02839336,-0.00985$ $135,-0.04445268,-0.01170939,0.00099157,0.00646555,-0.00010776,-0.00247$ $111,-0.00097858,0.00057803,0.00097510,0.00123121,-0.00286847,-0.003259$ $66,-0.00275727,0.03255116,-0.00512053,-0.00589587,-0.04771113,0.015902$ $25,0.04559726,-0.00008650,0.00003137,0.00016196,-0.00006103,0.00000356$ $, 0.00015015,0.00003332,-0.00005920,-0.00002839,-0.00011593,-0.00008835$ $,-0.00014553,0.00005031,0.00004185,-0.00003713,-0.00010506,0.00011665$, $0.00002685,-0.00006934,0.00004136,0.00004044,-0.00002097,-0.00001623,-$ $0.00000640,-0.00000651,-0.00002901,0.00003507,-0.00001538,0.00001722$,$0.00001321,0.00000097,0.00001614,0.00003608,0.00000498,0.00003879,0.00$ $002234,0.00656376,-0.00924340,-0.02028350,-0.06868044,0.02740018,0.059$ $05607,-0.00272774,0.01164017,0.02535911,-0.00436417,0.00104605,0.00203$ $098,-0.00100722,-0.00023249,-0.00058340,-0.00299729,-0.00135047,-0.002$ $87324,0.00101437,-0.00034186,-0.00063687,0.07087148,-0.00010896,0.0001$ $0987,-0.00008301,0.00014037,0.00000667,0.00004950,-0.00003897,-0.00003$ $728,-0.00003657,0.00003588,0.00002756,0.00000874,-0.00000751,0.0000055$ $6,0.00005833,-0.00004048,-0.00007219,-0.00001274,0.00001222,-0.0000161$ 
$4,-0.00000112,-0.00000069,0.00000058,-0.00000222,0.00000559,0.00000754$ ,-0.00000639,-0.00000586,-0.00000753,0.00000845,-0.00000239,-0.0000046 $0,-0.00000110,-0.00000885,-0.00000111,-0.00000427,0.00368951,-0.000001$ $93,-0.00708625,0.02574611,-0.08609253,-0.11054318,-0.00032122,0.002815$ $30,-0.00338932,0.00087302,0.00559014,-0.00205712,-0.00011337,-0.000594$ $85,0.00058848,-0.00151634,0.00430919,-0.00218493,0.00011002,-0.0019929$ $2,0.00144347,-0.02847322,0.07962029,-0.00017688,-0.00008904,0.00003493$ $, 0.00000521,0.00001499,-0.00002404,-0.00001837,0.00003595,-0.00003682$, $-0.00002011,0.00000873,0.00005966,-0.00000081,-0.00002499,-0.00001573$, $0.00006823,0.00002100,-0.00001654,0.00003656,0.00001748,-0.00001854,-0$ $.00000512,-0.00002754,-0.00000647,0.00001717,-0.00002261,0.00003319,0$. $00001046,0.00002812,-0.00001742,0.00000397,0.00001300,-0.00000091,0.00$ $002168,0.00002232,0.00001229,0.00813592,-0.00735317,-0.01275946,0.0555$ $4897,-0.11048284,-0.27594872,-0.00081211,-0.00309653,-0.00250637,0.001$ $40608,-0.00214013,0.00201098,-0.00018143,0.00062014,0.00036820,-0.0034$ $6348,-0.00226347,0.00045379,0.00031390,0.00141760,0.00048772,-0.061175$ $88,0.12158987,0.28861272,-0.00000289,0.00047542,0.00009799,0.00025623$, $-0.00009576,0.00021379,0.00002138,0.00034386,-0.00013708,-0.00064735,-$ $0.00027139,0.00009998,0.00000361,-0.00021446,-0.00005273,0.00015018,-0$ $.00005841,-0.00006686,-0.00001308,-0.00000931,0.00002812,0.00002607,-0$ $.00003897,-0.00000174,0.00003432,-0.00003894,0.00003878,0.00004884,0.0$ $0003894,-0.00000038,0.00001516,-0.00000371,-0.00003777,0.00006514,-0.0$ $0000915,0.00004165,-0.00290195,0.00118692,0.00229447,-0.01744565,-0.00$ $842714,-0.01884071,-0.20706635,-0.05551848,-0.12638122,0.00876523,0.00$ $087026,0.00215596,0.00063848,0.00126375,0.00252347,-0.00019219,0.00039$ $586,0.00061814,-0.00144775,-0.00012428,-0.00033673,0.00120467,-0.00013$ $095,-0.00008411,0.21817472,-0.00051380,-0.00055710,0.00018702,-0.00038$ $507,-0.00014760,-0.00003428,-0.00000711,0.00005436,0.00041519,-0.00013$ $127,0.00043115,-0.00013811,0.00019625,-0.00025113,-0.00006819,0.000244$ $37,0.00018169,-0.00005928,0.00007503,-0.00000017,-0.00001339,0.0000861$ $0,-0.00011449,-0.00009162,0.00002422,0.00001689,-0.00002952,-0.0000400$ $1,-0.00004034,0.00005463,-0.00002671,-0.00001321,0.00003935,-0.0000239$ $3,-0.00002135,-0.00001353,0.00100802,0.00574892,-0.00213924,0.00395834$ $, 0.00586725,0.00158416,-0.05680312,-0.05943137,-0.05360219,-0.00880447$ ,-0.00271642,-0.00685329,0.00064801,0.00410379,-0.00423166,0.00044179, $-0.00067004,0.00013432,0.00031758,0.00005555,0.00001355,-0.00018616,-0$ $.00326072,0.00181674,0.06035224,0.05141908,-0.00104780,0.00035770,-0.0$ $0028362,0.00013211,-0.00032248,0.00016062,0.00028357,0.00004752,0.0001$ $7669,0.00008777,0.00011873,0.00043826,-0.00003619,0.00015425,-0.000073$ $20,0.00006612,0.00003933,-0.00011914,-0.00005469,0.00003294,0.00005662$ ,-0.00000502,0.00005077,0.00001093,-0.00005759,0.00005389,-0.00001524, $0.00001930,-0.00000301,0.00001522,-0.00000037,-0.00003349,0.00000050,-$ $0.00005600,-0.00005682,-0.00002181,0.00192653,-0.00226813,0.00200436,0$ $.00826522,0.00134530,0.00835508,-0.12944596,-0.05367768,-0.15719556,-0$ $.01903978,-0.00668059,-0.01521800,0.00099910,-0.00429178,-0.00303741,0$ $.00097660,0.00028325,-0.00046676,0.00069347,0.00001317,0.00006488,-0.0$ $0025875,0.00181278,-0.00009826,0.13746265,0.06300506,0.16577507,0.0006$ $4253,-0.00067522,-0.00082945,-0.00005780,0.00010820,0.00018143,-0.0000$ $4468,-0.00000556,0.00003188,0.00001318,0.00004285,0.00005341,-0.000053$ $25,0.00002804,-0.00000288,-0.00007149,0.00010669,0.00009054,-0.0002776$ $4,0.00035415,0.00024151,0.00001096,-0.00006315,-0.00005321,0.00005161$, 
$-0.00003329,0.00004825,0.00004008,0.00003843,-0.00001741,0.00000636,-0$ $.00002271,-0.00000813,0.00003004,0.00003157,0.00002542,-0.00385349,0.0$ $0049230,0.00064520,-0.00115747,-0.00012013,-0.00020568,-0.00275709,-0$. $00156631,-0.00374400,0.00830928,-0.00886402,-0.01918372,-0.06666410,0$. $02353918,0.05063427,-0.00333101,0.01238549,0.02717508,-0.00119805,0.00$ $052277,0.00113348,0.00003516,0.00001204,0.00001913,0.00037286,-0.00039$ $402,-0.00082246,0.06870438,0.00051008,-0.00065221,0.00036940,0.0001260$ $9,0.00027629,-0.00005394,0.00009718,-0.00009865,0.00016567,-0.00000461$ ,-0.00003308,-0.00004910,0.00000980,-0.00002624,0.00008172,-0.00034653 ,-0.00012053,-0.00004318,-0.00030810,0.00013011,-0.00000873,0.00004474 ,-0.00000600,0.00002275,-0.00002524,0.00004082,-0.00000562,0.00001842, $-0.00002050,-0.00000143,-0.00013178,-0.00010840,0.00005569,-0.00001014$ ,-0.00002815,0.00000132,0.00067571,0.00582946,-0.00169564,-0.00030877, $-0.00054558,0.00046829,-0.00095626,0.00498899,-0.00284431,0.00085526$, $0.00273390,-0.00660103,0.02532147,-0.08462982,-0.10694993,-0.00000404$, $0.00318708,-0.00446707,0.00010114,0.00002187,-0.00016859,0.00005407,-0$ $.00065580,0.00027896,0.00011931,-0.00005753,-0.00021130,-0.02576730,0$. $07849972,0.00076096,0.00016849,-0.00041299,-0.00031047,0.00016225,-0.0$ $0011855,0.00012995,0.00003945,0.00026617,-0.00006571,-0.00001184,-0.00$ $012923,0.00001138,0.00017312,-0.00005603,-0.00006254,-0.00017579,0.000$ $30349,-0.00063903,0.00008226,0.00032856,-0.00001658,0.00001773,-0.0000$ $2716,0.00001447,0.00001514,-0.00000951,0.00003147,0.00000890,0.0000625$ $5,0.00000487,-0.00003547,-0.00003387,0.00001166,-0.00003294,0.00000966$ $, 0.00111285,-0.00181104,0.00287436,-0.00054635,0.00048740,0.00026349,-$ $0.00257164,-0.00284674,-0.00005404,0.00218920,-0.00675260,-0.01480223$, $0.05460016,-0.10693161,-0.26811949,-0.00021313,-0.00396528,-0.00403314$ $, 0.00021451,-0.00014849,-0.00025244,0.00013609,0.00025680,-0.00015455$, $0.00038586,-0.00024949,-0.00043461,-0.05515064,0.11989321,0.28468342,0$ $.00005291,-0.00006302,-0.00022642,-0.00020493,-0.00001688,0.00007071,0$ $.00004841,-0.00004154,0.00010767,-0.00004673,-0.00005746,-0.00012749,-$ $0.00001143,0.00005804,-0.00005203,-0.00015201,0.00008244,0.00010064,0$. $00001028,-0.00003347,-0.00000654,-0.00000458,0.00004738,-0.00000154,-0$ $.00002061,0.00002666,-0.00004912,-0.00000186,-0.00003996,0.00002142,-0$ $.00001818,-0.00003334,0.00000635,-0.00002423,-0.00003247,-0.00000541,0$ $.00569234,0.00295033,0.00666371,0.00088230,0.00088957,0.00187236,-0.00$ $001397,0.00043825,0.00097155,-0.00436698,0.00087571,0.00154429,-0.0158$ $1887,-0.00806535,-0.01802835,-0.21185312,-0.05847173,-0.13243062,0.001$ $25536,0.00028073,0.00069302,0.00047858,0.00014404,0.00035102,-0.000059$ $67,-0.00003628,-0.00008785,0.00124961,-0.00007049,-0.00002734,0.222927$ $38,0.00001329,0.00007927,0.00004039,0.00009076,0.00000932,-0.00002519$, $-0.00006156,-0.00005597,0.00006093,0.00009085,0.00005482,-0.00001876,0$ $.00002475,0.00001015,0.00009168,0.00003824,0.00002390,-0.00003446,0.00$ $002004,-0.00001220,-0.00001488,0.00001480,0.00006788,0.00001858,-0.000$ $04347,0.00008862,-0.00009199,-0.00002337,-0.00007060,0.00003762,-0.000$ $01190,-0.00004560,-0.00003972,-0.00006026,-0.00007019,-0.00004694,-0.0$ $0982080,0.00009192,-0.00688950,0.00091485,0.00361152,-0.00368357,0.000$ $38042,-0.00084990,0.00026054,0.00105536,0.00546028,-0.00196940,0.00412$ $566,0.00512398,0.00199227,-0.05703072,-0.05946812,-0.05522588,-0.00020$ $311,-0.00201322,0.00140538,-0.00042704,0.00024492,-0.00038456,-0.00010$ $562,-0.00059721,0.00023059,-0.00015000,-0.00325795,0.00165654,0.061168$ $84,0.05157439,0.00019622,0.00005951,0.00002309,-0.00001669,-0.00003121$ 
,-0.00008864,0.00004195,0.00004460,0.00005529,0.00002169,0.00002820,0. $00004508,-0.00005376,-0.00002153,-0.00005972,0.00005570,-0.00005811,0$. $00002895,0.00000418,-0.00002579,0.00003565,0.00000962,-0.00000426,0.00$ $000954,0 .,-0.00000594,-0.00000365,0.00001468,0.00000488,0.00000971,0.0$ $0000206,0.00000958,-0.00000597,0.00000684,-0.00001465,0.00000371,-0.02$ $182012,-0.00668009,-0.01180065,0.00244071,-0.00375323,-0.00288358,0.00$ $066759,0.00024173,-0.00038543,0.00185609,-0.00196116,0.00205774,0.0082$ $6076,0.00163162,0.00813216,-0.12869359,-0.05514684,-0.15894044,-0.0003$ $3747,0.00142390,0.00040080,-0.00101284,-0.00040819,-0.00046963,-0.0000$ $2153,0.00027377,-0.00009464,-0.00026204,0.00176288,-0.00015386,0.13863$ $997,0.06263033,0.16408451 \backslash 0.00000040,-0.00000581,-0.00000150,0.000000$ $11,-0.00000197,0.00000782,-0.00000019,0.00000286,0.00000407,-0.0000003$ $6,0.00000053,0.00000012,0.00000029,-0.00000216,-0.00000188,0.00000135$, $-0.00000007,-0.00000109,0.00000057,-0.00000572,0.00000467,-0.00000088$, $0.00000017,0.00000787,-0.00000210,0.00000423,0.00000544,0.00000146,-0$. $00000240,-0.00000475,0.00000240,-0.00000639,-0.00000251,-0.00000091,0$. $00000307,-0.00000083,-0.00000047,0.00000248,-0.00000286,-0.00000071,0$. $00000462,-0.00000301,-0.00000057,0.00000270,-0.00000152,-0.00000116,0$. $00000009,0.00000072,0.00000090,-0.00000301,0.00000062,0.00000049,-0.00$ $000129,-0.00000130,-0.00000064,0.00000436,-0.00000491,-0.00000141,0.00$ $000729,-0.00000452,-0.00000078,0.00000473,-0.00000155,0.00000131,-0.00$ 000555,0.00000177,0.00000091,-0.00000276,-0.00000087III@

Addition Product

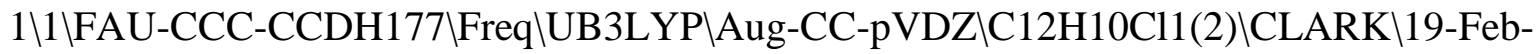
201610॥\#N Geom=AllCheck Guess=TCheck SCRF=Check GenChk UB3LYP/Aug-CCpVDZ Freq $\backslash p$-CHLOROPHENYL RADICAL addition product to benzenel $10,2 \backslash \mathrm{C}, 1$ $.3413277401,0.8256634308,-0.5862225046 \backslash \mathrm{C}, 2.140238401,-0.3937986826,-0$. 9841203803\C,3.2661514852,-0.7902605651,-0.3140564734\C,3.7574382313,$0.0665344056,0.8059811119 \backslash \mathrm{C}, 3.0650597804,1.0949956932,1.2431061338 \backslash \mathrm{C}, 1$ $.934501441,1.5350219359,0.6090215295 \backslash \mathrm{H}, 1.3499675271,1.5330006255,-1.44$ $14840101 \backslash \mathrm{H}, 1.7776036507,-0.9658677204,-1.8391470352 \backslash \mathrm{H}, 3.7993905798,-1$. 6828836836,-0.6463556362\H,3.4441125217,1.6477854123,2.1046652021\H,1. $4157212456,2.4268291586,0.9631207586 \backslash \mathrm{H}, 4.6545987027,-0.4000462655,1.32$ 56117104\C,-2.8334397117,-0.1507509894,0.0568821362\C,-2.4910808761,0. 7311433186,-0.9662942391\C,-1.1411736651,1.0411319691,-1.1673126875\C, $-0.1394559197,0.4840529711,-0.3638413104 \backslash \mathrm{C},-0.5165169172,-0.400358014$, $0.6579098843 \backslash \mathrm{C},-1.8569501927,-0.7229821709,0.8754356719 \backslash \mathrm{Cl},-4.53012741$ $75,-0.5530393468,0.3248567396 \backslash \mathrm{H},-3.2643208329,1.1685206298,-1.59541194$ $11 \backslash \mathrm{H},-0.8709677029,1.7315464921,-1.9680655121 \backslash \mathrm{H}, 0.2482681441,-0.845110$ $2238,1.2948608251 \mathrm{\downarrow H},-2.1443670851,-1.4098547223,1.6696672717 \backslash \backslash V e r s i o n=$ ES64L-G09RevD.01 $\backslash S t a t e=2-A \backslash H F=-923.5143174 \backslash S 2=0.786208 \backslash S 2-1=0 . \backslash S 2 A=0.7$ 50747\RMSD $=4.195 \mathrm{e}-09 \backslash \mathrm{RMSF}=2.454 \mathrm{e}-06 \backslash \mathrm{Z}$ eroPoint $=0.1796307 \backslash \mathrm{Thermal}=0.1904$ 408\ZPE $=112.7199395 \backslash$ Dipole $=0.7102321,0.1902657,-0.1386488 \backslash$ DipoleDeriv $=$ $0.0773535,0.0588281,-0.0581594,0.1227714,0.0780278,-0.1074389,-0.13564$ $69,-0.1157015,0.098772,-0.0946618,-0.0871581,0.0737144,-0.2400262,-0.0$ $686657,0.0801852,0.0883739,0.0363123,-0.0446942,-0.0773631,-0.0825741$, $0.0039856,0.0085204,0.0715114,0.1084562,0.0519483,0.0883966,-0.0215345$ $, 0.020206,-0.0736871,0.0994807,-0.0811276,-0.0699388,0.0023658,0.10850$ $81,0.0033252,-0.0493485,-0.0752744,-0.0123244,0.057421,-0.0747513,0.01$ 
$01768,0.1007809,-0.025292,0.1171374,0.0377141,-0.1162334,-0.0742347,0$. $0919106,-0.0542232,-0.0381714,0.0349106,0.238147,0.0959388,-0.0536489$, $0.0151424,0.0379663,-0.0531573,0.0223056,-0.0094605,0.1000981,-0.03418$ $87,0.1021167,-0.0572104,0.0708451,0.0030477,-0.068819,0.0095552,0.0241$ $159,-0.0833231,-0.0633724,-0.0735968,-0.0088703,0.0331711,0.0834621,0$. $0068638,0.1244878,-0.0305156,-0.0674978,-0.0172973,-0.0710135,0.068897$ $8,0.055505,-0.0122952,-0.0634502,0.0036638,0.0371773,-0.0938774,-0.108$ $2855,-0.0977165,-0.0211247,0.0551792,0.0857822,-0.007131,0.0781863,-0$. $0182619,-0.0560355,-0.0112624,-0.065618,0.0491698,-0.0310172,0.0602963$ ,-0.0832218,0.0695538,0.0631766,-0.0092349,-0.0944253,-0.0104309,0.048 $565,1.1805276,0.2552936,-0.1507841,0.3144784,0.0482074,-0.0728186,-0.2$ $22427,-0.080462,0.0231891,-0.2604602,-0.091412,0.0889846,-0.1200666,-0$ $.0851954,-0.0246401,0.123676,-0.0209388,-0.0514472,-0.0396358,0.027288$ $1,-0.0243932,-0.0418514,-0.0227264,-0.106948,0.0593023,-0.0980208,0.01$ $96698,0.3131069,0.0222393,0.0194993,0.0859277,-0.0573221,0.0243516,-0$. $0575657,0.0161354,-0.0733384,-0.1155654,-0.0253689,0.0264495,-0.020140$ $7,-0.0500397,-0.0421388,0.0201396,-0.0428054,-0.0282924,-0.2401455,-0$. $0032346,-0.0159696,-0.0234287,-0.0317113,-0.0687555,0.0084725,-0.06615$ $24,-0.0048712,-0.8828359,-0.1611295,0.0903662,-0.1886118,-0.1185131,0$. $0321258,0.1236391,0.0356744,-0.0990817,-0.0057799,0.0319057,-0.0559539$ $, 0.0258159,0.0945486,0.0445295,-0.0485814,0.0453162,0.0674748,0.052725$ $6,-0.0525406,0.0552072,-0.0340028,0.0361638,0.0943531,0.0327655,0.0919$ $61,0.0106704,0.014159,0.0383196,-0.057562,0.0465726,0.0740589,0.038437$ $3,-0.0675676,0.0373651,0.0469035,0.0510513,-0.0284697,0.0247183,-0.033$ 6086,0.0633573,0.0721146,0.03094,0.0727774,0.042436\Polar=223.109878,$0.2642962,127.2638594,13.4605161,-7.2206818,131.7975298 \mathrm{PG}=\mathrm{C} 01[\mathrm{X}(\mathrm{C} 12 \mathrm{H}$ 10Cl1)]\NImag=0\10.42231590,-0.02235533,0.48233697,0.02136027,-0.02206 $231,0.49596116,-0.10130280,0.05207172,0.01098815,0.51964719,0.04655811$ ,-0.15574142,-0.03743941,-0.12717110,0.41944844,0.00445446,-0.03234647 ,-0.09009627,0.24023594,0.15076428,0.48540324,-0.01390867,0.01581541,-$0.00668040,-0.27981057,0.08269315,-0.13367780,0.50314158,0.02375753,-0$ $.00335736,0.00982801,0.11344436,-0.12467614,0.03582307,-0.22096559,0.5$ $2227930,0.00153421,0.00398594,0.01040770,-0.10696274,0.00835083,-0.142$ $17111,0.14878068,0.16777937,0.41345611,-0.00084364,-0.00337909,0.00500$ $851,-0.01612436,-0.01235790,-0.03412763,-0.11026419,-0.02570862,-0.075$ $65190,0.51113625,-0.00341629,-0.00753045,-0.00094941,-0.03940210,0.027$ $87937,-0.01884913,0.02906283,-0.14148469,-0.05388341,-0.17755596,0.418$ $35327,0.00505326,-0.00094492,-0.00615372,-0.05955043,0.00569280,-0.039$ $98080,-0.02490025,-0.10407662,-0.17330895,0.21096884,0.12683094,0.4157$ $3465,-0.00890717,0.00001234,-0.01714031,-0.02433648,0.00939099,-0.0120$ $5653,0.04526339,0.00168422,0.03880293,-0.12341630,0.03828878,-0.028415$ $75,0.48249110,-0.00893670,0.01174018,-0.00201199,0.02109748,-0.0084684$ $5,0.01315488,-0.02411770,-0.02616813,-0.05153664,0.09321391,-0.1868683$ $6,-0.03718303,-0.12039591,0.45117137,-0.02413697,0.00396138,-0.0096900$ $9,-0.00127392,0.00228459,-0.00108536,0.01485275,-0.02679589,-0.0064386$ $5,0.02214911,-0.08739637,-0.11477939,0.22279021,0.19395999,0.50521803$, $-0.08911840,-0.01376141,-0.03806621,0.02257990,-0.00011408,0.02083964$, $-0.02120883,-0.00579850,-0.02331234,-0.03190497,0.06476866,0.02213008$, $-0.27560323,0.06303649,-0.14974835,0.53934374,-0.00684475,-0.10888227$, $-0.04865060,-0.01269324,-0.01625593,-0.02778269,0.00586295,0.00197155$, $0.00925088,0.03726883,-0.02701367,0.00123361,0.09221087,-0.11089116,0$. 
$03052495,-0.22294810,0.48375812,-0.03322493,-0.05392430,-0.14914170,0$. $00897858,-0.01400418,-0.00560755,-0.01247455,-0.00161398,-0.01465298,-$ $0.00274154,0.02583476,0.03069292,-0.12112407,0.00325657,-0.16016171,0$. $16938272,0.12647119,0.40139403,-0.03350039,0.00240137,-0.00152508,-0.0$ $0382623,0.01484667,-0.01230203,0.00039575,0.00158782,0.00201569,-0.000$ $88470,0.00022432,-0.00058995,0.00062562,-0.00172147,-0.00067498,-0.002$ $48107,0.00608467,-0.01375866,0.05422783,-0.00508427,-0.13581604,0.1102$ $5840,0.00746057,-0.01936330,0.01800445,-0.00201619,-0.00370123,-0.0033$ $4048,0.00058247,0.00055618,0.00131734,-0.00116623,-0.00084544,-0.00138$ $357,0.00019697,0.01263285,-0.00976115,0.00142031,0.15133591,0.00753789$ $, 0.11122436,-0.17788924,-0.00266754,-0.00193438,0.00962847,0.00186136$, $-0.00049828,0.00043445,-0.00102375,0.00127119,-0.00008884,0.00317080$, $0.00383216,-0.00265105,-0.00732326,0.01189209,-0.02371344,-0.00102809$, $-0.12385699,0.19870206,0.00536927,0.00807128,0.01335752,-0.07822654,-0$ $.04028421,-0.08157731,-0.00347834,-0.01411112,-0.02114666,-0.00122199$, $0.00466396,-0.00384632,-0.00054912,0.00018169,0.00027898,-0.00157522,0$ $.00321994,-0.00001305,0.00041925,0.00022691,-0.00042734,0.07838194,-0$. $00882526,-0.00943266,-0.01878896,-0.04176975,-0.12503239,-0.12613378,0$ $.00540674,0.00823257,0.00739021,0.00413955,0.00266392,-0.00093568,0.00$ $007338,-0.00047620,0.00038484,0.00425632,-0.00260971,-0.00010977,-0.00$ $055377,0.00082394,0.00056725,0.04048146,0.12667441,-0.00293234,-0.0015$ $4173,-0.00305533,-0.08250680,-0.12382874,-0.21925063,-0.00361784,-0.01$ $045131,-0.00745507,-0.00476329,-0.00043562,0.00339146,0.00035905,0.000$ $49860,0.00001522,0.00072764,-0.00126250,0.00150311,0.00031771,-0.00090$ $362,0.00018731,0.09112268,0.13577168,0.22660421,0.00038911,0.00261644$, $-0.00581205,-0.00927230,0.02230133,0.00861770,-0.11665466,0.11615006,0$ $.03447747,0.00813525,-0.00905609,-0.00364961,0.00151409,-0.00020934,-0$ $.00358508,-0.00046834,-0.00042704,-0.00022892,-0.00074516,0.00110326,0$ $.00048931,-0.00104007,-0.00215175,0.00214864,0.11808279,0.00282982,0.0$ $0476075,-0.00188964,0.00349074,-0.00471646,-0.00427528,0.11688698,-0.2$ $3642689,-0.07625590,0.00905062,-0.00892126,-0.00249699,-0.00060338,0.0$ $0157572,-0.00393017,-0.00053966,0.00021384,0.00024354,-0.00023785,0.00$ $045927,0.00032397,-0.00193922,-0.00095539,0.00231412,-0.12874413,0.245$ $92055,-0.00509082,-0.00288006,0.00241584,-0.00837330,0.01330162,0.0082$ $0943,0.03439565,-0.07652248,-0.07644896,0.01325988,-0.01924984,-0.0026$ $1177,-0.00387510,-0.00343352,-0.00156752,-0.00044625,0.00037056,-0.000$ $57350,0.00031949,-0.00040823,-0.00032697,0.00220310,0.00215649,-0.0014$ $5033,-0.03363746,0.08495640,0.07281987,-0.00002448,0.00508512,-0.00390$ $035,-0.00064001,0.00024031,0.00023754,0.00051846,0.00428578,-0.0003534$ $5,0.00844918,0.00759402,0.01204760,-0.08409093,-0.04214314,-0.08182566$ ,-0.00566221,-0.01393056,-0.02151392,-0.00053206,-0.00076937,-0.001057 $65,-0.00016151,-0.00031244,0.00029608,0.00027206,0.00047989,0.00067462$ $, 0.08294218,0.00444324,0.00283421,-0.00228669,0.00046098,-0.00033274,0$ $.00058653,0.00459356,-0.00177995,-0.00219074,-0.01067522,-0.00766578,-$ $0.01969177,-0.04217928,-0.12305781,-0.11799979,0.00446967,0.00848388,0$ $.00855041,-0.00026691,-0.00044182,-0.00041807,-0.00034510,-0.00018014$, $0.00024658,-0.00054037,-0.00057227,-0.00101277,0.04250640,0.12332288,-$ $0.00410268,-0.00124092,0.00475573,0.00032412,0.00044564,0.00014473,0.0$ $0004095,-0.00267633,0.00278392,-0.00466443,-0.00291775,-0.00418052,-0$. $08259344,-0.11782363,-0.22238266,-0.00402593,-0.00897369,-0.00860150,0$ $.00012753,0.00042245,0.00036104,0.00023387,0.00022938,-0.00033936,-0.0$ 
$0025838,-0.00016630,-0.00031533,0.09222302,0.12981384,0.23055881,0.005$ $75303,-0.01094311,-0.00400841,-0.00057384,-0.00073662,-0.00272264,-0.0$ $0043311,-0.00041063,-0.00015529,-0.00091819,0.00243861,-0.00558565,-0$. $00699934,0.02233194,0.00927829,-0.11081108,0.11715246,0.03406195,0.000$ $36685,0.00032765,-0.00025304,0.00065458,-0.00056817,-0.00028299,-0.000$ $18481,-0.00017889,0.00035532,-0.00104629,-0.00204872,0.00208291,0.1130$ $7980,0.00643525,-0.00798169,-0.00202213,-0.00154052,0.00081932,-0.0025$ $1329,-0.00047957,0.00006193,0.00031334,0.00338290,0.00390964,-0.000832$ $02,0.00329771,-0.00417786,-0.00590172,0.11810753,-0.23600536,-0.082790$ $48,-0.00009423,0.00017332,-0.00082450,0.00055148,-0.00046682,-0.000230$ $67,-0.00024770,-0.00029747,0.00028091,-0.00197069,-0.00108013,0.002333$ $20,-0.12845407,0.24465948,0.01169782,-0.01933111,-0.00489002,-0.003744$ $62,-0.00139059,-0.00292718,-0.00002076,0.00022248,-0.00063881,-0.00517$ $712,-0.00138632,0.00184162,-0.00883611,0.01187778,0.00847629,0.0356336$ $8,-0.08502776,-0.07569164,0.00045821,0.00080158,0.00088980,0.00075417$, $-0.00128420,-0.00014411,0.00033689,0.00026839,-0.00019874,0.00229995,0$ $.00219297,-0.00131928,-0.03335808,0.09207175,0.07392015,0.00006333,-0$. $00060780,0.00077635,0.00193533,-0.00049443,-0.00215797,-0.00431089,0.0$ $0515765,-0.00226136,-0.24105390,0.08197176,-0.12291822,-0.00730593,0.0$ $0142981,-0.00847464,0.00125356,0.00318709,0.00060185,0.00015802,0.0000$ $2979,-0.00002160,-0.00029474,0.00025207,-0.00015568,-0.00028944,-0.001$ $11564,0.00094751,-0.00026212,-0.00091042,0.00116863,-0.00046933,0.0001$ $3918,-0.00041986,0.25052080,-0.00048877,-0.00047499,0.00020943,-0.0002$ $1156,-0.00045119,-0.00418635,-0.01316969,0.00827794,-0.00756874,0.0820$ $3020,-0.07429086,0.03067118,0.01977270,-0.00234114,0.01304794,0.002758$ $33,-0.00288425,-0.00356083,0.00010511,0.00005384,0.00000408,-0.0006985$ $5,0.00028055,-0.00049491,-0.00078734,-0.00018585,0.00142646,-0.0012652$ $5,-0.00003556,0.00106648,0.00111455,-0.00031282,0.00061748,-0.08912113$ $, 0.07222135,0.00063224,0.00019402,-0.00040311,-0.00174847,-0.00443216$, $-0.00197121,-0.01920285,0.00919087,-0.00593857,-0.12298849,0.03066328$, $-0.11071258,0.00844758,-0.00371340,0.00767621,0.00036908,-0.00329617,0$ $.00114371,-0.00011280,-0.00000563,0.00003826,-0.00106126,0.00039605,-0$ $.00048305,0.00127917,0.00111368,-0.00025529,0.00086901,0.00138276,-0.0$ $0043305,0.00045577,-0.00027666,0.00028492,0.13302278,-0.03120018,0.110$ $90825,-0.00100637,0.00012438,-0.00022272,-0.00007613,0.00010618,0.0000$ $3718,0.00001570,-0.00010560,0.00010933,0.00005485,-0.00002605,0.000044$ $70,0.00001702,-0.00007454,0.00012929,-0.00006791,-0.00006254,-0.000109$ $16,-0.00004340,0.00003688,-0.00006866,-0.00007487,0.00004955,0.0000610$ $3,0.00009170,-0.00005058,-0.00000761,0.00008276,0.00002028,0.00005837$, $-0.00005083,-0.00007235,-0.00004512,0.00005020,-0.00002778,0.00003608$, $0.50575832,0.00037073,-0.00061261,0.00024681,0.00025283,0.00032470,-0$. $00025763,0.00008664,-0.00001075,-0.00011380,-0.00005507,0.00007361,-0$. $00015291,0.00004055,0.00002008,-0.00003473,0.00035759,0.00019987,-0.00$ $029305,0.00016490,0.00022673,-0.00007976,0.00003567,-0.00003155,0.0000$ $4744,-0.00008015,-0.00007399,0.00009856,-0.00007303,-0.00001908,0.0000$ $7381,-0.00006215,-0.00008871,0.00005137,0.00002972,0.00001156,0.000027$ $72,0.00059153,0.33576003,-0.00052068,0.00021538,-0.00077553,-0.0004129$ $5,-0.00034842,0.00027219,-0.00005387,-0.00004778,0.00005780,0.00007972$ $,-0.00014920,0.00014394,-0.00007934,-0.00014037,0.00002586,-0.00034499$ $,-0.00027548,0.00038942,-0.00022358,-0.00009612,0.00023108,0.00005267$, $0.00005499,-0.00009327,0.00009508,0.00008684,-0.00004519,0.00010434,0$. 
$00010134,-0.00009120,-0.00003219,0.00004397,-0.00006022,-0.00003354,0$. $00002031,0.00000238,0.04835436,-0.25446727,0.43966125,0.00055181,0.001$ $24143,-0.00237745,-0.00062000,-0.00057627,0.00025561,-0.00010326,0.000$ $08116,0.00009683,0.00016445,-0.00010689,0.00015980,-0.00007845,-0.0002$ $0040,-0.00011245,-0.00091759,-0.00016439,0.00058062,-0.00009639,-0.000$ $27090,0.00032085,-0.00005344,0.00008825,-0.00007887,0.00007474,0.00008$ $258,-0.00008078,0.00007196,0.00009604,-0.00010747,-0.00005646,0.000058$ $90,-0.00010448,-0.00001852,0.00000288,-0.00000063,-0.11586968,-0.00800$ $056,0.00307168,0.69928456,0.00100288,0.00347444,0.00480904,-0.00119321$ ,-0.00039491,-0.00023606,-0.00015110,0.00022892,0.00033827,0.00007511, $-0.00013837,0.00009046,-0.00011500,0.00034431,0.00021530,0.00119102,-0$ $.00040533,-0.00009620,-0.00004143,-0.00001215,-0.00003960,0.00000019,-$ $0.00005127,-0.00008119,0.00004414,0.00010803,-0.00016052,0.00007636,-0$ $.00007100,0.00004614,0.00004646,0.00004257,0.00000918,-0.00001652,-0.0$ $0000514,-0.00004544,-0.04880039,-0.17415086,0.12624001,0.00590153,0.36$ $099719,-0.00208952,0.00484033,0.00121311,-0.00100115,-0.00032660,-0.00$ $043595,0.00006345,0.00026206,0.00026352,-0.00006037,0.00006697,-0.0001$ $6055,0.00009820,0.00031071,0.00012335,0.00094447,-0.00010107,-0.000128$ $07,0.00004300,-0.00006948,0.00004918,-0.00006046,0.00003208,0.00004064$ ,-0.00007149,0.00001380,-0.00006908,-0.00004636,-0.00012068,0.00011291 ,-0.00000075,-0.00009088,-0.00005029,0.00002291,-0.00004294,0.00001402 $, 0.05245266,0.13150457,-0.21703286,0.06626819,-0.27845740,0.47585712,-$ $0.02712274,0.00913036,-0.01515033,-0.00139775,-0.00154275,-0.00189041$, $0.00002567,0.00099718,0.00059351,-0.00018098,0.00006334,-0.00009086,0$. $00023189,-0.00078148,-0.00085532,-0.00172795,0.00202493,0.00099690,0.0$ $0079714,0.00247501,-0.00287634,-0.00004305,0.00028545,0.00046120,-0.00$ $030021,0.00010384,-0.00016079,-0.00031435,0.00008939,-0.00015302,0.000$ $02118,-0.00051606,-0.00022582,-0.00004826,-0.00004269,0.00004068,0.001$ $46078,-0.05799169,0.06947511,-0.32007368,-0.02707871,-0.00048354,0.665$ $04501,-0.00463942,0.00627380,-0.00255542,-0.00114378,-0.00050207,-0.00$ $011690,-0.00004482,0.00037354,0.00015997,-0.00008675,0.00003222,-0.000$ $13684,-0.00016129,0.00001114,-0.00032888,-0.00158510,-0.00066229,0.000$ 29827,-0.00154193,0.00045727,-0.00090767,-0.00005771,0.00017198,-0.000 $00905,-0.00009599,0.00008002,-0.00003686,0.00004763,0.00011045,-0.0001$ $9519,0.00015561,0.00005749,0.00013164,-0.00004194,-0.00000721,0.000048$ $12,-0.02213113,-0.00001177,0.00561017,-0.06920987,-0.09767667,0.033874$ 49,0.04645704,0.37682821,0.00151445,-0.00077901,0.00579399,0.00168930, $0.00027403,-0.00077498,0.00020828,-0.00024671,0.00014077,0.00009089,-0$ $.00011732,0.00008246,0.00003987,0.00013199,0.00029688,0.00130440,0.000$ $32170,-0.00028295,0.00198531,-0.00039001,0.00059932,-0.00017354,0.0001$ $2711,0.00007584,-0.00006254,-0.00018230,0.00013038,0.00004132,-0.00001$ $851,0.00011397,0.00001114,-0.00004695,0.00012662,0.00003971,0.00004805$ - $0.00001601,0.02607221,0.00098344,0.00915586,0.05050930,0.03931085,-0$ $.10337000,0.01215830,-0.28669155,0.48355291,-0.15075557,-0.02651142,0$. $02137110,-0.02897217,0.01101560,0.00562562,0.00141268,-0.00081153,0.00$ $345279,-0.00110589,0.00051496,-0.00088722,0.00161856,-0.00257753,0.002$ $02965,-0.02233354,-0.01045613,-0.01327455,-0.00921163,-0.00232510,0.00$ $155071,0.00197700,-0.00099025,-0.00129206,0.00058809,0.00026120,-0.000$ $30604,0.00056445,0.00017807,-0.00008447,0.00170878,0.00157795,0.000875$ $72,0.00018182,0.00000544,0.00000374,-0.00318630,0.01178271,-0.01377401$ ,-0.04759772,0.00003293,-0.00706697,-0.22171148,0.07500172,-0.11090782 
$, 0.60061665,-0.01715039,-0.07645702,0.00843019,0.02537645,0.00814015,-$ $0.00149071,0.00034429,-0.00406062,-0.00256131,0.00012118,0.00064003,0$. $00037303,-0.00013718,-0.00108322,-0.00043927,-0.01419397,-0.00314821,0$ $.00011113,-0.02147404,-0.00746251,0.00695583,0.00010484,0.00018374,0.0$ $0015725,0.00010738,-0.00098888,0.00115185,0.00025880,0.00044911,-0.000$ $38111,-0.00002462,0.00086222,0.00028115,0.00009466,0.00013699,-0.00003$ $045,0.01247869,-0.03094056,0.03063318,0.02982567,0.02874967,-0.0226363$ $4,0.03273883,-0.12260703,0.07313659,0.01277452,0.37155315,0.01003578,0$ $.00722177,-0.07510528,0.00630281,0.00127308,-0.00065403,0.00044411,0.0$ $0002607,-0.00006018,-0.00040999,0.00042372,0.00036879,0.00068342,-0.00$ $349067,-0.00324330,-0.02680842,-0.00402399,0.00399577,0.02473414,0.009$ 42837,-0.00764636,0.00011903,0.00028236,0.00061295,-0.00022314,-0.0001 $1411,0.00024432,-0.00007882,0.00089425,-0.00116993,0.00007855,0.000221$ $55,0.00020212,-0.00010426,-0.00004199,0.00013911,-0.01461590,0.0305438$ $6,-0.04581291,-0.04312482,-0.02647970,0.03335153,-0.05975691,0.0785883$ $5,-0.16849457,0.03958889,-0.23748990,0.46650457,-0.01228761,-0.0202916$ 4,0.02272648,0.00099317,-0.00272569,0.00074599,-0.00029026,0.00016726, $0.00014303,0.00002642,-0.00008243,0.00015642,-0.00019610,-0.00016321,-$ $0.00022839,0.00076044,-0.00014128,0.00282264,-0.00572201,-0.00221313,0$ $.00179888,-0.00010854,0.00017046,0.00007449,0.00008419,0.00012899,-0.0$ $0014294,0.00007984,0.00011007,-0.00014844,-0.00007624,-0.00015705,-0.0$ $0019821,-0.00004742,-0.00000619,-0.00000707,-0.05223868,0.03298649,-0$. $04759176,-0.03151455,-0.02231815,0.02333049,0.05161156,0.00762050,-0.0$ $0353620,-0.13261535,-0.01324889,0.00757724,0.70618651,-0.00735387,-0.0$ $0232476,0.00418119,0.00048731,-0.00046820,-0.00023422,0.00054658,0.000$ $14738,0.00003227,0.00016439,-0.00047209,0.00009484,0.00004993,-0.00039$ $587,0.00050788,-0.00003254,0.00067679,0.00125625,-0.00074043,0.0007235$ $8,0.00035716,-0.00012323,0.00038749,0.00025355,-0.00000563,0.00002408$, $0.00005828,0.00004768,0.00009479,0.00002002,-0.00006140,0.00003995,-0$. $00001455,0.00001314,0.00006085,0.00000703,-0.00272263,0.02972226,-0.02$ $761107,-0.02214411,-0.01902708,0.01656615,0.03573933,-0.01252655,0.028$ $87644,-0.05200591,-0.16742331,0.11474470,0.01435168,0.35818991,0.00706$ $829,0.00251156,-0.00021391,-0.00014959,0.00112097,0.00057000,-0.000192$ $31,0.00042252,-0.00049023,-0.00014265,0.00006260,-0.00050597,-0.000609$ $86,0.00004141,-0.00004091,-0.00030316,-0.00052756,-0.00034195,0.000016$ $40,0.00016722,0.00089703,0.00006734,-0.00004894,0.00001210,-0.00005062$ $, 0.00004369,0.00005640,-0.00000218,0.00006011,-0.00000975,0.00004909,0$ $.00031381,0.00032729,-0.00003040,0.00000456,0.00005586,-0.00437343,-0$. $02300471,0.03447702,0.02312015,0.01654372,-0.02001972,-0.03756748,0.02$ $524900,-0.02788226,0.05448595,0.11974466,-0.20496832,0.05629856,-0.268$ $02709,0.46685698,0.00331089,-0.00024699,0.00001849,0.00036118,0.000237$ $41,-0.00047276,0.00004975,0.00023278,-0.00023937,-0.00005377,0.0000772$ $1,-0.00010828,0.00004430,0.00018784,-0.00030637,0.00027211,0.00047253$, $-0.00027141,0.00039027,0.00036669,-0.00040879,-0.00002332,-0.00008226$, $0.00012640,-0.00018539,-0.00004453,0.00006408,-0.00018316,-0.00006263$, $0.00003043,-0.00002946,-0.00011711,0.00012282,-0.00001574,0.00000442,-$ $0.00000218,-0.19921159,0.03924420,-0.06483841,0.05091388,0.03499275,-0$ $.03693156,-0.06070638,0.00657543,-0.01533132,0.00172264,-0.05302437,0$. $06346700,-0.32655135,-0.03192588,0.00441702,0.66189341,-0.00015949,0.0$ $0114659,0.00523262,0.00016208,-0.00024163,-0.00020249,-0.00010692,0.00$ $028795,-0.00007944,-0.00008284,0.00005797,-0.00000649,0.00000859,0.000$ 
$10558,0.00004761,0.00071715,0.00018243,0.00007189,-0.00081573,-0.00021$ $056,0.00028945,-0.00009252,-0.00005919,0.00007299,-0.00002777,0.000003$ $07,-0.00005893,-0.00002125,-0.00008922,0.00004076,-0.00000344,-0.00001$ $649,-0.00012742,0.00000496,-0.00003519,-0.00000541,0.08035241,-0.12557$ $951,0.08392304,0.00632315,-0.01111709,0.02504697,0.00761146,-0.0036849$ $6,0.00114167,-0.02299455,0.00184707,0.00352898,-0.07442789,-0.09935682$ $, 0.03644068,0.05250996,0.38259637,-0.00008748,0.00522073,-0.00084345,-$ $0.00073985,0.00008846,0.00006810,-0.00000113,0.00004261,0.00007901,0.0$ $0008553,0.00001432,0.00005539,0.00008354,-0.00000749,0.00026681,-0.000$ $20845,-0.00024073,-0.00026702,0.00102219,0.00044201,-0.00028747,0.0000$ $3329,-0.00012962,0.00001210,0.00000989,0.00002061,-0.00007588,0.000014$ $25,-0.00004635,0.00001417,0.00009023,0.00006180,-0.00002446,-0.0000028$ $7,-0.00000550,-0.00003393,-0.11459139,0.07861930,-0.17474096,-0.002233$ $32,0.02874598,-0.02617478,-0.01658606,0.00100791,-0.00691274,0.0271214$ $1,-0.00034473,0.01113142,0.05585885,0.04192431,-0.10484666,0.00496207$, $-0.29789529,0.49204245,0.00024894,0.00028191,-0.00021318,-0.00030729,-$ $0.00063783,-0.00009730,-0.00007859,0.00021017,0.00009699,-0.00001009,-$ $0.00003042,0.00003492,-0.00004039,-0.00014502,-0.00018437,-0.00038106$, $0.00016407,0.00056947,-0.00001470,-0.00001535,0.00001651,0.00007764,0$. $00000037,-0.00001207,-0.00005257,0.00011710,-0.00007001,-0.00004360,0$. $00003980,-0.00013200,0.00007502,0.00002082,0.00000413,-0.00005247,0.00$ $002330,-0.00003456,-0.14229408,-0.02540576,0.01544984,-0.02796335,-0.0$ $0734005,0.00470769,0.00313664,-0.00199854,0.00219141,-0.00121643,-0.00$ $084093,0.00095032,0.00139272,0.00395243,-0.00519245,-0.04113911,-0.010$ $86638,0.00726635,0.20903410,0.00003219,-0.00031373,-0.00051853,-0.0000$ $3588,-0.00013708,0.00000225,-0.00002349,0.00002788,0.00004988,-0.00000$ $523,-0.00000547,0.00001203,-0.00001996,-0.00003355,-0.00011376,-0.0001$ $7110,0.00008929,0.00014723,0.00004915,0.00003478,-0.00003394,0.0000154$ $7,0.00000496,0.00000321,-0.00001099,0.00002634,-0.00001359,-0.00001637$ $, 0.00001365,-0.00004176,0.00001971,0.00000404,0.00000918,-0.00001621,0$ $.00000188,-0.00000841,-0.02542303,-0.03604020,0.01162499,-0.02450644,-$ $0.00179648,0.00434089,0.00212986,0.00120902,0.00474070,-0.00059143,0.0$ $0018792,-0.00114043,-0.00028213,0.00189773,0.00318676,0.00642594,0.005$ $44543,-0.00034610,0.04264829,0.03156697,0.00008930,-0.00048634,-0.0001$ $4544,0.00011275,0.00012894,0.00006524,0.00003086,-0.00009544,-0.000005$ 47,0.00000437,0.00000876,-0.00000501,0.00001331,0.00004560,0.00001770, $0.00003623,-0.00001390,-0.00008747,-0.00006158,-0.00004222,0.00004466$, $-0.00001427,0.00000651,-0.00000204,0.00001451,-0.00002687,0.00001719,0$ $.00000657,-0.00000468,0.00002092,-0.00001059,0.00000407,0.00000150,0.0$ $0001329,-0.00000331,0.00000238,0.01547086,0.01162729,-0.03580100,0.025$ $48397,0.00655581,-0.00024376,-0.00280513,0.00420807,-0.00055592,0.0006$ $4815,-0.00117265,0.00081157,-0.00006732,0.00373305,0.00003396,-0.01366$ $240,-0.00257715,0.00583090,-0.02618125,-0.01830486,0.03067228,-0.00025$ $752,0.00058110,-0.00074579,-0.00019051,-0.00014615,-0.00006063,-0.0000$ $0015,0.00006479,0.00006237,-0.00003533,0.00000694,-0.00001084,0.000016$ $10,-0.00007149,-0.00005315,-0.00022294,0.00004447,0.00011814,0.0000594$ $9,0.00001251,-0.00000636,-0.00000738,0.00002622,0.00001518,-0.00001520$ $, 0.00002539,-0.00001914,-0.00001048,0.00001363,-0.00003165,-0.00000132$ ,-0.00001969,-0.00002196,-0.00000938,-0.00000001,-0.00000090,0.0065331 $6,-0.00506477,0.00653693,-0.19896064,0.08198250,-0.12035577,-0.0148814$ $6,0.01273761,-0.01780638,-0.00389662,-0.00252790,0.00166543,-0.0003592$ 
6,-0.00048395,0.00069664,0.00072980,-0.00177364,0.00152948,0.00121927, $-0.00016396,0.00071002,0.20879786,-0.00030544,0.00016805,-0.00015502,-$ $0.00008512,-0.00006198,0.00008291,0.00000283,0.00002596,-0.00001288,-0$ $.00000037,-0.00001039,-0.00000741,-0.00001028,-0.00000180,-0.00000813$, $0.00001417,0.00008026,0.00000008,0.00000869,-0.00000587,-0.00000216,0$. $00000323,-0.00000187,0.00000190,0.00001431,0.00001206,-0.00000974,-0.0$ $0001641,-0.00000479,0.00000272,-0.00000691,-0.00000847,-0.00001103,-0$. $00000134,-0.00000307,0.00000077,0.01485731,-0.00383668,0.01064870,0.08$ $014088,-0.09045470,0.07726043,-0.00603930,0.00639955,-0.00411503,-0.00$ $219960,0.00423418,0.00295222,-0.00057830,-0.00086932,-0.00049776,-0.00$ $217482,0.00118521,0.00495753,0.00052853,-0.00114578,-0.00186899,-0.085$ $38162,0.08694291,0.00032708,-0.00004070,0.00016396,-0.00003356,0.00000$ $576,0.00005553,0.00001097,-0.00000359,0.00001136,-0.00000201,-0.000006$ $48,-0.00000889,-0.00000084,-0.00001733,0.00002287,0.00005374,0.0000732$ $9,-0.00005420,-0.00000173,-0.00000167,-0.00000681,0.00000770,-0.000007$ $99,-0.00000393,0.00001232,0.00000499,-0.00000425,-0.00001330,-0.000006$ $70,0.00000788,-0.00000212,0.00000017,-0.00000337,0.00000071,0.00000094$ ,-0.00000334,-0.01757462,0.00807820,-0.00866661,-0.11812693,0.07749801 $,-0.14419762,0.00491923,-0.00169233,0.00632629,0.00126833,0.00290981,0$ $.00354981,0.00081085,-0.00048560,-0.00052665,0.00201487,0.00500935,-0$. $00028959,-0.00012800,-0.00195823,-0.00039280,0.12716541,-0.08706655,0$. $14588910,0.00119969,0.00157037,-0.00121949,-0.00004620,-0.00017329,0.0$ $0002567,-0.00003944,-0.00001307,-0.00006344,0.00005239,-0.00004656,0.0$ $0006079,-0.00004489,0.00009499,0.00002226,0.00005467,-0.00005932,0.000$ $17534,-0.00013456,-0.00037552,0.00040923,-0.00000684,0.00000851,-0.000$ $01802,0.00001647,0.00000343,-0.00001497,0.00001770,0.00000836,0.000004$ $78,0.00000170,0.00002279,0.00000269,0.00001298,0.00000068,0.00000181,-$ $0.00394829,-0.00102308,-0.00016436,-0.00398727,-0.01945232,0.02258018$, $-0.07329228,-0.04421608,0.04874201,0.00788231,0.01313806,-0.01539493,-$ $0.00265347,0.00164092,-0.00312960,-0.00106635,0.00023130,-0.00028457,-$ $0.00122010,-0.00087432,0.00087991,0.00102578,0.00045056,0.00012406,0.0$ $7572828,-0.00097560,-0.00225694,-0.00233489,0.00062247,0.00012425,0.00$ $015483,0.00001050,-0.00010716,-0.00014344,0.00000223,0.00000793,0.0000$ $1280,-0.00003993,-0.00017467,-0.00016613,-0.00047468,0.00046675,-0.000$ $08426,0.00057422,0.00050693,-0.00028200,0.00005815,-0.00010519,0.00000$ $892,-0.00001979,-0.00004305,0.00005829,-0.00004314,0.00002060,-0.00003$ $619,-0.00004139,-0.00005398,-0.00003207,-0.00000178,-0.00000037,-0.000$ $00879,-0.00125549,0.00484928,0.00234804,-0.00009024,-0.00080881,0.0051$ $7617,-0.04387940,-0.15556423,0.13869699,-0.00511871,-0.00422823,0.0092$ $5345,0.00226312,0.00330558,0.00380839,0.00033830,-0.00031923,-0.000817$ $32,-0.00016098,-0.00005675,0.00024352,0.00024429,-0.00215863,-0.002393$ $79,0.04831163,0.15733621,0.00186206,-0.00200719,-0.00141309,0.00030757$ $, 0.00001819,0.00050884,0.00007362,-0.00017518,-0.00011894,0.00000172,0$ $.00000646,0.00001067,0.00002013,-0.00012013,-0.00004616,-0.00056731,0$. $00000359,0.00006523,-0.00073974,-0.00040374,0.00060694,0.00004059,-0.0$ $0001614,-0.00004713,0.00005063,-0.00001839,0.00001967,0.00002961,0.000$ $05014,-0.00004523,-0.00003827,-0.00000522,-0.00010695,0.00000479,-0.00$ $000847,0.00000342,0.00011689,0.00237805,0.00405999,-0.00085412,0.00267$ $794,0.00012625,0.04833476,0.13865380,-0.19786902,0.00670093,0.01160899$ ,-0.00922332,-0.00388275,0.00372782,0.00119804,-0.00041393,-0.00083111 ,-0.00007806,0.00001658,0.00015148,-0.00001960,0.00037370,-0.00236715, 
$-0.00124930,-0.05196527,-0.15358285,0.20446959,-0.00026512,-0.00012263$ $, 0.00051485,0.00006691,-0.00032512,0.00012715,-0.00021444,0.00005962,-$ $0.00002312,-0.00000024,0.00002264,-0.00006878,-0.00022826,-0.00003448$, $-0.00002045,0.00007869,-0.00005743,0.00032570,0.00014759,0.00014891,-0$ $.00014302,-0.00000146,0.00000576,-0.00007160,-0.00001210,-0.00001550,0$ $.00000015,-0.00000207,0.00000939,0.00001758,-0.00000141,0.00009504,-0$. $00002694,0.00001524,0.00000386,0.00000058,-0.00313261,-0.00221575,0.00$ $133956,-0.00025626,-0.00044965,0.00066293,0.00058638,-0.00179988,0.001$ $42333,0.00936177,-0.00364165,0.00513159,-0.19371747,0.07925932,-0.1161$ $8585,-0.01628197,0.01264680,-0.01791015,-0.00146279,0.00020091,-0.0004$ $4292,-0.00001273,0.00009562,-0.00002297,0.00039314,-0.00031810,0.00042$ $234,0.20401172,0.00090789,-0.00152323,-0.00179264,0.00020506,0.0001959$ $1,0.00003052,-0.00023334,-0.00017457,0.00009802,-0.00010552,0.00021426$ $, 0.00011377,-0.00003528,0.00008740,-0.00043963,-0.00014304,-0.00003045$ $, 0.00004607,0.00011454,-0.00001542,-0.00013661,0.00003343,-0.00002034$, $-0.00012837,0.00006815,-0.00000568,-0.00006906,-0.00005125,-0.00000043$ ,-0.00003560,-0.00004607,-0.00011360,0.00006603,0.00002073,-0.00002213 $,-0.00000133,-0.00195426,0.00451913,0.00307877,-0.00054339,-0.00083489$ ,-0.00048497,-0.00225807,0.00155437,0.00562195,0.01490387,-0.00360256, $0.01082005,0.07893226,-0.09236136,0.07755820,-0.00665592,0.00638914,-0$ $.00387460,-0.00053217,0.00008339,-0.00006593,0.00010991,-0.00041594,-0$ $.00036780,0.00073405,-0.00029104,0.00057198,-0.08395397,0.08860165,-0$. $00073338,-0.00192568,-0.00086504,0.00011489,-0.00001895,-0.00001382,0$. $00008699,-0.00037898,0.00015767,0.00008614,0.00013038,0.00017291,0.000$ $22531,0.00007530,-0.00009063,-0.00019953,0.00003839,0.00020144,-0.0001$ $0123,-0.00013217,0.00000638,0.00004789,0.00005548,-0.00010775,0.000041$ $45,-0.00003000,0.00002498,-0.00005159,-0.00007761,0.00000964,-0.000009$ $19,-0.00010314,-0.00001402,-0.00001987,-0.00000353,-0.00002374,0.00102$ $291,0.00304498,0.00371791,0.00077664,-0.00047287,-0.00050511,0.0019775$ $7,0.00568113,-0.00018051,-0.01731358,0.00842730,-0.00868424,-0.1157900$ $2,0.07759996,-0.14555120,0.00545193,-0.00138396,0.00627581,0.00044426$, $0.00002865,0.00010830,-0.00004028,-0.00036966,-0.00028938,-0.00085106$, $0.00043624,-0.00053446,0.12473197,-0.08806528,0.14746494,0.00009850,-0$ $.00078383,0.00094004,0.00013949,-0.00010432,-0.00005949,-0.00002756,0$. $00004429,0.00000383,-0.00000023,0.00002474,-0.00003426,-0.00002547,-0$. $00000174,-0.00005043,0.00014123,0.00009197,0.00012087,-0.00039549,-0.0$ $0015185,0.00014989,-0.00001324,0.00000954,0.00002146,-0.00002822,0.000$ $00880,0.00000541,-0.00002530,-0.00001023,-0.00001028,-0.00000923,-0.00$ $002053,-0.00000699,-0.00001314,-0.00000251,0.00000070,0.00713626,0.014$ $02846,-0.01648961,-0.00289416,0.00161065,-0.00300621,-0.00111567,0.000$ $15869,-0.00020340,-0.00503206,-0.00109943,-0.00026114,-0.00275655,-0.0$ $1865313,0.02171015,-0.07424062,-0.04919545,0.05456819,0.00109231,0.000$ $61902,-0.00026863,0.00047949,0.00068337,-0.00080920,0.00005460,-0.0000$ $0569,0.00010549,0.00092748,0.00048240,0.00010177,0.07650758,0.00019792$ ,-0.00002982,0.00026196,0.00011423,-0.00008823,0.00002128,-0.00000404, $0.00003257,-0.00006220,-0.00001883,0.00001300,0.00002187,0.00003365,-0$ $.00007212,0.00000353,-0.00011169,0.00003300,-0.00004919,-0.00009398,-0$ $.00011026,-0.00003927,0.00000397,-0.00000083,-0.00000851,-0.00000098,0$ $.00000346,-0.00000382,-0.00000909,0.00000116,-0.00000597,-0.00000081,-$ $0.00002655,0.00000407,-0.00000337,0.00000480,-0.00000025,-0.00591085,-$ $0.00431099,0.00850595,0.00197101,0.00299963,0.00303474,0.00028413,-0.0$ 
$0031628,-0.00082412,-0.00146680,0.00501792,0.00257800,-0.00009489,-0.0$ $0004502,0.00482620,-0.04733096,-0.15853692,0.14256215,-0.00001336,-0.0$ $0106424,-0.00189926,-0.00021620,-0.00006889,0.00046821,0.00001789,-0.0$ $0044925,-0.00031285,0.00038628,-0.00223360,-0.00255597,0.05226678,0.15$ $924744,-0.00024831,0.00013527,-0.00003027,0.00011082,-0.00002577,0.000$ $04311,-0.00002790,-0.00001449,-0.00006440,0.00001847,0.00002748,0.0000$ $0217,-0.00000300,-0.00004248,0.00003821,-0.00006747,-0.00000410,-0.000$ $07993,0.00007978,-0.00004674,-0.00006581,0.00000138,0.00000064,-0.0000$ $2293,0.00000623,-0.00000408,0.00000473,-0.00000063,-0.00000459,0.00000$ $411,-0.00000404,-0.00000691,-0.00000122,0.00000174,-0.00000014,0.00000$ $535,0.00764274,0.01107846,-0.00907496,-0.00344231,0.00298826,0.0012361$ $6,-0.00035525,-0.00084024,-0.00005631,0.00018374,0.00262549,0.00415048$ $,-0.00075083,0.00243164,0.00091286,0.05231169,0.14232170,-0.20120116,0$ $.00049672,-0.00181764,-0.00036807,0.00027954,0.00058427,-0.00033005,0$. $00007696,-0.00031590,-0.00031740,0.00021808,-0.00254355,-0.00128007,-0$ $.05652815,-0.15652656,0.20649540 \backslash \backslash-0.00000105,0.00000014,0.00000337,0$. $00000045,-0.00000236,0.00000047,-0.00000020,-0.00000277,0.00000211,-0$. 00000106,-0.00000134,0.00000375,0.00000045,-0.00000228,0.00000335,0.00 $000042,-0.00000193,-0.00000072,0.00000356,-0.00000050,0.00000074,0.000$ $00069,-0.00000060,0.00000100,-0.00000086,-0.00000286,0.00000318,-0.000$ $00151,-0.00000221,0.00000306,0.00000097,-0.00000057,0.00000151,-0.0000$ $0182,-0.00000392,0.00000350,-0.00000163,0.00000472,-0.00000612,-0.0000$ $0055,0.00000099,-0.00000103,0.00000499,0.00000096,-0.00000085,0.000000$ $69,0.00000356,-0.00000330,-0.00000443,-0.00000153,0.00000092,0.0000019$ $2,0.00000152,-0.00000137,-0.00000077,0.00000390,-0.00000480,0.00000273$ $, 0.00000363,-0.00000390,0.00000267,0.00000122,-0.00000132,-0.00000205$, 0.00000018,-0.00000064,-0.00000362,0.00000204,-0.00000292lI!@ 


\section{Aniline addition}

p-Chlophenyl + aniline ortho-Complex

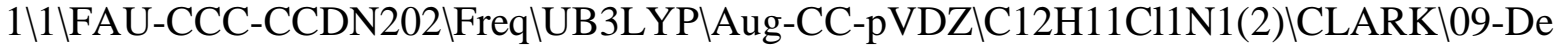
c-2014\0\\N Geom=AllCheck Guess=TCheck SCRF=Check GenChk UB3LYP/Aug-C C-pVDZ Freq \\Addition product p-Cl-Ph-radical + aniline (ortho) $\backslash 0,2 \backslash \mathrm{C}$ ,-5.9950411015,1.7778206676,1.7678192244\C,-4.6898325838,1.1482987979, $2.2273602021 \backslash \mathrm{C},-3.4829163632,1.8057799227,2.1153456589 \backslash \mathrm{C},-3.3898482985$ ,3.0997225728,1.5531798453\C,-4.5648305742,3.7524863886,1.0917263612\C ,-5.7924746449,3.1564910281,1.1811089396\H,-6.410446338,1.1144350583,0 $.9811945344 \mathrm{UN},-4.7902194296,-0.1635971247,2.6697125151 \mathrm{H},-2.5759392888$ $, 1.3049928534,2.4620918028 \backslash \mathrm{H},-4.4850317734,4.7501073031,0.6566467091 \backslash \mathrm{H}$ ,-6.6864531166,3.6682905308,0.8238751368\H,-2.4219692699,3.5931358301, $1.4811911463 \backslash \mathrm{C},-8.9601096262,1.8351252233,4.9335739219 \backslash \mathrm{C},-9.1896793482$ ,1.0565036708,3.8013562659\C,-8.2261054762,1.0413214728,2.7854873531\C ,-7.0478456994,1.7908872278,2.888066461\C,-6.8484146358,2.5677954069,4 $.0402042857 \backslash \mathrm{C},-7.7945878958,2.5949062573,5.0655563365 \backslash \mathrm{Cl},-10.163285574$ $1,1.8661844208,6.2233006474 \backslash \mathrm{H},-10.1048858001,0.473943395,3.7124614075 \backslash$ $\mathrm{H},-8.4053601646,0.4362563071,1.8950672643 \backslash \mathrm{H},-5.9403952106,3.1637218967$ ,4.1347457298\H,-7.6349190873,3.199041857,5.9571267358\H,-3.9736455572 ,-0.5457872796,3.1294309319〈H,-5.6568180161,-0.4187744336,3.127901682\ IVersion=EM64L-G09RevC.01 $\backslash$ State $=2-A \backslash H F=-978.8892229 \backslash S 2=0.779494 \backslash S 2-1=0$ .IS2A=0.750499\RMSD=2.846e-09\RMSF $=1.764 \mathrm{e}-05 \backslash$ ZeroPoint $=0.1967461 \backslash$ Therm $\mathrm{al}=0.2089091 \mathrm{ZPE}=123.4600162 \backslash \mathrm{Dipole}=0.3985491,-0.4844465,-0.0141901 \backslash \mathrm{Di}$ poleDeriv $=0.1217072,0.1841143,-0.0696348,-0.0223997,0.035675,0.1685884$ $,-0.0885193,0.1079053,0.2611324,0.0935212,0.2240312,-0.0952537,0.19172$ $63,1.1891398,-0.3856083,0.0748343,-0.3127817,-0.0113534,-0.0020266,-0$. $369036,0.189945,0.159706,-0.2123705,-0.0485033,-0.0443182,-0.0031346,-$ $0.1402569,0.0181904,0.0985403,-0.011383,-0.0558806,0.0186919,-0.073164$ $7,0.0750413,-0.063251,-0.1493426,0.0687957,-0.1295604,0.0778269,-0.088$ $0877,0.0579984,-0.0434437,0.0683832,-0.1020033,-0.1102243,-0.1063365,0$ $.1297452,0.0362217,0.0730648,-0.009444,-0.1904721,0.1125148,-0.0458347$ ,-0.2095434,-0.0577491,-0.0940223,-0.0408261,-0.1192742,-0.0577702,-0. $0804968,-0.0359487,-0.0866835,-0.0166409,-0.2501407,-0.0956203,0.07481$ $49,-0.0903865,-1.4530356,0.2974217,0.0202291,-0.0649879,-0.3101855,-0$. $0729217,0.0731857,-0.0392227,0.0828877,0.0048734,0.0733937,-0.083628,0$ $.0619103,0.1085036,0.0487952,-0.0118885,0.0094941,-0.0400101,-0.083311$ $6,0.113477,-0.014019,0.0889676,0.0939576,-0.0445895,0.046932,-0.059619$ $1,0.0401574,0.0218195,0.0554005,-0.0557537,0.0456442,0.1096099,-0.0787$ $938,-0.0651813,0.0072688,-0.0705101,0.0322825,0.0436268,-0.0057062,0.0$ $33412,0.1242156,0.6024429,0.0224661,-0.613045,-0.0113412,-0.0143761,0$. $0604123,-0.6771866,0.031561,0.6834595,-0.0951797,0.0717725,0.1232375,0$ $.088341,-0.0504834,-0.0156203,0.1416777,-0.0003716,-0.2323287,-0.03691$ $29,0.0382812,-0.0059909,0.1102024,-0.0428846,0.0379184,0.0795965,0.096$ $9155,0.0066647,0.1030341,-0.0035836,-0.1231798,-0.0298156,-0.0681336,-$ $0.0125673,-0.1917399,-0.0265728,0.0465953,-0.0684938,0.0489197,0.04534$ $87,0.0321597,-0.042538,0.0329384,0.0314143,0.0139512,-0.07133,-0.17549$ $03,0.0017627,0.1010739,0.0210271,-0.0330142,0.0661373,0.126398,0.08317$ $55,-0.073016,-0.5013121,-0.0062021,0.4085504,0.0040195,-0.0788007,-0.0$ 
$176437,0.4458606,-0.0076099,-0.5195706,-0.010302,-0.0694292,0.0133707$, $-0.067788,0.0790297,-0.0150891,0.0211558,-0.0133717,0.0856964,0.093481$ $4,-0.007245,0.011841,-0.0287322,0.0549483,-0.0746937,-0.0091971,-0.088$ $9708,-0.039823,-0.003134,-0.0620596,-0.0061672,-0.06742,0.057006,-0.00$ $74775,-0.015601,-0.0045442,0.0958704,0.0832778,-0.0202255,0.0065969,-0$ $.0201564,0.075901,-0.0656216,0.0165401,-0.0646369,-0.0010729,0.0980762$ ,-0.0371956,-0.0213917,-0.0632167,0.2494272,0.0357705,-0.0386111,0.135 $2403,0.1640641,0.1720608,0.0314985,-0.0198766,-0.0282728,0.26937,0.045$ $3171,0.0465831,0.1860717,0.1049186 \backslash$ Polar $=205.3764846,19.9572242,155.75$ 0163,-39.6966682,-8.6407827,160.3872279\PG=C01 [X(C12H11Cl1N1)] NImag= 0\0.45484454,0.02305577,0.49968666,0.04140249,0.01730855,0.45202447,$0.15404912,0.03075785,-0.02997913,0.65809632,0.02799684,-0.09767605,0$. $02047536,0.11027125,0.58778586,-0.02720572,0.01497524,-0.08062155,0.01$ $794997,-0.16651514,0.22217850,-0.02485441,-0.00677536,-0.00158411,-0.3$ $2222234,-0.11567975,0.01265187,0.69166214,0.00541585,0.01412360,-0.003$ 49990,-0.06606393,-0.14383594,0.02477811,0.00448849,0.55501592,-0.0079 $2937,-0.00356608,0.00755696,-0.00941602,0.02284175,-0.06435608,0.08415$ $603,-0.19039688,0.20208355,0.00162444,0.00683842,-0.00123125,0.0156173$ $6,-0.02791041,0.01267775,-0.11653027,-0.04511488,0.00988564,0.65400171$ $, 0.00296383,-0.00625937,-0.00092788,-0.06000929,-0.03501889,0.01282544$ $, 0.02471465,-0.24118905,0.08704691,0.08735787,0.52364932,0.00032328,0$. $00071503,-0.00756206,0.02762518,0.01347266,0.00063351,-0.02113325,0.07$ $971089,-0.08339246,0.04381693,-0.17467749,0.18371891,-0.00049717,-0.01$ $678840,0.00472422,-0.02956725,-0.00589653,0.00176201,0.02858352,0.0602$ $5564,-0.02436834,-0.19347012,0.01728063,-0.02819555,0.60240676,-0.0275$ $4872,-0.01367041,0.00500160,0.00046209,0.00042665,-0.00079132,0.027236$ $34,-0.02074584,0.01560934,0.09213508,-0.14841662,0.05515027,0.06641500$ $, 0.62284058,0.01079501,0.00625236,0.00422827,-0.00424107,-0.00205099,-$ $0.00029051,-0.00914152,0.02088910,-0.00491686,-0.06185157,0.04456163$,$0.08300117,0.04139767,-0.21634059,0.22132520,-0.08790180,-0.01142399,-$ $0.00128950,0.02240758,0.02914911,-0.00983507,-0.01315091,-0.02747079,0$ $.01096234,-0.07904768,0.02971319,-0.02619451,-0.32029221,-0.14159694,0$ $.02354309,0.74060727,-0.00290133,-0.17524527,0.04635611,0.01374763,-0$. $01247439,0.00521354,-0.00661066,-0.01337779,0.00485247,-0.00937756,0.0$ $3356807,-0.01191868,-0.10279187,-0.16437508,0.03728637,0.04296527,0.48$ $032776,-0.00606770,0.04508550,-0.08291697,-0.00235142,0.01028327,-0.00$ $742148,0.00090771,0.00108185,-0.00131775,-0.00779155,-0.00583951,0.011$ $66461,0.00587358,0.03211695,-0.06197018,0.06840147,-0.15455171,0.19967$ $901,-0.07055420,-0.04892348,-0.06792474,-0.02108041,-0.01489014,-0.021$ $16604,0.00104736,0.00576334,-0.00291606,-0.00039239,-0.00300870,0.0012$ $2520,0.00015847,0.00006688,-0.00006995,-0.00113486,-0.00779502,0.00015$ $260,0.08959307,-0.04577411,-0.12263842,-0.09985964,0.00048636,0.007152$ $45,0.00915613,0.00275615,0.00044418,-0.00033816,-0.00212214,-0.0007638$ $7,-0.00021291,0.00459500,-0.00296283,0.00039049,-0.01574886,-0.0228111$ $6,-0.01330462,0.05646500,0.13850822,-0.05924200,-0.09240400,-0.1586923$ $1,-0.00918078,-0.00447669,-0.00527464,0.00079953,0.00252676,-0.0020332$ $7,-0.00000020,-0.00138839,0.00035596,-0.00003184,0.00143958,-0.0010607$ $5,0.00642082,0.00653325,0.01226727,0.06428570,0.10531317,0.17958152,0$. $00214011,0.03207823,-0.01121212,-0.12187775,-0.01522409,0.00136827,-0$. $00132496,-0.04650926,0.01496426,-0.00632218,0.00293297,-0.00339659,0.0$ $0081717,0.00063203,-0.00010914,-0.00697780,-0.00007418,-0.00054245,0.0$ 
$0192098,0.00123119,0.00077579,0.78487553,0.00842407,-0.02221928,0.0083$ $9474,-0.01494337,-0.27778697,0.07662612,-0.01644300,-0.03375085,0.0126$ $7219,-0.00014347,0.00311523,0.00217806,-0.00478129,-0.00027208,-0.0007$ $1241,0.00427734,-0.00055640,0.00042679,-0.00024679,0.00208102,-0.00026$ $160,-0.01637010,0.48266672,-0.00224626,0.01490992,-0.00397218,0.005434$ $95,0.11290393,-0.06843427,0.00130272,0.01176472,0.00541029,-0.00008794$ $, 0.00073465,0.00456290,-0.00034488,0.00008163,-0.00081560,-0.00221917$, $0.00067097,0.00134245,0.00178860,-0.00012526,0.00096768,-0.01273626,-0$ $.28322141,0.27964089,-0.00122433,-0.00370638,0.00049161,-0.02066422,0$. $01585743,-0.01035819,-0.24553817,0.10043107,-0.07402102,0.00424816,-0$. $00195317,0.00119445,0.00204129,-0.00106567,0.00008428,-0.00004016,-0.0$ $0060754,0.00037551,-0.00076438,0.00032526,-0.00024050,0.00174031,0.001$ $35900,0.00010727,0.25991840,-0.00255462,0.00017119,0.00278973,-0.01229$ $505,0.00736233,-0.00333556,0.10129548,-0.10943769,0.04788054,0.0251475$ $6,-0.00764258,0.00766816,-0.00211687,-0.00365055,0.00351386,-0.0005386$ $4,-0.00034601,-0.00011581,0.00023708,-0.00004024,0.00008504,-0.0006454$ $3,0.00124376,-0.00233484,-0.10956130,0.11196997,0.00052554,0.00239835$, $0.00637468,0.00245028,0.00053332,0.00397122,-0.07493012,0.04691490,-0$. $06390092,-0.01086448,0.00452519,-0.00171223,0.00058058,0.00398127,0.00$ $364539,0.00025008,-0.00007099,-0.00051191,0.00089397,-0.00061807,0.000$ $33692,0.00141732,-0.00221631,-0.00276314,0.08142507,-0.05472462,0.0578$ $4830,-0.00383771,-0.00145025,-0.00110908,-0.00101877,-0.00016612,-0.00$ $000829,-0.00399222,0.00160004,-0.00197263,0.00629464,0.02347895,-0.009$ $91759,-0.05801578,-0.01884668,0.00511477,0.00122445,-0.02666629,0.0116$ $9268,-0.00008739,-0.00065641,0.00032035,0.00045523,0.00018930,0.000091$ $87,0.00008776,0.00127681,-0.00060443,0.05731353,-0.00194857,0.00299539$ $, 0.00258858,0.00011469,0.00053967,-0.00027101,0.00243544,0.00140311,0$. $00175506,-0.00396622,-0.00925441,0.00451019,-0.01905192,-0.28541122,0$. $10930602,0.00091921,-0.00983117,0.00576416,-0.00008526,0.00018662,-0.0$ $0018722,-0.00009054,-0.00016874,-0.00016625,-0.00017449,-0.00055127,0$. $00037383,0.02160350,0.30064634,-0.00067804,0.00285655,0.00786901,-0.00$ $021826,-0.00045931,-0.00003955,-0.00223993,0.00173945,0.00342003,0.002$ $51977,0.00834910,-0.00117057,0.00481164,0.10909134,-0.08380238,-0.0002$ $0955,0.00190657,0.00229291,0.00005093,0.00117359,-0.00079337,0.0000212$ $2,-0.00009946,-0.00037075,0.00006285,0.00056703,0.00003413,-0.00498457$ ,-0.12039956,0.07809513,0.00473848,-0.00404805,0.00325368,0.00036006,-$0.00168570,0.00067119,0.00020096,-0.00049005,0.00032442,-0.00278272,-0$ $.00425909,0.00054103,-0.01761157,0.01586794,-0.01028312,-0.24427757,0$. $10663995,-0.07942495,0.00021407,0.00040173,-0.00028081,0.00048179,0.00$ $001922,0.00013956,-0.00002821,0.00014376,0.00000812,0.00082466,0.00014$ $259,0.00076150,0.25783892,0.02232748,-0.01015856,0.00932457,-0.0039384$ $7,-0.00429583,0.00183532,0.00002430,-0.00049267,-0.00018112,-0.0034812$ $0,0.00057360,0.00261419,-0.01251861,0.00940998,-0.00405149,0.10825093$, $-0.11273736,0.05422254,0.00006826,0.00086323,-0.00068466,0.00210967,0$. $00008057,0.00063030,0.00011136,-0.00003589,-0.00023827,0.00051009,-0.0$ $0002174,-0.00236062,-0.11326276,0.11614827,-0.00812696,0.00404850,-0.0$ $0278174,0.00110648,0.00235203,0.00121736,0.00053464,0.00005770,-0.0006$ $1671,-0.00014330,0.00212337,0.00650076,0.00224536,0.00003855,0.0046146$ $1,-0.07977605,0.05339363,-0.06283407,0.00021916,0.00082661,0.00032316$, $-0.00072481,0.00000102,-0.00039469,0.00004337,-0.00023528,-0.00052656$, $0.00068592,-0.00234062,-0.00423770,0.08501772,-0.06020388,0.05745682,0$ 
$.00036445,0.00041392,0.00008141,0.00162478,0.00039942,-0.00052849,0.00$ $158886,0.00217244,-0.00111637,-0.28198510,-0.11728493,0.01303221,-0.01$ $644065,-0.01401519,0.00334461,-0.00205357,0.00360521,-0.00250734,0.000$ $07933,0.00002362,-0.00000444,-0.00001032,0.00005184,0.00001228,0.00067$ $084,-0.00015621,0.00054194,0.00083708,0.00029585,0.00035388,-0.0010308$ $9,-0.00064947,0.00010921,0.29621231,0.00030150,-0.00031882,-0.00025014$ $, 0.00090871,-0.00374596,0.00335632,-0.02480543,-0.00709720,0.00039561$, $-0.11689107,-0.11043113,0.02101261,0.01338706,0.00930911,-0.00103739,0$ $.00279612,-0.00056687,0.00213942,-0.00011495,0.00003422,0.00000890,-0$. $00150033,-0.00029227,0.00033162,0.00043934,0.00046171,-0.00137619,-0.0$ $0030536,0.00029606,-0.00125153,0.00074803,0.00039996,0.00011116,0.1251$ $4492,0.11226232,0.00009038,-0.00038820,-0.00086972,-0.00066230,0.00341$ $005,0.00228256,0.01090767,0.00446499,0.00230215,0.01239229,0.02093024$, $-0.03113282,-0.00835600,-0.00506978,0.00339696,-0.00213872,0.00231465$, $0.00205907,-0.00013039,0.00001738,0.00011655,0.00068285,0.00040970,0.0$ $0019160,0.00025714,-0.00141471,-0.00215339,0.00062430,-0.00120796,-0.0$ $0180861,-0.00053041,-0.00009899,0.00039146,-0.01320160,-0.02350850,0.0$ $2508681,-0.00153385,-0.00037006,0.00012087,0.00007230,-0.00027674,0.00$ $041636,-0.00000238,-0.00014748,0.00010777,-0.00003300,0.00030629,-0.00$ $004473,0.00026227,0.00015282,0.00011401,-0.00032398,0.00000621,0.00037$ $830,0.00001637,-0.00002086,0.00016049,0.00037757,0.00015894,0.00014727$ $, 0.00007760,-0.00004017,-0.00007296,0.00007692,0.00001927,-0.00008777$, $-0.00000088,-0.00005390,-0.00002043,0.00003412,0.00001361,0.00000781,0$ $.47044246,-0.00076526,-0.00077590,0.00002503,0.00018090,-0.00016416,0$. $00041024,-0.00002782,-0.00006383,0.00006503,-0.00007336,0.00019994,0.0$ $0003568,0.00024221,0.00004504,0.00013885,-0.00030187,0.00017511,0.0004$ $8556,-0.00009469,0.00014487,0.00022764,0.00011796,0.00026292,0.0002696$ $2,0.00003169,-0.00000852,-0.00009740,0.00002048,-0.00004149,-0.0001274$ $8,-0.00003521,-0.00006361,-0.00000643,-0.00002549,0.00001545,-0.000003$ $51,0.18634261,0.33826181,-0.00028194,-0.00005102,-0.00066275,-0.000011$ $33,-0.00030713,0.00057582,0.00003549,0.00020983,-0.00004055,0.00001127$ $, 0.00005630,0.00009858,0.00007369,-0.00000717,0.00001496,-0.00031501,0$ $.00026373,0.00059118,0.00000526,0.00005110,0.00035640,0.00003824,0.000$ $42877,0.00026156,-0.00004881,-0.00001677,-0.00013818,0.00000700,-0.000$ $10685,-0.00006580,-0.00000593,0.00000823,-0.00016315,-0.00006319,-0.00$ $004103,0.00001241,-0.02798765,0.18117659,0.47227452,-0.00014525,-0.004$ $53168,-0.00088014,0.00009456,-0.00060696,0.00048901,0.00019638,0.00013$ $080,0.00001954,0.00003536,0.00001657,0.00000413,0.00025594,-0.00026705$ $, 0.00022931,-0.00129235,0.00042039,0.00095080,0.00013421,0.00026591,0$. $00033698,0.00029839,0.00058895,0.00008788,0.00000296,0.00001691,-0.000$ $06757,-0.00005639,-0.00007660,-0.00012562,-0.00005611,-0.00010348,-0.0$ $0002531,-0.00000702,0.00002494,0.00000632,-0.11109625,-0.05888464,-0.0$ $5576756,0.61423769,-0.00435242,0.00318513,-0.00316139,-0.00096196,-0.0$ $0063557,0.00089215,-0.00005981,0.00053679,-0.00016016,0.00013453,-0.00$ $024278,0.00003085,-0.00034377,0.00020609,-0.00026958,0.00122318,-0.000$ $01878,-0.00042342,0.00000607,0.00002568,0.00009487,-0.00004470,-0.0001$ $0798,0.00005963,-0.00007402,-0.00007162,-0.00002306,0.00005556,0.00007$ $582,0.00011973,0.00002317,-0.00005528,0.00003815,0.00002815,-0.0000139$ $2,0.00002140,-0.03051649,-0.15756567,-0.13151968,0.22722158,0.36705270$ $, 0.00033704,-0.00304504,0.00211706,0.00038741,0.00075691,-0.00114432,0$ $.00013376,-0.00029448,0.00000185,-0.00009201,-0.00027389,-0.00005762,-$ 
$0.00004533,-0.00014493,0.00002503,-0.00025281,-0.00024329,-0.00035124$, $-0.00003918,0.00001894,-0.00019680,-0.00008778,-0.00036457,-0.00043748$ $, 0.00004591,0.00008758,0.00019813,-0.00007290,0.00004585,0.00009149,0$. $00004167,-0.00000430,0.00010105,0.00002729,0.00002526,-0.00000486,-0.0$ $0599217,-0.10288794,-0.23919928,-0.09705400,0.18388258,0.55346534,-0.0$ $1906807,-0.01187487,0.00456683,-0.00017467,-0.00110871,0.00080028,-0.0$ $0032059,0.00025043,-0.00043349,-0.00008273,-0.00002877,-0.00005691,0.0$ $0045918,-0.00069646,0.00044518,-0.00141568,0.00109227,-0.00135882,0.00$ $026570,-0.00114212,-0.00272528,0.00008167,-0.00029999,-0.00088774,-0.0$ $0016989,-0.00003576,0.00015487,-0.00023901,-0.00002796,0.00012128,0.00$ $018716,-0.00036138,0.00037284,0.00000658,-0.00001829,0.00002942,0.0405$ $1605,0.04525612,0.04485958,-0.21135960,-0.03543551,0.09107518,0.553405$ $25,-0.00276191,0.00393017,0.00157182,0.00123605,-0.00174955,-0.0002266$ $6,-0.00011938,0.00000423,-0.00008091,0.00010610,0.00026251,-0.00007606$ $, 0.00016963,0.00007316,-0.00005980,0.00027305,0.00018365,-0.00056765,0$ $.00152108,0.00023478,-0.00087009,0.00075730,-0.00000334,-0.00026979,-0$ $.00002692,0.00004371,0.00020447,0.00001408,0.00006675,-0.00002240,0.00$ $010818,0.00008742,0.00004699,0.00001213,0.00002720,-0.00003981,0.02015$ $530,0.01625123,-0.00457100,-0.00649683,-0.08664076,-0.03643166,0.18849$ $260,0.36228675,0.02089079,0.01056249,0.00164045,0.00195574,0.00239489$, $-0.00213973,-0.00003996,-0.00143813,0.00076136,-0.00005802,0.00076216$, $-0.00029107,0.00025574,0.00090744,-0.00017153,0.00210406,-0.00067876,-$ $0.00130002,0.00220505,0.00184001,0.00124199,-0.00027623,-0.00079475,0$. $00023499,0.00026877,0.00007637,0.00005821,0.00021166,0.00023347,0.0001$ $0715,-0.00023888,0.00008977,-0.00005721,0.00003546,-0.00001251,-0.0000$ $7014,0.00145385,-0.02978250,-0.04643836,0.14242998,-0.00715546,-0.2209$ $7535,-0.09892899,0.20887768,0.60615657,-0.10843123,0.00389805,0.046177$ $21,-0.03528419,0.00521129,0.01630478,0.00129332,0.00490184,-0.00077866$ ,-0.00080221,-0.00170590,0.00077227,0.00127327,0.00178746,0.00046988,-$0.00687030,-0.01356415,0.01084456,0.00338551,-0.00140245,-0.00592155,0$ $.00008614,-0.00172912,0.00179531,-0.00009639,-0.00063900,-0.00092099,0$ $.00032631,0.00002728,-0.00011619,0.00080037,0.00098043,-0.00140121,0.0$ $0014209,0.00000052,0.00007382,-0.02424560,-0.02379952,-0.02413095,-0.0$ $3952718,-0.00122791,0.04523963,-0.25169559,-0.12466160,-0.02870315,0.5$ $3654909,-0.00264121,-0.07097644,-0.00445707,0.00485629,-0.00142407,-0$. $00820173,0.00142585,-0.00068547,0.00050998,-0.00130981,0.00024133,-0.0$ $0014575,0.00292457,-0.00318005,0.00222695,-0.02357919,-0.00215264,0.02$ $195141,0.01140079,-0.00203602,-0.01651299,0.00295915,0.00119723,-0.000$ $50273,0.00006648,0.00035874,0.00033554,-0.00054035,-0.00094215,-0.0010$ $9760,-0.00015558,0.00051573,-0.00029967,-0.00003241,0.00008335,0.00004$ $144,-0.02457423,-0.03387801,-0.02233903,-0.02191827,0.01869414,0.04270$ $286,-0.09705275,-0.14642145,-0.04427244,0.17485650,0.37291100,0.034990$ $03,-0.01042583,-0.12267434,0.00432344,-0.00572963,0.01266888,-0.002970$ $93,0.00131049,-0.00238074,0.00106624,0.00029364,0.00033783,-0.00167193$ ,-0.00157773,-0.00106616,0.01132139,0.01213926,-0.01242703,0.01825255, $-0.00369666,-0.02435081,-0.00134305,0.00088960,-0.00029212,-0.00080291$ ,-0.00043410,-0.00038517,-0.00031239,-0.00000715,0.00041415,0.00008591 ,-0.00110043,0.00131135,-0.00009761,0.00000040,0.00017615,-0.02497373, $-0.02280970,-0.02108688,0.00967462,0.02189027,0.03556605,0.02216648,-0$ $.01549364,-0.11396330,-0.06204633,0.16574643,0.52661967,0.00467450,0.0$ $1300765,0.01974727,0.00187226,0.00055575,0.00105524,-0.00014996,0.0003$ 
$9510,-0.00000078,-0.00019120,-0.00028646,0.00001014,-0.00026305,-0.000$ $44799,-0.00008060,0.00104904,0.00131800,0.00034230,-0.00266047,0.00036$ $215,0.00458411,-0.00037480,0.00037371,0.00043266,-0.00002976,-0.000049$ $64,-0.00016337,-0.00002220,-0.00010781,-0.00007716,0.00003695,-0.00016$ $437,0.00008839,-0.00001490,0.00000064,0.00003992,-0.04272476,-0.024460$ $38,0.00861857,-0.00686509,0.00686144,0.01584398,0.01024515,-0.01439405$ $,-0.02828873,-0.11597280,-0.05140235,-0.03809473,0.60719157,0.00394576$ $, 0.00316950,-0.00229539,-0.00074874,0.00060688,-0.00019625,-0.00009270$ $, 0.00037025,-0.00060386,0.00004252,-0.00075984,0.00019450,-0.00057233$, $-0.00002143,0.00024122,-0.00012320,0.00000429,0.00066972,-0.00062539,0$ $.00107362,0.00051888,-0.00018412,-0.00030356,-0.00026029,-0.00007107,--$ $0.00000344,-0.00006926,-0.00001830,-0.00001378,-0.00010261,-0.00005677$ $, 0.00034166,-0.00017759,-0.00004201,0.00002238,0.00001819,0.00036172,0$ $.01938892,0.02142492,0.00709197,-0.00995067,-0.01936744,-0.03361813,-0$ $.01815210,-0.01040009,-0.02487940,-0.14989078,-0.11960719,0.21604340,0$ $.36404022,0.00427620,-0.01123791,-0.02211930,-0.00226950,0.00153912,-0$ $.00179418,-0.00008062,-0.00009259,0.00029898,-0.00015733,-0.00028593,-$ $0.00017790,-0.00065407,-0.00019895,-0.00049477,0.00019820,-0.00272355$, $-0.00001582,0.00274042,-0.00047443,-0.00265346,-0.00055096,-0.00068251$ $, 0.00020323,0.00002225,-0.00001120,0.00009670,0.00000306,0.00001422,0$. $00016699,0.00001775,0.00023316,-0.00013253,0.00002154,0.00000729,0.000$ $04776,0.05169331,0.04681288,0.03552726,0.01609366,-0.01958005,-0.05347$ $961,-0.06207471,-0.03000638,0.01892850,0.00814054,-0.09198784,-0.23648$ $982,-0.11167808,0.17537009,0.55743891,0.00298378,-0.00276256,-0.001640$ $49,-0.00043835,0.00012504,-0.00059928,0.00004852,0.00000373,-0.0000383$ $9,0.00017342,-0.00015224,0.00001461,-0.00029582,-0.00024717,-0.0001972$ $0,-0.00008420,-0.00013336,-0.00029453,0.00041138,-0.00031581,-0.000944$ $09,-0.00023244,-0.00035194,-0.00037710,-0.00005445,0.00002328,0.000120$ $16,-0.00010403,-0.00000871,0.00008505,0.00002830,0.00013768,0.00015042$ ,-0.00002150,-0.00000688,0.00000515,-0.24102287,-0.09989266,0.00609785 $, 0.01152380,-0.03341178,-0.06096414,-0.05140928,-0.01653718,0.02040628$ $, 0.03882726,0.04248096,0.04098662,-0.21240565,-0.03436072,0.09664061,0$ $.54629394,-0.00289490,0.00114796,-0.00419104,-0.00085339,0.00003248,0$. $00046027,-0.00001366,0.00002686,0.00000823,0.00009553,0.00004054,-0.00$ $008877,0.00002671,0.00021744,-0.00002899,0.00016817,-0.00012450,0.0001$ $6897,0.00055208,-0.00008684,-0.00097780,-0.00008097,0.00009179,-0.0001$ $0249,-0.00003636,-0.00003079,-0.00000030,0.00002496,0.00000959,0.00005$ $375,0.00001628,0.00000950,-0.00010281,0.00000694,-0.00001625,0.0000097$ $5,-0.12863942,-0.15160632,-0.01972679,-0.01351964,-0.01642193,-0.03028$ $230,-0.01728604,-0.00908667,0.00806320,0.02143029,0.01742988,-0.004352$ $61,-0.00444383,-0.08659803,-0.04058256,0.18833594,0.36784773,-0.002022$ $07,-0.00452417,-0.00043173,-0.00005919,0.00031222,0.00010742,0.0001117$ $4,-0.00009352,0.00028119,-0.00001730,0.00020386,-0.00007394,0.00024659$ $, 0.00017908,0.00024746,-0.00053571,-0.00012533,0.00081522,0.00061159,0$ $.00005321,-0.00043499,0.00023817,0.00009045,0.00051544,0.00009035,-0.0$ $0000619,-0.00007930,0.00004544,-0.00000880,-0.00014916,-0.00000740,0.0$ $0004741,-0.00008364,-0.00000097,0.00000820,-0.00002261,-0.04383496,-0$. $04911095,-0.10550148,-0.02643654,-0.00994283,0.01856600,0.01919487,0.0$ $0707988,-0.01041330,0.00481468,-0.02514976,-0.04197934,0.14814337,-0.0$ $1013129,-0.23188438,-0.09885448,0.22184729,0.62066804,0.00006747,0.000$ $13780,-0.00041035,0.00004585,-0.00026367,0.00041162,-0.00002358,0.0002$ 
$8265,-0.00012588,-0.00000421,-0.00010552,0.00006768,0.00004240,-0.0001$ $0915,0.00008435,-0.00023269,0.00036234,0.00016572,-0.00005982,0.000005$ $99,0.00006435,-0.00024047,0.00030941,0.00002390,-0.00008442,-0.0000066$ $6,-0.00005390,-0.00004773,-0.00008039,-0.00002075,0.00003224,-0.000003$ $84,-0.00004268,-0.00004167,-0.00002106,-0.00000434,-0.08837530,-0.0042$ $7906,0.05889658,-0.00510947,-0.00024141,0.00931321,0.00224304,-0.00105$ $474,0.00088175,-0.00005437,0.00126079,0.00143416,-0.00047613,-0.005305$ $85,-0.00412994,-0.02575403,0.00056053,0.03194975,0.11837126,0.00037101$ ,-0.00036557,0.00027626,0.00003640,0.00004201,-0.00006304,0.00004849,$0.00000620,0.00003707,0.00001957,0.00000821,0.00000564,-0.00002146,-0$. $00002548,-0.00002782,-0.00002963,0.00001637,-0.00000412,-0.00001242,0$. $00003405,0.00006929,0.00001139,0.00001322,0.00001069,0.00001014,0.0000$ $0341,0.00000251,-0.00000172,0.00000194,-0.00000202,-0.00000369,0.00000$ $694,-0.00000603,0.00000466,-0.00000186,-0.00000242,-0.00416344,-0.0302$ $1666,-0.00693554,0.01166836,0.00369017,-0.01333520,-0.00390871,0.00140$ $675,-0.00220163,0.00097296,0.00040109,0.00089054,-0.00233628,0.0011538$ $1,-0.00353475,-0.01154945,0.00425951,0.01146991,0.00619360,0.02161598$, $-0.00022443,0.00053759,0.00024289,-0.00006634,0.00021088,-0.00043360,0$ $.00001964,-0.00031502,0.00014175,-0.00000078,0.00011029,-0.00008925,-0$ $.00001677,0.00020848,-0.00007307,0.00037716,-0.00041067,-0.00017698,0$. $00001548,0.00003497,0.00003954,0.00025867,-0.00028573,-0.00000795,0.00$ $008755,0.00000550,0.00006056,0.00006062,0.00009866,0.00002414,-0.00004$ $034,-0.00000801,0.00004252,0.00004886,0.00002455,-0.00000452,0.0588518$ $4,-0.00680864,-0.09640624,0.03038876,-0.00116515,-0.02859157,-0.004161$ $30,-0.00515039,0.00012362,0.00112652,0.00059799,-0.00062449,0.00104381$ ,-0.00043949,0.00265812,0.01097627,-0.00092006,-0.00827368,-0.09849274 $, 0.01077444,0.13202832,-0.00021448,-0.00042338,-0.00047568,-0.00009484$ ,-0.00026163,0.00010657,0.00004618,0.00014873,-0.00005757,-0.00003938, $-0.00006312,0.00003363,0.00005606,-0.00004725,0.00003420,-0.00017067,0$ $.00004543,0.00016492,0.00001953,-0.00000370,-0.00002134,-0.00003091,0$. $00019392,0.00002316,-0.00002507,-0.00000332,-0.00000631,-0.00001018,-0$ $.00002155,-0.00001580,0.00000707,-0.00001728,0.00000357,-0.00000681,-0$ $.00000112,0.00000285,-0.00175082,-0.00124897,0.00310759,-0.25413374,-0$ $.13754173,-0.01799782,-0.01212319,-0.01136080,-0.00390111,0.00053349,-$ $0.00099849,0.00460305,0.00006011,0.00080543,0.00007139,0.00252494,-0.0$ $0160439,0.00028984,0.00044271,0.00165988,-0.00039289,0.26511924,0.0001$ $7562,0.00008878,-0.00017057,-0.00009122,0.00012134,0.00010454,0.000029$ $50,0.00003725,-0.00000065,0.00002215,-0.00004185,0.00001615,-0.0000474$ $5,0.00000223,0.00002612,0.00004039,-0.00001090,-0.00015475,-0.00000865$ $, 0.00000811,-0.00001297,0.00000590,-0.00007156,-0.00005737,0.00000443$, $-0.00000249,-0.00001319,-0.00001213,0.00000243,0.00001526,-0.00000088$, $-0.00000676,0.00000381,0.00000059,-0.00000510,-0.00000216,-0.01511053$, $-0.00516132,0.00043584,-0.13623664,-0.12614935,-0.02086893,0.00173654$, $0.00400926,-0.00087704,-0.00136267,0.00484598,-0.00215215,0.00086173$,$0.00064261,0.00014639,-0.00131826,0.00212351,-0.00471507,0.00121057,-0$ $.00115172,0.00154029,0.14897082,0.12475906,0.00062433,0.00048166,0.000$ $36784,0.00013094,0.00008634,-0.00024833,-0.00002585,-0.00012049,0.0000$ $6631,-0.00001398,0.00007437,-0.00004397,0.00003820,0.00005553,-0.00002$ $769,0.00009755,-0.00007625,-0.00001185,-0.00001443,-0.00001809,0.00001$ $811,-0.00001540,-0.00006041,0.00002445,0.00001575,0.00000562,0.0000194$ 4,0.00001859,0.00001504,0.00000085,-0.00000597,0.00002447,-0.00001186, 
$0.00000358,0.00000282,-0.00000333,-0.02124944,-0.01374024,0.00093936,-$ $0.01573293,-0.01957179,-0.05346696,0.01897272,0.01246105,0.00592557,0$. $00419216,-0.00238259,-0.00163418,0.00027314,0.00016424,-0.00118182,0.0$ $0074677,-0.00441981,-0.00299632,-0.00118275,0.00108482,0.00035708,0.01$ $554269,0.02480193,0.05188225,-0.00017173,0.00079211,-0.00278378,-0.000$ $13571,-0.00026557,0.00036600,-0.00005483,-0.00004308,0.00007260,0.0000$ $6937,0.00014123,-0.00004201,-0.00005961,0.00014201,-0.00005539,0.00039$ $446,0.00019124,0.00010385,-0.00009698,0.00033235,0.00090735,0.00007424$ $, 0.00004749,0.00015489,-0.00000965,-0.00003948,-0.00004436,0.00003304$, $0.00002407,-0.00002013,-0.00000811,0.00002720,-0.00005213,0.00000601,0$ $.00000803,-0.00000534,-0.00051666,-0.00212225,0.00387686,0.00637355,0$. $01219871,0.01840127,-0.06064256,-0.03322566,-0.03354242,0.00016289,-0$. $01360543,-0.02070923,-0.00309700,-0.00488133,0.00111443,-0.00114496,0$. $00016350,0.00034938,-0.00038461,0.00036838,0.00120415,-0.00005640,0.00$ $162900,-0.00126147,0.05952653,0.00276072,-0.00237434,0.00028719,0.0004$ $0151,0.00072174,-0.00023553,0.00010844,-0.00024119,0.00005253,-0.00002$ $845,0.00002495,0.00002649,0.00009098,-0.00006491,0.00009832,-0.0005778$ $7,-0.00002814,0.00027307,-0.00026359,0.00043993,0.00072771,-0.00003628$ ,-0.00013264,0.00004178,0.00006796,0.00001945,0.00000478,-0.00001085,$0.00003598,-0.00005428,-0.00003336,-0.00008993,0.00000506,0.00001076$,$0.00000264,0.00000106,-0.00196847,0.00504656,-0.00143657,-0.00112427,0$ $.00300422,0.00082310,-0.03319271,-0.12923289,-0.13189144,-0.00174667,-$ $0.00530026,-0.01421061,-0.00536572,0.00233373,-0.00149932,0.00012805$,$0.00052659,0.00086474,-0.00013998,0.00010475,0.00005074,0.00177477,-0$. $00220081,0.00153315,0.03866847,0.12925240,0.00042117,0.00251837,-0.000$ $25741,-0.00049672,-0.00055762,0.00029710,0.00021165,0.00045971,-0.0001$ $0699,0.00000132,-0.00024374,0.00006514,-0.00005798,0.00012867,-0.00005$ $930,0.00040987,-0.00012977,0.00017509,-0.00035554,0.00001270,0.0005079$ $9,0.00008457,0.00040251,0.00013404,-0.00001388,-0.00001612,-0.00003724$ $, 0.00003237,0.00001380,-0.00000550,-0.00000241,-0.00000884,-0.00004155$ $, 0.00000157,0.00000347,0.00000444,0.00416493,-0.00124947,0.00047068,-0$ $.00521389,-0.01291047,-0.01405319,-0.03392872,-0.13221425,-0.23658875$, $0.00145241,-0.00150888,-0.00036371,0.00037665,-0.00180549,0.00265671,0$ $.00017917,0.00077562,0.00008817,0.00031849,-0.00045340,-0.00098752,-0$. $00098856,0.00168109,-0.00008299,0.03363278,0.14506294,0.24882158,-0.00$ $101308,0.00131928,0.00030574,0.00025984,0.00001279,0.00032957,-0.00010$ $399,-0.00007449,-0.00025833,0.00000308,-0.00009501,0.00005503,0.000040$ $83,0.00013718,0.00021313,0.00004288,0.00029450,0.00020250,-0.00000906$, $-0.00002267,-0.00015635,-0.00005829,-0.00006998,-0.00004264,-0.0000273$ $7,0.00003469,0.00005458,0.00001843,0.00003280,-0.00000289,-0.00005424$, $-0.00002727,-0.00015782,-0.00001275,0.00000971,-0.00000029,0.00095378$, $-0.00145638,0.00417461,0.00010831,0.00080353,0.00008411,0.00252464,-0$. $00214027,0.00040218,-0.00016512,-0.00221853,0.00039393,-0.24677015,-0$. $13501358,-0.01748099,-0.01255778,-0.01166103,-0.00334059,-0.00082278,-$ $0.00037705,0.00029692,-0.00020777,0.00022718,-0.00016484,-0.00003645,0$ $.00003748,0.00014671,0.25818170,0.00065101,-0.00175033,0.00180873,0.00$ $017384,0.00024201,-0.00027410,0.00016822,-0.00001169,0.00036018,0.0000$ $0684,0.00020203,-0.00011526,-0.00003564,-0.00012774,-0.00021359,-0.000$ $09841,0.00030109,0.00011997,0.00002591,-0.00006074,0.00010317,0.000047$ $36,-0.00023302,0.00023770,0.00006534,-0.00000523,-0.00002870,-0.000026$ $95,-0.00001506,0.00010443,0.00003938,-0.00001676,0.00011084,-0.0000098$ 
$5,-0.00001777,0.00000698,-0.00158245,0.00509847,-0.00229858,0.00087020$ $,-0.00065551,0.00013640,-0.00169665,0.00245365,-0.00517404,-0.01503921$ $,-0.00533486,-0.00031410,-0.13507183,-0.12961489,-0.02108019,0.0018307$ $2,0.00414680,-0.00132986,0.00016202,0.00007564,-0.00014890,0.00019850$, $-0.00044185,0.00029357,-0.00074675,-0.00032112,-0.00001098,0.14841826$, $0.12848653,-0.00105349,0.00111001,-0.00008727,-0.00028861,-0.00076779$, $0.00015156,0.00023575,0.00016352,-0.00007929,0.00018152,-0.00009621,0$. $00000315,0.00026967,0.00032881,0.00001471,-0.00015932,-0.00014745,0.00$ $005473,-0.00006951,0.00013696,0.00021411,0.00014202,0.00051681,-0.0001$ $3329,-0.00004282,0.00006776,0.00003851,0.00004464,0.00001652,0.0000058$ $5,0.00002075,-0.00004156,-0.00006019,-0.00004146,-0.00000897,0.0000031$ $4,0.00385052,-0.00247113,-0.00088529,0.00018801,0.00016787,-0.00106282$ $, 0.00092681,-0.00485878,-0.00307118,-0.02190343,-0.01353495,0.00247284$ ,-0.01694422,-0.02084823,-0.05549881,0.01999519,0.01251131,0.00496245, $0.00119197,0.00038542,-0.00047935,-0.00017853,0.00028525,-0.00006592,-$ $0.00115233,-0.00077588,-0.00007018,0.01610315,0.02604818,0.05365129,0$. $00038088,0.00053543,0.00066480,0.00003495,-0.00006428,0.00004195,0.000$ $01298,0.00011276,-0.00003381,0.00003284,-0.00009726,0.00000423,-0.0001$ $0527,-0.00004499,-0.00001470,0.00017044,0.00014190,-0.00014972,-0.0002$ $2467,0.00009873,0.00023457,-0.00003096,0.00000599,-0.00006120,-0.00002$ $197,0.00001057,0.00000850,-0.00001029,-0.00000729,0.00001329,-0.000005$ $98,-0.00001041,0.00000706,-0.00000542,-0.00000237,0.00000835,-0.000677$ $34,-0.01442105,-0.02115943,-0.00316378,-0.00434826,0.00111680,-0.00105$ $992,0.00024539,0.00043579,-0.00108154,-0.00250426,0.00439458,0.0071110$ $1,0.01186472,0.01752078,-0.05777052,-0.03047890,-0.03064613,0.00031887$ $, 0.00104248,-0.00118903,0.00005787,-0.00073211,-0.00101365,-0.00007088$ $, 0.00029238,-0.00014749,-0.00029546,0.00173969,-0.00124051,0.05640235$, $-0.00013890,0.00010893,0.00008013,0.00016722,0.00004687,-0.00007895,-0$ $.00005514,-0.00009450,0.00001723,-0.00003093,0.00003736,-0.00005456,0$. $00006820,0.00002788,0.00006043,-0.00011422,-0.00010759,0.00003585,0.00$ $007492,-0.00007240,0.00006277,0.00000464,-0.00001930,0.00004939,0.0000$ $0987,-0.00000361,-0.00000091,0.00000173,-0.00000311,0.00000733,0.00000$ $577,-0.00000662,0.00001554,0.00000121,0.00000412,0.00000163,-0.0005419$ $9,-0.00537374,-0.01447987,-0.00460254,0.00208220,-0.00098130,0.0001021$ $7,-0.00048489,0.00085581,-0.00220808,0.00524030,-0.00151031,-0.0010712$ $4,0.00369432,0.00075726,-0.03179766,-0.13273830,-0.13695591,0.00150699$ $,-0.00105582,0.00109190,-0.00011013,-0.00013807,-0.00070853,0.00027105$ ,-0.00044268,0.00020373,0.00184542,-0.00227928,0.00176571,0.03662035,0 $.13157546,-0.00062058,-0.00068960,-0.00036305,-0.00014261,0.00010147,-$ $0.00001811,-0.00000568,-0.00007079,0.00007098,0.00002376,0.00005946,-0$ $.00001151,-0.00002728,0.00004332,-0.00002773,0.00004638,-0.00001479,0$. $00003927,0.00018532,0.00000212,-0.00032748,0.00003232,-0.00005792,0.00$ $001965,0.00001684,-0.00000151,-0.00000168,0.00001570,0.00001245,-0.000$ $01299,-0.00001710,0.00001230,-0.00002528,0.00000955,0.00000279,-0.0000$ $0371,0.00305579,-0.00023087,-0.00020472,0.00068033,-0.00120361,0.00244$ $318,0.00031670,0.00076955,0.00004763,0.00489592,-0.00132550,-0.0000260$ $5,-0.00507719,-0.01252549,-0.01267460,-0.03297104,-0.13822007,-0.24340$ $068,-0.00034647,0.00156334,0.00048287,0.00008180,-0.00005560,0.0001600$ $6,-0.00018082,0.00019251,-0.00020275,-0.00112697,0.00180582,-0.0000156$ $5,0.03116167,0.14980621,0.25406595,0.00171050,-0.00092737,-0.00000139$, $0.00533121,0.00111373,0.00048767,0.00137615,-0.00278311,0.00196696,-0$. 
$00067125,0.00059075,0.00048927,0.00003021,0.00005148,-0.00005739,-0.00$ $019654,-0.00045026,0.00021812,-0.00006706,-0.00013333,0.00001476,-0.31$ $582078,0.11136076,-0.13251768,-0.00024648,0.00026362,-0.00100698,-0.00$ $001089,-0.00001347,-0.00009942,0.00008739,0.00003148,-0.00002370,0.000$ $02228,-0.00007258,-0.00001792,0.00005932,0.00008145,0.00005218,0.00007$ $601,-0.00001072,-0.00010283,-0.00002170,0.00007785,-0.00000827,0.00004$ $387,0.00011993,-0.00016917,0.00007398,-0.00012976,-0.00017195,-0.00010$ $925,-0.00002530,0.00012309,0.00000250,-0.00000416,0.00000764,0.0000001$ 4,0.00000486,-0.00000302,0.00003354,-0.00000309,0.00005744,0.00000576, $0.00004365,0.00001687,-0.00000928,0.00000786,-0.00000059,0.32528438,-0$ $.00333322,-0.00453690,0.00250815,0.03142114,-0.01480829,0.01596520,0.0$ $0260846,0.00256970,-0.00227413,-0.00000540,-0.00093679,-0.00019566,-0$. $00012775,0.00017042,-0.00013072,0.00193835,0.00016691,0.00047892,0.000$ $03043,0.00023764,0.00022415,0.09870197,-0.09158094,0.08177937,-0.00148$ $709,0.00007328,0.00027652,-0.00012206,0.00004243,-0.00003467,-0.000178$ 42,-0.00051682,0.00024505,0.00001602,0.00011979,0.00003190,0.00013375, $-0.00000129,-0.00009692,0.00002929,0.00002030,-0.00001685,0.00001467,0$ $.00008437,-0.00005923,-0.00066215,0.00016266,0.00028191,0.00002690,0.0$ $0005140,-0.00010596,-0.00002739,-0.00002206,-0.00001853,-0.00008383,-0$ $.00000336,0.00008159,-0.00001251,0.00000217,-0.00000168,0.00004298,0$, $0.00003770,0.00000881,-0.00008168,0.00001117,0.00001355,-0.00000265,-0$ $.00000458,-0.12072558,0.10544593,0.00059964,0.00241026,0.00157412,-0.0$ $0859251,-0.00475177,-0.00530404,0.00046997,-0.00098902,-0.00025252,0.0$ $0001301,0.00045907,-0.00060467,0.00060049,-0.00007084,0.00005699,-0.00$ $093029,0.00010200,0.00059007,0.00073283,-0.00027917,0.00042883,-0.1578$ $1539,0.10398153,-0.11042685,0.00064145,0.00023577,0.00006697,0.0000652$ $9,-0.00003211,-0.00005558,-0.00000338,0.00015966,-0.00003017,-0.000019$ $35,0.00002975,0.00007135,-0.00007476,-0.00007312,-0.00008579,-0.000091$ $55,0.00000006,0.00015389,0.00000353,-0.00024192,0.00004340,0.00003692$, $-0.00014851,-0.00028512,-0.00003726,0.00012371,0.00035751,0.00012293,0$ $.00006028,-0.00010797,-0.00001163,0.00000075,-0.00000056,-0.00001198,0$ $.00000379,0.00000438,-0.00000028,0.00003127,-0.00004470,-0.00001483,-0$ $.00005650,0.00000425,0.00001606,-0.00001761,-0.00000056,0.14868349,-0$. $09135146,0.10736696,-0.00003271,0.00117133,-0.00101436,0.00079055,-0.0$ $0639233,0.00181094,0.00221223,0.00234417,0.00100717,0.00025405,-0.0004$ $4746,-0.00022814,0.00019112,-0.00015304,0.00023218,-0.00043314,0.00014$ $184,-0.00028612,-0.00011426,-0.00000699,-0.00015560,-0.34004744,-0.071$ $98088,0.13997854,0.00023761,0.00023239,0.00030446,-0.00003946,0.000046$ $07,0.00009466,0.00001785,0.00001355,-0.00008974,0.00004186,-0.00004370$ ,-0.00008097,-0.00058505,-0.00038481,-0.00008297,-0.00052493,0.0000145 $6,0.00065367,-0.00015194,-0.00038940,0.00004867,0.00080292,0.00018155$, $-0.00050099,-0.00029667,0.00056788,0.00069399,0.00058978,0.00015690,-0$ $.00045350,0.00014496,0.00000071,-0.00017927,-0.00000307,0.00000125,0.0$ $0002730,-0.00018749,0.00014978,-0.00028590,-0.00000495,-0.00013320,-0$. $00009266,0.00001108,-0.00001657,-0.00000575,-0.01698401,-0.00822089,0$. $01561763,0.35411113,-0.00077429,0.00035832,0.00001649,-0.03531651,-0.0$ $1146463,0.01359066,0.00334291,-0.00350198,0.00349784,-0.00145124,-0.00$ $096593,0.00014376,-0.00036173,0.00019567,-0.00006835,-0.00011068,0.000$ $02080,-0.00034237,-0.00014278,0.00000393,-0.00015876,-0.06082978,-0.06$ $325352,0.06256452,-0.00001534,0.00012240,-0.00007340,0.00004798,0.0000$ $2395,0.00002743,0.00005357,0.00006096,-0.00004569,0.00002784,-0.000525$ 
$87,0.00005671,0.00035572,0.00003017,-0.00012137,0.00002274,-0.00004342$ $, 0.00006265,0.00084001,0.00036606,0.00007475,0.00056120,0.00014077,-0$. $00002069,-0.00020453,-0.00029985,-0.00079628,-0.00019161,-0.00012532,-$ $0.00004404,-0.00029753,-0.00000717,0.00028419,-0.00007812,0.00002427,0$ $.00001529,-0.00011829,0.00004641,-0.00019129,-0.00002537,-0.00003871,0$ $.00003565,-0.00001276,0.00000513,0.00002900,0.01153131,0.00334377,-0.0$ $0958589,0.08314729,0.07548380,0.00042266,0.00016148,0.00001665,0.00661$ $140,-0.00605813,-0.00656356,-0.00032620,0.00123881,0.00099875,-0.00027$ $981,0.00061735,0.00137987,0.00089054,-0.00016055,0.00020813,-0.0002766$ $8,0.00005870,-0.00031555,-0.00067882,-0.00010035,-0.00093816,0.1687864$ $8,0.08173878,-0.10546085,0.00029768,-0.00033624,-0.00053945,-0.0000523$ $9,-0.00002032,-0.00000986,-0.00004262,-0.00021974,0.00003796,-0.000042$ $57,0.00002609,-0.00006867,-0.00018451,-0.00024777,-0.00024427,-0.00018$ $422,-0.00011089,0.00029202,0.00054936,0.00016973,0.00021051,-0.0004141$ $4,-0.00024556,0.00046235,-0.00015742,0.00010448,0.00021636,0.00035366$, $0.00003776,-0.00041888,0.00001789,-0.00000470,-0.00005263,-0.00001766$, $0.00001999,0.00000953,-0.00033387,0.00002718,-0.00031917,-0.00001561,-$ $0.00009116,-0.00006735,0.00002724,-0.00002293,-0.00001304,-0.01793716$, $-0.00763123,0.00647983,-0.15701324,-0.06895079,0.10469950 \backslash-0.00000220$ ,-0.00000713,0.00001202,-0.00000851,0.00000441,-0.00001133,0.00000751, $0.00001907,-0.00000487,0.00000339,-0.00000339,-0.00000371,-0.00000437$, $-0.00001133,0.00000478,0.00000290,0.00001918,-0.00000521,-0.00000361,-$ $0.00000055,0.00000027,-0.00000696,-0.00001979,-0.00001797,-0.00000440$, $-0.00000334,-0.00000552,0.00000046,0.00000046,-0.00000169,-0.00000258$, $-0.00000476,0.00000295,-0.00000639,0.00000024,-0.00000341,0.00008365$,$0.00000439,-0.00006715,-0.00004172,-0.00000356,0.00003147,0.00000028,0$ $.00000251,-0.00000423,0.00001213,0.00000224,0.00000280,0.00000686,-0.0$ $0000874,-0.00001409,-0.00002992,0.00000953,0.00004900,-0.00002364,0.00$ $000207,0.00003758,0.00001030,0.00000585,0.00000050,0.00000147,0.000002$ $86,0.00000108,0.00000575,-0.00000112,-0.00000252,0.00000721,-0.0000047$ $8,-0.00000647,0.00000714,0.00000096,-0.00000655,-0.00001476,0.00000352$ ,0.00001225॥I@

meta-Complex

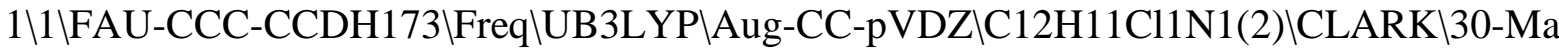
y-201610\\\#N Geom=AllCheck Guess=TCheck SCRF=Check GenChk UB3LYP/Aug-C C-pVDZ Freq $\mid$ p-CHLOROPHENYL RADICAL pre-addition complex with aniline (meta) \0,2\C,2.5435554466,1.8378781007,0.0722291149\C,2.7671339756,1. 1084950643,-1.0969244371\C,2.0648313308,-0.075867994,-1.3477916702\C, 1 $.1326297888,-0.5214306521,-0.4036528945 \backslash \mathrm{C}, 0.9019113021,0.200570503,0.7$ 688891113\C,1.6056761806,1.392890696,1.022681193\H,3.1014130449,2.7576 $420328,0.2561736876 \backslash \mathrm{H}, 3.497887808,1.4724056395,-1.820169946 \backslash \mathrm{H}, 2.242585$ $8947,-0.642507523,-2.2609700896 \backslash \mathrm{H}, 0.1776114498,-0.1628755233,1.5000403$ $71 \mathrm{~N}, 1.4241887222,2.0862283407,2.2231914203 \backslash \mathrm{H}, 0.5771732832,-1.44351122$ $2,-0.5797610719 \backslash \mathrm{C},-3.2836590498,2.9905519224,-2.0521058343 \backslash \mathrm{C},-3.163133$ $3796,4.2485549623,-2.6479061691 \backslash \mathrm{C},-1.8787672988,4.7730562995,-2.878194$ 9058\C,-0.8052323236,3.9962868172,-2.4930686889\C,-0.8818203125,2.7512 475098,-1.9036974451\C,-2.1700772395,2.2345002451,-1.6773216581\Cl,-4. $8978990798,2.3349790241,-1.7628912813 \backslash \mathrm{H},-4.0522656698,4.8116443093,-2$. $9288213313 \mathrm{UH},-1.7583421998,5.752358725,-3.3422664029 \mathrm{U},-0.0003289089,2$ 
$.1771447733,-1.6177353467 \backslash \mathrm{H},-2.2990597226,1.2561984646,-1.2164349018 \mathrm{LH}$ ,1.675933605,3.0658826271,2.2053077938\H,0.5413552053,1.9282929045,2.6 911940317\IVersion=ES64L-G09RevD.01 $\backslash$ State $=2-A \backslash H F=-978.8519955 \backslash S 2=0.757$ $653 \backslash \mathrm{S} 2-1=0 . \backslash \mathrm{S} 2 \mathrm{~A}=0.750033 \backslash \mathrm{RMSD}=4.873 \mathrm{e}-09 \backslash \mathrm{RMSF}=2.142 \mathrm{e}-06 \backslash \mathrm{ZeroPoint}=0.194$ 2756\Thermal $=0.2081592 \mathrm{ZPE}=121.9097815 \mathrm{VDipole}=0.1992698,0.4927447,0.29$ 50338\DipoleDeriv $=-0.1092523,0.2315564,0.190775,0.1033183,-0.0379189,0$ $.1029876,-0.0480847,-0.1777788,-0.3605191,0.001087,0.0864322,-0.090012$ $1,0.1538527,0.1084037,0.0740055,-0.0713502,0.1521259,0.1021468,-0.2218$ $039,-0.0336598,-0.0038362,-0.0441507,-0.1843084,0.0518424,-0.038733,0$. $0799248,-0.1386801,-0.0047077,0.1308645,-0.1069605,0.0521109,0.0610709$ $, 0.0963707,-0.1603524,0.0416508,0.143911,-0.0971666,0.0184151,-0.23164$ $3,0.1497392,-0.2245944,-0.3319082,-0.0066444,-0.0388348,-0.1976989,0.0$ $200957,-0.126263,-0.1575642,-0.0921541,0.3393419,0.6560477,-0.0851217$, $0.5966643,1.1304954,0.075735,-0.0889348,0.009872,-0.1001626,-0.0408922$ $,-0.0587218,0.0128527,-0.0601005,0.0606627,0.0334904,-0.0566703,0.0929$ $401,-0.0671141,0.0665536,0.0243004,0.0983071,0.0172222,0.0068364,0.104$ $4718,-0.0244812,0.0490837,-0.0237777,0.0419661,-0.0871511,0.0558219,-0$ $.0924746,-0.0309156,0.0335584,-0.0632454,0.1079511,-0.0560755,0.073775$ $4,0.0010522,0.10809,0.0021915,-0.0124041,-0.2540521,0.0561543,-0.19288$ $21,0.1344653,-0.3795549,-0.3200951,-0.023517,-0.5023088,-1.3079221,0.0$ $577691,-0.1038654,0.0068354,-0.093438,-0.0285881,-0.0419541,0.010364,-$ $0.0400849,0.0757266,0.9832712,0.3739148,-0.1465538,0.4046589,0.1188908$ ,-0.0631004,-0.1787515,-0.0732589,-0.0151201,-0.1195557,-0.075915,0.02 $70308,-0.0692178,-0.0571415,-0.0202354,0.0340572,-0.0223258,-0.0916435$ ,-0.0397935,0.0266841,-0.0129166,-0.0267237,0.0382435,-0.0539331,0.013 $2601,-0.0573686,-0.0528055,-0.1455582,-0.0492424,0.0121174,-0.0538749$, $-0.0309974,-0.0339703,0.0197002,-0.0292775,-0.0841238,0.0092986,-0.069$ $6597,0.0533043,0.0035172,-0.0065181,-0.0283197,-0.0099838,-0.0156338,-$ $0.0394805,-0.1905124,-0.0268145,0.000087,-0.059727,-0.022953,-0.026889$ $1,0.0202692,-0.0241244,-0.0764996,-0.6884093,-0.2117778,0.0781796,-0.2$ $365493,-0.1743445,0.0440436,0.1038643,0.0480747,-0.0885888,-0.0088401$, $0.0541281,-0.0287603,0.0318538,0.0674078,0.0300447,-0.0165566,0.029499$ $1,0.113621,0.073918,-0.0123336,0.0032024,-0.0098696,-0.0113605,0.07520$ $79,0.0043766,0.072421,0.1085745,0.0645957,-0.0203558,0.02942,-0.027232$ $6,0.0687985,-0.0035271,0.0316234,-0.0009592,0.1142861,0.1013658,-0.032$ $7715,0.0259788,-0.0104632,0.0026385,0.0512083,0.0147822,0.0509434,0.09$ $02541,0.2100676,0.0311098,0.1705994,-0.0146686,0.0947766,-0.0259074,0$. $0908397,0.0698911,0.2383016,0.1109275,-0.013269,0.1137519,-0.0483171,0$ $.1173046,-0.1113981,0.0208868,-0.0260783,0.3115852 \backslash$ Polar $=179.8178385,9$ $.372712,164.5720384,-8.2192323,-2.8994129,150.1523202 \backslash \mathrm{PG}=\mathrm{C} 01$ [X(C12H11 $\mathrm{Cl1N1})] \mathrm{N} I m a g=0 \backslash 10.39091184,0.22307195,0.56269868,-0.16903910,0.157559$ $26,0.56333871,-0.09326589,0.00775904,0.06808241,0.42296065,-0.02113142$ $,-0.16505954,-0.09321672,0.21325324,0.52698144,0.03612177,-0.13532926$, $-0.26614459,-0.20626797,0.11260394,0.58140827,0.02804861,-0.00917148,-$ $0.04135349,-0.14398344,-0.09786274,0.01705420,0.38140403,0.01277843,-0$ $.02709181,-0.05684667,-0.12689270,-0.25713669,-0.02477226,0.18815208,0$ $.53092845,-0.01817481,-0.02489788,0.00664882,-0.01493753,-0.06902834,-$ $0.11537019,-0.18699531,0.16754114,0.60141698,-0.01663952,0.00491850,0$. $02601976,-0.02227260,-0.04602312,-0.00656451,-0.18477837,-0.05248314,0$ $.13108031,0.40863555,0.00193580,-0.00344410,-0.00304440,-0.02374748,-0$ $.00912835,0.01914107,-0.08154348,-0.12682046,0.05302281,0.22726052,0.5$ 
$6926684,0.02363419,-0.00784991,-0.04694377,0.01768609,0.05258295,0.038$ $20162,0.09929192,0.00892127,-0.20524428,-0.16939123,0.13697327,0.55280$ $469,-0.01753656,-0.02022628,0.01263613,-0.00509469,-0.00723042,-0.0051$ $8528,0.00558512,0.01443045,0.01934023,-0.09362150,0.00769219,0.0677816$ $5,0.40367325,-0.03654157,-0.00502993,0.04464282,-0.01079862,-0.0398441$ $8,-0.02497978,0.03619054,0.04277025,-0.01637305,-0.02007344,-0.1636799$ $0,-0.09142011,0.20606444,0.51472432,-0.00604007,0.01931721,0.03584093$, $-0.00866416,-0.02871923,-0.02204692,0.04216934,0.01579430,-0.04041967$, $0.03801318,-0.13535227,-0.26698622,-0.21128667,0.12751109,0.59725196,-$ $0.17842811,-0.08432546,0.08038341,0.00503942,0.04353869,0.04175130,-0$. $03539020,-0.03217297,0.01246688,0.02838601,-0.00502623,-0.04855654,-0$. $14496062,-0.08706551,0.02517653,0.41692593,-0.05206641,-0.13318936,0.0$ $0491342,0.02152058,0.04726087,0.01959145,-0.03233194,-0.03271056,0.017$ $80392,0.01742529,-0.02814175,-0.05864976,-0.11841838,-0.23613550,-0.00$ $760503,0.19349445,0.54705211,0.11499202,0.05105702,-0.18307222,0.01834$ $329,-0.01403229,-0.04261302,0.01195951,0.01836593,-0.00168431,-0.02424$ $424,-0.02603466,0.00959441,-0.00750755,-0.05564612,-0.11377230,-0.1777$ $4351,0.13006100,0.57245729,-0.11761665,-0.12906192,-0.01453009,0.00493$ $499,0.00558091,0.00132648,0.00440102,-0.00117217,0.00307957,-0.0007645$ $4,0.00079839,0.00037883,0.00084176,-0.00233940,0.00519572,-0.00602359$, $-0.01824715,-0.00601839,0.11757369,-0.12910841,-0.24554570,-0.04358486$ ,-0.00887803,-0.01046544,0.00005170,-0.00074114,0.00242737,-0.00459971 $, 0.00062951,0.00011506,0.00041868,-0.00285237,0.00275742,0.00064296,-0$ $.00364536,-0.00663759,-0.00542411,0.14132096,0.25825837,-0.01456469,-0$ $.04391593,-0.05932944,-0.01490508,-0.02211134,-0.00029263,0.00368410,-$ $0.00362400,-0.00257662,0.00013871,0.00009394,-0.00114913,0.00462578,0$. $00005347,-0.00134613,0.01082561,0.01713868,0.00624883,0.01363903,0.049$ $91410,0.05754826,-0.00012243,-0.00305302,0.00192616,-0.17256981,-0.069$ $98233,0.13108514,-0.00603553,-0.00305631,0.01154993,0.00279220,-0.0051$ $1502,0.00039841,-0.00024234,0.00039006,-0.00076385,0.00494013,-0.00161$ $119,0.00209546,-0.00168763,0.00197592,-0.00154042,0.17549393,0.0110795$ $9,0.00815585,-0.01143167,-0.07051827,-0.08297146,0.05700997,-0.0169489$ $7,-0.00324106,0.01868776,-0.00480527,-0.00252341,-0.00314275,0.0004663$ $8,-0.00083342,-0.00045529,-0.00187269,0.00081279,-0.00500887,0.0017127$ $3,-0.00045918,0.00139831,0.07877092,0.08159012,0.01747890,0.01028049,-$ $0.01255648,0.13126405,0.05663273,-0.17581815,-0.00449673,-0.00316830,0$ $.00668308,0.00088038,-0.00267378,0.00256904,-0.00075088,-0.00039127,-0$ $.00040730,0.00196311,-0.00504613,-0.00122569,-0.00173254,0.00114880,-0$ $.00009566,-0.14382708,-0.05867005,0.18212389,0.00190236,-0.00487218,0$. $00156993,0.00504605,-0.00787069,-0.01432339,-0.05109034,0.01482886,0.0$ $4711277,-0.00022464,0.01225250,0.01672955,0.00071321,-0.00405155,0.004$ $13239,-0.00100519,-0.00041061,-0.00005438,0.00035099,-0.00062385,-0.00$ $054467,-0.00153321,0.00196534,-0.00131726,0.04739861,-0.00529096,-0.00$ $178889,-0.00094419,0.00640409,-0.00914028,-0.02213867,0.01494221,-0.12$ $641211,-0.12976440,-0.00211602,0.00783471,0.01008738,-0.00361693,0.001$ $78899,0.00047610,-0.00042402,-0.00071645,0.00061117,0.00008788,-0.0005$ $0359,-0.00124005,0.00172867,-0.00031610,0.00131330,-0.01368489,0.12926$ $559,0.00108309,-0.00169769,0.00305487,0.00174200,0.00015522,-0.0014293$ $4,0.04737082,-0.13004869,-0.25039243,0.00054402,-0.01198703,-0.0130058$ $6,0.00467480,0.00114224,0.00056437,-0.00005528,0.00061537,-0.00007246$, $0.00025210,-0.00010979,-0.00007284,-0.00159619,0.00089528,-0.00025287$, 
$-0.05234268,0.14028307,0.26239431,0.00256558,-0.00466788,0.00008506,-0$ $.00035503,0.00021468,-0.00089949,0.00495121,-0.00120016,0.00240847,-0$. $00119917,-0.00235747,0.00160533,-0.16561476,-0.07175334,0.12981579,-0$. $00705434,-0.00293254,0.01301116,-0.00055054,-0.00014509,0.00046642,-0$. $00048404,0.00033060,-0.00023260,0.00009384,-0.00032752,0.00023682,0.16$ $988674,-0.00416132,-0.00259552,-0.00308598,0.00036552,-0.00106225,-0.0$ $0060282,-0.00167896,0.00074931,-0.00458853,0.01191844,0.00842679,-0.01$ $115050,-0.07168216,-0.08244965,0.05606967,-0.01722332,-0.00379546,0.01$ $882242,-0.00087154,-0.00037867,0.00098538,0.00033065,-0.00018978,0.000$ $26141,0.00039468,0.00038938,-0.00069893,0.07912912,0.08140397,0.000522$ $47,-0.00234068,0.00225149,-0.00065802,-0.00027385,-0.00036147,0.001612$ $80,-0.00540335,-0.00153446,0.01755748,0.01125980,-0.01307368,0.1303453$ $1,0.05613901,-0.17405395,-0.00348903,-0.00418162,0.00443034,-0.0002776$ $3,-0.00004800,0.00022103,-0.00021323,0.00026231,-0.00020591,0.00105107$ $, 0.00041323,-0.00067308,-0.14315472,-0.05836645,0.18190684,0.00057262$, $0.01638743,0.03249610,-0.00118750,-0.00836126,0.00114933,0.00250085,0$. $00331558,-0.00228927,-0.00221983,-0.00275086,0.00631835,0.00777974,-0$. $01585432,-0.01939545,-0.08353047,-0.00665253,0.06789653,-0.00080123,0$. $00234109,-0.00171140,-0.00012818,0.00059048,0.00110501,-0.00007393,0.0$ $0040556,-0.00022624,-0.00028280,0.00087925,-0.00164077,0.47659408,-0.0$ $0056768,0.01180867,0.00638474,-0.00463490,-0.00347357,-0.00057671,0.00$ $408730,0.00121856,-0.00321275,-0.00755219,0.00008088,0.00526484,0.0015$ $6726,-0.02510997,-0.04323864,-0.02025735,-0.14728545,-0.11294515,0.000$ $64305,0.00084757,0.00149631,-0.00017715,0.00048166,0.00055030,0.000349$ $34,0.00008849,0.00003705,0.00255000,0.00065122,0.00102049,0.18484700,0$ $.64707016,0.01770655,-0.01659555,-0.04117705,0.00173690,0.00435496,0.0$ $0677371,-0.00039258,-0.00517390,-0.00612906,0.00320960,0.00291967,0.00$ $409776,-0.00370303,-0.02118801,-0.01123724,0.03633332,-0.08144626,-0.2$ $2509288,-0.00204571,0.00061646,0.00099166,0.00024491,-0.00024951,-0.00$ $065350,-0.00035190,0.00015694,-0.00003100,-0.00148005,0.00204629,0.000$ $63085,-0.27664331,0.12028081,0.41593778,-0.00058345,0.00082220,0.00010$ $868,0.00113156,-0.00294101,0.00557479,-0.00550708,-0.01747787,-0.00546$ $451,-0.11905534,-0.12814812,-0.01612301,0.00529732,0.00498064,0.001992$ $32,0.00451307,-0.00123245,0.00357300,-0.00043735,0.00028757,-0.0003360$ $4,-0.00043535,-0.00091801,-0.00023318,-0.00150525,0.00193647,-0.001504$ $36,-0.00169234,0.00170357,-0.00172756,0.00020811,0.00007731,-0.0001482$ $3,0.11817007,0.00071760,0.00017299,0.00018300,-0.00321320,0.00281696,0$ $.00063746,-0.00352399,-0.00453782,-0.00393672,-0.12766315,-0.25159829$, $-0.04315507,-0.00918631,-0.00954447,-0.00038630,-0.00098448,0.00247444$ $,-0.00401991,0.00028519,-0.00031645,0.00018215,-0.00019747,-0.00036424$ ,-0.00021962,0.00163286,-0.00048503,0.00118378,0.00194034,-0.00045678, $0.00107383,-0.00053291,-0.00070828,-0.00002069,0.14071691,0.26262141,0$ $.00011798,0.00012859,-0.00108222,0.00517223,0.00006118,-0.00115696,0.0$ $1044996,0.01788888,0.00759948,-0.01601458,-0.04302777,-0.06017378,-0.0$ $1367207,-0.02190077,-0.00035309,0.00351443,-0.00382000,-0.00246248,-0$. $00033545,0.00019670,-0.00012335,0.00060842,0.00091794,0.00021331,-0.00$ $177007,0.00080339,-0.00013805,-0.00157756,0.00141655,-0.00007237,-0.00$ $064945,-0.00118565,0.00019769,0.01425886,0.04865815,0.05788054,-0.0000$ $3181,-0.00006865,0.00014707,-0.00000239,-0.00012131,0.00001628,0.00006$ $870,-0.00000837,0.00018631,0.00012897,0.00011553,0.00017874,0.00038196$ ,-0.00007812,-0.00003023,-0.00000245,-0.00026253,-0.00035495,-0.000038 
$52,0.00001502,-0.00002909,-0.00003140,0.00002384,-0.00001985,-0.000046$ $35,0.00004266,-0.00003848,-0.00008089,0.00007323,-0.00007773,0.0001548$ 2,0.00001460,0.00040126,-0.00005578,0.00005073,-0.00003793,0.51952616, $0.00004919,-0.00009565,0.00003899,0.00003973,0.00004974,0.00011476,-0$. $00004285,-0.00004424,0.00009487,0.00006925,0.00001498,-0.00002228,0.00$ $009935,0.00002644,-0.00000051,0.00000870,-0.00012296,-0.00021375,-0.00$ 005079,-0.00000544,-0.00003727,-0.00004296,0.00001643,0.00000060,-0.00 $001915,0.00000812,-0.00001289,-0.00002408,0.00000095,-0.00001324,0.000$ $05421,0.00003081,0.00019653,-0.00002645,0.00003374,-0.00000326,-0.0501$ $5946,0.53248724,-0.00002244,0.00000872,0.00001875,0.00001402,0.0000315$ $5,0.00001738,-0.00003601,0.00002711,-0.00006131,-0.00002646,-0.0000310$ $5,-0.00001628,-0.00006956,-0.00001219,-0.00002265,0.00004226,0.0000843$ $1,0.00008503,0.00000462,-0.00000971,0.00000123,0.00003071,-0.00000501$, $-0.00000872,0.00000211,-0.00002436,0.00000704,0.00002909,-0.00001273,0$ $.00002229,-0.00002368,-0.00001568,-0.00007985,0.00002010,-0.00000113,0$ $.00000316,0.02950753,-0.19425716,0.21766700,-0.00004014,0.00012154,-0$. $00005044,0.00001013,0.00001062,-0.00008621,-0.00002529,0.00002174,-0.0$ $0013011,-0.00006781,-0.00004326,0.00002204,-0.00011094,-0.00002878,-0$. $00001770,-0.00002681,0.00013723,0.00024910,0.00003316,-0.00000089,0.00$ $002066,0.00002339,-0.00000866,0.00000360,0.00001988,-0.00001298,0.0000$ $1874,0.00002609,-0.00000674,0.00000735,-0.00003510,-0.00006383,-0.0001$ $9891,0.00001734,0.00000233,0.00000271,-0.10665837,0.02528768,-0.012845$ $78,0.69769370,-0.00002868,-0.00001838,0.00003806,-0.00001958,-0.000046$ $13,-0.00006642,0.00003776,0.00003727,0.00002311,-0.00001743,0.00000575$ $, 0.00005502,0.00009898,-0.00000604,-0.00000355,-0.00002044,-0.00007795$ ,-0.00006528,0.00002891,0.00005017,0.00002328,0.00002431,0.00000956,-0 $.00001257,0.00000203,-0.00001206,-0.00000420,-0.00001825,0.00000897,-0$ $.00001069,-0.00000750,0.00002492,0.00004796,-0.00000653,0.00002425,-0$. $00000949,-0.03173089,-0.27690775,0.10057765,-0.04235760,0.56844538,0.0$ $0002398,0.00000194,-0.00001795,-0.00000157,0.00001038,0.00001526,-0.00$ $001969,-0.00000889,-0.00001158,0.00001641,-0.00001365,-0.00001068,-0.0$ $0004469,0.00002877,-0.00000248,-0.00000256,0.00001845,0.00001501,0.000$ $00050,-0.00001141,-0.00000190,-0.00001356,-0.00000202,-0.00000389,0.00$ $000514,0.00001060,0.00000764,-0.00000175,-0.00000134,-0.00000278,-0.00$ $000627,-0.00001235,-0.00001808,-0.00000048,-0.00001581,0.00000361,0.01$ $427207,0.10141490,-0.11203268,0.02874739,-0.21168565,0.22517627,0.0000$ $6432,-0.00009933,-0.00005545,0.00000145,0.00002942,0.00004759,-0.00000$ $998,-0.00003222,0.00004057,-0.00000049,-0.00002844,-0.00009453,-0.0000$ $3940,0.00006099,0.00000520,-0.00003869,0.00002563,0.00004300,-0.000007$ $60,0.00003111,0.00001713,0.00001889,0.00000301,-0.00000501,-0.00000335$ ,-0.00000742,-0.00000670,0.00000973,-0.00001128,0.00002588,0.00001446, $-0.00000729,0.00000070,0.00000998,-0.00001282,0.00000411,0.01718110,-0$ $.07717598,0.03675535,-0.27862169,-0.03573149,0.01375411,0.59477636,-0$. $00006817,0.00005569,0.00002101,-0.00003915,-0.00004677,-0.00003482,0.0$ $0000345,0.00004129,0.00001922,0.00005385,0.00000819,0.00011882,0.00004$ $555,-0.00004557,-0.00004616,0.00003777,-0.00008226,-0.00008044,0.00003$ $072,-0.00002075,0.00001635,0.00004128,-0.00002393,0.00000839,0.0000111$ $6,-0.00000102,-0.00000547,0.00001149,0.00002161,-0.00002604,-0.0000227$ $0,0.00006074,0.00003850,0.00000568,0.00002840,-0.00000727,-0.02598172$, $-0.01069661,0.00782983,-0.10477016,-0.13176635,0.03309492,-0.01067721$, $0.61959718,0.00002016,-0.00006439,-0.00000083,0.00001135,0.00006284,0$. 
$00001266,-0.00004739,0.00000052,-0.00002650,-0.00001978,-0.00002914,-0$ $.00000659,-0.00002365,0.00004266,0.00003116,0.00002655,-0.00000517,0.0$ $0001763,-0.00002688,0.00002363,-0.00001658,0.00000197,0.00000542,0.000$ $01093,0.00000914,-0.00001339,-0.00001773,0.00000338,-0.00001457,0.0000$ $1494,0.00002438,-0.00002020,0.00001200,0.00000011,-0.00000096,-0.00000$ $684,0.01253333,0.00711791,0.00320592,0.04654027,0.03407248,-0.07433927$ $, 0.01199198,-0.23731381,0.23407544,0.00002744,0.00009539,-0.00003200,0$ $.00005255,0.00012959,-0.00005458,-0.00004520,0.00001536,-0.00016001,-0$ $.00010191,-0.00007169,0.00002248,-0.00013492,-0.00003903,0.00005959,0$. $00003649,0.00013363,0.00018402,-0.00002443,-0.00002000,-0.00004080,-0$. $00004122,-0.00000135,0.00003577,0.00000864,0.00000619,0.00001784,-0.00$ $000862,-0.00000387,-0.00003184,-0.00004279,-0.00004490,-0.00016593,-0$. $00001594,0.00000483,0.00000664,-0.01510364,0.02431023,-0.01161954,-0.0$ $6383041,-0.00800629,0.00263669,-0.24214647,0.12298456,-0.06119993,0.39$ $523732,-0.00003958,0.00009825,-0.00003204,0.00003458,0.00003485,-0.000$ $06938,-0.00005633,0.00001783,0.00000803,-0.00001353,-0.00000083,-0.000$ 06746,-0.00004252,-0.00001271,0.00004446,-0.00002897,-0.00004684,-0.00 $011713,0.00002991,-0.00002654,0.00000784,-0.00003022,0.00000575,0.0000$ $0435,0.00000946,0.00000437,0.00003532,-0.00000325,-0.00001058,-0.00001$ $012,-0.00008101,0.00009261,0.00002823,-0.00001330,0.00000740,0.0000175$ $1,0.02396351,-0.05285750,0.02257096,0.04164917,0.05085516,-0.01759645$, $0.09966349,-0.17447523,0.06086675,-0.10674777,0.51205973,-0.00006405,0$ $.00002709,0.00001728,0.00006577,-0.00011089,0.00002816,0.00008918,-0.0$ $0001118,0.00003551,0.00002346,0.00006328,-0.00004384,-0.00002115,0.000$ $00815,0.00001080,0.00002997,-0.00004799,-0.00006818,0.00003973,-0.0000$ $1900,0.00003174,-0.00004441,0.00000465,-0.00003370,-0.00002619,0.00003$ $311,0.00000621,0.00000152,0.00001237,-0.00000320,-0.00003307,0.0000526$ $9,0.00006288,-0.00000198,-0.00001115,-0.00000118,-0.01153801,0.0225129$ $7,-0.01677124,-0.02093738,-0.01827905,0.02080558,-0.05007546,0.0611599$ $8,-0.07974159,0.05557157,-0.21165900,0.17097690,-0.00022922,-0.0000362$ $0,0.00012649,-0.00025628,-0.00016436,-0.00002001,-0.00005214,0.0000112$ $1,0.00029231,0.00017865,0.00015225,0.00011864,0.00030420,-0.00000135,-$ $0.00010910,-0.00005951,-0.00038801,-0.00056543,0.00007333,0.00005542,0$ $.00003599,0.00005910,0.00004667,-0.00008181,-0.00000405,-0.00000050,-0$ $.00006518,-0.00001858,-0.00001807,0.00005006,-0.00002047,0.00021213,0$. $00035278,-0.00000584,-0.00002622,-0.00001079,-0.05652536,0.03399149,-0$ $.01710115,-0.01827748,-0.03068570,0.01430687,0.04133596,0.02649761,-0$. $01195105,-0.09778071,0.00621010,-0.00380953,0.65680120,-0.00026880,0.0$ $0046428,-0.00006964,-0.00004493,-0.00024771,-0.00047626,0.00005878,-0$. $00017181,0.00012567,0.00012800,-0.00009664,0.00011689,0.00009459,-0.00$ $018495,-0.00016517,-0.00026730,0.00000988,0.00031776,0.00005268,0.0000$ $2856,0.00003990,0.00004321,-0.00000140,0.00003926,0.00000291,0.0000141$ $5,0.00003950,-0.00000979,0.00002137,-0.00002097,0.00003312,-0.00003455$ ,-0.00008567,0.00003157,0.00008417,-0.00000227,-0.01714896,0.05052359, $-0.02127419,-0.02776640,-0.03678670,0.01660245,0.05263508,-0.02955795$, $0.01638780,-0.01768666,-0.29414151,0.11543738,-0.06233100,0.57095110$,$0.00004928,0.00010030,-0.00021560,0.00000220,-0.00040860,-0.00000005,0$ $.00028973,-0.00000098,0.00031236,0.00022443,-0.00011525,-0.00035406,-0$ $.00024036,0.00010695,0.00004154,-0.00017947,0.00012657,-0.00019626,-0$. $00000550,0.00004178,-0.00000957,-0.00005269,0.00002207,0.00001205,-0.0$ $0005121,0.00003681,-0.00000088,-0.00001315,-0.00001237,0.00003572,-0.0$ 
$0003015,0.00009926,0.00018843,0.00000571,0.00002305,-0.00000120,0.0073$ $4170,-0.02035731,0.01616035,0.01283688,0.01643091,-0.00866905,-0.02453$ $513,0.01599111,-0.00459970,0.00766928,0.11564516,-0.10515260,0.0376603$ $9,-0.21285118,0.22591086,-0.00007267,0.00004527,0.00002161,-0.00010802$ $, 0.00006074,-0.00008109,-0.00004821,-0.00011809,-0.00008381,0.00003870$ ,-0.00009011,-0.00006968,-0.00019155,0.00001668,0.00001786,0.00001180, $0.00023036,0.00036308,-0.00003469,-0.00000402,-0.00001469,0.00000981,-$ $0.00001503,-0.00000028,-0.00000922,0.00001276,0.00000400,0.00005598,-0$ $.00003747,0.00009117,0.00002612,-0.00014149,-0.00023470,0.00004118,-0$. $00006768,-0.00000796,-0.22971299,0.06810722,-0.03470535,0.03859511,0.0$ $5860889,-0.02727645,-0.06457949,0.00660122,-0.00417195,0.02273304,-0.0$ $7795548,0.03724137,-0.28279315,-0.03115225,0.01139913,0.63203597,0.000$ $06939,-0.00024033,0.00002747,-0.00007718,-0.00006949,0.00023577,0.0000$ $6598,-0.00004460,0.00007219,0.00011612,0.00002890,-0.00014934,-0.00012$ $894,0.00015369,-0.00002701,0.00011700,0.00007857,-0.00011120,-0.000037$ $38,-0.00002173,-0.00001118,-0.00004127,-0.00000220,-0.00001726,-0.0000$ $1330,0.00004251,-0.00000147,0.00004733,-0.00005807,0.00014823,0.000005$ $76,-0.00001178,0.00004611,0.00003488,-0.00010179,-0.00000553,0.1253081$ $4,-0.17541988,0.05467462,0.01802918,-0.02888880,0.01750315,0.01034468$, $0.00130984,-0.00083637,-0.02902208,-0.02060279,0.01506938,-0.10108214$, $-0.12788361,0.03114849,0.01070979,0.62312715,-0.00002538,0.00015597,0$. $00002695,-0.00001929,0.00003388,-0.00016460,0.00002217,-0.00006175,0.0$ $0003306,0.00001268,-0.00006057,0.00003429,-0.00002792,0.00003661,-0.00$ 001996,-0.00003969,-0.00009946,-0.00011752,0.00003713,0.00000589,0.000 $02559,-0.00000803,-0.00000736,0.00002275,-0.00002735,0.00003193,0.0000$ $1502,-0.00000969,-0.00002839,0.00000260,-0.00005782,0.00011190,0.00005$ $113,-0.00005092,0.00008688,-0.00001158,-0.06183273,0.05376002,-0.09054$ $056,-0.00793479,0.01814255,-0.00143116,-0.00574631,-0.00079357,-0.0002$ $6108,0.01379310,0.01430557,0.00538094,0.04459334,0.03215460,-0.0737260$ $9,0.00245064,-0.23613891,0.23591286,-0.00003062,0.00018962,-0.00007176$ ,-0.00001361,0.00005931,-0.00012851,-0.00003564,0.00001511,-0.00016943 ,-0.00010893,-0.00004984,-0.00000329,-0.00017025,-0.00004645,0.0000039 $3,-0.00004457,0.00017862,0.00032906,0.00005545,-0.00002476,0.00003540$, $0.00005181,-0.00002675,0.00001122,0.00002888,-0.00002699,0.00002514,0$. $00003288,-0.00001756,-0.00000658,-0.00008131,-0.00003411,-0.00032342,0$ $.00002268,-0.00000349,0.00002137,-0.13152151,-0.03796634,0.01656194,-0$ $.02088756,-0.00978383,0.00428519,0.00275803,-0.00165680,0.00077317,-0$. $00116586,-0.00129010,0.00058506,0.00001900,0.00580728,-0.00280548,-0.0$ 4080996,-0.01762315,0.00768699,0.19168213,-0.00001683,0.00006283,-0.00 $001596,-0.00001096,0.00000536,-0.00004221,0.00000778,0.00001761,-0.000$ $04117,-0.00004028,-0.00001658,0.00000752,-0.00003866,-0.00001602,0.000$ $00868,-0.00000247,0.00003503,0.00006517,0.00002303,-0.00000697,0.00001$ $411,0.00002191,-0.00001074,0.00000073,0.00000566,-0.00000851,0.0000065$ $4,0.00000486,-0.00000210,-0.00001559,-0.00002809,0.00000679,-0.0000750$ $0,0.00000076,0.00000712,0.00000544,-0.03781649,-0.05121561,0.01285454$, $-0.03368833,-0.01060452,0.00671680,0.00332015,-0.00243713,0.00322829,-$ $0.00117126,0.00072229,-0.00077549,0.00065020,-0.00009480,0.00203811,0$. $00618625,0.00578643,-0.00045912,0.06340274,0.05746932,0.00000983,-0.00$ $002928,0.00001084,-0.00000161,-0.00001147,0.00001959,0.00000451,-0.000$ $00916,0.00003429,0.00001017,-0.00000107,-0.00001371,0.00002283,0.00002$ $883,-0.00000654,0.00000180,-0.00002788,-0.00006079,-0.00001129,0.00000$ 
606,-0.00000488,-0.00000823,0.00000538,-0.00000162,-0.00000643,0.00000 $579,-0.00000382,-0.00000228,-0.00000651,0.00001180,0.00000959,0.000008$ $32,0.00006075,-0.00000033,-0.00000207,-0.00000728,0.01640408,0.0128086$ $4,-0.02895187,0.01568386,0.00707017,0.00139646,-0.00160661,0.00315292$, $0.00274037,0.00055941,-0.00077777,-0.00049775,-0.00035035,0.00212241,0$ $.00318585,-0.00357136,-0.00078514,0.00499476,-0.02759192,-0.02092249,0$ $.02117786,-0.00000183,0.00002603,0.00000066,-0.00000301,-0.00000002,-0$ $.00001019,0.00000427,-0.00000252,-0.00001172,-0.00000314,0.00000003,0$. $00000503,-0.00001015,-0.00000960,-0.00000393,-0.00000226,0.00001403,0$. $00003108,0.00000572,-0.00001062,0.00000376,0.00000602,-0.00000559,0.00$ $000272,-0.00000006,-0.00000011,0.00000197,0.00000296,0.00000160,-0.000$ $00318,-0.00000259,-0.00000333,-0.00002928,0.00000063,0.00000175,0.0000$ $0094,0.00344803,-0.00467340,0.00227123,-0.24427001,0.12387801,-0.06199$ $502,-0.01657036,0.01487629,-0.00738885,-0.00511965,-0.00231510,0.00091$ $099,0.00081428,-0.00083513,0.00045015,0.00137266,-0.00108078,0.0004141$ $5,0.00124491,-0.00059360,0.00031846,0.25756580,-0.00000543,0.00001312$, $0.00000420,0.00000031,-0.00000213,-0.00000371,0.00000083,0.00000493,-0$ $.00000476,0.00000183,0.00000866,0.00001971,0.00001905,-0.00001631,-0.0$ $0000077,-0.00000284,-0.00000512,-0.00000818,0.00000034,-0.00000897,-0$. $00000195,-0.00000078,-0.00000290,0.00000056,0.00000125,0.00000250,-0.0$ $0000011,-0.00000268,0.00000741,-0.00001110,0.00000416,-0.00000032,0.00$ $000391,-0.00000208,0.00000450,0.00000138,0.02347519,-0.00948352,0.0063$ $7701,0.12031461,-0.12870200,0.04653243,-0.01139339,0.00840118,-0.00212$ $649,-0.00301599,0.00163044,0.00221545,-0.00027500,-0.00049273,-0.00016$ $416,-0.00193287,-0.00319655,0.00371326,0.00045031,0.00027083,-0.001351$ $25,-0.12926132,0.13205444,0.00000266,0.00000762,-0.00001055,0.00000372$ $, 0.00000064,0.00000699,0.00000349,-0.00000004,-0.00000929,-0.00001072$, $0.00000937,-0.00000070,-0.00000472,-0.00001171,0.00000192,-0.00000225$, $0.00001517,0.00003808,-0.00000262,-0.00000520,-0.00000102,-0.00000125$, $0.00000027,0.00000106,0.00000106,-0.00000160,-0.00000047,0.00000234,0$. $00000179,-0.00000004,0.00000414,-0.00001373,-0.00002907,0.00000124,-0$. $00000743,0.00000212,-0.01114256,0.00593919,0.00018892,-0.06030568,0.04$ $661811,-0.05751072,0.00507797,-0.00176185,0.00513171,0.00128647,0.0022$ $3246,0.00538930,0.00015814,-0.00018772,-0.00079325,0.00085119,0.003762$ $17,0.00298816,-0.00016930,-0.00137305,-0.00196078,0.06493846,-0.053553$ $36,0.04978964,0.00000988,0.00003170,0.00000123,0.00001137,0.00002177,-$ $0.00000758,-0.00000729,-0.00001082,-0.00001851,-0.00001586,0.00000019$, $-0.00000992,-0.00003217,-0.00000405,0.00000835,0.00000551,0.00002273,0$ $.00002710,-0.00000794,-0.00001608,-0.00001278,-0.00000918,-0.00000026$, $0.00000502,0.00000090,0.00000285,0.00000035,0.00000112,-0.00000223,-0$. $00000264,-0.00000797,-0.00000706,-0.00002619,-0.00000115,-0.00000556,0$ $.00000392,-0.00344825,-0.00131045,0.00050909,0.00021105,-0.02589265,0$. $01221623,-0.05497790,-0.02655091,0.01232086,0.00570873,0.02076775,-0.0$ $0980598,-0.00344946,0.00372270,-0.00184815,-0.00125998,-0.00018108,0.0$ $0002585,-0.00096354,-0.00115971,0.00053589,0.00112938,0.00041403,-0.00$ $012069,0.05671680,0.00001456,-0.00002356,0.00000344,-0.00002293,-0.000$ $00371,0.00000941,0.00001363,0.00000088,0.00002375,0.00000258,0.0000096$ $1,0.00000201,0.00002199,-0.00000063,-0.00000761,-0.00000535,-0.0000049$ $9,-0.00002345,-0.00000229,0.00000565,0 ., 0.00000702,0.00000286,-0.00000$ $112,-0.00000203,-0.00000191,-0.00000471,-0.00000393,0.00000231,-0.0000$ $0116,0.00001073,-0.00000424,0.00001729,-0.00000142,-0.00000070,0.00000$ 
$075,-0.00177090,0.00262613,0.00187482,0.00119849,-0.00613697,0.0055065$ $5,-0.03074374,-0.27778290,0.11552634,0.00001512,-0.01235342,0.00568723$ $, 0.00195298,0.00086951,0.00202230,0.00031090,0.00029821,-0.00034792,-0$ $.00021573,-0.00037933,0.00016743,0.00014895,-0.00009558,-0.00186588,0$. $02939631,0.29341484,0.00002404,0.00001521,-0.00000778,-0.00001001,0.00$ $001921,0.00000378,-0.00000084,-0.00001470,-0.00001481,-0.00001821,0.00$ $000461,-0.00000006,-0.00000254,-0.00002377,-0.00000898,-0.00000865,0.0$ $0003975,0.00007162,-0.00001047,-0.00000871,-0.00000524,0.00000499,-0.0$ $0000051,0.00000324,0.00000103,-0.00000382,0.00000107,0.00000103,0.0000$ $0189,-0.00000212,0.00000692,-0.00003165,-0.00005563,0.00000246,-0.0000$ $0650,0.00000317,0.00068295,0.00184853,0.00568850,-0.00064122,0.0051303$ $6,0.00307321,0.01431187,0.11556625,-0.08873727,0.00008010,0.00606105,-$ $0.00309744,-0.00106486,0.00197756,0.00398303,-0.00015316,-0.00032922,-$ $0.00023182,0.00008787,0.00014855,-0.00009796,0.00000357,-0.00185829,-0$ $.00313377,-0.01346690,-0.12852748,0.08308424,-0.00008091,-0.00031839,--$ $0.00038938,-0.00036891,0.00021323,0.00040217,-0.00000084,0.00022157,0$. $00010046,-0.00009183,0.00007381,-0.00062600,-0.00033455,0.00031899,0.0$ $0022785,0.00005108,0.00001599,-0.00068050,0.00001727,0.00001114,0.0000$ $0748,-0.00003652,0.00003101,-0.00004649,0.00003576,-0.00008123,-0.0000$ $2882,0.00001903,-0.00005630,0.00007721,-0.00007232,0.00016434,0.000399$ $76,0.00000937,-0.00008591,0.00003555,-0.00227529,-0.00273107,0.0011474$ $5,-0.00014927,-0.00053672,0.00027573,0.00135682,-0.00228390,0.00097375$ $, 0.00647995,-0.00033014,0.00030715,-0.23964014,0.11955694,-0.05974470$, $-0.01803619,0.01562339,-0.00776326,-0.00118566,0.00021838,-0.00012898$, $-0.00004609,0.00003473,-0.00000604,0.00033495,-0.00025497,0.00013870,0$ $.25273654,0.00044648,-0.00048744,-0.00004931,0.00045304,0.00047929,0.0$ $0053468,-0.00004067,-0.00002886,-0.00047190,-0.00032361,-0.00039519,0$. $00006621,-0.00016096,0.00002429,0.00030418,0.00024385,0.00032993,0.000$ $39555,-0.00016179,-0.00002395,-0.00009134,-0.00012117,-0.00001162,0.00$ $002909,0.00000355,0.00001573,0.00004236,0.00001234,0.00001199,-0.00004$ $291,0.00001795,-0.00025887,-0.00042601,0.00003822,-0.00001870,0.000046$ $18,-0.00215831,0.00158222,0.00231064,-0.00106035,-0.00052059,0.0000657$ $3,-0.00034745,-0.00311665,0.00381186,0.02052048,-0.01239362,0.00626132$ $, 0.12408109,-0.12799537,0.04551752,-0.01154288,0.00942840,-0.00180732$, $-0.00095195,-0.00002379,-0.00001560,0.00005755,-0.00005983,-0.00017138$ $, 0.00077303,-0.00028932,0.00011224,-0.13009915,0.13384513,-0.00016081$, $-0.00024559,0.00008498,-0.00023185,0.00056793,0.00015655,-0.00029984,-$ $0.00000454,-0.00032997,-0.00035419,0.00032538,0.00039615,0.00041278,-0$ $.00006156,-0.00022753,-0.00004757,-0.00011143,-0.00035007,0.00003621,0$ $.00003278,0.00001227,0.00018251,-0.00001754,-0.00001521,0.00011596,-0$. $00011217,-0.00000728,-0.00002453,-0.00002763,0.00001168,0.00005232,-0$. $00005330,-0.00001428,0.00000090,-0.00009110,0.00001472,0.00091058,0.00$ $228340,0.00542847,0.00050984,0.00010437,-0.00037145,0.00011817,0.00372$ $582,0.00315351,-0.00963979,0.00602043,-0.00278620,-0.06192876,0.045491$ $39,-0.05840134,0.00509387,-0.00141873,0.00672895,0.00045486,0.00000205$ ,-0.00003043,-0.00002619,-0.00017034,-0.00034561,-0.00036114,0.0000944 $7,-0.00014206,0.06532188,-0.05447991,0.05030197,-0.00005093,0.00006745$ $, 0.00004324,-0.00001307,-0.00003414,-0.00007602,-0.00001216,0.00001224$ $, 0.00010195,0.00007651,0.00006699,0.00022480,0.00028507,-0.00008065,-0$ $.00008212,-0.00007402,-0.00026566,-0.00023675,0.00002845,0.00001500,0$. $00001330,0.00002000,0.00000718,-0.00000843,0.00000209,-0.00000097,-0.0$ 
$0000716,-0.00005038,0.00004645,-0.00009004,0.00004248,0.00009551,0.000$ $21363,-0.00004995,0.00008447,-0.00002193,0.00481034,0.02224952,-0.0104$ $6656,-0.00366779,0.00216526,-0.00118116,-0.00052771,0.00083846,-0.0003$ $8546,-0.00476422,-0.00345691,0.00147610,-0.00048974,-0.02465372,0.0116$ $2292,-0.05725887,-0.03118529,0.01443016,0.00103427,0.00070221,-0.00028$ $012,0.00042797,0.00118121,-0.00056068,0.00003997,-0.00005661,0.0000333$ $6,0.00126893,0.00033853,-0.00008719,0.05895715,-0.00000298,-0.00002176$ ,-0.00002917,0.00004404,-0.00001971,0.00000043,0.00000772,0.00002532,-$0.00006045,-0.00001516,0.00007096,0.00007132,0.00005247,-0.00007947,0$. $00003286,-0.00002353,0.00002647,0.00010632,0.00000794,0.00001249,0.000$ $00683,0.00000807,0.00000182,-0.00001198,0.00000614,-0.00000087,-0.0000$ $0944,0.00000609,0.00005379,-0.00002543,0.00003148,-0.00006184,-0.00004$ $699,0.00002828,-0.00003802,0.00000014,-0.00585856,-0.01058495,0.006433$ $55,0.00304180,0.00102062,0.00183757,0.00015225,0.00056176,-0.00072477$, $-0.00237122,0.00142103,0.00237080,0.00118094,-0.00527378,0.00459502,-0$ $.02784928,-0.28374718,0.11730605,-0.00032793,0.00046020,-0.00144870,-0$ $.00021136,-0.00033339,0.00036159,-0.00001259,-0.00015650,-0.00013449,0$ $.00028654,-0.00011823,-0.00186817,0.03187014,0.29678077,-0.00000251,0$. $00000058,-0.00002812,0.00000070,0.00002573,-0.00004581,-0.00002424,0.0$ $0004292,-0.00007458,-0.00003299,0.00009926,0.00010756,0.00006125,-0.00$ $011091,0.00006610,-0.00003908,-0.00003909,0.00007010,0.00000221,-0.000$ $00380,-0.00000804,-0.00000018,-0.00000269,0.00000416,0.00003983,-0.000$ $01655,-0.00000992,-0.00003996,0.00007076,-0.00010543,0.00003579,-0.000$ $03632,-0.00004051,0.00000427,-0.00004738,0.00003059,0.00282854,0.00690$ $976,-0.00005144,-0.00158507,0.00180887,0.00392974,-0.00006342,-0.00070$ $080,-0.00061697,0.00096418,0.00237540,0.00542856,-0.00060638,0.0042415$ $3,0.00222104,0.01284218,0.11715371,-0.08981584,0.00020326,-0.00143870$, $-0.00196497,0.00009854,0.00039139,0.00025213,0.00001777,-0.00013876,-0$ $.00037866,-0.00002459,-0.00185504,-0.00315085,-0.01462529,-0.12871512$, $0.08422242,-0.00022114,0.00204844,0.00158867,-0.00067011,0.00008927,-0$ $.00066035,-0.00041858,0.00025601,0.00085060,0.00107602,-0.00119256,-0$. $00044169,0.00198153,0.00100507,0.00171003,-0.00086334,0.00141644,-0.00$ $939868,-0.00008177,-0.00089394,0.00057876,-0.00000407,0.00000874,0.000$ $05720,0.00001630,-0.00010959,-0.00001053,-0.00006340,0.00037749,-0.000$ $18403,-0.05508737,-0.08374993,0.03375370,-0.00005287,0.00006722,-0.000$ $05476,-0.00011643,-0.00005300,0.00002094,0.00003524,0.00000533,-0.0000$ $0194,-0.00001106,0.00002383,-0.00001001,0.00003806,0.00005169,0.000010$ $36,-0.00000025,0.00000648,0.00003227,-0.00001595,-0.00002395,0.0000406$ $0,0.00007691,0.00002429,-0.00000999,0.00000494,-0.00000261,-0.00000199$ $, 0.00000778,-0.00000924,-0.00000436,-0.00000448,-0.00001202,-0.0000446$ $2,-0.00001983,-0.00000913,-0.00001096,0.06052005,-0.00061263,0.0022576$ $7,0.00223892,-0.00066425,-0.00040300,-0.00092736,0.00050762,0.00060225$ ,-0.00004019,-0.00162520,0.00034586,0.00040588,0.00168285,0.00033293,$0.00255694,-0.00221645,-0.01482044,0.00507987,0.00094418,-0.00014003,-$ $0.00035833,0.00000791,0.00000214,0.00007693,0.00019812,-0.00006683,0.0$ $0007744,0.00035773,-0.00006353,0.00031521,-0.11589065,-0.42001783,-0.0$ $0747758,0.00011205,-0.00009855,-0.00015277,0.00000416,0.00001241,-0.00$ $000133,0.00000598,-0.00000810,-0.00000097,0.00000148,-0.00001604,0.000$ $00251,0.00000887,0.00000191,-0.00000773,-0.00002837,0.00001127,-0.0000$ $1121,0.00000880,0.00000103,-0.00000063,-0.00000973,-0.00000708,0.00000$ 485,0.00000014,0.00000033,0.00000171,0.00000498,-0.00000051,0.00000977 
$, 0.00000490,0.00001239,0.00000032,-0.00000790,-0.00000923,-0.00000894$, $0.11193916,0.43378892,-0.00261757,-0.00023817,0.00135883,-0.00006630,0$ $.00023775,-0.00041014,-0.00012233,0.00020438,0.00040412,-0.00056012,-0$ $.00170596,0.00021323,0.00323007,-0.00107956,-0.00466041,-0.01225808,-0$ $.03578885,-0.00153686,0.00110847,0.00061118,-0.00009977,-0.00002220,0$. $00000862,0.00004866,0.00003849,0.00009331,-0.00000720,-0.00025021,0.00$ $000362,-0.00000358,0.03746478,0.01704550,-0.03947450,0.00002079,-0.000$ $16161,-0.00043604,-0.00011043,-0.00006652,0.00002162,0.00004673,0.0000$ $0585,-0.00000330,-0.00001604,0.00002909,-0.00002368,0.00002751,0.00004$ $441,0.00002193,-0.00001086,0.00002580,0.00002052,-0.00002033,-0.000018$ $83,0.00004687,0.00009484,0.00002990,-0.00001657,0.00000982,0.00000075$, $0.00000244,0.00000186,-0.00000786,-0.00000071,-0.00001717,-0.00006804$, $-0.00006671,-0.00000683,-0.00000486,-0.00000847,-0.02543057,0.00310048$ $, 0.04552874,0.00084922,0.00031625,0.00028510,0.00097619,-0.00147864,-0$ $.00016384,0.00047648,0.00056871,-0.00017533,-0.00014712,-0.00018365,0$. $00091983,0.00125125,-0.00026163,-0.00213479,-0.00240528,0.00020444,-0$. $00629306,-0.00023936,0.00028181,-0.00039245,-0.00005436,0.00008435,-0$. $00005482,-0.00012192,0.00013081,-0.00009007,-0.00019027,0.00055081,0.0$ $0021623,-0.34431944,-0.07736664,0.19139013,-0.00002362,-0.00000104,-0$. $00004457,-0.00004864,0.00000940,0.00000006,0.00000356,-0.00000590,0.00$ $000482,0.00000709,-0.00001220,-0.00000542,0.00000379,0.00002454,0.0000$ $0501,0.00001665,-0.00000839,0.00002049,0.00001057,0.00002489,0.0000363$ $5,0.00001205,-0.00000152,0.00000906,0.00000160,-0.00000288,-0.00000235$ $, 0.00000327,-0.00000413,0.00000233,0.00001330,-0.00000578,-0.00000340$, $-0.00001454,-0.00004217,-0.00004387,-0.00612620,0.00526626,-0.00053434$ $, 0.35006572,-0.00025214,-0.00115606,-0.00370472,-0.00031199,0.00149189$ $, 0.00008902,-0.00013313,-0.00060134,-0.00040261,0.00022766,-0.00037169$ $, 0.00049543,0.00124837,0.00041043,-0.00163702,0.01812430,0.00569260,-0$ $.00115166,0.00039761,0.00005835,0.00002074,0.00007896,-0.00012754,-0.0$ $0013485,-0.00011559,-0.00000091,0.00006725,-0.00115861,-0.00068667,0.0$ $0015909,-0.05874197,-0.06549637,0.00203151,-0.00000887,-0.00000626,-0$. $00002798,0.00007767,0.00002133,-0.00001080,-0.00001238,0.00000029,0.00$ $000389,0.00000078,-0.00001673,0.00000027,-0.00002214,-0.00003003,0.000$ $00563,-0.00000519,-0.00001439,-0.00002436,0.00000778,0.00003408,-0.000$ $04011,-0.00004748,-0.00002063,0.00000759,-0.00000187,0.00000103,0.0000$ $0287,-0.00000459,0.00000426,0.00000417,0.00000281,0.00001266,0.0000136$ $6,0.00000124,0.00000970,-0.00000582,-0.03126348,-0.00171794,0.01769962$ $, 0.07191228,0.06250993,-0.00097691,-0.00545567,-0.00206584,0.00163865$, $0.00053183,-0.00079012,-0.00021327,0.00036196,0.00071137,-0.00049354,0$ $.00016414,-0.00099612,-0.00059075,0.00345821,0.00176260,0.02990653,0.0$ 0646056,-0.02070328,-0.00010404,0.00011357,0.00005219,-0.00003731,-0.0 $0022468,-0.00041349,0.00000708,-0.00009692,0.00006469,0.00001958,-0.00$ $109539,0.00055251,0.15614364,0.00898992,-0.10497199,0.00002737,0.00005$ $515,0.00007801,-0.00019846,-0.00005687,0.00002091,0.00004413,-0.000011$ $85,0.00000420,0.00001103,-0.00000198,-0.00001164,0.00002975,0.00006141$ $, 0.00000114,-0.00001976,0.00001316,0.00004792,0.00001473,0.00000893,0$. $00005628,0.00011040,0.00003236,-0.00001360,0.00000683,-0.00000558,0.00$ $000280,0.00000698,-0.00000983,0.00000246,0.00004079,-0.00001880,-0.000$ $05026,-0.00004370,-0.00004291,-0.00003279,-0.00237865,0.00023011,-0.00$ 091903,-0.18295110,-0.01346081,0.12761106\\-0.00000159,-0.00000320,-0. $00000204,-0.00000165,-0.00000062,-0.00000139,0.00000133,0.00000495,0.0$ 
0000107,-0.00000176,-0.00000078,-0.00000233,0.00000113,-0.00000118,-0. $00000012,-0.00000151,-0.00000301,-0.00000119,0.00000117,-0.00000257,-0$ $.00000115,0.00000030,-0.00000240,-0.00000341,-0.00000073,-0.00000359,-$ $0.00000551,-0.00000033,-0.00000112,-0.00000039,0.00000131,-0.00000149$, $0.00000072,-0.00000265,0 .,-0.00000091,0.00000219,0.00000349,0.00000139$ $, 0.00000089,0.00000063,0.00000223,-0.00000218,-0.00000015,0.00000072,0$ $.00000535,0.00000612,0.00000269,0.00000127,-0.00000197,0.00000061,-0.0$ $0000286,0.00000187,0.00000167,-0.00000002,0.00000296,0.00000213,-0.000$ $00026,0.00000222,0.00000310,0.00000096,0.00000137,0.00000256,-0.000002$ $62,0.00000084,-0.00000032,-0.00000125,0.00000138,0.00000038,0.00000168$ ,-0.00000226,-0.00000025,0.00000183,-0.00000149,-0.00000026III@

para-Complex

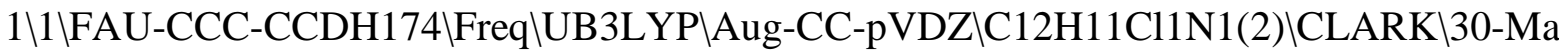
y-201610\॥N Geom=AllCheck Guess=TCheck SCRF=Check GenChk UB3LYP/Aug-C C-pVDZ Freq \p-CHLOROPHENYL RADICAL pre-addition complex with aniline (para) \0,2\C,1.9514476223,2.3314080345,0.9887093778\C,2.7656424479,2. 2587252971,-0.1473901655\C,2.7750972347,1.0770070026,-0.8972876932\C,1 $.9859258201,-0.0124385716,-0.5241571231 \backslash \mathrm{C}, 1.1651302438,0.0584398915,0$. 6170088084\C,1.1587044567,1.2473594405,1.3705037086\H,1.9322790968,3.2 427939137, 1.5876970244\H,3.3833144374,3.1064018913,-0.4414694396\H,3.4 $028525255,1.0004206764,-1.7858663792 \mathrm{WN}, 0.4214843581,-1.0513021899,1.02$ 97307496\H,0.5344856408,1.3153391224,2.2632591522ไH,2.0064504021,-0.92 88581195,-1.116407971\C,-3.0760118999,3.5252107467,-1.8132513575\C,-3. 4097285542,3.1432522742,-3.1151059537\C,-2.4902862522,2.381908459,-3.8 585562997\C,-1.2996578386,2.0574303991,-3.2410794425\C,-0.9325658798,2 $.4151767254,-1.9602208485 \backslash \mathrm{C},-1.8579823652,3.1765690555,-1.2241780535 \backslash \mathrm{C}$ $1,-4.2331858303,4.480184997,-0.8814999819 \backslash \mathrm{H},-4.3679460308,3.4326504396$ $,-3.5447643082 \backslash \mathrm{H},-2.7270990259,2.0720368164,-4.8767894448 \backslash \mathrm{H}, 0.02290957$ $82,2.1322964649,-1.5181245929 \backslash \mathrm{H},-1.6282718523,3.4920592161,-0.20740536$ $31 \backslash \mathrm{H}, 0.1968629882,-1.7214938796,0.3059862389 \backslash \mathrm{H},-0.3731271214,-0.850302$ 7222,1.6226522791॥Version=ES64L-G09RevD.01 \State=2-A $\backslash H F=-978.8519955 \backslash$ $\mathrm{S} 2=0.757653 \backslash \mathrm{S} 2-1=0 . \mathrm{SS} 2 \mathrm{~A}=0.750033 \backslash \mathrm{RMSD}=9.906 \mathrm{e}-09 \backslash \mathrm{RMSF}=1.607 \mathrm{e}-06 \backslash \mathrm{ZeroPoi}$ $\mathrm{nt}=0.1942756 \backslash$ Thermal $=0.2081592 \mathrm{ZPE}=121.9097682 \mathrm{VDipole}=-0.1194334,-0.57$ 41327,-0.1605037\DipoleDeriv $=0.0110957,0.0647631,-0.1730357,0.1474982$, $0.1008077,0.0007846,-0.1245565,0.0544823,0.0893918,-0.1572104,0.053263$ $, 0.0063583,0.0899753,-0.1571959,-0.0275823,-0.0203937,-0.0386893,-0.23$ $03129,-0.0108825,0.1197988,-0.0800937,0.0725249,0.1820283,0.0987334,-0$ $.1517284,0.037816,0.0399076,-0.2746666,-0.21728,-0.0112823,-0.0122719$, $-0.0877263,0.0399676,0.0715679,0.3613929,-0.1444665,0.4050653,0.649816$ $4,-0.2336729,0.5656373,0.9576996,-0.4044501,-0.1855098,-0.3779374,0.12$ $8468,-0.1858726,0.0016735,-0.062706,-0.1941149,-0.35503,0.2138955,-0.1$ $617448,-0.1110832,0.0202713,0.1064363,-0.0094667,0.0362051,-0.0191931$, $-0.0426877,-0.0877887,0.0299823,-0.0885832,0.0406416,0.0641358,-0.0901$ $769,0.0607048,-0.0966124,-0.0244595,0.0122186,0.0671694,0.0144333,0.07$ $56688,0.0543796,-0.0147835,0.0983666,-0.0122239,0.0752922,-0.0216801,0$ $.1117962,-0.0208142,-0.0227777,-0.4512916,-0.2441171,0.0155122,-0.4466$ $897,-1.0923913,0.4472475,0.1474417,0.5448078,-0.3978754,0.0445434,-0.0$ $269703,0.1231825,-0.0318972,0.0636453,-0.0122903,0.1173189,-0.0117099$, $-0.0133255,0.1085476,-0.0405681,0.0271213,-0.0341902,-0.0598614,-0.082$ 
$8404,0.0368803,-0.0855958,0.0461845,0.4975017,-0.4360358,-0.3815041,-0$ $.4387784,0.3147767,0.3180627,-0.4166023,0.3487799,0.2750092,-0.0641727$ $, 0.0218937,0.0751098,0.0122305,-0.1230188,-0.0271167,0.0755663,-0.0271$ $585,-0.0803719,-0.0363744,-0.0152844,0.0100546,0.0041513,-0.0624633,0$. $0443734,0.0621826,0.0230859,0.0439982,-0.0913066,0.0322823,0.0639101,0$ $.0318655,-0.1228039,-0.0064217,0.0697325,-0.015306,-0.0468064,0.016639$ $4,-0.0179573,0.0836869,-0.0252181,-0.0387088,-0.0208004,-0.0075377,0.0$ $141185,-0.015055,-0.1257962,0.0515103,0.0494032,0.0624664,-0.1215886,-$ $0.0003578,0.0825214,-0.0198426,-0.0427319,-0.40156,0.2635797,0.2133628$ $, 0.2644702,-0.2837375,-0.1864751,0.2426421,-0.2070848,-0.266456,-0.005$ $6369,0.0461184,-0.0491162,0.0535432,0.1022834,0.0014684,-0.0270755,-0$. $0086295,0.0747161,0.080674,0.0177931,-0.036466,0.0148295,0.113915,-0.0$ $530563,-0.0359279,-0.050642,-0.0229675,0.0744516,-0.0020497,0.0233129$, $0.0018373,0.0926484,-0.0365067,0.0277976,-0.0412506,0.0807852,0.100368$ $7,-0.0045752,-0.0019708,-0.0130286,0.1040825,-0.0499538,-0.0234213,-0$. $0393742,-0.0093215,0.1347222,-0.1311186,0.0674899,-0.0276698,0.2891274$ ,-0.0551266,0.0063982,-0.1116932,0.1191487,0.1062092,-0.0721088,0.0760 $669,0.0308587,0.1753665,-0.1043047,0.0055003,-0.1435222,0.258277$ Polar $=170.3989211,-8.4773156,159.3670632,-9.577847,13.778237,164.7715024 \backslash \mathrm{PG}$ $=\mathrm{C} 01[\mathrm{X}(\mathrm{C} 12 \mathrm{H} 11 \mathrm{Cl1N} 1)] \backslash \mathrm{NImag}=0 \backslash 10.36556738,0.13876445,0.64667209,-0.222$ $54527,0.13539871,0.51852613,-0.15992343,0.00777538,0.13626581,0.383734$ $11,-0.02694239,-0.11461054,0.00232244,0.17952987,0.64006103,0.11407616$ ,-0.04355187,-0.24233610,-0.23497074,0.08386939,0.48995458,0.01608977, $0.02182861,0.00120570,-0.08795713,0.00253464,0.03712964,0.38923854,0.0$ $4841200,0.02061825,-0.05528290,-0.03228191,-0.26520294,-0.08480825,0.1$ $3662060,0.59894833,0.01807056,-0.02053228,-0.02992987,0.01500417,-0.13$ $088867,-0.16327967,-0.25520037,0.10771346,0.54312599,-0.02895115,-0.01$ $451686,0.02574770,0.01732258,-0.03490587,-0.03532533,-0.15563587,-0.09$ $907737,0.07281576,0.35900346,-0.01741408,-0.01220439,0.01735770,-0.008$ $99728,-0.04872256,-0.02237096,-0.13407986,-0.25115855,0.05616863,0.155$ $81389,0.65867424,0.02288101,0.01286135,-0.02584664,-0.01874599,0.01071$ $418,0.03896193,0.05179968,0.01188798,-0.11765644,-0.22477350,0.1254125$ $1,0.49919416,0.01635601,-0.00723897,-0.02593186,-0.00889076,0.01656410$ $, 0.01694071,-0.02533759,-0.03085264,0.01469204,-0.14822157,0.01597439$, $0.12020957,0.39642464,-0.03415805,-0.05058468,0.01279736,0.01713181,-0$ $.01562908,-0.03038729,-0.00506674,0.03685339,0.02885751,-0.02263410,-0$ $.11941074,-0.00562338,0.14269745,0.62076257,-0.04251650,-0.02132159,0$. $04416304,0.01650346,-0.03062825,-0.04526199,0.03203252,0.06356933,-0.0$ $0191570,0.09645554,-0.05392653,-0.22692120,-0.22096919,0.10540562,0.51$ $930912,-0.15617356,-0.13256060,0.05181553,-0.02097155,-0.00593405,0.03$ $453238,-0.00889857,-0.01963412,-0.00449593,0.01531053,0.02143711,0.000$ $30013,-0.09168529,0.00974970,0.04260624,0.38388135,-0.10009658,-0.2497$ $3608,0.01235950,-0.03141412,0.03060504,0.05671746,-0.01538568,-0.05386$ $305,-0.01099079,0.04146373,0.02111979,-0.04507709,-0.02653156,-0.23594$ $134,-0.07298542,0.15449905,0.60183389,0.07423083,0.05750606,-0.1184696$ $5,0.01775390,0.02350600,-0.00169781,-0.00238381,-0.00707518,-0.0041694$ $1,0.01264110,-0.01835957,-0.02323587,0.01754654,-0.12208891,-0.1673465$ $9,-0.26080509,0.09271426,0.53007367,-0.04585443,-0.00127967,0.01144301$ $, 0.00406235,0.01549474,0.00925925,0.00175801,-0.00157843,0.00578430,-0$ $.00108100,-0.00012121,-0.00008166,0.00240815,-0.00454626,0.00419696,0$. $00356611,-0.01478312,-0.00992261,0.03917782,-0.00167994,-0.24977826,-0$ 
$.13230110,-0.00151012,0.00177639,0.00091541,-0.00122977,0.00265842,-0$. $00107262,-0.00003958,0.00007926,0.00083861,-0.00467349,-0.00062756,0.0$ $0109316,0.00228112,-0.01543183,-0.01442544,0.00407922,0.26229497,0.011$ $11518,-0.13235708,-0.13522495,-0.00133113,-0.02196990,-0.00825873,0.00$ $599285,-0.00046374,-0.00161846,0.00004350,0.00087251,-0.00048700,0.003$ $89022,0.00096865,0.00265822,0.00082395,0.00805521,0.00733019,-0.015994$ $06,0.14374553,0.13720995,-0.00674571,-0.01437364,0.00233594,-0.1337737$ $3,-0.13181853,0.05386757,0.00253075,0.00230681,0.00025327,0.00440763,-$ $0.00080437,0.00216316,-0.00041311,0.00058206,-0.00003855,0.00237846,-0$ $.00006508,0.00522820,-0.00178432,0.00133433,-0.00192023,0.13543372,0.0$ $0306935,0.00430750,-0.00221469,-0.13212862,-0.22507241,0.05460982,-0.0$ $1494394,-0.01529433,0.00976483,-0.00030163,-0.00172896,-0.00489030,0.0$ $0063559,-0.00033370,-0.00050747,-0.00065849,0.00235434,-0.00169122,0.0$ $0099875,0.00012931,0.00112292,0.14257826,0.23582248,0.01318327,0.02092$ $689,-0.00295018,0.05406912,0.05474990,-0.06907263,-0.01068214,-0.01350$ $490,0.00721066,0.00251456,-0.00412884,0.00052824,0.00001368,-0.0004450$ $9,-0.00106388,0.00488699,-0.00247118,-0.00164421,-0.00216128,0.0007353$ $2,-0.00048379,-0.06015352,-0.05741603,0.06783167,0.00472095,-0.0013672$ $0,0.00189453,0.00325920,-0.00139242,-0.00036165,-0.13864113,0.00581345$ $, 0.13957057,-0.00611061,0.00283651,0.01437141,0.00331413,-0.00474875,0$ $.00271346,-0.00050993,-0.00012162,-0.00075807,0.00004624,-0.00010603,-$ $0.00000460,-0.00186737,0.00129825,-0.00180710,0.13873681,-0.00183844,-$ $0.00358332,-0.00406778,0.01565331,0.00319723,-0.02110721,0.00601240,-0$ $.05624428,-0.02025870,-0.01404432,0.00501157,0.01989913,-0.00451200,-0$ $.00261994,-0.00011033,-0.00015741,-0.00110341,0.00017904,0.00078162,-0$ $.00004237,-0.00114706,0.00100770,0.00035936,0.00124852,-0.00379310,0.0$ $5447443,0.00174157,-0.00463177,0.00170413,0.01012928,0.00186610,-0.009$ $04534,0.14020732,-0.02012731,-0.23644596,0.00351954,-0.00267578,-0.003$ $45619,0.00283015,-0.00008997,0.00387562,-0.00083548,0.00015321,0.00009$ $092,0.00053454,0.00006074,-0.00058830,-0.00200130,0.00081707,-0.000600$ $26,-0.15402782,0.02329065,0.24598813,0.00160599,-0.00002905,0.00344588$ ,-0.00144947,-0.00277216,-0.00180765,0.00367191,0.00365262,0.00419541, $-0.01628957,-0.02853195,0.01650866,-0.13380639,-0.11880860,0.07232025$, $0.00345302,-0.00840443,0.00100503,0.00022252,0.00004120,0.00002154,-0$. $00019241,0.00005694,-0.00031172,-0.00042948,-0.00072912,0.00037951,0.4$ $9669784,0.00509286,0.00466667,-0.00631778,-0.00493509,-0.00376048,0.00$ $475638,0.00145704,0.00039242,-0.00583100,-0.01035959,0.00217349,0.0102$ $9468,-0.08221830,-0.21557415,0.02818461,-0.02766910,-0.04732611,0.0053$ $0372,-0.00053539,-0.00093798,0.00014888,0.00018520,0.00008687,0.000304$ $89,0.00027219,0.00050131,-0.00002362,0.16480774,0.52298214,0.00600016$, $-0.00399007,-0.00432390,-0.00029989,0.00575493,0.00280885,-0.00031328$, $-0.00949403,-0.00192367,0.03030901,0.03295533,-0.01469539,0.04825606,0$ $.01229991,-0.10653084,-0.01215556,-0.01684501,0.01529462,-0.00066849,--$ $0.00092805,0.00041685,-0.00038945,0.00025399,0.00008460,0.00077666,0.0$ $0087667,-0.00037591,-0.23200054,0.24152494,0.51993149,-0.00729709,0.00$ $354705,0.01425312,0.00306756,-0.00513305,0.00249636,-0.00050985,0.0000$ 2013,-0.00075076,0.00424974,-0.00122331,0.00158180,0.00174254,-0.00238 $326,0.00066696,-0.13186802,0.00423012,0.13873823,-0.00187334,0.0012599$ $6,-0.00222154,-0.00020166,0.00001875,0.00091841,-0.00052363,0.00025636$ $,-0.00031594,-0.00046347,0.00074319,-0.00276532,0.13406935,-0.01361219$ $, 0.00546500,0.02093114,-0.00439901,-0.00262111,-0.00034406,-0.00019986$ 
,-0.00125819,0.00017762,-0.00175583,-0.00356374,-0.00356530,0.01503223 $, 0.00219748,-0.02119814,0.00385404,-0.05577080,-0.02054003,0.00147823$, $0.00032688,0.00075673,-0.00085170,0.00016517,0.00092584,0.00024067,-0$. $00002218,0.00019032,0.00185582,0.00155038,0.00079126,-0.00184410,0.053$ $89358,0.00316799,-0.00240086,-0.00400809,0.00275633,0.00062932,0.00372$ $945,-0.00086932,-0.00018873,-0.00001638,0.00113252,-0.00427436,0.00154$ $469,0.01166060,0.00282537,-0.01036695,0.13900655,-0.02079270,-0.234484$ $29,-0.00197138,0.00106401,-0.00067838,0.00036413,-0.00024776,-0.000163$ $21,-0.00030104,0.00018744,-0.00032300,-0.00124206,0.00137266,-0.000080$ $66,-0.15301488,0.02287631,0.24523489,-0.00121721,-0.00014924,0.0000550$ $1,0.00222143,-0.00436959,0.00409397,0.00315505,-0.01471249,-0.01111456$ - $-0.04331082,-0.00103075,0.01442239,0.00330968,0.01513011,0.00943946,0$ $.00132333,-0.00158774,0.00521955,-0.00044312,0.00023641,-0.00042425,0$. $00026857,-0.00087540,-0.00031650,-0.00189089,0.00150495,-0.00200263,-0$ $.00059030,0.00226861,-0.00126626,-0.00000877,0.00088640,0.00044433,0.0$ $3785228,0.00007355,0.00009749,0.00060411,-0.00493663,-0.00085596,0.001$ $69585,0.00278049,-0.01608054,-0.01481744,-0.00101472,-0.24723414,-0.13$ $126014,-0.00285753,-0.00081765,0.00092908,-0.00090619,0.00249642,-0.00$ $073822,0.00023865,-0.00017138,0.00010043,-0.00000763,-0.00087156,-0.00$ $083372,0.00123404,0.00036019,0.00105478,0.00097872,0.00096190,0.001167$ 43,0.00003377,-0.00001824,0.00001592,0.00379366,0.26146600,0.00015897, $0.00095038,-0.00069951,0.00368977,0.00066699,0.00289213,-0.00021332,0$. $00837295,0.00710977,0.01459746,-0.13094590,-0.13193266,-0.00144939,-0$. $02289295,-0.00895061,0.00556558,-0.00005372,-0.00152430,-0.00040592,0$. $00010899,-0.00025280,0.00023795,0.00034305,0.00036644,-0.00218186,0.00$ $081351,-0.00070920,-0.00261456,0.00063494,0.00066483,-0.00011004,-0.00$ $104949,-0.00068327,-0.01812680,0.14259803,0.13406046,-0.00000565,-0.00$ $019545,0.00005086,0.00005232,-0.00014235,0.00005667,0.00008611,0.00014$ $590,0.00007213,-0.00007909,-0.00009460,0.00008444,0.00012578,0.0003654$ $6,0.00000999,0.00027284,-0.00016297,-0.00005114,-0.00004090,0.00003480$ $,-0.00004274,-0.00004078,0.00001122,-0.00004371,-0.00002550,0.00000722$ ,-0.00002627,0.00001767,-0.00023074,0.00015695,-0.00005763,0.00004622, $-0.00005730,-0.00000456,-0.00000037,-0.00001512,0.49438432,-0.00004350$ $, 0.00009831,0.00000141,0.00002969,0.00007586,-0.00007735,-0.00003621,0$ $.00003640,-0.00007557,-0.00000403,0.00006350,-0.00011255,-0.00014868,-$ $0.00030317,0.00005683,-0.00016428,0.00010754,0.00005471,0.00004245,-0$. $00001460,0.00003500,0.00002074,-0.00003129,0.00002190,0.00002789,-0.00$ $000087,0.00003027,0.00000191,0.00021724,-0.00014190,0.00002771,-0.0000$ $2385,0.00000996,0.00002622,-0.00003969,0.00001294,-0.12609957,0.233124$ $56,-0.00006305,0.00008764,0.00007898,0.00001255,0.00010777,-0.00014610$ $,-0.00001076,0.00003532,-0.00008710,-0.00009398,0.00002526,-0.00004708$ ,-0.00015290,-0.00034216,0.00012468,-0.00011527,0.00010626,-0.00002094 $, 0.00006389,-0.00002784,-0.00000417,-0.00000073,-0.00003731,0.00004478$ $, 0.00006947,0.00000614,0.00001329,0.00001120,0.00024896,-0.00015000,0$. $00005780,-0.00003224,0.00001042,0.00005299,-0.00003715,0.00001032,0.13$ $028387,0.11095876,0.54209488,-0.00002701,0.00004778,0.00002420,-0.0000$ $0508,0.00007887,-0.00008231,0.00002420,0.00002244,-0.00004274,-0.00006$ $372,-0.00003577,-0.00012997,-0.00012847,-0.00020376,0.00007506,-0.0001$ $0645,0.00007268,0.00002900,0.00001471,-0.00000050,0.00001070,0.0000172$ $1,-0.00000755,0.00000929,0.00000949,-0.00001185,0.00000743,0.00004077$, $0.00017281,-0.00009281,0.00002974,-0.00001698,0.00002099,0.00002234,0$. 
$00000074,0.00002230,-0.11913689,-0.01201979,-0.09344145,0.62492590,0.0$ $0001859,0.00001095,-0.00001161,0.00000471,-0.00001645,0.00004883,-0.00$ $000815,-0.00003980,0.00001409,0.00003093,0.00002102,0.00006763,0.00003$ $240,0.00010380,0.00002118,0.00002200,-0.00007237,-0.00005670,0.0000062$ $8,-0.00000173,-0.00001836,-0.00002033,-0.00000079,-0.00000354,-0.00000$ $548,0.00000742,0.00000088,-0.00000876,-0.00006844,0.00003219,0.0000103$ 2,0.00000302,0.00001382,-0.00000917,0.00001222,-0.00001546,0.01019073, $-0.08921799,-0.04779864,-0.19831501,0.27954266,0.00005616,0.00003379,-$ $0.00005488,-0.00002445,0.00001154,0.00003843,-0.00001955,-0.00005591,0$ $.00003407,0.00007502,0.00000297,-0.00002496,-0.00000299,0.00000402,-0$. $00004345,-0.00010848,-0.00000894,0.00000759,0.00000388,0.00002043,0.00$ $003290,0.00001123,0.00000584,-0.00001490,-0.00002454,0.00000085,-0.000$ $00213,0.00001352,0.00000296,0.00000531,0.00000189,-0.00000500,0.000029$ $18,-0.00001503,0.00002465,0.00000934,-0.04039215,-0.07377741,-0.287256$ $01,0.11209952,0.13891448,0.58685699,0.00000760,0.00011029,-0.00002765$, $0.00003484,0.00005355,0.00003161,-0.00000824,-0.00008178,-0.00001875,0$ $.00008130,0.00005220,0.00003748,-0.00010982,-0.00008575,0.00007694,-0$. $00004766,-0.00003392,-0.00006659,0.00002765,-0.00001414,-0.00000669,-0$ $.00004193,-0.00002765,0.00001918,-0.00000413,0.00002626,0.00000281,0.0$ $0003579,0.00004193,-0.00000483,0.00001853,-0.00000305,0.00000600,-0.00$ $000278,0.00000209,-0.00001173,0.04308489,0.00658197,0.05840008,-0.1874$ $4878,0.07560999,0.03017009,0.53606931,-0.00002145,0.00000353,-0.000039$ $81,0.00002979,-0.00001310,-0.00000160,0.00002226,0.00002032,0.00001425$ ,-0.00001996,-0.00003059,-0.00003154,-0.00003746,-0.00002446,-0.000024 $70,0.00001853,0.00002105,0.00003113,-0.00000635,0.00000766,0.00001837$, $-0.00001166,-0.00000732,0.00000304,-0.00002854,0.00001229,-0.00001110$, $0.00000756,0.00003036,-0.00000375,-0.00001587,0.00000661,-0.00000064,0$ $.00000609,0.00000519,0.00000583,-0.01338615,-0.00503084,-0.04301118,0$. $10247647,-0.13359268,-0.05870853,-0.15328722,0.26386634,-0.00003644,0$. $00007252,0.00001803,0.00000394,0.00008776,-0.00002384,-0.00000488,-0.0$ $0007739,-0.00006005,0.00003385,0.00001690,0.00004220,-0.00005664,-0.00$ $008758,0.00008384,-0.00003938,-0.00004604,-0.00004790,0.00001884,0.000$ $00036,-0.00000953,-0.00002726,-0.00002036,0.00000965,-0.00002974,0.000$ $01967,0.00000693,0.00005143,0.00004766,0.00000825,0.00001434,0.0000070$ $6,0.00001441,-0.00001227,0.00003573,-0.00002708,0.01082582,-0.01979800$ $,-0.02829092,0.09443868,-0.09010728,-0.16368259,0.11922425,0.16220760$, $0.64851816,-0.00005605,0.00003844,0.00009556,-0.00001743,0.00011468,-0$ $.00008000,-0.00008346,-0.00008722,-0.00011473,0.00008502,0.00005285,-0$ $.00001871,-0.00009745,-0.00035782,0.00001756,-0.00004790,0.00011837,0$. $00008738,-0.00000705,-0.00000922,0.00000255,0.00002072,0.00000518,0.00$ $000254,0.00000308,-0.00001805,0.00003220,0.00011070,0.00015202,-0.0000$ $3416,-0.00001702,0.00000672,-0.00003107,-0.00004902,0.00002809,-0.0000$ $3827,-0.03273101,-0.00173128,-0.03526950,-0.04902559,0.04417736,0.0335$ $9495,-0.27277014,0.05918805,-0.11924557,0.42617418,0.00001767,-0.00005$ $163,0.00007007,0.00000453,-0.00000563,0.00002206,-0.00006330,0.0000228$ $6,0.00004427,0.00005595,-0.00000282,0.00009032,-0.00002924,0.00002866$, $0.00007924,0.00009820,-0.00005773,-0.00011956,0.00002456,-0.00000353,-$ $0.00002861,0.00000615,0.00001537,-0.00000067,0.00005525,-0.00001905,0$. $00000033,0.00009259,-0.00000378,0.00003072,0.00001455,-0.00000903,0.00$ $001304,-0.00002156,0.00001116,-0.00002119,-0.00151465,-0.00519778,0.00$ $000523,0.02485706,0.00106180,0.00181656,0.06825276,-0.07584166,0.01753$ 
$593,-0.09137839,0.14967165,0.00001061,-0.00005661,-0.00003928,0.000093$ $00,-0.00006456,0.00003201,0.00004554,0.00000422,0.00013088,0.00000665$, $0.00003463,0.00008856,0.00004101,-0.00001904,-0.00000138,0.00003052,-0$ $.00000296,-0.00003434,0.00001727,0.00000797,-0.00000419,-0.00002486,0$. $00001684,-0.00002239,0.00000563,0.00002585,-0.00002135,0.00003607,-0.0$ $0004598,0.00004942,-0.00000816,-0.00001910,-0.00000897,-0.00000355,-0$. $00003001,0.00000504,-0.03489663,-0.00009872,-0.04680847,-0.01265710,0$. $02440934,0.05585772,-0.09746711,0.00690598,-0.14781918,0.19862931,0.08$ $411286,0.50242375,-0.00006436,-0.00021820,-0.00009210,-0.00018119,-0.0$ $0010246,0.00004594,-0.00020973,-0.00005160,0.00017697,0.00001225,-0.00$ $003014,0.00028033,0.00043402,0.00064746,-0.00023698,0.00016763,-0.0001$ $9123,-0.00006546,-0.00000156,0.00007796,0.00006720,0.00004842,0.000078$ $47,-0.00005747,0.00001495,0.00000362,-0.00010485,-0.00019010,-0.000297$ $43,0.00008380,-0.00000827,-0.00000923,0.00007676,0.00001819,-0.0000195$ $2,0.00000758,-0.03966913,0.01994225,-0.00621635,-0.00062740,0.00608151$ $, 0.01494526,0.00375646,-0.01580371,-0.03709189,-0.11115957,-0.00625263$ ,-0.08626613,0.60698521,-0.00030263,0.00004031,0.00004374,-0.00014119, $0.00028424,-0.00030284,-0.00017500,-0.00053560,-0.00013544,0.00041955$, $-0.00002595,0.00023605,0.00018403,-0.00034467,-0.00003444,-0.00009907$, $-0.00005531,0.00012531,-0.00000860,0.00004661,0.00006942,0.00007514,0$. $00002733,0.00001095,-0.00000134,0.00000279,0.00002040,0.00013639,0.000$ $22172,-0.00006209,0.00000725,0.00000005,-0.00001573,-0.00004191,0.0000$ $7481,0.00003388,0.03971869,-0.00350725,0.02286237,0.00495244,-0.014909$ $39,-0.02722842,-0.02588204,0.02012362,0.00969123,0.00300910,-0.0793232$ $9,-0.06529998,-0.17986649,0.26518506,0.00015976,0.00023926,-0.00030729$ $, 0.00005281,-0.00027209,-0.00001523,0.00002851,-0.00018381,0.00033384$, $0.00036164,0.00012567,-0.00000137,0.00008106,0.00009064,-0.00040812,-0$ $.00042023,-0.00006452,0.00011419,-0.00002107,0.00005447,0.00007399,0.0$ $0001424,0.00006959,-0.00003778,-0.00007787,0.00000008,-0.00001713,-0.0$ $0009307,-0.00013214,0.00007354,-0.00002267,-0.00002211,0.00006919,-0.0$ $0007473,0.00003514,-0.00001314,0.04128379,-0.00056192,0.05330905,0.012$ $21865,-0.02579805,-0.04827851,-0.06149795,0.02171114,-0.01671419,-0.06$ $383137,-0.07617670,-0.30650584,0.13500020,0.12562086,0.58141949,0.0000$ $1037,0.00003828,0.00004202,-0.00002013,0.00016723,-0.00000238,-0.00009$ $202,0.00003457,-0.00015310,-0.00010111,0.00004570,-0.00004622,-0.00020$ $403,-0.00047360,0.00010764,-0.00003313,0.00015702,0.00012949,0.0000422$ $4,-0.00001056,0.00002219,0.00001130,0.00000525,0.00001486,0.00004062,-$ $0.00000491,0.00001592,0.00016877,0.00021398,-0.00005523,0.00000664,-0$. $00003074,-0.00002903,-0.00002969,0.00002040,-0.00001046,-0.25704994,0$. $06551507,-0.06881488,0.00310600,-0.02623242,-0.06729893,-0.05256768,0$. $02730623,0.00433771,0.04782744,0.00478682,0.05347834,-0.19245404,0.077$ $29101,0.02838664,0.55170454,-0.00013486,-0.00005617,0.00005277,-0.0000$ $6706,-0.00004087,-0.00010814,0.00011680,-0.00003874,0.00000145,-0.0000$ $0989,-0.00001917,-0.00005175,0.00000690,0.00014232,0.00008037,0.000202$ $72,-0.00006923,0.00003605,-0.00002041,-0.00002137,-0.00001695,0.000043$ $47,0.00001613,0.00002178,0.00001024,-0.00001343,-0.00001748,-0.0000039$ $6,-0.00003764,0.00002012,-0.00003304,0.00007495,-0.00012488,0.00002081$ ,-0.00000216,-0.00000718,0.04320562,-0.08658706,-0.00322606,-0.0104272 $4,0.02210083,0.02291168,0.02574260,-0.01358754,-0.00076183,-0.01413479$ ,-0.00103583,-0.04771624,0.10447916,-0.13359911,-0.05551256,-0.1683925 $5,0.28091675,-0.00008446,-0.00003308,0.00016806,-0.00000374,0.00003688$ 
,-0.00002514,0.00009211,0.00010669,-0.00024876,-0.00020153,-0.00014696 ,-0.00017323,-0.00000802,0.00019209,0.00011406,0.00013639,-0.00001176, $0.00001463,-0.00011524,-0.00005466,-0.00010012,-0.00002711,-0.00001668$ $, 0.00002118,0.00000793,-0.00001481,0.00002826,-0.00010820,-0.00005896$, $-0.00004530,-0.00001697,0.00009146,-0.00010388,0.00005422,-0.00003608$, $0.00000349,-0.12191780,0.02282111,-0.15198606,-0.02952428,0.00439693$,$0.01688702,0.00095172,0.00074087,0.00257082,0.00784364,-0.02528348,-0$. $03931470,0.09336550,-0.08729871,-0.15830088,0.09714888,0.17710357,0.65$ $841219,-0.00005133,0.00005908,0.00003614,-0.00000526,0.00010703,-0.000$ $09134,-0.00001569,0.00003071,-0.00010764,-0.00006294,-0.00001583,-0.00$ $012519,-0.00016213,-0.00026484,0.00009468,-0.00009186,0.00009905,0.000$ $05380,0.00001606,-0.00002155,0.00001173,0.00002536,-0.00001364,0.00002$ $570,0.00004188,-0.00001138,0.00001510,0.00002812,0.00021903,-0.0001452$ $6,0.00002672,-0.00000616,-0.00000190,0.00003450,-0.00002851,0.00002087$ $,-0.08369272,0.04476058,0.03626787,-0.00304571,0.00573669,0.00485223,0$ $.00183928,0.00095373,-0.00053273,-0.00016961,0.00036274,0.00165268,-0$. $00130869,0.00019537,-0.00594905,-0.02526518,0.02407537,0.02248905,0.11$ $187309,0.00004145,-0.00005865,-0.00003363,-0.00000788,-0.00005340,0.00$ $006776,0.00000054,-0.00004383,0.00006527,0.00006177,0.00003857,0.00009$ $628,0.00009227,0.00012933,-0.00005956,0.00007433,-0.00003609,-0.000020$ $64,-0.00000718,0.00001072,-0.00000164,-0.00001278,0.00000812,-0.000016$ $94,-0.00003116,0.00000871,-0.00000825,0.00000542,-0.00013333,0.0001014$ $4,-0.00002434,-0.00000445,-0.00000555,-0.00003075,0.00002112,-0.000018$ $57,0.04476329,-0.06019492,-0.03647550,0.01502964,-0.00794284,-0.012239$ $77,-0.00097958,0.00366825,-0.00256668,0.00029242,-0.00115271,-0.000287$ $65,0.00220480,0.00373837,0.00146520,0.01475901,-0.00751392,-0.01180212$ ,-0.07445353,0.07329293,0.00004154,-0.00002934,-0.00003906,-0.00000684 ,-0.00004258,0.00007492,-0.00000305,-0.00004288,0.00006067,0.00006199, $0.00003002,0.00008437,0.00007946,0.00011522,-0.00007120,0.00004855,-0$. $00003402,-0.00003186,-0.00000015,0.00001245,-0.00000078,-0.00001364,0$. $00000763,-0.00001578,-0.00003235,0.00000753,-0.00000592,0.00000090,-0$. $00012580,0.00009861,-0.00001720,-0.00000980,0.00001168,-0.00003348,0.0$ $0002457,-0.00001562,0.03611364,-0.03633079,-0.06780528,0.02712196,-0.0$ 2315306,-0.01909989,-0.00517990,-0.00028403,-0.00246914,0.00156353,-0. $00025381,0.00036888,-0.00115744,-0.00088562,0.00072417,0.00034719,-0.0$ $0100241,0.00272969,-0.06079912,0.06046296,0.08515930,0.00000425,0.0000$ 0476,-0.00000940,-0.00000081,0.00000417,0.00000740,-0.00001547,-0.0000 $0304,-0.00000671,0.00000978,0.00001395,-0.00000459,-0.00000882,-0.0000$ $5321,-0.00001610,-0.00000996,0.00003560,0.00001954,-0.00000147,-0.0000$ $0345,0.00000381,0.00000524,0.00000446,0.00000183,0.00000983,-0.0000040$ $1,0.00000318,0.00000764,0.00001033,-0.00000548,-0.00000379,-0.00000579$ ,-0.00000224,-0.00000342,-0.00000754,0.00000252,-0.00376075,0.00373789 $, 0.00089230,-0.27294770,0.07251122,-0.10564878,-0.01161594,0.00388241$, $-0.00930798,-0.00106662,0.00440228,0.00175783,0.00076459,-0.00052941,0$ $.00065809,0.00220975,0.00063145,-0.00153754,0.00062208,-0.00113563,0.0$ $0101273,0.28580694,0.00001708,0.00000552,-0.00001791,-0.00000715,-0.00$ $000932,0.00001486,-0.00000373,-0.00000900,0.00001106,0.00001344,0.0000$ $0319,0.00000911,0.00002936,0.00001760,-0.00004544,-0.00001476,0.000006$ $73,0.00001261,-0.00000382,0.00000305,0.00000632,0.00000252,0.00000616$, $-0.00000244,-0.00000777,-0.00000198,-0.00000107,-0.00001490,-0.0000293$ $2,0.00001649,-0.00000465,-0.00000260,0.00000659,-0.00000910,0.00000544$ 
,-0.00000118,-0.00721684,0.00478427,-0.00467116,0.07392259,-0.06007393 $, 0.02536056,0.01410614,-0.00001312,0.00635708,0.00467166,0.00267594,-0$ $.00344552,-0.00075309,-0.00055611,-0.00017906,0.00096096,0.00301038,-0$ $.00295258,-0.00154788,-0.00152299,0.00078917,-0.08178450,0.05394860,-0$ $.00000117,0.00000665,-0.00003214,-0.00000648,0.00000168,0.00001536,-0$. $00002364,-0.00003048,0.00000734,0.00003566,0.00004187,0.00000051,-0.00$ $000339,-0.00008607,-0.00004122,-0.00000835,0.00005498,0.00004682,0.000$ 00096,-0.00000017,0.00001747,0.00001108,0.00001069,0.00000066,0.000000 $37,-0.00000396,0.00000159,0.00002144,0.00000704,0.00000684,-0.00001052$ $,-0.00001135,-0.00000352,-0.00001845,0.00000047,-0.00000178,-0.0252979$ $0,0.00818793,-0.00690502,-0.10235075,0.02368960,-0.09744466,0.01512406$ $,-0.00557170,0.00854463,0.00243072,-0.00380712,0.00027177,0.00013569,0$ $.00011542,-0.00057717,-0.00074083,-0.00338083,-0.00408695,0.00004463,0$ $.00126645,0.00045809,0.11160621,-0.02268075,0.09966688,-0.00000123,0.0$ $0001780,-0.00000670,-0.00000423,0.00001368,-0.00000933,0.00002135,-0.0$ $0000130,-0.00001616,-0.00001548,-0.00002969,-0.00002879,0.00000073,0.0$ $0000402,0.00000203,-0.00004604,-0.00000759,0.00001250,-0.00000225,0.00$ $000646,0.00000697,0.00000469,0.00000133,-0.00000480,-0.00001273,-0.000$ $00119,0.00000044,-0.00001323,0.00001442,-0.00001460,0.00000406,0.00000$ $168,0.00001125,0.00000332,0.00001121,0.00000330,-0.00037319,0.00383072$ $, 0.00107212,0.00754819,0.00707500,0.01931405,-0.06174819,-0.01229702,-$ $0.06192845,-0.00350900,-0.00814363,-0.02326730,-0.00343180,0.00263931$, $-0.00343353,-0.00096252,0.00053250,0.00052693,-0.00034418,0.00058101,0$ $.00100914,0.00007834,-0.00187029,0.00032706,0.06279075,0.00002292,-0.0$ $0001042,-0.00001099,-0.00001068,-0.00002037,-0.00000494,0.00002960,0.0$ $0001043,0.00002441,-0.00002205,-0.00000335,-0.00000323,0.00004528,0.00$ $006319,-0.00003435,-0.00001560,-0.00001451,0.00001188,-0.00000737,0.00$ $000592,0.00000255,0.00000403,0.00000504,-0.00000513,-0.00000884,-0.000$ $00013,-0.00000178,-0.00003218,-0.00004262,0.00000805,-0.00000165,-0.00$ $000230,0.00000759,0.00000297,-0.00000602,0.00000731,0.00399696,0.00356$ $358,-0.00256701,-0.00349199,0.00036092,-0.01630375,-0.01066319,-0.0621$ $7352,-0.07992071,-0.00003688,0.00294684,0.00765726,0.00334376,0.003419$ $33,0.00015424,0.00034014,-0.00056545,0.00005705,0.00021573,-0.00044238$ $,-0.00075067,-0.00175965,-0.00246290,0.00165970,0.00822572,0.05561299$, $-0.00000789,0.00000963,0.00000664,0.00000453,0.00001699,-0.00001370,0$. $00001362,0.00000566,-0.00000502,-0.00001129,-0.00003185,-0.00003103,-0$ $.00002832,-0.00001710,0.00004121,-0.00001073,-0.00000890,-0.00000302,0$ $.00000359,0.00000568,0.00000222,0.00000171,-0.00000136,0.00000124,-0.0$ $0000269,-0.00000284,-0.00000090,0.00000976,0.00004408,-0.00002769,0.00$ $000716,0.00000090,0.00000912,0.00000402,0.00000737,0.00000698,0.001464$ $91,-0.00271878,0.00170217,-0.00590535,-0.00397623,-0.01079853,-0.05799$ $525,-0.08182188,-0.29753210,-0.00396547,-0.00186086,-0.00917515,-0.001$ $78945,-0.00058443,0.00136721,0.00009857,0.00023377,0.00035490,0.000133$ $36,-0.00031891,-0.00064478,0.00056449,0.00152354,0.00029414,0.06718812$ $, 0.08954192,0.31479480,0.00019603,0.00027014,-0.00059939,-0.00010615,-$ $0.00040612,0.00011381,-0.00035187,0.00027552,0.00023347,-0.00012665,0$. $00034666,-0.00019075,0.00014824,0.00054165,-0.00038344,-0.00040897,-0$. $00008380,-0.00007592,0.00001455,0.00002333,0.00003119,0.00003256,0.000$ $05228,0.00000632,-0.00001409,0.00002243,-0.00005819,-0.00031601,-0.000$ $47404,0.00021519,0.00001853,-0.00003637,0.00008812,0.00005394,-0.00008$ $511,0.00000418,0.00091734,0.00348224,0.00173421,0.00025272,-0.00016931$ 
$, 0.00032755,0.00213219,0.00124418,-0.00041351,-0.00274818,-0.00022292$, $-0.00383785,-0.26950846,0.07169952,-0.10169759,-0.01249401,0.00495689$, $-0.00972681,-0.00066590,0.00027881,-0.00008504,-0.00013450,-0.00012458$ $, 0.00004664,0.00004172,-0.00000982,0.00014500,0.28290858,0.00040963,-0$ $.00020763,-0.00005685,0.00013551,-0.00034166,0.00033074,0.00053973,0.0$ $0079427,0.00006805,-0.00040989,-0.00039207,-0.00026998,-0.00013574,-0$. $00009751,0.00034937,0.00007381,0.00017345,-0.00000977,-0.00005996,-0.0$ $0002009,-0.00011399,-0.00013559,-0.00001627,-0.00005576,-0.00003466,0$. $00000901,0.00001892,-0.00006985,-0.00009400,-0.00002606,-0.00001051,-0$ $.00000737,-0.00004704,0.00005372,-0.00004282,0.00002825,0.00324286,0.0$ $0345964,-0.00343962,0.00006117,-0.00048550,-0.00032719,0.00042865,0.00$ $318826,-0.00355735,-0.00832800,0.00342426,-0.00209802,0.06998576,-0.05$ $994507,0.02437385,0.01555516,0.00099685,0.00626656,0.00071618,-0.00039$ 496,-0.00001625,-0.00013232,-0.00030198,0.00013710,-0.00040175,0.00006 $934,-0.00011029,-0.07961767,0.05274283,-0.00031779,-0.00050263,-0.0000$ $7212,-0.00016033,0.00006880,0.00009124,-0.00029991,0.00037929,-0.00019$ $550,-0.00049169,-0.00003149,0.00004879,0.00004558,0.00052710,-0.000026$ $53,0.00055141,0.00011977,-0.00030112,0.00000347,0.00004611,0.00000357$, $0.00009593,0.00001887,0.00002295,0.00019918,-0.00007680,-0.00004164,-0$ $.00006329,-0.00021654,0.00006788,-0.00001901,0.00001005,0.00002579,0.0$ $0008773,-0.00012655,0.00000012,0.00124139,-0.00318995,0.00034812,0.000$ $80980,-0.00058309,-0.00079164,-0.00217314,-0.00265082,-0.00391135,-0.0$ $2331117,0.00737203,-0.00940094,-0.10592359,0.02641059,-0.09654513,0.01$ $550397,-0.00606653,0.00960885,0.00101679,-0.00055961,-0.00018680,0.000$ $02026,0.00015396,-0.00003518,-0.00081314,0.00036080,-0.00019970,0.1136$ $2655,-0.02313024,0.10120947,0.00003580,-0.00012448,0.00009819,-0.00002$ $423,-0.00008355,0.00008931,0.00002319,0.00009224,-0.00001299,-0.000102$ $67,-0.00009656,-0.00000801,0.00012464,0.00040344,0.00002110,0.00019389$ ,-0.00008255,-0.00014243,-0.00008596,-0.00000646,-0.00008534,-0.000016 $57,-0.00000220,-0.00000154,0.00000465,-0.00000801,-0.00001373,-0.00010$ $527,-0.00020957,0.00005469,-0.00001010,0.00005260,-0.00000786,0.000024$ $04,-0.00002188,-0.00000048,-0.00219511,-0.00782925,-0.02477764,-0.0034$ $4496,0.00327407,-0.00192826,-0.00070625,-0.00021544,-0.00020245,-0.000$ $65374,0.00464465,0.00266318,0.00657367,0.00676017,0.01883197,-0.063826$ $51,-0.01077711,-0.05939863,0.00033679,-0.00146358,-0.00004939,0.000047$ $79,-0.00036759,-0.00131619,-0.00004433,-0.00017861,0.00007463,0.000152$ $93,-0.00188353,0.00038352,0.06368311,0.00010716,0.00022853,-0.00005970$ $, 0.00002913,-0.00000139,0.00005622,-0.00003930,-0.00004377,0.00004129$, $0.00003042,-0.00000659,0.00002211,-0.00000062,-0.00012663,-0.00010318$, $-0.00024232,0.00002854,-0.00008813,0.00000098,-0.00000482,-0.00001768$, $-0.00003660,-0.00000347,0.00000906,-0.00001164,0.00000388,0.00001104,-$ $0.00004736,0.00005403,-0.00003190,0.00005687,-0.00005055,0.00014860,-0$ $.00001060,0.00001923,0.00000717,0.00316020,0.00474638,0.00735012,0.002$ $90893,0.00317518,-0.00046303,0.00006199,-0.00062415,0.00085437,0.00420$ $012,0.00277206,-0.00405088,-0.00329138,-0.00041099,-0.01503087,-0.0120$ $9755,-0.06412108,-0.08404511,-0.00105968,-0.00150647,0.00144706,0.0001$ $8355,0.00050589,0.00038015,-0.00018485,-0.00031555,0.00008612,-0.00189$ $698,-0.00243006,0.00163342,0.00813327,0.05821000,-0.00002062,0.0000198$ $6,-0.00001318,-0.00001426,0.00000706,-0.00002995,-0.00001707,0.0000257$ $0,-0.00002327,0.00000201,-0.00003386,-0.00002333,0.00000168,0.00004761$ $, 0.00002423,-0.00005802,-0.00003856,0.00003020,-0.00000473,0.00000987$, 
$0.00003697,0.00003027,0.00000053,-0.00000099,-0.00001477,-0.00000768,0$ $.00000568,-0.00000649,0.00002429,-0.00003189,-0.00000923,0.00003903,-0$ $.00001190,0.00000358,0.00001770,0.00000423,0.00125739,-0.00545073,-0.0$ $0835925,-0.00272097,-0.00005550,0.00151816,0.00042628,0.00052831,0.000$ $81590,0.00166159,-0.00356887,-0.00001576,-0.00517320,-0.00333324,-0.00$ $979501,-0.06244827,-0.08244279,-0.30284224,0.00091580,0.00098662,0.000$ $70858,-0.00001793,-0.00026077,-0.00019815,0.00003424,0.00009397,-0.000$ $15723,0.00043630,0.00159191,0.00029340,0.06572703,0.09181175,0.3180415$ $4,0.00204437,0.00000760,-0.00019953,-0.00058941,-0.00010856,0.00063429$ $,-0.00030847,0.00003893,-0.00013703,0.00021656,-0.00080216,-0.00062449$ $,-0.00241076,-0.00398615,-0.01862549,-0.00108776,-0.00310086,0.0019344$ $9,-0.00017677,-0.00011424,0.00000726,-0.00002676,0.00011546,0.00004347$ $,-0.00000315,-0.00000214,0.00000110,-0.06447036,-0.09196809,-0.0483521$ $9,-0.00023071,0.00007434,-0.00043042,-0.00041688,0.00038850,0.00119129$ $,-0.00004870,0.00004342,0.00004415,0.00001513,-0.00001262,-0.00000672$, $-0.00001824,-0.00000566,-0.00001679,-0.00000343,-0.00003371,-0.0000239$ $8,0.00001586,-0.00002184,0.00002580,-0.00001540,0.00000259,0.00003102$, $0.00003444,-0.00002915,-0.00002640,0.00000174,0.00000277,-0.00000385,0$ $.00000971,0.00000890,0.00000027,0.00000344,0.00001600,-0.00001739,-0.0$ $0000434,0.00000890,0.00000503,0.05981865,0.00003731,-0.00113409,-0.001$ $29508,0.00041106,0.00077642,-0.00004641,0.00037343,-0.00112056,0.00065$ $704,0.00353129,0.00211912,-0.00104254,-0.00649120,-0.02457488,-0.02143$ $445,-0.00437655,-0.00057399,0.00335883,-0.00020167,-0.00031521,0.00021$ $528,-0.00012406,0.00008507,-0.00002204,0.00003555,0.00006491,-0.000069$ $67,-0.06740385,-0.19660178,-0.18512465,0.00007508,0.00014617,0.0000395$ $8,-0.00111861,0.00054095,0.00014581,0.00007383,-0.00007288,-0.00008610$ $,-0.00004738,0.00001647,0.00001512,0.00001084,-0.00000469,0.00001667,0$ $.00000863,0.00005083,0.00003723,0.00003246,0.00003024,0.00001069,0.000$ $02939,0.00000359,-0.00006131,-0.00006897,0.00005409,0.00004719,-0.0000$ $0168,0.00000758,0.00001056,-0.00001187,-0.00000001,-0.00001226,-0.0000$ $2602,-0.00007468,0.00001373,-0.00000415,-0.00002624,-0.00000616,0.0772$ $8155,0.22418062,0.00102701,0.00049380,0.00071880,-0.00021077,0.0002391$ $4,0.00039644,0.00014372,-0.00080264,-0.00006059,-0.00007742,0.00242177$ $, 0.00105837,0.00167773,0.01425083,0.00975434,0.00165448,0.00154030,-0$. $00066375,0.00002604,0.00018783,-0.00008887,-0.00014019,0.00001613,-0.0$ $0010948,-0.00001044,0.00002626,-0.00001750,-0.03655128,-0.21522087,-0$. $25350788,-0.00030610,0.00032896,-0.00005151,-0.00019438,-0.00051935,-0$ $.00044323,-0.00003059,0.00004254,0.00004039,0.00001941,-0.00001963,-0$. $00000526,-0.00002741,0.00000171,-0.00003791,-0.00001277,-0.00003138,-0$ $.00000295,0.00001546,-0.00001416,0.00001866,-0.00002201,-0.00000298,0$. $00002503,0.00004382,-0.00003310,-0.00003480,0.00000439,-0.00000186,-0$. $00000066,0.00000267,0.00001188,0.00000042,0.00001882,0.00006318,0.0000$ $0857,0.00000230,-0.00000643,0.00000180,0.04407676,0.20635572,0.2558396$ $4,-0.00027032,-0.00045631,0.00060273,0.00016559,-0.00055145,-0.0006443$ $4,0.00146621,0.00089599,0.00130280,-0.00054657,-0.00326570,0.00013785$, $-0.01291238,0.01051772,0.00544640,0.00143949,0.00137724,-0.00035847,-0$ $.00000426,0.00004423,-0.00002027,-0.00008028,0.00002958,-0.00016943,-0$ $.00016922,-0.00013995,-0.00003385,-0.28774467,0.04324599,0.21271080,-0$ $.00016005,-0.00075792,-0.00077703,-0.00031920,0.00030173,-0.00032383,-$ $0.00002972,0.00000586,-0.00000598,0.00000138,-0.00000057,0.00000454,0$. $00000193,0.00000119,-0.00000289,-0.00000887,-0.00001571,-0.00001498,0$. 
$00002303,-0.00001845,0.00001596,-0.00001230,-0.00002624,0.00002293,0.0$ $0000287,-0.00000921,0.00000337,0.00000128,0.00000414,-0.00000299,0.000$ $00553,0.00000251,0.00000270,0.00000203,0.00000129,0.00000488,0.0000195$ $3,0.00003736,0.00000435,0.00765123,-0.00202440,-0.01112926,0.29147773$, $0.00016011,-0.00019827,-0.00022912,0.00010197,0.00020107,0.00000613,-0$ $.00108461,0.00057811,0.00097925,-0.00230527,-0.00480011,-0.00050213,-0$ $.02556660,0.00671185,0.02264507,0.00012193,0.00130507,0.00237375,0.000$ $01396,0.00003153,0.00001722,0.00011555,-0.00002813,-0.00005181,-0.0001$ $6550,-0.00036212,0.00014307,0.05521927,-0.06922889,-0.07918598,0.00037$ $266,-0.00047488,-0.00111196,0.00003696,0.00006803,0.00027560,0.0000807$ $1,-0.00005510,-0.00004186,-0.00002582,0.00000560,-0.00000750,-0.000022$ $91,-0.00000979,-0.00000732,0.00001796,0.00002303,0.00002308,0.00000988$ ,0.00001826,-0.00001697,0.00001611,0.00004701,0.00000307,-0.00003933,0 $.00003072,0.00001725,-0.00000187,0.00000292,0.00000216,-0.00000564,0.0$ 0000196,-0.00000997,-0.00004092,-0.00000622,0.00001972,-0.00000590,-0. 00007196,-0.00000604,0.02219620,-0.00357703,-0.00932765,-0.04919890,0. $06978734,-0.00032760,0.00080063,-0.00103695,-0.00056406,-0.00079083,0$. $00021088,0.00134989,0.00003753,-0.00037737,0.00062123,0.00146032,0.002$ $98059,0.01063660,0.00369761,-0.01119866,-0.00290619,-0.00238933,0.0006$ $7224,-0.00000940,-0.00009708,0.00001773,0.00012218,-0.00001460,0.00005$ $742,0.00002533,0.00021582,-0.00006381,0.17677828,-0.06492800,-0.157817$ $95,0.00085228,-0.00033007,0.00030519,-0.00027435,0.00008739,0.00012002$ ,-0.00013050,0.00010030,0.00008930,0.00004072,-0.00001181,-0.00000527, $0.00000651,-0.00000694,-0.00000701,-0.00000874,-0.00004040,-0.00005314$ ,-0.00002579,-0.00004512,0.00001348,-0.00001207,-0.00002296,0.00006312 $, 0.00007800,-0.00006387,-0.00005804,0.00000217,-0.00000159,-0.00000566$ $, 0.00001197,0.00000395,0.00000778,0.00004114,0.00003045,-0.00002112,-0$ $.00000098,0.00003844,0.00002145,0.02046936,-0.00172279,-0.01283807,-0$. $20677597,0.06399294,0.17892386 \backslash \backslash-0.00000100,0.00000276,0.00000153,-0.0$ $0000032,0.00000379,-0.00000040,0.00000209,-0.00000121,-0.00000041,0.00$ $000084,0.00000142,0.00000073,0.00000096,0.00000308,0.00000020,0.000000$ 43,0.00000094,-0.00000049,-0.00000078,0.00000202,-0.00000073,-0.000001 $07,0.00000258,-0.00000091,0.00000043,0.00000317,-0.00000107,0.00000187$ ,0.00000063,0.00000093,0.00000111,0.00000133,-0.00000009,0.00000187,0. $00000144,0.00000063,-0.00000230,-0.00000227,-0.00000083,-0.00000193,-0$ $.00000343,0.00000068,0.00000062,-0.00000200,-0.00000015,-0.00000151,-0$ $.00000145,-0.00000033,0.00000046,0.00000060,-0.00000034,-0.00000006,-0$ $.00000101,-0.00000081,-0.00000159,-0.00000294,-0.00000011,-0.00000145$, $-0.00000390,-0.00000018,-0.00000083,-0.00000325,0.00000021,-0.00000184$ $,-0.00000257,0.00000115,-0.00000139,-0.00000081,-0.00000043,0.00000276$ ,0.00000068,0.00000081,0.00000261,0.00000040,0.00000042III@

ortho-Transition State

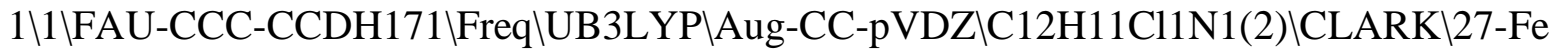
b-2015\0\\\# ub3lyp/aug-cc-pvdz freq name $=$ clark geom $=$ check guess $=$ read $\backslash$ Addition TS p-Cl-Ph-radical + aniline (ortho) $\backslash 10,2 \backslash \mathrm{C}, 2.0600550984,0.54$ $52465736,0.925759034 \backslash \mathrm{C}, 2.5343441466,0.9410846023,-0.3605598094 \backslash \mathrm{C}, 3.273$ $1729571,0.0280223853,-1.1252992053 \backslash \mathrm{C}, 3.5961805565,-1.2324811495,-0.613$ 2008601\C,3.191922997,-1.6045590091,0.6798686703\C,2.4592620355,-0.708 $0104827,1.4499934973 \backslash \mathrm{H}, 1.7011312552,1.3153322802,1.6067036331 \mathrm{~N}, 2.2742$ 
$797076,2.230198754,-0.8173773312 \backslash \mathrm{H}, 3.6145270013,0.314726929,-2.1215699$ 464\H,3.4680603723,-2.5788201695,1.0822003399\H,2.1585604567,-0.973826 2672,2.4633799601\H,4.1768944309,-1.9249603103,-1.2232868168\C,-2.8325 $301877,-0.1596961914,-0.0875769667 \backslash \mathrm{C},-2.4836906314,0.7779140264,0.8856$ $115255 \backslash \mathrm{C},-1.1226099509,0.9903572129,1.1644595887 \backslash \mathrm{C},-0.173406964,0.2665$ $062187,0.4592874596 \backslash \mathrm{C},-0.5121679313,-0.6725729075,-0.5025290273 \backslash \mathrm{C},-1.8$ $687102542,-0.8917357629,-0.7865936134 \backslash \mathrm{Cl},-4.5417798779,-0.4312688226,-$ $0.444068454 \backslash \mathrm{H},-3.258094689,1.3295337417,1.4176291432 \backslash \mathrm{H},-0.8424397893,1$ $.7161590665,1.9310603302 \backslash \mathrm{H}, 0.2533176711,-1.2382434591,-1.0357749903 \backslash \mathrm{H}$, $-2.1741648867,-1.6204383541,-1.536794763 \backslash \mathrm{H}, 2.3849957455,2.3716362633,-$ $1.8132861832 \backslash \mathrm{H}, 1.4208717308,2.6476488322,-0.4670682148 \backslash \mathrm{Version}=\mathrm{EM} 64 \mathrm{~L}-$ G09RevC.01 \State=2-A \HF=-978.8451964 \S2=0.775035 \S2-1=0. $\backslash S 2 A=0.750445 \backslash$ $\mathrm{RMSD}=8.624 \mathrm{e}-09 \backslash \mathrm{RMSF}=1.083 \mathrm{e}-05 \backslash \mathrm{ZeroPoint}=0.1943755 \backslash \mathrm{Thermal}=0.2069331 \backslash \mathrm{ZP}$ $\mathrm{E}=121.9724943 \backslash$ Dipole $=0.6560841,0.388086,-0.2284321 \backslash \mathrm{DipoleDeriv}=-0.1267$ $299,-0.1969454,0.0372695,0.1173807,-0.1398167,0.0778886,0.0007418,0.31$ $63931,-0.0702804,-0.0446734,-0.283845,0.1146214,0.1722121,1.2391479,-0$ $.4278688,-0.0629305,-0.4342705,0.0935485,-0.0112431,0.2074274,-0.19358$ $05,-0.2023596,-0.3605496,0.1711093,-0.1330108,-0.1824895,0.0619728,-0$. $1092384,-0.0849517,-0.0593274,0.2250905,0.1464772,-0.1329586,-0.259587$ $5,-0.0487524,0.1522886,-0.3037263,-0.0419334,0.0336684,0.0269056,-0.04$ $37291,-0.0602781,0.3278308,-0.0356706,-0.1741973,0.0204009,-0.0533671$, $-0.1446943,-0.3884056,0.0666673,-0.0469834,-0.099779,-0.0155509,0.0559$ $901,0.165533,0.0678874,0.0425057,0.0473366,-0.0223411,-0.0510025,0.043$ $2563,-0.0452256,0.0200802,-0.2969739,-0.1384589,-0.0465007,-0.2885141$, $-1.3965969,0.3757004,-0.0575016,0.1565169,-0.2190354,0.1003896,0.01133$ $63,0.0540104,-0.0021675,0.04254,0.0735699,0.0897016,0.068877,-0.068721$ $4,0.102666,0.055059,0.0108432,0.0858362,-0.0552116,0.067763,0.009483,0$ $.0639775,0.0497464,0.093684,0.0086304,0.0752487,0.0115982,0.0617744,0$. $0423518,0.0388146,0.0408034,-0.0520875,0.0501882,0.0707957,0.0676256,0$ $.0845992,0.0083313,-0.0465774,0.0688806,-0.053667,0.0251734,1.2108061$, $0.1630617,0.2129054,0.2033444,-0.0040452,0.0579731,0.2629881,0.0557932$ $, 0.0176963,-0.2240717,-0.0747321,-0.0810569,0.0018485,-0.0565737,0.029$ $9152,0.0213606,0.0320121,-0.0666064,-0.3303497,0.0240601,0.0340473,-0$. $2482856,0.0003199,0.1218254,-0.2671637,0.1177772,0.0348718,0.835155,0$. $0459752,-0.0166331,0.1321032,-0.0855315,-0.0151257,0.1758346,0.0079487$ ,-0.083708,-0.4525597,-0.031923,-0.0142785,0.0704755,-0.0369145,0.0399 $7,0.0550901,0.0416192,-0.0430865,-0.1959679,0.0091163,0.0041961,-0.114$ $9238,-0.008459,0.0741856,-0.1006541,0.0733993,-0.0100294,-0.9075135,-0$ $.0996822,-0.1257167,-0.1262828,-0.0999113,-0.0286481,-0.1724491,-0.029$ $4248,-0.1110356,-0.0633735,0.0544526,0.0531136,0.0358907,0.0753669,-0$. $0500848,0.0284105,-0.0494217,0.0774729,0.0591561,-0.0507838,-0.0549451$ $, 0.0060788,0.0179077,-0.1048145,-0.0113012,-0.1021633,0.0080959,0.0408$ $416,0.055927,0.0526059,0.0311095,0.0570524,-0.0398048,0.0205267,-0.039$ $1676,0.0601983,-0.0014044,-0.0294842,-0.0309603,-0.0391223,0.0501188,-$ $0.0760733,-0.0442816,-0.0761333,0.0466379,0.2305173,0.1546926,-0.03374$ $98,0.0891827,0.2368006,-0.070176,-0.0019904,0.0178533,0.0927853,0.1584$ $877,0.1576851,0.0087822,0.069069,0.3071759,0.0181436,0.06773,0.1189662$ ,0.1022294\Polar=246.8629672,-13.3356727, 158.2016436,-7.67259,6.552559 $3,145.7185316 \backslash \mathrm{PG}=\mathrm{C} 01[\mathrm{X}(\mathrm{C} 12 \mathrm{H} 11 \mathrm{Cl1N} 1)] \mathrm{NImag}=1 \| 0.12056620,-0.15167258$, $0.63749596,-0.15777228,-0.01130446,0.59077914,-0.07248095,0.00513672,0$ $.06651084,0.32110862,0.00137205,-0.12109948,0.06021148,-0.17227348,0.6$ 
$2514838,0.04265389,0.01693338,-0.24576764,-0.16890747,-0.06788604,0.56$ $825683,-0.00805964,-0.00489900,0.02777998,-0.13685969,0.08929042,0.066$ $93912,0.27568045,0.01471080,0.03682820,-0.03276292,0.06514275,-0.17929$ $095,-0.02700222,-0.15918267,0.59787473,0.02093013,-0.01364645,-0.02387$ $854,0.09421310,-0.08478503,-0.18191168,-0.18888864,-0.10602636,0.62814$ $028,0.00166242,-0.00405028,0.00142725,-0.00447639,0.02107920,-0.003565$ $14,-0.08436919,0.07574476,-0.00834288,0.29702936,-0.00788144,-0.021960$ $37,0.02160293,0.03773045,-0.04780257,-0.04139384,0.04969127,-0.2839927$ $7,0.10788469,-0.19123018,0.60721500,-0.01017671,0.02663230,-0.02996446$ ,-0.02056563,0.00205776,0.05090752,0.01627963,0.05311969,-0.14101132,-$0.18659577,-0.04215857,0.59035443,-0.01113131,0.03336930,-0.01336809$,$0.01468358,0.00676614,0.02066281,0.02182934,-0.02547910,-0.00469666,-0$ $.09319645,0.01609391,0.05492276,0.27871051,0.02890411,-0.03863442,-0.0$ $0095279,0.00277466,-0.00039375,-0.01128651,-0.00992769,-0.00121801,0.0$ $3197859,-0.00838034,-0.12744942,0.08848855,-0.19548778,0.64470306,0.01$ 465074,-0.02931776,0.04601900,0.02058581,-0.01890352,-0.04189853,-0.02 $192795,0.06769817,-0.01319921,0.08114632,0.03032599,-0.27362430,-0.151$ $87411,-0.10136397,0.57434090,-0.06158039,0.06086430,0.00427470,0.01344$ $320,-0.01902513,-0.01558481,-0.01341252,0.02063497,0.00089672,-0.00521$ $785,-0.01156268,0.04334166,-0.13871779,0.10912048,0.07016019,0.2897920$ $1,0.03518487,-0.26054627,0.04849104,-0.01505359,0.00249241,0.04751923$, $0.01640094,-0.03864968,-0.00097525,0.00710346,0.04034572,-0.05144374,0$ $.08625973,-0.20417998,-0.04222024,-0.16967491,0.55268180,-0.00084665,0$ $.09206452,-0.14153556,-0.01355603,0.03260880,-0.00373239,0.00563938,-0$ $.01248316,-0.00272138,0.02344349,-0.00775050,-0.03873554,0.09585887,-0$ $.09753440,-0.18077867,-0.18512179,-0.06769988,0.65430414,-0.04719018,0$ $.06667925,0.06401341,-0.00550242,0.00753726,0.01224194,0.00374691,-0.0$ $0210277,0.00293248,-0.00090651,-0.00002496,0.00119928,0.00606727,0.001$ $58830,-0.00305491,-0.00606877,0.01232716,0.00458778,0.06857624,0.07826$ $615,-0.19391099,-0.12242201,-0.00328881,0.00736898,0.00868922,-0.00014$ $571,0.00005172,0.00217495,-0.00056165,-0.00003578,0.00079747,0.0013798$ $3,-0.00342300,0.00325352,0.00854100,-0.01258174,-0.01908062,-0.0786287$ $0,0.20184702,0.07204211,-0.12283527,-0.16520520,0.00791545,-0.02170523$ $,-0.01057180,0.00190409,0.00249818,-0.00386286,-0.00056843,0.00111281$, $-0.00034152,0.00026218,0.00264279,-0.00028334,-0.00559206,0.01143080,0$ $.01059643,-0.07023018,0.12994076,0.17071656,0.00545205,-0.01218832,0.0$ $0663391,-0.07795922,0.08356067,0.01009444,0.00132944,0.02911661,-0.008$ $22096,0.00214625,0.00112195,0.00635198,0.00008199,-0.00091057,-0.00200$ $029,0.00050824,0.00268025,0.00325855,-0.00069537,-0.00098464,-0.000129$ $26,0.42351046,-0.00775981,-0.02041187,0.02051768,0.04466202,-0.2580768$ $1,0.05946465,0.01994764,-0.04632127,0.00463917,0.00136658,0.00967918,-$ $0.00528922,-0.00074313,-0.00477245,0.00572142,0.00433336,0.00217989,-0$ $.00164977,0.00092705,0.00134715,-0.00062954,-0.25825759,0.45672055,-0$. $00227860,0.04489140,-0.01647827,0.01821929,0.08716863,-0.12511352,0.00$ $577311,-0.02160351,0.01345321,0.00299417,0.00185534,-0.00577538,-0.002$ $45284,0.00170370,0.00280173,0.00624277,-0.00296505,-0.00454114,-0.0028$ $9135,-0.00038818,0.00161459,-0.19587927,-0.11838510,0.66532885,0.00251$ $412,0.00487185,0.00280888,-0.00127215,-0.00596262,0.01548593,-0.067165$ $94,-0.01460967,0.08811653,0.00448856,0.00230559,-0.00767469,0.00477341$ $, 0.00326494,0.00111536,-0.00064102,0.00005728,-0.00040146,-0.00030696$, $-0.00045229,0.00096945,-0.00172026,-0.00213038,-0.00076444,0.06189943$, 
$0.00372896,-0.00144888,-0.00170295,0.00705933,0.00744381,-0.01734633,-$ $0.01454838,-0.07260953,0.06799616,-0.01026914,-0.00253272,0.02625411,0$ $.00378144,-0.00381682,0.00190482,0.00024383,-0.00096113,-0.00039895,0$. $00028644,0.00029982,-0.00081889,-0.00149344,0.00131705,-0.00139700,0.0$ $1333400,0.07231203,0.00299097,-0.00216231,0.00079660,0.00402539,0.0088$ $0001,-0.01286653,0.08778129,0.06721817,-0.28271694,0.00412142,0.000522$ $32,-0.00730329,0.00085576,0.00290875,0.00267190,-0.00030155,-0.0005902$ $4,0.00025436,0.00029881,0.00024031,-0.00053446,-0.00209094,-0.00000331$ $, 0.00114704,-0.09659211,-0.07520444,0.29898542,0.00337505,0.00356695,0$ $.00485531,-0.00046953,0.00006818,0.00003630,0.00375922,0.00133073,0.00$ $329924,0.00411436,-0.00910938,0.00397873,-0.05709293,0.07213404,-0.019$ 75266,-0.00170538,0.01433872,-0.00720992,0.00006561,0.00100622,-0.0005 $1541,-0.00024250,-0.00052027,-0.00034641,0.00040207,-0.00025790,0.0003$ $2352,0.05260404,0.00364735,0.00044228,-0.00202234,-0.00014120,0.000173$ $74,-0.00056332,0.00089534,0.00257234,0.00181327,0.00237895,-0.00385116$ $, 0.00001945,0.07246898,-0.27881171,0.09655780,0.00286534,-0.01328497,0$ $.00967961,0.00036755,-0.00056354,0.00039422,-0.00018054,-0.00008238,-0$ $.00002946,0.00027377,-0.00018818,0.00004608,-0.07932878,0.29331428,0.0$ $0402495,-0.00175021,-0.00033686,0.00041480,-0.00046732,-0.00091907,0.0$ $0381720,0.00103734,-0.00388798,-0.00870605,0.02590709,-0.00554759,-0.0$ $1918586,0.09681221,-0.09245624,0.00478605,-0.01612315,0.00960856,0.000$ $30393,-0.00068805,0.00022222,-0.00032194,-0.00012605,0.00018608,-0.000$ $19846,0.00142617,-0.00040432,0.01828337,-0.10460529,0.09294361,0.00446$ $843,0.00369326,-0.00968876,0.00386169,0.00355965,0.00122243,-0.0006821$ $1,-0.00005687,-0.00038097,0.00363659,0.00485908,0.00299066,-0.00019477$ $,-0.00485896,0.01530380,-0.06192811,-0.01299702,0.07979408,-0.00222758$ $,-0.00068463,-0.00060536,0.00031377,-0.00018789,-0.00045793,-0.0005673$ $4,-0.00032829,-0.00027742,-0.00276093,-0.00153077,-0.00164845,0.055754$ $53,-0.00834863,-0.00275832,0.02540113,0.00410660,-0.00366778,0.0019679$ $3,0.00000181,-0.00102388,-0.00022125,0.00461388,-0.00116521,-0.0016862$ $5,0.00560319,0.00913909,-0.01898899,-0.01078943,-0.07093503,0.06741622$ $,-0.00143462,0.00066908,-0.00074233,-0.00054764,0.00006263,0.00143180$, $-0.00032981,-0.00006546,-0.00011128,-0.00189075,0.00016550,-0.00038374$ $, 0.00878057,0.06960434,0.00345894,0.00100904,-0.00720117,0.00047771,0$. $00219894,0.00223178,-0.00036279,-0.00038192,0.00045874,0.00359268,-0.0$ $0229372,0.00060261,0.00302324,0.00740749,-0.01103125,0.07866187,0.0659$ $7133,-0.29764982,-0.00118224,-0.00012907,0.00041293,0.00022334,-0.0000$ $9262,-0.00059431,-0.00022579,-0.00006541,-0.00019442,-0.00146570,-0.00$ $074094,0.00025261,-0.08711215,-0.07321890,0.31315218,-0.00058831,-0.00$ $059728,-0.00060730,0.00545875,0.00237316,0.00060121,-0.00024274,0.0036$ $0512,0.00510196,-0.12112084,0.10236329,0.09080929,-0.00109208,0.006859$ $24,0.00381888,0.00463476,0.00009219,0.00309978,-0.00048443,-0.00009793$ ,-0.00013251,-0.00021659,0.00014506,0.00032895,-0.00251532,-0.00143045 $,-0.00169203,-0.00220215,-0.00148610,-0.00129560,-0.00021312,0.0003672$ $8,0.00018930,0.11847520,-0.00032313,-0.00044451,0.00051631,0.00241740$, $-0.00285135,0.00399149,0.01456567,-0.01238339,-0.01712622,0.10211216,-$ $0.16739192,-0.09599348,-0.00477950,0.00821013,0.00595395,0.00078387,0$. $00153549,0.00276795,-0.00021739,-0.00006771,-0.00014790,0.00095872,-0$. $00058387,-0.00092278,-0.00163411,0.00034382,-0.00061136,-0.00120998,0$. $00008651,-0.00091433,-0.00025179,0.00030714,0.00037476,-0.11229723,0.1$ $7357792,-0.00046835,0.00051099,-0.00049014,0.00119277,0.00384056,0.001$ 
$12160,-0.00627208,0.00866875,0.00889181,0.09105864,-0.09644790,-0.1421$ $0577,0.01480617,-0.01983342,-0.00962819,0.00295138,0.00341690,-0.00407$ $308,-0.00021533,-0.00014916,-0.00003476,-0.00035329,0.00006176,0.00041$ $364,-0.00142466,-0.00098217,0.00012009,-0.00151916,-0.00044687,0.00042$ $802,0.00073242,-0.00098265,-0.00068701,-0.10022976,0.10216020,0.146053$ $35,0.00009129,-0.00010828,-0.00010065,0.00010653,-0.00065867,0.0003364$ $4,-0.00023262,0.00028297,0.00017632,0.00016017,-0.00045900,0.00031012$, $0.00026226,-0.00011607,-0.00058581,-0.00019572,0.00057224,0.00002481,-$ $0.00024807,-0.00000716,-0.00001353,0.00018551,0.00078379,-0.00012117,-$ $0.00000629,0.00001106,-0.00006856,0.00000048,-0.00006758,-0.00000590,-$ $0.00005322,0.00001648,0.00000825,0.00000804,-0.00003964,-0.00001992,0$. $50190974,0.00005901,-0.00025833,-0.00019302,0.00004792,-0.00022493,0.0$ $0000257,0.00005033,0.00007598,0.00010223,-0.00000300,0.00003065,0.0000$ $8428,0.00005492,0.00004313,0.00005782,0.00018220,0.00008128,-0.0000467$ $7,0.00012959,0.00013870,0.00006788,0.00008828,0.00014007,0.00012943,-0$ $.00002147,0.00001323,-0.00001658,-0.00001239,-0.00000204,-0.00000549,0$ $.00001260,-0.00004337,0.00002667,0.00000582,0.00000524,-0.00001130,-0$. $03456776,0.38118876,0.00006569,-0.00027034,-0.00021329,0.00009614,-0.0$ $0030400,0.00004524,-0.00000634,0.00014872,0.00003779,-0.00000877,0.000$ $00947,0.00016775,0.00012044,0.00009300,-0.00003309,0.00014987,0.000128$ $69,-0.00002243,0.00014015,0.00005147,0.00013152,0.00008658,0.00021597$, $0.00011050,-0.00001794,0.00000626,-0.00000797,-0.00001859,-0.00002769$, $0.00000832,-0.00002552,-0.00004810,-0.00000526,0.00000450,-0.00003280$, $-0.00000612,-0.01776795,0.25854544,0.38844921,0.00074985,0.00070629,0$. $00079526,-0.00036191,0.00085555,-0.00021266,0.00005872,-0.00044908,-0$. $00027770,0.00001219,0.00022056,-0.00028050,-0.00023435,-0.00006022,0.0$ $0017638,-0.00018354,-0.00025973,-0.00002375,0.00002400,-0.00034780,-0$. $00030541,-0.00000337,-0.00060507,-0.00002286,0.00004461,-0.00000331,0$. $00002810,0.00003349,0.00000745,0.00000550,-0.00002757,0.00007192,-0.00$ $004820,-0.00000505,0.00000319,-0.00000849,-0.11354518,-0.00407318,-0.0$ $0620384,0.68120507,0.00088045,0.00010085,-0.00087438,0.00063654,-0.000$ $55310,0.00038169,-0.00028622,0.00038916,0.00009442,0.00012076,-0.00051$ $807,0.00023530,0.00015235,-0.00010910,-0.00061991,-0.00040137,0.000616$ $93,0.00023283,0.00006226,-0.00002478,-0.00002915,-0.00001865,0.0005407$ $7,-0.00013808,-0.00001214,0.00000241,-0.00001516,0.00001916,-0.0000026$ $8,0.00001675,0.00002117,-0.00002216,0.00003888,-0.00000937,-0.00001142$ $, 0.00002312,-0.04919000,-0.18893569,-0.12896696,-0.04739434,0.40393440$ $, 0.00082714,-0.00081329,0.00018369,0.00042362,-0.00074414,0.00043775,-$ $0.00044222,0.00022903,0.00023897,0.00015694,-0.00063057,0.00018674,0.0$ $0013402,-0.00010470,-0.00048174,0.00039006,0.00050516,0.00014158,0.000$ $06000,-0.00004550,-0.00008566,-0.00031259,0.00070650,-0.00016674,0.000$ $03214,0.00004361,-0.00001209,-0.00000302,-0.00001300,-0.00001292,0.000$ $02434,-0.00003320,0.00007733,0.00000841,0.00002552,0.00001515,-0.05223$ $975,-0.12697783,-0.19702966,-0.02412846,0.28144287,0.41468321,-0.00556$ $284,0.00248981,0.00222913,-0.00197738,0.00278926,-0.00219380,0.0011753$ $5,-0.00096829,-0.00031110,-0.00040347,0.00177821,-0.00092428,-0.000680$ $20,0.00058568,0.00203499,-0.00016084,-0.00273368,-0.00016651,0.0009033$ $9,0.00037113,0.00043390,0.00072671,-0.00225251,0.00063321,-0.00006851$, $-0.00002364,0.00013432,-0.00003686,0.00014366,0.00004037,-0.00009269,0$ $.00003664,-0.00027046,-0.00009574,0.00000099,-0.00005382,-0.00105985,-$ $0.06190256,-0.06354737,-0.30264891,-0.00283671,-0.01390957,0.60470194$, 
$-0.00201349,0.00117936,0.00072261,-0.00053754,0.00103558,-0.00082167,0$ $.00047955,-0.00044739,-0.00024231,-0.00025636,0.00102880,-0.00032890,-$ $0.00035471,0.00009745,0.00090193,-0.00012101,-0.00123114,-0.00021556$,$0.00066766,0.00025222,0.00037992,-0.00039814,-0.00174728,0.00017124,0$. $00000297,-0.00004888,0.00005712,-0.00001123,0.00006847,-0.00001292,-0$. $00006687,0.00008081,-0.00004806,-0.00004907,0.00004030,-0.00002469,-0$. $02139151,0.00740973,-0.00079528,-0.05430043,-0.09204842,-0.03368619,-0$ $.00492248,0.41265393,-0.00222067,0.00119854,0.00113113,-0.00141780,0.0$ $0194108,-0.00079254,0.00110061,-0.00131423,-0.00050443,-0.00022456,0.0$ $0169974,-0.00103326,-0.00072894,0.00043692,0.00175998,0.00002485,-0.00$ $160574,-0.00074239,-0.00059909,0.00035761,0.00034764,-0.00000617,-0.00$ $171308,0.00014037,-0.00001528,-0.00007632,0.00007998,-0.00000923,0.000$ $07129,-0.00000265,0.00002538,0.00006405,-0.00010040,-0.00008299,0.0000$ $0274,-0.00001655,-0.02216833,-0.00214936,0.00405350,-0.06757644,-0.031$ $76182,-0.09753161,0.01700924,0.29487527,0.43301198,0.07052107,-0.00715$ $881,0.00204227,-0.02176556,-0.01228619,0.03015066,0.00169899,-0.007116$ $49,-0.00218090,-0.00262656,0.00145676,0.00669312,0.00669624,-0.0073366$ $9,-0.01305519,-0.02103118,0.03084110,-0.00928236,-0.01457384,-0.003659$ $16,-0.00400303,-0.00054551,0.00875779,-0.00436069,0.00059310,-0.000036$ $67,-0.00044952,0.00033819,-0.00045687,-0.00032331,0.00080241,-0.000322$ $84,0.00129710,0.00031583,-0.00012457,-0.00002898,-0.00756444,0.0117838$ $8,0.01216346,-0.05036126,0.00565891,0.00322461,-0.19782908,0.09921738$, $0.09558550,0.32392738,0.00833543,-0.01009806,-0.00359047,-0.00229157,-$ $0.00390207,0.00463653,-0.00001456,-0.00086844,0.00007815,-0.00012517,0$ $.00002491,0.00032575,0.00074603,-0.00025197,-0.00039646,0.00523152,0.0$ $0247922,0.00029031,-0.00710001,-0.00178568,-0.00235919,-0.00157354,0.0$ $0329861,-0.00058628,0.00009340,-0.00001373,-0.00011354,-0.00020122,-0$. $00011635,-0.00010210,-0.00013917,0.00005471,-0.00008703,0.00006847,-0$. $00000990,0.00001157,0.01217923,-0.03872503,-0.03150900,0.04442974,0.03$ $054065,0.01849827,0.07604890,-0.14399654,-0.09188735,-0.05968592,0.355$ $16993,0.01440880,-0.00508441,-0.00818792,0.00302319,0.00022783,0.00389$ $721,-0.00020987,0.00049265,-0.00050503,-0.00057387,-0.00080466,0.00274$ $317,0.00187851,-0.00214353,-0.00446451,-0.00598039,0.00780421,-0.00042$ $094,-0.00774828,-0.00225580,-0.00238193,0.00095488,-0.00003564,-0.0002$ 9276,-0.00012480,-0.00005135,-0.00009689,0.00012588,-0.00012354,-0.000 $08914,0.00026869,-0.00002557,0.00042098,0.00011460,-0.00000762,0.00005$ $374,0.01202187,-0.03176768,-0.03888190,0.04307020,0.01731884,0.0333481$ $7,0.07131849,-0.09006795,-0.14280894,-0.05356189,0.27505224,0.35918825$ ,-0.00668358,-0.00206451,-0.00245587,-0.00126937,0.00348535,-0.0031602 $5,0.00131545,-0.00147886,-0.00055862,-0.00046035,0.00226232,-0.0008849$ $9,-0.00076686,0.00075194,0.00224860,0.00072597,-0.00377427,0.00015384$, $-0.00092008,-0.00062584,-0.00067081,0.00037657,-0.00282617,0.00061170$, $-0.00012683,-0.00011657,0.00015399,-0.00008434,0.00014008,-0.00001876$, $-0.00008267,0.00017754,-0.00024867,-0.00013266,0.00000785,-0.00007665$, $-0.05020830,0.04190826,0.03992581,-0.03072505,-0.02288925,-0.02464893$, $0.04780005,0.00666809,0.00896055,-0.10048814,-0.02276126,-0.02466587,0$ $.64865166,-0.00012306,-0.00066216,0.00027344,0.00014933,0.00028322,-0$. $00009538,-0.00026641,0.00027829,-0.00028270,-0.00033520,-0.00112457,0$. $00056472,0.00080588,-0.00027383,-0.00080726,-0.00008096,0.00112601,0.0$ $0052217,-0.00029476,0.00012257,-0.00003969,0.00000692,-0.00002462,0.00$ $000175,0.00006800,0.00008148,-0.00003640,-0.00000203,-0.00000634,-0.00$ 
$006386,-0.00002823,0.00003049,-0.00006062,0.00005517,0.00003943,0.0000$ $1405,0.00148301,0.03005414,0.02328785,-0.02202954,-0.01722449,-0.01527$ $357,0.02954452,-0.02211486,-0.02519391,-0.04584441,-0.18893961,-0.1382$ $4780,-0.04941445,0.40736437,-0.00068316,-0.00041115,-0.00039424,0.0002$ $2869,0.00075337,0.00024266,0.00026192,-0.00012290,-0.00005544,-0.00037$ $331,-0.00030886,-0.00018099,0.00024628,-0.00026338,-0.00036446,0.00017$ $744,0.00026654,0.00047552,-0.00025781,-0.00006803,0.00007640,0.0004550$ $9,0.00035001,-0.00003662,-0.00000876,-0.00002821,-0.00000574,0.0000687$ $8,-0.00002407,0.00004582,-0.00005078,0.00000133,-0.00001017,0.00007395$ ,-0.00002407,-0.00000048,-0.00090368,0.02475710,0.03236858,-0.02342105 -0.01564352,-0.01988688,0.03241673,-0.02612738,-0.02119729,-0.0487058 $8,-0.13660946,-0.19426954,-0.02606083,0.27822876,0.40836457,0.00143508$ $,-0.00031145,-0.00001273,0.00031391,0.00005365,0.00006080,0.00000416,0$ $.00021454,0.00007926,-0.00021589,-0.00005449,-0.00001492,0.00002028,0$. $00018039,0.00023727,0.00046587,0.00027435,0.00006852,0.00021314,0.0001$ $5535,0.00013378,0.00007702,-0.00006825,0.00027270,-0.00002897,0.000014$ $87,0.00000673,-0.00003126,0.00001780,0.00000774,-0.00001586,-0.0000898$ 2,-0.00001893,0.00002091,0.00000810,0.00000321,-0.19404040,0.05512946, $0.04998338,0.04998203,0.03400282,0.03653248,-0.05919765,0.01291932,0.0$ $1077572,0.00109039,-0.06143183,-0.06275433,-0.30918854,-0.00209827,-0$. $01421478,0.64361496,-0.00059694,0.00021550,-0.00055033,-0.00038322,0.0$ $0084934,-0.00043611,0.00036119,-0.00041264,-0.00014424,-0.00024931,0.0$ $0073583,-0.00025365,-0.00028161,0.00033960,0.00060950,0.00011641,-0.00$ $061567,-0.00006603,-0.00009462,-0.00010128,-0.00010692,0.00030766,-0.0$ $0051831,0.00007955,-0.00003987,-0.00004611,0.00004130,-0.00003695,0.00$ $000561,0.00000712,-0.00005320,0.00000186,-0.00008805,-0.00001273,-0.00$ $002451,-0.00001664,0.10054190,-0.15296486,-0.08480557,0.00253553,-0.02$ $023003,-0.02880856,0.01494674,-0.00483897,-0.00095743,-0.02256219,0.00$ $757183,-0.00719263,-0.05490620,-0.09344338,-0.03479749,0.00680173,0.42$ $453727,-0.00054307,-0.00054520,0.00002606,-0.00013686,0.00051211,-0.00$ $045744,0.00039359,-0.00020424,0.00015629,-0.00017054,0.00062312,-0.000$ $32738,-0.00018797,0.00012472,0.00055251,0.00016557,-0.00072323,-0.0000$ $9163,-0.00018247,-0.00006277,-0.00010120,0.00005601,-0.00056343,0.0002$ $1462,-0.00005701,-0.00002132,0.00001108,-0.00005064,0.00002852,-0.0000$ $1706,-0.00000569,0.00005962,-0.00004633,-0.00002399,0.00002528,-0.0000$ $0426,0.09607659,-0.08669557,-0.14850175,0.00442351,-0.02718065,-0.0195$ $7645,0.01256535,-0.00148310,-0.00368098,-0.02332573,-0.00845878,0.0039$ $8185,-0.06718159,-0.03256404,-0.09734668,0.03048793,0.30077665,0.43344$ $790,-0.00024624,0.00011981,0.00014672,-0.00018267,0.00086289,-0.000416$ $59,0.00023499,-0.00037465,-0.00020846,-0.00010952,0.00043889,-0.000288$ $12,-0.00024839,0.00005870,0.00049478,0.00006377,-0.00060579,0.00005569$ $, 0.00024272,0.00003308,0.00005119,-0.00022408,-0.00090108,0.00013190,0$ $.00000480,-0.00001089,0.00007695,-0.00000582,0.00007233,0.00000772,0.0$ $0004921,-0.00001126,0.00000209,-0.00002393,0.00003549,0.00001945,-0.14$ $177186,-0.01608002,-0.02186062,-0.02871896,-0.00480172,-0.00634622,0.0$ $0256542,-0.00237224,-0.00259721,0.00004480,-0.00065765,-0.00048033,0.0$ $0075436,0.00476289,0.00457254,-0.04152168,-0.00740791,-0.00960117,0.20$ $933043,-0.00004335,-0.00003631,0.00010211,-0.00001050,0.00007855,-0.00$ $006442,0.00003604,-0.00003034,0.00001365,-0.00001241,0.00007976,-0.000$ 04664,-0.00004280,0.00000938,0.00006642,0.00000673,-0.00010255,0.00001 $118,0.00006944,0.00001768,0.00002240,-0.00005177,-0.00010774,0.0000211$ 
$6,-0.00000445,-0.00000344,0.00001158,-0.00000673,0.00001177,-0.0000000$ $4,0.00000763,-0.00000314,-0.00000348,-0.00000814,0.00000419,0.00000398$ ,-0.01610498,-0.03373085,-0.01132235,-0.02371834,0.00000373,-0.0056580 $7,0.00201240,0.00024574,-0.00433221,-0.00034384,0.00034800,0.00145344$, $0.00000785,0.00097073,-0.00354078,0.01166529,0.00558931,0.00159730,0.0$ $2709506,0.02826536,-0.00003895,0.00011992,-0.00003920,-0.00007765,0.00$ $018991,-0.00007240,0.00006563,-0.00009947,-0.00003672,-0.00003120,0.00$ $011175,-0.00007075,-0.00006637,0.00004404,0.00013429,-0.00000761,-0.00$ $013705,0.00000589,0.00008432,0.00002760,0.00002247,-0.00002340,-0.0001$ 7803,0.00002456,-0.00000617,-0.00000563,0.00001632,-0.00000276,0.00001 $988,0.00000095,0.00000899,-0.00000605,-0.00000222,-0.00001002,0.000009$ $98,0.00000173,-0.02180378,-0.01128771,-0.03589555,-0.02573308,-0.00485$ $085,-0.00158350,0.00200877,-0.00447036,0.00002918,-0.00031625,0.001394$ $00,0.00039474,-0.00012522,-0.00329970,0.00105518,0.00970463,0.00072168$ $, 0.00577031,0.03675917,0.01803224,0.03179950,-0.00028881,0.00020795,0$. $00019042,-0.00031195,0.00051803,-0.00023700,0.00021900,-0.00025723,-0$. $00009944,-0.00006910,0.00034834,-0.00017281,-0.00012798,0.00005682,0.0$ $0031687,-0.00003173,-0.00034538,-0.00006283,0.00004947,0.00000538,0.00$ $001450,0.00001673,-0.00047573,0.00006568,-0.00000750,-0.00001201,0.000$ $02236,-0.00000807,0.00002251,0.00000105,-0.00001236,0.00001240,-0.0000$ $2829,-0.00001226,0.00000512,-0.00000392,0.00636121,-0.00593581,-0.0059$ $3201,-0.19725780,0.10496758,0.10057108,-0.01506769,0.01514895,0.014660$ $14,-0.00444024,-0.00082963,-0.00122885,-0.00016493,-0.00081859,-0.0007$ $3804,0.00075530,-0.00149335,-0.00181019,0.00123379,-0.00053812,-0.0004$ $1167,0.20744031,-0.00011435,0.00005231,0.00001730,-0.00001439,0.000089$ $23,-0.00002712,0.00003813,-0.00005018,-0.00001170,0.00000344,0.0000523$ $8,-0.00004678,-0.00002772,0.00002531,0.00005530,-0.00000608,-0.0000361$ $1,-0.00003377,-0.00000344,0.00001770,-0.00001184,0.00000119,-0.0000505$ $1,0.00000406,-0.00000213,-0.00000136,0.00000600,0.00000391,-0.00000203$ ,-0.00000097,-0.00000726,0.00000128,-0.00000899,-0.00000413,-0.0000002 $1,-0.00000242,0.01604625,-0.00622699,-0.00903006,0.10204651,-0.1186544$ $4,-0.08138747,-0.00523357,0.00599110,0.00165349,-0.00092382,0.00382741$ $,-0.00257566,-0.00051847,-0.00048129,0.00047319,-0.00202261,0.00073200$ $,-0.00485432,0.00016819,-0.00093658,0.00184247,-0.11028232,0.11789806$, $-0.00020166,0.00004497,0.00004170,-0.00003328,0.00001852,-0.00005535,-$ $0.00000752,0.00001080,-0.00000483,-0.00000546,0.00002179,0.00001557,-0$ $.00001178,0.00000057,0.00002265,0.00001806,-0.00008158,0.00003013,0.00$ $000566,-0.00001409,0.00002212,0.00002009,-0.00002655,0.00000065,0.0000$ $0047,-0.00000133,0.00000278,-0.00000363,0.00000462,0.00000070,-0.00000$ $129,0.00000807,-0.00000694,-0.00000312,-0.00000066,-0.00000149,0.01650$ $412,-0.00996229,-0.00614822,0.09760592,-0.08134254,-0.11422084,-0.0060$ $0287,0.00253013,0.00655113,-0.00149623,-0.00234845,0.00376457,-0.00059$ $585,0.00028563,-0.00062049,-0.00223364,-0.00483606,0.00028667,0.000366$ $65,0.00183596,-0.00082410,-0.10508531,0.09179784,0.11318323,0.00068704$ $, 0.00062745,0.00066269,-0.00005688,-0.00017716,-0.00006871,-0.00009306$ $, 0.00006067,-0.00007454,0.00004100,-0.00010736,0.00001039,-0.00003827$, $-0.00005711,-0.00005974,-0.00005266,-0.00009156,-0.00007193,-0.0002239$ $7,-0.00027738,-0.00022854,0.00000911,0.00011232,-0.00004989,0.00003062$ $, 0.00000059,-0.00000813,0.00002648,-0.00001183,0.00000284,0.00000305,0$ $.00005230,0.00002930,0.00000814,-0.00000311,-0.00000550,-0.00385985,-0$ $.00011415,-0.00055785,-0.00493727,-0.02066814,-0.02183119,-0.07261337$, 
$-0.04572343,-0.04906740,0.00916764,0.01357651,0.01452546,-0.00229643,0$ $.00314213,0.00279296,-0.00124016,0.00024464,0.00020519,-0.00129767,-0$. $00082438,-0.00090059,0.00123220,0.00008601,0.00033347,0.07505337,-0.00$ $033749,-0.00049956,0.00032917,-0.00002336,0.00006502,-0.00005739,-0.00$ $017874,0.00006668,0.00019464,0.00006710,-0.00020763,0.00005411,0.00002$ $653,0.00004471,-0.00005887,0.00027634,-0.00000435,0.00008231,0.0005014$ $3,0.00016991,0.00003591,0.00005519,0.00017954,0.00002984,-0.00000938,0$ $.00002775,0.00001811,-0.00001778,-0.00000132,0.00000401,0.00009132,-0$. $00006751,0.00003863,-0.00000280,0.00000052,0.00001265,-0.00048050,0.00$ $457551,-0.00223007,0.00018949,0.00063034,-0.00443296,-0.04894714,-0.16$ $456728,-0.13693366,-0.00260317,-0.00940071,-0.00963692,0.00213738,0.00$ $237607,-0.00422388,0.00039345,-0.00020372,0.00059619,-0.00008138,-0.00$ $005743,-0.00018353,-0.00012765,-0.00175705,0.00229001,0.04960842,0.169$ $33429,-0.00012480,0.00025863,-0.00057425,0.00037930,-0.00046564,0.0000$ $6754,-0.00018446,0.00036647,0.00017334,0.00004827,-0.00028600,0.000118$ $71,0.00004695,0.00010561,-0.00024871,-0.00020583,0.00010236,0.00031034$ $, 0.00046735,0.00004878,0.00013133,0.00007101,0.00042003,-0.00000258,-0$ $.00002192,0.00000687,-0.00000270,-0.00000178,0.00001763,0.00001572,-0$. $00003747,-0.00000711,-0.00002117,-0.00000771,0.00000316,0.00000265,-0$. $00097087,-0.00218204,0.00431217,-0.00000810,-0.00542545,-0.00047010,-0$ $.05266436,-0.13677247,-0.17952477,-0.00235482,-0.00886125,-0.00972316$, $0.00187728,-0.00400223,0.00240275,0.00044581,0.00061411,-0.00008435,-0$ $.00017567,-0.00019406,-0.00012798,0.00012543,0.00227059,-0.00157356,0$. $05363905,0.15400348,0.18582859,0.00011064,-0.00014735,-0.00004815,0.00$ $035571,-0.00047830,-0.00010219,0.00003973,-0.00012971,0.00032011,-0.00$ $065491,0.00023559,-0.00012903,0.00006123,0.00020953,-0.00002508,0.0002$ $6892,-0.00007057,0.00009073,-0.00000287,-0.00001083,0.00000382,-0.0000$ $8330,0.00028094,-0.00004391,0.00002216,0.00002092,-0.00003340,0.000023$ $92,-0.00002627,0.00002588,0.00001378,0.00001479,0.00003613,0.00006395$, $-0.00002211,-0.00002064,-0.00311815,-0.00150048,-0.00193157,-0.0003476$ $4,-0.00047930,-0.00050779,0.00066419,-0.00207145,-0.00233962,0.0097029$ $3,-0.00308546,-0.00254941,-0.19227895,0.10091031,0.09430611,-0.0168389$ $7,0.01596027,0.01505626,-0.00151980,0.00034703,0.00027624,-0.00004372$, $0.00001848,0.00005390,0.00036987,-0.00028918,-0.00031014,0.20202504,0$. $00057714,-0.00022985,0.00042561,0.00013347,-0.00000364,-0.00015355,0.0$ $0029596,-0.00012900,0.00030430,0.00009842,0.00066260,-0.00031146,-0.00$ $029425,-0.00009715,0.00009137,-0.00025727,0.00008115,-0.00016141,0.000$ $02017,0.00001149,0.00002660,-0.00000833,-0.00025924,0.00012688,-0.0000$ $5599,-0.00002831,0.00000202,0.00004969,-0.00000600,0.00005836,0.000015$ $20,-0.00000055,0.00003984,0.00003661,-0.00001931,-0.00001958,-0.001130$ $12,0.00414668,-0.00271531,-0.00079373,-0.00050413,0.00024848,-0.001015$ $81,0.00079494,-0.00556806,0.01357761,-0.00998921,-0.00917474,0.1031624$ $8,-0.11944984,-0.07919105,-0.00502032,0.00642116,0.00099398,-0.0004349$ $8,0.00006563,0.00000153,0.00003644,-0.00038436,0.00039847,0.00062496,-$ $0.00024914,-0.00029449,-0.10964481,0.12095354,0.00060061,0.00036022,-0$ $.00028531,-0.00037123,-0.00024383,0.00020615,-0.00012393,-0.00004118,-$ $0.00015530,-0.00003636,0.00009055,0.00036518,0.00000017,0.00031249,0.0$ $0019562,0.00008034,-0.00015162,-0.00018675,0.00007333,0.00003053,0.000$ $03757,0.00003289,0.00012340,-0.00011186,0.00007155,0.00005538,-0.00000$ $163,-0.00002904,0.00000830,-0.00001500,-0.00001184,-0.00002669,-0.0000$ $1250,0.00003421,-0.00002579,-0.00001780,-0.00160657,-0.00284700,0.0039$ 
$2214,-0.00071868,0.00025179,-0.00057474,-0.00102949,-0.00564717,0.0005$ $8403,0.01429218,-0.01018379,-0.00939621,0.09659615,-0.07928301,-0.1109$ $8582,-0.00609160,0.00210841,0.00702863,-0.00053170,0.00003540,0.000060$ $15,0.00009122,0.00040963,-0.00037018,0.00061305,-0.00030921,-0.0002622$ $0,-0.10233409,0.09256134,0.11163200,-0.00042806,-0.00004380,-0.0001074$ $8,-0.00015512,0.00042217,-0.00025076,0.00020254,-0.00021076,-0.0000725$ $5,-0.00005499,0.00034101,-0.00014138,-0.00012224,0.00008745,0.00032065$ $, 0.00010352,-0.00037166,-0.00002104,-0.00002989,-0.00002324,-0.0000139$ $9,0.00003986,-0.00036126,0.00008138,-0.00003058,-0.00001858,0.00002214$ $,-0.00002505,0.00001946,-0.00000820,-0.00001407,0.00000036,-0.00003012$ ,-0.00002625,0.00000520,-0.00000414,0.00719808,0.01479673,0.01515926,-$0.00267905,0.00251132,0.00212103,-0.00098889,0.00042893,0.00049264,-0$. $00431766,-0.00118050,-0.00166603,-0.00391445,-0.01940943,-0.01997920,-$ $0.07571619,-0.05371963,-0.05613208,0.00106597,0.00042455,0.00058681,0$. $00047349,0.00074551,0.00078206,0.00005319,-0.00008918,-0.00004607,0.00$ $116608,0.00006955,0.00035231,0.07816483,0.00007198,0.00005942,-0.00004$ $379,-0.00015030,0.00014575,-0.00001388,0.00007931,-0.00008970,-0.00003$ $555,-0.00002312,0.00008001,-0.00005959,-0.00001671,0.00003112,0.000115$ $04,0.00004075,-0.00011103,-0.00001672,-0.00001170,-0.00000648,0.000006$ $41,0.00002552,-0.00011357,0.00002278,-0.00001339,-0.00000545,0.0000028$ $7,0.00000044,0.00000293,0.00000491,0.00000394,0.00000106,0.00000083,-0$ $.00000058,0.00000947,0.00000327,-0.00719174,-0.00672812,-0.01041612,0$. $00293773,0.00213120,-0.00313093,0.00031399,-0.00012510,0.00072496,-0.0$ $0076418,0.00400436,-0.00252801,0.00073189,0.00125488,-0.00312045,-0.05$ $081896,-0.17350338,-0.14194027,-0.00035070,-0.00073959,0.00192350,-0.0$ $0030647,-0.00012544,-0.00060222,-0.00005048,-0.00045437,0.00038695,-0$. $00004654,-0.00170005,0.00239228,0.05553881,0.17595904,0.00003946,-0.00$ $005973,0.00007176,0.00001167,0.00009644,-0.00007508,0.00005030,-0.0000$ $2158,0.00000152,-0.00001055,0.00007729,-0.00002502,-0.00000587,0.00000$ $424,0.00003753,-0.00006603,-0.00002689,-0.00000179,-0.00001495,0.00000$ $625,-0.00001152,-0.00002134,-0.00011188,0.00002053,0.00000046,-0.00000$ $217,0.00000198,-0.00001367,0.00000768,-0.00000441,-0.00000016,-0.00000$ $491,-0.00001563,-0.00000293,-0.00000161,0.00000333,-0.00711519,-0.0094$ $9355,-0.00670054,0.00260134,-0.00307992,0.00232605,0.00010629,0.000758$ $39,-0.00005326,-0.00115810,-0.00275065,0.00370544,0.00048208,-0.003986$ $92,0.00036517,-0.05318327,-0.14196581,-0.18120975,-0.00016860,0.001965$ $51,-0.00064393,-0.00030134,-0.00054055,-0.00011840,-0.00000691,0.00040$ $076,-0.00043307,0.00020451,0.00237200,-0.00166737,0.05853800,0.1563323$ $6,0.18441962,0.00286645,0.00200805,-0.00053122,-0.00070360,-0.00857781$ ,-0.00505092,-0.00096532,0.00141552,-0.00127728,-0.00076997,-0.0004746 $9,0.00008737,-0.00023440,0.00050782,-0.00006763,0.00102127,-0.00019520$ $, 0.00052502,-0.00067031,-0.00009646,-0.00012673,-0.03554995,0.02809694$ $, 0.03346158,0.00013582,0.00035027,0.00088609,0.00002358,-0.00000783,0$. $00007759,-0.00005173,-0.00001963,-0.00004666,0.00002567,0.00005545,0.0$ $0000092,-0.00011860,-0.00010288,-0.00008602,0.00014050,-0.00001767,-0$. $00001974,0.00010368,0.00013231,0.00018962,-0.00070301,-0.00019726,-0.0$ $0014733,0.00027424,0.00006454,-0.00000759,-0.00013471,0.00004921,-0.00$ $002196,0.00010020,0.00001673,0.00001201,0.00003814,-0.00000264,0.00000$ $758,0.00001273,-0.00001789,-0.00008436,0.00000342,-0.00007790,0.000023$ $05,0.00001606,-0.00000480,0.00001604,0.03702841,0.00354745,-0.00533898$ $, 0.00006733,-0.00687202,-0.00640938,0.03815101,-0.00283866,0.00202204$, 
$0.00090322,-0.00017106,-0.00063648,-0.00006729,-0.00000313,0.00030847$, $-0.00032205,-0.00054807,0.00025534,0.00177361,-0.00024970,0.00010519,0$ $.00012949,0.02358933,-0.04832351,0.04030683,0.00103489,-0.00019803,-0$. $00097942,0.00003591,0.00001063,-0.00011875,0.00013680,-0.00046020,0.00$ $012748,0.00000220,0.00008754,0.00000944,-0.00014158,-0.00005393,-0.000$ $03835,0.00004458,0.00000753,0.00001010,-0.00001385,0.00006473,0.000046$ $94,0.00015666,0.00006402,-0.00011063,0.00001021,0.00003692,-0.00001307$ ,-0.00002546,-0.00000369,-0.00003167,0.00012939,0.00001563,0.00002508, $0.00002156,0.00000130,-0.00000796,-0.00002141,-0.00001539,0.00002458,-$ $0.00000427,-0.00009631,0.00003541,-0.00000196,0.00000413,0.00000875,-0$ $.01567054,0.05675007,-0.00145090,0.00145866,0.00133909,-0.00100184,-0$. $00161134,-0.00983397,0.00068656,-0.00288986,0.00242318,0.00061374,0.00$ $098763,-0.00058117,-0.00033674,-0.00000878,0.00066426,0.00141706,-0.00$ $041787,-0.00010505,-0.00034623,-0.00022768,0.00025289,0.06506701,0.064$ $48328,-0.43242080,-0.00079776,0.00032686,-0.00017916,-0.00019417,-0.00$ $005439,-0.00004390,-0.00009572,0.00012800,-0.00003037,-0.00000466,-0.0$ $0006816,0.00000264,0.00004053,0.00002788,-0.00000686,-0.00000615,-0.00$ $000572,0.00001770,-0.00001695,-0.00005865,-0.00004766,0.00005558,-0.00$ $002163,0.00008687,-0.00002816,0.00002748,0.00002202,0.00000227,0.00000$ $449,-0.00000473,-0.00004727,-0.00001215,-0.00000785,-0.00000742,0.0000$ $0446,-0.00000460,-0.00000709,0.00000637,0.00000256,0.00000105,-0.00001$ $203,0.00001402,-0.00001067,-0.00000790,-0.00000302,-0.05815474,-0.0634$ $0125,0.44650826,-0.00025938,-0.00109935,0.00032177,-0.00416004,-0.0051$ $5429,-0.00073567,0.00078856,0.00015671,-0.00047516,0.00136111,-0.00034$ $304,0.00087386,0.00005909,0.00005133,-0.00020728,-0.00010000,0.0000410$ $5,0.00016372,0.00016252,0.00013409,-0.00059523,-0.31753355,0.16767564$, $0.14085290,-0.00045103,-0.00028242,-0.00021417,-0.00010168,-0.00008279$ $,-0.00008644,0.00002065,-0.00007133,0.00004001,-0.00003978,-0.00005666$ ,-0.00006028,-0.00026074,0.00006467,0.00006912,-0.00021356,-0.00002845 ,-0.00011640,-0.00015676,0.00026012,0.00022948,0.00134750,0.00056551,-$0.00001933,-0.00010675,-0.00024737,-0.00019563,0.00017723,-0.00002755$, $0.00000095,0.00018016,0.00002106,0.00004814,0.00002449,0.00000528,-0.0$ $0001633,0.00001516,-0.00014103,0.00009791,-0.00000326,0.00003582,0.000$ $02346,0.00001887,0.00000535,0.00001877,-0.00188857,-0.00214728,-0.0053$ $7733,0.32111978,-0.00007145,0.00196447,-0.00225867,0.03104278,-0.02089$ $821,-0.01458992,0.00015443,-0.00264196,0.00531961,0.00097851,-0.001266$ $18,-0.00094791,0.00000221,0.00048016,-0.00031402,-0.00077419,-0.000635$ $60,-0.00031195,0.00033443,0.00008176,0.00073778,0.13510086,-0.09407201$ ,-0.03152894,-0.00002897,0.00012662,-0.00003059,0.00005143,0.00006465, $0.00008831,0.00000971,0.00009460,-0.00001507,0.00002219,-0.00045957,0$. $00017956,-0.00026234,-0.00000894,0.00002514,-0.00001379,-0.00005947,0$. $00003043,-0.00021166,0.00022269,0.00042054,-0.00022427,0.00071366,-0.0$ $0051508,-0.00007541,-0.00027362,-0.00031253,0.00011033,-0.00009276,-0$. $00000891,0.00031355,0.00004248,0.00005483,0.00007121,0.00001847,-0.000$ $02365,-0.00000850,0.00001513,-0.00013851,0.00000876,0.00000887,-0.0000$ $1585,0.00003894,0.00001892,0.00001651,-0.00723366,0.00180236,0.0013423$ 2,-0.15933507,0.11475346,0.00011237,0.00271802,0.00016387,-0.01164997, $-0.00350934,0.00665365,-0.00004663,0.00346559,0.00047653,0.00060998,-0$ $.00075788,0.00116604,0.00009707,0.00042782,-0.00070814,-0.00087852,-0$. $00047474,-0.00038836,0.00031441,-0.00008751,-0.00117043,0.11605366,-0$. $02789967,-0.09992239,-0.00026952,0.00008225,0.00023860,0.00016463,-0.0$ 
$0001742,0.00002453,0.00002871,0.00000887,-0.00000019,-0.00009241,0.000$ $04543,-0.00004212,-0.00000736,-0.00016511,-0.00015409,0.00026918,-0.00$ $010235,-0.00002338,0.00015977,0.00040602,0.00034782,-0.00090158,-0.000$ $42137,-0.00006610,0.00037105,0.00003126,-0.00006384,-0.00027805,0.0000$ $6269,-0.00002591,0.00003710,0.00000188,-0.00001105,0.00006036,-0.00000$ $267,0.00002710,0.00012364,-0.00002969,-0.00031713,0.00000030,-0.000049$ $10,-0.00000282,0.00002741,-0.00000073,0.00000984,0.03033529,-0.0165288$ $2,-0.00806435,-0.13464087,0.04279657,0.10185230 \backslash 10.00002249,0.00002750$ $, 0.00003612,-0.00002897,0.00002310,-0.00000486,0.00000740,-0.00000761$, $-0.00000226,-0.00000716,0.00000501,-0.00000238,-0.00000164,-0.00000872$ ,-0.00000293,-0.00001717,-0.00001095,0.00001665,0.00002251,-0.00001371 $,-0.00000184,0.00000655,0.00000011,0.00000301,0.00000252,0.00000426,0$. $00000362,-0.00000134,-0.00000268,-0.00000271,-0.00000092,-0.00000672,-$ $0.00000024,0.00000140,0.00000171,-0.00000134,0.00000079,0.00000010,-0$. $00000440,0.00000212,-0.00000267,-0.00000121,0.00001810,0.00000245,0.00$ $000463,-0.00001214,-0.00002538,-0.00003553,-0.00001525,0.00000406,0.00$ $000103,-0.00000014,-0.00000060,-0.00000631,-0.00000059,0.00000318,-0.0$ $0000479,0.00000018,-0.00000064,-0.00000016,-0.00000116,-0.00000222,0.0$ $0000288,-0.00000003,0.00000153,-0.00000494,-0.00000091,0.00000475,-0.0$ $0000639,0.00000145,0.00000304,0.00000683,0.00000191,0.00000108,0.00000$ 749111@

meta-Transition State

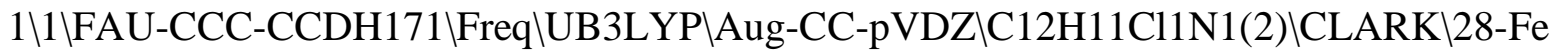
b-2015\0\\N Geom=AllCheck Guess=TCheck SCRF=Check GenChk UB3LYP/Aug-C C-pVDZ Freq \\Addition product p-Cl-Ph-radical + aniline (ortho) $\backslash 10,2 \mathrm{CC}$ ,1.8053988439,1.1585609181,-0.7799061924\C, 2.4633753241,-0.0434198248, $-1.1376090402 \backslash \mathrm{C}, 3.2502276388,-0.7312627476,-0.2080243474 \backslash \mathrm{C}, 3.445032668$ $7,-0.1697857907,1.0733430252 \backslash \mathrm{C}, 2.8720868751,1.0655565565,1.3987197614 \backslash$ C,2.077813949,1.7493852876,0.4842408606ไH,1.3804517159,1.7717391555,-1 $.572851955 \backslash \mathrm{H}, 2.3194752087,-0.4570314863,-2.1366929291 \mathrm{~N}, 3.8024702485,-$ $1.9809573515,-0.5168689706 \backslash \mathrm{H}, 3.0617801424,1.4971560054,2.3822623512 \backslash \mathrm{H}$, $1.6387185191,2.7120019314,0.7415880775 \backslash \mathrm{H}, 4.058333761,-0.6974456831,1.8$ 053052085\C,-2.9207153219,-0.1973784758,0.0808220148\C,-2.664839645,1. 0748860247,-0.4342829548\C,-1.3356110314,1.4440111412,-0.6982679989\C, $-0.3280308157,0.5281924694,-0.4425357511 \backslash \mathrm{C},-0.5689278473,-0.7356696482$ $, 0.0693643101 \backslash \mathrm{C},-1.8946684128,-1.1100633828,0.3380669349 \backslash \mathrm{Cl},-4.5911422$ $971,-0.6671434057,0.4158749703 \backslash \mathrm{H},-3.4864195061,1.7644603624,-0.6251791$ $95 \backslash \mathrm{H},-1.1232813416,2.437801954,-1.0973174744 \backslash \mathrm{H}, 0.2457446715,-1.4352187$ $578,0.2611011209 \backslash \mathrm{H},-2.1276378245,-2.0956080376,0.7398106812 \backslash \mathrm{H}, 3.892511$ $1274,-2.1826819592,-1.5043899411 \backslash \mathrm{H}, 4.6397933493,-2.2249772547,-0.00373$ 05665 \IVersion=EM64L-G09RevC.01 $\backslash$ State=2-A $\backslash H F=-978.8422328 \backslash S 2=0.779506 \backslash$ $\mathrm{S} 2-1=0 . \mathrm{S} 2 \mathrm{~A}=0.75062 \backslash \mathrm{RMSD}=9.451 \mathrm{e}-09 \backslash \mathrm{RMSF}=2.573 \mathrm{e}-06 \backslash$ ZeroPoint $=0.1941719 \backslash$ Thermal $=0.2066984 \mathrm{VPE}=121.8446908 \mathrm{VDipole}=1.4113512,-0.0305883,-0.31409$ 93\DipoleDeriv $=0.1656411,-0.1077489,-0.0537289,-0.0809827,0.1357289,0$. $0366847,-0.0649672,-0.0023638,0.1144883,-0.2802891,0.1969083,0.2037695$ $, 0.0059658,-0.3590843,-0.0246057,0.0007801,0.2092295,0.0471962,0.31879$ $69,-0.5290765,-0.1648406,-0.784436,1.1197932,0.2779759,-0.3872761,0.20$ $50343,-0.0175539,-0.1936599,-0.0678459,0.044894,0.2295533,-0.2147452,0$ $.017636,0.2596254,-0.2730719,-0.0177913,0.0407646,-0.0869057,0.1198672$ 
,-0.1923008,0.1024258,-0.0361963,0.1390994,0.093932,0.1340411,-0.25413 $03,-0.0891922,-0.0182956,-0.0146192,-0.1132852,0.0141823,0.0340711,0.0$ $696023,-0.2003542,0.1190272,0.0673965,-0.0387466,0.0633077,0.0235872,0$ $.0530621,-0.0495573,0.0435233,0.0038443,0.102709,0.0238746,-0.0429169$, $0.0427973,0.0330162,-0.090923,-0.0140659,-0.0672274,-0.0522062,-0.3801$ $801,0.0934336,0.1181726,0.6138029,-1.278472,-0.192037,0.1801296,-0.075$ $9799,-0.1596974,0.0962748,0.014213,-0.0396531,0.0029088,0.0541564,-0.0$ $712351,-0.0628792,-0.0789977,-0.0608133,0.0808281,0.1038587,-0.0078422$ $, 0.0683621,-0.0237634,-0.038471,-0.0087548,-0.0418277,0.0559796,0.0408$ $725,0.0762141,-0.0787739,0.1013469,0.0208261,0.043779,-0.0806158,0.038$ $8057,0.010817,1.1490005,0.2826422,-0.1950868,0.3367902,0.0706384,-0.06$ $49064,-0.2394809,-0.0741293,-0.0082991,-0.2017051,-0.1176893,0.0334587$ $, 0.0172844,-0.029604,-0.0016768,0.0112232,-0.0107682,-0.0941634,-0.332$ $8451,-0.0030892,-0.0470832,-0.3425884,0.0770022,-0.1023878,0.1418716,-$ $0.0586245,-0.0523178,0.7478959,0.102176,0.0790501,0.256788,-0.0395615$, $0.0356375,-0.1080691,-0.0059961,-0.0939625,-0.4613877,-0.0772037,-0.03$ $02878,0.0499543,-0.0109056,-0.0165337,0.0090339,-0.0220014,-0.0659787$, $-0.1679135,0.0004703,0.0149866,-0.1432624,0.0297899,-0.0565504,0.07754$ $64,-0.043405,-0.0626584,-0.8762864,-0.1735919,0.1099247,-0.2206575,-0$. $1408578,0.0364782,0.1545854,0.0408126,-0.1010941,-0.0676167,0.0663084$, $-0.0224463,0.0410397,0.0424493,0.0263499,0.0007802,0.0262387,0.1185398$ $, 0.0692177,-0.0558908,0.0318462,0.0033578,-0.0565424,0.0766965,0.00149$ $91,0.0662885,0.0865773,0.0454611,0.079105,-0.0072133,0.0409462,0.03466$ $23,0.0213827,-0.0185036,0.0190665,0.0920737,0.0073359,-0.0369921,0.012$ $9766,-0.0470906,-0.0115205,0.0517558,0.0327174,0.0560672,0.103921,0.13$ $76366,0.1309859,0.0057893,0.0086013,0.2574999,0.0633532,0.0011262,-0.0$ $102964,0.0849843,0.0945518,0.1076394,-0.0278204,-0.0568692,0.2767662,-$ $0.0594507,-0.0099191,-0.1039114,0.1344278 \backslash$ Polar $=251.1775403,-11.401057$ 9,165.9561245,7.4929508,-2.9205423,133.2804145\PG=C01 [X(C12H11Cl1N1)] WImag=1\0.15424843,-0.21975897,0.55379004,0.13515768,0.01546722,0.62 $392016,-0.08430594,0.10116361,0.00208993,0.31256711,0.07374748,-0.2498$ $0877,-0.03451534,-0.21094611,0.51860391,0.01742912,-0.07104646,-0.1260$ $0475,0.14473006,0.10420813,0.65048711,-0.02507833,0.03062335,0.0054359$ $8,-0.14329053,0.06126094,-0.10690933,0.34729146,0.02471801,-0.02563359$ $, 0.01508709,0.08564096,-0.14569758,0.07620813,-0.21383396,0.55535472,-$ $0.03117848,0.03743343,0.04172724,-0.06923664,0.02117355,-0.20853419,0$. $13587156,0.08145501,0.60152240,0.00100930,0.00548619,0.00731549,0.0044$ $8936,-0.00727189,-0.01725254,-0.08884478,0.00200598,-0.05866858,0.3204$ $2560,-0.00418069,-0.02778999,-0.02415950,-0.02076143,0.03759412,-0.003$ $73791,0.02526369,-0.12913191,-0.03353480,-0.23351808,0.56170462,0.0130$ $7216,-0.01872629,-0.02085677,-0.03717485,0.02757556,-0.04180095,-0.021$ $40235,-0.09156285,-0.25238332,0.16672239,0.05788818,0.59655843,-0.0076$ $2264,-0.01889146,-0.03517020,-0.01777593,0.01872659,-0.00837182,0.0201$ $4446,-0.00235536,0.04171385,-0.11109592,0.08653824,-0.01407888,0.31581$ $792,0.00892484,0.04404103,0.02463210,0.01929810,-0.02851478,0.01204402$ ,-0.01837830,-0.01551988,-0.06654431,0.10881565,-0.26601212,-0.0284520 $4,-0.18830713,0.53862474,-0.02400262,-0.00109807,-0.03976352,-0.007948$ $54,0.01019930,-0.00623259,0.01948431,-0.03238766,0.00674107,0.01773412$ ,-0.08344137,-0.12651504,0.16713310,0.11282098,0.65342838,-0.05894144, $0.01417462,-0.03938197,0.01466401,-0.00201879,0.01715911,-0.01167334,-$ $0.00395302,-0.02187267,-0.01257677,0.04320041,0.00863116,-0.15297229,0$ 
$.07109314,-0.11810993,0.32626696,0.01178295,-0.13965555,-0.09703671,-0$ $.00431475,-0.01003729,-0.02839571,0.00101567,0.00325542,0.00982910,0.0$ $2655270,-0.03080715,0.00941651,0.09517503,-0.15765279,0.08566034,-0.23$ $425519,0.60084656,-0.01601829,-0.05128280,-0.25305052,0.01959831,-0.04$ $183048,0.00355101,-0.01885523,0.00495871,-0.04592369,-0.01540935,0.049$ $44154,0.04062228,-0.08699516,0.03075139,-0.21099672,0.14758243,0.08585$ $250,0.55043304,-0.06305130,0.07175747,-0.08171247,-0.00880061,0.012365$ $49,-0.01129516,0.00493653,0.00427760,0.00378956,-0.00093237,0.00050181$ ,-0.00046534,0.00562086,-0.00198904,-0.00159080,-0.00405431,0.00225856 ,-0.00979583,0.08586331,0.08127535,-0.14071157,0.11461169,0.00772979,-$0.00755504,0.01941774,0.00298556,-0.00272176,-0.00124596,-0.00054085,-$ $0.00023373,-0.00110164,0.00104561,-0.00088594,-0.00300298,-0.00721114$, $0.01052450,-0.01346914,-0.07676177,0.14545464,-0.09184597,0.11278995,-$ $0.20464505,0.00471987,-0.00725929,0.00864273,0.00025992,-0.00245724,0$. $00066512,0.00084746,-0.00102874,-0.00008653,-0.00110776,-0.00299011,-0$ $.00401872,-0.00994614,0.01743431,-0.01233152,0.09215062,-0.12038304,0$. $21242765,0.00362116,0.00608760,0.01542522,-0.04458392,-0.00418174,-0.0$ $4228933,0.00198973,-0.00724027,-0.01622187,0.00192054,0.00445264,-0.00$ $303115,-0.00081564,0.00003011,0.00015191,0.00212884,0.00423989,-0.0013$ $5594,-0.00191706,-0.00118909,0.00033174,0.03914962,-0.00597847,-0.0058$ $7885,-0.02430735,-0.00221745,-0.08927332,-0.09988146,0.00329273,0.0076$ $3691,0.01347791,0.00412393,0.00048985,0.00158582,0.00004737,-0.0009550$ $8,0.00038718,0.00518032,-0.00262433,-0.00110128,-0.00182707,0.00033754$ $, 0.00071027,-0.00007429,0.08949049,-0.00142629,-0.00099526,-0.00401524$ ,-0.04142817,-0.09777800,-0.28721186,-0.00032482,-0.01197888,-0.015125 $81,-0.00376656,0.00233435,-0.00029626,-0.00000698,0.00078476,0.0002475$ $9,-0.00008956,-0.00167692,0.00240997,0.00105318,0.00029186,0.00045040$, $0.04585056,0.10853152,0.30343734,0.00391751,0.00070531,-0.00614828,-0$. $00929194,0.03291234,0.00374705,-0.10306780,0.11829000,-0.00211664,0.00$ $713294,-0.00081282,-0.00107820,0.00096330,0.00260370,-0.00313059,-0.00$ $063407,0.00017853,0.00240771,-0.00055906,0.00103029,0.00039434,-0.0013$ $9920,-0.00221009,0.00193561,0.45630213,-0.00132301,0.00807075,0.001731$ $74,0.02281503,-0.03183909,0.00207196,0.07631445,-0.22890890,-0.0390522$ $4,0.01073596,-0.02590258,-0.02025022,-0.00059363,0.00624124,0.00077091$ $, 0.00406445,-0.00592680,-0.00176759,0.00018450,-0.00027421,-0.00001956$ ,-0.00234689,0.00066784,-0.00006599,-0.26308985,0.37146528,0.00057026, $-0.00180521,-0.00621315,-0.01555504,0.02762334,0.01051984,-0.00320325$, $-0.06130276,-0.11597704,0.01737778,-0.04546370,-0.01168666,-0.00744521$ $, 0.00396884,-0.00580553,0.00265117,-0.00108296,0.00378988,-0.00101998$, $0.00075758,0.00030498,0.00020529,0.00120555,0.00133395,0.15002602,0.11$ $750533,0.70510099,0.00228621,0.00527156,-0.00397472,-0.00057994,-0.000$ $23962,0.00034195,0.00361998,0.00480838,-0.00130715,0.00515521,0.005660$ $30,0.01168808,-0.05202339,-0.01199745,-0.04987548,0.00059885,-0.007901$ $86,-0.01602610,-0.00002203,-0.00055297,-0.00104435,-0.00044945,-0.0003$ $9087,0.00020619,0.00026927,0.00003490,0.00060456,0.04544439,0.00430726$ $, 0.00084369,0.00156727,0.00027501,-0.00079074,0.00065003,0.00481617,-0$ $.00265824,-0.00122581,-0.00556814,-0.00598162,-0.02489766,-0.01144215$, $-0.09736963,-0.10300688,0.00305891,0.00973027,0.01380752,0.00017154,0$. $00020098,0.00055886,-0.00036259,-0.00016329,0.00006045,-0.00017677,-0$. $00015177,-0.00142824,0.00872385,0.09699655,-0.00416886,0.00179132,-0.0$ $0028774,0.00027178,0.00065051,0.00031786,-0.00118505,-0.00158929,0.002$ 
$87990,-0.00237935,-0.00064401,-0.00343015,-0.05015592,-0.10306688,-0.2$ $8190608,-0.00156995,-0.01059387,-0.01359748,-0.00021334,-0.00048160,-0$ $.00063152,0.00025507,0.00010929,-0.00014925,-0.00013191,-0.00015957,-0$ $.00038720,0.05622249,0.11237899,0.29660519,0.00393337,-0.00791585,-0.0$ $0264306,0.00301079,0.00128209,-0.00231924,-0.00037870,-0.00039570,-0.0$ $0036745,0.00287302,0.00275760,-0.00453878,-0.00356830,0.01515988,0.005$ $77595,-0.08572732,0.11238341,0.02040564,-0.00175309,-0.00076282,0.0005$ $3638,0.00058010,-0.00006806,-0.00023654,-0.00024199,-0.00015896,0.0003$ $3793,-0.00237879,-0.00194069,0.00132888,0.08351071,0.00505124,-0.00770$ $528,-0.00038538,0.00023669,0.00201920,-0.00268439,-0.00034612,0.000070$ 17,0.00020124,0.00338406,0.00192817,0.00179739,0.00403401,-0.00921428, $-0.00623134,0.11095990,-0.27323852,-0.06299355,-0.00138796,0.00009378$, $0.00009467,0.00046636,-0.00034364,-0.00005406,-0.00015683,0.00004360,0$ $.00006005,-0.00183560,-0.00023091,0.00087183,-0.12128180,0.28665799,0$. $01304371,-0.02389357,-0.00237377,-0.00316979,-0.00216331,-0.00382907,-$ $0.00038219,0.00034995,-0.00101605,-0.00416624,0.00082736,-0.00168299,-$ $0.00851609,0.01941562,0.00827908,0.01872558,-0.06515888,-0.06918134,0$. $00103645,0.00106831,0.00078787,0.00054009,-0.00132765,-0.00027686,0.00$ $031221,0.00001530,0.00023971,0.00175421,0.00053662,0.00035350,-0.01844$ $533,0.06946901,0.06843756,-0.00036820,-0.00059813,0.00056825,0.0041166$ $2,0.00051760,-0.00148793,-0.00006904,0.00490208,-0.00196110,-0.1266350$ $6,0.08425640,-0.11259310,-0.00426628,0.00474105,-0.00964635,0.00413202$ $, 0.00400890,0.00048697,-0.00047761,-0.00016961,0.00007427,-0.00016766$, $0.00004787,-0.00012338,-0.00068320,-0.00178877,0.00217634,-0.00219921$, $-0.00177617,0.00148744,-0.00011566,0.00035577,-0.00060432,0.12689560,-$ $0.00033975,-0.00071038,-0.00034388,0.00119121,0.00069809,-0.00337957,-$ $0.00601913,0.00750821,-0.00923413,0.08477429,-0.11496974,0.08484859,0$. $01463291,-0.00798896,0.02016367,0.00382916,-0.00286060,-0.00340328,-0$. $00025917,-0.00010068,0.00014392,-0.00045649,0.00038486,-0.00046778,-0$. $00185275,0.00051413,0.00073325,-0.00185821,0.00000264,0.00065831,0.000$ $88770,-0.00048430,0.00091415,-0.09448616,0.11751084,0.00068550,-0.0006$ $5788,-0.00038861,-0.00107820,-0.00410832,-0.00358931,-0.01681677,0.016$ $29714,-0.01302706,-0.11230574,0.08477342,-0.18091740,0.00520068,-0.004$ $90133,0.00779787,-0.00051753,-0.00272286,0.00168011,0.00016658,0.00016$ $274,-0.00004989,-0.00078442,0.00074122,-0.00084761,0.00038232,0.001591$ 75,0.00118407,0.00118021,0.00099516,0.00025187,0.00019596,-0.00033738, $0.00029938,0.12241434,-0.09071684,0.18804628,-0.00023681,0.00013515,0$. $00012285,0.00001783,0.00010116,-0.00002284,-0.00010746,0.00054627,0.00$ $045820,0.00019422,-0.00035017,-0.00011252,-0.00007533,0.00000276,-0.00$ $015656,0.00004255,-0.00005450,-0.00013393,-0.00021022,-0.00002356,0.00$ $001195,-0.00003303,-0.00003694,-0.00003757,0.00016654,-0.00053520,-0.0$ $0008203,-0.00000820,0.00000962,0.00005992,-0.00005995,-0.00007015,-0.0$ $0002877,0.00001247,-0.00004849,0.00001815,0.50533474,0.00000606,-0.000$ $35475,0.00043083,0.00008101,-0.00003716,0.00000615,-0.00011391,0.00040$ $209,0.00019515,-0.00011048,0.00010017,-0.00005671,0.00009443,0.0001002$ $7,-0.00015645,0.00011716,0.00001431,-0.00013875,0.00009919,0.00014416$, $-0.00016525,-0.00005438,-0.00000452,0.00006460,0.00000937,-0.00028157$, $-0.00007272,-0.00002844,0.00000580,0.00001460,-0.00001692,0.00012726,0$ $.00007306,0.00002183,-0.00000993,0.00001686,-0.05552855,0.57345007,0.0$ $0010848,0.00021002,-0.00014164,-0.00000986,-0.00013424,0.00001787,0.00$ $009294,-0.00012060,-0.00007717,-0.00005306,-0.00001246,0.00002025,0.00$ 
$004064,-0.00004683,-0.00003859,-0.00010676,-0.00001580,0.00006924,0.00$ $000852,-0.00000678,0.00008333,0.00003356,0.00000618,-0.00006979,-0.000$ $02563,0.00010606,0.00003425,0.00000760,0.00001293,-0.00000627,-0.00004$ $164,-0.00008134,-0.00003470,-0.00000414,0.00000903,-0.00000590,-0.0139$ $3211,-0.16795589,0.19164782,0.00086463,0.00104526,-0.00072906,-0.00029$ $638,-0.00023311,0.00010484,0.00002981,-0.00032509,-0.00021074,0.000028$ $63,-0.00003750,0.00002960,-0.00007419,0.00004455,0.00005408,0.00009798$ $,-0.00003378,0.00026345,0.00008291,-0.00033107,0.00032432,0.00007711,0$ $.00004428,-0.00007387,-0.00000644,0.00015907,0.00006424,0.00001914,-0$. $00000196,-0.00000614,-0.00010985,-0.00021753,-0.00008654,-0.00000926,-$ $0.00000881,0.00000015,-0.10875798,0.00810279,0.00094582,0.68654806,0.0$ $0059662,-0.00003609,0.00061272,0.00020031,0.00029557,-0.00029642,0.000$ $03517,0.00010898,0.00038015,0.00032325,-0.00046193,0.00008031,-0.00032$ $139,-0.00001937,-0.00014198,0.00059835,0.00012164,-0.00021228,0.000152$ $41,-0.00022369,0.00001019,0.00010971,-0.00004243,-0.00009577,-0.000003$ $39,-0.00007493,-0.00005246,0.00003289,0.00001381,0.00002889,0.00005496$ $, 0.00004490,0.00006130,-0.00000094,0.00000167,-0.00002634,-0.05193244$, $-0.28902988,0.09127189,-0.06387631,0.61431170,-0.00075956,0.00021378,0$ $.00053481,-0.00016201,-0.00003165,0.00005877,-0.00022670,-0.00018332,-$ $0.00038472,0.00002814,0.00036085,0.00018603,0.00005313,-0.00011147,0.0$ $0027946,0.00024654,0.00011513,0.00010189,0.00002900,-0.00006103,0.0001$ $1094,-0.00000338,0.00001278,0.00004693,0.00000787,0.00022662,0.0000527$ $7,-0.00001024,-0.00003976,0.00000826,0.00005079,0.00011433,-0.00002273$ $, 0.00000072,0.00000534,0.00000326,0.02415531,0.08608807,-0.10033835,-0$ $.02682541,-0.18209203,0.19980432,-0.00519625,0.00188950,-0.00138116,-0$ $.00097761,-0.00123417,0.00014798,-0.00028894,-0.00074941,-0.00143509,-$ $0.00047164,0.00099825,0.00028172,0.00055202,0.00019908,0.00039871,-0.0$ $0114919,0.00005841,0.00147072,0.00039246,0.00056455,-0.00035947,-0.000$ $21283,0.00012259,0.00030351,0.00001095,0.00098679,0.00017232,-0.000058$ $27,-0.00003495,-0.00011524,0.00003640,-0.00006177,-0.00004229,-0.00007$ $004,0.00001321,0.00003247,0.00706773,-0.08219683,0.03174008,-0.2961209$ $7,-0.01535157,0.02737462,0.60510938,-0.00148582,0.00088056,-0.00127025$ $,-0.00074147,-0.00053906,0.00051892,0.00025509,-0.00125022,-0.00121642$ $,-0.00033625,0.00083404,-0.00003124,0.00019204,-0.00001051,0.00039383$, $-0.00110895,-0.00015135,0.00076085,-0.00107510,0.00068205,0.00006982,-$ $0.00007863,0.00004654,0.00009005,-0.00002025,0.00117361,0.00026796,-0$. $00003439,-0.00004675,-0.00008597,-0.00009888,-0.00009812,-0.00022956,-$ $0.00006153,0.00001986,0.00000142,-0.02856077,0.00156500,0.00486264,-0$. $08492196,-0.12293891,0.03134143,-0.01245398,0.64683216,0.00119095,-0.0$ $0057276,0.00086230,0.00007496,0.00044911,-0.00053419,0.00021420,0.0004$ $2976,0.00126212,0.00025751,-0.00083517,-0.00028515,-0.00046034,0.00055$ $271,-0.00044089,0.00086022,0.00001460,-0.00006360,0.00006721,-0.000221$ $24,-0.00002661,-0.00001259,0.00002689,-0.00009767,-0.00005130,-0.00042$ $739,-0.00015713,-0.00001394,0.00001800,0.00003205,0.00005948,0.0001455$ $8,0.00008491,0.00003857,-0.00000141,-0.00001414,0.01081656,0.01030800$, $0.00228119,0.05361771,0.02465989,-0.07525113,-0.03854125,-0.19769270,0$ .20578760,0.07585616,0.01356600,0.00575243,-0.02860948,0.02478726,0.00 $399083,0.00531820,-0.00038439,0.01648889,-0.00249659,0.00234267,-0.005$ $71535,0.00464097,-0.01036104,0.00372988,-0.01865846,-0.01214060,-0.029$ $91446,-0.01431114,-0.00664140,0.00367056,0.00095647,-0.00013617,-0.001$ $19795,-0.00011926,-0.00227398,-0.00149009,0.00056188,-0.00006799,0.000$ 
$44302,0.00066026,0.00128272,0.00014920,0.00030936,-0.00006743,0.000020$ $47,-0.01038315,0.02008419,-0.00745898,-0.05471464,0.00126893,0.0063459$ $9,-0.21326418,0.13551892,-0.03706423,0.33313984,0.02230827,-0.00759550$ $, 0.00486240,0.00059499,0.00665412,0.00072129,0.00135859,0.00062533,0.0$ $0400373,-0.00108760,0.00108681,-0.00160416,0.00150790,-0.00387603,0.00$ $085549,-0.00710167,-0.00279501,-0.00957168,-0.00870675,-0.00434368,0.0$ $0293039,0.00008044,-0.00001441,-0.00022982,0.00010851,-0.00193458,-0.0$ $0059518,0.00023527,0.00003312,0.00016301,0.00010422,0.00043837,0.00000$ $334,0.00012105,0.00002355,-0.00001026,0.01996443,-0.06181634,0.0191302$ $7,0.05343343,0.04778509,-0.01821963,0.10318755,-0.20617181,0.04831531$, $-0.10552948,0.56174449,-0.01133743,0.00357536,-0.00925882,0.00516766$,$0.00504571,0.00112040,-0.00097235,0.00091124,-0.00313623,0.00021143,0$. $00028874,0.00062247,-0.00072493,-0.00010003,-0.00015088,-0.00512700,-0$ $.00136369,0.00194540,0.00791039,0.00324850,-0.00157237,-0.00031862,-0$. $00001941,0.00033440,0.00016898,-0.00066036,0.00017717,0.00016567,0.000$ $12861,-0.00007594,0.00007735,0.00008650,0.00004363,-0.00010795,-0.0000$ $2064,0.00005785,-0.00743758,0.01888088,-0.01354544,-0.01374225,-0.0129$ $7871,0.01977017,-0.02490732,0.04633870,-0.06938491,0.01395216,-0.17465$ $130,0.15275673,-0.00435502,-0.00483670,0.00086846,-0.00034006,-0.00187$ $534,0.00022400,0.00047200,-0.00206335,-0.00136900,-0.00051460,0.001348$ $08,-0.00011464,0.00055277,-0.00031996,0.00043831,-0.00111388,0.0003042$ $0,0.00183790,-0.00080553,-0.00072814,0.00064518,-0.00017017,0.00010829$ $, 0.00025663,0.00018528,0.00164712,0.00020485,-0.00004769,-0.00004472,-$ $0.00012722,-0.00009610,-0.00033542,-0.00015911,-0.00012616,0.00002375$, $-0.00000137,-0.05321695,0.04868694,-0.01310588,-0.02621218,-0.03026868$ $, 0.01359241,0.04420811,0.01426540,-0.00947452,-0.09679879,-0.01341126$, $0.00985179,0.65292251,-0.00109477,0.00082720,0.00173717,0.00012856,-0$. $00061766,-0.00094603,0.00094147,0.00085330,0.00146847,-0.00057613,-0.0$ $0090339,-0.00018261,0.00010656,0.00029541,-0.00032984,0.00041409,0.000$ $17348,-0.00036960,-0.00045537,-0.00007148,-0.00003449,-0.00004665,0.00$ $006412,-0.00009881,-0.00024462,-0.00080613,-0.00024412,0.00001342,0.00$ $000269,0.00003241,-0.00003958,-0.00015878,-0.00007477,0.00013643,0.000$ $02327,-0.00003681,-0.00470533,0.05036052,-0.01608145,-0.02830182,-0.03$ $190814,0.01398096,0.04504964,-0.03902429,0.01229256,-0.04572863,-0.299$ $17190,0.09821701,-0.06823209,0.61394675,0.00137769,-0.00064404,-0.0005$ $8406,-0.00003548,0.00105992,-0.00015055,-0.00016831,-0.00036079,-0.000$ $07693,-0.00002410,-0.00029229,-0.00012224,-0.00037908,0.00051538,-0.00$ $006259,0.00032134,-0.00010193,0.00058565,-0.00013672,0.00023542,-0.000$ $11921,0.00001056,-0.00003952,0.00014499,0.00008760,0.00041874,0.000083$ $05,0.00002306,0.00004427,0.00001635,-0.00006445,0.00005073,0.00007536$, $0.00003077,0.00003803,0.00000917,0.00768658,-0.02180718,0.01477129,0.0$ $1344933,0.01411376,-0.00967346,-0.02125097,0.01549255,0.00034071,0.021$ $78543,0.09568879,-0.09339979,-0.02318126,-0.18060721,0.20020158,0.0012$ $5476,0.00014742,0.00061663,0.00049026,0.00013508,-0.00010494,-0.000229$ $96,0.00038265,-0.00005869,-0.00022783,0.00008715,0.00012779,0.00002753$ $, 0.00017524,-0.00017323,0.00052512,0.00011770,-0.00041851,0.00012210,0$ $.00011543,-0.00019322,-0.00007946,-0.00002585,0.00008280,-0.00004953,-$ $0.00023175,-0.00004164,-0.00003190,-0.00001169,-0.00001617,0.00001719$, $0.00015293,0.00012050,0.00003352,0.00001345,0.00000507,-0.20823860,0.0$ $7600784,-0.01599963,0.04552074,0.05122380,-0.02318796,-0.06112382,0.01$ $438861,0.00036579,0.00949475,-0.08154927,0.03151426,-0.29957276,-0.014$ 
$10041,0.02735470,0.63722541,-0.00007124,-0.00091790,0.00018107,0.00061$ $045,0.00014372,0.00011677,-0.00031493,-0.00024036,-0.00080137,-0.00007$ $145,0.00050730,-0.00013444,0.00017141,-0.00011345,0.00031071,-0.000135$ $59,0.00000968,0.00042608,-0.00021088,-0.00020290,0.00013195,-0.0001403$ $0,-0.00002660,0.00016165,0.00003274,0.00036312,0.00012328,-0.00003872$, $-0.00002703,-0.00003056,0.00000475,-0.00007584,-0.00000215,-0.00004704$ $, 0.00000102,0.00003271,0.13614113,-0.20812719,0.04287830,0.00915198,-0$ $.03921596,0.01725200,0.01661918,-0.00412582,-0.00094827,-0.02994633,-0$ $.00367622,0.00923128,-0.08358864,-0.12297240,0.03186175,0.00222842,0.6$ $5395486,-0.00052554,0.00006845,0.00080520,0.00011346,0.00002165,0.0000$ $3635,0.00011597,0.00021482,-0.00008800,0.00032518,-0.00015361,0.000147$ $30,-0.00001326,-0.00003702,0.00015056,0.00034615,0.00021637,0.00012321$ $,-0.00004184,-0.00001182,0.00000847,0.00001056,-0.00002624,0.00000547$, $-0.00002806,-0.00006879,0.00003310,0.00000859,-0.00000947,0.00000963,0$ $.00002735,0.00002434,-0.00000867,0.00000096,-0.00003055,0.00000202,-0$. $03909260,0.04857711,-0.07981462,-0.00711420,0.01356778,0.00367507,-0.0$ $0110046,-0.00110015,-0.00220086,0.01190633,0.01467068,0.00590188,0.054$ $54164,0.02535550,-0.07560154,-0.04862288,-0.20364026,0.21026434,0.0002$ $1176,-0.00002271,-0.00009030,-0.00027100,-0.00021055,-0.00012538,0.000$ $30006,-0.00076226,-0.00038480,-0.00016147,0.00034122,0.00017506,0.0001$ $2962,-0.00017855,0.00017682,-0.00036408,0.00010516,0.00011522,0.000147$ $75,0.00006820,-0.00007089,0.00005615,0.00004093,-0.00000149,-0.0001493$ $4,0.00071516,0.00009109,-0.00000294,-0.00000863,-0.00007088,0.00005528$ $, 0.00002852,0.00001708,-0.00002945,0.00004779,-0.00002643,-0.13679899$, $-0.02705477,0.02101580,-0.02544355,-0.00753608,0.00567381,0.00245346,-$ $0.00275131,0.00118756,0.00003055,-0.00069391,0.00043729,0.00022912,0.0$ $0623873,-0.00211653,-0.04109111,-0.01281426,0.00918593,0.20112130,0.00$ $008349,-0.00001059,-0.00008380,-0.00010150,-0.00000379,-0.00005010,0.0$ $0007807,-0.00026731,-0.00012335,-0.00003961,0.00010730,0.00004694,0.00$ $002805,-0.00004783,0.00004754,-0.00011504,0.00001479,0.00002310,0.0000$ $7316,0.00003804,-0.00005507,0.00001247,0.00000616,0.00000826,-0.000029$ $05,0.00026236,0.00002775,0.00000041,-0.00000358,-0.00001929,0.00002340$ $, 0.00000968,0.00001583,-0.00001642,0.00001329,-0.00000985,-0.02702457$, $-0.04472847,0.01082757,-0.03273046,-0.00600097,0.00703552,0.00306991,-$ $0.00313180,0.00260823,-0.00061847,0.00140797,-0.00078660,0.00022167,-0$ $.00133051,0.00223013,0.01244037,0.00655327,-0.00196038,0.04569689,0.04$ $614760,0.00000775,-0.00003835,-0.00009142,-0.00002123,0.00005720,0.000$ $00161,-0.00003150,0.00011531,0.00015155,0.00001150,-0.00010296,-0.0000$ 4917,-0.00003648,0.00007471,-0.00007593,0.00005126,-0.00004385,-0.0000 $2636,-0.00005264,-0.00000738,0.00000121,-0.00000448,-0.00000177,-0.000$ $00124,0.00003386,-0.00015029,-0.00002563,0.00000582,0.00000412,0.00001$ $423,-0.00001171,-0.00001074,-0.00000530,0.00001066,-0.00000886,0.00000$ $280,0.02096874,0.01083616,-0.02934901,0.01542966,0.00471056,0.00118556$ ,-0.00099856,0.00322653,0.00332156,0.00010238,-0.00084624,-0.00070492, $0.00031991,0.00159689,0.00375449,-0.00057116,0.00037715,0.00443169,-0$. $03520258,-0.01747889,0.02174794,0.00001060,0.00013285,-0.00016551,-0.0$ 0017856,-0.00008601,0.00005301,0.00003925,-0.00022818,-0.00021410,-0.0 $0009908,0.00019931,0.00001469,0.00010168,-0.00005473,0.00009132,-0.000$ $21108,-0.00001954,0.00009765,0.00001619,0.00002638,-0.00000176,-0.0000$ $1071,0.00001732,0.00002228,-0.00001168,0.00020560,0.00003766,-0.000012$ $61,-0.00000764,-0.00002317,-0.00000899,-0.00002811,-0.00001704,-0.0000$ 
$1070,0.00000645,-0.00000066,0.00525350,-0.00654290,0.00233008,-0.21543$ $335,0.13958456,-0.03731159,-0.01572744,0.01859616,-0.00535726,-0.00423$ $594,-0.00131493,0.00157278,-0.00001986,-0.00102894,0.00033421,0.001114$ $95,-0.00170992,0.00099869,0.00123651,-0.00079047,-0.00005946,0.2267001$ $3,-0.00023448,0.00002959,-0.00003241,0.00000900,-0.00007502,0.00001853$ $,-0.00001727,-0.00001624,-0.00008834,-0.00003312,0.00005883,0.00001092$ ,0.00004064,-0.00003179,0.00002894,-0.00006918,0.00002151,0.00004474,0 $.00002103,0.00002368,0.00001593,-0.00000510,0.00000144,0.00000526,0.00$ $000762,0.00002633,0.00001246,-0.00000137,-0.00000139,-0.00000460,-0.00$ $000814,-0.00000708,-0.00000928,-0.00000270,-0.00000132,0.00000256,0.02$ $259184,-0.01272266,0.00410897,0.13574058,-0.16817913,0.03900733,-0.008$ $28668,0.00818729,-0.00076203,-0.00165469,0.00191694,0.00189370,-0.0007$ $2878,-0.00034544,-0.00013274,-0.00232177,-0.00329493,0.00350598,0.0002$ $4289,0.00056051,-0.00128726,-0.14652727,0.17437239,0.00002368,-0.00001$ $404,0.00006406,0.00002427,-0.00003050,-0.00000245,-0.00002800,0.000033$ $70,-0.00001536,-0.00002529,0.00002955,0.00002658,0.00005322,-0.0000621$ $1,0.00002621,-0.00009100,0.00002898,-0.00005389,0.00003302,-0.00000354$ $, 0.00002432,0.00001409,-0.00000816,-0.00000888,0.00001049,-0.00003137$, $-0.00000043,-0.00000453,-0.00000219,-0.00000272,0.00000479,0.00000303$, $0.00000814,0.00000277,0.00000005,-0.00000010,-0.00891936,0.00671325,0$. $00145493,-0.03584100,0.03867722,-0.04657562,0.00511875,-0.00319924,0.0$ $0499202,0.00157859,0.00196614,0.00547423,0.00023611,-0.00023176,-0.000$ $84048,0.00128905,0.00345262,0.00381579,-0.00043896,-0.00116803,-0.0021$ $9496,0.03794756,-0.04479735,0.03743236,0.00045298,0.00051512,-0.000758$ $57,0.00006319,-0.00012170,0.00016321,-0.00007294,0.00006236,0.00007083$ $, 0.00003245,-0.00011359,-0.00001194,-0.00005729,0.00004389,-0.00006352$ $, 0.00005717,-0.00000933,0.00019318,-0.00005951,-0.00004569,0.00042928$, $0.00002209,0.00001031,-0.00004865,0.00003939,-0.00017608,0.00000321,0$. $00002791,0.00001728,0.00001451,-0.00008543,-0.00007936,-0.00012339,0.0$ $0000714,-0.00001762,0.00000498,-0.00365215,-0.00047390,0.00118201,-0.0$ $0279407,-0.02745302,0.01113006,-0.06476760,-0.04744658,0.02104114,0.00$ $807570,0.01992511,-0.00831613,-0.00277135,0.00407787,-0.00069287,-0.00$ $126726,0.00014954,-0.00007074,-0.00117140,-0.00114275,0.00056408,0.001$ $14959,0.00015387,-0.00051794,0.06636127,-0.00085318,-0.00013359,0.0001$ $9395,0.00020159,-0.00003913,-0.00031137,-0.00006628,0.00015813,0.00012$ $323,0.00008647,-0.00013864,0.00000749,-0.00013350,0.00007320,-0.000169$ $05,0.00029936,0.00019347,0.00011883,0.00063526,0.00017753,-0.00030366$, $0.00000075,-0.00003083,0.00003034,-0.00001816,-0.00002239,-0.00005022$, $-0.00000456,0.00002187,-0.00001949,0.00001927,0.00003682,0.00006147,-0$ $.00001534,-0.00000167,-0.00000388,-0.00096195,0.00270329,0.00165693,0$. $00062452,-0.00429060,0.00345958,-0.05154802,-0.27944230,0.09823912,-0$. $00204984,-0.01604999,0.00610065,0.00286446,-0.00044338,0.00235711,0.00$ $042851,0.00031629,-0.00052738,-0.00018391,-0.00026301,0.00014540,-0.00$ $012010,0.00007202,-0.00151696,0.05127595,0.29772511,0.00045213,-0.0003$ $9989,-0.00025439,0.00005982,0.00010382,0.00013883,0.00010938,0.0000556$ $9,0.00004948,-0.00004376,-0.00004027,-0.00012615,0.00002638,-0.0000269$ $1,-0.00011637,-0.00020856,0.00001115,-0.00008266,-0.00017784,0.0001394$ $9,0.00000096,-0.00000358,-0.00002643,-0.00000060,-0.00001404,-0.000158$ $41,-0.00002037,0.00000903,0.00002530,-0.00001493,-0.00016646,-0.000198$ $88,-0.00009025,-0.00000156,-0.00001791,-0.00000130,0.00136984,0.001499$ 05,0.00602064,0.00068309,0.00606271,0.00250386,0.02217405,0.09801377,-- 
$0.07433005,-0.00000789,0.00417705,-0.00145196,-0.00011267,0.00223739,0$ $.00546656,-0.00022045,-0.00039504,-0.00064611,0.00016960,0.00024717,-0$ $.00000335,-0.00041714,-0.00157310,-0.00333851,-0.02375260,-0.10960572$, $0.06703051,0.00025525,0.00024675,0.00050054,0.00016505,-0.00028527,-0$. $00004734,-0.00035040,0.00019231,0.00053305,-0.00019397,-0.00023393,-0$. 00036298,-0.00004114,0.00004267,-0.00021216,0.00013101,0.00007960,-0.0 $0021854,-0.00005563,-0.00002076,0.00000304,-0.00001248,-0.00001324,-0$. $00005610,-0.00008772,-0.00019497,-0.00003907,0.00001899,0.00003321,0.0$ $0002924,0.00000831,0.00002691,-0.00001387,0.00001224,-0.00001568,0.000$ $00834,-0.00264547,-0.00219699,0.00167291,-0.00022932,-0.00060821,0.000$ $27140,0.00076200,-0.00236182,0.00132980,0.00827682,-0.00194629,0.00004$ $417,-0.20993442,0.13309031,-0.03481814,-0.01751879,0.01925588,-0.00526$ $604,-0.00144352,0.00036052,0.00002196,-0.00006359,0.00001711,-0.000069$ $33,0.00033120,-0.00037027,0.00007632,0.22142380,0.00107940,-0.00020503$ ,-0.00046580,0.00004693,0.00036499,0.00026434,-0.00038625,0.00055805,$0.00018796,0.00001433,0.00028774,-0.00029403,-0.00003176,-0.00011735,0$ $.00004123,-0.00027504,-0.00022354,-0.00013804,0.00008535,0.00002636,-0$ $.00003815,-0.00004790,-0.00002970,0.00004101,-0.00001157,-0.00036967,0$ $.00003759,-0.00000065,-0.00000317,0.00000372,0.00000318,0.00004742,0.0$ $0002962,-0.00001597,-0.00001707,0.00003930,-0.00172140,0.00180480,0.00$ $215084,-0.00102833,-0.00045078,-0.00013855,-0.00074583,-0.00344369,0.0$ $0389630,0.01970413,-0.01636639,0.00461791,0.13632883,-0.16621333,0.037$ $40521,-0.00838671,0.00846764,-0.00064746,-0.00068579,0.00007311,0.0000$ $6412,0.00006291,-0.00006238,-0.00024737,0.00086431,-0.00049740,0.00009$ $497,-0.14514376,0.17622193,-0.00063709,0.00015718,-0.00042471,-0.00037$ $447,-0.00030683,-0.00006767,0.00014493,-0.00011448,0.00031987,-0.00006$ $456,0.00021512,0.00004331,0.00013449,-0.00013650,-0.00019503,-0.000111$ $56,-0.00003534,-0.00016468,0.00021105,0.00008575,0.00001730,0.00016022$ $, 0.00006733,-0.00025660,-0.00007408,-0.00009025,-0.00013631,-0.0000218$ $4,-0.00003594,-0.00001459,-0.00001622,-0.00001728,-0.00000892,-0.00007$ $409,-0.00002178,-0.00002781,0.00155558,0.00202939,0.00578836,0.0003611$ $9,-0.00006921,-0.00078085,0.00100426,0.00396488,0.00451938,-0.00845649$ $, 0.00642006,-0.00108656,-0.03566640,0.03732739,-0.04711618,0.00526674$, $-0.00293588,0.00572708,0.00039573,-0.00003367,0.00006609,-0.00006143$,$0.00024921,-0.00061930,-0.00037369,0.00022751,-0.00004480,0.03716206$,$0.04485634,0.03782965,-0.00011811,-0.00024935,-0.00001205,-0.00000507$, $-0.00007197,0.00001273,0.00003389,-0.00030666,-0.00021378,-0.00004181$, $0.00023416,0.00000006,0.00006978,-0.00006539,0.00007600,-0.00013110,0$. $00002200,0.00012802,-0.00005383,-0.00002695,0.00001922,-0.00001896,0.0$ $0000493,0.00003499,0.00001512,0.00028722,0.00002828,-0.00001114,-0.000$ $01022,-0.00001719,-0.00000619,-0.00002064,-0.00000671,-0.00003483,0.00$ $000153,-0.00000042,0.00634149,0.02099942,-0.00844870,-0.00307543,0.003$ $15673,-0.00044232,-0.00102756,0.00048961,-0.00019927,-0.00408999,-0.00$ $184077,0.00181431,-0.00219237,-0.02571783,0.01048863,-0.06642901,-0.05$ $522540,0.02430955,0.00100267,0.00055135,-0.00054859,0.00042509,0.00107$ $178,-0.00040729,0.00004954,-0.00010814,-0.00002950,0.00112174,0.000190$ $00,-0.00055769,0.06822758,0.00015712,0.00000144,0.00002639,-0.00000952$ ,-0.00000720,0.00000913,0.00003587,-0.00008589,-0.00008404,-0.00000932 $, 0.00010037,0.00001251,0.00003366,-0.00004758,0.00004768,-0.00009554,-$ $0.00001973,-0.00003032,-0.00003829,-0.00001466,-0.00000140,-0.00000443$ $, 0.00000479,0.00000761,-0.00000095,0.00008192,0.00000938,-0.00000515,-$ 
$0.00000734,-0.00001216,0.00000448,-0.00000364,-0.00000003,-0.00001521$, $0.00000216,-0.00000051,-0.00808153,-0.01372560,0.00725805,0.00374850,0$ $.00013810,0.00169206,0.00014650,0.00044218,-0.00058949,-0.00127483,0.0$ $0182976,0.00205096,0.00101319,-0.00266537,0.00254991,-0.05146014,-0.28$ $721886,0.10196124,-0.00044492,0.00065673,-0.00126779,-0.00032214,-0.00$ $052871,0.00039912,-0.00005372,-0.00014104,-0.00023504,-0.00002374,0.00$ $007967,-0.00159925,0.05670928,0.30114825,-0.00009073,0.00011180,0.0000$ $7366,0.00013377,-0.00001888,0.00003968,-0.00007505,0.00013301,0.000024$ $76,0.00002748,-0.00003955,0.00001683,-0.00000991,-0.00002154,-0.000000$ $60,-0.00002228,-0.00003042,-0.00005773,-0.00000057,0.00000964,-0.00001$ $097,-0.00000059,-0.00000227,-0.00000730,-0.00000878,-0.00012105,-0.000$ $01866,-0.00000435,-0.00000253,0.00000111,0.00000624,-0.00000024,-0.000$ $00743,0.00000024,0.00000405,-0.00000154,0.00275405,0.00463216,0.001243$ $49,-0.00069856,0.00171657,0.00468683,-0.00010914,-0.00056578,-0.000664$ $26,0.00163833,0.00191815,0.00561382,0.00017450,0.00505254,0.00230005,0$ $.02288126,0.10233045,-0.07679219,-0.00014480,-0.00136468,-0.00214158,0$ $.00012111,0.00027765,0.00033365,-0.00005011,-0.00022114,-0.00068483,-0$ $.00050372,-0.00161953,-0.00338721,-0.02605023,-0.11221253,0.06944634,-$ $0.00062096,-0.00050165,-0.00011615,-0.00006522,0.00074332,0.00107645,-$ $0.00090581,-0.00737223,0.01266529,0.00000335,0.00342581,0.00042598,0.0$ $0196528,-0.00035754,-0.00000995,-0.00057921,0.00042038,-0.00024392,0.0$ $0006617,0.00002943,-0.00000639,-0.00018993,0.00035747,-0.00112005,-0.0$ $4196726,0.04398264,0.04160816,-0.00016766,0.00008776,0.00008134,-0.000$ $05031,-0.00005871,-0.00006961,-0.00023138,-0.00023677,0.00034261,-0.00$ $000426,0.00000843,0.00001198,0.00000314,0.00001868,-0.00003636,-0.0001$ $4338,-0.00013079,0.00005804,0.00039018,0.00026700,0.00001977,-0.000227$ $20,0.00012073,-0.00009350,0.00001352,-0.00008006,-0.00001042,-0.000024$ $44,-0.00001897,0.00000706,-0.00002109,-0.00000560,0.00000395,0.0000159$ $8,0.00000351,0.00002758,0.00004072,0.00004996,0.00014773,-0.00003679,-$ $0.00000492,0.00002625,0.03908568,-0.00009962,-0.00054493,0.00027303,-0$ $.00282360,0.00162264,-0.00090533,0.00122215,-0.01080763,-0.03734768,0$. $00424876,-0.00401990,0.00134129,0.00008340,-0.00018884,-0.00186210,-0$. $00033368,0.00046167,0.00005253,-0.00004202,0.00002644,-0.00002535,0.00$ $087637,0.00022402,0.00090940,0.03302537,-0.04394672,-0.05797897,0.0001$ $0298,-0.00046961,-0.00002046,0.00002743,0.00002083,0.00008982,-0.00020$ $472,-0.00001485,-0.00000997,0.00006305,0.00002154,-0.00001803,-0.00001$ $707,0.00002182,-0.00000904,-0.00014182,-0.00013496,0.00003963,0.000215$ $75,0.00013851,0.00002461,-0.00022526,0.00004773,-0.00007429,0.00001798$ ,-0.00004557,0.00001819,-0.00008481,-0.00003196,0.00001686,-0.00002467 $,-0.00000161,0.00000474,0.00002687,-0.00000226,0.00000939,0.00004586,0$ $.00004424,0.00005306,-0.00003754,-0.00000864,0.00002365,-0.03184180,0$. $05726390,-0.00034626,-0.00092537,-0.00033674,-0.00129829,0.00263084,0$. $00218354,0.00194468,0.00144059,-0.00538719,0.00111787,-0.00044310,0.00$ $138249,-0.00100797,0.00003806,-0.00012549,0.00002852,0.00015701,0.0004$ $1549,-0.00003225,0.00007148,0.00000623,0.00080127,-0.00009442,-0.00029$ $250,0.01447481,-0.08667765,-0.42848994,0.00008739,-0.00008425,0.000010$ $77,0.00017552,0.00005816,-0.00006674,0.00026491,0.00029980,0.00014970$, $0.00001213,0.00001201,-0.00000686,-0.00000980,-0.00000070,-0.00000443$, $-0.00000992,-0.00001091,-0.00001957,-0.00010390,0.00005916,0.00007662$, $0.00003450,-0.00003673,-0.00000669,-0.00000627,0.00001217,-0.00000152$, $-0.00002135,-0.00000392,0.00000174,-0.00000235,0.00000283,0.00000141$, 
$0.00000134,0.00000705,0.00000621,-0.00000886,0.00000250,-0.00000483,0$. $00000305,-0.00000066,0.00000075,-0.02413959,0.08566357,0.44344802,0.00$ $197286,-0.00017293,-0.00156995,-0.00021204,0.00219693,0.00104552,-0.00$ $981732,-0.00585671,-0.00477445,0.00102726,-0.00159954,-0.00048859,-0.0$ $0019785,0.00083447,-0.00032477,0.00014202,0.00002645,0.00079810,-0.000$ $18498,0.00013524,0.00015779,-0.00044129,-0.00041985,0.00019223,-0.3107$ $3393,0.11058021,-0.18728285,-0.00000860,-0.00004629,-0.00001330,-0.000$ $10474,-0.00009913,0.00013228,-0.00002521,0.00024502,0.00087371,-0.0000$ $0253,-0.00000058,-0.00000402,0.00000548,-0.00000434,0.00000573,0.00000$ $719,-0.00003735,-0.00000501,-0.00002954,-0.00001485,0.00008354,-0.0000$ $5471,-0.00004563,0.00001151,0.00002020,0.00002928,-0.00000431,-0.00002$ $293,-0.00001245,0.00000376,-0.00000280,-0.00000023,0.00000236,0.000013$ $41,-0.00000640,-0.00000116,0.00002933,0.00005163,-0.00005437,-0.000014$ $72,-0.00000426,0.00000559,0.00365089,-0.00407939,0.00804346,0.31498455$ $, 0.00138193,-0.00079193,0.00118575,0.00169259,-0.00316073,-0.00462041$, $0.02997717,-0.01359179,0.02094544,-0.00138866,0.00155338,0.00268238,-0$ $.00038147,-0.00080972,0.00009990,-0.00031705,0.00062538,0.00007099,0.0$ $0017004,-0.00039036,-0.00012893,-0.00012757,0.00003244,0.00012017,0.07$ $972333,-0.04847081,0.01774417,0.00000588,0.00005366,0.00002489,-0.0000$ $2497,0.00002220,-0.00007157,0.00008972,0.00045593,-0.00111207,0.000080$ $12,0.00003877,-0.00001624,-0.00003016,0.00002953,-0.00001929,-0.000100$ $12,-0.00015369,0.00005480,0.00010465,0.00012781,0.00003181,-0.00015359$ $, 0.00003773,-0.00000169,0.00002501,-0.00003589,0.00001103,-0.00009302$, $-0.00003100,0.00001936,-0.00002599,-0.00000283,0.00000421,0.00001087,0$ $.00001850,0.00000065,0.00002381,0.00002354,-0.00004572,-0.00003715,-0$. $00001112,0.00001046,-0.00890607,0.00032415,-0.00217750,-0.10169930,0.0$ $6410684,-0.00078196,0.00028147,0.00070347,0.00050869,-0.00253038,0.000$ $84200,0.00690701,0.00436772,0.00580926,0.00218278,-0.00222966,0.000612$ $79,0.00012453,0.00089849,-0.00050566,0.00055142,-0.00047706,-0.0001969$ $7,0.00007920,-0.00010404,-0.00003911,0.00023582,-0.00002597,0.00024644$ $,-0.16118134,0.02526234,-0.15393573,-0.00002214,-0.00005540,0.00000316$ ,-0.00013138,0.00001771,-0.00000431,-0.00095329,-0.00017155,-0.0006137 $0,-0.00003399,-0.00002438,-0.00000433,0.00001009,0.00001196,0.00002236$ $, 0.00003166,0.00005888,-0.00001367,-0.00014514,-0.00015700,-0.00012692$ $, 0.00008587,-0.00002406,0.00000447,-0.00000986,0.00000801,0.00001635,0$ $.00005340,0.00001958,-0.00001161,0.00001043,0.00000050,-0.00000100,-0$. $00001500,0.00000020,-0.00001042,-0.00003666,-0.00005542,0.00002501,0.0$ $0001892,0.00000918,-0.00000668,-0.03065624,0.00973144,-0.01294046,0.18$ $316718,-0.03481257,0.16012528 \backslash 10.00000369,-0.00000101,-0.00000717,0.00$ $000012,-0.00000469,0.00000045,0.00000018,-0.00000109,0.00000146,0.0000$ $0002,0.00000117,0.00000048,0.00000065,0.00000260,-0.00000226,-0.000002$ $09,-0.00000019,-0.00000232,0.00000108,-0.00000419,0.00000002,-0.000000$ $18,-0.00000521,0.00000130,-0.00000069,-0.00000260,0.00000443,0.0000005$ $8,0.00000441,-0.00000298,0.00000089,0.00000134,-0.00000542,-0.00000035$ ,0.00000264,0.00000186,-0.00000038,0.00000188,0.00000060,0.00000017,0. $00000068,-0.00000359,-0.00000077,-0.00000085,-0.00000304,-0.00000233,0$ $.00000203,-0.00000025,0.00000160,0.00000166,0.00000408,-0.00000031,0.0$ $0000147,0.00000194,-0.00000024,0.00000328,0.00000163,0.00000070,0.0000$ $0042,-0.00000373,0.00000092,-0.00000152,-0.00000508,-0.00000058,0.0000$ $0062,0.00000277,-0.00000084,0.00000283,0.00000453,-0.00000071,-0.00000$ 429,0.00000501,-0.00000115,-0.00000140,0.00000528III@ 
para-Transition State

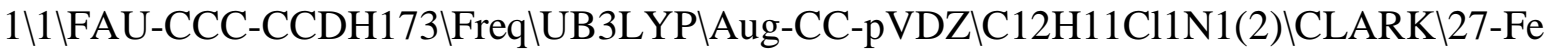
b-2015 \0\\NN Geom=AllCheck Guess=TCheck SCRF=Check GenChk UB3LYP/Aug-C C-pVDZ Freq \\Addition product p-Cl-Ph-radical + aniline (ortho) $\backslash 10,2 \backslash \mathrm{C}$ ,-1.6910407485,1.5363840465,0.0484037224\C,-2.2235406663,0.9815353136, $1.2427966781 \backslash \mathrm{C},-3.1007458185,-0.092252684,1.2040874034 \backslash \mathrm{C},-3.5414712928$ ,-0.6188003856,-0.0299375234\C,-3.0882751459,-0.0147256579,-1.22326023 $23 \backslash \mathrm{C},-2.2110690514,1.0590525704,-1.1843653777 \backslash \mathrm{H},-1.2008669316,2.506519$ $6454,0.0819060121 \backslash \mathrm{H},-1.9125657837,1.384647296,2.2065177509 \backslash \mathrm{H},-3.469240$ $0794,-0.5273406078,2.1348941302 \backslash \mathrm{H},-3.4470532185,-0.3894108242,-2.18366$ $62965 \backslash \mathrm{H},-1.8903443134,1.522731592,-2.1171570366 \mathrm{lN},-4.3697419927,-1.738$ 861964,-0.0699668304\C,2.9899296204,-0.2705098057,0.0143362323\C,2.806 $156427,1.1123928314,0.0574613229 \backslash \mathrm{C}, 1.4964691933,1.6209041187,0.0670995$ 704\C,0.4306401101,0.7347768732,0.0335451069\C,0.6071358665,-0.6395185 004,-0.0093468951\C,1.9110943663,-1.1580696365,-0.0193342822\Cl,4.6353 249447,-0.9178795191,0.001954958\H,3.6676851014,1.7789151045,0.0830046 $657 \backslash \mathrm{H}, 1.3444505737,2.7020189176,0.1007737827 \backslash \mathrm{H},-0.2467387605,-1.318777$ $1575,-0.0352593194 \backslash \mathrm{H}, 2.0882505202,-2.232691347,-0.052675452 \backslash \mathrm{H},-4.90825$ $66641,-1.9297220038,0.7644627156 \mathrm{lH},-4.8995922563,-1.8759152161,-0.9203$ $628061 \|$ Version=EM64L-G09RevC.01 $\backslash$ State $=2-A \backslash H F=-978.8436936 \backslash S 2=0.777263$ $\backslash \mathrm{S} 2-1=0 . \backslash \mathrm{S} 2 \mathrm{~A}=0.750513 \backslash \mathrm{RMSD}=1.642 \mathrm{e}-09 \backslash \mathrm{RMSF}=1.302 \mathrm{e}-06 \backslash \mathrm{ZeroPoint}=0.194207$ $2 \backslash$ Thermal $=0.2067971 \mathrm{ZPE}=121.8668473 \backslash$ Dipole $=-1.7932646,0.0130573,-0.008$ 7215 DipoleDeriv $=-0.1042143,-0.0557557,-0.0012737,-0.0582141,0.0262987$ $, 0.0070293,-0.001361,0.0070265,-0.2027729,0.1048965,0.1952633,-0.04060$ $81,0.3462782,-0.0014447,-0.128298,-0.0809108,0.0610103,0.111465,-0.354$ $1559,-0.0682762,0.0423355,-0.2164831,-0.2235932,0.0480869,-0.4078023,-$ $0.2529947,0.0808094,0.8234956,0.6564149,0.0256079,1.108469,0.9370523,0$ $.0382556,0.0401123,0.035954,-0.0818845,-0.3577471,-0.0677922,-0.051223$ $3,-0.2412708,-0.2353154,-0.0707363,0.3885753,0.2319631,0.0961065,0.103$ $49,0.1929424,0.0528807,0.339091,-0.0054295,0.1242761,0.1027093,-0.0665$ $121,0.1168647,0.1572157,-0.0849515,-0.0021728,-0.0996139,-0.0346308,-0$ $.0032597,-0.0026399,-0.0031798,0.0513468,0.0847587,-0.0563477,-0.04873$ $57,-0.0322768,0.0704257,-0.0390636,-0.0267826,-0.0497896,-0.0484389,0$. $064459,-0.0650624,0.0526527,-0.0899932,0.0535597,0.0690569,0.0703081,0$ $.0564238,-0.0473815,0.0657597,-0.0610608,-0.055477,-0.0846816,0.061186$ $9,-0.0629685,-0.074685,-0.050089,-0.0563081,0.0839963,-0.059933,0.0463$ $546,-0.0343989,0.0643149,0.0459005,0.0259869,0.0563716,-0.0415614,-0.7$ $356085,-0.2420591,-0.010951,-1.1235407,-1.220893,-0.0413137,-0.0391183$ $,-0.0368103,-0.1085935,1.1311192,-0.3750764,-0.0060051,-0.4686923,0.14$ $59589,0.0039945,-0.008998,0.0044704,-0.0532599,-0.2265965,0.1239548,0$. $0032831,-0.0022497,-0.057326,0.0011938,-0.0007181,0.0018352,-0.095384$, $-0.3059634,-0.0147223,-0.0015147,0.4603655,0.1550804,0.0105414,0.01364$ $21,0.0081298,-0.1020803,0.8251336,-0.012176,0.0041266,-0.3602002,-0.11$ $02514,-0.0033585,-0.0070448,-0.0015937,-0.0625813,-0.4987458,0.0375049$ ,-0.000924,-0.0543508,0.0042159,0.0025192,-0.0038104,0.0029847,-0.0834 $101,-0.1655862,0.0213356,0.0002847,0.1953452,0.0542747,0.0054684,0.005$ $8337,0.004586,-0.0861582,-0.8752017,0.2278266,0.0032603,0.3066603,-0.1$ $866014,-0.0018628,0.0057832,-0.0022627,-0.0797835,-0.0896088,-0.074178$ $2,-0.0034546,-0.0360182,0.0390111,-0.0029893,-0.0022331,-0.0031813,0.1$ 
271289,0.0673888,0.0569114,0.0015557,-0.0279096,-0.1115799,-0.0074944, $-0.0011523,-0.007067,0.1189502,0.03105,-0.077482,-0.0028095,-0.0371673$ $, 0.0262425,-0.0025495,-0.0015347,-0.0027542,0.100333,0.003127,0.031359$ $3,0.0003818,0.0620212,-0.0416848,-0.0050046,0.0013631,-0.0051624,0.125$ $1376,0.0838522,-0.1145276,0.0117931,0.0757692,0.2994725,-0.059499,-0.0$ 295223,-0.0511158,0.1067398,0.0836861,-0.1141123,-0.0193673,0.0730616, $0.2916561,0.0720754,0.0339995,0.0617573,0.1147161 \mathrm{Volar}=270.9735055,19$ $.9866004,164.7849031,1.3494468,1.2069707,130.1805478 \backslash \mathrm{PG}=\mathrm{C} 01$ [X(C12H11C $11 \mathrm{~N} 1)] \backslash \mathrm{NImag}=1 \| 0.18323495,0.28378514,0.54509121,0.00691603,-0.0003753$ $2,0.60241073,-0.07587888,-0.05840609,0.04853212,0.35905304,-0.03370870$ $,-0.12909487,0.03608479,0.27070232,0.45932254,0.04027268,0.08225440,-0$ $.25058799,0.02146469,0.04654922,0.67961699,-0.01893960,-0.01812178,0.0$ $3658197,-0.17680329,-0.14838213,-0.02183464,0.35749260,-0.03161892,-0$. $01204499,0.04660001,-0.14988341,-0.23498142,-0.02895541,0.28769052,0.4$ $6805191,0.00811907,0.02243600,0.02928174,0.01837327,0.02044342,-0.1220$ $5947,-0.02949923,-0.03239879,0.67889050,0.00440503,0.00390811,0.000462$ $15,-0.01135588,-0.01610841,-0.01829657,-0.09935252,-0.04074265,-0.0629$ $1832,0.36855504,0.01506169,-0.00062381,0.00201475,-0.01732304,-0.01576$ $572,-0.02473985,-0.04233707,-0.11419494,-0.07340910,0.26482738,0.47600$ $911,0.00081700,0.00195792,-0.06122619,-0.04968140,-0.06421855,0.027146$ $25,-0.01488096,-0.01710689,-0.26038813,0.00697790,-0.00441298,0.656708$ $52,-0.01846077,-0.01549143,-0.03812149,-0.01541337,-0.01863701,0.00981$ $201,0.02476337,0.02277317,0.01211830,-0.10014055,-0.04404981,0.0137371$ $1,0.35673595,-0.03053082,-0.00746474,-0.04925179,-0.01583612,-0.026330$ $67,0.01305960,0.02333924,0.03409514,0.01607165,-0.04494605,-0.12052514$ $, 0.02558315,0.28505208,0.46459846,-0.01056989,-0.02494381,0.02422156,0$ $.01105930,0.01715722,-0.01111831,-0.00988916,-0.01053602,-0.04876629,0$ $.06179641,0.08188181,-0.25326722,0.04446296,0.02162148,0.68310400,-0.0$ $7494951,-0.05444365,-0.05029739,0.01727733,0.01853664,0.00079410,-0.01$ $518697,-0.01495676,-0.01207400,-0.01203877,-0.02068278,0.04803583,-0.1$ $7673789,-0.14864101,-0.02847150,0.35935396,-0.03079810,-0.12202501,-0$. $02816806,0.01860819,0.03246895,-0.00494440,-0.01779063,-0.02433423,-0$. 01818266,-0.01787286,-0.02124359,0.06092836,-0.14920220,-0.23496768,-0 $.02919388,0.27216035,0.46597045,-0.04048922,-0.07458352,-0.25858148,0$. $00099143,0.00982045,-0.04079515,-0.01100746,-0.01405666,-0.01334182,0$. $01677955,0.02142805,0.03330736,0.01176728,0.02020135,-0.12214418,-0.00$ $738868,-0.05740587,0.67267071,-0.07726501,-0.12569452,-0.00412864,-0.0$ $0904451,-0.01271707,0.00311377,0.00507514,-0.00001878,0.00392940,-0.00$ $103995,-0.00129155,-0.00004099,0.00512621,0.00024010,-0.00382427,-0.00$ $887963,-0.01223563,-0.00404398,0.10257884,-0.13986237,-0.27480918,-0.0$ $0773176,-0.00146209,-0.00419974,-0.00513337,-0.00190593,0.00068478,0.0$ $0146715,0.00092354,0.00066114,0.00005915,-0.00182353,0.00083623,-0.001$ $15228,-0.00071067,-0.00293886,0.00463767,0.13510493,0.28735007,-0.0045$ $8009,-0.00765951,-0.05546177,0.01243586,0.02429123,0.00471727,0.001070$ $31,0.00122897,-0.00435821,0.00002954,0.00004792,-0.00104635,-0.0010911$ $6,-0.00089398,-0.00456086,-0.01263833,-0.02490400,0.00329100,0.0045621$ $0,0.00813570,0.05463159,0.00607927,0.00454056,0.01197757,-0.06664350,-$ $0.04031795,-0.07173080,-0.00157733,-0.00708352,-0.01777256,0.00260489$, $-0.00624146,0.00104041,-0.00084709,0.00012968,0.00019446,0.00242562,-0$ $.00342381,0.00194566,-0.00168721,0.00101290,-0.00024039,0.06189961,0.0$ $0410334,0.00774482,0.01072739,-0.04103995,-0.08691524,-0.09096607,-0.0$ 
$0636566,-0.00425102,-0.02198290,-0.00627745,-0.00019032,0.00080567,0.0$ $0021722,-0.00081816,0.00054176,-0.00343575,0.00175561,0.00265014,0.001$ $90134,-0.00030709,-0.00050005,0.04846888,0.08451625,-0.00911297,-0.011$ $20479,-0.01746048,-0.07015275,-0.09332649,-0.27755082,0.00136987,0.001$ $84905,0.00216211,0.00074159,0.00041815,0.00223242,0.00049410,0.0007631$ $7,0.00023094,0.00289209,0.00266412,-0.00312872,-0.00010176,0.00018962$, $0.00098177,0.07520831,0.09916852,0.29246888,0.00222634,-0.00675055,-0$. $00168276,-0.00375473,-0.00709053,0.01803597,-0.07316636,-0.04755762,0$. $07902495,0.00536144,0.00231398,-0.00882325,0.00280274,-0.00300723,-0.0$ $0186226,-0.00054394,0.00042367,-0.00007238,-0.00013046,-0.00047952,0.0$ $0119627,-0.00236382,0.00282940,-0.00022949,0.07037117,-0.00608582,-0.0$ $0007083,-0.00093975,-0.00683773,-0.00510929,0.02189767,-0.04850035,-0$. $09041151,0.09185679,0.00268523,0.00655144,-0.00954264,-0.00302105,0.00$ $192967,-0.00264993,0.00000400,-0.00100030,-0.00044358,-0.00057685,-0.0$ $0018138,0.00074305,0.00263196,-0.00114840,-0.00008343,0.05630086,0.089$ $38946,-0.00079641,-0.00058664,0.00209206,-0.00171477,-0.00157741,0.003$ $52514,0.07863956,0.09197671,-0.25941550,0.01186384,0.01411448,-0.01825$ $491,-0.00255562,-0.00320249,-0.00304509,-0.00061462,-0.00076608,0.0001$ $9365,0.00000401,-0.00018050,0.00002060,0.00009713,0.00012442,0.0009015$ $3,-0.08495778,-0.10031489,0.27353267,0.00220490,-0.00684917,0.00124911$ $,-0.00055105,0.00041099,0.00009093,0.00275877,-0.00315597,0.00172315,0$ $.00538846,0.00187510,0.00920105,-0.07153685,-0.04159230,-0.07988302,-0$ $.00358187,-0.00593627,-0.01851826,-0.00011775,-0.00040395,-0.00122573$, $-0.00041025,0.00040916,-0.00009063,0.00016546,0.00023884,0.00042232,0$. $06861224,-0.00613273,-0.00015490,0.00073262,-0.00003917,-0.00107257,0$. $00036222,-0.00320838,0.00153870,0.00290953,0.00332265,0.00673975,0.011$ $17283,-0.04255870,-0.07936472,-0.08076038,-0.00670342,-0.00377617,-0.0$ $2242887,-0.00056768,-0.00014437,-0.00075906,0.00041509,-0.00031682,-0$. $00003011,0.00025867,0.00034396,0.00065234,0.04986393,0.07732983,0.0004$ $0687,0.00037229,0.00219742,0.00060546,0.00068917,0.00027304,0.00241435$ $, 0.00346245,-0.00261025,-0.01142773,-0.01249374,-0.01847042,-0.0795588$ $3,-0.08087045,-0.27209188,0.00121602,0.00104900,0.00201897,-0.00004181$ $, 0.00016581,-0.00002904,-0.00001925,-0.00010189,-0.00003328,-0.0003937$ $0,-0.00055923,-0.00107209,0.08617896,0.08825923,0.28735147,0.00610301$, $0.00516444,-0.01142585,0.00247697,-0.00326865,-0.00210013,-0.00084003$, $0.00015024,-0.00019611,0.00262726,-0.00615709,-0.00143190,-0.00174068$, $-0.00818012,0.01723847,-0.06809633,-0.04589607,0.07106890,-0.00169126$, $0.00099861,0.00027702,0.00036941,0.00023244,-0.00051555,-0.00041163,0$. $00041291,0.00004177,-0.00236666,0.00263360,0.00003750,0.06343690,0.003$ $60759,0.00760886,-0.00908411,-0.00322038,0.00207620,-0.00234992,0.0002$ $5453,-0.00073088,-0.00060057,-0.00620821,-0.00009835,-0.00101834,-0.00$ $648705,-0.00550222,0.02142144,-0.04649162,-0.09939680,0.10185991,0.001$ $88695,-0.00032252,0.00043611,0.00020461,0.00016614,-0.00054424,0.00040$ $171,-0.00032435,0.00008741,0.00281192,-0.00113927,-0.00022597,0.054323$ $85,0.09795722,0.00960093,0.01285837,-0.01734859,-0.00304531,-0.0023641$ $1,-0.00350067,-0.00048965,-0.00082302,0.00013665,-0.00113579,-0.000630$ $37,0.00211820,-0.00182708,-0.00242292,0.00357686,0.06944867,0.10422730$ ,-0.26361732,0.00019519,-0.00026281,0.00100143,0.00054352,0.00062067,$0.00099400,0.00011260,0.00001572,-0.00002441,0.00037606,-0.00002004,0$. $00089517,-0.07421321,-0.11102701,0.27749112,-0.00280829,-0.00056512,-0$ $.00006088,0.00428106,-0.00014915,-0.00085829,-0.00933510,-0.02160349,-$ 
$0.00570907,-0.14416139,-0.15417728,-0.00505856,-0.00967221,-0.02230262$ $, 0.00419970,0.00431492,-0.00016515,0.00097710,0.00016059,0.00003032,-0$ $.00000020,-0.00011718,-0.00042566,-0.00002898,-0.00046701,0.00233652,0$ $.00075723,-0.00047039,0.00238007,-0.00062931,-0.00012833,-0.00043861,0$ $.00000034,0.46654786,-0.00431946,-0.00391579,-0.00032368,0.00219153,0$. $00539462,0.00124687,-0.02093623,-0.01981087,-0.01266312,-0.11209071,-0$ $.19765653,-0.00312080,-0.02283650,-0.02294499,0.01101420,0.00246904,0$. $00570969,-0.00034180,0.00128067,-0.00026643,-0.00001441,-0.00011122,0$. $00003523,-0.00002713,0.00244966,-0.00005903,0.00072949,0.00239229,-0.0$ $0001406,-0.00081829,-0.00017979,-0.00004132,0.00002681,0.29205692,0.29$ $266981,-0.00018059,-0.00030408,0.00550314,0.00434520,0.00456092,-0.008$ $04984,-0.02857916,-0.03798473,-0.00084592,-0.00371449,-0.00333687,-0.1$ $1812200,0.02707387,0.03633470,0.00262431,-0.00406744,-0.00368164,-0.00$ $839804,0.00003969,-0.00002085,0.00039125,-0.00107447,-0.00117152,-0.00$ $006522,-0.00095589,-0.00011597,0.00176266,0.00108781,0.00002658,0.0017$ $2109,0.00106413,0.00116825,0.00002246,0.00766628,-0.01436707,0.7891472$ $4,-0.00003877,0.00002530,0.00000070,-0.00027621,-0.00042386,0.00022966$ $, 0.00023979,0.00037000,0.00042287,-0.00048790,-0.00084099,-0.00002968$, $0.00024402,0.00039618,-0.00039535,-0.00027378,-0.00040797,-0.00025916$, $-0.00028431,0.00008637,0.00000084,-0.00005473,-0.00001432,0.00001299,0$ $.00001665,0.00005834,-0.00004119,0.00001616,0.00005555,0.00004494,-0.0$ $0005441,-0.00001380,-0.00001421,0.00042825,0.00105896,0.00003601,0.512$ $22142,-0.00012839,-0.00044883,-0.00001460,-0.00010792,0.00005745,-0.00$ $012824,-0.00022122,0.00008065,-0.00011845,0.00033619,0.00052652,0.0000$ $1699,-0.00022205,0.00007375,0.00012446,-0.00010945,0.00005041,0.000129$ $56,-0.00014434,0.00023086,0.00000363,0.00001366,-0.00005170,0.00003666$ $, 0.00001621,-0.00002337,0.00002125,0.00001626,-0.00002219,-0.00002280$, $0.00001524,-0.00005148,-0.00003810,-0.00017382,-0.00047323,-0.00001614$ $, 0.05373340,0.63167959,-0.00000425,-0.00001379,-0.00001310,-0.00000816$ $, 0.00001757,0.00001531,0.00000288,0.00001873,-0.00005366,0.00000802,0$. $00001108,0.00004665,-0.00001409,-0.00000659,-0.00005094,-0.00000157,-0$ $.00001944,0.00001994,-0.00000649,0.00000475,0.00009523,0.00001914,-0.0$ 0003506,-0.00002790,-0.00000250,-0.00000405,0.00000461,0.00000366,0.00 $000287,0.00000389,-0.00001853,0.00003344,-0.00002839,-0.00000342,-0.00$ $000992,0.00000406,0.00364665,0.01638408,0.12625774,0.00062682,-0.00122$ $145,-0.00004083,-0.00011591,0.00024605,-0.00014837,-0.00023692,0.00002$ $518,-0.00019940,0.00035238,0.00051039,0.00001936,-0.00023784,0.0000145$ $7,0.00019600,-0.00012369,0.00023470,0.00016289,0.00006068,0.00046651,0$ $.00001536,-0.00004258,-0.00002936,0.00007851,0.00002194,-0.00001338,0$. $00000386,0.00002227,-0.00001334,-0.00000455,-0.00004170,-0.00002419,-0$ $.00008103,-0.00020302,-0.00054388,-0.00001839,-0.10653815,-0.01902117$, $-0.00081400,0.69406794,-0.00113956,-0.00037603,-0.00004978,-0.00001908$ $, 0.00074898,-0.00015315,-0.00004742,-0.00040469,-0.00044885,0.00024312$ $, 0.00046391,0.00002451,-0.00004376,-0.00041911,0.00040791,-0.00006120$, $0.00072865,0.00020192,-0.00014179,-0.00016816,-0.00000514,-0.00002540$, $0.00002676,0.00007681,-0.00002458,0.00000151,0.00001130,-0.00002269,0$. $00000087,-0.00001191,-0.00002363,0.00003196,-0.00007702,-0.00030053,-0$ $.00084856,-0.00002847,0.04552546,-0.32698528,-0.00811701,0.04991145,0$. $67750775,-0.00003810,-0.00005009,0.00100208,-0.00063237,-0.00011253,-0$ $.00004504,0.00011979,0.00018053,0.00021215,0.00001086,0.00002547,-0.00$ $025722,-0.00012736,-0.00022052,0.00022735,0.00062896,0.00016387,-0.000$ 
$01698,-0.00000406,-0.00000208,-0.00002784,0.00001369,0.00000441,0.0000$ $3213,0.00002731,-0.00002186,0.00000628,-0.00002866,0.00002142,0.000006$ $60,-0.00001590,-0.00000471,0.00002596,-0.00001055,-0.00002948,-0.00000$ $742,0.00124194,-0.00844022,-0.06523956,0.00442594,0.01776360,0.1282151$ $0,-0.00530932,-0.00245017,-0.00010610,-0.00023280,0.00174286,-0.001210$ $65,-0.00038946,-0.00103262,-0.00093079,0.00071599,0.00146614,0.0000544$ $2,-0.00039539,-0.00108405,0.00085600,-0.00024838,0.00165768,0.00131757$ $, 0.00094867,-0.00067303,-0.00001636,-0.00010568,0.00015239,0.00023097$, $-0.00004762,-0.00010967,0.00006706,-0.00004694,-0.00010522,-0.00007422$ ,-0.00010392,0.00016649,-0.00022164,-0.00069748,-0.00209132,-0.0000710 $4,0.01334898,0.08792023,0.00282969,-0.28974029,0.03173432,-0.00012858$, $0.60715802,0.00338193,0.00194481,0.00007003,0.00005605,-0.00205065,0.0$ $0049199,0.00068500,0.00107530,0.00129961,-0.00130508,-0.00218909,-0.00$ $005434,0.00071631,0.00119039,-0.00123858,0.00004490,-0.00204191,-0.000$ $61723,0.00108604,0.00068628,0.00002906,0.00003780,-0.00000451,-0.00014$ $639,0.00003907,0.00006125,-0.00008949,0.00003800,0.00005477,0.00009483$ $, 0.00003230,-0.00001616,0.00014763,0.00105051,0.00314991,0.00010204,0$. $02988719,-0.01021661,-0.00041425,0.10592428,-0.14205704,-0.00201096,0$. $00433501,0.71774843,0.00007971,0.00004053,0.00028855,-0.00026499,-0.00$ $047753,-0.00008387,0.00030727,0.00055935,0.00034908,-0.00003443,-0.000$ $03968,-0.00070842,-0.00026957,-0.00051668,0.00023970,0.00026695,0.0003$ $6927,-0.00007669,0.00003956,0.00002034,-0.00005755,-0.00006270,-0.0000$ $3492,-0.00002810,-0.00000081,-0.00000698,-0.00002205,0.00000297,0.0000$ $1081,-0.00001629,0.00006400,0.00003741,-0.00001798,0.00002935,0.000085$ $80,0.00012838,0.00098121,-0.00012380,0.00748688,0.00223465,-0.00238266$ $,-0.06232816,0.00253009,0.01876564,0.12985788,0.07223610,-0.01079143,0$ $.00005179,-0.02612134,-0.01349210,0.02563130,0.00710719,0.01136893,0.0$ $0571768,-0.00473514,-0.00839583,-0.00031488,0.00714304,0.01167284,-0.0$ $0488517,-0.02577796,-0.01180044,-0.02669428,-0.01314854,0.00732955,0.0$ $0016534,0.00070970,-0.00065780,-0.00106449,0.00051462,0.00044742,-0.00$ $004617,0.00051203,0.00044385,0.00008006,0.00069654,-0.00072190,0.00102$ $503,0.00160040,0.00478890,0.00016283,-0.01280320,-0.02402808,-0.000792$ $33,-0.05674113,0.00663859,-0.00014448,-0.22809423,-0.13847561,-0.00528$ $176,0.34666928,-0.02818964,-0.01049747,-0.00029818,0.00409080,0.006580$ $70,-0.00783829,-0.00254066,-0.00381545,-0.00196179,0.00182817,0.003248$ $00,-0.00003166,-0.00267811,-0.00416202,0.00183414,0.00450682,0.0061914$ $2,0.00836684,0.01223669,-0.00727372,-0.00017807,-0.00016207,0.00027482$ $, 0.00029214,-0.00002038,-0.00026709,0.00001462,-0.00003362,-0.00026190$ $,-0.00003089,-0.00017530,0.00031062,-0.00029298,-0.00072004,-0.0020688$ $8,-0.00005927,-0.02422818,-0.06539591,-0.00196820,-0.04849243,0.056884$ $72,0.00111106,-0.10485671,-0.20451718,-0.00532022,0.11598803,0.6087618$ $7,-0.00050917,-0.00020993,-0.00562305,0.00780883,0.00219250,-0.0012197$ $8,-0.00188022,-0.00351487,-0.00256355,0.00001146,-0.00008328,0.0045292$ $5,0.00180948,0.00352968,-0.00225134,-0.00778495,-0.00183457,-0.0011745$ $9,0.00032354,-0.00020364,0.00025666,-0.00025628,0.00026363,0.00028939$, $-0.00020788,0.00005171,-0.00001358,0.00021153,-0.00006317,-0.00001590$, $0.00024995,-0.00026974,0.00026691,-0.00001327,-0.00003149,-0.00033025$, $-0.00079886,-0.00196720,-0.00747437,-0.00190034,0.00138710,0.01441807$, $-0.00421082,-0.00548918,-0.05413291,0.00500982,0.01729166,0.08466728,-$ $0.00522888,0.00462764,0.00012241,0.00080969,0.00254315,-0.00193492,-0$. $00081631,-0.00138105,-0.00130084,0.00151581,0.00260485,0.00009498,-0.0$ 
$0082505,-0.00145942,0.00119942,0.00078678,0.00241017,0.00209937,-0.000$ $87531,0.00109547,0.00002989,-0.00007761,0.00001005,0.00026536,-0.00014$ $148,-0.00006945,0.00012139,-0.00014067,-0.00006114,-0.00012798,-0.0000$ $7483,0.00002684,-0.00026554,-0.00115694,-0.00371847,-0.00012519,-0.055$ $62570,-0.04459047,-0.00173721,-0.02302778,0.03243835,0.00093557,0.0397$ $7403,-0.02181814,-0.00051772,-0.09228910,0.00502141,-0.00002507,0.6612$ $8061,0.00126587,-0.00103470,-0.00001537,0.00003037,0.00092711,0.000441$ $28,-0.00071401,-0.00056164,-0.00026446,0.00102713,0.00032630,0.0000384$ $1,-0.00069136,-0.00057101,0.00020896,0.00002531,0.00092805,-0.00039528$ $, 0.00052298,-0.00014111,-0.00000590,0.00009231,-0.00003427,-0.00011469$ ,-0.00005569,0.00000289,0.00001004,-0.00005889,0.00000599,-0.00001655, $0.00009125,-0.00004197,0.00011010,-0.00045164,-0.00096047,-0.00003578$, $0.01298464,0.06060000,0.00175026,0.03049821,-0.04188651,-0.00106845,-0$ $.05414595,-0.03886700,-0.00164868,0.03792901,-0.33792128,-0.00881149,0$ $.05519221,0.67147520,0.00001615,0.00000177,-0.00036171,-0.00014607,-0$. $00036890,0.00020501,0.00036781,0.00005804,0.00018146,0.00004387,0.0000$ 4626,-0.00071290,-0.00042286,-0.00012051,0.00019936,0.00015382,0.00043 $907,0.00022585,0.00001156,-0.00000303,0.00012676,0.00000450,-0.0000106$ 7,0.00004694,-0.00005589,0.00003196,0.00009936,0.00004984,-0.00003861, $0.00009557,-0.00000002,0.00000532,0.00005198,-0.00002063,-0.00005198,0$ $.00008581,0.00009669,0.00146233,0.00773525,0.00087404,-0.00105877,-0.0$ $0357872,-0.00154729,-0.00148669,0.00432722,0.00102286,-0.00897679,-0.0$ $5559657,0.00441825,0.01752222,0.13062111,0.00152719,-0.00005007,0.0000$ $0278,0.00040266,-0.00027936,0.00010501,0.00003705,-0.00021726,-0.00001$ $072,-0.00032967,-0.00011928,-0.00000381,0.00003750,-0.00021807,-0.0000$ $0467,0.00040444,-0.00027421,-0.00011866,0.00025066,-0.00027475,-0.0000$ $0746,0.00000449,0.00009277,-0.00004705,-0.00001713,-0.00001892,-0.0000$ 0296,-0.00001705,-0.00001909,0.00000177,0.00000378,0.00009034,0.000052 $97,0.00009323,0.00021173,0.00000701,-0.22033450,-0.07658526,-0.0032164$ $5,0.04181141,-0.06077758,-0.00176841,-0.06279925,-0.00969504,-0.000606$ $32,0.01559764,0.08546082,0.00272898,-0.29466388,0.03194164,-0.00014938$ $, 0.63853179,0.00025356,-0.00074417,-0.00004430,-0.00033681,-0.00064332$ $, 0.00031344,0.00024232,0.00062040,0.00041697,-0.00019614,-0.00075745,-$ $0.00001515,0.00024834,0.00064294,-0.00038582,-0.00033031,-0.00063438$,$0.00035769,0.00017349,-0.00017566,-0.00000423,0.00003369,-0.00003369$, $0.00007368,0.00007737,-0.00000347,-0.00003128,0.00007781,-0.00000579,0$ $.00003306,0.00003160,-0.00003372,0.00007165,0.00037270,0.00114158,0.00$ $003695,-0.14124211,-0.21020125,-0.00532876,-0.01579735,-0.03981027,-0$. $00161372,-0.01261916,-0.00216880,-0.00002535,0.03063533,-0.01628896,-0$ $.00081827,0.10629845,-0.14297217,-0.00205606,-0.01502261,0.73389863,0$. $00001242,-0.00004579,0.00068851,0.00003506,-0.00021860,0.00000353,0.00$ $003997,-0.00003153,0.00019786,-0.00000627,-0.00001454,-0.00032102,-0.0$ $0002568,0.00005780,0.00017476,-0.00005241,0.00017511,-0.00000687,0.000$ $00678,-0.00000646,-0.00001545,-0.00001950,0.00007153,0.00000343,0.0000$ $1462,-0.00000376,-0.00002108,-0.00000963,0.00000457,-0.00001886,0.0000$ $2161,-0.00007301,0.00000411,0.00001214,0.00003605,0.00004643,-0.005275$ $76,-0.00500519,-0.06528381,-0.00033586,-0.00183884,0.00831215,-0.00069$ $960,-0.00001060,-0.00335872,0.00098260,-0.00054393,0.01419569,0.002219$ $34,-0.00242854,-0.06177057,0.00207927,0.01923448,0.12827227,-0.0000813$ $0,-0.00011111,-0.00000316,0.00010258,0.00037099,-0.00031412,-0.0002749$ $9,-0.00024812,-0.00039143,0.00063044,0.00092112,0.00003259,-0.00027901$ 
,-0.00027218,0.00037206,0.00009930,0.00035011,0.00033812,0.00022501,-0 $.00009039,-0.00000171,0.00004969,0.00000074,-0.00001486,-0.00001883,-0$ $.00006568,0.00004121,-0.00001842,-0.00006289,-0.00004552,0.00004955,-0$ $.00000023,0.00001538,-0.00043053,-0.00113288,-0.00003841,-0.13331751,0$ $.03708777,0.00063009,-0.02341487,0.00983253,0.00017390,0.00256102,0.00$ $278247,0.00007860,0.00019177,0.00092120,0.00003581,-0.00013254,-0.0069$ $0721,-0.00024278,-0.04110517,0.01802915,0.00034586,0.19560975,0.000039$ $67,0.00006749,0.00000746,-0.00002695,-0.00015470,0.00012374,0.00012277$ $, 0.00012717,0.00015368,-0.00026106,-0.00040235,-0.00001512,0.00012341$, $0.00013824,-0.00014310,-0.00002528,-0.00014645,-0.00013395,-0.00013589$ $, 0.00007397,0.00000196,-0.00002217,-0.00000524,0.00000551,0.00001473,0$ $.00002592,-0.00002127,0.00001427,0.00002463,0.00002299,-0.00002201,-0$. $00000491,-0.00000630,0.00017315,0.00050142,0.00001683,0.03706709,-0.05$ $362695,-0.00078240,0.03710368,-0.01143082,-0.00031856,-0.00372132,-0.0$ $0427752,-0.00030016,0.00066392,0.00164626,0.00009070,-0.00013148,-0.00$ $156834,-0.00019099,-0.00923106,0.00645061,0.00001540,-0.06277559,0.060$ $93854,0.00000167,0.00000671,-0.00016041,0.00000521,-0.00000317,0.00000$ $737,-0.00001113,0.00002878,-0.00000933,-0.00000531,-0.00000910,0.00002$ $814,0.00001635,-0.00002213,-0.00001645,-0.00000594,-0.00000311,0.00000$ $237,-0.00000316,0.00000218,-0.00000878,0.00000111,-0.00000097,0.000003$ $82,-0.00000330,0.00000242,0.00000020,0.00000403,-0.00000149,0.00000109$ ,-0.00000206,0.00000041,0.00000362,0.00000335,0.00001022,0.00000265,0. $00062937,-0.00078232,-0.02325746,0.00104246,-0.00045504,0.00439397,-0$. $00012850,-0.00026762,0.00455290,0.00002759,0.00009197,-0.00109398,-0.0$ $0002690,-0.00022489,0.00439941,-0.00052243,0.00015187,0.00451819,-0.00$ $107902,0.00125122,0.01182127,-0.00017782,-0.00021744,-0.00000804,-0.00$ $003418,0.00026955,-0.00010350,-0.00010674,-0.00014295,-0.00019494,0.00$ $023590,0.00035638,0.00001239,-0.00010885,-0.00015521,0.00018435,-0.000$ $03540,0.00026261,0.00011995,0.00004462,-0.00002454,-0.00000075,-0.0000$ $1204,0.00000656,0.00002589,-0.00001062,-0.00001709,0.00001661,-0.00001$ $040,-0.00001598,-0.00001776,-0.00001183,0.00000826,-0.00002554,-0.0001$ $7778,-0.00051935,-0.00001744,0.00414053,0.00595636,0.00019420,-0.23140$ $380,-0.14093946,-0.00547249,-0.01630057,-0.01816395,-0.00068175,-0.004$ $05811,0.00210284,0.00001532,0.00004093,0.00107452,0.00003906,0.0013574$ $6,0.00165575,0.00003258,0.00128393,0.00060619,0.00003928,0.24390448,0$. $00024802,0.00007436,0.00000230,-0.00000657,-0.00008745,0.00005387,0.00$ $003259,0.00005054,0.00004586,-0.00005251,-0.00007808,-0.00000440,0.000$ $03172,0.00005211,-0.00004173,-0.00000628,-0.00008240,-0.00006065,0.000$ $00011,0.00001292,-0.00000080,0.00000579,-0.00000247,-0.00001486,0.0000$ $0260,0.00000161,-0.00000371,0.00000283,0.00000143,0.00000395,0.0000053$ $2,-0.00000298,0.00001478,0.00003687,0.00011219,0.00000420,-0.02549482$, $-0.01359969,-0.00066388,-0.13677365,-0.16319933,-0.00476821,0.01086389$ $, 0.00962560,0.00022384,0.00233536,0.00086756,-0.00016061,0.00077535,-0$ $.00037681,0.00002148,0.00235616,-0.00490631,-0.00031600,-0.00041234,0$. $00089125,0.00011249,0.14765041,0.17057739,0.00000679,-0.00000005,0.000$ $04164,-0.00000506,0.00001970,0.00002196,-0.00001994,-0.00001795,-0.000$ $00827,-0.00000077,-0.00000232,0.00005082,0.00002102,0.00002025,-0.0000$ $0774,0.00000405,-0.00002372,0.00001811,0.00000005,-0.00000091,0.000037$ $66,-0.00000482,0.00000665,-0.00000088,0.00000440,0.00000119,-0.0000018$ $8,-0.00000431,-0.00000114,-0.00000193,0.00000508,-0.00000671,-0.000000$ $56,0.00000036,0.00000140,-0.00001345,-0.00080746,-0.00050635,0.0032162$ 
$7,-0.00533986,-0.00478910,-0.03517367,0.00024290,0.00007844,0.00430943$ $, 0.00002264,-0.00016179,0.00627151,0.00002954,0.00002304,-0.00092830,0$ $.00005487,-0.00031949,0.00537530,0.00000683,0.00011759,-0.00270246,0.0$ $0580586,0.00542028,0.02381224,0.00066220,-0.00110882,-0.00002802,-0.00$ $007193,0.00014819,0.00001002,-0.00002804,0.00010703,-0.00000577,-0.000$ $03220,-0.00012252,-0.00000486,-0.00002849,0.00010498,0.00001406,-0.000$ $06844,0.00015045,-0.00000227,-0.00024038,0.00049863,0.00001434,-0.0000$ $0373,-0.00006020,0.00000414,0.00003458,0.00000835,-0.00001109,0.000034$ $33,0.00000784,0.00001193,-0.00000273,-0.00005982,-0.00000738,0.0000498$ $0,0.00017094,0.00000583,-0.00360228,0.00116404,-0.00001426,-0.00143503$ $, 0.02965008,0.00091125,-0.05971731,0.03698321,0.00105897,0.00686409,-0$ $.02294746,-0.00069673,-0.00313733,-0.00404962,-0.00017754,-0.00129548$, $-0.00010761,-0.00000625,-0.00113584,0.00133826,0.00003638,0.00120087$, $0.00036518,0.00001430,0.06156607,0.00042688,-0.00011642,0.00002490,-0$. $00007362,0.00023716,0.00020671,0.00001082,0.00001868,-0.00022491,0.000$ $14963,0.00019445,0.00000205,0.00000641,-0.00000479,0.00023702,-0.00004$ $965,0.00026226,-0.00019830,-0.00067429,0.00024183,0.00000143,-0.000040$ $19,-0.00002838,0.00003260,0.00001286,0.00002120,0.00000793,0.00001204$, $0.00002314,-0.00000665,-0.00003370,-0.00002638,-0.00003066,-0.00014492$ ,-0.00039007,-0.00001248,0.00174100,0.00197967,-0.00014004,-0.00054180 ,-0.00841794,-0.00043613,0.04186650,-0.32180919,-0.00889620,0.00027912 ,-0.01656534,-0.00052789,-0.00261851,-0.00097242,-0.00025315,-0.000382 $40,0.00051943,0.00003786,0.00028929,-0.00046364,-0.00001687,-0.0000668$ $0,0.00065453,0.00014728,-0.04051262,0.34512714,0.00002110,0.00001742,-$ $0.00083309,0.00034334,0.00019793,0.00012427,-0.00003180,-0.00013143,-0$ $.00019477,0.00000385,0.00000060,0.00015646,0.00003390,0.00014457,-0.00$ $017075,-0.00034916,-0.00018749,0.00009547,-0.00002313,0.00000725,0.000$ $09154,0.00009651,0.00000088,-0.00006300,-0.00001355,0.00002381,0.00000$ $498,0.00001465,-0.00002259,0.00000329,-0.00009832,0.00000089,-0.000066$ $14,-0.00000427,-0.00001087,-0.00002319,0.00000408,-0.00014301,0.006645$ $89,-0.00005048,-0.00028481,0.00517967,0.00121462,-0.00892081,-0.036208$ $39,0.00004298,-0.00064426,0.00003411,-0.00013187,-0.00026029,0.0065555$ $8,-0.00001497,0.00003922,-0.00072856,0.00000296,-0.00001161,0.00011130$ $, 0.00002384,0.00014579,-0.00397550,-0.00110134,0.01003268,0.02409412,-$ $0.00001877,0.00018508,0.00000971,0.00028691,-0.00009190,-0.00015631,0$. $00007491,-0.00006405,0.00018558,-0.00085375,-0.00008087,-0.00000992,0$. $00007604,-0.00004801,-0.00018674,0.00028118,-0.00010423,0.00015163,-0$. $00002351,-0.00000814,-0.00000028,0.00001998,-0.00004741,-0.00002658,0$. $00004092,0.00000702,-0.00003047,0.00004123,0.00000477,0.00003199,0.000$ 01976,-0.00004951,0.00002396,0.00014623,0.00028407,0.00001044,-0.00256 $496,0.00295757,0.00004761,-0.00009547,0.00069818,0.00002540,0.00117280$ $, 0.00230804,0.00004747,0.00746182,0.00048825,0.00005269,-0.22485484,-0$ $.13302650,-0.00518230,-0.01839729,-0.01889774,-0.00072033,-0.00150846$, $-0.00025772,-0.00001612,-0.00005359,-0.00004457,0.00000242,0.00029251$, $0.00031822,0.00001142,0.23727996,-0.00091619,0.00004757,0.00002092,0.0$ $0002049,-0.00024412,-0.00020678,0.00010424,0.00025518,0.00001661,0.000$ $06917,0.00035155,-0.00000676,0.00009811,0.00028014,0.00001128,-0.00000$ $658,-0.00027078,0.00018246,-0.00006287,0.00007364,0.00000259,-0.000015$ $91,-0.00000053,0.00002941,-0.00001010,0.00001054,0.00002347,-0.0000059$ $0,0.00001028,-0.00001845,-0.00001525,-0.00000189,-0.00002789,0.0001278$ $2,0.00002567,0.00000526,0.00239709,0.00070501,-0.00018020,0.00105074,-$ 
$0.00052795,0.00001166,0.00065907,-0.00543747,-0.00037288,-0.02290529,-$ $0.01700449,-0.00065874,-0.13678630,-0.16052089,-0.00464751,0.01102366$, $0.00951023,0.00019103,0.00086683,0.00002929,0.00000301,-0.00008065,0.0$ $0003957,0.00002698,-0.00098845,-0.00048479,-0.00002145,0.14574134,0.17$ $239119,-0.00002560,0.00002670,-0.00075500,-0.00039483,-0.00023540,0.00$ $010805,-0.00009431,0.00040592,-0.00013751,-0.00000494,-0.00000740,0.00$ 057285,0.00010301,-0.00037987,-0.00016369,0.00039692,0.00021004,0.0001 $4053,-0.00000195,0.00000280,-0.00001668,0.00000526,-0.00004918,-0.0000$ $2834,0.00006294,-0.00002243,-0.00006938,-0.00006227,0.00002758,-0.0000$ $6943,-0.00000577,0.00005032,-0.00002677,0.00000538,0.00000592,-0.00011$ $840,0.00002977,-0.00017729,0.00673191,0.00003668,0.00000984,-0.0007264$ $3,-0.00000504,-0.00036456,0.00635810,-0.00069249,-0.00054161,0.0000610$ $3,-0.00530205,-0.00462848,-0.03630051,0.00023275,0.00004100,0.00525046$ $, 0.00001969,-0.00000262,0.00007105,0.00000128,0.00002716,-0.00081945,-$ $0.00003019,-0.00001491,0.00003294,0.00571136,0.00546542,0.02392116,-0$. $00029728,0.00019609,0.00000401,0.00010855,0.00025468,-0.00013801,-0.00$ $010195,-0.00017822,-0.00019348,0.00023099,0.00037792,0.00001302,-0.000$ $10407,-0.00019085,0.00018089,0.00010809,0.00024587,0.00015553,-0.00003$ $967,0.00003414,0.00000098,-0.00001018,0.00001557,0.00001907,-0.0000283$ $6,-0.00000398,0.00002145,-0.00002807,-0.00000261,-0.00002191,-0.000009$ $97,0.00001666,-0.00001811,-0.00015291,-0.00055396,-0.00001840,0.005516$ $43,-0.02384853,-0.00074798,-0.00350761,-0.00296643,-0.00013892,-0.0011$ $1047,-0.00040988,-0.00001442,-0.00389846,0.00259218,0.00003046,-0.0005$ $3454,0.02757582,0.00085402,-0.06113959,0.04624714,0.00134332,0.0010326$ $4,-0.00071997,-0.00000438,0.00036828,-0.00121165,-0.00003913,0.0000682$ $3,0.00008353,0.00000736,0.00111269,-0.00035915,0.00001439,0.06251528,-$ $0.00008188,-0.00001209,-0.00000534,-0.00000622,-0.00011624,0.00004758$, $0.00004172,0.00008450,0.00010542,-0.00015201,-0.00018573,-0.00000864,0$ $.00004114,0.00008632,-0.00009765,0.00000124,-0.00010939,-0.00005221,0$. $00002456,-0.00001427,0.00000024,-0.00000204,-0.00000870,-0.00000756,0$. $00001585,0.00000616,-0.00001099,0.00001619,0.00000535,0.00001179,-0.00$ $000200,-0.00000969,0.00000719,0.00009964,0.00029451,0.00001048,0.00749$ $775,-0.01500601,-0.00054209,-0.00363309,0.00000168,-0.00018913,-0.0000$ $5984,0.00072677,0.00004963,0.00202611,0.00071528,-0.00017481,-0.001131$ $99,-0.00603715,-0.00033536,0.04204243,-0.33321100,-0.00928084,0.000377$ $33,0.00127900,0.00012764,0.00029344,-0.00060469,-0.00003297,0.00002498$ ,-0.00006531,0.00002572,-0.00017410,0.00075098,0.00015191,-0.04730924, $0.35149320,-0.00000485,-0.00000396,0.00014276,0.00010958,0.00005616,-0$ $.00003771,-0.00002446,-0.00006990,-0.00002946,-0.00000397,-0.00000591$, $0.00005915,0.00002634,0.00007537,-0.00002914,-0.00010823,-0.00005821,-$ $0.00004419,0.00000067,0.00000028,-0.00001786,0.00000174,-0.00000826,-0$ $.00000414,0.00000743,-0.00000095,-0.00000456,-0.00000665,0.00000156,-0$ $.00000405,-0.00000193,0.00000808,-0.00000335,0.00000249,0.00000715,-0$. $00001738,0.00025046,-0.00069905,0.00317171,-0.00016013,-0.00018580,0.0$ $0536129,-0.00000330,0.00004780,-0.00083835,0.00001241,-0.00017185,0.00$ $651495,-0.00006027,-0.00019164,0.00430797,0.00120937,-0.00925978,-0.03$ $551069,0.00003053,0.00012214,-0.00266448,0.00000880,-0.00004051,0.0004$ $7539,0.00000548,0.00002602,-0.00086823,0.00002030,0.00015098,-0.004040$ $56,-0.00131287,0.01020203,0.02407139,0.00008147,-0.00005604,-0.0007199$ $5,-0.00036970,-0.00053109,0.00018090,0.00089036,0.00090766,0.00083668$, $-0.00913553,0.00521584,0.01661090,-0.00127335,-0.00342227,-0.00119709$, 
$0.00185750,-0.00008865,0.00093085,-0.00000253,0.00007195,-0.00007929,0$ $.00003761,0.00001820,0.00001985,-0.00018303,-0.00019089,-0.00116814,-0$ $.00027254,0.00037072,0.00017639,-0.00024682,-0.00010302,-0.00003186,-0$ $.15559594,-0.07626643,0.18214027,0.00004168,-0.00001801,0.00000157,-0$. $00002858,-0.00006912,-0.00000091,-0.00016920,0.00023883,-0.00000447,0$. $00017866,-0.00009641,0.00004060,-0.00030090,-0.00007387,-0.00003596,0$. $00001987,0.00008641,-0.00000703,-0.00005866,0.00003155,-0.00000088,-0$. $00003742,0.00001015,0.00000267,0.00001323,-0.00002871,-0.00000051,0.00$ $003144,-0.00000456,0.00004802,-0.00004886,0.00002469,0.00000680,0.1535$ $1647,0.00069095,0.00042669,0.00019819,0.00018787,-0.00076660,-0.000134$ $78,0.00249519,0.00114399,0.00189119,-0.01672534,-0.01159898,0.02943783$ ,-0.00391834,-0.00197205,-0.00342368,-0.00128724,-0.00039026,0.0014680 $6,-0.00010704,0.00005051,-0.00008664,0.00000170,0.00005134,0.00002195$, $-0.00039972,0.00035356,-0.00094110,0.00023901,-0.00009009,0.00002539,-$ $0.00014097,-0.00045346,-0.00005811,-0.05381455,-0.03215571,0.03543721$, $-0.00011162,0.00006027,0.00000303,0.00006127,0.00007004,0.00000030,0.0$ $0019802,-0.00031352,-0.00000464,-0.00065528,0.00028803,-0.00002469,0.0$ $0037026,0.00010246,0.00002482,-0.00002745,-0.00009969,-0.00000171,0.00$ $012717,-0.00005760,0.00000083,0.00005167,-0.00001072,-0.00000039,-0.00$ $001755,0.00003380,0.00000129,-0.00004973,0.00000633,-0.00002072,0.0000$ $5872,-0.00003227,-0.00000784,0.06709622,0.04670585,-0.00068277,-0.0001$ $6402,0.00026470,0.00011493,0.00105316,-0.00058816,-0.00245713,-0.00221$ $730,0.00156206,-0.00004185,0.00149744,0.00401535,-0.00006684,-0.001753$ $13,0.00148516,0.00011703,0.00021304,-0.00000733,0.00028477,-0.00007004$ ,-0.00002526,0.00001429,-0.00008145,-0.00000007,0.00076827,-0.00009802 $,-0.00049455,0.00000280,-0.00020401,0.00028698,-0.00002939,0.00001068$, $0.00003011,0.16084808,0.05719934,-0.32858659,0.00007442,-0.00003007,-0$ $.00000365,-0.00004830,-0.00007745,0.00000130,-0.00017662,0.00024867,-0$ $.00000297,0.00048747,-0.00023608,-0.00001352,-0.00027547,-0.00005855,-$ $0.00001064,0.00000370,0.00008922,-0.00000057,-0.00007905,0.00003344,-0$ $.00000125,-0.00004229,0.00000869,0.00000196,0.00000609,-0.00003729,-0$. $00000018,0.00002795,0.00001094,0.00001215,-0.00003711,0.00002255,0.000$ $00515,-0.17862850,-0.06013069,0.33954784,0.00006691,-0.00010352,0.0007$ $1274,0.00186847,-0.00002750,-0.00091474,-0.00128359,-0.00350733,0.0009$ $4570,-0.00896039,0.00628878,-0.01636674,0.00087259,0.00093668,-0.00078$ $497,-0.00036654,-0.00050758,-0.00021147,-0.00000041,0.00006599,0.00008$ $391,-0.00024734,-0.00010449,0.00002237,-0.00027085,0.00037943,-0.00015$ $821,-0.00018693,-0.00026640,0.00115633,0.00003794,0.00001856,-0.000018$ $31,-0.15204785,-0.06405453,-0.18477846,0.00004250,-0.00001821,-0.00000$ $227,-0.00002908,-0.00006984,-0.00000384,-0.00017120,0.00024075,0.00001$ $813,0.00018431,-0.00009620,-0.00004485,-0.00030416,-0.00007654,0.00002$ $823,0.00001981,0.00008673,0.00001279,-0.00005953,0.00003181,0.00000232$ ,-0.00003785,0.00001040,-0.00000241,0.00001330,-0.00002908,-0.00000122 $, 0.00003224,-0.00000137,-0.00004803,-0.00004920,0.00002533,-0.00000575$ $, 0.01105478,0.00567678,0.01981552,0.14982204,0.00064793,0.00042802,-0$. $00018132,-0.00126246,-0.00028113,-0.00149835,-0.00394746,-0.00228528,0$ $.00314171,-0.01638185,-0.00956207,-0.03047061,0.00235298,0.00112388,-0$ $.00189484,0.00019336,-0.00070729,0.00012920,-0.00008955,0.00004023,0.0$ $0008988,-0.00014290,-0.00045443,0.00002565,0.00023927,-0.00010014,-0.0$ $0004762,-0.00035987,0.00028414,0.00098685,0.00000281,0.00004734,-0.000$ $01889,-0.04323192,-0.02742870,-0.01656855,-0.00010665,0.00005833,-0.00$ 
$000015,0.00005805,0.00006485,0.00000432,0.00018645,-0.00029685,-0.0000$ $1222,-0.00062320,0.00027042,0.00003660,0.00035221,0.00009975,-0.000014$ $26,-0.00002715,-0.00009373,-0.00000452,0.00012191,-0.00005524,-0.00000$ $305,0.00004888,-0.00001016,0.00000011,-0.00001713,0.00003139,0.0000005$ $6,-0.00004807,0.00000574,0.00001986,0.00005618,-0.00003121,0.00000609$, $0.00687683,-0.00135192,0.00477091,0.05510136,0.04020406,0.00072349,0.0$ $0017318,0.00027796,-0.00017856,-0.00023075,-0.00012737,-0.00021453,0.0$ $0147598,0.00180853,-0.00113860,-0.00230088,0.00180336,0.00260486,0.002$ $19831,0.00159987,-0.00010023,-0.00106606,-0.00065061,-0.00029084,0.000$ $07500,-0.00001711,0.00001747,-0.00004275,0.00003161,0.00000668,0.00018$ $317,0.00029532,-0.00078971,0.00014531,-0.00042116,-0.00001378,0.000084$ $68,0.00000359,-0.16210437,-0.03855321,-0.33686179,-0.00008093,0.000033$ $87,-0.00000251,0.00005178,0.00008078,0.00000695,0.00018725,-0.00026505$ ,-0.00001766,-0.00052636,0.00025341,-0.00000144,0.00029551,0.00006473, $-0.00000474,-0.00000520,-0.00009428,-0.00000647,0.00008642,-0.00003659$ ,-0.00000276,0.00004509,-0.00000938,0.00000181,-0.00000707,0.00003901, $0.00000217,-0.00003087,-0.00001136,0.00001195,0.00004022,-0.00002463,0$ $.00000442,-0.01912092,-0.00367512,-0.01747805,0.18054321,0.04150263,0$. $34974413 \backslash \backslash-0.00000254,0.00000275,0.00000210,-0.00000017,-0.00000038,-0$ $.00000027,0.00000033,-0.00000100,0.00000032,0.00000105,-0.00000206,-0$. $00000032,0.00000025,-0.00000135,-0.00000005,0.00000261,-0.00000088,-0$. $00000010,-0.00000096,-0.00000129,-0.00000028,-0.00000112,-0.00000024,-$ $0.00000002,0.00000021,-0.00000128,-0.00000004,0.00000010,-0.00000118,-$ $0.00000002,-0.00000104,-0.00000033,-0.00000008,0.00000115,-0.00000175$, $-0.00000004,0.00000022,0.00000170,0.00000006,-0.00000090,0.00000175,0$. $00000020,-0.00000289,0.00000263,-0.00000040,-0.00000210,-0.00000259,-0$ $.00000111,0.00000257,0.00000027,-0.00000015,0.00000055,0.00000109,0.00$ $000013,0.00000058,0.00000295,0.00000007,-0.00000101,0.00000245,0.00000$ $014,-0.00000143,0.00000126,-0.00000002,0.00000112,0.00000034,-0.000000$ $02,0.00000139,0.00000150,0.00000004,0.00000098,-0.00000217,-0.00000009$ ,0.00000105,-0.00000220,-0.00000005III@

ortho-Addition Product

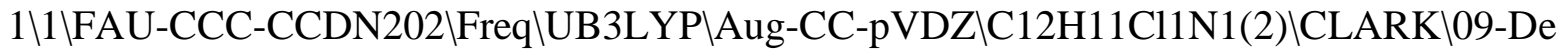
c-2014\0\\N Geom=AllCheck Guess=TCheck SCRF=Check GenChk UB3LYP/Aug-C C-pVDZ Freq \\Addition product p-Cl-Ph-radical + aniline (ortho) $\backslash 0,2 \mathrm{C}$ ,-5.9950411015,1.7778206676,1.7678192244\C,-4.6898325838,1.1482987979, $2.2273602021 \backslash \mathrm{C},-3.4829163632,1.8057799227,2.1153456589 \backslash \mathrm{C},-3.3898482985$ ,3.0997225728,1.5531798453\C,-4.5648305742,3.7524863886,1.0917263612\C ,-5.7924746449,3.1564910281,1.1811089396\H,-6.410446338,1.1144350583,0 $.9811945344 \mathrm{WN},-4.7902194296,-0.1635971247,2.6697125151 \backslash \mathrm{H},-2.5759392888$ $, 1.3049928534,2.4620918028 \mathrm{LH},-4.4850317734,4.7501073031,0.6566467091 \mathrm{VH}$ ,-6.6864531166,3.6682905308,0.8238751368\H,-2.4219692699,3.5931358301, $1.4811911463 \backslash \mathrm{C},-8.9601096262,1.8351252233,4.9335739219 \backslash \mathrm{C},-9.1896793482$ $, 1.0565036708,3.8013562659 \backslash \mathrm{C},-8.2261054762,1.0413214728,2.7854873531 \backslash \mathrm{C}$ ,-7.0478456994,1.7908872278,2.888066461\C,-6.8484146358,2.5677954069,4 $.0402042857 \backslash \mathrm{C},-7.7945878958,2.5949062573,5.0655563365 \backslash \mathrm{Cl},-10.163285574$ $1,1.8661844208,6.2233006474 \backslash \mathrm{H},-10.1048858001,0.473943395,3.7124614075 \backslash$ $\mathrm{H},-8.4053601646,0.4362563071,1.8950672643 \backslash \mathrm{H},-5.9403952106,3.1637218967$ ,4.1347457298\H,-7.6349190873,3.199041857,5.9571267358\H,-3.9736455572 
,-0.5457872796,3.1294309319\Н,-5.6568180161,-0.4187744336,3.127901682\

$\mid$ Version=EM64L-G09RevC.01 \State $=2-A \backslash H F=-978.8892229 \backslash S 2=0.779494 \backslash S 2-1=0$ . IS2A $=0.750499 \backslash \mathrm{RMSD}=2.846 \mathrm{e}-09 \backslash \mathrm{RMSF}=1.764 \mathrm{e}-05 \backslash$ ZeroPoint $=0.1967461 \backslash \mathrm{Therm}$ $\mathrm{al}=0.2089091 \mathrm{ZPE}=123.4600162 \backslash \mathrm{Dipole}=0.3985491,-0.4844465,-0.0141901 \mathrm{Di}$ poleDeriv $=0.1217072,0.1841143,-0.0696348,-0.0223997,0.035675,0.1685884$ ,-0.0885193,0.1079053,0.2611324,0.0935212,0.2240312,-0.0952537,0.19172 $63,1.1891398,-0.3856083,0.0748343,-0.3127817,-0.0113534,-0.0020266,-0$. $369036,0.189945,0.159706,-0.2123705,-0.0485033,-0.0443182,-0.0031346,-$ $0.1402569,0.0181904,0.0985403,-0.011383,-0.0558806,0.0186919,-0.073164$ $7,0.0750413,-0.063251,-0.1493426,0.0687957,-0.1295604,0.0778269,-0.088$ $0877,0.0579984,-0.0434437,0.0683832,-0.1020033,-0.1102243,-0.1063365,0$ $.1297452,0.0362217,0.0730648,-0.009444,-0.1904721,0.1125148,-0.0458347$ ,-0.2095434,-0.0577491,-0.0940223,-0.0408261,-0.1192742,-0.0577702,-0. $0804968,-0.0359487,-0.0866835,-0.0166409,-0.2501407,-0.0956203,0.07481$ $49,-0.0903865,-1.4530356,0.2974217,0.0202291,-0.0649879,-0.3101855,-0$. $0729217,0.0731857,-0.0392227,0.0828877,0.0048734,0.0733937,-0.083628,0$ $.0619103,0.1085036,0.0487952,-0.0118885,0.0094941,-0.0400101,-0.083311$ $6,0.113477,-0.014019,0.0889676,0.0939576,-0.0445895,0.046932,-0.059619$ $1,0.0401574,0.0218195,0.0554005,-0.0557537,0.0456442,0.1096099,-0.0787$ $938,-0.0651813,0.0072688,-0.0705101,0.0322825,0.0436268,-0.0057062,0.0$ $33412,0.1242156,0.6024429,0.0224661,-0.613045,-0.0113412,-0.0143761,0$. $0604123,-0.6771866,0.031561,0.6834595,-0.0951797,0.0717725,0.1232375,0$ $.088341,-0.0504834,-0.0156203,0.1416777,-0.0003716,-0.2323287,-0.03691$ $29,0.0382812,-0.0059909,0.1102024,-0.0428846,0.0379184,0.0795965,0.096$ $9155,0.0066647,0.1030341,-0.0035836,-0.1231798,-0.0298156,-0.0681336,-$ $0.0125673,-0.1917399,-0.0265728,0.0465953,-0.0684938,0.0489197,0.04534$ $87,0.0321597,-0.042538,0.0329384,0.0314143,0.0139512,-0.07133,-0.17549$ $03,0.0017627,0.1010739,0.0210271,-0.0330142,0.0661373,0.126398,0.08317$ $55,-0.073016,-0.5013121,-0.0062021,0.4085504,0.0040195,-0.0788007,-0.0$ $176437,0.4458606,-0.0076099,-0.5195706,-0.010302,-0.0694292,0.0133707$, $-0.067788,0.0790297,-0.0150891,0.0211558,-0.0133717,0.0856964,0.093481$ $4,-0.007245,0.011841,-0.0287322,0.0549483,-0.0746937,-0.0091971,-0.088$ $9708,-0.039823,-0.003134,-0.0620596,-0.0061672,-0.06742,0.057006,-0.00$ $74775,-0.015601,-0.0045442,0.0958704,0.0832778,-0.0202255,0.0065969,-0$ $.0201564,0.075901,-0.0656216,0.0165401,-0.0646369,-0.0010729,0.0980762$ ,-0.0371956,-0.0213917,-0.0632167,0.2494272,0.0357705,-0.0386111,0.135 $2403,0.1640641,0.1720608,0.0314985,-0.0198766,-0.0282728,0.26937,0.045$ $3171,0.0465831,0.1860717,0.1049186 \backslash$ Polar $=205.3764846,19.9572242,155.75$ 0163,-39.6966682,-8.6407827,160.3872279\PG=C01 [X(C12H11Cl1N1)]\NImag= 0\0.45484454,0.02305577,0.49968666,0.04140249,0.01730855,0.45202447,-$0.15404912,0.03075785,-0.02997913,0.65809632,0.02799684,-0.09767605,0$. $02047536,0.11027125,0.58778586,-0.02720572,0.01497524,-0.08062155,0.01$ $794997,-0.16651514,0.22217850,-0.02485441,-0.00677536,-0.00158411,-0.3$ $2222234,-0.11567975,0.01265187,0.69166214,0.00541585,0.01412360,-0.003$ 49990,-0.06606393,-0.14383594,0.02477811,0.00448849,0.55501592,-0.0079 $2937,-0.00356608,0.00755696,-0.00941602,0.02284175,-0.06435608,0.08415$ $603,-0.19039688,0.20208355,0.00162444,0.00683842,-0.00123125,0.0156173$ $6,-0.02791041,0.01267775,-0.11653027,-0.04511488,0.00988564,0.65400171$ $, 0.00296383,-0.00625937,-0.00092788,-0.06000929,-0.03501889,0.01282544$ $, 0.02471465,-0.24118905,0.08704691,0.08735787,0.52364932,0.00032328,0$. $00071503,-0.00756206,0.02762518,0.01347266,0.00063351,-0.02113325,0.07$ 
$971089,-0.08339246,0.04381693,-0.17467749,0.18371891,-0.00049717,-0.01$ $678840,0.00472422,-0.02956725,-0.00589653,0.00176201,0.02858352,0.0602$ $5564,-0.02436834,-0.19347012,0.01728063,-0.02819555,0.60240676,-0.0275$ $4872,-0.01367041,0.00500160,0.00046209,0.00042665,-0.00079132,0.027236$ $34,-0.02074584,0.01560934,0.09213508,-0.14841662,0.05515027,0.06641500$ $, 0.62284058,0.01079501,0.00625236,0.00422827,-0.00424107,-0.00205099$,$0.00029051,-0.00914152,0.02088910,-0.00491686,-0.06185157,0.04456163,-$ $0.08300117,0.04139767,-0.21634059,0.22132520,-0.08790180,-0.01142399,-$ $0.00128950,0.02240758,0.02914911,-0.00983507,-0.01315091,-0.02747079,0$ $.01096234,-0.07904768,0.02971319,-0.02619451,-0.32029221,-0.14159694,0$ $.02354309,0.74060727,-0.00290133,-0.17524527,0.04635611,0.01374763,-0$. $01247439,0.00521354,-0.00661066,-0.01337779,0.00485247,-0.00937756,0.0$ $3356807,-0.01191868,-0.10279187,-0.16437508,0.03728637,0.04296527,0.48$ $032776,-0.00606770,0.04508550,-0.08291697,-0.00235142,0.01028327,-0.00$ $742148,0.00090771,0.00108185,-0.00131775,-0.00779155,-0.00583951,0.011$ $66461,0.00587358,0.03211695,-0.06197018,0.06840147,-0.15455171,0.19967$ $901,-0.07055420,-0.04892348,-0.06792474,-0.02108041,-0.01489014,-0.021$ $16604,0.00104736,0.00576334,-0.00291606,-0.00039239,-0.00300870,0.0012$ $2520,0.00015847,0.00006688,-0.00006995,-0.00113486,-0.00779502,0.00015$ $260,0.08959307,-0.04577411,-0.12263842,-0.09985964,0.00048636,0.007152$ $45,0.00915613,0.00275615,0.00044418,-0.00033816,-0.00212214,-0.0007638$ $7,-0.00021291,0.00459500,-0.00296283,0.00039049,-0.01574886,-0.0228111$ $6,-0.01330462,0.05646500,0.13850822,-0.05924200,-0.09240400,-0.1586923$ $1,-0.00918078,-0.00447669,-0.00527464,0.00079953,0.00252676,-0.0020332$ $7,-0.00000020,-0.00138839,0.00035596,-0.00003184,0.00143958,-0.0010607$ $5,0.00642082,0.00653325,0.01226727,0.06428570,0.10531317,0.17958152,0$. $00214011,0.03207823,-0.01121212,-0.12187775,-0.01522409,0.00136827,-0$. $00132496,-0.04650926,0.01496426,-0.00632218,0.00293297,-0.00339659,0.0$ $0081717,0.00063203,-0.00010914,-0.00697780,-0.00007418,-0.00054245,0.0$ $0192098,0.00123119,0.00077579,0.78487553,0.00842407,-0.02221928,0.0083$ $9474,-0.01494337,-0.27778697,0.07662612,-0.01644300,-0.03375085,0.0126$ $7219,-0.00014347,0.00311523,0.00217806,-0.00478129,-0.00027208,-0.0007$ $1241,0.00427734,-0.00055640,0.00042679,-0.00024679,0.00208102,-0.00026$ $160,-0.01637010,0.48266672,-0.00224626,0.01490992,-0.00397218,0.005434$ $95,0.11290393,-0.06843427,0.00130272,0.01176472,0.00541029,-0.00008794$ $, 0.00073465,0.00456290,-0.00034488,0.00008163,-0.00081560,-0.00221917$, $0.00067097,0.00134245,0.00178860,-0.00012526,0.00096768,-0.01273626,-0$ $.28322141,0.27964089,-0.00122433,-0.00370638,0.00049161,-0.02066422,0$. $01585743,-0.01035819,-0.24553817,0.10043107,-0.07402102,0.00424816,-0$. $00195317,0.00119445,0.00204129,-0.00106567,0.00008428,-0.00004016,-0.0$ $0060754,0.00037551,-0.00076438,0.00032526,-0.00024050,0.00174031,0.001$ $35900,0.00010727,0.25991840,-0.00255462,0.00017119,0.00278973,-0.01229$ $505,0.00736233,-0.00333556,0.10129548,-0.10943769,0.04788054,0.0251475$ $6,-0.00764258,0.00766816,-0.00211687,-0.00365055,0.00351386,-0.0005386$ $4,-0.00034601,-0.00011581,0.00023708,-0.00004024,0.00008504,-0.0006454$ $3,0.00124376,-0.00233484,-0.10956130,0.11196997,0.00052554,0.00239835$, $0.00637468,0.00245028,0.00053332,0.00397122,-0.07493012,0.04691490,-0$. $06390092,-0.01086448,0.00452519,-0.00171223,0.00058058,0.00398127,0.00$ $364539,0.00025008,-0.00007099,-0.00051191,0.00089397,-0.00061807,0.000$ $33692,0.00141732,-0.00221631,-0.00276314,0.08142507,-0.05472462,0.0578$ $4830,-0.00383771,-0.00145025,-0.00110908,-0.00101877,-0.00016612,-0.00$ 
$000829,-0.00399222,0.00160004,-0.00197263,0.00629464,0.02347895,-0.009$ $91759,-0.05801578,-0.01884668,0.00511477,0.00122445,-0.02666629,0.0116$ $9268,-0.00008739,-0.00065641,0.00032035,0.00045523,0.00018930,0.000091$ $87,0.00008776,0.00127681,-0.00060443,0.05731353,-0.00194857,0.00299539$ $, 0.00258858,0.00011469,0.00053967,-0.00027101,0.00243544,0.00140311,0$. $00175506,-0.00396622,-0.00925441,0.00451019,-0.01905192,-0.28541122,0$. $10930602,0.00091921,-0.00983117,0.00576416,-0.00008526,0.00018662,-0.0$ $0018722,-0.00009054,-0.00016874,-0.00016625,-0.00017449,-0.00055127,0$. $00037383,0.02160350,0.30064634,-0.00067804,0.00285655,0.00786901,-0.00$ $021826,-0.00045931,-0.00003955,-0.00223993,0.00173945,0.00342003,0.002$ $51977,0.00834910,-0.00117057,0.00481164,0.10909134,-0.08380238,-0.0002$ $0955,0.00190657,0.00229291,0.00005093,0.00117359,-0.00079337,0.0000212$ $2,-0.00009946,-0.00037075,0.00006285,0.00056703,0.00003413,-0.00498457$ ,-0.12039956,0.07809513,0.00473848,-0.00404805,0.00325368,0.00036006,$0.00168570,0.00067119,0.00020096,-0.00049005,0.00032442,-0.00278272,-0$ $.00425909,0.00054103,-0.01761157,0.01586794,-0.01028312,-0.24427757,0$. $10663995,-0.07942495,0.00021407,0.00040173,-0.00028081,0.00048179,0.00$ $001922,0.00013956,-0.00002821,0.00014376,0.00000812,0.00082466,0.00014$ $259,0.00076150,0.25783892,0.02232748,-0.01015856,0.00932457,-0.0039384$ $7,-0.00429583,0.00183532,0.00002430,-0.00049267,-0.00018112,-0.0034812$ $0,0.00057360,0.00261419,-0.01251861,0.00940998,-0.00405149,0.10825093$, $-0.11273736,0.05422254,0.00006826,0.00086323,-0.00068466,0.00210967,0$. $00008057,0.00063030,0.00011136,-0.00003589,-0.00023827,0.00051009,-0.0$ $0002174,-0.00236062,-0.11326276,0.11614827,-0.00812696,0.00404850,-0.0$ $0278174,0.00110648,0.00235203,0.00121736,0.00053464,0.00005770,-0.0006$ $1671,-0.00014330,0.00212337,0.00650076,0.00224536,0.00003855,0.0046146$ $1,-0.07977605,0.05339363,-0.06283407,0.00021916,0.00082661,0.00032316$, $-0.00072481,0.00000102,-0.00039469,0.00004337,-0.00023528,-0.00052656$, $0.00068592,-0.00234062,-0.00423770,0.08501772,-0.06020388,0.05745682,0$ $.00036445,0.00041392,0.00008141,0.00162478,0.00039942,-0.00052849,0.00$ $158886,0.00217244,-0.00111637,-0.28198510,-0.11728493,0.01303221,-0.01$ $644065,-0.01401519,0.00334461,-0.00205357,0.00360521,-0.00250734,0.000$ $07933,0.00002362,-0.00000444,-0.00001032,0.00005184,0.00001228,0.00067$ $084,-0.00015621,0.00054194,0.00083708,0.00029585,0.00035388,-0.0010308$ $9,-0.00064947,0.00010921,0.29621231,0.00030150,-0.00031882,-0.00025014$ $, 0.00090871,-0.00374596,0.00335632,-0.02480543,-0.00709720,0.00039561$, $-0.11689107,-0.11043113,0.02101261,0.01338706,0.00930911,-0.00103739,0$ $.00279612,-0.00056687,0.00213942,-0.00011495,0.00003422,0.00000890,-0$. $00150033,-0.00029227,0.00033162,0.00043934,0.00046171,-0.00137619,-0.0$ 0030536,0.00029606,-0.00125153,0.00074803,0.00039996,0.00011116,0.1251 $4492,0.11226232,0.00009038,-0.00038820,-0.00086972,-0.00066230,0.00341$ $005,0.00228256,0.01090767,0.00446499,0.00230215,0.01239229,0.02093024$, $-0.03113282,-0.00835600,-0.00506978,0.00339696,-0.00213872,0.00231465$, $0.00205907,-0.00013039,0.00001738,0.00011655,0.00068285,0.00040970,0.0$ $0019160,0.00025714,-0.00141471,-0.00215339,0.00062430,-0.00120796,-0.0$ $0180861,-0.00053041,-0.00009899,0.00039146,-0.01320160,-0.02350850,0.0$ $2508681,-0.00153385,-0.00037006,0.00012087,0.00007230,-0.00027674,0.00$ $041636,-0.00000238,-0.00014748,0.00010777,-0.00003300,0.00030629,-0.00$ $004473,0.00026227,0.00015282,0.00011401,-0.00032398,0.00000621,0.00037$ $830,0.00001637,-0.00002086,0.00016049,0.00037757,0.00015894,0.00014727$ $, 0.00007760,-0.00004017,-0.00007296,0.00007692,0.00001927,-0.00008777$, 
$-0.00000088,-0.00005390,-0.00002043,0.00003412,0.00001361,0.00000781,0$ $.47044246,-0.00076526,-0.00077590,0.00002503,0.00018090,-0.00016416,0$. $00041024,-0.00002782,-0.00006383,0.00006503,-0.00007336,0.00019994,0.0$ $0003568,0.00024221,0.00004504,0.00013885,-0.00030187,0.00017511,0.0004$ $8556,-0.00009469,0.00014487,0.00022764,0.00011796,0.00026292,0.0002696$ $2,0.00003169,-0.00000852,-0.00009740,0.00002048,-0.00004149,-0.0001274$ $8,-0.00003521,-0.00006361,-0.00000643,-0.00002549,0.00001545,-0.000003$ $51,0.18634261,0.33826181,-0.00028194,-0.00005102,-0.00066275,-0.000011$ $33,-0.00030713,0.00057582,0.00003549,0.00020983,-0.00004055,0.00001127$ $, 0.00005630,0.00009858,0.00007369,-0.00000717,0.00001496,-0.00031501,0$ $.00026373,0.00059118,0.00000526,0.00005110,0.00035640,0.00003824,0.000$ $42877,0.00026156,-0.00004881,-0.00001677,-0.00013818,0.00000700,-0.000$ $10685,-0.00006580,-0.00000593,0.00000823,-0.00016315,-0.00006319,-0.00$ $004103,0.00001241,-0.02798765,0.18117659,0.47227452,-0.00014525,-0.004$ $53168,-0.00088014,0.00009456,-0.00060696,0.00048901,0.00019638,0.00013$ $080,0.00001954,0.00003536,0.00001657,0.00000413,0.00025594,-0.00026705$ $, 0.00022931,-0.00129235,0.00042039,0.00095080,0.00013421,0.00026591,0$. $00033698,0.00029839,0.00058895,0.00008788,0.00000296,0.00001691,-0.000$ $06757,-0.00005639,-0.00007660,-0.00012562,-0.00005611,-0.00010348,-0.0$ $0002531,-0.00000702,0.00002494,0.00000632,-0.11109625,-0.05888464,-0.0$ $5576756,0.61423769,-0.00435242,0.00318513,-0.00316139,-0.00096196,-0.0$ $0063557,0.00089215,-0.00005981,0.00053679,-0.00016016,0.00013453,-0.00$ $024278,0.00003085,-0.00034377,0.00020609,-0.00026958,0.00122318,-0.000$ $01878,-0.00042342,0.00000607,0.00002568,0.00009487,-0.00004470,-0.0001$ $0798,0.00005963,-0.00007402,-0.00007162,-0.00002306,0.00005556,0.00007$ $582,0.00011973,0.00002317,-0.00005528,0.00003815,0.00002815,-0.0000139$ $2,0.00002140,-0.03051649,-0.15756567,-0.13151968,0.22722158,0.36705270$ $, 0.00033704,-0.00304504,0.00211706,0.00038741,0.00075691,-0.00114432,0$ $.00013376,-0.00029448,0.00000185,-0.00009201,-0.00027389,-0.00005762,-$ $0.00004533,-0.00014493,0.00002503,-0.00025281,-0.00024329,-0.00035124$, $-0.00003918,0.00001894,-0.00019680,-0.00008778,-0.00036457,-0.00043748$ $, 0.00004591,0.00008758,0.00019813,-0.00007290,0.00004585,0.00009149,0$. $00004167,-0.00000430,0.00010105,0.00002729,0.00002526,-0.00000486,-0.0$ $0599217,-0.10288794,-0.23919928,-0.09705400,0.18388258,0.55346534,-0.0$ $1906807,-0.01187487,0.00456683,-0.00017467,-0.00110871,0.00080028,-0.0$ $0032059,0.00025043,-0.00043349,-0.00008273,-0.00002877,-0.00005691,0.0$ $0045918,-0.00069646,0.00044518,-0.00141568,0.00109227,-0.00135882,0.00$ $026570,-0.00114212,-0.00272528,0.00008167,-0.00029999,-0.00088774,-0.0$ $0016989,-0.00003576,0.00015487,-0.00023901,-0.00002796,0.00012128,0.00$ $018716,-0.00036138,0.00037284,0.00000658,-0.00001829,0.00002942,0.0405$ $1605,0.04525612,0.04485958,-0.21135960,-0.03543551,0.09107518,0.553405$ $25,-0.00276191,0.00393017,0.00157182,0.00123605,-0.00174955,-0.0002266$ $6,-0.00011938,0.00000423,-0.00008091,0.00010610,0.00026251,-0.00007606$ $, 0.00016963,0.00007316,-0.00005980,0.00027305,0.00018365,-0.00056765,0$ $.00152108,0.00023478,-0.00087009,0.00075730,-0.00000334,-0.00026979,-0$ $.00002692,0.00004371,0.00020447,0.00001408,0.00006675,-0.00002240,0.00$ $010818,0.00008742,0.00004699,0.00001213,0.00002720,-0.00003981,0.02015$ $530,0.01625123,-0.00457100,-0.00649683,-0.08664076,-0.03643166,0.18849$ $260,0.36228675,0.02089079,0.01056249,0.00164045,0.00195574,0.00239489$, $-0.00213973,-0.00003996,-0.00143813,0.00076136,-0.00005802,0.00076216$, $-0.00029107,0.00025574,0.00090744,-0.00017153,0.00210406,-0.00067876$,- 
$0.00130002,0.00220505,0.00184001,0.00124199,-0.00027623,-0.00079475,0$. $00023499,0.00026877,0.00007637,0.00005821,0.00021166,0.00023347,0.0001$ $0715,-0.00023888,0.00008977,-0.00005721,0.00003546,-0.00001251,-0.0000$ $7014,0.00145385,-0.02978250,-0.04643836,0.14242998,-0.00715546,-0.2209$ $7535,-0.09892899,0.20887768,0.60615657,-0.10843123,0.00389805,0.046177$ $21,-0.03528419,0.00521129,0.01630478,0.00129332,0.00490184,-0.00077866$ ,-0.00080221,-0.00170590,0.00077227,0.00127327,0.00178746,0.00046988,$0.00687030,-0.01356415,0.01084456,0.00338551,-0.00140245,-0.00592155,0$ $.00008614,-0.00172912,0.00179531,-0.00009639,-0.00063900,-0.00092099,0$ $.00032631,0.00002728,-0.00011619,0.00080037,0.00098043,-0.00140121,0.0$ $0014209,0.00000052,0.00007382,-0.02424560,-0.02379952,-0.02413095,-0.0$ $3952718,-0.00122791,0.04523963,-0.25169559,-0.12466160,-0.02870315,0.5$ $3654909,-0.00264121,-0.07097644,-0.00445707,0.00485629,-0.00142407,-0$. $00820173,0.00142585,-0.00068547,0.00050998,-0.00130981,0.00024133,-0.0$ $0014575,0.00292457,-0.00318005,0.00222695,-0.02357919,-0.00215264,0.02$ $195141,0.01140079,-0.00203602,-0.01651299,0.00295915,0.00119723,-0.000$ $50273,0.00006648,0.00035874,0.00033554,-0.00054035,-0.00094215,-0.0010$ $9760,-0.00015558,0.00051573,-0.00029967,-0.00003241,0.00008335,0.00004$ $144,-0.02457423,-0.03387801,-0.02233903,-0.02191827,0.01869414,0.04270$ $286,-0.09705275,-0.14642145,-0.04427244,0.17485650,0.37291100,0.034990$ $03,-0.01042583,-0.12267434,0.00432344,-0.00572963,0.01266888,-0.002970$ $93,0.00131049,-0.00238074,0.00106624,0.00029364,0.00033783,-0.00167193$ $,-0.00157773,-0.00106616,0.01132139,0.01213926,-0.01242703,0.01825255$, $-0.00369666,-0.02435081,-0.00134305,0.00088960,-0.00029212,-0.00080291$ ,-0.00043410,-0.00038517,-0.00031239,-0.00000715,0.00041415,0.00008591 ,-0.00110043,0.00131135,-0.00009761,0.00000040,0.00017615,-0.02497373, $-0.02280970,-0.02108688,0.00967462,0.02189027,0.03556605,0.02216648,-0$ $.01549364,-0.11396330,-0.06204633,0.16574643,0.52661967,0.00467450,0.0$ $1300765,0.01974727,0.00187226,0.00055575,0.00105524,-0.00014996,0.0003$ $9510,-0.00000078,-0.00019120,-0.00028646,0.00001014,-0.00026305,-0.000$ $44799,-0.00008060,0.00104904,0.00131800,0.00034230,-0.00266047,0.00036$ $215,0.00458411,-0.00037480,0.00037371,0.00043266,-0.00002976,-0.000049$ $64,-0.00016337,-0.00002220,-0.00010781,-0.00007716,0.00003695,-0.00016$ $437,0.00008839,-0.00001490,0.00000064,0.00003992,-0.04272476,-0.024460$ $38,0.00861857,-0.00686509,0.00686144,0.01584398,0.01024515,-0.01439405$ $,-0.02828873,-0.11597280,-0.05140235,-0.03809473,0.60719157,0.00394576$ $, 0.00316950,-0.00229539,-0.00074874,0.00060688,-0.00019625,-0.00009270$ $, 0.00037025,-0.00060386,0.00004252,-0.00075984,0.00019450,-0.00057233$, $-0.00002143,0.00024122,-0.00012320,0.00000429,0.00066972,-0.00062539,0$ $.00107362,0.00051888,-0.00018412,-0.00030356,-0.00026029,-0.00007107,-$ $0.00000344,-0.00006926,-0.00001830,-0.00001378,-0.00010261,-0.00005677$ $, 0.00034166,-0.00017759,-0.00004201,0.00002238,0.00001819,0.00036172,0$ $.01938892,0.02142492,0.00709197,-0.00995067,-0.01936744,-0.03361813,-0$ $.01815210,-0.01040009,-0.02487940,-0.14989078,-0.11960719,0.21604340,0$ $.36404022,0.00427620,-0.01123791,-0.02211930,-0.00226950,0.00153912,-0$ $.00179418,-0.00008062,-0.00009259,0.00029898,-0.00015733,-0.00028593,-$ $0.00017790,-0.00065407,-0.00019895,-0.00049477,0.00019820,-0.00272355$, $-0.00001582,0.00274042,-0.00047443,-0.00265346,-0.00055096,-0.00068251$ $, 0.00020323,0.00002225,-0.00001120,0.00009670,0.00000306,0.00001422,0$. $00016699,0.00001775,0.00023316,-0.00013253,0.00002154,0.00000729,0.000$ $04776,0.05169331,0.04681288,0.03552726,0.01609366,-0.01958005,-0.05347$ 
$961,-0.06207471,-0.03000638,0.01892850,0.00814054,-0.09198784,-0.23648$ $982,-0.11167808,0.17537009,0.55743891,0.00298378,-0.00276256,-0.001640$ $49,-0.00043835,0.00012504,-0.00059928,0.00004852,0.00000373,-0.0000383$ $9,0.00017342,-0.00015224,0.00001461,-0.00029582,-0.00024717,-0.0001972$ $0,-0.00008420,-0.00013336,-0.00029453,0.00041138,-0.00031581,-0.000944$ $09,-0.00023244,-0.00035194,-0.00037710,-0.00005445,0.00002328,0.000120$ $16,-0.00010403,-0.00000871,0.00008505,0.00002830,0.00013768,0.00015042$ $,-0.00002150,-0.00000688,0.00000515,-0.24102287,-0.09989266,0.00609785$ $, 0.01152380,-0.03341178,-0.06096414,-0.05140928,-0.01653718,0.02040628$ $, 0.03882726,0.04248096,0.04098662,-0.21240565,-0.03436072,0.09664061,0$ $.54629394,-0.00289490,0.00114796,-0.00419104,-0.00085339,0.00003248,0$. $00046027,-0.00001366,0.00002686,0.00000823,0.00009553,0.00004054,-0.00$ $008877,0.00002671,0.00021744,-0.00002899,0.00016817,-0.00012450,0.0001$ $6897,0.00055208,-0.00008684,-0.00097780,-0.00008097,0.00009179,-0.0001$ $0249,-0.00003636,-0.00003079,-0.00000030,0.00002496,0.00000959,0.00005$ $375,0.00001628,0.00000950,-0.00010281,0.00000694,-0.00001625,0.0000097$ $5,-0.12863942,-0.15160632,-0.01972679,-0.01351964,-0.01642193,-0.03028$ $230,-0.01728604,-0.00908667,0.00806320,0.02143029,0.01742988,-0.004352$ $61,-0.00444383,-0.08659803,-0.04058256,0.18833594,0.36784773,-0.002022$ $07,-0.00452417,-0.00043173,-0.00005919,0.00031222,0.00010742,0.0001117$ $4,-0.00009352,0.00028119,-0.00001730,0.00020386,-0.00007394,0.00024659$ $, 0.00017908,0.00024746,-0.00053571,-0.00012533,0.00081522,0.00061159,0$ $.00005321,-0.00043499,0.00023817,0.00009045,0.00051544,0.00009035,-0.0$ $0000619,-0.00007930,0.00004544,-0.00000880,-0.00014916,-0.00000740,0.0$ $0004741,-0.00008364,-0.00000097,0.00000820,-0.00002261,-0.04383496,-0$. $04911095,-0.10550148,-0.02643654,-0.00994283,0.01856600,0.01919487,0.0$ $0707988,-0.01041330,0.00481468,-0.02514976,-0.04197934,0.14814337,-0.0$ $1013129,-0.23188438,-0.09885448,0.22184729,0.62066804,0.00006747,0.000$ $13780,-0.00041035,0.00004585,-0.00026367,0.00041162,-0.00002358,0.0002$ $8265,-0.00012588,-0.00000421,-0.00010552,0.00006768,0.00004240,-0.0001$ $0915,0.00008435,-0.00023269,0.00036234,0.00016572,-0.00005982,0.000005$ $99,0.00006435,-0.00024047,0.00030941,0.00002390,-0.00008442,-0.0000066$ $6,-0.00005390,-0.00004773,-0.00008039,-0.00002075,0.00003224,-0.000003$ $84,-0.00004268,-0.00004167,-0.00002106,-0.00000434,-0.08837530,-0.0042$ $7906,0.05889658,-0.00510947,-0.00024141,0.00931321,0.00224304,-0.00105$ $474,0.00088175,-0.00005437,0.00126079,0.00143416,-0.00047613,-0.005305$ $85,-0.00412994,-0.02575403,0.00056053,0.03194975,0.11837126,0.00037101$ ,-0.00036557,0.00027626,0.00003640,0.00004201,-0.00006304,0.00004849,-$0.00000620,0.00003707,0.00001957,0.00000821,0.00000564,-0.00002146,-0$. $00002548,-0.00002782,-0.00002963,0.00001637,-0.00000412,-0.00001242,0$. $00003405,0.00006929,0.00001139,0.00001322,0.00001069,0.00001014,0.0000$ $0341,0.00000251,-0.00000172,0.00000194,-0.00000202,-0.00000369,0.00000$ $694,-0.00000603,0.00000466,-0.00000186,-0.00000242,-0.00416344,-0.0302$ $1666,-0.00693554,0.01166836,0.00369017,-0.01333520,-0.00390871,0.00140$ $675,-0.00220163,0.00097296,0.00040109,0.00089054,-0.00233628,0.0011538$ $1,-0.00353475,-0.01154945,0.00425951,0.01146991,0.00619360,0.02161598$, $-0.00022443,0.00053759,0.00024289,-0.00006634,0.00021088,-0.00043360,0$ $.00001964,-0.00031502,0.00014175,-0.00000078,0.00011029,-0.00008925,-0$ $.00001677,0.00020848,-0.00007307,0.00037716,-0.00041067,-0.00017698,0$. $00001548,0.00003497,0.00003954,0.00025867,-0.00028573,-0.00000795,0.00$ $008755,0.00000550,0.00006056,0.00006062,0.00009866,0.00002414,-0.00004$ 
$034,-0.00000801,0.00004252,0.00004886,0.00002455,-0.00000452,0.0588518$ $4,-0.00680864,-0.09640624,0.03038876,-0.00116515,-0.02859157,-0.004161$ $30,-0.00515039,0.00012362,0.00112652,0.00059799,-0.00062449,0.00104381$ ,-0.00043949,0.00265812,0.01097627,-0.00092006,-0.00827368,-0.09849274 $, 0.01077444,0.13202832,-0.00021448,-0.00042338,-0.00047568,-0.00009484$ ,-0.00026163,0.00010657,0.00004618,0.00014873,-0.00005757,-0.00003938, $-0.00006312,0.00003363,0.00005606,-0.00004725,0.00003420,-0.00017067,0$ $.00004543,0.00016492,0.00001953,-0.00000370,-0.00002134,-0.00003091,0$. $00019392,0.00002316,-0.00002507,-0.00000332,-0.00000631,-0.00001018,-0$ $.00002155,-0.00001580,0.00000707,-0.00001728,0.00000357,-0.00000681,-0$ $.00000112,0.00000285,-0.00175082,-0.00124897,0.00310759,-0.25413374,-0$ $.13754173,-0.01799782,-0.01212319,-0.01136080,-0.00390111,0.00053349,-$ $0.00099849,0.00460305,0.00006011,0.00080543,0.00007139,0.00252494,-0.0$ $0160439,0.00028984,0.00044271,0.00165988,-0.00039289,0.26511924,0.0001$ $7562,0.00008878,-0.00017057,-0.00009122,0.00012134,0.00010454,0.000029$ $50,0.00003725,-0.00000065,0.00002215,-0.00004185,0.00001615,-0.0000474$ $5,0.00000223,0.00002612,0.00004039,-0.00001090,-0.00015475,-0.00000865$ $, 0.00000811,-0.00001297,0.00000590,-0.00007156,-0.00005737,0.00000443$, $-0.00000249,-0.00001319,-0.00001213,0.00000243,0.00001526,-0.00000088$, $-0.00000676,0.00000381,0.00000059,-0.00000510,-0.00000216,-0.01511053$, $-0.00516132,0.00043584,-0.13623664,-0.12614935,-0.02086893,0.00173654$, $0.00400926,-0.00087704,-0.00136267,0.00484598,-0.00215215,0.00086173$,$0.00064261,0.00014639,-0.00131826,0.00212351,-0.00471507,0.00121057,-0$ $.00115172,0.00154029,0.14897082,0.12475906,0.00062433,0.00048166,0.000$ $36784,0.00013094,0.00008634,-0.00024833,-0.00002585,-0.00012049,0.0000$ $6631,-0.00001398,0.00007437,-0.00004397,0.00003820,0.00005553,-0.00002$ $769,0.00009755,-0.00007625,-0.00001185,-0.00001443,-0.00001809,0.00001$ $811,-0.00001540,-0.00006041,0.00002445,0.00001575,0.00000562,0.0000194$ $4,0.00001859,0.00001504,0.00000085,-0.00000597,0.00002447,-0.00001186$, $0.00000358,0.00000282,-0.00000333,-0.02124944,-0.01374024,0.00093936,-$ $0.01573293,-0.01957179,-0.05346696,0.01897272,0.01246105,0.00592557,0$. $00419216,-0.00238259,-0.00163418,0.00027314,0.00016424,-0.00118182,0.0$ $0074677,-0.00441981,-0.00299632,-0.00118275,0.00108482,0.00035708,0.01$ $554269,0.02480193,0.05188225,-0.00017173,0.00079211,-0.00278378,-0.000$ $13571,-0.00026557,0.00036600,-0.00005483,-0.00004308,0.00007260,0.0000$ $6937,0.00014123,-0.00004201,-0.00005961,0.00014201,-0.00005539,0.00039$ $446,0.00019124,0.00010385,-0.00009698,0.00033235,0.00090735,0.00007424$ $, 0.00004749,0.00015489,-0.00000965,-0.00003948,-0.00004436,0.00003304$, $0.00002407,-0.00002013,-0.00000811,0.00002720,-0.00005213,0.00000601,0$ $.00000803,-0.00000534,-0.00051666,-0.00212225,0.00387686,0.00637355,0$. $01219871,0.01840127,-0.06064256,-0.03322566,-0.03354242,0.00016289,-0$. $01360543,-0.02070923,-0.00309700,-0.00488133,0.00111443,-0.00114496,0$. $00016350,0.00034938,-0.00038461,0.00036838,0.00120415,-0.00005640,0.00$ $162900,-0.00126147,0.05952653,0.00276072,-0.00237434,0.00028719,0.0004$ $0151,0.00072174,-0.00023553,0.00010844,-0.00024119,0.00005253,-0.00002$ $845,0.00002495,0.00002649,0.00009098,-0.00006491,0.00009832,-0.0005778$ $7,-0.00002814,0.00027307,-0.00026359,0.00043993,0.00072771,-0.00003628$ ,-0.00013264,0.00004178,0.00006796,0.00001945,0.00000478,-0.00001085,$0.00003598,-0.00005428,-0.00003336,-0.00008993,0.00000506,0.00001076,-$ $0.00000264,0.00000106,-0.00196847,0.00504656,-0.00143657,-0.00112427,0$ $.00300422,0.00082310,-0.03319271,-0.12923289,-0.13189144,-0.00174667,-$ 
$0.00530026,-0.01421061,-0.00536572,0.00233373,-0.00149932,0.00012805,-$ $0.00052659,0.00086474,-0.00013998,0.00010475,0.00005074,0.00177477,-0$. $00220081,0.00153315,0.03866847,0.12925240,0.00042117,0.00251837,-0.000$ $25741,-0.00049672,-0.00055762,0.00029710,0.00021165,0.00045971,-0.0001$ $0699,0.00000132,-0.00024374,0.00006514,-0.00005798,0.00012867,-0.00005$ $930,0.00040987,-0.00012977,0.00017509,-0.00035554,0.00001270,0.0005079$ $9,0.00008457,0.00040251,0.00013404,-0.00001388,-0.00001612,-0.00003724$ $, 0.00003237,0.00001380,-0.00000550,-0.00000241,-0.00000884,-0.00004155$ $, 0.00000157,0.00000347,0.00000444,0.00416493,-0.00124947,0.00047068,-0$ $.00521389,-0.01291047,-0.01405319,-0.03392872,-0.13221425,-0.23658875$, $0.00145241,-0.00150888,-0.00036371,0.00037665,-0.00180549,0.00265671,0$ $.00017917,0.00077562,0.00008817,0.00031849,-0.00045340,-0.00098752,-0$. $00098856,0.00168109,-0.00008299,0.03363278,0.14506294,0.24882158,-0.00$ $101308,0.00131928,0.00030574,0.00025984,0.00001279,0.00032957,-0.00010$ $399,-0.00007449,-0.00025833,0.00000308,-0.00009501,0.00005503,0.000040$ $83,0.00013718,0.00021313,0.00004288,0.00029450,0.00020250,-0.00000906$, $-0.00002267,-0.00015635,-0.00005829,-0.00006998,-0.00004264,-0.0000273$ $7,0.00003469,0.00005458,0.00001843,0.00003280,-0.00000289,-0.00005424$, $-0.00002727,-0.00015782,-0.00001275,0.00000971,-0.00000029,0.00095378$, $-0.00145638,0.00417461,0.00010831,0.00080353,0.00008411,0.00252464,-0$. $00214027,0.00040218,-0.00016512,-0.00221853,0.00039393,-0.24677015,-0$. $13501358,-0.01748099,-0.01255778,-0.01166103,-0.00334059,-0.00082278,-$ $0.00037705,0.00029692,-0.00020777,0.00022718,-0.00016484,-0.00003645,0$ $.00003748,0.00014671,0.25818170,0.00065101,-0.00175033,0.00180873,0.00$ $017384,0.00024201,-0.00027410,0.00016822,-0.00001169,0.00036018,0.0000$ $0684,0.00020203,-0.00011526,-0.00003564,-0.00012774,-0.00021359,-0.000$ $09841,0.00030109,0.00011997,0.00002591,-0.00006074,0.00010317,0.000047$ $36,-0.00023302,0.00023770,0.00006534,-0.00000523,-0.00002870,-0.000026$ $95,-0.00001506,0.00010443,0.00003938,-0.00001676,0.00011084,-0.0000098$ $5,-0.00001777,0.00000698,-0.00158245,0.00509847,-0.00229858,0.00087020$ $,-0.00065551,0.00013640,-0.00169665,0.00245365,-0.00517404,-0.01503921$ ,-0.00533486,-0.00031410,-0.13507183,-0.12961489,-0.02108019,0.0018307 $2,0.00414680,-0.00132986,0.00016202,0.00007564,-0.00014890,0.00019850$, $-0.00044185,0.00029357,-0.00074675,-0.00032112,-0.00001098,0.14841826$, $0.12848653,-0.00105349,0.00111001,-0.00008727,-0.00028861,-0.00076779$, $0.00015156,0.00023575,0.00016352,-0.00007929,0.00018152,-0.00009621,0$. $00000315,0.00026967,0.00032881,0.00001471,-0.00015932,-0.00014745,0.00$ $005473,-0.00006951,0.00013696,0.00021411,0.00014202,0.00051681,-0.0001$ $3329,-0.00004282,0.00006776,0.00003851,0.00004464,0.00001652,0.0000058$ $5,0.00002075,-0.00004156,-0.00006019,-0.00004146,-0.00000897,0.0000031$ $4,0.00385052,-0.00247113,-0.00088529,0.00018801,0.00016787,-0.00106282$ $, 0.00092681,-0.00485878,-0.00307118,-0.02190343,-0.01353495,0.00247284$ ,-0.01694422,-0.02084823,-0.05549881,0.01999519,0.01251131,0.00496245, $0.00119197,0.00038542,-0.00047935,-0.00017853,0.00028525,-0.00006592,-$ $0.00115233,-0.00077588,-0.00007018,0.01610315,0.02604818,0.05365129,0$. $00038088,0.00053543,0.00066480,0.00003495,-0.00006428,0.00004195,0.000$ $01298,0.00011276,-0.00003381,0.00003284,-0.00009726,0.00000423,-0.0001$ $0527,-0.00004499,-0.00001470,0.00017044,0.00014190,-0.00014972,-0.0002$ $2467,0.00009873,0.00023457,-0.00003096,0.00000599,-0.00006120,-0.00002$ $197,0.00001057,0.00000850,-0.00001029,-0.00000729,0.00001329,-0.000005$ $98,-0.00001041,0.00000706,-0.00000542,-0.00000237,0.00000835,-0.000677$ 
$34,-0.01442105,-0.02115943,-0.00316378,-0.00434826,0.00111680,-0.00105$ $992,0.00024539,0.00043579,-0.00108154,-0.00250426,0.00439458,0.0071110$ $1,0.01186472,0.01752078,-0.05777052,-0.03047890,-0.03064613,0.00031887$ $, 0.00104248,-0.00118903,0.00005787,-0.00073211,-0.00101365,-0.00007088$ $, 0.00029238,-0.00014749,-0.00029546,0.00173969,-0.00124051,0.05640235$, $-0.00013890,0.00010893,0.00008013,0.00016722,0.00004687,-0.00007895,-0$ $.00005514,-0.00009450,0.00001723,-0.00003093,0.00003736,-0.00005456,0$. $00006820,0.00002788,0.00006043,-0.00011422,-0.00010759,0.00003585,0.00$ $007492,-0.00007240,0.00006277,0.00000464,-0.00001930,0.00004939,0.0000$ $0987,-0.00000361,-0.00000091,0.00000173,-0.00000311,0.00000733,0.00000$ $577,-0.00000662,0.00001554,0.00000121,0.00000412,0.00000163,-0.0005419$ $9,-0.00537374,-0.01447987,-0.00460254,0.00208220,-0.00098130,0.0001021$ $7,-0.00048489,0.00085581,-0.00220808,0.00524030,-0.00151031,-0.0010712$ $4,0.00369432,0.00075726,-0.03179766,-0.13273830,-0.13695591,0.00150699$ ,-0.00105582,0.00109190,-0.00011013,-0.00013807,-0.00070853,0.00027105 $,-0.00044268,0.00020373,0.00184542,-0.00227928,0.00176571,0.03662035,0$ $.13157546,-0.00062058,-0.00068960,-0.00036305,-0.00014261,0.00010147,-$ $0.00001811,-0.00000568,-0.00007079,0.00007098,0.00002376,0.00005946,-0$ $.00001151,-0.00002728,0.00004332,-0.00002773,0.00004638,-0.00001479,0$. $00003927,0.00018532,0.00000212,-0.00032748,0.00003232,-0.00005792,0.00$ $001965,0.00001684,-0.00000151,-0.00000168,0.00001570,0.00001245,-0.000$ $01299,-0.00001710,0.00001230,-0.00002528,0.00000955,0.00000279,-0.0000$ $0371,0.00305579,-0.00023087,-0.00020472,0.00068033,-0.00120361,0.00244$ $318,0.00031670,0.00076955,0.00004763,0.00489592,-0.00132550,-0.0000260$ $5,-0.00507719,-0.01252549,-0.01267460,-0.03297104,-0.13822007,-0.24340$ $068,-0.00034647,0.00156334,0.00048287,0.00008180,-0.00005560,0.0001600$ $6,-0.00018082,0.00019251,-0.00020275,-0.00112697,0.00180582,-0.0000156$ $5,0.03116167,0.14980621,0.25406595,0.00171050,-0.00092737,-0.00000139$, $0.00533121,0.00111373,0.00048767,0.00137615,-0.00278311,0.00196696,-0$. $00067125,0.00059075,0.00048927,0.00003021,0.00005148,-0.00005739,-0.00$ $019654,-0.00045026,0.00021812,-0.00006706,-0.00013333,0.00001476,-0.31$ $582078,0.11136076,-0.13251768,-0.00024648,0.00026362,-0.00100698,-0.00$ $001089,-0.00001347,-0.00009942,0.00008739,0.00003148,-0.00002370,0.000$ $02228,-0.00007258,-0.00001792,0.00005932,0.00008145,0.00005218,0.00007$ $601,-0.00001072,-0.00010283,-0.00002170,0.00007785,-0.00000827,0.00004$ $387,0.00011993,-0.00016917,0.00007398,-0.00012976,-0.00017195,-0.00010$ $925,-0.00002530,0.00012309,0.00000250,-0.00000416,0.00000764,0.0000001$ 4,0.00000486,-0.00000302,0.00003354,-0.00000309,0.00005744,0.00000576, $0.00004365,0.00001687,-0.00000928,0.00000786,-0.00000059,0.32528438,-0$ $.00333322,-0.00453690,0.00250815,0.03142114,-0.01480829,0.01596520,0.0$ $0260846,0.00256970,-0.00227413,-0.00000540,-0.00093679,-0.00019566,-0$. $00012775,0.00017042,-0.00013072,0.00193835,0.00016691,0.00047892,0.000$ $03043,0.00023764,0.00022415,0.09870197,-0.09158094,0.08177937,-0.00148$ $709,0.00007328,0.00027652,-0.00012206,0.00004243,-0.00003467,-0.000178$ 42,-0.00051682,0.00024505,0.00001602,0.00011979,0.00003190,0.00013375, $-0.00000129,-0.00009692,0.00002929,0.00002030,-0.00001685,0.00001467,0$ $.00008437,-0.00005923,-0.00066215,0.00016266,0.00028191,0.00002690,0.0$ $0005140,-0.00010596,-0.00002739,-0.00002206,-0.00001853,-0.00008383,-0$ $.00000336,0.00008159,-0.00001251,0.00000217,-0.00000168,0.00004298,0$, $0.00003770,0.00000881,-0.00008168,0.00001117,0.00001355,-0.00000265,-0$ $.00000458,-0.12072558,0.10544593,0.00059964,0.00241026,0.00157412,-0.0$ 
$0859251,-0.00475177,-0.00530404,0.00046997,-0.00098902,-0.00025252,0.0$ $0001301,0.00045907,-0.00060467,0.00060049,-0.00007084,0.00005699,-0.00$ $093029,0.00010200,0.00059007,0.00073283,-0.00027917,0.00042883,-0.1578$ $1539,0.10398153,-0.11042685,0.00064145,0.00023577,0.00006697,0.0000652$ $9,-0.00003211,-0.00005558,-0.00000338,0.00015966,-0.00003017,-0.000019$ $35,0.00002975,0.00007135,-0.00007476,-0.00007312,-0.00008579,-0.000091$ $55,0.00000006,0.00015389,0.00000353,-0.00024192,0.00004340,0.00003692$, $-0.00014851,-0.00028512,-0.00003726,0.00012371,0.00035751,0.00012293,0$ $.00006028,-0.00010797,-0.00001163,0.00000075,-0.00000056,-0.00001198,0$ $.00000379,0.00000438,-0.00000028,0.00003127,-0.00004470,-0.00001483,-0$ $.00005650,0.00000425,0.00001606,-0.00001761,-0.00000056,0.14868349,-0$. $09135146,0.10736696,-0.00003271,0.00117133,-0.00101436,0.00079055,-0.0$ $0639233,0.00181094,0.00221223,0.00234417,0.00100717,0.00025405,-0.0004$ $4746,-0.00022814,0.00019112,-0.00015304,0.00023218,-0.00043314,0.00014$ $184,-0.00028612,-0.00011426,-0.00000699,-0.00015560,-0.34004744,-0.071$ $98088,0.13997854,0.00023761,0.00023239,0.00030446,-0.00003946,0.000046$ $07,0.00009466,0.00001785,0.00001355,-0.00008974,0.00004186,-0.00004370$ ,-0.00008097,-0.00058505,-0.00038481,-0.00008297,-0.00052493,0.0000145 $6,0.00065367,-0.00015194,-0.00038940,0.00004867,0.00080292,0.00018155$, $-0.00050099,-0.00029667,0.00056788,0.00069399,0.00058978,0.00015690,-0$ $.00045350,0.00014496,0.00000071,-0.00017927,-0.00000307,0.00000125,0.0$ $0002730,-0.00018749,0.00014978,-0.00028590,-0.00000495,-0.00013320,-0$. $00009266,0.00001108,-0.00001657,-0.00000575,-0.01698401,-0.00822089,0$. $01561763,0.35411113,-0.00077429,0.00035832,0.00001649,-0.03531651,-0.0$ $1146463,0.01359066,0.00334291,-0.00350198,0.00349784,-0.00145124,-0.00$ $096593,0.00014376,-0.00036173,0.00019567,-0.00006835,-0.00011068,0.000$ $02080,-0.00034237,-0.00014278,0.00000393,-0.00015876,-0.06082978,-0.06$ $325352,0.06256452,-0.00001534,0.00012240,-0.00007340,0.00004798,0.0000$ $2395,0.00002743,0.00005357,0.00006096,-0.00004569,0.00002784,-0.000525$ $87,0.00005671,0.00035572,0.00003017,-0.00012137,0.00002274,-0.00004342$ $, 0.00006265,0.00084001,0.00036606,0.00007475,0.00056120,0.00014077,-0$. $00002069,-0.00020453,-0.00029985,-0.00079628,-0.00019161,-0.00012532,-$ $0.00004404,-0.00029753,-0.00000717,0.00028419,-0.00007812,0.00002427,0$ $.00001529,-0.00011829,0.00004641,-0.00019129,-0.00002537,-0.00003871,0$ $.00003565,-0.00001276,0.00000513,0.00002900,0.01153131,0.00334377,-0.0$ $0958589,0.08314729,0.07548380,0.00042266,0.00016148,0.00001665,0.00661$ $140,-0.00605813,-0.00656356,-0.00032620,0.00123881,0.00099875,-0.00027$ $981,0.00061735,0.00137987,0.00089054,-0.00016055,0.00020813,-0.0002766$ $8,0.00005870,-0.00031555,-0.00067882,-0.00010035,-0.00093816,0.1687864$ $8,0.08173878,-0.10546085,0.00029768,-0.00033624,-0.00053945,-0.0000523$ $9,-0.00002032,-0.00000986,-0.00004262,-0.00021974,0.00003796,-0.000042$ $57,0.00002609,-0.00006867,-0.00018451,-0.00024777,-0.00024427,-0.00018$ $422,-0.00011089,0.00029202,0.00054936,0.00016973,0.00021051,-0.0004141$ $4,-0.00024556,0.00046235,-0.00015742,0.00010448,0.00021636,0.00035366$, $0.00003776,-0.00041888,0.00001789,-0.00000470,-0.00005263,-0.00001766$, $0.00001999,0.00000953,-0.00033387,0.00002718,-0.00031917,-0.00001561,-$ $0.00009116,-0.00006735,0.00002724,-0.00002293,-0.00001304,-0.01793716$, $-0.00763123,0.00647983,-0.15701324,-0.06895079,0.10469950 \backslash 1-0.00000220$ ,-0.00000713,0.00001202,-0.00000851,0.00000441,-0.00001133,0.00000751, $0.00001907,-0.00000487,0.00000339,-0.00000339,-0.00000371,-0.00000437$, $-0.00001133,0.00000478,0.00000290,0.00001918,-0.00000521,-0.00000361$, 
0.00000055,0.00000027,-0.00000696,-0.00001979,-0.00001797,-0.00000440, $-0.00000334,-0.00000552,0.00000046,0.00000046,-0.00000169,-0.00000258$, $-0.00000476,0.00000295,-0.00000639,0.00000024,-0.00000341,0.00008365$,$0.00000439,-0.00006715,-0.00004172,-0.00000356,0.00003147,0.00000028,0$ $.00000251,-0.00000423,0.00001213,0.00000224,0.00000280,0.00000686,-0.0$ 0000874,-0.00001409,-0.00002992,0.00000953,0.00004900,-0.00002364,0.00 $000207,0.00003758,0.00001030,0.00000585,0.00000050,0.00000147,0.000002$ $86,0.00000108,0.00000575,-0.00000112,-0.00000252,0.00000721,-0.0000047$ $8,-0.00000647,0.00000714,0.00000096,-0.00000655,-0.00001476,0.00000352$ ,0.000012251I!@

meta-Addition Product

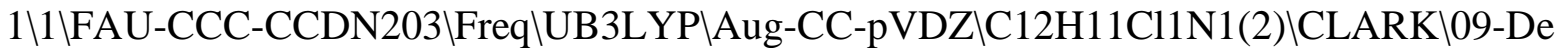
c-2014\0\\# ub31yp/aug-cc-pvdz geom=check guess=read freq name=clark \ Addition product p-Cl-Ph-radical + aniline (meta) $\backslash 0,2 \backslash \mathrm{C},-6.4281804961$ ,1.6681614897,1.5449915178\C,-5.1235867367,0.9727107265,1.8381700135\C ,-3.8968721828,1.568820655,1.6623948654IC,-3.815690458,2.9054386439,1. $1629701156 \backslash \mathrm{C},-4.9981827482,3.6189888785,0.8496213285 \backslash \mathrm{C},-6.2403314631,3$ $.0686679096,1.0192212466 \backslash \mathrm{H},-6.957998721,1.0890423356,0.7596276519 \backslash \mathrm{H},-5$ $.1823000424,-0.0476424265,2.2217517692 \backslash \mathrm{N},-2.7014555592,0.9239537647,2$. $0265425627 \backslash \mathrm{H},-4.9104160208,4.6380202023,0.4686389674 \backslash \mathrm{H},-7.1382016964,3$ $.6395460895,0.7817321647 \backslash \mathrm{H},-2.840788395,3.3772307793,1.0399298497 \backslash \mathrm{C},-9$ $.1066158858,1.6678039447,4.9560935963 \backslash \mathrm{C},-9.5303813596,1.0978402395,3.7$ $576517535 \backslash \mathrm{C},-8.6568464527,1.0973117176,2.6637544614 \backslash \mathrm{C},-7.3767897741,1$. 6557404835,2.7546036015\C,-6.9794474426,2.2210438522,3.975728337\C,-7. $8345084743,2.2323012705,5.0787313005 \backslash \mathrm{Cl},-10.1940975273,1.674487632,6.3$ $46341161 \mathrm{VH},-10.5240678583,0.6603066901,3.6794767457 \mathrm{VH},-8.9863982009,0$. $6495058772,1.7247386247 \backslash \mathrm{H},-5.9846623722,2.6573259512,4.0672369351 \backslash \mathrm{H},-7$ $.5210799255,2.6726410378,6.0238447357 \mathrm{H},-2.7706582597,-0.0830197131,2$. $1066266734 \backslash \mathrm{H},-1.8923866723,1.1949139687,1.4811968192 \mathrm{IV}$ Version=EM64L-G0

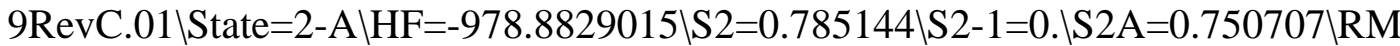
$\mathrm{SD}=8.272 \mathrm{e}-09 \backslash \mathrm{RMSF}=2.750 \mathrm{e}-06 \backslash$ ZeroPoint $=0.1961114 \backslash \mathrm{Thermal}=0.2084118 \backslash \mathrm{ZPE}=$ 123.0617438\Dipole $=0.8468535,-0.2719867,-0.9404151 \backslash$ DipoleDeriv $=0.09991$ $13,-0.030648,0.0351583,-0.0871232,-0.0024201,0.1697083,-0.0213997,0.07$ $43347,0.2517515,-0.371228,0.2670059,-0.0991586,-0.1047037,0.0573074,-0$ $.0599437,0.1791847,-0.1043026,-0.3040121,1.1732642,-0.634693,0.2296499$ $,-0.5621644,0.0187403,0.0230466,0.3247688,-0.0249969,-0.0016362,-0.037$ $0872,0.0376326,0.0277556,0.3470996,0.0625481,-0.1170947,-0.0967511,-0$. $0684863,-0.1257156,0.1655635,0.1085893,-0.0764794,-0.1412327,0.1255581$ ,-0.0340227,0.0696999,-0.0789242,-0.1089768,-0.1272937,0.0096002,0.084 $6158,0.1000301,-0.0496303,-0.1530792,0.077445,-0.0251434,-0.2319,-0.06$ $94669,-0.0851617,-0.0664456,-0.0685917,0.0143861,-0.0686362,-0.0582562$ - $-0.0494365,-0.0131294,0.034136,0.016397,-0.0166125,0.0128378,-0.08091$ $22,0.0684113,-0.0322263,0.0855762,0.1083011,-1.1321067,0.3374572,-0.11$ $58392,0.414239,-0.2674856,-0.0478451,-0.6424965,0.0979946,-0.3666364,0$ $.0491934,-0.0078329,0.017584,-0.0324094,-0.0858815,0.1011606,-0.01379$, $0.0762624,0.1058576,-0.0400306,0.0539132,-0.0379521,0.0560869,0.012755$ $1,0.0389838,-0.0299091,0.0311842,0.1199427,-0.0950892,-0.049085,0.0242$ $442,-0.0467946,0.0426914,0.0353516,-0.0029109,0.0285408,0.1244696,0.46$ $91859,0.0283648,-0.570575,-0.0065969,-0.0330054,0.0424491,-0.6723546,0$ 
$.0072106,0.798885,-0.0655593,0.0513915,0.1164014,0.064246,-0.0715348,-$ $0.0049492,0.1683564,0.0079082,-0.2616591,-0.0016885,0.0418579,0.012682$ $5,0.0723516,-0.0615374,0.0478132,0.0967224,0.0845717,0.0106235,0.13820$ $01,0.0316984,-0.17378,0.0288265,-0.0442314,-0.0435994,-0.2817834,-0.06$ $05375,0.1415565,-0.0612773,0.0302758,0.0600281,0.0290946,-0.0616898,0$. $0247641,0.0775224,0.024624,-0.0907823,-0.1187418,0.0147549,0.1031648,0$ $.0318532,-0.0595398,0.0453975,0.1354775,0.0634434,-0.0766754,-0.437568$ $6,-0.0167192,0.397623,-0.0062829,-0.0789596,-0.0069623,0.4450956,0.007$ $3232,-0.5993145,-0.0289366,-0.0610644,0.0115438,-0.0594139,0.099916,-0$ $.0120799,0.0204084,-0.0076337,0.0793405,0.0788776,-0.0190562,-0.011085$ $2,-0.027288,0.0828201,-0.0665338,-0.0386599,-0.0766147,-0.0638326,-0.0$ $270152,-0.0559655,-0.005336,-0.0650055,0.0723923,0.0017524,-0.0177901$, $-0.0072209,0.093297,0.0733791,-0.0253509,-0.0116243,-0.0191315,0.09962$ $61,-0.0543146,-0.0042622,-0.0493204,-0.0195102,0.132439,-0.0660129,0.0$ $210605,0.0018399,0.0808483,0.006955,0.1192942,-0.0555625,0.2483048,0.1$ 989394,0.022651,0.0433759,0.0682331,0.1272386,0.0632672,0.1986145,0.01 $92055,0.1814509 \backslash$ Polar $=218.5097223,12.6206101,137.0545282,-40.2074364,-$ 7.5406701,167.6400818 $\mathrm{PG}=\mathrm{C} 01[\mathrm{X}(\mathrm{C} 12 \mathrm{H} 11 \mathrm{Cl1N} 1)] \mathrm{NImag}=0 \backslash 10.47712056,0.01$ $675397,0.48165985,0.04642064,0.01123967,0.44165723,-0.16537236,0.05603$ $610,-0.02587494,0.64311874,0.04498592,-0.11094022,0.02255513,0.0722203$ $5,0.58735722,-0.02520224,0.02218171,-0.06708759,-0.00929345,-0.1775976$ $0,0.17823245,-0.03123733,-0.00845419,0.00118445,-0.30924340,-0.1094702$ $9,0.03332119,0.68637607,0.00418939,0.00831190,-0.00603133,-0.06190667$, $-0.13988488,0.03016354,-0.01090716,0.52983935,-0.00398204,-0.00362221$, $0.00264282,0.01463500,0.02930340,-0.06255484,0.01774068,-0.14579245,0$. $20205823,0.00112268,0.00420790,-0.00134645,0.01108352,-0.02638271,0.01$ $013846,-0.11855394,-0.04667612,0.01386086,0.68236836,0.00229572,-0.002$ $89645,-0.00129461,-0.05962748,-0.04248338,0.01699306,0.04319696,-0.202$ $36678,0.05688808,0.04263262,0.51834683,-0.00023882,-0.00142609,-0.0074$ $7872,0.02209816,0.01728224,0.00204984,-0.01761437,0.05365971,-0.077189$ $99,0.01263871,-0.15705612,0.15608060,-0.00342923,-0.01424932,0.0033293$ $7,-0.03121305,-0.01374594,0.00435324,0.04401521,0.06950872,-0.02357521$ $,-0.20901624,0.02083900,-0.01609501,0.60704961,-0.02635692,-0.01139566$ $, 0.00794281,-0.00094057,0.00085354,-0.00050059,0.02799324,-0.02316689$, $0.01041627,0.10330063,-0.15974034,0.04534380,0.05085889,0.64281432,0.0$ $0929869,0.00697735,0.00553571,-0.00115292,-0.00098805,0.00029991,-0.00$ $848017,0.01113742,-0.00387370,-0.04503374,0.04239747,-0.06702933,-0.00$ 201801,-0.19644309,0.19447827,-0.08509653,-0.01031483,0.00211404,0.015 $58756,0.03329943,-0.01176713,-0.01314344,-0.02883412,0.01043779,-0.077$ $39682,0.03683432,-0.01628464,-0.31726963,-0.13565646,0.04241201,0.7440$ $3820,0.00103199,-0.18543872,0.04299382,0.01462382,-0.01668286,0.005819$ $95,-0.01285952,-0.01717754,0.00530665,-0.00352228,0.03763932,-0.010273$ $56,-0.08755855,-0.16118276,0.03874876,0.00755933,0.50250407,-0.0029730$ $0,0.04337716,-0.07842765,-0.00559518,0.00813673,-0.00243766,0.00422344$ $, 0.00561725,-0.00285338,-0.00126780,-0.00963024,0.01250733,0.02529538$, $0.03774417,-0.06510783,0.01319282,-0.14157907,0.17843155,-0.08704477,-$ $0.05680789,-0.08466567,-0.02255290,-0.00998167,-0.01699217,-0.00007046$ $, 0.00245587,-0.00287087,-0.00138339,0.00017484,-0.00020183,0.00269941$, $-0.00028327,0.00006054,-0.00314151,-0.00804431,0.00001754,0.10976066$,$0.05282868,-0.10424903,-0.08812799,0.00586490,0.00980280,0.00905340,0$. $00151667,0.00155722,0.00030060,-0.00123477,-0.00076203,-0.00003150,0.0$ 
$0317536,-0.00384586,0.00064992,-0.01825607,-0.02121334,-0.01559822,0.0$ $6305282,0.11787446,-0.07457730,-0.08232446,-0.15194302,-0.00671280,-0$. $00075371,-0.00136706,0.00116069,0.00114253,-0.00176757,-0.00030470,0.0$ $0005015,-0.00013467,0.00132884,0.00071865,-0.00106867,0.00565912,0.004$ $75735,0.00945495,0.08164792,0.09269797,0.17587801,0.00331823,0.0249407$ $0,-0.00804493,-0.05656825,-0.01471538,0.00477293,0.00215635,-0.0263768$ $7,0.00978970,-0.00498273,-0.00134644,0.00017744,-0.00044620,0.00024151$ $,-0.00018319,-0.00541035,0.00104458,-0.00051828,0.00071838,-0.00066649$ ,-0.00025036,0.05770692,-0.00281121,-0.00971977,0.00331127,-0.01738051 $,-0.29338143,0.09975194,0.00366144,-0.01056236,0.00623356,-0.00277155$, $0.00178596,0.00175672,-0.00026519,0.00022527,-0.00040106,0.00300450,0$. $00112225,0.00044564,0.00047828,0.00017096,0.00037825,0.01705164,0.3099$ $4633,0.00157450,0.00470383,-0.00228456,0.00592940,0.10155531,-0.063296$ $76,-0.00265814,0.00482860,0.00406420,0.00057759,0.00174944,0.00569521$, $0.00020543,-0.00018878,-0.00069878,-0.00127484,0.00004799,0.00152656,0$ $.00079937,-0.00036669,0.00057888,-0.00537017,-0.11027229,0.05758987,0$. $00323528,-0.00333626,0.00193224,-0.05564142,0.01700804,-0.01110256,-0$. $23395135,0.06455182,-0.04942450,-0.00176895,0.00675606,-0.00295213,-0$. $00095533,-0.00339664,0.00135147,-0.00245759,0.00030208,0.00006231,-0.0$ $0138081,0.00113800,-0.00086026,0.00199222,0.00113880,-0.00002138,0.645$ $55846,-0.00236683,-0.00027392,0.00159370,-0.01406446,0.01120224,-0.001$ $82662,0.08223375,-0.13569031,0.05468747,0.04175580,-0.03112037,0.01780$ $327,-0.00926634,-0.00304390,0.00262534,0.00361413,0.00130727,-0.000697$ $70,-0.00002134,-0.00056718,-0.00000161,-0.00105495,0.00117146,-0.00117$ $665,0.00766264,0.68049443,0.00378986,0.00227255,0.00706588,-0.00254516$ ,-0.00258032,0.01016809,-0.01132292,0.03052641,-0.06040086,-0.01567263 $, 0.01633218,-0.00175486,0.00397016,0.00107611,0.00270070,-0.00290891,-$ $0.00049861,-0.00042330,0.00074230,-0.00021376,-0.00018218,0.00030712$,$0.00193018,-0.00377470,-0.15702483,-0.16533063,0.19174013,-0.00411513$, $-0.00059553,0.00003572,-0.00081317,0.00001871,0.00001935,-0.00387225,0$ $.00213768,-0.00129115,0.00585941,0.02388187,-0.00887735,-0.05949772,-0$ $.02017615,0.00697189,0.00152841,-0.02792997,0.01039301,-0.00008185,-0$. $00063941,0.00020525,0.00015008,0.00001614,0.00002821,0.00041806,0.0012$ $5719,-0.00032980,0.05893634,-0.00168497,0.00288494,0.00243771,0.000133$ $48,0.00048012,-0.00042656,0.00260708,0.00163023,0.00161643,-0.00380582$ ,-0.01096461,0.00495686,-0.02050078,-0.29654151,0.09714546,0.00104379, $-0.00914347,0.00472731,-0.00010237,0.00017364,-0.00011646,0.00000110,-$ $0.00013861,-0.00015672,-0.00018114,-0.00082036,0.00030889,0.02251235,0$ $.31258447,0.00044203,0.00296763,0.00819338,-0.00009236,-0.00053438,-0$. $00049297,-0.00118517,0.00153334,0.00486044,0.00171055,0.00591648,0.000$ $68872,0.00645715,0.09671479,-0.07372805,0.00002173,0.00370845,0.002254$ $77,0.00010085,0.00136559,-0.00068911,0.00003389,-0.00018729,-0.0005004$ $9,-0.00000818,0.00033125,-0.00005018,-0.00747633,-0.10745798,0.0657616$ $5,0.00502877,-0.00434253,0.00257366,0.00051581,-0.00130871,0.00059680$, $-0.00003852,-0.00051460,0.00012597,-0.00261565,-0.00383155,0.00137660$, $-0.01814582,0.01731781,-0.00717217,-0.24722493,0.12105995,-0.05161731$, $0.00025091,0.00052531,-0.00028133,0.00039674,-0.00019561,0.00005345,0$. $00013768,0.00019125,-0.00001704,0.00095687,-0.00009088,0.00013516,0.26$ $090158,0.02293607,-0.01071416,0.00572425,-0.00326913,-0.00423686,0.001$ $98676,-0.00028518,0.00017751,-0.00034319,-0.00283076,0.00005965,0.0025$ $7159,-0.01287228,0.00937417,-0.00199242,0.12393990,-0.12983796,0.04206$ 
$629,-0.00007493,0.00086036,-0.00069469,0.00154810,-0.00062317,0.000524$ $38,0.00029668,0.00005424,-0.00009620,0.00028704,0.00027399,-0.00208757$ ,-0.12935787,0.13365837,-0.00757109,0.00359666,-0.00202619,0.00120488, $0.00214565,0.00059508,0.00012241,-0.00020934,-0.00041751,0.00061086,0$. $00246730,0.00629492,0.00368196,-0.00123553,0.00535035,-0.05183088,0.04$ $147285,-0.04471576,0.00019989,0.00098552,0.00041995,-0.00059341,0.0004$ $3081,0.00046714,-0.00005670,-0.00019046,-0.00029964,0.00013593,-0.0020$ $7672,-0.00451865,0.05476116,-0.04681706,0.03748209,0.00046662,0.000265$ $25,0.00002441,0.00146214,0.00030745,-0.00027160,-0.00102485,0.00259273$ ,-0.00100624,-0.28071384,-0.11017408,0.02733779,-0.01659345,-0.0138593 $3,0.00418473,-0.00218601,0.00383263,-0.00177254,0.00000839,-0.00000991$ ,-0.00002817,0.00007647,0.00002273,0.00001499,0.00159964,0.00066586,-0 $.00016690,0.00089650,0.00016175,0.00013884,-0.00103741,-0.00063693,0.0$ $0021995,0.29654644,0.00050323,-0.00013402,-0.00029679,0.00105941,-0.00$ 486688,0.00294126,-0.02604590,-0.00581883,0.00208715,-0.11016383,-0.10 $772467,0.02544564,0.01460143,0.01007985,-0.00196735,0.00318134,-0.0010$ $0636,0.00198619,-0.00004104,-0.00001474,0.00002260,-0.00139137,-0.0005$ $7266,0.00021991,-0.00098963,0.00134349,-0.00089999,-0.00034104,0.00045$ $824,-0.00118402,0.00082288,0.00035271,-0.00003979,0.11753711,0.1085729$ $3,-0.00026092,-0.00037840,-0.00096846,-0.00041502,0.00302277,0.0019807$ $1,0.01006117,0.00367316,0.00266734,0.02599342,0.02532288,-0.03121647,-$ $0.00548924,-0.00317229,0.00370653,-0.00157944,0.00197644,0.00314028,-0$ $.00013529,-0.00007249,0.00012734,0.00049258,0.00023641,-0.00012435,-0$. $00021614,-0.00174941,-0.00142124,0.00028211,-0.00120213,-0.00220879,-0$ $.00035414,-0.00008934,0.00029052,-0.02886664,-0.02794544,0.02376899,-0$ $.00133125,-0.00019935,-0.00000698,-0.00046982,-0.00004610,0.00045195,0$ $.00023188,0.00018149,0.00033581,0.00018129,0.00009126,-0.00000884,0.00$ $014117,0.00011592,0.00017850,-0.00010504,-0.00002946,0.00060819,-0.000$ $09339,0.00007557,0.00019076,-0.00005134,-0.00002693,-0.00001474,-0.000$ $01904,0.00002169,-0.00026356,0.00008035,0.00000285,-0.00012027,-0.0000$ $1694,-0.00012055,0.00001394,0.00003988,0.00001552,0.00002174,0.5220811$ $1,-0.00048634,-0.00052465,-0.00013579,-0.00036043,-0.00014645,0.000460$ $51,0.00046142,-0.00008694,0.00023597,0.00003409,0.00018516,-0.00000701$ $, 0.00017011,-0.00003770,0.00013554,-0.00002655,0.00009204,0.00046067,-$ $0.00005084,0.00025607,0.00011993,0.00004474,-0.00006812,-0.00008010,-0$ $.00028760,0.00011695,-0.00029311,0.00001626,-0.00002724,-0.00010821,-0$ $.00002786,-0.00007900,0.00004682,-0.00001786,-0.00000478,0.00000403,0$. $17080539,0.24065220,-0.00024511,-0.00002057,-0.00064160,-0.00049099,-0$ $.00011813,0.00073513,0.00088492,-0.00045604,0.00041337,0.00000680,0.00$ $037098,-0.00007155,0.00022311,-0.00005463,0.00009886,-0.00015002,0.000$ $20573,0.00064668,-0.00000043,0.00017035,0.00031377,0.00002739,-0.00009$ $198,-0.00009604,-0.00087119,0.00032597,-0.00062670,0.00000866,-0.00010$ $298,-0.00008859,-0.00002910,-0.00002105,-0.00011598,-0.00005755,-0.000$ $01714,0.00001048,0.02112869,0.13771958,0.51673104,-0.00146801,-0.00365$ $932,-0.00125397,0.00005271,-0.00000122,0.00061826,0.00022033,-0.000101$ $34,-0.00004263,0.00006069,0.00011604,-0.00000299,0.00019824,-0.0003104$ $9,0.00011221,-0.00078262,0.00043408,0.00077163,0.00009366,0.00022322,0$ $.00023032,-0.00008053,0.00000810,-0.00005718,-0.00016076,0.00008605,-0$ $.00011105,-0.00005570,-0.00004902,-0.00009285,-0.00007146,-0.00015661$, $0.00005134,-0.00000735,0.00000255,0.00000306,-0.13237603,-0.05820575,-$ $0.09163940,0.66342286,-0.00387348,0.00469242,-0.00269466,-0.00075502,-$ 
$0.00045498,0.00097522,-0.00040000,0.00022807,0.00002415,0.00000884,-0$. $00000430,-0.00005519,-0.00018369,0.00036484,-0.00028593,0.00125439,-0$. $00008535,-0.00076488,0.00016982,0.00009762,0.00017079,-0.00005422,-0.0$ $0001235,-0.00001311,-0.00003597,-0.00008530,0.00000410,0.00007838,0.00$ $007392,0.00014008,0.00002174,-0.00004652,0.00000554,0.00001746,0.00000$ $006,0.00001018,-0.03964971,-0.11461981,-0.10241327,0.20461080,0.260274$ $51,-0.00073453,-0.00239812,0.00192779,0.00090997,0.00043450,-0.0011146$ $9,-0.00074977,0.00014328,-0.00051963,-0.00019381,-0.00037700,0.0000949$ $2,-0.00015943,0.00002244,-0.00016974,-0.00021644,-0.00007527,-0.000668$ $03,-0.00005888,-0.00019148,-0.00022933,-0.00000636,0.00015274,0.000081$ $15,0.00075603,-0.00025781,0.00066961,-0.00006635,0.00003656,0.00013318$ $, 0.00007617,0.00008366,0.00002012,0.00002769,0.00002010,-0.00000609,-0$ $.03505377,-0.07867548,-0.26014371,-0.04878761,0.13835502,0.61080987,-0$ $.01902959,-0.00832745,0.00529476,0.00042063,-0.00143502,0.00017669,-0$. $00079659,0.00067167,-0.00047431,-0.00003561,-0.00006548,0.00011388,0.0$ $0038215,-0.00050554,0.00008185,-0.00131134,0.00117421,-0.00150641,0.00$ $026917,-0.00091067,-0.00293209,-0.00012824,0.00041737,0.00005721,0.000$ $16703,-0.00027294,0.00036924,-0.00022374,-0.00001896,0.00015871,0.0002$ $0317,-0.00029136,0.00030371,0.00001335,0.00001538,0.00001733,0.0519589$ $5,0.03230115,0.04164024,-0.19834351,-0.03000212,0.07985387,0.59480360$, $-0.00219550,0.00390745,0.00076729,0.00085665,-0.00079999,-0.00091824,0$ $.00006088,0.00061773,-0.00031102,0.00009292,-0.00021729,0.00011466,-0$. $00022212,0.00013817,0.00002487,0.00029637,0.00011731,-0.00051771,0.001$ $08450,0.00008285,-0.00053045,-0.00022734,0.00017359,0.00006036,0.00025$ $631,-0.00013259,0.00033903,0.00004091,0.00002818,-0.00004208,0.0001120$ $7,0.00018713,0.00003384,0.00000294,0.00000038,-0.00001999,0.01567595,0$ $.01263321,-0.00318616,-0.01083074,-0.07545231,-0.02713502,0.17715242,0$ $.25995437,0.02434940,0.00864549,0.00007477,0.00121283,0.00134979,-0.00$ $205826,0.00046500,-0.00044773,0.00031913,0.00028339,-0.00005588,-0.000$ $03573,-0.00011350,0.00083571,-0.00006243,0.00203305,-0.00094186,-0.000$ $89255,0.00273107,0.00160897,0.00138165,0.00010608,-0.00033860,0.000070$ $75,0.00046224,0.00007315,0.00010172,0.00023240,0.00023068,0.00006002,-$ $0.00022686,0.00010475,-0.00003485,0.00003224,-0.00002653,-0.00006146$,$0.00805106,-0.02373208,-0.05426805,0.13836095,-0.00312087,-0.24552009$, $-0.04235063,0.15909362,0.66868621,-0.10061866,0.00196947,0.04521542,-0$ $.03086671,0.00312859,0.02027443,-0.00011677,0.00447327,0.00075888,-0.0$ $0097302,-0.00023010,-0.00009884,0.00251386,0.00097565,0.00082637,-0.00$ $708352,-0.01288774,0.01018187,0.00375033,-0.00175823,-0.00916793,0.001$ $53712,-0.00116510,-0.00069132,-0.00058584,-0.00052596,-0.00090390,0.00$ $027099,-0.00001110,-0.00016846,0.00068088,0.00088188,-0.00130176,0.000$ $10734,0.00002608,0.00005470,-0.03579092,-0.02176927,-0.02942487,-0.036$ $58110,0.00101040,0.05773872,-0.28232478,-0.10237223,-0.03817210,0.5773$ $0149,-0.00385897,-0.06625331,0.00018687,0.00674771,0.00126682,-0.00925$ $380,0.00128337,0.00080376,-0.00134399,-0.00035228,-0.00086783,0.000594$ $72,0.00138124,-0.00417372,0.00139239,-0.02044527,-0.00170193,0.0238700$ $1,0.00811562,-0.00090926,-0.01614758,0.00013881,0.00056967,-0.00024141$ $, 0.00105533,-0.00025236,0.00108447,-0.00075011,-0.00083398,-0.00115065$ $,-0.00021528,0.00044918,-0.00039098,-0.00002258,0.00008822,0.00005909$, $-0.02203547,-0.02175905,-0.01737423,-0.01271766,0.01284078,0.03399767$, $-0.08280646,-0.11094707,-0.03333164,0.16166384,0.27959267,0.03352224,-$ $0.00701044,-0.13091485,0.00619366,-0.00604061,0.00781849,-0.00161171,-$ 
$0.00025059,-0.00107353,0.00045595,0.00096852,0.00000563,-0.00081830,-0$ $.00133531,-0.00094063,0.00925986,0.01227615,-0.01300318,0.01555942,-0$. $00173342,-0.02775335,-0.00109028,0.00097112,0.00066565,-0.00187563,0.0$ $0006504,-0.00082154,-0.00034140,-0.00012211,0.00042805,0.00007508,-0.0$ $0119344,0.00151744,-0.00010831,-0.00002047,0.00015849,-0.03064054,-0.0$ $1763181,-0.02234516,0.01645521,0.01740950,0.03852628,0.02028304,-0.009$ $88586,-0.12001978,-0.01646885,0.12307834,0.57843226,0.00791648,0.00813$ $386,0.01837947,0.00095734,0.00026507,0.00115532,0.00031032,-0.00070186$ $, 0.00096586,-0.00025496,0.00043141,-0.00033454,0.00002030,-0.00046613$, $0.00009373,0.00068337,0.00132470,0.00054804,-0.00174714,0.00028607,0.0$ $0460333,0.00005156,-0.00009771,0.00004571,-0.00072217,0.00030288,-0.00$ $076643,-0.00001638,-0.00010328,-0.00010285,0.00000917,-0.00014118,0.00$ $004184,-0.00004560,0.00000507,0.00005907,-0.03951989,-0.01426603,0.015$ $13793,-0.00325071,0.00319498,0.00991336,-0.00386718,-0.01495084,-0.030$ $24711,-0.13333615,-0.05101082,-0.07283410,0.65525925,0.00306504,0.0026$ $0276,-0.00081665,-0.00044502,0.00063529,-0.00091623,-0.00002060,-0.000$ $08366,-0.00032184,-0.00010703,-0.00042148,0.00009465,-0.00042208,0.000$ $01210,0.00033369,0.00005869,0.00041862,0.00043499,-0.00042901,0.000916$ $48,0.00036561,-0.00004682,0.00015125,0.00000856,-0.00020396,0.00001993$ ,-0.00017000,-0.00002987,0.00001722,-0.00007427,-0.00011849,0.00033231 ,-0.00016166,-0.00002712,0.00003753,-0.00000009,0.00223487,0.01368735, $0.01661092,0.00310822,-0.00672348,-0.01504107,-0.02780530,-0.00635457$, $-0.00767407,-0.03322754,-0.11119475,-0.09297430,0.19308886,0.25784859$, $0.00043407,-0.00868739,-0.02498790,-0.00147640,0.00075978,-0.00136040$, $-0.00111753,-0.00016824,-0.00019346,-0.00032001,-0.00042936,-0.0001461$ $5,-0.00049697,0.00004516,-0.00034140,-0.00034701,-0.00281477,-0.000108$ $42,0.00245073,-0.00047200,-0.00321685,0.00029531,-0.00025379,0.0001190$ $2,0.00066337,-0.00020619,0.00051931,0.00000425,0.00002930,0.00018948,-$ $0.00001883,0.00040595,-0.00016221,0.00003570,0.00003141,0.00003518,0.0$ $6456449,0.03677348,0.03814111,0.00955031,-0.01509710,-0.06039809,-0.06$ $885443,-0.02325546,0.02176047,-0.01977362,-0.07107011,-0.25950751,-0.0$ $6139533,0.13247395,0.61638388,0.00135275,-0.00256438,-0.00267164,0.000$ $31136,0.00015793,-0.00025669,-0.00043839,0.00005156,-0.00044352,-0.000$ $03159,-0.00013590,0.00007503,-0.00028095,-0.00019582,-0.00025305,-0.00$ $033053,-0.00029089,-0.00040168,0.00043742,-0.00036933,-0.00117130,0.00$ $005003,0.00006747,0.00004246,0.00033465,-0.00016250,0.00042011,-0.0001$ $0167,-0.00000820,0.00010125,0.00005747,0.00019442,0.00006393,-0.000011$ $58,-0.00001104,-0.00001768,-0.27607949,-0.08621027,0.00296908,-0.00292$ $676,-0.02726745,-0.06806499,-0.05255594,-0.01237811,0.02288971,0.05057$ $435,0.03058264,0.03791184,-0.19824203,-0.02929904,0.08365712,0.5896193$ $5,-0.00238469,0.00328011,-0.00311520,-0.00037508,0.00003900,0.00015661$ ,-0.00024716,0.00000764,-0.00013705,0.00009204,-0.00004965,-0.00010777 ,-0.00000439,0.00027656,0.00002653,0.00016781,-0.00023292,-0.00024104, $0.00016755,-0.00004362,-0.00052801,0.00006163,0.00001068,0.00010559,0$. $00006832,-0.00003485,0.00010490,0.00004234,0.00001752,0.00005982,0.000$ $02071,-0.00001820,-0.00009003,0.00000556,-0.00000573,0.00001902,-0.104$ $93865,-0.11255672,-0.01361519,-0.01414407,-0.00474207,-0.02423628,-0.0$ $1325340,-0.00672167,0.00672617,0.01661049,0.01335728,-0.00290394,-0.00$ $958194,-0.07526378,-0.03020413,0.17729374,0.25879930,-0.00297356,-0.00$ $345632,-0.00120525,-0.00070139,0.00030928,0.00077411,0.00061325,0.0001$ $1798,0.00035036,0.00011289,0.00013404,-0.00001142,0.00021767,0.0000880$ 
$5,0.00025458,-0.00036607,-0.00023381,0.00089250,0.00050341,0.00012331$, $-0.00062488,0.00006017,-0.00014659,-0.00015077,-0.00015703,0.00007325$, $-0.00027320,0.00004843,0.00000256,-0.00015559,-0.00001700,-0.00001082$, $-0.00009039,-0.00000990,-0.00001812,-0.00001417,-0.05422950,-0.0372135$ $2,-0.10996183,-0.02821700,-0.00764192,0.02096249,0.02122339,0.00572539$ ,-0.01194808,-0.00346356,-0.01983510,-0.04927007,0.14253891,-0.0057054 $7,-0.25704842,-0.03629328,0.16804888,0.68627452,0.00009954,0.00007748$, $-0.00029663,-0.00009877,-0.00021128,0.00040665,0.00039252,-0.00022946$, $0.00014121,-0.00006932,0.00020800,-0.00004881,0.00016172,-0.00013309,0$ $.00006346,-0.00026993,0.00032131,0.00017582,-0.00001093,0.00003327,0.0$ $0008003,0.00002742,0.00000295,-0.00004885,-0.00050660,0.00016017,-0.00$ $028824,-0.00004444,-0.00006856,-0.00002489,0.00002963,0.00001233,-0.00$ $003748,-0.00004379,-0.00001275,-0.00000623,-0.07929613,-0.00489252,0.0$ $5531496,-0.00074211,-0.00041937,0.00515752,0.00118248,-0.00144202,0.00$ $029988,0.00050163,0.00113681,0.00151403,-0.00182999,-0.00427420,-0.004$ $88372,-0.02327008,-0.00002873,0.03476430,0.10344317,0.00031778,-0.0005$ $2723,0.00026268,0.00003998,0.00009132,-0.00001407,0.00005617,-0.000020$ $07,0.00003983,0.00001003,0.00001173,0.00001220,-0.00002336,-0.00002947$ ,-0.00003088,-0.00007676,0.00000105,0.00001080,-0.00002596,0.00000010, $0.00003465,-0.00000723,-0.00000121,-0.00000470,-0.00000192,0.00000969$, $-0.00000875,-0.00000401,-0.00000282,-0.00000349,-0.00000174,0.00000640$ - $0.00000480,0.00000284,-0.00000016,-0.00000321,-0.00489376,-0.0270169$ $6,-0.00459723,0.00748383,0.00407014,-0.01034461,-0.00334910,0.00297325$ ,-0.00162566,0.00090789,-0.00007897,0.00065160,-0.00229579,0.00266354, $-0.00267792,-0.00786101,0.00423806,0.00937864,0.00727093,0.01710335,-0$ $.00005872,0.00050146,0.00028232,0.00018593,0.00028707,-0.00053392,-0.0$ $0055844,0.00029275,-0.00024056,0.00009132,-0.00028953,0.00006928,-0.00$ $021099,0.00025036,-0.00009000,0.00046669,-0.00041612,-0.00026065,-0.00$ $000033,0.00000635,0.00007012,-0.00003350,-0.00001945,0.00005922,0.0007$ 0814,-0.00022171,0.00043916,0.00006779,0.00010158,0.00003656,-0.000041 $51,-0.00002796,0.00004496,0.00005933,0.00001632,0.00000074,0.05540493$, $-0.00456964,-0.10686853,0.02917701,-0.00029454,-0.03340572,-0.00542771$ $,-0.00400122,-0.00044117,0.00114385,0.00039623,-0.00048493,0.00104139$, $-0.00027673,0.00234076,0.01055063,-0.00055892,-0.01069837,-0.09306121$, $0.00696336,0.14992012,-0.00028366,-0.00028551,-0.00047603,-0.00008769$, $-0.00012574,0.00010435,0.00006573,0.00003614,0.00003477,-0.00004066,0$. $00001816,-0.00001049,0.00005058,-0.00004613,0.00002847,-0.00011814,0.0$ $0007370,0.00009934,0.00003109,0.00000379,-0.00002884,-0.00001469,0.000$ $02126,-0.00001468,-0.00008995,0.00001541,-0.00004350,-0.00001172,-0.00$ $002295,-0.00001319,0.00000693,-0.00001702,0.00000251,-0.00000709,0.000$ $00037,0.00000295,-0.00577098,-0.00270118,0.00331998,-0.29191711,-0.112$ $78992,-0.01923091,-0.01186144,-0.00768729,-0.00386480,0.00087012,-0.00$ $122864,0.00393365,0.00029881,0.00068713,0.00014910,0.00207578,-0.00180$ $235,-0.00110616,0.00068141,0.00142962,-0.00004211,0.30618280,0.0001474$ $6,0.00005373,-0.00012536,-0.00004838,0.00006038,0.00009861,0.00000452$, $-0.00004789,0.00003478,-0.00001347,0.00004236,-0.00001569,0.00005634,0$ $.00000551,0.00001516,-0.00007450,-0.00001272,-0.00008400,-0.00000813,-$ $0.00000837,-0.00000571,0.00000997,-0.00000420,-0.00000125,-0.00003042$, $0.00001884,-0.00004159,-0.00000945,0.00000139,0.00001840,-0.00000743,-$ $0.00000659,0.00000312,-0.00000029,-0.00000242,0.00000010,-0.01182679,-$ $0.00129308,0.00064173,-0.11196407,-0.08630692,-0.01466958,0.00093066,0$ 
$.00373810,-0.00084571,-0.00131959,0.00580144,-0.00156604,0.00075376,-0$ $.00090078,0.00006394,-0.00163263,0.00364840,-0.00360608,0.00113594,-0$. $00194884,0.00113438,0.12275828,0.08069176,0.00076558,0.00039495,0.0002$ $8927,0.00009675,0.00004281,-0.00020898,-0.00001246,0.00003571,-0.00002$ $770,0.00003017,-0.00004693,0.00000713,-0.00004910,0.00004470,-0.000001$ $44,0.00013187,-0.00008981,-0.00005840,-0.00002151,-0.00001455,0.000027$ $46,0.00000784,-0.00001389,0.00000894,0.00008463,-0.00002100,0.00006250$ $, 0.00001409,0.00001823,0.00000061,-0.00000454,0.00002432,-0.00001096,0$ $.00000356,-0.00000087,-0.00000482,-0.02446584,-0.01091676,0.00100485,-$ $0.01668141,-0.01360850,-0.05516369,0.02228234,0.01002996,0.00594271,0$. $00340060,-0.00175472,-0.00283151,0.00026951,0.00012966,-0.00115265,-0$. $00051351,-0.00337829,-0.00410465,-0.00092565,0.00080539,0.00073713,0.0$ $1769645,0.01749911,0.05486548,0.00029771,0.00081073,-0.00273867,-0.000$ $05537,-0.00001721,0.00036385,-0.00009300,0.00010648,-0.00000611,0.0000$ $7370,-0.00003424,0.00003861,-0.00011888,0.00012631,-0.00006643,0.00033$ $951,0.00009579,0.00013483,0.00004689,0.00037471,0.00108991,-0.00005328$ ,-0.00003373,-0.00004988,0.00010996,-0.00005013,0.00003356,0.00003160, $0.00001776,-0.00002242,-0.00000787,0.00001307,-0.00004798,0.00001403,0$ $.00000600,-0.00000259,-0.00038062,-0.00188723,0.00345356,0.00857223,0$. $00804900,0.01792246,-0.07985659,-0.04136509,-0.06997466,-0.00392799,-0$ $.01120689,-0.02395340,-0.00442221,-0.00416190,0.00076598,-0.00098536,0$ $.00021028,0.00059530,-0.00024728,0.00021957,0.00119577,0.00024647,0.00$ $153027,-0.00084369,0.08067134,0.00214616,-0.00294885,0.00057938,0.0005$ $1007,0.00055183,-0.00012908,0.00003143,-0.00019311,-0.00000825,0.00004$ $250,0.00001125,0.00001854,0.00006152,-0.00019387,0.00011020,-0.0006903$ $7,0.00005058,0.00028441,-0.00025727,0.00033884,0.00045176,0.00006247,-$ $0.00003744,-0.00001170,0.00012625,0.00000223,0.00006221,-0.00003854,-0$ $.00003924,-0.00006492,-0.00004083,-0.00015809,0.00001510,0.00001036,-0$ $.00000272,0.00000165,-0.00176885,0.00580120,-0.00101374,-0.00087876,0$. $00332634,0.00081068,-0.04172416,-0.08751331,-0.10322153,-0.00262520,-0$ $.00097681,-0.01154216,-0.00437524,0.00408399,-0.00096673,0.00015596,-0$ $.00073428,0.00065854,-0.00010105,0.00014750,0.00003493,0.00164907,-0.0$ $0305759,0.00116465,0.04754516,0.08226267,0.00102131,0.00170899,0.00015$ $815,-0.00028128,0.00007477,0.00015544,0.00007940,0.00001761,0.00007734$ ,-0.00007013,0.00002738,-0.00001982,0.00008533,0.00010599,-0.00004576, $0.00024583,-0.00008319,0.00013917,-0.00017593,0.00003170,0.00058797,-0$ $.00006336,-0.00000020,-0.00003792,-0.00007117,0.00003082,-0.00008674,0$ $.00002814,0.00000873,-0.00001122,0.00000041,-0.00000869,-0.00002589,0$. $00000125,0.00000773,0.00000244,0.00379292,-0.00085146,-0.00047724,-0.0$ $0906137,-0.01034100,-0.01671771,-0.07031199,-0.10320478,-0.25892136,0$. $00107898,-0.00100830,-0.00072627,-0.00009349,-0.00143605,0.00216826,0$. $00043467,0.00061012,0.00025195,0.00020860,-0.00038054,-0.00118739,-0.0$ $0052885,0.00129211,0.00047140,0.07362774,0.11324450,0.27445198,-0.0007$ $0685,0.00134216,0.00069718,0.00014899,-0.00011718,0.00041098,0.0000215$ 9,0.00013986,-0.00001788,0.00007711,-0.00019195,0.00000643,-0.00002742 $, 0.00012933,0.00009788,0.00008907,0.00019880,-0.00000097,-0.00006447,0$ $.00000880,-0.00009725,-0.00001790,0.00002455,0.00000051,-0.00000822,-0$ $.00000633,0.00004934,0.00001882,0.00003644,0.00000196,-0.00003903,-0.0$ $0002762,-0.00009800,-0.00000904,-0.00000056,0.00000735,0.00107775,-0.0$ $0135595,0.00339529,0.00035808,0.00064385,0.00008963,0.00190550,-0.0021$ $9251,-0.00113745,-0.00486664,-0.00324490,0.00060481,-0.28755725,-0.109$ 
$51686,-0.02091125,-0.01234620,-0.00782611,-0.00345673,-0.00075049,-0.0$ $0024847,0.00026190,-0.00016832,0.00020061,-0.00007695,-0.00022069,-0.0$ $0010475,0.00013888,0.30297645,0.00059907,-0.00238997,0.00118615,0.0003$ $3110,0.00026559,0.00021998,0.00012392,-0.00030065,0.00044167,-0.000096$ $83,0.00045020,-0.00022192,0.00020689,-0.00019188,-0.00023462,-0.000212$ $55,0.00002535,0.00019917,0.00005410,-0.00006198,0.00004529,0.00000847$, $-0.00020506,-0.00017771,-0.00003880,0.00008904,-0.00008860,-0.00001341$ ,-0.00003360,0.00008871,0.00002727,-0.00000110,0.00009646,-0.00001269, $-0.00001502,0.00001435,-0.00154772,0.00612322,-0.00164550,0.00072637,-$ $0.00087754,0.00005926,-0.00206577,0.00431150,-0.00399606,-0.01163055,-$ $0.00098742,-0.00007097,-0.10917869,-0.08643435,-0.01508265,0.00092321$, $0.00392449,-0.00111654,0.00006668,0.00009141,-0.00010258,0.00020112,-0$ $.00057531,0.00021663,-0.00057391,-0.00016443,0.00001157,0.12049066,0.0$ $8023491,-0.00075199,0.00073300,0.00004449,-0.00012628,0.00006974,0.000$ $35622,0.00069168,-0.00024119,-0.00007887,0.00014275,0.00013038,0.00000$ $501,0.00016215,0.00025731,0.00002118,-0.00002455,-0.00003903,-0.000043$ $60,-0.00008292,0.00009204,0.00028090,-0.00001333,0.00006407,-0.0000325$ $5,-0.00032360,0.00012007,-0.00025546,0.00003476,0.00001515,0.00000621$, $0.00004636,-0.00006059,0.00003533,-0.00003503,-0.00001173,-0.00000410$, $0.00309667,-0.00184243,-0.00208898,0.00016716,0.00015044,-0.00107269,-$ $0.00041405,-0.00373628,-0.00427081,-0.02527346,-0.01071498,0.00294329$, $-0.02043991,-0.01506819,-0.05771640,0.02337385,0.01003097,0.00485865,0$ $.00130760,0.00030409,-0.00059053,-0.00009889,0.00019809,0.00002634,-0$. $00134425,-0.00059385,-0.00008889,0.02060255,0.01876266,0.05696158,0.00$ $042278,0.00028328,0.00067375,0.00004487,-0.00004200,-0.00005031,0.0000$ $3853,-0.00004681,0.00006582,-0.00001688,0.00001285,-0.00004581,-0.0000$ $3098,-0.00001665,-0.00000898,0.00009678,0.00014345,-0.00009874,-0.0001$ 2664,0.00007755,0.00024906,-0.00001577,0.00002306,0.00000797,-0.000075 $40,0.00002293,-0.00006591,-0.00000654,-0.00000977,0.00001258,-0.000003$ $82,-0.00000660,0.00000632,-0.00000425,0.00000255,0.00001054,-0.0044574$ $2,-0.01131741,-0.02472355,-0.00422644,-0.00361258,0.00093822,-0.000931$ $31,0.00027246,0.00066536,-0.00098072,-0.00214770,0.00395397,0.00924865$ $, 0.00784171,0.01705919,-0.07719069,-0.04013098,-0.06971750,0.00059021$, $0.00104854,-0.00082776,-0.00014680,-0.00059715,-0.00118252,-0.00001884$ $, 0.00024944,-0.00008856,0.00011913,0.00159637,-0.00072856,0.07766929,-$ $0.00022319,0.00012290,0.00006697,0.00021496,0.00002430,-0.00004135,-0$. $00011918,0.00000649,-0.00007107,-0.00001158,-0.00002830,0.00001086,0.0$ $0002783,0.00000757,0.00003756,-0.00006878,-0.00008362,0.00002141,0.000$ $04489,-0.00009213,0.00002960,0.00000139,-0.00002053,-0.00000695,0.0000$ $8889,-0.00003487,0.00007682,-0.00000322,-0.00000004,0.00000871,0.00000$ $713,-0.00000795,0.00001223,0.00000078,0.00000009,-0.00000171,-0.002145$ $72,-0.00134708,-0.01135292,-0.00378920,0.00346748,-0.00067843,0.000212$ $37,-0.00071073,0.00066540,-0.00192024,0.00612772,-0.00119491,-0.000681$ $65,0.00394508,0.00083291,-0.04101873,-0.08718187,-0.10566931,0.0013745$ $2,-0.00178046,0.00081994,-0.00018321,0.00009974,-0.00055982,0.00024632$ $,-0.00055704,0.00015234,0.00160434,-0.00328032,0.00134073,0.04634094,0$ $.08133087,-0.00069133,-0.00047009,-0.00047633,-0.00013364,0.00004423,0$ $.00003365,-0.00003950,0.00009052,-0.00002539,0.00003891,-0.00004903,0$. $00002670,-0.00004965,0.00004877,-0.00001065,0.00002917,-0.00008531,0.0$ $0005165,0.00016058,0.00000143,-0.00034433,0.00000468,-0.00001677,-0.00$ $000318,0.00010029,-0.00002813,0.00006989,0.00001294,0.00001376,-0.0000$ 
$1172,-0.00001298,0.00000144,-0.00001924,0.00001150,-0.00000043,-0.0000$ $0631,0.00302725,0.00001529,-0.00050573,0.00041373,-0.00087797,0.002093$ $07,0.00050815,0.00059660,0.00014424,0.00440321,-0.00090430,-0.00103913$ ,-0.00879442,-0.00988337,-0.01513235,-0.07229100,-0.10674174,-0.269099 40,0.00007627,0.00122976,0.00097360,0.00006924,-0.00004366,0.00013643, $-0.00012650,0.00013697,-0.00012985,-0.00056410,0.00139449,0.00058863,0$ $.07386064,0.11553842,0.28271883,-0.00017160,-0.00056648,0.00006261,0.0$ $0160573,0.00155479,-0.00113700,0.00530916,0.03392221,-0.00272369,-0.00$ $559859,0.00137441,-0.00096320,0.00092150,0.00131784,-0.00055340,0.0001$ $7043,-0.00014003,-0.00000025,-0.00007452,0.00016104,-0.00002071,-0.000$ $57794,-0.00132256,0.00045272,-0.06682677,-0.03313602,0.01561807,-0.000$ $36579,0.00018885,-0.00009777,-0.00002652,-0.00012709,0.00002446,0.0001$ $7703,0.00023333,-0.00005253,-0.00005362,-0.00001139,0.00003578,-0.0000$ $0727,0.00001282,0.00001169,0.00004045,-0.00001676,-0.00004233,-0.00003$ $941,0.00002033,0.00008955,0.00001165,0.00002451,0.00002386,0.00002234$, $0.00000788,-0.00001408,0.00003367,-0.00000126,-0.00004218,0.00000614,-$ $0.00000139,-0.00000245,-0.00001164,0.00000716,-0.00000790,-0.00000146$, $-0.00000532,-0.00000092,0.00000091,0.00000084,-0.00000705,0.06488374,0$ $.00019665,0.00014711,0.00086482,-0.00253879,0.00239362,-0.00030344,-0$. $00341952,-0.01462781,-0.00201695,0.00321489,0.00011213,0.00006703,-0.0$ $0109251,-0.00024869,0.00082804,0.00009740,0.00015050,-0.00008701,-0.00$ $002163,-0.00002320,-0.00004901,0.00051874,-0.00013533,-0.00083254,-0.0$ $2644188,-0.43768604,0.05112701,0.00022359,-0.00008086,0.00000204,0.000$ $03993,-0.00001167,-0.00014897,-0.00006496,0.00017149,-0.00033739,-0.00$ $000791,-0.00001724,-0.00003570,-0.00000576,-0.00000794,0.00003752,-0.0$ $0000733,-0.00001155,0.00001837,-0.00010521,0.00009044,-0.00007735,0.00$ $000185,0.00002827,0.00004162,0.00001261,-0.00000186,-0.00002251,-0.000$ $02280,-0.00000158,0.00002783,-0.00000744,0.00000054,0.00000250,-0.0000$ $0224,0.00000469,-0.00000705,0.00000460,-0.00002124,-0.00000122,0.00000$ $317,0.00000109,-0.00000088,0.02700627,0.45566692,-0.00015993,-0.000002$ $05,-0.00113618,0.00226172,-0.00110729,-0.00025862,-0.00504106,0.015771$ $28,-0.00740513,-0.00200140,-0.00035844,0.00218817,-0.00075085,0.001231$ $70,0.00082624,0.00070749,0.00014160,-0.00013783,-0.00007992,-0.0000348$ $8,0.00007411,0.00010861,0.00023411,0.00069390,0.01423328,0.00876990,-0$ $.00878911,-0.00015432,0.00001689,-0.00018361,-0.00001749,-0.00002889,-$ $0.00002715,-0.00002358,-0.00023144,-0.00031282,0.00005019,0.00005203,0$ $.00011226,0.00001085,0.00001406,-0.00012733,-0.00001035,-0.00004325,-0$ $.00004869,0.00012578,-0.00024016,0.00030186,0.00015692,0.00006732,-0.0$ $0013741,-0.00008081,-0.00001115,0.00001890,0.00006507,0.00000084,-0.00$ $010358,0.00001402,0.00000455,-0.00001371,-0.00001720,-0.00002433,0.000$ $01134,-0.00000815,-0.00004311,0.00006683,0.00002372,-0.00002728,-0.000$ $02147,-0.00926963,-0.04441961,0.01867212,-0.00047642,-0.00111788,-0.00$ $056183,-0.00232650,0.00465421,-0.00109130,-0.02655737,-0.00872388,0.01$ $696580,0.00262543,-0.00322630,-0.00008497,-0.00112881,-0.00003396,-0.0$ $0083974,0.00079463,0.00004231,0.00004794,-0.00029355,0.00015649,-0.000$ $01219,0.00016570,0.00013183,0.00041164,-0.28840276,-0.07706195,0.16713$ $100,0.00005867,-0.00004765,0.00009004,0.00005237,0.00010563,0.00009220$ $, 0.00027641,0.00098488,0.00038985,0.00001951,0.00004133,0.00008582,0.0$ $0001867,-0.00002892,-0.00007248,-0.00008063,-0.00002382,0.00005690,-0$. $00001588,-0.00008268,-0.00006980,0.00004477,0.00002580,-0.00005837,-0$. $00004424,-0.00003079,0.00004503,0.00003646,0.00000318,-0.00005025,0.00$ 
$000238,0.00000587,-0.00000376,-0.00000381,0.00000745,0.00001057,-0.000$ $00854,0.00002102,0.00003222,0.00000135,-0.00000049,-0.00000640,0.00057$ $237,0.00241826,-0.00014295,0.31466978,-0.00009767,0.00074280,0.0009258$ $6,0.00307301,-0.00114612,0.00112504,0.00899785,0.00684782,-0.01364800$, $0.00257679,0.00100289,-0.00053764,-0.00048077,-0.00035965,-0.00024226$, $0.00030484,0.00037117,-0.00018231,0.00035138,-0.00034064,0.00016655,0$. $00021327,0.00015481,-0.00027336,-0.06550499,-0.08608778,0.06883708,0.0$ $0001824,0.00000250,0.00003958,-0.00009269,-0.00004473,0.00005345,-0.00$ $059970,-0.00093599,0.00063182,0.00001647,0.00003743,0.00007120,0.00001$ $476,-0.00001731,-0.00007331,-0.00003417,-0.00000215,0.00000611,-0.0000$ $9948,-0.00005150,0.00013347,0.00006261,-0.00002345,-0.00007165,-0.0000$ $3426,-0.00001183,0.00001616,0.00003543,0.00000023,-0.00005419,0.000003$ $34,0.00000253,-0.00000347,-0.00001890,-0.00000434,-0.00000310,0.000009$ $20,0.00002417,0.00004075,0.00000933,-0.00000836,-0.00000999,-0.0305039$ $8,-0.00589179,0.02026759,0.08178006,0.08573929,-0.00119775,0.00049570$, $0.00170648,0.00016352,0.00234688,-0.00042738,-0.02015706,-0.00515417,0$ $.00116890,-0.00048194,-0.00016119,-0.00062474,0.00051646,0.00004345,-0$ $.00010393,0.00003845,-0.00032531,0.00009280,0.00031213,-0.00023018,0.0$ $0012593,-0.00001656,-0.00029457,-0.00062039,0.20461128,0.08520868,-0.1$ $3437727,0.00001376,-0.00000444,0.00002593,-0.00001051,-0.00002149,-0.0$ $0005346,0.00025445,-0.00011822,0.00046002,-0.00001097,0.00003353,0.000$ $09213,0.00002542,-0.00005012,-0.00006194,-0.00008245,0.00000189,0.0000$ $4162,-0.00029413,0.00010218,-0.00006600,0.00005779,0.00000065,-0.00004$ $784,-0.00003052,-0.00001985,0.00001844,0.00004632,0.00000202,-0.000068$ $61,0.00000360,0.00000057,-0.00000172,-0.00002778,0.00000279,0.00000060$ $, 0.00000404,0.00004385,0.00004821,0.00001105,-0.00001012,-0.00001159,-$ $0.00138367,-0.00467716,-0.00426309,-0.18236496,-0.07721537,0.136946881$ $1-0.00000180,-0.00000298,0.00000073,-0.00000059,-0.00000043,-0.0000002$ $1,-0.00000362,-0.00000309,0.00000088,0.00000437,-0.00000477,0.00000115$ $, 0.00000112,-0.00000214,-0.00000061,0.00000279,-0.00000005,-0.00000023$ $, 0.00000011,0.00000067,0 .,-0.00000218,-0.00000183,0.00000086,0.0000007$ $9,-0.00000546,0.00000184,0.00000467,-0.00000255,-0.00000005,0.00000334$ $, 0.00000115,-0.00000060,0.00000206,-0.00000507,0.00000057,-0.00000495$, $0.00000392,0.00000498,0.00000002,0.00000555,-0.00000142,-0.00000099,0$. $00000297,-0.00000071,0.00000139,0.00000367,-0.00000016,-0.00000025,0.0$ $0000053,-0.00000131,-0.00000024,0.00000282,-0.00000275,0.00000083,0.00$ $000677,-0.00000424,-0.00000209,0.00000603,-0.00000152,-0.00000192,0.00$ $000352,-0.00000012,0.00000033,0.00000051,0.00000051,0.00000025,0.00000$ $244,-0.00000040,-0.00000286,-0.00000547,0.00000175,-0.00000058,-0.0000$ 0670,0.00000106111@

para-Addition Product

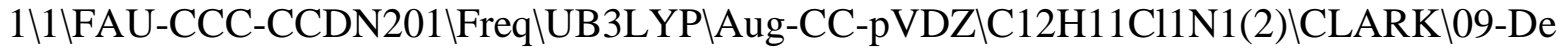
c-2014\0\\N Geom=AllCheck Guess=TCheck SCRF=Check GenChk UB3LYP/Aug-C C-pVDZ Freq \\Addition product p-Cl-Ph-radical + aniline (para) \} \backslash 0 , 2 \backslash \mathrm { C } \text { , } $-6.0710601814,1.4800431289,1.5614320511 \backslash \mathrm{C},-4.7846495191,0.7998309572,1$ $.9775583342 \backslash \mathrm{C},-3.5510920705,1.3513510032,1.7776271497 \backslash \mathrm{C},-3.3838273735$, $2.6164139834,1.1434202835 \backslash \mathrm{C},-4.5485940684,3.3234594003,0.7262728348 \backslash \mathrm{C}$, $-5.8045860496,2.8162973887,0.9025089697 \mathrm{H},-6.5900055818,0.8272104624,0$ $.8292618244 \backslash \mathrm{H},-4.8715159238,-0.172438696,2.4635148075 \backslash \mathrm{H},-2.6632161757$, 
$0.8089812859,2.1120858609 \mathrm{lH},-4.4290968685,4.3002548751,0.2509431253 \mathrm{lH}$, $-6.6714288355,3.386075346,0.5664385036 \backslash \mathrm{N},-2.1159516268,3.1885935453,1$. $013686654 \backslash \mathrm{C},-8.8224493029,1.8646914237,4.8909889042 \backslash \mathrm{C},-9.189508721,1.0$ 936535778,3.7897775564\C,-8.2946100043,0.9756312732,2.720387557\C,-7.0 $486222487,1.6134740437,2.7387026837 \backslash \mathrm{C},-6.709482315,2.3823140681,3.8622$ $29729 \backslash \mathrm{C},-7.586661793,2.5137965371,4.9402098759 \backslash \mathrm{Cl},-9.9386029274,2.0248$ $87957,6.249196947 \backslash \mathrm{H},-10.1566259174,0.5943271452,3.7675794662 \backslash \mathrm{H},-8.5797$ $54061,0.3722947054,1.8568508176 \mathrm{lH},-5.7438503373,2.8871106582,3.8961660$ $311 \mathrm{\downarrow H},-7.3181698405,3.111775222,5.8096057242 \backslash \mathrm{H},-1.3360876162,2.5431977$ $371,1.0195337658 \backslash \mathrm{H},-2.0210383426,3.8971623701,0.2969702192 \mathrm{IV}$ ersion=EM 64L-G09RevC.01 \State=2-A $\backslash H F=-978.8857521 \backslash S 2=0.778824 \backslash S 2-1=0 . \mid S 2 A=0.750$ 49\RMSD $=2.904 \mathrm{e}-09 \backslash \mathrm{RMSF}=2.994 \mathrm{e}-06 \backslash$ ZeroPoint $=0.1962854 \backslash$ Thermal $=0.2086374$ $\backslash \mathrm{ZPE}=123.1709452 \backslash$ Dipole $=1.1050884,0.0032392,-1.041679 \backslash$ DipoleDeriv $=0.05$ $19671,0.0441662,-0.0059207,0.0018812,0.0464887,0.1631212,-0.0860938,0$. $1214574,0.2678635,0.1801345,-0.1905292,0.0918034,0.0871846,-0.1158901$, $-0.0080917,0.1181369,-0.0585339,-0.3079686,-0.1728514,0.128259,-0.0094$ $487,-0.2374617,0.2373801,-0.2400663,0.1570677,-0.1812892,-0.0153035,1$. $073842,0.6171419,-0.3203383,0.6952068,-0.0995312,0.061421,-0.1720328,0$ $.1377016,-0.0625398,0.0364635,-0.4168579,0.2237839,-0.0499975,0.113862$ $8,-0.0670522,0.0607017,-0.1240493,-0.1036516,-0.08469,0.2595947,-0.068$ $6725,0.0832132,0.0495716,-0.2463958,0.0955619,-0.098876,-0.2068153,-0$. $1128888,-0.1189638,-0.0429072,-0.1203674,-0.0208853,-0.0695424,-0.0446$ $92,-0.0699761,-0.0117245,0.0462053,-0.0142578,-0.0090716,-0.0179485,-0$ $.049428,0.0833159,-0.0195321,0.0907486,0.0904192,-0.1098181,0.0727989$, $-0.0207628,0.0487247,0.0404393,0.0564678,-0.0636592,0.0464983,0.113811$ $4,0.0316741,-0.0417002,0.0368484,-0.0589175,-0.0720733,0.1239353,0.000$ $841,0.0932706,0.0853871,-0.0370836,0.0508492,-0.0494051,0.0485964,0.01$ $62666,0.0595273,-0.0506361,0.045903,0.1076742,-1.1891388,-0.4454633,0$. $1919978,-0.6928801,-0.446656,0.0188598,-0.2723759,-0.2164135,-0.253478$ $9,0.5118135,-0.0291561,-0.5846042,-0.0749128,-0.0135826,0.134494,-0.67$ $18272,0.0896963,0.7577076,-0.076484,0.0696179,0.1123193,0.0880472,-0.0$ $66843,-0.0283744,0.1476726,-0.0100493,-0.2549952,-0.0077655,0.0488025$, $-0.0025143,0.1029584,-0.0271665,0.0529049,0.0971214,0.1022517,-0.00737$ $84,0.0920014,-0.0106957,-0.1385129,-0.0553791,-0.0619327,-0.0020341,-0$ $.2245124,-0.0460738,0.0936542,-0.0649904,0.0385451,0.0567367,0.0366055$ ,-0.0507015,0.0234743,0.0536868,0.0222633,-0.0907874,-0.1449625,0.0247 $693,0.1013175,0.0393044,-0.040239,0.0510354,0.130478,0.0667283,-0.0854$ $408,-0.4593746,0.0215486,0.4045733,0.0455685,-0.0866385,-0.0647591,0.4$ $50045,-0.0414969,-0.5795887,-0.0268391,-0.0670081,0.0205165,-0.0628077$ $, 0.0888963,-0.0134234,0.0286156,-0.0092114,0.0832618,0.0851983,-0.0178$ $947,-0.0032429,-0.0335035,0.0555487,-0.0843908,-0.0324628,-0.0991315,-$ $0.038122,-0.024536,-0.0600963,0.0038531,-0.0697822,0.0638386,0.000614$, $-0.0135833,-0.0079882,0.0966718,0.076752,-0.0273982,-0.0040466,-0.0219$ $31,0.0775548,-0.071791,0.0062231,-0.0665901,-0.0042317,0.1869603,0.023$ $6831,0.0348722,0.0998502,0.1213263,0.0206136,0.184659,0.0850394,0.1981$ $905,0.1384107,0.0402448,-0.0191745,0.1187479,0.240394,0.0461367,0.1205$ $971,0.1281206,0.1273853 \backslash$ Polar $=226.1447415,23.5613799,138.4389449,-48.1$ $382522,-4.060609,166.7783355 \backslash \mathrm{PG}=\mathrm{C} 01[\mathrm{X}(\mathrm{C} 12 \mathrm{H} 11 \mathrm{Cl1N} 1)] \mathrm{NImag}=0 \backslash 10.472492$ 17,0.02849792,0.48320917,0.04315892,0.01381618,0.44644911,-0.15503930, $0.04393868,-0.03175102,0.64306127,0.03708778,-0.11013065,0.02562363,0$. $10023532,0.55234315,-0.02944591,0.02511052,-0.07976924,-0.01872160,-0$. 
$21177232,0.22752464,-0.03060228,-0.00345452,-0.00068188,-0.36096159,-0$ $.11728312,0.03932472,0.73822964,0.00566547,0.01489701,-0.00553446,-0.0$ $7880973,-0.13250268,0.03773082,-0.00275663,0.50448003,-0.00503962,-0.0$ $0527001,0.00840699,0.01749413,0.03488960,-0.06151890,0.04360957,-0.195$ $62140,0.21524123,0.00267005,0.00648141,-0.00219876,0.01511471,-0.02273$ $040,0.01179911,-0.12196363,-0.04778852,0.02117093,0.66097283,0.0066103$ $7,-0.00895620,0.00035804,-0.06975207,-0.04108075,0.02130108,0.04690248$ ,-0.19473312,0.07355474,0.05357952,0.49017989,-0.00199022,0.00044724,-$0.00973535,0.03708993,0.02342081,-0.00205632,-0.03012736,0.06722859,-0$ $.09053676,0.00734419,-0.18712972,0.20490981,-0.00139148,-0.01743693,0$. $00724260,-0.03206150,-0.01728113,0.00718001,0.03720739,0.05953726,-0.0$ $2799172,-0.18014180,-0.00387417,-0.00741954,0.64036041,-0.02614683,-0$. $00863908,0.00612081,0.00534400,0.00337858,-0.00154690,0.01831242,-0.02$ $869534,0.01753043,0.09362016,-0.14637908,0.05007431,0.07274036,0.58838$ $558,0.01243077,0.00627156,0.00273282,-0.00444005,-0.00253014,0.0011613$ $7,-0.00743792,0.02034898,-0.00613288,-0.05350926,0.04636162,-0.0806466$ $6,-0.00131907,-0.23104258,0.22917493,-0.08827033,-0.01347304,0.0030960$ $5,0.02256078,0.03423530,-0.01549095,-0.01761912,-0.02850684,0.01355036$ $,-0.07355250,0.04658013,-0.02718596,-0.34163407,-0.13346576,0.04579952$ $, 0.74708867,-0.00769886,-0.16911836,0.04861963,0.01040827,-0.01602489$, $0.00743523,-0.00583084,-0.00851953,0.00396093,-0.00129519,0.03296626,-$ $0.01163724,-0.09312458,-0.14780265,0.04467564,0.01903141,0.46703232,-0$ $.00126565,0.04810084,-0.08749059,-0.00405949,0.00993091,-0.00637752,0$. $00198249,0.00298854,-0.00133892,-0.00356284,-0.01036978,0.01248884,0.0$ $2754749,0.04366353,-0.06556953,0.02720897,-0.17151072,0.20883400,-0.08$ $606386,-0.06189582,-0.07758474,-0.02292831,-0.01222260,-0.01698525,0.0$ $0097837,0.00456822,-0.00351457,0.00069728,-0.00159006,0.00117064,0.000$ $66598,-0.00016626,-0.00035805,-0.00273641,-0.00926325,0.00081309,0.107$ $79517,-0.05692967,-0.12238324,-0.09027136,0.00636748,0.01319634,0.0083$ $7647,0.00038732,-0.00286423,0.00139613,-0.00188465,0.00320641,-0.00173$ $243,0.00623791,-0.00383560,0.00066605,-0.01897735,-0.02145708,-0.01145$ $579,0.06852862,0.13605835,-0.06828647,-0.08556945,-0.14118471,-0.00834$ $821,-0.00293277,-0.00255512,0.00128876,0.00268373,-0.00244994,0.000613$ $93,-0.00201651,0.00071223,-0.00096667,0.00149952,-0.00115014,0.0088420$ $0,0.00827782,0.01188744,0.07356819,0.09669932,0.16142720,0.00360977,0$. $02303058,-0.00998209,-0.05684910,-0.01926835,0.00797893,0.00110445,-0$. $02565641,0.01269984,-0.00425410,-0.00196672,0.00020391,-0.00141776,0.0$ $0022050,-0.00019248,-0.00458547,0.00139956,-0.00101071,0.00067272,-0.0$ $0067038,-0.00023263,0.05875833,-0.00296412,-0.00803279,0.00448158,-0.0$ $2177344,-0.27461673,0.12252067,0.00171494,-0.00796615,0.00639226,-0.00$ $246479,0.00366170,0.00198877,0.00057712,0.00014473,-0.00045017,0.00243$ $527,0.00062783,0.00083576,0.00052991,0.00017140,0.00040660,0.02282625$, $0.28726043,0.00227325,0.00687706,-0.00200615,0.00892063,0.12371686,-0$. $08945157,-0.00110727,0.00458544,0.00205028,0.00054694,0.00183221,0.006$ $69461,-0.00048888,-0.00031033,-0.00059323,-0.00161729,0.00054726,0.001$ $35080,0.00062071,-0.00030612,0.00039943,-0.00917073,-0.13424557,0.0857$ $3534,-0.00149746,-0.00364084,0.00109414,-0.02049295,0.01610674,-0.0097$ $7210,-0.23353953,0.10954626,-0.06845595,0.00230261,-0.00330488,0.00171$ $545,0.00146577,-0.00129331,0.00040137,0.00001948,-0.00037914,0.0002820$ $8,-0.00070910,0.00034480,-0.00018427,0.00106281,0.00003268,0.00056783$, $0.24930335,-0.00231032,0.00142742,0.00410790,-0.01096071,0.00973888,-0$ 
$.00384307,0.10910598,-0.12253745,0.05188173,0.02411026,-0.00799180,0.0$ $0701074,-0.00203444,-0.00332456,0.00373737,-0.00082922,-0.00033318,-0$. $00020632,0.00035562,-0.00031073,0.00004011,0.00016032,-0.00036001,-0.0$ $0275749,-0.11784890,0.12314454,0.00122148,0.00380040,0.00778704,0.0041$ $5128,-0.00178116,0.00453434,-0.06914196,0.05170958,-0.06574853,-0.0120$ $4854,0.00538643,-0.00044501,0.00073109,0.00386854,0.00224648,0.0005220$ $5,-0.00015820,-0.00056913,0.00106307,-0.00088019,0.00028940,0.00029455$ $,-0.00279813,-0.00433467,0.07455263,-0.05862720,0.05854264,-0.00405963$ ,-0.00061530,-0.00047236,-0.00082196,-0.00016016,0.00011123,-0.0039990 $5,0.00224397,-0.00161248,0.00621264,0.02108262,-0.01030732,-0.06080272$ $,-0.02396022,0.01059790,0.00046217,-0.02735104,0.01342006,-0.00012386$, $-0.00063294,0.00031639,0.00014277,-0.00000613,0.00005513,0.00026032,0$. $00120729,-0.00061640,0.06020013,-0.00146917,0.00379325,0.00274996,0.00$ $031270,0.00037290,-0.00047993,0.00299400,0.00101386,0.00166523,-0.0063$ $3988,-0.01127250,0.00683139,-0.02420692,-0.27012782,0.11496650,0.00009$ $053,-0.00819230,0.00566597,-0.00010471,0.00024163,-0.00021534,-0.00004$ $179,-0.00018137,-0.00019544,-0.00009935,-0.00063971,0.00038670,0.02817$ $165,0.28492918,0.00027977,0.00351205,0.00798991,-0.00008238,-0.0005207$ 7,-0.00043076,-0.00194566,0.00153719,0.00335797,0.00350162,0.00849024, $-0.00107033,0.00997822,0.11446596,-0.09088832,0.00018427,0.00396361,0$. $00149781,0.00009501,0.00122343,-0.00085373,0.00005662,-0.00022923,-0.0$ $0046942,0.00007297,0.00039574,-0.00013507,-0.01213183,-0.12737840,0.08$ $586373,0.00589132,-0.00460518,0.00354350,0.00037482,-0.00135227,0.0007$ $3461,0.00003948,-0.00051302,0.00024348,-0.00249177,-0.00381111,0.00137$ $609,-0.01843008,0.01691902,-0.00983765,-0.23341435,0.11538324,-0.07048$ $651,0.00024610,0.00042816,-0.00038070,0.00046461,-0.00018836,0.0001075$ $8,-0.00002320,0.00010673,-0.00004855,0.00094869,0.00001178,0.00031595$, $0.24674028,0.02111765,-0.00992217,0.00788337,-0.00250718,-0.00349802,0$ $.00247813,-0.00091033,-0.00119659,0.00017212,-0.00332585,0.00213124,0$. $00243889,-0.01052806,0.00845832,-0.00267298,0.11801458,-0.12708932,0.0$ $5710296,0.00001120,0.00068513,-0.00073776,0.00146999,-0.00065738,0.000$ $32552,0.00009836,-0.00003528,-0.00025542,0.00029816,-0.00015267,-0.002$ $79682,-0.12330224,0.13029596,-0.00922192,0.00524004,-0.00240451,0.0010$ $6522,0.00264317,0.00052970,0.00050010,0.00000426,-0.00071328,0.0010345$ $0,0.00259976,0.00648442,0.00378157,-0.00095522,0.00516858,-0.07113561$, $0.05602876,-0.06042283,0.00023971,0.00071857,0.00031663,-0.00070576,0$. $00018994,-0.00034696,0.00000092,-0.00026638,-0.00045731,0.00033861,-0$. $00268555,-0.00443080,0.07516847,-0.06331389,0.05471472,-0.00218342,-0$. $00120874,-0.00001139,-0.00063241,-0.00036206,0.00077400,-0.00673735,-0$ $.00517810,0.00129498,-0.28000008,-0.08539301,0.00447392,-0.04493481,-0$ $.01633423,0.00288227,0.00238552,0.00546585,-0.00244354,-0.00114720,-0$. $00046874,0.00004458,0.00027621,0.00013247,-0.00025360,0.00205066,0.000$ $67805,-0.00071902,0.00211565,-0.00049369,0.00044112,-0.00088878,-0.000$ $41100,0.00005862,0.67457153,-0.00153407,-0.00088625,-0.00059782,0.0077$ $1127,-0.00138066,0.00199211,-0.04407930,-0.02202924,0.00806876,-0.0702$ $9083,-0.12262636,0.03650939,0.02554130,0.01156798,-0.00123009,-0.00296$ $465,-0.00349702,0.00332451,-0.00077472,-0.00048936,0.00015752,-0.00119$ $879,-0.00040492,0.00053530,-0.00043806,0.00111933,-0.00119614,0.000757$ $70,0.00081789,-0.00208924,0.00121431,0.00045234,0.00002587,-0.08634619$ $, 0.57252054,-0.00059810,-0.00088309,-0.00089170,-0.00395269,0.00175856$ $, 0.00116308,0.02361566,0.01341567,-0.00022404,0.03273688,0.05082350,-0$ 
$.04232469,-0.01387719,-0.00377854,0.00434617,0.00158800,0.00320202,0.0$ $0027849,-0.00053690,-0.00014114,0.00006911,0.00081277,0.00068402,0.000$ $33640,0.00043015,-0.00187173,-0.00131159,-0.00042889,-0.00127194,-0.00$ $106613,-0.00044295,0.00016421,0.00063908,-0.06751119,-0.29580899,0.283$ $70219,-0.00136544,-0.00031614,0.00023340,-0.00001622,0.00009077,0.0003$ $5930,0.00005179,0.00015946,0.00008544,-0.00022128,-0.00008459,0.000136$ 93,0.00009005,0.00002117,0.00007883,-0.00008694,0.00020764,0.00031388, $-0.00001505,0.00000306,0.00018453,-0.00009783,0.00003575,-0.00000696,0$ $.00006755,-0.00005028,-0.00012549,0.00006207,0.00001557,-0.00010853,-0$ $.00001527,-0.00007301,-0.00002120,0.00040925,0.00026130,0.00008171,0.5$ $0285205,-0.00049242,-0.00066151,0.00001004,-0.00008462,0.00008833,0.00$ $048317,0.00003945,0.00005511,0.00002041,0.00022771,0.00011472,0.000089$ $41,-0.00008478,-0.00003153,0.00016235,0.00004128,0.00031605,0.00033216$ ,-0.00007748,0.00019176,0.00018845,0.00000795,-0.00007268,-0.00008887, $0.00000575,-0.00002203,-0.00009087,0.00000592,-0.00007021,-0.00013039$, $-0.00004274,-0.00005068,-0.00000244,-0.00008927,-0.00006238,-0.0000236$ $7,0.18253646,0.30984869,-0.00002905,-0.00009930,-0.00061733,0.00007044$ $, 0.00016217,0.00047338,-0.00017880,-0.00002994,0.00009377,0.00063967,0$ $.00034562,0.00004107,-0.00013036,-0.00003615,0.00003734,-0.00007604,0$. $00029886,0.00041395,-0.00000130,0.00008774,0.00031504,0.00001191,-0.00$ $008459,-0.00004882,-0.00006417,-0.00003426,-0.00013387,-0.00001239,-0$. $00010098,-0.00005737,-0.00001328,0.00000804,-0.00015967,-0.00057492,-0$ $.00035444,-0.00013764,-0.01495837,0.17448759,0.46701062,-0.00080057,-0$ $.00452882,-0.00080943,0.00030094,0.00029782,0.00022902,-0.00009500,-0$. $00015849,0.00011512,0.00014184,0.00024709,-0.00021139,0.00027886,-0.00$ $020379,0.00027530,-0.00107937,0.00042333,0.00085436,0.00017084,0.00028$ $681,0.00033305,-0.00009698,-0.00000428,-0.00006534,0.00003732,-0.00001$ $675,0.00000706,-0.00006151,-0.00006976,-0.00010852,-0.00007482,-0.0001$ $0097,0.00001772,-0.00013443,-0.00008677,-0.00000138,-0.12497017,-0.069$ $37474,-0.07304880,0.64329375,-0.00408429,0.00358051,-0.00307466,-0.001$ $07500,-0.00057337,0.00097503,0.00003004,0.00060058,-0.00022494,0.00010$ $658,-0.00037281,0.00011019,-0.00035840,0.00029828,-0.00029652,0.001190$ $63,-0.00019533,-0.00059133,0.00006120,0.00006550,0.00007745,-0.0000549$ $1,0.00003339,0.00001524,-0.00008029,-0.00007321,-0.00003131,0.00006180$ $, 0.00010405,0.00013574,0.00003644,-0.00005509,0.00003339,-0.00001498,0$ $.00000821,0.00000947,-0.04179241,-0.15200659,-0.12482326,0.21085962,0$. $33351971,-0.00002984,-0.00267581,0.00225713,0.00053481,0.00010409,-0.0$ $0112223,0.00017962,-0.00034781,-0.00000076,-0.00041978,-0.00001914,-0$. $00010682,0.00033233,-0.00011602,-0.00000087,-0.00055322,-0.00028211,-0$ $.00020524,-0.00005621,-0.00012643,-0.00017708,0.00002947,0.00011115,0$. $00001968,0.00002284,0.00012027,0.00018721,-0.00005540,0.00003273,0.000$ $09744,0.00005305,0.00001420,0.00008183,0.00011759,0.00008092,0.0000353$ $4,-0.02124843,-0.09869061,-0.22986617,-0.08991593,0.18253600,0.5582789$ $2,-0.01938077,-0.00896361,0.00618749,-0.00000198,-0.00168420,0.0006480$ $6,-0.00006751,0.00055909,-0.00045461,-0.00009113,-0.00007386,0.0001354$ $2,0.00048992,-0.00053346,0.00019051,-0.00133254,0.00108991,-0.00124262$ $, 0.00025282,-0.00126137,-0.00273881,-0.00004318,0.00041965,-0.00002474$ $,-0.00018652,-0.00006261,0.00007648,-0.00022984,-0.00000081,0.00015345$ $, 0.00015351,-0.00034300,0.00035934,-0.00000204,-0.00000705,0.00001357$, $0.04851638,0.04119563,0.03808085,-0.20116278,-0.02353110,0.08735201,0$. $57727549,0.00005192,0.00522271,0.00056030,0.00107636,-0.00078443,-0.00$ 
$102005,0.00020206,0.00006134,0.00004369,-0.00038153,-0.00000495,-0.000$ $08362,0.00018691,0.00019063,-0.00013943,0.00049095,-0.00004710,-0.0005$ $9982,0.00153938,0.00027078,-0.00071074,-0.00026114,0.00010487,0.000007$ $76,0.00004056,0.00004288,0.00021578,0.00005806,0.00004530,-0.00001048$, $0.00009611,0.00014438,0.00004453,0.00035632,0.00023410,0.00007622,0.01$ $698919,0.01007643,-0.01025793,0.00481574,-0.08513996,-0.04759420,0.179$ $39646,0.33789057,0.02315398,0.00910983,0.00013943,0.00121118,0.0011713$ $3,-0.00193643,0.00052539,-0.00059915,0.00041846,-0.00039775,-0.0003123$ $5,-0.00003664,-0.00016050,0.00081145,-0.00026328,0.00223089,-0.0007494$ $4,-0.00132629,0.00251945,0.00194274,0.00111176,0.00015448,-0.00029271$, $0.00016203,0.00031296,0.00011830,0.00002839,0.00023755,0.00023557,0.00$ $007724,-0.00025470,0.00011719,-0.00007557,0.00067332,0.00039866,0.0001$ $4026,-0.00745844,-0.03323894,-0.04838374,0.14069452,-0.02072165,-0.234$ $46913,-0.08175958,0.21119222,0.60938581,-0.10322830,0.00694405,0.04619$ $491,-0.03252937,0.00640860,0.01873273,0.00131466,0.00490660,-0.0010163$ $8,-0.00060832,-0.00190290,0.00089469,0.00103300,0.00132092,0.00062404$, $-0.00776338,-0.01181113,0.01194377,0.00404193,-0.00282139,-0.00870223$, $0.00155822,-0.00116293,-0.00032679,-0.00015573,-0.00059189,-0.00101760$ $, 0.00029205,-0.00000573,-0.00019786,0.00074555,0.00081872,-0.00132560$, $0.00030280,0.00031110,0.00014381,-0.03232798,-0.02619907,-0.02515855,-$ $0.03714383,0.00638830,0.05367201,-0.27092285,-0.11466935,-0.02152976,0$ $.56177339,0.00067106,-0.07258860,-0.00897733,0.00950459,0.00070521,-0$. $00990809,-0.00051061,-0.00283976,0.00117942,-0.00134993,0.00276521,-0$. $00117652,0.00411714,-0.00387719,0.00226824,-0.02188942,0.00212206,0.02$ $196532,0.01020053,-0.00398675,-0.01798142,0.00005119,0.00062870,-0.000$ $26593,0.00009500,0.00039786,0.00048707,-0.00069780,-0.00094107,-0.0010$ $3687,-0.00016810,0.00031269,-0.00021652,-0.00007425,-0.00014227,0.0000$ $7167,-0.02659193,-0.03001491,-0.01826099,-0.01350166,0.02122043,0.0398$ $4048,-0.08671499,-0.12904798,-0.03605676,0.16760675,0.34690966,0.03447$ $153,-0.01490550,-0.12882329,0.00468877,-0.00547252,0.01115681,-0.00231$ $744,0.00194503,-0.00241203,0.00192887,-0.00066019,0.00103525,-0.003032$ $95,-0.00100312,-0.00113345,0.01279392,0.01088511,-0.01496103,0.0157076$ $7,-0.00562907,-0.02503500,-0.00119993,0.00085394,0.00040235,-0.0008429$ $6,-0.00054709,-0.00035968,-0.00033947,0.00001803,0.00053780,0.00000255$ ,-0.00111490,0.00151095,-0.00057438,-0.00030040,-0.00015093,-0.0259510 $8,-0.01866684,-0.01738806,0.01633764,0.02101815,0.03083335,0.03104234$, $-0.00955840,-0.11348888,-0.05119971,0.16380076,0.53161196,0.00765333,0$ $.01205067,0.01732165,0.00134729,0.00041848,0.00100117,-0.00046853,0.00$ $015830,0.00021712,0.00040079,0.00017484,-0.00009099,-0.00052448,-0.000$ $47139,0.00000531,0.00120680,0.00112010,0.00033866,-0.00208054,0.000836$ $55,0.00463811,0.00003524,-0.00008419,0.00004202,-0.00004516,-0.0001048$ $5,-0.00012158,-0.00004997,-0.00010818,-0.00008180,0.00002083,-0.000117$ $50,0.00008080,-0.00055033,-0.00038632,-0.00011278,-0.04032319,-0.01595$ $686,0.01506465,-0.00422849,0.00553541,0.01153439,0.00050923,-0.0184239$ $2,-0.02786673,-0.12775840,-0.05982389,-0.05523776,0.63460527,0.0035171$ $8,0.00140744,-0.00450193,-0.00040228,0.00084349,-0.00075752,-0.0005805$ $8,-0.00055857,-0.00032312,-0.00000021,-0.00009155,-0.00011746,-0.00014$ $808,0.00009664,0.00042334,-0.00022689,-0.00038038,0.00046687,-0.000260$ $47,0.00105048,0.00004707,0.00001567,0.00006143,-0.00001964,-0.00004673$ $, 0.00005936,0.00000948,-0.00000809,0.00000451,-0.00004066,-0.00007810$, $0.00036303,-0.00022183,-0.00027266,-0.00008073,-0.00006925,0.00814773$, 
$0.02195015,0.01993019,0.00529287,-0.01183709,-0.02155729,-0.03706392,-$ $0.01367053,-0.00492291,-0.03416261,-0.14604303,-0.11541045,0.19816608$, $0.33003316,0.00126866,-0.01258150,-0.02285958,-0.00226734,0.00036439$,$0.00109652,-0.00006225,-0.00014723,0.00020784,-0.00041233,-0.00028588$, $-0.00016895,-0.00047657,-0.00014988,-0.00036651,-0.00043707,-0.0027241$ $6,0.00022937,0.00258202,-0.00099717,-0.00314081,0.00034999,-0.00033546$ $, 0.00014836,0.00003393,0.00007567,0.00008921,0.00004141,0.00004546,0.0$ $0014812,0.00002382,0.00023561,-0.00013839,0.00009616,0.00005181,0.0000$ $6651,0.06036873,0.04278460,0.03040669,0.01106255,-0.02180436,-0.054440$ $04,-0.06290983,-0.02255925,0.02475035,-0.00703469,-0.09110755,-0.23196$ $586,-0.10038927,0.17786031,0.56484193,0.00167047,-0.00302459,-0.002029$ $32,-0.00019074,-0.00013779,-0.00033625,0.00006471,-0.00009995,-0.00011$ $686,-0.00003238,-0.00007750,-0.00009199,-0.00006033,-0.00015049,-0.000$ $14715,-0.00037584,-0.00023410,-0.00007351,0.00040304,-0.00042225,-0.00$ $109689,0.00006902,0.00004580,0.00004553,-0.00005324,0.00005344,0.00012$ $910,-0.00008213,-0.00001123,0.00009177,0.00006094,0.00013973,0.0001150$ $7,-0.00000873,-0.00000604,0.00000124,-0.26464513,-0.09286817,0.0148747$ $2,0.00147404,-0.03686686,-0.06216775,-0.05188122,-0.01168066,0.0238648$ $4,0.04735877,0.03843300,0.03427992,-0.20021629,-0.02189734,0.09034811$, $0.57135321,-0.00325736,0.00175397,-0.00393351,-0.00049630,0.00005956,0$ $.00043856,-0.00010498,0.00000409,-0.00000925,-0.00001413,0.00002804,-0$ $.00011213,0.00017863,0.00025656,-0.00000138,0.00000484,-0.00023746,0.0$ $0016683,0.00043864,-0.00012190,-0.00084010,0.00009026,0.00000644,0.000$ $09763,-0.00002599,-0.00005118,-0.00004422,0.00003817,0.00003071,0.0000$ $3530,0.00002048,-0.00002396,-0.00009920,0.00003794,0.00002119,0.000011$ $35,-0.12066285,-0.13223981,-0.01256840,-0.01747031,-0.01223520,-0.0233$ $4496,-0.01255447,-0.00619372,0.00690954,0.01844643,0.01129968,-0.00989$ $437,0.00677003,-0.08545194,-0.05162112,0.18144063,0.34094324,-0.002516$ $72,-0.00420878,0.00000129,-0.00028615,0.00043768,0.00042606,0.00030284$ $, 0.00018621,0.00026694,0.00006097,-0.00005498,-0.00006281,0.00010095,0$ $.00015927,0.00019935,-0.00032104,0.00009305,0.00085084,0.00054210,-0.0$ $0000654,-0.00043878,0.00000872,-0.00010981,-0.00013349,0.00008366,-0.0$ $0003951,-0.00008567,0.00002526,-0.00003125,-0.00015455,-0.00000729,0.0$ $0002035,-0.00008657,0.00009471,0.00005308,0.00002965,-0.03732009,-0.03$ $887762,-0.10240462,-0.02577699,-0.00496867,0.02404060,0.02219452,0.006$ $02629,-0.01318473,-0.00328228,-0.02882456,-0.04379636,0.14420624,-0.02$ $444760,-0.24393981,-0.07640831,0.22309183,0.62233923,0.00004100,0.0001$ $8292,-0.00039475,0.00005725,-0.00012416,0.00044182,-0.00013029,0.00004$ $957,-0.00003678,0.00046475,0.00022389,-0.00003689,-0.00002603,-0.00008$ $200,0.00008513,-0.00013325,0.00035647,0.00015955,-0.00004919,0.0000157$ $5,0.00008035,0.00003779,-0.00001105,-0.00003864,-0.00009211,-0.0000314$ $9,-0.00004825,-0.00005781,-0.00007988,-0.00001821,0.00003241,-0.000005$ $65,-0.00004243,-0.00047171,-0.00029899,-0.00010344,-0.08098955,0.00100$ $682,0.05635289,-0.00191874,0.00026107,0.00638827,0.00156176,-0.0013323$ $7,0.00065678,0.00028194,0.00123879,0.00137860,-0.00138413,-0.00516346$, $-0.00406905,-0.02371717,0.00380781,0.03378201,0.10653657,0.00033237,-0$ $.00039378,0.00033148,0.00002734,0.00007201,-0.00011981,0.00007202,-0.0$ $0000101,0.00005843,-0.00006362,-0.00005375,0.00000146,-0.00001185,-0.0$ $0000456,-0.00004542,-0.00001321,-0.00003666,-0.00001126,-0.00000670,0$. $00003036,0.00005917,-0.00001061,0.00000194,0.00000169,0.00002489,0.000$ $01213,0.00000830,0.00000631,0.00001229,0.00000126,-0.00000779,0.000007$ 
$95,-0.00000157,0.00006919,0.00005651,0.00001871,0.00103052,-0.03002685$ $,-0.01325720,0.01195397,0.00222750,-0.01522954,-0.00414475,0.00172532$, $-0.00204511,0.00107769,0.00019200,0.00067962,-0.00227278,0.00182816,-0$ $.00298161,-0.00792868,0.00505703,0.00876169,-0.00267846,0.02189933,-0$. $00011932,0.00046566,0.00025098,-0.00007676,0.00013781,-0.00050634,0.00$ $016690,-0.00009342,0.00001984,-0.00058756,-0.00027694,0.00000780,0.000$ 06930,0.00020362,-0.00007997,0.00027717,-0.00047099,-0.00020926,0.0000 $3737,0.00003984,0.00003962,-0.00004344,0.00000108,0.00004632,0.0001131$ $0,0.00003242,0.00006127,0.00008345,0.00010849,0.00002326,-0.00004477,-$ $0.00000436,0.00005048,0.00059092,0.00037115,0.00014642,0.05641261,-0.0$ $1322496,-0.10177688,0.02834390,-0.00415724,-0.03035230,-0.00462825,-0$. $00471482,0.00042860,0.00108921,0.00052796,-0.00074367,0.00135715,-0.00$ $023944,0.00282349,0.01171800,-0.00236181,-0.01114939,-0.09473184,0.021$ $44568,0.14186634,-0.00020973,-0.00038773,-0.00048111,-0.00004945,-0.00$ $007764,0.00007865,-0.00004525,0.00001801,0.00002154,0.00006743,0.00009$ $941,-0.00001194,0.00007220,-0.00004478,0.00003600,-0.00015355,0.000050$ $79,0.00014869,0.00001596,-0.00001441,-0.00001878,-0.00001321,0.0000156$ $7,-0.00001301,-0.00002911,-0.00000258,-0.00000727,-0.00001152,-0.00002$ $606,-0.00001240,0.00000767,-0.00001563,0.00000074,-0.00011920,-0.00007$ $315,-0.00002733,-0.00450902,-0.00207648,0.00353807,-0.27833777,-0.1247$ $8425,-0.00406611,-0.01184583,-0.00929183,-0.00277788,0.00080676,-0.000$ $77887,0.00430629,0.00021973,0.00075741,0.00002637,0.00227445,-0.001884$ $79,-0.00047570,0.00053942,0.00148415,-0.00033150,0.29147326,0.00021481$ $, 0.00011387,-0.00010798,-0.00009273,0.00005998,0.00009025,0.00006861,0$ $.00007315,-0.00004724,0.00005855,-0.00009366,0.00005100,-0.00009624,0$. $00002925,0.00001222,0.00005356,-0.00002852,-0.00014340,-0.00000849,0.0$ $0000833,-0.00001170,0.00000938,-0.00000582,0.00000058,0.00000732,-0.00$ $000232,-0.00001490,-0.00000744,0.00000686,0.00001721,-0.00000295,-0.00$ $000342,0.00000111,-0.00001755,-0.00000837,-0.00000425,-0.01557392,-0.0$ $0385273,0.00120788,-0.12354299,-0.10316415,-0.01088574,0.00339493,0.00$ 494967,-0.00081163,-0.00102646,0.00500292,-0.00259930,0.00083297,-0.00 $080958,0.00005331,-0.00159543,0.00219742,-0.00469183,0.00104447,-0.001$ $43776,0.00149152,0.13532284,0.09993202,0.00066258,0.00046212,0.0003164$ $0,0.00011271,0.00001291,-0.00021964,0.00000032,-0.00007319,0.00004583$, $-0.00009741,0.00001517,-0.00004239,0.00005560,0.00003996,-0.00003140,0$ $.00008801,-0.00007645,-0.00001963,-0.00001433,-0.00000664,0.00001347,0$ $.00000865,-0.00001582,0.00001040,0.00001888,0.00000773,0.00002583,0.00$ $001811,0.00001738,-0.00000083,-0.00000508,0.00002320,-0.00000858,0.000$ $08371,0.00004903,0.00002101,-0.02180427,-0.01157262,0.00227260,-0.0017$ $4685,-0.00971138,-0.05169362,0.02107605,0.01120315,0.00477264,0.003842$ $92,-0.00283433,-0.00205308,0.00016475,0.00012329,-0.00118598,0.0000488$ $7,-0.00441788,-0.00286314,-0.00116002,0.00107515,0.00035738,0.00098252$ $, 0.01438827,0.05016513,0.00003458,0.00052914,-0.00293762,-0.00013639,-$ $0.00010574,0.00041911,-0.00001569,0.00007740,0.00000729,-0.00007323,-0$ $.00007813,0.00001239,-0.00010412,0.00013070,-0.00006528,0.00040783,0.0$ $0020108,0.00012407,0.00008453,0.00050257,0.00102277,-0.00006048,-0.000$ $00878,-0.00004466,0.00000721,-0.00003623,-0.00004916,0.00003608,0.0000$ $1959,-0.00002723,-0.00000930,0.00000962,-0.00006184,0.00015997,0.00011$ $724,0.00005507,-0.00031480,-0.00162711,0.00379683,0.00794335,0.0112336$ $6,0.01692027,-0.07277745,-0.04718112,-0.05417402,-0.00261704,-0.014727$ $65,-0.02135259,-0.00392995,-0.00439928,0.00139317,-0.00102992,0.000264$ 
$94,0.00049961,-0.00027888,0.00038759,0.00116033,0.00010299,0.00152989$, $-0.00115964,0.07280756,0.00249439,-0.00255114,0.00054860,0.00044700,0$. $00054223,-0.00020583,0.00012685,-0.00021519,0.00011313,-0.00001649,0.0$ $0003302,-0.00003297,0.00013837,-0.00011910,0.00013419,-0.00064223,0.00$ $006211,0.00030512,-0.00020728,0.00043561,0.00071743,0.00005555,-0.0000$ $5676,-0.00001728,0.00005000,0.00002843,-0.00000558,-0.00002317,-0.0000$ $3670,-0.00006342,-0.00003280,-0.00011227,0.00001480,0.00000290,0.00000$ $331,0.00001628,-0.00145616,0.00527661,-0.00174307,-0.00186865,0.001965$ $66,-0.00113661,-0.04729342,-0.12820411,-0.12777962,-0.00265666,-0.0038$ $2422,-0.01233545,-0.00481821,0.00315430,-0.00126869,0.00018415,-0.0005$ $1311,0.00083836,-0.00009187,0.00007876,-0.00008664,0.00167379,-0.00245$ $663,0.00161555,0.05371932,0.12730319,0.00075837,0.00241646,-0.00030046$ ,-0.00041588,-0.00000225,0.00018581,0.00004961,0.00016037,-0.00002116, $0.00010435,-0.00000116,-0.00000490,-0.00000457,0.00013815,-0.00005660$, $0.00041234,-0.00017600,0.00014042,-0.00031125,0.00004092,0.00054591,-0$ $.00006729,0.00001819,-0.00004250,-0.00001463,-0.00002017,-0.00004435,0$ $.00003258,0.00000622,-0.00000370,0.00000405,0.00000281,-0.00004238,-0$. $00015411,-0.00008690,-0.00003033,0.00412470,-0.00157117,0.00006176,-0$. $00770468,-0.01355239,-0.01464578,-0.05437025,-0.12786877,-0.22513526,0$ $.00134507,-0.00088464,0.00111505,0.00059876,-0.00167093,0.00256237,0.0$ $0033561,0.00074479,0.00005405,0.00025551,-0.00054472,-0.00108812,-0.00$ $089112,0.00174923,-0.00002504,0.05590018,0.14084035,0.23694365,-0.0009$ $6340,0.00133128,0.00048709,0.00031479,0.00005486,0.00046166,-0.0002020$ $7,-0.00021011,-0.00000928,0.00003761,0.00008842,-0.00011544,0.00021974$ $, 0.00016970,0.00019261,-0.00005440,0.00032453,0.00018129,-0.00000135,-$ $0.00001829,-0.00014792,-0.00002158,-0.00000060,-0.00000878,-0.00002868$ $, 0.00003955,0.00005113,0.00002421,0.00003128,-0.00000352,-0.00005183,-$ $0.00002418,-0.00013484,-0.00004183,0.00000558,-0.00000638,0.00114069$,$0.00104972,0.00380469,0.00025764,0.00070971,-0.00001641,0.00214583,-0$. $00239054,-0.00040642,-0.00341301,-0.00325495,0.00069179,-0.27319158,-0$ $.12206480,-0.00552783,-0.01237815,-0.00946498,-0.00236800,-0.00077556$, $-0.00027078,0.00031870,-0.00018552,0.00020680,-0.00012818,-0.00017951$, $-0.00004036,0.00015791,0.28742317,0.00058336,-0.00191682,0.00167238,-0$ $.00015777,-0.00003939,0.00008443,0.00053824,0.00043411,0.00019024,0.00$ $029423,-0.00008208,0.00003180,-0.00037480,-0.00012196,-0.00031469,0.00$ $008425,0.00033091,0.00015634,0.00002658,-0.00004006,0.00011363,0.00001$ 495,-0.00012095,-0.00010903,0.00007415,-0.00000920,-0.00001297,-0.0000 2006,-0.00001874,0.00010481,0.00004983,-0.00002264,0.00012034,-0.00012 $048,-0.00013720,-0.00002003,-0.00124469,0.00521640,-0.00260526,0.00078$ $038,-0.00075324,0.00002716,-0.00206588,0.00264426,-0.00518443,-0.01553$ $744,-0.00368022,0.00072109,-0.12195407,-0.10461334,-0.01178941,0.00360$ $519,0.00503691,-0.00130157,0.00022602,0.00010847,-0.00020212,0.0001966$ $9,-0.00047420,0.00029447,-0.00076936,-0.00030208,0.00003442,0.13416034$ $, 0.10109290,-0.00092898,0.00094232,-0.00042058,-0.00002302,0.00003188$, $0.00002860,0.00009680,-0.00022111,-0.00012937,0.00026883,0.00023294,-0$ $.00009441,0.00048953,0.00030960,0.00002013,-0.00016716,-0.00019937,0.0$ $0001248,-0.00006180,0.00016479,0.00023847,-0.00000999,0.00012490,0.000$ $01869,-0.00003018,0.00004309,0.00003943,0.00003277,0.00001639,-0.00001$ $109,0.00002707,-0.00003628,-0.00003675,-0.00023974,-0.00014208,-0.0000$ $9113,0.00345603,-0.00278807,-0.00125693,0.00009742,0.00009722,-0.00108$ $863,0.00019355,-0.00490297,-0.00291488,-0.02238848,-0.01092301,0.00447$ 
$416,-0.00532405,-0.01168083,-0.05378216,0.02218810,0.01108905,0.003697$ $03,0.00125573,0.00027263,-0.00058335,-0.00014308,0.00028571,-0.0000600$ $7,-0.00120701,-0.00065039,-0.00001227,0.00345871,0.01606257,0.05181747$ $, 0.00043238,0.00046946,0.00060176,0.00002420,-0.00005474,-0.00002268,-$ $0.00000597,0.00004196,-0.00000249,0.00008269,-0.00002152,0.00001583,-0$ $.00009523,-0.00002313,-0.00001715,0.00015332,0.00009571,-0.00013482,-0$ $.00016847,0.00012126,0.00024162,-0.00001044,0.00001960,0.00000474,-0.0$ $0002022,0.00001485,0.00000592,-0.00000550,-0.00000831,0.00001612,-0.00$ $000608,-0.00000320,0.00000072,-0.00007869,-0.00004515,-0.00003388,-0.0$ $0323444,-0.01505256,-0.02207841,-0.00382197,-0.00382525,0.00144248,-0$. $00096925,0.00033777,0.00057651,-0.00095472,-0.00185464,0.00432543,0.00$ $872487,0.01088120,0.01602920,-0.07007020,-0.04562210,-0.05313489,0.000$ $41330,0.00094813,-0.00110294,-0.00007995,-0.00078110,-0.00104471,-0.00$ $004709,0.00026221,-0.00014094,-0.00007334,0.00160443,-0.00107557,0.069$ $80749,-0.00023480,0.00004859,0.00003821,0.00018503,0.00003845,-0.00006$ $225,-0.00006555,-0.00012369,0.00002661,-0.00007264,0.00007326,-0.00005$ $818,0.00010022,0.00001481,0.00005280,-0.00013623,-0.00009488,0.0000655$ 2,0.00008574,-0.00010808,0.00001407,0.00000396,-0.00002573,-0.00000381 $, 0.00001154,0.00000333,-0.00000023,0.00000651,0.00000011,0.00000561,0$. $00000348,-0.00000225,0.00001326,0.00003900,0.00001027,0.00001473,-0.00$ $158379,-0.00405686,-0.01238131,-0.00406186,0.00279721,-0.00081772,0.00$ $024850,-0.00045675,0.00080878,-0.00158586,0.00544525,-0.00196801,-0.00$ $166080,0.00265072,-0.00107267,-0.04690035,-0.12961007,-0.13210951,0.00$ $139596,-0.00133608,0.00111962,-0.00017623,-0.00004281,-0.00060472,0.00$ $024292,-0.00048574,0.00020593,0.00167628,-0.00257608,0.00183047,0.0524$ $8150,0.12784065,-0.00070948,-0.00062268,-0.00035020,-0.00019439,0.0000$ $3659,0.00003373,0.00005865,0.00004445,0.00002403,-0.00008081,-0.000098$ $56,0.00001928,-0.00005604,0.00004357,-0.00002416,0.00004362,-0.0000340$ $5,0.00003868,0.00016998,-0.00002209,-0.00036273,0.00000685,-0.00000940$ $, 0.00000027,0.00002627,0.00000165,-0.00000528,0.00002142,0.00001429,-0$ $.00001642,-0.00001285,0.00001357,-0.00002611,0.00012926,0.00009799,0.0$ $0003993,0.00324820,0.00038410,0.00097331,0.00098941,-0.00104829,0.0023$ $7716,0.00042246,0.00073223,-0.00004218,0.00482788,-0.00171392,-0.00049$ $493,-0.00753985,-0.01295696,-0.01314996,-0.05555311,-0.13332382,-0.233$ $58595,-0.00026363,0.00154226,0.00051284,0.00009142,-0.00003079,0.00022$ $068,-0.00017738,0.00018761,-0.00019559,-0.00094268,0.00189808,0.000064$ $30,0.05549912,0.14489395,0.24399260,0.00035589,0.00012431,0.00002039,-$ $0.00107805,-0.00069805,-0.00002865,0.00333126,0.00214114,-0.00228937,-$ $0.02542842,0.02158194,-0.00155785,-0.00313124,-0.00378716,0.00328311,-$ $0.00063305,0.00114410,-0.00083227,0.00002025,-0.00001615,-0.00003163,0$ $.00006386,0.00008816,0.00003729,0.00014398,-0.00080835,0.00099520,0.00$ $016339,0.00011229,0.00008918,-0.00038488,-0.00028665,-0.00007435,-0.27$ $696601,0.18606623,0.00226370,-0.00004183,0.00000814,0.00005586,0.00001$ $476,0.00000513,-0.00002564,-0.00000712,-0.00002631,-0.00003079,-0.0000$ $1639,-0.00003374,0.00005881,0.00002970,0.00000878,-0.00001635,0.000010$ $82,-0.00000023,-0.00000462,0.00004114,-0.00000422,-0.00004994,0.000009$ $10,0.00000128,-0.00000484,-0.00000862,-0.00000176,0.00000367,-0.000006$ $72,0.00001667,0.00001810,0.00000408,-0.00000047,-0.00000683,0.29801183$ ,-0.00008825,0.00036182,0.00008689,0.00070167,-0.00020635,0.00008055,-$0.00220284,0.00116436,-0.00089178,-0.01579545,0.01309541,-0.00330158,-$ $0.00249474,-0.00161048,0.00073560,-0.00080941,0.00062087,-0.00001158,0$ 
$.00012062,-0.00002534,0.00005817,0.00000760,-0.00004104,-0.00008321,0$. $00067972,-0.00052175,0.00020789,-0.00016216,0.00007811,-0.00026585,-0$. $00021719,-0.00011906,-0.00003331,0.20192965,-0.21660403,0.03441794,-0$. $00001171,0.00002490,0.00002699,0.00000687,-0.00001631,-0.00002320,-0.0$ $0000181,0.00000316,-0.00001957,-0.00004223,-0.00003327,0.00010763,0.00$ 002763,-0.00000233,-0.00002600,-0.00000053,-0.00000961,0.00001698,0.00 $002165,-0.00000680,-0.00002810,-0.00000032,0.00000162,-0.00000134,-0.0$ $0000877,-0.00000519,-0.00000038,-0.00000304,0.00001350,0.00000725,0.00$ $000211,-0.00000252,-0.00000619,-0.19991134,0.21647608,0.00032753,0.000$ $20795,0.00018860,0.00014325,0.00055180,-0.00096139,0.00120237,-0.00181$ $806,0.00026372,-0.00558281,-0.00383475,-0.00378360,0.00055716,0.000711$ $80,0.00170780,-0.00078360,-0.00008310,0.00082841,0.00016163,0.00008634$ ,-0.00001333,0.00001931,0.00002531,0.00009818,-0.00029214,0.00085268,0 $.00009570,0.00013199,-0.00032289,-0.00014930,-0.00003700,-0.00007321,-$ $0.00011047,0.03008528,0.01229965,-0.01852822,-0.00005404,0.00002001,0$. $00007617,0.00002147,-0.00000401,-0.00001396,-0.00000769,-0.00004290,-0$ $.00010512,-0.00004807,-0.00001141,0.00006645,0.00011405,0.00004862,-0$. $00001661,-0.00001102,-0.00000789,-0.00001703,0.00006553,-0.00001082,-0$ $.00008852,0.00001857,0.00000049,-0.00001237,-0.00003043,-0.00000096,0$. $00002648,0.00000999,0.00000616,0.00004746,0.00002039,-0.00001123,-0.00$ $002334,-0.00352786,-0.02370660,0.02114049,0.00020500,-0.00002921,0.000$ $15256,0.00063327,-0.00139326,0.00052925,-0.00586887,0.00000510,0.00003$ $029,-0.00030659,-0.02524019,0.02457750,0.00287233,-0.00213614,0.000441$ $40,-0.00086001,0.00028613,-0.00020646,-0.00002166,0.00005730,-0.000039$ $07,-0.00040569,-0.00020460,-0.00005493,0.00015192,-0.00014347,0.000142$ $78,-0.00061466,0.00130607,-0.00055812,0.00006415,-0.00002355,-0.000038$ $58,-0.06737455,-0.01345595,0.02589055,-0.00001566,0.00000090,0.0000338$ $6,0.00001308,-0.00001185,-0.00002442,-0.00000296,0.00000098,0.00000471$ $,-0.00006976,0.00005435,0.00003415,-0.00000203,-0.00001142,-0.00002154$ $, 0.00000200,0.00001968,0.00000049,0.00001709,-0.00000071,-0.00002180,0$ $.00000013,-0.00000009,0.00000162,-0.00000164,-0.00000546,-0.00000800,0$ $.00000484,-0.00001076,0.00001640,-0.00000076,-0.00000206,0.00000252,0$. $00550227,0.01825229,-0.02250390,0.06607874,0.00036589,0.00036485,-0.00$ $000994,-0.00014787,-0.00060118,0.00061876,-0.00271512,0.00199891,0.001$ $24388,-0.00466433,-0.01451655,0.00914785,0.00269989,0.00174758,-0.0020$ $9307,-0.00031847,-0.00102373,-0.00017117,0.00011157,0.00001030,-0.0000$ $2429,-0.00023460,-0.00016053,-0.00013981,0.00003972,0.00023825,-0.0001$ $8366,-0.00029366,0.00018244,0.00067397,0.00005227,0.00008978,0.0001181$ $2,-0.01192643,-0.21846288,0.19918829,-0.00005715,0.00000965,0.00008200$ $, 0.00002534,0.00000132,-0.00001982,-0.00000386,-0.00005688,-0.00008134$ $,-0.00005351,0.00001760,0.00003625,0.00009189,0.00003621,-0.00001920,0$ .,-0.00000689,-0.00001255,0.00006816,-0.00001251,-0.00008401,0.0000159 $9,0.00000488,-0.00001302,-0.00002350,-0.00000099,0.00002166,-0.0000059$ $4,0.00003073,0.00003330,0.00001153,-0.00000113,-0.00002360,-0.00572375$ ,-0.01263574,0.01511704,0.02268593,0.24274651,0.00017442, $0.00003588,0$. $00033399,-0.00086338,0.00001774,0.00078446,0.00009673,-0.00008310,0.00$ $082968,-0.00285294,-0.00624139,-0.00127767,-0.00214178,-0.00070179,0.0$ $0014207,0.00113078,0.00011392,-0.00036156,0.00013375,0.00013266,-0.000$ $00434,-0.00002096,-0.00006330,-0.00004630,-0.00007279,-0.00021214,-0.0$ $0029677,0.00051268,-0.00007860,0.00014455,-0.00002888,0.00000546,-0.00$ $003619,0.03064146,0.23779036,-0.22625722,-0.00002740,0.00000478,0.0000$ 
$5540,0.00001075,0.00000213,-0.00002603,0.00001027,-0.00003881,-0.00005$ $283,-0.00008303,0.00003893,0.00006214,0.00007998,0.00002863,-0.0000230$ $6,-0.00000872,-0.00000484,-0.00000213,0.00004175,-0.00001047,-0.000056$ $86,0.00000838,0 .,-0.00000561,-0.00002423,-0.00000493,0.00001295,0.0000$ $0013,0.00002161,0.00002340,0.00000863,0.00000010,-0.00001997,0.0016556$ $6,-0.00734719,-0.00071619,-0.02838127,-0.22340563,0.22679410 \backslash 1-0.00000$ $330,-0.00000604,0.00000541,-0.00000043,0.00000241,-0.00000147,0.000001$ $60,-0.00000043,-0.00000071,-0.00000921,-0.00000728,-0.00000122,0.00000$ $175,0.00000209,-0.00000029,0.00000053,0.00000199,-0.00000002,0.0000029$ $1,-0.00000008,-0.00000166,0.00000080,0.00000036,0.00000145,0.00000015$, $0.00000058,0.00000060,0.00000011,0.00000030,0.00000146,0.00000010,0.00$ $000103,0.00000151,0.00000669,0.00001012,0.00000775,-0.00000565,-0.0000$ $0307,0.00000566,0.00000009,0.00000266,-0.00000053,0.00000193,-0.000000$ $52,-0.00000230,0.00000234,0.00000467,-0.00000329,-0.00000087,-0.000002$ $98,-0.00000337,-0.00000136,-0.00000058,-0.00000210,0.00000144,-0.00000$ $033,-0.00000398,-0.00000027,-0.00000139,-0.00000246,-0.00000083,-0.000$ $00103,0.00000045,-0.00000055,0.00000011,0.00000246,-0.00000022,-0.0000$ $0021,-0.00000024,0.00000092,-0.00000117,0.00000004,0.00000132,-0.00000$ 121,-0.00000315।II@ 


\section{Anisole addition}

Chlorophenyl + Anisole

ortho-Transitions State

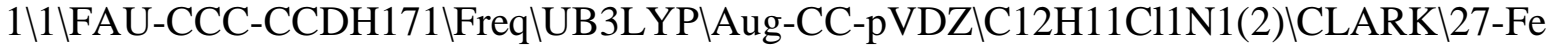
b-2015\0\\\# ub3lyp/aug-cc-pvdz freq name $=$ clark geom $=$ check guess $=$ read $\backslash \backslash$ Addition TS p-Cl-Ph-radical + aniline (ortho) \\0,2\C,2.0600550984,0.54 52465736,0.925759034\C,2.5343441466,0.9410846023,-0.3605598094\C,3.273 $1729571,0.0280223853,-1.1252992053 \backslash \mathrm{C}, 3.5961805565,-1.2324811495,-0.613$ 2008601\C,3.191922997,-1.6045590091,0.6798686703\C,2.4592620355,-0.708 $0104827,1.4499934973 \backslash \mathrm{H}, 1.7011312552,1.3153322802,1.6067036331 \backslash \mathrm{N}, 2.2742$ $797076,2.230198754,-0.8173773312 \backslash \mathrm{H}, 3.6145270013,0.314726929,-2.1215699$ $464 \backslash \mathrm{H}, 3.4680603723,-2.5788201695,1.0822003399 \backslash \mathrm{H}, 2.1585604567,-0.973826$ $2672,2.4633799601 \backslash \mathrm{H}, 4.1768944309,-1.9249603103,-1.2232868168 \backslash \mathrm{C},-2.8325$ $301877,-0.1596961914,-0.0875769667 \backslash \mathrm{C},-2.4836906314,0.7779140264,0.8856$ $115255 \backslash \mathrm{C},-1.1226099509,0.9903572129,1.1644595887 \backslash \mathrm{C},-0.173406964,0.2665$ $062187,0.4592874596 \backslash \mathrm{C},-0.5121679313,-0.6725729075,-0.5025290273 \backslash \mathrm{C},-1.8$ $687102542,-0.8917357629,-0.7865936134 \mathrm{ICl},-4.5417798779,-0.4312688226,-$ $0.444068454 \backslash \mathrm{H},-3.258094689,1.3295337417,1.4176291432 \backslash \mathrm{H},-0.8424397893,1$ $.7161590665,1.9310603302 \backslash \mathrm{H}, 0.2533176711,-1.2382434591,-1.0357749903 \backslash \mathrm{H}$, $-2.1741648867,-1.6204383541,-1.536794763 \backslash \mathrm{H}, 2.3849957455,2.3716362633,-$ $1.8132861832 \backslash \mathrm{H}, 1.4208717308,2.6476488322,-0.4670682148 \backslash \mathrm{Version}=\mathrm{EM} 64 \mathrm{~L}-$ G09RevC.01 \State=2-A $\backslash H F=-978.8451964 \backslash S 2=0.775035 \backslash S 2-1=0 . \backslash S 2 A=0.750445 \backslash$ $\mathrm{RMSD}=8.624 \mathrm{e}-09 \backslash \mathrm{RMSF}=1.083 \mathrm{e}-05 \backslash \mathrm{ZeroPoint}=0.1943755 \backslash$ Thermal $=0.2069331 \backslash \mathrm{ZP}$ $\mathrm{E}=121.9724943 \backslash \mathrm{Dipole}=0.6560841,0.388086,-0.2284321 \mathrm{DipoleDeriv}=-0.1267$ $299,-0.1969454,0.0372695,0.1173807,-0.1398167,0.0778886,0.0007418,0.31$ $63931,-0.0702804,-0.0446734,-0.283845,0.1146214,0.1722121,1.2391479,-0$ $.4278688,-0.0629305,-0.4342705,0.0935485,-0.0112431,0.2074274,-0.19358$ $05,-0.2023596,-0.3605496,0.1711093,-0.1330108,-0.1824895,0.0619728,-0$. $1092384,-0.0849517,-0.0593274,0.2250905,0.1464772,-0.1329586,-0.259587$ $5,-0.0487524,0.1522886,-0.3037263,-0.0419334,0.0336684,0.0269056,-0.04$ $37291,-0.0602781,0.3278308,-0.0356706,-0.1741973,0.0204009,-0.0533671$, $-0.1446943,-0.3884056,0.0666673,-0.0469834,-0.099779,-0.0155509,0.0559$ $901,0.165533,0.0678874,0.0425057,0.0473366,-0.0223411,-0.0510025,0.043$ $2563,-0.0452256,0.0200802,-0.2969739,-0.1384589,-0.0465007,-0.2885141$, $-1.3965969,0.3757004,-0.0575016,0.1565169,-0.2190354,0.1003896,0.01133$ $63,0.0540104,-0.0021675,0.04254,0.0735699,0.0897016,0.068877,-0.068721$ $4,0.102666,0.055059,0.0108432,0.0858362,-0.0552116,0.067763,0.009483,0$ $.0639775,0.0497464,0.093684,0.0086304,0.0752487,0.0115982,0.0617744,0$. $0423518,0.0388146,0.0408034,-0.0520875,0.0501882,0.0707957,0.0676256,0$ $.0845992,0.0083313,-0.0465774,0.0688806,-0.053667,0.0251734,1.2108061$, $0.1630617,0.2129054,0.2033444,-0.0040452,0.0579731,0.2629881,0.0557932$ $, 0.0176963,-0.2240717,-0.0747321,-0.0810569,0.0018485,-0.0565737,0.029$ $9152,0.0213606,0.0320121,-0.0666064,-0.3303497,0.0240601,0.0340473,-0$. $2482856,0.0003199,0.1218254,-0.2671637,0.1177772,0.0348718,0.835155,0$. $0459752,-0.0166331,0.1321032,-0.0855315,-0.0151257,0.1758346,0.0079487$ $,-0.083708,-0.4525597,-0.031923,-0.0142785,0.0704755,-0.0369145,0.0399$ $7,0.0550901,0.0416192,-0.0430865,-0.1959679,0.0091163,0.0041961,-0.114$ 
$9238,-0.008459,0.0741856,-0.1006541,0.0733993,-0.0100294,-0.9075135,-0$ $.0996822,-0.1257167,-0.1262828,-0.0999113,-0.0286481,-0.1724491,-0.029$ $4248,-0.1110356,-0.0633735,0.0544526,0.0531136,0.0358907,0.0753669,-0$. $0500848,0.0284105,-0.0494217,0.0774729,0.0591561,-0.0507838,-0.0549451$ $, 0.0060788,0.0179077,-0.1048145,-0.0113012,-0.1021633,0.0080959,0.0408$ $416,0.055927,0.0526059,0.0311095,0.0570524,-0.0398048,0.0205267,-0.039$ $1676,0.0601983,-0.0014044,-0.0294842,-0.0309603,-0.0391223,0.0501188,-$ $0.0760733,-0.0442816,-0.0761333,0.0466379,0.2305173,0.1546926,-0.03374$ $98,0.0891827,0.2368006,-0.070176,-0.0019904,0.0178533,0.0927853,0.1584$ $877,0.1576851,0.0087822,0.069069,0.3071759,0.0181436,0.06773,0.1189662$ ,0.1022294\Polar=246.8629672,-13.3356727,158.2016436,-7.67259,6.552559 $3,145.7185316 \mathrm{PG}=\mathrm{C} 01[\mathrm{X}(\mathrm{C} 12 \mathrm{H} 11 \mathrm{Cl} 1 \mathrm{~N} 1)] \mathrm{NImag}=1 \| 0.12056620,-0.15167258$, $0.63749596,-0.15777228,-0.01130446,0.59077914,-0.07248095,0.00513672,0$ $.06651084,0.32110862,0.00137205,-0.12109948,0.06021148,-0.17227348,0.6$ $2514838,0.04265389,0.01693338,-0.24576764,-0.16890747,-0.06788604,0.56$ $825683,-0.00805964,-0.00489900,0.02777998,-0.13685969,0.08929042,0.066$ $93912,0.27568045,0.01471080,0.03682820,-0.03276292,0.06514275,-0.17929$ $095,-0.02700222,-0.15918267,0.59787473,0.02093013,-0.01364645,-0.02387$ $854,0.09421310,-0.08478503,-0.18191168,-0.18888864,-0.10602636,0.62814$ $028,0.00166242,-0.00405028,0.00142725,-0.00447639,0.02107920,-0.003565$ $14,-0.08436919,0.07574476,-0.00834288,0.29702936,-0.00788144,-0.021960$ $37,0.02160293,0.03773045,-0.04780257,-0.04139384,0.04969127,-0.2839927$ $7,0.10788469,-0.19123018,0.60721500,-0.01017671,0.02663230,-0.02996446$ ,-0.02056563,0.00205776,0.05090752,0.01627963,0.05311969,-0.14101132,-$0.18659577,-0.04215857,0.59035443,-0.01113131,0.03336930,-0.01336809,-$ $0.01468358,0.00676614,0.02066281,0.02182934,-0.02547910,-0.00469666,-0$ $.09319645,0.01609391,0.05492276,0.27871051,0.02890411,-0.03863442,-0.0$ $0095279,0.00277466,-0.00039375,-0.01128651,-0.00992769,-0.00121801,0.0$ $3197859,-0.00838034,-0.12744942,0.08848855,-0.19548778,0.64470306,0.01$ $465074,-0.02931776,0.04601900,0.02058581,-0.01890352,-0.04189853,-0.02$ $192795,0.06769817,-0.01319921,0.08114632,0.03032599,-0.27362430,-0.151$ $87411,-0.10136397,0.57434090,-0.06158039,0.06086430,0.00427470,0.01344$ $320,-0.01902513,-0.01558481,-0.01341252,0.02063497,0.00089672,-0.00521$ $785,-0.01156268,0.04334166,-0.13871779,0.10912048,0.07016019,0.2897920$ $1,0.03518487,-0.26054627,0.04849104,-0.01505359,0.00249241,0.04751923$, $0.01640094,-0.03864968,-0.00097525,0.00710346,0.04034572,-0.05144374,0$ $.08625973,-0.20417998,-0.04222024,-0.16967491,0.55268180,-0.00084665,0$ $.09206452,-0.14153556,-0.01355603,0.03260880,-0.00373239,0.00563938,-0$ $.01248316,-0.00272138,0.02344349,-0.00775050,-0.03873554,0.09585887,-0$ $.09753440,-0.18077867,-0.18512179,-0.06769988,0.65430414,-0.04719018,0$ $.06667925,0.06401341,-0.00550242,0.00753726,0.01224194,0.00374691,-0.0$ $0210277,0.00293248,-0.00090651,-0.00002496,0.00119928,0.00606727,0.001$ $58830,-0.00305491,-0.00606877,0.01232716,0.00458778,0.06857624,0.07826$ $615,-0.19391099,-0.12242201,-0.00328881,0.00736898,0.00868922,-0.00014$ $571,0.00005172,0.00217495,-0.00056165,-0.00003578,0.00079747,0.0013798$ $3,-0.00342300,0.00325352,0.00854100,-0.01258174,-0.01908062,-0.0786287$ $0,0.20184702,0.07204211,-0.12283527,-0.16520520,0.00791545,-0.02170523$ $,-0.01057180,0.00190409,0.00249818,-0.00386286,-0.00056843,0.00111281$, $-0.00034152,0.00026218,0.00264279,-0.00028334,-0.00559206,0.01143080,0$ $.01059643,-0.07023018,0.12994076,0.17071656,0.00545205,-0.01218832,0.0$ $0663391,-0.07795922,0.08356067,0.01009444,0.00132944,0.02911661,-0.008$ 
$22096,0.00214625,0.00112195,0.00635198,0.00008199,-0.00091057,-0.00200$ $029,0.00050824,0.00268025,0.00325855,-0.00069537,-0.00098464,-0.000129$ $26,0.42351046,-0.00775981,-0.02041187,0.02051768,0.04466202,-0.2580768$ $1,0.05946465,0.01994764,-0.04632127,0.00463917,0.00136658,0.00967918,-$ $0.00528922,-0.00074313,-0.00477245,0.00572142,0.00433336,0.00217989,-0$ $.00164977,0.00092705,0.00134715,-0.00062954,-0.25825759,0.45672055,-0$. $00227860,0.04489140,-0.01647827,0.01821929,0.08716863,-0.12511352,0.00$ $577311,-0.02160351,0.01345321,0.00299417,0.00185534,-0.00577538,-0.002$ $45284,0.00170370,0.00280173,0.00624277,-0.00296505,-0.00454114,-0.0028$ $9135,-0.00038818,0.00161459,-0.19587927,-0.11838510,0.66532885,0.00251$ $412,0.00487185,0.00280888,-0.00127215,-0.00596262,0.01548593,-0.067165$ $94,-0.01460967,0.08811653,0.00448856,0.00230559,-0.00767469,0.00477341$ $, 0.00326494,0.00111536,-0.00064102,0.00005728,-0.00040146,-0.00030696$, $-0.00045229,0.00096945,-0.00172026,-0.00213038,-0.00076444,0.06189943$, $0.00372896,-0.00144888,-0.00170295,0.00705933,0.00744381,-0.01734633,-$ $0.01454838,-0.07260953,0.06799616,-0.01026914,-0.00253272,0.02625411,0$ $.00378144,-0.00381682,0.00190482,0.00024383,-0.00096113,-0.00039895,0$. $00028644,0.00029982,-0.00081889,-0.00149344,0.00131705,-0.00139700,0.0$ $1333400,0.07231203,0.00299097,-0.00216231,0.00079660,0.00402539,0.0088$ $0001,-0.01286653,0.08778129,0.06721817,-0.28271694,0.00412142,0.000522$ $32,-0.00730329,0.00085576,0.00290875,0.00267190,-0.00030155,-0.0005902$ $4,0.00025436,0.00029881,0.00024031,-0.00053446,-0.00209094,-0.00000331$ $, 0.00114704,-0.09659211,-0.07520444,0.29898542,0.00337505,0.00356695,0$ $.00485531,-0.00046953,0.00006818,0.00003630,0.00375922,0.00133073,0.00$ $329924,0.00411436,-0.00910938,0.00397873,-0.05709293,0.07213404,-0.019$ $75266,-0.00170538,0.01433872,-0.00720992,0.00006561,0.00100622,-0.0005$ $1541,-0.00024250,-0.00052027,-0.00034641,0.00040207,-0.00025790,0.0003$ $2352,0.05260404,0.00364735,0.00044228,-0.00202234,-0.00014120,0.000173$ $74,-0.00056332,0.00089534,0.00257234,0.00181327,0.00237895,-0.00385116$ $, 0.00001945,0.07246898,-0.27881171,0.09655780,0.00286534,-0.01328497,0$ $.00967961,0.00036755,-0.00056354,0.00039422,-0.00018054,-0.00008238,-0$ $.00002946,0.00027377,-0.00018818,0.00004608,-0.07932878,0.29331428,0.0$ $0402495,-0.00175021,-0.00033686,0.00041480,-0.00046732,-0.00091907,0.0$ $0381720,0.00103734,-0.00388798,-0.00870605,0.02590709,-0.00554759,-0.0$ $1918586,0.09681221,-0.09245624,0.00478605,-0.01612315,0.00960856,0.000$ $30393,-0.00068805,0.00022222,-0.00032194,-0.00012605,0.00018608,-0.000$ $19846,0.00142617,-0.00040432,0.01828337,-0.10460529,0.09294361,0.00446$ $843,0.00369326,-0.00968876,0.00386169,0.00355965,0.00122243,-0.0006821$ $1,-0.00005687,-0.00038097,0.00363659,0.00485908,0.00299066,-0.00019477$ $,-0.00485896,0.01530380,-0.06192811,-0.01299702,0.07979408,-0.00222758$ ,-0.00068463,-0.00060536,0.00031377,-0.00018789,-0.00045793,-0.0005673 $4,-0.00032829,-0.00027742,-0.00276093,-0.00153077,-0.00164845,0.055754$ $53,-0.00834863,-0.00275832,0.02540113,0.00410660,-0.00366778,0.0019679$ $3,0.00000181,-0.00102388,-0.00022125,0.00461388,-0.00116521,-0.0016862$ $5,0.00560319,0.00913909,-0.01898899,-0.01078943,-0.07093503,0.06741622$ $,-0.00143462,0.00066908,-0.00074233,-0.00054764,0.00006263,0.00143180$, $-0.00032981,-0.00006546,-0.00011128,-0.00189075,0.00016550,-0.00038374$ $, 0.00878057,0.06960434,0.00345894,0.00100904,-0.00720117,0.00047771,0$. $00219894,0.00223178,-0.00036279,-0.00038192,0.00045874,0.00359268,-0.0$ $0229372,0.00060261,0.00302324,0.00740749,-0.01103125,0.07866187,0.0659$ $7133,-0.29764982,-0.00118224,-0.00012907,0.00041293,0.00022334,-0.0000$ 
$9262,-0.00059431,-0.00022579,-0.00006541,-0.00019442,-0.00146570,-0.00$ $074094,0.00025261,-0.08711215,-0.07321890,0.31315218,-0.00058831,-0.00$ $059728,-0.00060730,0.00545875,0.00237316,0.00060121,-0.00024274,0.0036$ $0512,0.00510196,-0.12112084,0.10236329,0.09080929,-0.00109208,0.006859$ $24,0.00381888,0.00463476,0.00009219,0.00309978,-0.00048443,-0.00009793$ ,-0.00013251,-0.00021659,0.00014506,0.00032895,-0.00251532,-0.00143045 $,-0.00169203,-0.00220215,-0.00148610,-0.00129560,-0.00021312,0.0003672$ $8,0.00018930,0.11847520,-0.00032313,-0.00044451,0.00051631,0.00241740$, $-0.00285135,0.00399149,0.01456567,-0.01238339,-0.01712622,0.10211216,-$ $0.16739192,-0.09599348,-0.00477950,0.00821013,0.00595395,0.00078387,0$. $00153549,0.00276795,-0.00021739,-0.00006771,-0.00014790,0.00095872,-0$. $00058387,-0.00092278,-0.00163411,0.00034382,-0.00061136,-0.00120998,0$. $00008651,-0.00091433,-0.00025179,0.00030714,0.00037476,-0.11229723,0.1$ $7357792,-0.00046835,0.00051099,-0.00049014,0.00119277,0.00384056,0.001$ $12160,-0.00627208,0.00866875,0.00889181,0.09105864,-0.09644790,-0.1421$ $0577,0.01480617,-0.01983342,-0.00962819,0.00295138,0.00341690,-0.00407$ $308,-0.00021533,-0.00014916,-0.00003476,-0.00035329,0.00006176,0.00041$ $364,-0.00142466,-0.00098217,0.00012009,-0.00151916,-0.00044687,0.00042$ $802,0.00073242,-0.00098265,-0.00068701,-0.10022976,0.10216020,0.146053$ $35,0.00009129,-0.00010828,-0.00010065,0.00010653,-0.00065867,0.0003364$ $4,-0.00023262,0.00028297,0.00017632,0.00016017,-0.00045900,0.00031012$, $0.00026226,-0.00011607,-0.00058581,-0.00019572,0.00057224,0.00002481,-$ $0.00024807,-0.00000716,-0.00001353,0.00018551,0.00078379,-0.00012117,-$ $0.00000629,0.00001106,-0.00006856,0.00000048,-0.00006758,-0.00000590,-$ $0.00005322,0.00001648,0.00000825,0.00000804,-0.00003964,-0.00001992,0$. $50190974,0.00005901,-0.00025833,-0.00019302,0.00004792,-0.00022493,0.0$ $0000257,0.00005033,0.00007598,0.00010223,-0.00000300,0.00003065,0.0000$ $8428,0.00005492,0.00004313,0.00005782,0.00018220,0.00008128,-0.0000467$ $7,0.00012959,0.00013870,0.00006788,0.00008828,0.00014007,0.00012943,-0$ $.00002147,0.00001323,-0.00001658,-0.00001239,-0.00000204,-0.00000549,0$ $.00001260,-0.00004337,0.00002667,0.00000582,0.00000524,-0.00001130,-0$. $03456776,0.38118876,0.00006569,-0.00027034,-0.00021329,0.00009614,-0.0$ $0030400,0.00004524,-0.00000634,0.00014872,0.00003779,-0.00000877,0.000$ $00947,0.00016775,0.00012044,0.00009300,-0.00003309,0.00014987,0.000128$ $69,-0.00002243,0.00014015,0.00005147,0.00013152,0.00008658,0.00021597$, $0.00011050,-0.00001794,0.00000626,-0.00000797,-0.00001859,-0.00002769$, $0.00000832,-0.00002552,-0.00004810,-0.00000526,0.00000450,-0.00003280$, $-0.00000612,-0.01776795,0.25854544,0.38844921,0.00074985,0.00070629,0$. $00079526,-0.00036191,0.00085555,-0.00021266,0.00005872,-0.00044908,-0$. $00027770,0.00001219,0.00022056,-0.00028050,-0.00023435,-0.00006022,0.0$ $0017638,-0.00018354,-0.00025973,-0.00002375,0.00002400,-0.00034780,-0$. $00030541,-0.00000337,-0.00060507,-0.00002286,0.00004461,-0.00000331,0$. $00002810,0.00003349,0.00000745,0.00000550,-0.00002757,0.00007192,-0.00$ $004820,-0.00000505,0.00000319,-0.00000849,-0.11354518,-0.00407318,-0.0$ $0620384,0.68120507,0.00088045,0.00010085,-0.00087438,0.00063654,-0.000$ $55310,0.00038169,-0.00028622,0.00038916,0.00009442,0.00012076,-0.00051$ $807,0.00023530,0.00015235,-0.00010910,-0.00061991,-0.00040137,0.000616$ $93,0.00023283,0.00006226,-0.00002478,-0.00002915,-0.00001865,0.0005407$ $7,-0.00013808,-0.00001214,0.00000241,-0.00001516,0.00001916,-0.0000026$ $8,0.00001675,0.00002117,-0.00002216,0.00003888,-0.00000937,-0.00001142$ $, 0.00002312,-0.04919000,-0.18893569,-0.12896696,-0.04739434,0.40393440$ 
$, 0.00082714,-0.00081329,0.00018369,0.00042362,-0.00074414,0.00043775,-$ $0.00044222,0.00022903,0.00023897,0.00015694,-0.00063057,0.00018674,0.0$ $0013402,-0.00010470,-0.00048174,0.00039006,0.00050516,0.00014158,0.000$ 06000,-0.00004550,-0.00008566,-0.00031259,0.00070650,-0.00016674,0.000 $03214,0.00004361,-0.00001209,-0.00000302,-0.00001300,-0.00001292,0.000$ $02434,-0.00003320,0.00007733,0.00000841,0.00002552,0.00001515,-0.05223$ $975,-0.12697783,-0.19702966,-0.02412846,0.28144287,0.41468321,-0.00556$ $284,0.00248981,0.00222913,-0.00197738,0.00278926,-0.00219380,0.0011753$ $5,-0.00096829,-0.00031110,-0.00040347,0.00177821,-0.00092428,-0.000680$ $20,0.00058568,0.00203499,-0.00016084,-0.00273368,-0.00016651,0.0009033$ $9,0.00037113,0.00043390,0.00072671,-0.00225251,0.00063321,-0.00006851$, $-0.00002364,0.00013432,-0.00003686,0.00014366,0.00004037,-0.00009269,0$ $.00003664,-0.00027046,-0.00009574,0.00000099,-0.00005382,-0.00105985,-$ $0.06190256,-0.06354737,-0.30264891,-0.00283671,-0.01390957,0.60470194$, $-0.00201349,0.00117936,0.00072261,-0.00053754,0.00103558,-0.00082167,0$ $.00047955,-0.00044739,-0.00024231,-0.00025636,0.00102880,-0.00032890,-$ $0.00035471,0.00009745,0.00090193,-0.00012101,-0.00123114,-0.00021556,-$ $0.00066766,0.00025222,0.00037992,-0.00039814,-0.00174728,0.00017124,0$. $00000297,-0.00004888,0.00005712,-0.00001123,0.00006847,-0.00001292,-0$. $00006687,0.00008081,-0.00004806,-0.00004907,0.00004030,-0.00002469,-0$. $02139151,0.00740973,-0.00079528,-0.05430043,-0.09204842,-0.03368619,-0$ $.00492248,0.41265393,-0.00222067,0.00119854,0.00113113,-0.00141780,0.0$ $0194108,-0.00079254,0.00110061,-0.00131423,-0.00050443,-0.00022456,0.0$ $0169974,-0.00103326,-0.00072894,0.00043692,0.00175998,0.00002485,-0.00$ $160574,-0.00074239,-0.00059909,0.00035761,0.00034764,-0.00000617,-0.00$ $171308,0.00014037,-0.00001528,-0.00007632,0.00007998,-0.00000923,0.000$ $07129,-0.00000265,0.00002538,0.00006405,-0.00010040,-0.00008299,0.0000$ $0274,-0.00001655,-0.02216833,-0.00214936,0.00405350,-0.06757644,-0.031$ $76182,-0.09753161,0.01700924,0.29487527,0.43301198,0.07052107,-0.00715$ $881,0.00204227,-0.02176556,-0.01228619,0.03015066,0.00169899,-0.007116$ $49,-0.00218090,-0.00262656,0.00145676,0.00669312,0.00669624,-0.0073366$ $9,-0.01305519,-0.02103118,0.03084110,-0.00928236,-0.01457384,-0.003659$ $16,-0.00400303,-0.00054551,0.00875779,-0.00436069,0.00059310,-0.000036$ $67,-0.00044952,0.00033819,-0.00045687,-0.00032331,0.00080241,-0.000322$ $84,0.00129710,0.00031583,-0.00012457,-0.00002898,-0.00756444,0.0117838$ $8,0.01216346,-0.05036126,0.00565891,0.00322461,-0.19782908,0.09921738$, $0.09558550,0.32392738,0.00833543,-0.01009806,-0.00359047,-0.00229157,-$ $0.00390207,0.00463653,-0.00001456,-0.00086844,0.00007815,-0.00012517,0$ $.00002491,0.00032575,0.00074603,-0.00025197,-0.00039646,0.00523152,0.0$ $0247922,0.00029031,-0.00710001,-0.00178568,-0.00235919,-0.00157354,0.0$ $0329861,-0.00058628,0.00009340,-0.00001373,-0.00011354,-0.00020122,-0$. $00011635,-0.00010210,-0.00013917,0.00005471,-0.00008703,0.00006847,-0$. $00000990,0.00001157,0.01217923,-0.03872503,-0.03150900,0.04442974,0.03$ $054065,0.01849827,0.07604890,-0.14399654,-0.09188735,-0.05968592,0.355$ $16993,0.01440880,-0.00508441,-0.00818792,0.00302319,0.00022783,0.00389$ $721,-0.00020987,0.00049265,-0.00050503,-0.00057387,-0.00080466,0.00274$ $317,0.00187851,-0.00214353,-0.00446451,-0.00598039,0.00780421,-0.00042$ $094,-0.00774828,-0.00225580,-0.00238193,0.00095488,-0.00003564,-0.0002$ $9276,-0.00012480,-0.00005135,-0.00009689,0.00012588,-0.00012354,-0.000$ $08914,0.00026869,-0.00002557,0.00042098,0.00011460,-0.00000762,0.00005$ $374,0.01202187,-0.03176768,-0.03888190,0.04307020,0.01731884,0.0333481$ 
$7,0.07131849,-0.09006795,-0.14280894,-0.05356189,0.27505224,0.35918825$ ,-0.00668358,-0.00206451,-0.00245587,-0.00126937,0.00348535,-0.0031602 $5,0.00131545,-0.00147886,-0.00055862,-0.00046035,0.00226232,-0.0008849$ $9,-0.00076686,0.00075194,0.00224860,0.00072597,-0.00377427,0.00015384$, $-0.00092008,-0.00062584,-0.00067081,0.00037657,-0.00282617,0.00061170$, $-0.00012683,-0.00011657,0.00015399,-0.00008434,0.00014008,-0.00001876$, $-0.00008267,0.00017754,-0.00024867,-0.00013266,0.00000785,-0.00007665$, $-0.05020830,0.04190826,0.03992581,-0.03072505,-0.02288925,-0.02464893$, $0.04780005,0.00666809,0.00896055,-0.10048814,-0.02276126,-0.02466587,0$ $.64865166,-0.00012306,-0.00066216,0.00027344,0.00014933,0.00028322,-0$. $00009538,-0.00026641,0.00027829,-0.00028270,-0.00033520,-0.00112457,0$. $00056472,0.00080588,-0.00027383,-0.00080726,-0.00008096,0.00112601,0.0$ $0052217,-0.00029476,0.00012257,-0.00003969,0.00000692,-0.00002462,0.00$ $000175,0.00006800,0.00008148,-0.00003640,-0.00000203,-0.00000634,-0.00$ $006386,-0.00002823,0.00003049,-0.00006062,0.00005517,0.00003943,0.0000$ $1405,0.00148301,0.03005414,0.02328785,-0.02202954,-0.01722449,-0.01527$ $357,0.02954452,-0.02211486,-0.02519391,-0.04584441,-0.18893961,-0.1382$ $4780,-0.04941445,0.40736437,-0.00068316,-0.00041115,-0.00039424,0.0002$ $2869,0.00075337,0.00024266,0.00026192,-0.00012290,-0.00005544,-0.00037$ $331,-0.00030886,-0.00018099,0.00024628,-0.00026338,-0.00036446,0.00017$ $744,0.00026654,0.00047552,-0.00025781,-0.00006803,0.00007640,0.0004550$ $9,0.00035001,-0.00003662,-0.00000876,-0.00002821,-0.00000574,0.0000687$ $8,-0.00002407,0.00004582,-0.00005078,0.00000133,-0.00001017,0.00007395$ $,-0.00002407,-0.00000048,-0.00090368,0.02475710,0.03236858,-0.02342105$ ,-0.01564352,-0.01988688,0.03241673,-0.02612738,-0.02119729,-0.0487058 $8,-0.13660946,-0.19426954,-0.02606083,0.27822876,0.40836457,0.00143508$ $,-0.00031145,-0.00001273,0.00031391,0.00005365,0.00006080,0.00000416,0$ $.00021454,0.00007926,-0.00021589,-0.00005449,-0.00001492,0.00002028,0$. $00018039,0.00023727,0.00046587,0.00027435,0.00006852,0.00021314,0.0001$ $5535,0.00013378,0.00007702,-0.00006825,0.00027270,-0.00002897,0.000014$ $87,0.00000673,-0.00003126,0.00001780,0.00000774,-0.00001586,-0.0000898$ 2,-0.00001893,0.00002091,0.00000810,0.00000321,-0.19404040,0.05512946, $0.04998338,0.04998203,0.03400282,0.03653248,-0.05919765,0.01291932,0.0$ $1077572,0.00109039,-0.06143183,-0.06275433,-0.30918854,-0.00209827,-0$. $01421478,0.64361496,-0.00059694,0.00021550,-0.00055033,-0.00038322,0.0$ $0084934,-0.00043611,0.00036119,-0.00041264,-0.00014424,-0.00024931,0.0$ $0073583,-0.00025365,-0.00028161,0.00033960,0.00060950,0.00011641,-0.00$ $061567,-0.00006603,-0.00009462,-0.00010128,-0.00010692,0.00030766,-0.0$ $0051831,0.00007955,-0.00003987,-0.00004611,0.00004130,-0.00003695,0.00$ $000561,0.00000712,-0.00005320,0.00000186,-0.00008805,-0.00001273,-0.00$ $002451,-0.00001664,0.10054190,-0.15296486,-0.08480557,0.00253553,-0.02$ $023003,-0.02880856,0.01494674,-0.00483897,-0.00095743,-0.02256219,0.00$ $757183,-0.00719263,-0.05490620,-0.09344338,-0.03479749,0.00680173,0.42$ $453727,-0.00054307,-0.00054520,0.00002606,-0.00013686,0.00051211,-0.00$ $045744,0.00039359,-0.00020424,0.00015629,-0.00017054,0.00062312,-0.000$ $32738,-0.00018797,0.00012472,0.00055251,0.00016557,-0.00072323,-0.0000$ $9163,-0.00018247,-0.00006277,-0.00010120,0.00005601,-0.00056343,0.0002$ $1462,-0.00005701,-0.00002132,0.00001108,-0.00005064,0.00002852,-0.0000$ $1706,-0.00000569,0.00005962,-0.00004633,-0.00002399,0.00002528,-0.0000$ $0426,0.09607659,-0.08669557,-0.14850175,0.00442351,-0.02718065,-0.0195$ $7645,0.01256535,-0.00148310,-0.00368098,-0.02332573,-0.00845878,0.0039$ 
$8185,-0.06718159,-0.03256404,-0.09734668,0.03048793,0.30077665,0.43344$ $790,-0.00024624,0.00011981,0.00014672,-0.00018267,0.00086289,-0.000416$ $59,0.00023499,-0.00037465,-0.00020846,-0.00010952,0.00043889,-0.000288$ $12,-0.00024839,0.00005870,0.00049478,0.00006377,-0.00060579,0.00005569$ $, 0.00024272,0.00003308,0.00005119,-0.00022408,-0.00090108,0.00013190,0$ $.00000480,-0.00001089,0.00007695,-0.00000582,0.00007233,0.00000772,0.0$ $0004921,-0.00001126,0.00000209,-0.00002393,0.00003549,0.00001945,-0.14$ $177186,-0.01608002,-0.02186062,-0.02871896,-0.00480172,-0.00634622,0.0$ $0256542,-0.00237224,-0.00259721,0.00004480,-0.00065765,-0.00048033,0.0$ $0075436,0.00476289,0.00457254,-0.04152168,-0.00740791,-0.00960117,0.20$ $933043,-0.00004335,-0.00003631,0.00010211,-0.00001050,0.00007855,-0.00$ $006442,0.00003604,-0.00003034,0.00001365,-0.00001241,0.00007976,-0.000$ 04664,-0.00004280,0.00000938,0.00006642,0.00000673,-0.00010255,0.00001 $118,0.00006944,0.00001768,0.00002240,-0.00005177,-0.00010774,0.0000211$ $6,-0.00000445,-0.00000344,0.00001158,-0.00000673,0.00001177,-0.0000000$ $4,0.00000763,-0.00000314,-0.00000348,-0.00000814,0.00000419,0.00000398$ ,-0.01610498,-0.03373085,-0.01132235,-0.02371834,0.00000373,-0.0056580 $7,0.00201240,0.00024574,-0.00433221,-0.00034384,0.00034800,0.00145344$, $0.00000785,0.00097073,-0.00354078,0.01166529,0.00558931,0.00159730,0.0$ $2709506,0.02826536,-0.00003895,0.00011992,-0.00003920,-0.00007765,0.00$ $018991,-0.00007240,0.00006563,-0.00009947,-0.00003672,-0.00003120,0.00$ $011175,-0.00007075,-0.00006637,0.00004404,0.00013429,-0.00000761,-0.00$ $013705,0.00000589,0.00008432,0.00002760,0.00002247,-0.00002340,-0.0001$ $7803,0.00002456,-0.00000617,-0.00000563,0.00001632,-0.00000276,0.00001$ $988,0.00000095,0.00000899,-0.00000605,-0.00000222,-0.00001002,0.000009$ $98,0.00000173,-0.02180378,-0.01128771,-0.03589555,-0.02573308,-0.00485$ $085,-0.00158350,0.00200877,-0.00447036,0.00002918,-0.00031625,0.001394$ $00,0.00039474,-0.00012522,-0.00329970,0.00105518,0.00970463,0.00072168$ $, 0.00577031,0.03675917,0.01803224,0.03179950,-0.00028881,0.00020795,0$. $00019042,-0.00031195,0.00051803,-0.00023700,0.00021900,-0.00025723,-0$. $00009944,-0.00006910,0.00034834,-0.00017281,-0.00012798,0.00005682,0.0$ $0031687,-0.00003173,-0.00034538,-0.00006283,0.00004947,0.00000538,0.00$ $001450,0.00001673,-0.00047573,0.00006568,-0.00000750,-0.00001201,0.000$ 02236,-0.00000807,0.00002251,0.00000105,-0.00001236,0.00001240,-0.0000 $2829,-0.00001226,0.00000512,-0.00000392,0.00636121,-0.00593581,-0.0059$ $3201,-0.19725780,0.10496758,0.10057108,-0.01506769,0.01514895,0.014660$ $14,-0.00444024,-0.00082963,-0.00122885,-0.00016493,-0.00081859,-0.0007$ $3804,0.00075530,-0.00149335,-0.00181019,0.00123379,-0.00053812,-0.0004$ $1167,0.20744031,-0.00011435,0.00005231,0.00001730,-0.00001439,0.000089$ $23,-0.00002712,0.00003813,-0.00005018,-0.00001170,0.00000344,0.0000523$ $8,-0.00004678,-0.00002772,0.00002531,0.00005530,-0.00000608,-0.0000361$ $1,-0.00003377,-0.00000344,0.00001770,-0.00001184,0.00000119,-0.0000505$ $1,0.00000406,-0.00000213,-0.00000136,0.00000600,0.00000391,-0.00000203$ ,-0.00000097,-0.00000726,0.00000128,-0.00000899,-0.00000413,-0.0000002 $1,-0.00000242,0.01604625,-0.00622699,-0.00903006,0.10204651,-0.1186544$ $4,-0.08138747,-0.00523357,0.00599110,0.00165349,-0.00092382,0.00382741$ $,-0.00257566,-0.00051847,-0.00048129,0.00047319,-0.00202261,0.00073200$ $,-0.00485432,0.00016819,-0.00093658,0.00184247,-0.11028232,0.11789806$, $-0.00020166,0.00004497,0.00004170,-0.00003328,0.00001852,-0.00005535$,$0.00000752,0.00001080,-0.00000483,-0.00000546,0.00002179,0.00001557,-0$ $.00001178,0.00000057,0.00002265,0.00001806,-0.00008158,0.00003013,0.00$ 
$000566,-0.00001409,0.00002212,0.00002009,-0.00002655,0.00000065,0.0000$ $0047,-0.00000133,0.00000278,-0.00000363,0.00000462,0.00000070,-0.00000$ $129,0.00000807,-0.00000694,-0.00000312,-0.00000066,-0.00000149,0.01650$ $412,-0.00996229,-0.00614822,0.09760592,-0.08134254,-0.11422084,-0.0060$ $0287,0.00253013,0.00655113,-0.00149623,-0.00234845,0.00376457,-0.00059$ $585,0.00028563,-0.00062049,-0.00223364,-0.00483606,0.00028667,0.000366$ $65,0.00183596,-0.00082410,-0.10508531,0.09179784,0.11318323,0.00068704$ $, 0.00062745,0.00066269,-0.00005688,-0.00017716,-0.00006871,-0.00009306$ $, 0.00006067,-0.00007454,0.00004100,-0.00010736,0.00001039,-0.00003827$, $-0.00005711,-0.00005974,-0.00005266,-0.00009156,-0.00007193,-0.0002239$ $7,-0.00027738,-0.00022854,0.00000911,0.00011232,-0.00004989,0.00003062$ $, 0.00000059,-0.00000813,0.00002648,-0.00001183,0.00000284,0.00000305,0$ $.00005230,0.00002930,0.00000814,-0.00000311,-0.00000550,-0.00385985,-0$ $.00011415,-0.00055785,-0.00493727,-0.02066814,-0.02183119,-0.07261337$, $-0.04572343,-0.04906740,0.00916764,0.01357651,0.01452546,-0.00229643,0$ $.00314213,0.00279296,-0.00124016,0.00024464,0.00020519,-0.00129767,-0$. $00082438,-0.00090059,0.00123220,0.00008601,0.00033347,0.07505337,-0.00$ $033749,-0.00049956,0.00032917,-0.00002336,0.00006502,-0.00005739,-0.00$ $017874,0.00006668,0.00019464,0.00006710,-0.00020763,0.00005411,0.00002$ $653,0.00004471,-0.00005887,0.00027634,-0.00000435,0.00008231,0.0005014$ $3,0.00016991,0.00003591,0.00005519,0.00017954,0.00002984,-0.00000938,0$ $.00002775,0.00001811,-0.00001778,-0.00000132,0.00000401,0.00009132,-0$. $00006751,0.00003863,-0.00000280,0.00000052,0.00001265,-0.00048050,0.00$ 457551,-0.00223007,0.00018949,0.00063034,-0.00443296,-0.04894714,-0.16 $456728,-0.13693366,-0.00260317,-0.00940071,-0.00963692,0.00213738,0.00$ $237607,-0.00422388,0.00039345,-0.00020372,0.00059619,-0.00008138,-0.00$ $005743,-0.00018353,-0.00012765,-0.00175705,0.00229001,0.04960842,0.169$ $33429,-0.00012480,0.00025863,-0.00057425,0.00037930,-0.00046564,0.0000$ $6754,-0.00018446,0.00036647,0.00017334,0.00004827,-0.00028600,0.000118$ $71,0.00004695,0.00010561,-0.00024871,-0.00020583,0.00010236,0.00031034$ $, 0.00046735,0.00004878,0.00013133,0.00007101,0.00042003,-0.00000258,-0$ $.00002192,0.00000687,-0.00000270,-0.00000178,0.00001763,0.00001572,-0$. $00003747,-0.00000711,-0.00002117,-0.00000771,0.00000316,0.00000265,-0$. $00097087,-0.00218204,0.00431217,-0.00000810,-0.00542545,-0.00047010,-0$ $.05266436,-0.13677247,-0.17952477,-0.00235482,-0.00886125,-0.00972316$, $0.00187728,-0.00400223,0.00240275,0.00044581,0.00061411,-0.00008435,-0$ $.00017567,-0.00019406,-0.00012798,0.00012543,0.00227059,-0.00157356,0$. $05363905,0.15400348,0.18582859,0.00011064,-0.00014735,-0.00004815,0.00$ $035571,-0.00047830,-0.00010219,0.00003973,-0.00012971,0.00032011,-0.00$ $065491,0.00023559,-0.00012903,0.00006123,0.00020953,-0.00002508,0.0002$ $6892,-0.00007057,0.00009073,-0.00000287,-0.00001083,0.00000382,-0.0000$ $8330,0.00028094,-0.00004391,0.00002216,0.00002092,-0.00003340,0.000023$ $92,-0.00002627,0.00002588,0.00001378,0.00001479,0.00003613,0.00006395$, $-0.00002211,-0.00002064,-0.00311815,-0.00150048,-0.00193157,-0.0003476$ $4,-0.00047930,-0.00050779,0.00066419,-0.00207145,-0.00233962,0.0097029$ $3,-0.00308546,-0.00254941,-0.19227895,0.10091031,0.09430611,-0.0168389$ $7,0.01596027,0.01505626,-0.00151980,0.00034703,0.00027624,-0.00004372$, $0.00001848,0.00005390,0.00036987,-0.00028918,-0.00031014,0.20202504,0$. $00057714,-0.00022985,0.00042561,0.00013347,-0.00000364,-0.00015355,0.0$ 0029596,-0.00012900,0.00030430,0.00009842,0.00066260,-0.00031146,-0.00 $029425,-0.00009715,0.00009137,-0.00025727,0.00008115,-0.00016141,0.000$ 
$02017,0.00001149,0.00002660,-0.00000833,-0.00025924,0.00012688,-0.0000$ $5599,-0.00002831,0.00000202,0.00004969,-0.00000600,0.00005836,0.000015$ $20,-0.00000055,0.00003984,0.00003661,-0.00001931,-0.00001958,-0.001130$ $12,0.00414668,-0.00271531,-0.00079373,-0.00050413,0.00024848,-0.001015$ $81,0.00079494,-0.00556806,0.01357761,-0.00998921,-0.00917474,0.1031624$ $8,-0.11944984,-0.07919105,-0.00502032,0.00642116,0.00099398,-0.0004349$ $8,0.00006563,0.00000153,0.00003644,-0.00038436,0.00039847,0.00062496,-$ $0.00024914,-0.00029449,-0.10964481,0.12095354,0.00060061,0.00036022,-0$ $.00028531,-0.00037123,-0.00024383,0.00020615,-0.00012393,-0.00004118,-$ $0.00015530,-0.00003636,0.00009055,0.00036518,0.00000017,0.00031249,0.0$ $0019562,0.00008034,-0.00015162,-0.00018675,0.00007333,0.00003053,0.000$ $03757,0.00003289,0.00012340,-0.00011186,0.00007155,0.00005538,-0.00000$ $163,-0.00002904,0.00000830,-0.00001500,-0.00001184,-0.00002669,-0.0000$ $1250,0.00003421,-0.00002579,-0.00001780,-0.00160657,-0.00284700,0.0039$ $2214,-0.00071868,0.00025179,-0.00057474,-0.00102949,-0.00564717,0.0005$ $8403,0.01429218,-0.01018379,-0.00939621,0.09659615,-0.07928301,-0.1109$ $8582,-0.00609160,0.00210841,0.00702863,-0.00053170,0.00003540,0.000060$ $15,0.00009122,0.00040963,-0.00037018,0.00061305,-0.00030921,-0.0002622$ $0,-0.10233409,0.09256134,0.11163200,-0.00042806,-0.00004380,-0.0001074$ $8,-0.00015512,0.00042217,-0.00025076,0.00020254,-0.00021076,-0.0000725$ $5,-0.00005499,0.00034101,-0.00014138,-0.00012224,0.00008745,0.00032065$ $, 0.00010352,-0.00037166,-0.00002104,-0.00002989,-0.00002324,-0.0000139$ $9,0.00003986,-0.00036126,0.00008138,-0.00003058,-0.00001858,0.00002214$ ,-0.00002505,0.00001946,-0.00000820,-0.00001407,0.00000036,-0.00003012 ,-0.00002625,0.00000520,-0.00000414,0.00719808,0.01479673,0.01515926,$0.00267905,0.00251132,0.00212103,-0.00098889,0.00042893,0.00049264,-0$. $00431766,-0.00118050,-0.00166603,-0.00391445,-0.01940943,-0.01997920,-$ $0.07571619,-0.05371963,-0.05613208,0.00106597,0.00042455,0.00058681,0$. $00047349,0.00074551,0.00078206,0.00005319,-0.00008918,-0.00004607,0.00$ $116608,0.00006955,0.00035231,0.07816483,0.00007198,0.00005942,-0.00004$ $379,-0.00015030,0.00014575,-0.00001388,0.00007931,-0.00008970,-0.00003$ $555,-0.00002312,0.00008001,-0.00005959,-0.00001671,0.00003112,0.000115$ $04,0.00004075,-0.00011103,-0.00001672,-0.00001170,-0.00000648,0.000006$ $41,0.00002552,-0.00011357,0.00002278,-0.00001339,-0.00000545,0.0000028$ $7,0.00000044,0.00000293,0.00000491,0.00000394,0.00000106,0.00000083,-0$ $.00000058,0.00000947,0.00000327,-0.00719174,-0.00672812,-0.01041612,0$. $00293773,0.00213120,-0.00313093,0.00031399,-0.00012510,0.00072496,-0.0$ $0076418,0.00400436,-0.00252801,0.00073189,0.00125488,-0.00312045,-0.05$ $081896,-0.17350338,-0.14194027,-0.00035070,-0.00073959,0.00192350,-0.0$ $0030647,-0.00012544,-0.00060222,-0.00005048,-0.00045437,0.00038695,-0$. $00004654,-0.00170005,0.00239228,0.05553881,0.17595904,0.00003946,-0.00$ $005973,0.00007176,0.00001167,0.00009644,-0.00007508,0.00005030,-0.0000$ $2158,0.00000152,-0.00001055,0.00007729,-0.00002502,-0.00000587,0.00000$ $424,0.00003753,-0.00006603,-0.00002689,-0.00000179,-0.00001495,0.00000$ $625,-0.00001152,-0.00002134,-0.00011188,0.00002053,0.00000046,-0.00000$ $217,0.00000198,-0.00001367,0.00000768,-0.00000441,-0.00000016,-0.00000$ 491,-0.00001563,-0.00000293,-0.00000161,0.00000333,-0.00711519,-0.0094 $9355,-0.00670054,0.00260134,-0.00307992,0.00232605,0.00010629,0.000758$ $39,-0.00005326,-0.00115810,-0.00275065,0.00370544,0.00048208,-0.003986$ $92,0.00036517,-0.05318327,-0.14196581,-0.18120975,-0.00016860,0.001965$ $51,-0.00064393,-0.00030134,-0.00054055,-0.00011840,-0.00000691,0.00040$ 
$076,-0.00043307,0.00020451,0.00237200,-0.00166737,0.05853800,0.1563323$ $6,0.18441962,0.00286645,0.00200805,-0.00053122,-0.00070360,-0.00857781$ ,-0.00505092,-0.00096532,0.00141552,-0.00127728,-0.00076997,-0.0004746 $9,0.00008737,-0.00023440,0.00050782,-0.00006763,0.00102127,-0.00019520$ $, 0.00052502,-0.00067031,-0.00009646,-0.00012673,-0.03554995,0.02809694$ $, 0.03346158,0.00013582,0.00035027,0.00088609,0.00002358,-0.00000783,0$. $00007759,-0.00005173,-0.00001963,-0.00004666,0.00002567,0.00005545,0.0$ $0000092,-0.00011860,-0.00010288,-0.00008602,0.00014050,-0.00001767,-0$. $00001974,0.00010368,0.00013231,0.00018962,-0.00070301,-0.00019726,-0.0$ $0014733,0.00027424,0.00006454,-0.00000759,-0.00013471,0.00004921,-0.00$ $002196,0.00010020,0.00001673,0.00001201,0.00003814,-0.00000264,0.00000$ $758,0.00001273,-0.00001789,-0.00008436,0.00000342,-0.00007790,0.000023$ 05,0.00001606,-0.00000480,0.00001604,0.03702841,0.00354745,-0.00533898 $, 0.00006733,-0.00687202,-0.00640938,0.03815101,-0.00283866,0.00202204$, $0.00090322,-0.00017106,-0.00063648,-0.00006729,-0.00000313,0.00030847$, $-0.00032205,-0.00054807,0.00025534,0.00177361,-0.00024970,0.00010519,0$ $.00012949,0.02358933,-0.04832351,0.04030683,0.00103489,-0.00019803,-0$. $00097942,0.00003591,0.00001063,-0.00011875,0.00013680,-0.00046020,0.00$ $012748,0.00000220,0.00008754,0.00000944,-0.00014158,-0.00005393,-0.000$ $03835,0.00004458,0.00000753,0.00001010,-0.00001385,0.00006473,0.000046$ $94,0.00015666,0.00006402,-0.00011063,0.00001021,0.00003692,-0.00001307$ ,-0.00002546,-0.00000369,-0.00003167,0.00012939,0.00001563,0.00002508, $0.00002156,0.00000130,-0.00000796,-0.00002141,-0.00001539,0.00002458$,$0.00000427,-0.00009631,0.00003541,-0.00000196,0.00000413,0.00000875,-0$ $.01567054,0.05675007,-0.00145090,0.00145866,0.00133909,-0.00100184,-0$. $00161134,-0.00983397,0.00068656,-0.00288986,0.00242318,0.00061374,0.00$ $098763,-0.00058117,-0.00033674,-0.00000878,0.00066426,0.00141706,-0.00$ $041787,-0.00010505,-0.00034623,-0.00022768,0.00025289,0.06506701,0.064$ $48328,-0.43242080,-0.00079776,0.00032686,-0.00017916,-0.00019417,-0.00$ $005439,-0.00004390,-0.00009572,0.00012800,-0.00003037,-0.00000466,-0.0$ $0006816,0.00000264,0.00004053,0.00002788,-0.00000686,-0.00000615,-0.00$ $000572,0.00001770,-0.00001695,-0.00005865,-0.00004766,0.00005558,-0.00$ $002163,0.00008687,-0.00002816,0.00002748,0.00002202,0.00000227,0.00000$ $449,-0.00000473,-0.00004727,-0.00001215,-0.00000785,-0.00000742,0.0000$ $0446,-0.00000460,-0.00000709,0.00000637,0.00000256,0.00000105,-0.00001$ $203,0.00001402,-0.00001067,-0.00000790,-0.00000302,-0.05815474,-0.0634$ $0125,0.44650826,-0.00025938,-0.00109935,0.00032177,-0.00416004,-0.0051$ $5429,-0.00073567,0.00078856,0.00015671,-0.00047516,0.00136111,-0.00034$ $304,0.00087386,0.00005909,0.00005133,-0.00020728,-0.00010000,0.0000410$ $5,0.00016372,0.00016252,0.00013409,-0.00059523,-0.31753355,0.16767564$, $0.14085290,-0.00045103,-0.00028242,-0.00021417,-0.00010168,-0.00008279$ ,-0.00008644,0.00002065,-0.00007133,0.00004001,-0.00003978,-0.00005666 $,-0.00006028,-0.00026074,0.00006467,0.00006912,-0.00021356,-0.00002845$ ,-0.00011640,-0.00015676,0.00026012,0.00022948,0.00134750,0.00056551,-$0.00001933,-0.00010675,-0.00024737,-0.00019563,0.00017723,-0.00002755$, $0.00000095,0.00018016,0.00002106,0.00004814,0.00002449,0.00000528,-0.0$ $0001633,0.00001516,-0.00014103,0.00009791,-0.00000326,0.00003582,0.000$ $02346,0.00001887,0.00000535,0.00001877,-0.00188857,-0.00214728,-0.0053$ $7733,0.32111978,-0.00007145,0.00196447,-0.00225867,0.03104278,-0.02089$ $821,-0.01458992,0.00015443,-0.00264196,0.00531961,0.00097851,-0.001266$ $18,-0.00094791,0.00000221,0.00048016,-0.00031402,-0.00077419,-0.000635$ 
$60,-0.00031195,0.00033443,0.00008176,0.00073778,0.13510086,-0.09407201$ ,-0.03152894,-0.00002897, $0.00012662,-0.00003059,0.00005143,0.00006465$, $0.00008831,0.00000971,0.00009460,-0.00001507,0.00002219,-0.00045957,0$. $00017956,-0.00026234,-0.00000894,0.00002514,-0.00001379,-0.00005947,0$. $00003043,-0.00021166,0.00022269,0.00042054,-0.00022427,0.00071366,-0.0$ $0051508,-0.00007541,-0.00027362,-0.00031253,0.00011033,-0.00009276,-0$. $00000891,0.00031355,0.00004248,0.00005483,0.00007121,0.00001847,-0.000$ $02365,-0.00000850,0.00001513,-0.00013851,0.00000876,0.00000887,-0.0000$ $1585,0.00003894,0.00001892,0.00001651,-0.00723366,0.00180236,0.0013423$ $2,-0.15933507,0.11475346,0.00011237,0.00271802,0.00016387,-0.01164997$, $-0.00350934,0.00665365,-0.00004663,0.00346559,0.00047653,0.00060998,-0$ $.00075788,0.00116604,0.00009707,0.00042782,-0.00070814,-0.00087852,-0$. $00047474,-0.00038836,0.00031441,-0.00008751,-0.00117043,0.11605366,-0$. $02789967,-0.09992239,-0.00026952,0.00008225,0.00023860,0.00016463,-0.0$ $0001742,0.00002453,0.00002871,0.00000887,-0.00000019,-0.00009241,0.000$ $04543,-0.00004212,-0.00000736,-0.00016511,-0.00015409,0.00026918,-0.00$ $010235,-0.00002338,0.00015977,0.00040602,0.00034782,-0.00090158,-0.000$ 42137,-0.00006610,0.00037105,0.00003126,-0.00006384,-0.00027805,0.0000 $6269,-0.00002591,0.00003710,0.00000188,-0.00001105,0.00006036,-0.00000$ $267,0.00002710,0.00012364,-0.00002969,-0.00031713,0.00000030,-0.000049$ $10,-0.00000282,0.00002741,-0.00000073,0.00000984,0.03033529,-0.0165288$ $2,-0.00806435,-0.13464087,0.04279657,0.10185230 \backslash 10.00002249,0.00002750$ $, 0.00003612,-0.00002897,0.00002310,-0.00000486,0.00000740,-0.00000761$, $-0.00000226,-0.00000716,0.00000501,-0.00000238,-0.00000164,-0.00000872$ ,-0.00000293,-0.00001717,-0.00001095,0.00001665,0.00002251,-0.00001371 ,-0.00000184,0.00000655,0.00000011,0.00000301,0.00000252,0.00000426,0. $00000362,-0.00000134,-0.00000268,-0.00000271,-0.00000092,-0.00000672,-$ $0.00000024,0.00000140,0.00000171,-0.00000134,0.00000079,0.00000010,-0$. $00000440,0.00000212,-0.00000267,-0.00000121,0.00001810,0.00000245,0.00$ $000463,-0.00001214,-0.00002538,-0.00003553,-0.00001525,0.00000406,0.00$ $000103,-0.00000014,-0.00000060,-0.00000631,-0.00000059,0.00000318,-0.0$ $0000479,0.00000018,-0.00000064,-0.00000016,-0.00000116,-0.00000222,0.0$ $0000288,-0.00000003,0.00000153,-0.00000494,-0.00000091,0.00000475,-0.0$ $0000639,0.00000145,0.00000304,0.00000683,0.00000191,0.00000108,0.00000$ 749111@

meta-Transition State

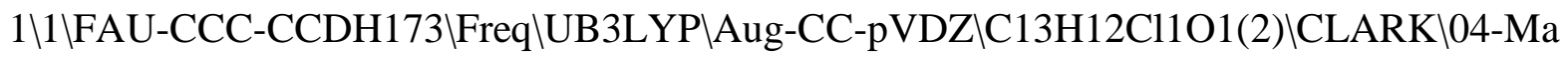
r-2015\0\\N Geom=AllCheck Guess=TCheck SCRF=Check GenChk UB3LYP/Aug-C C-pVDZ Freq \\Addition TS p-Cl-Ph-radical + anisole (meta) \\0,2\C,-1.32 $33409682,1.4849349403,0.7500227315 \backslash \mathrm{C},-2.1009403134,0.3765794675,1.1563$ $286325 \backslash \mathrm{C},-2.9660305132,-0.2473358521,0.2530653223 \backslash \mathrm{C},-3.124123747,0.268$ 0852276,-1.0459631165\C,-2.4165987926,1.4255346684,-1.4184098358\C,-1. $5475155641,2.051277151,-0.5380011649 \mathrm{UH},-0.8343191554,2.0831892734,1.51$ $69039853 \backslash \mathrm{H},-2.0158072105,-0.0248772882,2.1649234883 \backslash \mathrm{O},-3.6326023361,-1$ $.3439190661,0.7306598534 \backslash \mathrm{H},-2.5685555248,1.8380317807,-2.4163946064 \backslash \mathrm{H}$, $-1.0120237349,2.9515786435,-0.8358657994 \backslash \mathrm{H},-3.798537973,-0.1979346777$, $-1.7597012075 \backslash \mathrm{C}, 3.2494741553,-0.3259353191,-0.0402852759 \backslash \mathrm{C}, 3.097323605$ $9,1.0051469796,0.3531113534 \backslash \mathrm{C}, 1.8046178997,1.4960640088,0.5974898938 \mathrm{IC}$ $, 0.7276885048,0.6381988713,0.4437737038 \backslash \mathrm{C}, 0.8651224908,-0.6830454437,0$ 
$.0535362699 \backslash \mathrm{C}, 2.1544146982,-1.1795225717,-0.194501424 \backslash \mathrm{Cl}, 4.8736313812$, $-0.9477913108,-0.3511722947 \backslash \mathrm{H}, 3.9706235249,1.646474627,0.4661677906 \mathrm{H}$, $1.6736725544,2.5361101825,0.9023411787 \backslash \mathrm{H},-0.0010549641,-1.3362852888,-$ $0.0584234031 \backslash \mathrm{H}, 2.3063279799,-2.2137501239,-0.5015168329 \backslash \mathrm{C},-4.593370786$ $8,-1.9705334553,-0.1120540195 \backslash \mathrm{H},-5.394842843,-1.2702122211,-0.39362691$ 19\Н,-5.0159470091,-2.789933651,0.4775338194\Н,-4.125758359,-2.3793125 $52,-1.0211691303 \|$ Version=EM64L-G09RevC.01 $\backslash$ State $=2-A \backslash H F=-1038.0102801 \backslash$ $\mathrm{S} 2=0.77898 \backslash \mathrm{S} 2-1=0 . \mathrm{IS} 2 \mathrm{~A}=0.750612 \backslash \mathrm{RMSD}=4.926 \mathrm{e}-09 \backslash \mathrm{RMSF}=9.042 \mathrm{e}-06 \backslash$ ZeroPoin $\mathrm{t}=0.2098788 \backslash \mathrm{Thermal}=0.2235312 \mathrm{ZPE}=131.7009315 \backslash$ Dipole $=-1.1655274,0.1166$ $302,-0.2765936$ DipoleDeriv $=0.1977993,0.0716277,-0.0071736,0.0215184,0$. $0874188,-0.0083075,-0.0303677,0.0058303,0.1055966,-0.2992055,-0.141764$ $7,0.1449231,-0.0138422,-0.3031775,-0.0247626,-0.0092293,-0.1425801,0.0$ $395952,0.6368708,0.6060971,-0.1437603,0.962236,1.0033363,-0.2004634,-0$ $.4852791,-0.301727,0.0762647,-0.2317488,0.0547806,-0.0047613,-0.236227$ $3,-0.1901694,-0.05497,0.2111533,0.2027605,-0.0754483,0.0456975,0.08756$ $74,0.1273395,0.1812936,0.0478998,0.0263876,0.1050188,-0.1102571,0.1375$ $228,-0.2203176,0.1159444,-0.0400882,0.0211645,-0.1052643,-0.0373323,0$. $0230939,-0.0743302,-0.1776652,0.1033633,-0.0739939,-0.0451522,-0.07312$ $8,0.0315173,-0.0481774,-0.054339,-0.0343069,0.0124699,0.107529,-0.0238$ $33,-0.034338,-0.0297421,0.073006,0.0657859,-0.0166021,0.0477711,-0.011$ $0204,-1.041621,-0.6940629,-0.0310594,-1.0090263,-1.1634723,0.0718657,0$ $.0731734,0.1343119,-0.4009133,0.0913491,-0.0229342,-0.0336355,-0.01683$ $92,0.0572566,0.0670688,-0.0513186,0.0820105,-0.0623345,0.0607043,-0.11$ $26338,0.0007805,-0.0773273,-0.0015825,0.0426982,-0.0002241,0.0445354,0$ $.0563189,0.0470875,-0.0648455,-0.0666425,-0.0658042,0.0660617,-0.02598$ $85,-0.0826124,-0.0408422,0.0373569,1.0976977,-0.3745562,-0.1734898,-0$. $4300594,0.1395163,0.072209,-0.2161271,0.0856945,-0.0142598,-0.1890977$, $0.13466,0.0232988,-0.00252,-0.0376672,-0.0064516,0.0168872,0.0026397,-$ $0.0945034,-0.2980484,0.0312578,-0.0398982,0.3780678,0.0544975,0.090800$ $1,0.1145141,0.0337513,-0.0651593,0.6921544,-0.1537767,0.0681449,-0.287$ $789,-0.004303,-0.0393354,-0.1091017,0.0072163,-0.0875974,-0.4527134,0$. $1185697,-0.0370094,-0.0104288,-0.0050963,0.0156022,0.0182914,0.0135416$ $,-0.0688295,-0.1629603,0.0132326,0.0195288,0.159719,0.0234455,0.041976$ $5,0.0684594,0.0277024,-0.0720671,-0.8382871,0.2347876,0.094768,0.28552$ $27,-0.1818164,-0.0406552,0.1407918,-0.0493894,-0.0948858,-0.0723344,-0$ $.0580142,-0.0155457,-0.0313674,0.046465,-0.0166815,0.005905,-0.0180201$ $, 0.1233785,0.0753312,0.0475415,0.0234021,-0.0121547,-0.0684573,-0.0625$ $887,-0.0014726,-0.0510851,0.0986736,0.0277791,-0.0819111,0.0010459,-0$. $0462098,0.0384202,-0.0185164,-0.0145204,-0.0135244,0.0954617,0.0150999$ $, 0.0353682,0.0064176,0.0458387,-0.0267641,-0.0402028,0.025203,-0.04682$ $59,0.1129334,0.7181056,0.2963855,0.0904719,0.2626577,0.4985632,0.08008$ $57,0.358579,0.2947024,0.3640328,-0.0899362,0.0018002,-0.0328179,0.1334$ $645,-0.0423427,0.0372581,-0.1088403,-0.0498997,0.0277916,0.0179597,-0$. $0704738,0.0096637,-0.1114537,-0.0482151,0.046403,0.0553292,0.0893135,0$ $.0228265,-0.038258,0.0231795,0.0955872,0.0024364,0.0109237,-0.0337073$, $-0.0363654,-0.1389932,-0.0855391 \backslash$ Polar $=264.5191728,8.295782,172.79495$, $8.8243862,2.384489,136.0305998 \mathrm{PG}=\mathrm{C} 01[\mathrm{X}(\mathrm{C} 13 \mathrm{H} 12 \mathrm{Cl1O} 1)] \mathrm{NImag}=1 \backslash 0.2044$ $7643,0.26238783,0.50790343,0.12568905,-0.02733344,0.62106951,-0.107660$ $65,-0.11866636,0.00978091,0.36832921,-0.08909743,-0.22981568,0.0394910$ $1,0.23565885,0.46849273,0.02715873,0.07527651,-0.13310245,0.12269370,-$ $0.12062937,0.66617992,-0.02897784,-0.03093526,0.00767769,-0.15896101,-$ 
$0.05848495,-0.11493759,0.38625024,-0.02446867,-0.02159377,-0.01026690$, $-0.07996256,-0.12511188,-0.07001084,0.21802572,0.47433744,-0.02852551$, $-0.03124034,0.04505726,-0.07309730,-0.01901514,-0.20773291,0.13266227$, $-0.08398466,0.61146605,0.00127428,-0.00794597,0.01003751,0.00908492,0$. $00974919,-0.01648316,-0.09740451,-0.00896844,-0.06282618,0.38026964,0$. $00240362,-0.02610510,0.02416718,0.02360333,0.03476556,0.00549051,-0.03$ $335043,-0.12228161,0.03152609,0.25246399,0.48493274,0.01312278,0.01852$ $011,-0.02349753,-0.04110675,-0.02602330,-0.04089723,-0.01864184,0.0864$ $9364,-0.26349683,0.16411214,-0.06783222,0.61223081,-0.00739498,0.02282$ $521,-0.03856091,-0.02219763,-0.02024516,-0.01083160,0.02474962,0.00236$ $575,0.04937929,-0.13191593,-0.09360990,-0.00851904,0.36482390,-0.00554$ $235,0.04143280,-0.02313898,-0.02085113,-0.02475047,-0.01249598,0.01794$ $197,-0.01237644,0.06480864,-0.11628006,-0.22876746,0.03031698,0.211398$ $55,0.48083410,-0.02462320,0.00064902,-0.03834709,-0.00821298,-0.008227$ $22,-0.00596259,0.02245479,0.03029956,0.00182142,0.03038844,0.08234506$, $-0.12823950,0.15532651,-0.11887920,0.66242315,-0.06291828,-0.02269775$, $-0.02988435,0.01580621,0.00106179,0.02145390,-0.01328429,0.00234354,-0$ $.02444712,-0.02010892,-0.04399011,0.00968554,-0.17819775,-0.07864457,-$ $0.12841182,0.38774593,-0.01922766,-0.13036076,0.09405355,0.00331130,-0$ $.00771273,0.02735934,-0.00094510,0.00074327,-0.01012355,-0.02817888,-0$ $.02471500,-0.00742139,-0.10075728,-0.14684147,-0.07703266,0.26369083,0$ $.54286099,-0.01135178,0.04784003,-0.25577046,0.02386772,0.03927737,0.0$ $0250319,-0.02101387,-0.00560177,-0.04563325,-0.01855356,-0.04490052,0$. $04285656,-0.09150793,-0.02717739,-0.20997358,0.13375167,-0.09829672,0$. $55845736,-0.07959064,-0.08181591,-0.09052761,-0.01120103,-0.01299893,-$ $0.01257438,0.00449384,-0.00454749,0.00355902,-0.00100128,-0.00057564,-$ $0.00010602,0.00561668,0.00151530,-0.00128769,-0.00372124,-0.00129593,-$ $0.00825473,0.10327140,-0.09249998,-0.13316744,-0.10824814,-0.00747298$, $-0.00603273,-0.01767346,-0.00333013,-0.00115408,0.00130400,0.00055108$, $-0.00014220,0.00097923,-0.00169731,-0.00079801,0.00291435,0.00835147,0$ $.00983673,0.01347253,0.08572554,0.13836740,-0.10022240,-0.10540457,-0$. $19538884,0.00597476,0.00740807,0.00933882,0.00053757,0.00236104,0.0003$ $7310,0.00100656,0.00091861,-0.00011098,-0.00076426,0.00295619,-0.00430$ $576,-0.01188753,-0.01714455,-0.01192907,0.10102632,0.11297484,0.202412$ $59,0.00366258,-0.00617523,0.01776263,-0.04444761,-0.00195471,-0.028013$ $01,0.00339613,0.00709033,-0.01867395,0.00043127,-0.00434023,-0.0027760$ $9,-0.00079445,0.00001186,0.00017097,0.00118626,-0.00469747,-0.00110839$ $,-0.00162420,0.00139991,0.00021157,0.03868673,0.00457049,-0.00426814,0$ $.02144451,-0.00315341,-0.08504494,0.10115848,-0.00261412,0.00849029,-0$ $.01231865,-0.00393896,0.00095953,-0.00114300,-0.00008885,-0.00099445,-$ $0.00036819,-0.00550506,-0.00123199,0.00148337,0.00220635,-0.00005852,-$ $0.00070831,0.00568415,0.08271803,-0.00124695,0.00081423,-0.00336923,-0$ $.02858429,0.09839099,-0.30001157,0.00060165,0.01107713,-0.01473671,-0$. $00366296,-0.00185790,-0.00070221,-0.00002017,-0.00077502,0.00037918,-0$ $.00001552,0.00157564,0.00229047,0.00097682,-0.00036708,0.00042699,0.03$ $056334,-0.10932194,0.31549265,0.00408384,0.00070857,-0.00713374,-0.023$ $03732,-0.04144540,0.00712555,-0.10645528,-0.09608796,0.02557989,0.0045$ $0833,-0.00628917,0.00415071,0.00223621,-0.00320124,-0.00324149,-0.0015$ $2901,-0.00158352,0.00354154,-0.00084320,-0.00119861,0.00048155,-0.0007$ $9143,0.00243745,0.00188147,0.28650774,0.00141948,0.00630075,-0.0034755$ $9,-0.03073332,-0.03378659,0.00311423,-0.08017582,-0.18785166,0.0785984$ 
$0,-0.01567105,-0.02028569,0.01664153,-0.00132200,0.00335417,0.00182053$ ,-0.00212255,-0.00385444,0.00314517,-0.00069647,-0.00057173,0.00015161 $, 0.00228858,-0.00026546,0.00018007,0.21608420,0.34599186,-0.00247135,-$ $0.00021615,-0.00501865,-0.01338535,-0.02136631,0.01774412,0.00223975,0$ $.05126974,-0.12449081,0.02169731,0.03834793,-0.01142681,-0.00736111,-0$ $.00293260,-0.00556395,0.00267394,0.00128985,0.00197399,-0.00093409,-0$. $00073200,0.00036756,0.00158902,-0.00055507,0.00090883,0.08419020,-0.03$ $140037,0.28570659,0.00123834,-0.00555945,-0.00393847,-0.00059494,0.000$ $27028,0.00028065,0.00215682,-0.00502794,-0.00095658,0.00495265,-0.0066$ $5743,0.01441290,-0.05047725,0.00644827,-0.04119722,0.00100528,0.008192$ $51,-0.01802546,0.00000912,0.00054394,-0.00114390,-0.00030562,0.0003717$ 9,0.00014540,-0.00005806,-0.00047744, $0.00093930,0.04516622,-0.00456383$ $, 0.00185912,-0.00106725,-0.00026181,-0.00082426,-0.00062019,-0.0052021$ $1,-0.00131585,0.00112088,0.00409639,-0.00534884,0.02350187,0.00597930$, $-0.09123570,0.10084482,-0.00240998,0.00906681,-0.01286264,-0.00015341$, $0.00015463,-0.00045610,0.00034091,-0.00021940,-0.00008254,-0.00016300$, $-0.00091276,0.00155961,-0.00225249,0.08975594,-0.00417526,-0.00135021$, $-0.00017310,0.00020312,-0.00065820,0.00036173,-0.00060118,0.00181888,0$ $.00278009,-0.00254308,0.00115824,-0.00438508,-0.04142873,0.10083989,-0$ $.28906825,-0.00056468,0.00982023,-0.01331437,-0.00017816,0.00046193,-0$ $.00060109,0.00019370,-0.00012036,-0.00012640,0.00010003,0.00026937,-0$. $00038450,0.04659024,-0.11003659,0.30435604,0.00427322,0.00676872,-0.00$ $300584,0.00278413,-0.00135707,-0.00204649,-0.00018889,0.00036657,-0.00$ $055182,0.00232831,-0.00319559,-0.00464393,-0.00558348,-0.01562601,0.00$ $706935,-0.11122238,-0.12700435,0.03249892,-0.00142784,0.00088554,0.000$ $47013,0.00048916,0.00001695,-0.00019510,-0.00038021,0.00011858,0.00032$ $602,-0.00207588,0.00222855,0.00120215,0.11100968,-0.00567505,-0.006958$ 21,0.00096377,-0.00045701,0.00225663,0.00270779,0.00030890,0.00025656, $-0.00015783,-0.00372612,0.00253064,-0.00131321,-0.00489990,-0.00747059$ $, 0.00636504,-0.12568689,-0.24275770,0.06814841,0.00152699,-0.00018530$, $-0.00008776,-0.00049814,-0.00023676,0.00001413,0.00027968,-0.00010174$, $-0.00016448,0.00215645,-0.00080383,-0.00106004,0.13768559,0.25337101,0$ $.01549058,0.02251203,-0.00333607,-0.00289019,0.00236311,-0.00408396,-0$ $.00064451,-0.00032063,-0.00113296,-0.00420031,-0.00051217,-0.00138190$, $-0.01033056,-0.01760742,0.00885066,0.03113739,0.07065065,-0.07505091,0$ $.00081200,-0.00108482,0.00081007,0.00068328,0.00116159,-0.00021563,0.0$ $0041686,0.00003685,0.00017623,0.00165059,-0.00078081,0.00032679,-0.031$ 42683,-0.07557102,0.07467320,-0.00020650,0.00052541,0.00066948,0.00364 $716,-0.00088681,-0.00113654,0.00070750,-0.00267979,-0.00139555,-0.1513$ $1881,-0.08647438,-0.12519918,-0.00639356,-0.00507842,-0.01102995,0.002$ $88489,-0.00440032,0.00084461,-0.00038914,0.00019723,0.00005483,-0.0001$ $2703,-0.00012170,0.00001232,-0.00170270,0.00098462,0.00017095,-0.00163$ $340,0.00197746,0.00124127,-0.00018684,-0.00039278,-0.00064830,0.156191$ $08,0.00035397,-0.00081048,0.00039704,-0.00173585,0.00102964,0.00340591$ $, 0.00765488,0.00908069,0.00676856,-0.08672150,-0.09904051,-0.07504308$, $-0.01487109,-0.00649739,-0.01816235,-0.00445361,-0.00175534,0.00322978$ $, 0.00027215,-0.00014464,-0.00013953,0.00044954,0.00027732,0.00051291,0$ $.00028717,-0.00098028,-0.00181466,0.00208417,-0.00040210,-0.00089566,-$ $0.00087718,-0.00030803,-0.00073823,0.09877194,0.10327713,0.00068670,0$. $00063192,-0.00040992,-0.00071437,0.00405131,-0.00410911,-0.01846692,-0$ $.01546651,-0.01111397,-0.12415121,-0.07446822,-0.18065767,0.00652459,0$ 
$.00477022,0.00862480,0.00009850,0.00293374,0.00143802,0.00013621,-0.00$ $017940,-0.00003413,-0.00087625,-0.00072691,-0.00071497,0.00101689,-0.0$ $0088317,0.00125940,0.00102143,-0.00107217,0.00028346,0.00023967,0.0003$ $7039,0.00032976,0.13388245,0.07982660,0.18509411,-0.00029019,-0.000065$ $90,0.00004349,0.00005067,-0.00010120,-0.00000936,-0.00027491,-0.000561$ $08,0.00042202,0.00023935,0.00032621,-0.00005933,-0.00007947,0.00000244$ $,-0.00013095,0.00007408,0.00003960,-0.00007805,-0.00020624,0.00005313$, $0.00003196,-0.00002347,0.00001663,-0.00002683,0.00054864,0.00061040,-0$ $.00006054,0.00000035,-0.00000630,0.00005882,-0.00005048,0.00009157,-0$. $00003892,0.00000500,0.00002626,0.00001984,0.51476569,0.00001843,-0.000$ $43298,-0.00041009,-0.00003195,-0.00000469,0.00002182,0.00022528,0.0003$ $8343,-0.00023723,0.00005772,0.00007421,0.00001898,-0.00007680,0.000056$ $88,0.00012247,-0.00003686,0.00001278,0.00018315,-0.00006097,0.00015224$ $, 0.00016721,0.00006350,0.00000184,-0.00006840,-0.00022468,-0.00031695$, $0.00005596,0.00002415,-0.00000208,-0.00001348,0.00002461,0.00012411,-0$ $.00008004,-0.00001329,0.00000019,-0.00001932,0.06221086,0.59362224,0.0$ $0010290,-0.00020723,-0.00009130,0.00000506,0.00011482,0.00004148,0.000$ $11165,0.00010948,-0.00005551,-0.00004060,-0.00002745,-0.00004438,-0.00$ $000783,0.00004617,-0.00002578,-0.00004452,-0.00001297,0.00005707,0.000$ $02220,-0.00000313,0.00005871,0.00003541,-0.00001239,-0.00006204,-0.000$ 09616,-0.00009268,0.00000590,0.00001584,-0.00001647,-0.00000661,-0.000 $03443,0.00007941,-0.00003424,-0.00000460,0.00000807,-0.00000044,-0.014$ $02139,0.12883966,0.16368182,0.00074441,-0.00127068,-0.00064943,-0.0002$ $7767,0.00027113,0.00013636,0.00010388,0.00032487,-0.00018163,-0.000019$ $85,0.00001292,-0.00004640,-0.00003506,-0.00007671,0.00003658,0.0000944$ $6,0.00002696,0.00029016,0.00011067,0.00033013,0.00030531,0.00006029,-0$ $.00003616,-0.00007364,-0.00013160,-0.00016551,0.00003585,0.00000046,0$. $00000502,-0.00001129,-0.00008834,0.00023376,-0.00011217,-0.00000357,0$. $00001883,-0.00000261,-0.10649802,-0.02374711,-0.00342834,0.69570663,-0$ $.00079572,-0.00004101,-0.00036964,-0.00027868,0.00033690,0.00020181,0$. $00002235,0.00015929,-0.00026395,-0.00035293,-0.00043934,-0.00009512,0$. $00030915,-0.00001606,0.00014240,-0.00058826,0.00015618,0.00017313,-0.0$ $0018126,-0.00022499,-0.00002323,-0.00011690,-0.00001700,0.00009561,-0$. $00006667,-0.00009907,0.00001298,-0.00002855,0.00001745,-0.00003084,-0$. $00005272,0.00002349,-0.00007265,0.00000412,0.00001768,0.00001773,0.038$ $15120,-0.30645361,-0.07223072,0.05899632,0.63625105,-0.00072204,-0.000$ $12995,0.00068591,-0.00009503,0.00009975,0.00001883,-0.00018110,0.00008$ $675,-0.00030228,-0.00001096,-0.00028290,0.00016536,0.00009270,0.000094$ $34,0.00023558,0.00018842,-0.00010373,0.00006906,0.00005823,0.00009623$, $0.00012664,-0.00000754,-0.00000973,0.00002970,-0.00006736,-0.00014921$, $0.00006061,-0.00002465,0.00004395,0.00000983,0.00004718,-0.00014709,-0$ $.00001039,0.00001066,-0.00001643,0.00000273,0.01432607,-0.06742413,-0$. $08545948,-0.02923623,0.14076954,0.16979654,-0.00575352,-0.00166143,-0$. $00070285,-0.00097049,0.00141836,-0.00009876,-0.00001295,0.00067357,-0$. $00098186,-0.00047187,-0.00082037,0.00005979,0.00039587,-0.00029328,0.0$ $0030974,-0.00101037,0.00005371,0.00136746,0.00036819,-0.00058899,-0.00$ $040517,-0.00021667,-0.00006591,0.00024731,-0.00045810,-0.00078151,0.00$ $012048,-0.00005098,0.00002901,-0.00010079,0.00005604,0.00003728,-0.000$ $00790,-0.00005599,0.00002906,0.00002311,0.01610299,0.08358822,0.023148$ $53,-0.28699108,0.02970654,0.02671743,0.60713705,0.00230628,0.00132833$, $0.00093492,0.00081060,-0.00093642,-0.00046200,-0.00058937,-0.00122670$, 
$0.00119042,0.00051669,0.00086406,0.00020968,-0.00019256,0.00001049,-0$. $00037359,0.00121134,-0.00021882,-0.00081151,0.00111646,0.00063629,-0.0$ $0008423,0.00009855,-0.00000998,-0.00008666,0.00072194,0.00115831,-0.00$ $025616,0.00003138,-0.00003951,0.00009364,0.00013266,-0.00013187,0.0002$ $7287,0.00003446,-0.00004502,0.00000661,0.02830774,-0.00836160,-0.00688$ $824,0.10140815,-0.13684217,-0.02950099,0.01585452,0.67894538,0.0012088$ $7,0.00063508,0.00064011,0.00003003,-0.00050591,-0.00039612,0.00007307$, $-0.00021658,0.00094200,0.00032622,0.00065767,-0.00020225,-0.00048369,-$ $0.00045055,-0.00031439,0.00070748,-0.00008869,-0.00009416,-0.00005826$, $0.00015772,-0.00003482,-0.00000356,-0.00005416,-0.00008238,0.00017306$, $0.00030167,-0.00006615,-0.00000375,-0.00001017,0.00004197,0.00003490,-$ $0.00014978,0.00007949,0.00001376,-0.00001554,-0.00001451,0.00711483,-0$ $.01176193,0.00336488,0.04677118,-0.02342047,-0.07122012,-0.03384285,0$. $15491134,0.17311794,0.07313226,-0.01986150,0.00405461,-0.03071800,-0.0$ $2153492,0.00563603,0.00424929,0.00052439,0.01355716,-0.00267600,-0.002$ $82435,-0.00407698,0.00623238,0.01010198,0.00380297,-0.01706321,0.01238$ $368,-0.02881317,-0.01299127,0.00793208,0.00335119,0.00096040,-0.000162$ $87,-0.00101880,0.00097948,0.00180841,-0.00082542,0.00056333,0.00004651$ $, 0.00039801,0.00052064,-0.00131422,0.00026709,0.00022235,-0.00004189,0$ $.00006437,-0.01414423,-0.02487418,-0.00655202,-0.05838419,0.00777335,0$ $.00850679,-0.23189546,-0.13761475,-0.02449018,0.35149002,-0.03145424,-$ $0.00502355,-0.00405107,0.00321283,0.00948915,-0.00175886,-0.00135359,0$ $.00017778,-0.00449725,0.00147991,0.00159124,0.00121923,-0.00287109,-0$. $00494584,-0.00125561,0.00847355,-0.00419432,0.01225401,0.01014260,-0.0$ $0639574,-0.00330189,-0.00018102,0.00003485,0.00029047,-0.00089842,-0.0$ $0148882,0.00054372,-0.00028040,0.00002965,-0.00018584,-0.00021486,0.00$ $064293,-0.00011554,-0.00012913,0.00006360,-0.00000431,-0.02479671,-0.0$ $6140024,-0.01336216,-0.04607407,0.05401964,0.01530225,-0.10384217,-0.1$ $9576362,-0.03133560,0.12673920,0.57410810,-0.01088017,-0.00250588,-0.0$ $0905962,0.00550275,0.00413342,0.00076359,-0.00075760,-0.00073394,-0.00$ $257441,0.00016928,-0.00029000,0.00069148,-0.00057590,0.00040196,-0.000$ $21691,-0.00541294,0.00216027,0.00161362,0.00736506,-0.00387730,-0.0013$ $3376,-0.00029858,0.00010710,0.00029820,0.00022521,0.00039118,-0.000099$ $90,0.00016761,-0.00016298,-0.00008101,0.00014297,-0.00013961,0.0000597$ $8,-0.00007867,0.00004418,0.00003945,-0.00650349,-0.01317849,-0.0102286$ $0,-0.00693505,0.01056044,0.01701412,-0.01522442,-0.02927219,-0.0611761$ $0,0.01144207,0.13037337,0.12326343,-0.00331803,0.00490729,0.00065262,-$ $0.00027083,0.00192756,0.00016245,0.00090739,0.00200289,-0.00115733,-0$. $00065985,-0.00120994,-0.00043714,0.00041277,0.00016021,0.00031299,-0.0$ $0100457,-0.00018249,0.00170299,-0.00071979,0.00085068,0.00058230,-0.00$ $015948,0.00001591,0.00022223,-0.00070042,-0.00160258,0.00027009,-0.000$ $04546,0.00003335,-0.00012141,-0.00006916,0.00033552,-0.00017186,-0.000$ $08779,0.00006691,-0.00000909,-0.05589333,-0.04140574,-0.00663489,-0.02$ $148497,0.03041209,0.00999855,0.03895367,-0.02115756,-0.00907301,-0.094$ $10273,-0.00345976,0.00268029,0.66162302,0.00172965,0.00018010,-0.00174$ $481,-0.00030971,-0.00066009,0.00094426,-0.00064117,0.00042882,-0.00139$ $342,0.00031939,-0.00076502,0.00016547,-0.00004402,0.00025867,0.0002918$ $3,-0.00038780,0.00020128,0.00026064,0.00047217,-0.00020189,-0.00002168$ $, 0.00004974,0.00008113,0.00014109,-0.00008150,-0.00054799,0.00024274,-$ $0.00002559,-0.00001506,-0.00002385,0.00000411,-0.00018102,0.00008314,-$ $0.00007458,0.00003320,0.00003122,0.01370577,0.05637106,0.01285516,0.02$ 
$849489,-0.03887372,-0.01279043,-0.05310916,-0.03636727,-0.00699019,0.0$ $2970906,-0.31938772,-0.07809127,0.06665871,0.63554009,0.00139060,0.000$ $40037,-0.00031215,-0.00013330,-0.00099055,-0.00014132,-0.00005874,0.00$ $019983,0.00003129,0.00006985,0.00027794,-0.00022050,-0.00054647,-0.000$ $44945,-0.00001456,0.00043696,0.00002258,0.00036964,-0.00022286,-0.0002$ $2093,-0.00013922,-0.00003793,0.00005262,0.00009605,-0.00000280,-0.0001$ $6626,0.00012306,0.00004622,-0.00004415,-0.00000386,-0.00007164,-0.0000$ $3548,0.00009616,0.00000788,0.00000830,0.00000792,0.00929647,0.01789408$ $, 0.01156268,0.01003999,-0.01290121,-0.00753697,-0.01808972,-0.01005079$ $, 0.00299052,0.01180762,-0.07542146,-0.07693002,-0.02563821,0.13884148$, $0.16988855,0.00120946,-0.00027348,0.00061203,0.00037308,-0.00018034,-0$ $.00014222,-0.00029892,-0.00026987,0.00000382,-0.00013788,-0.00006868,0$ $.00021705,0.00001145,-0.00010252,-0.00015838,0.00039184,-0.00014418,-0$ $.00051279,0.00010979,-0.00014996,-0.00019677,-0.00005821,0.00001678,0$. $00006172,0.00004417,0.00014820,-0.00005405,-0.00001951,0.00001566,-0.0$ $0000985,0.00000887,-0.00017659,0.00014021,0.00001673,-0.00002670,-0.00$ $000047,-0.22516941,-0.07706623,-0.00890377,0.04015525,-0.05944676,-0.0$ $1955555,-0.06320638,-0.00997810,0.00254885,0.01857781,0.08233855,0.022$ $89859,-0.28952523,0.02851564,0.02661587,0.63530104,-0.00031193,-0.0007$ $2550,-0.00022670,-0.00049100,0.00013679,-0.00008582,0.00013175,-0.0001$ $2533,0.00062518,0.00024400,0.00044759,0.00017900,-0.00018486,-0.000029$ $78,-0.00022719,0.00009419,-0.00003666,-0.00031632,0.00019102,-0.000233$ $31,-0.00010318,0.00014120,-0.00005144,-0.00016117,0.00012360,0.0002208$ $3,-0.00009646,0.00005120,-0.00002980,0.00003127,0.00000220,-0.00005545$ ,-0.00000257,0.00001805,-0.00001225,-0.00001858,-0.13893935,-0.1997166 $4,-0.02723869,-0.01610694,-0.03646195,-0.01127883,-0.01218039,-0.00186$ $445,0.00114417,0.02902317,-0.01471601,-0.01021297,0.10028838,-0.136696$ $20,-0.02982028,-0.00063663,0.68975120,-0.00060155,0.00011979,0.0009147$ 4,0.00017466,0.00003576,0.00002681,0.00002821,-0.00022361,-0.00009699, $0.00023855,0.00015553,0.00017845,0.00007745,-0.00003627,0.00009564,0.0$ $0036675,-0.00023955,0.00021554,-0.00007520,0.00004888,0.00002740,-0.00$ $000339,0.00002968,0.00002760,0.00002831,0.00006432,0.00002113,-0.00002$ $426,0.00001325,0.00002248,0.00001616,-0.00001400,-0.00001270,0.0000182$ $3,-0.00001331,-0.00000688,-0.02648169,-0.03241045,-0.07199082,-0.00734$ $304,-0.00790471,0.00660662,0.00135907,0.00136793,-0.00244932,0.0078877$ $0,-0.01529212,0.00773693,0.04742821,-0.02391954,-0.07163847,-0.0421417$ $9,0.16053955,0.17666152,0.00022715,-0.00006535,-0.00004078,-0.00022475$ $, 0.00023927,-0.00011723,0.00046135,0.00073943,-0.00036917,-0.00019965$, $-0.00030006,0.00008473,0.00011246,0.00014516,0.00013566,-0.00032885,-0$ $.00007115,0.00011372,0.00012779,-0.00007986,-0.00007512,0.00005677,-0$. $00002182,-0.00002610,-0.00059471,-0.00074569,0.00007624,-0.00000371,0$. $00000041,-0.00006677,0.00004885,-0.00003175,0.00001822,-0.00001609,-0$. $00001756,-0.00002440,-0.13220927,0.03549404,0.01905849,-0.02197712,0.0$ $0915622,0.00478895,0.00242360,0.00233052,0.00078976,0.00006514,0.00087$ $455,0.00036665,-0.00025943,-0.00646373,-0.00154496,-0.04059345,0.01708$ $912,0.00860778,0.19287263,-0.00010494,0.00003641,0.00006091,0.00010514$ ,-0.00004247,0.00005654,-0.00017298,-0.00031675,0.00016758,0.00007395, $0.00012598,-0.00002557,-0.00003920,-0.00005497,-0.00005454,0.00013140$, $0.00001224,-0.00003675,-0.00008062,0.00005426,0.00006329,-0.00001639$,$0.00000049,0.00000035,0.00023934,0.00033339,-0.00003902,0.00000021,0.0$ $0000079,0.00002467,-0.00002687,0.00001548,-0.00001952,0.00001449,0.000$ 
$00390,0.00000929,0.03546817,-0.05171257,-0.01096629,0.03514973,-0.0103$ $1991,-0.00710698,-0.00363121,-0.00357361,-0.00195385,0.00076568,0.0014$ $3042,0.00052692,-0.00031247,-0.00116915,-0.00158083,-0.00885578,0.0063$ $7030,0.00125025,-0.05970271,0.05752823,0.00001274,0.00004965,-0.000125$ $82,-0.00002353,-0.00006364,-0.00000489,-0.00006132,-0.00010228,0.00013$ $163,0.00002228,0.00009179,-0.00002679,-0.00002748,-0.00006141,-0.00006$ $625,0.00003453,0.00004200,-0.00002026,-0.00004612,0.00000499,-0.000001$ $96,-0.00000629,-0.00000214,0.00000229,0.00011690,0.00013642,-0.0000159$ $7,0.00000486,-0.00000380,0.00001178,-0.00000881,0.00001147,-0.00000687$ $, 0.00000620,0.00000352,0.00000247,0.01902037,-0.01096030,-0.02802935,0$ $.01223277,-0.00497107,0.00204275,-0.00087309,-0.00253323,0.00381243,0$. $00010049,0.00060515,-0.00081612,0.00030516,-0.00103136,0.00409776,0.00$ $117529,-0.00086584,0.00420006,-0.03188947,0.01792915,0.01950551,0.0000$ $2162,-0.00019307,-0.00009826,-0.00018386,0.00011393,0.00003651,0.00010$ $001,0.00021323,-0.00016343,-0.00011288,-0.00017042,-0.00002211,0.00008$ $421,0.00003182,0.00007135,-0.00018603,0.00003427,0.00008151,0.00000909$ $,-0.00002392,-0.00000860,-0.00001054,-0.00000498,0.00001220,-0.0001334$ $1,-0.00019243,0.00002857,-0.00000990,0.00000659,-0.00002198,-0.0000072$ $2,0.00002886,-0.00001637,-0.00000631,0.00000532,-0.00000045,0.00382894$ $, 0.00534169,0.00147389,-0.23687407,-0.13828841,-0.02326352,-0.01635368$ $,-0.01709516,-0.00325587,-0.00392120,0.00185799,0.00143945,0.00013020$, $0.00103686,0.00021597,0.00140584,0.00136563,0.00067686,0.00122714,0.00$ $067122,-0.00011544,0.24953555,0.00024364,0.00003720,0.00002260,0.00000$ $274,-0.00008103,-0.00001362,-0.00000238,-0.00003397,0.00008452,0.00004$ $563,0.00005659,0.00000120,-0.00003828,-0.00001492,-0.00002915,0.000075$ $80,0.00000796,-0.00005284,-0.00001665,0.00002671,-0.00001159,0.0000089$ $7,-0.00000151,-0.00000677,0.00001780,0.00004064,-0.00001035,0.00000117$ ,-0.00000253,0.00000613,0.00000843,-0.00000997,0.00001161,0.00000211,$0.00000360,-0.00000068,-0.02471156,-0.01231864,-0.00245237,-0.13440277$ $,-0.15385116,-0.02315329,0.01064584,0.00918145,0.00053239,0.00219153,0$ $.00130339,-0.00165314,0.00070756,-0.00045112,0.00006725,0.00197979,-0$. $00418190,-0.00285615,-0.00032055,0.00068417,0.00099997,0.14499486,0.15$ $980995,0.00000965,0.00001480,0.00006269,0.00003490,0.00002910,-0.00000$ $228,-0.00003149,-0.00003939,-0.00002260,-0.00003308,-0.00002266,0.0000$ $2612,0.00005289,0.00005959,0.00002689,-0.00009402,-0.00002547,-0.00005$ $262,0.00003910,0.00000099,0.00002688,0.00001345,0.00000558,-0.00000497$ $, 0.00002373,0.00003293,-0.00000576,-0.00000274,0.00000097,-0.00000326$, $0.00000368,-0.00000115,0.00000988,0.00000140,-0.00000214,0.00000143,-0$ $.00714142,-0.00484255,0.00249031,-0.02216557,-0.02283989,-0.03974196,0$ $.00478143,0.00274698,0.00465270,0.00141788,-0.00169917,0.00578817,0.00$ $016861,0.00014432,-0.00086283,0.00089554,-0.00281448,0.00438358,-0.000$ $38423,0.00089638,-0.00238461,0.02326954,0.02724979,0.02948396,0.000487$ $43,-0.00060704,-0.00073173,0.00005867,0.00010249,0.00016472,-0.0000955$ $7,-0.00006062,0.00008476,0.00005083,0.00010791,-0.00000260,-0.00005307$ $,-0.00002431,-0.00006727,0.00003323,-0.00001297,0.00020032,-0.00003638$ $, 0.00012525,0.00045391,0.00001486,-0.00002094,-0.00004743,0.00012967,0$ $.00017508,-0.00001503,0.00002951,-0.00001793,0.00001111,-0.00013117,0$. $00008872,-0.00017162,0.00000665,0.00001803,0.00000532,-0.00346995,0.00$ $101439,0.00114804,-0.00059908,0.02798095,0.00830602,-0.05809164,0.0303$ $4922,0.01067095,0.00646722,-0.02248045,-0.00680759,-0.00330600,-0.0039$ $2149,-0.00032986,-0.00129988,-0.00001400,0.00000337,-0.00104585,0.0012$ 
$3891,0.00045367,0.00111871,-0.00023192,-0.00046854,0.05941767,0.000914$ $30,-0.00021229,-0.00014585,-0.00020530,-0.00000015,0.00032646,0.000102$ $86,0.00013611,-0.00009827,-0.00010656,-0.00013902,-0.00003643,0.000133$ $03,0.00007677,0.00014997,-0.00030954,0.00020485,-0.00017721,-0.0006373$ $8,0.00025747,0.00032837,-0.00000366,-0.00002452,-0.00003305,0.00000012$ ,-0.00000470,0.00003281,0.00000875,0.00002200,0.00001584,-0.00002334,0 $.00005728,-0.00006697,0.00002133,0.00000505,0.00000353,0.00150949,0.00$ $226375,-0.00141016,-0.00092695,-0.00714530,-0.00334402,0.03442676,-0.3$ $0275849,-0.07865823,0.00025179,-0.01530279,-0.00419399,-0.00268557,-0$. $00027980,-0.00167207,-0.00032405,0.00042308,0.00043135,0.00025244,-0.0$ $0040505,-0.00014959,0.00003366,0.00034322,0.00120517,-0.03285428,0.323$ $05966,0.00043844,0.00037037,-0.00027951,0.00002300,-0.00015030,0.00008$ $828,0.00010123,-0.00003274,0.00009916,-0.00002229,0.00005283,-0.000110$ $43,0.00000698,0.00001478,-0.00015112,-0.00015058,-0.00004980,-0.000016$ $53,-0.00009482,-0.00009193,-0.00004181,0.00000214,0.00003607,0.0000026$ $9,0.00003610,0.00011145,-0.00005653,0.00000890,-0.00002630,-0.00001977$ $,-0.00018245,0.00022851,-0.00011507,-0.00000291,0.00002139,-0.00000305$ $, 0.00130868,-0.00127566,0.00626757,0.00036809,-0.00570743,0.00322836,0$ $.01139256,-0.07845921,-0.05794666,-0.00047731,-0.00241367,-0.00056277$, $0.00013505,-0.00157799,0.00581515,-0.00015058,0.00031882,-0.00072654,0$ $.00014439,-0.00024006,0.00002027,-0.00039586,0.00125634,-0.00359516,-0$ $.01247510,0.08748922,0.04881995,0.00012375,-0.00019014,0.00044810,0.00$ $040218,0.00020053,-0.00020520,-0.00036520,-0.00008272,0.00055343,-0.00$ $019562,0.00031677,-0.00030795,-0.00004119,-0.00010705,-0.00022132,0.00$ $016413,-0.00009333,-0.00017264,-0.00005446,0.00005006,0.00001556,-0.00$ $006572,0.00000816,-0.00001886,0.00007173,0.00022101,-0.00003917,0.0000$ $1060,-0.00002615,0.00002780,0.00000171,-0.00002083,-0.00001104,0.00002$ $413,0.00000322,0.00000782,-0.00227615,0.00262763,0.00144005,-0.0001200$ $0,0.00060635,0.00020827,0.00100058,0.00203573,0.00093203,0.00676165,0$. $00006071,-0.00048088,-0.23031782,-0.13192026,-0.02108321,-0.01822118,-$ $0.01772528,-0.00306993,-0.00142143,-0.00024894,0.00007239,-0.00007683$, $-0.00001511,-0.00006169,0.00027876,0.00030364,0.00004085,0.24337528,-0$ $.00125857,0.00003437,0.00048693,0.00008866,0.00042854,-0.00030896,0.00$ $045357,0.00052419,0.00011326,0.00010448,0.00023998,0.00029715,-0.00001$ $623,-0.00018447,-0.00005401,0.00024203,-0.00017740,0.00013220,-0.00006$ $509,0.00001294,0.00003526,0.00007385,-0.00002140,-0.00008458,-0.000130$ $54,-0.00034734,0.00002980,-0.00000361,0.00000488,-0.00001076,-0.000003$ $20,0.00005063,-0.00003238,0.00000327,-0.00000928,-0.00004407,0.0021301$ $8,0.00112031,-0.00184766,0.00101594,-0.00056085,0.00007641,0.00041913$, $-0.00433750,-0.00313695,-0.02228417,-0.01577349,-0.00261104,-0.1352914$ $0,-0.15227323,-0.02182282,0.01079590,0.00939504,0.00042635,0.00081385$, $0.00003809,-0.00007728,-0.00006271,-0.00001294,0.00019415,-0.00096376$, $-0.00048321,-0.00004442,0.14415881,0.16187352,-0.00042840,-0.00011241$, $-0.00056083,-0.00035908,0.00029198,0.00001967,0.00010517,0.00009716,0$. $00028694,-0.00013180,-0.00017402,-0.00002629,0.00016545,0.00018046,-0$. $00022836,-0.00021779,0.00006212,-0.00015699,0.00021153,-0.00013028,0.0$ $0001799,0.00020570,-0.00007518,-0.00022129,-0.00007357,-0.00001675,-0$. $00010308,-0.00002040,0.00003013,-0.00000473,-0.00000731,0.00000773,-0$. $00001163,-0.00004519,0.00002293,-0.00000956,0.00136550,-0.00172279,0.0$ $0610487,0.00023797,0.00002512,-0.00079542,0.00073105,-0.00323279,0.005$ $16852,-0.00708712,-0.00447979,-0.00005597,-0.02152968,-0.02157914,-0.0$ 
$4016641,0.00488964,0.00248568,0.00537307,0.00033802,0.00000849,0.00006$ $606,-0.00005075,0.00019079,-0.00066027,-0.00030537,-0.00016549,-0.0000$ $0607,0.02246464,0.02694566,0.02953826,-0.00006404,0.00019574,0.0000081$ $6,-0.00002151,0.00009310,0.00000662,0.00012336,0.00025433,-0.00017386$, $-0.00008588,-0.00020142,-0.00003831,0.00006771,0.00004683,0.00005391,-$ $0.00012163,-0.00000531,0.00012013,-0.00005220,0.00003698,0.00001730,-0$ $.00001716,0.00000619,0.00002452,-0.00013517,-0.00023947,0.00003262,-0$. $00001090,0.00000829,-0.00001743,-0.00000593,0.00002198,-0.00001047,-0$. $00002189,0.00001176,-0.00000120,0.00522015,-0.02327571,-0.00684005,-0$. $00359494,-0.00294602,-0.00011206,-0.00106473,-0.00038647,-0.00011197,-$ $0.00377344,0.00238487,0.00158522,-0.00021322,0.02632752,0.00786106,-0$. $05910224,0.03779625,0.01282540,0.00098994,-0.00057376,-0.00045577,0.00$ $035955,-0.00118075,-0.00032837,0.00005841,0.00009701,-0.00003513,0.001$ $06846,-0.00026479,-0.00049674,0.06037964,-0.00017515,0.00001544,-0.000$ $02655,0.00002095,-0.00001515,-0.00000863,-0.00006453,-0.00011888,0.000$ $08647,0.00004195,0.00011283,0.00000851,-0.00004935,-0.00005495,-0.0000$ $4678,0.00009833,-0.00001977,0.00001482,0.00004272,-0.00002554,-0.00000$ $039,0.00000829,-0.00000103,-0.00000605,0.00006903,0.00011676,-0.000010$ $14,0.00000466,-0.00000408,0.00001315,-0.00000426,-0.00000632,0.0000033$ $9,0.00001205,-0.00000184,-0.00000098,0.00671184,-0.01355650,-0.0053108$ $4,-0.00354408,0.00034483,-0.00116572,-0.00003964,0.00059515,0.00049419$ $, 0.00180848,0.00119707,-0.00177190,-0.00129053,-0.00523786,-0.00255313$ $, 0.03389560,-0.31181596,-0.08157273,0.00048753,0.00104007,0.00098529,0$ $.00025292,-0.00052116,-0.00028831,0.00003720,-0.00011540,0.00017513,-0$ $.00004699,0.00037976,0.00126473,-0.03825052,0.32771665,-0.00009949,-0$. $00007836,0.00006636,0.00012360,-0.00000035,0.00004313,-0.00008600,-0.0$ $0010542,0.00001206,0.00003553,0.00001163,0.00002599,-0.00000171,0.0000$ $3724,0.00001207,-0.00002539,0.00002822,-0.00005392,-0.00000467,-0.0000$ $1139,-0.00000918,-0.00000176,-0.00000253,-0.00000201,0.00003426,0.0000$ $8850,-0.00003009,-0.00000126,0.00000463,0.00000038,0.00000721,0.000000$ $35,-0.00000609,-0.00000563,-0.00000200,0.00000080,0.00168929,-0.002966$ $32,0.00236156,-0.00031588,-0.00118805,0.00496631,-0.00004392,0.0004674$ $1,-0.00076428,0.00150813,-0.00167833,0.00587881,-0.00003611,-0.0048552$ $7,0.00292941,0.01176934,-0.08185856,-0.05955664,-0.00015240,0.00109532$ ,-0.00236017,0.00007144,-0.00017518,0.00038461,-0.00005221,0.00016233, $-0.00070892,-0.00046384,0.00128866,-0.00364257,-0.01395036,0.08974496$, $0.05044933,0.00044268,-0.00120123,-0.00105390,0.00284859,0.00023624,0$. $00161380,-0.02611158,-0.02077676,-0.00544421,0.00245529,0.00467637,-0$. $00037606,-0.00107675,-0.00095301,-0.00159013,0.00115484,0.00090307,-0$. $00002995,-0.00007546,0.00010188,0.00015012,-0.00043843,0.00029707,0.00$ $003181,-0.12675217,-0.05038512,-0.05445297,0.00018614,0.00039984,-0.00$ $023526,-0.00012370,0.00008277,-0.00000701,-0.00096606,-0.00064476,-0.0$ $0052892,-0.00030678,0.00014669,0.00006648,0.00011824,0.00002552,0.0000$ $2196,0.00018731,-0.00030801,-0.00006675,-0.00048665,0.00047548,-0.0000$ $8647,0.00018472,-0.00003627,-0.00004230,0.00001261,0.00003914,0.000004$ $19,0.00033226,-0.00012299,-0.00006093,0.00006642,-0.00000818,-0.000004$ $85,-0.00003549,0.00001845,-0.00002484,-0.00012313,0.00008211,0.0000520$ $0,0.00003772,-0.00003486,-0.00000987,0.51008986,-0.00189835,-0.0003001$ $8,-0.00049761,-0.00035293,0.00019002,0.00415597,-0.02934608,-0.0293521$ $2,-0.01826074,0.00140798,0.00052187,-0.00027133,-0.00104797,0.00002798$ ,-0.00117678,0.00048564,0.00068110,-0.00033079,0.00001248,-0.00012700, 
$0.00009300,0.00021685,-0.00017782,-0.00034126,-0.03721129,-0.09355974$, $-0.01428274,0.00015111,0.00028400,-0.00017231,0.00013425,-0.00012319$,$0.00013007,-0.00144675,-0.00171546,-0.00033315,-0.00019493,0.00010374$, $0.00002803,0.00006344,0.00001864,0.00002457,0.00015977,-0.00027290,-0$. $00007940,-0.00041237,0.00024707,-0.00001741,0.00029358,0.00000504,-0.0$ $0005955,-0.00001353,-0.00000982,-0.00000594,0.00021853,-0.00009008,-0$. $00004000,0.00004804,-0.00000887,-0.00000840,-0.00003921,0.00001868,-0$. $00000178,-0.00010102,0.00008872,0.00010757,0.00006078,-0.00003007,-0.0$ $0000989,-0.02536305,0.56768605,-0.00028432,0.00037479,0.00077877,0.001$ $66145,0.00421409,-0.00109247,0.00691584,-0.00271195,0.01263631,0.00541$ $134,0.00587668,-0.00053015,-0.00096428,-0.00057519,-0.00062405,0.00092$ $473,0.00093333,0.00001288,0.00028266,0.00035003,-0.00009407,0.00025347$ ,-0.00019505,0.00013116,-0.07342083,-0.03758926,-0.14002259,0.00012908 $, 0.00025626,-0.00009647,0.00010826,-0.00008263,-0.00010918,0.00096024$, $0.00152319,-0.00088073,-0.00020735,0.00009720,0.00005450,0.00006111,0$. $00002482,0.00001927,0.00019221,-0.00032100,-0.00008503,-0.00076322,0.0$ $0045878,-0.00005079,0.00036677,0.00006602,-0.00004471,-0.00003383,-0.0$ $0000593,-0.00004186,0.00025814,-0.00010745,-0.00005311,0.00005809,-0.0$ $0001431,-0.00000448,-0.00005302,-0.00000451,-0.00001127,-0.00010323,0$. $00012018,0.00014608,0.00007922,-0.00004773,0.00000557,-0.05960144,-0.0$ $5658966,0.54039097,0.00066469,0.00043683,-0.00046433,-0.00091733,-0.00$ $157377,-0.00031152,0.00281547,0.00590705,-0.00181630,0.00004049,0.0000$ $4310,-0.00023869,0.00006116,-0.00063546,0.00016482,0.00003720,-0.00005$ $389,0.00054268,-0.00009305,-0.00001249,0.00001034,-0.00000566,0.000160$ $07,0.00017710,-0.02581080,0.00263307,-0.01517044,-0.00006425,-0.000047$ $65,0.00002406,-0.00001593,0.00005502,0.00007691,-0.00066590,-0.0001250$ $3,0.00109644,0.00003285,-0.00001128,-0.00001155,-0.00000684,0.00001270$ $, 0.00000063,0.00002181,-0.00001476,-0.00001088,-0.00011445,0.00000633$, $0.00005431,0.00005579,0.00003355,0.00001232,-0.00000636,-0.00002968,-0$ $.00000258,-0.00003306,0.00001031,0.00000501,-0.00000084,-0.00000268,0$. $00000007,0.00000101,0.00000143,0.00000081,0.00000711,-0.00000927,-0.00$ $001332,0.00000416,0.00000015,-0.00000184,-0.17399445,0.11819676,-0.038$ $68078,0.19583018,0.00047415,0.00020418,-0.00044443,-0.00028917,-0.0009$ $0467,-0.00059029,-0.00013878,0.00333016,-0.00006948,0.00074287,0.00045$ $420,0.00099088,-0.00038467,-0.00090198,-0.00051210,0.00039811,0.000113$ $66,0.00049073,-0.00004847,0.00004360,0.00000655,-0.00006775,0.00005393$ $, 0.00006123,-0.01751376,0.01270095,-0.00660524,-0.00000588,0.00004110$, $0.00000600,-0.00008204,0.00003476,0.00002618,-0.00135456,-0.00020970,0$ $.00015183,-0.00002631,0.00000664,0.00000547,0.00001090,-0.00000383,0.0$ $0000895,0.00000153,0.00000596,-0.00001474,-0.00012335,0.00007354,0.000$ $00256,-0.00002396,0.00000026,-0.00000545,0.00000645,0.00001335,0.00000$ $596,0.00002201,-0.00000511,-0.00000470,0.00000281,0.00000032,0.0000011$ $0,0.00000037,-0.00000021,-0.00000349,-0.00001343,-0.00000049,-0.000000$ $79,-0.00000825,-0.00000056,0.00000332,0.12430138,-0.16528022,0.0411183$ $9,-0.12855846,0.16972362,0.00024035,0.00004876,-0.00007898,-0.00017912$ ,-0.00066083,-0.00019028,0.00212706,0.00294224,-0.00070550,0.00016528, $-0.00059725,0.00068230,-0.00004880,0.00015043,-0.00005249,-0.00021309$, $-0.00023260,0.00007198,-0.00004836,-0.00003140,0.00000317,-0.00005304$, $0.00010691,0.00010300,-0.02436322,0.00681160,-0.01053732,-0.00000215,-$ $0.00003627,0.00002957,-0.00001877,-0.00001074,0.00001030,0.00071359,0$. $00017303,-0.00030469,0.00004845,-0.00002081,-0.00001478,-0.00001500,-0$ 
$.00000721,-0.00000348,-0.00004061,0.00007250,0.00001697,0.00015807,-0$. $00008582,0.00000572,-0.00008273,-0.00000704,0.00000039,0.00001043,0.00$ $000236,0.00001458,-0.00005653,0.00002316,0.00001077,-0.00001352,0.0000$ $0287,0.00000220,0.00001096,-0.00000148,0.00000395,0.00001877,-0.000025$ $39,-0.00002741,-0.00001669,0.00000855,-0.00000078,-0.03812839,0.037704$ $24,-0.06089416,0.05382021,-0.04180591,0.06755148,0.00001868,0.00000574$ ,-0.00041899,-0.00052984,-0.00110720,0.00058380,-0.00129066,0.00038725 ,-0.00337943,0.00009515,0.00002274,-0.00077131,0.00012852,-0.00015352, $0.00017540,-0.00001475,0.00002362,0.00007496,-0.00009518,-0.00007884,0$ $.00004450,-0.00000483,0.00000860,-0.00002659,-0.01708082,-0.02561072,0$ $.00846089,0.00000166,-0.00005697,0.00000936,-0.00000792,0.00001627,0.0$ $0003519,0.00004930,0.00037283,0.00033920,0.00002386,-0.00001195,-0.000$ $00770,-0.00000755,-0.00000671,0.00000554,-0.00002608,0.00004246,0.0000$ $0481,0.00008719,-0.00003583,-0.00000974,-0.00006416,-0.00000467,-0.000$ $00496,0.00000621,0.00000412,-0.00000089,-0.00003241,0.00001563,0.00000$ $549,-0.00000792,0.00000363,0.00000177,0.00000793,0.00000137,0.00000256$ $, 0.00001484,-0.00002460,0.00001257,-0.00001281,0.00000481,0.00000689$,$0.08211613,-0.06876036,0.05518983,0.01048046,0.01631356,-0.01235740,0$. 09466547,-0.00001166,-0.00001687,-0.00026884,-0.00042324,-0.00052884,0 $.00051716,-0.00059418,0.00146500,-0.00249419,-0.00017670,0.00021484,-0$ $.00011197,-0.00005374,0.00011730,0.00008285,-0.00005173,-0.00007418,-0$ $.00000434,-0.00002948,-0.00007624,0.00003119,-0.00003397,0.00002159,-0$ $.00002299,-0.01193645,-0.01593308,0.00400508,-0.00002314,-0.00001060,0$ $.00002020,0.00000942,-0.00001639,-0.00000013,0.00020289,-0.00016747,-0$ $.00008522,0.00005015,-0.00002135,-0.00000652,-0.00001805,-0.00000546,-$ $0.00000137,-0.00004280,0.00005395,0.00001747,0.00011657,-0.00004568,-0$ $.00001946,-0.00007182,-0.00000589,0.00001099,0.00000233,0.00001083,-0$. $00000247,-0.00005036,0.00001924,0.00000871,-0.00001269,0.00000277,0.00$ $000252,0.00000869,-0.00000386,0.00000154,0.00001335,-0.00001517,-0.000$ $00727,-0.00001374,0.00000670,0.00000374,-0.07367826,-0.19776859,0.1129$ $3182,-0.00717194,-0.01310645,0.01169492,0.08883464,0.21551548,-0.00001$ $576,0.00004135,-0.00036620,-0.00061617,-0.00092675,0.00054301,-0.00261$ $271,-0.00134806,-0.00224961,-0.00042117,0.00020594,-0.00099223,0.00003$ $706,-0.00016615,0.00044060,0.00010587,0.00012788,0.00008252,-0.0000509$ $2,-0.00006735,0.00003725,-0.00000975,0.00003891,-0.00000615,-0.0148352$ $0,-0.02302718,0.01076782,-0.00003118,-0.00003389,0.00000449,0.00003876$ $, 0.00001741,0.00003506,0.00063037,0.00028811,0.00049594,-0.00002544,0$. $00001326,0.00000157,0.00000987,0.00000656,-0.00000034,0.00002530,-0.00$ $003493,-0.00001118,-0.00006155,0.00001752,0.00002722,0.00003536,0.0000$ $0113,-0.00001654,-0.00000341,-0.00000535,0.00001117,0.00002351,-0.0000$ $0916,-0.00000416,0.00000598,-0.00000157,-0.00000078,-0.00000230,0.0000$ $0591,0.00000056,-0.00001247,0.00000481,-0.00000288,0.00000516,-0.00000$ $168,-0.00000441,0.06373499,0.12284740,-0.13662740,0.00316449,0.0031813$ $1,-0.00284698,-0.05674938,-0.12002915,0.14260021,0.00009385,0.00042440$ ,-0.00022508,-0.00117033,-0.00037484,-0.00014577,0.00320672,0.00227497 ,-0.00166042,0.00057843,0.00010115,0.00152409,-0.00069238,-0.00023475, $-0.00030108,0.00013294,-0.00000573,0.00042395,0.00000602,-0.00000181,-$ $0.00002202,0.00014607,-0.00008181,0.00000535,0.00748459,-0.01556996,-0$ $.02808169,0.00002937,-0.00000566,0.00001620,0.00004479,-0.00007008,-0$. $00004028,0.00002679,-0.00091086,-0.00038664,-0.00000069,-0.00000047,-0$ $.00000069,-0.00000032,-0.00000728,-0.00000136,-0.00001711,0.00002802,0$ 
$.00000098,-0.00003831,0.00002516,0.00001203,-0.00006529,-0.00003711,0$. $00000615,0.00001451,0.00001707,0.00000339,-0.00000530,0.00000471,0.000$ $00012,-0.00000259,0.00000134,0.00000236,0.00000351,0.00000021,0.000002$ $10,-0.00002618,-0.00000383,-0.00001273,-0.00001140,0.00000339,0.000006$ $66,-0.10550990,0.04473862,0.10125987,-0.00832344,0.00636468,0.01832425$ $,-0.00428822,0.00515589,0.00763068,0.10838384,0.00026373,0.00049983,-0$ $.00030652,-0.00113796,-0.00087317,-0.00007878,0.00307370,0.00334601,-0$ $.00223929,0.00017193,0.00013794,-0.00022685,-0.00052074,-0.00023422,0$. $00037144,0.00022670,0.00005275,0.00046345,-0.00002009,-0.00000290,-0.0$ $0000821,0.00015924,-0.00001367,0.00006156,-0.00293670,-0.00930964,-0.0$ $1690849,0.00000188,-0.00006998,-0.00002128,0.00008131,-0.00001715,0.00$ $001356,0.00028308,-0.00168503,0.00049831,-0.00000830,0.00000441,-0.000$ $00028,0.00000600,0.00001028,0.00000206,0.00001356,-0.00002944,-0.00000$ $896,-0.00012730,0.00002936,0.00003015,0.00003318,0.00001340,0.00001896$ $,-0.00000151,-0.00000657,0.00000225,0.00000749,-0.00000279,-0.00000148$ $, 0.00000410,-0.00000103,-0.00000076,0.00000168,0.00000685,-0.00000166$, $-0.00001471,0.00000579,-0.00002385,0.00000456,-0.00000020,-0.00000467$, $0.04172649,-0.08105686,-0.07010090,0.01074997,-0.00638703,-0.01621883$, $-0.01020590,0.01036841,0.01885372,-0.04183542,0.08521465,0.00009679,0$. $00027972,0.00001239,-0.00067819,-0.00068293,0.00005461,0.00245892,0.00$ 440980,-0.00129296,-0.00059991,-0.00031834,0.00009167,0.00031754,0.000 $06872,0.00024461,-0.00019632,-0.00028774,0.00005421,-0.00002525,-0.000$ $04712,-0.00000250,0.00018487,-0.00001119,0.00006676,-0.00588789,-0.015$ 09536,-0.02129310,-0.00004100,-0.00006406,0.00003990,-0.00001196,0.000 $02043,0.00005021,0.00030829,0.00060945,0.00067934,0.00003897,-0.000021$ $82,-0.00000961,-0.00000895,-0.00000396,-0.00000776,-0.00006352,0.00009$ $323,0.00002995,0.00020559,-0.00012601,0.00000544,-0.00013223,-0.000042$ $50,0.00001898,0.00002510,0.00001288,0.00000289,-0.00005709,0.00002773$, $0.00001257,-0.00001520,0.00000555,0.00000216,0.00001652,0.00000634,0.0$ $0000528,0.00002173,-0.00005049,-0.00004172,-0.00003093,0.00001238,0.00$ $000267,0.09606891,-0.07235198,-0.21291297,-0.00242796,0.00399618,0.007$ $15049,0.00877360,-0.00627427,-0.01191857,-0.09834043,0.08583535,0.2389$ $5503 \backslash \backslash-0.00005419,0.00001523,0.00000340,0.00001732,0.00001420,-0.00000$ 046,-0.00000290,-0.00000257,-0.00000711,0.00000237,0.00000629,0.000006 $78,-0.00000707,-0.00000529,0.00000205,0.00000939,-0.00000682,0.0000235$ $4,0.00000952,-0.00001055,-0.00000287,0.00000276,-0.00000233,0.00000172$ $, 0.00000097,0.00000064,0.00000207,-0.00000286,0.00000393,0.00000503,-0$ $.00000085,0.00000137,0.00000475,-0.00000265,0.00000357,0.00000358,-0.0$ $0000024,-0.00000109,-0.00000612,0.00000201,-0.00000178,-0.00000390,-0$. $00000183,-0.00000235,-0.00000131,0.00003315,-0.00001284,-0.00000618,-0$ $.00000149,0.00000177,-0.00000250,-0.00000025,-0.00000127,-0.00000625,-$ $0.00000062,-0.00000126,-0.00000953,0.00000129,-0.00000210,-0.00000432$, $0.00000226,-0.00000152,0.00000009,-0.00000072,-0.00000115,-0.00000351$, $-0.00000136,-0.00000035,-0.00000783,-0.00000125,0.00000100,0.00000199$, $-0.00000071,0.00000213,0.00000445,0.00000014,0.00000066,0.00000152,-0$. 00000220,0.00000247,0.000000901I!@

para-Trsnaition State

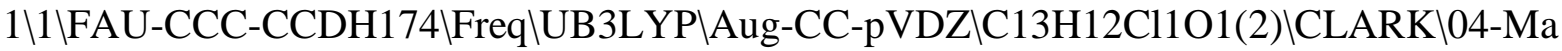

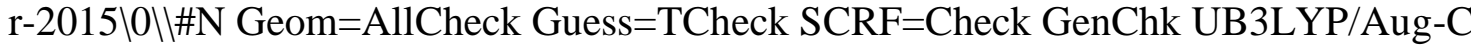


C-pVDZ Freq \\Addition TS p-Cl-Ph-radical + anisole (para) \\0,2\C,1.222 $5033907,1.8128015833,0.0572068368 \mathrm{IC}, 1.7931720043,1.2595670779,-1.11683$ $80318 \mathrm{IC}, 2.7366422082,0.236232225,-1.049222008 \backslash \mathrm{C}, 3.1874643348,-0.211940$ 4799,0.2046037466\C,2.7052522534,0.3976191166,1.3822515413\C,1.7661682 $62,1.4117214658,1.3091656205 \backslash \mathrm{H}, 0.6672887876,2.7458679272,-0.0074716894$ $\mathrm{UH}, 1.4623743693,1.6134715302,-2.0930996956 \mathrm{H}, 3.1248226959,-0.190987598$ 2,-1.9707592006\H,3.104147602,0.0649755629,2.3400946028\H,1.4137836196 $, 1.8868595794,2.2244656602 \backslash \mathrm{O}, 4.1043670929,-1.2060494494,0.3892829957 \backslash \mathrm{C}$ ,-3.2703406695,-0.379613594,-0.0903242637\C,-3.10457118,0.8767837073,$0.6762894545 \backslash \mathrm{C},-1.8501713239,1.5026731033,-0.5921225683 \backslash \mathrm{C},-0.821185061$ $3,0.8556542274,0.0748090742 \backslash C,-0.9773187966,-0.3917544519,0.6578746507$ $\backslash \mathrm{C},-2.2268515968,-1.0260319324,0.576559759 \backslash \mathrm{Cl},-4.8456930539,-1.1742771$ $822,-0.1940519952 \backslash \mathrm{H},-3.9380555643,1.3568307976,-1.1880418761 \backslash \mathrm{H},-1.7107$ $414198,2.484404438,-1.0497169098 \backslash \mathrm{H},-0.1529237197,-0.8820329441,1.17819$ 03764\H,-2.3887161742,-2.0058347572,1.024722568\C,4.6439865088,-1.8508 911242,-0.7605299505\Н,5.1902505294,-1.1386089205,-1.3976158567\H,3.85 413287,-2.3423749346,-1.3488478676 $\mathrm{H}, 5.3385590309,-2.6054489733,-0.379$ $1500648 \|$ Version=EM64L-G09RevC.01 $\backslash$ State $=2-A \backslash H F=-1038.0116246 \backslash S 2=0.7779$ $08 \mathrm{IS} 2-1=0 . \mathrm{IS} 2 \mathrm{~A}=0.750548 \backslash \mathrm{RMSD}=7.897 \mathrm{e}-09 \backslash \mathrm{RMSF}=2.077 \mathrm{e}-06 \backslash \mathrm{ZeroPoint}=0.2101$ $038 \backslash$ Thermal $=0.2237064 \backslash \mathrm{ZPE}=131.842125 \backslash \mathrm{Dipole}=1.2148229,0.112399,-0.4310$ 56|DipoleDeriv $=-0.0538511,0.0216229,0.012491,0.0454128,0.044533,-0.018$ $9222,0.0222967,-0.0069669,-0.176819,0.0766574,-0.1774679,-0.0420224,-0$ $.3163779,-0.0511882,0.1152923,-0.0481146,-0.0635799,0.1033605,-0.32106$ $83,0.0578835,0.0605349,0.1726969,-0.2044426,-0.0666337,-0.3474816,0.18$ $23418,-0.0025103,1.0744973,-0.7177159,-0.0567671,-1.0689722,0.852739,0$ $.0575703,0.1826555,-0.1353029,0.0140899,-0.310035,0.0327893,-0.0043788$ $, 0.2100499,-0.1980329,0.0213047,0.3220578,-0.1374136,0.0691646,0.07363$ $43,-0.1616824,0.0271301,-0.2792231,-0.0614003,-0.077597,0.0974445,0.07$ $41505,0.1117779,0.133602,0.0910317,-0.0123855,0.1074513,-0.0126533,0.0$ $087056,-0.0122047,0.0168346,0.0531267,0.0746569,0.063112,-0.0517162,0$. $0391925,0.0732245,0.0282234,-0.0354716,0.043018,-0.0471945,0.0828155,0$ $.0470563,0.0388114,0.0651416,0.0831395,-0.0508904,0.0709132,-0.0591135$ $,-0.0148886,0.087053,0.0432628,-0.0411407,0.0570874,0.0948516,0.040311$ $9,-0.0586222,0.0305213,-0.01925,0.0760487,0.0633705,0.0490062,0.040689$ $, 0.0669237,-0.0438885,0.027833,-0.058239,-0.0342081,-1.3674372,0.73121$ $38,0.1685845,1.2342266,-1.037238,-0.2001715,0.142338,-0.1362438,-0.472$ $0175,1.0404983,0.460556,0.0706416,0.5449096,0.2151095,0.009312,0.07509$ $79,0.0127547,-0.0335419,-0.2001187,-0.1138296,0.0555564,-0.0132564,-0$. $0813791,-0.0350053,-0.0458054,-0.0425612,-0.056542,-0.3033958,0.003288$ $, 0.026769,-0.4142489,0.0633988,-0.0841829,0.1462422,-0.0829677,-0.0452$ $866,0.7537315,0.0867524,-0.0086505,0.4193458,-0.0681064,-0.0085915,0.0$ $48589,-0.0047729,-0.066408,-0.4754778,-0.0609997,0.0531991,-0.016999,-$ $0.0192673,-0.0280981,-0.1116068,-0.0483721,-0.0523587,-0.1561852,-0.03$ $08629,-0.0183791,-0.1803,0.0054557,-0.0660613,0.0458583,-0.0570427,-0$. $056517,-0.8114076,-0.2815367,-0.038053,-0.3620374,-0.2323069,-0.010618$ $1,-0.0487094,-0.011336,-0.0845957,-0.0703259,0.0401409,-0.060579,0.003$ $3719,0.0688199,0.0380982,-0.0621394,0.0426485,0.0882092,0.0729157,-0.0$ $55889,0.0069267,0.0174308,-0.064096,0.0793477,-0.0167299,0.0779276,0.0$ $759715,0.0397113,0.0528233,-0.0687919,0.034345,0.0535121,0.0277421,-0$. $0262668,0.0391607,0.0664169,0.0147475,-0.0370205,0.0026616,-0.0664786$, $-0.0147997,0.0634363,-0.0045653,0.0577561,0.0930737,0.5583891,-0.15436$ 
$99,-0.1228903,-0.2913129,0.4977406,0.144019,-0.5449785,0.3654739,0.518$ $948,-0.0140736,-0.0173026,0.0484858,-0.097739,-0.0495216,0.0899382,0.1$ $60284,-0.0348346,-0.042158,-0.0482946,-0.0907824,-0.0903558,-0.0077743$ $, 0.001533,-0.0381052,0.0647646,-0.1135663,-0.0334721,-0.0272877,0.1045$ $562,-0.0046878,0.1233685,-0.0265487,0.0054643,-0.0436784,0.0497255,0.0$ 436288\Polar $=271.8201941,-8.6267024,164.3573057,6.7975258,-7.8384422,1$ 45.3047146\PG=C01 [X(C13H12Cl1O1)] $\mathrm{NImag}=1 \| 0.22086881,-0.30559176,0.5$ $0629596,0.01716966,0.00478099,0.60655781,-0.08325640,0.06587342,0.0511$ $6923,0.38561361,0.04016571,-0.12729338,-0.03846663,-0.26816627,0.41509$ $284,0.04410866,-0.08534436,-0.24986000,0.02792598,-0.03491957,0.684294$ $87,-0.02034597,0.01845623,0.03816591,-0.18775664,0.14133833,-0.0272186$ $2,0.38185575,0.02977506,-0.01072472,-0.04308119,0.14293103,-0.20693761$ $, 0.02973903,-0.28177874,0.42008169,0.00912164,-0.02041412,0.03070507,0$ $.01695745,-0.01892109,-0.12046041,-0.02790682,0.04242893,0.69687528,0$. $00403722,-0.00326404,0.00209847,-0.01174211,0.01421154,-0.02129418,-0$. $10341420,0.04221329,-0.06372823,0.37448005,-0.01393806,-0.00329845,-0$. $00502482,0.01422489,-0.01044288,0.02421388,0.04323747,-0.10757893,0.06$ $257723,-0.24796256,0.42371981,-0.00144077,-0.00467251,-0.05935135,-0.0$ $5542241,0.06418558,0.02228890,-0.01351972,0.00750855,-0.27553519,0.011$ $87662,0.01376766,0.67113208,-0.02201791,0.01281833,-0.03957499,-0.0172$ $9291,0.01905053,0.00920063,0.02882181,-0.02296451,0.01381333,-0.099989$ $91,0.03757108,0.01414152,0.40316364,0.03028840,-0.00248946,0.04692962$, $0.01687448,-0.02507999,-0.01120815,-0.02393048,0.03286103,-0.01771470$, $0.04171122,-0.11290894,-0.02995693,-0.29444760,0.43651746,-0.01147250$, $0.02576435,0.02243375,0.01223911,-0.01686990,-0.01002846,-0.01114465,0$ $.00937018,-0.04740836,0.06427342,-0.08422828,-0.25593212,0.06059668,-0$ $.01426009,0.68936943,-0.08005470,0.05413626,-0.05320487,0.01992160,-0$. $01904954,0.00186348,-0.01784963,0.01598281,-0.01411893,-0.01472835,0.0$ $2262967,0.05016172,-0.20082443,0.15410117,-0.03537689,0.39930832,0.030$ $61876,-0.11279748,0.02050849,-0.01906907,0.02986379,0.00236396,0.01777$ $510,-0.02198808,0.01764468,0.01973960,-0.02202061,-0.05498795,0.157121$ $19,-0.22133142,0.03079245,-0.28061628,0.44125865,-0.04568269,0.0659024$ $5,-0.26138264,0.00254211,-0.01224729,-0.04035013,-0.01327766,0.0145718$ $3,-0.01456008,0.01583073,-0.01499796,0.03828601,0.00693900,-0.01686151$ ,-0.12529327,-0.00307393,0.06579054,0.66660242,-0.09637860,0.13701585, $-0.00995328,-0.00967359,0.01237022,0.00238131,0.00471104,0.00077214,0$. $00379391,-0.00080525,0.00106299,0.00038008,0.00506037,-0.00018165,-0.0$ $0414505,-0.00951979,0.01161400,-0.00387378,0.12161820,0.15108267,-0.25$ $573251,0.01367969,0.00158447,-0.00372895,0.00573985,0.00221210,0.00085$ $705,-0.00133516,-0.00100187,0.00087373,0.00010040,0.00219950,0.0008656$ $4,0.00062413,-0.00003639,-0.00139704,-0.00455785,-0.14573770,0.2678523$ $6,-0.01044224,0.01414865,-0.05657628,0.01404685,-0.02313257,0.00547707$ $, 0.00128682,-0.00136871,-0.00414402,0.00003491,-0.00012657,-0.00135378$ ,-0.00124829,0.00082039,-0.00472499,-0.01415103,0.02397696,0.00276094, $0.01027530,-0.01488913,0.05572895,0.00656322,-0.00423566,0.01298422,-0$ $.07061568,0.03885981,-0.07755814,-0.00242110,0.00687986,-0.01908086,0$. $00159624,0.00589637,0.00103884,-0.00080911,-0.00008771,0.00019787,0.00$ $188033,0.00346486,0.00194169,-0.00158449,-0.00114056,-0.00023732,0.067$ $22880,-0.00443712,0.00708260,-0.01071599,0.03958461,-0.07605709,0.0804$ $4517,0.00688506,-0.00321199,0.02103328,0.00600475,0.00042474,-0.001033$ $66,-0.00021186,-0.00079976,-0.00051992,0.00350048,0.00208131,-0.002535$ 
$41,-0.00202514,-0.00055630,0.00051650,-0.04692644,0.07281514,-0.009651$ $84,0.00986114,-0.01747764,-0.07599632,0.08300810,-0.28347999,0.0013045$ $5,-0.00153440,0.00155051,0.00049179,-0.00025542,0.00227200,0.00057712$, $-0.00066711,0.00024577,0.00300032,-0.00269077,-0.00292767,0.00006819,-$ $0.00016804,0.00092857,0.08141016,-0.08782981,0.29882959,0.00132275,0.0$ $0650215,-0.00175033,-0.00484543,0.00750795,0.01883448,-0.07813476,0.05$ $104505,0.08598706,0.00700443,-0.00450683,-0.00859218,0.00224318,0.0030$ $6552,-0.00197846,-0.00042860,-0.00040180,-0.00015448,-0.00015377,0.000$ $49464,0.00111602,-0.00188494,-0.00287024,-0.00011059,0.07890373,0.0060$ $4349,0.00055948,0.00064045,0.00685033,-0.00478247,-0.02034520,0.051568$ $99,-0.08835148,-0.09375636,-0.00434669,0.00768138,0.00727843,0.0030285$ $3,0.00224838,0.00238488,0.00011233,-0.00100819,0.00064159,0.00053322,-$ $0.00009551,-0.00064718,-0.00265074,-0.00133952,0.00006615,-0.06135527$, $0.08967073,-0.00098936,0.00071594,0.00189649,-0.00195623,0.00177500,0$. $00470259,0.08492394,-0.09345005,-0.26517813,0.01250471,-0.01381596,-0$. $01688347,-0.00278386,0.00288469,-0.00347630,-0.00070684,0.00078873,0.0$ $0020453,-0.00006437,0.00020260,0.00009287,0.00009115,-0.00004144,0.000$ $88534,-0.09267357,0.10261925,0.27652024,0.00161054,0.00693001,0.001039$ $98,-0.00039010,-0.00035851,0.00012807,0.00173603,0.00292406,0.00148453$ $, 0.00688549,-0.00159434,0.01006769,-0.08109542,0.04117353,-0.09226647$, $-0.00398822,0.00487625,-0.01895139,-0.00014777,0.00032685,-0.00123444$, $-0.00031076,-0.00037599,-0.00008088,0.00014697,-0.00013395,0.00053134$, $0.07838453,0.00638776,0.00088621,-0.00063747,0.00012214,-0.00110089,-0$ $.00035561,0.00301247,0.00130474,-0.00269431,-0.00396629,0.00751808,-0$. $01135157,0.04184213,-0.07180609,0.07312953,0.00651812,-0.00196649,0.02$ $066820,0.00050558,-0.00007682,0.00062997,-0.00037324,-0.00032642,0.000$ $02995,-0.00019264,0.00030552,-0.00072716,-0.04834343,0.06771874,0.0002$ $7921,-0.00064528,0.00203638,0.00067028,-0.00058254,0.00046487,0.002352$ $25,-0.00330517,-0.00286808,-0.01210619,0.01098604,-0.01861848,-0.09067$ $355,0.07245735,-0.27783882,0.00143059,-0.00110656,0.00277152,0.0000094$ $2,-0.00019079,0.00005505,-0.00000348,0.00009057,-0.00003388,-0.0004536$ $4,0.00056617,-0.00093645,0.09893817,-0.07961145,0.29184345,0.00669350$, $-0.00569466,-0.01156623,0.00195800,0.00305601,-0.00225040,-0.00085670$, $-0.00012103,-0.00020368,0.00242315,0.00593919,-0.00192702,-0.00289066$, $0.00897086,0.01835972,-0.07438143,0.05061268,0.07611441,-0.00142099,-0$ $.00105063,0.00032695,0.00042902,-0.00020394,-0.00055191,-0.00034423,-0$ $.00040435,0.00005848,-0.00206210,-0.00282010,0.00013046,0.07074832,-0$. $00330083,0.00677086,0.00776818,0.00294438,0.00249399,0.00189145,-0.000$ $17567,-0.00070824,0.00057163,0.00604174,0.00142152,0.00120528,0.006816$ $26,-0.00530769,-0.02016332,0.05086991,-0.10060812,-0.10315348,-0.00190$ $943,-0.00050190,-0.00036391,-0.00014851,0.00014961,0.00047538,-0.00039$ $291,-0.00036562,-0.00007555,-0.00293913,-0.00155821,0.00039755,-0.0591$ $0379,0.09886190,0.01067164,-0.01310077,-0.01704535,-0.00332804,0.00208$ $856,-0.00361960,-0.00047236,0.00075837,0.00016111,-0.00143165,0.000547$ $54,0.00190827,-0.00221733,0.00270538,0.00447510,0.07477961,-0.10530253$ ,-0.25640580,0.00027954,0.00028807,0.00100365,0.00060213,-0.00062473,-$0.00100556,0.00011486,0.00000020,-0.00002216,0.00048626,0.00007145,0.0$ $0077340,-0.07985628,0.11237801,0.26944110,-0.00279291,0.00126684,-0.00$ $180401,0.00495582,0.00228451,-0.00116275,-0.01238134,0.02193519,-0.008$ $05846,-0.14710064,0.11757273,-0.03629051,-0.02053323,0.02891908,0.0071$ $3487,0.00550074,-0.00092916,0.00166409,0.00026656,-0.00003866,-0.00005$ 
$262,-0.00066285,0.00069535,0.00000504,-0.00139231,-0.00118017,0.000294$ $32,-0.00087556,-0.00219975,-0.00058322,-0.00031416,0.00070215,0.000052$ $71,0.26623735,0.00229329,-0.00251723,0.00230963,0.00128709,0.00267791$, $0.00057154,0.02115194,-0.01431468,0.00808830,0.11812263,-0.16679046,0$. $03607332,0.03033411,-0.02787913,-0.00737960,-0.00195477,0.00460925,-0$. $00250377,-0.00088212,-0.00031877,0.00011954,0.00057437,-0.00065728,-0$. 00001053,-0.00107760,-0.00128696,-0.00030081,-0.00212626,-0.00050920,0 $.00108223,0.00076850,-0.00044688,-0.00004913,-0.22219986,0.31214090,0$. $00027468,0.00041120,0.00370783,0.00313284,-0.00400961,-0.00745404,-0.0$ $2770346,0.02978162,0.00100800,-0.01033509,0.00754325,-0.11317400,0.030$ $39056,-0.03307632,0.00938745,-0.00207569,0.00152918,-0.00830855,-0.000$ $01342,-0.00005702,0.00035528,-0.00110465,0.00125077,0.00002767,0.00139$ $013,-0.00127306,0.00139832,-0.00028965,0.00064530,0.00198114,0.0011998$ $7,-0.00122458,0.00010210,-0.04470519,0.06268428,0.34515386,-0.00001290$ ,-0.00002691,-0.00007716,-0.00022925,0.00028947,0.00013162,0.00021911, $-0.00024825,0.00038818,-0.00066982,0.00069076,-0.00012212,0.00031793$,$0.00024819,-0.00026606,-0.00018066,0.00029066,-0.00025090,-0.00024123$, $-0.00006630,-0.00002648,-0.00008376,-0.00001838,0.00006322,0.00000630$, $-0.00003737,-0.00003434,-0.00000805,-0.00005803,0.00003934,-0.00003412$ $, 0.00000709,0.00003182,0.00088967,-0.00095421,-0.00008336,0.48504754,0$ $.00002113,-0.00050258,-0.00007075,-0.00001455,0.00023760,0.00015622,0$. $00022718,-0.00003062,0.00014754,-0.00046106,0.00043550,-0.00021926,0.0$ $0008275,-0.00004339,-0.00023347,-0.00013933,0.00005234,0.00000849,0.00$ $002248,0.00018983,0.00001631,0.00002264,0.00001695,-0.00012107,-0.0000$ $2537,-0.00001726,-0.00001634,-0.00000912,-0.00000756,0.00003820,-0.000$ $02710,-0.00005898,-0.00006276,0.00047119,-0.00048816,-0.00006409,0.000$ $14491,0.52120287,-0.00011478,0.00017351,0.00001888,-0.00000002,-0.0000$ $2572,-0.00003740,-0.00006715,-0.00012087,-0.00001637,0.00005399,-0.000$ $00052,0.00009457,0.00000354,-0.00007832,0.00001032,0.00000839,0.000167$ $41,-0.00010364,-0.00012895,-0.00010811,0.00004516,0.00001573,0.0000053$ $5,0.00006201,0.00002141,0.00000262,-0.00000015,-0.00000053,-0.00001108$ $, 0.00000090,-0.00000577,-0.00000270,0.00001104,0.00005828,-0.00005736$, $-0.00000025,0.11628819,-0.20108683,0.26588403,0.00048529,0.00111977,-0$ $.00036624,0.00034992,-0.00036707,-0.00015212,-0.00028949,0.00018707,-0$ $.00027777,0.00037173,-0.00033070,0.00033077,-0.00004641,-0.00012224,0$. $00011642,-0.00022776,-0.00012903,0.00004532,0.00013246,-0.00044028,0.0$ $0008897,-0.00008883,-0.00004688,0.00016620,0.00000801,0.00000625,0.000$ $00429,0.00003738,0.00001114,0.00001668,0.00003236,0.00007032,0.0000261$ $2,-0.00037895,0.00038891,0.00004742,-0.10381246,0.01259593,-0.01915443$ $, 0.65387974,0.00143137,0.00026514,0.00051581,-0.00023965,0.00050506,0$. $00015656,0.00000681,-0.00025731,0.00038631,-0.00022083,0.00017263,-0.0$ $0002842,0.00015527,-0.00020282,-0.00007780,0.00024802,0.00045495,-0.00$ $031475,0.00006083,-0.00010756,0.00016036,0.00000487,-0.00005453,0.0000$ $2889,0.00004011,0.00001148,-0.00001513,-0.00001793,-0.00000829,-0.0000$ $3312,0.00003555,0.00005942,0.00011345,0.00044431,-0.00049511,0.0000031$ $5,-0.04287254,-0.27635725,0.09355011,0.02657005,0.56215771,-0.00002696$ $, 0.00089463,0.00074597,-0.00061110,-0.00048020,-0.00006469,0.00007437$, $0.00020377,-0.00010196,0.00022718,-0.00032003,0.00005534,-0.00001480,0$ $.00016988,0.00024075,-0.00001763,-0.00041498,0.00004490,-0.00007875,0$. $00003715,-0.00010955,-0.00008084,-0.00004184,0.00009204,-0.00002911,0$. $00001190,0.00000410,-0.00001452,-0.00002215,0.00000682,-0.00004466,-0$. 
$00000351,-0.00005777,-0.00048115,0.00051042,0.00001342,0.00918551,0.11$ $106185,-0.11884909,0.15597880,-0.21247205,0.28604037,-0.00574558,0.001$ $46314,-0.00178528,0.00026389,-0.00205703,-0.00121907,-0.00029904,0.001$ $02391,-0.00095249,0.00090410,-0.00099778,0.00040984,-0.00041545,0.0005$ $8137,0.00029856,-0.00088918,-0.00125115,0.00142957,0.00058156,0.000649$ $52,-0.00002846,0.00001879,-0.00002333,0.00007024,-0.00009370,0.0000603$ $4,0.00004969,0.00000850,0.00012896,-0.00006694,-0.00010506,-0.00012989$ $,-0.00028858,-0.00140694,0.00155043,-0.00000135,0.01392103,-0.07407699$ $, 0.03980838,-0.27291174,-0.05631260,-0.03909594,0.57270418,-0.00386371$ $, 0.00164403,-0.00074931,-0.00015162,-0.00192452,-0.00071054,-0.0005753$ $4,0.00072326,-0.00108389,0.00156517,-0.00142274,0.00051274,-0.00061748$ $, 0.00099508,0.00086106,-0.00010867,-0.00169601,0.00074037,-0.00060585$, $0.00039657,-0.00059173,-0.00015921,0.00002930,0.00024407,-0.00004519,0$ $.00005647,0.00006323,0.00000294,0.00004620,-0.00005321,-0.00003599,-0$. $00013631,-0.00011546,-0.00217958,0.00239068,0.00007788,-0.02439223,-0$. $02025984,0.00635754,-0.11981984,-0.14593318,0.00462624,0.05415890,0.60$ $351171,0.00001929,-0.00036339,-0.00009297,-0.00015608,0.00054360,0.000$ $09739,0.00046957,-0.00046782,0.00043101,-0.00048116,0.00045063,-0.0006$ $5833,-0.00017267,0.00011647,-0.00003543,0.00010357,0.00023468,-0.00000$ $402,0.00076952,-0.00004389,0.00039979,0.00004113,-0.00008550,-0.000009$ $31,0.00001538,-0.00000902,-0.00001356,0.00000425,0.00004229,0.00001740$ $, 0.00002485,-0.00006419,-0.00000848,0.00062417,-0.00070127,0.00002012$, $0.01461455,-0.01016739,0.01754332,-0.00613004,0.02544376,-0.07685267,0$ $.11491658,-0.22595048,0.28111757,0.07047798,0.01671129,-0.00318206,-0$. $02686172,0.01150493,0.02636827,0.00612286,-0.00960894,0.00486759,-0.00$ $475867,0.00769431,0.00162436,0.00833258,-0.01175626,-0.00521009,-0.025$ $69982,0.00882978,-0.02557619,-0.01159195,-0.00773215,0.00036849,0.0006$ $7234,0.00072541,-0.00110213,0.00049595,-0.00034280,-0.00002688,0.00050$ $103,-0.00031885,0.00002682,0.00055817,0.00075876,0.00082978,0.00274475$ ,-0.00341031,-0.00040540,-0.01266231,0.02054065,-0.01211307,-0.0519254 $4,-0.01582583,-0.01381855,-0.21607933,0.09771366,-0.10241973,0.3282381$ $8,0.03490668,-0.00707170,0.00196282,-0.00624980,0.00784942,0.00946434$, $0.00289700,-0.00361948,0.00222835,-0.00269918,0.00346301,0.00038659,0$. $00364538,-0.00537678,-0.00227742,-0.00743123,0.00622617,-0.01074575,-0$ $.01270771,-0.00831310,0.00045816,0.00011145,0.00021793,-0.00037429,0.0$ $0010236,-0.00022752,-0.00000645,0.00002825,-0.00030332,0.00003418,0.00$ $030627,0.00048345,0.00041303,0.00176269,-0.00196113,-0.00013264,0.0205$ $3318,-0.04746178,0.02717298,0.03195074,0.05137863,-0.00872787,0.068380$ $29,-0.14780380,0.07034255,-0.06918778,0.47907027,-0.00121474,0.0007779$ $6,-0.00712514,0.00785894,-0.00065449,-0.00136968,-0.00154709,0.0029036$ $1,-0.00200539,-0.00058862,0.00049993,0.00360747,0.00193370,-0.00293517$ ,-0.00250949,-0.00764974,0.00105897,-0.00085680,0.00024847,0.00113114, $-0.00003345,-0.00020673,-0.00025240,0.00017831,-0.00017943,-0.00003992$ ,-0.00000284,0.00027865,0.00010499,0.00001130,0.00027533,0.00023908,0. $00022947,0.00065048,-0.00068971,-0.00012115,-0.01210129,0.02746038,-0$. $02532497,-0.03811723,-0.02444169,0.01416734,-0.08705724,0.07862535,-0$. $12363764,0.11762345,-0.22078228,0.23686012,-0.00397268,-0.00421349,0.0$ $0222507,0.00035712,-0.00212910,-0.00172319,-0.00049997,0.00084440,-0.0$ $0094260,0.00169991,-0.00166668,0.00045815,-0.00047607,0.00116604,0.001$ $04995,0.00047284,-0.00207213,0.00167926,-0.00076467,-0.00103425,0.0000$ $7728,-0.00013787,-0.00009549,0.00029468,-0.00006713,0.00005883,0.00007$ 
$255,-0.00022276,-0.00002526,-0.00010344,-0.00009395,-0.00015363,-0.000$ $20373,-0.00242161,0.00269919,-0.00000285,-0.05126403,0.02963565,-0.034$ $04074,-0.02117464,-0.02978594,0.00968749,0.03707078,0.02307106,-0.0009$ $2333,-0.09105550,-0.00728936,-0.00891068,0.62116963,-0.00141230,-0.001$ $25372,-0.00146853,-0.00032916,0.00055341,0.00022702,0.00054756,-0.0001$ $5612,0.00034155,-0.00049250,-0.00022078,-0.00013103,0.00071389,-0.0006$ $0819,0.00003845,-0.00021706,-0.00009575,0.00104813,-0.00043908,-0.0003$ $4709,0.00011261,-0.00005468,-0.00002813,0.00020444,-0.00001431,-0.0000$ $2187,0.00003556,0.00008916,0.00003814,-0.00001080,-0.00007557,0.000113$ $27,-0.00000603,0.00018549,-0.00004513,0.00004650,-0.01995632,0.0520237$ $0,-0.02925999,-0.02823409,-0.04159013,0.01021231,0.05105215,-0.0208836$ $8,0.02953130,-0.03606274,-0.28519235,0.10597319,0.01783092,0.55591378$, $-0.00091352,-0.00019684,0.00069004,-0.00013102,-0.00066086,-0.00034561$ ,-0.00007781,-0.00005840,-0.00005580,0.00108916,-0.00040849,-0.0004388 $4,-0.00071835,0.00092632,0.00057539,0.00017867,-0.00137270,0.00017870$, $0.00027860,-0.00018128,0.00013852,0.00004593,0.00005523,0.00001817,-0$. $00003398,0.00003440,0.00003311,-0.00011503,-0.00000210,-0.00002768,0.0$ $0000705,-0.00003984,0.00000655,-0.00096622,0.00102029,0.00003023,-0.00$ $908478,-0.01270525,0.01097214,0.00831805,0.00962337,-0.00554354,-0.015$ $52524,0.02032455,-0.01079745,0.00658411,0.11489681,-0.11138466,0.15121$ $854,-0.21244535,0.28623715,0.00127890,-0.00016904,-0.00054569,0.000388$ $68,0.00048339,0.00024418,0.00006364,0.00015552,0.00003087,-0.00020571$, $0.00003801,-0.00024978,-0.00021123,-0.00003575,-0.00006171,0.00025890$, $0.00027484,0.00009476,0.00014086,0.00023105,0.00001596,0.00005061,-0.0$ $0001538,-0.00015716,-0.00003335,-0.00000162,0.00001186,0.00003855,0.00$ $006719,-0.00002261,-0.00003132,-0.00008920,-0.00003313,0.00014896,-0.0$ $0012911,0.00001002,-0.21084003,0.04688532,-0.07111124,0.03884067,0.056$ $58150,-0.01911482,-0.05854208,0.00071420,-0.01854364,0.01661907,-0.072$ $59182,0.03817136,-0.27509725,-0.05617408,-0.04025078,0.59947806,0.0003$ $0476,-0.00009676,0.00075307,-0.00006114,-0.00047212,-0.00007113,-0.000$ $14232,0.00026725,-0.00027856,0.00035479,-0.00038120,0.00004360,-0.0004$ $8507,0.00060779,0.00031086,0.00054733,-0.00026285,0.00015197,-0.000080$ $45,-0.00013024,0.00003831,-0.00000997,-0.00004252,0.00001336,-0.000047$ $91,0.00000062,0.00001995,-0.00008012,-0.00002323,-0.00003464,-0.000058$ $74,-0.00007945,-0.00008661,-0.00073656,0.00080775,-0.00001747,0.102318$ $45,-0.15745257,0.08005114,0.01790302,-0.02028799,0.02062241,0.00332366$ ,-0.00045707,-0.00051111,-0.02555078,-0.02443326,0.01094389,-0.1202542 $0,-0.14647024,0.00466281,0.07352541,0.62106658,0.00071428,0.00104699,0$ $.00023674,-0.00045064,0.00044017,0.00040956,0.00004315,-0.00006385,0.0$ $0027298,-0.00022667,0.00003434,-0.00028508,0.00001444,-0.00036945,-0.0$ $0001513,-0.00044556,0.00015144,-0.00022666,0.00018970,0.00017215,-0.00$ $003691,0.00000417,0.00001761,-0.00006408,0.00001651,-0.00001184,-0.000$ $00445,0.00005510,0.00000749,0.00002000,0.00006397,0.00010257,0.0000481$ $3,0.00022936,-0.00023801,0.00003887,-0.09953394,0.06215369,-0.12876836$ $, 0.00083947,0.03288297,-0.00822287,-0.01933769,-0.00088138,-0.00914018$ $, 0.01394633,-0.00495961,0.02048433,-0.00797375,0.02537351,-0.07635498$, $0.11556275,-0.22802683,0.28089771,-0.00000330,0.00012647,0.00004121,0$. $00000854,-0.00033979,-0.00022635,-0.00021204,0.00018012,-0.00027943,0$. $00074685,-0.00078983,0.00011423,-0.00020354,0.00018938,0.00028175,0.00$ $002402,-0.00027537,0.00032110,0.00016629,0.00011450,0.00001182,0.00004$ $806,0.00000609,-0.00002241,-0.00000599,0.00004534,0.00003765,0.0000029$ 
$5,0.00003458,-0.00001940,0.00004925,0.00002246,0.00000963,-0.00093798$, $0.00102145,0.00010093,-0.12484045,-0.04581928,-0.00950392,-0.02080411$, $-0.01183129,-0.00214334,0.00265752,-0.00249326,0.00070345,0.00006121,-$ $0.00068336,0.00053462,0.00015753,0.00528461,-0.00398977,-0.03762017,-0$ $.02121436,-0.00283248,0.18111944,-0.00005125,0.00002741,-0.00008345,0$. $00004371,-0.00017244,-0.00012210,-0.00012008,0.00008348,-0.00015724,0$. $00039838,-0.00039760,0.00007612,-0.00012274,0.00015188,0.00013097,0.00$ $006247,-0.00015131,0.00017314,0.00012184,0.00008412,0.00001163,0.00003$ $047,-0.00000249,-0.00001626,-0.00000896,0.00001924,0.00002042,-0.00000$ $685,0.00001617,-0.00001632,0.00001781,-0.00000165,0.00000680,-0.000484$ $15,0.00053574,0.00004649,-0.04577539,-0.05825144,0.00303580,-0.0350902$ $3,-0.01417587,-0.00184900,0.00294339,-0.00235779,0.00448553,-0.0004435$ $5,0.00088729,-0.00121274,-0.00042028,0.00041810,0.00192698,0.00211829$, $0.00425035,0.00081875,0.07697035,0.06897104,-0.00004650,-0.00008227,-0$ $.00012373,0.00005223,-0.00000644,-0.00002890,-0.00001047,-0.00000537,-$ $0.00002348,0.00004975,-0.00002865,-0.00000234,-0.00000151,0.00001398,0$ $.00001535,0.00003400,-0.00004409,0.00004801,0.00000274,-0.00002463,-0$. $00000887,-0.00000287,0.00000396,0.00000048,-0.00000445,-0.00000007,0.0$ $0000395,0.00000938,0.00000596,0.00000138,0.00000200,0.00000323,0.00000$ $528,-0.00004675,0.00004801,0.00001789,-0.00942437,0.00307924,-0.027892$ 49,0.00969012,0.00569322,0.00468190,-0.00210450,0.00261299,0.00244285, $0.00062377,-0.00117926,-0.00021193,-0.00118786,0.00379285,0.00214291,-$ $0.01470367,-0.00670907,0.00318561,0.01544368,-0.00422490,0.01890466,-0$ $.00016560,0.00018069,-0.00004625,-0.00002939,-0.00028512,-0.00010486,-$ $0.00006855,0.00013318,-0.00016226,0.00023897,-0.00025558,0.00008975,-0$ $.00005653,0.00011014,0.00012110,-0.00008681,-0.00019633,0.00010738,0.0$ $0003777,0.00002194,-0.00001691,-0.00001073,-0.00000679,0.00002643,-0.0$ $0001736,0.00001127,0.00001246,-0.00000579,0.00000804,-0.00001049,-0.00$ $000299,-0.00000819,-0.00001920,-0.00036749,0.00040679,0.00001941,0.003$ $98976,-0.00489548,0.00278944,-0.21886279,0.09658901,-0.10825609,-0.014$ $76961,0.01289626,-0.01260806,-0.00320689,-0.00307532,-0.00165594,0.000$ $00724,-0.00081344,0.00074666,0.00167276,-0.00185554,-0.00023961,0.0009$ $8617,-0.00004979,0.00123277,0.22961761,-0.00021769,0.00008447,-0.00002$ $157,0.00004014,-0.00012595,-0.00003188,-0.00004017,0.00006482,-0.00006$ $538,0.00009175,-0.00006635,0.00005381,-0.00001991,0.00003977,0.0000345$ $0,-0.00001343,-0.00006885,0.00004989,-0.00000429,0.00002058,-0.0000105$ $3,-0.00001513,0.00000049,0.00000965,0.00000140,0.00000447,0.00000220,-$ $0.00000357,0.00000170,-0.00000541,-0.00001024,-0.00000651,-0.00001785$, $-0.00011409,0.00011775,-0.00000442,0.02200111,-0.00779319,0.01269215,0$ .09303544,-0.10747972,0.06671822,-0.01202345,0.00896374,-0.00626376,-0 $.00327807,0.00124899,0.00149159,-0.00057293,-0.00065010,-0.00028877,-0$ $.00240456,-0.00335620,0.00363491,0.00084616,0.00025445,-0.00127364,-0$. $09938239,0.10932209,0.00016092,0.00007246,0.00003034,0.00004349,-0.000$ $03311,0.00005751,-0.00002970,0.00003516,-0.00002940,0.00005322,-0.0000$ $1241,0.00005022,0.00001868,-0.00003500,-0.00000248,-0.00003867,0.00001$ $882,-0.00003714,-0.00003141,0.00001078,0.00002298,0.00000087,-0.000002$ $18,-0.00000124,0.00000389,0.00000107,-0.00000116,-0.00000081,0.0000031$ $8,-0.00000189,-0.00000426,0.00000217,-0.00000873,-0.00004022,0.0000314$ $3,-0.00000741,-0.01097492,0.00413560,-0.00235088,-0.10641494,0.0677815$ $4,-0.10363668,-0.00007463,0.00167681,0.00341857,-0.00137965,0.00151472$ $, 0.00512266,0.00051534,-0.00026443,-0.00057006,0.00005236,0.00380740,0$ 
$.00339752,0.00078727,-0.00155811,-0.00163642,0.11668983,-0.07541996,0$. $09938923,0.00046019,0.00104212,0.00030585,-0.00007502,-0.00010061,-0.0$ $0006895,0.00000896,-0.00001009,0.00006155,-0.00006306,0.00008150,-0.00$ $002387,0.00001816,-0.00011720,0.00007015,0.00003285,-0.00013169,-0.000$ $08958,-0.00008249,-0.00042101,-0.00020973,-0.00008343,-0.00000159,0.00$ $012205,0.00002888,0.00000409,-0.00001107,0.00002358,-0.00000969,0.0000$ 0806,0.00000684,0.00004982,0.00001836,0.00010985,-0.00014704,0.0000029 $0,-0.00279309,-0.00229997,-0.00191206,-0.00064029,-0.02594710,0.011536$ $84,-0.05751810,-0.03317379,0.00974662,0.00620507,0.02052298,-0.0085523$ $5,-0.00246049,0.00224844,-0.00405047,-0.00127171,0.00000853,-0.0001435$ $8,-0.00101294,-0.00128837,0.00028888,0.00077771,0.00092030,0.00105159$, $0.05808561,-0.00064257,-0.00010555,-0.00055220,0.00031177,0.00013868,-$ $0.00011544,0.00000184,-0.00001026,0.00005227,-0.00020656,0.00010393,-0$ $.00008632,-0.00005251,0.00003831,-0.00021920,-0.00003769,0.00017444,0$. $00029108,0.00050758,0.00017938,0.00011137,0.00012422,0.00002881,-0.000$ $13933,-0.00000555,-0.00000760,0.00000091,0.00000161,0.00002666,0.00000$ $147,0.00001271,-0.00001578,-0.00000793,0.00029178,-0.00029706,-0.00002$ $293,-0.00276515,0.00245863,0.00129220,-0.00027822,-0.00907023,0.007090$ $60,-0.03692038,-0.27243117,0.10947393,0.00056600,-0.01020144,0.0056510$ $7,0.00099920,0.00122348,0.00302390,0.00029753,0.00027728,-0.00045801,--$ $0.00038198,-0.00054114,0.00021793,0.00066413,-0.00016973,-0.00174883,0$ $.03776293,0.28867358,0.00018402,0.00027606,-0.00041828,0.00011809,-0.0$ $0014244,0.00010471,-0.00001548,-0.00004611,-0.00017162,0.00001973,-0.0$ $0000643,0.00005653,0.00000867,-0.00007867,0.00001099,-0.00012926,-0.00$ $017221,0.00000983,-0.00033895,-0.00038007,-0.00004366,0.00008539,0.000$ 07689,-0.00006061,0.00000466,-0.00002910,0.00000607,0.00002984,-0.0000 $0232,0 ., 0.00000172,0.00004901,-0.00000864,-0.00004089,0.00005883,-0.00$ $001285,-0.00165168,0.00151394,0.00538584,-0.00190469,-0.00122365,0.005$ $01360,0.01211114,0.11052291,-0.08776959,0.00180876,0.01210409,-0.00567$ $642,-0.00353914,0.00333033,0.00357680,-0.00024423,-0.00057984,-0.00053$ $600,-0.00013587,-0.00007336,0.00009493,0.00116850,-0.00164880,-0.00275$ $014,-0.00803575,-0.12372819,0.08396596,0.00039724,0.00004354,-0.000446$ $65,-0.00012110,0.00044361,0.00022863,0.00009917,-0.00000849,0.00017451$ ,-0.00066503,0.00014172,0.00025242,-0.00018914,-0.00004866,-0.00026758 $, 0.00025839,-0.00036103,0.00027800,-0.00002657,-0.00003906,-0.00002239$ ,-0.00000418,0.00002161,-0.00001352,0.00002434,0.00000003,-0.00002814, $-0.00001253,-0.00003484,0.00000239,-0.00000050,0.00006915,0.00004599,0$ $.00027297,-0.00025374,-0.00007966,-0.00170443,-0.00362759,-0.00091892$, $-0.00020062,-0.00049185,0.00043257,0.00148830,-0.00261700,-0.00024489$, $0.00647608,0.00070416,0.00158223,-0.21149063,0.09049957,-0.10370141,-0$ $.01661584,0.01305514,-0.01366297,-0.00135004,0.00001018,-0.00048306,-0$ $.00011628,0.00011983,0.00015534,0.00026408,-0.00022522,0.00022806,0.22$ $255815,0.00097014,-0.00001932,-0.00010551,-0.00013811,-0.00001476,0.00$ $012565,0.00003671,-0.00018248,-0.00008675,-0.00012942,0.00047959,0.000$ $10308,-0.00025620,0.00053153,-0.00014548,0.00039224,-0.00024510,-0.000$ $11608,-0.00006612,0.00000632,0.00003302,0.00001629,0.00000567,-0.00004$ $223,0.00001395,0.00001475,-0.00003135,-0.00001853,0.00002361,0.0000218$ $7,-0.00012026,-0.00021900,-0.00009938,0.00004073,-0.00019232,-0.000053$ $66,-0.00313671,0.00120213,0.00176008,-0.00085670,-0.00076069,-0.000209$ $58,-0.00123539,-0.00340262,0.00441967,0.02060846,-0.01060528,0.0124664$ $2,0.09348818,-0.10564955,0.06612211,-0.01244376,0.00906708,-0.00609048$ 
- $-0.00092111,-0.00004880,-0.00022136,0.00014656,-0.00011017,-0.0003059$ $8,0.00089159,-0.00031294,0.00048220,-0.09825156,0.11051202,-0.00018535$ ,-0.00047374,-0.00070158,0.00005974,0.00016542,0.00007401,0.00006284,-$0.00012168,-0.00008142,-0.00012575,0.00011868,0.00023777,0.00018034,-0$ $.00019053,-0.00009547,0.00023973,0.00007837,0.00026015,-0.00020206,-0$. $00008488,0.00001206,0.00000160,0.00000764,0.00002409,0.00001192,0.0000$ $0541,-0.00001011,-0.00005874,-0.00002739,-0.00003760,-0.00005808,-0.00$ $009661,-0.00004765,0.00029759,-0.00032713,-0.00002831,-0.00115450,0.00$ $174119,0.00552657,0.00063992,-0.00016929,-0.00054594,-0.00116589,0.004$ $04462,0.00396500,-0.00847125,0.00615176,-0.00564662,-0.10558876,0.0654$ $4148,-0.10360145,-0.00054093,0.00194679,0.00378145,0.00002423,0.000083$ $69,0.00005232,0.00010944,-0.00031820,-0.00057659,-0.00036849,0.0000869$ $6,-0.00016015,0.11524345,-0.07636651,0.10033858,-0.00021766,-0.0001730$ $6,0.00008789,0.00002642,-0.00023544,-0.00011305,-0.00005909,0.00010834$ ,-0.00013041,0.00028245,-0.00024423,0.00005319,-0.00010811,0.00014423, $0.00013530,0.00012021,-0.00017176,0.00013562,-0.00004476,-0.00003307,0$ $.00000216,-0.00000719,-0.00000869,0.00001851,-0.00001869,0.00000182,0$. $00001410,-0.00003023,-0.00000488,-0.00002429,-0.00001297,-0.00002106,-$ $0.00002167,-0.00035152,0.00040942,0.00000032,0.00520637,0.02086871,-0$. $00996822,-0.00287737,0.00144657,-0.00338833,-0.00108613,0.00032981,-0$. $00023820,-0.00303248,-0.00355599,-0.00135438,-0.00012795,-0.02416624,0$ $.01100430,-0.05849358,-0.04085672,0.01321194,0.00075132,0.00104210,0.0$ $0057133,0.00036971,0.00102643,-0.00055403,-0.00000367,0.00003225,0.000$ $24906,0.00071550,0.00095110,0.00104870,0.05895777,0.00007532,-0.000019$ $98,0.00008930,0.00003149,-0.00011997,-0.00006155,-0.00005142,0.0000821$ $5,-0.00009970,0.00014302,-0.00016020,0.00005646,-0.00000194,0.00006879$ $, 0.00007625,-0.00003637,-0.00007413,0.00006210,-0.00002148,-0.00002211$ $, 0.00000204,0.00000047,-0.00000482,0.00000375,-0.00000652,0.00000449,0$ $.00000756,-0.00001524,0.00000261,-0.00000764,-0.00000207,-0.00001318,-$ $0.00000796,-0.00022427,0.00025236,0.00000117,-0.00595652,-0.00922839,0$ $.00448865,0.00198818,0.00167689,0.00248924,0.00004908,0.00047133,-0.00$ $061066,-0.00308128,0.00118389,0.00161514,0.00046851,-0.00707912,0.0059$ $2584,-0.03734825,-0.28224270,0.11421965,0.00015869,0.00053136,-0.00165$ $520,-0.00025374,-0.00027166,0.00029781,0.00008851,-0.00022506,-0.00029$ $124,0.00076098,-0.00010136,-0.00174310,0.04319314,0.29533839,-0.000096$ $55,-0.00002557,0.00011470,0.00007492,-0.00002162,-0.00006703,-0.000013$ $58,0.00004082,-0.00002111,-0.00006488,0.00001063,0.00005153,0.00001985$ ,-0.00004263,-0.00000130,-0.00005772,0.00002095,0.00001939,0.00002099, $-0.00000778,-0.00000716,-0.00000209,0.00000027,-0.00000461,0.00000899$, $0.00000390,-0.00000670,-0.00000346,0.00000448,-0.00000787,0.00000327,0$ $.00000028,-0.00000331,0.00003057,-0.00003047,-0.00002357,0.00376254,0$. $01308026,-0.00221778,-0.00366003,0.00232537,0.00301348,-0.00003057,-0$. $00057908,-0.00060545,-0.00167508,0.00160927,0.00519214,-0.00156495,-0$. $00209288,0.00468637,0.01136401,0.11311693,-0.08916284,0.00103975,-0.00$ $135347,-0.00164603,0.00011344,0.00070881,0.00012754,0.00022440,-0.0003$ $0397,-0.00060685,0.00116323,-0.00168084,-0.00277496,-0.01065851,-0.124$ $80268,0.08393248,0.00086842,-0.00017858,-0.00006944,-0.00030271,0.0005$ 2664,0.00143029,0.00016804,-0.00180287,0.00008686,-0.02261999,0.020332 $39,0.02322352,0.00089342,0.00194749,-0.00319722,0.00026027,0.00119655$, $0.00104258,-0.00029006,-0.00015526,0.00005904,0.00021853,-0.00019807,0$ $.00007334,-0.00115085,0.00104519,0.00075335,-0.00026944,-0.00028408,0$. 
$00027904,-0.00026419,-0.00002286,-0.00007746,-0.08355051,0.02913741,0$. $02401518,-0.00026103,-0.00013189,-0.00000896,0.00011469,-0.00007906,0$. $00009786,0.00025531,0.00043411,-0.00013081,-0.00068364,-0.00041118,-0$. $00008112,0.00041781,-0.00000151,0.00008857,-0.00000896,0.00009526,-0.0$ $0004315,0.00024286,0.00011449,0.00001457,0.00007641,0.00001849,0.00001$ $217,-0.00002004,-0.00006470,0.00001922,-0.00006011,0.00000846,0.000019$ $12,0.00004543,0.00004581,0.00000682,0.56623034,-0.00083480,0.00031345$, $-0.00008637,0.00079455,-0.00010858,-0.00146685,-0.00217792,0.00077228$, $0.00004526,0.01962209,-0.02775506,-0.02504169,0.00198160,0.00107998,0$. $00347871,0.00146347,0.00004640,-0.00116778,0.00007904,-0.00000504,-0.0$ $0006421,-0.00023470,0.00023722,-0.00009618,0.00112378,-0.00139114,-0.0$ $0080394,-0.00029994,-0.00025209,-0.00019584,-0.00005051,-0.00025204,0$. $00011336,0.03015215,-0.09250690,-0.03244192,0.00025778,0.00014737,0.00$ $000330,-0.00011874,0.00011103,-0.00012447,-0.00040463,-0.00059652,0.00$ $017381,0.00108884,0.00063786,0.00010535,-0.00065231,0.00000770,-0.0002$ $3996,0.00003019,-0.00019094,0.00004348,-0.00028293,-0.00014950,-0.0000$ $1849,-0.00010639,-0.00002698,-0.00000737,0.00003585,0.00007040,-0.0000$ $1161,0.00012021,0.00003839,0.00008695,-0.00010294,-0.00005985,0.000027$ $56,0.00063817,0.56101569,-0.00099535,0.00066003,0.00051630,0.00110976$, $-0.00070796,-0.00144959,-0.00618817,0.00683987,0.00176056,0.00912617,-$ $0.00919182,0.00680691,-0.00312047,0.00337435,0.00112848,0.00010487,0.0$ $0025576,-0.00018339,0.00026375,0.00007146,-0.00008655,-0.00029929,0.00$ $031549,-0.00008883,-0.00092611,0.00104620,-0.00074742,0.00006005,-0.00$ $003099,0.00011300,-0.00025161,0.00028203,0.00007845,0.04524084,-0.0556$ $6802,-0.18233339,0.00041826,0.00023288,0.00001685,-0.00019297,0.000196$ $74,-0.00022339,-0.00062480,-0.00098330,0.00028881,0.00138087,0.0008438$ $9,0.00013419,-0.00100347,-0.00002869,-0.00035597,0.00004331,-0.0003040$ $4,0.00007842,-0.00046028,-0.00023665,-0.00003428,-0.00017652,-0.000046$ $75,-0.00000958,0.00004991,0.00013790,-0.00001931,0.00014524,0.00006643$ $, 0.00011837,-0.00015496,-0.00009842,0.00003247,0.05410785,-0.06249330$, $0.49237385,-0.00009130,-0.00008792,-0.00046121,-0.00008475,0.00063060$, $-0.00027926,0.00015107,-0.00029295,0.00037904,0.00251480,-0.00389952,0$ $.00084765,-0.00061695,0.00112541,0.00037533,0.00058711,-0.00041825,0.0$ $0022149,0.00000404,-0.00003681,-0.00005846,-0.00005066,0.00001869,0.00$ $001647,-0.00138877,0.00018316,-0.00062113,-0.00001977,-0.00009949,-0.0$ $0011156,-0.00003677,0.00000015,0.00000397,-0.00865245,-0.00009826,0.01$ $450611,0.00002320,0.00000537,0.00000286,-0.00001328,0.00001470,-0.0000$ $1250,-0.00002637,-0.00004785,-0.00000260,0.00005145,0.00004116,0.00002$ $378,-0.00002791,-0.00000613,-0.00000254,-0.00000529,-0.00000820,0.0000$ $0573,-0.00001430,-0.00000428,-0.00000197,-0.00000780,-0.00000088,0.000$ $00036,-0.00000158,0.00001153,-0.00000053,0.00000715,-0.00000756,-0.000$ $00629,0.00000091,-0.00000261,-0.00000280,-0.10953542,-0.08307275,0.065$ $88849,0.11572986,-0.00010360,0.00001824,0.00057696,0.00021712,-0.00082$ $025,-0.00058735,-0.00016405,0.00044164,0.00146571,-0.00050299,0.002950$ $68,-0.00042968,0.00002411,-0.00090517,-0.00044774,-0.00044213,0.000294$ $22,-0.00033505,0.00009729,0.00003771,-0.00002100,0.00001281,0.00004482$ $, 0.00000715,0.00129327,-0.00008853,-0.00048900,0.00006419,0.00005354,0$ $.00001894,0.00003032,0.00004458,-0.00001391,0.01273925,0.01347796,-0.0$ $1464970,0.00002799,0.00001332,0.00000409,-0.00001501,0.00001153,-0.000$ $01264,-0.00002241,-0.00004586,0.00000688,0.00003794,0.00003147,-0.0000$ $0945,-0.00002440,-0.00000474,0.00000730,-0.00000306,-0.00001397,0.0000$ 
$0475,-0.00002073,-0.00000727,-0.00000258,-0.00000748,-0.00000169,-0.00$ $000125,-0.00000116,0.00001199,-0.00000161,-0.00000659,-0.00000657,-0.0$ $0000929,0.00000274,-0.00000210,-0.00000395,-0.08691083,-0.16979517,0.0$ $9885955,0.08956555,0.17454380,0.00037062,-0.00010136,0.00017308,-0.000$ $05353,-0.00005421,0.00009897,0.00012310,-0.00057622,0.00054270,-0.0040$ $0871,0.00473980,-0.00016193,0.00047691,-0.00121663,-0.00043742,-0.0005$ $0890,0.00019930,-0.00003368,-0.00004272,-0.00001311,0.00002447,0.00003$ $036,-0.00005669,0.00002213,-0.00024563,0.00014626,0.00040077,0.0000782$ $6,0.00016482,0.00012393,0.00006151,-0.00001957,-0.00001779,0.02185524$, $0.00659080,-0.02871835,-0.00008329,-0.00004388,-0.00000618,0.00003476$, $-0.00004025,0.00004386,0.00012090,0.00019075,-0.00005617,-0.00030953,-$ $0.00019285,-0.00003675,0.00018864,-0.00000465,0.00006753,-0.00000591,0$ $.00005935,-0.00001284,0.00008769,0.00004416,0.00000629,0.00003277,0.00$ $000933,0.00000241,-0.00001067,-0.00002447,0.00000523,-0.00003163,-0.00$ $000908,-0.00002099,0.00002737,0.00001862,-0.00000572,0.06583160,0.0933$ $2761,-0.12160435,-0.08115740,-0.10168426,0.14377078,0.00003829,-0.0000$ $6357,-0.00048432,-0.00070451,0.00029980,0.00056816,0.00035717,-0.00034$ $298,-0.00142140,0.00244266,-0.00032853,0.00032099,-0.00072825,0.000021$ $94,0.00041607,0.00019200,-0.00041351,0.00032201,0.00001235,0.00004437$, $0.00002392,0.00004538,-0.00000431,-0.00001464,0.00000869,0.00111390,0$. $00053104,0.00003961,0.00007334,-0.00002264,0.00003610,0.00001267,0.000$ $00234,0.01448366,0.00996195,0.01101372,-0.00002760,-0.00002292,0.00000$ 076,0.00001957,-0.00001226,0.00001995,0.00005404,0.00007384,-0.0000270 $3,-0.00011669,-0.00006834,0.00001967,0.00008600,0.00000147,0.00003165$, $-0.00001053,0.00001390,-0.00001494,0.00003021,0.00001826,0.00000468,0$. $00001389,0.00000288,0.00000005,-0.00000143,-0.00001083,0.00000084,-0.0$ $0001701,0.00000310,-0.00001076,0.00001055,0.00000958,-0.00000273,-0.19$ $453025,-0.08499286,-0.09920421,-0.01000580,-0.00456356,-0.00821878,0.2$ $0082107,0.00003246,0.00002020,0.00053085,0.00063067,-0.00029604,0.0002$ $1099,-0.00026877,0.00027805,-0.00021376,-0.00419952,0.00309417,-0.0008$ $8353,0.00112741,-0.00076928,-0.00046716,-0.00044705,0.00062460,-0.0002$ $7867,-0.00007980,-0.00001347,0.00005534,0.00000194,-0.00005314,-0.0000$ $1657,-0.00000838,-0.00144313,0.00061959,-0.00010144,-0.00001837,0.0001$ $3690,-0.00000948,-0.00003236,-0.00000099,-0.00343725,-0.01105170,-0.01$ $636528,-0.00000325,0.00000085,-0.00000322,0.00000515,-0.00001573,0.000$ $01394,0.00003990,0.00004798,-0.00000481,-0.00006525,-0.00003852,-0.000$ $01575,0.00004497,0.00000192,0.00003555,0.00000449,0.00002528,-0.000005$ $56,0.00000553,0.00000309,0.00000146,0.00000903,0.00000107,-0.00000179$, $0.00000039,-0.00001069,-0.00000007,-0.00000976,0.00000273,-0.00002734$, $0.00000554,0.00000423,-0.00000245,-0.08086571,-0.09669755,-0.05321425$, $-0.01632746,-0.00792211,-0.00966545,0.08853449,0.10285962,0.00028257,-$ $0.00016992,0.00016804,-0.00003725,-0.00006063,0.00006110,0.00055094,-0$ $.00018064,0.00061479,-0.00433000,0.00430649,-0.00011846,0.00111130,-0$. $00053030,-0.00049449,-0.00021321,0.00041770,-0.00005617,-0.00006385,-0$ $.00003295,0.00002642,0.00004810,-0.00003226,0.00002116,-0.00005168,0.0$ $0027528,0.00036556,-0.00015023,-0.00005708,0.00016396,-0.00000095,-0.0$ $0006124,-0.00001259,-0.01040774,-0.02212137,-0.02744896,-0.00006253,-0$ $.00003882,-0.00000376,0.00002775,-0.00003795,0.00003895,0.00012804,0.0$ $0019524,-0.00005918,-0.00035294,-0.00019705,-0.00004009,0.00021786,-0$. $00000041,0.00011518,-0.00001441,0.00006638,-0.00002074,0.00007776,0.00$ $004466,0.00000498,0.00003314,0.00001063,0.00000184,-0.00001121,-0.0000$ 
$2058,0.00000281,-0.00003013,-0.00003115,-0.00005350,0.00004102,0.00002$ $125,-0.00000765,-0.09371547,-0.05348138,-0.10991223,0.01190976,0.00803$ $806,0.01056192,0.10391138,0.06835218,0.13036243,-0.00004683,0.00001257$ $, 0.00003053,0.00013770,0.00006542,-0.00004281,0.00027386,0.00008783,0$. $00011682,0.00113469,-0.00059845,0.00219704,-0.00034585,0.00058547,-0.0$ $0027963,0.00004182,0.00002229,0.00022148,-0.00000554,0.00000238,-0.000$ $00612,0.00000425,0.00002758,-0.00000691,-0.00023336,-0.00021416,0.0000$ $0487,0.00002332,0.00001912,0.00001873,-0.00008258,0.00003023,-0.000005$ $32,-0.01148989,0.01312584,0.00069567,0.00004430,0.00002673,-0.00000223$ , $-0.00001764,0.00000790,-0.00001147,-0.00003968,-0.00007332,0.00002532$ $, 0.00011770,0.00005848,0.00001594,-0.00008375,-0.00000407,-0.00003039$, $0.00000738,-0.00000819,0.00000807,-0.00004737,-0.00002584,-0.00000191$, $-0.00001341,-0.00000312,-0.00000213,0.00000555,0.00000628,-0.00000120$, $0.00001264,0.00001391,-0.00000175,-0.00001524,-0.00001106,0.00000019$, $0.15624433,0.11594373,-0.06434118,0.01150958,-0.01132455,0.00535698,-0$ $.01254917,0.01538113,-0.00889802,0.16790186,-0.00000330,-0.00004521,0$. $00001793,0.00007307,0.00006519,0.00003406,0.00007015,0.00025455,-0.000$ $19002,-0.00076646,0.00110542,-0.00252907,0.00066772,-0.00049756,0.0002$ $9307,-0.00003435,0.00002530,-0.00028442,-0.00002224,-0.00001690,0.0000$ $0438,0.00003613,-0.00000062,0.00000999,-0.00023070,-0.00015021,0.00001$ $755,0.00000748,0.00001509,-0.00003271,0.00003855,-0.00008672,0.0000118$ $1,0.01426006,-0.01525222,-0.00062809,-0.00004564,-0.00002809,-0.000002$ $22,0.00001707,-0.00000808,0.00001576,0.00004999,0.00007475,-0.00002182$ ,-0.00011542,-0.00006893,-0.00001444,0.00008250,0.00000318,0.00001438, $-0.00000131,0.00002336,0.00000053,0.00004759,0.00002358,0.00000318,0.0$ $0001454,0.00000270,0.00000149,-0.00000775,-0.00000691,0.00000146,-0.00$ $001438,-0.00001285,-0.00000327,0.00001190,0.00000816,-0.00000216,0.115$ $56612,-0.17469040,0.06982163,0.01281460,-0.01236207,0.00821187,-0.0093$ $6183,0.01139830,-0.00467416,-0.13315410,0.19022718,-0.00008404,0.00010$ $378,0.00012352,0.00014162,-0.00020955,0.00048398,-0.00016469,0.0001987$ $3,-0.00126119,0.00099129,-0.00137877,-0.00433426,0.00109958,-0.0011769$ $1,0.00040074,-0.00013607,0.00009338,-0.00045676,-0.00002490,0.00001726$ $, 0.00005344,0.00003693,-0.00004692,-0.00000441,-0.00034168,0.00038757$, $0.00076898,-0.00002542,0.00002306,-0.00001376,0.00009126,-0.00009732,0$ $.00001978,0.02650218,-0.02911146,0.00438865,0.00000850,0.00000413,-0.0$ $0000034,-0.00000058,-0.00000752,0.00000533,0.00001573,0.00002202,-0.00$ $000142,-0.00001009,-0.00000807,-0.00000431,0.00002368,0.00000358,0.000$ $00725,-0.00000174,0.00001109,-0.00000020,-0.00000354,-0.00000152,-0.00$ $000035,0.00000368,0.00000124,-0.00000102,0.00000065,-0.00000418,0.0000$ $0080,-0.00000113,-0.00000511,-0.00000802,0.00000527,0.00000331,-0.0000$ $0019,-0.07383735,0.08033602,-0.08639839,-0.01146277,0.00970904,-0.0047$ $3191,-0.00776577,0.01120985,-0.00428130,0.06493941,-0.07007672,0.09524$ $537 \backslash 0.00000075,0.00000018,-0.00000278,0.00000006,-0.00000279,-0.00000$ $272,-0.00000044,-0.00000241,-0.00000145,0.00000014,-0.00000013,-0.0000$ $0079,0.00000090,0.00000222,-0.00000179,0.00000080,0.00000265,-0.000003$ $35,0.00000081,0.00000072,-0.00000415,-0.00000070,-0.00000360,-0.000003$ $02,-0.00000116,-0.00000399,-0.00000079,0.00000134,0.00000370,-0.000001$ $24,0.00000174,0.00000378,-0.00000349,-0.00000018,-0.00000002,0.0000004$ $6,-0.00000055,0.00000112,0.00000299,-0.00000006,0.00000058,0.00000049$, $-0.00000016,0.00000006,-0.00000094,-0.00000075,-0.00000031,-0.00000083$ $, 0.00000106,0.00000200,0.00000229,0.00000052,0.00000170,0.00000406,-0$. 
$00000039,0.00000134,0.00000547,-0.00000027,-0.00000063,0.00000043,0.00$ $000017,-0.00000100,-0.00000270,0.00000049,0.00000250,0.00000232,0.0000$ $0012,0.00000286,0.00000559,-0.00000091,-0.00000223,0.00000131,-0.00000$ $109,-0.00000351,-0.00000027,-0.00000136,-0.00000306,0.00000263,-0.0000$ 0089,-0.00000174,0.00000225।II@

ortho-Addition Product

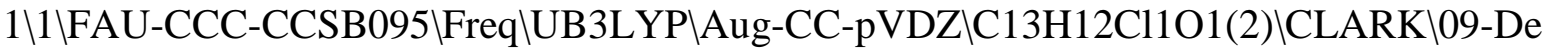
c-2014\0\\N Geom=AllCheck Guess=TCheck SCRF=Check GenChk UB3LYP/Aug-C C-pVDZ Freq I\Addition product p-Cl-Ph-radical + anisol (ortho, trans) \ $\backslash 0,2 \backslash \mathrm{C}, 1.3002598145,-0.3453965195,1.0345084754 \backslash \mathrm{C}, 2.2304323621,0.486629$ 9443,0.1774611606\C,3.2436453596,-0.0550063155,-0.5741530781\C,3.50226 $82367,-1.4532971844,-0.5422661487 \backslash \mathrm{C}, 2.7251444814,-2.2990921988,0.28901$ 70726\C,1.7014125614,-1.8024461681, $1.0508042377 \backslash \mathrm{H}, 1.3622889508,0.05817$ $80968,2.0638916316 \backslash \mathrm{O}, 1.9290564715,1.80891271,0.263691656 \backslash \mathrm{H}, 3.874856143$ $3,0.5808271816,-1.1916745525 \backslash \mathrm{H}, 2.9525233603,-3.365663738,0.3260375351 \backslash$ $\mathrm{H}, 1.110449305,-2.4622946871,1.6863369725 \backslash \mathrm{H}, 4.3099336942,-1.8645158087$, $-1.1455279907 \backslash \mathrm{C},-2.836322455,0.1264862043,-0.1744761027 \backslash \mathrm{C},-2.433877513$ $1,0.5961221183,1.0744328833 \backslash \mathrm{C},-1.0964269408,0.4425218257,1.4550460335 \backslash$ C,-0.167237023,-0.1724452715,0.6093553838\C,-0.6032326871,-0.639133320 $3,-0.6390556563 \backslash \mathrm{C},-1.9322072221,-0.4919740738,-1.040658805 \backslash \mathrm{Cl},-4.52001$ $98459,0.3132159285,-0.6701466216 \mathrm{lH},-3.1519383886,1.0754184978,1.737489$ 306\H,-0.7768091342,0.8157193629,2.4289018872\H,0.1043386005,-1.127409 $0735,-1.3094300327 \backslash \mathrm{H},-2.2660030793,-0.8544917753,-2.0114338956 \backslash \mathrm{C}, 2.695$ $9012969,2.7299011579,-0.5076412457 \backslash \mathrm{H}, 2.6033301112,2.5139921192,-1.5825$ $167007 \backslash \mathrm{H}, 3.7565810177,2.7021874659,-0.2157121631 \backslash \mathrm{H}, 2.279332522,3.71841$ $35213,-0.292915242 \backslash \backslash$ Version=EM64L-G09RevC.01 $\backslash$ State $=2-A \backslash H F=-1038.056903$ 9\S2=0.780436 $\backslash \mathrm{S} 2-1=0 . \mathrm{S} 2 \mathrm{~A}=0.750537 \backslash \mathrm{RMSD}=7.106 \mathrm{e}-09 \backslash \mathrm{RMSF}=1.933 \mathrm{e}-05 \backslash \mathrm{ZeroP}$ oint $=0.2123693 \backslash$ Thermal $=0.2257274 \mathrm{ZPE}=133.2637535 \backslash$ Dipole $=1.0290826,0.38$ $51379,-0.0336012$ DipoleDeriv $=0.1485561,-0.0815073,0.088067,0.0293623$,$0.048702,0.0677096,0.1371712,0.1107012,0.192475,0.1588864,-0.3769241,-$ $0.0712176,-0.0318943,1.3943923,-0.0126006,-0.2396861,0.2020031,0.02818$ $,-0.152784,0.280093,-0.0846051,-0.1827563,-0.2249365,0.0484065,0.00610$ $72,-0.2419455,-0.1064843,-0.0331169,-0.0877304,-0.1092227,0.0360006,0$. $0983899,-0.0464817,-0.1418521,0.029587,-0.0640244,-0.0727285,0.0385579$ ,-0.0747316,0.0756055,0.0848576,-0.0403177,-0.0703785,-0.0760212,-0.06 $01211,-0.1650381,-0.0795526,-0.1113373,-0.1428981,0.0313337,-0.0726193$ $,-0.1477957,0.0649323,-0.1193281,0.0123255,0.021979,0.0418471,0.037975$ $6,0.0174355,-0.0986734,0.0221898,-0.0897676,-0.0994584,-0.4751059,0.02$ $17155,0.1147424,-0.1298311,-1.8496364,0.2000557,0.1986511,0.1541149,-0$ $.3349316,0.0479189,-0.0634706,0.0724892,-0.0486991,0.0059149,0.0646568$ $, 0.1085644,0.0640167,0.0720731,0.0801468,0.0394504,0.0263932,0.0676457$ $,-0.1136112,0.0193096,0.0604412,0.0110486,0.1035756,0.0528314,-0.04139$ $91,0.0917108,-0.0335019,-0.0124563,0.0567926,0.0845742,0.046182,0.0476$ $872,-0.005425,0.0548525,0.0989166,0.0488495,0.0211501,-0.0153782,0.110$ $5924,-0.020621,0.0559329,1.1666801,-0.1403913,0.301831,-0.1224691,-0.0$ $159206,-0.0026638,0.3781217,-0.0087227,0.1127282,-0.2592966,-0.0305165$ $,-0.1228292,-0.0333664,-0.0624163,0.0440588,-0.1429941,0.0417576,-0.06$ $43424,-0.056385,0.011578,0.0535499,-0.0379453,-0.0551149,0.0777893,-0$. $0562322,0.0824918,0.069815,0.2868529,-0.012351,0.0043913,0.033548,-0.0$ 
$486408,-0.020358,0.0770127,-0.0242995,-0.0914137,-0.1074964,-0.0234633$ ,-0.0214229,-0.0003647,-0.065671,0.0361764,-0.0144934,0.0283997,-0.017 $9292,-0.2456501,0.046089,0.0108031,0.028464,-0.0712443,0.04946,-0.0156$ $147,0.0570521,0.0255933,-0.8748083,0.087737,-0.1845244,0.0892849,-0.08$ $74383,0.0129885,-0.230428,0.0172283,-0.1454743,0.0029189,0.0488175,0.0$ $491026,0.0497523,0.0951772,-0.0457358,0.0392567,-0.0433716,0.0528778,0$ $.052826,-0.0157164,-0.0809066,-0.0035207,0.0889262,-0.0561609,-0.05308$ $75,-0.0560899,-0.0389928,0.0290181,0.0455775,0.0538237,0.0467004,0.071$ $2197,-0.0409921,0.0682389,-0.0377549,0.0371974,0.0497461,-0.002922,-0$. $0420303,-0.0019146,0.104157,-0.0474838,-0.0528037,-0.047048,-0.0012256$ $, 0.4274787,0.3803351,-0.0829967,0.1929242,0.7169709,-0.125088,-0.11255$ $42,-0.3677424,0.3889806,0.023144,-0.0731383,-0.0216471,-0.0177039,0.01$ $53845,-0.0808559,0.0107182,0.0840345,-0.1267038,-0.1451968,-0.1236753$, $-0.0202311,0.0414749,0.0226832,0.0239889,-0.0492229,0.0533379,0.015675$ $5,0.0537021,0.0759757,0.0200346,0.0092775,-0.1122041,0.0040165,0.02550$ $34,-0.0335033,0.0676382 \backslash$ Polar $=228.6313977,-17.859673,164.3374034,-6.84$ $21386,4.5931869,146.8994647 \backslash \mathrm{PG}=\mathrm{C} 01[\mathrm{X}(\mathrm{C} 13 \mathrm{H} 12 \mathrm{Cl1O} 1)] \mathrm{NImag}=0 \backslash 10.4157030$ 7,0.00154514,0.49069451,-0.02772246,0.02967087,0.51629059,-0.11716538, $-0.03480837,0.04850924,0.49168768,-0.03020988,-0.10401642,0.03389715,-$ $0.08974925,0.59463413,0.04706346,0.03086155,-0.11378564,-0.26559408,0$. $01294708,0.36239252,-0.01048400,0.00336278,0.01435336,-0.24022273,0.07$ $743951,0.14267888,0.47047335,-0.00858518,0.01406886,0.00438751,0.03869$ $740,-0.13803456,-0.02089357,-0.00874075,0.61445671,0.01548296,-0.00309$ $032,-0.00522908,0.14891123,-0.05109555,-0.16687528,-0.29909444,-0.0734$ $5446,0.36553171,-0.00161483,-0.00427203,-0.00391507,0.00017455,0.02910$ $195,-0.00177890,-0.09220670,0.05802490,0.02697545,0.44535278,-0.001242$ $58,-0.00527189,0.00067370,0.05595243,-0.03058857,-0.03863212,-0.002231$ $61,-0.25679299,0.03591113,-0.08249190,0.56671206,-0.00459675,0.0035686$ $6,-0.00430212,-0.00651342,-0.01841944,0.01164668,0.03705773,-0.0104302$ $9,-0.07540359,-0.27719846,-0.01826330,0.33809961,-0.00383197,0.0144687$ $6,0.00620013,-0.01764885,0.00300065,0.01244555,0.02895620,-0.04098773$, $-0.01112746,-0.12265147,-0.00811156,0.06027613,0.41876559,0.02416306,-$ $0.01077702,-0.01659556,-0.00391014,0.00097902,0.00240178,-0.01374596$,$0.03644175,0.01988245,-0.07174450,-0.18201516,0.08684038,-0.09813619,0$ $.69882242,0.00287182,-0.00830244,0.00476906,0.01446245,-0.00275362,-0$. $01168737,-0.01631453,0.04207231,0.01086651,0.07351817,0.03415478,-0.12$ $260452,-0.23352034,-0.04042743,0.32338345,-0.07910022,0.02664728,0.011$ $02731,0.01768011,-0.01774957,-0.01274346,-0.01243168,0.02262369,0.0049$ $3372,-0.03812053,-0.03706177,0.04824486,-0.23214453,0.10942810,0.13282$ $193,0.49481197,0.01843764,-0.19167357,0.01065548,-0.00635406,-0.020136$ $48,0.00846116,0.00579858,-0.01030947,-0.00323429,-0.00391591,0.0340701$ $9,-0.00175664,0.07435756,-0.15691334,-0.03947621,-0.04240883,0.5373669$ $8,0.01349489,0.00516519,-0.07701357,-0.01484390,0.01846218,0.00514440$, $0.00804607,-0.01767471,-0.00491110,0.04183484,0.02704199,-0.03091698,0$ $.13763153,-0.06839198,-0.15102838,-0.29983190,-0.05890597,0.38578072,-$ $0.03740231,-0.00648390,-0.00840629,-0.00310017,0.00551593,0.02302888,0$ $.00028728,-0.00367984,0.00015905,-0.00045956,0.00140091,0.00020686,0.0$ $0009744,0.00079541,-0.00048586,-0.00059709,0.00694415,0.00845213,0.056$ $29437,-0.00970921,-0.07418128,-0.07930585,0.00246431,0.00609162,0.0151$ $8147,-0.00180254,-0.00102899,0.00128262,0.00107255,-0.00007530,-0.0010$ $8940,-0.00288834,-0.00401000,0.00174933,0.00471540,-0.01522285,-0.0277$ 
$2403,0.00419906,0.08310806,-0.01722241,-0.07410373,-0.24667318,0.00757$ $009,-0.00453836,-0.02117676,-0.00175233,0.00487092,-0.00047282,0.00049$ $796,-0.00181549,-0.00051153,-0.00179369,0.00023929,0.00037802,0.002666$ 49,-0.00504185,0.00441533,0.01279990,0.08637879,0.27303307,0.00641914, $-0.02246344,-0.00235991,-0.09121104,0.03773397,0.04039311,-0.00134567$, $0.03676548,0.00426003,-0.00088511,-0.00233314,0.00691226,-0.00012385,-$ $0.00037082,-0.00017923,-0.00323299,-0.00049276,0.00347834,0.00065735,-$ $0.00068932,-0.00074831,0.22364293,-0.00153054,-0.04169660,0.00491272,0$ $.06510988,-0.27709799,-0.02403331,0.01488725,-0.03297106,-0.00787507,0$ $.00191084,-0.00127395,-0.00019226,0.00383452,0.00062632,-0.00381755,-0$ $.00597838,-0.00198168,0.00493023,0.00120058,0.00241235,-0.00087849,0.0$ $1242667,0.51796256,-0.00522852,0.02419577,0.00167169,0.03855317,-0.000$ $26725,-0.06748553,0.00615433,-0.02330393,0.00777499,0.00623333,0.00184$ $925,0.00138872,-0.00024025,0.00031609,-0.00041264,0.00458711,0.0011934$ $3,-0.00182430,-0.00126904,-0.00014482,0.00168129,-0.13084688,-0.068133$ $74,0.18671657,0.00139340,0.00335452,0.00463615,-0.01017560,-0.01580344$ $, 0.01323838,-0.14327502,-0.09342312,0.10630597,0.00577791,0.00462312,-$ $0.00400871,0.00234914,0.00197501,0.00144487,-0.00021452,0.00050808,-0$. $00032763,-0.00091164,-0.00082577,0.00073159,-0.00030090,-0.00078662,-0$ $.00278486,0.14562238,0.00239245,-0.00054219,-0.00061011,0.00684937,0.0$ $1083137,-0.00646891,-0.09425203,-0.15069548,0.09554352,-0.01877663,-0$. $01237596,0.01778315,0.00242168,-0.00474106,-0.00049340,0.00059505,-0.0$ $0029417,-0.00049450,0.00004032,0.00009714,-0.00008965,-0.00150612,-0.0$ $0030146,0.00073344,0.10056381,0.16232527,0.00401233,-0.00163471,0.0042$ $6255,0.00902960,0.01240600,-0.00572340,0.10742384,0.09321303,-0.134259$ $69,-0.00035342,0.00011648,0.00180890,0.00122292,0.00005610,0.00381046$, $-0.00008357,-0.00048705,-0.00032664,-0.00025048,-0.00033884,0.00022638$ $,-0.00258296,0.00033159,-0.00154633,-0.11665012,-0.10451310,0.13411921$ $, 0.00102624,0.00125610,0.00666094,-0.00062977,-0.00012312,0.00052561,0$ $.00020097,-0.00146208,0.00407641,0.00642051,-0.01700087,0.00000984,-0$. $05968694,0.05828073,0.00791202,-0.00205633,0.02381166,-0.00111171,-0.0$ $0037008,0.00139667,-0.00022360,0.00003610,-0.00004164,-0.00044690,0.00$ $026207,-0.00083692,0.00018152,0.05664373,0.00144685,0.00216070,0.00013$ $604,-0.00028677,0.00080688,0.00012430,-0.00199526,0.00018240,0.0027703$ $2,0.00598246,-0.01356529,-0.00184549,0.05853338,-0.32219509,0.01239521$ $, 0.00078938,-0.00851163,0.00160568,0.00010457,-0.00010104,-0.00002827$, $0.00001793,-0.00023323,-0.00008374,0.00029656,-0.00076306,-0.00004016$, $-0.06409214,0.34106695,0.00593116,0.00101303,0.00381374,0.00083691,-0$. $00016756,-0.00056111,0.00408678,0.00233835,0.00069214,-0.00530805,0.01$ $712505,0.00304683,0.00870902,0.01239832,-0.04626351,0.00337435,-0.0177$ $8426,0.00427859,-0.00014804,0.00061212,-0.00024455,-0.00042170,0.00003$ $639,-0.00015343,-0.00003987,0.00095161,0.00023920,-0.01289321,-0.01371$ $732,0.03891850,0.00461359,0.00626494,-0.00560576,0.00002115,0.00184426$ $, 0.00104060,-0.00031671,0.00047630,-0.00033047,0.00036524,0.00344607,0$ $.00494137,-0.00715013,-0.01592497,0.01322560,-0.12719816,-0.08961084,0$ $.10289248,0.00025365,0.00004650,0.00034749,0.00043296,0.00036821,-0.00$ $057648,-0.00020252,-0.00011812,-0.00025316,-0.00155333,-0.00047359,-0$. $00293089,0.12971191,-0.01562398,-0.01454025,0.01722700,0.00443032,-0.0$ $0326230,-0.00226580,-0.00002314,-0.00046127,-0.00010412,0.00296228,0.0$ $0030123,-0.00096742,0.00778266,0.00958821,-0.00757569,-0.09054558,-0.1$ $5825571,0.10492064,-0.00036328,0.00087037,-0.00044331,-0.00156206,-0.0$ 
$0123781,0.00152847,-0.00012181,0.00005503,-0.00002018,-0.00083733,0.00$ $087343,-0.00063422,0.09437660,0.16520015,-0.00034003,-0.00202822,0.001$ $97575,0.00090726,-0.00063914,0.00168640,-0.00068084,-0.00022110,-0.000$ $04013,0.00535118,-0.00202278,0.00317936,0.00884152,0.01255792,-0.00536$ $135,0.10218738,0.10275502,-0.13596696,-0.00031741,0.00100335,0.0004743$ $6,-0.00022238,0.00012354,-0.00011045,-0.00026795,0.00001975,-0.0003397$ $0,-0.00299237,-0.00081398,-0.00261318,-0.11338691,-0.11093531,0.137104$ $02,-0.00006600,-0.00042057,-0.00079033,0.00228197,0.00063954,0.0006050$ $7,-0.00063109,-0.00081297,0.00316110,-0.20101336,0.08355702,0.13346596$ $,-0.00784092,0.00887522,0.00733392,0.00093125,-0.00260886,0.00286330,0$ $.00015950,0.00001453,-0.00001076,-0.00001437,0.00017201,0.00025831,-0$. $00056516,-0.00008459,-0.00163171,-0.00056918,-0.00049501,-0.00156669,-$ $0.00044088,0.00039874,0.00054456,0.20770892,-0.00015158,-0.00024026,0$. $00000768,-0.00027449,-0.00495451,0.00143198,0.02191242,-0.00627508,-0$. $01658300,0.08305433,-0.09717463,-0.05590131,-0.01386792,0.01042742,0.0$ $1026503,-0.00207068,-0.00213265,0.00290936,0.00006154,0.00005755,-0.00$ $002957,0.00146714,-0.00128046,-0.00098166,-0.00046409,0.00110302,-0.00$ $021028,0.00004515,0.00073549,-0.00072800,-0.00076053,0.00038137,0.0006$ $2835,-0.08913736,0.09886268,-0.00074407,0.00001666,-0.00049059,0.00080$ $222,0.00096721,0.00233176,-0.00109822,0.00206647,0.00340075,0.13346544$ -0.05619712,-0.12558785,0.01178620,-0.00887168,-0.00535300,0.00264487 $, 0.00347219,0.00059947,0.00014161,0.00002645,0.00001165,-0.00004798,0$. $00025675,0.00036866,-0.00150545,-0.00045529,-0.00124066,-0.00166616,-0$ $.00034026,-0.00104094,0.00079490,-0.00037148,-0.00026160,-0.14449025,0$ $.05938972,0.12697538,-0.00099059,0.00028380,0.00027109,-0.00016253,-0$. $00016595,-0.00000792,0.00001135,0.00024226,-0.00007492,0.00002171,-0.0$ $0018219,0.00004112,0.00007622,-0.00008880,-0.00019225,-0.00008952,0.00$ $017627,0.00009175,0.00001054,0.00004252,0.00006227,0.00045619,0.000391$ $69,-0.00027301,0.00006473,0.00001528,-0.00004993,0.00005589,-0.0000707$ $7,-0.00002855,-0.00003729,0.00005264,-0.00003994,0.00004030,-0.0000157$ $3,-0.00004165,0.48611422,0.00051836,-0.00073447,-0.00025338,0.00004061$ ,-0.00004695,0.00014933,0.00005541,-0.00010287,0.00006315,0.00003849,0 $.00015742,0.00007450,-0.00004950,-0.00001768,0.00012015,0.00025748,0.0$ $0006507,0.00017594,0.00015873,0.00009182,0.00007452,0.00000822,-0.0000$ $2523,0.00014619,-0.00005763,-0.00000225,-0.00002140,-0.00006264,0.0000$ $0341,-0.00006069,0.00003769,-0.00006932,-0.00000259,0.00000510,0.00001$ $466,-0.00000522,-0.08621388,0.23574865,0.00080492,-0.00037915,-0.00101$ $421,0.00027127,-0.00046610,0.00050702,0.00006383,0.00002808,0.00007663$ $, 0.00004794,0.00017254,0.00017098,-0.00001718,0.00003664,0.00016264,0$. $00058841,0.00009485,0.00035980,0.00021827,-0.00001358,0.00034546,-0.00$ $008933,0.00040418,0.00021104,-0.00011850,-0.00001000,-0.00012402,-0.00$ $011172,-0.00004427,-0.00008741,-0.00004967,0.00002151,-0.00012786,0.00$ $003310,-0.00002000,-0.00002038,0.00678276,0.19589898,0.55880314,0.0008$ $0278,0.00229929,0.00191426,-0.00047016,0.00089930,-0.00044112,-0.00001$ $744,-0.00010277,-0.00015889,0.00003605,-0.00011369,-0.00013479,0.00001$ $498,0.00021143,-0.00017615,-0.00123201,-0.00029439,-0.00036157,-0.0000$ $9654,-0.00010914,-0.00040108,0.00033998,-0.00065654,-0.00021672,0.0000$ $8932,0.00001475,0.00011094,0.00008443,0.00003892,0.00014562,-0.0000189$ $7,0.00005005,0.00010765,-0.00001633,-0.00000480,0.00000047,-0.11761214$ $, 0.00378208,-0.02083898,0.67156714,0.00238184,0.00500660,-0.00394887,0$ $.00146419,-0.00111992,0.00026360,-0.00000414,0.00056066,-0.00007371,-0$ 
$.00004342,-0.00018000,0.00009219,0.00008338,0.00031014,-0.00033699,-0$. $00121743,0.00000309,0.00034427,0.00005240,-0.00002591,0.00005049,-0.00$ $020028,0.00005514,0.00003133,0.00001196,-0.00005347,-0.00012793,0.0000$ $0801,0.00003409,0.00013042,-0.00001001,-0.00004582,0.00000177,-0.00001$ $060,-0.00002123,0.00002016,-0.02213598,-0.10279889,-0.09546909,-0.1293$ $5429,0.25976228,0.00110502,-0.00387410,-0.00076638,-0.00041155,0.00020$ $579,-0.00026716,-0.00020643,-0.00004741,0.00003054,0.00002326,-0.00021$ $727,-0.00016023,-0.00008792,-0.00018340,0.00001307,0.00066157,0.000046$ $60,-0.00010154,-0.00000405,0.00006171,0.00005396,0.00002183,0.00004143$ ,-0.00009457,0.00006973,0.00002472,0.00013834,0.00008040,-0.00000099,-$0.00000717,0.00002273,-0.00008806,0.00004425,-0.00002108,0.00002205,0$. $00002403,-0.07798740,-0.08168852,-0.28587594,0.00822845,0.21425263,0.6$ $0359969,-0.02459465,0.01377158,0.01442177,-0.00160809,0.00265687,-0.00$ $048518,-0.00001714,-0.00108810,0.00058589,-0.00010469,0.00034092,0.000$ $09611,0.00020805,0.00107832,-0.00021171,-0.00206067,-0.00147427,0.0009$ $5842,0.00077058,0.00111677,0.00328020,-0.00007138,-0.00057552,0.000433$ $77,-0.00024972,0.00008212,0.00010595,-0.00026169,0.00012823,0.00017251$ $, 0.00006541,0.00043178,-0.00032387,-0.00004490,0.00001501,-0.00004391$, $-0.00466420,-0.03432278,-0.08160289,-0.31485303,0.04817922,-0.02981954$ $, 0.64704484,0.00536924,0.00248211,-0.00174317,-0.00051796,-0.00153350$, $-0.00017653,-0.00004519,0.00030739,-0.00003814,-0.00013052,-0.00003491$ $,-0.00001836,0.00002974,-0.00001787,-0.00003460,-0.00011789,0.00015638$ $,-0.00023115,-0.00135482,0.00002883,-0.00045534,-0.00081902,0.00009966$ $, 0.00008032,0.00015349,-0.00004537,0.00013968,-0.00000455,0.00001147,-$ $0.00002595,-0.00010449,0.00005930,0.00015453,-0.00001769,0.00002660,0$. $00000184,-0.01182668,0.01760675,0.01626681,0.02089575,-0.07839483,-0.0$ $2388917,-0.09358491,0.25486202,-0.00509935,0.00245779,0.00773287,-0.00$ $199139,0.00131318,-0.00015599,0.00018688,-0.00089185,0.00001479,-0.000$ $10742,0.00054023,-0.00000469,-0.00021897,0.00032283,0.00040256,-0.0019$ $5441,0.00035429,-0.00103964,-0.00211872,0.00101167,0.00103624,-0.00000$ $786,-0.00093577,-0.00026254,-0.00001155,0.00004178,0.00019417,0.000053$ $75,0.00015120,0.00019690,0.00014509,-0.00012513,0.00005183,-0.00005446$ ,-0.00000628,-0.00002826,-0.03114666,0.00370315,-0.00205717,-0.0889893 $7,-0.00888841,-0.12839255,0.06768116,0.21029997,0.62668450,-0.14662114$ $, 0.00328229,-0.03306456,-0.03390547,-0.00868330,0.00735788,0.00182426$, $-0.00302825,-0.00281983,-0.00105729,0.00126216,0.00077494,0.00117294,-$ $0.00178765,-0.00267171,-0.01558926,0.02010728,-0.00198172,-0.00960155$, $0.00067545,-0.00333625,0.00005154,0.00379231,-0.00162519,0.00065588,0$. $00009272,0.00052277,0.00056886,-0.00012074,-0.00004496,0.00142363,-0.0$ $0166686,0.00082261,0.00013243,-0.00000023,-0.00006883,-0.00190839,0.00$ $482725,0.01364641,-0.04202495,0.01448120,0.00491737,-0.20600025,0.0827$ $6767,0.10625021,0.58060280,0.00996985,-0.06873587,0.00052023,-0.012428$ $91,0.00345663,-0.00689449,-0.00070396,-0.00192291,0.00127623,0.0010578$ $9,0.00066373,-0.00103830,-0.00168644,-0.00351933,0.00279993,0.03256763$ ,-0.00735059,0.00583144,-0.00982245,0.00101027,-0.00512469,-0.00194771 $, 0.00100814,0.00086605,0.00011692,0.00029946,0.00071052,-0.00039949,-0$ $.00046548,-0.00141210,-0.00007315,0.00078521,-0.00037930,0.00001618,0$. $00008008,-0.00000850,0.00548447,-0.02037488,-0.02654524,0.03321264,0.0$ $0737672,0.01863436,0.05569840,-0.11711785,-0.08307050,-0.09024509,0.27$ $924502,-0.01945272,-0.00230638,-0.08491952,0.01829492,-0.00591798,0.00$ $703876,0.00029138,0.00369000,-0.00160011,0.00019184,-0.00068902,0.0004$ 
$9949,-0.00019574,-0.00109370,-0.00058990,0.00060912,0.00237308,0.00124$ $953,-0.03092103,0.00115146,-0.01397491,0.00013398,0.00031164,0.0005313$ $5,0.00002400,-0.00036770,-0.00106138,0.00017170,-0.00022827,-0.0001796$ $7,-0.00012009,-0.00043805,0.00043685,0.00014673,-0.00002606,0.00017227$ $, 0.01446942,-0.02706511,-0.05810078,0.04679232,0.00796563,0.04992641,0$ $.04650485,-0.06860757,-0.19101779,0.01864521,0.18220917,0.57908164,-0$. $01400665,-0.00889211,-0.02852444,0.00156954,0.00103991,-0.00290016,-0$. $00028527,-0.00024321,0.00006761,-0.00002227,0.00000491,-0.00003060,-0$. $00006933,0.00026915,-0.00015884,0.00041983,-0.00232001,-0.00129584,-0$. $00567199,0.00052666,-0.00265239,-0.00005951,-0.00063669,0.00001415,0.0$ $0009812,0.00001947,0.00015034,0.00006194,0.00005361,0.00013702,-0.0000$ $7126,0.00028101,-0.00007139,-0.00002263,0.00000261,0.00000690,-0.04630$ $414,0.03685892,0.05121579,-0.03372384,-0.00718089,-0.03177292,0.052783$ $64,-0.00825231,0.00651449,-0.13510410,0.00417561,-0.02648620,0.6772104$ $4,-0.00072352,0.00403188,0.00362157,0.00021863,0.00010832,0.00005557,-$ $0.00024839,0.00047257,-0.00046310,-0.00004612,-0.00066043,0.00003640,0$ $.00056372,0.00006555,0.00007068,0.00054147,0.00036890,0.00063860,0.001$ $37149,0.00088218,0.00040518,-0.00018992,0.00003342,-0.00017374,0.00004$ $165,0.00001564,-0.00006959,-0.00003745,0.00000489,-0.00007908,-0.00000$ $880,0.00032969,-0.00008909,0.00002771,0.00001534,-0.00001168,0.0138849$ $9,0.00717757,0.00723769,-0.00662688,-0.00408153,-0.00552471,0.00934939$ ,-0.00463858,-0.02160912,-0.02005689,-0.10180542,-0.08666832,-0.126126 $82,0.26172183,-0.01032044,-0.00034525,-0.00504735,0.00038525,0.0017503$ $3,-0.00067136,0.00035078,0.00016164,0.00001822,0.00004392,-0.00032693$, $-0.00049686,0.00044093,-0.00029611,-0.00074723,-0.00055983,-0.00156152$ $, 0.00021562,-0.00062438,-0.00002512,0.00054327,0.00082645,-0.00057316$, $-0.00006944,-0.00001988,-0.00001840,-0.00005157,0.00002555,-0.00005223$ ,-0.00000169,-0.00006524,0.00019764,-0.00004020,0.00004174,-0.00000441 $, 0.00005958,0.00144803,0.01978764,0.05140308,-0.03135908,-0.00578462,-$ $0.03313263,0.04584119,-0.03211164,-0.03621643,-0.08124688,-0.07308692$, $-0.26886552,0.02014655,0.20501202,0.59105834,0.00369443,0.00011568,-0$. $00087791,0.00013895,0.00007660,0.00053731,0.00012785,-0.00013523,0.000$ $21914,0.00004437,0.00022154,-0.00001503,-0.00003487,0.00019724,0.00045$ $068,0.00039823,-0.00010984,0.00038733,0.00032914,0.00012316,0.00047793$ ,-0.00007382,-0.00003804,0.00042258,-0.00018430,-0.00000561,-0.0000660 $6,-0.00017224,0.00003751,-0.00004226,-0.00005742,-0.00003174,-0.000166$ $78,-0.00001445,0.00000656,0.00000908,-0.18333739,0.05390114,0.05393472$ $, 0.05153119,0.00985633,0.04464291,-0.05653317,0.01902313,0.01162786,-0$ $.00379376,-0.03102153,-0.07470503,-0.31963591,0.04755724,-0.03623091,0$ $.64189673,0.00026748,0.00352177,-0.00394858,0.00078631,-0.00013756,0.0$ $0000582,0.00004292,0.00008007,-0.00008534,-0.00013855,-0.00006243,-0.0$ $0002510,0.00005278,0.00020842,0.00008068,-0.00019674,-0.00016376,0.000$ $05654,-0.00071860,0.00010714,-0.00042053,0.00005001,0.00023718,-0.0001$ $4629,0.00002597,-0.00001090,-0.00005105,0.00001620,-0.00000052,0.00005$ $499,-0.00005785,0.00001347,-0.00006232,0.00001229,-0.00000887,0.000013$ $74,0.08037847,-0.11849183,-0.07414582,-0.00846168,-0.00309964,-0.03104$ $538,0.01925546,-0.00998942,-0.00363441,-0.01215727,0.01739610,0.015538$ $44,0.02023047,-0.07690463,-0.02329393,-0.09176909,0.25106868,-0.000787$ $94,-0.00390737,-0.00337436,0.00039287,0.00031931,-0.00033810,-0.000019$ $31,-0.00013677,0.00023169,-0.00010471,0.00013211,0.00007677,-0.0000617$ $3,0.00006601,0.00013126,0.00074244,-0.00034471,0.00017273,-0.00122175$, 
$0.00002431,-0.00050978,0.00003088,-0.00009071,0.00013224,-0.00002885,0$ $.00000687,-0.00000018,-0.00003710,0.00001195,-0.00005611,-0.00003130,0$ $.00010087,0.00002348,0.00000503,0.00000875,-0.00002427,0.11117971,-0.0$ $8805099,-0.19852377,0.00462435,-0.02140112,-0.03485541,0.01341982,-0.0$ $0369060,-0.00505343,-0.03245435,0.00482848,0.00111655,-0.09544778,-0.0$ $0872267,-0.13241090,0.07694256,0.21479771,0.64214245,0.00029394,-0.000$ $05812,0.00038223,-0.00007927,0.00069090,-0.00045343,-0.00004626,-0.000$ 42335,0.00009327,-0.00002241,0.00012231,-0.00006865,-0.00002805,0.0001 $9645,-0.00003957,-0.00041631,-0.00050099,-0.00012945,-0.00003828,0.000$ $00826,-0.00002009,-0.00038478,-0.00072601,0.00027904,-0.00004582,0.000$ $00475,0.00012661,-0.00002472,0.00009452,0.00009776,0.00007245,-0.00001$ $747,0.00002210,-0.00004485,0.00001779,0.00003882,-0.13955279,0.0142177$ $2,-0.03108195,-0.02876152,0.00393326,-0.00922178,0.00317375,-0.0010817$ $2,-0.00317752,-0.00125396,-0.00003978,-0.00106496,0.00174060,0.0031116$ $7,0.00531354,-0.03894464,0.00467054,-0.01306313,0.20476253,-0.00024845$ ,-0.00054692,0.00027727,-0.00005126,-0.00003417,0.00004281,-0.00001629 $, 0.00003371,0.00004643,0.00000269,-0.00001170,0.00001138,-0.00000171,-$ $0.00004859,-0.00002651,0.00008714,0.00007502,-0.00001942,0.00003604,0$. $00001237,0.00004387,0.00002920,0.00011287,-0.00003448,0.00000096,0.000$ $00261,-0.00000617,0.00000435,-0.00001092,-0.00001639,-0.00000767,0.000$ $01401,-0.00000528,0.00000100,-0.00000296,-0.00000460,0.01414054,-0.028$ $17808,-0.00254962,-0.00700130,0.00494756,-0.00250786,0.00157667,0.0032$ $8095,-0.00266016,-0.00016319,-0.00017818,0.00113573,0.00041942,0.00266$ $642,-0.00395013,0.01582182,0.00246924,0.00420202,-0.02343725,0.0189772$ $2,0.00007283,0.00038565,0.00013286,-0.00000647,0.00015677,-0.00015691$, $-0.00001748,-0.00013467,0.00003703,-0.00001925,0.00003577,-0.00002338$, $-0.00000430,0.00011167,0.00001612,-0.00017875,-0.00016215,-0.00000231$, $0.00007153,0.00000825,0.00005583,-0.00011989,-0.00018986,0.00009278,-0$ $.00001440,-0.00000083,0.00003664,-0.00001344,0.00003584,0.00003339,0.0$ $0002057,-0.00001091,0.00000258,-0.00001840,0.00000637,0.00001025,-0.03$ $112157,-0.00248065,-0.04581820,-0.03340272,0.00331917,-0.00620095,0.00$ $265668,-0.00414514,-0.00273414,-0.00070572,0.00110159,0.00120875,-0.00$ $056944,-0.00243015,-0.00116478,0.01119277,-0.00176050,0.00669144,0.052$ $32463,0.00317207,0.04688994,-0.00024359,0.00042050,0.00083111,-0.00017$ $744,0.00034680,-0.00003969,0.00004569,-0.00018216,-0.00001137,-0.00004$ $247,0.00008792,0.00000123,0.00000621,0.00006680,-0.00002674,-0.0002288$ $3,-0.00006269,-0.00004093,0.00004135,-0.00000753,0.00001189,0.00001002$ ,-0.00024266,0.00001503,-0.00001453,0.00000498,0.00002949,-0.00000223, $0.00001978,0.00002709,0.00000192,0.00002624,-0.00000778,-0.00000729,0$. $00000046,0.00000118,0.00697083,-0.00459389,-0.00804098,-0.17803154,0.0$ $8769827,0.11513135,-0.01334981,0.01291350,0.01899023,-0.00372883,0.001$ $05770,-0.00327780,-0.00049664,-0.00049576,-0.00067240,0.00068772,-0.00$ $004644,-0.00275273,0.00107671,-0.00103512,-0.00023107,0.18619163,-0.00$ $012703,0.00002885,-0.00015293,0.00008811,0.00010283,0.00008785,0.00000$ $745,0.00001707,0.00000347,-0.00001164,-0.00002199,-0.00000060,0.000028$ $80,-0.00000417,0.00002706,-0.00006612,0.00002443,-0.00010719,-0.000002$ $88,0.00000366,-0.00000133,0.00002197,-0.00005425,-0.00002220,-0.000012$ $88,-0.00000073,-0.00001120,0.00001983,-0.00000282,0.00000323,0.0000048$ $5,-0.00001188,0.00000092,0.00000181,-0.00000419,-0.00000263,0.00808778$ ,-0.00094187,-0.00500172,0.08655502,-0.09532559,-0.08538175,0.00088786 $, 0.00236467,-0.00230453,0.00127956,0.00590709,-0.00155274,-0.00052194$, 
$-0.00085824,0.00052594,-0.00028791,0.00406528,-0.00326744,-0.00059831$, $-0.00187024,0.00154719,-0.09468011,0.09012746,-0.00040242,0.00017643,0$ $.00026808,-0.00005411,-0.00000215,-0.00008365,-0.00001054,-0.00001078$, $0.00002674,0.00001239,0.00001274,-0.00000943,-0.00001776,0.00001576,0$. $00002055,-0.00001013,-0.00004219,0.00001559,0.00000865,-0.00000262,-0$. $00000999,-0.00000997,0.00003421,-0.00001131,0.00000707,-0.00000015,0.0$ $0000911,-0.00000701,0.00000288,0.00000173,-0.00000188,0.00001107,-0.00$ $000523,-0.00000298,0.00000081,-0.00000147,0.01984430,-0.01196439,-0.01$ 215954,0.11262013,-0.08460396,-0.15991648,-0.00733031,0.00413421,0.008 $84213,-0.00281557,-0.00169834,0.00172934,-0.00083308,0.00058658,-0.000$ $39967,-0.00332218,-0.00311952,-0.00316453,0.00069938,0.00130977,0.0005$ $8391,-0.12002883,0.09386166,0.16498983,0.00082725,-0.00020829,0.002205$ $19,-0.00008051,0.00011523,-0.00011083,-0.00010360,0.00016441,0.0000264$ $6,0.00004437,-0.00015742,-0.00004809,-0.00003321,-0.00008416,0.0000259$ $1,0.00016893,-0.00015755,-0.00012809,-0.00014188,-0.00004611,-0.000519$ $86,0.00007248,0.00015582,-0.00008516,0.00001768,0.00001815,0.00002426$, $0.00001825,-0.00001464,-0.00000222,0.00000470,-0.00002615,0.00002197,0$ $.00000626,-0.00000284,0 .,-0.00354391,0.00202719,-0.00115069,-0.0051686$ $2,-0.01048188,-0.02713604,-0.07911929,-0.02365350,-0.07418826,0.008419$ $22,0.00683746,0.01723935,-0.00191704,0.00305021,0.00238444,-0.00103623$ $, 0.00016135,0.00038240,-0.00121800,-0.00018692,-0.00117850,0.00081497$, $-0.00092071,0.00061851,0.08158082,-0.00209045,-0.00307513,0.00100567,-$ $0.00056870,0.00084706,0.00017954,-0.00005795,-0.00031034,0.00006153,0$. $00002157,0.00006445,-0.00000532,-0.00001923,-0.00012928,0.00012951,0.0$ $0064499,-0.00002104,-0.00015700,0.00044915,0.00034260,0.00052157,0.000$ $17957,-0.00021983,-0.00006249,-0.00004315,0.00002543,0.00004218,-0.000$ $02468,-0.00001981,-0.00006243,0.00003662,-0.00006811,-0.00002724,-0.00$ $000550,-0.00000246,0.00000739,0.00195782,0.00570863,-0.00168322,0.0021$ $3632,0.00463173,0.00347873,-0.02366608,-0.07353348,-0.09299415,-0.0045$ $1268,-0.00051312,-0.01205465,0.00341598,0.00378374,-0.00342339,0.00015$ $301,-0.00086853,0.00053094,0.00028049,0.00013995,0.00014080,-0.0010526$ $4,-0.00309457,0.00169841,0.02329308,0.06698981,-0.00131870,0.00212732$, $-0.00035426,0.00051522,-0.00046988,0.00000801,-0.00011540,0.00019519,0$ $.00014136,-0.00000290,-0.00008824,0.00000839,0.00001200,0.00018658,-0$. $00004307,-0.00021348,-0.00011884,0.00042785,0.00094594,0.00003492,0.00$ $072737,-0.00023477,0.00024735,0.00018173,-0.00003484,-0.00001237,-0.00$ $004740,-0.00004631,0.00002014,0.00000855,-0.00002403,0.00000595,-0.000$ $05466,-0.00000512,0.00000308,0.00000467,-0.00154148,-0.00156703,0.0028$ $4368,-0.00016413,-0.00336947,-0.00397408,-0.07330590,-0.09268354,-0.27$ $518748,-0.00844054,-0.00611825,-0.01333669,0.00316777,-0.00363130,-0.0$ $0000853,0.00058420,0.00048365,0.00035615,-0.00018261,-0.00013155,-0.00$ $021815,0.00031209,0.00181037,-0.00010864,0.08059498,0.10298703,0.28958$ $110,-0.00040039,-0.00063823,-0.00008335,0.00003002,-0.00039561,-0.0004$ $2239,-0.00004767,0.00005080,0.00022311,-0.00007339,0.00007193,0.000027$ $91,-0.00027545,0.00012347,-0.00013837,0.00001667,-0.00025415,-0.000337$ $79,0.00014678,0.00002130,0.00018661,-0.00005925,0.00034435,0.00002009$, $-0.00002017,0.00000545,-0.00000379,0.00001371,-0.00002920,0.00001802,0$ $.00001213,-0.00002775,0.00011094,0.00000983,-0.00001316,-0.00000852,-0$ $.00290343,0.00121030,-0.00293145,-0.00036045,-0.00049180,-0.00063000,0$ $.00055500,0.00020179,-0.00280010,0.00992923,-0.00421222,-0.00587021,-0$ $.17280657,0.08492358,0.10995118,-0.01477303,0.01329707,0.01883556,-0.0$ 
$0139945,0.00053582,0.00033703,-0.00003235,-0.00011219,0.00013439,0.000$ $39063,-0.00032581,-0.00048169,0.18135489,-0.00011697,-0.00247673,0.001$ $51052,-0.00033838,0.00028531,-0.00013216,0.00016075,-0.00020628,0.0004$ $0662,-0.00004988,0.00040514,-0.00010048,-0.00011138,-0.00015545,-0.000$ $18095,0.00020033,0.00025961,0.00018986,0.00001602,-0.00010766,0.000057$ $64,0.00006326,-0.00036762,0.00006374,-0.00008485,-0.00001821,0.0000004$ $5,0.00007562,-0.00004958,0.00005005,0.00003225,-0.00005168,0.00013790$, $0.00000417,-0.00001456,0.00000600,0.00132966,0.00608825,-0.00175481,-0$ $.00052494,-0.00081458,0.00051387,-0.00011324,0.00470179,-0.00373906,0$. $00742499,-0.00089637,-0.00570125,0.08504425,-0.09771355,-0.08560929,0$. $00082558,0.00250460,-0.00246245,0.00005269,0.00004009,-0.00008459,-0.0$ $0009112,-0.00057849,0.00025141,0.00035928,-0.00009229,-0.00024424,-0.0$ $9316161,0.09248703,0.00121974,0.00103253,-0.00005485,0.00016859,-0.000$ $60965,0.00001068,-0.00016140,0.00010777,0.00004160,-0.00018870,-0.0001$ $3573,0.00014200,-0.00013160,0.00035287,0.00018202,0.00014201,0.0000675$ $3,-0.00000137,0.00013068,0.00005564,0.00010083,-0.00014061,0.00049689$, $-0.00005930,0.00006438,0.00004130,0.00004546,-0.00000703,0.00000871,0$. $00006360,-0.00004682,-0.00000768,-0.00010364,0.00003576,-0.00000474,-0$ $.00001820,-0.00255618,-0.00182003,0.00188380,-0.00079774,0.00048865,-0$ $.00038920,-0.00341625,-0.00360894,-0.00335523,0.01982320,-0.01212153,-$ $0.01176620,0.10955493,-0.08508632,-0.16107348,-0.00807718,0.00413964,0$ $.00857456,-0.00066834,0.00016337,0.00010435,0.00015970,0.00025891,-0.0$ $0009764,0.00098156,-0.00060545,-0.00074117,-0.11673921,0.09502268,0.16$ $644864,0.00004459,-0.00050900,-0.00113525,0.00012059,0.00015862,-0.000$ $08509,0.00000490,-0.00012968,0.00002167,0.00001739,0.00008840,0.000024$ $67,-0.00004685,0.00004854,0.00005276,0.00016881,-0.00016964,0.00001781$ ,-0.00042247,-0.00001365,-0.00018066,-0.00003396,-0.00010714,0.0000675 $1,-0.00002539,-0.00000376,0.00000321,-0.00002130,0.00001569,-0.0000021$ $2,-0.00000540,0.00001671,-0.00001329,-0.00001438,0.00000377,-0.0000032$ $2,0.00749424,0.00742126,0.01857829,-0.00218433,0.00294074,0.00232584,-$ $0.00108784,0.00012192,0.00031665,-0.00457191,0.00241574,-0.00124419,-0$ $.00386213,-0.01008800,-0.02614604,-0.08013364,-0.02575736,-0.08048289$, $0.00096884,-0.00049417,0.00087510,0.00050415,0.00037143,0.00090106,0.0$ 0002266,-0.00017952,-0.00001512,0.00071641,-0.00096590,0.00069282,0.08 $234274,0.00008660,0.00022078,0.00012344,-0.00016106,0.00004155,0.00005$ $762,0.00002022,-0.00005846,-0.00004454,-0.00000024,0.00002334,-0.00003$ $126,-0.00000684,-0.00000750,0.00007655,0.00009186,-0.00008447,-0.00005$ $716,0.00001087,-0.00009261,0.00009166,0.00000518,-0.00005443,0.0000309$ $4,-0.00000859,-0.00000067,0.00000007,0.00000696,-0.00000445,0.00000609$ $, 0.00000102,-0.00001480,0.00001647,0.00000154,0.00000290,0.00000164,-0$ $.00536351,-0.00090148,-0.01198795,0.00312440,0.00322179,-0.00286130,0$. $00026915,-0.00085545,0.00055331,0.00218455,0.00591065,-0.00173370,0.00$ $184722,0.00490262,0.00325716,-0.02458634,-0.07185368,-0.09291225,-0.00$ $089572,-0.00184378,0.00126847,-0.00019641,0.00012616,-0.00063467,-0.00$ $016038,-0.00055044,0.00024799,-0.00105941,-0.00322080,0.00184553,0.024$ $79143,0.06507415,0.00030096,-0.00025253,-0.00020808,0.00005888,0.00006$ $600,-0.00004358,0.00003131,-0.00003910,0.00004218,-0.00003244,0.000016$ $60,-0.00002620,0.00004030,0.00002243,-0.00001838,-0.00008666,0.0000159$ $0,0.00002419,-0.00011445,0.00005596,-0.00007641,-0.00002073,-0.0000657$ $3,0.00001755,-0.00000315,0.00000317,0.00000416,-0.00001110,0.00000691$, $0.00000273,0.00000605,0.00000547,-0.00001667,-0.00000164,0.00000114,0$. 
$00001157,-0.00932363,-0.00518387,-0.01290178,0.00288513,-0.00294077,0$. $00030484,0.00044872,0.00051634,0.00043480,-0.00178583,-0.00158569,0.00$ $270397,-0.00020049,-0.00313164,-0.00304045,-0.07785439,-0.09349333,-0$. $28132867,-0.00001077,0.00148241,0.00048866,-0.00035741,-0.00031775,-0$. $00052968,0.00001459,0.00024091,-0.00017591,0.00053914,0.00184220,-0.00$ $000826,0.08546333,0.10270924,0.29435394,0.00149840,0.00312955,0.001063$ $36,0.00555625,-0.00481858,-0.00652107,-0.00070686,0.00691053,-0.001947$ $46,-0.00058269,-0.00016911,0.00086745,-0.00015893,0.00000674,0.0001128$ $6,0.00055032,0.00075565,-0.00003303,-0.00027591,-0.00048888,0.00042376$ ,-0.11837896,-0.051261 14,0.05610364,-0.00062943,0.00127615,0.00000377, $-0.00008796,0.00002881,-0.00007607,0.00002593,0.00019499,-0.00010484,0$ $.00002464,0.00029694,0.00004833,-0.00029887,-0.00003567,-0.00015569,0$. $00009394,0.00001631,0.00007736,0.00010908,0.00005084,0.00014726,-0.000$ $17756,-0.00020404,-0.00023198,0.00017055,-0.00002404,0.00000123,-0.000$ $04458,0.00000111,-0.00002671,0.00030314,-0.00003270,0.00008861,0.00003$ $815,-0.00000091,-0.00000531,-0.00006025,-0.00000651,0.00002515,-0.0000$ $5141,-0.00000881,-0.00006732,0.00002715,0.00001318,0.00001980,0.537690$ $26,0.00360893,-0.00211715,-0.00210966,-0.01938266,-0.04949667,0.022214$ $47,-0.00127756,0.00406766,0.00034245,-0.00161761,-0.00071995,0.0013815$ $7,-0.00005963,-0.00018172,0.00018230,-0.00086639,0.00045935,0.00083776$ ,-0.00002045,-0.00019312,0.00047478,-0.02888123,-0.12342355,0.03232707 ,-0.00051763,-0.00254691,0.00070595,-0.00003526,0.00003826,-0.00001105 $, 0.00014788,-0.00025687,-0.00017736,-0.00013658,0.00045863,0.00003261$, $-0.00045467,0.00002078,-0.00006906,0.00006348,0.00005998,0.00004298,-0$ $.00002099,0.00018243,0.00014008,-0.00024664,-0.00009937,0.00000274,0.0$ $0002173,0.00002727,-0.00023170,0.00011031,-0.00009543,-0.00004595,0.00$ $047681,-0.00005897,0.00013664,0.00006666,0.00000942,-0.00002559,-0.000$ $10308,-0.00000532,0.00008754,-0.00006674,0.00000031,-0.00009437,0.0000$ $3777,0.00002176,0.00003316,-0.06590080,0.55018919,0.00102312,-0.001805$ $76,0.00225709,-0.00459137,0.00966859,-0.00054021,-0.00108093,-0.006580$ $14,-0.00047010,0.00123079,0.00025241,-0.00034729,0.00004199,0.00013204$ ,-0.00021558,-0.00004509,-0.00067316,0.00073895,-0.00023519,0.00022628 $, 0.00035064,0.05363851,0.05232903,-0.11222174,0.00020099,-0.00084906,-$ $0.00054347,-0.00009280,-0.00007152,-0.00012403,0.00003740,-0.00018760$, $0.00007785,0.00014112,-0.00027234,0.00011693,0.00026041,-0.00001813,0$. $00005693,0.00000392,-0.00003962,-0.00001620,-0.00012921,-0.00016201,-0$ $.00015632,0.00080040,0.00034668,-0.00019987,-0.00009972,0.00011452,0.0$ $0020220,-0.00012403,0.00007315,-0.00004651,-0.00030330,0.00003981,-0.0$ $0010315,-0.00004659,-0.00000585,0.00001078,0.00006334,0.00002811,-0.00$ $006386,0.00009839,-0.00012778,0.00010191,-0.00006605,-0.00003965,-0.00$ $002596,0.03184569,0.05813229,0.53071682,-0.00020581,-0.00021746,-0.000$ $11934,-0.00015988,0.00277846,0.00014234,0.00082878,-0.00048580,-0.0009$ $3499,-0.00018248,0.00005001,0.00019041,0.00005758,-0.00011158,-0.00001$ $059,-0.00012468,-0.00002880,-0.00003058,0.00001723,0.00003261,-0.00011$ $403,-0.00128208,-0.00281335,0.02577504,-0.00049391,0.00044438,-0.00003$ $505,-0.00000142,-0.00003121,0.00003125,0.00000633,-0.00002672,0.000026$ $19,0.00000422,-0.00001195,-0.00004196,0.00000684,-0.00001243,0.0000043$ $3,0.00000158,0.00000224,-0.00000826,-0.00004678,0.00000558,0.00000738$, $0.00016122,0.00002593,0.00009342,0.00000653,0.00003896,-0.00002278,-0$. $00004582,0.00002275,-0.00003381,-0.00004648,0.00000931,-0.00001638,-0$. $00001005,-0.00000164,0.00000020,0.00001643,-0.00000755,-0.00002108,0.0$ 
$0002678,-0.00002410,0.00001699,-0.00001349,-0.00001369,-0.00000157,-0$. $05172264,-0.00600629,-0.03213241,0.05271906,-0.00052751,-0.00039423,-0$ $.00021378,-0.00116126,0.00537216,0.00245701,0.00100424,0.00058149,-0.0$ $0075115,-0.00042857,-0.00081107,-0.00021598,0.00036984,0.00004568,-0.0$ $0029954,-0.00031676,0.00015803,0.00007366,0.00009659,-0.00005154,-0.00$ $022029,-0.00221373,-0.00273207,0.02976544,0.00104506,-0.00100054,0.000$ $98259,0.00006328,0.00001722,0.00005167,0.00001547,-0.00002028,0.000015$ $48,0.00001144,0.00000264,0.00000874,-0.00001460,-0.00000609,-0.0000089$ $6,0.00000582,-0.00000987,-0.00001378,0.00001010,0.00000704,0.00002571$, $0.00009562,-0.00009028,0.00005547,-0.00001493,0.00002570,0.00000050,0$. $00000056,0.00000476,-0.00000912,0.00000357,-0.00000265,0.00000082,0.00$ $000146,0.00000426,-0.00000194,0.00000195,-0.00001383,-0.00000138,-0.00$ $000500,-0.00000684,-0.00001017,-0.00000303,0.00000360,0.00000085,-0.00$ $568283,-0.06310262,-0.06260676,0.01003254,0.06439305,0.00015550,0.0004$ $8246,-0.00000607,0.00123157,-0.00529508,0.00002483,0.00022915,0.000563$ $28,-0.00019554,-0.00044434,0.00001862,0.00031850,-0.00010343,-0.000061$ $28,0.00004937,0.00016163,0.00007117,0.00003313,-0.00009813,-0.00016044$ $, 0.00024537,0.00799947,0.01064987,-0.02129487,-0.00061510,-0.00011651$, $0.00059321,-0.00003299,-0.00001209,0.00002856,-0.00002297,0.00003904,-$ $0.00001737,-0.00000405,0.00007400,-0.00000987,-0.00005926,-0.00000460$, $-0.00001478,-0.00000624,0.00001052,0.00002100,0.00002926,0.00001512,-0$ $.00000145,-0.00023687,-0.00008032,-0.00001212,0.00004874,-0.00002956,-$ $0.00003081,0.00002997,-0.00001923,0.00002989,0.00007239,-0.00001013,0$. $00002781,0.00001197,0.00000411,-0.00000424,-0.00003430,-0.00000527,0.0$ $0004283,-0.00003154,0.00004297,-0.00001842,0.00002022,0.00001156,0.000$ $00755,-0.02858728,-0.05607988,-0.28668562,0.02329432,0.05521563,0.3175$ $1965,0.00003568,-0.00041851,0.00010757,-0.00051900,0.00506341,0.000309$ $21,-0.00012338,-0.00059866,0.00023413,0.00039981,0.00006598,-0.0003735$ $4,-0.00001951,0.00005139,-0.00006438,-0.00005514,-0.00008291,0.0001014$ $2,0.00000082,0.00011586,-0.00005561,-0.02066162,-0.01285774,0.00605812$ $, 0.00075758,0.00032459,-0.00034870,0.00002991,0.00000347,-0.00002353,0$ $.00001278,-0.00004214,0.00000872,0.00000433,-0.00007936,0.00000660,0.0$ $0012436,-0.00001649,0.00000308,0.00002375,-0.00000107,0.00000604,-0.00$ $002711,-0.00005350,-0.00006929,0.00023887,0.00011173,-0.00002535,0.000$ $00904,0.00001426,0.00008844,-0.00004022,0.00002388,0.00000858,-0.00009$ $796,0.00001159,-0.00002466,-0.00001750,-0.00000399,0.00000088,0.000019$ $03,0.00001337,-0.00002504,0.00000098,-0.00000209,0.00000625,-0.0000064$ $9,0.00000250,-0.00000545,-0.27934437,0.01325310,-0.07277850,-0.0015404$ $5,0.00188571,-0.00117528,0.30994213,0.00017985,-0.00027641,0.00054985$, $-0.00310832,0.00544021,0.00011358,0.00071839,0.00064862,-0.00090874,0$. $00033901,-0.00074386,0.00055650,0.00036200,0.00009227,-0.00036525,-0.0$ $0023168,0.00013090,0.00030475,-0.00001466,-0.00006224,-0.00004782,-0.0$ 2837393,-0.00781286,-0.00097146,-0.00056856,-0.00149772,-0.00107876,-0 $.00003020,-0.00000428,-0.00006667,0.00001296,-0.00004535,-0.00001826,-$ $0.00001674,-0.00001414,-0.00001908,0.00002165,-0.00000276,-0.00001948$, $0.00000522,-0.00000843,0.00000185,0.00003108,-0.00002930,0.00000679,0$. $00017176,-0.00005968,-0.00005298,0.00001382,-0.00000864,0.00004817,-0$. $00002817,0.00001253,-0.00000485,-0.00002859,0.00000322,-0.00000841,-0$. $00000263,-0.00000279,0.00000457,0.00000830,0.00000137,-0.00001229,-0.0$ $0000964,-0.00000219,-0.00001155,-0.00000212,-0.00000074,-0.00000188,0$. $01888868,-0.05000011,0.00434729,-0.00664149,0.00114604,-0.00365507,-0$. 
$00752775,0.05177769,-0.00014828,0.00004853,-0.00025068,0.00068713,-0.0$ $0263661,0.00042607,-0.00125825,0.00020608,0.00071026,0.00025726,0.0001$ $4829,-0.00026198,-0.00010743,0.00008194,0.00008302,0.00007328,-0.00000$ $222,-0.00014122,0.00008246,0.00000469,-0.00003896,0.02564462,0.0055278$ $6,0.00365459,0.00028621,-0.00021595,-0.00027922,0.00002658,0.00003615$, $-0.00000652,0.00000184,0.00003136,0.00000490,-0.00003645,-0.00001252,-$ $0.00000268,-0.00002449,-0.00000848,0 ., 0.00001255,0.00001928,-0.0000032$ 4,0.00000839,0.00003435,0.00003724,-0.00014991,0.00000356,-0.00002768, $0.00000239,-0.00002722,-0.00004189,0.00003196,-0.00000538,0.00001133,0$ $.00004805,-0.00000577,0.00001616,0.00000686,-0.00000292,-0.00000067,-0$ $.00000638,0.00001040,0.00000370,-0.00001849,0.00002747,-0.00001159,0.0$ $0001185,0.00001097,0.00000364,-0.07680325,0.00299329,-0.07170919,-0.02$ $862675,0.00196652,-0.00687054,0.07264170,-0.00750225,0.07219868,0.0003$ $7502,-0.00081218,0.00002097,-0.00194494,-0.00140321,0.00221705,-0.0004$ $9943,0.00015311,0.00076261,0.00038457,-0.00019982,-0.00015079,0.000040$ $54,0.00013769,-0.00004273,-0.00025364,-0.00005038,0.00020384,0.0000234$ $3,0.00000888,-0.00002507,0.00600061,-0.02634213,-0.00138219,0.00022045$ $, 0.00041831,-0.00063861,0.00001458,0.00001766,-0.00003932,0.00002829,-$ $0.00008803,-0.00001629,0.00000214,-0.00002899,0.00001358,-0.00004223,-$ $0.00000103,-0.00000412,-0.00001327,0.00002243,-0.00000689,-0.00001595$, $0.00003212,0.00001438,0.00015753,0.00003663,-0.00001293,-0.00001095,-0$ $.00002240,-0.00002135,0.00000686,0.00000852,-0.00001009,0.00001358,-0$. $00000052,0.00000270,-0.00000623,-0.00000620,0.00000512,-0.00000113,-0$. $00002151,0.00001582,-0.00000007,0.00000442,0.00000176,-0.00000288,-0.0$ $0000447,0.00000150,-0.09356742,0.10780757,0.02233911,0.00202338,-0.004$ $27505,-0.00183243,-0.00914634,0.02583177,0.00735656,0.09621353,0.00051$ $959,-0.00091965,-0.00029480,-0.00299998,0.00146251,0.00246037,-0.00026$ $513,0.00013430,0.00021163,0.00009854,-0.00001746,-0.00004379,0.0000370$ $1,0.00000956,-0.00003334,-0.00036195,-0.00008656,0.00027504,0.00000714$ $, 0.00003657,0.00003002,0.00327487,-0.02978356,0.00119828,0.00000103,0$. $00013517,-0.00009423,0.00000382,-0.00000798,-0.00000561,0.00003839,-0$. $00013003,0.00000698,0.00000817,-0.00004235,-0.00000002,0.00004293,0.00$ $001165,0.00000680,-0.00004403,0.00000993,-0.00000300,-0.00003304,0.000$ $01316,-0.00006262,0.00022400,-0.00000978,0.00007013,-0.00003584,0.0000$ $3749,-0.00004628,-0.00001393,-0.00000205,0.00001767,-0.00007441,0.0000$ $1192,-0.00001923,-0.00002191,-0.00000938,0.00000579,0.00001118,-0.0000$ $1717,0.00001128,0.00001665,0.00000074,0.00003415,-0.00000904,-0.000005$ $52,-0.00000642,0.09706451,-0.26323728,-0.05225085,0.00299128,-0.003519$ $77,-0.00166888,0.00074338,0.00131863,-0.00073144,-0.10122320,0.2946069$ $1,-0.00015460,0.00082751,0.00031564,0.00241843,0.00111880,-0.00166507$, $0.00085578,-0.00008187,-0.00017970,-0.00023013,0.00018632,0.00027044,-$ $0.00002043,-0.00009995,0.00005682,0.00024081,0.00003033,-0.00018188,-0$ $.00003115,-0.00006433,0.00000983,-0.00530696,0.02615199,0.00112324,-0$. $00057846,-0.00043338,0.00005586,-0.00003084,-0.00002673,0.00001488,-0$. $00003044,0.00008596,-0.00000261,-0.00000178,0.00003185,-0.00000719,0.0$ $0001798,0.00000152,0.00000164,0.00000283,-0.00000553,-0.00000239,0.000$ $02409,-0.00002135,0.00003510,-0.00011228,0.00003595,-0.00004275,0.0000$ $1660,-0.00003153,0.00001047,-0.00000927,0.00001236,-0.00000454,-0.0000$ $1227,0.00000111,-0.00000653,0.00000510,0.00000204,0.00000438,0.0000054$ $6,0.00001791,-0.00002148,0.00001452,0.00001366,-0.00000091,0.00000112$, $-0.00000623,0.00000027,0.02373176,-0.06122307,-0.06116099,0.01256771,-$ 
$0.02626722,-0.00378000,-0.00461141,0.00881148,0.00249648,-0.02877219,0$ $.05093240,0.06266100 \-0.00004960,0.00000350,-0.00000548,0.00005043,0$. $00001617,-0.00000879,-0.00003576,0.00007564,0.00003350,-0.00001904,-0$. $00007966,0.00001573,0.00004287,0.00002223,-0.00003530,-0.00002088,-0.0$ $0001233,0.00002417,0.00000955,0.00000355,-0.00000816,0.00000744,-0.000$ $01895,0.00000371,0.00000121,-0.00001085,-0.00001668,-0.00000429,-0.000$ $00250,0.00000880,-0.00000372,-0.00000108,-0.00000267,0.00000315,0.0000$ 0991,0.00000273,-0.00000153,-0.00000353,0.00000370,0.00000017,0.000002 $03,-0.00000204,-0.00000937,0.00000177,-0.00000666,0.00003507,-0.000008$ $73,0.00002027,-0.00001042,0.00000218,-0.00000939,0.00000413,-0.0000014$ $8,-0.00000345,-0.00000022,0.00000193,-0.00000191,-0.00000037,0.0000011$ $3,-0.00000197,0.00000132,0.00000237,-0.00000102,0.00000086,0.00000080$, $0.00000016,0.00000117,0.00000016,-0.00000064,-0.00003186,-0.00001244,0$ $.00000337,0.00001187,0.00000763,-0.00000572,0.00001494,-0.00000179,-0$. 00000092,0.00000286,0.00000233,-0.00000535।I।@

meta-Addition Product

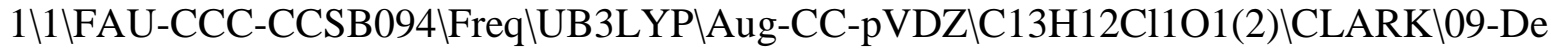
c-2014\0\\#N Geom=AllCheck Guess=TCheck SCRF=Check GenChk UB3LYP/Aug-C C-pVDZ Freq \\Addition product p-Cl-Ph-radical + anisol (meta, trans) \\ 0,2\C,-6.5799513461,1.6189748253,1.479965558\C,-5.3635742821,0.7530517 $02,1.6732551345 \backslash \mathrm{C},-4.082728657,1.1955026301,1.4192400807 \backslash \mathrm{C},-3.84307330$ 29,2.5153181988,0.9594889987\C,-4.9494614251,3.3924169965,0.7634716448 \C,-6.2365413372,3.0061205641,0.9988465437\Н,-7.2169445137,1.144524197 $5,0.7035427426 \backslash \mathrm{H},-5.5066465782,-0.2663307035,2.030251976 \backslash \mathrm{O},-3.07890449$ $59,0.2845990487,1.6423871994 \backslash \mathrm{H},-4.7499283954,4.4068143814,0.4138300962$ $\mathrm{VH},-7.0616862732,3.7016200522,0.8444173695 \backslash \mathrm{H},-2.8379971735,2.872890185$ $8,0.7563226578 \backslash \mathrm{C},-9.042996288,1.7563169267,5.0462439548 \backslash \mathrm{C},-9.572080148$ 9,1.2208504408,3.8739162375\C,-8.7695964918,1.1784374197,2.7278326985 C,-7.4563209591,1.6612516692,2.7420036028\C,-6.9521692001,2.1935040197 ,3.9381344287\C,-7.7353353818,2.2459582403,5.0920242896\Cl,-10.0409897 $738,1.8166602512,6.5002863556 \mathrm{LH},-10.5925878698,0.8426083171,3.85539598$ $65 \backslash \mathrm{H},-9.1821799676,0.7586712346,1.8089986919 \backslash \mathrm{H},-5.9304936391,2.5724051$ 643,3.9695529884\H,-7.339292939,2.6602854534,6.0176659763\C,-1.7343065 $455,0.6808591779,1.4003621813 \backslash \mathrm{H},-1.1206222602,-0.1928275203,1.64143183$ $47 \mathrm{\backslash H},-1.4384327632,1.5218030223,2.0467044106 \backslash \mathrm{H},-1.577478273,0.95463801$ $1,0.3454190467 \backslash \backslash$ Version=EM64L-G09RevC.01 $\backslash$ State $=2-A \backslash H F=-1038.0502155 \backslash S 2$ $=0.786441 \backslash \mathrm{S} 2-1=0 . \backslash \mathrm{S} 2 \mathrm{~A}=0.750719 \backslash \mathrm{RMSD}=5.836 \mathrm{e}-09 \backslash \mathrm{RMSF}=1.156 \mathrm{e}-05 \backslash$ ZeroPoint $=0.2117509 \backslash \mathrm{Thermal}=0.2251888 \mathrm{ZPE}=132.8757189 \mathrm{\backslash Dipole}=0.8393517,0.236907$ $3,-0.7887734 \backslash$ DipoleDeriv $=0.0742919,0.0028813,0.0514587,-0.0693519,0.01$ $36494,0.1338874,-0.0304491,0.0640514,0.2539989,-0.2990646,0.2571734,-0$ $.0597417,-0.0403506,0.0580212,-0.0302788,0.1464841,-0.1099301,-0.29747$ $37,1.2020952,-0.7517563,0.0560335,-0.8834112,0.2928429,0.0845864,0.216$ $1555,-0.0148627,0.0277653,-0.0954551,-0.0301397,0.0299268,0.3042227,-0$ $.0250321,-0.1180993,-0.0965908,-0.0422614,-0.1302005,0.1621568,0.11054$ $7,-0.085232,-0.1638517,0.1575988,-0.0116816,0.0306621,-0.0906332,-0.11$ 20705,-0.1032142,-0.0115764,0.0589248,0.1136958,-0.0660219,-0.1568123, $0.0721367,-0.0241801,-0.2498061,-0.0880702,-0.0725895,-0.0741284,-0.06$ $12038,0.0431702,-0.0533303,-0.0603492,-0.0397248,-0.0045652,0.0567087$, $-0.0049385,-0.0037405,-0.0088151,-0.0266591,0.0534456,-0.0079507,0.062$ 
$5131,0.1218488,-1.7760092,0.4313053,0.1704239,0.6895306,-0.5928472,-0$. $0290254,-0.1168269,0.078935,-0.2378299,0.0337193,-0.0213158,0.034003,-$ $0.0354092,-0.0860439,0.09025,-0.0032452,0.0699949,0.1116425,-0.0283006$ $, 0.0629107,-0.0207303,0.0648747,-0.0029494,0.0343476,-0.0146998,0.0241$ $574,0.127785,-0.0545965,-0.0246447,0.0325056,-0.0471722,0.0753134,0.02$ $74336,0.0283582,0.0205799,0.1246394,0.4049938,0.004562,-0.5441402,-0.0$ 26054,-0.0316874,0.0709475,-0.6507661,0.043006,0.8789543,-0.0472861,0. $0499851,0.1020049,0.0629371,-0.0769207,-0.0158858,0.1554773,-0.0056804$ $,-0.2842825,0.0207184,0.0455357,0.0097516,0.0767467,-0.067929,0.038278$ $9,0.105642,0.0710356,0.0082407,0.1170502,0.0085028,-0.1700304,-0.00703$ $9,-0.0442057,-0.0184903,-0.2743059,-0.0373736,0.1520842,-0.0462893,0.0$ $32002,0.0609013,0.0299589,-0.0653133,0.0193038,0.0693789,0.0173102,-0$. $0918202,-0.1098424,0.0217616,0.1119964,0.0341364,-0.0629052,0.0385627$, $0.1449888,0.0531565,-0.0913672,-0.3893899,0.0020282,0.3832153,0.010793$ $8,-0.0771945,-0.0279404,0.4349569,-0.0177345,-0.6487802,-0.0321406,-0$. $0527048,0.0162976,-0.0519316,0.1069871,-0.0089296,0.0265612,-0.0059986$ $, 0.0769794,0.0732911,-0.023859,-0.0230857,-0.0302515,0.0880813,-0.0615$ $884,-0.052111,-0.0697943,-0.060108,-0.0411819,-0.0502783,0.004904,-0.0$ $57717,0.0789955,0.0032345,-0.0115749,-0.0024502,0.0930932,0.0699864,-0$ $.0265721,-0.0206491,-0.0215102,0.1029378,-0.0504868,-0.0111773,-0.0469$ $608,-0.0174925,1.0251859,-0.0726572,-0.1095019,0.2294061,0.2514399,-0$. $01944,-0.1442999,0.0293277,0.3460512,-0.050319,0.0641083,0.0001794,0.1$ $312091,-0.0425548,0.0261312,-0.0324824,0.037782,0.0760056,-0.0438055,0$ $.0232524,0.0095287,-0.1489238,-0.0505388,-0.0795573,-0.0246364,-0.0923$ $693,-0.0309553,-0.0352326,0.0264766,-0.0210753,-0.0945192,0.0497655,0$. $0611372,0.1006641,0.0281042,-0.1423366 \mathrm{P}$ Polar $=228.5777045,5.46465,141.1$ $745843,-45.5855392,-3.6279293,177.7080621 \mathrm{PG}=\mathrm{C} 01[\mathrm{X}(\mathrm{C} 13 \mathrm{H} 12 \mathrm{Cl1O})] \mathrm{NIma}$ $\mathrm{g}=0 \backslash \backslash 0.48392398,0.01577666,0.48044140,0.04225458,0.00514896,0.43689265$ ,-0.15636869,0.06397745,-0.01548319,0.64229585,0.05173158,-0.12822201, $0.02139868,0.04298817,0.59080161,-0.01366456,0.02016058,-0.06285137,-0$ $.05862825,-0.16667182,0.17295064,-0.02668703,-0.00122468,0.00149398,-0$ $.30080069,-0.08495203,0.04857663,0.63694446,0.01205109,0.00944895,-0.0$ $0598345,-0.03765652,-0.11678295,0.02392404,-0.02289628,0.54131592,-0.0$ $0243165,-0.00278156,0.00355755,0.03223868,0.02678520,-0.06550094,-0.03$ 209034,-0.13062682,0.18747342,0.00212234,0.00314766,-0.00164384,0.0054 $8100,-0.02915817,0.01029405,-0.13340379,-0.06377779,0.02772230,0.68359$ $918,-0.00011291,-0.00482999,-0.00091880,-0.07012547,-0.03488847,0.0207$ $9646,0.03728977,-0.21012750,0.04830852,0.03121990,0.52500243,-0.000695$ $30,-0.00103079,-0.00681579,0.02399417,0.01714028,0.00121505,-0.0062822$ $6,0.05688896,-0.07384932,-0.06012781,-0.14507992,0.14917442,-0.0097407$ $7,-0.01507639,0.00440547,-0.03520650,-0.01231457,0.00718321,0.06022310$ $, 0.06389805,-0.02588008,-0.18833518,0.02273800,0.00397428,0.63298561,-$ $0.02680238,-0.00681864,0.00897463,0.00393481,0.00275156,-0.00164651,0$. $01672288,-0.03750832,0.01063147,0.10594073,-0.17198927,0.03100694,0.05$ $071977,0.63902107,0.00982954,0.00616724,0.00540121,0.00149630,-0.00021$ $671,-0.00025060,-0.01051439,0.00733483,-0.00114790,-0.02354029,0.03825$ $416,-0.06110109,-0.05854467,-0.17509310,0.18357208,-0.08728864,-0.0210$ $3016,0.00796284,0.02256285,0.03074539,-0.01174144,-0.02280766,-0.03048$ $461,0.01150356,-0.06781178,0.04962142,-0.00977577,-0.35817411,-0.11169$ $347,0.06140730,0.75481133,-0.00963173,-0.18673347,0.03781448,0.0103716$ $8,-0.02216530,0.00556220,-0.01311885,-0.01193546,0.00497714,0.01071566$ 
$, 0.03040834,-0.00778630,-0.06833406,-0.13835928,0.03432546,-0.02725643$ $, 0.52025084,0.00301126,0.03862156,-0.07669309,-0.00577630,0.00609170,-$ $0.00084690,0.00598205,0.00651664,-0.00344516,0.00338962,-0.01136614,0$. $01242850,0.04807591,0.03835208,-0.06880865,-0.04596510,-0.12492522,0.1$ $7250239,-0.10926283,-0.05635689,-0.09972511,-0.02408921,-0.00476218,-0$ $.01504082,0.00083753,0.00259918,-0.00255167,-0.00175963,0.00019326,0.0$ $0003552,0.00316730,-0.00110025,-0.00009658,-0.00664932,-0.00974526,-0$. $00153063,0.13440392,-0.05259290,-0.08406181,-0.07052702,0.01067900,0.0$ $0944064,0.01093119,0.00158406,0.00072400,0.00062262,-0.00097084,-0.000$ $23366,-0.00031610,0.00249676,-0.00431455,0.00042880,-0.02112681,-0.016$ $78764,-0.01576973,0.06167122,0.09481334,-0.08838313,-0.06639333,-0.148$ $61588,-0.00545890,0.00094644,0.00010063,0.00115347,0.00072579,-0.00183$ $099,-0.00044336,0.00005170,-0.00003600,0.00141910,0.00051210,-0.001089$ $12,0.00611802,0.00320030,0.00885144,0.09732329,0.07492294,0.17268186,0$ $.00558869,0.02281811,-0.00691194,-0.06066145,-0.03485191,0.01468179,0$. 00094693,-0.02794700,0.00989895,-0.00534862,-0.00035511,0.00111322,-0. $00049339,0.00040436,-0.00021021,-0.00478060,0.00183503,-0.00008678,0.0$ $0072048,-0.00074902,-0.00025632,0.05989564,-0.00412100,-0.01068752,0.0$ $0355357,-0.03899617,-0.30157245,0.09596890,0.00270799,-0.00699011,0.00$ $463717,-0.00184064,0.00142287,0.00156854,-0.00014228,0.00028703,-0.000$ $31090,0.00327133,0.00054696,0.00020747,0.00039458,0.00013975,0.0003952$ $2,0.03878101,0.31691766,0.00141335,0.00260945,-0.00152187,0.01614491,0$ $.09646474,-0.05983477,-0.00067901,0.00697561,0.00513255,0.00137473,0.0$ $0129362,0.00464829,0.00002813,-0.00024908,-0.00064587,-0.00060354,-0.0$ $0001472,0.00158386,0.00078147,-0.00043682,0.00063784,-0.01687485,-0.10$ $490425,0.05336584,0.00109761,-0.00422089,0.00233141,-0.06478147,0.0353$ $2564,-0.00533091,-0.17423571,0.10274226,-0.02358785,-0.00015924,0.0035$ $9512,-0.00055847,-0.00415690,-0.00121943,0.00093714,-0.00039732,0.0016$ $8128,-0.00043461,-0.00160812,0.00118051,-0.00076100,0.00213735,0.00091$ $263,-0.00074508,0.52614022,-0.00410138,0.00049802,0.00211600,0.0070638$ $6,0.01182079,-0.00183258,0.05902121,-0.17587536,0.04037536,0.03759521$, $-0.03150919,0.00975093,-0.00758284,-0.00257242,0.00333213,0.00308250,-$ $0.00009689,-0.00043404,0.00033010,-0.00088838,0.00024463,-0.00006656,0$ $.00014929,-0.00147670,-0.05366319,0.29536617,0.00228790,0.00172362,0.0$ $0461610,0.00360924,-0.00360757,0.00967764,-0.00901211,0.03659977,-0.05$ $246110,-0.01197067,0.01253930,0.00384614,0.00323202,0.00281039,0.00391$ $272,-0.00095995,-0.00025701,-0.00043745,0.00056045,-0.00050480,-0.0000$ $6722,-0.00049684,-0.00151517,-0.00350286,-0.01998881,-0.07325154,0.088$ $82822,-0.00417077,0.00048410,0.00127869,-0.00078198,0.00017782,-0.0000$ $0249,-0.00315690,0.00259856,-0.00025013,0.00716258,0.02220895,-0.00766$ $307,-0.06612209,-0.04579462,0.01760222,-0.00172516,-0.02916752,0.00992$ $485,-0.00013967,-0.00048891,0.00011512,0.00013082,-0.00004883,-0.00002$ $090,-0.00015808,0.00167510,-0.00055067,0.06709616,-0.00064281,0.002847$ $01,0.00238546,0.00025772,0.00051235,-0.00042276,0.00334205,0.00078493$, $0.00101727,-0.00573822,-0.01374293,0.00585538,-0.04609261,-0.29504703$, $0.08871192,0.00025461,-0.00578998,0.00302161,-0.00007852,0.00025410,-0$ $.00011176,-0.00003710,-0.00009516,-0.00010740,0.00022100,-0.00122580,0$ $.00041333,0.04964046,0.31108369,0.00160742,0.00284015,0.00860432,-0.00$ $008186,-0.00051613,-0.00054071,-0.00024087,0.00098391,0.00449586,0.001$ $60936,0.00362725,0.00154443,0.01730972,0.08837651,-0.06779954,0.000619$ $87,0.00522138,0.00206122,0.00029821,0.00147663,-0.00070230,-0.00003040$ 
,-0.00015269,-0.00041779,-0.00012445,0.00026558,-0.00015969,-0.0198829 $6,-0.09803088,0.05926996,0.00705971,-0.00662645,0.00245956,-0.00003651$ ,-0.00181661,0.00080229,-0.00009774,-0.00067443,0.00017927,-0.00316850 ,-0.00309767,0.00212230,-0.01736333,0.02105228,-0.00500592,-0.21707212 $, 0.13640779,-0.02841709,0.00025082,0.00066075,-0.00034746,0.00054109,-$ $0.00028045,0.00010316,0.00007955,0.00014854,-0.00011719,0.00088186,-0$. $00030101,-0.00054247,0.22906474,0.02113503,-0.01335735,0.00358067,-0.0$ 0364406,-0.00387290,0.00192787,-0.00051008,0.00012632,-0.00013637,-0.0 $0203591,0.00085920,0.00232922,-0.00956458,0.00834684,-0.00030364,0.139$ $38151,-0.16834226,0.03370884,-0.00009272,0.00072792,-0.00063034,0.0013$ $9335,-0.00062335,0.00030246,0.00036801,-0.00000101,-0.00017629,0.00003$ $669,0.00043204,-0.00194691,-0.14625601,0.17469335,-0.00646818,0.004491$ $37,-0.00124523,0.00119323,0.00197465,0.00065468,0.00017811,-0.00003016$ $,-0.00019063,0.00149805,0.00234975,0.00673164,0.00464618,-0.00294050,0$ $.00533099,-0.02875089,0.03339980,-0.03711462,0.00029785,0.00093069,0.0$ $0040775,-0.00045560,0.00043038,0.00049974,-0.00006510,-0.00023943,-0.0$ 0044936,-0.00051378,-0.00192620,-0.00490998,0.02923876,-0.03794345,0.0 $2901848,0.00058394,0.00025915,-0.00012797,0.00129564,-0.00017877,0.000$ $23261,-0.00154820,0.00043695,0.00019519,-0.30381130,-0.09014588,0.0536$ $2971,-0.01616180,-0.01016543,0.00490526,-0.00149094,0.00383260,-0.0008$ $5873,0.00002614,-0.00001575,-0.00000743,-0.00011171,0.00013709,-0.0000$ $4151,-0.00078165,-0.00056060,0.00007416,0.00088250,-0.00010041,-0.0002$ $5239,-0.00090234,-0.00044696,0.00025030,0.32469932,0.00033568,-0.00022$ $581,-0.00027661,0.00024779,-0.00548107,0.00285655,-0.02630946,-0.00077$ $543,0.00359475,-0.08906837,-0.08743812,0.02908606,0.01803482,0.0096990$ $2,-0.00389050,0.00357582,-0.00197849,0.00156106,-0.00003259,0.00000369$ $, 0.00003171,-0.00142833,-0.00038510,0.00024440,0.00094382,0.00146301,-$ $0.00124552,-0.00045850,0.00062714,-0.00099581,0.00095533,0.00030543,-0$ $.00005940,0.09206553,0.08642477,-0.00032026,-0.00032354,-0.00097904,0$. $00020112,0.00298567,0.00235590,0.00898462,0.00117354,0.00206368,0.0532$ $2781,0.02910440,-0.03684303,-0.00451280,-0.00136509,0.00333650,-0.0006$ $4859,0.00153092,0.00307074,-0.00015514,-0.00001404,0.00013366,0.000455$ $76,0.00010223,-0.00007898,-0.00035681,-0.00110161,-0.00158892,-0.00011$ $461,-0.00099052,-0.00203656,-0.00020787,0.00002587,0.00034323,-0.05650$ $446,-0.03139409,0.03075175,-0.00139331,-0.00015855,0.00003729,-0.00028$ 045,0.00000085,0.00052491,0.00016817,0.00019916,0.00038357,0.00010802, $0.00001159,-0.00001920,0.00025943,0.00007970,0.00015906,-0.00007408,-0$ $.00000375,0.00053074,-0.00005098,0.00004117,0.00023281,-0.00007092,0.0$ $0002024,-0.00001193,0.00009873,0.00009301,-0.00018329,0.00004439,-0.00$ $003589,-0.00010939,-0.00007683,-0.00005283,-0.00000471,0.00001133,0.00$ $001093,-0.00000386,0.54529061,-0.00041145,-0.00051749,-0.00002557,-0.0$ $0021741,-0.00004662,0.00036697,0.00035950,-0.00018880,0.00025168,-0.00$ $003366,0.00011386,-0.00007376,0.00017742,-0.00001597,0.00014497,-0.000$ $03255,0.00008665,0.00035515,-0.00004861,0.00020703,0.00012535,0.000028$ $14,-0.00003620,-0.00007887,-0.00029042,0.00021666,-0.00011435,-0.00000$ $980,-0.00006279,-0.00009539,-0.00004149,-0.00004705,0.00002351,-0.0000$ 0947,-0.00000092,0.00001001,0.16135110,0.22030978,-0.00019666,-0.00006 $385,-0.00058564,-0.00024034,-0.00005308,0.00066503,0.00082504,-0.00065$ $018,0.00028948,-0.00007896,0.00028678,-0.00003887,0.00023847,-0.000136$ $58,0.00005716,-0.00012769,0.00025332,0.00055554,0.00004943,0.00011350$, $0.00030066,0.00001512,-0.00009065,-0.00007776,-0.00112979,0.00062068,-$ 
$0.00020652,-0.00004027,-0.00010324,-0.00007194,-0.00005480,0.00003492$, $-0.00012380,-0.00004925,-0.00001857,0.00000603,0.02794272,0.12421288,0$ $.51523506,-0.00194828,-0.00367457,-0.00103524,0.00016850,0.00014745,0$. $00040025,0.00008058,-0.00032999,-0.00007655,0.00017709,0.00020860,-0.0$ $0004539,0.00002812,-0.00033239,0.00008359,-0.00082321,0.00044732,0.000$ $88105,0.00014457,0.00016629,0.00023562,-0.00009148,0.00001382,-0.00003$ $477,-0.00018754,0.00012972,-0.00003573,-0.00003294,-0.00001260,-0.0000$ $9631,-0.00003286,-0.00011362,-0.00000088,0.00000489,0.00000162,0.00002$ $989,-0.14728501,-0.06092423,-0.10484435,0.68118001,-0.00355529,0.00530$ $869,-0.00214504,-0.00085072,-0.00046973,0.00100015,-0.00026543,0.00032$ $645,-0.00008118,0.00001702,-0.00002010,0.00002946,-0.00016076,0.000348$ $37,-0.00032307,0.00118283,-0.00023315,-0.00078249,0.00010492,0.0000183$ $0,0.00007967,-0.00005441,-0.00003092,0.00001080,-0.00003519,-0.0001068$ $3,0.00002289,0.00010376,0.00008592,0.00013619,0.00002607,-0.00004391,0$ $.00002655,0.00001494,-0.00000290,0.00000525,-0.04394734,-0.10777028,-0$ $.09301994,0.18861059,0.23567913,-0.00075113,-0.00194951,0.00189923,0.0$ $0062764,0.00016935,-0.00119058,-0.00054319,0.00044247,-0.00039781,-0.0$ $0021570,-0.00037698,0.00008048,-0.00015859,0.00009022,-0.00016182,-0.0$ $0007111,-0.00007288,-0.00057747,-0.00005839,-0.00013839,-0.00022119,0$. $00006005,0.00010825,0.00006181,0.00084762,-0.00053557,0.00030401,-0.00$ $003708,0.00001889,0.00012985,0.00007224,0.00002278,0.00005842,0.000025$ $13,0.00001167,0.00000458,-0.04676412,-0.07182490,-0.25234121,-0.043703$ $16,0.12667144,0.61816341,-0.01774820,-0.00644218,0.00621152,0.00024271$ ,-0.00164561,-0.00011940,-0.00031959,0.00102307,-0.00041742,-0.0000768 $7,-0.00020636,0.00014285,0.00033522,-0.00053631,0.00005132,-0.00096854$ $, 0.00139555,-0.00137550,0.00022271,-0.00071909,-0.00290659,-0.00003677$ $, 0.00038424,0.00006782,0.00024521,-0.00043098,0.00023311,-0.00022364,0$ $.00001195,0.00016972,0.00012983,-0.00038122,0.00030694,0.00001548,0.00$ $001631,-0.00000087,0.05643931,0.02816431,0.03479167,-0.18735951,-0.024$ $07248,0.07447651,0.61098896,-0.00063305,0.00447837,0.00039766,0.000436$ $28,-0.00073629,-0.00093644,0.00017953,0.00052077,-0.00020937,0.0000933$ $8,-0.00019922,0.00006581,-0.00021556,0.00022096,0.00004100,0.00033342$, $-0.00008048,-0.00038353,0.00098868,0.00004830,-0.00054222,-0.00019082$, $0.00019296,0.00004011,0.00029947,-0.00017345,0.00017921,0.00004798,0.0$ $0001774,-0.00005739,0.00016888,0.00012619,-0.00002354,0.00000242,0.000$ $00540,-0.00001698,0.01323571,0.01019405,-0.00582726,-0.00650430,-0.073$ $12185,-0.03034649,0.16596928,0.23814143,0.02578754,0.00774717,-0.00192$ $547,0.00132990,0.00146917,-0.00178302,-0.00003758,-0.00065722,0.000363$ $92,0.00039290,0.00009217,-0.00004648,-0.00006963,0.00098374,-0.0000424$ $1,0.00207413,-0.00128007,-0.00133838,0.00289928,0.00153245,0.00150574$, $0.00004361,-0.00030990,0.00005500,0.00071217,0.00001977,-0.00005065,0$. $00030604,0.00024096,0.00007000,-0.00020414,0.00018206,-0.00006095,0.00$ $000269,-0.00002908,-0.00008940,-0.01629325,-0.02444460,-0.05608619,0.1$ $3454624,-0.00850710,-0.25918261,-0.02793796,0.14823031,0.67525436,-0.0$ $9559261,0.00230975,0.04466762,-0.02790809,0.00599373,0.02078913,0.0002$ $1557,0.00379197,0.00056721,-0.00102489,0.00014510,-0.00011873,0.003058$ $42,-0.00011406,0.00086782,-0.01041275,-0.01143398,0.01223458,0.0047290$ $6,-0.00214276,-0.01279610,0.00132393,-0.00105477,-0.00082750,-0.000498$ $85,-0.00045330,-0.00046370,0.00012339,-0.00018555,-0.00033580,0.000681$ $85,0.00083678,-0.00127762,0.00008616,0.00004964,0.00008070,-0.04245872$ $,-0.02149423,-0.02935695,-0.03193028,0.00444585,0.06407797,-0.29379432$ 
,-0.09211385,-0.03023309,0.59610897,-0.00198143,-0.06784444,-0.0027091 $5,0.00952439,0.00063274,-0.01262087,0.00129703,-0.00003641,-0.00099828$ ,-0.00002773,-0.00029880,0.00051060,0.00084548,-0.00458039,0.00116486, $-0.01889409,0.00105826,0.02436238,0.00583820,-0.00101567,-0.01388223,0$ $.00010168,0.00060856,-0.00020024,0.00093167,-0.00019694,0.00040405,-0$. $00095570,-0.00071879,-0.00117489,-0.00028589,0.00050656,-0.00029329,-0$ $.00002618,0.00006369,0.00005014,-0.02187337,-0.01891156,-0.01417019,-0$ $.00779295,0.01302626,0.03113895,-0.07447607,-0.10073597,-0.02684483,0$. $15103399,0.26156153,0.03166607,-0.00822685,-0.13546576,0.00766896,-0.0$ $0750231,0.00527013,-0.00077005,-0.00015420,-0.00146813,0.00046236,0.00$ $048369,0.00011611,-0.00144114,-0.00130940,-0.00101763,0.00956024,0.011$ $92234,-0.01208605,0.01399782,-0.00265331,-0.02845532,-0.00096057,0.001$ $03312,0.00086917,-0.00237165,0.00052879,-0.00014635,-0.00038469,-0.000$ $13192,0.00042278,-0.00001276,-0.00125240,0.00149543,-0.00005470,-0.000$ $00931,0.00017775,-0.03046762,-0.01453829,-0.01878242,0.02171055,0.0158$ $8769,0.03388168,0.03008235,-0.00496350,-0.11935291,-0.01051426,0.11371$ 212,0.58021986,0.00940298,0.00731045,0.01642780,0.00124554,0.00016180, $0.00090494,0.00005254,-0.00069598,0.00077926,-0.00032266,0.00033393,-0$ $.00026760,-0.00018447,-0.00053066,-0.00001224,0.00094080,0.00104003,0$. $00058672,-0.00134545,0.00035094,0.00446030,0.00003093,-0.00015946,0.00$ $006780,-0.00075297,0.00045093,-0.00031113,-0.00004970,-0.00006488,-0.0$ $0008384,0.00001515,-0.00008420,0.00005139,-0.00002677,0.00000637,0.000$ $06176,-0.03445139,-0.00926761,0.02058297,-0.00212149,0.00193608,0.0052$ $7899,-0.01253321,-0.01581516,-0.02852555,-0.14529377,-0.05340564,-0.08$ $585126,0.66965921,0.00231038,0.00194998,-0.00203984,-0.00060082,0.0006$ $0245,-0.00076089,0.00005475,-0.00003887,-0.00035203,-0.00017079,-0.000$ $43167,-0.00000170,-0.00029241,0.00011912,0.00041845,0.00002721,0.00031$ $652,0.00052653,-0.00024394,0.00100259,0.00021037,-0.00000794,0.0001490$ $3,-0.00001515,-0.00014326,0.00007459,-0.00000771,-0.00003049,-0.000002$ $40,-0.00006901,-0.00007998,0.00034655,-0.00015499,-0.00001437,0.000032$ $72,0.00001673,0.00558559,0.01368908,0.01499310,0.00180397,-0.00681057$, $-0.01513782,-0.02747333,-0.00367383,-0.00459083,-0.03723980,-0.1058250$ $5,-0.08561649,0.17784350,0.23562403,-0.00217301,-0.00893163,-0.0261522$ $5,-0.00176465,0.00088786,-0.00120201,-0.00095104,0.00023707,-0.0000986$ $9,-0.00042648,-0.00030305,-0.00012374,-0.00047569,0.00008023,-0.000252$ $24,-0.00070348,-0.00275285,-0.00027859,0.00233256,-0.00055068,-0.00369$ $034,0.00029097,-0.00033393,0.00009442,0.00076385,-0.00048929,0.0001914$ $9,0.00001870,-0.00000136,0.00015825,0.00001623,0.00035260,-0.00012031$, $0.00003007,0.00000908,0.00001774,0.07122572,0.03353152,0.03327715,0.00$ $504491,-0.01533001,-0.06176075,-0.06841010,-0.01880490,0.02777732,-0.0$ $3084095,-0.06585943,-0.25403855,-0.05550770,0.12286448,0.62564910,0.00$ $083150,-0.00261534,-0.00304737,0.00004009,0.00004212,-0.00029202,-0.00$ $028204,0.00010535,-0.00041048,0.00000622,-0.00010836,0.00005679,-0.000$ $25372,-0.00008363,-0.00017499,-0.00035097,-0.00020870,-0.00017947,0.00$ $032339,-0.00031436,-0.00124856,0.00007704,0.00005066,0.00002474,0.0002$ $3076,-0.00026399,0.00017254,-0.00007592,0.00000450,0.00007730,0.000097$ $64,0.00011202,0.00007149,0.00000070,-0.00000194,-0.00000360,-0.2894162$ $3,-0.07809737,0.01274731,-0.01106386,-0.02700723,-0.06751508,-0.051133$ $05,-0.00978717,0.02561217,0.05500403,0.02661866,0.03154803,-0.18658135$ $,-0.02320102,0.07723845,0.60615340,-0.00254181,0.00358994,-0.00291116$, $-0.00041807,0.00000838,0.00029838,-0.00020588,0.00007132,-0.00014047,0$ 
$.00009773,0.00000725,-0.00000248,0.00007715,0.00019156,0.00002432,0.00$ $013446,-0.00022513,-0.00013809,0.00020054,-0.00001084,-0.00056886,0.00$ $005974,-0.00002170,0.00009891,-0.00000318,-0.00004356,0.00001126,0.000$ $04853,0.00004681,0.00005725,0.00000670,-0.00002736,-0.00009427,-0.0000$ $0334,-0.00001171,-0.00000774,-0.09515079,-0.10191866,-0.00787373,-0.01$ $508803,-0.00217191,-0.01950467,-0.01029023,-0.00550854,0.00613189,0.01$ $401456,0.01089532,-0.00554844,-0.00543458,-0.07292165,-0.03340106,0.16$ $724338,0.23703898,-0.00326910,-0.00298836,-0.00087989,-0.00044061,0.00$ $040951,0.00075010,0.00045919,-0.00009965,0.00029171,0.00013012,0.00014$ 472,-0.00003971,0.00028761,0.00009636,0.00024922,-0.00036956,-0.000241 $02,0.00090153,0.00044263,0.00002762,-0.00051639,-0.00000431,-0.0000898$ $2,-0.00016472,-0.00015788,0.00016726,-0.00013685,0.00003555,0.00000258$ $,-0.00016230,-0.00001336,0.00001465,-0.00011318,-0.00000617,0.00000173$ ,-0.00001654,-0.04572231,-0.02918494,-0.10745267,-0.02681247,-0.004637 $83,0.02686877,0.02402432,0.00539417,-0.01482596,-0.01116645,-0.0211063$ $8,-0.05121055,0.13777537,-0.01126590,-0.27116550,-0.02058689,0.1566021$ $6,0.69187868,0.00008821,0.00013471,-0.00029685,-0.00007972,-0.00018371$ $, 0.00035181,0.00038779,-0.00031916,0.00007722,-0.00007242,0.00014964,-$ $0.00003428,0.00011610,-0.00012784,0.00004351,-0.00017170,0.00031468,0$. $00019372,-0.00000560,0.00002492,0.00007812,0.00003554,-0.00002505,-0.0$ $0002991,-0.00064863,0.00029532,-0.00006450,-0.00004886,-0.00005449,-0$. $00002121,0.00002304,0.00001004,-0.00003912,-0.00002853,-0.00001472,0.0$ $0000240,-0.07352862,-0.00262517,0.05326106,0.00165143,-0.00029064,0.00$ $242537,0.00059701,-0.00154553,0.00010310,0.00078097,0.00101334,0.00153$ $891,-0.00241500,-0.00392519,-0.00478431,-0.02083178,0.00124854,0.03588$ $776,0.09326842,0.00029798,-0.00055496,0.00022932,0.00006935,0.00008310$ ,-0.00004636,0.00002148,-0.00000267,0.00002439,0.00001264,-0.00000626, $0.00001113,-0.00003129,-0.00001639,-0.00003119,-0.00005090,-0.00000679$ ,-0.00000275,-0.00001167,0.00000585,0.00004089,-0.00000935,-0.00000023 ,-0.00000245,0.00004167,-0.00000486,-0.00000077,-0.00000152,0.00000310 ,-0.00000275,-0.00000413,0.00000745,-0.00000180,0.00000230,-0.00000050 ,-0.00000233,-0.00261818,-0.02668755,-0.00697694,0.006886660,0.00375682 ,-0.01077290,-0.00326775,0.00329140,-0.00141801,0.00087354,-0.00020814 $, 0.00053025,-0.00215990,0.00306327,-0.00228335,-0.00590885,0.00456555$, $0.00784372,0.00351661,0.01648603,0.00001069,0.00043970,0.00036384,0.00$ $014250,0.00028721,-0.00052626,-0.00061674,0.00045228,-0.00016124,0.000$ $12227,-0.00023331,0.00005020,-0.00016367,0.00029367,-0.00006464,0.0003$ $6621,-0.00050305,-0.00030994,-0.00000781,0.00000801,0.00006565,-0.0000$ $4750,0.00001359,0.00004215,0.00099164,-0.00046198,0.00012759,0.0000928$ $7,0.00008909,0.00003497,-0.00003524,-0.00002078,0.00004799,0.00004196$, $0.00001708,-0.00000795,0.05336334,-0.00696936,-0.11416131,0.02701950,-$ $0.00176587,-0.03549763,-0.00579476,-0.00358789,-0.00009635,0.00112819$, $0.00038667,-0.00071328,0.00127075,-0.00010323,0.00256951,0.01116093,-0$ $.00112921,-0.01348719,-0.08940905,0.01111295,0.16178832,-0.00024856,-0$ $.00023893,-0.00043232,-0.00010286,-0.00011232,0.00008147,0.00009828,0$. $00001900,0.00003224,-0.00004959,0.00000423,-0.00000323,0.00005482,-0.0$ $0004933,0.00002137,-0.00011387,0.00007569,0.00011327,0.00002499,0.0000$ $0020,-0.00003530,-0.00000363,0.00000645,-0.00000705,-0.00012501,0.0000$ $4866,-0.00000577,-0.00001475,-0.00002688,-0.00001241,0.00000022,-0.000$ $00769,0.00000203,-0.00000628,-0.00000290,0.00000518,-0.00787220,-0.002$ $98912,0.00384648,-0.30594316,-0.10052044,-0.00548453,-0.01094527,-0.00$ 
$617408,-0.00289211,0.00115961,-0.00107349,0.00356657,0.00041672,0.0006$ $1210,0.00004233,0.00173428,-0.00187790,-0.00165700,0.00083194,0.001363$ $24,0.00006348,0.32113721,0.00015006,0.00006923,-0.00010902,-0.00000787$ $, 0.00007785,0.00007917,-0.00001986,-0.00005104,0.00002707,-0.00001217$, $0.00003026,-0.00001800,0.00003945,0.00001490,0.00001001,-0.00004963,-0$ $.00001049,-0.00010596,-0.00000537,-0.00000367,-0.00000288,0.00001216,-$ $0.00000236,-0.00000121,-0.00002491,0.00001287,-0.00001718,-0.00000401$, $0.00000189,0.00002368,-0.00000673,-0.00000372,0.00000264,-0.00000114,--$ $0.00000285,0.00000071,-0.01130433,-0.00040760,0.00106163,-0.09979743,-$ $0.07373360,-0.00751833,0.00167888,0.00403482,-0.00071249,-0.00118219,0$ $.00596079,-0.00165709,0.00066516,-0.00098107,0.00000593,-0.00170748,0$. $00390428,-0.00336845,0.00109474,-0.00204290,0.00108348,0.10944163,0.06$ $683193,0.00082550,0.00036794,0.00024921,0.00007415,0.00005432,-0.00019$ $680,-0.00004007,0.00002410,-0.00000699,0.00003825,-0.00002724,0.000000$ $71,-0.00003949,0.00006445,-0.00000296,0.00012957,-0.00011930,-0.000084$ $80,-0.00002653,-0.00000534,0.00003471,0.00000731,-0.00001435,0.0000048$ $3,0.00010985,-0.00004362,0.00001621,0.00002218,0.00001990,0.00000217,-$ $0.00000048,0.00002609,-0.00000968,0.00000303,0.00000105,-0.00000524,-0$ $.02459621,-0.00933393,0.00223365,-0.00290580,-0.00655542,-0.05390750,0$ $.02390234,0.00906091,0.00476841,0.00306266,-0.00181933,-0.00326088,0.0$ $0019118,0.00007250,-0.00117922,-0.00109164,-0.00316119,-0.00400689,-0$. $00084782,0.00074760,0.00081248,0.00319025,0.00983122,0.05373266,0.0004$ $0406,0.00087400,-0.00261237,-0.00005751,-0.00002523,0.00035173,-0.0001$ $1927,0.00010348,-0.00000321,0.00008316,-0.00002276,0.00002178,-0.00008$ $320,0.00015811,-0.00006337,0.00035983,0.00003430,0.00016927,0.00016695$ $, 0.00037060,0.00120213,-0.00004109,-0.00000853,-0.00004687,0.00013357$, $-0.00007547,-0.00000530,0.00003528,0.00001162,-0.00003071,-0.00001580$, $0.00001855,-0.00004355,0.00000890,0.00000227,-0.00000430,-0.00016309,-$ $0.00160402,0.00334917,0.00939813,0.00699929,0.01638133,-0.09461218,-0$. $04672387,-0.08656458,-0.00625191,-0.01092063,-0.02453176,-0.00482924,-$ $0.00370232,0.00109814,-0.00086521,0.00025449,0.00071455,-0.00015802,0$. $00020871,0.00113049,0.00034412,0.00140078,-0.00073715,0.09665445,0.001$ $90488,-0.00319431,0.00043162,0.00058387,0.00048008,-0.00019657,0.00001$ $077,-0.00014191,0.00002105,-0.00000166,-0.00001983,0.00001345,0.000071$ $19,-0.00017992,0.00009361,-0.00064303,0.00010040,0.00031697,-0.0001501$ $8,0.00028534,0.00049047,0.00005091,-0.00004955,-0.00001598,0.00014584$, $-0.00003896,0.00002185,-0.00004626,-0.00003676,-0.00006115,-0.00005717$ ,-0.00010424,0.00000975,0.00000943,-0.00000294,0.00000066,-0.00151269, $0.00601577,-0.00107697,-0.00112671,0.00302109,-0.00000821,-0.04687708$, $-0.08085840,-0.09462261,-0.00318504,-0.00003433,-0.01000134,-0.0039190$ $5,0.00454733,-0.00079459,0.00021834,-0.00075943,0.00062524,-0.00008470$ $, 0.00013718,-0.00001192,0.00149883,-0.00324246,0.00109008,0.05299610,0$ $.07476958,0.00109010,0.00164584,0.00036328,-0.00026681,0.00009154,0.00$ $012053,0.00010836,-0.00000885,0.00006719,-0.00009685,0.00000836,-0.000$ $02973,0.00010241,0.00010409,-0.00004345,0.00023470,-0.00013419,0.00012$ $336,-0.00014787,0.00001092,0.00051136,-0.00005212,-0.00000254,-0.00002$ $159,-0.00009599,0.00006405,-0.00003428,0.00002848,-0.00000844,-0.00000$ $938,-0.00001531,-0.00000530,-0.00002664,-0.00000251,0.00000079,0.00001$ $136,0.00365217,-0.00095863,-0.00095250,-0.01125821,-0.01007545,-0.0171$ $9217,-0.08703101,-0.09478178,-0.25082804,0.00146063,-0.00041813,0.0006$ $8955,0.00024718,-0.00113311,0.00207372,0.00057146,0.00056462,0.0002112$ 
$8,0.00010881,-0.00038879,-0.00126719,-0.00044947,0.00119847,0.00055517$ $, 0.09171209,0.10404952,0.26578895,-0.00055370,0.00117184,0.00069264,0$. $00014318,-0.00001909,0.00041826,0.00007697,0.00004677,-0.00009535,0.00$ $002685,-0.00013599,0.00000433,0.00006065,0.00014723,0.00012036,0.00016$ $539,0.00017038,0.00001637,-0.00005278,0.00001526,-0.00008096,-0.000025$ $77,0.00001946,-0.00001828,-0.00002333,0.00002310,0.00003334,0.00002812$ $, 0.00003428,0.00001093,-0.00005911,-0.00001369,-0.00010692,-0.00000933$ $, 0.00000252,0.00000857,0.00131686,-0.00120035,0.00311736,0.00045824,0$. $00057296,0.00001326,0.00152128,-0.00226679,-0.00177688,-0.00715033,-0$. $00349989,0.00138214,-0.30105987,-0.09787980,-0.00743240,-0.01150003,-0$ $.00638076,-0.00261979,-0.00067249,-0.00019506,0.00027490,-0.00015032,0$ $.00019441,-0.00005309,-0.00034279,-0.00012529,0.00016149,0.31758983,0$. $00074493,-0.00247443,0.00121430,0.00035863,0.00021315,0.00002177,-0.00$ $000665,-0.00033735,0.00039918,-0.00005759,0.00043450,-0.00015637,0.000$ $11612,-0.00015794,-0.00032422,-0.00023442,0.00008511,0.00026545,0.0000$ $2018,-0.00008386,0.00007955,-0.00002548,-0.00012807,-0.00015046,-0.000$ $04653,0.00004562,-0.00004513,-0.00001233,-0.00003099,0.00009499,0.0000$ $6790,-0.00003102,0.00012641,-0.00000397,-0.00001181,0.00000232,-0.0013$ $5796,0.00627243,-0.00170475,0.00063685,-0.00094726,0.00002598,-0.00210$ $316,0.00457494,-0.00373839,-0.01124886,-0.00023335,0.00050941,-0.09763$ $566,-0.07445151,-0.00817650,0.00163711,0.00426542,-0.00103173,0.000101$ $74,0.00009435,-0.00011849,0.00019327,-0.00059917,0.00020525,-0.0005560$ $1,-0.00009967,0.00003416,0.10780223,0.06704450,-0.00067490,0.00068986$, $0.00022625,-0.00018285,-0.00001934,0.00022469,0.00058709,-0.00037146,-$ $0.00017295,0.00020152,0.00008222,-0.00003140,0.00021978,0.00022981,0.0$ $0002862,-0.00009498,-0.00000346,-0.00008983,-0.00005486,0.00010091,0.0$ $0027736,-0.00000476,0.00009700,0.00000614,-0.00041618,0.00024894,-0.00$ $009166,0.00004394,0.00001848,-0.00000285,0.00002538,-0.00004951,-0.000$ $01741,-0.00002603,-0.00000837,0.00000982,0.00275227,-0.00185610,-0.002$ $50881,0.00015349,0.00007829,-0.00106137,-0.00106889,-0.00349581,-0.004$ $19159,-0.02502388,-0.00890659,0.00450089,-0.00716838,-0.00823430,-0.05$ $625782,0.02491856,0.00902016,0.00360851,0.00132796,0.00025127,-0.00067$ $941,-0.00006782,0.00020004,0.00003819,-0.00135104,-0.00051174,0.000011$ $66,0.00636693,0.01117857,0.05550249,0.00038828,0.00024287,0.00062419,0$ $.00005209,-0.00005147,-0.00003583,0.00003345,-0.00001826,0.00003831,-0$ $.00003048,-0.00001528,-0.00003624,-0.00003205,-0.00001530,0.00000227,0$ $.00011559,0.00012966,-0.00011138,-0.00009643,0.00007612,0.00023880,-0$. $00001232,0.00001389,0.00001113,-0.00005894,0.00003334,-0.00001989,-0.0$ $0001262,-0.00000937,0.00001299,-0.00000795,-0.00001024,0.00001015,-0.0$ $0000129,0.00000299,0.00000547,-0.00654682,-0.01087588,-0.02505122,-0.0$ $0455616,-0.00321131,0.00127543,-0.00081217,0.00030325,0.00075477,-0.00$ $069357,-0.00181402,0.00381075,0.01003072,0.00682380,0.01559952,-0.0920$ $8772,-0.04598623,-0.08702126,0.00066362,0.00097218,-0.00078787,-0.0002$ $5720,-0.00057083,-0.00119564,-0.00000616,0.00023050,-0.00008457,0.0002$ $2324,0.00147042,-0.00059763,0.09370140,-0.00020038,0.00014043,0.000033$ $97,0.00019143,-0.00000559,-0.00003371,-0.00010173,0.00003811,-0.000064$ $60,-0.00000986,-0.00002618,0.00000271,0.00004290,0.00000438,0.00004883$ $,-0.00009350,-0.00007785,0.00002348,0.00005431,-0.00010058,0.00001102$, $-0.00000483,-0.00001455,-0.00000899,0.00009344,-0.00006924,0.00003633$, $-0.00000374,-0.00000070,0.00000519,0.00000576,-0.00000943,0.00001362,0$ $.00000116,0.00000514,-0.00000129,-0.00257220,-0.00049452,-0.00984726,-$ 
$0.00337277,0.00388819,-0.00049878,0.00026523,-0.00075594,0.00060299,-0$ $.00163548,0.00634184,-0.00124083,-0.00095936,0.00361490,0.00005424,-0$. $04674873,-0.08096190,-0.09730127,0.00126142,-0.00195785,0.00076859,-0$. $00019409,0.00014028,-0.00048108,0.00022032,-0.00058181,0.00014375,0.00$ $150408,-0.00345529,0.00123477,0.05227397,0.07433725,-0.00077085,-0.000$ $44958,-0.00047696,-0.00012623,0.00006293,0.00003559,-0.00005368,0.0001$ $0040,-0.00001092,0.00004506,-0.00004193,0.00002346,-0.00002925,0.00005$ $133,-0.00000381,0.00001595,-0.00009223,0.00004964,0.00014115,-0.000010$ $20,-0.00036280,-0.00000412,-0.00000971,-0.00000719,0.00014194,-0.00005$ $941,0.00001956,0.00001251,0.00001651,-0.00001389,-0.00001332,0.0000047$ $4,-0.00001732,0.00000855,0.00000337,-0.00001113,0.00340032,0.00049783$, $0.00084850,0.00073442,-0.00069080,0.00197643,0.00059952,0.00052633,0.0$ $0006693,0.00431596,-0.00100915,-0.00154912,-0.01092376,-0.00962954,-0$. $01554575,-0.08968743,-0.09831894,-0.26055484,0.00017107,0.00113451,0.0$ $0101278,0.00009302,-0.00000853,0.00020269,-0.00012274,0.00013309,-0.00$ $010624,-0.00045682,0.00129887,0.00065914,0.09254898,0.10650243,0.27376$ $508,-0.00084440,-0.00048520,0.00024318,0.00359774,0.00161175,-0.000844$ $84,-0.04782440,0.00630379,0.00125536,0.00588923,-0.00204810,0.00008256$ ,-0.00231832,-0.00137350,0.00074846,0.00145866,0.00000591,-0.00017816, $0.00023013,-0.00024984,0.00023512,0.00000064,-0.00015984,-0.00003470,-$ $0.19593315,-0.02852605,0.02098609,0.00047010,-0.00053010,0.00017247,-0$ $.00003998,-0.00014306,-0.00000541,-0.00191713,-0.00062931,0.00035567,--$ $0.00009134,0.00013018,0.00052873,0.00010857,-0.00004678,-0.00035047,-0$ $.00019885,-0.00011935,-0.00011806,-0.00022952,-0.00020533,0.00065120,0$ $.00025389,-0.00004307,-0.00031587,-0.00007653,-0.00000841,0.00011667,0$ $.00029067,-0.00001428,-0.00043259,0.00004420,0.00001829,-0.00003530,-0$ $.00006173,-0.00001387,0.00003141,-0.00000030,0.00014383,0.00019570,0.0$ $0001092,-0.00002582,-0.00005769,0.46398760,0.00071228,0.00086501,0.000$ $34970,0.00239918,-0.00430781,0.00168957,0.02706832,0.00948578,-0.00751$ $388,0.00535152,-0.00274819,0.00005458,-0.00053831,0.00024102,0.0001469$ $3,0.00059353,0.00021653,-0.00015785,0.00029638,-0.00029210,0.00009664$, $0.00012824,0.00017714,-0.00037740,-0.05916192,-0.10134353,0.02007028,0$ $.00007380,-0.00003801,0.00002892,-0.00001196,-0.00007244,-0.00006080,0$ $.00227754,-0.00166569,0.00038753,-0.00005227,0.00002788,0.00011726,0.0$ $0003130,-0.00000104,-0.00009249,-0.00000936,-0.00003276,-0.00012127,-0$ $.00003695,-0.00010733,0.00041040,0.00012592,0.00002499,-0.00008068,-0$. $00000358,-0.00000851,0.00001740,0.00007636,-0.00001239,-0.00012506,0.0$ $0000769,0.00000464,-0.00001228,-0.00003515,-0.00002362,-0.00000612,0.0$ $0002432,0.00002681,0.00006903,0.00001415,-0.00000035,-0.00002444,-0.05$ $202323,0.59236013,0.00014406,0.00042482,0.00214498,-0.00093357,0.00191$ $425,0.00079707,-0.00577813,-0.00585636,-0.00367042,-0.00237946,0.00064$ $491,-0.00142368,0.00044091,0.00022595,0.00035029,-0.00030946,-0.000055$ $78,0.00005697,0.00017580,-0.00014938,0.00005043,-0.00010968,-0.0003677$ $2,-0.00076280,0.03119726,0.01754028,-0.06430536,-0.00005512,0.00005736$ $, 0.00003001,-0.00002278,-0.00001449,-0.00019076,-0.00060519,0.00063138$ ,-0.00011519,-0.00003428,-0.00005362,-0.00013104,-0.00000824,-0.000018 $80,0.00008768,0.00003880,0.00005791,0.00006278,-0.00032708,0.00031406$, $-0.00016730,-0.00004130,0.00002373,0.00006213,0.00004978,0.00000061,-0$ $.00002645,-0.00007636,0.00000471,0.00009443,-0.00001912,-0.00001121,0$. $00001436,-0.00001736,0.00000703,-0.00003302,0.00002896,-0.00002292,0.0$ $0000670,0.00001069,0.00000413,0.00000831,0.02527561,-0.00610237,0.5617$ 
$3948,-0.00014793,-0.00029019,0.00014931,-0.00135354,0.00130895,-0.0003$ $4104,-0.00226747,-0.00378109,0.00152781,-0.00032796,-0.00110009,0.0004$ $3444,0.00008100,0.00029993,-0.00009803,0.00004693,-0.00006289,0.000019$ $08,-0.00010226,0.00004916,-0.00002358,-0.00001914,-0.00004972,0.000030$ $92,-0.03562518,0.02661339,-0.00590305,-0.00005044,0.00005109,-0.000010$ $84,0.00002740,0.00002430,-0.00001035,0.00051829,0.00015073,-0.00012565$ $, 0.00001416,-0.00001320,-0.00005502,-0.00000980,-0.00000896,0.00003160$ $, 0.00000065,0.00001965,0.00005298,-0.00002998,0.00004141,-0.00015293,-$ $0.00002548,0.00000106,0.00002968,-0.00000042,-0.00000723,-0.00000117,-$ $0.00003678,0.00000233,0.00005479,-0.00000866,-0.00000058,0.00000777,0$. $00000437,0.00001187,-0.00000805,-0.00000070,-0.00000210,-0.00002355,-0$ $.00000212,0.00000550,0.00000877,-0.11870200,0.11351828,-0.03216876,0.1$ $4851179,-0.00005994,-0.00003496,0.00002999,-0.00051808,0.00037560,-0.0$ $0008396,-0.00256644,0.00054857,0.00016440,-0.00039738,-0.00075607,0.00$ $040213,-0.00000964,0.00040624,-0.00009588,0.00018052,-0.00011190,0.000$ $02625,0.00000975,0.00001075,0.00000187,0.00002315,-0.00000585,0.000020$ $43,-0.00922433,0.01104286,-0.00270513,-0.00001921,0.00001397,0.0000074$ 2,0.00004458,0.00000614,-0.00001606,0.00064269,0.00018234,-0.00024443, $-0.00001639,-0.00000072,0.00004164,0.00001847,0.00000189,-0.00001621,-$ $0.00002373,-0.00002072,-0.00000414,-0.00002837,0.00002184,0.00002413,0$ $.00000727,-0.00001525,-0.00001168,0.00000160,0.00001387,-0.00000343,0$. $00002629,0.00000059,-0.00003574,0.00000585,0.00000207,-0.00000349,-0.0$ $0000176,-0.00000003,0.00001010,-0.00000172,0.00000399,0.00001809,-0.00$ $000022,-0.00000607,-0.00000833,0.12656123,-0.23317722,0.05096200,-0.12$ $887548,0.24273003,0.00003116,0.00004294,-0.00001887,0.00030420,-0.0001$ $9859,0.00013568,0.00106684,0.00028242,0.00021937,0.00019082,0.00048476$ $, 0.00019673,0.00000049,-0.00011157,0.00019610,-0.00007168,0.00003720,-$ $0.00001822,0.00000795,-0.00000709,0.00001296,-0.00001141,0.00001245,0$. $00002306,0.00605884,-0.00565075,0.00207032,0.00001343,0.00000125,0.000$ $01505,-0.00001641,-0.00001976,-0.00002392,-0.00028376,-0.00021512,-0.0$ $0029519,0.00000832,0.00000677,-0.00000638,0 .,-0.00000604,0.00000414,-0$ $.00000217,0.00000467,-0.00000820,0.00001340,-0.00001202,0.00001324,-0$. $00000337,0.00001004,0.00000528,-0.00000234,-0.00000207,-0.00000669,-0$. $00000347,-0.00000044,0.00000448,0.00000007,0.00000106,0.00000095,-0.00$ $000009,-0.00000016,0.00000160,-0.00000365,-0.00000591,-0.00000188,0.00$ $000106,-0.00000299,-0.00000168,-0.03649544,0.05203872,-0.06362157,0.03$ $452822,-0.05521093,0.06105879,0.00031366,0.00007391,0.00040430,-0.0023$ $1645,0.00013511,0.00063635,0.00675570,-0.00430573,-0.00082465,0.000454$ $43,-0.00013801,0.00020152,-0.00081251,0.00038919,0.00030387,0.00032698$ $, 0.00023667,-0.00012037,0.00001702,-0.00003760,-0.00001650,0.00011245$, $-0.00000868,-0.00028299,-0.02754313,-0.02887098,-0.01624113,-0.0000544$ $4,0.00003439,-0.00004219,0.00000580,0.00000272,-0.00007807,-0.00075541$ $, 0.00059481,-0.00110158,-0.00000947,-0.00001849,-0.00003769,-0.0000044$ $1,0.00000452,0.00003943,0.00001497,0.00000554,0.00001960,-0.00004479,0$ $.00004623,-0.00007303,-0.00004822,-0.00002317,0.00004193,0.00002244,0$. $00001438,-0.00001900,-0.00001850,0.00000283,0.00003225,-0.00000333,-0$. $00000108,0.00000375,0.00000660,0.00000723,-0.00000154,-0.00000494,0.00$ $000252,-0.00001990,-0.00000346,0.00000128,0.00000639,-0.05899955,-0.03$ $796989,-0.03241872,0.00591615,0.01166427,0.01063699,0.07388507,0.00016$ 490,-0.00003686,0.00003721,-0.00017582,0.00051948,-0.00017983,0.001748 $86,-0.00150946,0.00139692,-0.00066172,0.00028851,0.00019896,0.00063822$ 
$, 0.00001939,-0.00030499,-0.00040940,-0.00010433,0.00006277,-0.00001602$ $, 0.00006350,-0.00002549,0.00006898,0.00001101,-0.00007511,-0.01248772$, $-0.00462594,-0.00128800,-0.00004638,0.00005303,-0.00000125,0.00002091$, $0.00004775,0.00003432,0.00117387,0.00004026,0.00074792,0.00000701,-0.0$ $0001565,-0.00006919,-0.00001245,0.00000244,0.00005691,0.00002224,0.000$ $02990,0.00002568,0.00001952,0.00004688,-0.00013882,-0.00006155,-0.0000$ 0806,0.00004816,0.00001993,-0.00000755,-0.00001724,-0.00003795,0.00000 $459,0.00006401,-0.00000474,-0.00000321,0.00000605,0.00001419,0.0000056$ $0,-0.00000087,-0.00000586,-0.00000624,-0.00003617,-0.00000736,0.000004$ $84,0.00001265,-0.04308433,-0.19621565,-0.11741531,-0.00531184,-0.01686$ $395,-0.01602986,0.05537747,0.21212923,0.00005016,-0.00002131,-0.000053$ $70,-0.00027571,0.00018829,-0.00027906,0.00043968,-0.00047141,0.0004964$ $3,-0.00058628,-0.00106458,0.00039902,0.00024125,0.00040690,-0.00011293$ $, 0.00005876,-0.00011664,0 ., 0.00001503,0.00002729,-0.00001672,0.0000523$ $0,0.00002584,0.00004172,0.00091572,0.00794737,0.00891079,-0.00005188,0$ $.00000396,-0.00001270,0.00005658,0.00001577,-0.00000076,-0.00001432,0$. $00068717,-0.00056873,0.00000361,0.00000201,0.00000575,0.00000145,-0.00$ $000005,0.00000471,-0.00000313,-0.00000779,-0.00001555,-0.00000447,0.00$ $001884,0.00000275,0.00000897,0.00000488,0.00000955,-0.00000535,-0.0000$ $0217,-0.00000772,0.00000603,0.00000185,-0.00000645,0.00000368,-0.00000$ $049,-0.00000218,0.00000106,-0.00000099,0.00000678,0.00000229,-0.000008$ $90,0.00000354,0.00000375,-0.00000522,-0.00000317,-0.03601309,-0.118459$ 48,-0.14406696,0.00233573,0.00659410,0.00509790,0.03866686,0.12745500, $0.14674664,0.00019076,-0.00014025,-0.00032818,-0.00230008,-0.00029869$, $-0.00018784,0.00672944,-0.00303186,0.00222044,0.00059279,-0.00010752,-$ $0.00018613,-0.00093143,0.00008962,-0.00031869,0.00032440,0.00026892,-0$ $.00009436,-0.00010274,0.00004093,-0.00004672,0.00015536,0.00018205,0.0$ $0021074,-0.02248779,-0.01200865,0.03514086,-0.00003723,0.00004679,0.00$ $001011,0.00001436,0.00003786,0.00007568,-0.00058060,0.00094914,0.00064$ $147,0.00001845,-0.00000612,-0.00003256,-0.00001018,0.00000816,0.000019$ $98,0.00000882,0.00000608,0.00001570,0.00004241,-0.00005962,-0.00005953$ ,-0.00000746,0.00000935,0.00001260,-0.00000265,-0.00000374,-0.00000642 $,-0.00001810,0.00000144,0.00002847,-0.00000171,0.00000005,0.00000144,0$ $.00000777,0.00000296,0.00000102,-0.00000482,-0.00000504,-0.00001695,-0$ $.00000184,0.00000169,0.00000230,-0.04910513,-0.00285618,0.01794273,0.0$ $0358911,0.00255703,-0.01596801,0.00278732,0.00304706,-0.00590870,0.061$ $13077,0.00007304,-0.00004299,-0.00005055,0.00012739,0.00023142,-0.0001$ $5149,0.00076920,-0.00108948,-0.00032963,-0.00022895,0.00069823,-0.0004$ $4972,0.00045998,-0.00011137,0.00024668,-0.00038555,-0.00003071,0.00004$ $588,-0.00005709,0.00004768,-0.00000951,0.00002299,0.00004085,0.0000543$ $1,-0.00796139,-0.00191754,0.00990388,-0.00000793,0.00002761,-0.0000407$ $8,-0.00002551,0.00000432,-0.00002953,0.00081660,-0.00086928,-0.0006822$ $6,0.00002047,-0.00001078,-0.00005332,-0.00002054,0.00000049,0.00003719$ $, 0.00002390,0.00002021,0.00002218,0.00003475,0.00002288,-0.00009743,-0$ $.00003871,0.00000278,0.00003381,0.00000410,-0.00000023,-0.00001109,-0$. $00003420,0.00000220,0.00004956,-0.00000493,-0.00000168,0.00000513,0.00$ $000895,0.00000580,-0.00000400,-0.00000454,-0.00001286,-0.00002460,-0.0$ $0000135,0.00000248,0.00000779,-0.00504551,-0.06338330,0.05725486,-0.00$ $259429,-0.00436800,0.02455104,0.00278057,0.00613050,-0.02322022,0.0112$ $6856,0.06460079,-0.00021062,0.00003911,-0.00003782,0.00063427,-0.00053$ $625,0.00004966,-0.00224559,0.00224794,-0.00003796,0.00081048,0.0008592$ 
$0,-0.00007635,-0.00048996,-0.00043902,0.00010975,0.00014212,0.00011016$ ,-0.00005608,0.00003696,-0.00005067,0.00003222,-0.00010166,-0.00005209 ,-0.00003022,0.01111712,0.00235476,0.00111750,0.00007307,-0.00003944,0 $.00000076,-0.00004918,-0.00002928,0.00002619,-0.00056423,-0.00059544,0$ $.00035316,-0.00000671,0.00002368,0.00005306,0.00001615,-0.00000930,-0$. $00003308,-0.00003099,-0.00001320,-0.00000029,0.00002070,-0.00003764,0$. $00005492,0.00000078,-0.00002828,-0.00001992,-0.00000434,-0.00000503,0$. $00001424,0.00003003,-0.00000175,-0.00003556,0.00000469,0.00000570,-0.0$ $0000325,0.00000265,-0.00000216,0.00000844,-0.00000800,0.00002757,0.000$ $00298,-0.00000673,-0.00000001,-0.00000330,0.02400338,0.05765716,-0.286$ $99108,-0.00028906,0.00013686,-0.00505304,0.00028412,0.00543558,-0.0165$ $7895,-0.03316945,-0.06705815,0.30713404 \backslash 0.00001435,-0.00000729,-0.000$ $02677,0.00003531,-0.00000431,0.00000355,-0.00002769,-0.00001901,0.0000$ $0632,-0.00001898,0.00004423,-0.00000381,0.00004719,-0.00002165,-0.0000$ $0109,-0.00003041,0.00000240,0.00000637,-0.00000161,-0.00000041,0.00001$ 036,-0.00000740,0.00000082,-0.00000816,0.00000414,-0.00000067,0.000004 $55,-0.00000555,0.00000671,0.00000577,0.00000453,0.00000245,0.00000003$, $0.00000194,-0.00000618,-0.00001030,-0.00000116,0.00000141,0.00000904,0$ $.00000248,0.00000088,-0.00000270,-0.00000234,0.00000284,0.00000024,-0$. $00000708,-0.00000140,0.00001500,0.00000119,0.00000434,0.00000181,0.000$ $00288,-0.00000127,-0.00000128,0.00000337,-0.00000079,-0.00000121,0.000$ $00008,0.00000059,0.00000315,0.00000006,0.00000084,0.00000075,0.0000014$ $1,-0.00000155,-0.00000020,0.00000248,-0.00000129,0.00000182,0.00000281$ ,-0.00000283,0.00000028,-0.00000932,-0.00000293,-0.00000875,-0.0000117 5,0.00000406,-0.00000612,-0.00000094,0.,0.00000138III@

para-Addition Product

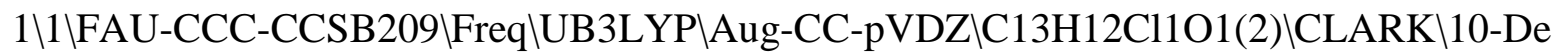
c-2014\0\\N Geom=AllCheck Guess=TCheck SCRF=Check GenChk UB3LYP/Aug-C C-pVDZ Freq \\Addition product p-Cl-Ph-radical + anisol (para, trans) \\ $0,2 \backslash \mathrm{C},-6.2811564878,1.6696615864,1.3125314765 \backslash \mathrm{C},-4.9879119946,0.900291$ 4807,1.460960321\C,-3.7544329177,1.4348702753,1.1744259915\C,-3.630688 $8881,2.7624859353,0.6972701779 \backslash \mathrm{C},-4.8032191644,3.5548504283,0.50802855$ 99\C,-6.0440877665,3.0656408345,0.7805621664\H,-6.9293715552,1.1288085 $432,0.5927470367 \backslash \mathrm{H},-5.0583154922,-0.1238739602,1.8280397341 \backslash \mathrm{H},-2.87060$ $40661,0.8158758801,1.3194441038 \backslash \mathrm{H},-4.676272406,4.5705634065,0.13161539$ $88 \backslash \mathrm{H},-6.9219130841,3.6938025142,0.6253514039 \backslash \mathrm{O},-2.4514178981,3.3790356$ $113,0.3851268637 \backslash \mathrm{C},-8.503134452,1.7255416124,5.0363604799 \backslash \mathrm{C},-9.0229014$ $858,1.0340058206,3.9438822213 \backslash \mathrm{C},-8.3006322194,1.0238415012,2.745219769$ 5\C,-7.0752480901,1.6902836113,2.6281408336\C,-6.5789288203,2.37656929 $33,3.7465538179 \backslash \mathrm{C},-7.2834817561,2.4008978794,4.9513762058 \backslash \mathrm{Cl},-9.401953$ $7121,1.75031152,6.5549763664 \backslash \mathrm{H},-9.9755560276,0.5138512716,4.0269449326$ $\mathrm{H},-8.7067892521,0.4848655752,1.8875935395 \backslash \mathrm{H},-5.6271870768,2.903323002$ 3,3.6754332164\H,-6.8944618596,2.9367118361,5.8156614379\C,-1.24265154 $22,2.6428944581,0.5382873488 \backslash \mathrm{H},-1.2340353217,1.7518053171,-0.108145741$ $1 \mathrm{H},-1.0899423432,2.339725226,1.5855480783 \backslash \mathrm{H},-0.439626637,3.3213482317$ ,0.2344702731॥Version=EM64L-G09RevA.02\State $=2-A \backslash H F=-1038.0527466 \backslash \mathrm{S} 2=$ $0.781265 \backslash \mathrm{S} 2-1=0 . \mathrm{IS} 2 \mathrm{~A}=0.750554 \backslash \mathrm{RMSD}=2.308 \mathrm{e}-09 \backslash \mathrm{RMSF}=6.122 \mathrm{e}-06 \backslash$ ZeroPoint $=$ $0.2121072 \backslash$ Thermal $=0.2255282 \backslash \mathrm{ZPE}=133.0992881 \mathrm{Wipole}=0.8680482,-0.305219$ 7,-0.6458898\DipoleDeriv $=0.0272977,0.0618804,0.0498546,0.0275764,0.012$ 
$0239,0.1101932,-0.023869,0.0869219,0.2779418,0.1935945,-0.2050077,0.00$ $10607,0.037018,-0.0652574,0.0112835,0.0575397,-0.0303963,-0.3123725,-0$ $.2312321,0.1577397,0.0079626,-0.2055927,0.1716844,-0.1261659,0.0977581$ ,-0.1224249,-0.0867569,1.3029241,0.5102857,-0.4215127,0.7603274,-0.001 $6281,-0.1157028,-0.3626657,-0.0335125,0.0878732,-0.0489914,-0.3157867$, $0.1301975,-0.0909583,0.1328058,-0.0273613,0.0300329,-0.0577123,-0.1303$ $186,-0.0140138,0.1930129,-0.0586828,0.0866391,0.0561712,-0.2194574,0.0$ $499358,-0.0850448,-0.2290761,-0.1176214,-0.101521,-0.0539045,-0.109047$ $6,0.0123506,-0.0503626,-0.0454945,-0.0486798,-0.0078597,0.0318935,-0.0$ $05643,0.0002977,-0.0137984,-0.0639319,0.0631844,-0.0057196,0.0744323,0$ $.1098469,-0.0559863,0.0690194,0.0159571,0.0758477,0.0478323,0.0274129$, $-0.0348316,0.020138,0.1377097,0.0601128,-0.0216594,0.0314302,-0.030959$ $9,-0.0443007,0.0842979,-0.0031151,0.0610926,0.1206154,-0.0439209,0.060$ $8011,-0.0206581,0.0596973,0.0046314,0.0382326,-0.0178456,0.0245107,0.1$ 246826,-1.9218544,-0.2690152,0.4199995,-0.3195808,-0.4916542,0.1727785 $, 0.2497529,0.1170482,-0.3127479,0.3305269,0.0271189,-0.5196645,-0.0014$ $638,-0.0259156,0.0565129,-0.6145755,0.0126618,0.9522575,-0.0447166,0.0$ $627593,0.0847418,0.0705365,-0.0602472,-0.0192917,0.1242779,-0.0013135$, $-0.2914415,0.0082639,0.0648401,-0.0023071,0.1040448,-0.0402466,0.03322$ $32,0.1101628,0.0832694,-0.0105436,0.055493,-0.0030144,-0.1382069,-0.04$ $14504,-0.0542627,-0.0009819,-0.2401205,-0.0526799,0.1474461,-0.0559002$ $, 0.0390072,0.0516031,0.0377694,-0.0514874,0.0214913,0.0565975,0.019838$ $8,-0.1005846,-0.1086314,0.0256223,0.1064771,0.0348656,-0.0474797,0.052$ $7005,0.1334401,0.0666574,-0.1157424,-0.3349499,-0.014605,0.3641341,-0$. $0015986,-0.0799218,-0.015025,0.4189598,0.0054268,-0.7045738,-0.0135338$ ,-0.0696486,0.0293122,-0.0678154,0.0885075,-0.0023824,0.0393335,0.0014 $927,0.0733672,0.0766484,-0.0324496,-0.0202446,-0.0408835,0.0694009,-0$. $0691697,-0.0524668,-0.0845018,-0.0445255,-0.0250653,-0.0634679,0.01761$ $85,-0.0698961,0.0628626,0.0059958,-0.0021102,0.00118,0.0941119,0.07656$ $76,-0.0345437,-0.0177662,-0.0309216,0.0867719,-0.0593518,-0.0070359,-0$ $.0543625,-0.0096076,0.9804331,-0.0333314,-0.1141742,-0.4208621,0.29981$ $82,0.0837319,0.0887202,0.0098998,0.3027595,-0.0186333,-0.0438047,-0.02$ $40375,0.1440823,-0.0630731,-0.1151954,0.0034477,-0.0901283,-0.0207155$, $-0.0198395,-0.0070449,0.0381574,0.1189036,0.0397206,0.0170189,-0.10464$ $74,0.0434486,-0.119814,-0.0888653,-0.0515436,0.0423551,-0.1124789,0.00$ $48252,0.0423905,0.0545386,0.0327377,0.0680683 \backslash$ Polar $=224.3215085,22.448$ $454,142.8530824,-51.5646357,-7.0698165,183.2322233 \backslash \mathrm{PG}=\mathrm{C} 01$ [X(C13H12Cl1 O1)]WNImag $=0 \backslash 10.48473256,0.02750023,0.48321358,0.04358779,0.01205903,0$ $.43468089,-0.15859299,0.05251619,-0.01436852,0.61755717,0.04434691,-0$. $11911199,0.01657874,0.06632243,0.59052864,-0.01197879,0.01456202,-0.06$ $638735,-0.06640879,-0.17219050,0.18292323,-0.02908241,-0.00272817,0.00$ $356665,-0.33251163,-0.09924001,0.05869783,0.71191748,0.00700221,0.0148$ $6257,-0.00512720,-0.05228164,-0.12605885,0.03221178,-0.06383949,0.5534$ $6145,0.00074583,-0.00328305,0.00709048,0.04111124,0.03562994,-0.061052$ $07,-0.02645625,-0.14964624,0.16497384,0.00177859,0.00571314,-0.0025486$ $5,0.01987576,-0.02329570,0.00697552,-0.13050554,-0.03758943,0.01948330$ $, 0.61785864,0.00577058,-0.00784864,-0.00076569,-0.07874295,-0.04428425$ $, 0.02450501,0.06721171,-0.21981664,0.05367714,0.01246402,0.54110619,-0$ $.00289894,-0.00094245,-0.00882602,0.02685915,0.01985951,-0.00132962,-0$ $.01716340,0.06283681,-0.07139427,-0.04533878,-0.15475869,0.16404020,-0$ $.00196542,-0.01643969,0.00534684,-0.03256825,-0.01581706,0.00840722,0$. 
$03890261,0.05968297,-0.02366336,-0.16872272,0.01188361,0.00481836,0.65$ $578795,-0.02714933,-0.00860833,0.00812392,0.00718820,0.00436852,-0.002$ $31353,0.01717181,-0.03388991,0.01219415,0.10527304,-0.16055782,0.02870$ $909,0.06165626,0.64127570,0.01024148,0.00660454,0.00295127,-0.00000806$ ,-0.00059069,-0.00011045,-0.00879707,0.00930857,0.00263057,-0.02893143 $, 0.03640537,-0.06652716,-0.06443832,-0.19357443,0.19193418,-0.08813713$ ,-0.01188833,0.00410385,0.02098478,0.03480861,-0.01398543,-0.01747962, $-0.02735005,0.01090001,-0.07126959,0.04540129,-0.00988107,-0.35508573$, $-0.13118338,0.07028532,0.76066495,-0.00470632,-0.18090414,0.03891868,0$ $.01078716,-0.01999943,0.00532632,-0.00632590,-0.00853961,0.00372232,-0$ $.00080164,0.03248141,-0.00856934,-0.09187821,-0.15415877,0.04561070,-0$ $.00298719,0.50643882,0.00004672,0.03970441,-0.07891912,-0.00596286,0.0$ $0433240,-0.00183164,0.00369435,0.00535416,-0.00210508,0.00635543,-0.01$ $259448,0.01166042,0.05741967,0.04904975,-0.07013229,-0.05107555,-0.137$ $23706,0.18163732,-0.11474122,-0.06399153,-0.09492812,-0.02576470,-0.00$ $857305,-0.01422158,0.00043651,0.00452249,-0.00302601,0.00029754,-0.000$ $71416,0.00009505,0.00165853,-0.00035765,-0.00050253,-0.00326107,-0.009$ $01104,0.00097383,0.13781163,-0.06082247,-0.09897081,-0.07459282,0.0100$ $1707,0.01208722,0.00937704,0.00021767,-0.00311770,0.00114275,-0.001860$ $27,0.00299805,-0.00115988,0.00540350,-0.00447881,-0.00005203,-0.022034$ $04,-0.01884791,-0.01333674,0.07176260,0.10969542,-0.08499275,-0.070374$ $55,-0.13711403,-0.00406631,0.00046248,0.00142456,0.00104588,0.00163382$ ,-0.00173998,0.00026782,-0.00111354,0.00008861,-0.00014439,0.00122594, $-0.00093682,0.00837063,0.00550995,0.00971998,0.09181713,0.07915975,0.1$ $5891969,0.00357671,0.02425511,-0.00736602,-0.05596934,-0.01464210,0.00$ $778035,0.00112738,-0.02669172,0.00971287,-0.00413350,-0.00105683,0.001$ $36054,-0.00119506,0.00020372,-0.00011953,-0.00475537,0.00169295,0.0000$ $9882,0.00070242,-0.00068814,-0.00035116,0.05806520,-0.00322742,-0.0100$ $0074,0.00416270,-0.01730145,-0.30222501,0.09783707,0.00191653,-0.00821$ $073,0.00442312,-0.00208241,0.00317479,0.00145028,0.00057339,0.00020097$ ,-0.00036146,0.00291453,0.00026408,0.00029459,0.00048136,0.00016813,0. $00034059,0.01761712,0.31709042,0.00143748,0.00238907,-0.00013626,0.008$ $52631,0.09897775,-0.06150367,-0.00058019,0.00629759,0.00243240,0.00147$ $284,0.00134857,0.00576100,-0.00009658,-0.00021834,-0.00062246,-0.00048$ $804,0.00022730,0.00157908,0.00068906,-0.00025812,0.00042620,-0.0098337$ $6,-0.10698581,0.05585664,-0.00136872,-0.00311176,0.00189409,-0.0201524$ $6,0.01756257,-0.00419277,-0.23903948,0.13181210,-0.02930635,0.00462765$ ,-0.00164051,0.00036931,0.00127935,-0.00107802,0.00063018,0.00009009,-$0.00048256,0.00015422,-0.00051906,0.00023488,-0.00002217,0.00111149,-0$ $.00025062,-0.00037275,0.25859603,-0.00199165,0.00056061,0.00316463,-0$. $01065898,0.01065425,-0.00159072,0.13053692,-0.14987946,0.02989988,0.02$ $577593,-0.00869312,0.00185871,-0.00183090,-0.00430387,0.00321930,-0.00$ $101189,-0.00024635,-0.00003253,0.00012314,-0.00018045,-0.00004662,-0.0$ $0017226,0.00033499,-0.00197081,-0.14032497,0.15104722,0.00224047,0.003$ $09081,0.00769225,0.00574006,-0.00351204,0.00393730,-0.02935436,0.02960$ $070,-0.04172117,-0.00911528,0.00428698,0.00178573,0.00080689,0.0032959$ $4,0.00324892,0.00030834,-0.00012093,-0.00063676,0.00102384,-0.00093797$ $, 0.00003642,-0.00054481,-0.00205959,-0.00462945,0.02983191,-0.03323611$ $, 0.03285384,-0.00384151,0.00006851,0.00112141,-0.00079050,-0.00014661$, $0.00013215,-0.00405799,0.00242862,-0.00030464,0.00662610,0.02290310,-0$ $.00848039,-0.05986799,-0.02675685,0.01150516,0.00089344,-0.02770279,0$. 
$01033553,-0.00009540,-0.00047183,0.00014949,0.00012301,-0.00003346,-0$. $00002874,0.00014997,0.00129577,-0.00043967,0.05789658,-0.00064926,0.00$ $318004,0.00254983,0.00031011,0.00054217,-0.00043043,0.00336241,-0.0000$ $3667,0.00098702,-0.00607994,-0.01341914,0.00656208,-0.02555585,-0.2980$ $2412,0.09679357,0.00041502,-0.00659526,0.00354200,-0.00010253,0.000248$ $11,-0.00012938,-0.00006825,-0.00010745,-0.00012028,-0.00022676,-0.0006$ $6910,0.00031153,0.03001673,0.31396085,0.00170832,0.00304074,0.00884669$ ,-0.00009528,-0.00035760,-0.00039529,-0.00057049,0.00090596,0.00304711 $, 0.00218326,0.00420181,0.00196340,0.01061178,0.09678363,-0.07175004,0$. $00048143,0.00554465,0.00158273,0.00006283,0.00147086,-0.00076868,-0.00$ $003596,-0.00015887,-0.00042801,0.00012173,0.00016186,0.00004531,-0.013$ $53274,-0.10679681,0.06429256,0.00585382,-0.00487961,0.00228782,0.00051$ $317,-0.00121399,0.00069566,-0.00005128,-0.00053596,0.00009855,-0.00148$ $946,-0.00344359,0.00219495,-0.01890207,0.01850999,-0.00468488,-0.23769$ $228,0.13035127,-0.03087287,0.00026187,0.00059472,-0.00032953,0.0004315$ $0,-0.00020536,-0.00000446,-0.00002029,0.00008431,-0.00011018,0.0009625$ $1,-0.00030693,-0.00052258,0.25125295,0.02235013,-0.01182672,0.00376684$ $,-0.00251729,-0.00400222,0.00234143,-0.00096753,-0.00090555,0.00011281$ ,-0.00269796,0.00153966,0.00258203,-0.01071257,0.00894481,-0.00053841, $0.13323711,-0.14623739,0.03182037,-0.00010546,0.00073305,-0.00066316,0$ $.00152374,-0.00074194,0.00010325,0.00005269,0.00001199,-0.00022539,-0$. $00002081,0.00029536,-0.00228381,-0.13972334,0.15109638,-0.00757093,0.0$ $0486272,-0.00031892,0.00096041,0.00226022,0.00086176,0.00037022,0.0000$ $1932,-0.00066427,0.00166054,0.00268506,0.00699310,0.00554367,-0.002804$ $58,0.00561602,-0.03111717,0.03125408,-0.03866870,0.00028741,0.00072733$ $, 0.00035427,-0.00061978,0.00023465,-0.00011024,-0.00006667,-0.00023280$ ,-0.00054470,-0.00053599,-0.00222042,-0.00521053,0.03165562,-0.0363214 $1,0.03009499,-0.00180259,-0.00193844,0.00004123,-0.00335705,-0.0048907$ $7,0.00256544,-0.00704256,-0.00219090,0.00198368,-0.23466341,-0.0995356$ $0,0.05212009,-0.05752624,-0.01944915,0.01190820,0.00185036,0.00767455$, $-0.00233230,-0.00162709,-0.00023766,0.00004973,-0.00059282,-0.00002793$ ,-0.00003597,-0.00047677,-0.00058627,0.00006629,0.00204496,-0.00092612 ,-0.00005834,-0.00158525,-0.00048492,0.00022237,0.54351345,-0.00091907 ,-0.00062582,-0.00024487,0.00641766,-0.00459924,0.00227245,-0.04149873 ,-0.01464027,0.01006526,-0.05843688,-0.13852951,0.04551960,0.02021584, $0.01453176,-0.00600108,-0.00010652,-0.00368368,0.00204038,-0.00065025$, $-0.00018924,0.00007650,-0.00181224,-0.00054836,0.00047725,0.00089496,0$ $.00145984,-0.00130748,-0.00062888,0.00107376,-0.00108761,0.00111444,0$. $00053059,-0.00003161,-0.00484710,0.28671346,0.00027423,0.00010534,-0.0$ $0057844,-0.00174729,0.00307876,0.00196785,0.01558459,0.00677289,0.0004$ $8791,0.03805437,0.04889061,-0.04260208,-0.00242904,-0.00282085,0.00300$ 139,0.00003343,0.00118605,0.00085447,0.00029508,0.00000728,0.00010810, $0.00072584,0.00042285,0.00049032,-0.00045042,-0.00112383,-0.00151698,-$ $0.00010850,-0.00102775,-0.00156123,-0.00019330,0.00014937,0.00085571,-$ $0.03885128,-0.07902292,0.08904640,-0.00128058,-0.00041337,0.00024077,0$ $.00001702,0.00020885,0.00051245,0.00009373,0.00019378,0.00004977,-0.00$ $008530,-0.00021785,0.00024434,-0.00002582,0.00010445,0.00002898,-0.000$ $07876,0.00008819,0.00033961,0.00003072,-0.00001868,0.00023890,-0.00011$ $847,0.00004397,-0.00000502,0.00007270,-0.00004238,-0.00012376,0.000056$ $26,0.00001515,-0.00009856,-0.00002671,-0.00001554,-0.00005397,0.000434$ $62,0.00015291,-0.00008372,0.49216552,-0.00049124,-0.00071632,0.0001461$ 
$8,-0.00004450,0.00008423,0.00048497,0.00012503,0.00013998,0.00006894,0$ $.00020522,-0.00000302,0.00003375,-0.00012366,0.00001929,0.00014625,0.0$ $0007442,0.00020262,0.00026087,-0.00005328,0.00014765,0.00020753,-0.000$ $04121,-0.00002220,-0.00010374,0.00002483,-0.00001693,-0.00010087,-0.00$ $000981,-0.00004864,-0.00012583,-0.00004487,-0.00002964,0.00000442,-0.0$ $0009182,-0.00000990,0.00001525,0.21039588,0.29343043,0.00001180,-0.000$ $14886,-0.00059192,0.00014327,0.00008169,0.00047658,-0.00013574,0.00008$ $416,0.00005823,0.00079783,0.00036975,-0.00013314,-0.00015168,-0.000092$ $66,0.00003821,0.00001100,0.00027905,0.00034104,0.00006955,0.00006980,0$ $.00029827,-0.00001980,-0.00007148,-0.00006665,-0.00005756,-0.00001065$, $-0.00011919,-0.00003045,-0.00005926,-0.00006230,-0.00004591,0.00004550$ $,-0.00013313,-0.00098986,-0.00024503,0.00017978,-0.00506331,0.12978159$ $, 0.49518116,-0.00071603,-0.00505130,-0.00059346,0.00054721,0.00031309$, $-0.00010934,-0.00008193,-0.00028516,0.00014943,0.00007563,0.00029209$, $0.00017241,0.00031287,-0.00033315,0.00019659,-0.00106891,0.00040622,0$. $00103247,0.00022534,0.00020979,0.00028898,-0.00012427,0.00005131,-0.00$ $003305,0.00001615,0.00001964,0.00000650,-0.00008180,-0.00004836,-0.000$ $11084,-0.00006475,-0.00007738,-0.00000267,-0.00015425,-0.00002170,0.00$ $003652,-0.13962925,-0.07615720,-0.09179328,0.60801117,-0.00446336,0.00$ $387802,-0.00214412,-0.00094404,-0.00065073,0.00105479,-0.00000458,0.00$ $046118,-0.00018204,0.00009091,-0.00021836,0.00007580,-0.00027705,0.000$ $27856,-0.00023479,0.00097108,-0.00012053,-0.00056981,0.00004057,0.0000$ $0077,0.00004510,-0.00003019,0.00002616,0.00002722,-0.00008167,-0.00006$ $294,-0.00000556,0.00008267,0.00009464,0.00010986,0.00003850,-0.0000616$ $0,0.00001301,-0.00006011,-0.00003365,0.00002672,-0.05713367,-0.1377326$ $2,-0.11211494,0.24731641,0.31800086,-0.00002935,-0.00173226,0.00186428$ $, 0.00027877,0.00007824,-0.00122554,0.00008986,-0.00044840,0.00000254,-$ $0.00056339,-0.00001623,-0.00003705,0.00026719,-0.00004679,-0.00009761$, $-0.00030209,-0.00025652,-0.00015188,-0.00009592,-0.00010866,-0.0002122$ $3,0.00010622,0.00005501,0.00001995,0.00003235,0.00007061,0.00022368,-0$ $.00000897,0.00002081,0.00010512,0.00008953,-0.00003413,0.00007023,0.00$ $031533,0.00004191,-0.00004834,-0.03853740,-0.08140001,-0.22917771,-0.0$ $8002882,0.12519626,0.60956679,-0.01454664,-0.00820424,0.00765502,0.000$ $15939,-0.00167731,0.00002087,0.00004709,0.00051118,-0.00027538,-0.0002$ $0253,-0.00005944,0.00008739,0.00052495,-0.00055699,0.00006412,-0.00103$ $276,0.00118607,-0.00097569,0.00021987,-0.00061695,-0.00261807,0.000081$ $46,0.00031537,0.00004925,-0.00012682,-0.00004230,0.00009141,-0.0001782$ $5,0.00003064,0.00017723,0.00011145,-0.00041253,0.00030359,0.00015872,-$ $0.00000008,-0.00000283,0.05020617,0.03417276,0.02585189,-0.16709466,-0$ $.03380326,0.07490396,0.54784084,-0.00185016,0.00428368,0.00121386,0.00$ $063464,-0.00086755,-0.00101479,0.00016426,0.00024588,0.00006236,-0.000$ $29857,-0.00006682,0.00001975,0.00011884,0.00016532,-0.00011311,0.00023$ $695,-0.00006394,-0.00062282,0.00108664,0.00017436,-0.00114036,-0.00029$ $200,0.00016407,0.00007205,0.00000217,0.00000728,0.00016905,0.00002088$, $-0.00001537,0.00002163,0.00010883,0.00008133,0.00007204,0.00042749,0.0$ $0014778,-0.00008175,0.01765991,0.01474542,-0.00702909,-0.01426646,-0.0$ $8200976,-0.03174964,0.21791305,0.31562178,0.02507530,0.01160645,-0.004$ $12534,0.00086593,0.00160053,-0.00164752,0.00061480,-0.00052129,0.00017$ $335,-0.00027709,-0.00060892,0.00027223,-0.00038922,0.00118509,-0.00017$ $559,0.00245774,-0.00091991,-0.00201557,0.00263812,0.00208338,0.0013949$ 5,0.00012906,-0.00034923,0.00010966,0.00028621,0.00007836,-0.00003509, 
$0.00026658,0.00018618,0.00009753,-0.00025043,0.00021518,-0.00010082,0$. $00090238,0.00029708,-0.00021610,-0.02091054,-0.03400297,-0.05453332,0$. $12981208,-0.00004330,-0.27202462,-0.05751635,0.15442478,0.66120427,-0$. $08987593,-0.00034732,0.04112812,-0.02755643,0.00496653,0.02381385,0.00$ $071484,0.00436451,-0.00045526,-0.00091563,-0.00110710,0.00030437,0.001$ $98175,0.00074805,0.00059995,-0.00680301,-0.01112329,0.01045751,0.00478$ $995,-0.00231994,-0.01396431,0.00125897,-0.00094061,-0.00058670,-0.0003$ $7722,-0.00048134,-0.00092064,0.00014711,-0.00009212,-0.00022525,0.0006$ $4467,0.00074012,-0.00105538,0.00037971,0.00004841,0.00008214,-0.038942$ $78,-0.02701129,-0.02389117,-0.02508671,0.00385682,0.06257221,-0.263188$ $01,-0.11656031,-0.00750237,0.54000480,-0.00358134,-0.07150765,-0.00340$ $307,0.00830000,0.00237467,-0.01234632,-0.00038841,-0.00315514,0.000818$ $14,-0.00142678,0.00257546,-0.00044784,0.00399393,-0.00437530,0.0012820$ $9,-0.01948990,-0.00048163,0.02654335,0.00564461,-0.00178789,-0.0158587$ $3,0.00015632,0.00053944,-0.00021968,0.00029773,0.00035684,0.00054229,-$ $0.00079352,-0.00075060,-0.00125393,-0.00013897,0.00049565,-0.00034708$, $0.00015658,0.00015837,0.00001734,-0.02723164,-0.02832066,-0.01568008,-$ $0.00977912,0.01625888,0.04215099,-0.09744120,-0.13052892,-0.02359397,0$ $.19754365,0.33116636,0.02820841,-0.00898974,-0.14062591,0.01115114,-0$. $00781168,0.00447131,-0.00244442,0.00135688,-0.00168016,0.00235855,-0.0$ $0021016,0.00035224,-0.00327033,-0.00135168,-0.00089789,0.00999521,0.01$ $358771,-0.01396902,0.01225910,-0.00309778,-0.02763612,-0.00127142,0.00$ $107502,0.00074610,-0.00069077,-0.00030093,-0.00005489,-0.00032399,-0.0$ $0005773,0.00047208,-0.00001323,-0.00128579,0.00147924,-0.00133652,-0.0$ $0035400,0.00021308,-0.02457496,-0.01632722,-0.01249007,0.02392517,0.01$ $983785,0.02370134,0.04652930,0.00792764,-0.11943617,-0.03901100,0.1187$ $7347,0.56717170,0.00933262,0.00904666,0.01343808,0.00115303,0.00019741$ $, 0.00077875,-0.00031324,0.00023259,0.00031007,0.00029216,0.00009058,-0$ $.00021416,-0.00067776,-0.00051249,-0.00006243,0.00134631,0.00064559,0$. $00030245,-0.00101857,0.00028262,0.00425996,0.00006042,-0.00016878,0.00$ $003013,-0.00005887,-0.00004812,-0.00014446,-0.00005630,-0.00004351,-0$. $00008028,0.00003648,-0.00006035,0.00007215,-0.00065974,-0.00026038,0.0$ $0017290,-0.02723656,-0.01172063,0.02303654,-0.00202682,0.00183650,0.00$ $335842,-0.01199378,-0.01963657,-0.02361909,-0.13779672,-0.06699312,-0$. $07414960,0.59661595,0.00301286,0.00279490,-0.00251954,-0.00030852,0.00$ $076752,-0.00042515,-0.00050062,-0.00030409,-0.00030021,-0.00005260,-0$. $00013937,-0.00025582,-0.00010631,0.00002497,0.00051654,-0.00006355,-0$. $00006520,0.00057015,-0.00042313,0.00111959,0.00064937,-0.00002426,0.00$ $009403,-0.00002325,-0.00005130,0.00003290,0.00001994,-0.00004439,0.000$ $01458,-0.00006885,-0.00009622,0.00034077,-0.00015595,-0.00024570,-0.00$ $003454,0.00006264,0.00464319,0.01664411,0.01860968,0.00166129,-0.00846$ $365,-0.01798123,-0.03257471,-0.01249387,-0.00377658,-0.04977632,-0.132$ $85085,-0.10305548,0.23498744,0.31552866,-0.00319321,-0.01203466,-0.026$ $37639,-0.00247777,0.00073651,-0.00085357,-0.00008266,-0.00042218,0.000$ $10941,-0.00077818,-0.00010022,-0.00016800,-0.00027047,-0.00009578,-0.0$ $0027321,-0.00066164,-0.00278006,-0.00015942,0.00218077,-0.00055289,-0$. $00423426,0.00038165,-0.00037318,0.00005978,0.00004065,0.00004113,0.000$ $16986,0.00003035,0.00002540,0.00010308,0.00003405,0.00022218,-0.000109$ $80,0.00035473,0.00002329,-0.00000486,0.06951808,0.04559651,0.02271563$, $0.00309154,-0.01836259,-0.06008071,-0.05973010,-0.02455624,0.03604567$, $-0.02439058,-0.07430783,-0.23493657,-0.08902725,0.12175318,0.61861777$, 
$0.00116124,-0.00335466,-0.00235700,-0.00020755,-0.00015535,-0.00042638$ ,-0.00001386,-0.00014906,-0.00003496,-0.00009789,0.00012344,-0.0002361 $5,0.00019875,-0.00017227,-0.00005346,-0.00043592,-0.00014434,0.0001912$ $2,0.00022492,-0.00028951,-0.00117704,0.00009093,0.00001005,0.00002602$, $-0.00005693,0.00003900,0.00010022,-0.00008468,-0.00003168,0.00007204,0$ $.00006613,0.00007565,0.00011653,0.00000574,-0.00001449,0.00000953,-0.2$ $6129846,-0.10230518,0.03156526,-0.01047613,-0.03252473,-0.05929937,-0$. $04359189,-0.01347921,0.02750110,0.04914445,0.03207152,0.02282654,-0.16$ $567435,-0.03248758,0.07639960,0.54378551,-0.00363571,0.00224885,-0.003$ $38721,-0.00043413,-0.00005616,0.00044223,-0.00022879,0.00000203,-0.000$ $08111,0.00002705,0.00006500,-0.00003354,0.00024761,0.00022516,0.000028$ $88,-0.00003133,-0.00030041,0.00012060,0.00033546,-0.00004073,-0.000951$ $48,0.00011553,-0.00002804,0.00009482,-0.00004946,-0.00003982,-0.000030$ $68,0.00003788,0.00001038,0.00005167,0.00001087,-0.00000478,-0.00008607$ $, 0.00000135,0.00002321,-0.00001435,-0.12162631,-0.13377847,0.00411033$, $-0.01901925,-0.01105790,-0.02506420,-0.01393263,-0.00799915,0.01009630$ $, 0.01858855,0.01536971,-0.00710327,-0.01300642,-0.08167636,-0.03527229$ $, 0.22100697,0.31847646,-0.00287136,-0.00339469,0.00013218,-0.00018474$, $0.00042558,0.00060486,0.00029561,0.00014546,0.00027740,0.00014192,-0.0$ $0006293,-0.00002555,0.00007425,0.00023939,0.00018191,-0.00019517,-0.00$ $011477,0.00083401,0.00044166,-0.00001226,-0.00036157,-0.00004435,-0.00$ $006374,-0.00014505,0.00008857,-0.00002363,-0.00013708,0.00003948,-0.00$ $002918,-0.00015469,-0.00001739,0.00005515,-0.00012396,0.00008982,0.000$ $05923,-0.00002809,-0.02199373,-0.02692716,-0.10460581,-0.02187576,-0.0$ $0345464,0.03494981,0.02586010,0.00930473,-0.01961310,-0.01588673,-0.02$ $966480,-0.04978649,0.13187137,-0.00330115,-0.28301259,-0.05029337,0.16$ $575879,0.67377425,-0.00006516,0.00032221,-0.00031238,0.00008041,-0.000$ $16344,0.00033482,-0.00013494,0.00004640,-0.00003314,0.00043446,0.00020$ $084,-0.00010397,-0.00002371,-0.00005358,0.00006165,-0.00005316,0.00028$ $064,0.00015719,-0.00002302,0.00001266,0.00008305,0.00002557,-0.0000096$ $0,-0.00003475,-0.00005750,-0.00002161,-0.00003326,-0.00003796,-0.00004$ $217,-0.00002555,0.00002051,-0.00000107,-0.00003701,-0.00056956,-0.0001$ $4945,0.00010225,-0.06482563,-0.00556390,0.05099495,0.00256316,-0.00051$ $109,0.00149154,0.00105573,-0.00209568,0.00038392,0.00060690,0.00116029$ $, 0.00129445,-0.00157189,-0.00486468,-0.00377132,-0.01654118,0.00027294$ $, 0.03460134,0.07905279,0.00038080,-0.00043674,0.00018903,0.00004658,0$. $00006940,-0.00007277,0.00005208,-0.00000974,0.00004533,-0.00001708,-0$. $00001900,-0.00001459,-0.00000651,-0.00001101,-0.00002604,-0.00003318,0$ $.00000645,0.00001136,0.00000204,0.00002561,0.00006714,-0.00000871,-0.0$ $0000018,-0.00000095,0.00001315,0.00000402,0.00000775,-0.00000291,0.000$ $00164,-0.00000231,-0.00000359,0.00000607,-0.00000439,0.00001621,0.0000$ $1330,-0.00000172,-0.00554278,-0.02878215,-0.00528446,0.00755669,0.0038$ $1887,-0.01375981,-0.00402135,0.00209553,-0.00128747,0.00109962,0.00015$ $117,0.00063080,-0.00291700,0.00190306,-0.00274111,-0.00782869,0.004260$ $47,0.01216412,0.00823960,0.01965750,-0.00001660,0.00033341,0.00036560$, $-0.00014420,0.00024202,-0.00052612,0.00022464,-0.00011350,0.00001175,-$ $0.00077013,-0.00034256,0.00014428,0.00011118,0.00023282,-0.00006028,0$. $00021317,-0.00053200,-0.00030216,0.00002435,0.00002782,0.00003019,-0.0$ $0004179,0.00000046,0.00005341,0.00010096,0.00002263,0.00005647,0.00007$ $193,0.00007916,0.00004201,-0.00003884,-0.00001226,0.00006441,0.0009979$ $1,0.00025780,-0.00016860,0.05102925,-0.00525056,-0.12050670,0.02400509$ 
,-0.00077207,-0.03649430,-0.00500019,-0.00441514,0.00063019,0.00094749 $, 0.00050275,-0.00105644,0.00179195,0.00046093,0.00296691,0.01208525,-0$ $.00088080,-0.01746874,-0.08547356,0.00824297,0.17264384,-0.00017428,-0$ $.00030435,-0.00039288,-0.00003909,-0.00008592,0.00002968,-0.00003669,0$ $.00001558,0.00003815,0.00004284,0.00010311,-0.00003836,0.00008817,-0.0$ $0005253,0.00002261,-0.00012403,0.00003585,0.00016669,0.00001263,-0.000$ $00707,-0.00002443,-0.00001420,0.00001004,-0.00000773,-0.00002130,0.000$ $00010,0.00000268,-0.00001221,-0.00001609,-0.00001650,0.00000440,-0.000$ $01056,0.00000218,-0.00013469,-0.00003786,0.00002463,-0.00683381,-0.004$ $25538,0.00461276,-0.27097388,-0.12905395,0.01954998,-0.00861678,-0.007$ $56467,-0.00127674,0.00203986,-0.00152272,0.00354532,0.00019368,0.00080$ $055,-0.00008799,0.00214128,-0.00243253,-0.00138211,0.00026476,0.001671$ $74,-0.00021541,0.28283348,0.00011690,0.00008350,-0.00016260,-0.0000690$ $2,0.00003598,0.00010149,0.00007243,0.00008862,-0.00002475,0.00004218,-$ $0.00006179,-0.00001081,-0.00003439,0.00000981,0.00003203,0.00002950,-0$ $.00000657,-0.00014015,-0.00000311,0.00000449,-0.00000477,0.00000469,-0$ $.00000155,-0.00000039,-0.00000368,0.00000301,-0.00002359,-0.00001093,0$ $.00000680,0.00002063,-0.00000503,-0.00001150,0.00000837,-0.00003949,-0$ $.00001830,0.00001409,-0.01359540,-0.00340386,0.00291159,-0.12817317,-0$ $.10762702,0.00419149,0.00117987,0.00396418,-0.00109346,-0.00173855,0.0$ $0531857,-0.00168952,0.00081443,-0.00078008,-0.00003063,-0.00220382,0.0$ $0280876,-0.00396274,0.00138810,-0.00153205,0.00113540,0.14075603,0.104$ $27852,0.00074201,0.00054095,0.00027984,0.00003128,0.00000779,-0.000198$ $57,0.00004837,-0.00002238,0.00003345,-0.00011060,-0.00001742,-0.000009$ $69,0.00003220,0.00004030,-0.00002679,0.00013694,-0.00009586,-0.0001077$ $0,-0.00002143,-0.00000591,0.00002178,0.00001314,-0.00002037,0.00000900$ $, 0.00001273,0.00001172,0.00000838,0.00001542,0.00001906,0.00001201,-0$. $00001031,0.00002394,-0.00000191,0.00013462,0.00003919,-0.00002635,-0.0$ $2138340,-0.01207232,0.00417708,0.02193640,0.00558041,-0.05501087,0.023$ $15500,0.01297770,0.00261690,0.00313511,-0.00194623,-0.00359082,0.00001$ $141,0.00003083,-0.00116311,-0.00082296,-0.00363265,-0.00333947,-0.0010$ $3766,0.00064227,0.00076034,-0.02385947,-0.00279413,0.05471557,-0.00029$ $856,0.00134128,-0.00276006,-0.00015367,-0.00015641,0.00044183,-0.00005$ $188,0.00007505,0.00000166,-0.00004139,-0.00008492,0.00005468,-0.000105$ $09,0.00022045,-0.00005956,0.00050273,0.00009424,0.00011607,0.00018613$, $0.00030794,0.00107995,-0.00008290,0.00001785,-0.00004271,0.00000102,-0$ $.00003676,-0.00004195,0.00004497,0.00000966,-0.00003362,-0.00001841,0$. $00002548,-0.00005975,0.00017029,0.00007306,-0.00003303,0.00091049,-0.0$ $0223587,0.00350941,0.00871615,0.00804019,0.01401285,-0.09104829,-0.059$ $49918,-0.07821062,-0.00531403,-0.01304000,-0.02141327,-0.00333617,-0.0$ $0484053,0.00193404,-0.00089836,0.00032315,0.00064952,-0.00009495,0.000$ $22179,0.00102093,-0.00025150,0.00189633,-0.00100198,0.09162250,0.00235$ $078,-0.00268958,-0.00017408,0.00043833,0.00053230,-0.00022390,0.000121$ $22,-0.00026911,0.00007251,-0.00005025,0.00003767,-0.00002218,0.0001084$ $1,-0.00011787,0.00009173,-0.00053933,0.00005505,0.00040909,0.00006629$, $0.00043690,0.00074881,0.00007462,-0.00005691,-0.00003574,0.00005775,0$. $00002854,0.00000449,-0.00002332,-0.00002354,-0.00006986,-0.00005868,-0$ $.00009264,0.00001103,0.00005522,0.00000327,-0.00000070,-0.00208832,0.0$ $0540059,-0.00090542,-0.00097672,0.00323505,0.00089107,-0.05957868,-0.1$ $1038495,-0.11209125,-0.00464682,-0.00313847,-0.01266040,-0.00518363,0$. $00312969,-0.00041389,0.00023654,-0.00062548,0.00075958,-0.00011464,0.0$ 
$0012664,0.00005614,0.00199459,-0.00262903,0.00107439,0.06759954,0.1077$ $8399,0.00085059,0.00200826,0.00032827,-0.00031985,0.00004290,0.0001519$ $3,0.00004407,0.00010728,-0.00000244,0.00007656,0.00008802,-0.00008858$, $0.00005909,0.00007269,-0.00001860,0.00025780,-0.00020233,0.00009103,-0$ $.00019103,-0.00012692,0.00028590,-0.00006618,0.00002126,-0.00002540,-0$ $.00000445,-0.00000447,-0.00002800,0.00002655,0.00000243,-0.00000443,0$. $00000360,-0.00000196,-0.00002380,-0.00021500,-0.00006757,0.00005470,0$. $00383474,-0.00073838,-0.00132339,-0.01126724,-0.01366107,-0.01666976,-$ $0.07843816,-0.11227637,-0.22500404,0.00201301,0.00081777,0.00298744,0$. $00111188,-0.00081265,0.00205679,0.00051207,0.00067513,-0.00000326,0.00$ $010000,-0.00047495,-0.00132360,-0.00072717,0.00124410,0.00050399,0.082$ $21606,0.12306190,0.23805894,-0.00106717,0.00142925,0.00061546,0.000321$ $19,0.00004238,0.00036104,-0.00020757,-0.00020274,-0.00016430,0.0000708$ $1,0.00005714,-0.00001138,0.00020532,0.00021478,0.00023712,0.00003108,0$ $.00024374,0.00017803,-0.00001502,0.00001695,-0.00010979,-0.00001858,0$. $00001621,-0.00001095,-0.00001638,0.00003195,0.00005850,0.00003977,0.00$ $002622,0.00000746,-0.00009372,-0.00000460,-0.00015417,-0.00008600,0.00$ $001637,0.00001174,0.00223511,-0.00178323,0.00318719,0.00023831,0.00078$ $427,-0.00013889,0.00210345,-0.00304498,-0.00138016,-0.00617442,-0.0050$ $9023,0.00202079,-0.26613909,-0.12654884,0.01741562,-0.00905334,-0.0078$ $5504,-0.00100171,-0.00054539,-0.00026446,0.00030198,-0.00023177,0.0002$ $6067,-0.00008501,-0.00032186,-0.00015515,0.00019122,0.27914263,0.00099$ $138,-0.00197001,0.00160303,0.00000880,-0.00005991,0.00001843,0.0004974$ $5,0.00019195,0.00022480,0.00020599,0.00010727,-0.00010802,-0.00025158$, $-0.00013090,-0.00020606,0.00001061,0.00034739,0.00019669,0.00000663,-0$ $.00007150,0.00011415,-0.00002863,-0.00006429,-0.00010691,0.00006189,-0$ $.00000493,-0.00003524,-0.00000827,-0.00002190,0.00010976,0.00007940,-0$ $.00004064,0.00011061,-0.00013907,-0.00011767,0.00004987,-0.00187564,0$. $00551511,-0.00172318,0.00077030,-0.00073309,-0.00005889,-0.00274077,0$. $00327512,-0.00431814,-0.01352422,-0.00340403,0.00220555,-0.12673678,-0$ $.10939372,0.00276052,0.00121243,0.00409359,-0.00150174,0.00008980,0.00$ $008791,-0.00015719,0.00024801,-0.00050648,0.00021955,-0.00065740,-0.00$ $025714,0.00011338,0.13977398,0.10590581,-0.00081179,0.00068697,0.00018$ $010,0.00001109,-0.00003874,0.00000674,-0.00000639,-0.00010614,0.000013$ $58,0.00039405,0.00024203,-0.00014870,0.00051706,0.00030568,-0.00014707$ $,-0.00028576,-0.00025681,-0.00019928,-0.00001763,0.00013844,0.00026237$ ,-0.00000744,0.00012312,0.00003980,-0.00001638,0.00003666,0.00001881,0 $.00003802,0.00001929,0.00001432,-0.00001499,-0.00002218,-0.00004312,-0$ $.00039227,-0.00007092,0.00003651,0.00280895,-0.00187064,-0.00266643,-0$ $.00001278,-0.00004252,-0.00110749,-0.00074297,-0.00390064,-0.00348149$, $-0.02167983,-0.01161524,0.00652521,0.01763011,0.00311224,-0.05642461,0$ $.02417828,0.01299058,0.00130481,0.00125410,0.00040159,-0.00080421,-0.0$ $0010505,0.00021661,0.00002263,-0.00120257,-0.00069605,0.00011109,-0.02$ $078699,-0.00086819,0.05585550,0.00038051,0.00030567,0.00051109,0.00001$ $777,-0.00000709,-0.00000986,-0.00001021,0.00001296,-0.00001987,0.00006$ $564,-0.00003827,0.00000669,-0.00012187,0.00001471,-0.00001258,0.000136$ 29,0.00010684,-0.00012524,-0.00011185,0.00009853,0.00021933,-0.0000046 $3,0.00001706,0.00000521,-0.00000128,-0.00000249,0.00002244,0.00000144$, $-0.00001194,0.00000528,-0.00000190,-0.00000437,-0.00000343,-0.00007505$ ,-0.00001784,0.00000308,-0.00568697,-0.01309881,-0.02193129,-0.0032095 $1,-0.00417713,0.00202304,-0.00081292,0.00032835,0.00065131,0.00048342$, 
$-0.00246829,0.00404538,0.00940245,0.00785528,0.01321095,-0.08840863,-0$ $.05872784,-0.07839524,0.00025377,0.00134614,-0.00090715,-0.00019990,-0$ $.00069018,-0.00103329,-0.00009916,0.00031938,-0.00012702,-0.00042517,0$ $.00198201,-0.00088055,0.08841209,-0.00015329,0.00010655,0.00009056,0.0$ $0017705,0.00001029,-0.00006727,-0.00004742,-0.00009868,-0.00000026,-0$. $00006088,0.00004284,-0.00001290,0.00007049,0.00000556,0.00005192,-0.00$ $011680,-0.00006959,0.00004681,0.00008202,-0.00009462,0.00001943,-0.000$ $00149,-0.00001747,-0.00000729,0.00000507,-0.00000312,0.00000174,0.0000$ $0206,0.00000150,0.00000503,0.00000640,-0.00000794,0.00001133,0.0000613$ $7,0.00000103,-0.00000645,-0.00375836,-0.00345812,-0.01286034,-0.004378$ $38,0.00272823,-0.00007654,0.00032030,-0.00057368,0.00073286,-0.0023177$ $0,0.00558168,-0.00098679,-0.00082059,0.00382509,0.00084092,-0.05962572$ ,-0.11196219,-0.11641127,0.00164686,-0.00143546,0.00076193,-0.00027330 $,-0.00002391,-0.00061586,0.00029474,-0.00049522,0.00013906,0.00204755$, $-0.00275160,0.00126895,0.06686168,0.10867531,-0.00081109,-0.00063902,-$ $0.00039185,-0.00017154,0.00005219,0.00005781,0.00006795,0.00002615,0.0$ $0001841,-0.00009706,-0.00009725,0.00004365,-0.00002395,0.00004890,-0.0$ $0001265,0.00001509,-0.00007010,0.00006225,0.00012978,-0.00001248,-0.00$ $040506,0.00000463,-0.00001084,-0.00000318,0.00001580,0.00000105,-0.000$ $00756,0.00001599,0.00001427,-0.00001625,-0.00001461,0.00001588,-0.0000$ $2218,0.00017827,0.00006774,-0.00002775,0.00398532,0.00216373,0.0029334$ 2,0.00152136,-0.00037084,0.00185339,0.00054684,0.00065078,-0.00010727, $0.00453579,-0.00077068,-0.00204665,-0.01092184,-0.01314676,-0.01502641$ ,-0.08088233,-0.11782895,-0.23324906,-0.00000167,0.00127142,0.00095870 $, 0.00013614,0.00006186,0.00031365,-0.00016601,0.00011774,-0.00011699,-$ $0.00075109,0.00136483,0.00060500,0.08273210,0.12707917,0.24459601,0.00$ $075204,0.00033732,0.00007319,-0.00159647,0.00281870,-0.00094620,0.0052$ $8522,-0.00116020,-0.00028718,-0.05148845,0.01154945,-0.00000455,0.0008$ $2745,-0.00276584,0.00113038,-0.00066474,0.00041355,-0.00020515,0.00047$ $374,0.00021762,-0.00006591,0.00047105,0.00030129,-0.00009388,-0.002054$ $55,0.00063683,-0.00006159,-0.00007518,0.00019630,-0.00005951,-0.000013$ $75,-0.00013973,0.00007335,-0.16837019,0.04635211,-0.00674866,-0.000179$ $60,0.00004888,0.00041281,0.00008835,0.00000798,-0.00012533,-0.00008226$ ,-0.00016390,-0.00034222,-0.00021127,0.00008003,0.00048753,0.00019366, $0.00007671,-0.00017100,0.00001090,0.00001649,-0.00001596,0.00023311,-0$ $.00000040,-0.00040600,0.00005468,0.00001057,-0.00004812,-0.00005754,-0$ $.00001361,0.00008020,0.00002247,0.00002606,0.00017954,0.00002740,-0.00$ $003003,-0.00006009,0.49578416,-0.00047406,-0.00005980,-0.00004927,0.00$ $059080,-0.00107080,0.00034188,-0.00675741,0.00025966,0.00005243,-0.011$ $70868,0.01266720,-0.00487589,-0.00256378,-0.00229289,0.00212061,-0.001$ $19634,0.00096834,0.00010476,-0.00030555,-0.00014534,0.00007647,-0.0001$ $8262,-0.00010893,0.00004228,-0.00162112,-0.00096007,0.00047961,-0.0000$ $3538,0.00017052,-0.00021212,-0.00039812,-0.00016728,-0.00005080,0.0776$ $5685,-0.12785197,0.01767342,0.00008930,-0.00000020,-0.00021201,-0.0000$ $4864,-0.00002689,0.00004205,0.00005264,0.00011088,0.00023222,0.0001348$ $5,-0.00012337,-0.00028401,-0.00008949,-0.00003951,0.00010469,0.0000082$ $1,-0.00002192,0.00002116,-0.00012083,-0.00000150,0.00022549,-0.0000364$ $4,-0.00000078,0.00003240,0.00002112,0.00000657,-0.00005248,-0.00003522$ ,-0.00000121,-0.00010585,-0.00001487,0.00002403,0.00003165,0.07975970, $0.55528633,0.00010712,-0.00000345,0.00015504,-0.00010585,0.00010929,-0$ $.00020827,0.00182844,-0.00057439,-0.00144642,0.00803467,-0.00665254,-0$ 
$.00233722,0.00100459,0.00203886,0.00210099,0.00054207,-0.00008816,0.00$ $073967,0.00003426,0.00006232,0.00004252,0.00003168,0.00002492,0.000075$ $28,0.00071858,0.00030020,-0.00010432,-0.00000803,-0.00022703,-0.000301$ $68,0.00009937,-0.00004088,-0.00027142,-0.01748266,0.02019391,-0.063517$ $11,-0.00001295,0.00001798,0.00003868,-0.00000465,-0.00003428,-0.000054$ $00,0.00001459,-0.00000705,-0.00006043,-0.00008533,0.00002728,0.0001091$ $3,0.00009440,0.00007931,-0.00002559,0.00001761,0.00000353,-0.00000738$, $0.00001764,-0.00000304,-0.00003534,0.00000945,0.00000256,0.00000398,-0$ $.00003036,-0.00001127,0.00001081,0.00001477,-0.00001941,0.00006296,0.0$ $0001245,-0.00001595,-0.00002375,-0.02222503,-0.00417424,0.56557241,0.0$ $0000239,-0.00010698,0.00004452,-0.00097919,-0.00058677,0.00007805,0.00$ $104530,0.00010931,-0.00000017,0.00621004,0.00240894,-0.00014619,-0.001$ $95472,-0.00010212,-0.00051738,0.00079398,0.00030783,-0.00023833,-0.000$ $06645,0.00000678,-0.00002319,-0.00003108,0.00000348,0.00002537,-0.0009$ $1872,-0.00017177,0.00114713,0.00009807,-0.00007021,0.00012963,-0.00002$ $347,0.00002602,0.00004471,-0.01405717,0.03232385,0.01759573,0.00000608$ ,-0.00000171,-0.00002033,0.00000098,-0.00000785,0.00000771,-0.00000285 $, 0.00002315,0.00002096,0.00000403,-0.00000879,-0.00005183,-0.00002091$, $-0.00000183,0.00000250,0.00000674,-0.00000325,0.00000256,-0.00001073,0$ $.00000032,0.00001942,-0.00000224,-0.00000086,0.00000393,0.00000338,0.0$ $0000015,-0.00000360,-0.00000152,-0.00000024,-0.00000819,-0.00000536,0$. $00000093,0.00000351,-0.04844200,-0.01302121,-0.00752445,0.05528945,0.0$ $0018898,0.00004589,-0.00004237,-0.00037841,0.00034384,-0.00015166,0.00$ $064760,-0.00013862,0.00030127,-0.00348519,-0.00096634,0.00105496,0.000$ $46648,0.00032709,0.00001922,-0.00004551,-0.00011880,0.00008363,0.00007$ $344,0.00005695,-0.00003556,0.00004938,0.00004981,0.00003277,-0.0007321$ $9,0.00048329,0.00033192,-0.00010067,0.00004483,-0.00008536,0.00004818$, $0.00000888,-0.00000795,0.01452572,-0.01729548,-0.00644873,-0.00003224$, $0.00000160,0.00007045,0.00001160,0.00000465,-0.00002239,-0.00000743,-0$ $.00003472,-0.00005852,-0.00004832,0.00003222,0.00007657,0.00002248,0.0$ $0000221,-0.00002872,0.00000444,0.00001213,-0.00000345,0.00004135,0.000$ $00064,-0.00006904,0.00001119,0.00000097,-0.00000880,-0.00000713,-0.000$ $00048,0.00001616,0.00000838,0.00000269,0.00002824,0.00000264,-0.000007$ $64,-0.00000984,-0.00758538,-0.20865391,-0.12196756,-0.00141084,0.23179$ $478,0.00000865,-0.00004704,-0.00001613,-0.00007637,0.00067041,-0.00005$ $985,0.00016584,-0.00137067,0.00048156,-0.00063228,-0.00050856,0.000133$ $35,0.00033107,0.00015083,-0.00022074,-0.00017222,-0.00010401,0.0000691$ $7,-0.00001147,0.00002885,-0.00001769,0.00003376,-0.00001521,-0.0000130$ $3,0.00013604,0.00062662,-0.00060331,-0.00004223,0.00003178,0.00000311$, $0.00001065,0.00001897,0.00000430,0.00263805,0.00559836,0.00738352,-0.0$ $0000504,-0.00000303,-0.00001287,0.00000994,0.00000664,0.00002500,-0.00$ $001150,0.00000373,0.00000434,-0.00000251,0.00001214,-0.00003489,-0.000$ $03148,-0.00001090,0.00000052,-0.00000436,-0.00000515,0.00000169,-0.000$ $00351,0.00000175,0.00000557,-0.00000082,-0.00000240,-0.00000211,0.0000$ $0991,0.00000308,0.00000269,-0.00000140,-0.00000360,-0.00000905,-0.0000$ $0111,0.00000181,0.00000465,-0.00414139,-0.12354958,-0.14301960,0.00381$ $519,0.13391964,0.14600677,-0.00002816,-0.00012391,0.00000851,-0.000916$ $60,-0.00054175,0.00048375,0.00096696,0.00020125,-0.00020947,0.00616491$ $, 0.00186011,-0.00244667,-0.00193400,0.00029276,0.00077074,0.00082465,0$ $.00039063,-0.00010411,-0.00004070,0.00000871,0.00001611,-0.00004186,-0$ $.00001784,-0.00001816,-0.00100552,-0.00092334,-0.00056269,0.00009829,-$ 
$0.00015486,-0.00007893,-0.00003506,-0.00001145,-0.00005398,-0.01914631$ $, 0.01575152,-0.03033599,0.00000150,-0.00000475,-0.00001563,0.00000491$, $0.00000349,0.00001135,0.00000146,0.00000450,0.00002324,0.00001053,0.00$ $000679,-0.00002498,-0.00001709,-0.00002482,0.00001369,0.00000244,0.000$ $00669,0.00000572,-0.00000791,0.00000043,0.00001416,-0.00000298,-0.0000$ $0088,0.00000247,0.00000540,0.00000459,-0.00000735,-0.00001226,0.000008$ $53,-0.00001379,-0.00000427,0.00000232,0.00000499,-0.04979813,0.0028127$ $2,-0.01885498,0.00146081,-0.00129806,-0.00179155,0.05876730,0.00014829$ $, 0.00009320,0.00002658,-0.00016052,-0.00001969,0.00010355,0.00029698,0$ $.00057437,-0.00047923,-0.00271964,-0.00077987,0.00020470,0.00022082,0$. $00001175,-0.00032053,0.00002469,-0.00008210,-0.00006062,0.00007983,0.0$ $0001671,-0.00001430,0.00001108,0.00001959,-0.00004297,-0.00070059,-0.0$ $0034056,-0.00032958,-0.00004528,0.00007050,0.00006333,0.00003826,0.000$ $00627,0.00002474,0.01293743,-0.00885357,0.01812014,-0.00002074,0.00000$ $469,0.00006172,0.00000635,0.00000242,-0.00002180,-0.00000601,-0.000037$ $30,-0.00006576,-0.00004959,0.00001977,0.00011073,0.00005209,0.00001656$ ,-0.00002315,-0.00000376,0.00000160,-0.00000754,0.00003089,-0.00000143 $,-0.00005927,0.00000839,0.00000225,-0.00000657,-0.00001255,-0.00000164$ $, 0.00000981,0.00000986,0.00001114,0.00003124,0.00000628,-0.00000268,-0$ $.00001300,0.00481245,-0.06398809,0.05959770,-0.00323621,0.00745468,-0$. $02381381,-0.01373572,0.06953544,-0.00012404,-0.00002154,-0.00013191,0$. $00045559,-0.00059999,0.00028060,-0.00070073,0.00127160,-0.00019031,0.0$ $0178739,0.00085726,-0.00009223,-0.00024311,-0.00035219,0.00006500,0.00$ $003220,0.00013110,0.00003165,-0.00002537,-0.00004647,0.00000785,-0.000$ $05146,-0.00000458,0.00000682,0.00056095,-0.00065983,0.00034901,0.00008$ $029,-0.00005657,-0.00000766,-0.00003149,-0.00001963,-0.00000191,-0.008$ $28016,0.00437502,0.00379305,0.00001945,-0.00000880,-0.00003470,-0.0000$ $1500,0.00001292,0.00000676,0.00001256,-0.00000162,0.00004677,0.0000684$ $1,-0.00002675,-0.00002513,-0.00003331,-0.00003112,0.00001600,-0.000005$ $49,0.00000542,0.00000255,-0.00001630,-0.00000135,0.00003196,-0.0000063$ $7,-0.00000152,0.00000179,0.00000964,0.00000341,-0.00001095,-0.00000206$ $, 0.00000501,-0.00002794,-0.00000331,0.00000567,0.00000899,-0.02483334$, $0.06177739,-0.28580040,-0.00022692,0.00536747,-0.01638904,0.03200919,-$ $0.07231344,0.30461267,0.00001402,0.00004809,-0.00003735,-0.00007098,-0$ $.00039413,0.00016987,0.00008371,0.00106535,-0.00034823,-0.00080537,0.0$ $0432123,-0.00137851,-0.00109123,-0.00107489,0.00050475,-0.00017655,0.0$ $0045796,-0.00012770,-0.00004767,-0.00002547,0.00000209,-0.00004404,-0$. $00002408,0.00001176,0.00032739,-0.00019655,0.00000597,-0.00000271,0.00$ $004432,-0.00001655,-0.00014106,-0.00006346,0.00001816,-0.03663111,-0.0$ $1395964,0.00797800,0.00001877,-0.00000871,-0.00005823,-0.00001132,0.00$ $000329,0.00001924,0.00000787,0.00002201,0.00004609,0.00003116,-0.00001$ $743,-0.00007060,-0.00002890,-0.00000140,0.00003395,0.00000013,-0.00000$ $783,-0.00000431,-0.00002846,-0.00000053,0.00005420,-0.00000643,-0.0000$ $0317,0.00000320,0.00001067,0.00000174,-0.00001041,-0.00000090,-0.00001$ $064,-0.00002585,-0.00000208,0.00000511,0.00000770,-0.18119012,-0.12251$ $643,0.05375261,0.00159517,-0.00097051,-0.00022985,0.00468170,0.0020069$ $0,-0.00043722,0.21350833,-0.00004537,-0.00007409,0.00001923,0.00008643$ $, 0.00060678,-0.00015343,0.00013296,-0.00121691,0.00049672,0.00286640,-$ $0.00139185,0.00044486,0.00095544,0.00056034,-0.00021270,-0.00004647,-0$ $.00024284,0.00010881,-0.00001639,0.00001199,-0.00000458,0.00003641,0.0$ $0000732,0.00000438,-0.00066458,0.00041592,-0.00021771,-0.00002050,-0.0$ 
$0000389,0.00001152,0.00006907,0.00003902,-0.00003586,0.02217829,0.0123$ $7482,-0.00604357,0.00001734,-0.00001299,-0.00003783,0.00000123,0.00001$ $047,0.00003906,-0.00000598,-0.00000659,-0.00000763,-0.00000627,0.00002$ $573,-0.00001604,-0.00001054,0.00000259,-0.00000890,-0.00001821,-0.0000$ 0595,0.00000247,-0.00002083,0.00000099,0.00002265,-0.00000181,-0.00000 $253,-0.00000199,0.00000887,0.00000582,-0.00000099,0.00000480,-0.000008$ $96,-0.00000772,0.00000159,-0.00000017,0.00000239,-0.13580295,-0.163979$ $10,0.05136141,-0.01648014,-0.01345115,0.00835937,-0.00451767,-0.003734$ $03,0.00033317,0.13129888,0.17006928,0.00001265,0.00001661,-0.00002728$, $0.00000317,-0.00012571,0.00016502,-0.00004741,0.00041924,0.00015416,-0$ $.00088120,0.00029157,0.00048108,-0.00023064,-0.00002613,0.00024117,0.0$ $0001992,0.00004995,-0.00000420,0.00001926,0.00000533,0.00000477,-0.000$ $00509,0.00000873,0.00001794,0.00018600,-0.00026041,-0.00028748,0.00001$ $600,0.00001044,0.00003372,-0.00001481,-0.00002389,-0.00004465,-0.00459$ $378,-0.00297188,0.00182313,-0.00000425,0.00000355,0.00001926,0.0000072$ $4,0.00000597,0.00000320,-0.00001189,-0.00001147,-0.00001939,-0.0000008$ $8,-0.00000539,0.00003835,0.00001332,-0.00000017,-0.00001232,-0.0000084$ $0,-0.00000653,-0.00000158,0.00000720,-0.00000058,-0.00001910,0.0000007$ $4,0.00000124,-0.00000301,-0.00000107,-0.00000023,-0.00000032,-0.000003$ $02,0.00001042,-0.00000002,0.00000159,0.00000375,0.00000070,0.05840412$, $0.05020774,-0.07169854,-0.01418033,-0.01236236,0.00627207,0.02115435,0$ $.01921732,-0.00654930,-0.05986278,-0.05445710,0.06941265 \backslash 10.00001525,-$ $0.00000650,-0.00001265,-0.00001609,0.00000032,0.00000490,0.00000606,0$. $00000606,-0.00000426,-0.00000557,-0.00001107,0.00000344,-0.00000197,0$. $00000352,0.00000028,0.00000390,0.00000771,-0.00000468,-0.00000342,0.00$ $000255,0.00000237,0.00000330,0.00000019,0.00000114,0.00000071,0.000000$ $92,0.00000135,-0.00000003,0 ., 0.00000142,-0.00000202,-0.00000385,0.0000$ $0192,0.00001936,-0.00000542,-0.00000575,0.00000688,-0.00000123,-0.0000$ $1454,-0.00000205,0.00000053,0.00000603,0.00000480,0.00000495,-0.000004$ $55,-0.00000602,-0.00001311,0.00001230,0.00000071,0.00000309,-0.0000033$ $5,-0.00000141,0.00000273,0.00000493,-0.00000472,0.00000104,0.00000740$, $0.00000040,-0.00000016,0.00000048,-0.00000102,-0.00000019,0.00000074,0$ $.00000017,0.00000100,-0.00000021,0.00000068,0.00000022,-0.00000080,-0$. $00001856,0.00001267,0.00000417,0.00000029,-0.00000168,0.00000068,0.000$ 00382,-0.00000257,-0.00000417,-0.00000348,-0.00000170,0.00000138III@ 


\section{Cyanobenzene addition}

P-Chlrophenyl Radical + Cyanobenzene

ortho-Transition State

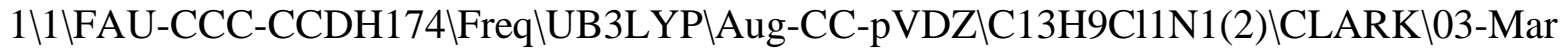
-2015\0\॥N Geom=AllCheck Guess=TCheck SCRF=Check GenChk UB3LYP/Aug-CC -pVDZ Freq \\Addition TS p-Cl-Ph-radical + aniline (ortho) \0,2\C,1.967 8809379,-0.0316097203,1.0951790576\C,2.5070989444,0.7157129057,0.00558 75135\C,3.1822813319,0.0620177902,-1.0352691027\C,3.3930654251,-1.3167 766171,-0.9722590735\C,2.9542841835,-2.0463039488,0.1468743356\C,2.276 $2788819,-1.4102914911,1.18015694 \mathrm{H}, 1.6226001188,0.4999484792,1.9784227$ 856\C,2.3360558689,2.137271431,-0.0256242104\H,3.5548332831,0.64031021 $06,-1.8794955657 \backslash \mathrm{H}, 3.1640486364,-3.1142962482,0.2118574092 \backslash \mathrm{H}, 1.9508579$ $151,-1.973903809,2.0537704591 \backslash \mathrm{H}, 3.9243417323,-1.8196739772,-1.77974997$ 03\C,-2.9097193067,-0.0644530117,-0.1075100931\C,-2.3415042132,1.08327 $33692,0.4501222677 \backslash \mathrm{C},-0.9813567523,1.0702271021,0.7994175482 \backslash \mathrm{C},-0.2645$ $729882,-0.0924791298,0.5760354648 \backslash C,-0.804862725,-1.2381637475,0.02138$ $33215 \backslash \mathrm{C},-2.1644309785,-1.2248563011,-0.3294605989 \backslash \mathrm{Cl},-4.618505743,-0.0$ $47695832,-0.5460457705 \backslash \mathrm{H},-2.946909428,1.9750992065,0.6063351929 \backslash \mathrm{H},-0.5$ $229556709,1.9650354121,1.2214559267 \backslash \mathrm{H},-0.2069319012,-2.1345028784,-0.1$ $454061232 \backslash \mathrm{H},-2.6359483077,-2.1032569619,-0.7684593638 \backslash \mathrm{N}, 2.1917757553,3$ $.2926537675,-0.0324723502 \|$ Version=EM64L-G09RevC.01 $\backslash$ State $=2-A \backslash H F=-1015$ $.7281584 \backslash \mathrm{S} 2=0.779236 \mathrm{~S} 2-1=0 . \mathrm{SS} 2 \mathrm{~A}=0.750618 \backslash \mathrm{RMSD}=2.171 \mathrm{e}-09 \backslash \mathrm{RMSF}=2.131 \mathrm{e}-0$ 6VZeroPoint $=0.1764258 \backslash$ Thermal $=0.1892551 \mathrm{ZPE}=110.708836 \backslash \mathrm{Dipole}=0.74436$, $-1.7496044,0.1737284 \backslash$ DipoleDeriv $=0.3027935,0.0175947,-0.0053612,-0.053$ $3052,-0.0175386,0.0688232,0.0424849,-0.0262919,0.0325229,-0.0258248,0$. $0270533,-0.0462724,-0.0750711,-0.0863746,0.0397429,-0.0900737,-0.05688$ $62,0.0561026,-0.0749643,-0.0508626,-0.0329661,0.138533,0.0574658,-0.09$ $21538,-0.0555655,0.0260427,0.0152353,-0.1173287,-0.040921,-0.0930818,-$ $0.1070878,-0.1031418,0.1229821,0.053428,0.0754293,0.0049541,-0.0177734$ $,-0.0427753,-0.0518415,-0.0855689,0.1126355,-0.0354146,-0.1168969,-0.0$ 203391,0.0007229,-0.2065932,0.0511369,-0.0989994,0.0778744,-0.0591631, $-0.1387699,-0.0470483,-0.0534109,-0.0113428,0.1184083,0.0207502,0.0330$ $21,0.0232775,0.0360206,-0.0397237,0.042,-0.0293604,0.0369443,0.0829183$ - $0.0340511,0.0497424,0.1143445,0.4713838,-0.0271527,0.0384643,0.00066$ $15,0.0501857,0.1059057,-0.0056683,0.0449528,-0.0183612,0.0423526,0.055$ $2881,0.0606862,0.0487673,0.0296415,0.1089769,0.0251838,0.022006,0.0518$ $277,-0.0680836,0.0173241,0.0195675,0.0139515,0.0855245,0.097932,-0.016$ $7184,0.0696972,-0.0044625,0.0270369,0.0536035,0.0511434,0.0480513,0.00$ $79847,0.076202,0.0428188,0.0737137,0.0390144,0.0342869,-0.0372331,0.07$ $64681,-0.0419057,0.0097763,1.2220316,-0.0172997,0.2725349,-0.012574,-0$ $.0054557,0.0079615,0.3245133,0.0095697,0.0312734,-0.2042745,-0.0846714$ ,-0.0424348,0.0640876,0.0225044,0.0116618,-0.0138771,0.0082491,-0.0916 $273,-0.3856577,0.0534659,0.0649783,-0.1886245,0.0293526,0.0474106,-0.1$ $474652,0.0284541,-0.0531784,0.6334011,-0.0076771,-0.0294662,-0.0184215$ ,-0.0183725,0.0033729,0.1432288,0.0054611,-0.0892768,-0.4248814,-0.053 $3281,0.0201808,0.1808872,0.0035502,0.0185624,-0.0177344,0.0237013,-0.0$ $645939,-0.2036073,0.0649111,-0.0028008,-0.0701981,0.0220187,0.0551002$, 
$-0.0678412,0.0583006,-0.0646846,-0.8900363,0.0075095,-0.153373,0.00643$ $81,-0.0821251,-0.0042798,-0.2085099,-0.0036416,-0.1093446,-0.0185717,0$ $.0604964,0.0060499,0.0482329,0.0238735,-0.0253973,-0.0206261,-0.017742$ $8,0.1199767,0.0744313,-0.0505525,-0.0520168,-0.0201567,0.022056,-0.046$ $8071,-0.0281382,-0.0356863,0.0948115,0.0630383,0.0898992,-0.002033,0.0$ $466913,0.006718,-0.0237513,0.0090504,-0.0179673,0.0975238,-0.0069885,-$ $0.0444165,-0.0307414,-0.0326655,0.0189539,-0.0460689,-0.048857,-0.0510$ $835,0.1018798,-0.309537,-0.0118776,-0.0154885,-0.1047117,-0.4899544,0$. 0149193,0.0015985,0.0076761,-0.2910118\Polar $=235.8355704,-12.8536405,1$ 93.2425654,-4.656302,6.686968,128.3062179\PG=C01 [X(C13H9Cl1N1)]WNImag $=1 \backslash 0.11376934,-0.06802473,0.64170821,-0.20324640,0.02515505,0.5925086$ $3,-0.07606814,-0.02547444,0.07405829,0.26659970,-0.01339460,-0.1548427$ $9,0.08587390,-0.06281407,0.64133552,0.04476443,0.05086115,-0.20634712$, $-0.20383495,-0.01043668,0.49907287,-0.00783109,-0.01298514,0.02854139$, $-0.12261430,0.05729593,0.09304958,0.26793533,0.01035144,0.05212755,-0$. $01273540,0.03225338,-0.14185824,-0.03487495,-0.04675343,0.66794710,0.0$ 2734866,0.01361866,-0.03711845,0.10181497,-0.08011997,-0.21887394,-0.2 $3204539,-0.08326400,0.57611764,-0.00132254,-0.00542068,-0.00048016,0.0$ $0409306,0.01739949,0.00201783,-0.08162636,0.05459682,0.01938428,0.2727$ $3258,-0.00580995,-0.04266806,0.02245722,0.04034793,-0.03644656,-0.0626$ $0282,0.02093262,-0.32420667,0.04715857,-0.09681853,0.64942108,-0.01105$ $836,0.02319457,-0.01354682,-0.00585018,-0.01835281,0.02364419,0.029913$ $56,-0.01363847,-0.11061176,-0.23710203,-0.00332895,0.57746568,-0.00843$ $376,0.03485059,-0.00339836,-0.01609876,0.00360910,0.01816578,0.0196862$ $0,-0.02847696,-0.01369704,-0.09505932,-0.00322612,0.06410368,0.2660180$ $1,0.01749271,-0.02094308,-0.02618717,-0.00150582,0.00097126,0.00202504$ ,-0.00727923,-0.02978843,0.02715576,-0.03587263,-0.17456142,0.11404170 ,-0.10895669,0.71269004,0.02157571,-0.05657105,0.02774248,0.01931112,$0.00894346,-0.04204758,-0.02100960,0.06595130,0.02306611,0.07701465,0$. $05249931,-0.22881167,-0.20468457,-0.03840122,0.53388540,-0.05866966,0$. $04010132,0.02199725,0.01267030,-0.01275261,-0.01047759,-0.01171395,0.0$ $1647234,0.00653537,-0.00528078,-0.02039747,0.03224256,-0.12946237,0.07$ $876619,0.10046411,0.27595879,0.01833644,-0.29634221,-0.00402125,-0.013$ $06673,-0.01823466,0.04155180,0.01491765,-0.03797450,-0.01788675,0.0012$ $0750,0.05815115,-0.02168355,0.04713273,-0.17185881,-0.03861601,-0.0793$ $9544,0.61944489,0.01007558,0.04150395,-0.11222110,-0.01238989,0.026589$ $00,0.01844644,0.00818427,-0.02304290,-0.01238316,0.02431478,0.02141506$ ,-0.04667251,0.11237706,-0.09947689,-0.23190880,-0.23172760,-0.0735010 4,0.60938792,-0.04695811,0.04362351,0.07970229,-0.00497966,0.00309903, $0.01321369,0.00404236,-0.00332358,0.00222830,-0.00090563,-0.00034724,0$ $.00080702,0.00639107,0.00239537,-0.00194269,-0.00369575,0.00910091,0.0$ $0683017,0.06672645,0.05049710,-0.12539682,-0.11493658,-0.00613452,0.01$ $028902,0.01573444,-0.00106474,-0.00187135,0.00284732,-0.00026671,-0.00$ $054372,0.00062501,0.00078074,-0.00519520,0.00151513,0.00922653,-0.0080$ $4451,-0.02460258,-0.05116044,0.12847003,0.09188664,-0.11384843,-0.2446$ $6624,0.00507762,-0.01366833,-0.01163640,0.00213418,0.00323499,-0.00165$ $330,-0.00072943,0.00037697,0.00055622,0.00137544,0.00154994,0.00062516$ ,-0.00257761,0.00569197,0.00700092,-0.08947238,0.11956865,0.25288193,0 $.00651049,-0.01159525,-0.00245425,-0.06430542,0.02453083,0.01011190,-0$ $.00160327,0.02109338,0.00013203,0.00428791,0.00072689,0.00626397,-0.00$ $076158,0.00008378,-0.00053739,0.00339620,0.00029948,0.00436025,-0.0025$ 
$4058,-0.00051852,-0.00059742,0.11263729,-0.01483827,-0.03610793,0.0134$ $9045,0.02379767,-0.27443574,0.00766932,0.01547146,-0.04281484,-0.01677$ $115,0.00258822,0.01207890,-0.00694471,-0.00264448,-0.00148489,0.005844$ $73,-0.00046887,-0.00137634,0.00279837,0.00172026,0.00139621,-0.0007521$ $8,-0.17270554,1.50310745,-0.00608703,0.02652329,0.00387661,0.01208975$, $0.00563956,-0.07771664,0.00122936,-0.02569271,0.00641784,0.00578716,-0$ $.00289503,-0.00167476,0.00057881,0.00015144,-0.00233310,0.00475232,0.0$ $0360833,-0.00241306,-0.00131653,0.00055739,-0.00004369,-0.01461043,-0$. $01416781,0.10957885,0.00383800,0.00349203,0.00397128,-0.00209429,-0.00$ $825727,0.01177832,-0.07501407,-0.04992631,0.08430581,0.00528967,0.0042$ $6914,-0.00386179,0.00528362,0.00199009,0.00259305,-0.00047621,-0.00021$ $651,-0.00065891,0.00004582,-0.00060111,0.00069395,-0.00285478,-0.00138$ $485,-0.00182427,0.06891533,0.00240881,-0.00083930,-0.00239624,0.006315$ $02,0.01016753,-0.01255691,-0.04999356,-0.13769153,0.11937683,-0.011169$ $43,-0.00945613,0.02429564,0.00239882,-0.00521615,-0.00048824,0.0003095$ $3,-0.00051649,-0.00067230,0.00034500,0.00047463,-0.00057240,-0.0001996$ $0,0.00108459,-0.00110471,0.05090383,0.14181626,0.00395877,-0.00259770$, $-0.00047264,0.00674760,0.01388686,-0.01239213,0.08510685,0.11714629,-0$ $.22509837,0.00054854,-0.00137683,0.00318789,0.00240900,-0.00006531,0.0$ $0316525,-0.00041246,-0.00071646,-0.00007451,0.00052080,0.00058736,-0.0$ $0044214,-0.00190657,0.00084767,-0.00050592,-0.09449439,-0.12679844,0.2$ $3298702,0.00421494,0.00183290,0.00605562,-0.00047990,-0.00016669,-0.00$ $027465,0.00432430,-0.00005211,0.00404308,0.00516155,-0.01025721,0.0013$ $3428,-0.05233916,0.05684561,0.00394266,0.00049173,0.01531658,-0.001335$ $95,-0.00005922,0.00101552,-0.00027104,-0.00085875,-0.00009157,-0.00060$ $899,0.00000116,-0.00045438,-0.00000052,0.04441832,0.00238310,0.0010860$ $9,-0.00170670,-0.00026949,0.00059492,-0.00014523,-0.00028977,0.0007790$ $8,0.00345759,0.00407559,-0.01105161,-0.00191548,0.05694432,-0.32666519$ $, 0.01803167,0.00089460,-0.00957476,0.00355274,0.00010097,-0.00044143,0$ $.00022346,-0.00023371,0.00000961,-0.00016172,0.00015402,-0.00059866,-0$ $.00019280,-0.06220497,0.34466420,0.00516428,-0.00169351,-0.00148805,0$. $00034387,-0.00033989,-0.00118987,0.00419940,0.00307652,-0.00245621,-0$. $00530054,0.02405688,0.00325003,0.00469294,0.01862673,-0.05518585,0.004$ $61140,-0.02289925,0.00571144,0.00021153,-0.00092926,0.00006194,-0.0005$ $7883,0.00007932,-0.00017922,-0.00018606,0.00128476,0.00008996,-0.00916$ $627,-0.02030647,0.05140558,0.00468176,0.00505269,-0.00641918,0.0041926$ $6,0.00211578,0.00207208,-0.00084104,0.00018422,-0.00028909,0.00451868$, $0.00323592,0.00439645,-0.00048738,-0.00875033,0.01172743,-0.06535165,-$ $0.04083584,0.07681699,-0.00235212,-0.00024792,-0.00088287,0.00057190,0$ $.00029173,-0.00022015,-0.00072293,-0.00018459,-0.00042546,-0.00296458$, $-0.00074723,-0.00202335,0.05882625,-0.01034702,-0.01135016,0.02452871$, $0.00297923,-0.00425509,-0.00015438,0.00016441,-0.00066780,-0.00071193$, $0.00304864,-0.00024547,-0.00203567,0.00496290,0.01087936,-0.01305746$,$0.03919651,-0.13253321,0.12062329,-0.00075310,0.00096420,-0.00045952$,$0.00057426,-0.00068566,0.00134542,-0.00023748,0.00001498,-0.00006145$,$0.00104754,0.00064314,-0.00017784,0.04060657,0.13692012,0.00062848,-0$. $00101070,0.00204738,0.00151078,-0.00015660,0.00267093,-0.00031975,-0.0$ $0070337,0.00006847,0.00457655,-0.00276782,-0.00035483,0.00570735,0.014$ $17149,-0.01305131,0.07631332,0.11941859,-0.23538058,-0.00153212,0.0000$ $7613,0.00004319,0.00011197,-0.00005818,0.00012449,-0.00039108,-0.00000$ $328,-0.00025236,-0.00206003,-0.00052975,-0.00010735,-0.08535862,-0.128$ 
$92732,0.24487870,-0.00057174,-0.00038272,-0.00067180,0.00556846,0.0011$ $0268,0.00153705,0.00131361,0.00090533,0.00389953,-0.10831633,0.0675415$ $6,0.11208883,-0.00103871,0.00563215,0.00622475,0.00477255,-0.00088501$, $0.00306461,-0.00063422,-0.00004894,-0.00020921,0.00031755,0.00015341,0$ $.00040418,-0.00274119,-0.00052347,-0.00202246,-0.00235995,-0.00078991$, $-0.00173483,-0.00018631,0.00029534,0.00030531,0.10400995,-0.00024843$,$0.00066185,0.00045499,0.00116260,-0.00486575,0.00162102,0.01507827,-0$. $00930513,-0.02343267,0.06716923,-0.11694773,-0.09527169,-0.00854002,0$. $01075448,0.01302071,-0.00036593,-0.00093298,0.00370212,-0.00012509,0.0$ $0004639,-0.00010608,0.00082757,-0.00037675,-0.00121704,-0.00069974,0.0$ $0087085,-0.00020761,-0.00050663,0.00062894,-0.00064922,-0.00045342,0.0$ $0040529,0.00069837,-0.07303998,0.12070288,-0.00064292,0.00061239,-0.00$ $028402,0.00188068,0.00153525,0.00296318,-0.00119675,0.00301310,0.00441$ $812,0.11186937,-0.09536132,-0.20846597,0.01111412,-0.01354286,-0.01245$ $704,0.00324764,0.00403884,-0.00143910,-0.00031041,-0.00010988,-0.00013$ $465,0.00013397,0.00003073,0.00039755,-0.00188309,-0.00051357,-0.000369$ $09,-0.00175676,-0.00032073,0.00009090,0.00053000,-0.00065213,-0.000705$ $88,-0.12285686,0.10116011,0.21609723,-0.00058580,0.00004292,-0.0001389$ $1,-0.00004288,0.00012311,0.00012055,0.00007115,-0.00024401,0.00004937$, $0.00006813,0.00009521,-0.00019669,-0.00000131,0.00011877,0.00008986,0$. $00012800,-0.00008187,-0.00003115,-0.00011056,0.00003594,0.00002143,-0$. $00004689,-0.00022307,-0.00001561,-0.00001961,0.00001236,-0.00003153,-0$ $.00003113,-0.00005297,-0.00001792,-0.00004023,0.00000881,0.00000371,0$. $00000608,-0.00001276,-0.00002293,0.47597548,-0.00006642,-0.00033022,-0$ $.00030001,-0.00001245,0.00003572,0.00007721,0.00010402,-0.00011561,-0$. $00000382,-0.00007755,0.00018832,0.00002267,-0.00002117,0.00013751,-0.0$ $0004642,-0.00010318,-0.00011797,0.00002647,0.00016189,0.00005411,0.000$ $15524,0.00008814,-0.00010713,-0.00000793,-0.00000981,0.00000632,0.0000$ $1026,-0.00000870,-0.00001646,0.00001753,-0.00002514,0.00006993,-0.0000$ $5623,0.00000631,0.00000486,0.00000366,-0.03959323,0.59561426,-0.000244$ $23,-0.00008346,-0.00014379,0.00006324,0.00003806,0.00004016,0.00002378$ ,-0.00011167,-0.00003576,0.00005676,0.00009902,-0.00007051,-0.00006775 $, 0.00007395,0.00002407,-0.00000024,-0.00003974,0.00007812,-0.00001433$, $-0.00000720,0.00003369,0.00006368,-0.00012550,0.00003007,-0.00000137,0$ $.00000313,-0.00002029,-0.00000739,-0.00000548,0.00001040,-0.00003749,0$ $.00002074,-0.00005513,-0.00000472,0.00000662,-0.00000779,0.07722541,0$. $15735948,0.20338046,0.00122999,0.00063838,0.00049447,0.00017419,-0.000$ $10907,-0.00010469,-0.00015003,0.00020508,0.00006537,0.00003614,-0.0002$ $1880,0.00002573,0.00002099,-0.00011393,-0.00011529,-0.00011513,0.00008$ $906,-0.00011041,-0.00000803,-0.00027436,-0.00030874,-0.00011242,0.0001$ $4770,0.00001583,0.00001152,-0.00001480,0.00000006,0.00003310,-0.000018$ $38,0.00000832,0.00006531,-0.00007159,0.00010760,-0.00000131,-0.0000132$ $5,0.00001385,-0.13017692,-0.03130458,-0.02852069,0.63634269,-0.0002349$ $4,-0.00034776,-0.00056737,0.00054223,0.00041351,0.00023423,-0.00004339$ ,-0.00037610,-0.00012632,0.00018338,0.00008890,-0.00046915,-0.00015701 $, 0.00009723,0.00040506,0.00064094,-0.00015208,0.00002860,0.00017316,-0$ $.00014443,0.00000221,0.00031610,-0.00082769,0.00002708,0.00003546,-0.0$ $0000597,0.00000489,-0.00001349,0.00000975,-0.00000305,0.00006545,-0.00$ $008453,0.00003951,0.00000728,0.00001764,0.00000157,-0.08989566,-0.2620$ $1597,-0.09346992,-0.08477239,0.65301008,0.00042873,-0.00048435,0.00065$ $142,-0.00003628,0.00013423,0.00009354,-0.00009575,0.00002718,0.0001093$ 
$6,-0.00002908,-0.00015306,-0.00006695,0.00010550,-0.00008413,-0.000033$ $15,0.00018167,0.00014267,0.00001905,0.00003375,0.00001888,0.00013175,-$ $0.00006289,-0.00035088,-0.00009541,0.00000907,0.00000659,0.00003084,-0$ $.00002834,0.00000164,-0.00001570,0.00001536,-0.00002228,0.00004637,0.0$ $0000055,-0.00000853,0.00000393,-0.04929688,-0.07807170,-0.10609026,0.1$ $0333149,0.16372333,0.21252403,-0.00361981,0.00302458,0.00065286,-0.000$ $63813,-0.00073585,-0.00164374,-0.00003425,0.00147779,0.00038100,-0.000$ $32917,-0.00053610,0.00115788,0.00035798,-0.00004306,-0.00072566,-0.001$ $95399,-0.00046298,0.00013149,-0.00018848,0.00057790,0.00042427,-0.0007$ $0863,0.00150019,-0.00005228,-0.00006530,-0.00000548,0.00007607,0.00003$ $874,0.00012274,0.00003532,-0.00016062,0.00022774,-0.00022235,-0.000072$ $72,-0.00003118,-0.00004108,-0.01985705,-0.07382913,-0.03332240,-0.3028$ $0581,0.03911782,-0.04914686,0.58780609,0.00081499,0.00038868,0.0004445$ $8,-0.00051559,-0.00037215,0.00000969,-0.00024126,0.00051050,0.00012041$ ,-0.00018911,-0.00032576,0.00062186,0.00033076,-0.00020015,-0.00037648 $,-0.00045153,0.00019359,-0.00022211,-0.00077015,0.00061601,-0.00004568$ ,-0.00073071,0.00121222,0.00003948,0.00001118,0.00001697,0.00003112,-0 $.00002668,0.00003364,-0.00004196,-0.00001939,-0.00002640,0.00001181,-0$ $.00001727,-0.00002481,0.00000924,-0.02189256,0.03242588,0.00313320,-0$. $03062162,-0.10918405,-0.02478741,-0.00638135,0.65603643,-0.00053502,0$. $00125975,0.00064170,-0.00089113,-0.00028138,-0.00029549,0.00002939,0.0$ $0003471,-0.00015020,-0.00006445,0.00009227,0.00047888,0.00008246,0.000$ $00316,0.00014087,-0.00052501,-0.00022445,-0.00031483,-0.00003402,0.000$ $22733,-0.00003429,-0.00013488,0.00128406,-0.00000111,0.00000854,0.0000$ $2844,0.00000791,0.00001905,0.00003768,0.00002170,-0.00004911,-0.000046$ $06,-0.00012366,-0.00004384,-0.00000477,-0.00002676,-0.01446022,-0.0107$ $5939,-0.00065163,-0.07343832,-0.00634763,-0.08183178,0.11545534,0.1822$ $8786,0.21865516,0.07034918,-0.00764157,0.00100121,-0.02005557,-0.01645$ $101,0.02219094,0.00231345,-0.00759783,-0.00485920,-0.00169105,0.000499$ $73,0.00491141,0.00524757,-0.00026807,-0.01302627,-0.01820407,0.0301626$ $8,0.00135293,-0.01415240,-0.00163879,-0.00588951,-0.00072576,0.0047641$ $5,0.00037096,0.00029667,-0.00013415,-0.00034478,0.00041946,-0.00034721$ ,-0.00031471,0.00065680,-0.00036408,0.00087724,0.00020187,-0.00004871, $0.00001645,-0.00417329,0.00453627,0.00242921,-0.03425967,0.02438725,-0$ $.00417921,-0.14875719,0.12092067,0.01809032,0.29245330,0.00103783,-0.0$ $1015520,-0.00174978,-0.00419703,0.00106888,0.00174881,0.00051925,-0.00$ $231599,-0.00110949,-0.00007753,0.00197777,-0.00071002,-0.00068305,-0.0$ $0097827,0.00211221,0.00676341,-0.00121892,-0.00008472,-0.00352925,-0.0$ $0048365,-0.00206275,-0.00036918,-0.00087467,0.00038304,0.00008829,-0.0$ $0000592,-0.00001938,-0.00030105,0.00001724,-0.00007119,-0.00017574,0.0$ $0008084,-0.00009191,0.00002479,0.00003212,-0.00001937,0.00483197,-0.06$ $810932,-0.02060240,0.07591582,0.02435130,0.02425336,0.08889582,-0.2724$ $8814,-0.05400046,-0.04037902,0.60197045,0.01395298,-0.00547722,-0.0096$ $8347,0.00308259,-0.00149503,0.00427980,0.00050431,-0.00019021,0.000036$ $46,-0.00072489,-0.00057614,0.00109803,0.00125900,0.00001245,-0.0036065$ $3,-0.00331637,0.00669324,0.00189748,-0.00886594,-0.00067365,-0.0026169$ $0,0.00005627,0.00030911,0.00002305,-0.00010985,-0.00007951,-0.00004022$ $, 0.00004918,-0.00010769,-0.00010274,0.00030305,-0.00011382,0.00031935$, $0.00012377,-0.00002526,0.00008479,0.00237039,-0.02059706,-0.01358087,0$ $.01489917,0.01039436,0.02014325,0.00638378,-0.04622512,-0.06725604,0.0$ $4327970,0.17454567,0.15816286,-0.00213106,-0.00240830,-0.00170621,-0.0$ 
$0090083,-0.00033222,-0.00096109,-0.00004496,0.00076022,0.00021313,-0.0$ $0063669,-0.00031835,0.00136594,0.00097297,-0.00063426,-0.00103322,-0.0$ $0184782,-0.00090674,-0.00022208,-0.00117262,-0.00031320,-0.00061754,-0$ $.00057935,0.00116408,0.00003610,-0.00003782,-0.00001964,0.00006386,-0$. $00015160,0.00011885,-0.00003331,-0.00012830,0.00012373,-0.00011335,-0$. $00004193,-0.00004513,0.00000136,-0.03413731,0.07095912,0.01460620,-0.0$ $4023509,-0.02662404,-0.01920287,0.05332200,-0.01198653,0.00814860,-0.1$ $2336924,-0.05595667,-0.03863378,0.59928156,-0.00002256,0.00204716,-0.0$ $0093028,0.00050102,-0.00019824,0.00101560,0.00018242,-0.00042238,0.000$ $22877,-0.00025034,-0.00014041,-0.00069737,0.00027585,0.00013541,-0.000$ $48708,0.00046305,-0.00060568,0.00053392,-0.00010132,0.00003397,0.00022$ $147,0.00004671,-0.00034268,0.00004713,0.00000976,-0.00002109,-0.000043$ $44,0.00018970,-0.00012577,0.00001239,0.00006548,0.00006127,0.00028216$, $0.00008758,0.00000103,0.00003354,0.01864019,0.02999444,0.01321622,-0.0$ $2494551,-0.01525766,-0.01149710,0.01896321,-0.04928080,-0.01381010,-0$. $08748595,-0.26805911,-0.10058885,-0.08037784,0.65229503,-0.00104499,0$. $00095992,-0.00030364,-0.00033998,-0.00032001,0.00027008,0.00022098,-0$. $00043843,-0.00011390,0.00009386,0.00020090,0.00005030,0.00009551,-0.00$ $014958,-0.00010535,-0.00033538,-0.00077346,-0.00038485,-0.00001381,-0$. $00025700,-0.00031732,-0.00005888,0.00052833,0.00006860,-0.00006653,-0$. $00001160,-0.00003912,0.00000644,0.00000989,0.00000762,0.00001320,0.000$ $00777,0.00021401,-0.00003264,0.00001235,0.00000504,-0.00398367,0.02718$ $068,0.01553740,-0.01922338,-0.01139485,-0.01147621,0.01937145,-0.02259$ $220,0.00124673,-0.04957939,-0.09272176,-0.09883077,0.09520732,0.169316$ $15,0.21114716,0.00109699,-0.00010304,-0.00058114,0.00020748,-0.0000181$ $6,0.00039720,0.00010679,-0.00020412,-0.00007973,-0.00001146,0.00030572$ ,-0.00011511,-0.00028286,0.00017156,0.00024971,0.00042902,-0.00011156, $0.00015359,0.00003722,0.00000818,0.00010322,0.00010888,-0.00018686,-0$. $00003297,-0.00001762,-0.00000362,0.00001116,0.00003085,0.00002704,0.00$ $002828,-0.00007421,0.00005043,-0.00012487,0.00000329,0.00001798,-0.000$ $01975,-0.15343132,0.06357353,-0.00048394,0.05438761,0.02417597,0.02091$ $534,-0.04867815,0.02856981,-0.00243669,-0.01860673,-0.07693867,-0.0354$ $5190,-0.30374125,0.04181317,-0.04856864,0.62466267,0.00024661,-0.00057$ $601,-0.00026732,-0.00017443,-0.00027660,-0.00041050,-0.00001038,0.0003$ $6378,-0.00009527,-0.00005573,-0.00010324,0.00062533,0.00014377,-0.0000$ $1201,-0.00001752,0.00012197,0.00026980,0.00001291,-0.00035915,-0.00012$ $172,-0.00021861,-0.00015284,0.00031414,-0.00003952,-0.00003621,-0.0000$ $0299,0.00000004,-0.00008815,0.00007741,-0.00002268,-0.00013198,0.00003$ $631,-0.00017241,-0.00002728,-0.00000038,-0.00003589,0.12208527,-0.2646$ $3023,-0.03996249,-0.01675379,-0.04837234,-0.02440032,0.02946712,-0.016$ $44065,0.00242919,-0.02513971,0.02590543,-0.00146360,-0.02718246,-0.108$ $62512,-0.02501148,-0.00574779,0.64856012,0.00080298,-0.00051770,0.0009$ $5504,-0.00063185,-0.00029274,0.00001657,-0.00004629,0.00008706,0.00005$ $010,-0.00022102,-0.00000034,0.00021233,0.00005238,-0.00000161,-0.00002$ $520,-0.00015542,0.00029648,0.00009001,0.00005190,0.00002611,0.00005846$ ,-0.00005834,0.00003025,0.00000092,0.00001816,-0.00000658,0.00002382,-$0.00003710,-0.00000233,-0.00003133,0.00001958,0.00004474,-0.00001040$,$0.00001636,-0.00000659,-0.00000639,0.02045244,-0.05527535,-0.07958165$, $0.00628956,-0.01410645,0.00491590,-0.00142961,0.00263140,-0.00175175,-$ $0.01749568,-0.01557214,0.00192947,-0.07331125,-0.00666671,-0.08199736$, $0.12838646,0.18625615,0.22758577,0.00050120,-0.00004807,0.00005563,-0$. 
$00011777,-0.00006503,-0.00017944,-0.00005985,0.00018986,-0.00000308,-0$ $.00007961,-0.00011734,0.00021566,0.00011880,-0.00013886,-0.00021155,-0$ $.00029616,-0.00003775,0.00008485,0.00010628,0.00003417,0.00010979,0.00$ $001828,0.00033256,0.00000370,0.00000668,-0.00000291,0.00003335,0.00001$ $006,0.00004154,0.00001234,0.00005538,-0.00001288,0.00004527,-0.0000199$ $3,0.00001162,0.00002413,-0.14380684,0.00228012,-0.03050312,-0.03320015$ $, 0.00047909,-0.00979231,0.00239943,-0.00407499,-0.00188559,-0.00064608$ $,-0.00008212,-0.00020179,0.00177824,0.00511451,0.00122705,-0.03693235$, $0.00056240,-0.01067568,0.21120118,0.00000980,-0.00000718,0.00005127,-0$ $.00000426,-0.00000618,-0.00001482,-0.00000294,0.00001806,0.00000615,0$. $00000257,-0.00001228,0.00000848,-0.00000631,0.00001433,0.00002117,0.00$ $000298,0.00003869,0.00002407,0.00002462,0.00000159,0.00003066,0.000017$ $30,-0.00004996,-0.00000496,0.00000026,-0.00000036,-0.00000300,-0.00000$ $904,0.00000988,-0.00000464,-0.00000618,-0.00000231,-0.00001702,-0.0000$ $0473,0.00000137,-0.00000026,0.00229324,-0.03749147,-0.00429666,-0.0239$ $4991,0.00332689,-0.00673629,0.00151393,-0.00276562,-0.00210047,-0.0001$ $6721,0.00158727,0.00079559,-0.00039724,-0.00281714,-0.00273162,0.02492$ $414,0.00289307,0.00587741,-0.00371494,0.03381024,0.00009632,0.00007019$ ,-0.00010307,0.00000420,-0.00002119,-0.00004721,0.00000339,0.00003497, $-0.00003092,-0.00000487,-0.00000720,0.00005456,0.00002225,-0.00001642$, $-0.00001094,-0.00002541,-0.00005206,-0.00002225,0.00004373,-0.00000261$ $, 0.00000962,0.00000533,0.00009311,-0.00000185,-0.00000524,-0.00000296$, $0.00000528,0.00000390,0.00001237,0.00000254,0.00000527,-0.00000561,0.0$ $0000282,-0.00000761,0.00000407,0.00000495,-0.03058990,-0.00429387,-0.0$ $3336314,-0.01842713,-0.00034304,-0.00024614,0.00002021,-0.00363137,0.0$ $0309356,0.00000515,0.00083571,-0.00053446,-0.00083698,-0.00123676,0.00$ $373701,-0.00187280,-0.00050002,0.00401753,0.05066357,0.00688883,0.0275$ $8522,0.00016944,0.00014196,0.00007549,-0.00016591,-0.00013899,-0.00012$ $350,-0.00000940,0.00013653,0.00001742,-0.00005776,-0.00005378,0.000193$ $21,0.00007785,-0.00004336,-0.00011716,-0.00022625,0.00004425,0.0000038$ $5,-0.00000801,0.00002635,0.00001780,-0.00007248,0.00030298,0.00000198$, $-0.00001335,0.00000056,0.00000975,-0.00000115,0.00001544,0.00000047,-0$ $.00001177,0.00000865,-0.00001188,-0.00000763,-0.00000406,-0.00000115,0$ $.00811671,-0.01210312,-0.00301147,-0.14060069,0.13390201,0.01968199,-0$ $.01158051,0.02357503,0.00414714,-0.00430133,0.00082625,-0.00258157,-0$. $00043415,-0.00103508,-0.00022906,-0.00009119,-0.00234433,-0.00221480,0$ $.00087159,-0.00092559,0.00058912,0.14676487,-0.00019938,0.00005135,-0$. $00002034,-0.00002799,0.00001469,-0.00001517,0.00000820,-0.00000655,-0$. $00001114,-0.00000346,0.00001898,0.00000616,-0.00000982,0.00000239,0.00$ $002793,0.00004392,-0.00005547,-0.00001598,0.00001673,0.00001165,-0.000$ $00895,-0.00001575,0.00000627,-0.00000168,-0.00000330,-0.00000022,-0.00$ $000136,-0.00000233,0.00000134,-0.00000057,-0.00000751,0.00000104,-0.00$ $000224,-0.00000146,-0.00000141,0.00000151,0.01633033,-0.01534652,-0.00$ $237618,0.13048157,-0.24699841,-0.04061649,-0.00274339,0.00364479,-0.00$ $096625,0.00017627,0.00226482,-0.00137485,-0.00065935,0.00011564,0.0001$ $5155,-0.00284951,-0.00185502,-0.00318287,0.00009724,0.00066187,0.00117$ $790,-0.14114343,0.25803409,0.00000574,0.00001315,0.00004769,0.00004205$ ,-0.00004320,-0.00003864,-0.00001655,0.00007818,0.00003917,0.00000244, $-0.00004320,0.00004332,0.00001026,-0.00001173,-0.00005418,-0.00006655$, $0.00003993,0.00001829,-0.00004304,0.00001758,0.00002542,0.00001776,0.0$ $0006550,0.00000458,0.00000450,-0.00000038,0.00000133,-0.00000035,0.000$ 
$00449,-0.00000014,-0.00000334,0.00001179,-0.00000445,-0.00000335,-0.00$ $000118,0.00000035,0.00708468,-0.00995613,0.00177955,0.01844427,-0.0394$ $7359,-0.04454775,-0.00530460,0.00590000,0.00524451,-0.00262817,-0.0013$ $6865,0.00512735,-0.00008029,0.00016549,-0.00080141,-0.00245820,-0.0030$ $3637,0.00333858,0.00093945,0.00091101,-0.00207499,-0.01753812,0.045839$ $00,0.03540119,0.00009974,0.00027326,0.00051220,-0.00007128,0.00013534$, $-0.00012536,-0.00006912,0.00005502,0.00014902,0.00002391,-0.00008983,-$ $0.00003737,-0.00003442,-0.00006690,-0.00003583,0.00004833,-0.00005944$, $-0.00004608,0.00015044,0.00004301,-0.00021702,-0.00035180,0.00029187,0$ $.00023712,0.00000602,0.00001550,-0.00000128,0.00001899,-0.00001059,-0$. $00000077,0.00001585,-0.00002306,0.00003073,-0.00000357,-0.00001470,-0$. $00000224,-0.00337231,0.00128692,-0.00212689,-0.00894625,-0.02492099,-0$ $.01221763,-0.10370795,-0.09467077,-0.05003424,0.01086083,0.01269883,0$. $00704339,-0.00097989,0.00431504,-0.00009469,-0.00097984,0.00046726,0.0$ $0012453,-0.00127011,-0.00075435,-0.00061051,0.00096821,-0.00027290,0.0$ $0114969,0.10719892,-0.00103963,-0.00004705,-0.00038245,0.00028098,0.00$ $025572,-0.00022053,-0.00002797,-0.00001476,0.00008586,0.00004593,0.000$ $00156,-0.00006959,-0.00008439,0.00009711,0.00016696,0.00013978,-0.0002$ $0478,0.00021499,0.00047775,-0.00000527,0.00042875,0.00000515,-0.000257$ $75,0.00002482,-0.00001479,0.00001409,0.00001812,-0.00001800,0.00001992$ $, 0.00002357,-0.00000955,-0.00002378,-0.00005327,-0.00001722,0.00000567$ ,0.00000134,0.00099298,0.00242344,-0.00110814,0.00268011,0.00389869,0. $00018741,-0.09792028,-0.24131944,-0.09564651,-0.00916415,-0.01815246,-$ $0.00867220,0.00296718,-0.00223566,-0.00195166,0.00075213,0.00004354,0$. $00049898,0.00026776,0.00004148,0.00009098,-0.00042480,0.00015436,0.001$ $27097,0.10102434,0.25582966,-0.00047657,0.00045206,-0.00009797,0.00026$ $664,0.00001581,-0.00007266,0.00007645,-0.00007256,-0.00014700,0.000037$ $82,0.00012819,-0.00017701,-0.00012365,0.00008984,0.00016933,0.00005822$ ,-0.00029039,-0.00004919,0.00008445,-0.00000598,-0.00006867,0.00036137 ,-0.00047608,0.00004043,-0.00000012,-0.00001755,-0.00002486,0.00001490 $, 0.00000699,0.00001027,0.00000141,0.00003009,0.00000396,0.00000684,0.0$ $0001543,0.00000442,-0.00238737,-0.00106750,0.00577507,-0.00304831,-0.0$ $0716113,0.00196726,-0.05025599,-0.09399546,-0.08015824,-0.00068281,-0$. $00310818,-0.00114777,-0.00057991,-0.00182130,0.00502416,0.00029141,0.0$ $0040943,-0.00059485,-0.00026933,-0.00021599,-0.00010995,0.00113246,0.0$ $0137731,-0.00330442,0.05598709,0.10524745,0.07347707,0.00072328,0.0001$ $2456,-0.00015021,-0.00004098,-0.00013205,0.00035711,-0.00002666,-0.000$ $10279,0.00002834,-0.00015158,-0.00006339,0.00022869,-0.00030335,0.0002$ $7970,-0.00001936,-0.00008146,-0.00030147,-0.00030326,-0.00003750,-0.00$ $001803,-0.00002325,0.00000945,-0.00005126,0.00003784,0.00002048,0.0000$ $1721,-0.00000291,-0.00005164,0.00000443,-0.00003380,-0.00009165,0.0000$ $0724,-0.00004011,0.00001156,-0.00000330,-0.00001439,-0.00305538,-0.000$ $52798,-0.00274419,-0.00073725,-0.00052021,-0.00019153,-0.00027837,-0.0$ $0306313,-0.00259442,0.01059472,-0.00903437,-0.00056114,-0.13683586,0.1$ $2692654,0.01895088,-0.01244488,0.02500795,0.00449009,-0.00136357,0.000$ $76416,-0.00010511,-0.00006998,0.00000466,0.00013365,0.00039652,-0.0005$ $5593,-0.00010580,0.14291183,0.00092941,-0.00061949,0.00057281,-0.00030$ $154,-0.00012897,-0.00010878,-0.00001333,0.00006059,-0.00013385,0.00001$ $969,0.00008267,0.00027394,0.00007225,0.00024709,0.00028922,0.00007875$, $0.00060907,-0.00010858,0.00010789,0.00002041,0.00004785,0.00000909,-0$. $00008827,-0.00003280,-0.00000330,-0.00000283,0.00000004,0.00002853,-0$. 
$00002543,0.00001701,-0.00005674,0.00004012,-0.00012716,-0.00001470,0.0$ $0000919,-0.00001850,-0.00011952,0.00257708,-0.00158403,-0.00092168,-0$. $00000167,0.00009146,-0.00151768,-0.00211906,-0.00325903,0.01196883,-0$. $01923922,-0.00376024,0.12995275,-0.24087595,-0.04045783,-0.00205116,0$. $00333372,-0.00107229,-0.00018819,0.00001737,-0.00005862,0.00005504,-0$. $00007965,0.00019996,0.00064401,-0.00051869,-0.00007162,-0.13841905,0.2$ $5654340,0.00041917,-0.00005049,-0.00064597,0.00024725,0.00006974,0.000$ $05123,0.00005039,0.00006779,-0.00017552,0.00005794,0.00006127,-0.00008$ $018,-0.00003610,0.00009971,0.00025595,0.00011292,-0.00011016,-0.000042$ $84,-0.00021703,-0.00006385,-0.00002704,0.00005157,-0.00004519,-0.00001$ $998,0.00000883,0.00001164,-0.00000492,-0.00000820,0.00001704,0.0000371$ $3,-0.00020991,0.00006733,-0.00028694,0.00004695,0.00001325,-0.00002127$ $,-0.00259883,-0.00156329,0.00545332,-0.00032798,0.00015287,-0.00081082$ ,-0.00225800,-0.00353999,0.00381561,0.00723931,-0.00922077,-0.00089643 $, 0.01995819,-0.04110743,-0.04444820,-0.00529457,0.00589353,0.00573791$, $-0.00042995,0.00019413,0.00001017,0.00013807,0.00018423,-0.00049427,0$. $00033197,-0.00037162,0.00001836,-0.01847481,0.04781836,0.03583407,0.00$ $015700,-0.00016571,-0.00004217,-0.00013921,-0.00009614,-0.00012135,-0$. $00000859,0.00009847,-0.00000428,-0.00003163,-0.00001420,0.00020121,0.0$ $0008889,-0.00006661,-0.00010265,-0.00011742,0.00003180,0.00001609,-0.0$ $0009264,-0.00000120,-0.00001942,-0.00004896,0.00019057,-0.00000043,-0$. $00001116,-0.00000546,0.00000998,-0.00003103,0.00003004,-0.00001254,-0$. $00001632,0.00001546,-0.00002238,-0.00002712,-0.00000229,0.00000022,0.0$ $0834778,0.01475939,0.00665637,-0.00103809,0.00355156,-0.00037926,-0.00$ $067225,0.00076670,0.00039226,-0.00425030,0.00009477,-0.00282945,-0.008$ $40289,-0.02327957,-0.01166340,-0.10561087,-0.09997596,-0.05437820,0.00$ $081307,0.00042238,0.00105308,0.00056751,0.00075608,0.00031412,-0.00001$ $642,-0.00012657,0.00009951,0.00094251,-0.00022007,0.00122614,0.1095556$ $9,0.00015777,0.00003948,-0.00001272,-0.00003268,-0.00002306,0.00003002$ $, 0.00001120,-0.00002283,-0.00000876,0.00000231,0.00001010,-0.00002602$, $0.00000675,0.00001849,0.00001676,0.00000823,0.00000994,-0.00000652,-0$. $00001463,0.00000027,0.00000231,0.00001521,0.00001634,0.00000134,-0.000$ $00187,-0.00000070,-0.00000383,0.00000357,-0.00000785,-0.00000094,0.000$ $00354,-0.00000178,0.00000558,0.00000058,0.00000413,0.00000423,-0.01371$ $999,-0.01535380,-0.01030950,0.00413191,-0.00196621,-0.00146639,0.00045$ $947,0.00011096,0.00047299,0.00065736,0.00222974,-0.00137091,0.00305513$ $, 0.00404719,0.00078967,-0.09633921,-0.24053323,-0.09902312,-0.00063695$ $, 0.00069650,0.00103401,-0.00061228,-0.00063752,-0.00053115,-0.00008730$ $,-0.00006855,0.00018232,-0.00042494,0.00008463,0.00137817,0.10336892,0$ $.25135332,0.00013093,-0.00010176,0.00003140,0.00000344,0.00001703,-0.0$ $0002064,-0.00000707,0.00004719,0.00002354,-0.00004347,-0.00002865,0.00$ $007437,0.00004115,-0.00002915,-0.00006336,-0.00008808,0.00003153,0.000$ $05681,-0.00002018,-0.00000637,-0.00000014,-0.00002163,-0.00001285,-0.0$ $0000607,0.00000220,0.00000471,0.00000109,-0.00000186,0.00001275,0.0000$ $0281,-0.00000865,-0.00000160,-0.00000700,0.00000988,-0.00000110,-0.000$ $00178,-0.00361737,-0.00287157,0.00146787,-0.00010712,-0.00165432,0.004$ $47166,0.00032018,0.00056541,-0.00059808,-0.00264172,-0.00133141,0.0051$ $6022,-0.00225502,-0.00621188,0.00152608,-0.05305929,-0.09999925,-0.084$ $95741,0.00067908,0.00130686,-0.00200497,-0.00017535,-0.00017246,0.0002$ $8722,0.00010948,0.00013904,-0.00053502,0.00114482,0.00145966,-0.003258$ $41,0.05959989,0.10883459,0.07835283,-0.00114962,0.00105094,0.00217082$, 
$0.01530718,0.00605245,-0.00193013,0.00185375,-0.00209874,0.00038215,-0$ $.00071172,0.00031358,-0.00122857,0.00003904,-0.00003617,0.00036818,-0$. $00040225,-0.00027775,-0.00094117,0.00039587,0.00004306,-0.00002890,-0$. $05228729,0.13987791,-0.00077641,0.00035336,-0.00001556,0.00014657,0.00$ $016491,0.00005156,0.00009476,-0.00015548,0.00012834,-0.00002178,-0.000$ $18225,-0.00011267,-0.00008348,0.00017549,-0.00007326,-0.00003445,0.000$ $08530,-0.00039560,-0.00004454,0.00018417,0.00066350,0.00075385,-0.0002$ $0116,0.00002665,-0.00003091,0.00045386,-0.00009605,0.00004776,-0.00016$ $805,0.00007785,-0.00001648,-0.00008779,-0.00003269,-0.00002994,0.00011$ $537,0.00002458,-0.00000602,0.00001522,-0.00023080,-0.00048870,-0.00004$ $026,-0.00003929,-0.00003331,0.00004244,-0.00001211,0.00000575,0.036199$ $91,0.00955992,0.00378661,-0.00681536,0.00635096,-0.02920274,-0.0010375$ $6,-0.00598031,0.00764606,0.00961918,-0.00013767,-0.00531037,0.00142810$ $, 0.00129745,0.00085892,-0.00289722,-0.00090201,0.00102624,0.00070811,-$ $0.00086313,-0.00013040,0.00020845,0.13903572,-1.15939543,0.00697758,0$. $00043848,-0.00010786,-0.00044504,0.00001613,-0.00006802,-0.00005423,0$. $00003018,-0.00024042,-0.00002637,-0.00000433,-0.00036804,0.00012899,0$. $00022454,0.00010284,0.00013197,-0.00018597,0.00052089,0.00009218,-0.00$ $114185,-0.00064263,-0.00071094,-0.00124574,0.00059500,0.00004096,-0.00$ $093910,0.00027653,-0.00034570,0.00016406,-0.00024780,-0.00002176,-0.00$ $029083,0.00003389,-0.00007039,-0.00020624,-0.00000252,-0.00005146,-0.0$ $0027958,0.00006294,0.00034880,0.00008143,0.00007386,0.00004375,-0.0001$ $3236,-0.00000555,0.00000362,-0.14488974,1.18073801,0.00209613,-0.00178$ $775,-0.00138001,-0.00256982,0.00067186,0.01912934,0.00146986,0.0019654$ $0,-0.00149831,-0.00106203,0.00059571,0.00041718,-0.00018524,-0.0000351$ 4,0.00061944,-0.00080906,-0.00069880,0.00060031,0.00005487,-0.00020636 $, 0.00021250,-0.00064855,0.00663357,-0.03557217,0.00008897,0.00021292,0$ $.00023618,0.00008833,0.00002605,0.00005414,0.00002640,-0.00025282,-0.0$ $0007978,-0.00010857,0.00022272,-0.00015072,-0.00002886,0.00001709,-0.0$ $0000936,0.00003633,0.00010312,0.00010578,-0.00000729,-0.00034828,-0.00$ $015277,-0.00012336,-0.00003443,0.00003409,-0.00001379,0.00002548,-0.00$ $001606,0.00004322,0.00002666,0.00002392,0.00005797,0.00001009,0.000026$ $65,-0.00000471,0.00000096,-0.00003830,-0.00012532,0.00007672,-0.000002$ $70,-0.00000712,0.00002301,-0.00000095,0.00000666,0.00000786,-0.0000030$ $1,0.00172497,-0.00725564,0.01744462 \backslash \backslash-0.00000204,0.00000281,-0.0000000$ $8,0.00000099,-0.00000101,-0.00000077,-0.00000167,0.00000412,-0.0000004$ $6,0.00000073,0.00000249,-0.00000311,0.00000124,0.00000091,-0.00000263$, $0.00000257,-0.00000464,-0.00000082,0.00000161,-0.00000032,0.00000118,-$ $0.00000114,0.00000092,0.00000309,-0.00000109,0.00000394,0.00000010,0.0$ $0000119,0.00000084,-0.00000499,0.00000099,-0.00000349,-0.00000383,-0.0$ $0000013,0.00000389,-0.00000353,-0.00000061,-0.00000052,0.00000152,0 .,-$ $0.00000122,0.00000342,0.00000086,-0.00000168,0.00000158,-0.00000381,-0$ $.00000076,-0.00000008,0.00000159,-0.00000083,-0.00000024,0.00000036,-0$ $.00000104,-0.00000086,-0.00000034,-0.00000099,0.00000207,-0.00000060,-$ $0.00000191,0.00000429,-0.00000035,-0.00000208,0.00000388,0.00000075,0$. $00000013,-0.00000288,0.00000038,-0.00000012,-0.00000129,-0.00000147,0$. 00000055,0.00000445!II@

meta-Transition State 


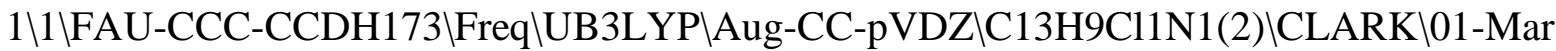
-2015\0\\N Geom=AllCheck Guess=TCheck SCRF=Check GenChk UB3LYP/Aug-CC -pVDZ Freq \\Addition TS p-Cl-Ph-radical + aniline (meta) \0,2\C,-0.848 $1978462,-1.9412538555,0.000170636 \mathrm{lC},-1.3797269065,-1.4434816294,-1.219$ $1823025 \backslash \mathrm{C},-2.292871966,-0.3953483765,-1.2219852104 \backslash \mathrm{C},-2.735544553,0.12$ 99289014,0.0000517327\C,-2.2928322525,-0.3951720036,1.2221502139\C,-1. $3796871578,-1.4433056319,1.2194690653 \backslash \mathrm{H},-0.3223735164,-2.893682754,0.0$ $002310038 \backslash \mathrm{H},-1.0473687177,-1.8681675011,-2.1659578964 \backslash \mathrm{H},-2.67897012,0$. $0064343835,-2.1571282299 \backslash \mathrm{H},-2.6789007359,0.0067451986,2.1572477126 \backslash \mathrm{H},-$ $1.0472988714,-1.8678553605,2.1662951793 \backslash \mathrm{Cl},-3.8942744523,1.4515699892$, $-0.0000245883 \backslash \mathrm{C}, 3.7832521708,0.0100567215,-0.0000981837 \backslash \mathrm{C}, 3.614492557$, $-1.3747238426,-0.0000083036 \backslash \mathrm{C}, 2.3242022464,-1.9317106716,0.0000760893 \backslash$ C,1.2379144743,-1.0710718889,0.0000677655\C,1.3565406463,0.3051614843, $-0.000019138 \backslash \mathrm{C}, 2.6571000448,0.855237093,-0.0001037757 \backslash \mathrm{H}, 4.7812556914,0$ $.4451314914,-0.0001642038 \mathrm{LH}, 4.4895611088,-2.0261098116,-0.0000040752 \backslash \mathrm{H}$ ,2.1985173256,-3.0161304008,0.0001451502\H,0.4847234678,0.9592913722,$0.0000232809 \backslash \mathrm{C}, 2.83106019,2.2821083528,-0.0001956366 \mathrm{WN}, 2.9668111723,3$. 4376127402,-0.0002697237\\Version=EM64L-G09RevC.01 \State=2-A 7277126\S2=0.779995 \S2-1=0. IS2A=0.750638\RMSD=9.641e-09\RMSF=1.912e-06 ZZeroPoint $=0.1763758 \backslash$ Thermal $=0.1892256 \mathrm{ZPE}=110.6774877 \mathrm{Dipole}=-0.00517$ $15,-2.2891441,0.0001432 \backslash$ DipoleDeriv $=0.0635599,0.0036352,-0.0000027,0.0$ $393651,0.0698079,-0.000011,-0.0000048,-0.0000082,-0.0581981,-0.0362474$ ,-0.1301937,-0.0567307,-0.1760971,-0.0794412,0.0136996,0.0621161,0.057 $3665,0.0560194,-0.2140005,0.0694599,0.0610263,0.1443025,-0.1693282,-0$. $0696413,0.1637708,-0.0485047,0.0169371,0.6399097,-0.5727341,0.0000311$, $-0.7479523,0.621865,-0.0000286,0.0000481,-0.0000325,0.0411543,-0.21400$ $94,0.0694511,-0.0610287,0.1442856,-0.1693112,0.069663,-0.1637871,0.048$ $5294,0.0169286,-0.0362469,-0.1301859,0.0567529,-0.176109,-0.079452,-0$. $0136732,-0.0620882,-0.0573438,0.0560305,0.1211747,0.0949333,-0.0000081$ $, 0.0935499,-0.0105223,0.0000034,-0.000008,0.000003,0.0602667,0.0740195$ $, 0.0657462,0.0455909,0.0469483,0.0654891,-0.034352,0.0252599,-0.041575$ $6,-0.0269599,0.090376,0.0371845,-0.0346275,0.0475945,0.0891219,0.04161$ $75,-0.0407651,0.0295653,0.0125303,0.0903783,0.0371888,0.0346199,0.0475$ $982,0.0891124,-0.0416305,0.0407552,-0.0295772,0.012538,0.0740179,0.065$ $7399,-0.045603,0.0469456,0.0655003,0.034337,-0.0252687,0.0415599,-0.02$ $69686,-0.5519126,0.3398137,-0.0000164,0.5484186,-0.4362249,0.0000151,-$ $0.000032,0.0000196,-0.0875338,-0.0763338,-0.0272934,-0.0000015,0.09753$ $15,-0.0329433,-0.0000078,-0.0000073,-0.0000024,-0.0956702,0.0109105,-0$ $.1040129,0.0000003,0.0136989,0.0179163,-0.0000063,-0.0000041,-0.000004$ $4,-0.1223732,-0.3009391,-0.0269282,0.0000043,-0.4119791,0.0811877,-0.0$ $000016,0.0000327,-0.0000085,-0.0843808,0.7499716,0.1285916,-0.0000251$, $0.4147102,-0.0960887,-0.0000063,-0.0000584,-0.000001,-0.0597575,-0.484$ $467,0.0193257,0.0000077,-0.0924348,-0.0309175,0.0000023,0.0000215,-0.0$ $000019,-0.0492148,0.2640854,-0.0033919,-0.0000071,-0.1423347,0.0163701$ $, 0.0000042,0.0000014,0.0000025,0.0431918,-0.0176354,-0.039504,0.000008$ $5,-0.0518602,0.0579333,0.0000067,0.0000088,0.0000061,0.1358428,-0.1033$ $017,0.0776947,0.0000029,0.0417988,0.0043125,0.0000053,0.0000066,0.0000$ $045,0.1275503,0.0969622,-0.0463073,0.0000043,0.0250431,-0.0834047,0.00$ $00118,-0.000001,0.0000129,0.1212978,0.0710799,0.0650533,-0.0000014,0.0$ $434823,0.0519785,0.000002,-0.0000015,0.0000002,0.103266,-0.0262634,0.0$ $314372,0.0000023,0.1383235,0.4371392,-0.000023,-0.000002,-0.0000202,0$. 
$1184482,-0.2850886,-0.0247038,0.0000007,-0.1348292,-0.4801005,0.000013$ $6,0.0000072,0.0000106,-0.310945 \backslash$ Polar $=241.7753302,-17.5903242,197.3901$ $511,-0.0016976,-0.0039088,128.5715213 \backslash \mathrm{PG}=\mathrm{C} 01[\mathrm{X}(\mathrm{C} 13 \mathrm{H} 9 \mathrm{Cl1N} 1)] \mathrm{NImag}=1 \rrbracket$ $0.20101906,-0.29121954,0.52786822,0.00002768,0.00001076,0.60952169,-0$. $07805281,0.05752605,-0.04963018,0.37317742,0.03184027,-0.12298197,0.03$ $217154,-0.27032630,0.44107297,-0.04111869,0.07654911,-0.25832333,-0.01$ $806317,0.05248684,0.67850681,-0.02047721,0.01593858,-0.03776296,-0.184$ $01134,0.14334334,0.01779355,0.37108568,0.03130753,-0.00839064,0.046364$ $75,0.14239269,-0.21945350,-0.02486716,-0.27497753,0.43424667,-0.007500$ $26,0.02421146,0.02871289,-0.02830224,0.02906450,-0.12511287,0.04668789$ ,-0.03686912,0.70563855,0.00526478,-0.00273092,-0.00000080,-0.01188469 $, 0.02110448,0.01499930,-0.09442330,0.03957956,0.06912969,0.27805407,-0$ $.01543898,-0.00384045,-0.00000377,0.02184169,-0.01874160,-0.01720117,0$ $.03816719,-0.10550237,-0.08247296,-0.18594246,0.33253418,0.00000026,-0$ $.00000404,-0.05898113,0.05340231,-0.06336273,0.02681528,0.02307940,-0$. $02980655,-0.27317096,0.00001903,0.00002421,0.62227319,-0.02047565,0.01$ $594324,0.03776227,-0.01756750,0.01822318,-0.01066684,0.02855922,-0.024$ $24112,-0.01321140,-0.09442614,0.03816644,-0.02309064,0.37108259,0.0313$ $0714,-0.00840077,-0.04636044,0.01707116,-0.02480282,0.01306036,-0.0242$ $3883,0.03578774,0.01487555,0.03957030,-0.10548629,0.02978116,-0.274982$ $82,0.43425731,0.00749718,-0.02420663,0.02872153,-0.01481434,0.01960864$ $,-0.01161208,0.01321225,-0.01488759,-0.05266996,-0.06914113,0.08244738$ $,-0.27318389,-0.04663745,0.03691747,0.70563075,-0.07804997,0.05753051$, $0.04961571,0.02017704,-0.01963646,0.00009636,-0.01756664,0.01707288,0$. $01481214,-0.01188672,0.02183445,-0.05340406,-0.18401095,0.14239767,0.0$ $2828337,0.37317807,0.03184547,-0.12299801,-0.03219225,-0.01963661,0.03$ $093157,-0.00598421,0.01822408,-0.02480753,-0.01960735,0.02110450,-0.01$ $872998,0.06336865,0.14333961,-0.21945389,-0.02905565,-0.27032539,0.441$ $05815,0.04110801,-0.07657046,-0.25831040,-0.00009530,0.00597457,-0.039$ $87938,0.01066432,-0.01305901,-0.01160821,-0.01500122,0.01720703,0.0268$ $0588,-0.01781224,0.02487598,-0.12511267,0.01811199,-0.05244350,0.67852$ $071,-0.08846732,0.13125584,-0.00000888,-0.00865332,0.01253230,-0.00348$ $623,0.00513309,0.00037001,-0.00422592,-0.00104215,0.00102757,-0.000000$ $18,0.00513327,0.00037057,0.00422561,-0.00865273,0.01253205,0.00348481$, $0.11344101,0.14564161,-0.26568354,0.00001280,0.00124913,-0.00272177,-0$ $.00492569,0.00215722,0.00073657,0.00095823,-0.00118784,0.00108194,-0.0$ $0000012,0.00215740,0.00073623,-0.00095908,0.00125123,-0.00272448,0.004$ $92664,-0.14103061,0.27768795,-0.00001007,0.00001292,-0.05591083,-0.013$ $29523,0.02384683,0.00423684,-0.00146127,0.00144129,-0.00445400,0.00000$ $009,-0.00000021,-0.00136857,0.00146065,-0.00144207,-0.00445385,0.01329$ $545,-0.02384621,0.00423899,0.00000933,-0.00001370,0.05473330,0.0061323$ $3,-0.00501724,-0.01216881,-0.07027843,0.04431423,0.07575482,-0.0023128$ $0,0.00805839,0.01821498,0.00245051,0.00549735,-0.00106897,-0.00081348$, $-0.00019655,-0.00022574,0.00226812,0.00344222,-0.00202668,-0.00164346$, $-0.00104241,0.00025848,0.06636801,-0.00404377,0.00736300,0.00979706,0$. $04502349,-0.09070340,-0.09537524,0.00693205,-0.00454975,-0.02114638,0$. $00565814,0.00070756,0.00058493,-0.00014466,-0.00075130,0.00056414,0.00$ $328735,0.00207624,0.00247419,-0.00197889,-0.00042824,-0.00043097,-0.05$ $269958,0.08826281,0.00970348,-0.01159226,-0.01728378,0.07399862,-0.097$ $40092,-0.27148955,-0.00175882,0.00228134,0.00338317,-0.00077257,0.0002$ $1733,0.00199204,-0.00039276,0.00069623,0.00017848,-0.00305087,0.002465$ 
46,-0.00310638,0.00014830,0.00019716,0.00096362,-0.07904497,0.10340877 $, 0.28527948,0.00180735,0.00669932,0.00130239,-0.00323314,0.00657011,-0$ $.01781540,-0.07968107,0.04665656,-0.08909442,0.00550068,-0.00250087,0$. $01005359,0.00229897,0.00309465,0.00193564,-0.00051307,-0.00043060,0.00$ $010254,-0.00020953,0.00034009,-0.00112325,-0.00208630,-0.00279276,0.00$ $028964,0.07651826,0.00624595,0.00040509,-0.00065763,0.00689679,-0.0032$ $9531,0.02029344,0.04728561,-0.08564123,0.09094227,-0.00340614,0.006416$ $89,-0.01084941,0.00318483,0.00165968,-0.00285669,-0.00005537,-0.000951$ $46,-0.00037094,0.00040535,-0.00018265,0.00066385,-0.00262462,-0.001285$ $73,-0.00004431,-0.05537423,0.08330622,0.00071453,-0.00081452,0.0017327$ $8,0.00189770,-0.00167556,0.00418860,-0.08717497,0.08931615,-0.26948600$ ,-0.01113360,0.01199329,-0.01821557,0.00238333,-0.00301495,-0.00277194 $, 0.00051792,-0.00054161,0.00040462,0.00004370,-0.00020476,0.00011636,0$ $.00002104,0.00015017,0.00076680,0.09342393,-0.09620129,0.28287592,0.00$ $180726,0.00669917,-0.00130336,-0.00051309,-0.00043060,-0.00010245,0.00$ $229882,0.00309446,-0.00193624,0.00550075,-0.00250273,-0.01005403,-0.07$ $967549,0.04666656,0.08908171,-0.00323266,0.00657273,0.01781470,-0.0002$ $0949,0.00034026,0.00112322,-0.00038362,-0.00040685,0.00008528,0.000374$ $80,-0.00012392,-0.00042176,0.07651231,0.00624578,0.00040528,0.00065763$ ,-0.00005544,-0.00095131,0.00037117,0.00318457,0.00166053,0.00285597,-$0.00340418,0.00641660,0.01084588,0.04729530,-0.08566737,-0.09097027,0$. $00689586,-0.00329797,-0.02029263,0.00040534,-0.00018271,-0.00066382,-0$ $.00040777,-0.00035030,-0.00001295,-0.00012384,0.00052186,0.00056730,-0$ $.05538464,0.08333412,-0.00071549,0.00081448,0.00173263,-0.00051789,0.0$ $0054183,0.00040453,-0.00238394,0.00301421,-0.00277264,0.01113335,-0.01$ $199675,-0.01821547,0.08716209,-0.08934423,-0.26946568,-0.00189845,0.00$ $167643,0.00419075,-0.00004374,0.00020480,0.00011640,0.00002781,-0.0000$ $7229,-0.00003397,0.00042173,-0.00056749,-0.00081237,-0.09340942,0.0962$ $3180,0.28285399,0.00613239,-0.00501511,0.01216882,0.00226829,0.0034424$ 2,0.00202598,-0.00081346,-0.00019654,0.00022578,0.00245055,0.00549754, $0.00106817,-0.00231333,0.00805567,-0.01821596,-0.07028312,0.04430633,-$ $0.07576744,-0.00164349,-0.00104248,-0.00025827,0.00040465,-0.00018911$, $0.00053044,-0.00038363,-0.00040778,-0.00002774,-0.00208631,-0.00262460$ ,-0.00002056,0.06637305,-0.00404553,0.00736327,-0.00980046,0.00328770, $0.00207553,-0.00247504,-0.00014462,-0.00075148,-0.00056398,0.00565823$, $0.00070744,-0.00058497,0.00693298,-0.00454705,0.02114731,0.04501583,-0$ $.09067565,0.09534770,-0.00197888,-0.00042819,0.00043127,-0.00018921,0$. $00020438,-0.00056490,-0.00040687,-0.00035029,0.00007235,-0.00279281,-0$ $.00128575,-0.00014979,-0.05269143,0.08823301,-0.00970343,0.01158895,-0$ $.01728417,0.00305026,-0.00246634,-0.00310585,0.00039280,-0.00069609,0$. $00017861,0.00077172,-0.00021727,0.00199213,0.00175800,-0.00228046,0.00$ $338099,-0.07401144,0.09737338,-0.27151243,-0.00014793,-0.00019691,0.00$ $096361,-0.00053047,0.00056474,-0.00091517,-0.00008521,0.00001301,-0.00$ $003398,-0.00028915,0.00004469,0.00076682,0.07905936,-0.10337866,0.2853$ $0420,-0.00263926,0.00007788,0.00000007,0.00465305,0.00013682,0.0036702$ $5,-0.01442730,0.02110864,0.00038905,-0.08029584,0.06730467,-0.00000428$ $,-0.01442791,0.02110926,-0.00039153,0.00465296,0.00013625,-0.00367055$, $-0.00002547,-0.00003021,0 .,-0.00058435,0.00078387,-0.00019390,-0.00081$ $550,-0.00184426,-0.00016561,-0.00081549,-0.00184424,0.00016598,-0.0005$ $8435,0.00078392,0.00019381,0.10511285,0.00205461,-0.00116020,0.0000001$ $9,-0.00007114,0.00385502,-0.00417168,0.02119072,-0.01983632,-0.0003217$ 
$9,0.06665698,-0.09848211,0.00000301,0.02118814,-0.01983332,0.00032443$, $-0.00007083,0.00385543,0.00417056,-0.00071975,-0.00039518,0.00000006,0$ $.00074498,-0.00073530,0.00023113,-0.00172405,-0.00044578,0.00038906,-0$ $.00172410,-0.00044572,-0.00038870,0.00074488,-0.00073522,-0.00023104,-$ $0.10797825,0.13455589,-0.00000009,0.00000023,0.00170722,-0.00106116,0$. $00113642,-0.00385787,0.01802187,-0.02046746,0.00301313,-0.00000422,0.0$ $0000305,-0.03914100,-0.01802435,0.02047004,0.00301070,0.00106087,-0.00$ $113756,-0.00385821,0.00000007,0.00000004,0.00026318,0.00060771,-0.0006$ $8818,0.00005628,0.00042530,-0.00043592,0.00121618,-0.00042495,0.000436$ $30,0.00121638,-0.00060780,0.00068827,0.00005622,0.00000672,-0.00000525$ $, 0.03635409,-0.00003673,0.00016379,-0.00000007,-0.00024563,-0.00001932$ $, 0.00015924,-0.00005165,-0.00009314,0.00004630,0.00028053,-0.00028664$, $0.00000007,-0.00005164,-0.00009313,-0.00004631,-0.00024568,-0.00001933$ , $0.00015920,-0.00002674,0.00002796,-0.00000001,0.00000887,0.00002656$, $0.00000241,-0.00000094,0.00000799,0.00000084,-0.00000094,0.00000795,-0$ $.00000087,0.00000891,0.00002656,-0.00000239,-0.00018568,0.00021839,-0$. $00000001,0.71511976,0.00017373,-0.00026040,0.00000014,0.00008302,-0.00$ $007496,0.00001645,0.00008954,0.00014659,0.00002715,-0.00008097,0.00014$ $773,-0.00000009,0.00008946,0.00014653,-0.00002714,0.00008320,-0.000075$ $01,-0.00001647,0.00017941,0.00026837,0 ., 0.00001063,-0.00005097,0.00003$ $018,-0.00002833,0.00000941,-0.00000050,-0.00002831,0.00000950,0.000000$ $54,0.00001054,-0.00005096,-0.00003027,-0.00002829,0.00003162,0 ., 0.0436$ $1635,0.68006568,-0.00000002,0 .,-0.00014638,-0.00011486,-0.00001253,0.0$ $0008905,0.00007175,0.00008685,-0.00005101,0 ., 0 ., 0.00002914,-0.00007176$ ,-0.00008686,-0.00005100,0.00011487,0.00001257,0.00008905,-0.00000001, $0 ., 0.00004425,0.00002690,-0.00003144,-0.00008226,-0.00000584,-0.000022$ $00,0.00001239,0.00000585,0.00002199,0.00001238,-0.00002690,0.00003143$, $-0.00008225,0 .,-0.00000002,0.00000662,-0.00002602,-0.00003439,0.132360$ $30,0.00067362,0.00121622,0 .,-0.00023202,-0.00009658,0.00002489,-0.0001$ $2043,-0.00015535,0.00005833,0.00016693,-0.00013050,0.00000002,-0.00012$ $044,-0.00015536,-0.00005832,-0.00023200,-0.00009658,-0.00002488,-0.000$ $02335,-0.00044817,0.00000004,-0.00005626,0.00002333,-0.00007184,0.0000$ $3372,0.00000400,0.00000189,0.00003372,0.00000405,-0.00000187,-0.000056$ $30,0.00002334,0.00007180,-0.00007111,0.00007273,-0.00000002,-0.1252731$ $9,0.00619665,0.00000231,0.68670593,0.00117814,-0.00036756,0 ., 0.0001528$ $0,0.00059500,-0.00010787,-0.00006026,-0.00021747,-0.00033311,-0.000027$ $22,-0.00008119,-0.00000005,-0.00006021,-0.00021740,0.00033319,0.000152$ $69,0.00059501,0.00010777,0.00015916,-0.00019987,0 ., 0.00003268,0.000029$ $63,0.00005076,0.00002341,-0.00000803,0.00002245,0.00002341,-0.00000808$ ,-0.00002247,0.00003273,0.00002962,-0.00005072,0.00030629,-0.00032897, $0.00000003,-0.05569481,-0.33928934,0.00001869,-0.04293572,0.68402960$,$0.00000021,0.00000002,0.00118879,0.00068198,-0.00000140,-0.00011911,-0$ $.00010979,0.00001215,0.00016970,0 ., 0 .,-0.00010089,0.00010980,-0.000012$ $09,0.00016964,-0.00068191,0.00000130,-0.00011915,0 ., 0.00000003,0.00001$ $759,-0.00002903,0.00002334,0.00008604,-0.00002114,-0.00000990,-0.00000$ $445,0.00002113,0.00000991,-0.00000444,0.00002903,-0.00002332,0.0000860$ $3,0 ., 0.00000002,-0.00001383,0.00000600,0.00001639,-0.06284292,-0.00001$ $966,-0.00003148,0.13143202,-0.00530081,0.00208484,-0.00000005,-0.00057$ $562,-0.00133581,0.00116305,-0.00007392,0.00065164,0.00056145,0.0002330$ $3,-0.00036014,0.00000028,-0.00007386,0.00065155,-0.00056163,-0.0005759$ $9,-0.00133588,-0.00116278,0.00075285,0.00090202,-0.00000013,-0.0001180$ 
$0,-0.00013468,-0.00019729,-0.00004462,0.00009649,-0.00004511,-0.000044$ $65,0.00009644,0.00004505,-0.00011795,-0.00013466,0.00019740,-0.0006784$ $5,0.00072869,-0.00000007,0.01634821,-0.08109048,0.00000451,-0.28293913$ , $0.03670460,0.00001101,0.60756416,-0.00284814,0.00194527,0.00000008,-$ $0.00058157,-0.00140871,0.00026496,-0.00027463,0.00041718,0.00075860,0$. 00067997,-0.00051036,-0.00000014,-0.00027465,0.00041696,-0.00075855,-0 $.00058148,-0.00140872,-0.00026475,-0.00134099,0.00063134,0.00000002,-0$ $.00005323,0.00002448,-0.00011023,-0.00000569,0.00002537,-0.00006071,-0$ $.00000567,0.00002545,0.00006074,-0.00005329,0.00002451,0.00011014,-0.0$ $0098827,0.00107540,-0.00000005,-0.03382301,-0.01307841,0.00000255,-0.1$ $1046267,-0.14923198,0.00000967,-0.00519970,0.72541601,0.00000035,-0.00$ $000013,0.00043639,0.00032009,-0.00045981,0.00000586,-0.00032007,0.0006$ $8060,0.00036604,-0.00000006,-0.00000003,-0.00079137,0.00032013,-0.0006$ $8060,0.00036627,-0.00031997,0.00046005,0.00000587,0.00000003,-0.000000$ $08,-0.00012978,0.00007040,-0.00004560,-0.00008794,-0.00000568,-0.00002$ $810,-0.00001828,0.00000568,0.00002808,-0.00001830,-0.00007038,0.000045$ $58,-0.00008796,0.00000008,-0.00000010,0.00005716,0.00000164,0.00000446$ $, 0.00724107,0.00001521,0.00000663,-0.06296540,-0.00001847,-0.00003527$, $0.12868584,0.07318753,0.01254547,-0.00000199,-0.02559069,0.01224715,-0$ $.02619408,0.00702047,-0.01139618,-0.00643205,-0.00542569,0.00821268,-0$ $.00000105,0.00702059,-0.01139527,0.00643372,-0.02558915,0.01225069,0.0$ $2619302,-0.01281282,-0.00800973,0.00000091,0.00074124,0.00065522,0.000$ $91398,0.00051128,-0.00033759,0.00001609,0.00051134,-0.00033747,-0.0000$ $1596,0.00074109,0.00065511,-0.00091427,0.00209917,-0.00242762,0.000000$ $21,-0.01144948,0.02347111,-0.00000126,-0.05940236,-0.00887764,0.000003$ $54,-0.23460731,0.13755676,-0.00000106,0.35240756,0.03154436,-0.0089394$ $6,-0.00000051,-0.00507464,0.00645106,-0.00888523,0.00275797,-0.0041310$ $6,-0.00234164,-0.00220348,0.00313165,0.00000025,0.00275791,-0.00413034$ $, 0.00234173,-0.00507331,0.00645229,0.00888433,-0.01232453,-0.00828740$, $0.00000076,0.00021668,0.00029741,0.00027902,0.00003912,-0.00025036,0.0$ $0000615,0.00003906,-0.00025043,-0.00000614,0.00021673,0.00029734,-0.00$ $027900,0.00095994,-0.00097958,0.00000003,0.02452340,-0.05822263,0.0000$ $0210,0.04613680,0.05976485,-0.00000460,0.10255098,-0.20075300,0.000004$ $77,-0.12642502,0.61342295,-0.00000343,0.00000003,-0.00648613,-0.007403$ $61,0.00099262,-0.00110688,0.00120666,-0.00259648,-0.00206068,0.0000003$ $0,-0.00000013,0.00325827,-0.00120737,0.00259707,-0.00206167,0.00740503$ $,-0.00099422,-0.00110906,0.00000104,0.00000057,0.00038406,0.00023485,0$ $.00021465,0.00030227,0.00021827,0.00009067,0.00003052,-0.00021831,-0.0$ $0009057,0.00003058,-0.00023488,-0.00021466,0.00030232,-0.00000011,0.00$ $000019,-0.00005693,-0.00000120,0.00000210,-0.00610260,0.00000010,-0.00$ $000231,0.01294136,0.00000079,0.00000347,-0.05253982,-0.00000335,-0.000$ $02667,0.08336172,-0.00451493,-0.00453847,0.00000032,0.00005601,-0.0018$ $3286,0.00166100,-0.00036413,0.00047942,0.00066835,0.00076065,-0.000571$ $33,0.00000018,-0.00036412,0.00047936,-0.00066852,0.00005597,-0.0018330$ $1,-0.00166068,-0.00082380,-0.00109863,0.00000009,-0.00008570,0.0000058$ $8,-0.00021463,-0.00008616,0.00002725,-0.00006666,-0.00008619,0.0000272$ 7,0.00006666,-0.00008570,0.00000592,0.00021463,-0.00108929,0.00120125, $-0.00000009,-0.05467160,0.03294007,0.00000066,-0.02093754,-0.03242403$, $0.00000254,0.03813381,0.02532408,-0.00000286,-0.09279110,0.00453183,0$. $00000121,0.65507761,-0.00206178,-0.00145431,0.00000031,0.00003264,0.00$ $053213,0.00075366,0.00070284,-0.00028101,-0.00012201,-0.00076287,-0.00$ 
$021897,-0.00000013,0.00070286,-0.00028106,0.00012211,0.00003276,0.0005$ $3204,-0.00075379,-0.00043899,-0.00021823,0.00000006,-0.00007985,-0.000$ $03642,-0.00013409,0.00002817,0.00000224,0.00001132,0.00002818,0.000002$ $29,-0.00001128,-0.00007989,-0.00003636,0.00013402,0.00027619,-0.000240$ $28,0.00000003,-0.01041219,0.05703328,-0.00000235,-0.03003114,-0.042470$ $53,0.00000344,0.05648564,-0.03679393,0.00000016,-0.02716735,-0.3489054$ $7,0.00001843,-0.06727614,0.68648715,0.00000023,0.00000003,-0.00030234$, $0.00021983,-0.00038125,0.00026589,-0.00034824,0.00009128,0.00022841,0$. $00000003,-0.00000004,-0.00070976,0.00034810,-0.00009107,0.00022849,-0$. $00021965,0.00038148,0.00026588,0 ., 0.00000011,0.00009826,0.00001639,-0$. $00000625,0.00005559,0.00004462,0.00003759,0.00006939,-0.00004459,-0.00$ $003765,0.00006934,-0.00001634,0.00000619,0.00005561,-0.00000003,-0.000$ $00009,0.00004947,0.00000315,-0.00000415,0.00931213,0.00000258,0.000003$ $45,-0.00391758,-0.00000462,0.00000141,0.00330244,0.00000298,0.00001751$ ,-0.05254349,-0.00001687,-0.00003075,0.12570903,0.00155709,-0.00010756 $, 0.00000001,0.00048096,0.00035178,-0.00019713,0.00007857,0.00023810,-0$ $.00005682,-0.00041806,0.00016732,-0.00000006,0.00007857,0.00023808,0.0$ $0005684,0.00048092,0.00035180,0.00019702,0.00013168,0.00027883,-0.0000$ $0002,-0.00000219,-0.00009651,0.00005528,-0.00003066,0.00000585,-0.0000$ $0234,-0.00003065,0.00000583,0.00000233,-0.00000217,-0.00009653,-0.0000$ $5523,0.00020034,-0.00017965,0.00000002,-0.24620409,0.06526854,0.000003$ $36,0.03941419,0.06326595,-0.00000511,-0.06286833,0.00745427,0.00000188$ $, 0.01775531,-0.08381744,0.00000488,-0.27435516,-0.02750777,0.00000993$, $0.62428636,0.00019349,-0.00068572,-0.00000033,0.00020417,-0.00030268,0$ $.00012978,-0.00007636,0.00030743,0.00020072,-0.00008263,0.00000335,0.0$ $0000047,-0.00007617,0.00030756,-0.00020098,0.00020357,-0.00030253,-0.0$ $0012965,-0.00012666,-0.00012857,-0.00000009,-0.00003500,-0.00004390,-0$ $.00003058,-0.00005980,-0.00000065,-0.00003812,-0.00005987,-0.00000090$, $0.00003797,-0.00003476,-0.00004395,0.00003087,-0.00029079,0.00033015,-$ $0.00000006,0.11611247,-0.17498034,0.00000197,0.01795132,-0.04305762,0$. $00000219,0.00989136,0.00136750,-0.00000072,-0.02832235,-0.02659506,0.0$ $0000347,-0.08632997,-0.12693770,0.00000747,-0.00485338,0.65403616,-0.0$ $0000023,0.00000001,0.00091092,-0.00008295,-0.00012297,-0.00013387,-0.0$ $0007223,-0.00025163,0.00011936,0.00000006,-0.00000004,-0.00009526,0.00$ $007222,0.00025164,0.00011934,0.00008296,0.00012295,-0.00013404,0 ., 0.00$ $000002,0.00001159,-0.00002847,0.00006788,0.00005541,-0.00000516,0.0000$ $0852,-0.00003423,0.00000518,-0.00000854,-0.00003425,0.00002849,-0.0000$ $6787,0.00005543,0 .,-0.00000003,-0.00000762,0.00000038,0.00000409,-0.06$ 400940,-0.00000233,0.00000042,0.00634104,0.00000171,-0.00000062,-0.004 $71801,0.00000147,0.00000561,0.01199547,0.00001372,0.00000528,-0.062686$ $56,-0.00001912,-0.00003074,0.14140605,-0.00003289,-0.00002575,0.0 .000$ $00023,-0.00000269,0.00003741,0.00001478,0.00001583,0.00000996,0.000025$ $71,-0.00005824,0.00000004,0.00001478,0.00001583,-0.00000999,0.00000023$ ,-0.00000269,-0.00003741,0.00000665,-0.00000815,0.,-0.00000068,-0.0000 $0197,-0.00000241,-0.00000334,-0.00000181,0.00000264,-0.00000335,-0.000$ $00180,-0.00000264,-0.00000069,-0.00000197,0.00000240,-0.00002138,0.000$ $02369,0 .,-0.29859794,-0.10385656,0.00001667,0.00083242,0.00177076,0 ., 0$ $.00182504,-0.00017190,0.00000022,0.00055214,0.00073248,-0.00000015,-0$. $00179347,0.00385798,0.00000012,-0.01722588,-0.01206193,0.00000154,0.31$ $378292,-0.00001652,-0.00004300,-0.00000002,-0.00001217,-0.00000583,0.0$ $0001024,-0.00000121,0.00003218,-0.00000349,-0.00000801,-0.00000519,0.0$ 
$0000001,-0.00000120,0.00003218,0.00000348,-0.00001218,-0.00000583,-0.0$ $0001024,0.00005858,0.00005260,0 ., 0.00000309,-0.00000392,0.00000274,-0$. $00000515,0.00000141,-0.00000167,-0.00000515,0.00000141,0.00000167,0.00$ $000310,-0.00000392,-0.00000274,-0.00001450,0.00001479,0 .,-0.10611975,-$ $0.10257533,0.00000819,-0.02824628,-0.00607935,0.00000169,0.00011581,-0$ $.00555167,0.00000074,0.00071623,-0.00111404,-0.00000004,0.00344540,-0$. $00177178,0.00000031,0.01870332,0.00962579,-0.00000115,0.11104115,0.105$ $50433,0.00000003,0 .,-0.00021855,0.00001678,-0.00000784,0.00001636,0.00$ $000283,0.00006225,-0.00001132,0 ., 0 ., 0.00001334,-0.00000283,-0.00006226$ - $-0.00001131,-0.00001679,0.00000784,0.00001638,0 ., 0 ., 0.00000600,-0.000$ $00892,-0.00000999,0.00000016,-0.00000119,0.00000650,-0.00000037,0.0000$ 0119,-0.00000650,-0.00000036,0.00000891,0.00000999,0.00000016,0.,0.,-0 $.00000339,0.00001678,0.00000803,-0.03525424,0.00000183,0.00000055,0.00$ $363390,0.00000019,0.00000075,0.00694408,-0.00000014,-0.00000004,-0.001$ $24726,0.00000008,0.00000027,0.00566573,-0.00000029,0.00000011,0.002960$ $22,-0.00001814,-0.00000917,0.02454319,-0.00004894,0.00023237,0 .,-0.000$ $12360,-0.00016037,0.00008124,-0.00003236,0.00004274,0.00009725,0.00011$ $506,-0.00010072,-0.00000001,-0.00003236,0.00004272,-0.00009724,-0.0001$ $2360,-0.00016039,-0.00008121,0.00003421,0.00003518,0 .,-0.00001202,-0.0$ $0000310,-0.00002336,-0.00000515,0.00000955,-0.00000875,-0.00000514,0.0$ $0000955,0.00000875,-0.00001202,-0.00000310,0.00002335,-0.00016714,0.00$ $018392,0 ., 0.00598287,-0.00477202,0.00000019,-0.23675829,0.13558164,-0$. $00000018,-0.01737424,0.01815287,-0.00000021,-0.00423622,-0.00223450,0$. $00000056,0.00007849,-0.00112740,0.00000002,0.00170964,-0.00153214,0.00$ $000029,0.00078408,0.00016591,-0.00000020,0.24887026,-0.00026172,0.0000$ $6694,0 .,-0.00001735,-0.00007348,0.00005045,-0.00001421,0.00003027,0.00$ $003775,0.00002799,-0.00002313,0.00000002,-0.00001421,0.00003027,-0.000$ 03776,-0.00001735,-0.00007349,-0.00005044,-0.00000735,0.00002089,0.,-0 $.00000939,-0.00000303,-0.00001017,-0.00000245,0.00000112,-0.00000411,-$ $0.00000245,0.00000112,0.00000411,-0.00000939,-0.00000303,0.00001017,-0$ $.00004509,0.00005177,0.0 .02537383,-0.01292650,-0.00000002,0.13450735$, $-0.15647353,0.00000184,-0.01201389,0.00929664,0.00000017,-0.00244969,0$ $.00085960,0.00000042,-0.00062770,-0.00053983,0 .,-0.00174171,-0.0045956$ $7,0.00000076,-0.00014981,0.00081646,-0.00000028,-0.14405582,0.16366189$ $, 0 .,-0.00000004,0.00001133,-0.00003185,0.00003629,0.00002637,0.0000448$ $5,-0.00004643,-0.00002882,0 ., 0 ., 0.00008350,-0.00004485,0.00004644,-0.0$ $0002882,0.00003187,-0.00003624,0.00002636,0 ., 0 ., 0.00003323,0.00000243$, $0.00000523,-0.00000072,-0.00000381,-0.00000038,-0.00000031,0.00000381$, $0.00000037,-0.00000032,-0.00000243,-0.00000524,-0.00000072,0 .,-0.00000$ $002,-0.00000658,-0.00000163,0.00000119,0.00352224,-0.00000009,0.000001$ $79,-0.03668243,0.00000160,-0.00000100,0.00432272,0.00000055,0.00000042$ $, 0.00623107,0.00000003,0.00000003,-0.00088486,0.00000030,0.00000071,0$. $00682972,-0.00000018,-0.00000031,-0.00405773,-0.00000028,-0.00000260,0$ $.02483126,0.00062045,0.00117839,-0.00000015,-0.00005154,-0.00016850,-0$ $.00003921,-0.00003085,-0.00009095,0.00004004,-0.00003297,0.00006475,0$. $00000003,-0.00003085,-0.00009096,-0.00004006,-0.00005150,-0.00016851,0$ $.00003925,-0.00030078,-0.00043577,0.00000001,-0.00000581,0.00005853,-0$ $.00000998,0.00003367,-0.00000219,0.00000475,0.00003367,-0.00000218,-0$. $00000475,-0.00000581,0.00005853,0.00000996,0.00007511,-0.00009097,0 .,-$ $0.00500981,-0.00172068,0.00000058,0.00066730,-0.02910991,0.00000188,-0$ $.05838446,-0.03076819,0.00000267,0.00587605,0.02374367,-0.00000163,-0$. 
$00345004,0.00397497,0.00000021,-0.00129003,0.00009933,0.00000004,-0.00$ $001001,-0.00138662,0.00000008,0.00113493,0.00039084,-0.00000021,0.0597$ $9656,-0.00073855,-0.00018652,-0.00000002,0.00014194,0.00010455,0.00027$ $563,-0.00008952,0.00012524,-0.00012699,0.00000328,-0.00006681,0 .,-0.00$ $008951,0.00012527,0.00012697,0.00014193,0.00010450,-0.00027565,0.00070$ $936,0.00035973,-0.00000007,0.00003435,-0.00002356,0.00002267,-0.000014$ $13,0.00002538,0.00000568,-0.00001413,0.00002536,-0.00000569,0.00003438$ ,-0.00002356,-0.00002265,0.00008281,-0.00009106,0.,-0.00263248,0.00149 $719,0.00000041,0.00141828,-0.00866744,0.00000078,-0.03550726,-0.326471$ $34,0.00001848,0.00044382,-0.01558365,0.00000098,0.00217744,-0.00074650$ $, 0.00000032,0.00051515,0.00059403,-0.00000012,0.00015343,-0.00045910,0$ $.00000002,0.00000081,0.00067450,-0.00000027,0.03359667,0.34914535,0.00$ $000010,-0.00000005,-0.00081147,-0.00032305,0.00014606,0.00010295,0.000$ $00956,-0.00008497,-0.00015588,0 ., 0.00000003,0.00007571,-0.00000953,0.0$ $0008491,-0.00015589,0.00032299,-0.00014605,0.00010305,-0.00000004,0 ., 0$ $.00012600,-0.00010094,0.00000390,-0.00006914,0.00001216,0.00002689,0.0$ $0000707,-0.00001216,-0.00002688,0.00000708,0.00010092,-0.00000391,-0.0$ $0006915,0 ., 0.00000001,-0.00001257,0.00000064,0.00000034,0.00687862,0.0$ $0000013,0.00000206,0.00534538,0.00000284,0.00001827,-0.03642203,-0.000$ $00027,-0.00000006,0.00019064,0.00000026,0.00000030,0.00674594,0 .,-0.00$ $000002,-0.00062959,-0.00000002,0.00000008,-0.00008253,-0.00000019,-0.0$ $0000029,-0.00375460,-0.00000336,-0.00002050,0.02368288,-0.00003543,-0$. $00028944,-0.00000001,0.00019052,0.00001809,0.00013168,0.00023061,0.000$ 04916,-0.00026816,-0.00097532,0.00009884,-0.00000008,0.00023052,0.0000 $4919,0.00026820,0.00019059,0.00001794,-0.00013167,-0.00002597,0.000007$ $68,0 ., 0.00002190,0.00005111,0.00002477,0.00001551,-0.00000632,0.000015$ $55,0.00001553,-0.00000633,-0.00001555,0.00002190,0.00005110,-0.0000247$ $8,0.00012843,-0.00010371,0.00000002,-0.00323371,-0.00382790,0.00000059$ $, 0.00023749,-0.00044678,-0.00000001,0.00077254,-0.00241095,0.00000037$, $0.00723996,-0.00008748,-0.00000028,-0.23634991,0.13247123,0.00000004,-$ $0.01812666,0.01777574,-0.00000016,-0.00077148,0.00084633,0 .,-0.0000511$ $7,0.00004035,-0.00000005,0.00031877,-0.00030261,0.00000002,0.24914230$, $0.00107932,0.00005943,-0.00000014,-0.00008146,-0.00004111,-0.00028553$, $-0.00009440,0.00007193,-0.00005651,-0.00024950,0.00053056,0.00000003,-$ $0.00009451,0.00007194,0.00005647,-0.00008145,-0.00004118,0.00028557,0$. $00001427,0.00006160,0 ., 0.00001037,-0.00000200,0.00002673,0.00001816,0$. $00003503,0.00002148,0.00001818,0.00003504,-0.00002149,0.00001036,-0.00$ $000202,-0.00002674,0.00004586,-0.00010078,0 .,-0.00288496,-0.00019245,0$ $.00000048,-0.00093288,-0.00058772,0 .,-0.00056661,-0.00509432,0.0000007$ 4,0.02275097,-0.01589489,0.00000001,0.13840456,-0.15467465,0.00000166, $-0.01224622,0.00967038,0.00000021,-0.00056504,0.00045066,0.00000002,0$. $00007345,0.00002626,-0.00000006,0.00093613,-0.00039757,-0.00000002,-0$. $14506396,0.16505320,0.00000003,0.00000010,-0.00075751,0.00026545,-0.00$ $015319,0.00008160,0.00015697,0.00031706,-0.00019435,0.00000006,0.00000$ $004,0.00062089,-0.00015694,-0.00031724,-0.00019432,-0.00026557,0.00015$ $313,0.00008170,0.00000002,-0.00000005,-0.00001275,-0.00000873,-0.00004$ $840,-0.00003041,-0.00006974,-0.00003589,-0.00004819,0.00006971,0.00003$ $592,-0.00004816,0.00000870,0.00004843,-0.00003044,0.00000003,0.0000000$ $5,-0.00005670,0.00000057,0.00000043,0.00583140,-0.00000011,0.00000011$, $-0.00094212,0.00000023,0.00000073,0.00672058,-0.00000160,0.00000090,0$. $00006541,-0.00000040,0.00000177,-0.03512690,0.00000174,-0.00000088,0.0$ 
0485993,0.00000004,-0.00000004,0.00038857,-0.00000003,-0.00000007,-0.0 $0095587,-0.00000010,0.00000008,0.00002789,-0.00000025,-0.00000266,0.02$ $295805,-0.00006593,-0.00019562,-0.00000002,0.00003722,-0.00014969,0.00$ $007902,-0.00004691,0.00006416,0.00009614,0.00006675,-0.00003352,0.0000$ $0002,-0.00004690,0.00006416,-0.00009616,0.00003716,-0.00014970,-0.0000$ $7899,-0.00010444,-0.00006139,0 .,-0.00002185,-0.00002104,-0.00001436,-0$ $.00001628,0.00000234,-0.00000434,-0.00001629,0.00000232,0.00000433,-0$. $00002182,-0.00002104,0.00001439,-0.00015108,0.00017070,0 ., 0.01102995,0$ $.02439362,-0.00000184,-0.00325484,0.00483203,0.00000016,-0.00262639,0$. $00079998,0.00000001,-0.00472748,-0.00435036,0.00000079,-0.00136563,-0$. $03435616,0.00000218,-0.08488226,-0.02333194,0.00000244,0.00035912,0.00$ $096555,-0.00000022,0.00041651,0.00139475,-0.00000008,0.00008395,-0.000$ $04842,-0.00000004,0.00062721,-0.00068013,-0.00000006,0.13531543,-0.000$ $13303,-0.00008230,0.00000058,-0.00000860,0.00007935,-0.00010222,0.0000$ $8240,-0.00009859,-0.00011850,-0.00032873,0.00037102,-0.00000079,0.0000$ $8214,-0.00009873,0.00011889,-0.00000772,0.00007915,0.00010205,-0.00015$ $311,-0.00013434,0.00000015,-0.00003511,0.00000134,-0.00002950,0.000021$ $87,-0.00000176,0.00002575,0.00002198,-0.00000144,-0.00002554,-0.000035$ 43,0.00000141,0.00002912,0.00028688,-0.00036963,0.00000009,0.00968791, $-0.04390412,0.00000238,0.00688266,0.00434470,0 ., 0.00041238,-0.00093970$ ,-0.00000005,-0.00511427,0.00609585,0.00000041,-0.02211956,-0.03301075 $, 0.00000284,-0.02086354,-0.27600855,0.00001390,-0.00121453,0.00125588$, $-0.00000029,-0.00028727,-0.00048885,0.00000011,-0.00009113,-0.00002820$ - $-0.00000015,0.00166348,0.00106804,-0.00000039,0.16547567,1.51825634,0$ $.00000002,0 ., 0.00018512,-0.00013889,0.00004550,-0.00001190,0.00008147$, $-0.00004482,-0.00004349,0.00000002,-0.00000002,0.00004913,-0.00008148$, $0.00004486,-0.00004349,0.00013889,-0.00004549,-0.00001191,0 ., 0.0000000$ $2,-0.00002863,0.00000206,-0.00001974,-0.00002526,-0.00001811,-0.000008$ $81,0.00000237,0.00001811,0.00000879,0.00000237,-0.00000205,0.00001973$, $-0.00002526,-0.00000002,0 .,-0.00000434,-0.00000097,0.00000176,0.001560$ $64,0.00000003,0 ., 0.00764969,0.00000008,-0.00000004,-0.00083003,0.00000$ $074,0.00000029,0.00763969,0.00000149,0.00000345,0.00261785,0.00000237$, $0.00001394,-0.05868352,-0.00000008,-0.00000033,-0.00373845,0.00000003$, $0.00000002,0.00071178,-0.00000005,-0.00000009,-0.00141936,-0.00000028$, $-0.00000025,-0.00353195,-0.00001197,-0.00009214,0.08307316,-0.00000399$ $, 0.00004800,0.00000002,-0.00002734,0.00003501,0.00000540,0.00001211,-0$ $.00003095,-0.00000984,-0.00005915,0.00004566,-0.00000004,0.00001210,-0$ $.00003096,0.00000986,-0.00002730,0.00003500,-0.00000541,0.00003210,0.0$ $0000675,0 ., 0.00000932,0.00000741,-0.00000445,0.00001515,0.00001220,0.0$ $0000696,0.00001516,0.00001222,-0.00000695,0.00000930,0.00000742,0.0000$ $0443,0.00005770,-0.00008961,0 .,-0.00349365,-0.00117233,0.00000028,0.00$ $071191,-0.00117048,-0.00000001,0.00077408,-0.00012391,-0.00000001,0.00$ $096860,0.00083980,-0.00000015,-0.00094809,0.00270808,-0.00000006,0.019$ $26251,-0.00515769,0.00000017,0.00026301,-0.00023573,0.00000002,-0.0001$ $4382,-0.00029553,0.00000002,0.00001401,-0.00001701,0 ., 0.00018586,0.000$ $17948,-0.00000002,-0.05062520,-0.13422532,0.00000859,0.03298560,0.0001$ $5017,-0.00000166,-0.00000027,-0.00003799,-0.00013875,0.00011894,-0.000$ $08455,0.00009861,0.00015165,0.00037153,-0.00036046,0.00000040,-0.00008$ $444,0.00009867,-0.00015185,-0.00003839,-0.00013866,-0.00011884,0.00007$ $994,0.00007792,-0.00000007,0.00002501,0.00000097,0.00002040,-0.0000227$ 1,0.00000356,-0.00002336,-0.00002276,0.00000342,0.00002327,0.00002515, 
$0.00000094,-0.00002023,-0.00033538,0.00040936,-0.00000006,-0.00771440$, $0.00632552,0 .,-0.00074549,-0.00210233,0.00000004,-0.00028413,0.0005760$ $5,0 ., 0.00075498,-0.00273564,-0.00000002,0.00960194,0.00402864,-0.00000$ $041,-0.00533451,-0.02255850,0.00000241,0.00051368,-0.00016874,0.000000$ $03,-0.00009387,-0.00033719,0 ., 0.00002472,-0.00003578,0.00000004,-0.000$ $59474,-0.00010535,0.00000005,-0.13476625,-1.17638614,0.00007337,0.1386$ $1248,1.19344553,0 ., 0.00000003,-0.00004527,0.00002169,-0.00000710,0.000$ $01256,-0.00003385,0.00000938,0.00001034,-0.00000003,0.00000003,-0.0000$ $3464,0.00003387,-0.00000943,0.00001035,-0.00002171,0.00000709,0.000012$ $61,0 .,-0.00000002,0.00000512,0.00000382,0.00001546,0.00000525,0.000019$ $15,0.00000602,0.00000269,-0.00001915,-0.00000600,0.00000270,-0.0000038$ $3,-0.00001545,0.00000525,0.00000003,0 ., 0.00001241,0.00000068,-0.000000$ $26,0.00163279,-0.00000007,0.00000013,-0.00134886,-0.00000003,-0.000000$ $04,0.00015461,-0.00000010,0.00000002,-0.00141886,-0.00000052,-0.000000$ 26,0.00143202,0.00000019,0.00000250,0.01550184,-0.00000004,0.00000005, $0.00043744,0 ., 0.00000002,-0.00022777,0 ., 0.00000003,0.00028901,0.000000$ $06,0 ., 0.00029367,0.00000864,0.00007330,-0.03509423,-0.00000882,-0.0000$ $7550,0.01834893 \backslash \backslash-0.00000141,0.00000142,-0.00000001,-0.00000096,0.0000$ $0200,0.00000019,0.00000021,0.00000282,0.00000004,0.00000197,0.00000280$ $, 0 ., 0.00000019,0.00000284,-0.00000004,-0.00000094,0.00000201,-0.000000$ $16,-0.00000265,0.00000087,0 .,-0.00000151,0.00000161,0.00000005,0.00000$ $054,0.00000338,-0.00000002,0.00000054,0.00000338,0.00000003,-0.0000015$ $1,0.00000161,-0.00000004,0.00000224,0.00000464,0 ., 0.00000073,-0.000003$ $53,0 .,-0.00000242,-0.00000334,0 .,-0.00000210,-0.00000211,0 .,-0.0000008$ $3,-0.00000046,0 ., 0.00000111,-0.00000113,0 ., 0.00000147,-0.00000243,-0.0$ $0000001,0.00000105,-0.00000479,0 .,-0.00000170,-0.00000445,0 .,-0.000003$ $00,-0.00000170,0 ., 0.00000154,-0.00000011,0 ., 0.00000313,-0.00000258,0$. 0.00000431,-0.00000276,0.III@

para-Transition State

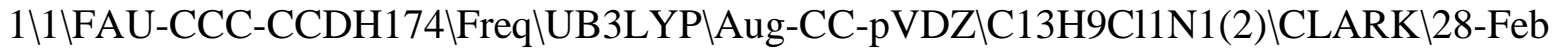
-2015\0\\N Geom=AllCheck Guess=TCheck SCRF=Check GenChk UB3LYP/Aug-CC -pVDZ Freq \\Addition TS p-Cl-Ph-radical + aniline (para) \0,2\C,-1.072 $964545,1.7565637956,0.002807255 \backslash \mathrm{C},-1.6234552258,1.2772480979,1.2211536$ 46\C,-2.5726122928,0.2615129729,1.2218052459\C,-3.0316806994,-0.246689 $3625,-0.0012897933 \backslash \mathrm{C},-2.5716212494,0.2655096483,-1.2223268197 \backslash \mathrm{C},-1.622$ $4663003,1.2812388828,-1.2175692761 \mathrm{\downarrow H},-0.5111321221,2.6882016155,0.0046$ $033008 \mathrm{\backslash H},-1.2771524949,1.6889138179,2.168678347 \backslash \mathrm{H},-2.9740748758,-0.126$ $8803999,2.1561606125 \backslash \mathrm{H},-2.972283127,-0.1198461161,-2.1582805832 \backslash \mathrm{H},-1.2$ 753982046,1.6959961434,-2.1634652941\Cl,-4.2340149815,-1.5300249216,-0 $.0038612664 \backslash \mathrm{C}, 3.4508922647,-0.4062145034,-0.0004685051 \backslash \mathrm{C}, 3.3562800225$, $0.9975722751,-0.0032336795 \backslash \mathrm{C}, 2.0956210039,1.6079819464,-0.0021218321 \backslash \mathrm{C}$ $, 0.9719040278,0.7961981438,0.0017728976 \mathrm{lC}, 1.0295184533,-0.5884484105,0$ $.0045411975 \backslash \mathrm{C}, 2.2874856684,-1.2004482115,0.0034051658 \backslash \mathrm{C}, 4.743590089,-1$ $.0337465751,-0.0015986483 \mathrm{UH}, 4.2644719225,1.6001023828,-0.0062085404 \mathrm{\backslash H}$, $2.0212437138,2.6967066135,-0.0042328793 \backslash \mathrm{H}, 0.123579411,-1.1954101346,0$. $0075452326 \backslash \mathrm{H}, 2.3765146269,-2.2866518238,0.005485459 \mathrm{~N}, 5.7902319149,-1$. 541714877,-0.0025112421॥Version=EM64L-G09RevC.01 \State=2-A $\backslash H F=-1015.7$ $280688 \mathrm{IS} 2=0.780608 \mathrm{IS} 2-1=0 . \mathrm{IS} 2 \mathrm{~A}=0.75067 \backslash \mathrm{RMSD}=6.576 \mathrm{e}-09 \backslash \mathrm{RMSF}=3.739 \mathrm{e}-06 \mathrm{Z}$ eroPoint $=0.1764684 \backslash$ Thermal $=0.1893087 \mathrm{ZPE}=110.7356094 \backslash \mathrm{Dipole}=-1.4280068$ 
, 1.2847501,0.0021378\DipoleDeriv $=0.1003688,-0.0032437,0.0001002,-0.051$ $5063,0.0746107,0.0002499,-0.0001273,0.0002421,-0.0583075,-0.0427685,0$. $1269765,0.056526,0.1713637,-0.0871977,0.0109629,-0.0569393,0.0544191,0$ $.0555434,-0.2174759,-0.0688677,-0.063888,-0.1359531,-0.1661041,-0.0657$ $937,-0.1624138,-0.0449634,0.0178079,0.6895847,0.5842143,0.0011688,0.75$ $21121,0.5894019,0.0011032,0.0019329,0.0010079,0.0387957,-0.2171121,-0$. $0694499,0.0635573,-0.1360125,-0.1665873,0.0652016,0.161386,0.044405,0$. $0182401,-0.0430801,0.1273477,-0.0562087,0.1709423,-0.086873,-0.0113396$ $, 0.0573804,-0.0546288,0.0553145,0.11581,-0.0945235,-0.0000897,-0.09932$ $5,-0.0057381,-0.0000999,-0.0000524,-0.0000688,0.0605133,0.0723624,-0.0$ $647128,-0.0463674,-0.0484179,0.0699807,-0.0320999,-0.0276192,-0.040853$ $,-0.0261681,0.0875132,-0.0379673,0.0368845,-0.0489212,0.0917432,0.0407$ $091,0.0428814,0.0296118,0.0118806,0.087573,-0.0378422,-0.0369721,-0.04$ $87881,0.0919908,-0.040468,-0.0430307,-0.0293567,0.0115833,0.0723064,-0$ $.0649503,0.0462534,-0.0485535,0.0697581,0.0324195,0.027597,0.0410541,-$ $0.0258666,-0.59273,-0.3448307,-0.0007328,-0.555814,-0.4244685,-0.00072$ $75,-0.001092,-0.000703,-0.08949,-0.1280313,0.0467639,-0.0000099,0.0730$ $737,0.0384677,-0.0001164,0.0001742,-0.0001559,0.0266806,0.0470158,0.06$ $50983,-0.0005335,-0.2652732,0.058785,-0.000124,0.0002887,-0.0003109,-0$ $.0796598,-0.5165938,-0.0002575,0.0000891,0.4122357,0.1190606,-0.000403$ $6,-0.0000082,-0.000444,-0.1027502,0.7805293,-0.0602413,-0.0000367,-0.3$ $419238,0.0369845,-0.0003702,-0.0010606,-0.0002126,-0.044121,-0.5428392$ $, 0.1597662,-0.0004952,0.1598165,-0.1337504,-0.0000102,0.0008287,-0.000$ $2604,-0.0840698,0.230336,-0.0985306,-0.0000744,0.196786,0.0632338,-0.0$ $004267,-0.0008705,-0.0000554,-0.0711434,0.5354501,-0.1849486,-0.000151$ $3,-0.2454463,0.1290297,0.0003234,-0.0002601,0.0003129,0.123225,-0.0804$ $222,-0.0576106,0.0003806,-0.0146778,0.0434601,0.000254,0.0004503,0.000$ $2572,0.1291238,0.0866195,0.0392439,0.0000589,-0.0247823,-0.0807486,0.0$ $003875,0.0001078,0.0003071,0.1225632,0.0378114,-0.0690308,0.0003998,-0$ $.0488297,0.0482551,0.0001346,0.0001403,0.0002104,0.1017894,0.0069254,0$ $.0230681,0.0000608,0.0438396,-0.0368143,0.0003404,0.0001643,0.0002668$, $0.1270766,-0.5691529,0.0845287,0.0000804,0.1340549,-0.33648,-0.0001064$ $, 0.0001423,-0.0000817,-0.3185609$ Polar $=282.3771584,8.0787551,166.74811$ $52,-0.0310022,-0.0542936,128.7149373 \backslash \mathrm{PG}=\mathrm{C} 01[\mathrm{X}(\mathrm{C} 13 \mathrm{H} 9 \mathrm{Cl1N} 1)] \mathrm{NImag}=1 \backslash 10$ $.22288744,0.30343031,0.50567258,0.00033150,-0.00006797,0.60959881,-0.0$ $8136276,-0.05946804,0.05094402,0.39231323,-0.03342067,-0.11988951,0.03$ $026127,0.27192709,0.42150260,0.04385151,0.07522397,-0.25812938,0.01986$ $570,0.05204538,0.67864619,-0.02225293,-0.01540619,0.03940135,-0.193970$ $82,-0.14412087,-0.01899821,0.39028415,-0.03086949,-0.00680593,0.045050$ $31,-0.14321370,-0.20924260,-0.02451654,0.27650438,0.41497017,0.0082612$ $6,0.02387769,0.02895072,0.02900031,0.02787297,-0.12516578,-0.04810893$, $-0.03494184,0.70550616,0.00584363,0.00231496,0.00003055,-0.01329763,-0$ $.02121940,-0.01559318,-0.09679371,-0.03992077,-0.07194843,0.28969819,0$ $.01513009,-0.00452760,0.00009553,-0.02193392,-0.01718557,-0.01670100,-$ $0.03842880,-0.10263224,-0.07998839,0.18723988,0.31940436,0.00006117,0$. $00009195,-0.05918364,-0.05563588,-0.06170321,0.02657826,-0.02384260,-0$ $.02893217,-0.27339467,0.00016614,-0.00043020,0.62246080,-0.02220202,-0$ $.01525571,-0.03949738,-0.01883149,-0.01847279,0.01098097,0.03023689,0$. $02438334,0.01390567,-0.09686609,-0.03857881,0.02387432,0.39018345,-0.0$ $3080169,-0.00657634,-0.04518417,-0.01727039,-0.02364054,0.01256306,0.0$ $2441658,0.03399513,0.01462234,-0.04019084,-0.10298720,0.02945868,0.276$ 
$32228,0.41473109,-0.00841641,-0.02400954,0.02868392,0.01548965,0.01913$ $130,-0.01145478,-0.01376390,-0.01431993,-0.05282971,0.07198157,0.08052$ 496,-0.27297889,0.04873341,0.03421874,0.70583547,-0.08127013,-0.059251 $09,-0.05099089,0.02152293,0.01995665,-0.00009632,-0.01880359,-0.017207$ $18,-0.01554190,-0.01335735,-0.02213063,0.05552961,-0.19394588,-0.14312$ $642,-0.02952763,0.39230049,-0.03325386,-0.11954406,-0.02982408,0.01996$ $559,0.02967311,-0.00582818,-0.01842141,-0.02353769,-0.01918608,-0.0213$ $1898,-0.01744722,0.06154798,-0.14415296,-0.20921964,-0.02827055,0.2720$ $2582,0.42182401,-0.04379226,-0.07480350,-0.25853182,0.00020960,0.00607$ $385,-0.03979143,-0.01103536,-0.01261396,-0.01157792,0.01548620,0.01653$ $820,0.02688853,0.01847775,0.02412224,-0.12518093,-0.01923468,-0.052683$ $68,0.67824241,-0.09950075,-0.13756718,-0.00024659,-0.00912968,-0.01228$ $882,0.00325708,0.00502073,-0.00054308,0.00426502,-0.00100307,-0.000929$ $36,-0.00000283,0.00502105,-0.00052721,-0.00425593,-0.00911076,-0.01225$ $507,-0.00330854,0.12440438,-0.15186904,-0.25501889,-0.00039402,-0.0010$ $0205,-0.00221070,-0.00506969,-0.00233901,0.00083823,0.00078511,0.00126$ $371,0.00106790,0.00000478,-0.00233401,0.00084389,-0.00076790,-0.000955$ $23,-0.00214862,0.00504865,0.14677887,0.26686477,-0.00026976,-0.0003854$ $0,-0.05599258,0.01416287,0.02329681,0.00427403,0.00152290,0.00138650,-$ $0.00444790,0.00000342,0.00000459,-0.00135928,-0.00152517,-0.00136931,-$ $0.00445933,-0.01417736,-0.02333056,0.00420006,0.00027215,0.00041001,0$. $05479800,0.00651371,0.00504909,0.01250410,-0.07334976,-0.04483031,-0.0$ $7892667,-0.00282614,-0.00805822,-0.01893679,0.00205050,-0.00553724,0.0$ $0106043,-0.00080565,0.00017501,0.00025184,0.00201288,-0.00343150,0.002$ $12000,-0.00152527,0.00109025,-0.00027082,0.06996456,0.00404302,0.00705$ $171,0.00941449,-0.04548595,-0.08724476,-0.09254365,-0.00696362,-0.0040$ $6189,-0.02050410,-0.00569584,0.00110810,0.00057084,0.00015688,-0.00074$ $471,0.00055304,-0.00329393,0.00229650,0.00238522,0.00201987,-0.0005491$ $8,-0.00040841,0.05316919,0.08427963,-0.01009622,-0.01118237,-0.0172733$ $9,-0.07728036,-0.09458128,-0.27187215,0.00185895,0.00219536,0.00335038$ $, 0.00076927,0.00017869,0.00200313,0.00043347,0.00067034,0.00017084,0.0$ $0312736,0.00235903,-0.00308260,-0.00014544,0.00019445,0.00096342,0.082$ $51097,0.10039145,0.28567530,0.00136268,-0.00672274,-0.00134936,-0.0037$ $3447,-0.00657278,0.01850609,-0.08322367,-0.04694574,0.09254141,0.00567$ $979,0.00251189,-0.01042624,0.00206069,-0.00309301,-0.00202654,-0.00047$ $694,0.00041333,-0.00010989,-0.00023974,-0.00033415,0.00113924,-0.00189$ $529,0.00281447,-0.00029643,0.08070337,-0.00629322,0.00085369,-0.000600$ $71,-0.00690325,-0.00283887,0.01965439,-0.04756058,-0.08237255,0.087658$ $82,0.00344191,0.00617679,-0.01041300,-0.00318342,0.00189263,-0.0027955$ $4,0.00002599,-0.00098144,-0.00037478,-0.00040142,-0.00015774,0.0006323$ $2,0.00265437,-0.00146034,-0.00002640,0.05565827,0.07947139,-0.00074624$ ,-0.00080462,0.00173051,-0.00196855,-0.00159234,0.00420490,0.09052438, $0.08616062,-0.26903258,0.01158086,0.01162957,-0.01821448,-0.00247471$,$0.00294337,-0.00277330,-0.00053642,-0.00051701,0.00040518,-0.00004995$, $-0.00020214,0.00011473,-0.00001965,0.00015148,0.00076818,-0.09703678$,$0.09283749,0.28239289,0.00135985,-0.00672686,0.00132712,-0.00047743,0$. $00041387,0.00010967,0.00205766,-0.00310284,0.00202156,0.00567818,0.002$ $48644,0.01045136,-0.08306360,-0.04657076,-0.09253617,-0.00372195,-0.00$ $651394,-0.01853402,-0.00023861,-0.00033025,-0.00113994,-0.00035676,0.0$ $0040994,-0.00008744,0.00038899,0.00012015,0.00043933,0.08053830,-0.006$ $29500,0.00084773,0.00059204,0.00002409,-0.00098413,0.00037019,-0.00319$ 
$385,0.00187395,0.00280834,0.00347011,0.00618205,0.01049610,-0.04719183$ ,-0.08180469,-0.08709227,-0.00689228,-0.00278133,-0.01967981,-0.000400 $84,-0.00015566,-0.00063387,0.00040961,-0.00038114,-0.00000858,0.000121$ $05,0.00052191,0.00055508,0.05526375,0.07886535,0.00072635,0.00079612,0$ $.00173761,0.00053561,0.00051317,0.00040695,0.00246795,0.00295494,-0.00$ $275076,-0.01154990,-0.01154953,-0.01822032,-0.09052163,-0.08559067,-0$. $26975980,0.00193899,0.00156480,0.00413181,0.00004802,0.00020096,0.0001$ $1279,-0.00002817,-0.00007456,-0.00003191,-0.00043843,-0.00055024,-0.00$ $081273,0.09704687,0.09222460,0.28317470,0.00651496,0.00508166,-0.01246$ $879,0.00201701,-0.00342163,-0.00212678,-0.00080506,0.00017519,-0.00025$ $161,0.00205102,-0.00553325,-0.00107837,-0.00283782,-0.00811817,0.01890$ $395,-0.07347893,-0.04516730,0.07894232,-0.00152490,0.00108772,0.000271$ $61,0.00042212,0.00018384,-0.00055231,-0.00035689,0.00040963,0.00002900$ - $-0.00189562,0.00265402,0.00002674,0.07009909,0.00401788,0.00704608,-0$ $.00933085,-0.00328133,0.00231178,-0.00237120,0.00015839,-0.00074053,-0$ $.00055598,-0.00569312,0.00111069,-0.00057840,-0.00697400,-0.00412429,0$ $.02047467,-0.04581670,-0.08785938,0.09311052,0.00201845,-0.00054952,0$. $00040569,0.00018291,0.00018297,-0.00054624,0.00040975,-0.00038128,0.00$ $007382,0.00281364,-0.00145837,-0.00015682,0.05352237,0.08493920,0.0101$ $2865,0.01126381,-0.01726676,-0.00313199,-0.00234372,-0.00310271,-0.000$ $43614,-0.00067302,0.00016656,-0.00078717,-0.00018727,0.00200015,-0.001$ $88582,-0.00222401,0.00342160,0.07729303,0.09515060,-0.27112982,0.00014$ $968,-0.00020043,0.00096411,0.00055352,0.00054977,-0.00091554,0.0000886$ $7,0.00000781,-0.00003196,0.00030359,0.00002048,0.00076843,-0.08251198$, $-0.10100770,0.28488011,-0.00264653,-0.00001259,-0.00000178,0.00462011$, $-0.00020548,-0.00380069,-0.01583154,-0.02111712,-0.00034725,-0.0843850$ $4,-0.06757709,-0.00013144,-0.01584813,-0.02113592,0.00026252,0.0046203$ $1,-0.00021647,0.00380772,-0.00001296,0.00002276,-0.00000015,-0.0006319$ $3,-0.00079329,0.00020211,-0.00069371,0.00185918,0.00017584,-0.00069486$ $, 0.00185965,-0.00017061,-0.00063252,-0.00079282,-0.00020553,0.11200295$ ,-0.00193107,-0.00108322,-0.00000564,0.00001498,0.00382755,-0.00405753 ,-0.02125121,-0.01841253,-0.00025980,-0.06676325,-0.09341801,-0.000115 $29,-0.02131251,-0.01847982,0.00017364,0.00001689,0.00381973,0.00408325$ $, 0.00069492,-0.00041549,-0.00000078,-0.00076157,-0.00068344,0.00023061$ $, 0.00174219,-0.00055639,0.00037334,0.00174127,-0.00055805,-0.00037909$, $-0.00076340,-0.00068506,-0.00023366,0.10836193,0.12681114,-0.00000514$, $-0.00000423,0.00170712,0.00110891,0.00111410,-0.00386012,-0.01871977,-$ $0.01987705,0.00297344,-0.00013025,-0.00011420,-0.03917424,0.01863546,0$ $.01978856,0.00305513,-0.00110272,-0.00108837,-0.00384946,0.00000091,-0$ $.00000148,0.00026424,-0.00063344,-0.00066718,0.00005650,-0.00043727,-0$ $.00042596,0.00123054,0.00044169,0.00042018,0.00122491,0.00063063,0.000$ $66417,0.00005842,0.00020990,0.00019194,0.03636009,-0.00001520,-0.00020$ $297,-0.00000095,-0.00035475,0.00001531,-0.00014354,-0.00003910,0.00012$ $250,-0.00002328,0.00032512,0.00030005,0.00000044,-0.00004769,0.0001218$ $8,0.00002279,-0.00035511,0.00001685,0.00014704,-0.00000588,-0.00004763$ ,-0.00000162,0.00002551,-0.00004101,-0.00001573,-0.00000213,-0.0000098 $7,0.00000602,-0.00000175,-0.00000942,-0.00000540,0.00002536,-0.0000395$ $0,0.00001363,-0.00021411,-0.00024077,-0.00000129,0.65821569,-0.0001296$ $3,-0.00031541,-0.00000200,-0.00005124,-0.00007562,0.00002393,-0.000084$ $72,0.00012824,0.00003903,-0.00000352,0.00009799,-0.00000222,-0.0000923$ $4,0.00012807,-0.00003951,-0.00004998,-0.00007296,-0.00001999,-0.000250$ 
$25,0.00035199,-0.00000045,-0.00002181,-0.00004115,0.00004773,0.0000254$ $9,0.00001265,-0.00000166,0.00002590,0.00001307,0.00000262,-0.00002254$, $-0.00003910,-0.00005073,0.00008532,0.00009112,-0.00000044,-0.01083285$, $0.64484017,0.00000016,0.00000139,-0.00006075,0.00004988,0.00001323,-0$. $00003582,-0.00001551,0.00003714,-0.00004056,0.00000020,-0.00000130,0.0$ $0006984,0.00001411,-0.00003595,-0.00004147,-0.00004704,-0.00001370,-0$. $00003750,0.00000048,-0.00000131,0.00009202,0.00000117,-0.00002297,-0.0$ $0001625,0.00000113,-0.00000525,0.00000227,-0.00000072,0.00000475,0.000$ $00291,-0.00000154,0.00002349,-0.00001491,-0.00000014,0.00000010,-0.000$ $00246,-0.00094858,-0.00102084,0.14583013,0.00055609,-0.00124348,0.0000$ $0226,-0.00018668,0.00013220,-0.00001498,-0.00010149,0.00013461,-0.0000$ $5810,0.00010709,0.00007596,-0.00000211,-0.00009654,0.00013430,0.000059$ $75,-0.00018236,0.00013217,0.00001273,-0.00001572,0.00043752,0.00000199$ ,-0.00007164,-0.00001203,0.00008507,0.00002800,0.00000560,-0.00001189, $0.00002768,0.00000474,0.00001085,-0.00007273,-0.00001148,-0.00008588,-$ $0.00005400,-0.00004191,0.00000051,-0.11234297,-0.02315278,0.00013771,0$ $.70907619,-0.00071945,-0.00037931,0.00000297,-0.00024052,0.00056763,-0$ $.00011920,0.00008129,-0.00021333,-0.00032684,0.00010087,0.00003189,0.0$ $0000204,0.00008491,-0.00021172,0.00032802,-0.00023870,0.00056413,0.000$ $11861,-0.00014147,-0.00022161,0.00000219,-0.00000589,0.00001895,0.0000$ $4859,-0.00002481,-0.00000267,0.00001484,-0.00002518,-0.00000300,-0.000$ $01591,-0.00000546,0.00001814,-0.00004669,-0.00040705,-0.00041066,-0.00$ $000076,0.02886129,-0.31078213,0.00045449,0.03674127,0.66160039,-0.0000$ $0268,0.00000303,0.00112612,-0.00065745,-0.00000540,-0.00004168,0.00011$ $167,0.00008206,0.00019139,-0.00000055,-0.00000206,-0.00015927,-0.00011$ $310,-0.00008108,0.00019006,0.00066628,0.00000113,-0.00003703,0.0000017$ $6,0.00000231,-0.00000537,0.00003834,-0.00000655,0.00003972,0.00002795$, $-0.00002582,0.00000413,-0.00002861,0.00002641,0.00000258,-0.00003791,0$ $.00000577,0.00003879,0.00000168,0.00000161,-0.00002290,0.00003448,0.00$ $055743,-0.06567227,-0.00117634,-0.00117589,0.13348141,-0.00475258,-0.0$ $0195869,0.00001517,-0.00065239,0.00121564,-0.00108939,-0.00005241,-0.0$ $0053565,-0.00053510,0.00025909,0.00039856,0.00000080,-0.00005008,-0.00$ $053963,0.00053734,-0.00064353,0.00121420,0.00109118,0.00072379,-0.0007$ $9395,-0.00000355,-0.00009261,0.00013498,0.00018844,-0.00003313,-0.0001$ $0676,0.00004898,-0.00003384,-0.00010615,-0.00004961,-0.00009084,0.0001$ $3540,-0.00018644,-0.00072048,-0.00072894,-0.00000102,0.01615938,0.0767$ $9537,-0.00017413,-0.28316819,0.04815199,0.00031110,0.61263145,0.002752$ $58,0.00197372,-0.00000145,0.00064298,-0.00140365,0.00021839,0.00022018$ $, 0.00039014,0.00070332,-0.00060615,-0.00040678,-0.00000120,0.00022586$, $0.00038835,-0.00070108,0.00064217,-0.00140121,-0.00022621,0.00125777,0$ $.00061915,-0.00000366,0.00006779,0.00000738,-0.00011717,0.00001120,0.0$ $0002257,-0.00005665,0.00001128,0.00002221,0.00005614,0.00006931,0.0000$ $0803,0.00011933,0.00100903,0.00102178,0.00000289,0.02922338,-0.0184516$ $4,0.00000313,0.12036677,-0.15764692,-0.00003500,0.00752281,0.72664054$, $0.00000534,-0.00000288,0.00048876,-0.00048886,-0.00048310,-0.00006016$, $0.00038959,0.00068895,0.00039416,-0.00000050,0.00000399,-0.00086356,-0$ $.00038768,-0.00069381,0.00039047,0.00048907,0.00048726,-0.00005495,-0$. $00000707,0.00000049,-0.00005946,-0.00009709,-0.00003047,-0.00002979,-0$ $.00000066,-0.00001425,-0.00002109,0.00000070,0.00001476,-0.00002170,0$. $00009711,0.00002992,-0.00002921,-0.00000045,-0.00000098,0.00007634,-0$. $00007394,-0.00009272,0.00855379,0.00015829,0.00010468,-0.06476408,-0.0$ 
$0094316,-0.00125978,0.12883371,0.07144573,-0.01608231,0.00000908,-0.02$ $583267,-0.01099476,0.02593783,0.00725813,0.01090929,0.00634187,-0.0055$ $5503,-0.00791116,-0.00002601,0.00724639,0.01092371,-0.00629387,-0.0258$ $0810,-0.01090769,-0.02600009,-0.01179129,0.00795063,0.00001013,0.00068$ $809,-0.00064957,-0.00090946,0.00052051,0.00031049,-0.00001489,0.000520$ $11,0.00031053,0.00001660,0.00068808,-0.00065211,0.00090947,0.00211767$, $0.00231036,0.00000463,-0.01285468,-0.02597761,0.00006036,-0.06248195,0$ $.01486450,0.00011941,-0.24394793,-0.13570456,0.00065894,0.36371747,-0$. $03498972,-0.00686900,0.00001217,0.00640491,0.00671235,-0.00992374,-0.0$ $0318079,-0.00453849,-0.00258935,0.00246184,0.00334518,0.00000049,-0.00$ $318698,-0.00454617,0.00258038,0.00641834,0.00668158,0.00994160,0.01254$ $852,-0.00914801,-0.00000775,-0.00023071,0.00035793,0.00030291,-0.00008$ $471,-0.00025959,0.00001509,-0.00008659,-0.00025869,-0.00001629,-0.0002$ $2865,0.00035686,-0.00029966,-0.00109754,-0.00107790,-0.00000182,-0.025$ $80437,-0.05492577,0.00014565,-0.04157877,0.05994154,-0.00001631,-0.100$ $46757,-0.19059714,0.00048537,0.13845667,0.59431710,-0.00004033,0.00000$ $069,-0.00644417,0.00780106,0.00087969,-0.00111247,-0.00150461,-0.00278$ 246,-0.00225863,0.00000448,-0.00000300,0.00364207,0.00149147,0.0027829 $5,-0.00223609,-0.00779243,-0.00085170,-0.00110535,0.00002245,-0.000003$ $83,0.00033472,-0.00022608,0.00023754,0.00024640,-0.00021534,0.00009136$ $, 0.00002227,0.00021473,-0.00009161,0.00002096,0.00022675,-0.00023805,0$ $.00024450,-0.00000251,-0.00000228,-0.00007291,0.00006386,0.00015080,-0$ $.00861220,0.00023745,-0.00012555,0.01429572,0.00058068,0.00054110,-0.0$ $5275588,-0.00085085,-0.00130905,0.08427399,-0.00386529,0.00470493,-0.0$ $0001427,0.00003789,0.00188063,-0.00168857,-0.00036502,-0.00051855,-0.0$ $0070319,0.00090183,0.00064248,0.00000706,-0.00036506,-0.00051591,0.000$ $69444,0.00003278,0.00187394,0.00169907,-0.00082510,0.00116360,0.000001$ $26,-0.00007602,-0.00001465,0.00021969,-0.00011682,-0.00001276,0.000081$ $66,-0.00011389,-0.00001435,-0.00008177,-0.00007566,-0.00001467,-0.0002$ $2130,-0.00129720,-0.00136529,-0.00000249,-0.05650247,-0.02760022,0.000$ $18169,-0.01865021,0.03114290,-0.00004209,0.03512926,-0.02822010,-0.000$ $00661,-0.09276001,-0.01776080,0.00012125,0.68301196,0.00167373,-0.0014$ $8199,-0.00002089,0.00012041,0.00046262,0.00070546,-0.00072685,-0.00025$ $346,-0.00004709,0.00072771,-0.00030820,-0.00000067,-0.00072801,-0.0002$ $4693,0.00004632,0.00012172,0.00047346,-0.00068479,0.00043168,-0.000229$ 40,-0.00000199,0.00007556,-0.00004784,-0.00011205,-0.00001714,0.000016 $18,-0.00000966,-0.00001651,0.00001677,0.00001126,0.00007541,-0.0000484$ $5,0.00011262,-0.00015275,-0.00008113,-0.00000115,0.01930772,0.05529981$ $,-0.00013658,0.02901626,-0.04517318,0.00003101,-0.05913073,-0.03137606$ $, 0.00019117,0.01660425,-0.34044542,0.00056189,0.05545881,0.66602177,0$. $00000571,0.00000175,-0.00032068,-0.00025532,-0.00051505,0.00025298,0.0$ $0039270,0.00014009,0.00025853,-0.00000620,0.00000018,-0.00080293,-0.00$ $039302,-0.00013914,0.00025548,0.00026618,0.00049753,0.00025387,-0.0000$ $0351,-0.00000229,0.00010438,-0.00002269,0.00001294,0.00006137,-0.00004$ $163,0.00003009,0.00008650,0.00004283,-0.00003143,0.00008761,0.00002071$ ,-0.00001001,0.00006144,0.00000316,0.00000339,0.00004848,0.00008391,-0 $.00004099,0.00864083,-0.00002794,0.00002117,-0.00473011,0.00006103,0.0$ $0012967,0.00387440,0.00004526,0.00062556,-0.05368699,-0.00118220,-0.00$ $122913,0.12894989,0.00125919,0.00006481,0.00000696,0.00056914,-0.00042$ $232,0.00024522,0.00009112,-0.00021215,0.00006212,-0.00053019,-0.000261$ $65,0.00000223,0.00009792,-0.00021474,-0.00006180,0.00057204,-0.0004245$ 
$2,-0.00025168,0.00013351,-0.00026986,0.00000025,-0.00001163,0.00010066$ ,-0.00004509,-0.00001151,-0.00001009,-0.00001756,-0.00001238,-0.000009 $71,0.00001681,-0.00001134,0.00009907,0.00004788,0.00035315,0.00036387$, $0.00000120,-0.23535290,-0.06808289,0.00046515,0.03660383,-0.06770027,0$ $.00008682,-0.06391297,-0.00356485,0.00012244,0.02481637,0.08388024,-0$. $00020151,-0.29207508,0.04604461,0.00033757,0.64496002,-0.00029398,-0.0$ $0071740,0.00000564,-0.00017580,-0.00033633,0.00020630,0.00008940,0.000$ $32166,0.00019841,0.00013447,-0.00003378,-0.00000089,0.00009030,0.00031$ $892,-0.00019553,-0.00017149,-0.00033806,-0.00020962,0.00022084,-0.0002$ 4870,0.00000110,0.00003300,-0.00003546,-0.00006576,0.00006068,-0.00001 $075,-0.00002493,0.00006042,-0.00001124,0.00002402,0.00003283,-0.000036$ $20,0.00006582,0.00029534,0.00032055,0.00000098,-0.12010849,-0.18471937$ $, 0.00047855,-0.02448028,-0.03545398,0.00013719,-0.00682746,0.00014833$, $0.00000308,0.02802496,-0.02798962,0.00002885,0.11832866,-0.15489519,-0$ $.00003176,-0.01813381,0.72691850,-0.00000206,0.00000740,0.00078601,0.0$ $0004404,-0.00015178,0.00003204,0.00002547,-0.00013884,0.00015815,0.000$ $00070,0.00000165,-0.00024201,-0.00002104,0.00013379,0.00016083,-0.0000$ $4133,0.00015540,0.00003652,-0.00000140,0.00000161,0.00000373,-0.000002$ $74,0.00005732,-0.00000697,0.00001118,-0.00000296,-0.00003082,-0.000011$ $97,0.00000385,-0.00003088,0.00000243,-0.00005659,-0.00000838,-0.000000$ $58,-0.00000102,0.00001679,0.00057119,0.00037362,-0.06576258,-0.0000047$ $2,0.00022436,0.00873995,0.00012770,-0.00000145,-0.00450703,-0.00008080$ $,-0.00007997,0.01385428,0.00019273,0.00010518,-0.06371945,-0.00094083$, $-0.00119833,0.13328986,-0.00036908,0.00020979,0.00000074,0.00019428,-0$ $.00008670,0.00008331,0.00007046,0.00000535,0.00010188,-0.00031216,-0.0$ $0028723,-0.00000112,0.00007276,0.00000401,-0.00010110,0.00019542,-0.00$ $008669,-0.00008451,-0.00009745,0.00006447,0.00000055,-0.00005042,0.000$ $03269,0.00002052,-0.00000021,0.00002167,-0.00001724,-0.00000045,0.0000$ $2144,0.00001690,-0.00005038,0.00003257,-0.00002036,0.00030248,0.000317$ $47,0.00000088,-0.24490898,0.07867290,0.00018885,-0.01230997,0.02509960$ ,-0.00002866,0.00803305,-0.00056733,-0.00000118,-0.00221810,-0.0007063 $1,0.00000356,0.00047026,-0.00773089,0.00002797,-0.04900895,0.01037287$, $0.00007344,1.27098398,0.00015032,-0.00014848,-0.00000129,-0.00009387,0$ $.00004566,-0.00004964,-0.00003817,0.00004058,-0.00006067,0.00019156,0$. $00015727,0.00000081,-0.00003917,0.00003953,0.00005986,-0.00009410,0.00$ $004565,0.00005052,-0.00000122,0.00002495,0.00000015,0.00001723,-0.0000$ $2035,-0.00000981,0.00000952,-0.00001314,0.00000412,0.00000931,-0.00001$ $291,-0.00000393,0.00001705,-0.00002009,0.00000933,-0.00013874,-0.00015$ $131,-0.00000049,0.07860083,-0.12087864,-0.00001810,0.03943122,-0.02131$ $534,-0.00002535,-0.00182929,-0.00610330,0.00003290,-0.00055074,-0.0030$ $2965,0.00000475,-0.00645368,0.00138559,0.00002272,-0.00388065,0.015738$ $34,-0.00002096,-0.56009522,0.38886555,0.00000156,0.00000006,-0.0002603$ $5,-0.00002711,-0.00000390,0.00004630,-0.00001461,0.00006624,-0.0000328$ 9,0.00000032,0.00000109,0.00003017,0.00001495,-0.00006679,-0.00003336, $0.00002295,0.00000408,0.00004470,0.00000002,-0.00000081,-0.00001093,0$. $00001147,-0.00001525,-0.00001015,0.00000109,0.00000926,0.00000204,-0.0$ $0000094,-0.00000944,0.00000262,-0.00001082,0.00001517,-0.00000980,-0.0$ 0000063,-0.00000053,0.00000006,0.00018631,-0.00001917,-0.05846196,-0.0 $0005481,0.00000181,0.00164903,0.00000317,0.00002950,0.00713970,0.00000$ $215,0.00000480,-0.00130502,0.00002666,0.00002400,0.00689371,0.00010558$ ,-0.00004721,0.00180440,-0.00106742,0.00042189,0.08530975,0.00002086,- 
$0.00023496,0.00000002,-0.00013076,0.00014250,-0.00004965,-0.00002164,-$ $0.00002493,-0.00008492,0.00009732,0.00007157,0.00000015,-0.00002420,-0$ $.00002563,0.00008478,-0.00012982,0.00014290,0.00005124,0.00000876,-0.0$ $0002342,-0.00000059,-0.00001307,0.00000169,0.00001838,-0.00000526,-0.0$ $0000805,0.00000899,-0.00000526,-0.00000775,-0.00000870,-0.00001332,0.0$ $0000213,-0.00001879,-0.00015242,-0.00016041,-0.00000064,0.00484203,0.0$ $0463979,-0.00001331,-0.25341571,-0.13280811,0.00069151,-0.01642906,-0$. $01639347,0.00007561,-0.00402133,0.00239562,0.00001576,0.00021615,0.001$ $02632,-0.00000516,0.00144163,0.00117798,0.00000642,0.00089185,0.000552$ $03,-0.00001033,0.26549151,0.00024004,0.00007120,-0.00000020,0.00003044$ ,-0.00007763,0.00005539,0.00000932,0.00003381,0.00003801,-0.00003385,$0.00002945,0.00000026,0.00001043,0.00003402,-0.00003814,0.00002978,-0$. $00007784,-0.00005567,0.00000141,0.00002566,0.00000003,0.00000704,-0.00$ $000204,-0.00001539,0.00000370,-0.00000053,-0.00000366,0.00000364,-0.00$ $000058,0.00000356,0.00000722,-0.00000220,0.00001546,0.00005979,0.00006$ $194,0.00000018,-0.02626179,-0.01197119,0.00008223,-0.12950709,-0.14415$ $701,0.00047189,0.01321032,0.00993062,-0.00003451,0.00269434,0.00046127$ $, 0.00000665,0.00054956,-0.00042555,-0.00000230,0.00212287,-0.00507418$, $0.00001955,-0.00160037,0.00081025,-0.00000697,0.13949099,0.15013612,-0$ $.00000115,0.00000154,0.00000347,0.00006375,0.00003506,0.00001557,-0.00$ 005886,-0.00005864,-0.00003550,0.00000011,0.00000021,0.00010085,0.0000 $5846,0.00005886,-0.00003537,-0.00006483,-0.00003526,0.00001445,0.00000$ $037,-0.00000041,0.00003515,-0.00000850,0.00001170,0.00000083,0.0000033$ $7,0.00000027,-0.00000122,-0.00000316,-0.00000041,-0.00000059,0.0000089$ $8,-0.00001193,0.00000165,-0.00000007,-0.00000003,-0.00000568,0.0000507$ $3,0.00002246,0.00335895,0.00068422,0.00047946,-0.03674015,0.00001504,0$ $.00002196,0.00530301,0.00001279,0.00000719,0.00625610,-0.00000154,-0.0$ $0000377,-0.00093705,0.00000496,0.00002204,0.00616091,-0.00000481,-0.00$ $001169,-0.00384934,-0.00075055,-0.00052914,0.02448024,0.00058316,-0.00$ $120657,-0.00000708,-0.00006001,0.00018900,0.00003748,-0.00002170,0.000$ $09301,-0.00004736,-0.00002616,-0.00006230,0.00000024,-0.00002342,0.000$ $09190,0.00004645,-0.00006133,0.00018718,-0.00003469,-0.00029760,0.0004$ $4485,0.00000295,-0.00000684,-0.00006361,0.00001041,0.00003415,0.000002$ $97,-0.00000444,0.00003427,0.00000294,0.00000466,-0.00000777,-0.0000627$ $0,-0.00001202,0.00007028,0.00008236,-0.00000008,-0.00424515,0.00197712$ $, 0.00001631,0.00123907,0.02895684,-0.00005177,-0.05558949,0.01797394,0$ $.00000087,0.00481791,-0.02485739,0.00004446,-0.00378457,-0.00361066,0$. $00002839,-0.00125632,-0.00000365,0.00000076,0.00016195,0.00142181,-0.0$ $0000231,0.00111282,-0.00039540,-0.00000944,0.05736607,0.00071814,-0.00$ $014698,-0.00000505,-0.00014658,0.00008977,0.00029525,0.00010761,0.0001$ $2794,-0.00011881,-0.00001185,-0.00007857,-0.00000004,0.00010363,0.0001$ $2629,0.00011720,-0.00014905,0.00009071,-0.00029111,-0.00065885,0.00036$ $016,0.00000158,-0.00003295,-0.00002207,0.00002036,0.00001500,0.0000243$ $2,0.00000471,0.00001492,0.00002462,-0.00000389,-0.00003419,-0.00002153$ ,-0.00002246,-0.00008468,-0.00008266,-0.00000059,0.00233316,0.00160756 $, 0.00000504,-0.00126574,-0.01015403,0.00003756,0.02282529,-0.32989297$, $0.00055910,-0.00154863,-0.01440386,0.00003519,-0.00193524,-0.00027776$, $0.00001669,-0.00052433,0.00050659,-0.00000074,0.00000229,-0.00042235,0$ $.00000112,-0.00008649,0.00074856,-0.00001119,-0.02005201,0.35230887,-0$ $.00000032,0.00000463,-0.00081463,0.00032208,0.00014529,0.00011353,-0.0$ $0000989,-0.00009323,-0.00015923,0.00000083,-0.00000007,0.00009276,0.00$ 
$000948,0.00009286,-0.00015940,-0.00032348,-0.00014787,0.00011200,0.000$ $00132,-0.00000276,0.00010461,0.00010346,-0.00000163,-0.00006944,-0.000$ $00999,0.00002572,0.00000747,0.00000977,-0.00002619,0.00000794,-0.00010$ $462,0.00000339,-0.00007057,-0.00000023,0.00000003,-0.00001835,0.000014$ $72,0.00000648,0.00667082,0.00001146,-0.00002177,0.00539312,-0.00000907$ $, 0.00056871,-0.03634295,-0.00000840,0.00007539,-0.00016396,0.00002646$, $0.00002045,0.00672951,0.00000302,-0.00000284,-0.00080326,0.00000074,-0$ $.00000149,0.00054057,-0.00000978,-0.00000944,-0.00413627,-0.00002365,-$ $0.00063764,0.02412700,-0.00012338,0.00039912,0.00000510,0.00020155,-0$. $00004502,-0.00013192,0.00018627,0.00001291,0.00027078,-0.00095524,-0.0$ $0003438,-0.00000887,0.00018681,0.00001236,-0.00026860,0.00019508,-0.00$ $005063,0.00012416,-0.00002814,0.00000916,0.00000029,0.00001423,-0.0000$ 4176,-0.00001257,0.00002742,-0.00000199,-0.00002171,0.00002710,-0.0000 $0235,0.00002233,0.00001479,-0.00004227,0.00001197,0.00013819,0.0001249$ $9,0.00000051,-0.00267273,0.00347611,0.00001132,0.00012699,0.00047247,-$ $0.00000273,0.00106445,0.00212293,0.00000705,0.00558451,-0.00126861,-0$. $00000606,-0.24864217,-0.12738497,0.00067451,-0.01848766,-0.01676931,0$. $00007907,-0.00091776,-0.00079975,0.00000473,-0.00006859,-0.00002601,-0$ $.00000196,0.00026515,0.00022822,-0.00000151,0.26289798,-0.00104241,-0$. $00005690,0.00000302,0.00004100,0.00002985,-0.00025591,0.00009215,0.000$ $05875,-0.00011888,0.00035761,0.00055682,0.00000422,0.00008703,0.000051$ $12,0.00011502,0.00004460,0.00003343,0.00024612,-0.00001745,0.00006280$, $0.00000108,-0.00000613,-0.00001068,0.00002211,-0.00001486,0.00003603,0$ $.00002244,-0.00001489,0.00003517,-0.00002280,-0.00000697,-0.00000939,-$ $0.00002247,-0.00005181,-0.00010535,0.00000012,0.00323986,-0.00002513,0$ $.00000737,0.00097017,-0.00077861,-0.00000221,0.00032564,-0.00527402,0$. $00002022,-0.02456730,-0.01520851,0.00007959,-0.13134908,-0.14015871,0$. $00047382,0.01314792,0.01035848,-0.00003535,0.00054237,0.00065251,-0.00$ $000023,-0.00007346,0.00005791,-0.00000144,-0.00101645,-0.00039922,0.00$ $000384,0.13950706,0.14957698,-0.00000062,-0.00001231,-0.00071914,-0.00$ $030103,-0.00005696,0.00012508,-0.00016554,0.00027398,-0.00020675,-0.00$ $000830,-0.00000099,0.00068871,0.00016916,-0.00027276,-0.00020466,0.000$ $29098,0.00006382,0.00011863,0.00000224,-0.00000170,-0.00001094,0.00002$ $657,-0.00006389,-0.00003127,0.00006789,-0.00003046,-0.00005268,-0.0000$ $6566,0.00002949,-0.00005184,-0.00002438,0.00005990,-0.00002900,-0.0000$ $0131,-0.00000170,-0.00006039,0.00000944,0.00000791,0.00673990,-0.00000$ $118,-0.00000040,-0.00075688,0.00001070,0.00001966,0.00652474,0.0000416$ $1,0.00004069,-0.00024449,0.00068434,0.00046613,-0.03640478,0.00001865$, $0.00002010,0.00546536,0.00000200,0.00000121,0.00045444,-0.00000157,-0$. $00000213,-0.00098053,0.00000127,-0.00000017,0.00007006,-0.00074514,-0$. $00052911,0.02393907,-0.00015599,0.00019362,-0.00000046,0.00001947,0.00$ $014358,-0.00010953,-0.00003188,-0.00006248,-0.00009038,0.00011403,0.00$ $010113,0.00000036,-0.00003300,-0.00006302,0.00008961,0.00001877,0.0001$ $4382,0.00011074,-0.00005021,0.00004225,-0.00000034,-0.00001305,0.00001$ $129,0.00001380,-0.00002020,0.00000255,0.00001587,-0.00002006,0.0000028$ $0,-0.00001550,-0.00001311,0.00001152,-0.00001400,-0.00015935,-0.000189$ $99,-0.00000054,0.00489227,-0.02616079,0.00005066,-0.00415909,-0.002354$ $29,0.00002514,-0.00097072,-0.00035729,0.00000146,-0.00359856,0.0031082$ $6,0.00001364,0.00144955,0.02712493,-0.00004961,-0.05852419,0.02416804$, $-0.00000761,0.00137483,-0.00123420,-0.00000775,0.00024674,-0.00130978$, $0.00000315,0.00007951,0.00008877,-0.00000256,0.00108432,-0.00031605,-0$ 
$.00000954,0.05873788,-0.00017009,-0.00002054,0.00000052,0.00003600,-0$. $00005000,0.00002328,0.00000044,0.00002845,0.00004285,-0.00008396,-0.00$ $003776,-0.00000018,0.00000071,0.00002845,-0.00004285,0.00003572,-0.000$ $04991,-0.00002376,0.00004547,-0.00002802,0.00000006,-0.00000003,-0.000$ $00715,-0.00000683,0.00001274,-0.00000232,-0.00000511,0.00001258,-0.000$ $00228,0.00000517,-0.00000004,-0.00000723,0.00000683,0.00009525,0.00009$ $902,0.00000022,0.00466132,-0.01201973,0.00002325,-0.00322991,0.0005620$ $1,0.00001736,0.00015235,0.00070844,-0.00000302,0.00245038,0.00009299,0$ $.00000767,-0.00211495,-0.00786536,0.00003176,0.02087775,-0.33948122,0$. $00058490,0.00090235,0.00031949,-0.00001045,0.00028771,-0.00054982,0.00$ $000184,0.00002325,-0.00005903,-0.00000225,-0.00014168,0.00080269,-0.00$ $001004,-0.02351390,0.35720498,0.00000044,-0.00000046,0.00013803,0.0001$ $3943,0.00005761,-0.00005502,-0.00002659,-0.00007679,-0.00002655,0.0000$ $0063,-0.00000001,0.00004740,0.00002580,0.00007699,-0.00002670,-0.00013$ $903,-0.00005779,-0.00005654,-0.00000021,-0.00000012,-0.00001688,-0.000$ $00281,-0.00000491,-0.00000193,0.00000570,-0.00000363,-0.00000074,-0.00$ $000555,0.00000361,-0.00000064,0.00000284,0.00000493,-0.00000182,-0.000$ $00007,0.00000003,-0.00000906,-0.00001384,0.00008141,0.00325803,0.00002$ $693,0.00001584,0.00615023,-0.00000068,-0.00000262,-0.00086128,0.000015$ $24,0.00000835,0.00650835,0.00001214,-0.00002498,0.00525203,-0.00000104$ $, 0.00057871,-0.03702820,-0.00001097,-0.00000627,-0.00377734,-0.0000002$ $9,0.00000481,0.00053644,-0.00000222,-0.00000255,-0.00101935,-0.0000102$ $4,-0.00000963,-0.00418821,-0.00001748,-0.00064206,0.02475191,0.0001693$ $4,-0.00010891,-0.00000042,-0.00012432,0.00008507,-0.00010544,-0.000064$ $71,-0.00000870,-0.00009465,0.00027589,0.00024779,0.00000115,-0.0000652$ $7,-0.00000872,0.00009384,-0.00012490,0.00008525,0.00010597,0.00008490$, $-0.00005720,-0.00000029,0.00003448,-0.00002325,-0.00001740,0.00000434$, $-0.00002138,0.00000907,0.00000430,-0.00002119,-0.00000897,0.00003443,-$ $0.00002336,0.00001733,-0.00025037,-0.00026092,-0.00000059,-0.01456974$, $0.01681516,0.00002179,-0.00045568,-0.00988674,0.00002573,-0.00286022,0$ $.00129376,0.00000075,0.00144867,-0.00014490,-0.00000189,-0.00173511,0$. $00168939,-0.00000266,0.00829720,0.00387448,-0.00002005,-0.97250740,0.4$ $5484792,0.00081577,0.00006076,0.00066340,-0.00000106,-0.00038401,0.000$ $09356,0.00000015,-0.00011919,0.00012143,-0.00000057,-0.00026796,-0.000$ $33945,0.00000185,0.98311458,-0.00007679,0.00006486,0.00000035,0.000059$ $13,-0.00004302,0.00005130,0.00003635,0.00000419,0.00005093,-0.00016311$ $,-0.00014666,-0.00000069,0.00003716,0.00000456,-0.00005031,0.00005909$, $-0.00004295,-0.00005187,-0.00003559,0.00002204,0.00000006,-0.00001470$, $0.00000904,0.00001026,0.00000065,0.00000944,-0.00000649,0.00000071,0.0$ $0000924,0.00000636,-0.00001466,0.00000887,-0.00001003,0.00013511,0.000$ $14818,0.00000041,0.01679452,0.01174718,-0.00002381,-0.00305930,0.00359$ $886,0.00000093,0.00094226,0.00067243,-0.00000637,-0.00026366,0.0010310$ $2,-0.00000118,0.00214474,-0.00036159,-0.00000524,-0.00298997,-0.005249$ $87,0.00001937,0.45485890,-0.25600982,-0.00039828,-0.00008455,-0.000003$ $33,0.00000133,-0.00011645,-0.00005437,0.00000034,0.00024342,-0.0002860$ $1,-0.00000028,0.00041925,0.00033384,-0.00000045,-0.46891251,0.24453386$ ,-0.00000045,-0.00000078,0.00004620,0.00000965,-0.00000299,-0.00001432 $, 0.00000557,-0.00001172,0.00000993,-0.00000083,-0.00000063,-0.00000937$ ,-0.00000493,0.00001167,0.00001000,-0.00000841,0.00000321,-0.00001282, $-0.00000004,0.00000053,-0.00000330,-0.00000644,0.00000777,0.00000533$,$0.00000517,-0.00000355,-0.00000002,0.00000494,0.00000375,-0.00000064,0$ 
$.00000605,-0.00000769,0.00000472,0.00000050,0.00000040,0.00000354,0.00$ $002361,-0.00002269,0.01553922,0.00000873,0.00001394,0.00162751,0.00000$ $082,-0.00000752,-0.00120793,-0.00000100,-0.00000171,0.00029880,-0.0000$ $0443,-0.00000275,-0.00120904,-0.00000736,0.00000609,0.00163076,0.00081$ $604,-0.00039858,-0.03616635,0.00000143,-0.00000044,0.00045409,0.000000$ $81,0.00000041,-0.00021192,-0.00000053,-0.00000092,-0.00018445,0.000000$ $84,0.00000083,0.00042785,-0.00083941,0.00041335,0.01896221 \backslash-0.0000134$ 7,0.00001063,0.00000269,0.00000329,-0.00000189,0.00000190,-0.00000031, $-0.00000103,0.00000068,0.00000356,-0.00000437,-0.00000033,-0.00000098$, $-0.00000132,-0.00000047,0.00000498,0.00000085,-0.00000367,-0.00000455$, $0.00000363,0.00000237,-0.00000070,-0.00000162,0.00000028,0.00000036,-0$ $.00000202,-0.00000007,0.00000033,-0.00000184,0.00000002,-0.00000058,-0$ $.00000140,-0.00000018,0.00000055,-0.00000188,-0.00000001,0.00000028,0$. $00000219,-0.00000022,-0.00000105,0.00000070,0.00000100,-0.00000793,0.0$ $0000537,-0.00000202,0.00000485,-0.00001596,-0.00000575,0.00000818,-0.0$ $0000145,0.00000466,0.00000021,-0.00000041,0.00000140,0.00000088,0.0000$ $0292,-0.00000018,-0.00000123,0.00000275,0.00000009,-0.00000157,0.00000$ $166,-0.00000067,0.00000137,-0.00000004,-0.00000134,0.00000222,0.000001$ 08,-0.00000012,0.00000131,0.00000346,-0.00000006III@

ortho-Addition Product

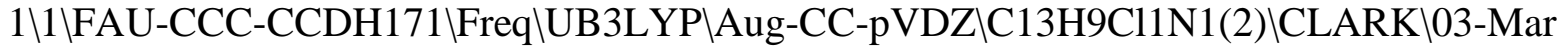
-2015\0\॥N Geom=AllCheck Guess=TCheck SCRF=Check GenChk UB3LYP/Aug-CC -pVDZ Freq \\Addition product p-Cl-Ph-radical + aniline (ortho) $\backslash \backslash 0,2 \backslash \mathrm{C}$, $1.4403463325,-0.7064766808,0.693400042 \backslash \mathrm{C}, 2.26511505,0.5733936015,0.637$ $2714952 \backslash \mathrm{C}, 3.363225596,0.7068116281,-0.1993753908 \backslash \mathrm{C}, 3.7891650772,-0.349$ 9635931,-1.0213882068\C,3.0963370919,-1.5973844067,-0.9956634036\C,2.0 $077659967,-1.7848290116,-0.19618716 \mathrm{VH}, 1.4670860049,-1.0760657132,1.736$ $2395752 \backslash \mathrm{C}, 1.8855680846,1.6419055069,1.4950664038 \backslash \mathrm{H}, 3.9071510564,1.6513$ $132232,-0.2083559077 \backslash \mathrm{H}, 3.4488227705,-2.4141649457,-1.6269265837 \backslash \mathrm{H}, 1.48$ $56635962,-2.7419242263,-0.1889419363 \backslash \mathrm{H}, 4.6545453667,-0.217740801,-1.66$ $88944228 \backslash \mathrm{C},-2.7411597183,-0.0142386945,-0.2001572767 \backslash \mathrm{C},-1.7834067619,0$ $.1828009489,-1.1975969007 \backslash \mathrm{C},-0.4388166748,-0.0412570394,-0.898538555 \backslash \mathrm{C}$ ,-0.0431907502,-0.4568576587,0.3809994827\C,-1.0235661165,-0.642271781 $9,1.3618257497 \backslash \mathrm{C},-2.3760171335,-0.4240588002,1.081090437 \backslash \mathrm{Cl},-4.4416416$ $375,0.2612695569,-0.5693405716 \mathrm{UH},-2.0878285855,0.5065382716,-2.1912746$ $685 \backslash \mathrm{H}, 0.3101392773,0.1118429771,-1.6757635749 \backslash \mathrm{H},-0.7355697164,-0.95547$ $39563,2.3660187509 \backslash \mathrm{H},-3.1346509772,-0.5669063616,1.8482096254 \backslash \mathrm{N}, 1.5676$ $157705,2.4988109569,2.2210519973 \backslash \backslash$ Version=EM64L-G09RevC.01\State $=2-\mathrm{A} \backslash \mathrm{H}$ $\mathrm{F}=-1015.7727319 \backslash \mathrm{S} 2=0.788714 \backslash \mathrm{S} 2-1=0 . \backslash \mathrm{S} 2 \mathrm{~A}=0.750901 \backslash \mathrm{RMSD}=0.000 \mathrm{e}+00 \backslash \mathrm{RMSF}=3$ $.907 \mathrm{e}-06 \backslash \mathrm{Z}$ eroPoint $=0.1784846 \backslash \mathrm{Thermal}=0.1911062 \mathrm{ZPE}=112.0007983 \backslash \mathrm{Dipole}=$ $0.9697319,-1.4716769,-0.9253321 \backslash$ DipoleDeriv $=0.0788872,-0.0460023,0.087$ $5552,-0.0043808,0.0548875,-0.0705888,0.1842098,-0.048192,0.1277312,0.0$ $101164,0.1886818,0.0172059,0.2063278,-0.0206073,-0.0109618,-0.0882167$, $-0.127714,-0.0338536,-0.073909,-0.0225049,-0.0490619,-0.0129895,0.0841$ $09,0.0052266,-0.0046792,0.0760418,-0.0412437,0.058256,0.1382625,-0.086$ $725,0.0323125,-0.0423734,0.019613,-0.1700104,-0.0781891,-0.0521274,-0$. $1137189,-0.0991068,-0.0778495,0.0501627,0.0659614,0.0823639,0.0368504$, $0.1577982,-0.0072529,-0.0543533,0.0965434,-0.095146,-0.0592231,-0.0294$ $532,-0.011841,-0.2506243,-0.0075138,-0.0943496,0.0425931,-0.0256164,0$. 
$0414446,0.0076418,0.0675639,0.0398582,0.013825,0.0590047,-0.0662779,0$. $1068726,-0.1441211,-0.0418489,-0.0840469,0.2575183,0.1640974,0.0196493$ $, 0.2049207,0.2359613,0.0673392,-0.0540809,0.019547,-0.079029,-0.010835$ $3,-0.0138065,0.053913,-0.0205985,0.1061957,0.0722559,0.0229626,0.04916$ $06,0.0226245,-0.0213952,-0.0855962,0.095035,-0.0839637,0.0537742,0.063$ $0324,-0.0664173,0.0352998,-0.0602721,-0.0273101,-0.0180236,0.0267554,-$ $0.0225872,0.078312,-0.0006054,-0.0234688,0.0886041,-0.0319997,0.058216$ $4,-0.0099845,0.0939557,-0.0113381,0.0558112,1.2258406,-0.1714326,0.228$ $3127,-0.2133792,-0.0025666,-0.0600616,0.3014063,-0.0594138,0.0876326,-$ $0.2795562,0.0051136,0.0233648,0.0166743,-0.0700639,-0.0415308,0.003705$ $2,-0.0358145,0.0390453,-0.0853307,0.0023064,-0.0405159,-0.0038721,-0.0$ $732288,-0.0226444,0.0302915,-0.0236964,-0.0046043,0.2509694,0.0217635$, $0.0061092,-0.0283405,-0.04931,0.010978,0.0604716,0.019257,-0.0649695,-$ $0.0039899,-0.0273706,0.0484757,0.0218726,-0.0751045,-0.032361,-0.08879$ $83,-0.0527603,0.0773335,-0.2715763,0.040143,-0.1427612,0.0603577,-0.08$ $99314,0.0103943,-0.1752596,0.004828,-0.03949,-0.8847422,0.0973133,-0.1$ 457032,0.1290944,-0.0817118,0.0297599,-0.1743342,0.0277398,-0.1163677, $0.0515536,0.0145829,-0.0334307,0.0197933,0.1127241,0.040562,-0.0377415$ $, 0.0383938,-0.0002251,0.0043767,-0.0075632,0.0728749,-0.016756,0.09285$ $52,0.0134584,0.0779869,0.0214462,0.0266599,0.0486617,0.0329593,-0.0665$ $283,0.0261188,0.0977312,0.0421486,-0.0460649,0.0500272,-0.0338724,0.00$ $50101,-0.009652,0.0558724,-0.0006657,0.1217545,0.0163082,0.0493541,0.0$ $200821,0.0428144,-0.317983,0.0367045,0.0057438,0.0019741,-0.4194299,-0$ $.0973682,-0.0116803,-0.1077581,-0.3766375 \backslash$ Polar $=227.9208672,-8.304523$, $149.8747482,-16.552945,16.5448673,157.8374969 / \mathrm{PG}=\mathrm{C} 01$ [X(C13H9Cl1N1)]LN Imag=0\\0.41305390,0.00553129,0.46081970,-0.02778891,-0.01053590,0.524 $19705,-0.09708436,-0.05097994,0.00761663,0.44698106,-0.03687827,-0.147$ $54997,-0.00362835,0.06094525,0.48246766,0.01415247,0.00301170,-0.07304$ $457,-0.22761794,0.11991837,0.38197552,-0.01265700,-0.01137050,0.013230$ $72,-0.22449314,-0.04332872,0.12011737,0.45304040,-0.02029347,0.0034517$ $9,0.01277063,-0.06862882,-0.10023530,0.03296931,0.15601382,0.63769669$, $0.00741689,0.00741851,0.00412398,0.11079935,0.01213569,-0.13612462,-0$. $20020614,0.10996359,0.33599993,-0.00107916,0.00155146,-0.00585739,-0.0$ $1438995,0.02407770,0.02954909,-0.10632863,0.04825917,0.06337104,0.4886$ $8552,0.00101680,-0.00702184,0.00058388,0.06107203,0.00536778,-0.051421$ $56,-0.02222011,-0.21424600,-0.05453615,0.09487384,0.51236785,-0.005772$ $48,-0.00020867,-0.00445375,0.04609729,-0.02103640,-0.04005917,0.031000$ $04,-0.11069816,-0.13006277,-0.26743701,0.09889170,0.37242018,-0.007339$ $83,0.00466711,0.01603857,-0.02573041,-0.00142266,0.01800853,0.04479957$ $,-0.01568842,-0.03892797,-0.12472225,-0.02949175,0.04401603,0.47548314$ $, 0.01607512,0.00967974,-0.01157441,-0.02143002,0.00107341,0.01931877,0$ $.01808296,-0.05010956,-0.03936654,-0.09465909,-0.21389900,0.00894154,0$ $.04407684,0.58144442,0.01994084,-0.00295507,-0.00870639,0.00924225,0.0$ $0283645,-0.00950737,-0.02371084,-0.01124062,0.01966401,0.01564235,-0.0$ $4375121,-0.08685869,-0.26759363,0.16032749,0.40516203,-0.08703250,0.02$ $641601,0.03114101,0.02099470,-0.00878186,-0.02061821,-0.01987239,0.012$ $21570,0.02072125,-0.02769857,-0.06216123,0.00207463,-0.27584549,-0.011$ $49103,0.17598429,0.53475163,0.01783735,-0.14954210,-0.05075774,0.00750$ $435,-0.02806943,-0.01929497,-0.00215555,0.00195614,0.00308342,-0.03219$ 266,-0.01099477,0.01820207,-0.04450648,-0.10739978,0.01314751,0.152310 $58,0.56168798,0.02774159,-0.05671572,-0.11462779,-0.01358962,-0.003963$ 
$54,0.00895782,0.01448002,-0.00853646,-0.01697168,0.01500350,0.04215001$ $, 0.01471498,0.16027490,-0.01392537,-0.18831154,-0.25248863,0.05936930$, $0.36034008,-0.03377650,-0.00037151,-0.00134134,-0.00293133,-0.01051779$ $, 0.01575058,-0.00052057,-0.00179819,-0.00078121,-0.00054149,-0.0007168$ $3,0.00025684,0.00070367,0.00227612,-0.00015038,-0.00248883,-0.00141754$ $, 0.01481050,0.05444963,0.00304390,-0.07054195,0.07178952,-0.00536793,-$ $0.01197461,0.02412826,0.00082108,-0.00401672,-0.00149579,-0.00047329,0$ $.00049702,0.00062815,0.00083182,-0.00248294,-0.00171518,0.00123416,0.0$ $0935075,-0.02160417,-0.00136713,0.07905221,-0.01074643,0.07506612,-0.2$ $5419109,0.00341664,0.00511571,0.00180475,-0.00113413,0.00065340,0.0001$ $1379,0.00054081,0.00114774,-0.00020208,-0.00299103,-0.00380630,-0.0006$ $0446,0.00562865,0.00099773,-0.02011210,0.00486549,-0.08095510,0.281061$ $01,0.00927248,-0.00734902,-0.01198377,-0.07815376,0.03080305,0.0384257$ $6,-0.01610863,0.02866470,0.02971492,0.00020191,-0.00964145,0.00190404$, $0.00189197,0.00220378,-0.00106618,-0.00319733,-0.00312115,0.00241592,-$ $0.00004306,-0.00089516,0.00129819,0.20257004,0.00304098,-0.03116076,-0$ $.01833667,0.01648268,-0.19233213,-0.08453669,0.03239238,-0.00866208,-0$ $.03235351,-0.00693711,0.02127436,0.01159896,-0.00749160,-0.00798670,0$. $00269467,-0.00133723,-0.00319994,-0.00169003,0.00141917,0.00050978,-0$. $00256560,-0.25896426,0.84979873,-0.00674765,-0.00840950,0.00046813,0.0$ $3261790,-0.09838894,-0.12776183,0.03155397,-0.02923563,-0.03748084,0.0$ $0311693,0.01453876,0.01229200,-0.00569096,-0.00524093,0.00155867,0.003$ $24277,0.00034847,-0.00136670,0.00102422,0.00089902,-0.00064252,-0.2433$ $7857,0.62597541,0.61398790,0.00068802,-0.00030908,0.00575678,-0.009556$ $34,-0.02215383,0.00066093,-0.12055323,-0.12594939,0.00952688,0.0082620$ $4,0.00943485,0.00078489,0.00190117,0.00090595,0.00364127,-0.00029233,0$ $.00054849,-0.00003475,-0.00069137,-0.00109130,-0.00014976,-0.00135779$, $0.00008314,-0.00293526,0.12181934,-0.00070214,0.00249556,-0.00183043,0$ $.00013038,0.00026413,-0.00160142,-0.12714900,-0.26752005,-0.00221971,-$ $0.01287450,-0.01430766,0.00232307,0.00131784,-0.00083674,-0.00434615,0$ $.00065705,0.00027692,-0.00006142,0.00009644,0.00030389,0.00011099,0.00$ $189638,0.00013897,0.00062164,0.13679022,0.27972502,0.00478906,-0.00309$ $439,0.00249651,0.00983547,0.01708379,0.00374552,0.01058861,-0.00365941$ ,-0.04838358,-0.00986409,-0.01496213,0.00500412,0.00370357,-0.00392716 $, 0.00126525,0.00022524,0.00000095,-0.00075449,-0.00011708,-0.00032221$, $-0.00023501,-0.00177760,0.00187665,-0.00241446,-0.01580995,0.00547932$, $0.04058603,0.00077845,-0.00365129,0.00584393,-0.00061857,-0.00026773,0$ $.00007359,0.00042330,-0.00387469,0.00167694,0.00787379,-0.01162566,-0$. $00892407,-0.07869966,0.06362189,0.06030120,-0.00514323,0.01984719,0.01$ $530618,-0.00056938,0.00121228,0.00079679,-0.00015162,0.00015789,-0.000$ $52210,0.00023551,-0.00062434,-0.00051343,0.07709429,-0.00288150,0.0018$ $2859,-0.00119817,-0.00056814,0.00010435,0.00068138,-0.00405766,-0.0029$ $7448,0.00012693,0.01051601,-0.01442347,-0.01592043,0.06404638,-0.21342$ $009,-0.12602105,-0.00288316,0.00663215,0.00339817,0.00020633,-0.000343$ $38,-0.00015276,0.00024912,-0.00003790,0.00009729,0.00054440,-0.0008184$ $6,-0.00081706,-0.06657140,0.22200139,0.00547018,-0.00017400,0.00537722$ $, 0.00013189,0.00037973,-0.00020795,0.00156168,-0.00016963,0.00336861,0$ $.00022944,0.00165853,0.00322580,0.06115700,-0.12554590,-0.14009300,0.0$ 0521386,-0.01541643,-0.00725008,-0.00022715,0.00068052,0.00030104,-0.0 $0046484,0.00029062,-0.00036796,0.00000723,0.00014443,0.00007389,-0.069$ $89407,0.13719049,0.14007676,0.00557875,0.01107746,-0.00013671,-0.00030$ 
$685,0.00154112,0.00207991,-0.00044947,0.00031938,0.00002930,-0.0000819$ $8,-0.00052115,0.00643113,-0.00641169,-0.02335421,0.00013392,-0.1117873$ $2,-0.12332796,0.01155395,0.00035085,-0.00016693,0.00039505,0.00067032$, $0.00077801,0.00010340,-0.00023008,0.00008119,-0.00039611,-0.00135804,0$ $.00128621,-0.00279575,0.11326009,-0.00995426,-0.01482390,0.00159948,0$. $00237077,-0.00029533,-0.00285240,0.00047908,0.00020071,-0.00012266,-0$. $00155014,0.00272140,-0.00128543,-0.00000720,0.00003059,-0.00263319,-0$. $12439484,-0.27282333,-0.00261191,-0.00009112,0.00027104,-0.00054067,-0$ $.00078094,-0.00110217,0.00003167,0.00012989,-0.00010814,0.00023319,0.0$ $0116391,0.00031111,0.00164958,0.13330782,0.28477384,-0.00866654,-0.016$ $34135,0.00187007,0.00252622,-0.00215713,-0.00107083,-0.00004870,-0.000$ $17761,-0.00075014,0.00641743,-0.00193354,0.00249522,0.01047190,0.01640$ $445,0.00491048,0.01061092,-0.00510179,-0.04223727,-0.00044906,0.000937$ $21,0.00089973,-0.00055135,-0.00110397,0.00028982,-0.00040823,0.0002521$ $4,-0.00036421,-0.00299692,0.00153461,-0.00260651,-0.01732109,0.0065093$ $3,0.03652562,-0.00005538,0.00024828,-0.00093313,0.00280957,0.00025579$, $0.00174601,-0.00314981,-0.00346212,0.00437244,-0.22858086,-0.03331529$, $0.14817377,-0.00629877,0.00137603,0.00891938,0.00114071,-0.00312349,0$. $00070593,0.00015406,-0.00001334,0.00002815,-0.00014762,0.00006979,0.00$ $060570,-0.00065814,0.00092126,-0.00163821,-0.00027861,0.00046230,-0.00$ $131684,-0.00052851,0.00006894,0.00032748,0.23565341,0.00026574,-0.0003$ $2354,0.00019371,0.00054395,-0.00296165,-0.00404173,0.01856875,0.006514$ $42,-0.01399550,-0.03364847,-0.05901121,0.01426116,-0.02049253,0.002167$ $13,0.01597413,-0.00292704,-0.00477377,-0.00120221,-0.00006417,0.000051$ $17,0.00001310,0.00080870,0.00068248,-0.00063439,0.00058914,0.00044417$, $0.00123068,0.00087689,0.00068286,0.00048372,-0.00108193,-0.00012641,0$. $00089096,0.03653541,0.05706058,-0.00082999,0.00018259,-0.00053183,0.00$ $187136,-0.00377001,0.00164591,0.01406750,0.00364795,-0.00589773,0.1479$ $9367,0.01408231,-0.14312389,-0.00061680,-0.00176036,0.00362083,0.00070$ $546,-0.00102883,0.00262656,0.00014347,-0.00001143,0.00002268,0.0008931$ $1,0.00013637,-0.00008514,-0.00174110,0.00097029,-0.00129894,-0.0011451$ $1,0.00077765,-0.00091022,-0.00015381,-0.00004930,-0.00005523,-0.160940$ $03,-0.01302937,0.14406168,-0.00093888,-0.00017972,0.00029640,-0.000152$ $18,-0.00016186,-0.00001104,0.00010742,0.00007405,-0.00012117,-0.000032$ $74,0.00007335,0.00002152,0.00004033,-0.00001052,-0.00020463,-0.0000545$ $8,0.00016931,0.00024901,-0.00005122,-0.00002865,0.00007856,-0.00019325$ $, 0.00001265,-0.00009265,0.00005083,0.00002940,-0.00004812,0.00005919,-$ $0.00002063,-0.00006711,-0.00005198,0.00007421,0.00002222,0.00003974,0$. $00001463,-0.00002679,0.50621694,-0.00017141,-0.00056148,0.00019557,-0$. $00020257,0.00019907,-0.00016159,-0.00001023,-0.00008131,0.00000038,0.0$ $0002769,0.00004536,-0.00011616,-0.00005215,-0.00007424,0.00001993,-0.0$ $0012134,0.00002996,-0.00009764,-0.00005242,0.00014928,-0.00005712,-0.0$ $0004117,0.00005871,0.00003069,0.00003832,-0.00001352,0.00004646,0.0000$ $2272,0.00003197,0.00002455,0.00007148,-0.00008384,0.00002083,-0.000003$ 04,0.00002104,0.00001697,-0.02544312,0.16926071,0.00103182,0.00013306, $-0.00107354,0.00038435,-0.00040990,0.00038764,0.00000677,0.00003864,0$. $00021317,-0.00010159,-0.00024171,0.00019106,0.00025226,0.00004286,-0.0$ $0000969,0.00012617,-0.00006546,0.00046047,0.00026459,-0.00007296,0.000$ $24901,-0.00003807,-0.00000197,-0.00017981,-0.00013603,0.00006218,-0.00$ $007525,-0.00011783,0.00005403,-0.00011463,0.00000770,0.00006583,-0.000$ $05500,0.00002952,0.00000905,-0.00000654,-0.04292172,-0.13661566,0.6090$ 
$6592,0.00383325,0.00047782,-0.00023054,0.00023642,-0.00025460,0.000504$ $14,-0.00009179,-0.00007732,0.00032787,-0.00001268,-0.00016480,0.000072$ 45,0.00012396,0.00005632,0.00033521,0.00002019,-0.00032142,0.00018788, $0.00037852,-0.00015925,0.00043515,0.00009162,0.00011862,-0.00002852,-0$ $.00016047,0.00001823,-0.00001159,-0.00016197,0.00003896,-0.00000165,-0$ $.00003339,0.00007362,-0.00013462,-0.00001773,-0.00000261,0.00000204,-0$ $.19572866,-0.00878693,0.07467429,0.66247079,0.00039530,0.00458452,0.00$ $281255,0.00031908,-0.00024945,-0.00004332,0.00008621,0.00022284,-0.000$ $18886,0.00005888,0.00000789,-0.00000845,-0.00000953,0.00009070,0.00015$ $331,-0.00053885,0.00003942,-0.00010344,0.00027762,0.00003166,0.0001781$ $5,0.00003348,-0.00002768,0.00021116,0.00001580,0.00000508,-0.00005549$, $0.00002803,-0.00007210,0.00005915,-0.00004518,0.00000742,-0.00011722,-$ $0.00001287,-0.00002179,0.00000599,-0.02695391,-0.07466471,0.04134586,-$ $0.05807714,0.18539155,-0.00075208,0.00296763,-0.00424150,0.00082676,0$. $00031577,0.00000850,-0.00015178,0.00006110,0.00008102,-0.00009080,-0.0$ $0012447,0.00005642,-0.00000832,0.00001844,0.00025138,0.00053921,-0.000$ $42202,-0.00025985,-0.00128099,0.00034785,-0.00050256,-0.00005495,-0.00$ $011923,-0.00000201,-0.00001237,0.00001526,-0.00005073,-0.00003092,0.00$ $000724,-0.00001625,-0.00008071,0.00007872,0.00000145,0.00000862,-0.000$ $00196,-0.00002946,0.13613236,0.03483857,-0.23048797,0.01541745,-0.1664$ $1732,0.69022286,-0.01419897,0.01021167,-0.02879081,0.00119929,0.002364$ $66,-0.00149044,-0.00023049,-0.00014890,-0.00008025,0.00004286,0.000102$ $29,-0.00014568,-0.00023078,0.00010181,0.00010007,0.00084159,-0.0009472$ $2,-0.00277579,-0.00567759,0.00141040,-0.00211938,-0.00027552,-0.000066$ $66,0.00004490,0.00008662,-0.00007031,0.00011527,0.00005810,-0.00004542$ $, 0.00010807,-0.00006237,0.00016574,0.00013714,-0.00003073,0.00000771,-$ $0.00000734,-0.05071378,-0.01122530,0.05866172,-0.32789628,0.04930907,-$ $0.07665570,0.70282354,0.00409462,-0.00013274,0.00405583,-0.00034622,0$. $00024381,-0.00001203,-0.00036417,0.00015254,-0.00005006,-0.00020039,-0$ $.00043741,0.00001152,0.00028405,-0.00005410,0.00047404,0.00024794,0.00$ $067135,0.00081208,0.00077417,0.00084329,0.00024072,0.00037635,-0.00014$ $938,-0.00039992,0.00004294,0.00002560,0.00004139,-0.00004061,0.0000873$ $6,0.00002324,0.00007723,0.00014193,0.00005879,0.00000809,0.00003571,-0$ $.00000742,0.00421382,0.01216430,-0.01893324,0.03037398,-0.07252127,0.0$ $2803556,-0.04313747,0.18195079,-0.00865403,0.00202637,-0.00226366,0.00$ $045089,0.00092512,0.00020126,0.00055587,0.00024504,-0.00033335,0.00006$ $814,0.00010326,-0.00036526,0.00063639,0.00011692,-0.00052990,0.0001932$ $8,-0.00092660,-0.00000318,-0.00007876,0.00002441,0.00078116,-0.0003350$ $2,0.00002100,-0.00000932,0.00001556,0.00002951,-0.00007227,-0.00001031$ $, 0.00002331,-0.00006872,-0.00005421,0.00032227,0.00021906,0.00004653,0$ $.00000933,0.00004414,0.00515686,-0.01323755,0.05076756,-0.01261753,0.0$ $2136644,-0.12983671,-0.04878444,-0.14547065,0.64583207,-0.15001772,0.0$ $1780812,-0.02740930,-0.02700829,-0.01294798,-0.00246324,0.00097416,-0$. $00071148,-0.00299920,-0.00094013,-0.00006360,0.00055262,0.00150989,0.0$ $0184754,-0.00265969,-0.02230214,0.01483934,0.00903147,-0.00921176,0.00$ $179881,-0.00234959,0.00066560,0.00156983,0.00122535,0.00051830,-0.0001$ $4745,0.00043728,0.00065618,-0.00018089,0.00032217,0.00152966,-0.001612$ $21,-0.00028901,0.00011963,-0.00001719,0.00001622,-0.00276022,-0.005216$ $24,0.01609452,-0.00072515,0.01065180,-0.03316210,-0.13301349,0.0284800$ $9,-0.07365910,0.59709593,0.01344414,-0.06943690,0.00678725,-0.02500877$ $, 0.00658471,-0.00297029,0.00006364,-0.00414956,-0.00172105,-0.00029923$ 
,0.00060999,0.00057076,0.00027510,-0.00224668,-0.00106378,0.02241752,-$0.00371668,0.00299803,0.01154551,-0.00205036,0.00419551,-0.00106356,-0$ $.00085450,-0.00071714,0.00000918,-0.00032358,0.00123433,-0.00033300,0$. $00046959,-0.00100970,0.00005342,0.00097068,0.00003570,-0.00003051,0.00$ $012332,-0.00005110,-0.00545847,-0.01116516,0.01595553,0.02421909,0.004$ $32286,0.00236789,0.01048318,-0.08826581,0.07106751,-0.03413835,0.21342$ $258,-0.01301387,0.00541955,-0.07992752,0.00287639,-0.00117691,0.001170$ $23,-0.00002082,0.00125123,-0.00033355,0.00027935,0.00044700,0.00041635$ -0.00086192,-0.00342081,-0.00189975,0.02023280,-0.00168487,0.00408069 $,-0.03081019,0.00765039,-0.01155849,0.00014304,-0.00139233,-0.00042876$ $, 0.00017827,0.00009754,-0.00022794,-0.00002766,0.00036917,-0.00118927$, $0.00001933,-0.00005359,0.00023706,0.00014862,-0.00003744,0.00015619,0$. $01723823,0.01554861,-0.06625755,-0.07941991,0.00741128,0.01033277,-0.0$ $1415925,0.06503103,-0.28321615,-0.03335785,-0.12925380,0.62603070,-0.0$ $2748595,-0.00159425,0.01782695,-0.00100844,0.00134333,0.00093252,-0.00$ $020174,-0.00065375,0.00024152,-0.00011330,-0.00044201,-0.00000818,0.00$ $048744,0.00115995,0.00040614,-0.00227491,-0.00208725,-0.00020690,0.000$ $92534,-0.00129457,0.00342334,-0.00055236,0.00009249,0.00009922,-0.0002$ $9096,0.00003556,0.00010027,-0.00028095,-0.00002992,0.00017077,0.000049$ $54,0.00053297,0.00006121,-0.00004818,0.00003551,-0.00005538,-0.0005996$ $9,0.02607781,-0.08658989,-0.06020421,0.00030318,0.01871686,0.05262800$, $-0.01869586,0.04756384,-0.21685871,-0.00448152,0.06863319,0.66635921,0$ $.00493581,0.00420412,-0.00223425,0.00020043,-0.00043188,-0.00035598,0$. $00004511,0.00022767,0.00007633,-0.00008037,-0.00005975,-0.00007341,0.0$ $0023269,0.00004494,-0.00038297,0.00093240,-0.00034315,0.00038983,0.000$ $45388,0.00018359,-0.00083396,-0.00009418,-0.00043689,-0.00044134,0.000$ $15405,-0.00002573,0.00004765,-0.00006447,0.00005718,-0.00017585,-0.000$ $10876,0.00006608,0.00017151,0.00002720,0.00001413,0.00003204,0.0100870$ $2,0.00345023,0.00840278,0.00059396,-0.00338469,-0.00038658,-0.00566767$ $, 0.00520811,0.01096514,-0.02378542,-0.07367144,0.03815581,-0.05510362$, $0.18528077,-0.00349527,-0.00014649,0.00680457,-0.00180910,-0.00001831$, $-0.00088317,-0.00032135,-0.00023585,0.00026140,-0.00004437,-0.00016033$ $,-0.00006159,-0.00004834,0.00023235,0.00046277,-0.00184827,0.00027340$, $-0.00080044,-0.00235331,-0.00002596,0.00110598,-0.00007567,0.00034274$, $0.00039664,0.00010259,-0.00010388,0.00024478,0.00001338,-0.00000824,0$. $00022674,0.00001870,-0.00008379,0.00003155,-0.00005486,0.00001851,-0.0$ $0004236,-0.03298800,0.00296511,0.00853486,0.01869862,-0.00067703,-0.00$ $803622,0.00426992,0.01572957,-0.04684445,0.13355170,0.03187995,-0.2216$ $6285,0.00672831,-0.15644682,0.67986541,0.00017340,-0.00012725,0.002775$ $32,-0.00059570,0.00057179,-0.00036477,-0.00003986,-0.00012912,-0.00015$ $688,0.00015193,0.00016145,-0.00016632,-0.00020110,0.00013194,0.0001476$ $1,-0.00070437,-0.00012906,-0.00063447,-0.00010779,0.00010564,-0.000322$ $17,-0.00006006,0.00019572,0.00036965,0.00009558,-0.00005254,0.00010796$ $, 0.00008101,-0.00006426,0.00013980,-0.00006449,-0.00003286,0.00009308$, $-0.00001889,0.00000343,-0.00000097,-0.11736125,0.00799944,-0.00883717$, $0.05242741,-0.00551804,0.00315190,-0.03286345,0.01225792,-0.03034915,-$ $0.04658030,-0.01049425,0.05335161,-0.32311927,0.04662279,-0.07040472,0$ $.69910215,-0.00017600,0.00651258,0.00246029,0.00160952,-0.00050873,-0$. $00024432,0.00003453,0.00046872,0.00027352,0.00001572,-0.00013040,0.000$ $05489,0.00007005,0.00046294,0.00010763,-0.00143566,-0.00037303,-0.0000$ $1888,0.00005059,-0.00004838,-0.00000739,0.00008665,-0.00011065,-0.0000$ 
$7973,-0.00004250,0.00003817,-0.00017293,-0.00003892,-0.00007841,0.0001$ $1790,-0.00005199,0.00002276,-0.00000394,-0.00000566,-0.00003216,-0.000$ $02254,0.02590141,-0.08792059,0.06928258,-0.01792126,0.00640450,0.01509$ $501,0.01171467,-0.00763858,0.01123017,0.00337496,0.01205786,-0.0176931$ $8,0.02810421,-0.07284790,0.02587741,-0.04044132,0.17749273,0.00274227$, $0.00226727,-0.00171825,0.00025609,-0.00002003,-0.00014226,0.00002826,0$ $.00003302,-0.00017632,0.00007462,0.00024182,-0.00010860,-0.00032244,0$. $00000727,0.00003748,-0.00007820,0.00004025,-0.00002110,-0.00001618,-0$. $00001055,0.00000708,0.00021945,-0.00013828,-0.00008016,0.00008877,-0.0$ $0004560,0.00002203,0.00007407,-0.00004813,0.00009434,0.00001917,-0.000$ $11564,-0.00003982,-0.00002233,-0.00001514,0.00003397,-0.07010322,0.076$ $05999,-0.30184948,0.04553360,0.01075088,-0.04389600,-0.02981479,0.0111$ $8664,-0.03021836,0.00775509,-0.01303586,0.04894051,-0.00616636,0.01907$ $741,-0.12787682,-0.06111518,-0.14727885,0.66251362,0.00036864,-0.00008$ $949,0.00030459,-0.00021193,0.00055608,-0.00012389,-0.00010772,-0.00025$ $527,-0.00001276,0.00005154,0.00004230,-0.00012464,-0.00009771,0.000164$ $72,0.00018813,-0.00030635,-0.00041731,-0.00053439,0.00000468,0.0000259$ $7,-0.00001707,0.00003275,0.00013853,0.00018796,-0.00001668,-0.00005746$ $, 0.00011805,-0.00002426,-0.00001023,0.00013434,0.00006938,-0.00004619$, $-0.00001654,-0.00003720,-0.00001549,0.00002658,-0.14528657,0.01863235$, $-0.02248277,-0.04126987,0.00741507,-0.00960556,0.00168278,-0.00156790$, $0.00622914,-0.00159524,0.00052160,-0.00105369,0.00348794,0.00096269,-0$ $.00294933,-0.02884268,0.00549040,-0.00710544,0.21259531,-0.00010755,-0$ $.00065093,-0.00031753,-0.00002625,-0.00006448,0.00006386,0.00001645,0$. $00001047,0.00002565,-0.00001281,0.00000867,0.00002649,0.00001224,-0.00$ $004112,-0.00009885,0.00013189,0.00011143,0.00008459,-0.00002405,0.0000$ $0609,-0.00000914,0.00000324,-0.00001841,-0.00002759,0.00000322,0.00000$ $468,-0.00000874,0.00000972,-0.00000022,-0.00003265,-0.00001223,0.00001$ $289,0.00001127,0.00000694,-0.00000110,-0.00000481,0.01886273,-0.028479$ $37,0.00791451,-0.00019940,0.00459779,0.00032586,0.00030228,0.00415205$, $0.00140563,0.00035051,-0.00055703,-0.00057651,-0.00085133,0.00420501,0$ $.00276346,0.01284375,0.00245650,0.00314622,-0.03107160,0.01887164,-0.0$ $0005832,-0.00023726,0.00019797,-0.00010899,0.00015824,-0.00000996,-0.0$ $0003480,-0.00009968,0.00001018,0.00000774,0.00001838,-0.00003399,-0.00$ $003628,0.00005265,0.00005671,-0.00006899,-0.00010489,-0.00011426,0.000$ $08697,-0.00002439,0.00005751,0.00002490,0.00001452,0.00002244,-0.00000$ $580,-0.00001589,0.00002751,-0.00000889,0.00000048,0.00003313,0.0000154$ $7,-0.00000587,-0.00000841,-0.00001300,-0.00000399,0.00000425,-0.022510$ $26,0.00787541,-0.04350598,0.01604622,-0.00220948,0.00682523,-0.0000540$ $2,0.00202890,-0.00226270,-0.00084204,-0.00055649,0.00161462,0.00332511$ $, 0.00209986,-0.00358727,-0.03271092,0.00574422,-0.00377952,0.03777338$, $-0.01277999,0.04278007,-0.00004299,0.00038628,-0.00116923,0.00013228,0$ $.00014581,-0.00003218,-0.00004059,-0.00003284,0.00004513,0.00000819,-0$ $.00003403,0.00000674,-0.00001808,0.00002866,0.00005630,0.00014507,-0.0$ $0015636,-0.00011247,-0.00039087,0.00008877,-0.00016653,-0.00002611,0.0$ $0002083,0.00003475,-0.00002402,-0.00000478,-0.00000243,-0.00002244,0.0$ $0001131,0.00000412,-0.00000868,0.00002508,-0.00000289,-0.00001061,0.00$ $000060,-0.00000163,0.00745452,-0.00643882,0.02025818,-0.07669174,0.025$ $72379,-0.07323527,-0.00331871,0.00870692,-0.02719059,-0.00489159,0.001$ $24444,0.00001789,-0.00109386,-0.00010039,0.00026287,-0.00279195,-0.000$ $15401,0.00344831,0.00106023,-0.00051511,0.00047424,0.07910001,-0.00007$ 
$985,0.00017925,0.00023002,-0.00008437,-0.00009947,0.00003868,0.0000049$ $1,0.00001226,-0.00004827,0.00000683,0.00000784,0.00001785,-0.00001027$, $-0.00004741,0.00002882,0.00010220,0.00000820,-0.00008376,0.00003233,-0$ $.00012160,-0.00000410,-0.00004804,0.00003399,-0.00002690,-0.00000870,-$ $0.00000203,-0.00000599,0.00000917,-0.00000287,0.00000009,0.00000258,-0$ $.00002403,0.00001282,-0.00000002,0.00000348,-0.00000454,0.00239754,0.0$ $0216741,0.00344788,0.02485961,-0.06296680,0.08401745,0.00057360,0.0025$ $9717,0.00419531,0.00139392,0.00666723,0.00138188,-0.00015940,-0.000890$ $35,-0.00045661,-0.00035661,0.00492824,0.00146173,-0.00019747,-0.002311$ $68,-0.00105614,-0.02799623,0.05420338,0.00027562,0.00009147,-0.0001306$ $9,-0.00004678,0.00000420,-0.00000699,0.00001271,-0.00003256,-0.0000041$ $8,-0.00001962,0.00001637,-0.00003693,0.00002762,0.00000682,0.00004514$, $0.00000811,-0.00003979,-0.00005315,-0.00009656,-0.00001641,-0.00002219$ ,-0.00001760,0.00002942,0.00001655,-0.00000483,0.00000359,0.00000302,-$0.00000294,-0.00000501,0.00000422,0.00000449,-0.00000178,-0.00000554,0$ $.00000015,-0.00000392,0.00000899,-0.00961027,0.00652105,-0.01549677,-0$ $.07059612,0.08361554,-0.29521658,0.00078901,0.00139236,-0.00119569,-0$. $00051058,0.00150050,0.00244844,0.00049739,-0.00051335,0.00044561,0.003$ $93871,0.00144378,-0.00082345,-0.00053637,-0.00094975,0.00086972,0.0763$ $8289,-0.09178577,0.30952020,0.00007579,-0.00020019,-0.00037409,-0.0000$ $2936,0.00013815,-0.00009332,-0.00010820,-0.00013107,-0.00004048,-0.000$ $06062,0.00004904,0.00005172,-0.00027126,0.00013907,-0.00003686,-0.0000$ $1489,0.00010657,-0.00031311,0.00014189,-0.00005985,0.00016676,0.000051$ $92,-0.00012156,-0.00005826,-0.00001253,0.00000642,0.00000026,0.0000016$ $3,-0.00001796,-0.00000957,0.00000446,-0.00006989,0.00004134,0.00000990$ $,-0.00000737,0.00000496,-0.00323434,0.00167161,-0.00199715,-0.01604660$ ,-0.00455805,0.02212923,-0.18808958,-0.02521698,0.13782125,0.00934577, $0.00140552,-0.00668047,0.00055983,0.00121538,-0.00210314,-0.00033771,0$ $.00013705,-0.00079828,-0.00146705,-0.00000029,0.00053177,0.00089408,-0$ $.00051402,-0.00008042,0.19822565,-0.00051456,-0.00267956,-0.00104512,-$ $0.00016620,0.00012471,0.00003963,0.00011287,-0.00020982,0.00017068,0.0$ $0012476,0.00037797,0.00001740,-0.00020581,-0.00012174,-0.00037241,0.00$ $032929,-0.00003231,0.00021807,-0.00005326,-0.00010099,-0.00007510,-0.0$ $0012917,0.00011395,0.00000158,-0.00008142,-0.00001732,-0.00003121,0.00$ $006890,-0.00003005,-0.00002557,0.00006555,-0.00013338,0.00013580,-0.00$ $001072,-0.00001202,0.00000770,0.00156114,0.00662659,0.00180309,0.00402$ $413,0.00453873,-0.00338371,-0.02511203,-0.04381407,0.03404473,-0.00694$ $150,0.00234862,0.00584727,0.00144075,0.00530412,0.00321380,0.00022663$, $-0.00118828,-0.00021102,0.00030317,0.00003940,-0.00007246,-0.00056007$, $-0.00389472,-0.00140409,0.02589679,0.03341715,0.00091015,-0.00111935,0$ $.00026254,-0.00019988,-0.00002040,0.00001691,-0.00017566,-0.00021117,0$ $.00004943,-0.00013873,0.00003273,0.00013531,-0.00020570,0.00008245,0.0$ $0014542,0.00017964,0.00012162,0.00015563,0.00016706,-0.00007567,0.0000$ $8319,0.00012785,-0.00015991,-0.00018592,-0.00001060,-0.00000963,0.0000$ $4660,0.00003393,-0.00005526,0.00004938,-0.00000558,-0.00004749,-0.0000$ $2197,0.00002181,0.00000163,-0.00001536,-0.00160742,0.00182662,0.001714$ $66,-0.00698145,-0.00045290,0.00784944,0.13737031,0.03411933,-0.1991858$ $5,0.02154574,0.00316757,-0.01499367,-0.00287399,0.00333986,-0.00379642$ $,-0.00089791,-0.00025625,-0.00017576,-0.00054508,0.00003009,0.00010860$ $, 0.00005256,-0.00139673,0.00040679,-0.14779657,-0.03899291,0.20809873$, $0.00123301,-0.00107741,0.00152439,-0.00013953,0.00010452,0.00006000,0$. 
$00003171,0.00002523,-0.00002764,0.00001138,0.00006385,-0.00001455,-0.0$ $0004265,-0.00010899,-0.00001081,0.00009937,0.00005565,-0.00011065,-0.0$ $0027796,0.00022336,-0.00041424,0.00000465,-0.00006125,-0.00000673,0.00$ $000628,-0.00001276,0.00001219,0.00001804,-0.00000812,-0.00000007,-0.00$ $000065,-0.00002569,-0.00001051,0.00000888,-0.00000055,0.00000088,-0.00$ $397552,0.00113814,-0.00014122,-0.00105347,-0.00009831,0.00035333,-0.00$ $253917,-0.00017891,0.00354139,0.00784020,-0.00586059,0.01906188,-0.075$ $90403,0.02311700,-0.06680740,-0.00437984,0.00886767,-0.02840577,-0.001$ $20112,0.00046005,-0.00111935,0.00005762,-0.00004894,-0.00006768,0.0004$ $0948,-0.00035408,0.00104729,0.07882507,-0.00006912,-0.00360204,-0.0015$ $8880,-0.00068534,0.00015083,0.00033921,-0.00007533,-0.00019600,-0.0001$ $1313,0.00001966,-0.00000305,-0.00000086,0.00002375,-0.00020554,-0.0000$ $7885,0.00064164,0.00032612,-0.00021673,-0.00014586,0.00033775,-0.00005$ $619,-0.00014850,0.00022386,0.00027327,-0.00001106,-0.00002956,0.000072$ $93,0.00001896,0.00001142,-0.00005939,0.00005713,-0.00005425,-0.0000255$ 7,0.,-0.00000701,-0.00000263,0.00128784,0.00650864,0.00137765,-0.00011 $670,-0.00095367,-0.00051092,-0.00046018,0.00570112,0.00175273,0.002169$ 46,0.00244671,0.00417462,0.02268975,-0.06078656,0.07878339,0.00060747, $0.00232632,0.00467430,0.00016319,0.00002106,0.00019803,-0.00004573,-0$. $00063274,-0.00016500,0.00010337,0.00011352,0.00019880,-0.02577086,0.05$ $209077,-0.00191580,-0.00089689,0.00019841,-0.00009209,0.00011857,0.000$ $33915,-0.00002885,-0.00012919,-0.00002599,-0.00001954,0.00001268,0.000$ $01301,-0.00002065,0.00000352,0.00003308,0.00025559,-0.00014544,0.00014$ $565,0.00089926,-0.00030484,0.00070370,0.00000233,-0.00010004,-0.000131$ $50,-0.00005285,-0.00000490,-0.00000257,-0.00005265,0.00002271,-0.00003$ $537,0.00004049,-0.00000344,-0.00006035,-0.00000078,-0.00000606,0.00001$ $225,-0.00042568,0.00126232,0.00239112,0.00047659,-0.00047682,0.0004519$ $2,0.00438316,0.00164337,-0.00125201,-0.00872779,0.00654978,-0.01587440$ ,-0.06612079,0.07934982,-0.29267996,0.00081678,0.00155545,-0.00202804, $-0.00002913,0.00007204,-0.00012889,-0.00009521,-0.00015673,-0.00010068$ $,-0.00049406,0.00036051,-0.00083202,0.07148865,-0.08750287,0.30843394$, $-0.00041614,-0.00017061,0.00088351,-0.00013635,0.00011326,-0.00002200$, $-0.00002739,-0.00005304,0.00000573,-0.00001663,-0.00002903,-0.00001610$ $, 0.00001625,0.00008779,0.00004198,-0.00020527,-0.00010613,-0.00011966$, $0.00006265,-0.00001975,0.00002262,-0.00003881,0.00007610,0.00008411,-0$ $.00001402,-0.00000834,0.00002193,-0.00000510,-0.00000662,0.00003231,-0$ $.00000415,0.00002415,0.00000644,-0.00000658,-0.00000119,-0.00000080,0$. $00693394,0.00203466,-0.00843325,0.00058210,0.00119535,-0.00217292,-0.0$ $0038978,0.00012413,-0.00081523,-0.00379206,0.00176866,-0.00230515,-0.0$ $1465596,-0.00434861,0.02173795,-0.19417817,-0.02506091,0.14380872,0.00$ $115011,-0.00017704,-0.00068612,0.00050304,0.00009414,-0.00036261,-0.00$ $000156,-0.00009738,0.00007930,0.00098909,-0.00046253,-0.00024480,0.203$ $60068,0.00014301,0.00014984,-0.00015335,0.00005965,-0.00005571,0.00009$ $545,0.00000167,0.00000701,-0.00002530,0.00001742,0.00003179,0.00000097$ ,-0.00004357,-0.00003797,0.00002683,0.00002280,0.00006060,-0.00005959, $-0.00000475,-0.00002321,-0.00000208,-0.00002605,0.00004941,0.00004108$, $-0.00001738,0.00000980,-0.00001558,0.00002043,-0.00000432,0.00000600,0$ $.00000764,-0.00000998,-0.00001160,0.00000181,-0.00000203,-0.00000086,-$ $0.00663735,0.00238499,0.00637368,0.00135184,0.00455703,0.00286907,0.00$ $017817,-0.00114021,-0.00026370,0.00162016,0.00621293,0.00181242,0.0038$ $1813,0.00441923,-0.00349229,-0.02425700,-0.04228230,0.03193254,-0.0004$ 
$7382,-0.00241542,-0.00098169,-0.00030646,0.00022018,0.00032064,-0.0000$ $8852,-0.00065173,-0.00020066,-0.00051479,-0.00375041,-0.00122286,0.025$ $07420,0.03229140,-0.00045513,0.00001760,0.00022635,-0.00000821,0.00003$ $799,-0.00000239,-0.00000193,-0.00001576,0.00002571,0.00000371,0.000010$ $32,-0.00000756,-0.00000939,-0.00001127,0.00003975,-0.00003836,-0.00000$ $418,-0.00006752,0.00000642,-0.00000747,-0.00000180,-0.00002701,0.00001$ $934,0.00002096,-0.00000830,-0.00000182,-0.00000080,0.00000563,-0.00000$ $191,0.00000944,-0.00000159,0.00000354,-0.00000275,-0.00000317,0.000001$ $17,-0.00000334,0.02147177,0.00308769,-0.01493697,-0.00270109,0.0029329$ $3,-0.00354751,-0.00100435,-0.00019734,-0.00022032,-0.00192461,0.001711$ $66,0.00162127,-0.00654985,-0.00065467,0.00828786,0.14130454,0.03250819$ ,-0.19879057,0.00030921,-0.00108299,0.00082938,0.00098771,0.00018089,-$0.00067547,0.00005953,-0.00019978,-0.00004082,0.00003153,-0.00130276,0$ $.00034290,-0.15146922,-0.03702765,0.20691602,-0.00293030,-0.00090525,0$ $.00277642,0.01019316,0.01375478,0.01077613,0.00560409,-0.00368416,-0.0$ 0489554,-0.00069016,0.00353711,0.00116534,-0.00104771,-0.00128261,0.00 $035210,0.00122723,0.00079076,-0.00084041,0.00010842,0.00014097,-0.0006$ $8586,-0.11514734,0.21833084,0.18514989,0.00019425,-0.00027675,0.000123$ $44,0.00003432,-0.00001511,0.00009420,-0.00013340,0.00014916,0.00018010$ ,-0.00006827,0.00000810,-0.00020550,0.00023194,0.00005052,0.00000480,$0.00007027,-0.00000079,0.00001874,0.00016790,-0.00031375,0.00026922,-0$ $.00055853,-0.00028277,-0.00046981,0.00019520,-0.00022694,-0.00008091,0$ $.00021541,-0.00012919,-0.00010023,-0.00003901,-0.00000537,-0.00002805$, $0.00001720,0.00002708,0.00001567,-0.00004668,0.00007221,-0.00009708,-0$ $.00002085,0.00020896,-0.00004218,0.00005010,0.00005275,0.00002214,0.10$ $251329,0.00200408,0.00412203,-0.00027801,0.02225648,-0.01175234,-0.033$ $06835,-0.01599220,0.00147963,0.01296185,0.00337891,-0.00878336,-0.0061$ $9853,0.00358411,0.00396973,-0.00113147,-0.00158696,0.00011728,0.001470$ $79,-0.00050754,0.00011663,0.00134632,0.21717720,-0.62680504,-0.4994680$ $3,0.00037507,0.00027080,-0.00027505,0.00003411,-0.00001222,-0.00009943$ ,-0.00005589,0.00005432,-0.00000169,-0.00000477,-0.00052781,-0.0001166 $6,0.00013512,-0.00005402,0.00003167,-0.00012476,0.00006016,0.00000479$, $-0.00004410,0.00017845,0.00002430,-0.00014224,0.00020062,0.00069163,-0$ $.00024016,0.00021316,-0.00022410,-0.00012567,-0.00001191,0.00003978,-0$ $.00021387,0.00004209,-0.00004250,-0.00003765,-0.00003491,-0.00002828,0$ $.00011679,-0.00011878,0.00007762,0.00006578,-0.00003730,-0.00000068,-0$ $.00005127,-0.00002093,-0.00000374,-0.23000057,0.63733370,0.00442559,0$. $00223273,-0.00131224,0.01452314,-0.02443971,-0.01143210,-0.01136850,0$. $00280780,0.01263353,0.00127531,-0.00680441,-0.00559017,0.00274667,0.00$ $302352,-0.00073223,-0.00194219,-0.00059170,0.00146926,-0.00057317,-0.0$ $0044320,0.00088163,0.18460114,-0.50050147,-0.45791617,0.00054666,0.000$ $13089,0.00036498,0.00012127,-0.00005543,0.00001941,0.00009354,-0.00008$ $646,-0.00020201,-0.00021178,-0.00011545,-0.00023740,0.00007115,-0.0000$ $5500,0.00007371,0.00001151,-0.00009292,0.00004237,-0.00009732,0.000186$ $28,0.00003265,-0.00017806,0.00035723,0.00052107,-0.00015531,0.00039114$ ,-0.00039659,-0.00023634,0.00002696,0.00007546,-0.00013472,0.00001889, $-0.00000633,-0.00002325,0.00001213,-0.00000038,0.00006897,-0.00004687$, $0.00012516,0.00001507,-0.00022125,0.00008664,-0.00007675,-0.00002147,-$ $0.00002183,-0.19350263,0.52428779,0.46152156 \backslash 10.00001078,0.00000077,-0$ $.00000786,-0.00000278,-0.00000584,0.00000149,-0.00000063,0.00000360,0$. $00000089,-0.00000217,-0.00000149,-0.00000298,0.00000076,0.00000382,-0$. 
$00000358,-0.00000423,0.00000073,-0.00000164,-0.00000083,-0.00000168,-0$ $.00000095,0.00000029,0.00000293,-0.00000170,-0.00000047,0.00000012,-0$. $00000056,-0.00000099,0.00000217,-0.00000409,0.00000131,0.00000055,-0.0$ $0000320,-0.00000146,0.00000252,-0.00000277,-0.00000226,-0.00000115,0.0$ $0000834,0.00000454,0.00000077,0.00000040,-0.00000173,0.00000248,-0.000$ $00346,-0.00001031,-0.00000377,0.00001604,0.00000999,-0.00000105,-0.000$ $00507,-0.00000219,0.00000005,-0.00000316,0.00000054,-0.00000046,0.0000$ $0449,-0.00000139,0.00000217,0.00000284,-0.00000090,0.00000192,0.000000$ $88,0.00000072,-0.00000216,0.00000061,0.00000144,-0.00000228,0.00000236$ $, 0.00000199,-0.00000474,0.0000026811 \backslash @$

meta-Addition Product

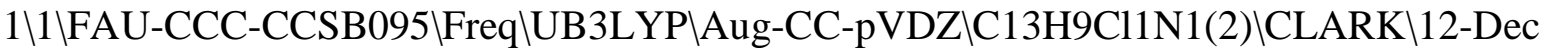
-2014\0\\#N Geom=AllCheck Guess=TCheck SCRF=Check GenChk UB3LYP/Aug-CC -pVDZ Freq \\Addition product p-Cl-Ph-radical + cyanobenzene (meta) $\backslash 0$, 2lC $, 1.3911966676,-0.960685311,-0.0003559111 \backslash \mathrm{C}, 1.9798843077,-0.35882629$ 7,1.2549346221\C,2.9422297639,0.6113695039,1.23532627\C,3.438722076,1. $105181464,0.0004954723 \backslash \mathrm{C}, 2.942200249,0.6124152301,-1.234741674 \backslash \mathrm{C}, 1.979$ $8526781,-0.3577616776,-1.2551482579 \backslash \mathrm{H}, 1.6242549551,-2.0452575152,-0.00$ $08196161 \backslash \mathrm{H}, 1.6040930273,-0.7291666309,2.2091411474 \backslash \mathrm{H}, 3.3370689302,1.01$ $54437316,2.1669288649 \backslash \mathrm{H}, 3.33701963,1.0172762992,-2.1660110622 \backslash \mathrm{H}, 1.6040$ $410119,-0.7272956379,-2.2096593808 \mathrm{lCl}, 4.6749643502,2.3425241627,0.0010$ $045673 \backslash \mathrm{C},-2.9616578462,-0.7274589199,-0.0001083061 \backslash \mathrm{C},-2.3249240386,-1$. 9663925796,-0.0005190154\C,-0.9272109775,-2.0410623359,-0.0006076178\C ,-0.1431354265,-0.8804805038,-0.0002843166\C,-0.7809893532,0.365536778 $7,0.0001266629 \backslash \mathrm{C},-2.182801182,0.4447166229,0.0002111483 \backslash \mathrm{H},-4.048038038$ $9,-0.6566014045,-0.0000367761 \backslash \mathrm{H},-2.9190758767,-2.8800730796,-0.0007737$ $275 \backslash \mathrm{H},-0.4412166354,-3.0179488356,-0.0009340342 \backslash \mathrm{H},-0.191431033,1.28128$ $77328,0.0003813228 \backslash \mathrm{C},-2.8259396974,1.7307553737,0.0006329713 \backslash \mathrm{N},-3.3491$ 975774,2.7695615349,0.0009845756।IVersion=EM64L-G09RevC.01 \State $=2-A \backslash H$ $\mathrm{F}=-1015.7696979 \backslash \mathrm{S} 2=0.784459 \backslash \mathrm{S} 2-1=0 . \backslash \mathrm{S} 2 \mathrm{~A}=0.750699 \backslash \mathrm{RMSD}=0.000 \mathrm{e}+00 \backslash \mathrm{RMSF}=3$ $.366 \mathrm{e}-06 \mathrm{ZZeroPoint}=0.1784354 \backslash \mathrm{Thermal}=0.1910505 \mathrm{ZPE}=111.9699122 \backslash \mathrm{Dipole}=$ $0.4463017,-2.0853089,-0.0007255 \backslash$ DipoleDeriv $=0.1526533,-0.1075152,-0.00$ $00483,-0.2054714,0.1239392,0.0000718,-0.0001256,0.0000636,-0.0235725,-$ $0.1533359,0.1199289,0.0329644,0.2282674,-0.0268671,0.0395992,0.164817$, $0.0243772,0.0210029,-0.19171,-0.0639123,0.0032384,-0.1002475,-0.143955$ $8,0.0175288,-0.0916616,-0.0118646,0.1057912,0.6687472,0.5902638,0.0002$ $398,0.716131,0.5680316,0.0002488,0.0003029,0.0002534,-0.0436687,-0.191$ $7089,-0.0639072,-0.0032834,-0.1003327,-0.1439525,-0.0177345,0.0915664$, $0.0116518,0.1057873,-0.1533219,0.1199556,-0.0328583,0.2284228,-0.02681$ $06,-0.0396474,-0.1645992,-0.0244153,0.0209558,0.0130289,-0.0417869,-0$. $0000162,-0.0233613,-0.1510238,-0.0000953,-0.0000056,-0.0000896,0.07252$ $91,0.0806672,-0.0609111,0.0418038,-0.0634442,0.0539199,0.0262014,0.034$ $1602,0.0321491,-0.0431597,0.087001,-0.0370622,-0.0422795,-0.0769001,0$. $0824611,-0.0441605,-0.0382004,-0.0342682,-0.0004011,0.087002,-0.037096$ $5,0.0422457,-0.0769269,0.0823956,0.0442323,0.038134,0.0343392,-0.00033$ $61,0.0806669,-0.0608733,-0.0418568,-0.0634197,0.0539694,-0.0261165,-0$. $0342159,-0.0320681,-0.0432089,-0.5243233,-0.37378,-0.000153,-0.468397$, $-0.4297551,-0.0001332,-0.0001932,-0.0001349,-0.1022951,-0.0421834,0.03$ $39965,0.0000025,-0.1295835,-0.036815,0.0000184,-0.000043,0.0000156,-0$. 
$0900048,-0.1017318,0.0847381,0.0000215,0.1456451,0.0856334,0.0000586,0$ $.0000454,0.0000607,-0.1219025,-0.0755154,-0.0375747,-0.0000217,0.03548$ $82,0.0843654,0.0000591,0.0000093,0.0000582,-0.0934275,0.4120802,0.0798$ $344,0.0000289,-0.0749558,-0.1556556,-0.0000358,-0.0000128,-0.00004,-0$. $045975,-0.1891251,-0.0900886,-0.0000307,0.099298,-0.024301,0.000008,0$. $0000351,0.0000133,-0.0526068,0.159944,0.0460273,0.0000124,0.0733232,0$. $0628291,0.0000022,0.0000162,0.0000082,0.0420366,-0.0347158,0.0057114,0$ $.000009,0.0161296,0.078504,-0.000018,0.0000107,-0.0000172,0.1342617,0$. $0028536,-0.069986,-0.0000175,-0.064248,-0.0383301,-0.0000489,-0.000013$ $1,-0.0000489,0.1281743,0.0484486,0.0966875,0.0000365,0.0660445,-0.0310$ $435,-0.0000491,0.0000238,-0.0000519,0.1181815,0.0878375,-0.0443087,-0$. $0000107,-0.0707477,0.0116468,-0.0000289,-0.0000281,-0.000027,0.1043253$ $, 0.1346335,-0.1568233,-0.000046,-0.2296193,0.3298076,0.0000682,-0.0000$ $659,0.000066,0.1177146,-0.3578927,0.0684825,0.0000193,0.1389055,-0.408$ $9929,-0.0000287,0.0000434,-0.0000305,-0.3102018 \backslash$ Polar $=221.6826704,24.8$ $425208,189.4409964,0.0095607,0.019964,125.8899313 \backslash \mathrm{PG}=\mathrm{C} 01$ [X(C13H9Cl1N1 )]WImag=0\\0.41621245,0.01011539,0.52328026,0.00000108,0.00001935,0.4 $6661494,-0.08564974,-0.02316054,-0.03449167,0.42115128,-0.02421118,-0$. $09217584,-0.03832040,0.29482444,0.42368935,-0.02710972,-0.03149234,-0$. $16987808,-0.05137999,-0.04703737,0.58544832,-0.00638448,-0.01441413,-0$ $.00822595,-0.22001706,-0.17174082,0.00512920,0.39538859,-0.01406670,-0$ $.00812064,-0.00796734,-0.17239507,-0.22429108,0.00520799,0.28259527,0$. 40156042,-0.01820384,-0.01591501,0.00657307,-0.03071239,-0.03028392,-0 $.11019592,0.06079693,0.06356308,0.65482759,-0.00303690,0.00706997,0.00$ $000292,-0.01637882,-0.02583032,0.02220861,-0.08375632,-0.03332566,0.06$ $251654,0.27835877,0.00664400,-0.00233094,0.00000251,-0.02578367,-0.016$ $25721,0.02269761,-0.03350442,-0.08380347,0.06178686,0.19336585,0.27802$ $374,0.00000262,0.00000275,-0.00845031,0.06025719,0.05986003,-0.0008071$ $8,-0.00189437,-0.00279309,-0.22612713,0.00008449,-0.00010812,0.5267117$ $6,-0.00638391,-0.01442086,0.00821407,-0.01452220,-0.01373101,-0.000776$ $92,0.03072937,0.02576275,-0.01360193,-0.08375839,-0.03350783,0.0018628$ $4,0.39538817,-0.01408206,-0.00814139,0.00795548,-0.01400461,-0.0146774$ $0,-0.00051439,0.02575103,0.03150760,-0.01414247,-0.03327278,-0.0837537$ $6,0.00291439,0.28264605,0.40166779,0.01819196,0.01590241,0.00659304,-0$ $.01463287,-0.01423124,0.00162543,0.01362135,0.01421421,-0.05415739,-0$. $06254776,-0.06166533,-0.22617497,-0.06055142,-0.06378318,0.65472201,-0$ $.08564927,-0.02318804,0.03446971,0.01549050,0.01601564,-0.00783690,-0$. $01452201,-0.01401690,0.01462157,-0.01638040,-0.02573276,-0.06027841,-0$ $.22001781,-0.17242228,0.03056792,0.42115548,-0.02423367,-0.09223635,0$. $03838739,0.01600886,0.01577169,-0.00937089,-0.01373155,-0.01468989,0.0$ $1421757,-0.02581231,-0.01618715,-0.05987216,-0.17173682,-0.22431182,0$. $03019141,0.29478275,0.42361153,0.02708905,0.03155974,-0.16982229,0.007$ $84902,0.00941184,-0.03323327,0.00076607,0.00050105,0.00163792,-0.02223$ $036,-0.02270987,-0.00087527,-0.00527278,-0.00530058,-0.11017520,0.0516$ $3236,0.04689302,0.58552675,-0.04514942,0.04426042,0.00001892,0.0012084$ $2,-0.01465900,-0.00261015,-0.00003799,0.00027298,-0.00147888,-0.000284$ $35,-0.00012093,0 .,-0.00003803,0.00027179,0.00147893,0.00120850,-0.0146$ $6064,0.00259778,0.06572074,0.05586207,-0.26030174,-0.00009214,-0.00414$ $244,-0.01413443,-0.00721530,0.00284686,0.00126863,-0.00309527,-0.00086$ $943,-0.00111920,-0.00000144,0.00284793,0.00126634,0.00310004,-0.004141$ $41,-0.01416435,0.00720362,-0.05064880,0.29149255,0.00002421,-0.0000917$ 
$4,-0.04476096,0.00086331,-0.02789345,-0.00036899,0.00123997,0.00038623$ ,-0.00445492,-0.00000032,-0.00000149,0.00241744,-0.00123764,-0.0003813 $8,-0.00445265,-0.00086707,0.02788194,-0.00033905,-0.00002192,0.0001031$ $8,0.04939213,0.00418908,0.00427761,-0.01073762,-0.07553148,-0.04597159$ $, 0.08493514,-0.00273471,-0.00833273,0.02000730,0.00171471,-0.00540425$, $-0.00089813,-0.00052109,0.00003418,-0.00029200,-0.00030991,-0.00192001$ -0.00209664,0.00036502,-0.00045888,-0.00006313,0.07383064,0.00407695, $0.00629091,-0.01188840,-0.04576713,-0.07526330,0.08316893,-0.00857195$, $-0.00285397,0.02025995,-0.00568551,0.00170840,-0.00093107,0.00013383$,$0.00057013,-0.00040299,-0.00212102,-0.00039858,-0.00195059,0.00047709$, $0.00057092,0.00105422,0.05427058,0.07297160,0.00860324,0.00947963,-0.0$ $1669306,0.08626099,0.08558718,-0.27313054,-0.00190717,-0.00185777,0.00$ $226709,-0.00045719,-0.00070051,0.00234096,-0.00038535,-0.00043547,0.00$ $001397,-0.00291539,-0.00307961,-0.00226181,0.00029443,-0.00035149,0.00$ $038127,-0.09090622,-0.08947397,0.28625569,0.00280718,-0.00672478,0.001$ $20419,-0.00400671,-0.00811374,-0.01925895,-0.08244590,-0.04684948,-0.0$ $8829681,0.00569556,0.00320637,0.00995025,0.00135421,-0.00261777,0.0024$ $7875,-0.00058188,0.00008794,0.00024826,-0.00061387,-0.00043142,-0.0013$ $2459,-0.00237803,0.00341445,0.00007886,0.07984689,-0.00642815,0.003333$ $24,0.00178927,-0.00781723,-0.00423208,-0.01989796,-0.04722849,-0.08437$ $794,-0.09061897,0.00316798,0.00584043,0.01003918,-0.00255915,0.0012380$ $9,0.00223276,0.00008021,-0.00042916,0.00019557,0.00023991,0.00004638,0$ $.00059162,0.00331606,-0.00234273,0.00016989,0.05602721,0.08172184,0.00$ $103081,0.00089511,0.00154266,0.00140613,0.00137355,0.00430607,-0.08684$ $045,-0.08893138,-0.26618065,-0.01159013,-0.01173285,-0.01803756,0.0026$ $8986,0.00260005,-0.00263288,0.00048960,0.00052161,0.00030873,-0.000051$ $95,0.00000773,0.00004483,-0.00001750,0.00005377,0.00101372,0.09364850$, $0.09590889,0.27926341,0.00280716,-0.00672375,-0.00120995,-0.00058189,0$ $.00008811,-0.00024818,0.00135406,-0.00261572,-0.00248102,0.00569565,0$. $00321507,-0.00994823,-0.08244235,-0.04692215,0.08825347,-0.00400632,-0$ $.00813003,0.01925233,-0.00061382,-0.00043255,0.00132424,-0.00026510,0$. $00033984,0.00002344,0.00039357,0.00018797,-0.00052700,0.07984312,-0.00$ $642738,0.00333563,-0.00178762,0.00008060,-0.00042855,-0.00019616,-0.00$ $255690,0.00124220,-0.00222944,0.00315794,0.00583891,-0.01001894,-0.047$ 29976,-0.08452973,0.09077314,-0.00781556,-0.00424760,0.01989083,0.0002 $3985,0.00004688,-0.00059164,0.00031981,-0.00026069,0.00007200,0.000187$ $52,0.00041107,-0.00048557,0.05610417,0.08188399,-0.00103622,-0.0008934$ $5,0.00154041,-0.00048950,-0.00052224,0.00030812,-0.00269208,-0.0025967$ $3,-0.00263684,0.01159231,0.01175295,-0.01803610,0.08679664,0.08908571$, $-0.26603247,-0.00141258,-0.00138061,0.00432137,0.00005218,-0.00000772$, $0.00004430,0.00007894,0.00006911,-0.00000114,0.00052712,0.00048666,-0$. $00086821,-0.09359691,-0.09607674,0.27910511,0.00418930,0.00426831,0.01$ $074079,-0.00030978,-0.00192171,0.00209496,-0.00052107,0.00003397,0.000$ $29203,0.00171475,-0.00540502,0.00089366,-0.00273525,-0.00831586,-0.020$ $01435,-0.07553487,-0.04590146,-0.08497757,0.00036500,-0.00045892,0.000$ $06266,0.00048269,0.00033285,0.00062790,-0.00026511,0.00031988,-0.00007$ $866,-0.00237807,0.00331600,0.00002038,0.07383428,0.00408461,0.00628892$ $, 0.01190793,-0.00212343,-0.00040283,0.00195223,0.00013352,-0.00057090$, $0.00040251,-0.00568588,0.00170708,0.00093058,-0.00857387,-0.00283836,-$ $0.02026396,-0.04569617,-0.07512125,-0.08300093,0.00047732,0.00057158,-$ $0.00105416,0.00033340,0.00046587,0.00063917,0.00033985,-0.00026057,-0$. 
$00006934,0.00341456,-0.00234259,-0.00005668,0.05419553,0.07282094,-0.0$ $0860029,-0.00946039,-0.01669127,0.00291356,0.00308132,-0.00225768,0.00$ $038548,0.00043496,0.00001466,0.00045241,0.00070008,0.00234231,0.001900$ $03,0.00185359,0.00225200,-0.08630353,-0.08541885,-0.27326921,-0.000294$ $00,0.00035162,0.00038080,-0.00062766,-0.00063794,-0.00102764,-0.000023$ $15,-0.00007224,-0.00000125,-0.00007591,-0.00017280,0.00101352,0.090956$ $28,0.08929263,0.28640280,-0.00069532,-0.00050980,-0.00000023,0.0028804$ $3,0.00114275,0.00415025,-0.01758518,-0.01989624,-0.00108441,-0.0885934$ $5,-0.07588275,-0.00003132,-0.01758561,-0.01989759,0.00106802,0.0028803$ $9,0.00114628,-0.00414941,-0.00018039,-0.00013035,-0.00000005,-0.000414$ $94,-0.00094029,-0.00017291,-0.00022782,0.00134447,-0.00018956,-0.00022$ $782,0.00134432,0.00019069,-0.00041496,-0.00094045,0.00017211,0.1200762$ $7,0.00009585,-0.00147167,-0.00000067,0.00081964,0.00324932,0.00412891$, $-0.01989638,-0.01750282,-0.00100383,-0.07609617,-0.08916670,-0.0000211$ $4,-0.01987963,-0.01748691,0.00098769,0.00081844,0.00325172,-0.00412357$ ,-0.00040186,-0.00014582,-0.00000006,-0.00088407,-0.00049440,-0.000162 $87,0.00139689,-0.00023030,-0.00013272,0.00139720,-0.00023009,0.0001315$ $5,-0.00088352,-0.00049394,0.00016244,0.11334260,0.12058866,0.00000006$, $-0.00000065,0.00005730,-0.00128694,-0.00126848,-0.00306834,0.01965215$, $0.01969416,0.00219840,-0.00003145,-0.00002110,-0.03659501,-0.01966853$, $-0.01971033,0.00218298,0.00128750,0.00127380,-0.00307068,-0.00000017,-$ $0.00000008,0.00003357,0.00066014,0.00068515,0.00003635,0.00035910,0.00$ $041508,0.00117058,-0.00035789,-0.00041625,0.00117030,-0.00066088,-0.00$ $068557,0.00003592,0.00004686,0.00003426,0.03573498,-0.00037767,-0.0008$ $1926,-0.00000057,-0.00050609,0.00023626,0.00053352,-0.00004347,0.00002$ $292,-0.00025301,0.00022513,0.00025009,0.00000015,-0.00004345,0.0000226$ $8,0.00025294,-0.00050600,0.00023678,-0.00053313,0.00005253,-0.00012468$ ,-0.00000002,0.00003120,0.00000025,-0.00002826,0.00001811,-0.00010597, $-0.00000335,0.00001810,-0.00010595,0.00000326,0.00003122,0.00000020,0$. $00002824,-0.00016974,-0.00016205,-0.00000007,0.74224878,-0.00047457,-0$ $.00068322,0.00000030,-0.00039749,0.00035305,-0.00011939,-0.00008928,0$. $00014330,0.00006373,0.00014369,0.00030129,0.00000012,-0.00008930,0.000$ $14324,-0.00006361,-0.00039756,0.00035259,0.00011945,-0.00027590,0.0003$ $0516,0.00000018,0.00001600,-0.00011197,0.00003859,0.00013802,-0.000054$ $98,-0.00000061,0.00013799,-0.00005499,0.00000056,0.00001607,-0.0001118$ $8,-0.00003878,-0.00005481,-0.00004834,-0.00000003,-0.02392479,0.659458$ $96,-0.00000009,0.00000011,-0.00108540,-0.00013035,0.00005655,0.0001501$ $1,-0.00001358,-0.00005986,-0.00004800,0.00000002,0.00000011,-0.0000283$ $9,0.00001359,0.00005996,-0.00004802,0.00013006,-0.00005641,0.00015018$, $-0.00000005,0.00000010,0.00006341,-0.00000176,0.00003101,-0.00008143,0$ $.00002844,-0.00004181,0.00002005,-0.00002837,0.00004176,0.00002010,0.0$ $0000176,-0.00003100,-0.00008146,0 .,-0.00000001,0.00000874,-0.00003577$, $0.00016212,0.13247346,0.00138603,-0.00154429,0.00000031,-0.00070588,0$. $00031931,0.00009874,-0.00011626,0.00004661,-0.00005861,0.00021316,0.00$ $030319,0.00000007,-0.00011625,0.00004657,0.00005877,-0.00070623,0.0003$ $1887,-0.00009886,-0.00019826,0.00034046,0.00000018,-0.00003923,-0.0001$ $2657,-0.00000770,0.00012337,-0.00007033,0.00001045,0.00012334,-0.00007$ $033,-0.00001052,-0.00003911,-0.00012654,0.00000743,-0.00004203,-0.0000$ $3127,-0.00000002,-0.17100953,0.05925477,0.00002310,0.68112675,-0.00164$ $580,-0.00378396,-0.00000339,-0.00023685,-0.00004221,0.00026957,0.00007$ $516,0.00000303,-0.00012024,-0.00006531,-0.00021367,-0.00000007,0.00007$ 
$518,0.00000289,0.00012002,-0.00023622,-0.00004145,-0.00026917,-0.00002$ $322,0.00014819,0.00000009,-0.00003775,0.00001079,-0.00012473,-0.000071$ $07,-0.00000644,0.00000174,-0.00007106,-0.00000636,-0.00000174,-0.00003$ $778,0.00001065,0.00012476,0.00000068,0.00001257,0.00000002,0.12244156$, $-0.30188936,-0.00007920,0.03219946,0.72291180,-0.00000022,-0.00000381$, $0.00775847,0.00174299,0.00041974,-0.00062933,-0.00006693,-0.00007705,0$ $.00055354,0.00000002,-0.00000005,-0.00018750,0.00006687,0.00007660,0.0$ $0055362,-0.00174286,-0.00041925,-0.00062974,0 ., 0.00000019,0.00000275,-$ $0.00000315,-0.00001283,0.00002597,-0.00003064,0.00017652,-0.00002149,0$ $.00003059,-0.00017649,-0.00002165,0.00000315,0.00001278,0.00002602,0$, $0.00000003,-0.00002399,0.00004250,-0.00007637,-0.06309220,-0.00001531$, $0.00017968,0.13526633,-0.02312911,-0.02429051,-0.00000607,-0.00203510$, $-0.00071256,0.00239309,0.00003582,-0.00026829,-0.00138682,0.00004971,0$ $.00015422,-0.00000001,0.00003596,-0.00026947,0.00138694,-0.00203630,-0$ $.00071020,-0.00239349,0.00103273,-0.00376011,-0.00000136,-0.00000476,0$ $.00013701,-0.00048676,-0.00024168,-0.00029542,0.00002219,-0.00024167,-$ $0.00029548,-0.00002244,-0.00000484,0.00013657,0.00048696,-0.00026808,-$ $0.00021791,-0.00000009,-0.02179179,0.07876250,0.00002559,-0.33347985,-$ $0.02269482,0.00000553,0.68167379,-0.00247147,0.00361561,-0.00000083,0$. $00177687,-0.00045298,0.00058433,0.00020835,0.00023378,0.00009121,-0.00$ 024993,-0.00014354,0.00000007,0.00020818,0.00023374,-0.00009131,0.0017 $7710,-0.00045219,-0.00058436,0.00265073,0.00089890,0.00000006,-0.00012$ $104,0.00007591,-0.00014834,-0.00010689,0.00014985,0.00003901,-0.000106$ $81,0.00014987,-0.00003885,-0.00012095,0.00007581,0.00014846,0.00025899$ $, 0.00022366,0.00000008,0.03229376,0.02718332,0.00000475,0.04331280,-0$. $12596603,-0.00002109,-0.03199430,0.71145640,0.00000016,0.00000008,0.00$ $520087,-0.00032172,0.00026403,-0.00032588,-0.00001570,-0.00014100,0.00$ $021821,-0.00000010,0 .,-0.00000497,0.00001583,0.00014099,0.00021811,0.0$ $0032319,-0.00026378,-0.00032653,0.00000070,0.00000071,-0.00017127,0.00$ $015740,0.00014565,0.00005346,0.00010800,-0.00005841,-0.00003884,-0.000$ $10805,0.00005856,-0.00003879,-0.00015748,-0.00014564,0.00005341,0.0000$ $0010,0.00000006,0.00002323,0.00001132,0.00000225,0.00717263,0.00002557$ - $-0.00001790,-0.06381325,-0.00003483,0.00018020,0.13596381,-0.15841266$ $, 0.01744589,0.00001055,-0.02148745,-0.00815702,-0.01483426,0.00105606$, $0.00352664,-0.00023932,-0.00154996,-0.00203142,-0.00000098,0.00105611$, $0.00352686,0.00024258,-0.02148516,-0.00816971,0.01482666,-0.01666822,0$ $.00244718,0.00000086,0.00178823,-0.00001345,0.00172017,0.00060440,-0.0$ $0001895,-0.00002439,0.00060437,-0.00001893,0.00002432,0.00178828,-0.00$ $001211,-0.00172016,0.00070462,0.00071451,0.00000029,0.00147748,-0.0026$ $9503,-0.00000130,-0.03066417,-0.02529420,-0.00000616,-0.18321081,-0.12$ $447802,-0.00003315,0.59486473,0.00295930,-0.07625194,-0.00000062,-0.01$ $662801,-0.00104417,-0.00092757,0.00039766,0.00046204,-0.00316408,-0.00$ $039858,-0.00028203,-0.00000095,0.00039817,0.00045957,0.00316840,-0.016$ $65169,-0.00104576,0.00092558,0.03278313,-0.00805466,-0.00000366,0.0003$ $8449,0.00018961,0.00006573,0.00008547,-0.00080243,-0.00002880,0.000085$ $75,-0.00080360,0.00002805,0.00038446,0.00018951,-0.00006622,-0.0000683$ $4,0.00015633,0.00000002,-0.00379579,-0.06830161,-0.00001919,-0.0721177$ $0,0.04049886,0.00001413,-0.05593169,-0.26351848,-0.00005930,0.00691833$ $, 0.67040407,0.00000379,-0.00000120,-0.06870607,-0.02708657,-0.00013872$ $, 0.00131722,0.00024970,0.00023104,-0.00428847,0.00000004,-0.00000078,0$ $.00154220,-0.00024974,-0.00022735,-0.00428608,0.02707378,0.00013687,0$. 
$00131683,0.00001426,-0.00000335,0.00062742,-0.00007701,-0.00018195,0.0$ $0072012,0.00040598,-0.00129219,0.00011065,-0.00040594,0.00129149,0.000$ $11175,0.00007715,0.00018162,0.00072040,-0.00000005,0.00000003,0.000115$ $51,-0.00000125,-0.00001942,-0.00516870,-0.00002060,0.00001170,0.005526$ $56,-0.00001229,-0.00005559,-0.06504517,-0.00001372,0.00015125,0.174120$ $75,-0.02018237,0.02746822,0.00000917,0.00039890,0.00248439,0.00194071$, $-0.00029146,-0.00002939,-0.00027217,0.00040255,0.00062390,0.00000035,-$ $0.00029135,-0.00002965,0.00027195,0.00039865,0.00248580,-0.00193826,-0$ $.00612569,0.00149758,0.00000082,-0.00008372,-0.00006466,-0.00032296,0$. $00013486,-0.00016890,0.00002757,0.00013477,-0.00016877,-0.00002772,-0$. $00008373,-0.00006484,0.00032278,-0.00026524,-0.00025183,-0.00000011,-0$ $.03331296,-0.06836015,-0.00001913,-0.04588023,0.03023699,0.00001133,0$. $06044761,0.01441791,0.00000175,-0.16040423,0.04658472,0.00001799,0.683$ $44693,0.00721863,0.00339296,0.00000020,-0.00010649,-0.00002121,-0.0010$ $9872,-0.00044098,-0.00049572,0.00005591,-0.00009494,-0.00036592,-0.000$ $00013,-0.00044109,-0.00049561,-0.00005640,-0.00010645,-0.00002175,0.00$ $109852,-0.00084604,0.00084457,-0.00000011,0.00004680,0.00010500,0.0002$ $4126,-0.00000409,-0.00002603,-0.00001796,-0.00000407,-0.00002611,0.000$ $01792,0.00004678,0.00010526,-0.00024126,-0.00015199,-0.00012394,-0.000$ $00006,-0.02473100,0.03785998,0.00001018,0.02892771,-0.02135756,-0.0000$ $0687,-0.02915226,-0.05542573,-0.00001755,0.10757347,-0.28522401,-0.000$ $07263,0.03530513,0.71462653,0.00000297,-0.00000045,0.00214517,-0.00033$ $127,0.00020460,0.00050740,-0.00024801,0.00035951,0.00009889,-0.0000001$ $5,0.00000002,-0.00033822,0.00024772,-0.00036012,0.00009846,0.00033153$, $-0.00020491,0.00050818,0.00000007,-0.00000023,0.00098794,0.00000416,0$. $00002329,0.00029615,0.00001660,-0.00003997,0.00004352,-0.00001658,0.00$ $003991,0.00004353,-0.00000407,-0.00002351,0.00029591,-0.00000002,-0.00$ $000005,0.00006336,-0.00000575,0.00001255,0.00846353,0.00001128,-0.0000$ $0703,-0.00355915,-0.00001167,-0.00001965,0.00615499,0.00003704,-0.0000$ $6919,-0.06452359,-0.00001257,0.00017672,0.13202429,0.00286878,0.002112$ $49,0.00000105,0.00064988,-0.00056207,0.00009053,0.00013236,-0.00025298$ ,-0.00003950,-0.00019047,-0.00028704,-0.00000012,0.00013231,-0.0002528 $4,0.00003937,0.00064985,-0.00056166,-0.00009114,0.00012666,-0.00048403$ ,-0.00000034,-0.00009870,0.00016651,-0.00004775,-0.00020322,0.00006559 ,-0.00002141,-0.00020317,0.00006553,0.00002146,-0.00009879,0.00016643, $0.00004805,0.00006950,0.00004417,0.00000003,-0.18356718,-0.06722477,-0$ $.00001468,0.05751050,-0.02775567,-0.00001087,-0.05213311,-0.02526093,-$ $0.00000539,-0.02157897,0.07757973,0.00002513,-0.31786126,-0.02572586,0$ $.00000252,0.64975287,0.00141791,-0.00461028,-0.00000351,-0.00059225,-0$ $.00011859,-0.00019351,0.00002803,0.00010689,-0.00001358,0.00015378,-0$. $00002157,-0.00000001,0.00002803,0.00010722,0.00001361,-0.00059214,-0.0$ $0011861,0.00019368,0.00129410,-0.00043140,-0.00000024,0.00006933,0.000$ $01949,0.00006049,0.00000162,-0.00001073,0.00000841,0.00000165,-0.00001$ $067,-0.00000842,0.00006932,0.00001948,-0.00006047,0.00003117,0.0000338$ $8,0.00000001,-0.12045013,-0.24120007,-0.00004830,0.01867707,-0.0584635$ $7,-0.00002071,-0.02688927,-0.01354717,-0.00000118,0.02992601,0.0232431$ $7,0.00000472,0.02805474,-0.11500792,-0.00001769,-0.00798271,0.65834360$ $, 0.00000054,-0.00000312,0.00602773,0.00055938,0.00000370,-0.00032639,-$ $0.00001740,0.00021523,0.00015553,0.00000006,-0.00000005,0.00007990,0.0$ $0001746,-0.00021525,0.00015532,-0.00055952,-0.00000352,-0.00032625,0.0$ $0000019,-0.00000022,0.00023547,-0.00000049,-0.00009419,0.00000649,-0.0$ 
$0001639,0.00009069,-0.00001947,0.00001640,-0.00009065,-0.00001956,0.00$ $000051,0.00009418,0.00000654,0 ., 0.00000001,-0.00002262,-0.00003096,-0$. $00005066,-0.06330967,0.00000338,-0.00001871,0.00678382,-0.00000612,-0$. $00000148,-0.00531519,0.00001040,0.00000186,0.00503361,0.00001953,-0.00$ $001481,-0.06201104,-0.00002524,0.00015717,0.14329893,-0.00002914,0.000$ 04220,0.,0.00000036,-0.00000953,0.00000458,0.00001740,-0.00001955,-0.0 $0001282,0.00002284,0.00005248,0.00000002,0.00001740,-0.00001954,0.0000$ $1281,0.00000034,-0.00000954,-0.00000459,0.00000592,-0.00002104,-0.0000$ $0001,-0.00000068,0.00000102,0.00000325,-0.00000626,0.00000859,0.000007$ $82,-0.00000626,0.00000860,-0.00000781,-0.00000067,0.00000102,-0.000003$ $25,-0.00002513,-0.00002402,0 .,-0.34306849,0.01710274,0.00001946,-0.008$ $30441,-0.00264871,-0.00000028,0.00104344,0.00260219,0.00000110,0.00055$ $008,-0.00000073,-0.00000009,0.00064798,-0.00310856,-0.00000070,-0.0119$ $1063,0.00435898,0.00000199,0.36064554,0.00008097,0.00007293,0.00000030$ ,-0.00001479,0.00004438,-0.00000655,0.00000853,0.00001010,0.00001751,0 $.00002054,0.00001858,0.00000001,0.00000854,0.00001009,-0.00001748,-0.0$ $0001483,0.00004434,0.00000655,-0.00010452,0.00006904,0.00000003,0.0000$ $0095,-0.00000814,0.00000063,0.00000792,0.00000076,0.00000470,0.0000079$ $1,0.00000076,-0.00000470,0.00000094,-0.00000813,-0.00000065,-0.0000031$ $3,-0.00000061,0 ., 0.01940115,-0.05859082,-0.00000775,0.02755132,0.00343$ $643,-0.00000134,0.00184340,-0.00494728,-0.00000388,0.00004133,-0.00113$ $154,-0.00000007,-0.00226734,-0.00441099,-0.00000305,-0.02660413,0.0045$ $7768,0.00000172,-0.01905731,0.05850428,-0.00000007,0.00000035,-0.00093$ $853,-0.00006278,-0.00005430,0.00005319,0.00000618,-0.00007524,-0.00003$ $330,0 ., 0.00000002,-0.00001549,-0.00000621,0.00007528,-0.00003323,0.000$ $06276,0.00005427,0.00005323,-0.00000003,-0.00000002,0.00001244,-0.0000$ $1170,0.00000665,0.00000876,-0.00001222,-0.00001527,-0.00000219,0.00001$ 223,0.00001527,-0.00000219,0.00001170,-0.00000667,0.00000875,0.,0.,-0. $00000294,0.00002010,-0.00000762,-0.03598075,0.00000902,-0.00000003,0.0$ $0374759,0.00000082,-0.00000394,0.00739351,-0.00000002,-0.00000004,-0.0$ $0090181,-0.00000048,-0.00000297,0.00597309,-0.00000739,0.00000031,0.00$ $287927,-0.00002135,0.00001114,0.02478863,-0.00013925,-0.00109928,-0.00$ $000027,-0.00023378,0.00003313,0.00014316,-0.00001073,-0.00001208,-0.00$ $009817,0.00005162,0.00006232,0.00000002,-0.00001071,-0.00001215,0.0000$ $9819,-0.00023389,0.00003324,-0.00014314,0.00005359,0.00000001,0 .,-0.00$ $000128,-0.00000490,-0.00003266,-0.00000513,-0.00003669,-0.00000636,-0$. $00000513,-0.00003671,0.00000633,-0.00000128,-0.00000492,0.00003265,-0$. $00006671,-0.00006060,-0.00000003,0.01068911,0.01340381,0.00000379,-0.1$ $4113431,-0.12957527,-0.00003518,-0.01153975,-0.02709265,-0.00000765,-0$ $.00508069,0.00154636,0.00000098,-0.00077755,0.00088834,0.00000025,-0.0$ $0020415,0.00329000,0.00000136,0.00076208,-0.00022740,-0.00000029,0.146$ $46059,0.00036476,0.00012092,0.00000005,-0.00002672,-0.00000899,0.00001$ $865,0.00000921,0.00000164,-0.00002528,0.00000964,0.00002699,0.00000003$ $, 0.00000920,0.00000162,0.00002529,-0.00002668,-0.00000901,-0.00001871$, $0.00000943,0.00000651,0 ., 0.00000468,0.00000006,-0.00000265,-0.00000206$ $,-0.00000256,0.00000113,-0.00000207,-0.00000255,-0.00000113,0.00000467$ $, 0.00000006,0.00000265,-0.00001728,-0.00001595,0 .,-0.01685202,-0.01771$ $715,-0.00000580,-0.12954525,-0.25582608,-0.00006170,0.00359863,0.00455$ $427,0.00000014,0.00092311,0.00187034,-0.00000165,0.00082363,0.00010012$ $, 0.00000038,0.00340131,-0.00284109,-0.00000324,0.00011371,0.00089087,0$ $.00000156,0.13802592,0.26922796,0.00000013,0.00000015,-0.00007746,0.00$ 
$004946,-0.00007708,0.00006286,-0.00001674,-0.00001234,0.00001173,-0.00$ $000002,0.00000004,-0.00004586,0.00001677,0.00001232,0.00001174,-0.0000$ 4955,0.00007699,0.00006291,0.,0.,-0.00000032,-0.00000699,-0.00000492,-$0.00000513,-0.00001934,0.00001062,0.00000197,0.00001934,-0.00001062,0$. $00000196,0.00000699,0.00000493,-0.00000513,0 ., 0 .,-0.00000234,-0.000005$ $55,-0.00000709,0.00375959,-0.00003513,-0.00006173,-0.03710743,0.000001$ $77,0.00000147,0.00372260,0.00000089,-0.00000158,0.00699405,0.00000029$, $0.00000033,-0.00120673,0.00000133,-0.00000325,0.00709602,-0.00000019,0$ $.00000156,-0.00418406,0.00003705,0.00006898,0.02542432,0.00110628,-0.0$ $0194622,-0.00000093,0.00000794,0.00010365,0.00010592,-0.00004568,-0.00$ $004126,0.00006554,-0.00003284,-0.00003400,0 .,-0.00004564,-0.00004119,-$ $0.00006562,0.00000799,0.00010381,-0.00010579,0.00004231,0.00031091,0.0$ $0000020,0.00000201,0.00000233,0.00000827,0.00000925,-0.00000466,0.0000$ $0550,0.00000925,-0.00000465,-0.00000550,0.00000199,0.00000232,-0.00000$ $829,0.00008517,0.00008572,0.00000003,-0.00577133,-0.00091211,0.0000003$ $0,-0.00868505,0.02893853,0.00000953,-0.11135544,0.10992027,0.00003770$, $0.00910714,-0.01493957,-0.00000491,-0.00125599,-0.00341012,-0.00000068$ $,-0.00077129,-0.00072322,-0.00000022,-0.00046696,0.00142193,0.00000047$ $, 0.00099408,-0.00016647,-0.00000028,0.11650576,0.00230300,0.00044532,0$ $.00000116,-0.00017643,0.00024727,-0.00013760,0.00001738,0.00005853,0.0$ $0007931,-0.00001830,-0.00004150,-0.00000004,0.00001738,0.00005864,-0.0$ $0007914,-0.00017679,0.00024701,0.00013771,-0.00125459,0.00078541,0.000$ $00031,0.00001103,-0.00006157,-0.00000939,0.00005651,-0.00001524,0.0000$ $0621,0.00005652,-0.00001528,-0.00000623,0.00001101,-0.00006159,0.00000$ $935,0.00002784,0.00004125,0.00000002,-0.00012467,0.00201034,-0.0000015$ $9,-0.00197185,0.00193246,-0.00000046,0.10955829,-0.28021639,-0.0000806$ $9,0.01382143,-0.01881436,-0.00000773,-0.00420965,-0.00322147,-0.000002$ $96,-0.00092885,0.00042908,0.00000046,-0.00018733,0.00001842,0 ., 0.00022$ $706,0.00070710,0.00000143,-0.11796598,0.29638562,0.00000040,0.00000170$ ,-0.00397857,-0.00062138,-0.00040208,0.00030256,-0.00003541,0.00002682 ,-0.00028813,0.,-0.00000003,0.00004158,0.00003541,-0.00002652,-0.00028 $812,0.00062124,0.00040194,0.00030291,-0.00000037,-0.00000003,0.0002082$ $6,-0.00004940,-0.00004495,-0.00008209,0.00000164,-0.00007361,0.0000112$ $8,-0.00000160,0.00007357,0.00001133,0.00004939,0.00004496,-0.00008200$, $0 ., 0.00000001,-0.00000672,0.00000055,-0.00000151,0.00707329,-0.0000000$ $2,-0.00000190,0.00375595,0.00003763,-0.00008087,-0.03729768,0.00000415$ $,-0.00000639,0.00406947,-0.00000097,-0.00000285,0.00670318,-0.00000029$ $, 0.00000044,-0.00087906,-0.00000003,-0.00000007,0.00003440,-0.00000016$ $, 0.00000145,-0.00392416,-0.00004096,0.00008985,0.02534607,-0.00021888$, $0.00049443,0.00000008,0.00001138,0.00029988,0.00014453,-0.00017398,-0$. $00010755,-0.00008034,-0.00018774,-0.00018437,-0.00000008,-0.00017412,-$ $0.00010766,0.00008023,0.00001144,0.00030016,-0.00014433,0.00020770,-0$. $00020469,-0.00000006,0.00000460,-0.00005828,0.00006851,-0.00001497,-0$. $00000833,0.00000432,-0.00001498,-0.00000832,-0.00000432,0.00000457,-0$. $00005816,-0.00006851,0.00008839,0.00008667,0.00000004,-0.00521410,0.00$ $216169,0.00000122,-0.00024181,0.00070041,0.00000011,-0.00112709,0.0031$ $8111,0.00000137,0.01233300,0.01205750,0.00000346,-0.14036804,-0.127879$ $51,-0.00003477,-0.01241975,-0.02660925,-0.00000752,-0.00055130,-0.0012$ $6720,-0.00000032,0.00005450,-0.00007391,-0.00000006,0.00048691,0.00082$ $156,0.00000022,0.14671393,-0.00116003,0.00022746,0.00000116,-0.0001148$ $7,0.00019818,0.00016283,0.00011797,0.00013036,0.00008641,0.00030788,0$. 
$00021883,0 ., 0.00011808,0.00013035,-0.00008629,-0.00011512,0.00019842,-$ $0.00016267,-0.00012994,0.00008420,0.00000006,0.00004008,-0.00005003,-0$ $.00002205,-0.00002257,0.00005289,-0.00001959,-0.00002265,0.00005289,0$. $00001967,0.00004008,-0.00005007,0.00002199,-0.00014230,-0.00014464,-0$. $00000005,0.00115624,0.00142535,-0.00000160,0.00091721,0.00013904,0.000$ $00041,0.00377644,-0.00350377,-0.00000342,-0.01664224,-0.01743209,-0.00$ $000567,-0.12987593,-0.25882543,-0.00006268,0.00448525,0.00491374,0.000$ $00030,0.00023353,0.00025417,-0.00000006,-0.00009108,-0.00001046,0.0000$ $0028,-0.00080871,-0.00090575,-0.00000024,0.13743895,0.27192560,-0.0000$ $0054,0.00000103,-0.00324806,-0.00020474,0.00008123,0.00011797,0.000082$ $42,-0.00040563,-0.00008765,0.00000005,0.00000002,0.00018685,-0.0000822$ $8,0.00040577,-0.00008735,0.00020475,-0.00008129,0.00011788,0.00000004$, $0.00000009,-0.00012828,-0.00007168,0.00011651,-0.00009485,-0.00008226$, $-0.00000506,-0.00003643,0.00008224,0.00000511,-0.00003639,0.00007172$, $0.00011658,-0.00009506,-0.00000007,-0.00000006,-0.00004511,0.00000084$, $-0.00000167,0.00643105,0.00000021,0.00000045,-0.00125679,0.00000151,-0$ $.00000339,0.00705576,-0.00000524,-0.00000699,0.00389975,-0.00003555,-0$ $.00006235,-0.03643258,0.00000221,0.00000151,0.00347560,0.00000012,0 ., 0$ $.00042947,-0.00000007,0.00000029,-0.00093473,-0.00000027,-0.00000030,-$ $0.00003114,0.00003670,0.00006983,0.02447496,-0.00023496,0.00144159,0.0$ $0000051,0.00024548,0.00005742,0.00000999,-0.00001413,-0.00002825,0.000$ $02827,-0.00009489,-0.00010661,-0.00000005,-0.00001413,-0.00002823,-0.0$ $0002829,0.00024546,0.00005743,-0.00000993,-0.00052473,0.00016039,0.000$ $00007,-0.00004867,0.00000604,-0.00002583,0.00000164,0.00000190,0.00000$ $932,0.00000165,0.00000191,-0.00000931,-0.00004866,0.00000603,0.0000258$ $4,0.00005275,0.00006065,0.00000003,0.01576185,-0.00333696,-0.00000172$, $0.00106682,-0.00618146,-0.00000153,-0.00248655,-0.00065107,-0.00000014$ $,-0.00623347,-0.00231016,-0.00000020,-0.02239308,0.03909414,0.00001308$ ,-0.12194888,0.07975004,0.00002719,0.00035930,-0.00125411,-0.00000057, $0.00067131,-0.00088030,-0.00000029,0.00006951,0.00004888,-0.00000004,0$ $.00079363,0.00059232,-0.00000008,0.40466969,0.00035721,0.00005745,-0.0$ $0000015,-0.00006104,0.00007747,0.00019290,-0.00005898,-0.00007867,-0.0$ $0012581,0.00025711,0.00024364,0.00000008,-0.00005895,-0.00007874,0.000$ $12577,-0.00006110,0.00007760,-0.00019283,0.00000656,-0.00006817,0.0000$ $0002,0.00003028,0.00001753,0.00003655,-0.00004353,-0.00003066,-0.00001$ $551,-0.00004354,-0.00003066,0.00001549,0.00003028,0.00001757,-0.000036$ $47,-0.00022560,-0.00023991,-0.00000009,0.01161755,-0.04859360,-0.00001$ $584,-0.00806723,-0.00027747,-0.00000203,-0.00060387,-0.00139135,-0.000$ $00007,-0.00026225,0.00720801,-0.00000022,0.02478926,-0.01119628,-0.000$ $00519,0.07885212,-0.24195043,-0.00005945,0.00090363,0.00135556,0.00000$ $152,0.00064166,-0.00091612,-0.00000047,0.00008443,0.00004582,0.0000003$ $8,-0.00155663,0.00093488,0.00000164,-0.57288651,1.25548660,0.00000009$, $-0.00000017,0.00041689,-0.00015363,-0.00014629,0.00002125,0.00006741,0$ $.00008388,-0.00007295,0.00000009,0.00000004,0.00009463,-0.00006744,-0$. $00008387,-0.00007294,0.00015359,0.00014632,0.00002123,-0.00000002,0.00$ $000002,-0.00018692,0.00000644,0.00002716,-0.00005880,-0.00001089,0.000$ $01626,-0.00000083,0.00001086,-0.00001628,-0.00000083,-0.00000642,-0.00$ $002708,-0.00005882,-0.00000007,-0.00000007,-0.00000525,0.00000285,-0.0$ $0001519,0.00161476,-0.00000213,-0.00000208,0.00756334,-0.00000008,-0.0$ $0000017,-0.00099082,0.00000059,-0.00000022,0.00812245,0.00000867,-0.00$ $000580,0.00217448,0.00002714,-0.00005995,-0.05852436,0.00000009,0.0000$ 
$0162,-0.00372432,0.00000018,-0.00000040,0.00054953,-0.00000004,0.00000$ $040,-0.00122177,-0.00000070,0.00000152,-0.00417444,-0.00019407,0.00039$ $293,0.08383910,0.00010684,-0.00015506,-0.00000008,-0.00007860,0.000015$ $59,0.00007212,-0.00001324,-0.00000329,-0.00002813,0.00010009,0.0001075$ $9,0.00000005,-0.00001324,-0.00000332,0.00002813,-0.00007859,0.00001566$ ,-0.00007211,0.00004564,-0.00000464,0.,0.00002411,-0.00000501,0.000007 $08,0.00000153,-0.00001750,-0.00000131,0.00000154,-0.00001750,0.0000012$ $9,0.00002411,-0.00000501,-0.00000708,-0.00007509,-0.00007303,-0.000000$ $03,-0.00517170,-0.00290455,-0.00000053,-0.00018736,0.00190721,0.000000$ $49,0.00076685,0.00004578,0,0.00084130,0.00067718,0.00000011,0.0035646$ $4,-0.00289753,-0.00000097,0.01129686,0.01668716,0.00000548,0.00029726$, $0.00041933,0.00000014,-0.00029256,0.00029485,0.00000010,-0.00000536,0$. $00003656,0.00000002,-0.00000330,-0.00009857,0 .,-0.26989694,0.46635915$, $0.00015750,0.25874523,-0.00037964,-0.00035362,-0.00000004,0.00003864,-$ $0.00007984,-0.00017621,0.00004495,0.00005658,0.00011385,-0.00024926,-0$ $.00024121,-0.00000010,0.00004494,0.00005669,-0.00011380,0.00003862,-0$. $00007999,0.00017614,0.00014211,0.00001450,0 .,-0.00002495,-0.00001060,-$ $0.00002227,0.00003763,0.00002728,0.00001242,0.00003765,0.00002728,-0.0$ $0001240,-0.00002494,-0.00001062,0.00002223,0.00022182,0.00023864,0.000$ $00009,0.00351687,0.00782140,0.00000173,0.00145602,-0.00123255,-0.00000$ $003,0.00011910,0.00071380,0.00000015,0.00092857,-0.00197486,-0.0000001$ $9,-0.00928118,-0.00078878,-0.00000032,0.01694444,-0.01346212,-0.000009$ $99,-0.00032285,-0.00021089,-0.00000019,0.00010190,-0.00015848,0 ., 0.000$ $02423,-0.00005911,-0.00000008,0.00058148,0.00003590,-0.00000018,0.4663$ $8491,-0.96067004,-0.00031258,-0.48038105,0.97034066,-0.00000011,-0.000$ $00007,-0.00014049,0.00000376,0.00003180,0.00000110,-0.00002311,-0.0000$ $0074,0.00001472,-0.00000009,-0.00000007,-0.00003366,0.00002314,0.00000$ $076,0.00001464,-0.00000374,-0.00003185,0.00000119,0.00000006,0 ., 0.0000$ $3134,0.00001156,-0.00001760,0.00001648,0.00001956,-0.00000754,0.000002$ $34,-0.00001953,0.00000756,0.00000234,-0.00001158,0.00001758,0.00001650$ $, 0.00000008,0.00000008,0.00001177,0.00000142,0.00000211,0.00152314,0.0$ $0000039,-0.00000012,-0.00122142,0 ., 0.00000021,0.00013780,0.00000019,-0$ $.00000020,-0.00142885,-0.00000299,-0.00000058,0.00148922,0.00000531,-0$ $.00000953,0.01551938,-0.00000009,-0.00000022,0.00043652,0.00000004,0$, $-0.00020591,0.00000002,-0.00000009,0.00021916,0.00000021,-0.00000013,0$ $.00049954,0.00015778,-0.00031311,-0.03532463,-0.00016226,0.00032176,0$. $01841779 \backslash 0.00001044,0.00000197,-0.00000055,0.00000078,0.00000259,0.00$ $000628,-0.00000058,0.00000113,-0.00000411,-0.00001333,-0.00001160,0.00$ $000011,-0.00000077,0.00000095,0.00000389,0.00000110,0.00000268,-0.0000$ $0587,-0.00000069,0.00000025,-0.00000008,-0.00000052,-0.00000083,-0.000$ $00212,0.00000050,0.00000224,0.00000057,0.00000053,0.00000226,-0.000000$ $59,-0.00000050,-0.00000085,0.00000210,0.00000619,0.00000872,-0.0000000$ $2,0.00000011,0.00000209,0.00000054,0.00000434,-0.00000302,-0.00000012$, $-0.00000266,-0.00000220,0.00000004,-0.00000499,0.00000373,0.00000027,0$ $.00000344,-0.00000256,0.00000022,-0.00000269,-0.00000178,-0.00000028,0$ $.00000025,-0.00000185,0.00000002,0.00000071,-0.00000106,-0.00000002,0$. $00000162,-0.00000013,0 .,-0.00000096,0.00000005,0 .,-0.00000156,-0.00000$ 171,-0.00000067,-0.00000077,-0.00000107,0.00000039lII@

para-Addition Product 


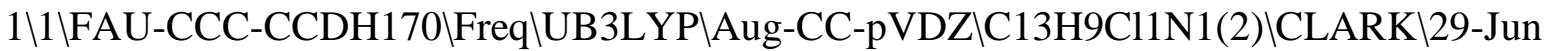
-2015\0\\N Geom=AllCheck Guess=TCheck SCRF=Check GenChk UB3LYP/Aug-CC -pVDZ Freq \\Addition product p-Cl-Ph-radical + cyanobenzene (para) $\backslash 0$, 2\C,-0.5368918844,0.3331401557,-0.4982463807\C,-0.8362933856,0.0040658 $839,0.8320732031 \backslash \mathrm{C},-2.155941413,-0.0319930348,1.2856289365 \backslash \mathrm{C},-3.188225$ $2691,0.2660189986,0.3929557494 \backslash C,-2.9208355996,0.5965255497,-0.9346852$ 449\C, $-1.5918178564,0.6271611479,-1.3699342896 \mathrm{\backslash H}, 0.8683208585,0.651529$ $9301,-2.0517640285 \backslash \mathrm{H},-0.0295582611,-0.2281746646,1.5277160915 \backslash \mathrm{H},-2.383$ $6822058,-0.2880214467,2.3189195617 \backslash \mathrm{H},-3.7368543933,0.826201247,-1.6173$ $713567 \backslash \mathrm{H},-1.3822800883,0.8857721794,-2.4089385969 \backslash \mathrm{Cl},-4.8570561168,0.2$ $232115734,0.9556458736 \backslash \mathrm{C}, 3.4540392354,-0.1476546432,0.3860836563 \backslash \mathrm{C}, 2.8$ $985629367,1.1745049573,0.3638075624 \backslash \mathrm{C}, 1.7196297853,1.4296279382,-0.267$ 7738691\C,0.9202006732,0.3682648606,-0.9809331204\C, $1.5682614564,-0.99$ $10640385,-0.9007824604 \backslash \mathrm{C}, 2.7491408225,-1.2158688818,-0.261366103 \backslash \mathrm{C}, 4.6$ $832978731,-0.3971434053,1.0462126451 \backslash \mathrm{H}, 3.4373999034,1.9778957753,0.865$ $4349887 \backslash \mathrm{H}, 1.3118759597,2.4409335663,-0.2684753617 \backslash \mathrm{H}, 1.0455279586,-1.81$ $81156178,-1.3821736518 \backslash \mathrm{H}, 3.1749427362,-2.21848177,-0.2318127045 \backslash \mathrm{N}, 5.69$ 65343914,-0.6029217318,1.5909940068॥Version=EM64L-G09RevC.01।State $=2-$ $\mathrm{A} \backslash \mathrm{HF}=-1015.7744763 \backslash \mathrm{S} 2=0.783314 \backslash \mathrm{S} 2-1=0 . \mathrm{S} 2 \mathrm{~A}=0.750797 \backslash \mathrm{RMSD}=0.000 \mathrm{e}+00 \backslash \mathrm{RMS}$ $\mathrm{F}=4.755 \mathrm{e}-06 \mathrm{ZZeroPoint}=0.1787803 \backslash$ Thermal $=0.1913038 \mathrm{ZPE}=112.1863146 \mathrm{Wipo}$ le $=-1.1844621,0.3671377,-1.1206403 \backslash$ DipoleDeriv $=0.3421443,-0.0254283,0$. $0011014,0.0070674,-0.0600855,0.00347,-0.1230668,0.0113414,-0.0726437,-$ $0.1220171,-0.006591,0.0346782,0.0016724,-0.0773207,-0.0222108,0.003057$ $7,-0.0202507,-0.0061004,-0.2638263,0.0092891,0.0073842,0.0031631,-0.07$ $42733,-0.033142,0.0309329,-0.0345075,0.0418616,1.1945612,0.0113822,-0$. $3383202,0.0408971,-0.0346423,-0.0272672,-0.4511935,-0.0202393,0.146044$ $1,-0.2628303,-0.0251925,0.1376232,-0.0410339,-0.0831376,-0.0133382,0.1$ $981251,-0.0170936,-0.0712563,-0.0227311,0.0050953,-0.0389882,-0.032675$ $3,-0.0902568,-0.0483087,0.1058272,-0.0575232,0.0896619,0.0056895,0.023$ $3931,-0.0743767,0.0135365,0.0563037,0.0443525,-0.0366556,0.0420113,-0$. $0830954,0.0029633,0.0220124,-0.0604128,0.0279113,0.0950141,0.0214329,-$ $0.0830944,0.0228671,0.0346461,0.0535618,-0.0030182,0.0292244,-0.005605$ $3,0.1189779,0.0328411,0.0390575,0.0321833,-0.0052413,-0.0093111,0.0228$ $627,-0.0542522,0.0196059,0.1217109,0.0235142,-0.0417412,0.0227254,0.05$ $22326,0.0629064,-0.0145144,0.0689509,-0.0077123,0.108325,0.0420902,0.0$ $428783,0.0437717,-0.0586773,-0.880788,-0.00283,0.2059694,-0.0190391,-0$ $.0665269,0.0105173,0.2679826,0.0066204,-0.1543517,0.0784313,0.0043872$, $0.0287025,0.0034097,0.2555443,0.0497154,0.0322688,0.0495108,0.0718578$, $-0.1373876,0.1582885,0.0527899,-0.1208103,-0.0100126,0.0038933,-0.0489$ $645,0.1198338,-0.0570008,0.0116418,-0.1178577,0.1066175,0.1372691,0.03$ $52685,0.0284666,0.3078092,-0.0283695,-0.0974729,-0.0413844,0.0319902,-$ $0.1131392,0.0700456,0.000564,-0.0312955,-0.2589456,-0.0222757,0.143605$ $3,-0.0028219,0.0542109,0.1510946,-0.2684724,0.0289235,0.0186243,0.2075$ $368,0.0595175,-0.0764819,-0.139726,-0.1458571,-0.0249201,0.1510203,0.0$ $332363,0.0449491,0.0096481,-0.0669414,-0.0981535,0.4221453,-0.0543944$, $0.1073345,-0.0660631,0.0186634,-0.0519977,0.1518222,-0.0547982,0.17404$ $13,0.0490496,-0.0395881,-0.0475215,-0.0199268,0.0084636,-0.0446515,-0$. $0873559,-0.0500291,0.0944583,0.0654655,0.0488556,-0.0372244,0.050062,-$ $0.0415619,-0.0085911,-0.0363518,-0.0197368,0.0891749,0.054718,-0.03485$ $14,-0.0618625,-0.03663,-0.0151281,-0.0460737,-0.0604301,-0.0351219,0.0$ 735357,0.059168,0.0491078,-0.0219058,0.0512343,-0.0241595,-0.0139072,- 
$0.0653211,-0.0032647,0.1169462,-0.5196222,0.0292483,-0.0585472,0.04107$ $36,-0.30389,0.0169167,-0.1038259,0.0197687,-0.3475906 \backslash$ Polar $=275.569243$ $9,-15.4218376,128.3896879,23.0702796,-7.4412325,148.4117603 \backslash \mathrm{PG}=\mathrm{C} 01$ [X( $\mathrm{C} 13 \mathrm{H} 9 \mathrm{Cl1N} 1)] \mathrm{NImag}=0 \backslash 0.60714920,-0.03191611,0.20635912,0.01815855,-0$. $11996203,0.62744596,-0.12841577,0.00272258,0.00428562,0.71076679,-0.01$ $296802,-0.08171648,0.05950496,-0.04218956,0.17385776,0.06428695,0.0557$ $4670,-0.29560961,0.02394675,-0.13489311,0.64621873,0.00817799,-0.02104$ $158,0.08032026,-0.31982780,0.00657698,0.03653775,0.66040300,-0.0089705$ $9,0.00872599,-0.00239346,-0.01032294,-0.06621263,0.01917912,-0.0245612$ $2,0.17179082,0.03415691,0.00049404,-0.00251609,0.10116903,0.01513551,-$ $0.14392955,-0.03254089,-0.14808479,0.70552330,-0.00576869,0.00571733,-$ $0.02213684,-0.05489670,0.00261411,0.00483207,-0.21209416,0.04406721,-0$ $.13339824,0.51472854,0.00547669,-0.01140059,0.01603582,0.01664939,0.00$ $960222,-0.01356062,0.02782821,-0.07906122,0.04610529,-0.03381279,0.162$ $02622,-0.02120390,0.01597693,-0.06289465,-0.04883821,-0.01020340,0.057$ $80295,-0.07129106,0.04221693,-0.20965708,0.03641817,-0.12816659,0.6077$ $2608,-0.05071834,0.01515778,-0.04416972,-0.02838254,-0.00669056,0.0316$ $6990,0.04810994,0.00065335,-0.01188727,-0.11259694,0.00397291,-0.00378$ $283,0.70852181,0.00317402,0.00928507,-0.01000912,-0.00652196,-0.004430$ $13,0.00709966,0.01182680,0.00467427,0.01303937,-0.01214792,-0.08113822$ $, 0.06508847,-0.04585290,0.17019682,0.00165242,-0.01287817,0.05570858,0$ $.03101211,0.00714198,-0.03771102,-0.05462281,0.01571432,-0.03819182,0$. $05786668,0.06123161,-0.31282214,0.03697299,-0.14032429,0.65826257,-0.2$ $3285685,0.02664286,-0.06180150,0.04815042,0.01227550,-0.05662769,-0.06$ $271478,0.00700320,-0.01259525,0.00848955,-0.02290163,0.08718755,-0.316$ $14169,0.00801230,0.03013212,0.66585945,0.04370709,-0.07841393,0.039376$ $96,0.00092392,0.00335140,0.01613934,0.00689672,-0.00413132,0.00109774$, $-0.00883587,0.00796714,-0.00215313,-0.00874981,-0.06652391,0.01830982$, $-0.02675733,0.17396102,-0.12703539,0.04346541,-0.20116820,-0.01321644$, $0.01341591,-0.04051381,-0.01220617,0.00107341,-0.00460269,0.03341520,0$ $.00120621,-0.00575356,0.09420790,0.01430148,-0.13951281,-0.02480066,-0$ $.14322556,0.68841632,-0.00696883,-0.00774054,0.03136998,-0.00546425,0$. $00017581,0.00088582,0.00042527,-0.00032385,0.00116727,-0.00007696,0.00$ $007684,-0.00023881,-0.00007615,-0.00001589,0.00006776,0.00081861,-0.00$ $067325,0.00236450,0.05428721,-0.00014328,-0.00019120,0.00244425,-0.000$ $37999,0.00104606,0.00010512,0.00012565,0.00009250,0.00012400,0.0000263$ $4,0.00015597,-0.00002227,-0.00008788,-0.00003516,-0.00001236,0.0009071$ $6,0.00000325,-0.00050481,-0.00362274,0.06558182,0.00232127,0.00426065$, $-0.01641226,0.00301061,-0.00002797,0.00043695,-0.00054857,0.00023108,-$ $0.00062171,-0.00004633,-0.00003455,0.00029586,0.00034348,-0.00002977,-$ $0.00001308,-0.00368266,-0.00012541,0.00129380,0.01254709,-0.06323546,0$ $.28763750,0.00810414,-0.00154247,0.00480238,-0.20945354,0.04532039,-0$. $13212317,-0.01711856,0.00646133,-0.01965383,-0.00296524,-0.00008148,0$. $00275640,-0.00017223,-0.00026668,0.00077438,0.00085905,-0.00016273,0.0$ $0194707,0.00012082,0.00003088,-0.00018347,0.22123507,0.00587179,0.0020$ $6149,0.00411662,0.04526505,-0.05107396,0.04345840,-0.00123561,0.004168$ $45,-0.00017231,0.00001861,0.00689144,0.00143794,-0.00029617,-0.0011137$ $6,-0.00027097,-0.00036220,0.00572379,0.00274437,0.00002847,-0.00013703$ $,-0.00006153,-0.04913226,0.04096508,-0.02355642,0.00589164,-0.01338697$ $,-0.13192852,0.04344805,-0.17156538,0.00980216,-0.00201293,0.00940834$, $0.00237362,0.00146106,0.00110795,0.00088905,-0.00027796,-0.00034992,0$. 
$00271532,0.00269135,-0.00454365,-0.00017415,-0.00006182,0.00012714,0.1$ $4107281,-0.04892700,0.17855803,-0.00486800,0.00048708,0.00102718,-0.00$ $129913,-0.00692373,0.02776011,-0.06698714,-0.01310420,0.05747719,0.006$ $50149,0.00566300,-0.02236774,-0.00334132,0.00132101,-0.00297841,-0.001$ $13581,0.00004786,-0.00014994,-0.00036537,-0.00004229,0.00021964,0.0009$ $1966,-0.00025599,-0.00029153,0.06881541,0.00035286,0.00687405,0.001266$ $89,0.00047970,0.00392053,0.00045835,-0.01240530,-0.05345748,0.06926407$ ,-0.00223090,0.00206779,0.00605137,0.00144562,0.00481339,0.00161480,0. $00010617,-0.00091246,-0.00040652,-0.00001779,-0.00011267,-0.00002908,-$ $0.00028578,-0.00404076,-0.00126680,0.01296588,0.04362278,0.00154449,0$. $00123488,0.00213893,-0.00053803,0.00223036,-0.00432913,0.05479046,0.06$ $942964,-0.31466587,0.00782088,0.00416215,-0.01427377,-0.00345550,0.001$ $64614,-0.00014078,-0.00038204,-0.00039229,0.00057769,0.00012674,-0.000$ $02328,-0.00006231,-0.00017477,-0.00127445,0.00052464,-0.06016764,-0.07$ $621796,0.33028783,-0.00349998,-0.00017978,0.00317473,-0.00022920,-0.00$ $026178,0.00077179,0.00092962,-0.00024352,0.00203013,0.00579129,-0.0019$ $1797,0.00678775,-0.21607099,0.04712902,-0.13694978,-0.01568837,0.00629$ $483,-0.01941962,0.00006438,0.00000095,-0.00002535,-0.00001617,-0.00001$ $669,-0.00010822,0.00044395,-0.00008045,0.00027145,0.22697648,-0.000061$ $34,0.00655278,0.00135915,-0.00031497,-0.00109592,-0.00025649,-0.000389$ $25,0.00491379,0.00243512,0.00599447,0.00208320,0.00403344,0.04645181,-$ $0.04979441,0.04349248,-0.00117927,0.00386788,-0.00037044,-0.00000294,-$ $0.00002518,-0.00000563,-0.00002240,-0.00068195,-0.00017998,0.00027650$, $0.00021428,0.00021608,-0.05029792,0.04011180,0.00272192,0.00138914,0.0$ $0094089,0.00097452,-0.00026991,-0.00038355,0.00258645,0.00240020,-0.00$ $426899,-0.02346350,0.00592842,-0.01353655,-0.13435885,0.04332841,-0.16$ $898842,0.00915277,-0.00215859,0.00976113,-0.00001055,-0.00000671,0.000$ $00116,-0.00008521,-0.00018145,-0.00001707,-0.00109405,0.00030142,-0.00$ $060321,0.14375766,-0.04861650,0.17569378,0.00688186,0.00527700,-0.0209$ $0431,-0.00315443,0.00140007,-0.00312478,-0.00109733,0.00006509,-0.0002$ $4334,-0.00392765,0.00041134,0.00104311,-0.00228169,-0.00718806,0.02889$ $909,-0.06678055,-0.01129958,0.05050949,-0.00040569,-0.00011235,0.00058$ $758,0.00036729,0.00028853,-0.00115196,0.00006601,-0.00005574,0.0000288$ $4,0.00100988,-0.00025600,-0.00025852,0.06911630,-0.00204085,0.00234407$ $, 0.00625373,0.00164859,0.00554850,0.00193881,0.00010626,-0.00096733,-0$ $.00041455,0.00032728,0.00666582,0.00119083,0.00052173,0.00343929,0.000$ $59634,-0.01115846,-0.05334081,0.06784008,0.00022478,0.00028994,-0.0001$ $8228,-0.00012113,0.00009286,0.00032308,-0.00006571,-0.00065965,-0.0001$ $5682,-0.00032794,-0.00382996,-0.00118013,0.01161395,0.04406780,0.00707$ $673,0.00449922,-0.01505431,-0.00407710,0.00199892,-0.00050897,-0.00039$ $198,-0.00040656,0.00057465,0.00135989,0.00117237,0.00219176,-0.0005807$ $6,0.00244022,-0.00556662,0.04996864,0.06787837,-0.30778690,-0.00070123$ ,-0.00010148,0.00081221,0.00041543,0.00022380,-0.00078731,0.00006618,-$0.00015900,-0.00011151,0.00001533,-0.00119675,0.00041408,-0.05493466$,$0.07511654,0.32551754,-0.00126690,-0.00033123,0.00140586,0.00118137,0$. $00190098,-0.00645161,-0.04144322,-0.00107549,0.01513657,-0.14226077,-0$ $.00168959,0.03468664,-0.02558380,-0.00070397,0.00990558,0.00324748,-0$. $00053017,0.00242942,-0.00000879,-0.00000564,0.00002352,-0.00144325,-0$. $00010021,0.00074060,0.00106221,-0.00034027,0.00041782,0.00113188,-0.00$ $010194,-0.00053589,-0.00111250,0.00002959,0.00018100,0.20694485,-0.000$ $24758,-0.00050981,-0.00063806,0.00023508,0.00405363,0.00205477,0.00571$ 
$917,0.00471648,-0.00156484,-0.00171084,-0.02532527,0.00466619,-0.00748$ 246,0.00429869,0.00287901,0.00110474,0.00431811,0.00207069,0.00002118, $0.00000453,-0.00001876,0.00018794,0.00005662,-0.00005814,-0.00006181,-$ $0.00238025,-0.00095720,-0.00036046,-0.00248723,-0.00087516,-0.00025007$ $, 0.00010780,0.00009796,0.00295800,0.01378244,0.00108464,-0.00061648,0$. $00140526,-0.00007675,0.00165490,-0.00170423,-0.01084939,0.00006183,0.0$ $0702540,0.03477276,0.00465822,-0.05033573,0.03582102,0.00125926,-0.008$ $79748,-0.00382347,0.00246115,-0.00356745,-0.00007816,-0.00001264,0.000$ $06781,-0.00036101,0.00001152,0.00009562,-0.00064745,-0.00089069,0.0009$ $2532,0.00045261,-0.00093696,0.00073529,0.00125049,0.00003046,-0.000298$ $33,-0.05791669,-0.00743324,0.05429201,-0.00155505,0.00023283,-0.000585$ $66,0.00012641,0.00004178,-0.00031701,-0.00006512,-0.00002550,0.0001192$ $8,0.00007346,-0.00002991,0.00010739,0.00015156,0.00002024,-0.00011893$, $-0.00029689,-0.00004278,0.00020159,-0.00120943,0.00051395,-0.00162879$, $-0.00004612,-0.00002589,0.00021301,-0.00003187,-0.00000257,0.00002184$, $-0.00004066,0.00000528,0.00000015,0.00008003,-0.00000486,-0.00000721,-$ $0.00000427,-0.00000347,0.00001195,0.50815746,0.00028784,-0.00028019,-0$ $.00008818,-0.00007016,-0.00052153,-0.00002318,0.00004592,0.00002254,-0$ $.00000508,-0.00002534,0.00006156,-0.00005541,-0.00005077,-0.00003267,0$ $.00004505,0.00003479,-0.00012234,-0.00004206,0.00030053,0.00007358,0.0$ $0042171,0.00002739,0.00040290,0.00009524,0.00000565,0.00001733,0.00000$ 493,0.00000559,0.00004168,0.00001069,-0.00002295,-0.00002608,-0.000006 $12,-0.00000351,-0.00000936,0.00000187,-0.05433996,0.52229640,-0.000800$ $79,-0.00008028,0.00021301,0.00010898,-0.00004678,-0.00037836,-0.000153$ $09,0.00001065,0.00001940,0.00008595,-0.00005077,0.00021965,0.00015871$, $0.00002702,-0.00016593,-0.00009120,-0.00002187,-0.00002351,-0.00081195$ $, 0.00037096,-0.00102241,0.00001067,0.00010678,0.00001752,-0.00000906,0$ $.00000705,-0.00000566,-0.00000310,0.00001103,0.00000367,0.00006180,-0$. $00000981,-0.00000699,0.00001275,0.00000157,-0.00001818,0.21407805,0.05$ $954118,0.25563115,0.00160649,-0.00117422,-0.00017357,-0.00041659,-0.00$ $012027,-0.00065344,0.00004161,0.00005106,0.00011000,0.00007525,-0.0000$ $1300,-0.00018267,-0.00013681,0.00010132,0.00023134,0.00016651,-0.00030$ $144,0.00018868,0.00054045,-0.00117190,0.00259672,-0.00011403,0.0001466$ $3,0.00023335,-0.00002305,0.00001386,-0.00000187,-0.00000178,0.00002827$ $, 0.00001608,-0.00003797,-0.00006595,0.00003480,-0.00013313,-0.00001183$ $, 0.00006368,-0.09767092,0.00734682,-0.02324766,0.56834843,-0.00235429$, $-0.00208612,-0.00341464,-0.00017073,0.00006823,0.00018159,-0.00000142$, $0.00010873,0.00001632,0.00004661,-0.00003462,-0.00006685,-0.00014962,0$ $.00044411,0.00005227,-0.00106867,0.00019130,0.00047704,-0.00263046,-0$. $00221527,-0.00364225,-0.00000049,-0.00009408,-0.00000263,-0.00002231,-$ $0.00005330,0.00001532,-0.00009402,-0.00002444,0.00000090,0.00006671,-0$ $.00025137,-0.00001766,-0.00023410,-0.00002791,0.00009943,0.05889037,-0$ $.22156992,-0.00371885,0.00969821,0.59597687,0.00228832,-0.00149077,-0$. $00189268,-0.00008936,0.00036406,-0.00036002,-0.00028658,0.00021756,0.0$ $0028889,0.00017504,-0.00001870,0.00001300,-0.00005412,0.00011331,0.000$ $00495,-0.00039578,-0.00022478,0.00044763,-0.00003067,-0.00120882,-0.00$ $057490,-0.00001172,-0.00040207,0.00009938,-0.00005396,0.00005045,0.000$ $02587,-0.00002553,0.00002147,0.00003255,-0.00001854,-0.00006296,0.0000$ $2662,-0.00015129,-0.00007080,0.00006123,-0.01157489,-0.03408722,-0.069$ $16266,0.26588741,0.11465856,0.30119708,-0.02878488,-0.02018059,-0.0149$ $0605,0.00133072,0.00005164,-0.00051985,0.00011658,0.00054561,-0.000599$ 
$19,-0.00011530,0.00014087,-0.00019877,-0.00048364,0.00142998,0.0002783$ $0,-0.00150825,-0.00060964,0.00194479,-0.00526525,-0.00292212,-0.007432$ $99,0.00000835,-0.00036692,-0.00013763,0.00014465,-0.00011860,-0.000034$ $56,-0.00017899,0.00000872,0.00003962,-0.00005485,-0.00061986,-0.000288$ $84,-0.00021283,-0.00008902,0.00007151,-0.02723085,0.06448414,-0.005572$ $42,-0.32562290,0.02004322,-0.15568260,0.58343079,-0.01116040,0.0010619$ $7,0.00297483,0.00099525,0.00053626,-0.00095044,0.00025697,-0.00005043$, $-0.00038552,-0.00019945,0.00001160,-0.00002922,0.00015369,-0.00059156$, $0.00008809,0.00245118,-0.00047334,-0.00035930,0.00132360,0.00710916,-0$ $.00067171,-0.00005608,0.00000102,0.00014296,0.00013289,0.00000343,-0.0$ $0005082,0.00015514,0.00004993,-0.00001832,-0.00000661,0.00043632,-0.00$ $006888,0.00047901,0.00006901,-0.00017059,0.03764526,-0.01686539,0.0166$ $0934,0.05676659,-0.11460775,0.01975670,-0.12315267,0.60171118,-0.00334$ $429,0.00722853,0.00615619,0.00270238,0.00057178,-0.00035764,-0.0004556$ $5,-0.00018071,-0.00017351,-0.00010032,-0.00009985,0.00065738,0.0006917$ $0,-0.00021062,-0.00025752,0.00006884,0.00047052,-0.00090226,-0.0189328$ $8,-0.02159439,-0.01610503,0.00031876,0.00016196,0.00008369,0.00008345$, $-0.00008020,-0.00005108,0.00011261,-0.00008238,-0.00007594,0.00011621$, $-0.00018875,0.00019383,0.00051894,0.00001416,-0.00018349,-0.01136097,0$ $.03177222,0.00844234,-0.14650560,-0.00176724,-0.13966294,0.23153931,0$. $03755786,0.28120086,-0.15017975,-0.00074031,0.02258114,-0.01066341,-0$. $00178443,0.00978908,0.00341235,0.00013807,-0.00001645,-0.00093486,0.00$ $021590,-0.00071886,0.00002838,0.00113695,-0.00261219,-0.02846047,0.000$ $09065,0.00746675,-0.03307827,0.00087063,-0.00598878,-0.00013301,0.0000$ $4313,-0.00086875,0.00004740,0.00008374,-0.00028444,-0.00040302,-0.0000$ $9915,0.00050648,0.00132689,-0.00077277,0.00166853,0.00020064,-0.000073$ $81,0.00005425,0.00060050,-0.00193895,0.00584572,-0.01527616,-0.0164372$ $6,-0.01853326,-0.10458319,-0.03346991,-0.03292755,0.43274537,-0.004486$ $32,-0.06819933,0.00321722,-0.00711548,0.00175880,0.00162116,0.00022029$ $, 0.00485055,0.00258738,0.00006883,-0.00052649,0.00014523,0.00113063,0$. $00656737,0.00244759,0.00577823,0.00426949,-0.00145334,0.00368607,-0.05$ $914629,0.05631519,-0.00025852,-0.00282955,-0.00087458,-0.00028425,0.00$ $018107,0.00013154,0.00025391,0.00013270,-0.00007004,0.00015641,-0.0037$ $4520,-0.00122002,0.00004137,-0.00067778,-0.00026598,-0.00207842,-0.005$ $83422,-0.00004305,-0.00416489,0.01074322,-0.00147768,-0.04219854,-0.15$ $030070,-0.04606188,-0.00661130,0.47689716,0.03687925,0.00232501,-0.085$ $34075,0.03018039,0.00034477,-0.00680074,-0.00033735,0.00260937,-0.0043$ $5041,-0.00015622,0.00011124,-0.00087548,-0.00258990,0.00244568,-0.0014$ $4963,-0.01425633,-0.00009903,0.00801999,-0.01675411,0.05698971,-0.2581$ $1416,0.00028628,-0.00094587,0.00047797,0.00112522,0.00004318,-0.000238$ $41,-0.00084321,0.00001396,0.00027755,-0.00188487,-0.00099698,0.0002121$ $4,-0.00038442,-0.00023927,0.00026004,0.00637640,-0.00007509,-0.0072085$ $2,-0.01614873,-0.00703423,-0.00221975,-0.03415989,-0.04124450,-0.09803$ $976,0.03508589,-0.01050973,0.50553867,-0.02412606,0.02827844,-0.002618$ $18,0.00113092,0.00008474,-0.00036077,0.00005119,-0.00022528,-0.0007503$ $5,-0.00009595,-0.00000882,-0.00024725,-0.00070028,-0.00136626,-0.00047$ $593,-0.00177019,-0.00027666,0.00207762,-0.00420229,0.00653898,-0.00429$ $624,0.00005228,0.00039487,0.00004280,0.00013851,0.00009871,0.00002971$, $-0.00019897,-0.00000252,0.00004168,0.00002872,0.00071631,0.00007642,-0$ $.00027287,0.00007041,0.00013973,-0.03819579,-0.04885580,-0.03750475,-0$ $.01832435,0.01666169,-0.00641828,0.02219681,-0.01445874,0.00967976,-0$. 
09472200,0.04413086,-0.00856560,0.59819098,0.01490080,0.00197457,-0.00 $275905,-0.00226558,0.00040679,0.00134840,-0.00002802,-0.00029315,0.000$ $39588,0.00023225,0.00008744,-0.00026348,-0.00045612,-0.00034671,-0.000$ $18940,-0.00201184,-0.00025005,0.00039001,0.00780438,-0.00963793,0.0055$ $4653,-0.00009744,0.00011713,-0.00010267,-0.00018062,-0.00005957,0.0000$ $4481,-0.00017035,-0.00000064,0.00004814,-0.00002022,0.00019253,0.00010$ $056,-0.00063916,0.00001066,0.00024675,-0.02215143,0.01877265,-0.010724$ $25,0.00703642,-0.00386726,0.00321923,0.00139640,-0.03143590,-0.0047603$ $7,0.03577079,-0.18553928,-0.00219518,-0.00670515,0.55841528,0.00249889$ $,-0.00806721,0.00057695,0.00178764,-0.00050094,-0.00001650,-0.00051154$ $, 0.00016614,0.00013492,0.00001101,-0.00020962,0.00057211,0.00053498,0$. $00001938,-0.00028822,-0.00113741,-0.00011997,-0.00087236,-0.01387548,0$ $.02778748,-0.00041815,0.00028405,-0.00016937,-0.00008054,0.00001264,0$. $00006726,0.00001651,0.00004264,0.00010833,-0.00000433,0.00014141,0.000$ $19408,0.00035914,0.00022871,0.00002786,-0.00006747,-0.03118819,-0.0259$ $1617,-0.01622833,-0.00835513,0.00900928,-0.00446890,0.01305468,-0.0171$ $2755,0.00444120,-0.01119787,0.00270768,-0.07266896,0.26574658,0.086528$ $63,0.30974445,0.00187812,0.00040688,0.00062690,-0.00035271,0.00048672$, $-0.00049864,0.00004303,-0.00008286,0.00007035,0.00006876,0.00008604,-0$ $.00015347,-0.00012634,-0.00013557,0.00017687,0.00033437,0.00018393,0.0$ $0024652,0.00084669,-0.00082001,0.00303265,-0.00013821,-0.00024490,0.00$ $012174,-0.00002026,-0.00001115,-0.00001243,0.00000533,-0.00003434,-0.0$ $0000203,-0.00004151,0.00001722,0.00004851,-0.00010003,-0.00000641,0.00$ $005008,-0.10607370,-0.00747941,-0.03143142,0.04133049,-0.02093744,0.01$ $551698,-0.02037030,0.00046762,-0.01101086,-0.01150163,0.01348571,-0.01$ $021797,-0.32227673,0.04637244,-0.14690309,0.55093245,0.00029706,-0.004$ $29091,0.00205635,0.00026479,0.00011487,0.00019668,0.00015668,0.0002456$ $2,-0.00005345,-0.00013307,-0.00005834,0.00005887,0.00021653,0.00040779$ $, 0.00014804,0.00111385,0.00020722,-0.00058881,0.00180865,-0.00407230,0$ $.00137470,-0.00000998,-0.00019018,-0.00019736,0.00004736,-0.00001031,-$ $0.00003384,0.00009578,-0.00000877,-0.00002176,-0.00007290,-0.00021652$, $-0.00012046,0.00028686,-0.00002565,-0.00014655,-0.05906609,-0.19487589$ $,-0.06555920,0.00019138,-0.04462463,-0.01101086,-0.00904688,0.00251151$ $,-0.00423666,0.02552470,-0.00033556,0.01107027,0.08355248,-0.12529395$, $0.03228824,-0.12760360,0.65446512,0.00310193,-0.00016120,0.00005218,-0$ $.00000569,-0.00006297,-0.00048078,-0.00026794,-0.00020719,0.00015292,0$ $.00012131,-0.00003792,0.00003401,0.00004917,0.00011385,0.00003393,0.00$ $019691,0.00015258,0.00026306,0.00118630,-0.00078093,0.00097449,0.00002$ $752,0.00022767,0.00022438,-0.00004433,-0.00006307,-0.00001937,0.000017$ $85,-0.00004145,0.00000831,-0.00005685,-0.00006961,-0.00000805,-0.00000$ $828,0.00004206,0.00002681,-0.04292880,-0.03519999,-0.08745752,0.020201$ $18,-0.02437317,0.01349417,-0.01334888,0.00157410,-0.00880102,-0.006944$ $14,0.00546557,0.00509110,-0.13944468,0.01088041,-0.13232470,0.22459571$ $, 0.04374256,0.26011982,0.00059946,-0.00015529,0.00049904,0.00006547,-0$ $.00000429,0.00002525,0.00001632,-0.00001166,0.00003368,-0.00028404,-0$. $00001419,0.00012051,0.00013592,-0.00000160,-0.00003460,0.00008744,0.00$ $007222,-0.00028364,0.00092584,-0.00033543,0.00111177,-0.00007863,-0.00$ $001866,0.00007870,0.00003130,0.00000455,-0.00002303,0.00005003,-0.0000$ $0364,0.00000034,-0.00008125,0.00000253,0.00000772,0.00029615,0.0000072$ $0,-0.00009720,-0.21322063,0.03126338,-0.08947575,-0.03673728,0.0290627$ $9,-0.01564998,0.01298234,-0.00260436,0.00457342,-0.00117265,0.00009130$ 
,-0.00022413,0.01261923,-0.00212024,0.00447283,-0.04234264,-0.01176278 ,-0.02812237,1.10305166,-0.00007201,0.00023547,-0.00011083,-0.00002545 $, 0.00009734,0.00000439,-0.00000354,-0.00002977,0.00000291,0.00005018,-$ $0.00000230,-0.00003538,-0.00002653,-0.00003196,-0.00000228,-0.00004145$ $, 0.00004160,0.00007259,-0.00010372,0.00026894,-0.00018279,0.00000818,-$ $0.00003644,-0.00003331,-0.00001123,0.00000731,0.00000411,-0.00001133,-$ $0.00000642,0.00000058,0.00001756,-0.00000761,-0.00000111,-0.00005916,0$ $.00000429,0.00002359,0.03120761,-0.09158890,0.00836181,0.03258930,-0.0$ $0395156,0.01778441,0.00065596,-0.00561043,-0.00172350,-0.00000528,-0.0$ $0072963,0.00027587,-0.00553760,-0.00444908,-0.00502096,-0.01515711,0.0$ $1435842,-0.00615876,-0.20612409,0.15921527,0.00018098,-0.00009142,0.00$ $050935,0.00010420,-0.00000017,0.00007183,0.00000436,0.00000474,-0.0000$ $4798,-0.00012732,-0.00001956,0.00009646,0.00006226,-0.00000835,-0.0000$ $1693,0.00015104,0.00004523,-0.00015239,0.00022658,-0.00012720,0.000647$ $47,-0.00002309,-0.00002706,0.00006509,0.00003703,0.00000029,-0.0000024$ $0,0.00002922,-0.00000122,-0.00000949,-0.00004956,0.00000279,-0.0000051$ $9,0.00015687,0.00000768,-0.00006010,-0.08926252,0.00835079,-0.09803958$ $,-0.01512701,0.01574627,-0.00337482,0.00562478,-0.00366337,0.00614876$, $0.00014339,0.00025216,-0.00165763,0.00402245,-0.00311826,0.00535003,-0$ $.02915612,-0.00409007,-0.01607837,0.55324902,-0.10124701,0.37481132,0$. $00053153,0.00008153,-0.00026127,0.00006420,0.00001683,-0.00001576,-0.0$ $0016897,0.00001999,0.00003245,0.00007695,-0.00003339,0.00010913,0.0000$ $6953,0.00004872,-0.00007157,-0.00029994,0.00008586,0.00005022,-0.00071$ $312,-0.00102045,-0.00057254,-0.00002008,-0.00007489,-0.00001810,-0.000$ $02315,-0.00000559,0.00000442,-0.00001316,-0.00001560,-0.00000166,0.000$ $01711,-0.00003365,0.00004072,-0.00003984,-0.00000760,0.00001662,0.0082$ $8652,0.01039478,0.00570669,-0.12068965,-0.10170246,-0.07245525,-0.0095$ $5401,-0.02098625,-0.01282316,-0.00026643,0.00324185,-0.00506896,-0.000$ $42881,0.00037285,0.00021317,0.00022106,0.00313687,-0.00166560,0.000403$ $11,0.00030083,0.00146735,0.12294166,0.00012398,0.00041158,0.00030452,0$ $.00006748,0.00005231,0.00002101,-0.00003324,-0.00003178,-0.00000784,0$. $00000831,-0.00000773,0.00003108,0.00004654,-0.00004628,-0.00001540,0.0$ $0006347,-0.00000535,-0.00005574,-0.00012420,-0.00011141,-0.00000442,-0$ $.00000831,-0.00003625,-0.00003634,-0.00000540,0.00000120,-0.00000551,0$ $.00000590,-0.00000275,-0.00000189,0.00000895,0.00002389,0.00001844,0.0$ $0002711,0.00000583,-0.00000661,-0.01486512,-0.01625286,-0.01307617,-0$. $10077959,-0.21180698,-0.09944969,0.00480913,0.00810450,0.00373483,0.00$ $255177,0.00099277,-0.00065344,0.00048295,0.00011125,0.00039631,0.00379$ $170,-0.00326755,0.00051010,-0.00175092,0.00061267,-0.00043832,0.106481$ $30,0.22113456,-0.00060558,-0.00107347,-0.00100727,-0.00015607,0.000006$ $06,-0.00000563,0.00006270,0.00007755,-0.00002670,0.00002379,-0.0000090$ $1,-0.00015044,-0.00012094,0.00015224,0.00012826,-0.00011164,-0.0001782$ $2,0.00019643,0.00050345,0.00089402,0.00032351,0.00001326,-0.00000853,0$ $.00004244,0.00000277,0.00000154,0.00000934,-0.00002929,0.00001569,0.00$ $001244,-0.00001180,-0.00005071,-0.00004298,-0.00011382,-0.00000938,0.0$ $0004183,-0.00004777,0.00186106,0.00293348,-0.07267690,-0.10006796,-0.1$ $0260085,-0.00665564,-0.01188962,-0.00286105,-0.00483859,0.00031182,0.0$ $0724724,0.00023031,0.00031760,-0.00030139,-0.00146978,-0.00006388,0.00$ $331004,0.00096526,0.00070458,-0.00127943,0.08189311,0.10771792,0.09808$ $355,0.00188814,-0.00008989,-0.00009902,-0.00010245,-0.00001262,0.00015$ $339,-0.00000571,0.00001966,0.00009142,-0.00007816,-0.00006290,-0.00000$ 
$405,-0.00007240,0.00005640,-0.00002748,-0.00001082,0.00017622,-0.00005$ $012,0.00045595,0.00027833,-0.00039883,-0.00000080,-0.00004713,-0.00000$ $216,-0.00001129,-0.00000383,-0.00000115,-0.00000629,-0.00000861,0.0000$ $0074,-0.00000871,-0.00006400,-0.00003174,0.00007402,0.00000514,-0.0000$ $2584,-0.00169305,0.00035543,-0.00560134,-0.00557521,0.02633383,-0.0008$ $4888,-0.08896506,0.09950791,-0.00991847,0.00639414,-0.01562193,0.00076$ $220,-0.00192825,-0.00205557,-0.00256989,-0.00036553,-0.00034614,0.0002$ $0838,-0.00009594,0.00137566,-0.00009065,-0.00066281,-0.00063959,0.0028$ $1472,0.09045320,0.00156999,0.00079276,-0.00024134,-0.00014348,0.000223$ $00,0.00034192,-0.00004374,-0.00003343,0.00007166,-0.00007966,-0.000029$ $17,0.00006627,0.00001503,-0.00002478,-0.00012806,-0.00052641,0.0001503$ $8,0.00002072,0.00005480,0.00023058,-0.00048105,0.00005440,-0.00011013$, $-0.00005962,-0.00002311,-0.00002043,0.00000464,-0.00002395,-0.00000396$ $, 0.00000676,0.00000749,-0.00006520,-0.00001348,0.00003298,0.00000418,-$ $0.00000633,0.00065973,0.00236982,-0.00115889,-0.00021332,-0.00194259,-$ $0.00202868,0.10158219,-0.29947398,-0.00248132,0.00840870,-0.01489532,0$ $.00162260,-0.00325440,-0.00072081,-0.00266490,-0.00014257,0.00028553,0$ $.00014553,0.00029504,-0.00009150,0.00001702,-0.00060221,0.00076569,0.0$ $0124812,-0.10807244,0.31357104,-0.00011969,-0.00001688,0.00019687,-0.0$ $0008537,0.00004604,0.00016929,0.00014163,-0.00012991,-0.00002769,-0.00$ $001808,0.00004838,-0.00008679,-0.00010251,-0.00001148,0.00000639,0.000$ $03600,0.00016286,0.00005552,0.00058362,0.00099785,0.00076626,-0.000044$ $05,0.00011248,-0.00001300,0.00000827,0.00001495,-0.00000240,-0.0000094$ $7,-0.00000808,-0.00000058,-0.00001562,-0.00003095,-0.00007141,-0.00000$ $108,0.00000573,-0.00000414,-0.00580906,-0.00115296,0.00468528,-0.00725$ $315,0.01362055,0.00530443,-0.00919691,-0.00472655,-0.03908709,0.005940$ $97,-0.01391679,0.00209867,-0.00300915,-0.00154447,-0.00043907,0.000348$ $90,-0.00001622,-0.00073456,-0.00033224,0.00067070,0.00052303,0.0029337$ $3,0.00117273,-0.00388612,0.01540721,0.00547063,0.03090541,0.00170501,-$ $0.00000295,-0.00002903,-0.00008015,-0.00000795,0.00011582,-0.00001169$, $-0.00006311,0.00005992,-0.00005936,0.00006450,0.00002336,-0.00007687,-$ $0.00003661,-0.00003765,0.00003354,-0.00011292,-0.00012341,0.00041078,-$ $0.00006238,-0.00043656,-0.00000149,0.00003067,0.00002254,-0.00000821,0$ $.00000330,-0.00000043,-0.00000213,0.00000703,0.00000398,-0.00000152,0$. $00006221,0.00000321,0.00006957,-0.00000019,-0.00002596,-0.00141253,0.0$ $0280415,-0.00486301,-0.00031771,0.00026289,0.00037832,-0.00114075,0.00$ $290732,-0.00096582,0.00668955,0.01069683,0.00678884,-0.11451910,-0.100$ $08249,-0.06953428,-0.00825472,-0.02146522,-0.01316649,-0.00027327,-0.0$ $0110635,-0.00075203,-0.00010652,-0.00016117,0.00028401,0.00073456,0.00$ $062038,0.00025761,0.11757353,-0.00143563,0.00069184,0.00047792,0.00021$ $403,0.00035731,-0.00024546,-0.00004057,-0.00004695,-0.00009516,0.00008$ $665,-0.00001052,-0.00001870,0.00003822,-0.00007770,0.00008504,0.000464$ $71,0.00017127,0.00006610,-0.00030120,0.00062300,0.00044014,-0.00003258$ ,-0.00006563,0.00002823,0.00001622,-0.00000961,-0.00000818,0.00002540, $-0.00000726,-0.00000956,-0.00000848,-0.00009383,0.00000561,-0.00003570$ $, 0.00000589,0.00001384,0.00263954,0.00134230,0.00012174,0.00001344,0.0$ $0007272,0.00028233,0.00420050,-0.00330024,0.00153057,-0.01327011,-0.01$ $609690,-0.01234710,-0.10231216,-0.21851212,-0.10008257,0.00529967,0.00$ $680131,0.00314326,-0.00002593,0.00048901,-0.00002456,-0.00011744,0.000$ $02655,0.00004072,-0.00088668,-0.00090419,-0.00086247,0.10603396,0.2278$ $5676,-0.00088357,0.00030950,0.00047832,0.00000727,0.00007046,0.0000122$ 
$1,0.00015734,0.00009726,-0.00001083,0.00002161,-0.00000067,-0.00012374$ $,-0.00009586,-0.00002309,0.00006284,0.00027788,-0.00010653,-0.00000473$ $, 0.00034976,-0.00114954,0.00041849,-0.00008290,-0.00013368,-0.00005706$ $, 0.00001615,-0.00001996,-0.00001559,0.00000422,0.00000572,-0.00000108$, $-0.00001600,0.00002277,-0.00005067,-0.00001944,0 .,-0.00000044,-0.00519$ $346,0.00014847,0.00543116,0.00042184,0.00042989,-0.00056714,-0.0008948$ $4,0.00043566,0.00135164,0.00136345,0.00320769,0.00300621,-0.06966214,-$ $0.09787849,-0.09449868,-0.00758294,-0.01244849,-0.00075697,-0.00050986$ ,-0.00067825,0.00011967,0.00032987,0.00007126,-0.00050917,-0.00007758, $0.00007699,0.00046554,0.07909882,0.10631607,0.08950032,0.00052941,0.00$ $001134,-0.00027711,0.00005890,-0.00000690,-0.00002407,-0.00016590,-0.0$ $0001464,0.00002456,0.00007316,-0.00003446,0.00010793,0.00006462,-0.000$ $01433,-0.00008959,-0.00031282,-0.00007103,0.00000777,-0.00056630,0.001$ $25318,0.00001824,-0.00001057,0.00007016,0.00002271,-0.00002216,0.00000$ $494,0.00000777,-0.00001126,0.00001581,0.00000617,0.00001873,0.00000935$ $, 0.00005028,-0.00003879,0.00000321,0.00001893,0.00825404,-0.01514020,-$ $0.00003671,-0.00052880,-0.00212113,-0.00328514,-0.00053136,-0.00032923$ $,-0.00000058,-0.00061523,-0.00010319,-0.00601130,-0.00687401,0.0260604$ $6,-0.00066894,-0.09476191,0.10354735,-0.01098687,0.00049891,-0.0009991$ $4,0.00123201,0.00043852,-0.00079905,-0.00001911,-0.00009078,-0.0000470$ $9,0.00035407,-0.00066809,-0.00070706,0.00289950,0.09538304,0.00009916$, $-0.00027809,0.00014616,0.00001597,0.00004920,0.00001085,0.00002006,0.0$ $0000064,0.00001975,-0.00003138,-0.00002575,0.00002195,0.00001751,0.000$ $05296,-0.00001635,0.00002610,-0.00004757,-0.00008759,-0.00002613,0.000$ $23960,0.00003294,-0.00000244,-0.00004527,-0.00000970,0.00000593,0.0000$ $0050,0.00000023,0.00001085,0.00000391,-0.00000247,-0.00000508,-0.00000$ $652,-0.00000418,0.00003679,0.00000359,-0.00001572,0.01015610,-0.016216$ $04,0.00185543,-0.00280465,-0.00094590,-0.00275955,-0.00043617,0.000417$ $33,-0.00003508,0.00041572,0.00233983,-0.00140074,0.00006381,-0.0009248$ $3,-0.00090398,0.10284018,-0.29748584,0.00300322,0.00107049,0.00026429$, $0.00113840,0.00056319,-0.00079608,0.00006498,0.00001231,-0.00004808,0$. $00014324,-0.00061229,0.00086272,0.00107715,-0.11083259,0.31225881,-0.0$ $0043645,0.00155553,-0.00031613,-0.00016679,0.00004472,0.00000336,0.000$ $06619,-0.00006943,-0.00006052,0.00001968,0.00007349,-0.00012885,-0.000$ $15166,-0.00017686,0.00003493,-0.00010635,0.00005012,0.00025125,0.00040$ $780,-0.00095683,-0.00017448,0.00001290,-0.00003919,0.00004135,0.000005$ $29,-0.00000691,0.00000867,-0.00002984,-0.00001610,0.00000362,-0.000007$ $14,0.00007277,-0.00001405,-0.00011050,0.00000290,0.00004275,0.00555940$ $,-0.01307886,0.00292966,-0.00336724,-0.00219460,0.00173654,-0.00003112$ $, 0.00004689,-0.00050422,-0.00558834,-0.00240946,0.00624844,-0.00608455$ $, 0.01467090,0.00348683,-0.01155980,0.00368135,-0.04284721,0.00166506,0$ $.00000013,-0.00102721,0.00029283,-0.00063671,0.00018218,0.00033912,0.0$ $0017694,-0.00044961,0.00280503,0.00101611,-0.00397798,0.01673306,-0.00$ $196267,0.03451716,-0.00004225,0.00007933,-0.00028935,-0.00013655,0.000$ $02455,-0.00005787,-0.00002651,0.00000031,0.00000606,0.00027634,0.00001$ $445,-0.00012033,-0.00011975,-0.00000502,0.00004931,-0.00006899,-0.0000$ $5610,0.00022790,-0.00049531,0.00018040,-0.00057149,0.00004531,0.000019$ $63,-0.00009096,-0.00003383,-0.00000136,0.00001146,-0.00004185,0.000001$ $99,0.00000172,0.00007284,-0.00000461,0.00000014,-0.00028721,-0.0000065$ $3,0.00009365,-0.03698311,0.01057063,-0.02646708,0.00949712,-0.01314193$ $, 0.00131975,-0.00560783,0.00318127,-0.00205923,0.00054486,-0.00005946$, 
$0.00011854,-0.00577297,-0.00096253,-0.00308741,0.01106593,0.00926632,0$ $.00728468,-0.83747687,0.16307869,-0.43210324,-0.00006369,0.00069802,0$. $00006355,-0.00032674,-0.00012835,-0.00001562,-0.00028245,0.00014120,0$. $00007090,-0.00012137,-0.00060345,-0.00026657,0.86638487,-0.00000485,-0$ $.00000412,0.00008133,0.00003845,0.00000643,0.00002304,0.00000376,0.000$ $00514,-0.00000548,-0.00005097,-0.00000018,0.00002892,0.00002148,0.0000$ $0750,-0.00000917,0.00002301,-0.00000277,-0.00004985,0.00007760,-0.0000$ $3449,0.00010973,-0.00000972,-0.00002579,0.00001921,0.00001129,-0.00000$ $719,-0.00000445,0.00000879,-0.00000308,-0.00000218,-0.00001505,0.00000$ $125,-0.00000322,0.00005747,0.00000150,-0.00002103,0.01062331,0.0187946$ $1,0.00746535,-0.00316224,0.00049853,-0.00217552,0.00039264,0.00068651$, $0.00046054,-0.00004748,0.00012329,-0.00011050,0.00192916,0.00124301,0$. $00148257,-0.00078274,-0.00464102,-0.00184468,0.16306360,-0.06905900,0$. $08729120,-0.00014508,0.00012223,-0.00006313,-0.00020956,-0.00002938,-0$ $.00004940,0.00022036,-0.00017572,0.00016979,0.00024423,0.00032659,0.00$ $015577,-0.17228745,0.05216613,0.00003284,0.00006070,-0.00022359,-0.000$ $11116,0.00002677,-0.00006038,-0.00000627,-0.00000481,0.00002361,0.0001$ $2919,0.00001359,-0.00007842,-0.00005123,-0.00000297,0.00002987,-0.0000$ $7510,-0.00003066,0.00012150,-0.00017869,0.00008516,-0.00028416,0.00002$ $132,0.00001196,-0.00007262,-0.00003705,-0.00000142,0.00000668,-0.00002$ $456,-0.00000049,0.00000476,0.00003993,-0.00000566,0.00001132,-0.000151$ $26,-0.00000574,0.00005767,-0.02666576,0.00747711,-0.00080866,0.0037078$ $9,-0.00800733,0.00215230,-0.00289280,0.00216994,-0.00161331,0.00007358$ $,-0.00010768,0.00048620,-0.00264920,-0.00020222,-0.00200452,0.00516338$ $, 0.00397053,0.00572333,-0.43204979,0.08728852,-0.26659148,-0.00013646$, $0.00040988,0.00016028,-0.00002997,-0.00000612,-0.00037421,0.00009322,0$ $.00012606,-0.00027234,-0.00008356,-0.00031626,0.00001352,0.45588150,-0$ $.09294885,0.26359263 \backslash \backslash-0.00000853,-0.00001590,-0.00000141,-0.00000258$, $-0.00000029,-0.00000117,0.00000135,0.00000633,-0.00000281,0.00000415,0$ $.00000007,-0.00000010,0.00000257,-0.00000244,0.00000368,-0.00000062,0$. $00000420,0.00000483,-0.00000339,-0.00000065,0.00000215,0.00000022,0.00$ $000603,0.00000115,0.00000027,-0.00000422,-0.00000067,-0.00000007,0.000$ $00207,0.00000219,-0.00000073,-0.00000052,0.00000094,0.00000065,-0.0000$ $0135,0.00000080,0.00000696,0.00000226,-0.00000563,-0.00000336,-0.00000$ $124,0.00001656,-0.00000503,-0.00000053,-0.00000343,0.00000845,0.000006$ $80,-0.00000822,-0.00000987,0.00000002,0.00000498,0.00001119,-0.0000005$ $2,-0.00001018,-0.00000477,-0.00000176,-0.00000197,0.00000383,0.0000012$ $0,-0.00000662,-0.00000051,-0.00000009,-0.00000016,-0.00000025,0.000000$ $66,-0.00000088,-0.00000228,-0.00000085,0.00000443,0.00000234,0.0000006$ 9,0.00000153III@ 


\section{Nitrobenzene addition}

Chlorophenyl + Nitrobenzene

ortho-Transition State

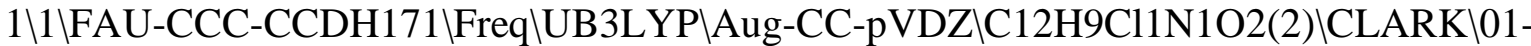 $\mathrm{M}$}

ar-2015\0\\N Geom=AllCheck Guess=TCheck SCRF=Check GenChk UB3LYP/AugCC-pVDZ Freq \\Addition TS p-Cl-Ph-radical + nitrobenzene (ortho) \10,2\

C, $1.7561470054,-0.2964596652,1.1263771194 \backslash \mathrm{C}, 2.3651878801,0.3470881569$, $0.0228642105 \backslash \mathrm{C}, 2.9851145194,-0.3639568305,-1.003485833 \backslash \mathrm{C}, 3.076375132,-$

$1.7529536157,-0.9017337331 \backslash \mathrm{C}, 2.5698853771,-2.4122970425,0.2337325395 \backslash \mathrm{C}$ ,1.9406680619,-1.6953026526,1.2446198565\H,1.4480358341,0.3000280461,1 $.9789416534 \mathrm{WN}, 2.3329365747,1.8089307174,-0.0449011215 \mathrm{\downarrow H}, 3.4017936418,0$ $.1756151498,-1.8501462946 \backslash \mathrm{H}, 2.686684882,-3.4922326517,0.3275521292 \backslash \mathrm{H}, 1$ $.5590525348,-2.2070663805,2.1271455757 \backslash \mathrm{H}, 3.5653403996,-2.3211811008,-1$ $.6922605391 \backslash \mathrm{C},-3.1153821591,-0.0729631956,-0.1398823426 \backslash \mathrm{C},-2.535513240$ $6,1.0038882185,0.5349167441 \backslash \mathrm{C},-1.1825453704,0.9310683772,0.9057942525 \backslash$ C,-0.4885126922,-0.2211281516,0.581305456\C,-1.0375678639,-1.299385318 7,-0.0867800817\C,-2.3896372134,-1.2232644174,-0.4582581691\Cl,-4.8144 $222747,0.0221696209,-0.6043929649 \backslash \mathrm{H},-3.126578635,1.8892689067,0.765405$ $3632 \backslash \mathrm{H},-0.7086820182,1.7672673845,1.4181723732 \backslash \mathrm{H},-0.4547467014,-2.1893$ $90768,-0.3244472508 \backslash \mathrm{H},-2.8698467652,-2.0461927065,-0.9861695629 \backslash \mathrm{O}, 2.85$ $11978566,2.357352984,-1.016589556 \backslash O, 1.7906802347,2.4134139355,0.883598$ $1761 \|$ Version=EM64L-G09RevC.01 $\backslash$ State $=2-A \backslash H F=-1128.0140129 \backslash S 2=0.776515 \backslash$ $\mathrm{S} 2-1=0 . \mathrm{IS} 2 \mathrm{~A}=0.750486 \backslash \mathrm{RMSD}=7.754 \mathrm{e}-09 \backslash \mathrm{RMSF}=3.884 \mathrm{e}-06 \backslash \mathrm{ZeroPoint}=0.180507 \backslash$ Thermal $=0.1939954 \mathrm{ZPE}=113.2698512 \mathrm{WDipole}=0.4846843,-1.8626553,0.263691$ 4\DipoleDeriv $=0.4195815,0.1625803,0.0200228,-0.1021147,0.0266735,0.080$ 4906,0.0821093,-0.0959355,-0.0155974,-0.0018866,0.0448431,-0.0302423,$0.3504408,-0.6358577,0.0936696,-0.0885454,0.0029673,0.0283137,-0.11583$ $58,-0.0992735,0.0067278,0.2765889,0.1611391,-0.1224537,0.0550275,0.130$ $5084,-0.043539,-0.143335,-0.0543511,-0.0896066,-0.2437549,-0.186173,0$. $1705258,0.0924818,0.1141339,0.0077522,0.0354662,-0.008039,-0.0622281,-$ $0.067166,0.1633927,-0.040683,-0.227739,-0.0691776,0.0219526,-0.2160838$ $, 0.0626264,-0.1143792,0.1806699,-0.0457736,-0.1471606,-0.0168404,-0.05$ $8319,0.0020869,0.1074364,-0.01515,0.0229868,0.0138677,0.0805615,-0.029$ $0872,0.0163423,-0.027292,0.0782843,0.4977526,-0.0034824,-0.4980705,0.5$ $722889,1.9087633,-0.1112698,-0.6386556,-0.1530647,1.1263484,0.1251096$, $0.0018208,0.0255755,-0.0091733,0.1016129,0.03368,0.030824,0.0184294,0$. $0747522,0.1169218,0.0089915,0.0207033,0.0343401,-0.0672473,0.0193928,0$ $.0155463,0.0140096,0.0886821,0.0950372,-0.0209943,0.0732243,-0.0070155$ $, 0.0345036,0.044135,0.0555611,0.0424015,0.0105946,0.0850712,0.0387278$, $0.0667494,0.0353213,0.0268022,-0.0420442,0.0723544,-0.0463068,0.015353$ $9,1.2171923,-0.0737726,0.2861066,-0.0691132,-0.0015676,-0.0014818,0.34$ $41182,-0.0022826,0.0442951,-0.2052162,-0.0786115,-0.0548431,0.0800781$, $0.0234857,0.0299524,-0.004189,0.0207428,-0.091476,-0.3992343,0.066889$, $0.0725983,-0.1834251,-0.0003549,0.0361166,-0.1892503,0.0304889,-0.0500$ $073,0.5855226,-0.1344139,-0.0164785,-0.0506046,-0.0014977,0.0062694,0$. $1492678,0.0006206,-0.0792663,-0.4448731,-0.0431308,0.0091929,0.2017107$ 
,-0.02042,0.0224234,-0.0142494,0.0191137,-0.0598844,-0.2009277,0.07109 $25,0.0043542,-0.0716087,0.0076674,0.0614108,-0.0732062,0.0673166,-0.05$ $40208,-0.8867318,0.0455141,-0.1605444,0.0445506,-0.0819374,0.0023827,-$ $0.2226003,0.0056431,-0.1132521,-0.0197666,0.062141,0.0089739,0.0477198$ $, 0.0271796,-0.0330471,-0.0187284,-0.0244021,0.1147809,0.1092629,-0.033$ $3902,-0.0563913,-0.0024785,0.0544167,-0.04187,-0.023566,-0.0330903,0.0$ $969389,0.0702505,0.0829019,0.0058624,0.0470389,0.0103114,-0.0291933,0$. $011714,-0.0219144,0.0934639,-0.009505,-0.0375616,-0.036525,-0.0231467$, $0.0317367,-0.0516119,-0.053885,-0.0563427,0.0912061,-0.4692035,-0.1886$ $231,0.2628404,-0.3682488,-0.8186452,0.3204394,0.4791457,0.3755821,-0.7$ $204903,-0.3520054,0.1426659,0.2333905,0.0141158,-0.7987721,-0.2709859$, $0.1669626,-0.2538305,-0.6672722 \backslash$ Polar $=238.5770664,-12.3735209,189.3973$ 973,-8.6214018,7.4313634,137.4823835\PG=C01 [X(C12H9Cl1N1O2)]\NImag=1\ $10.12197713,-0.02865586,0.65780256,-0.21130251,0.04121580,0.59946092,-$ $0.08783997,-0.02751463,0.09263994,0.28509550,-0.01914676,-0.14385890,0$ $.07818019,-0.02786491,0.52782086,0.06312120,0.04185062,-0.22513815,-0$. $25369108,0.01053605,0.58193100,-0.00879884,-0.00747640,0.02646364,-0.1$ $1880847,0.05676105,0.09797979,0.26992321,0.01515526,0.04936078,-0.0169$ $2232,0.02955345,-0.14567310,-0.04245258,-0.01707104,0.68096814,0.02659$ $477,0.00834139,-0.03550521,0.10260176,-0.08948623,-0.23077429,-0.24326$ $466,-0.06668825,0.58986591,-0.00213865,-0.00860268,0.00209063,0.008025$ $73,0.01323858,-0.00504060,-0.07776402,0.03435891,0.02253452,0.26132803$ $,-0.00928040,-0.03857172,0.02354316,0.03694987,-0.04627600,-0.05929210$ $, 0.00055396,-0.33244970,0.05103810,-0.07064215,0.66649920,-0.00690386$, $0.02366741,-0.01431679,-0.00998772,-0.01470604,0.02579226,0.02854641,-$ $0.00963537,-0.11175874,-0.23578286,0.01584673,0.57405338,-0.00359244,0$ $.03273143,-0.00683643,-0.01600492,0.00602547,0.01783120,0.01626050,-0$. $03197432,-0.01090904,-0.09982368,-0.00814683,0.07501479,0.25502258,0.0$ $1572205,-0.02733724,-0.02448444,0.00083507,-0.00053998,-0.00078975,-0$. $01190784,-0.02421277,0.02950011,-0.03976229,-0.16572523,0.10397775,-0$. $07533708,0.72793323,0.01474761,-0.05489042,0.03018675,0.01906305,-0.01$ $237033,-0.04013984,-0.01515215,0.06648596,0.02087558,0.08285481,0.0438$ $2198,-0.23252800,-0.20709609,-0.02485845,0.53371888,-0.05672523,0.0189$ $8365,0.02177723,0.00969619,-0.01384082,-0.00839209,-0.00810900,0.01355$ $038,0.00491930,-0.00649481,-0.01333362,0.03061705,-0.12178041,0.077078$ $45,0.09653564,0.26990886,-0.00025934,-0.29958727,-0.00145590,-0.018168$ $67,-0.01477647,0.04372736,0.01337753,-0.04070061,-0.01841859,0.0069871$ $2,0.05905879,-0.02558824,0.04582388,-0.18669881,-0.04846681,-0.0521900$ $6,0.62788563,0.01378595,0.04526576,-0.11365253,-0.01055969,0.02779139$, $0.01656496,0.00608573,-0.02208155,-0.01100647,0.02621399,0.01586140,-0$ $.04557108,0.10331679,-0.10851026,-0.22768564,-0.23903627,-0.05528965,0$ $.60941481,-0.04398281,0.04108422,0.07221796,-0.00407184,0.00492323,0.0$ $1486235,0.00310247,-0.00356228,0.00233912,-0.00101264,-0.00046845,0.00$ $062020,0.00666022,0.00127921,-0.00156425,-0.00152245,0.00829458,0.0039$ $2587,0.06140605,0.04833006,-0.14029104,-0.12766616,-0.00322955,0.01158$ $820,0.01110988,-0.00171132,-0.00132246,0.00304173,-0.00029655,-0.00086$ $244,0.00064951,-0.00006172,-0.00480741,0.00140654,0.00839394,-0.009351$ $32,-0.02274775,-0.05131476,0.14573242,0.08327548,-0.12696613,-0.237347$ $85,0.00517901,-0.01493788,-0.01217058,0.00223187,0.00295671,-0.0018239$ $0,-0.00067188,0.00038872,0.00045187,0.00160635,0.00195884,0.00034488,-$ $0.00249927,0.00652163,0.00873569,-0.08093954,0.13491156,0.24424998,0.0$ 
$0370890,-0.01379349,-0.00442610,-0.08590680,-0.00124038,0.01000731,0.0$ $0033669,0.01413007,-0.00609198,0.00459581,0.00229893,0.00683612,-0.001$ $53220,0.00006182,0.00031667,0.00404365,0.00010884,0.00614295,-0.003202$ $71,-0.00052121,0.00067327,0.39408702,-0.02179245,-0.02780552,0.0162017$ $6,-0.00128808,-0.11300052,0.00239597,0.01303843,-0.04405058,-0.0183482$ $6,0.00249505,0.01498498,-0.00745364,-0.00311789,-0.00089196,0.00717022$ ,-0.00160494,-0.00097401,0.00045107,0.00293361,0.00181926,-0.00191917, $-0.01156386,0.62931128,-0.00803575,0.02381659,0.00776993,0.01243783,0$. $00118676,-0.10133654,-0.00654903,-0.01987773,0.01219104,0.00712180,-0$. $00541716,-0.00299729,0.00124661,0.00003517,-0.00375573,0.00584601,0.00$ $420105,-0.00421100,-0.00055623,0.00038978,-0.00287061,-0.29419414,-0.0$ $0533729,0.75460374,0.00359010,0.00302976,0.00320932,-0.00125236,-0.006$ $86881,0.01184090,-0.08631621,-0.05438569,0.09596671,0.00543230,0.00326$ $196,-0.00225948,0.00568478,0.00137719,0.00271470,-0.00064277,-0.000266$ $83,-0.00078810,0.00014608,-0.00053474,0.00070292,-0.00409164,-0.001907$ $29,-0.00033000,0.07957798,0.00234340,-0.00185129,-0.00290528,0.0059790$ $0,0.01214178,-0.01138366,-0.05437943,-0.12420895,0.11264774,-0.0115856$ $9,-0.00794930,0.02323895,0.00143090,-0.00499564,-0.00040195,0.00026084$ ,-0.00057514,-0.00059938,0.00041549,0.00065651,-0.00072041,0.00004316, $0.00131163,-0.00094777,0.05703526,0.12864063,0.00334456,-0.00286235,-0$ $.00064719,0.00880045,0.01232191,-0.01250475,0.09656931,0.11013316,-0.2$ $3241916,0.00025291,-0.00134695,0.00415360,0.00262911,-0.00043008,0.003$ $02788,-0.00057506,-0.00060896,-0.00005792,0.00060513,0.00056595,-0.000$ $39050,-0.00042942,0.00258411,-0.00358942,-0.10667394,-0.12094699,0.239$ $60485,0.00433060,0.00179896,0.00578941,-0.00008104,-0.00020367,0.00017$ $636,0.00407739,-0.00029662,0.00431634,0.00463622,-0.01164309,0.0014577$ $6,-0.04527743,0.03247358,0.00453488,0.00199347,0.01435996,-0.00141457$, $-0.00000264,0.00084306,-0.00036166,-0.00111252,0.00014863,-0.00040147$, $-0.00009976,-0.00045498,-0.00009104,0.03629862,0.00217174,0.00046567,-$ $0.00212777,-0.00004829,0.00076738,-0.00013294,-0.00045407,0.00077327,0$ $.00286780,0.00273484,-0.00959722,-0.00174455,0.03260823,-0.33362594,0$. $02508744,-0.00008562,-0.01112021,0.00415469,0.00003180,-0.00046349,0.0$ $0028098,-0.00019426,-0.00003922,-0.00008200,0.00010160,-0.00044212,-0$. $00021398,-0.03573190,0.35210983,0.00486696,-0.00216992,-0.00129869,0.0$ $0073680,-0.00057656,-0.00121609,0.00438442,0.00278880,-0.00258220,-0.0$ $0323405,0.02453568,0.00273941,0.00523125,0.02565223,-0.05637611,0.0026$ $4682,-0.02260239,0.00624133,0.00006259,-0.00083855,0.00010560,-0.00035$ $852,0.00026363,-0.00070595,-0.00016110,0.00113311,0.00012643,-0.009948$ $51,-0.02754027,0.05253330,0.00410078,0.00351454,-0.00441845,0.00466778$ $, 0.00130718,0.00225815,-0.00082407,0.00025301,-0.00035746,0.00477451,0$ $.00287287,0.00409036,-0.00080910,-0.00756406,0.01073208,-0.07493597,-0$ $.04532665,0.08999488,-0.00251162,0.00002106,-0.00097008,0.00060996,0.0$ $0021172,-0.00010355,-0.00060069,-0.00008262,-0.00034342,-0.00303727,-0$ $.00047989,-0.00200521,0.06860201,-0.01191143,-0.01080364,0.02524829,0$. $00250678,-0.00432088,-0.00071969,0.00013433,-0.00080010,-0.00062993,0$. $00262991,-0.00090321,-0.00233713,0.00636166,0.01098244,-0.01460927,-0$. $04365471,-0.11950805,0.11044267,-0.00048834,0.00117950,-0.00045557,-0$. $00075233,0.00008938,0.00158040,-0.00014142,0.00000386,-0.00000161,-0.0$ $0077533,0.00072682,-0.00003162,0.04582733,0.12374128,0.00083933,-0.000$ $89680,0.00164635,0.00168640,0.00011211,0.00257398,-0.00045504,-0.00060$ $238,0.00009902,0.00426144,-0.00309455,-0.00020506,0.00684659,0.0128793$ 
$5,-0.01279502,0.08922541,0.10940541,-0.23975175,-0.00158862,0.00017384$ $,-0.00002935,0.00010402,-0.00052253,0.00014056,-0.00030966,0.00001902$, $-0.00020776,-0.00210520,-0.00036822,-0.00016260,-0.09924757,-0.1177756$ $8,0.24932280,-0.00050941,-0.00033961,-0.00060388,0.00574493,0.00033104$ $, 0.00180919,0.00266322,-0.00016524,0.00177329,-0.09783748,0.06916318,0$ $.10212349,-0.00130393,0.00683691,0.00713842,0.00440304,-0.00127244,0.0$ $0339711,-0.00052947,0.00001194,-0.00019209,0.00070895,-0.00019453,0.00$ $046137,-0.00297600,-0.00026622,-0.00209513,-0.00237572,-0.00055414,-0$. $00172000,-0.00018783,0.00035219,0.00033924,0.09262003,-0.00031429,-0.0$ $0063548,0.00049213,0.00002438,-0.00463064,0.00218167,0.01417247,-0.011$ $87399,-0.02313954,0.06865930,-0.13377237,-0.10649624,-0.00733030,0.010$ $79933,0.01193042,-0.00076875,-0.00064490,0.00329675,-0.00003164,0.0000$ $1562,-0.00008544,0.00095424,0.00027428,-0.00147641,-0.00048761,0.00106$ $465,-0.00006480,-0.00026659,0.00067569,-0.00052405,-0.00039478,0.00040$ $834,0.00062632,-0.07407388,0.13933135,-0.00056730,0.00073558,-0.000298$ $30,0.00218528,0.00133071,0.00271591,-0.00139502,0.00363344,0.00513328$, $0.10199254,-0.10657977,-0.20255791,0.01000053,-0.01495988,-0.01213616$, $0.00357764,0.00363977,-0.00172391,-0.00024958,-0.00005888,-0.00010531$, $0.00024384,0.00034812,0.00056746,-0.00197673,-0.00042381,-0.00048171,-$ $0.00172315,-0.00017506,0.00006852,0.00048499,-0.00072275,-0.00067498$,$0.11230409,0.11326045,0.20954337,-0.00078769,0.00011686,-0.00017265,-0$ $.00008760,0.00062362,0.00013158,0.00014897,-0.00044092,-0.00008756,0.0$ $0009847,0.00030975,-0.00027113,-0.00008023,0.00009435,0.00026635,0.000$ $12630,-0.00022287,-0.00008343,-0.00007133,0.00002145,0.00003875,-0.000$ $36461,-0.00114114,0.00063441,-0.00004690,-0.00000805,-0.00000117,-0.00$ $003753,-0.00005020,-0.00001359,-0.00003690,0.00001020,-0.00000504,0.00$ $000305,-0.00001376,-0.00002181,0.47172655,-0.00001327,-0.00025868,-0.0$ $0019891,0.00013962,-0.00003546,-0.00003478,0.00011542,0.00001562,0.000$ $08609,-0.00008922,0.00010755,0.00005504,0.00007030,0.00012074,-0.00008$ $946,-0.00018963,-0.00010171,-0.00001214,0.00022619,0.00002577,0.000102$ $31,0.00029992,-0.00015901,-0.00050368,-0.00001202,-0.00000159,0.000009$ $73,-0.00000557,-0.00001994,0.00001034,0.00001532,0.00005204,-0.0000467$ $5,0.00000692,-0.00000164,-0.00000128,-0.04822209,0.56448066,-0.0003408$ $4,-0.00010745,-0.00017164,0.00012529,0.00019922,0.00000917,0.00008729$, $-0.00013747,-0.00006522,0.00005213,0.00015866,-0.00008995,-0.00009278$, $0.00008033,0.00007275,0.00002728,-0.00005314,0.00002890,0.00004181,-0$. $00001133,0.00007473,0.00012559,-0.00049922,-0.00019416,-0.00000562,-0$. $00001104,0.00001337,-0.00000625,-0.00000359,0.00001426,-0.00004092,0.0$ $0002888,-0.00006372,-0.00000525,0.00000144,-0.00001144,0.08128913,0.18$ $920310,0.23959938,0.00117263,0.00057958,0.00036751,-0.00003151,-0.0001$ $2836,0.00008406,-0.00014526,0.00008665,-0.00004211,0.00004242,-0.00018$ $549,-0.00000228,-0.00002792,-0.00006288,-0.00005945,-0.00005998,0.0000$ $4266,-0.00008591,-0.00004036,-0.00023297,-0.00025039,-0.00030473,0.000$ $42501,0.00035064,0.00002219,0.00000974,-0.00000467,0.00002962,-0.00001$ $731,0.00000895,0.00002833,-0.00005590,0.00010694,-0.00000027,-0.000005$ $84,0.00000883,-0.13092131,-0.02923363,-0.03360719,0.62762474,-0.000965$ $84,-0.00011969,-0.00049597,0.00040425,0.00142726,0.00024893,0.00009595$ ,-0.00081676,-0.00037103,0.00027028,0.00053965,-0.00060817,-0.00041563 $, 0.00005371,0.00074324,0.00076020,-0.00050854,-0.00012168,0.00028611,-$ $0.00023293,-0.00003822,0.00069217,-0.00253316,-0.00080402,0.00003011,-$ $0.00002314,0.00001082,-0.00000519,0.00002012,0.00001176,0.00003924,-0$. 
$00009310,0.00003282,-0.00000178,0.00002196,-0.00000121,-0.08525510,-0$. $24177127,-0.10740376,-0.09768823,0.62157949,0.00018291,-0.00049733,0.0$ $0058484,-0.00023611,0.00062157,0.00022318,-0.00006583,-0.00023915,0.00$ $002067,0.00001069,0.00002500,-0.00019059,0.00002577,-0.00008502,0.0001$ 4002,0.00028557,-0.00004293,-0.00003435,0.00008742,-0.00005861,0.00004 $495,0.00002431,-0.00089155,-0.00011871,0.00000305,0.00001708,0.0000003$ $5,-0.00003209,-0.00000858,-0.00001429,0.00002545,-0.00003969,0.0000560$ $9,0.00000373,0.00000435,0.00001460,-0.05965228,-0.09068706,-0.12537605$ $, 0.10544987,0.19855614,0.25109075,-0.00167370,0.00269767,0.00072119,-0$ $.00049917,-0.00296980,-0.00161923,-0.00031082,0.00237777,0.00091258,-0$ $.00055675,-0.00143210,0.00165837,0.00089225,-0.00008641,-0.00166645,-0$ $.00214950,0.00056228,0.00043787,-0.00047007,0.00064566,0.00039409,-0.0$ $0110641,0.00438823,0.00025802,-0.00002662,0.00001963,0.00001173,0.0000$ $3570,0.00010515,-0.00000288,-0.00012413,0.00024916,-0.00022682,-0.0000$ $7294,-0.00002645,-0.00000558,-0.02090207,-0.06886706,-0.04029407,-0.29$ $885610,0.04759307,-0.04924185,0.58173448,0.00160501,0.00010831,0.00043$ $709,0.00004490,-0.00191028,-0.00021714,-0.00045219,0.00109134,0.000461$ $98,-0.00024411,-0.00089961,0.00066312,0.00053014,-0.00010163,-0.000835$ $74,-0.00047533,0.00060036,0.00009040,-0.00075431,0.00071247,0.00009062$ ,-0.00096561,0.00340581,0.00100131,0.00001941,0.00003585,-0.00000634,$0.00001688,0.00002252,-0.00004290,0.00000593,-0.00001651,0.00004765,-0$ $.00001826,-0.00001153,0.00003800,-0.01933129,0.03479262,0.00681254,-0$. $01968839,-0.10663675,-0.02624438,-0.01737768,0.62032685,0.00037925,0.0$ $0089348,0.00066186,-0.00061816,-0.00191318,-0.00039658,-0.00015535,0.0$ $0072356,0.00010533,-0.00019778,-0.00049759,0.00070356,0.00031327,0.000$ $06241,-0.00032256,-0.00052676,0.00038276,-0.00012959,-0.00026733,0.000$ $26017,0.00005800,-0.00087385,0.00390945,0.00125352,0.00001991,0.000028$ $93,0.00002770,0.00002440,0.00005607,0.00000344,-0.00007857,-0.00001560$ ,-0.00012891,-0.00003531,-0.00001432,-0.00002405,-0.01684592,-0.008021 $88,-0.00199738,-0.08027202,-0.00630444,-0.08794773,0.12495700,0.219153$ $09,0.26355930,0.06442358,-0.01308414,0.00184572,-0.02047952,-0.0129787$ $6,0.02183767,0.00221275,-0.00804837,-0.00516901,-0.00121578,0.00146721$ $, 0.00340876,0.00423791,-0.00051974,-0.01115813,-0.01401992,0.02907790$, $0.00038061,-0.01305509,-0.00127727,-0.00580353,-0.00036591,0.00185071$, $-0.00026295,0.00020446,-0.00037808,-0.00028682,0.00041408,-0.00038629$, $-0.00022434,0.00066378,-0.00037655,0.00082732,0.00019161,-0.00001645,0$ $.00001929,-0.00400742,0.00336214,0.00246932,-0.03281567,0.02592236,-0$. $00204830,-0.14463161,0.11655813,0.02675624,0.29025389,-0.00391683,-0.0$ $0820334,-0.00176163,-0.00213602,0.00213220,-0.00014199,0.00030370,-0.0$ $0150745,-0.00062010,0.00019712,0.00169270,-0.00091140,-0.00112415,-0.0$ $0090209,0.00256375,0.00718142,-0.00358480,-0.00001225,-0.00280153,-0.0$ $0036428,-0.00168831,-0.00061205,-0.00080090,0.00020812,0.00003785,0.00$ $003361,0.00000717,-0.00031384,0.00008598,-0.00005263,-0.00016117,0.000$ $16468,-0.00011157,0.00001139,0.00002435,-0.00003453,0.00382865,-0.0637$ $7840,-0.02531301,0.07509381,0.01862134,0.02546810,0.08592035,-0.265935$ $17,-0.07222015,-0.04098151,0.56763766,0.01263435,-0.00600810,-0.008650$ $97,0.00278958,-0.00077969,0.00437749,0.00052013,-0.00077785,-0.0002192$ $4,-0.00045617,-0.00003372,0.00071601,0.00082131,-0.00016107,-0.0030059$ $3,-0.00285630,0.00630748,0.00127528,-0.00809367,-0.00021217,-0.0028664$ $8,0.00015789,-0.00112666,-0.00004580,-0.00008597,-0.00005634,-0.000054$ $23,0.00006745,-0.00012288,-0.00007547,0.00033910,-0.00017210,0.0003504$ 
$4,0.00010135,-0.00000536,0.00007897,0.00241056,-0.02547224,-0.01783723$ $, 0.02143131,0.01054703,0.02371465,0.01240498,-0.06391141,-0.07838120,0$ $.04590374,0.21270784,0.19605841,-0.00140019,-0.00216683,-0.00225179,-0$ $.00056279,-0.00234874,-0.00114065,-0.00038783,0.00174240,0.00073450,-0$ $.00070631,-0.00128978,0.00175687,0.00134209,-0.00065856,-0.00199379,-0$ $.00232818,-0.00010809,0.00011970,-0.00145012,-0.00015914,-0.00057159,-$ $0.00116148,0.00465486,0.00065497,-0.00000861,0.00002673,0.00000778,-0$. $00011641,0.00013607,-0.00006911,-0.00013303,0.00013604,-0.00010419,-0$. $00005904,-0.00004102,0.00001659,-0.03259504,0.06979068,0.02055327,-0.0$ 4017256,-0.02378937,-0.02249962,0.05284214,-0.01466117,0.00760319,-0.1 $2389838,-0.05244068,-0.04595355,0.59091053,-0.00002967,0.00203167,-0.0$ $0096575,0.00032786,0.00072441,0.00122569,0.00029466,-0.00068907,-0.000$ $04330,-0.00025909,0.00015801,-0.00077220,0.00026631,0.00014050,-0.0001$ $3419,0.00047707,-0.00094865,0.00056403,-0.00002549,0.00012525,0.000223$ $35,0.00015297,-0.00186393,0.00006786,-0.00001181,-0.00002073,-0.000034$ $89,0.00016575,-0.00014463,0.00003049,0.00006999,0.00004914,0.00018745$, $0.00009336,-0.00000023,0.00001055,0.01991434,0.02415220,0.01413391,-0$. $02231811,-0.01186170,-0.01175221,0.01514705,-0.04560581,-0.01821885,-0$ $.08257742,-0.24669210,-0.11540833,-0.09268536,0.62285983,-0.00081802,0$ $.00123259,-0.00078003,-0.00042863,-0.00044426,0.00046462,0.00026272,-0$ $.00045559,-0.00007723,0.00001645,0.00017253,-0.00002619,0.00030219,-0$. $00014452,-0.00024392,-0.00070120,-0.00090297,-0.00029959,-0.00010617,-$ $0.00023916,-0.00019258,-0.00022593,0.00072733,0.00029626,-0.00006704,-$ $0.00002623,-0.00001058,0.00002352,-0.00001692,0.00001177,-0.00004814,0$ $.00003677,0.00017610,-0.00002315,0.00000473,0.00001980,-0.00240088,0.0$ $2940822,0.01976359,-0.02238881,-0.01171757,-0.01408383,0.02165134,-0.0$ $2791113,-0.00231810,-0.05985825,-0.10658404,-0.11993937,0.09765552,0.2$ $0385167,0.24953308,0.00095462,-0.00016155,-0.00044949,0.00039594,0.000$ $05861,0.00025020,0.00008946,-0.00011745,-0.00000593,-0.00002820,0.0002$ $9369,-0.00010721,-0.00024047,0.00017847,0.00024487,0.00047018,-0.00019$ $025,0.00012957,0.00012610,-0.00000593,0.00005932,0.00050843,-0.0004777$ $2,-0.00082831,-0.00001921,-0.00001141,0.00000528,0.00002823,0.00002473$ $, 0.00003490,-0.00007215,0.00003642,-0.00014300,0.00001137,0.00001110,-$ $0.00001594,-0.15011742,0.06238721,0.00335021,0.05390277,0.02026351,0.0$ $2342669,-0.04729751,0.02951172,-0.00035388,-0.02004879,-0.07227794,-0$. $04307259,-0.30057509,0.05085328,-0.04871303,0.61908509,0.00031978,-0.0$ $0060107,-0.00038809,-0.00011680,-0.00083118,-0.00047027,-0.00010312,0$. $00060005,0.00004755,-0.00010616,-0.00040003,0.00070557,0.00029239,0.00$ $001594,-0.00023867,0.00009882,0.00046263,0.00011726,-0.00042332,-0.000$ $08393,-0.00020031,-0.00026193,0.00119975,0.00006098,-0.00002934,-0.000$ $00160,-0.00000926,-0.00008882,0.00008539,-0.00004106,-0.00011899,0.000$ $04999,-0.00014947,-0.00001907,0.00000079,-0.00001998,0.11815045,-0.258$ $10673,-0.05473096,-0.01883065,-0.04443460,-0.02987728,0.03018990,-0.01$ $793799,0.00140107,-0.02244506,0.02921118,0.00134306,-0.01555497,-0.106$ $17278,-0.02625173,-0.02185998,0.61117606,0.00087200,-0.00069665,0.0007$ $9142,-0.00046761,-0.00054527,-0.00005644,-0.00012268,0.00034234,0.0001$ $8859,-0.00024828,-0.00019024,0.00036832,0.00010911,-0.00002461,-0.0002$ $6746,-0.00019370,0.00042445,0.00019583,-0.00006386,-0.00000690,-0.0000$ $0577,0.00001128,0.00041665,-0.00027887,0.00000488,-0.00000154,-0.00000$ $961,-0.00003870,0.00002394,-0.00003303,0.00002961,0.00003672,-0.000000$ $03,-0.00001656,0.00000067,-0.00000750,0.02918541,-0.07146955,-0.089711$ 
$35,0.00537741,-0.01847316,0.00121004,0.00050340,0.00164910,-0.00097265$ ,-0.02037907,-0.01416715,-0.00071362,-0.07956002,-0.00618865,-0.087196 $23,0.13820111,0.21936687,0.27064678,0.00073035,-0.00011811,0.00008730$, $-0.00007704,-0.00060725,-0.00019002,-0.00012732,0.00040972,0.00013990$, $-0.00012208,-0.00033266,0.00027059,0.00019644,-0.00014065,-0.00038622$, $-0.00034013,0.00015457,0.00015134,0.00007750,0.00003333,0.00008488,0.0$ $0026187,0.00134285,-0.00060998,0.00002820,0.00001767,0.00000156,0.0000$ $1765,0.00003626,0.00000616,0.00005289,-0.00001928,0.00004564,-0.000012$ $59,0.00001082,0.00002348,-0.14288854,0.00748175,-0.03215791,-0.0331238$ $4,0.00217482,-0.01041771,0.00243446,-0.00399102,-0.00238592,-0.0007717$ $9,-0.00001974,-0.00015903,0.00181094,0.00491780,0.00157900,-0.03616746$ $, 0.00248463,-0.01114730,0.20938432,-0.00003692,-0.00002790,0.00006013$, $-0.00000912,0.00007516,0.00000837,-0.00000346,-0.00001723,-0.00000552$, $0.00000861,0.00001680,-0.00000208,-0.00002056,0.00001267,0.00002952,0$. $00002577,0.00003807,0.00001920,0.00002068,-0.00000951,0.00002215,0.000$ $03340,-0.00021412,-0.00003883,0.00000027,-0.00000567,-0.00000112,-0.00$ $000978,0.00000913,-0.00000376,-0.00000718,-0.00000197,-0.00001515,-0.0$ $0000580,0.00000078,-0.00000036,0.00750435,-0.03697108,-0.00380899,-0.0$ 2114022,0.00441301,-0.00649461,0.00141485,-0.00209586,-0.00253588,-0.0 $0012554,0.00137675,0.00095519,-0.00029877,-0.00247332,-0.00332147,0.02$ $575704,0.00179416,0.00635230,-0.01238262,0.03293179,0.00016360,0.00005$ $292,-0.00006723,0.00000994,-0.00016046,-0.00005637,-0.00002535,0.00009$ $298,0.00000329,-0.00001031,-0.00006425,0.00007851,0.00004619,-0.000014$ $72,-0.00005264,-0.00003512,-0.00000704,0.00000371,0.00004010,-0.000001$ $56,0.00000962,0.00011029,0.00032962,-0.00024527,0.00000185,0.00000065$, $-0.00000365,0.00000389,0.00001359,-0.00000105,0.00000520,-0.00000620,0$ $.00000130,-0.00000661,0.00000386,0.00000298,-0.03222385,-0.00380276,-0$ $.03537402,-0.02119559,0.00051162,-0.00136539,0.00006261,-0.00422986,0$. $00242388,-0.00003272,0.00100176,-0.00034028,-0.00091993,-0.00170193,0$. $00328497,-0.00022504,-0.00063391,0.00436851,0.05340332,0.00604369,0.03$ $079515,0.00038413,0.00007778,0.00006495,-0.00013365,-0.00052057,-0.000$ $12874,-0.00006269,0.00028594,0.00009520,-0.00008269,-0.00019750,0.0002$ $4030,0.00014829,-0.00003910,-0.00023706,-0.00025559,0.00018314,0.00005$ $332,-0.00004921,0.00003743,0.00002156,-0.00017499,0.00095602,0.0000729$ $4,-0.00000280,0.00000767,0.00000399,-0.00000011,0.00001476,-0.00000459$ ,-0.00000960,0.00001245,-0.00001294,-0.00000736,-0.00000434,0.00000142 $, 0.00815024,-0.01204691,-0.00412495,-0.13612829,0.13026745,0.02975862$, $-0.01132518,0.02321397,0.00599503,-0.00425204,0.00129628,-0.00266488,-$ $0.00047672,-0.00101738,-0.00030548,-0.00008105,-0.00208426,-0.00254763$ $, 0.00081186,-0.00105170,0.00056260,0.14192213,-0.00015932,0.00003158,-$ $0.00003684,-0.00003289,-0.00000553,-0.00001054,0.00000271,-0.00000915$, $-0.00001295,0.00000608,0.00001137,-0.00000401,-0.00001309,0.00001544,0$ $.00004287,0.00004132,-0.00004155,-0.00001340,0.00000668,0.00001426,-0$. $00000557,-0.00003131,0.00005196,0.00000911,-0.00000242,0.00000041,0.00$ $000127,-0.00000229,-0.00000167,-0.00000213,-0.00000270,-0.00000041,-0$. $00000404,-0.00000075,-0.00000006,-0.00000301,0.01516532,-0.01406937,-0$ $.00353900,0.12691102,-0.24293312,-0.05748642,-0.00193585,0.00274734,-0$ $.00144335,0.00058393,0.00252972,-0.00155468,-0.00063551,0.00009497,0.0$ $0027372,-0.00255460,-0.00111200,-0.00361442,-0.00005231,0.00051622,0.0$ $0143928,-0.13760671,0.25300522,0.00004682,0.00001708,0.00004917,0.0000$ $3518,-0.00010982,-0.00004339,-0.00001409,0.00010804,0.00005239,-0.0000$ 
$2433,-0.00007769,0.00006586,0.00003846,-0.00001110,-0.00008516,-0.0000$ $8776,0.00005493,0.00002928,-0.00004255,0.00002242,0.00002957,-0.000041$ $95,0.00014451,0.00013797,0.00000414,0.00000170,0.00000049,-0.00000144$, $0.00000490,-0.00000205,-0.00000397,0.00001486,-0.00000309,-0.00000069$, $-0.00000128,-0.00000062,0.00848916,-0.01190529,0.00053190,0.02817043,-$ $0.05624295,-0.05296557,-0.00576739,0.00616124,0.00584919,-0.00279193,-$ $0.00146481,0.00474130,-0.00015005,0.00025678,-0.00081099,-0.00282414,-$ $0.00346202,0.00256867,0.00102696,0.00114825,-0.00181887,-0.02787943,0$. $06431293,0.04510633,0.00024120,0.00015555,0.00042190,-0.00008195,-0.00$ $002473,-0.00002046,-0.00009240,0.00004814,0.00009648,-0.00002551,-0.00$ $010313,0.00005816,0.00000110,-0.00010948,-0.00005217,0.00001864,-0.000$ $08083,-0.00011035,-0.00014033,0.00032524,-0.00023823,-0.00106779,0.001$ $10383,0.00187544,0.00001026,0.00003536,0.00002576,0.00001577,-0.000022$ $78,-0.00000689,0.00001931,-0.00002551,0.00003852,0.00000557,-0.0000229$ $4,-0.00000788,-0.00318008,0.00157882,-0.00221161,-0.00879102,-0.022546$ $89,-0.01443973,-0.10650075,-0.09302075,-0.06292322,0.01068793,0.011637$ $59,0.00831481,-0.00087367,0.00439963,0.00028452,-0.00095567,0.00043079$ $, 0.00019497,-0.00116413,-0.00063093,-0.00062769,0.00092717,-0.00041524$ $, 0.00123979,0.11025278,-0.00106433,-0.00004860,-0.00028532,0.00008500$, $0.00044572,-0.00019177,-0.00002895,-0.00011467,0.00006690,0.00003606,0$ $.00007012,-0.00002024,-0.00006033,0.00004312,0.00018091,0.00014948,-0$. $00020637,0.00011170,0.00064215,-0.00012403,0.00028995,0.00008807,-0.00$ $043070,-0.00023689,-0.00000400,0.00002045,0.00002126,-0.00002318,0.000$ $01746,0.00001033,-0.00001196,-0.00003720,-0.00004849,-0.00001641,-0.00$ $000279,-0.00000355,0.00145716,0.00252744,-0.00137443,0.00352701,0.0055$ $8009,0.00097646,-0.09475915,-0.21942489,-0.11025475,-0.00965555,-0.016$ $78244,-0.01063115,0.00307889,-0.00199278,-0.00248428,0.00067597,-0.000$ $04854,0.00058238,0.00040374,0.00007699,0.00018514,-0.00060922,-0.00014$ $744,0.00155812,0.09790919,0.23136885,-0.00063674,0.00054185,-0.0000888$ $9,0.00025751,0.00029606,-0.00016760,0.00010288,-0.00014860,-0.00016153$ $, 0.00008340,0.00023322,-0.00018985,-0.00016550,0.00006684,0.00023558,0$ $.00003172,-0.00037071,-0.00004206,0.00007120,0.00002641,0.00005122,0.0$ $0078100,-0.00112830,-0.00113520,0.00000224,-0.00004215,-0.00001971,0.0$ $0001453,0.00000866,0.00001295,0.00000756,0.00002210,0.00000222,-0.0000$ $0492,0.00001007,-0.00000178,-0.00245460,-0.00128984,0.00547627,-0.0030$ $3759,-0.00711730,0.00149519,-0.06280530,-0.10851686,-0.10326461,-0.001$ $33333,-0.00451240,-0.00198089,-0.00040754,-0.00226156,0.00441731,0.000$ $39362,0.00046936,-0.00048367,-0.00023489,-0.00019177,-0.00014538,0.001$ $19926,0.00169076,-0.00307629,0.06940282,0.12142390,0.09860220,0.000518$ $29,0.00019472,-0.00016497,-0.00006842,0.00006363,0.00036266,0.00000990$ $,-0.00009613,0.00004385,-0.00030158,-0.00006618,0.00029692,-0.00016927$ $, 0.00031691,-0.00008540,-0.00009906,-0.00039886,-0.00036378,-0.0000193$ $0,-0.00002398,-0.00002652,0.00007054,-0.00046117,-0.00003142,0.0000195$ $0,0.00000248,0.00000568,-0.00005369,-0.00002208,-0.00003236,-0.0000392$ $2,0.00001295,0.00000393,0.00001022,-0.00000274,-0.00001299,-0.00296002$ ,-0.00010317,-0.002961 12,-0.00077325,-0.00047025,-0.00023624,-0.000294 $97,-0.00276557,-0.00301135,0.01084872,-0.00957487,-0.00141252,-0.13241$ $791,0.12372461,0.02806414,-0.01192372,0.02471254,0.00641105,-0.0013235$ $2,0.00078480,-0.00005590,-0.00007693,-0.00001099,0.00013634,0.00039944$ ,-0.00054984,-0.00014192,0.13787395,0.00081517,-0.00070500,0.00073891, $-0.00028887,-0.00017264,-0.00016484,-0.00003353,0.00011518,-0.00005991$ 
$, 0.00007285,0.00005686,0.00027642,0.00009238,0.00024080,0.00019852,0.0$ $0005440,0.00077200,-0.00011168,0.00009546,-0.00001669,0.00002617,0.000$ $08092,-0.00011189,-0.00023258,-0.00000026,-0.00001624,0.00000936,0.000$ $04807,-0.00004843,0.00003548,-0.00003325,0.00002217,-0.00007098,-0.000$ $01717,0.00001065,-0.00001623,0.00026445,0.00288440,-0.00179713,-0.0008$ $6118,-0.00000867,0.00022678,-0.00130363,-0.00127824,-0.00369129,0.0107$ $2879,-0.01793197,-0.00505214,0.12657974,-0.23790064,-0.05623894,-0.001$ $23920,0.00247814,-0.00151323,-0.00012676,-0.00001279,-0.00004469,0.000$ $04287,-0.00010910,0.00020421,0.00061297,-0.00045390,-0.00009155,-0.135$ $04709,0.25219204,0.00049629,-0.00008474,-0.00047471,0.00009946,0.00008$ $504,0.00005359,0.00005293,0.00005133,-0.00023967,0.00009499,0.00004308$ $, 0.00000261,-0.00009966,0.00016823,0.00031667,0.00017985,-0.00009158,-$ $0.00011247,-0.00016860,-0.00003735,-0.00002153,0.00007240,-0.00021839$, $-0.00001685,0.00001782,0.00000724,0.00000443,-0.00003445,0.00002463,0$. $00003167,-0.00018394,0.00007262,-0.00028926,0.00006043,0.00000445,-0.0$ $0002725,-0.00278844,-0.00171301,0.00505712,-0.00040721,0.00022133,-0.0$ $0083112,-0.00251016,-0.00396681,0.00294569,0.00831510,-0.01108430,-0.0$ $0201724,0.02922618,-0.05688799,-0.05261407,-0.00564795,0.00614496,0.00$ $627041,-0.00046860,0.00021647,0.00000140,0.00014092,0.00019458,-0.0004$ $0969,0.00038634,-0.00042990,-0.00002452,-0.02810071,0.06545092,0.04536$ $151,0.00025386,-0.00018279,-0.00006549,-0.00009352,-0.00037281,-0.0001$ $4168,-0.00005428,0.00023116,0.00006292,-0.00003423,-0.00014752,0.00025$ $353,0.00012724,-0.00006502,-0.00021706,-0.00014121,0.00013170,0.000062$ $78,-0.00011590,0.00001064,-0.00002115,-0.00006698,0.00063944,-0.000028$ $15,-0.00000583,0.00000002,0.00000234,-0.00002636,0.00003331,-0.0000178$ $1,-0.00001409,0.00001623,-0.00001982,-0.00003146,0.00000097,-0.0000016$ $8,0.00829032,0.01366054,0.00790532,-0.00087366,0.00357039,-0.00016292$, $-0.00063685,0.00070226,0.00049049,-0.00415675,0.00054918,-0.00298786,-$ $0.00866518,-0.02166488,-0.01398314,-0.10734791,-0.09484321,-0.06597998$ $, 0.00077373,0.00023804,0.00113776,0.00056641,0.00070040,0.00037630,-0$. $00002492,-0.00012243,0.00008901,0.00088482,-0.00039437,0.00132776,0.11$ $133220,0.00012535,0.00005414,-0.00001016,-0.00004609,0.00002540,0.0000$ $5283,0.00001958,-0.00004798,-0.00002782,0.00001785,0.00003527,-0.00004$ $011,-0.00000534,0.00001855,0.00004039,0.00002376,-0.00001930,-0.000024$ $88,-0.00000125,-0.00000303,0.00000036,0.00004129,-0.00003680,-0.000039$ $85,-0.00000176,-0.00000197,-0.00000128,0.00000848,-0.00000769,0.000003$ $99,-0.00000079,-0.00000313,0.00000742,-0.00000125,0.00000224,0.0000017$ $8,-0.01356332,-0.01396971,-0.01215500,0.00414102,-0.00178620,-0.001951$ $15,0.00042875,-0.00004727,0.00055371,0.00107072,0.00250028,-0.00152372$ $, 0.00356691,0.00489299,0.00140952,-0.09144079,-0.21670614,-0.11210576$, $-0.00074569,0.00041319,0.00121240,-0.00060955,-0.00056901,-0.00063308$, $-0.00008363,-0.00008512,0.00019067,-0.00059083,-0.00016914,0.00165185$, $0.09768565,0.22548775,0.00017657,-0.00013027,0.00002832,0.00002491,-0$. $00008345,-0.00004539,-0.00002238,0.00009643,0.00005563,-0.00005042,-0$. $00006807,0.00008719,0.00006893,-0.00003275,-0.00010612,-0.00010731,0.0$ $0008351,0.00007183,-0.00003135,0.00000079,-0.00000075,-0.00002713,0.00$ $013871,-0.00001686,0.00000503,0.00000539,-0.00000132,-0.00000391,0.000$ $00938,0.00000104,-0.00000681,-0.00000216,-0.00000933,0.00000720,-0.000$ $00467,0.00000022,-0.00482252,-0.00403006,0.00011938,0.00017728,-0.0021$ $2785,0.00405244,0.00031295,0.00063680,-0.00049507,-0.00273867,-0.00152$ $218,0.00475753,-0.00224439,-0.00622540,0.00076805,-0.06442290,-0.11315$ 
$723,-0.10712997,0.00070438,0.00152058,-0.00176092,-0.00022805,-0.00023$ $422,0.00021526,0.00009570,0.00016166,-0.00044734,0.00123905,0.00171357$ ,-0.00294079,0.07188142,0.12334435,0.10278329,0.00532791,-0.00023860,0 $.00017745,0.01262276,-0.01841030,0.01371503,-0.00117937,0.00576149,0.0$ $0386953,-0.00025469,-0.00258215,-0.00024912,0.00066478,0.00044929,-0.0$ $0141234,-0.00098187,0.00061404,-0.00027983,0.00004330,0.00041415,-0.00$ $066258,-0.15295709,-0.07379632,0.14248047,0.00084132,0.00017295,-0.001$ $47218,0.00016906,0.00003655,-0.00014622,-0.00018922,-0.00013872,-0.000$ $07467,-0.00002054,-0.00011024,0.00000112,0.00031929,-0.00006179,0.0000$ $6957,-0.00000719,0.00006387,0.00000748,-0.00038061,-0.00002985,-0.0001$ $0768,-0.00061620,0.00026122,-0.00005011,-0.00042954,0.00010028,-0.0000$ $5614,-0.00010147,-0.00014964,-0.00010941,-0.00029258,0.00000163,-0.000$ $08888,-0.00004683,0.00000224,-0.00000857,0.00041048,0.00010593,-0.0000$ $5771,-0.00000919,-0.00003203,0.00000155,-0.00007187,0.00000082,-0.0000$ $2212,0.14515367,0.00708309,-0.00534323,-0.00518677,-0.02248940,-0.0385$ $2537,0.04497803,-0.00142789,-0.00198965,0.00237722,-0.00083517,-0.0010$ $6808,0.00167859,0.00005473,-0.00030499,-0.00013382,-0.00110976,-0.0008$ $3774,0.00191314,-0.00051693,0.00013594,-0.00009274,-0.07876567,-0.2223$ $1928,0.15136157,-0.00034120,-0.00425510,0.00093862,-0.00002342,-0.0001$ $1848,0.00001494,0.00011170,-0.00085955,0.00006878,0.00005452,-0.000149$ $35,-0.00010785,0.00053070,-0.00002526,0.00015613,-0.00012588,0.0005264$ $1,0.00016658,-0.00120681,-0.00072924,-0.00085418,0.00023549,-0.0000685$ $3,0.00040847,-0.00136610,0.00049377,-0.00021947,0.00000879,-0.00031162$ $,-0.00017644,-0.00059434,0.00006636,-0.00016326,-0.00026803,-0.0000093$ $9,-0.00001395,0.00007359,0.00003745,0.00012171,0.00014660,0.00004118,0$ $.00009303,-0.00019388,0.00000046,-0.00004575,0.09254745,0.22958367,-0$. $00442370,0.00158468,0.00278748,0.01305968,0.03799831,-0.00881828,0.004$ $56051,-0.00970963,-0.00601326,-0.00073047,0.00450078,-0.00020741,-0.00$ $122074,-0.00085887,0.00264820,-0.00003570,-0.00169586,-0.00032522,0.00$ $060537,-0.00098891,0.00130621,0.14393647,0.14305510,-0.34294869,-0.001$ $55971,0.00008538,0.00252504,-0.00008898,-0.00002598,0.00037086,-0.0001$ $5252,0.00016793,-0.00008301,-0.00001094,0.00018932,-0.00003381,-0.0005$ $9495,0.00021795,-0.00002800,-0.00004984,-0.00003667,-0.00005559,0.0009$ $7447,0.00009115,0.00010201,0.00174196,-0.00020117,-0.00014984,0.000887$ $99,-0.00034120,0.00011175,0.00032109,0.00031945,0.00031656,0.00064969$, $-0.00001384,0.00022040,0.00016962,0.00000756,-0.00005318,-0.00108741,0$ $.00002432,0.00032831,0.00000032,0.00012831,-0.00006607,0.00017782,0.00$ $000828,0.00004150,-0.21727355,-0.18153817,0.43189906,-0.00426515,-0.00$ $263003,0.00281762,0.00983175,0.02419426,0.01409688,0.00415604,-0.00178$ $629,-0.00107300,-0.00053438,0.00267964,-0.00168604,-0.00059618,-0.0004$ $5018,0.00193147,-0.00007423,-0.00123330,-0.00147653,0.00068617,0.00028$ $119,-0.00105899,-0.15551598,0.08315703,0.13849045,0.00053223,-0.000633$ $82,-0.00029275,0.00017408,0.00001177,-0.00003371,0.00000555,0.00005389$ ,-0.00003221,-0.00043793,0.00007835,-0.00027380,0.00022149,-0.00029045 ,-0.00024769,0.00025022,-0.00129550,-0.00025888,-0.00012327,0.00160094 $, 0.00211432,0.00019616,0.00004179,-0.00010391,0.00151235,-0.00031574,0$ $.00028463,-0.00059608,0.00035602,-0.00002543,-0.00016918,-0.00006683,-$ $0.00010037,0.00025549,0.00003628,0.00007471,-0.00009142,-0.00125328,-0$ $.00115673,-0.00010532,-0.00011071,-0.00006228,0.00013246,-0.00003991,0$ $.00003454,-0.00801430,0.00841781,0.06014353,0.15256942,0.00387582,-0.0$ $0380507,-0.00122617,0.02638317,-0.04259141,-0.04231334,-0.00382054,-0$. 
$00244018,0.00737480,0.00071052,-0.00475637,-0.00043964,0.00131944,-0.0$ $0023279,-0.00267265,0.00017375,0.00136794,-0.00055486,0.00014889,-0.00$ $407259,-0.00066358,0.08971631,-0.23721672,-0.14892447,0.00010180,0.000$ $43910,-0.00007474,0.00001464,-0.00013022,-0.00006495,-0.00006584,-0.00$ $012190,0.00014643,0.00008511,-0.00090705,0.00010976,0.00025708,0.00011$ $082,0.00017130,-0.00024144,0.00097659,0.00029392,-0.00177458,-0.001212$ $70,-0.00143923,0.00199333,0.00054102,0.00074054,-0.00150821,0.00064364$ $,-0.00015879,0.00027625,-0.00032586,-0.00002185,-0.00033753,0.00008838$ ,-0.00005685,-0.00034046,-0.00001067,-0.00008798,-0.00122386,-0.000288 $22,0.00056943,0.00022452,0.00012192,0.00008430,-0.00018301,0.00002225$, $-0.00004709,-0.00498226,0.04602962,0.00703175,-0.11080291,0.24777046,0$ $.00750108,0.00609439,-0.00552883,0.01425692,-0.03454617,-0.00184188,-0$ $.00062607,0.00231572,0.00405538,-0.00113316,-0.00350693,0.00142630,0.0$ $0097176,0.00098426,-0.00206690,-0.00062298,0.00084644,0.00204195,-0.00$ $180738,0.00030876,0.00236291,0.13712562,-0.14069124,-0.31249353,-0.000$ $27614,0.00128138,0.00091337,-0.00015712,0.00003066,0.00020696,-0.00000$ $676,-0.00013223,0.00003502,-0.00019621,-0.00019453,-0.00006038,-0.0000$ $0553,0.00018589,0.00015937,-0.00013016,0.00098030,0.00033542,-0.001120$ $88,-0.00145151,-0.00131505,-0.00141869,0.00044689,0.00020771,-0.001350$ $34,0.00038466,-0.00025458,0.00039255,-0.00034587,-0.00005893,-0.000016$ $59,0.00005751,0.00003737,-0.00024598,-0.00000751,-0.00010847,-0.000709$ $58,0.00036774,0.00060807,0.00007324,0.00007655,0.00004014,-0.00014070$, $0.00003527,-0.00002781,0.06174892,-0.01574441,-0.08387503,-0.21210583$, $0.18222398,0.39520142 \backslash 10.00000366,-0.00000022,0 .,-0.00000205,0.0000018$ $1,0.00000534,0.00000173,0.00000537,0.00000314,0.00000257,0.00000495,-0$ $.00000048,0.00000225,0.00000183,-0.00000363,-0.00000163,0.00000031,-0$. $00000074,-0.00000260,-0.00000398,0.00000236,-0.00000265,0.00000273,0.0$ $0000791,0.00000179,0.00000810,0.00000475,0.00000298,0.00000162,-0.0000$ $0579,-0.00000074,-0.00000441,-0.00000394,0.00000362,0.00000777,-0.0000$ $0196,0.00000021,-0.00000284,-0.00000335,-0.00000126,-0.00000402,-0.000$ $00011,0.00000008,-0.00000463,0.00000207,-0.00000277,-0.00000536,-0.000$ 00006,-0.00000098,0.00000065,-0.00000610,0.00000138,-0.00000132,-0.000 $00626,0.00000045,-0.00000305,-0.00000492,-0.00000276,-0.00000514,0.000$ $00204,-0.00000342,-0.00000410,0.00000475,0.00000201,0.00000010,-0.0000$ $0533,0.00000271,-0.00000011,-0.00000837,-0.00000162,0.00000528,0.00000$ 939,-0.00000297,-0.00000132,0.00000931III@

meta-Transition State

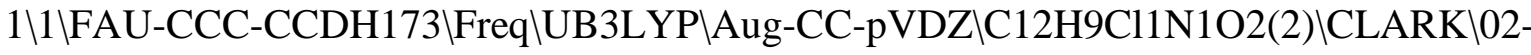 $\mathrm{M}$}

ar-2015\0\\N Geom=AllCheck Guess=TCheck SCRF=Check GenChk UB3LYP/Aug-

CC-pVDZ Freq \\Addition TS p-Cl-Ph-radical + nitrobenzene (meta) \\0,2\C ,-1.1522836253,1.4524544305,1.0468466009\C,-1.8617756668,0.2263349876, $1.1201086892 \backslash \mathrm{C},-2.7253597025,-0.110440613,0.0869941082 \backslash \mathrm{C},-2.9765306936$ $, 0.7420854648,-0.9957304219 \backslash \mathrm{C},-2.3443980191,1.9925176303,-1.0151045701$ IC,-1.4701085843,2.3618462463,0.003667974\H,-0.6471449998,1.8207659933 $, 1.9370019269 \backslash \mathrm{H},-1.7191365948,-0.4615739283,1.9489394926 \backslash \mathrm{N},-3.42715711$ $49,-1.4111159663,0.1447254194 \backslash \mathrm{H},-2.5578376064,2.6869245404,-1.82756663$ $24 \backslash \mathrm{H},-1.000304706,3.3448123706,-0.0100177222 \backslash \mathrm{H},-3.6654148235,0.4337234$ $65,-1.777219405 \backslash C, 3.3420385975,-0.3301210658,-0.1811772964 \backslash \mathrm{C}, 3.0311269$ 
353,-0.1327914613,1.1661843074\C, $1.7755263052,0.3974906431,1.503689868$ $1 \backslash \mathrm{C}, 0.8979564757,0.7089007534,0.4790464799 \backslash \mathrm{C}, 1.1868297009,0.5173341602$ ,-0.8605434287\C,2.440419002,-0.0143544204,-1.2008148773\Cl,4.91789561 $3,-0.9969032007,-0.6079576246 \backslash \mathrm{H}, 3.7546679944,-0.3893901334,1.938973156$ $5 \backslash \mathrm{H}, 1.5202952632,0.5515441557,2.5534356323 \backslash \mathrm{H}, 0.4717421899,0.7708344765$ $,-1.6437388764 \backslash \mathrm{H}, 2.712099505,-0.1811686015,-2.2423392935 \backslash \mathrm{O},-3.21776404$ $94,-2.1333913701,1.1158684833 \backslash 0,-4.1803503957,-1.694113557,-0.78368099$ $01 \|$ Version=EM64L-G09RevC.01 $\backslash$ State $=2-A \backslash H F=-1128.0108602 \backslash S 2=0.781051 \backslash S 2$ $-1=0 . \mathrm{IS} 2 \mathrm{~A}=0.750698 \backslash \mathrm{RMSD}=8.492 \mathrm{e}-09 \backslash \mathrm{RMSF}=4.195 \mathrm{e}-06 \backslash \mathrm{ZeroPoint}=0.1802945 \backslash \mathrm{T}$ hermal $=0.1938573 \backslash \mathrm{ZPE}=113.1365102 \backslash$ Dipole $=0.4120724,1.7961462,0.1068742 \backslash$ DipoleDeriv $=0.3208538,-0.0898387,0.1196462,-0.0756858,-0.0950897,0.112$ $7888,0.0255712,0.0981057,0.0369448,-0.0776739,0.1446568,-0.0249144,0.0$ $029894,0.0266787,-0.1465543,0.0827763,0.1368972,-0.1137388,-0.056097,-$ $0.1920258,0.032184,-0.2303132,-0.2556506,-0.0216136,0.0746,-0.0495287$, $0.1111471,-0.1246362,0.0126415,0.0442283,0.1412032,0.0146551,0.1038532$ $,-0.2287651,-0.0454703,-0.0816589,-0.1584753,-0.042065,0.025021,-0.147$ $3952,-0.0130208,-0.1610728,0.0580383,-0.1464996,0.029943,-0.0909805,0$. $1215175,0.0788799,0.1210307,0.0331249,0.0131818,0.0822242,-0.0588001,-$ $0.04223,0.0919776,-0.0339446,-0.0703754,-0.0417515,0.053093,-0.005045$, $-0.0614237,-0.021004,0.0000017,0.1188319,0.0031976,-0.0345916,0.027588$ $6,0.0997653,0.0340636,-0.0250422,0.0251559,0.0824039,0.9951999,0.50384$ $08,0.458868,0.8529367,1.3940552,-0.2599567,0.4430128,-0.2627941,1.1829$ $223,0.0973522,-0.0061978,-0.0370778,-0.001261,0.0140448,0.072922,-0.05$ $3842,0.0842669,0.0142577,0.0728828,-0.083321,-0.0235665,-0.058656,-0.0$ $140459,0.0089657,-0.0153598,0.0216182,0.0854275,0.1125706,-0.0104705,-$ $0.0408838,-0.001363,0.1160761,-0.0119616,-0.0260495,-0.0024828,0.08284$ $26,1.0560885,-0.4155265,-0.2394636,-0.4668251,0.1329482,0.1038399,-0.3$ $049803,0.1059201,0.0607477,-0.2003634,0.0246192,0.1096444,0.0593942,-0$ $.0974838,-0.0430902,-0.0047306,-0.0180331,-0.0278398,-0.3030602,0.0141$ $202,-0.043431,0.0972849,-0.0958085,0.021945,0.3387121,-0.0365995,0.109$ $5037,0.5303456,-0.0747925,-0.0344655,-0.2601382,-0.0391456,-0.0181211$, $-0.1302004,0.0016856,-0.0405283,-0.434152,0.0361482,0.04404,0.1244409$, $-0.0892596,-0.0094317,-0.1009527,0.0206056,-0.0088491,-0.1723178,0.044$ $174,-0.0110592,0.0640829,-0.0984017,0.0156039,0.1260078,-0.0158367,0.0$ $587884,-0.7914289,0.2496725,0.1402701,0.3017319,-0.1704549,-0.0599862$, $0.1913855,-0.0670723,-0.1224063,-0.0272057,0.0427,-0.0633729,0.0615812$ $, 0.1103967,0.0217377,-0.0316833,0.0108245,0.0358216,0.0778417,0.030186$ $6,0.0648258,0.0127223,0.1076867,-0.0310185,0.0100392,-0.02206,-0.05874$ $7,0.0514566,0.0356749,-0.0745009,0.0110552,0.0908328,0.0236919,-0.0491$ $415,0.0068737,0.0275476,0.0186917,0.0303033,0.0349946,0.0496883,0.1112$ $409,-0.0225044,0.0434525,-0.0187068,-0.0126554,-0.4077747,-0.1180435,-$ $0.0887137,-0.2295084,-0.7668779,0.3912058,-0.0823876,0.3011862,-0.7244$ $56,-0.6999272,-0.2272272,-0.3661859,-0.4148329,-0.5693594,-0.1334433,-$ $0.3612612,-0.0482515,-0.6851901 \backslash$ Polar $=249.4187537,-9.3975903,155.41919$ 32,14.5684867,-6.5879715,162.6708939\PG=C01 [X(C12H9Cl1N1O2)]\NImag=1। $10.19966547,0.19062225,0.54772751,0.21961960,-0.05294496,0.59047828,-0$ $.09725445,-0.10183629,-0.02697325,0.36412969,-0.07844954,-0.25622830,-$ $0.00033940,0.15312714,0.56400332,-0.00129233,0.04167492,-0.11126305,0$. $20768859,-0.15617162,0.58740833,-0.02516257,-0.03641509,-0.00191993,-0$ $.16609908,-0.02722913,-0.13651737,0.35463545,-0.01660376,-0.00544123,-$ $0.02330249,-0.05700497,-0.11266405,-0.04053344,0.10875942,0.46944137,-$ 
$0.03313195,-0.04656156,0.02606840,-0.11157846,0.00684108,-0.23215242,0$ $.23402670,-0.11554206,0.58528830,0.00341775,-0.00615919,0.00557589,0.0$ $0337710,0.01180680,-0.01866060,-0.09410893,0.02336702,-0.06905122,0.38$ $678933,-0.00168440,-0.04317935,0.02048257,0.03429775,0.03984959,0.0300$ $4145,-0.00812891,-0.16400333,0.07287214,0.18087058,0.56540941,0.017354$ $90,0.02011775,-0.01331591,-0.03703611,-0.00776851,-0.04476325,-0.04256$ $634,0.12060686,-0.22367864,0.24451004,-0.07568285,0.55836995,-0.010076$ $26,0.03334721,-0.03025127,-0.02126512,-0.01589901,-0.01611090,0.022833$ $66,-0.01448122,0.03816579,-0.12585949,-0.07792443,-0.03596057,0.351528$ $62,0.00297327,0.04690601,-0.00140862,-0.01961181,-0.02075678,-0.020506$ $79,0.00860207,-0.04229884,0.05096601,-0.11473281,-0.27195448,-0.008141$ $47,0.13498412,0.59722660,-0.02680245,0.02346610,-0.03584735,-0.0104477$ $9,-0.01126660,-0.01176934,0.01896850,0.01578374,0.02152023,-0.00851710$ $, 0.04569700,-0.10712138,0.22027154,-0.14881960,0.56037116,-0.06897863$, $-0.00457228,-0.04661575,0.01838876,-0.00008681,0.02166173,-0.01471146$, $0.00668656,-0.01911362,-0.01766716,-0.04628632,-0.00209145,-0.17259003$ ,-0.03486660,-0.13997697,0.37466833,-0.00365072,-0.18750314,0.11755514 $, 0.00116048,-0.02523750,0.02103676,0.00278111,-0.00272182,0.00180895,-$ $0.02241936,-0.00574333,-0.02334901,-0.06878726,-0.13030291,-0.05171157$ $, 0.18331009,0.63250495,-0.02831086,0.06938556,-0.20594072,0.02280304,0$ $.03196558,0.02100742,-0.01678297,0.01010928,-0.03964345,-0.02087949,-0$ $.06220260,0.02344156,-0.11316794,0.00080752,-0.22358457,0.21157095,-0$. $07907863,0.49090318,-0.08394160,-0.05366436,-0.11485405,-0.01042992,-0$ $.00900983,-0.01364467,0.00446647,-0.00477093,0.00201603,-0.00105502,-0$ $.00039448,-0.00059154,0.00582555,0.00204998,-0.00130874,-0.00494949,0$. $00047562,-0.00980320,0.10722077,-0.06039934,-0.08408640,-0.07575745,-0$ $.01012212,-0.00325903,-0.02198052,-0.00315941,-0.00302350,0.00125377,0$ $.00050646,-0.00046169,0.00078399,-0.00127950,-0.00280649,0.00329448,0$. $01177017,0.00922996,0.01943315,0.05497615,0.08635590,-0.12792435,-0.07$ $385837,-0.24404512,0.00459898,0.00495911,0.00638608,-0.00090353,0.0014$ $6483,0.00050450,0.00106758,0.00057711,0.00059215,-0.00167328,0.0032906$ $7,-0.00175684,-0.00862990,-0.01075744,-0.01049087,0.12570640,0.0793370$ $5,0.25327950,0.00577200,-0.01060266,0.01289506,-0.05025072,0.02011204$, $-0.03857867,0.00280414,0.01204727,-0.01492506,0.00055913,-0.00329043$,$0.00425039,-0.00094829,-0.00019454,0.00012504,0.00167922,-0.00426291,-$ $0.00232537,-0.00161698,0.00117282,0.00056396,0.04331179,0.00517188,-0$. $00961823,0.01862812,0.01926701,-0.16644660,0.14674733,-0.00097719,0.00$ $938665,-0.00500377,-0.00336214,0.00112198,-0.00123097,-0.00018232,-0.0$ $0063922,-0.00067663,-0.00474422,-0.00203717,0.00058152,0.00152734,0.00$ $051014,-0.00084366,-0.01701262,0.17146244,-0.00033999,-0.00155687,0.00$ $444583,-0.03972669,0.14329468,-0.22464260,-0.00279392,0.01619426,-0.01$ $270184,-0.00455884,-0.00176130,-0.00040944,0.00047207,-0.00062046,0.00$ $013216,-0.00218898,0.00019506,0.00247871,0.00158878,-0.00038356,-0.000$ $09319,0.04439029,-0.15781012,0.23160653,0.00288290,-0.00085470,-0.0085$ $5645,-0.01657498,-0.02324085,-0.00179186,-0.09367181,-0.01072479,-0.00$ $745727,0.00378358,-0.00613904,0.01336927,0.00700985,0.00328859,-0.0040$ $3154,-0.00141264,0.00016540,-0.00115863,0.00008435,-0.00106071,0.00038$ $116,-0.00242326,0.00176892,0.00175464,0.46212962,-0.00079548,0.0072862$ $6,0.00079321,-0.02080617,-0.01795428,-0.01674185,-0.00967859,-0.107363$ $93,0.00723567,-0.00964616,-0.03412760,0.01244051,0.00251328,0.00598083$ $, 0.00635982,0.00051216,-0.00045211,-0.00071036,-0.00002721,0.00097067$, 
$0.00042675,0.00384237,-0.00083285,0.00170019,0.10552508,0.57404611,-0$. $00927105,-0.00111867,-0.00226997,-0.00299987,-0.02043198,0.01212022,-0$ $.00913410,0.00598661,-0.10150464,0.01682726,0.01625934,0.00919648,-0.0$ $0387084,0.00732237,-0.00335997,-0.00118426,-0.00005939,-0.00370400,-0$. $00067515,-0.00103722,0.00018975,-0.00010552,-0.00089115,-0.00364579,0$. $26770723,-0.13915362,0.77162199,0.00169030,-0.00416905,-0.00535658,-0$. $00056517,0.00007470,0.00026520,0.00270565,-0.00410876,-0.00264320,0.00$ $554915,-0.01017409,0.01101064,-0.05579442,0.03034301,-0.04965151,-0.00$ $056048,0.01327067,-0.01450620,-0.00005101,0.00087448,-0.00083919,-0.00$ $037255,0.00030884,0.00029835,0.00073741,-0.00051368,0.00046602,0.05066$ $256,-0.00322306,0.00188720,-0.00062187,-0.00041824,-0.00032014,-0.0008$ $4962,-0.00458951,-0.00243174,-0.00012200,0.00638020,-0.01285679,0.0211$ $7233,0.02967015,-0.16955683,0.13811968,-0.00277450,0.00856253,-0.00711$ $308,-0.00014556,0.00012580,-0.00033336,0.00025927,-0.00016376,-0.00007$ $658,0.00066383,0.00021249,0.00156598,-0.02850392,0.17513192,-0.0053174$ $3,-0.00062390,-0.00014143,0.00002539,-0.00092312,-0.00028979,-0.002286$ $96,0.00065674,0.00332976,-0.00057236,-0.00148823,0.00303098,-0.0501139$ $3,0.13781026,-0.20915344,-0.00243959,0.01590107,-0.01161552,-0.0002412$ $4,0.00062778,-0.00047814,0.00032267,-0.00017336,-0.00021207,-0.0004095$ $9,-0.00023793,0.00046640,0.05675998,-0.14971680,0.21627111,0.00616003$, $0.00825998,-0.00124127,0.00324557,-0.00039882,-0.00313931,-0.00037784$, $0.00020058,-0.00052562,0.00234976,-0.00195365,-0.00576940,-0.00371886$, $-0.01784453,0.00130666,-0.09726278,-0.11728252,-0.00640364,-0.00166640$ $, 0.00062327,0.00087949,0.00022731,0.00036307,0.00006474,-0.00078098,0$. $00069178,0.00027829,-0.00203519,0.00150184,0.00191819,0.09371224,-0.00$ $930500,-0.01288533,-0.00177737,0.00016914,-0.00022367,0.00382078,0.000$ $60028,0.00069290,0.00037208,-0.00225553,0.00226167,-0.00013957,-0.0017$ $2849,-0.00293563,0.00292395,-0.11600134,-0.28565975,0.00667692,0.00103$ $663,0.00041129,-0.00040982,-0.00043601,-0.00057783,-0.00026733,0.00030$ $707,-0.00045270,-0.00011652,0.00133201,0.00006737,-0.00102827,0.127053$ $13,0.29921535,0.01059855,0.02043470,0.00370114,-0.00351247,0.00402463$, $-0.00166504,-0.00046648,0.00050501,-0.00119016,-0.00573831,0.00007922$, $-0.00113844,-0.00986139,-0.02087553,0.00475041,-0.00738963,0.00831255$, $-0.05190723,0.00158480,-0.00110088,0.00021942,0.00068014,0.00076566,0$. $00001129,0.00028613,-0.00046799,-0.00077495,0.00221986,-0.00079339,-0$. $00038712,0.01195243,-0.01005555,0.04764267,-0.00055491,0.00027376,0.00$ $085995,0.00351838,-0.00034345,-0.00109646,0.00052605,-0.00204126,-0.00$ $446621,-0.16174345,-0.05620164,-0.14383083,-0.00289237,-0.00173206,-0$. $00923061,0.00378126,-0.00379400,-0.00123874,-0.00040777,0.00011455,0.0$ $0013900,0.00033385,-0.00027493,-0.00027502,-0.00321304,0.00069614,0.00$ $043554,-0.00182712,0.00138061,0.00190631,-0.00033994,0.00001954,-0.000$ $36036,0.16278684,0.00017936,-0.00095006,0.00013936,-0.00094993,-0.0020$ $3477,0.00432074,0.01188753,0.00998283,0.01303786,-0.05728338,-0.073933$ $83,-0.05465593,-0.01613961,-0.00145855,-0.01943512,-0.00384564,-0.0034$ $8250,0.00201796,0.00019354,-0.00003363,-0.00015218,0.00057826,0.000652$ $89,0.00091539,0.00250609,0.00051869,0.00019457,0.00163090,0.00035418,-$ $0.00074614,-0.00067511,-0.00021019,-0.00094936,0.06425492,0.07300153,0$ $.00054903,0.00014354,-0.00013242,-0.00133664,0.00451796,-0.00109009,-0$ $.01523103,-0.00772756,-0.01201170,-0.14251887,-0.05301222,-0.20524387$, $0.00240702,0.00228309,0.00590606,-0.00162500,0.00215526,0.00267283,0.0$ $0026345,-0.00015931,-0.00014851,-0.00089593,-0.00032241,-0.00040778,-0$ 
$.00112047,-0.00270498,-0.00340920,0.00166421,-0.00104655,-0.00061391,0$ $.00026099,0.00015199,0.00016564,0.15588816,0.05684771,0.21088097,-0.00$ $050672,0.00013590,0.00000926,-0.00003331,0.00002484,-0.00005200,0.0000$ $2195,0.00011436,-0.00004917,0.00016085,-0.00009548,0.00032939,0.000141$ $02,0.00017268,-0.00002258,0.00004519,-0.00002041,-0.00011375,-0.000148$ $16,0.00006094,-0.00001611,-0.00010702,-0.00007947,0.00004907,-0.000553$ $50,-0.00080346,-0.00005628,-0.00000905,-0.00000422,0.00005722,-0.00004$ $212,0.00000083,0.00000114,-0.00003956,-0.00002955,-0.00000781,0.453483$ $28,0.00032764,-0.00012960,-0.00006115,-0.00005149,0.00001500,0.0000145$ $2,-0.00001626,-0.00014516,0.00002944,-0.00007788,0.00003126,-0.0001542$ $4,-0.00002897,-0.00007802,0.00001276,-0.00002969,0.00003983,0.00011264$ $, 0.00008460,0.00002733,-0.00000367,-0.00001705,0.00002818,-0.00000369$, $0.00023084,0.00036058,0.00000151,0.00000850,0.00000632,-0.00002051,0.0$ $0002494,-0.00004174,-0.00001167,0.00001866,0.00001255,0.00001315,-0.13$ $172331,0.18175123,-0.00001762,-0.00029977,-0.00045985,0.00009763,0.000$ $18685,0.00002110,-0.00032954,-0.00010701,0.00009167,0.00010672,-0.0000$ $4883,0.00008536,-0.00007362,-0.00014496,0.00009072,0.00016694,0.000052$ $48,-0.00009862,-0.00013465,0.00013432,0.00017425,-0.00003796,-0.000100$ $38,0.00004654,0.00031275,0.00054888,-0.00002078,0.00000774,0.00002645$, $-0.00002215,-0.00000930,-0.00007355,-0.00000226,0.00000052,0.00000986$, $0.00000516,0.04233360,0.00962297,0.64128185,0.00092534,-0.00020370,-0$. $00110414,0.00031154,0.00012269,0.00015578,-0.00028972,-0.00005982,-0.0$ $0009502,0.00003321,-0.00011320,0.00008111,-0.00001122,0.00004982,0.000$ $13836,-0.00022229,0.00007015,-0.00000776,0.00008710,0.00012243,0.00043$ $151,-0.00009702,-0.00006436,0.00004783,0.00020392,0.00050289,-0.000121$ $98,0.00003791,-0.00002288,0.00001040,0.00002041,-0.00006982,-0.0000261$ $8,0.00001054,0.00000007,0.00001685,-0.10630112,0.01733502,0.00334072,0$ $.60641361,-0.00069221,0.00134373,0.00015642,0.00036866,-0.00005048,0.0$ $0002464,0.00009785,0.00028133,-0.00009281,0.00006406,-0.00001046,0.000$ $04424,-0.00010686,0.00005790,-0.00010870,-0.00003907,-0.00021158,0.000$ $12246,0.00009212,-0.00003302,-0.00002645,0.00012030,0.00002546,-0.0000$ $8015,-0.00016934,-0.00036325,0.00014851,0.00001495,-0.00001612,-0.0000$ $2056,0.00000661,0.00003027,0.00001787,0.00000572,-0.00000712,-0.000019$ $39,0.02032725,-0.07452652,-0.01466718,-0.19328558,0.20729193,-0.000243$ $14,-0.00010549,-0.00048607,-0.00070603,0.00012265,0.00022545,0.0004268$ $0,0.00025918,0.00002794,-0.00033919,-0.00013715,-0.00047822,0.00017331$ $, 0.00006223,0.00016425,-0.00071859,0.00027511,0.00013927,-0.00019041,0$ $.00014104,-0.00015470,-0.00006035,-0.00000208,-0.00004398,-0.00010830$, $0.00004364,-0.00008858,0.00000949,-0.00001354,0.00002286,-0.00003120,0$ $.00010848,-0.00003956,0.00002497,0.00002657,0.00003869,0.06273622,-0.0$ $3877171,-0.31744736,0.05701155,0.00539611,0.68870803,-0.00382880,0.001$ $20849,-0.00287302,-0.00090319,0.00127271,0.00068157,0.00048453,0.00039$ $270,-0.00035217,-0.00058092,-0.00039355,-0.00067230,0.00026271,0.00009$ $564,0.00021128,-0.00198883,0.00001223,0.00075133,0.00000060,-0.0002470$ $8,-0.00063881,-0.00002852,-0.00000685,-0.00003872,0.00012042,0.0004428$ $8,-0.00013921,0.00000589,0.00000201,-0.00012684,-0.00007742,0.00027377$ $,-0.00007774,-0.00000462,0.00008657,0.00006334,0.00119299,0.00666661,0$ $.08144596,-0.27031385,0.08579310,0.00925634,0.54019844,0.00177747,-0.0$ $0042966,0.00139276,0.00087470,-0.00040068,-0.00020829,-0.00050660,-0.0$ $0024779,-0.00035671,0.00024750,-0.00018800,0.00055967,0.00023954,0.000$ $39202,0.00020899,0.00047220,-0.00009130,-0.00059833,-0.00024644,0.0001$ 
$8817,0.00019368,0.00006408,0.00000437,-0.00003454,0.00002696,-0.000177$ $15,0.00014252,-0.00001457,0.00001078,0.00007792,0.00001786,-0.00013367$ ,-0.00005237,-0.00000602,-0.00005854,-0.00004749,0.00369454,0.00385576 ,-0.03385201,0.08895839,-0.09779190,-0.00649766,-0.16856231,0.19671019 $, 0.00049232,0.00024618,0.00168078,0.00094674,-0.00034423,-0.00053169,0$ $.00009986,0.00001267,-0.00007612,0.00044910,0.00035086,0.00087806,-0.0$ $0017610,0.00025540,-0.00021247,0.00082374,-0.00069387,-0.00045760,0.00$ $111997,-0.00059038,0.00070590,0.00020024,0.00005409,0.00011532,-0.0005$ $9685,-0.00129915,0.00019111,-0.00000384,-0.00005065,0.00002778,0.00003$ $554,-0.00004461,-0.00002127,-0.00001335,-0.00006875,-0.00008278,0.0286$ $5580,-0.01191009,0.00612251,0.07885607,-0.03510241,-0.12704429,-0.0005$ $0468,0.02876723,0.72452825,0.07084746,-0.01533113,-0.00467696,-0.02883$ $348,-0.02439571,-0.00106596,0.00422852,-0.00088570,0.01203064,-0.00303$ $645,-0.00231305,-0.00528284,0.00704135,0.00821254,0.00690265,-0.017687$ $63,0.01961847,-0.02245228,-0.01201395,0.00543091,0.00613731,0.00077750$ $, 0.00002596,-0.00066059,-0.00010527,-0.00209580,0.00114988,0.00041582$, $0.00005657,0.00037910,0.00043434,-0.00096930,-0.00009295,0.00012123,-0$ $.00009322,0.00004664,-0.00786459,-0.00069416,-0.01670470,-0.04324241,0$ $.02341340,-0.00604446,-0.17851553,0.04469443,-0.13084001,0.28873173,-0$ $.02617844,-0.00171970,-0.00123821,0.00064760,0.00766886,0.00022469,-0$. $00075692,-0.00055038,0.00004255,0.00136236,0.00361078,-0.00059710,-0.0$ $0403044,-0.00560809,-0.00349681,0.01048232,-0.00652534,0.00969872,0.00$ $783439,-0.00302324,-0.00311353,-0.00007795,-0.00003495,0.00009057,0.00$ $074349,0.00122936,-0.00027033,-0.00027059,0.00002538,-0.00017733,-0.00$ $034990,0.00062666,0.00013407,-0.00004922,0.00014288,0.00006849,-0.0005$ $2113,-0.00660422,0.00374324,0.02026547,0.00474560,0.00409014,0.0461530$ $6,-0.07043150,0.04431971,-0.08252929,0.11991557,-0.01853037,0.00331534$ ,-0.01269183,0.00534695,0.00582020,0.00249948,-0.00153587,0.00086466,$0.00400888,0.00060532,-0.00119393,0.00208471,-0.00055616,-0.00031678,-$ $0.00117766,-0.00247667,-0.00246531,0.00552739,0.00919640,-0.00434210,-$ $0.00494173,-0.00006076,0.00003478,0.00015919,-0.00004971,0.00039913,-0$ $.00037536,0.00016576,-0.00012415,-0.00019393,-0.00003452,0.00018918,0$. $00007929,-0.00009550,0.00004599,0.00003484,-0.01674433,0.00373104,-0.0$ $7081527,-0.05812597,0.02560810,0.04951144,-0.09850857,0.03193016,-0.24$ $113908,0.08572869,-0.00521850,0.64261386,-0.00257543,0.00063824,0.0042$ $2867,-0.00048823,0.00149499,0.00022249,0.00022743,-0.00016350,0.000386$ $62,-0.00075774,-0.00013727,-0.00130357,0.00038232,-0.00041675,0.000428$ $47,-0.00083077,-0.00002936,0.00104317,-0.00111388,0.00061871,0.0008425$ $0,-0.00019368,-0.00006649,0.00008772,0.00066426,0.00137317,-0.00012214$ $,-0.00016598,0.00013254,-0.00004613,-0.00008604,0.00019332,-0.00000252$ $, 0.00000636,0.00007587,0.00010173,-0.04225166,0.01751534,-0.05297004,-$ $0.02612815,0.01098632,0.03064080,0.04215051,-0.01607428,-0.00982256,-0$ $.09500247,0.01858433,0.02515222,0.57401231,0.00150613,-0.00075667,-0.0$ $0085629,0.00080459,-0.00027526,0.00015114,-0.00049082,0.00011776,-0.00$ $075401,0.00008205,-0.00037894,0.00082632,0.00005015,0.00045686,-0.0001$ $1046,0.00035762,-0.00014399,-0.00113001,0.00012453,-0.00010456,-0.0005$ $2495,0.00000342,0.00010077,-0.00001487,-0.00013501,-0.00040797,-0.0000$ $7015,0.00006780,-0.00002406,0.00005724,0.00005813,0.00002140,0.0000182$ $0,0.00001739,-0.00002545,-0.00004857,0.02024661,0.00001515,0.02468893$, $0.01158146,-0.00800217,-0.01412272,-0.01741194,0.01162987,0.00102683,0$ $.01970528,-0.06309772,-0.02534029,-0.18044991,0.20089596,0.00089621,-0$ 
$.00175718,0.00027902,-0.00009832,0.00043232,0.00038368,-0.00035726,0.0$ $0004420,0.00017920,0.00053135,-0.00001822,-0.00066369,-0.00085206,-0.0$ $0057396,-0.00065382,0.00018012,0.00111361,0.00021262,0.00030937,-0.000$ $04439,-0.00000546,0.00007072,0.00012033,-0.00010785,0.00027362,0.00034$ $121,0.00011867,-0.00014044,0.00005919,-0.00001391,0.00005074,0.0000440$ $8,0.00010958,-0.00005187,0.00002892,0.00004169,-0.00048359,0.00258814$, $0.05435162,0.02964426,-0.01389887,-0.03315261,-0.04100359,0.01412674,-$ $0.04860648,0.05665060,-0.03804543,-0.33179612,0.06135385,0.00468407,0$. $69076398,0.00117925,0.00057153,0.00015963,0.00037334,-0.00044475,-0.00$ $014937,0.00015392,0.00001413,-0.00018895,-0.00008480,0.00021376,0.0000$ $1129,-0.00021750,-0.00006924,-0.00021241,0.00041750,-0.00030056,0.0000$ $3251,0.00012680,-0.00013058,-0.00013442,0.00007565,0.00013677,-0.00001$ $944,-0.00010232,-0.00017431,0.00003266,0.00001338,-0.00001977,-0.00005$ $352,-0.00003568,0.00009088,0.00006857,0.00002465,0.00000662,-0.0000194$ $7,-0.17843154,0.04273348,-0.07208692,0.04418916,-0.01712799,-0.0462153$ $3,-0.05178198,0.01970770,-0.01705697,0.00352211,0.00783640,0.08223120$, $-0.27225128,0.08676695,0.00755973,0.57009173,-0.00084651,0.00106592,0$. $00010092,0.00019821,0.00031667,0.00004365,0.00009747,0.00029593,-0.000$ $02299,0.00017660,-0.00026757,0.00024142,0.00021749,0.00001904,-0.00005$ $690,0.00009990,-0.00009742,0.00020452,-0.00008495,0.00006873,0.0000902$ 2,0.00001167,-0.00006974,0.00000106,-0.00012684,-0.00019134,0.00001611 $,-0.00000307,-0.00000603,-0.00000176,-0.00003668,0.00002436,0.00003407$ $,-0.00000586,-0.00001970,-0.00000602,0.03971362,-0.08062910,0.02018823$ ,-0.01513516,0.01523834,0.01586901,0.01898025,-0.00996507,0.00718227,0 $.00567213,0.00838963,-0.03522075,0.09072883,-0.09805014,-0.00670842,-0$ $.18245431,0.20466829,-0.00043099,0.00024106,-0.00070336,0.00040071,-0$. $00019575,-0.00011964,-0.00020158,-0.00018078,-0.00022114,0.00033180,0$. $00018879,0.00053216,0.00003798,0.00020918,0.00018029,-0.00016942,-0.00$ $011514,0.00003283,0.00028202,-0.00012164,-0.00024872,0.00000934,0.0000$ $0981,-0.00003384,0.00001771,-0.00016083,-0.00000748,0.00009716,-0.0000$ $7495,0.00002273,0.00007863,-0.00011994,-0.00002766,-0.00000679,-0.0000$ $3981,-0.00002771,-0.13130279,0.04447648,-0.23886460,-0.00491721,-0.000$ $64748,-0.04813571,-0.01847192,0.00725386,-0.00542010,0.03066938,-0.013$ $18362,-0.00213591,0.07711805,-0.03540456,-0.12688849,-0.01384470,0.037$ $86523,0.72931491,0.00045804,-0.00015390,-0.00003828,-0.00008830,0.0001$ $0654,0.00001967,-0.00008574,-0.00022462,0.00013046,-0.00013542,0.00013$ $331,-0.00032290,-0.00005718,-0.00015537,0.00007425,-0.00019091,-0.0000$ $3355,0.00014373,0.00009125,-0.00008653,-0.00007247,0.00005322,0.000073$ $29,-0.00003922,0.00059217,0.00097674,-0.00001974,0.00000090,-0.0000023$ $3,-0.00003630,0.00004032,-0.00003705,-0.00000371,0.00002891,0.00002763$ $, 0.00001599,-0.12799039,0.04379516,0.02438511,-0.02340851,0.01194220,0$ $.00732590,0.00288836,0.00070493,0.00295194,-0.00065056,0.00011128,0.00$ $076621,0.00120963,0.00086165,-0.00587435,-0.03509516,0.01692751,0.0102$ $3868,0.18400525,-0.00016460,-0.00014399,0.00005741,0.00001593,-0.00004$ $283,-0.00000230,0.00002796,0.00005393,-0.00007427,0.00003049,-0.000037$ $62,0.00013635,0.00003311,0.00008225,0.00000759,0.00002462,0.00003976,-$ $0.00013321,-0.00005730,0.00002465,0.00001347,-0.00002352,-0.00003383,0$ $.00002031,-0.00023012,-0.00040703,0.00000831,-0.00000556,0.00000190,0$. $00001922,-0.00001008,0.00001453,-0.00000430,-0.00001098,-0.00001480,-0$ $.00000791,0.04386852,-0.04248735,-0.01116154,0.01317014,-0.00090466,-0$ $.00351967,0.00048498,0.00414074,-0.00169621,-0.00010007,-0.00086577,-0$ 
$.00017808,0.00127895,0.00401174,0.00209479,0.01556355,-0.00182939,-0.0$ $0406284,-0.07250529,0.04259440,-0.00012665,0.00001683,0.00003891,0.000$ 02984,-0.00001773,-0.00000045,0.00003414,0.00004868,-0.00005045,0.0000 $2579,-0.00001759,0.00010192,0.00003698,0.00005410,0.00001000,0.0000292$ $6,0.00000564,-0.00002935,-0.00005569,0.00005387,0.00004152,-0.00002480$ $,-0.00002429,0.00000260,-0.00016344,-0.00024858,-0.00003075,0.00000880$ $,-0.00000933,0.00001640,-0.00000577,0.00000359,-0.00000838,0.00000001$, $-0.00001122,-0.00000635,0.02433379,-0.01112133,-0.04587686,0.03190278$, $-0.01361129,-0.00578436,-0.00261682,0.00067603,-0.00419042,0.00062051$, $-0.00015999,0.00172321,-0.00013234,-0.00031665,-0.00243343,-0.01446292$ $, 0.00602299,0.00688984,-0.04043240,0.01846011,0.04755272,0.00013872,-0$ $.00004296,-0.00020345,-0.00018898,0.00007006,0.00006841,0.00003848,0.0$ $0000869,0.00004986,-0.00009987,-0.00002958,-0.00017562,0.00002123,-0.0$ $0003439,0.00004509,-0.00020224,0.00007445,0.00005009,-0.00000389,0.000$ $00595,-0.00002091,-0.00001973,0.00000766,-0.00001116,0.00008562,0.0002$ $4701,-0.00007760,-0.00000136,0.00000502,-0.00001378,-0.00000517,0.0000$ $1398,-0.00000279,0.00000555,0.00001441,0.00001427,0.00605794,-0.000728$ $21,0.00764708,-0.17700394,0.05099162,-0.13784773,-0.01259212,0.0059053$ $2,-0.01937318,-0.00299421,0.00395452,0.00112214,-0.00017321,-0.0002701$ $7,0.00105883,0.00135621,0.00169500,0.00202312,0.00060904,-0.00128678,0$ $.00075982,0.18423076,-0.00011328,0.00009546,0.00009048,-0.00000142,0.0$ $0000360,-0.00006153,0.00001066,0.00001261,0.00002752,0.00002392,0.0000$ $4944,0.00004713,-0.00004461,-0.00002634,-0.00005502,0.00011027,-0.0000$ $5005,0.00004415,0.00004340,0.00001354,0.00001303,0.00000817,0.00000281$ ,-0.00000298,-0.00004901,-0.00005720,-0.00001833,0.00000293,-0.0000039 $0,0.00000517,0.00000695,-0.00001222,0.00000846,-0.00000110,-0.00000286$ ,-0.00000443,-0.00220114,0.00413929,-0.00407718,0.05117125,-0.05380316 $, 0.04795610,0.00744413,0.00177467,0.00804477,0.00385160,0.00469765,-0$. $00083941,-0.00024168,-0.00078736,-0.00025999,0.00172271,0.00439290,-0$. $00132205,-0.00133807,-0.00207258,-0.00015793,-0.05831414,0.04502144,0$. $00018100,-0.00004323,0.00005711,0.00002820,-0.00004549,-0.00001220,-0$. $00000810,-0.00001200,-0.00000472,0.00001915,0.00001062,0.00003596,-0.0$ $0000253,0.00001745,0.00000837,0.00002883,0.00000461,-0.00004285,0.0000$ $0053,-0.00001017,0.00001965,0.00001057,0.00000312,0.00000395,-0.000005$ $28,-0.00002484,0.00000907,0.00000391,-0.00000294,0.00000079,0.00000424$ $,-0.00001115,0.00000135,0.00000069,-0.00000262,-0.00000280,-0.02118142$ $, 0.00769187,-0.01563437,-0.13428017,0.04644165,-0.20080768,0.00746948$, $-0.00287150,0.00823254,0.00152972,-0.00088208,0.00136981,0.00071295,-0$ $.00026541,-0.00018230,0.00256942,-0.00154165,-0.00428975,-0.00032198,0$ $.00029531,0.00103979,0.14442756,-0.04948891,0.21023944,0.00031345,-0.0$ $0083688,-0.00056369,0.00001704,0.00012357,0.00000762,-0.00004898,-0.00$ $004600,-0.00004169,0.00004264,-0.00000116,0.00008648,-0.00000474,0.000$ $00842,0.00009905,0.00006167,0.00010750,0.00004621,0.00002821,0.0003662$ $9,0.00008588,-0.00011708,-0.00007923,0.00001992,-0.00006208,-0.0000978$ $0,-0.00007049,0.00002201,-0.00000108,0.00001551,0.00001102,-0.00003258$ ,-0.00002083,-0.00000663,-0.00000644,0.00000841,-0.00235279,0.00374658 $, 0.00039527,-0.00301596,0.00458991,0.02737756,-0.06685645,0.01601215,0$ $.06117091,0.00776319,-0.00390368,-0.01841948,-0.00127585,0.00276946,-0$ $.00393617,-0.00120046,0.00011737,-0.00023200,-0.00102639,0.00050005,0$. $00107261,0.00046537,-0.00184087,-0.00016457,0.06689546,0.00003885,-0.0$ $0041064,0.00061366,-0.00019422,-0.00001153,-0.00009288,-0.00001065,-0$. 
$00018793,0.00005089,-0.00005361,-0.00000194,-0.00008501,0.00007117,-0$. $00004732,0.00003894,0.00008976,0.00010906,-0.00021167,0.00004132,-0.00$ $008372,-0.00018862,-0.00014167,0.00000432,0.00004919,0.00002731,0.0001$ $9543,-0.00016597,-0.00002913,0.00001123,0.00000638,-0.00000346,0.00000$ $530,0.00003968,-0.00000575,0.00000456,0.00001234,0.00381976,0.00518864$ ,-0.00026745,0.00346471,0.00308220,-0.01170198,0.01589920,-0.04323823, $-0.03824327,-0.00305596,0.00170459,0.00673449,0.00296136,0.00482295,0$. $00117611,0.00001787,-0.00085440,0.00009988,0.00042483,-0.00015115,-0.0$ $0045741,-0.00183225,-0.00327686,0.00030991,-0.02120467,0.03374936,0.00$ $107539,-0.00061225,-0.00011996,-0.00034440,-0.00016026,0.00010149,0.00$ $006399,0.00002713,0.00011579,-0.00008857,-0.00006848,-0.00014572,0.000$ $13658,-0.00000076,0.00023068,-0.00010477,0.00041912,-0.00010331,-0.000$ $62572,0.00046691,0.00023387,-0.00014019,-0.00007103,-0.00001039,0.0000$ $0102,0.00009800,-0.00008490,0.00001575,0.00001101,0.00003006,-0.000011$ $13,-0.00002639,-0.00004118,0.00001858,0.00000535,0.00001185,0.00078674$ ,-0.00048738,0.00215790,-0.00046448,-0.00034195,-0.00406006,0.06507281 $,-0.03972985,-0.31046834,0.00332612,-0.00221468,-0.01849918,-0.0026805$ $9,0.00078527,-0.00155751,-0.00051150,0.00025215,0.00044789,0.00012426$, $-0.00005470,-0.00023978,0.00006264,0.00019069,0.00063570,-0.06624752,0$ $.04179112,0.33184324,0.00028415,0.00012866,0.00004712,-0.00005728,-0.0$ $0025319,-0.00021952,0.00003380,-0.00020474,0.00002118,-0.00053691,-0.0$ $0013246,0.00000240,-0.00016604,0.00033586,-0.00006101,0.00015010,-0.00$ $002812,0.00031070,0.00001699,0.00001926,0.00000484,0.00000195,-0.00001$ $334,-0.00000089,-0.00009723,-0.00023312,0.00000562,-0.00000347,-0.0000$ $0024,0.00005170,0.00002523,-0.00007846,-0.00004299,-0.00001732,-0.0000$ $1418,0.00000320,-0.00164242,0.00354071,0.00204983,-0.00048367,-0.00009$ $273,0.00062239,0.00132578,0.00200114,0.00277231,0.00811624,-0.00287751$ $, 0.00328726,-0.17194722,0.04931639,-0.13114069,-0.01376750,0.00663837$, $-0.02051801,-0.00124540,0.00051634,-0.00041732,-0.00013211,-0.00020877$ ,-0.00002996,0.00031415,-0.00010395,0.00037761,0.17932945,-0.00037171, $-0.00079229,-0.00007185,-0.00007242,0.00012471,0.00011096,-0.00003762$, $-0.00029796,0.00003423,-0.00002562,0.00033630,-0.00023278,0.00002047,-$ $0.00011819,0.00018520,-0.00031822,0.00021141,-0.00022845,0.00018963,-0$ $.00004445,-0.00005060,-0.00001721,0.00002370,0.00000764,0.00010462,0.0$ $0024695,0.00006841,0.00005481,-0.00002981,-0.00000609,0.00007939,-0.00$ $018844,-0.00009611,-0.00003193,0.00000212,0.00000899,0.00352592,0.0052$ $6013,-0.00110002,-0.00012082,-0.00080039,-0.00019083,0.00213646,0.0049$ $1661,-0.00168030,-0.00423043,0.00201386,-0.00208797,0.04935681,-0.0530$ $7356,0.04552697,0.00807530,0.00218914,0.00842425,0.00054017,-0.0001811$ $4,0.00018003,-0.00018479,-0.00056040,0.00003990,-0.00014401,0.00014622$ ,-0.00019281,-0.05660736,0.04413296,-0.00116107,0.00053819,-0.00013892 $, 0.00033658,0.00018748,-0.00001047,-0.00008514,-0.00016158,-0.00019281$ $, 0.00002587,0.00012450,0.00034638,0.00014458,0.00003151,0.00040514,-0$. $00005939,-0.00045763,0.00018371,0.00000005,0.00004173,0.00000272,-0.00$ $002559,-0.00002412,0.00000780,0.00016125,0.00029233,-0.00002319,-0.000$ $01830,0.00006296,-0.00000683,0.00005997,-0.00011637,-0.00006421,-0.000$ $02046,-0.00001566,0.00000421,0.00162465,-0.00104804,0.00131240,0.00104$ $433,-0.00029500,-0.00028318,0.00121838,-0.00119259,-0.00456456,-0.0181$ $7894,0.00669120,-0.01915537,-0.13440853,0.04657695,-0.19796199,0.00708$ $338,-0.00266495,0.00828393,0.00057252,-0.00022143,0.00008302,-0.000075$ $23,0.00005185,0.00001797,-0.00081690,0.00031666,-0.00056385,0.14287987$ 
,-0.04884430,0.21164271,0.00006067,0.00001573,0.00014939,-0.00013570,0 $.00007317,0.00003045,0.00004260,-0.00000602,0.00010707,-0.00005992,-0$. $00001849,-0.00022800,-0.00001219,-0.00006455,0.00003466,-0.00002707,0$. $00002095,0.00006671,-0.00008263,0.00002813,0.00003600,-0.00000841,0.00$ $000462,0.00000212,0.00010615,0.00020957,0.00002694,-0.00002491,0.00002$ $758,-0.00001376,-0.00001283,0.00002340,0.00000349,-0.00001435,0.000022$ $48,0.00001720,0.00667594,-0.00244418,-0.01974978,-0.00164085,0.0026519$ $3,-0.00312708,-0.00091290,-0.00002739,-0.00061890,-0.00284677,0.003935$ $17,0.00175622,-0.00292150,0.00429225,0.02559115,-0.06855301,0.01734602$ $, 0.06876178,0.00047661,-0.00131367,-0.00055297,0.00047506,-0.00008648$, $-0.00099999,-0.00006205,-0.00024859,0.00009574,0.00041139,-0.00184776$, $-0.00018271,0.06915706,-0.00005445,0.00012489,-0.00011909,-0.00002783$, $-0.00006097,-0.00001141,-0.00002545,0.00000605,0.00002200,0.00007599,0$ $.00003903,0.00006376,-0.00005186,-0.00000088,-0.00006381,0.00012503,-0$ $.00001756,0.00002957,0.00002298,-0.00001476,-0.00001730,0.00000844,-0$. $00000926,0.00000042,-0.00002598,-0.00003478,0.00000610,0.00001247,-0.0$ $0001025,0.00000970,0.00000186,-0.00000872,-0.00000425,-0.00000299,0.00$ $000084,-0.00000466,-0.00093125,0.00421555,0.00706532,0.00261664,0.0041$ $6827,0.00091118,-0.00006654,-0.00081695,0.00030559,0.00400378,0.004746$ $77,-0.00087246,0.00289802,0.00256674,-0.01080849,0.01718159,-0.0448334$ $4,-0.04337987,-0.00125776,-0.00214700,0.00042010,-0.00002744,0.0004044$ $4,0.00035901,-0.00025000,-0.00058071,-0.00001311,-0.00186429,-0.003294$ $03,0.00033203,-0.02239042,0.03552471,-0.00024001,0.00004073,-0.0000040$ $4,0.00005393,0.00002401,0.00000016,0.00000061,0.00000649,-0.00004065,0$ $.00000158,0.00000339,0.00008239,-0.00001326,0.00001726,-0.00001652,0.0$ $0004198,-0.00002208,0.00001338,0.00003815,-0.00000850,-0.00001412,-0.0$ $0000125,-0.00000595,0.00000041,-0.00004590,-0.00009724,0.00000051,-0.0$ $0000062,0.00000252,0.00000467,0.00000366,-0.00000311,-0.00000465,0.000$ $00410,-0.00000784,-0.00000978,0.00903766,-0.00469695,-0.01651319,-0.00$ $366383,0.00111104,-0.00097973,-0.00028096,0.00026181,0.00058856,0.0011$ $1717,-0.00082827,0.00130445,-0.00102054,0.00013877,-0.00225641,0.06526$ $517,-0.04185350,-0.31855182,0.00041646,0.00000047,0.00110535,0.0003868$ $1,-0.00020420,-0.00068191,0.00004446,0.00000721,-0.00005161,-0.0000035$ $8,0.00024649,0.00066851,-0.07117795,0.04577671,0.33535471,0.00088972,0$ $.00081726,0.00132007,-0.00281554,-0.00481044,-0.00212353,0.01811518,-0$ $.01178998,0.00868207,-0.00029059,-0.00220603,-0.00300218,-0.00051047,-$ $0.00063181,0.00143953,0.00012443,-0.00052495,0.00054696,-0.00004539,0$. $00008875,-0.00006633,-0.00025180,-0.00231422,0.00164083,-0.10569471,0$. $01496765,-0.05892544,-0.00038817,-0.00017531,0.00016921,0.00012687,-0$. $00014487,0.00009604,0.00029477,-0.00063649,0.00037651,0.00018200,-0.00$ $008434,-0.00003951,0.00000963,0.00000271,-0.00001710,-0.00011605,0.000$ $03334,0.00014305,0.00009489,-0.00014113,-0.00000452,-0.00018747,0.0000$ $7064,-0.00006407,0.00000564,0.00001872,0.00001640,-0.00016205,0.000065$ $50,0.00004399,-0.00002439,0.00001707,0.00000452,0.00003729,0.00002351$, $0.00002591,0.00002596,-0.00000826,-0.00003741,-0.00003181,0.00001287,0$ $.00001206,0.08839413,0.00123245,-0.00031831,0.00022473,0.00049826,-0.0$ $0194741,0.00295350,-0.00442277,-0.03925841,0.04515312,-0.00634963,-0.0$ $0156344,-0.00549205,-0.00035985,-0.00324265,0.00089110,0.00033457,-0.0$ $0002974,0.00111628,-0.00011295,-0.00019957,-0.00007369,-0.00180764,-0$. $00315940,0.00028508,0.01580581,-0.27650110,0.20339366,-0.00021692,-0.0$ $0086723,-0.00012165,-0.00016983,-0.00001924,0.00007323,0.00010658,0.00$ 
$068791,-0.00000542,0.00036010,-0.00016135,-0.00020384,-0.00021446,0.00$ $018337,-0.00013088,-0.00036261,0.00020808,0.00066592,0.00083158,-0.000$ $69829,-0.00013210,-0.00073156,0.00028549,-0.00011231,0.00005691,0.0001$ $0374,0.00014551,-0.00045238,0.00019217,0.00010729,-0.00014813,0.000021$ $12,0.00001705,0.00004447,-0.00013363,-0.00006850,0.00011062,-0.0001199$ $6,-0.00010526,-0.00010787,0.00001627,0.00005002,-0.02052594,0.28978884$ $, 0.00179164,0.00094668,0.00074913,-0.00721840,-0.00455438,-0.00484832$, $0.00209376,0.03351639,-0.00706976,0.00060719,0.00085160,0.00309534,0.0$ $0131920,0.00080308,0.00024285,-0.00033221,-0.00062011,0.00014370,0.000$ $00997,0.00022880,0.00003279,0.00123130,-0.00084443,0.00230816,-0.05959$ $805,0.20172277,-0.34922431,0.00024360,0.00015418,-0.00008903,0.0001612$ $6,-0.00011144,0.00025483,-0.00022585,-0.00132826,0.00100442,-0.0000860$ $5,0.00005221,0.00010364,0.00014201,-0.00013467,0.00003436,0.00007710,-$ $0.00005953,-0.00031472,-0.00042686,0.00032588,0.00005734,0.00026813,-0$ $.00003373,0.00000200,-0.00004709,-0.00006560,-0.00001274,0.00014994,-0$ $.00005803,-0.00001822,0.00007019,-0.00000511,-0.00000991,0.00003037,0$. $00010751,0.00007332,-0.00002317,0.00004014,0.00004994,0.00001677,-0.00$ $000876,-0.00001337,0.11280244,-0.26537979,0.44097153,-0.00180588,-0.00$ $117683,0.00036344,0.00262202,-0.00384772,0.00364102,-0.01676171,-0.028$ $47466,-0.03199681,-0.00004583,0.00210229,-0.00367146,-0.00092216,-0.00$ $076152,0.00021762,-0.00003405,0.00006661,-0.00021454,-0.00042201,-0.00$ $029400,0.00004472,0.00083929,0.00052200,0.00023711,-0.25370844,-0.0876$ $2893,-0.20015575,-0.00004209,-0.00019899,0.00005576,0.00008816,-0.0000$ $5552,0.00010722,-0.00037732,-0.00179072,0.00105822,0.00031277,-0.00013$ $255,-0.00013625,-0.00008368,0.00004505,0.00004360,-0.00012906,0.000005$ $38,0.00028886,0.00069892,-0.00022418,-0.00021595,-0.00030227,0.0000753$ $7,-0.00016817,-0.00000810,0.00005421,0.00001263,-0.00031769,0.00012784$ $, 0.00009279,-0.00004276,0.00002751,0.00000321,0.00005794,0.00000427,0$. $00003573,0.00006137,-0.00006543,-0.00008175,-0.00005859,0.00001660,0.0$ $0002409,0.00221796,0.01660119,-0.05305717,0.26816319,-0.00129680,-0.00$ $184407,-0.00173604,-0.00033256,-0.00406746,0.00599518,-0.03641944,-0.0$ $0963532,-0.03121714,-0.00262872,-0.00400108,0.00048914,-0.00010226,0.0$ $0070973,-0.00118813,-0.00081058,-0.00039029,-0.00089948,-0.00017820,-0$ $.00075212,-0.00001229,-0.00034388,0.00029914,-0.00014141,-0.08840760,-$ $0.15172410,-0.07178069,0.00001325,-0.00011046,0.00011418,-0.00016482$, $0.00002198,-0.00003597,-0.00238667,-0.00312899,-0.00108560,0.00034356$, $-0.00014374,-0.00016380,-0.00014132,0.00007243,-0.00006771,-0.00029389$ $, 0.00013916,0.00049239,0.00117717,-0.00035839,-0.00034124,-0.00052600$, $0.00020317,-0.00015344,0.00002537,0.00004065,0.00009519,-0.00037839,0$. $00016306,0.00010407,-0.00009484,0.00002392,0.00001149,0.00005861,-0.00$ $004578,-0.00000463,0.00010511,-0.00010348,-0.00010047,-0.00009555,0.00$ $001576,0.00004073,0.02787574,0.03673275,0.03445462,0.10500272,0.137927$ $49,0.00058162,-0.00108534,0.00006140,-0.00037815,-0.00015749,0.0030049$ $5,-0.02459425,-0.01851982,-0.00257840,0.00023991,0.00809279,-0.0049148$ $3,-0.00048121,-0.00252120,0.00064103,0.00061268,0.00049482,0.00086148$, $0.00000915,-0.00026075,-0.00000469,0.00093253,0.00107957,0.00099028,-0$ $.19917147,-0.07055474,-0.32539032,-0.00007658,-0.00022548,-0.00000626$, $0.00011631,0.00000993,0.00033649,0.00163419,-0.00017739,0.00242056,0.0$ $0016134,-0.00005629,-0.00008941,-0.00001682,-0.00002412,0.00011381,0.0$ $0005778,-0.00009049,0.00005912,0.00013815,-0.00000340,-0.00003467,-0.0$ $0007841,0.00003894,-0.00016199,-0.00001055,0.00003069,-0.00001590,-0.0$ 
$0015376,0.00006114,0.00006354,0.00001555,0.00001771,-0.00000299,0.0000$ $5308,0.00006029,0.00006397,0.00002111,-0.00009110,-0.00004308,-0.00004$ $453,0.00000172,0.00001469,-0.06303949,0.01674896,-0.08752297,0.2834718$ $4,0.06713105,0.41213417 \backslash 10.00000298,-0.00000380,0.00001772,-0.00000264$ $, 0.00000518,0.00000733,-0.00000185,0.00000073,0.00000207,0.00000004,0$. $00000375,0.00000032,-0.00000079,-0.00000002,0.00000184,0.00001163,-0.0$ $0000047,-0.00000290,-0.00001266,0.00000140,-0.00000327,0.00000418,-0.0$ $0000207,-0.00000115,0.00000062,0.00000114,0.00000091,0.00000003,0.0000$ $0132,0.00000135,0.00000166,0.00000010,0.00000002,-0.00000210,0.0000025$ $0,0.00000243,-0.00000047,-0.00000020,-0.00000274,-0.00000014,-0.000001$ $90,-0.00000131,-0.00000501,0.00000628,0.00000149,0.00000970,-0.0000057$ $2,-0.00001416,0.00000345,-0.00000050,-0.00000293,-0.00000249,-0.000003$ $55,-0.00000301,-0.00000097,-0.00000239,-0.00000253,0.00000018,-0.00000$ $260,-0.00000218,-0.00000095,-0.00000173,-0.00000020,-0.00000130,0.0000$ $0082,-0.00000037,-0.00000091,-0.00000121,-0.00000163,-0.00000090,0.000$ 00133,0.00000142,-0.00000129,0.00000163,0.00000150॥II@

para-Transition State

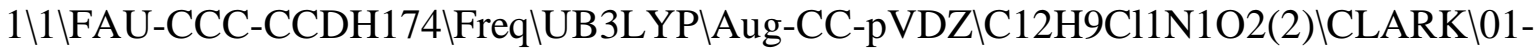 $\mathrm{M}$}

ar-2015\0\\N Geom=AllCheck Guess=TCheck SCRF=Check GenChk UB3LYP/AugCC-pVDZ Freq \\Addition TS p-Cl-Ph-radical + nitrobenzene (para) \\0,2\C ,-0.9435817326,1.9225680769,-0.0030206306\C,-1.4921959346,1.4613323078 ,1.2204409284\C,-2.4764151863,0.4826428233,1.2265847601\C,-2.95181614, $-0.0073279349,0.0017842062 \backslash \mathrm{C},-2.4777863083,0.4780143777,-1.225413707 \backslash \mathrm{C}$ ,-1.4936214935,1.4567749593,-1.2240851503\H,-0.3185094799,2.8128155038 ,-0.0050722684\H,-1.1224087362,1.8624732183,2.1630451414\H,-2.89586906 $09,0.1005129308,2.1537183497 \backslash \mathrm{H},-2.8982980012,0.0923844993,-2.150620828$ $6 \backslash \mathrm{H},-1.1249209686,1.8543965805,-2.1686034515 \backslash \mathrm{N},-3.9961237578,-1.037236$ $8855,0.0043221121 \backslash \mathrm{C}, 3.5199487178,-0.4357479719,0.0009481102 \backslash \mathrm{C}, 3.500800$ $4137,0.960534261,0.0070682346 \backslash \mathrm{C}, 2.2625720347,1.625054849,0.0057110748 \backslash$ C,1.1144739628,0.8544501002,-0.0017795028\C,1.107594638,-0.5276473638, $-0.0079068094 \backslash \mathrm{C}, 2.3441141195,-1.191668932,-0.0065053877 \backslash \mathrm{Cl}, 5.073189974$ $,-1.2700162005,0.0026500643 \backslash \mathrm{H}, 4.434158243,1.5219446238,0.0128219261 \backslash \mathrm{H}$, $2.2346829011,2.7155795735,0.010411655 \backslash \mathrm{H}, 0.1795241896,-1.0998071161,-0$. $0137565477 \backslash \mathrm{H}, 2.3924394667,-2.2797561415,-0.0111323069 \backslash \mathrm{O},-4.4045623555$, $-1.4398768741,1.0940721837 \backslash \mathrm{O},-4.4052055055,-1.4445762646,-1.0834481557$ IIVersion=EM64L-G09RevC.01 $\backslash$ State $=2-A \backslash H F=-1128.013139 \backslash S 2=0.777943 \backslash \mathrm{S} 2-1=$ $0 . \mathrm{SS} 2 \mathrm{~A}=0.750519 \backslash \mathrm{RMSD}=7.071 \mathrm{e}-09 \backslash \mathrm{RMSF}=3.342 \mathrm{e}-06$ ZeroPoint $=0.1804807 \backslash$ Ther $\mathrm{mal}=0.194048 \backslash \mathrm{ZPE}=113.2533362 \backslash \mathrm{Dipole}=1.087944,1.6295371,-0.0035777 \backslash \mathrm{Dipo}$ leDeriv $=0.5927069,0.1269606,-0.0006633,-0.0768522,0.0795692,-0.0000393$ ,-0.0003389,-0.0005149,0.0610898,-0.3100405,0.0287933,0.1450575,-0.129 $6159,-0.2073836,0.1048947,0.1835086,0.1540245,0.035125,0.1794661,0.104$ $7508,-0.1047956,0.2639073,0.0439754,-0.1118529,0.1739567,0.1153045,-0$. 0507176,-0.6600924,-0.2790789,0.0005444,-0.71024,-0.2372941,0.0013523, $0.0012716,0.0009507,0.1044054,0.1782862,0.1053052,0.1045116,0.262472,0$ $.0439054,0.1098956,-0.1744982,-0.1159304,-0.0509275,-0.3085017,0.02800$ $93,-0.1447171,-0.1299509,-0.208368,-0.102988,-0.1822734,-0.1531352,0.0$ $36499,0.042025,-0.1070568,0.00023,-0.0547238,0.0299981,0.0000626,-0.00$ $01509,0.0000734,0.0662291,0.0784279,-0.0591538,-0.0442091,-0.0464083,0$ 
$.0786209,-0.0256497,-0.0326563,-0.0389433,-0.0170802,0.1211821,-0.0137$ $218,0.0226343,-0.0135318,0.1238217,0.0259067,0.0084571,0.0106585,0.058$ $0729,0.1207408,-0.0138141,-0.0224808,-0.0140708,0.1237763,-0.0261169,-$ $0.0091163,-0.0108128,0.0584192,0.0782717,-0.0587986,0.044068,-0.046278$ $, 0.078844,0.0253186,0.0331618,0.0387401,-0.017409,1.6953497,0.8489973$, $-0.0017479,1.4739167,1.0523596,0.0003631,-0.0028575,0.0008066,1.484567$ $4,1.0445659,-0.4794047,0.0007583,-0.5858859,0.2511933,-0.0003898,0.000$ $8853,-0.0000537,-0.0433276,-0.1950327,0.131458,0.0007937,0.000693,-0.0$ $831327,0.0007708,0.0003079,0.0006949,-0.0889232,-0.3041134,-0.0126229$, $0.0002757,0.5058924,0.0843043,0.0009664,0.0010471,0.0005322,-0.0866304$ $, 0.5120075,-0.1151871,0.0001986,-0.3140416,-0.0077761,0.000743,0.00073$ $89,0.0002939,-0.062949,-0.5264295,0.0735974,0.0005931,0.045766,-0.0185$ $711,-0.0009239,-0.0011383,0.0002888,-0.0689263,-0.1248759,0.0426105,-0$ $.0001556,0.2087215,0.0356612,0.0015043,0.0000883,0.0003468,-0.0775222$, $-0.7876397,0.2854591,-0.0004468,0.3777882,-0.2352263,0.0001265,-0.0009$ $849,0.0001681,-0.0640699,-0.0739736,-0.0518692,-0.0008308,0.0030289,0$. $0606879,-0.000537,-0.0005858,-0.0006017,0.1283625,0.0882771,0.0394214$, $-0.0000311,-0.024874,-0.0750021,-0.0011162,-0.000072,-0.0006281,0.1256$ $597,0.0154483,-0.0638221,-0.0007179,-0.0483605,0.0566284,-0.0001826,-0$ $.0002051,-0.0002161,0.1022769,0.0103476,0.0221678,-0.0000738,0.0410831$ -0.0199672,-0.0008645,-0.0004062,-0.000542,0.1259464,-0.7329412,-0.29 $08043,0.2522286,-0.4933462,-0.5243752,0.2488355,0.4901069,0.2490249,-0$ $.8803356,-0.7334625,-0.2921966,-0.2510242,-0.4950892,-0.5262492,-0.250$ $0794,-0.4882466,-0.2505298,-0.8778349 \backslash$ Polar $=284.755597,19.972841,167.3$ $144329,0.0464879,0.1461907,139.2747342 \backslash \mathrm{PG}=\mathrm{C} 01$ [X(C12H9Cl1N1O2)]\NImag= $1 \backslash 0.27345316,0.31041771,0.47901916,-0.00035502,0.00011678,0.61802882$, $-0.08945972,-0.05940593,0.04893569,0.41513575,-0.03558212,-0.11696013$, $0.02952903,0.27658146,0.40700245,0.05136307,0.07363979,-0.26319886,0.0$ $2567374,0.05088805,0.68543051,-0.02306144,-0.01461340,0.04203724,-0.20$ $981726,-0.14689339,-0.02024974,0.42373869,-0.02922737,-0.00585980,0.04$ $438091,-0.14620184,-0.20428945,-0.02399613,0.28212754,0.40906497,0.010$ $47571,0.02426392,0.02891004,0.02747888,0.02480635,-0.12690310,-0.04703$ $604,-0.03226179,0.70918348,0.00931767,0.00132120,-0.00003142,-0.016874$ $47,-0.02436481,-0.01463030,-0.09978238,-0.04001274,-0.06783518,0.33451$ $131,0.01729658,-0.00623368,-0.00010236,-0.02338064,-0.01929180,-0.0143$ $0802,-0.03902655,-0.09943333,-0.07045351,0.20046379,0.33478143,-0.0001$ $0024,-0.00008666,-0.05261067,-0.05073201,-0.05105087,0.02610907,-0.026$ $93277,-0.03049347,-0.28115802,-0.00017008,0.00068381,0.73073083,-0.023$ $15297,-0.01480130,-0.04192791,-0.02085491,-0.01751413,0.01138629,0.030$ $48865,0.02353421,0.01289985,-0.09966127,-0.03882800,0.02687144,0.42382$ $168,-0.02931887,-0.00612733,-0.04421683,-0.01751873,-0.02115487,0.0119$ $2844,0.02349628,0.03041594,0.01238405,-0.03969974,-0.09905736,0.029799$ $08,0.28232422,0.40938133,-0.01026432,-0.02411547,0.02923619,0.01702116$ $, 0.01800127,-0.01088909,-0.01309547,-0.01269417,-0.05267190,0.06777249$ $, 0.06976646,-0.28162472,0.04631995,0.03311574,0.70882404,-0.08958987,-$ $0.05965727,-0.04892500,0.02625432,0.02108908,-0.00240889,-0.02088358,-$ $0.01760669,-0.01694654,-0.01681691,-0.02316842,0.05088085,-0.20982265$, $-0.14630379,-0.02686140,0.41513078,-0.03582160,-0.11734030,-0.03006125$ $, 0.02107953,0.02787235,-0.00604304,-0.01757622,-0.02126820,-0.01796636$ $,-0.02426221,-0.01903306,0.05127926,-0.14685107,-0.20434139,-0.0243651$ $9,0.27645236,0.40666492,-0.05147199,-0.07413548,-0.26272923,0.00225457$ 
$, 0.00575943,-0.04142066,-0.01131508,-0.01187774,-0.01074970,0.01476542$ $, 0.01451369,0.02580431,0.02088169,0.02445007,-0.12691795,-0.02634466,-$ $0.05012262,0.68591804,-0.12335317,-0.14589373,0.00030941,-0.00786075$, $0.01145732,0.00217524,0.00476300,-0.00122688,0.00395024,-0.00092065,-0$ $.00108061,0.00000958,0.00476747,-0.00124548,-0.00396942,-0.00789480,-0$ $.01149299,-0.00211528,0.14469048,-0.15899570,-0.23746084,0.00043149,-0$ $.00159443,-0.00116003,-0.00509761,-0.00296950,0.00113539,0.00060318,0$. $00177320,0.00164119,-0.00000708,-0.00297810,0.00112808,-0.00062336,-0$. $00165341,-0.00122564,0.00512013,0.15625533,0.24709252,0.00033444,0.000$ $41917,-0.05688938,0.01548733,0.02217761,0.00450090,0.00184918,0.001548$ $14,-0.00454812,-0.00000578,-0.00000831,-0.00153305,-0.00184387,-0.0015$ $6328,-0.00453291,-0.01546703,-0.02214115,0.00458719,-0.00034209,-0.000$ $44811,0.05534133,0.00671379,0.00493920,0.01271907,-0.07845803,-0.04597$ $969,-0.08430535,-0.00380043,-0.00815825,-0.01958978,0.00140218,-0.0059$ $3669,0.00147324,-0.00079236,0.00014225,0.00033938,0.00192028,-0.003503$ $12,0.00221196,-0.00141655,0.00125790,-0.00028051,0.07591347,0.00399148$ $, 0.00672919,0.00936046,-0.04655370,-0.08483494,-0.09023739,-0.00717276$ ,-0.00393839,-0.01972326,-0.00615398,0.00133670,0.00108853,0.00015617, $-0.00081634,0.00052485,-0.00338157,0.00260640,0.00235169,0.00205188,-0$ $.00078855,-0.00037684,0.05464054,0.08158747,-0.01060853,-0.01077837,-0$ $.01754564,-0.08291403,-0.09213213,-0.27120147,0.00197805,0.00216238,0$. $00397569,0.00075778,0.00033782,0.00174313,0.00047626,0.00057800,0.0001$ $3578,0.00310060,0.00234564,-0.00288936,-0.00009049,0.00016520,0.000964$ $57,0.08827696,0.09755489,0.28449958,0.00118389,-0.00671618,-0.00100912$ $,-0.00307616,-0.00658631,0.01821080,-0.08859265,-0.04621387,0.09647102$ $, 0.00819953,0.00340567,-0.00876671,0.00165449,-0.00317684,-0.00240701$, $-0.00071487,0.00031587,-0.00027310,-0.00032698,-0.00019339,0.00105798$, $-0.00170125,0.00290508,-0.00028098,0.08584297,-0.00619360,0.00158956$, $0.00048871,-0.00698309,-0.00166047,0.01799902,-0.04672991,-0.08134518$, $0.08636369,0.00440629,0.00837122,-0.00846813,-0.00339829,0.00173887,-0$ $.00275226,0.00009603,-0.00105615,-0.00039897,-0.00021074,-0.00019847,0$ $.00055223,0.00276847,-0.00169706,-0.00005143,0.05534834,0.07724428,-0$. $00074297,-0.00105633,0.00136800,-0.00242614,-0.00196796,0.00581141,0.0$ $9486418,0.08520148,-0.27253541,0.01103109,0.01029827,-0.01837156,-0.00$ $267339,-0.00292295,-0.00257136,-0.00046191,-0.00037736,0.00033085,-0.0$ $0013528,-0.00020195,0.00020478,0.00002015,0.00018524,0.00070083,-0.102$ $67562,-0.09266420,0.28459591,0.00118857,-0.00671443,0.00103615,-0.0007$ $1327,0.00031376,0.00027373,0.00165675,-0.00316440,0.00240778,0.0081938$ $0,0.00342958,0.00873022,-0.08881065,-0.04667861,-0.09650372,-0.0030986$ $0,-0.00665279,-0.01817679,-0.00032697,-0.00019683,-0.00105783,-0.00034$ $596,0.00038163,-0.00006603,0.00057326,0.00004981,0.00055205,0.08607950$ ,-0.00619722,0.00159490,0.00049872,0.00009645,-0.00104927,0.00040170,$0.00338034,0.00175294,0.00274055,0.00437134,0.00836456,0.00835978,-0.0$ $4718547,-0.08201137,-0.08702894,-0.00699474,-0.00171474,-0.01796567,-0$ $.00020821,-0.00019846,-0.00055220,0.00040633,-0.00042047,0.00000568,0$. $00004999,0.00071334,0.00058853,0.05584290,0.07795641,0.00076145,0.0010$ $6036,0.00135444,0.00045390,0.00038236,0.00033115,0.00268551,0.00290859$ ,-0.00260791,-0.01108259,-0.01039819,-0.01835051,-0.09489292,-0.085878 $48,-0.27166635,0.00246968,0.00200505,0.00589400,0.00014024,0.00020578$, $0.00020591,-0.00004402,-0.00007834,-0.00001004,-0.00055095,-0.00059510$ $,-0.00081282,0.10268907,0.09338334,0.28365214,0.00671246,0.00489866,-0$ 
$.01276070,0.00191943,-0.00351851,-0.00220839,-0.00079725,0.00014444,-0$ $.00033435,0.00140407,-0.00594745,-0.00146117,-0.00378272,-0.00807814,0$ $.01964013,-0.07826599,-0.04556229,0.08424817,-0.00141802,0.00126396,0$. $00028146,0.00043045,0.00014208,-0.00055475,-0.00034681,0.00040678,0.00$ $004497,-0.00170025,0.00276752,-0.00003050,0.07570930,0.00401986,0.0067$ $3414,-0.00945475,-0.00339201,0.00259034,-0.00236715,0.00015242,-0.0008$ $2304,-0.00052357,-0.00615889,0.00133223,-0.00107761,-0.00715341,-0.003$ $87141,0.01975791,-0.04613917,-0.08415050,0.08958387,0.00205432,-0.0007$ $8915,0.00037985,0.00014206,0.00021156,-0.00051878,0.00038059,-0.000421$ $69,0.00008136,0.00290644,-0.00169689,-0.00017639,0.05419435,0.08085893$ $, 0.01056568,0.01068538,-0.01754945,-0.00309467,-0.00235993,-0.00286668$ $,-0.00047393,-0.00057512,0.00014364,-0.00073333,-0.00032807,0.00175000$ ,-0.00194301,-0.00212519,0.00389019,0.08286437,0.09147859,-0.27207736, $0.00008458,-0.00015773,0.00096325,0.00055284,0.00051322,-0.00087797,0$. $00006365,-0.00000377,-0.00001235,0.00027262,0.00005815,0.00070141,-0.0$ $8823932,-0.09685071,0.28543384,-0.01000109,0.00173495,0.00000206,0.010$ $24112,0.00401778,-0.00560759,-0.02109626,-0.01919289,-0.01542117,-0.09$ $753360,-0.01825907,0.00002645,-0.02106384,-0.01911141,0.01552388,0.010$ $25215,0.00404168,0.00557250,0.00020588,-0.00049211,0.00000140,0.000529$ $69,-0.00033976,0.00035135,-0.00110250,0.00249764,0.00225997,-0.0011026$ $8,0.00249226,-0.00226775,0.00053040,-0.00034031,-0.00035132,0.43113967$ ,-0.00849651,0.00015800,0.00000015,0.00182609,0.01112087,-0.00545436,$0.01842206,-0.02113183,-0.01444255,-0.01796618,-0.09722569,-0.00001704$ - $-0.01834521,-0.02101734,0.01460651,0.00184637,0.01115444,0.00537956,0$ $.00140765,-0.00139155,0.00000204,-0.00030860,0.00059921,0.00032000,0.0$ $0258071,-0.00136120,0.00235413,0.00258447,-0.00136710,-0.00236569,-0.0$ $0030296,0.00060321,-0.00032335,0.20083930,0.42721607,0.00000870,-0.000$ $00881,-0.00415398,-0.00398531,-0.00442368,-0.00731240,-0.01781132,-0.0$ $1686081,0.01414938,0.00001944,-0.00000191,-0.10754968,0.01793418,0.016$ $98799,0.01396964,0.00397123,0.00437521,-0.00734793,-0.00000106,0.00000$ $237,-0.00052983,-0.00122770,-0.00131148,0.00026896,-0.00048641,-0.0004$ $3111,-0.00336981,0.00047029,0.00042055,-0.00334815,0.00121839,0.001308$ $31,0.00026202,-0.00027693,0.00097983,0.92147619,-0.00086332,0.00007306$ ,-0.00000666,0.00017280,0.00007406,-0.00006443,-0.00026025,-0.00011287 ,-0.00010152,0.00075962,0.00069854,-0.00000955,-0.00024586,-0.00010581 $, 0.00011467,0.00014254,0.00006872,0.00005868,-0.00018010,0.00008155,0$. $00000917,-0.00005731,0.00000453,0.00003281,-0.00002179,0.00000685,0.00$ $002451,-0.00001644,0.00001375,-0.00001404,-0.00004799,0.00000087,-0.00$ $003778,-0.00116717,-0.00110812,-0.00000479,0.53180561,0.00020444,-0.00$ $050294,-0.00000478,-0.00026864,-0.00009565,-0.00002090,-0.00000848,0.0$ $0028731,0.00007200,-0.00024703,-0.00019218,0.00000362,-0.00000259,0.00$ $028491,-0.00007141,-0.00027385,-0.00009854,0.00002084,-0.00013680,0.00$ $021661,0.00000397,0.00001098,-0.00005945,0.00002208,0.00003413,-0.0000$ $0088,-0.00000824,0.00003567,0.00000290,0.00001205,0.00001419,-0.000063$ $07,-0.00002079,0.00066445,0.00064444,-0.00000204,0.06255921,0.61794031$ $,-0.00000775,-0.00000665,0.00000743,0.00001811,0.00001368,0.00000441,0$ $.00000412,0.00004434,-0.00003414,-0.00000030,0.00000078,0.00000340,0.0$ $0000056,-0.00004174,-0.00003041,-0.00001962,-0.00001359,0.00001256,0.0$ $0000045,0.00000407,0.00010338,0.00000851,-0.00003511,-0.00002554,0.000$ $00728,-0.00000664,0.00000475,-0.00000884,0.00000431,0.00000271,-0.0000$ $0767,0.00003612,-0.00003076,-0.00000154,-0.00000102,0.00000675,0.00170$ 
$056,0.00238777,0.12799221,0.00096633,-0.00130365,0.00000475,-0.0004298$ $1,-0.00001681,0.00005820,0.00012469,0.00032910,0.00009475,-0.00040927$, $-0.00039094,0.00000315,0.00012039,0.00032740,-0.00009619,-0.00042025,-$ $0.00001525,-0.00005968,-0.00003174,0.00039415,-0.00000791,-0.00005243$, $-0.00002142,0.00007264,0.00003363,0.00000569,-0.00002658,0.00003103,-0$ $.00000071,0.00001871,-0.00005607,-0.00002094,-0.00006608,0.00067878,0$. $00066495,-0.00000314,-0.10797710,-0.04421401,-0.00035655,0.70737966,0$. $00016146,-0.00019339,-0.00000704,-0.00055044,0.00005338,0.00023959,0.0$ $0047748,0.00025448,0.00004038,-0.00090978,-0.00097931,0.00000537,0.000$ $48887,0.00024392,-0.00005567,-0.00057893,0.00006356,-0.00023937,-0.000$ $37427,-0.00010385,0.00000161,-0.00000838,0.00002224,0.00005272,-0.0000$ $3645,-0.00000324,0.00000826,-0.00002890,0.00001053,0.00000766,-0.00000$ $371,0.00002272,-0.00006241,0.00083689,0.00085833,-0.00000410,0.0193920$ $2,-0.32617024,-0.00106485,0.04662579,0.66590509,0.00000921,-0.00000935$ $, 0.00094759,-0.00029737,0.00011710,-0.00007007,-0.00007599,-0.00015678$ $, 0.00003975,-0.00000937,-0.00000436,0.00017334,0.00009169,0.00016085,0$ $.00002215,0.00029009,-0.00010576,-0.00006307,-0.00000650,-0.00000198,-$ $0.00002907,-0.00000901,0.00002988,0.00003075,0.00001803,-0.00002418,-0$ $.00000515,-0.00002184,0.00002233,0.00000151,0.00000085,-0.00003214,0.0$ $0002779,0.00000593,0.00000372,0.00001662,-0.00006641,-0.00129702,-0.06$ $559791,0.00223214,0.00253419,0.12789588,-0.00217900,-0.00136127,-0.000$ $02275,-0.00134307,0.00023877,-0.00028581,0.00077539,0.00037037,0.00017$ $115,-0.00186208,-0.00169067,-0.00000320,0.00076309,0.00037356,-0.00017$ $014,-0.00134407,0.00023674,0.00028847,0.00012243,-0.00062323,0.0000023$ $0,-0.00008462,0.00015492,0.00018554,-0.00002714,-0.00007823,0.00000524$ $,-0.00002948,-0.00008592,-0.00001144,-0.00008949,0.00015515,-0.0001839$ $8,0.00187902,0.00199179,-0.00000319,0.02679739,0.08263531,0.00042856,-$ $0.26976874,0.04702384,-0.00050820,0.60607837,0.00007565,0.00190907,0.0$ $0000779,0.00170142,-0.00018459,-0.00051427,-0.00094308,-0.00088778,-0$. $00030817,0.00248251,0.00261285,0.00000659,-0.00093164,-0.00087424,0.00$ $029538,0.00168967,-0.00018825,0.00051685,0.00152541,0.00035195,0.00000$ $638,0.00002610,0.00001178,-0.00010975,0.00000308,0 ., 0.00001018,0.00000$ $555,0.00000374,-0.00000706,0.00002288,0.00001100,0.00010471,-0.0030021$ $8,-0.00315710,0.00000518,0.02561365,-0.02248851,-0.00004562,0.12283653$ ,-0.16031899,-0.00002163,0.02154990,0.72589355,-0.00000811,0.00000758, $0.00036021,-0.00051068,-0.00038684,-0.00006784,0.00046769,0.00068520,0$ $.00035261,0.00000674,-0.00000173,-0.00073389,-0.00047612,-0.00068143,0$ $.00036902,0.00051133,0.00037376,-0.00007817,0.00001233,-0.00000071,0.0$ $0001767,-0.00006804,-0.00004585,-0.00001784,0.00000227,-0.00001271,-0$. $00001652,-0.00000086,0.00001317,-0.00002083,0.00007261,0.00004933,-0.0$ $0001848,-0.00000620,-0.00000262,-0.00005879,0.00018740,0.00015816,0.00$ $720794,-0.00017682,-0.00027456,-0.06147623,0.00177219,0.00272745,0.126$ $32190,0.05720605,-0.01466125,-0.00002281,-0.02034880,-0.00864032,0.020$ $61211,0.00550546,0.00827206,0.00520495,-0.00403609,-0.00513747,0.00003$ $834,0.00554603,0.00826093,-0.00526742,-0.02040799,-0.00870606,-0.02054$ $626,-0.00938091,0.00720314,-0.00000669,0.00058421,-0.00051223,-0.00077$ $143,0.00042541,0.00005275,-0.00002422,0.00043334,0.00006614,0.00003858$ $, 0.00058910,-0.00050993,0.00076050,0.00128084,0.00094046,-0.00000797,-$ $0.01930324,-0.02984093,-0.00016140,-0.06340051,0.02077824,-0.00019189$, $-0.25584621,-0.13573988,-0.00132251,0.38398616,-0.02910018,-0.00413469$ $,-0.00002555,0.00527176,0.00519025,-0.00826232,-0.00226368,-0.00332790$ 
,-0.00194559,0.00088657,0.00145159,0.00001684,-0.00226753,-0.00334226, $0.00192922,0.00529240,0.00523397,0.00826008,0.01020716,-0.00846840,0.0$ $0000293,-0.00022812,0.00031548,0.00028400,-0.00007351,-0.00016485,-0.0$ $0000637,-0.00007518,-0.00016784,0.00000218,-0.00024002,0.00031865,-0.0$ $0028426,0.00036332,0.00074868,0.00000939,-0.03002739,-0.05968963,-0.00$ $035105,-0.03597101,0.06065014,0.00009235,-0.10181669,-0.18345175,-0.00$ $094709,0.14716061,0.59137730,0.00004062,-0.00001015,-0.00498818,0.0056$ $1717,0.00005017,-0.00090147,-0.00067354,-0.00162018,-0.00133665,-0.000$ $00464,0.00000777,0.00153935,0.00068883,0.00161303,-0.00135475,-0.00562$ $593,-0.00006649,-0.00090955,-0.00002522,0.00000046,0.00015512,-0.00018$ $103,0.00019745,0.00026621,-0.00021621,0.00009217,0.00003371,0.00021737$ ,-0.00009120,0.00003440,0.00017812,-0.00019548,0.00026912,-0.00000207, $-0.00000578,-0.00002037,-0.00017995,-0.00034107,-0.00734078,-0.0004299$ $9,0.00028600,0.01313210,-0.00116625,-0.00104712,-0.05268620,0.00173619$ $, 0.00273618,0.08210845,-0.00106399,0.00370365,0.00002532,-0.00127189,0$ $.00059772,-0.00063056,0.00089845,0.00098676,0.00042426,-0.00243324,-0$. $00280839,0.00000240,0.00088206,0.00098274,-0.00041741,-0.00122985,0.00$ $059563,0.00062381,-0.00095146,0.00120129,-0.00000635,-0.00004947,-0.00$ $003296,0.00020012,-0.00007614,0.00003592,-0.00000804,-0.00009012,0.000$ $02492,-0.00000978,-0.00005678,-0.00003164,-0.00018531,0.00310126,0.003$ $31565,0.00001065,-0.05700415,-0.02941053,-0.00036418,-0.01531241,0.029$ $10837,0.00010268,0.03056517,-0.03001134,-0.00003985,-0.09306980,-0.028$ $80558,-0.00027739,0.67635675,0.00120440,-0.00163047,0.00003907,0.00022$ $789,0.00031563,0.00057133,-0.00095571,-0.00013405,-0.00014010,0.001122$ $71,0.00013520,-0.00004162,-0.00096297,-0.00012274,0.00019942,0.0001901$ $0,0.00027728,-0.00061719,0.00057511,-0.00036862,0.00000900,0.00009499$, $-0.00005838,-0.00015505,0.00000178,0.00001784,0.00002326,0.00000488,0$. $00001270,-0.00002167,0.00010876,-0.00005911,0.00014713,-0.00069416,-0$. $00073679,-0.00001956,0.02714881,0.06259938,0.00035836,0.02867772,-0.04$ $928694,-0.00010746,-0.06397055,-0.03031591,-0.00037651,0.00499249,-0.3$ $4501742,-0.00126075,0.06139450,0.66536141,-0.00000452,0.00000412,-0.00$ $045963,-0.00025968,-0.00053527,0.00025430,0.00034367,0.00025009,0.0002$ $5451,0.00000475,-0.00000808,-0.00069686,-0.00034134,-0.00025284,0.0002$ $4318,0.00023840,0.00056486,0.00024384,0.00000615,0.00000023,0.00011029$ ,-0.00001137,-0.00001370,0.00004603,-0.00002495,0.00002203,0.00008902, $0.00002541,-0.00001733,0.00009389,0.00001550,0.00000570,0.00005050,0.0$ $0000582,0.00000544,-0.00019298,-0.00012365,0.00012361,0.00747446,0.000$ $08781,-0.00010445,-0.00242832,-0.00018623,-0.00025391,0.00444182,-0.00$ $013206,-0.00136866,-0.05369423,0.00222861,0.00258282,0.12710569,0.0009$ $8524,-0.00006849,-0.00000307,0.00058613,-0.00013481,0.00001057,-0.0000$ $9454,-0.00048047,-0.00011674,0.00005348,0.00037622,-0.00000515,-0.0001$ $1357,-0.00047950,0.00011694,0.00061464,-0.00012775,-0.00000091,0.00026$ $454,-0.00031370,-0.00000822,0.00001319,0.00008366,-0.00005755,-0.00001$ $048,-0.00002042,-0.00001171,-0.00001574,-0.00003179,-0.00000018,0.0000$ $0409,0.00008709,0.00006119,-0.00029576,-0.00036121,0.00000280,-0.24560$ $997,-0.07231406,-0.00096720,0.03264181,-0.06944070,-0.00021640,-0.0653$ $8267,-0.00324463,-0.00024577,0.02885939,0.08229926,0.00043104,-0.27615$ $979,0.04679581,-0.00053018,0.63597293,-0.00106532,-0.00042075,-0.00001$ $473,0.00023818,0.00009672,-0.00008302,-0.00026300,-0.00016831,-0.00013$ $409,0.00098856,0.00090917,0.00002783,-0.00026842,-0.00017960,0.0000920$ $0,0.00027070,0.00011024,0.00009822,0.00023739,-0.00022836,-0.00000784$, 
$0.00001839,-0.00003208,-0.00004836,0.00007248,-0.00001789,-0.00002247$, $0.00006766,-0.00001587,0.00001841,0.00000807,-0.00002798,0.00005586,-0$ $.00077957,-0.00084985,0.00002735,-0.13602965,-0.18540694,-0.00101553,-$ $0.02495912,-0.02975372,-0.00024963,-0.00471849,0.00079170,-0.00000873$, $0.02561975,-0.03181854,-0.00010053,0.12296747,-0.16027632,0.00000743$,$0.00406923,0.73869594,0.00000129,-0.00000789,0.00062803,0.00027223,-0$. $00003426,-0.00000652,-0.00013251,-0.00036004,0.00000071,-0.00000194,0$. $00000771,0.00008955,0.00012073,0.00034822,0.00000472,-0.00027633,0.000$ $03163,-0.00001764,0.00000403,-0.00000338,-0.00000979,-0.00001687,0.000$ $08654,0.00001090,0.00000523,-0.00000003,-0.00003134,0.00000028,0.00000$ $104,-0.00003412,0.00001836,-0.00008424,0.00001621,0.00000043,-0.000000$ $24,-0.00006051,-0.00123296,-0.00077939,-0.06591183,-0.00001689,-0.0004$ $0743,0.00830397,-0.00024324,0.00000694,-0.00220328,0.00017788,0.000107$ $45,0.01289829,-0.00022235,-0.00028900,-0.06123650,0.00176583,0.0026783$ $9,0.12852065,0.00067205,-0.00015494,-0.00000207,-0.00033495,-0.0001244$ $9,-0.00002399,0.00021970,0.00027428,0.00011973,-0.00065346,-0.00065866$ $, 0.00000387,0.00022610,0.00027159,-0.00012516,-0.00034457,-0.00012156$, $0.00002520,0.00007096,-0.00006774,0.00000273,0.00004436,-0.00002121,-0$ $.00003580,0.00002006,-0.00001143,-0.00001900,0.00002274,-0.00000602,0$. $00002476,0.00004673,-0.00002150,0.00003303,0.00119931,0.00117041,-0.00$ $000382,-0.12814491,0.04788607,-0.00014599,-0.01733217,0.01109784,-0.00$ $003162,0.00227918,0.00171232,0.00000186,-0.00017426,0.00129795,0.00000$ $541,-0.00091573,-0.00654671,-0.00004795,-0.03980667,0.02274990,-0.0000$ $5423,0.18366948,-0.00038166,0.00008817,0.00000204,0.00020345,0.0000803$ $8,-0.00000004,-0.00010737,-0.00014574,-0.00007964,0.00036491,0.0003691$ $8,-0.00000191,-0.00011115,-0.00014256,0.00008218,0.00020926,0.00007878$ ,-0.00000024,-0.00006580,0.00007722,-0.00000203,-0.00002681,0.00000653 $, 0.00001847,-0.00000410,0.00000369,0.00000536,-0.00000570,0.00000043,-$ $0.00000895,-0.00002805,0.00000702,-0.00001651,-0.00065019,-0.00064817$, $0.00000236,0.04788070,-0.06495612,-0.00001945,0.03757505,-0.01748627,0$ $.00003412,-0.00419965,-0.00398557,-0.00005321,0.00107312,0.00122151,0$. $00001353,-0.00043493,-0.00077296,-0.00002082,-0.00379387,0.00506225,-0$ $.00001375,-0.07933034,0.07892169,-0.00000028,0.00000101,-0.00014697,-0$ $.00004984,-0.00002276,0.00001152,0.00002274,0.00007028,0.00001544,-0.0$ 0000041,-0.00000051,-0.00003227,-0.00002355,-0.00007106,0.00001811,0.0 $0004991,0.00002117,0.00001007,0.00000077,0.00000046,-0.00001131,0.0000$ $0570,-0.00000470,0.00000126,-0.00000222,0.00000434,-0.00000061,0.00000$ $339,-0.00000407,-0.00000170,-0.00000481,0.00000575,0.00000208,0.000001$ $84,0.00000178,-0.00002049,-0.00015102,-0.00001714,-0.02416284,0.000089$ $95,-0.00005786,0.00475612,-0.00002759,-0.00002826,0.00437732,0.0000098$ $0,0.00001320,-0.00075737,-0.00002387,-0.00004608,0.00424043,-0.0001735$ $7,0.00007823,0.00489531,0.00024896,0.00002279,0.01200378,0.00030964,-0$ $.00021638,-0.00000201,-0.00025200,-0.00002184,0.00005247,0.00013928,0$. $00014261,0.00005964,-0.00033350,-0.00035216,-0.00000422,0.00013576,0.0$ $0014412,-0.00005329,-0.00025060,-0.00002410,-0.00005181,-0.00003692,-0$ $.00000476,0.00000108,-0.00001070,0.00000195,0.00001512,-0.00000162,-0$. $00000400,-0.00000367,-0.00000237,-0.00000636,0.00000186,-0.00000988,0$. $00000125,-0.00001453,0.00042539,0.00043601,-0.00000223,0.00213327,0.00$ $409435,0.00001552,-0.26431294,-0.13006625,-0.00137812,-0.01698442,-0.0$ $1450145,-0.00014037,-0.00360739,0.00261640,-0.00002383,0.00038005,0.00$ $093670,0.00000696,0.00154182,0.00087268,-0.00001250,0.00127921,0.00077$ 
$344,0.00001622,0.27834770,-0.00000787,0.00008539,0.00000191,0.00011548$ $, 0.00001139,-0.00001357,-0.00006247,-0.00008109,-0.00002579,0.00017868$ $, 0.00020105,-0.00000461,-0.00007135,-0.00007853,0.00003240,0.00012911$, $0.00000920,0.00001386,0.00004622,0.00000875,-0.00000246,0.00000572,0.0$ $0000016,-0.00001159,0.00000617,0.00000343,-0.00000485,0.00000279,-0.00$ $000318,-0.00000220,0.00000285,0.00000057,0.00001537,-0.00022266,-0.000$ $23733,0.00000167,-0.02702732,-0.01092526,-0.00016485,-0.12625116,-0.13$ $217015,-0.00087027,0.01452182,0.00974065,0.00007160,0.00307224,0.00025$ $972,-0.00001527,0.00057238,-0.00061571,0.00000727,0.00148501,-0.005252$ $20,-0.00004334,-0.00035514,0.00105539,0.00001368,0.13547324,0.13751936$ $, 0.00000316,0.00000085,0.00001710,0.00007276,0.00001930,0.00001684,-0$. $00007867,-0.00006067,-0.00004513,0.00000168,-0.00000004,0.00011273,0.0$ $0007565,0.00006056,-0.00004506,-0.00007271,-0.00002053,0.00001280,-0.0$ $0000111,-0.00000088,0.00002869,-0.00000556,0.00000795,0.00000276,0.000$ 00363,-0.00000005,0.00000056,-0.00000311,0.00000109,0.00000095,0.00000 $595,-0.00000849,0.00000371,0 ., 0.00000031,0.00003127,-0.00012827,-0.000$ $05141,0.00349171,-0.00136050,-0.00089496,-0.03530758,-0.00000929,-0.00$ $002555,0.00454588,-0.00002410,-0.00001310,0.00621326,0.00000979,-0.000$ $00166,-0.00089969,-0.00000020,-0.00003995,0.00509465,0.00001050,0.0000$ $2064,-0.00262240,0.00149335,0.00098543,0.02365826,0.00041529,-0.001112$ $64,0.00000809,-0.00001956,0.00022640,0.00002126,-0.00004698,0.00004314$ ,-0.00008458,0.00012029,0.00010720,0.00000283,-0.00004347,0.00004392,0 $.00008169,-0.00001962,0.00023063,-0.00002357,-0.00035354,0.00040911,-0$ $.00000506,-0.00001545,-0.00006106,0.00001576,0.00002745,-0.00000970,0$. $00000268,0.00002824,-0.00000697,-0.00000053,-0.00001340,-0.00006282,-0$ $.00001452,-0.00016437,-0.00017769,0.00000140,-0.00324266,0.00187383,-0$ $.00002401,0.00216242,0.02824926,0.00011380,-0.05364556,0.00589700,-0.0$ $0003788,0.00390965,-0.02514426,-0.00010093,-0.00394475,-0.00350925,-0$. $00005172,-0.00128815,0.00018014,-0.00000078,-0.00084814,0.00130676,0.0$ $0000282,0.00115446,-0.00041236,0.00001539,0.05543567,0.00087186,-0.000$ $24481,0.00000665,-0.00022700,-0.00006667,0.00032084,0.00021588,0.00024$ $338,0.00001035,-0.00030839,-0.00035404,-0.00001146,0.00021516,0.000249$ $40,0.00000895,-0.00022909,-0.00006940,-0.00032836,-0.00053857,0.000401$ $56,0.00000090,-0.00002500,-0.00001511,0.00001808,0.00000698,0.00002037$ $, 0.00000148,0.00000675,0.00001769,-0.00000256,-0.00001910,-0.00001743$, $-0.00001548,0.00031553,0.00030489,-0.00000593,0.00226487,0.00150781,-0$ $.00000843,-0.00186545,-0.01110847,-0.00007887,0.01024021,-0.33271993,-$ $0.00126416,-0.00175763,-0.01306198,-0.00006920,-0.00213094,0.00009104$, $-0.00003770,-0.00010505,0.00048494,-0.00000215,0.00027537,-0.00060496$, $-0.00000301,-0.00014980,0.00074859,0.00001980,-0.00719532,0.35476547,0$ $.00000031,-0.00000874,-0.00072411,0.00016999,0.00009760,0.00012670,0.0$ $0007715,0.00002793,-0.00007438,-0.00000002,-0.00000108,-0.00009219,-0$. $00007640,-0.00002182,-0.00007397,-0.00016723,-0.00009407,0.00012877,-0$ $.00000218,0.00000622,0.00013320,0.00010980,-0.00001418,-0.00007094,-0$. $00000810,0.00002378,0.00000878,0.00000751,-0.00002419,0.00000870,-0.00$ $010824,0.00001038,-0.00006980,-0.00000047,-0.00000105,0.00000664,-0.00$ $002243,-0.00001414,0.00666115,-0.00002139,0.00002420,0.00534098,-0.000$ $01951,-0.00128319,-0.03533544,0.00000906,-0.00014595,0.00025711,-0.000$ $04678,-0.00003257,0.00569532,-0.00000292,0.00000436,-0.00075674,-0.000$ $00205,0.00000178,0.00003687,0.00001817,0.00002038,-0.00407700,0.000080$ $65,0.00142789,0.02339485,-0.00015610,0.00036741,-0.00000626,0.00023905$ 
,-0.00000988,-0.00018742,0.00013230,0.00000077,0.00025123,-0.00065578, $-0.00000373,0.00001253,0.00014412,0.00000381,-0.00025437,0.00023586,-0$ $.00000854,0.00019803,-0.00003066,0.00002563,0.00000122,0.00001170,-0.0$ $0003831,-0.00000503,0.00000212,-0.00001061,-0.00000482,0.00000634,-0.0$ $0000557,0.00001071,0.00001392,-0.00003837,0.00000252,-0.00017999,-0.00$ $000765,-0.00000188,-0.00195110,0.00315813,-0.00001323,0.00000548,0.000$ $58561,-0.00000068,0.00134263,0.00178894,-0.00000472,0.00427409,-0.0020$ $0896,0.00001098,-0.25832867,-0.12377094,-0.00134747,-0.01852926,-0.015$ $47603,-0.00014716,-0.00132425,-0.00010689,-0.00000405,-0.00007731,-0.0$ $0001920,0.00000030,0.00020997,0.00022547,0.00000189,0.27336862,-0.0010$ 0236,-0.00010391,-0.00000112,0.00004084,0.00009739,-0.00025355,0.00010 $181,-0.00003851,-0.00010882,0.00018258,0.00034231,-0.00000649,0.000109$ $50,-0.00003038,0.00011714,0.00004782,0.00010637,0.00026290,0.00000880$, $0.00003967,-0.00000441,-0.00000671,-0.00000415,0.00002609,-0.00000046$, $0.00004689,0.00000306,-0.00000168,0.00004434,-0.00000719,-0.00000718,-$ $0.00000710,-0.00002183,0.00022913,0.00035613,0.00000144,0.00276946,-0$. $00002764,-0.00002571,0.00097419,-0.00066811,0.00000225,0.00010539,-0.0$ 0539806,-0.00004308,-0.02510254,-0.01341113,-0.00015904,-0.12771170,-0 $.13095561,-0.00088459,0.01443144,0.01031143,0.00007305,0.00097216,-0.0$ $0004578,0.00000284,-0.00006665,0.00003731,0.00000511,-0.00102649,-0.00$ $037567,-0.00000741,0.13548436,0.13918496,-0.00000390,0.00002107,-0.000$ $54089,-0.00031816,-0.00002262,0.00011266,-0.00012735,0.00028536,-0.000$ $14037,0.00001322,0.00000240,0.00051212,0.00012500,-0.00027970,-0.00013$ $681,0.00033782,0.00000105,0.00013435,-0.00000356,0.00000358,-0.0000149$ $9,0.00003372,-0.00006661,-0.00003955,0.00006736,-0.00002058,-0.0000553$ $2,-0.00007321,0.00001967,-0.00005659,-0.00003863,0.00007274,-0.0000455$ $3,-0.00000158,-0.00000298,0.00036341,-0.00001023,-0.00001625,0.0067001$ $7,-0.00000141,0.00000184,-0.00077764,-0.00001566,-0.00004646,0.0055123$ $0,-0.00008341,-0.00008016,0.00030721,-0.00136656,-0.00087338,-0.035447$ $34,-0.00002303,-0.00003630,0.00546382,0.00000022,-0.00000090,-0.000003$ $54,0.00000344,0.00000280,-0.00056533,-0.00000325,0.00000415,0.00012467$ $, 0.00146924,0.00100704,0.02311042,0.00015968,0.00011685,-0.00000087,-0$ $.00014614,-0.00002656,0.00001023,0.00013170,0.00013698,0.00005471,-0.0$ $0034557,-0.00038242,0.00000009,0.00013612,0.00013648,-0.00005411,-0.00$ $015525,-0.00002674,-0.00001145,-0.00008226,0.00005889,0.00000257,-0.00$ $000624,0.00000882,0.00000970,-0.00001622,0.00000964,0.00000751,-0.0000$ $1420,0.00001311,-0.00000335,-0.00000343,0.00000826,-0.00001226,0.00044$ $123,0.00049353,-0.00000245,0.00347910,-0.02595281,-0.00010753,-0.00422$ $671,-0.00240892,-0.00004642,-0.00099555,-0.00032407,-0.00000062,-0.003$ $42282,0.00324459,-0.00002174,0.00167271,0.02675063,0.00010507,-0.05443$ $844,0.01288550,-0.00001084,0.00095527,-0.00059040,0.00000973,0.0002852$ $6,-0.00131219,-0.00000795,0.00005779,0.00006171,0.00000313,0.00108081$, $-0.00036422,0.00001777,0.05571745,-0.00025895,0.00000068,0.00000138,0$. $00011176,0.00001402,-0.00002702,-0.00007779,-0.00005827,-0.00002457,0$. $00014247,0.00018696,-0.00000874,-0.00007227,-0.00005365,0.00003639,0.0$ $0009701,0.00001263,0.00002254,0.00005245,-0.00002955,0.00000221,-0.000$ $00146,-0.00000541,-0.00000435,0.00000604,-0.00000445,0.00000482,0.0000$ $0870,-0.00000400,-0.00000229,0.00000223,-0.00000636,0.00000090,-0.0002$ $1147,-0.00022854,-0.00000937,0.00501741,-0.01232158,-0.00004962,-0.002$ $97012,0.00058298,-0.00003503,0.00006499,0.00072780,0.00000948,0.002483$ $28,0.00013625,-0.00001818,-0.00198405,-0.00876908,-0.00007064,0.009176$ 
$73,-0.34248411,-0.00131505,0.00052487,0.00135590,0.00002112,0.00018775$ ,-0.00045881,-0.00000368,0.00000740,-0.00005758,0.00000309,-0.00023099 $, 0.00083212,0.00002200,-0.01220718,0.36047770,0.00000208,-0.00000026,0$ $.00014694,0.00010204,0.00005720,-0.00004640,-0.00001975,-0.00006470,-0$ $.00002167,0.00000161,-0.00000382,0.00003067,0.00001752,0.00007509,-0.0$ $0001612,-0.00010574,-0.00006562,-0.00004729,-0.00000053,0.00000015,-0$. $00000253,-0.00000137,-0.00000506,-0.00000109,0.00000357,-0.00000442,0$. $00000469,-0.00000403,0.00000387,0.00000190,0.00000400,0.00000552,-0.00$ $000098,-0.00000002,0.00000218,0.00008285,0.00002833,-0.00015811,0.0034$ $6973,-0.00004709,-0.00002760,0.00509154,-0.00000039,0.00000494,-0.0008$ $4142,-0.00002416,-0.00002198,0.00642905,-0.00002244,0.00004422,0.00453$ $654,-0.00002997,-0.00131136,-0.03573517,0.00001439,0.00001466,-0.00263$ $090,0.00000167,-0.00000857,0.00036641,0.00000223,0.00000563,-0.0006226$ $4,0.00001899,0.00001975,-0.00412607,0.00005963,0.00143845,0.02402205,0$ $.00179550,-0.00044506,0.00111797,-0.00073704,-0.00034106,0.00133453,-0$ $.00041104,-0.00170392,0.00176801,-0.01080380,-0.02927784,0.03585226,0$. $00006509,-0.00308522,-0.00562221,-0.00254741,-0.00191938,0.00059641,-0$ $.00015906,0.00007992,-0.00000202,-0.00007155,-0.00005833,-0.00010247,-$ $0.00225984,-0.00206268,0.00051618,0.00031049,0.00002868,0.00011668,-0$. $00070170,-0.00017529,-0.00001429,-0.15402611,-0.07699927,0.12333102,0$. $00040436,-0.00024831,0.00000083,-0.00021029,-0.00020731,-0.00000370,-0$ $.00051121,0.00088237,0.00001380,0.00020168,-0.00031049,-0.00011768,-0$. $00094939,0.00009312,-0.00000349,0.00010062,0.00022547,0.00000169,-0.00$ $041194,0.00022441,-0.00000175,-0.00012572,0.00006395,0.00000319,0.0000$ $6283,-0.00007358,0.00000383,0.00008309,-0.00008552,0.00000903,-0.00013$ $115,0.00006534,0.00000377,0.13465073,0.00169318,-0.00034442,0.00105232$ $,-0.00009696,-0.00083459,0.00133265,-0.00177476,-0.00047814,0.00150935$ $,-0.02922778,-0.00998214,0.03526718,-0.00334481,0.00023265,-0.00531707$ ,-0.00131345,-0.00285219,0.00065971,-0.00024396,0.00015790,0.00003075, $-0.00007322,-0.00005881,-0.00010344,-0.00207172,-0.00220010,0.00059104$ $,-0.00004811,0.00037834,0.00012455,-0.00019379,-0.00069642,-0.00001749$ ,-0.07707977,-0.15169174,0.12120451,0.00037073,-0.00024342,0.00000601, $-0.00020291,-0.00021035,0.00000178,-0.00055789,0.00095780,0.00000116,0$ $.00032022,-0.00042489,-0.00012788,-0.00106160,0.00015612,-0.00001450,0$ $.00012467,0.00023090,-0.00002168,-0.00040560,0.00022934,-0.00000383,-0$ $.00013377,0.00006977,0.00000388,0.00007134,-0.00006999,0.00000731,0.00$ $004977,-0.00016559,0.00007510,-0.00016389,0.00007861,0.00001805,0.1053$ $1442,0.13180521,-0.00573715,0.00015581,0.00026684,0.00315299,0.0039122$ $7,-0.00027205,-0.00748708,-0.00791853,-0.00817274,0.02856817,0.0281245$ $8,-0.01307715,-0.00117337,-0.00134869,0.00401093,0.00213292,0.00288484$ $, 0.00242591,0.00053712,-0.00058954,0.00039293,0.00011817,0.00015363,0$. $00001247,-0.00011591,-0.00015556,0.00348019,-0.00090363,-0.00101897,0$. $00114869,0.00013285,0.00014534,0.00002227,0.11643664,0.11474692,-0.408$ $92214,-0.00040123,0.00020652,-0.00000589,0.00025925,0.00040613,-0.0000$ $0729,0.00087407,-0.00128169,0.00002106,0.00142142,-0.00004613,0.000172$ $22,0.00132780,-0.00034933,0.00009192,-0.00016016,-0.00037868,0.0000367$ $5,0.00040748,-0.00022633,0.00001109,0.00016978,-0.00010084,-0.00001754$ ,-0.00005820,0.00013879,-0.00001340,0.00003468,0.00007032,-0.00019768, $0.00018989,-0.00008308,-0.00004548,-0.14919862,-0.14653245,0.53584354$, $0.00180134,-0.00044678,-0.00112008,-0.00255077,-0.00192696,-0.00057979$ $, 0.00007000,-0.00306005,0.00563400,-0.01083989,-0.02944785,-0.03572216$ 
,-0.00040678,-0.00170515,-0.00176865,-0.00074370,-0.00034947,-0.001334 $32,-0.00016021,0.00007883,0.00000251,-0.00070097,-0.00017546,0.0000144$ $2,0.00031164,0.00002848,-0.00011519,-0.00225733,-0.00206407,-0.0005031$ $2,-0.00007019,-0.00005839,0.00010333,-0.15417137,-0.07760191,-0.123149$ $89,0.00039867,-0.00025272,0.00000131,-0.00020381,-0.00021159,-0.000000$ $90,-0.00050936,0.00088529,-0.00000967,0.00019660,-0.00030743,0.0001206$ $8,-0.00094885,0.00008988,-0.00000352,0.00010716,0.00022649,0.00000067$, $-0.00041411,0.00022581,0.00000113,-0.00012648,0.00006609,-0.00000387,0$ $.00006157,-0.00007487,-0.00000377,0.00008300,-0.00008196,-0.00000688,-$ $0.00013314,0.00006584,-0.00000434,0.03638289,0.01004968,0.00947212,0.1$ $3482408,0.00171270,-0.00035079,-0.00105159,-0.00132088,-0.00286268,-0$. $00064301,-0.00333094,0.00025459,0.00534399,-0.02937327,-0.01025305,-0$. $03526219,-0.00174042,-0.00045375,-0.00154226,-0.00010951,-0.00085177,-$ $0.00132781,-0.00024638,0.00016062,-0.00003018,-0.00019686,-0.00069757$, $0.00002224,-0.00004407,0.00038215,-0.00012376,-0.00207396,-0.00220364$, $-0.00056622,-0.00007556,-0.00006023,0.00010334,-0.07765738,-0.15272006$ $,-0.12224737,0.00037238,-0.00024161,-0.00000560,-0.00020708,-0.0002143$ $6,-0.00000414,-0.00056017,0.00095881,0.00000236,0.00031282,-0.00042228$ $, 0.00013155,-0.00106333,0.00015348,0.00000889,0.00012582,0.00023922,0$. $00002091,-0.00040741,0.00023065,0.00000263,-0.00013298,0.00007152,-0.0$ $0000395,0.00007243,-0.00006911,-0.00000653,0.00004578,-0.00016729,-0.0$ $0007285,-0.00016558,0.00007572,-0.00001821,0.01001556,0.03595612,0.009$ $08468,0.10604829,0.13308532,0.00573906,-0.00015169,0.00026534,-0.00212$ $266,-0.00287508,0.00244714,0.00117972,0.00137544,0.00397400,-0.0284370$ $4,-0.02812330,-0.01276795,0.00748227,0.00790289,-0.00819258,-0.0031642$ $0,-0.00391801,-0.00025478,-0.00053743,0.00058900,0.00039178,-0.0001288$ $9,-0.00014182,0.00002054,0.00090648,0.00102059,0.00114876,0.00013402,0$ $.00018008,0.00347721,-0.00011283,-0.00015288,0.00001334,-0.11625165,-0$ $.11578587,-0.40774666,0.00039882,-0.00020792,-0.00000267,-0.00025114,-$ $0.00040767,-0.00001776,-0.00087066,0.00128031,0.00002718,-0.00142254,0$ $.00004440,0.00017394,-0.00133458,0.00035732,0.00008381,0.00016455,0.00$ $036313,0.00003986,-0.00040646,0.00022584,0.00001099,-0.00016869,0.0001$ $0185,-0.00001785,0.00005642,-0.00013619,-0.00001314,-0.00003521,-0.000$ $06775,-0.00019350,-0.00019042,0.00008925,-0.00004458,-0.00960295,-0.00$ $974701,-0.11720544,0.14897599,0.14818507,0.53438304 \backslash 10.00000462,0.0000$ $0542,-0.00000594,0.00000086,0.00000852,-0.00000321,0.00000001,0.000000$ $36,0.00000011,0.00000148,0.00000143,-0.00000225,-0.00000287,-0.0000009$ $8,0.00000113,-0.00000653,0.00000523,0.00000295,-0.00000844,0.00000179$, $0.00000154,0.00000348,-0.00000337,-0.00000018,-0.00000014,0.00000035,-$ $0.00000029,0.00000045,-0.00000077,0.00000017,0.00000417,-0.00000433,-0$ $.00000007,-0.00000096,0.00000062,-0.00000020,-0.00000003,-0.00000015,0$ $.00000039,-0.00000067,-0.00000142,-0.00000171,-0.00000597,0.00000408,0$ $.00000236,0.00000021,-0.00000981,0.00000739,0.00001256,-0.00000457,-0$. $00000440,-0.00000239,-0.00000192,-0.00000162,0.00000011,-0.00000018,0$. $00000041,-0.00000035,-0.00000010,0.00000003,-0.00000018,0.00000043,0.0$ $0000101,0.00000054,-0.00000014,0.00000182,0.00000046,-0.00000034,0.000$ $00028,-0.00000028,0.00000009,0.00000067,-0.00000012,-0.00000024,-0.000$ 00040III@

ortho-Addition Product 


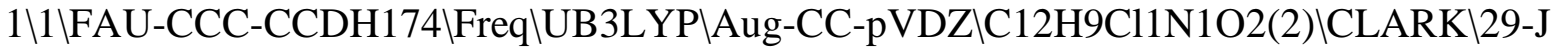
an-2015\0\\N Geom=AllCheck Guess=TCheck SCRF=Check GenChk UB3LYP/AugCC-pVDZ Freq \\Addition product p-Cl-Ph-radical + nitrobenzene (ortho) \ $\backslash 0,2 \backslash \mathrm{C},-5.9968047413,1.6451419418,1.8007722959 \backslash \mathrm{C},-4.6711169967,1.10924$ $19043,2.2716568538 \backslash \mathrm{C},-3.5004270902,1.8370840584,2.2545126529 \backslash \mathrm{C},-3.4794$ $337563,3.1305046449,1.705739238 \backslash \mathrm{C},-4.6620997172,3.6865480116,1.1336150$ $175 \backslash \mathrm{C},-5.8391795879,2.9971354139,1.1503628991 \backslash \mathrm{H},-6.4112827522,0.938458$ $8417,1.0613303397 \mathrm{~W},-4.6341500625,-0.2637636424,2.7226219407 \backslash \mathrm{H},-2.5946$ $649277,1.3779914766,2.6450570268 \backslash \mathrm{H},-4.6183935228,4.6744757556,0.674119$ $1153 \mathrm{\downarrow H},-6.7407433541,3.4317439887,0.7181586158 \mathrm{lH},-2.548130652,3.694864$ $9747,1.6917601368 \backslash \mathrm{C},-8.9728943496,1.9758407751,4.9356209385 \backslash \mathrm{C},-9.24822$ $52477,1.1987014063,3.81056095 \backslash \mathrm{C},-8.2777288056,1.0845675398,2.811650119$ 7\C,-7.047027985,1.7392271643,2.9261273401\C,-6.7955555685,2.514165609 7,4.066039164\C,-7.7508825014,2.6352593431,5.0775009424\Cl,-10.1882608 $126,2.1267365865,6.2021500102 \backslash \mathrm{H},-10.2026114943,0.6832915627,3.72091641$ 64\Н,-8.481410905,0.45981488,1.9421323113\H,-5.8430054822,3.0330671457 ,4.1749870457\H,-7.5516480831,3.2343835601,5.9641409259\O,-3.594726163 $5,-0.6988165333,3.2313724395 \backslash 0,-5.6601834247,-0.9365632241,2.556424828$ $7 \backslash$ Version $=E M 64 L-G 09 R e v C .01 \backslash$ State $=2-A \backslash H F=-1128.0581736 \backslash S 2=0.781062 \backslash S 2-$ $1=0 . \backslash \mathrm{S} 2 \mathrm{~A}=0.750552 \backslash \mathrm{RMSD}=4.134 \mathrm{e}-09 \backslash \mathrm{RMSF}=3.877 \mathrm{e}-06 \backslash \mathrm{ZeroPoint}=0.1827025 \backslash \mathrm{Th}$ ermal $=0.1959259 \backslash \mathrm{ZPE}=114.6475181 \backslash \mathrm{Dipole}=0.2595671,1.8682146,-1.1320239 \backslash$ DipoleDeriv $=-0.1129515,0.0783412,-0.0286241,-0.1315688,0.0485739,0.114$ $1432,0.0013249,0.0378886,0.1516796,0.2719112,-0.2806672,0.0351764,0.11$ $5248,-0.7735753,0.2834839,0.0960229,0.2134264,-0.1696047,-0.1576432,0$. $2021046,-0.0076648,-0.1510478,0.2383985,-0.1277262,0.0290973,-0.050222$ $5,-0.0041238,0.2268118,-0.0500918,0.0841248,0.2163254,-0.2033769,0.019$ $2403,0.0257193,0.0163129,-0.1117745,-0.1925,0.0514616,-0.017449,-0.145$ $5942,0.1548032,-0.0763844,0.0346165,-0.1033951,-0.0700246,0.1170538,-0$ $.0091025,0.1024669,0.2567801,-0.073028,-0.1520882,-0.0090162,-0.013900$ $1,-0.103785,0.0451611,-0.0319272,-0.0540987,-0.037923,0.04075,-0.05960$ $52,0.001913,-0.0373293,0.0442542,1.3440321,-0.0095229,0.2799796,-0.125$ $8864,2.0605216,-0.5034241,0.4522054,-0.6034888,0.4664699,0.0616631,0.0$ $092689,-0.0130494,0.0114868,0.1024245,0.0334035,-0.0485391,0.0124839,0$ $.1297599,0.0668957,-0.0006465,-0.004337,-0.0350482,-0.0432991,0.100015$ $5,-0.0315594,0.0772348,0.104132,-0.0075528,0.0293601,-0.0574392,0.0335$ $386,0.0458845,0.0304361,-0.0422597,0.0378542,0.0953942,-0.0274692,-0.0$ $531199,-0.0061488,-0.0534999,0.0397508,0.0260611,-0.0112118,0.0250899$, $0.1121912,0.6446003,-0.0396095,-0.6099034,-0.0709161,-0.0010987,0.1209$ $403,-0.6806901,0.1003359,0.6766186,-0.0934551,0.0933533,0.1322981,0.10$ $09787,-0.0491772,-0.0314484,0.1476344,-0.0336072,-0.2498018,0.0054846$, $0.0144017,-0.0466129,0.0922802,0.002926,0.0655781,0.0839892,0.0714643$, $-0.0085941,0.1960189,-0.0186766,-0.1492226,-0.0718955,-0.0290633,0.019$ $3435,-0.2199838,0.0037734,0.076329,-0.0706377,0.0392074,0.0456458,-0.0$ $170274,-0.0563347,0.0514516,0.0021906,0.012635,-0.0482771,-0.2092487,0$ $.015506,0.1219119,0.0255078,-0.0414972,0.0542295,0.1416965,0.065996,-0$ $.0765576,-0.511461,0.0368768,0.4025739,0.0483146,-0.0771384,-0.0592254$ $, 0.4498981,-0.0531201,-0.4984624,-0.0090422,-0.0535817,0.0102449,-0.05$ $51423,0.089012,-0.01901,0.0218266,-0.0142525,0.088442,0.09464,-0.00433$ $11,0.0150566,-0.0269846,0.0573978,-0.0787876,-0.0003007,-0.0694621,-0$. $0223847,-0.0333988,-0.0628533,0.0013855,-0.0683834,0.0663994,-0.003206$ $4,-0.0049964,0.0014335,0.0868684,0.0744379,-0.0200506,0.0045029,-0.015$ 
$3408,0.0791356,-0.0656539,0.0098869,-0.065458,0.0091208,-0.9231914,0.5$ $496864,-0.2826647,0.3651422,-0.7934427,0.214532,-0.3667617,0.3475263,-$ $0.3969523,-0.8001589,-0.4853871,0.0418472,-0.259344,-0.8849462,0.04370$ $12,-0.0827025,0.0207806,-0.280917 \backslash$ Polar $=213.9983452,11.6698915,172.576$ $5271,-32.6324714,-11.3498976,160.1777918 \backslash \mathrm{PG}=\mathrm{C} 01$ [X(C12H9Cl1N1O2)]।NIma $\mathrm{g}=0 \backslash 10.46743786,0.02680189,0.50337141,0.06271135,0.02709792,0.44389954$ $,-0.16879436,0.03383363,-0.04191229,0.66504325,0.03475040,-0.09076472$, $0.01685935,0.08827678,0.47406406,-0.03785566,0.01330738,-0.07737911,0$. $08620123,-0.09917633,0.19327246,-0.02657905,-0.01388859,-0.00281114,-0$ $.30444884,-0.10503453,-0.01555428,0.68115701,-0.00556417,0.00941904,-0$ $.00237888,-0.07186628,-0.12402378,0.01233123,-0.00919033,0.56041905,-0$ $.00538805,-0.00285158,0.00719936,-0.02913990,0.00523578,-0.05954694,0$. $13269497,-0.18010025,0.22984291,-0.00019253,0.00395480,-0.00009859,0.0$ $1807452,-0.02571598,0.01294770,-0.12010580,-0.03157333,-0.00085673,0.6$ $3997921,0.00792836,-0.00091790,-0.00144539,-0.06425882,-0.06820582,0.0$ $1424229,0.04916692,-0.24527203,0.09239495,0.10477365,0.54036379,-0.001$ $87251,-0.00211152,-0.00785225,0.02828496,0.02392956,0.00312508,-0.0339$ $7983,0.07597034,-0.09586783,0.08971710,-0.16163607,0.21095599,0.000583$ $72,-0.01143535,0.00328029,-0.03325410,-0.01952120,0.00061844,0.0372975$ $9,0.06376641,-0.01964409,-0.20549825,-0.00058766,-0.04070217,0.5954588$ $1,-0.02537687,-0.01364587,0.00434039,-0.00065640,0.00328736,-0.0026855$ $1,0.02805143,-0.01756175,0.01720936,0.07281357,-0.13431458,0.05603432$, $0.10130645,0.63253891,0.01040250,0.00917899,0.00845269,-0.00726813,-0$. $00792038,-0.00136524,-0.00514014,0.02625305,-0.00781476,-0.07393426,0$. $03718106,-0.09314811,0.07596494,-0.21649428,0.24350781,-0.08413687,-0$. $01031323,-0.00504817,0.01788735,0.03231672,-0.01058630,-0.01508331,-0$. $02422398,0.00669530,-0.06699483,0.02224274,-0.02922489,-0.31351684,-0$. $16198049,0.00816791,0.73007655,0.00132818,-0.17564970,0.05059860,0.017$ $18708,-0.01415269,0.00978057,-0.00983109,-0.01497489,0.00328740,-0.010$ $96280,0.03524437,-0.01499460,-0.12156346,-0.19223486,0.03340798,0.0743$ $6926,0.48849087,-0.01017571,0.05225442,-0.09106247,-0.00409776,0.01538$ $300,-0.00840867,0.00039577,-0.00088142,-0.00051254,-0.01436584,-0.0062$ $9078,0.00672192,-0.00907937,0.02419463,-0.06817896,0.11326418,-0.15221$ $649,0.24291468,-0.07322170,-0.05826254,-0.07120784,-0.01752809,-0.0147$ $9899,-0.01974705,-0.00196972,0.00271417,-0.00188532,-0.00110600,0.0005$ $3220,-0.00069549,0.00294388,-0.00011845,0.00065243,-0.00294566,-0.0067$ $3110,0.00113585,0.09351844,-0.05503041,-0.14265427,-0.10944803,0.00046$ $536,0.00966847,0.00711082,0.00221794,0.00030557,-0.00010516,-0.0003659$ $3,-0.00056603,-0.00000010,0.00278392,-0.00336073,0.00144914,-0.0136824$ $1,-0.02106312,-0.01429392,0.06562306,0.15902568,-0.06501919,-0.1027721$ $2,-0.14900743,-0.00638201,-0.00477925,-0.00555746,-0.00011047,0.001281$ $85,-0.00162336,-0.00034851,0.00009878,-0.00008744,0.00113692,0.0016815$ $4,-0.00153006,0.00693206,0.00886669,0.01142971,0.06702228,0.11561124,0$ $.16892780,0.00523259,0.02756590,-0.00647838,-0.10190901,0.00156677,-0$. $00360101,0.01319684,-0.02201119,0.01078960,-0.00627361,-0.00675986,-0$. $00074311,-0.00315805,-0.00033501,-0.00053788,-0.00496388,0.00113767,-0$ $.00352573,-0.00172693,-0.00047107,-0.00294932,0.81554480,0.00232094,-0$ $.01775305,0.00909144,0.02369625,-0.11832973,0.02176001,-0.02975842,-0$. $05293724,0.01017423,0.00176919,0.02187045,-0.00593037,0.00397247,-0.00$ $427528,0.00292305,0.00223711,0.00453201,-0.00374992,-0.00283711,0.0001$ $9269,-0.00163125,0.06085286,0.59228941,0.00106741,0.01145335,-0.006007$ 
$44,-0.01247935,0.01140623,-0.08144448,0.01539032,0.01749809,-0.0025108$ $8,-0.00335453,-0.00720842,0.01130789,-0.00305957,0.00078975,-0.0021045$ $5,-0.00219246,0.00039568,0.00130432,0.00081051,-0.00006094,0.00065953$, $0.18788004,-0.09462183,0.31210994,-0.00046096,-0.00372803,-0.00060931$, $-0.02025908,0.01140846,-0.01033882,-0.25920277,0.09852284,-0.08955309$, $0.00615878,-0.00342448,0.00173509,0.00128920,-0.00155396,-0.00024479,0$ $.00027646,-0.00040568,0.00049073,-0.00083973,0.00044238,-0.00023133,-0$ $.00333568,0.00285772,-0.00083453,0.27152437,-0.00237886,-0.00124573,0$. $00261854,-0.01283854,0.01227494,-0.00503477,0.09885627,-0.10364023,0.0$ 4738392,0.02307969,-0.00469992,0.00899718,-0.00145610,-0.00327751,0.00 $360181,-0.00062320,-0.00057257,-0.00018200,-0.00023714,0.00004565,-0.0$ $0010913,-0.00059477,0.00060171,-0.00265491,-0.10567088,0.10332683,-0.0$ $0022660,0.00229866,0.00603708,0.00136573,0.00115219,0.00561654,-0.0915$ $4345,0.04462006,-0.07850539,-0.00914189,0.00533934,-0.00032847,0.00006$ $816,0.00365986,0.00484180,0.00017330,-0.00041563,-0.00058585,0.0007343$ $2,-0.00065348,0.00031717,0.00074472,-0.00137469,-0.00443495,0.09818697$ $,-0.05501728,0.07089202,-0.00305671,-0.00180514,-0.00265373,-0.0011987$ $2,-0.00018554,-0.00021572,-0.00385602,0.00151186,-0.00291690,0.0048702$ $6,0.02325868,-0.01057319,-0.05606241,-0.01115086,0.00000076,0.00177447$ ,-0.02623213,0.01250629,-0.00006051,-0.00088599,0.00054096,-0.00050021 ,-0.00012987,0.00007801,0.00020296,0.00110730,-0.00030099,0.05574783,-$0.00224681,0.00304117,0.00231135,-0.00018892,0.00043282,-0.00016900,0$. $00160016,0.00170656,0.00192392,-0.00264685,-0.00674520,0.00367559,-0.0$ $1223256,-0.28481701,0.11491691,0.00150811,-0.01095029,0.00712021,-0.00$ $009982,0.00017947,-0.00012826,0.00015591,0.00007381,-0.00039255,-0.000$ $19468,-0.00031417,0.00007788,0.01389677,0.29838742,-0.00143192,0.00312$ $887,0.00773476,-0.00040720,-0.00041257,0.00010559,-0.00314410,0.001763$ $86,0.00296941,0.00233725,0.01052088,-0.00163006,-0.00007729,0.11478147$ ,-0.09253956,-0.00026975,-0.00015871,0.00348506,-0.00001280,0.00102632 ,-0.00080522,0.00014805,-0.00020054,-0.00075480,0.00021008,0.00047184, $-0.00017571,0.00134432,-0.12645118,0.08620194,0.00442012,-0.00300221,0$ $.00344997,0.00044286,-0.00109228,0.00043508,0.00013598,-0.00023595,0.0$ $0026766,-0.00217910,-0.00459636,-0.00034551,-0.01657060,0.01290388,-0$. $01185156,-0.24925046,0.09110756,-0.09839240,0.00027195,0.00017483,-0.0$ $0032165,0.00028512,-0.00057145,0.00007613,-0.00002937,0.00010746,0.000$ $08807,0.00057376,0.00046724,0.00117690,0.26162587,0.02234241,-0.008586$ $11,0.01062168,-0.00314414,-0.00296325,0.00191773,-0.00015517,-0.000771$ $69,-0.00019685,-0.00374737,0.00012175,0.00294636,-0.01563931,0.0098812$ $7,-0.00539781,0.09372727,-0.09638349,0.05468963,0.00009350,0.00112880$, $-0.00075939,0.00148017,-0.00030038,0.00072071,0.00010763,-0.00003102,-$ $0.00024266,0.00076501,-0.00011974,-0.00238319,-0.09576268,0.09784160,-$ $0.00945923,0.00361426,-0.00356343,0.00159972,0.00225904,0.00140782,0.0$ $0050298,-0.00044791,-0.00034798,-0.00133975,0.00259762,0.00661695,0.00$ $080163,0.00189850,0.00625150,-0.09820863,0.05274425,-0.08363685,0.0002$ $7058,0.00094797,0.00048378,-0.00036981,-0.00003000,0.00014915,0.000064$ $72,-0.00019897,-0.00050419,0.00126681,-0.00233960,-0.00403212,0.106001$ $03,-0.06105761,0.07647223,0.00036622,0.00049106,0.00014946,0.00173349$, $0.00098409,-0.00110536,0.00318854,0.00208746,-0.00141374,-0.26685759,-$ $0.12924710,-0.00247797,-0.01399859,-0.01500310,0.00202956,-0.00276581$, $0.00259538,-0.00305018,0.00003486,-0.00000217,-0.00003587,0.00024312,-$ $0.00017465,-0.00010705,0.00047690,0.00005873,0.00087464,0.00071391,0.0$ 
$0036311,0.00061213,-0.00090987,-0.00067074,0.00018950,0.27774861,0.000$ $39953,-0.00025364,-0.00030435,0.00130239,-0.00324069,0.00373278,-0.024$ $68658,-0.01007958,0.00034704,-0.12870361,-0.12957546,0.01121223,0.0112$ $7682,0.00981921,0.00062056,0.00247690,-0.00008151,0.00248271,-0.000062$ $35,-0.00000021,0.00003918,-0.00168386,0.00037367,-0.00004116,0.0004325$ $2,0.00049950,-0.00148434,-0.00009340,0.00017069,-0.00114430,0.00069620$ $, 0.00028350,-0.00012100,0.13850156,0.13295093,0.00021047,-0.00045761,-$ $0.00099467,-0.00144372,0.00358644,0.00314055,0.01072812,0.00655521,0.0$ $0419700,-0.00242457,0.01138808,-0.03681715,-0.01004026,-0.00623760,0.0$ $0488636,-0.00298860,0.00266979,0.00190611,-0.00010556,-0.00001252,0.00$ $012863,0.00056171,0.00054004,0.00081970,0.00059220,-0.00154546,-0.0025$ $7342,0.00083468,-0.00107769,-0.00147503,-0.00047871,-0.00050572,-0.000$ $23151,0.00423946,-0.01531823,0.02749119,-0.00119178,-0.00033182,0.0000$ $6897,-0.00043014,-0.00005813,0.00012081,0.00017335,0.00027128,0.000055$ $39,0.00015726,-0.00012741,0.00019130,-0.00005868,0.00018428,0.00017235$ ,-0.00005027,-0.00015877,0.00021893,-0.00014802,0.00003787,0.00008776, $-0.00095128,-0.00018102,-0.00031467,0.00004188,0.00001756,-0.00004581$, $0.00006661,0.00001892,-0.00007306,-0.00002896,-0.00011214,0.00004695,0$ $.00001743,-0.00000065,0.00000348,0.50534252,-0.00076554,-0.00076777,0$. $00017061,-0.00013096,-0.00005570,0.00017539,0.00003160,0.00016711,0.00$ $005917,0.00017800,-0.00002077,0.00021298,-0.00019225,-0.00006321,0.000$ $08511,0.00015985,0.00035106,0.00017524,-0.00011211,0.00021405,0.000100$ $42,0.00000455,-0.00047191,-0.00000581,0.00004133,-0.00001533,-0.000069$ $11,0.00003465,-0.00002425,-0.00011916,-0.00003387,-0.00010513,0.000046$ $01,-0.00002274,0.00000098,0.00000112,0.17065790,0.31400493,-0.00069122$ ,-0.00018142,-0.00018067,0.00013588,0.00014360,0.00039493,-0.00022231, $-0.00000341,-0.00004473,0.00030075,0.00014036,0.00019469,-0.00037613,-$ $0.00009520,0.00007183,0.00023038,0.00044884,0.00005237,-0.00004095,0.0$ $0008812,0.00019425,0.00044586,-0.00097607,0.00019848,0.00003778,-0.000$ $06013,-0.00009382,0.00002506,-0.00009632,-0.00010548,-0.00000807,-0.00$ $000432,-0.00010670,-0.00003908,-0.00002533,0.00000264,-0.01602549,0.18$ $635706,0.46511769,-0.00150681,-0.00375125,-0.00012996,0.00017006,0.000$ $37581,0.00034791,-0.00009705,-0.00019167,0.00004769,0.00014246,0.00016$ $818,0.00000132,-0.00000003,-0.00029392,0.00010044,-0.00069748,0.000571$ $56,0.00047314,-0.00006158,0.00010547,0.00006758,-0.00058540,-0.0012625$ $5,0.00002695,0.00002981,-0.00003453,-0.00003760,-0.00001659,-0.0000646$ $7,-0.00012351,-0.00007291,-0.00014269,0.00007977,0.00001035,0.00002037$ $, 0.00002599,-0.11759996,-0.06147192,-0.06259649,0.65310159,-0.00441587$ $, 0.00331386,-0.00262744,-0.00055298,-0.00022185,0.00090402,-0.00029602$ $,-0.00001525,-0.00010421,-0.00019565,0.00015812,-0.00019178,0.00022355$ $, 0.00030837,-0.00018573,0.00078633,-0.00020655,-0.00052688,0.00003198$, $-0.00005447,0.00005984,-0.00039451,-0.00040793,0.00004413,-0.00005734$, $-0.00006395,-0.00004138,0.00002235,0.00008525,0.00016867,0.00003245,-0$ $.00005826,0.00000520,0.00003404,-0.00000111,-0.00000115,-0.03228087,-0$ $.15318151,-0.12718093,0.20043567,0.34102416,-0.00031806,-0.00253567,0$. $00270508,0.00069370,0.00020317,-0.00080100,0.00011349,-0.00047004,0.00$ $007607,-0.00038841,0.00001464,-0.00034872,0.00055137,-0.00000673,-0.00$ $009310,-0.00086291,-0.00031086,0.00004453,-0.00005421,-0.00018223,-0.0$ $0020055,0.00024985,0.00111214,0.00010917,-0.00004654,0.00011711,0.0001$ $4097,-0.00007439,0.00003773,0.00012922,0.00003853,0.00006244,0.0000242$ $5,0.00001108,0.00002918,-0.00000265,-0.01127467,-0.10209999,-0.2338690$ 
$9,-0.08895406,0.19652042,0.54359523,-0.02076515,-0.00888256,0.00437479$ $, 0.00009275,-0.00120493,0.00110156,-0.00050607,-0.00012361,-0.00039471$ $,-0.00048186,0.00044844,-0.00035785,0.00127019,-0.00021175,0.00039622$, $-0.00195031,0.00038714,-0.00088833,0.00053476,-0.00119502,-0.00252798$, $-0.00041568,0.00060913,0.00002837,-0.00015095,-0.00008383,0.00007952$, $0.00019705,0.00000104,0.00015535,0.00020079,-0.00027596,0.00026056,0.0$ $0000745,0.00000033,0.00002130,0.04537007,0.04474563,0.04516533,-0.2197$ $5653,-0.02313694,0.09087141,0.58626234,0.00099358,0.00438919,0.0003168$ $5,0.00058270,-0.00082333,-0.00098685,0.00054745,0.00047970,-0.00001009$ $, 0.00019689,-0.00045764,0.00013148,-0.00036483,0.00018018,-0.00010711$, $0.00058713,-0.00015749,-0.00073677,0.00153984,0.00038686,-0.00050102,-$ $0.00030453,0.00037780,-0.00035364,-0.00002776,0.00012127,0.00025227,0$. $00001824,0.00006406,0.00001330,0.00011711,0.00017199,0.00003721,0.0000$ $0959,0.00000227,-0.00002824,0.01925397,0.01135277,-0.00838153,0.008045$ $21,-0.08654767,-0.04883301,0.16648086,0.35248553,0.02378995,0.00820038$ ,-0.00094440,0.00053135,0.00023901,-0.00192206,0.00098747,0.00023036,0 $.00034536,0.00011699,-0.00045101,0.00030633,-0.00019418,0.00084119,0.0$ $0006120,0.00189676,-0.00103979,-0.00076434,0.00212531,0.00181563,0.001$ $61234,0.00090278,0.00228393,-0.00055770,0.00016809,0.00018147,0.000041$ $69,0.00013298,0.00019322,0.00009886,-0.00018689,-0.00000880,-0.0000624$ $6,0.00005094,-0.00004859,-0.00009663,-0.00003220,-0.02953109,-0.045047$ $89,0.14467001,-0.02201014,-0.21931794,-0.08877560,0.23091562,0.5961759$ $2,-0.10378512,0.00723744,0.04358184,-0.03383579,0.00361491,0.01526857$, $0.00107040,0.00545364,-0.00094830,-0.00086978,-0.00134169,0.00075216,0$ $.00235072,0.00138780,0.00109524,-0.00735008,-0.01437317,0.01240842,0.0$ $0306509,-0.00130425,-0.00500810,-0.00034255,0.00070020,-0.00017520,-0$. $00000484,-0.00057950,-0.00090932,0.00033981,-0.00004817,-0.00032290,0$. $00083719,0.00079191,-0.00129955,0.00005852,-0.00001269,0.00001833,-0.0$ $2868858,-0.02518380,-0.02557656,-0.04189483,0.00469542,0.04976925,-0.2$ $6777757,-0.11873768,-0.03650966,0.55897959,-0.00178373,-0.06476813,-0$. $00495957,0.00509642,0.00049432,-0.00691626,0.00042306,-0.00074737,0.00$ $002971,0.00013494,0.00022945,0.00008441,0.00092084,-0.00371275,0.00105$ $704,-0.02024910,0.00003209,0.02050724,0.01212986,-0.00244448,-0.017984$ $61,0.00030080,-0.00171702,-0.00009795,-0.00002544,0.00022006,0.0002680$ $3,-0.00025660,-0.00091368,-0.00126145,-0.00020992,0.00044756,-0.000197$ $31,-0.00007426,0.00007016,0.00015095,-0.02511449,-0.03032552,-0.020089$ $87,-0.01691293,0.02101787,0.03884534,-0.08734336,-0.13102423,-0.043274$ $76,0.16350341,0.34655017,0.03184906,-0.01308594,-0.11570172,0.00488337$ $,-0.00413025,0.01199187,-0.00293384,-0.00045204,-0.00203129,-0.0000355$ $6,0.00109984,-0.00030563,-0.00020489,-0.00065596,-0.00125104,0.0123201$ $0,0.01061426,-0.01631999,0.01706243,-0.00376935,-0.02327913,-0.0008631$ $9,-0.00203125,0.00138375,-0.00044493,-0.00064426,-0.00036474,-0.000336$ $34,0.00015270,0.00072391,-0.00000065,-0.00093647,0.00157256,-0.0000866$ $9,0.00004681,0.00020076,-0.02644841,-0.02102298,-0.02143456,0.01031334$ $, 0.02110945,0.03498125,0.01891228,-0.01810455,-0.11081204,-0.04612422$, $0.17504425,0.51960665,0.00313560,0.01205911,0.02115193,0.00233848,0.00$ $065736,0.00152691,-0.00075351,-0.00042513,0.00037623,-0.00004374,0.000$ $36438,-0.00024923,-0.00031757,-0.00043326,0.00026787,0.00082880,0.0022$ $3179,0.00022048,-0.00249259,0.00053640,0.00422992,0.00054414,-0.000426$ $29,0.00022237,0.00004000,-0.00008219,-0.00010306,0.00001526,-0.0001025$ $6,-0.00012412,0.00001869,-0.00018561,0.00006518,-0.00001890,0.00002139$ 
$, 0.00005250,-0.04531139,-0.01984088,0.00926740,-0.00645585,0.00719570$, $0.01480624,0.00804451,-0.01689909,-0.02822735,-0.12168637,-0.05446590$, $-0.04947740,0.64253102,0.00295150,0.00168510,-0.00281434,-0.00032594,0$ $.00075069,-0.00046845,-0.00044381,-0.00022058,-0.00032750,0.00021226,-$ $0.00047209,0.00010214,-0.00106309,-0.00019450,0.00022402,0.00030039,0$. $00054021,0.00043298,-0.00025322,0.00066302,0.00019500,0.00035071,-0.00$ $025834,0.00036631,-0.00004400,0.00001858,-0.00002986,-0.00001081,0.000$ $00487,-0.00010232,-0.00011215,0.00036115,-0.00018226,-0.00001433,0.000$ $04028,0.00002563,0.00625955,0.02202379,0.02058233,0.00701433,-0.011767$ $02,-0.02121330,-0.03710044,-0.01486759,-0.00762878,-0.02567577,-0.1477$ $4225,-0.12118561,0.19009254,0.33555379,0.00371370,-0.01096818,-0.02218$ $434,-0.00162506,-0.00013238,-0.00091025,-0.00034245,0.00005478,0.00025$ $994,-0.00032295,-0.00025110,-0.00029468,-0.00023758,-0.00036569,-0.000$ $45668,0.00013650,-0.00263399,0.00024297,0.00261563,-0.00033193,-0.0022$ $5877,0.00156260,0.00180925,0.00002021,-0.00000671,-0.00000285,-0.00007$ 096,-0.00003027,-0.00001561,0.00011903,0.00003166,0.00027789,-0.000153 $24,-0.00001424,0.00000875,0.00001577,0.05403583,0.04201579,0.03549130$, $0.01506824,-0.02104910,-0.05266069,-0.06533848,-0.02559963,0.01789567$, $0.00181564,-0.09809611,-0.23697046,-0.09371664,0.19262793,0.55172489,0$ $.00290724,-0.00242911,-0.00255290,-0.00017569,-0.00000479,-0.00029711$, $0.00006695,-0.00006400,-0.00006519,-0.00012600,0.00005981,-0.00010148$, $0.00004342,-0.00034818,-0.00035026,-0.00032942,-0.00020383,-0.00000786$ $, 0.00054629,-0.00039877,-0.00081939,0.00039808,0.00036401,0.00025508,-$ $0.00006132,-0.00001663,0.00006878,-0.00010583,-0.00003091,0.00006930,0$ $.00003723,0.00021878,0.00003756,-0.00001909,-0.00000717,-0.00000078,-0$ $.26237976,-0.09176286,0.00264868,0.00921465,-0.03575386,-0.06293984,-0$ $.05535082,-0.01356219,0.01991840,0.04389951,0.04055209,0.04201199,-0.2$ $1616286,-0.02164366,0.09217401,0.58158752,-0.00206114,0.00180420,-0.00$ 445406,-0.00059494,0.00004011,0.00034741,0.00003694,0.00005372,-0.0001 $6081,0.00015290,-0.00001486,0.00000783,0.00002408,0.00035362,0.0001116$ $3,-0.00000117,-0.00056764,0.00003269,0.00032762,-0.00004126,-0.0006544$ $8,0.00016212,0.00010952,0.00004465,-0.00000163,-0.00006084,-0.00002631$ $, 0.00002063,-0.00000271,0.00003106,0.00002282,-0.00000191,-0.00010179$, $-0.00000550,-0.00001913,0.00000781,-0.12107666,-0.13472181,-0.02151733$ $,-0.01529031,-0.01270208,-0.02495616,-0.01354951,-0.00623403,0.0055871$ $6,0.01849075,0.01184454,-0.00805766,0.00971308,-0.08543221,-0.05006816$ $, 0.16805269,0.34397078,-0.00153609,-0.00430256,-0.00042280,-0.00070369$ $, 0.00028748,0.00003872,0.00040521,0.00031568,0.00012616,0.00016675,-0$. $00005951,0.00018630,-0.00005542,0.00011082,0.00015048,-0.00039087,-0.0$ $0018873,0.00067470,0.00045975,-0.00001645,-0.00054663,-0.00055427,-0.0$ $0047282,-0.00028042,0.00007849,0.00000745,-0.00003407,0.00006584,0.000$ $00124,-0.00014112,0.00000008,0.00000095,-0.00005229,0.00000558,-0.0000$ $0073,-0.00001202,-0.04885446,-0.04619150,-0.10605503,-0.02739503,-0.00$ $798193,0.01831850,0.02000474,0.00646693,-0.01004815,0.00228393,-0.0267$ $9869,-0.04172295,0.14576120,-0.02404584,-0.22559962,-0.08046196,0.2358$ $1819,0.61196238,-0.00000990,0.00003201,-0.00024429,0.00032865,0.000138$ $67,0.00031439,-0.00024741,-0.00006438,-0.00006348,0.00010300,0.0001813$ $1,0.00001372,-0.00005257,-0.00017567,0.00005106,-0.00014067,0.00051115$ $,-0.00000068,0.00001262,0.00002517,0.00005608,0.00050541,-0.00073138,0$ $.00037459,-0.00001342,-0.00005442,-0.00005233,-0.00003053,-0.00007545$, $-0.00005009,0.00002724,0.00003229,-0.00003660,-0.00003142,-0.00000862$, 
$-0.00000122,-0.09147847,0.00199603,0.05887960,-0.00462877,0.00061768,0$ $.00888383,0.00213167,-0.00095214,0.00056068,-0.00011703,0.00093589,0.0$ $0145962,-0.00092062,-0.00510211,-0.00427240,-0.02750623,0.00330276,0.0$ $3255801,0.12284618,0.00034372,-0.00034049,0.00034141,0.00000363,0.0000$ $8591,-0.00008579,0.00006293,-0.00000788,0.00004141,-0.00001507,-0.0000$ $0108,-0.00000940,0.00001121,-0.00000230,-0.00003448,-0.00003381,-0.000$ $06216,0.00000475,-0.00004327,0.00000255,0.00004256,-0.00014250,-0.0000$ $0332,-0.00003476,0.00000789,0.00001173,0.00001544,0.00000226,0.0000061$ $3,0.00000214,-0.00000908,0.00000519,0.00000409,0.00000773,-0.00000019$, $-0.00000301,0.00188286,-0.03054139,-0.01269816,0.01296992,0.00247745,-$ $0.01449749,-0.00397657,0.00155384,-0.00236279,0.00108757,0.00025590,0$. $00074941,-0.00214389,0.00183003,-0.00310850,-0.00890088,0.00526636,0.0$ $0833915,-0.00378993,0.02198848,0.00000621,0.00056922,0.00002769,-0.000$ $32998,-0.00016152,-0.00033087,0.00027184,0.00004559,0.00004875,-0.0001$ $1239,-0.00018993,-0.00002740,0.00007976,0.00026543,-0.00003295,0.00021$ $826,-0.00056115,-0.00001168,-0.00006295,0.00000599,0.00006437,-0.00058$ $942,0.00070765,-0.00042433,0.00001283,0.00005094,0.00004895,0.00003883$ $, 0.00009502,0.00005511,-0.00003104,-0.00004702,0.00003679,0.00003597,0$ $.00001060,-0.00000192,0.05907249,-0.01277666,-0.09522589,0.03029583,-0$ $.00421482,-0.02790796,-0.00470179,-0.00493843,0.00030774,0.00134297,0$. $00089319,-0.00053081,0.00090073,-0.00067723,0.00256209,0.01088213,-0.0$ $0180930,-0.00753483,-0.09824846,0.02067365,0.12925491,-0.00047481,-0.0$ $0037915,-0.00024852,0.00003396,0.00006086,0.00011405,-0.00006928,-0.00$ $002892,-0.00001797,0.00001059,0.00005466,-0.00000970,-0.00000448,-0.00$ $006370,0.00001947,-0.00006667,0.00013671,0.00001672,0.00003280,-0.0000$ $0940,-0.00006342,-0.00006678,-0.00035730,0.00007768,-0.00001375,-0.000$ $01609,0.00000476,-0.00000576,-0.00001632,-0.00001348,0.00000057,-0.000$ $01237,0.00000902,-0.00000523,0.00000119,0.00000289,-0.00272325,-0.0012$ $4638,0.00295075,-0.27366084,-0.12680071,-0.01941330,-0.01284170,-0.010$ $37255,-0.00379058,0.00033417,-0.00079717,0.00430392,0.00017788,0.00076$ $097,0.00015549,0.00232984,-0.00153751,0.00001817,0.00070904,0.00148577$ ,-0.00022088,0.28622429,0.00029489,0.00013132,-0.00019905,-0.00004249, $-0.00000305,0.00004756,0.00000894,-0.00000933,0.00000777,-0.00005924,0$ $.00002111,-0.00003249,0.00011026,0.00004774,0.00004254,-0.00008552,-0$. $00010810,-0.00001503,-0.00000416,-0.00001695,0.00002280,0.00008563,0.0$ $0016387,-0.00003389,0.00001022,-0.00000712,-0.00001382,-0.00000654,0.0$ $0000317,0.00001368,-0.00001144,0.00000138,-0.00000274,0.00000132,-0.00$ $000115,-0.00000028,-0.01537415,-0.00391899,0.00015161,-0.12590674,-0.1$ $0823288,-0.01988946,0.00326203,0.00502038,-0.00072471,-0.00077817,0.00$ $504648,-0.00264721,0.00083553,-0.00078169,-0.00001168,-0.00138040,0.00$ $210218,-0.00468169,0.00098564,-0.00130654,0.00160592,0.13725557,0.1048$ $3859,0.00082909,0.00036452,0.00010862,-0.00002053,-0.00002121,-0.00020$ $740,0.00009662,0.00005864,0.00003060,0.00003562,-0.00007974,0.00003324$ ,-0.00009537,0.00003875,-0.00001175,0.00014934,-0.00007858,-0.00002341 ,-0.00002102,0.00001665,0.00004528,0.00001676,0.00015945,-0.00004907,0 $.00000986,0.00001709,0.00002457,0.00001284,0.00001441,-0.00000066,0.00$ $000424,0.00002042,-0.00001952,0.00000692,-0.00000144,-0.00000551,-0.02$ $207055,-0.01221059,0.00091489,-0.01728889,-0.01851272,-0.05306866,0.01$ $983279,0.01078617,0.00582220,0.00390545,-0.00282622,-0.00115834,0.0003$ $0647,0.00014497,-0.00111635,0.00051782,-0.00451393,-0.00289818,-0.0010$ $7564,0.00117925,0.00028903,0.01724992,0.02415073,0.05150015,0.00043371$ 
$, 0.00061551,-0.00243800,-0.00014645,0.00010051,0.00021386,0.00003410,0$ $.00003511,0.00001788,0.00004776,-0.00000466,0.00002247,-0.00006981,0.0$ $0012485,-0.00008067,0.00032367,0.00002950,0.00006768,-0.00033535,0.000$ $20270,0.00085633,-0.00068851,-0.00058768,-0.00015158,-0.00000788,-0.00$ $001959,-0.00004847,0.00002696,0.00001687,-0.00001308,-0.00000988,0.000$ $01797,-0.00003051,0.00000224,0.00000669,-0.00000371,-0.00083780,-0.001$ $69236,0.00382265,0.00704123,0.01252807,0.01830257,-0.06376793,-0.03659$ $017,-0.03783330,-0.00120456,-0.01520717,-0.02142108,-0.00395768,-0.004$ $15563,0.00100435,-0.00105101,0.00024473,0.00039355,-0.00037347,0.00044$ $033,0.00115842,0.00016844,0.00146522,-0.00115593,0.06355840,0.00229566$ ,-0.00217048,0.00096576,0.00033946,0.00038241,-0.00012532,0.00018900,$0.00012530,0.00003078,0.00000718,-0.00001781,0.00004272,0.00004681,-0$. $00011446,0.00008570,-0.00055198,0.00002375,0.00017721,-0.00045567,0.00$ $036486,0.00049216,0.00023603,0.00039759,-0.00005798,0.00004508,0.00003$ $914,0.00000570,-0.00000333,-0.00003947,-0.00006528,-0.00007713,-0.0001$ $1593,0.00006145,0.00001128,-0.00000266,0.00000706,-0.00159747,0.005096$ $78,-0.00184320,-0.00172730,0.00265547,-0.00071172,-0.03771267,-0.13747$ $126,-0.13700404,-0.00099106,-0.00414989,-0.01227277,-0.00443963,0.0029$ $6434,-0.00137769,0.00022701,-0.00055318,0.00077428,-0.00007881,0.00003$ $934,0.00003564,0.00154849,-0.00236052,0.00172411,0.04270986,0.13604811$ $, 0.00010946,0.00217737,-0.00008327,-0.00006313,0.00024716,0.00010555$,$0.00016126,-0.00006393,0.00002826,-0.00000719,0.00013211,-0.00007665,0$ $.00006638,0.00008420,-0.00005915,0.00031786,0.00002977,0.00005461,-0.0$ $0020978,-0.00001391,0.00042088,-0.00050345,-0.00091229,0.00001256,0.00$ $000132,-0.00003779,-0.00002953,0.00002496,0.00002796,0.00001126,0.0000$ $2061,0.00000036,-0.00003909,-0.00000300,0.00000864,0.00000267,0.004177$ $86,-0.00185222,0.00068043,-0.00570776,-0.01302245,-0.01341530,-0.03914$ $651,-0.13577516,-0.23032160,0.00185318,-0.00153439,-0.00031602,0.00032$ $510,-0.00196084,0.00281719,0.00025712,0.00080962,0.00006432,0.00027665$ ,-0.00048757,-0.00096511,-0.00097471, $0.00194845,-0.00028050,0.03841525$ $, 0.14942357,0.24156721,-0.00050994,0.00127134,-0.00004921,0.00013156,0$ $.00008163,0.00040282,-0.00016530,0.00006859,0.00001521,0.00017933,-0.0$ $0013298,0.00019912,-0.00017243,0.00017267,0.00020494,0.00001828,0.0000$ $9981,-0.00007966,-0.00005665,-0.00001568,-0.00006297,-0.00006071,-0.00$ $001803,-0.00001314,-0.00002060,0.00003203,0.00002995,0.00001098,0.0000$ $3883,-0.00001227,-0.00002650,-0.00004754,-0.00010658,0.00000261,-0.000$ $00204,-0.00001666,0.00058853,-0.00083851,0.00399121,0.00024190,0.00070$ $192,0.00013077,0.00230951,-0.00186235,-0.00007084,-0.00188225,-0.00180$ $165,0.00110663,-0.26688217,-0.12325151,-0.02192636,-0.01410474,-0.0107$ $4290,-0.00363135,-0.00093604,-0.00028766,0.00030502,-0.00016648,0.0001$ $7576,-0.00014660,-0.00008575,-0.00005787,0.00011994,0.28143390,0.00067$ $288,-0.00188058,0.00161885,0.00001836,0.00011623,-0.00026077,0.0002875$ $2,-0.00001692,0.00027625,-0.00018529,0.00034835,-0.00016247,0.00034086$ $,-0.00011788,-0.00021641,-0.00025915,0.00015454,0.00028324,0.00001082$, $-0.00007340,0.00010432,-0.00033916,-0.00043883,-0.00009914,0.00006221$, $0.00000191,-0.00000930,-0.00001645,-0.00002142,0.00008363,0.00002075,-$ $0.00000742,0.00007633,-0.00001738,-0.00000811,0.00001813,-0.00113631,0$ $.00509250,-0.00262767,0.00078153,-0.00069588,0.00018398,-0.00155035,0$. $00252067,-0.00528019,-0.01527726,-0.00410340,-0.00019380,-0.12309458,-$ $0.10757369,-0.02151523,0.00332284,0.00492386,-0.00115436,0.00020076,0$. $00007962,-0.00014900,0.00017518,-0.00045423,0.00030532,-0.00079170,-0$. 
$00016349,-0.00003602,0.13486638,0.10467646,-0.00088563,0.00119987,-0.0$ $0011788,0.00005303,0.00010427,-0.00016731,0.00025110,-0.00017711,-0.00$ $020197,0.00027980,0.00004481,0.00004803,0.00003259,0.00038219,0.000016$ $69,-0.00002857,-0.00016897,-0.00040258,-0.00015690,0.00006566,0.000211$ $21,-0.00011360,-0.00086380,0.00029973,-0.00002734,0.00002944,0.0000771$ $6,0.00003549,0.00003009,0.00001969,0.00002197,-0.00004007,0.00001866,-$ $0.00003521,-0.00001089,0.00002053,0.00370334,-0.00273124,-0.00073629,0$ $.00024811,0.00011336,-0.00114911,0.00065616,-0.00497758,-0.00293594,-0$ $.02278341,-0.01113687,0.00299323,-0.02149774,-0.02173317,-0.05648050,0$ $.02075374,0.01074599,0.00532443,0.00120772,0.00025888,-0.00044569,-0.0$ $0015430,0.00028558,-0.00007058,-0.00118587,-0.00066799,-0.00000265,0.0$ $2055605,0.02671553,0.05398402,0.00020459,0.00044507,0.00082480,0.00019$ $621,0.00003659,-0.00000891,-0.00009714,-0.00005670,0.00003983,0.000000$ $98,0.00003718,-0.00006987,-0.00001090,-0.00004858,-0.00001616,0.000096$ $47,0.00020402,-0.00010155,-0.00017360,0.00007334,0.00023140,0.00005710$ ,-0.00014323,0.00009973,-0.00001630,0.00000400,0.00000764,-0.00000800, $-0.00000899,0.00001167,-0.00000400,-0.00000951,0.00001174,-0.00000765$, $0.00000257,0.00001086,-0.00155414,-0.01500270,-0.02221612,-0.00392218$, $-0.00379918,0.00105695,-0.00105433,0.00027852,0.00047846,-0.00143783$,$0.00180123,0.00408734,0.00768737,0.01194890,0.01774363,-0.06252116,-0$. $03542900,-0.03973577,0.00049083,0.00089001,-0.00110023,0.00001282,-0.0$ $0074753,-0.00107660,-0.00001139,0.00022908,-0.00013929,0.00006246,0.00$ $150091,-0.00106388,0.06209381,-0.00024026,0.00010281,-0.00004691,0.000$ $07461,-0.00006840,0.00002468,0.00001261,0.00000837,-0.00001689,-0.0000$ $0086,-0.00004180,-0.00000118,-0.00000488,0.00002878,0.00005583,-0.0000$ $5585,-0.00010028,0.00000094,0.00007383,-0.00003176,0.00000814,-0.00001$ $713,0.00017037,-0.00010544,0.00000234,0.00000782,-0.00001186,0.0000007$ $0,-0.00000039,0.00000664,0.00000650,-0.00000767,0.00001286,0.00000185$, $0.00000345,0.00000035,-0.00065506,-0.00407039,-0.01259561,-0.00403528$, $0.00273952,-0.00119139,0.00011404,-0.00051236,0.00083425,-0.00147692,0$ $.00528864,-0.00197592,-0.00153951,0.00259970,-0.00077100,-0.03662704,-$ $0.13029035,-0.13615912,0.00137835,-0.00131894,0.00122078,-0.00010993,-$ $0.00008072,-0.00059653,0.00022237,-0.00044038,0.00022451,0.00154956,-0$ $.00247528,0.00190924,0.04128864,0.12859392,-0.00034641,-0.00056625,-0$. $00045373,-0.00024954,-0.00000756,-0.00004440,0.00007149,0.00006431,0.0$ $0004060,0.00000260,-0.00005225,0.00000514,-0.00001076,0.00004523,-0.00$ $002458,0.00001493,-0.00010026,0.00008669,0.00014698,-0.00000458,-0.000$ $26245,-0.00009467,0.00009864,-0.00005438,0.00000632,0.00000769,-0.0000$ $0432,0.00001116,0.00000899,-0.00001077,-0.00001325,-0.00000331,-0.0000$ $2147,0.00000679,0.00000017,0.00000070,0.00299525,-0.00073947,-0.000138$ $56,0.00053273,-0.00128737,0.00248283,0.00032665,0.00070355,0.00005708$, $0.00449923,-0.00155853,0.00028525,-0.00580889,-0.01201940,-0.01237449$, $-0.04190710,-0.13727416,-0.24179533,-0.00027447,0.00163366,0.00035045$, $0.00006569,-0.00005816,0.00013009,-0.00015490,0.00019711,-0.00021024,-$ $0.00094439,0.00192990,-0.00007996,0.04101808,0.14907394,0.25197481,-0$. $00120386,-0.00046527,-0.00103324,-0.00487718,0.04324377,-0.02260274,-0$ $.01238654,-0.01682029,0.00261454,0.00276049,0.00933465,-0.00297702,0.0$ $0335391,-0.00128958,0.00150301,-0.00122609,-0.00016593,-0.00003917,-0$. $00014005,0.00036494,0.00089025,-0.36063472,0.09921101,-0.13234097,0.00$ $342174,0.00030749,0.00044152,0.00038034,-0.00000299,0.00005226,0.00007$ $175,0.00013770,-0.00027398,-0.00016841,0.00020559,0.00007309,0.0006449$ 
$7,0.00004724,-0.00037035,0.00009410,0.00006625,-0.00016100,-0.00028812$ $, 0.00021307,0.00021866,0.00067779,0.00011127,-0.00069187,-0.00032748,-$ $0.00042026,-0.00027287,-0.00031290,-0.00000505,0.00046907,-0.00046781$, $0.00007215,0.00053524,-0.00006029,0.00000418,0.00003299,0.00038262,-0$. $00003970,0.00021093,0.00002603,0.00015758,-0.00016470,-0.00009308,0.00$ $006775,0.00008879,0.46298475,-0.00271096,-0.00477673,0.00191105,0.0386$ $4221,-0.02863171,0.02784489,0.00233973,-0.00240844,0.00238113,0.000166$ $00,-0.00099660,0.00001872,0.00081866,0.00024505,-0.00002013,0.00167634$ ,-0.00117337,0.00106553,0.00040756,-0.00009639,0.00022961,0.11962730,$0.17770230,0.07521920,0.00115618,-0.00378111,0.00181568,0.00009591,-0$. $00012242,0.00011982,-0.00006360,-0.00074814,0.00030246,-0.00005272,-0$. $00010167,0.00001876,-0.00022473,0.00008507,0.00038658,0.00024482,0.000$ $07718,-0.00023904,0.00001573,-0.00013715,-0.00067766,-0.00085971,0.000$ $26144,0.00117089,0.00025179,0.00019572,-0.00043341,0.00006658,0.000008$ $11,-0.00005704,0.00037750,-0.00002932,-0.00039109,0.00010649,-0.000036$ $72,-0.00005245,0.00003216,-0.00010998,0.00016608,-0.00002177,0.0000048$ $1,0.00026894,0.00006427,-0.00006519,-0.00005250,-0.13393693,0.17871145$ $, 0.00033346,0.00249395,0.00264444,-0.02073056,0.03000147,0.00003107,-0$ $.00505985,-0.00431305,0.00141387,0.00048327,0.00318039,-0.00182930,0.0$ $0115328,-0.00042759,0.00033801,-0.00093962,0.00004848,-0.00021838,-0.0$ $0006072,-0.00032612,0.00013326,-0.13879079,0.06899558,-0.14346704,0.00$ $050872,0.00140132,-0.00060082,0.00006220,0.00007108,0.00005269,0.00001$ $715,0.00025927,-0.00017758,-0.00001406,0.00005189,-0.00003377,0.000360$ $69,0.00004207,-0.00015946,0.00007714,-0.00002195,-0.00012813,-0.000209$ $81,0.00038267,0.00032495,-0.00013738,0.00039069,-0.00083983,-0.0002110$ $3,-0.00032457,-0.00019045,-0.00024048,-0.00004898,0.00025813,-0.000321$ $44,0.00003488,0.00037060,-0.00006093,0.00001972,0.00002237,0.00016289$, $-0.00001080,0.00010318,0.00002504,0.00009231,-0.00016203,-0.00007343,0$ $.00006820,0.00005484,0.19795390,-0.09631537,0.12922157,0.00116793,0.00$ 436650,-0.00207784,-0.01961569,-0.04999696,0.00229963,0.00924707,0.008 $00859,-0.00132684,-0.00175556,-0.00807014,0.00346256,-0.00184611,0.002$ $09484,-0.00160486,0.00018659,-0.00126885,0.00260605,0.00184550,0.00030$ $773,0.00182866,-0.35039419,-0.16141192,-0.05136414,0.00095455,0.001115$ $75,-0.00045855,0.00037518,-0.00010724,-0.00004173,0.00013254,0.0001905$ $9,-0.00008308,0.00017830,-0.00008306,0.00012872,0.00102985,0.00040008$, $0.00026143,0.00091066,0.00031253,-0.00099165,-0.00091996,0.00080913,-0$ $.00092824,-0.00073561,0.00193438,0.00075899,-0.00003103,-0.00027443,-0$ $.00195125,-0.00082386,-0.00025578,0.00064854,-0.00020028,0.00007467,0$. $00024061,0.00012491,-0.00009881,-0.00005558,0.00052834,-0.00015233,0.0$ $0073943,0.00006447,0.00024902,0.00029803,0.00000907,-0.00003043,0.0000$ $1769,-0.09261196,-0.02821880,-0.03428765,0.45217929,-0.00084933,-0.001$ $08295,-0.00018577,-0.05491850,-0.05011261,0.00238227,0.00945206,0.0002$ $5314,0.00309053,-0.00326706,-0.00603777,0.00170411,-0.00154163,0.00100$ $220,-0.00081807,-0.00032828,-0.00093023,0.00008879,0.00053146,-0.00177$ $460,0.00048540,-0.18047283,-0.24655759,-0.01216966,0.00003708,0.000540$ $34,-0.00011540,0.00004229,-0.00008465,0.00016652,0.00012130,-0.0000440$ $5,0.00007193,0.00013749,-0.00084955,0.00021815,-0.00015451,-0.00010534$ $, 0.00007802,0.00035288,0.00029917,0.00001095,0.00028316,-0.00087375,-0$ $.00127989,-0.00032321,-0.00010998,0.00117841,0.00011945,0.00007868,-0$. $00006218,0.00027048,0.00016502,-0.00001122,0.00031770,0.00001107,-0.00$ $026346,0.00016163,-0.00005822,-0.00008710,0.00025650,-0.00017698,0.000$ 
$41675,0.00002200,0.00011160,0.00035384,0.00004354,-0.00003946,-0.00003$ $938,-0.00039860,0.04132842,-0.00574553,0.23010495,0.26504809,0.0001836$ $5,0.00249368,-0.00285366,0.00310387,0.00570371,0.01885500,-0.00090626$, $0.00224499,0.00242939,0.00034336,-0.00071095,-0.00088789,0.00002329,0$. $00061090,0.00003210,0.00022390,-0.00002457,0.00120804,0.00122454,0.001$ $08119,0.00053029,-0.04358735,-0.00686060,-0.08628440,0.00023416,0.0006$ $0176,0.00026830,0.00012036,0.00001191,-0.00003601,0.00000065,0.0000657$ $9,-0.00008528,0.00017042,-0.00009018,-0.00056832,-0.00017209,-0.000091$ $18,-0.00011535,-0.00014293,0.00003491,0.00035579,0.00015403,-0.0006724$ $3,-0.00021819,0.00120037,-0.00053825,-0.00070069,-0.00006509,0.0000121$ 7,0.00057242,0.00014974,0.00009910,0.00003930,0.00000516,0.00000128,0. $00002297,-0.00002074,0.00001385,-0.00001109,0.00007920,0.00011354,-0.0$ $0006903,-0.00002665,0.00001657,-0.00006087,-0.00002032,0.00003150,0.00$ $000618,-0.04415634,-0.01468065,0.01283782,0.08188107,0.01053197,0.0547$ $3320 \backslash 0.00001043,-0.00000705,-0.00000226,0.00000048,-0.00000076,0.0000$ $0195,-0.00000935,-0.00000706,-0.00000178,-0.00000004,-0.00000027,-0.00$ $000277,0.00000797,0.00000274,0.00000038,-0.00000611,-0.00000038,0.0000$ $0204,-0.00000452,0.00000129,-0.00000018,-0.00000071,-0.00000200,-0.000$ $00755,0.00000118,-0.00000658,-0.00000526,0.00000091,0.00000080,0.00000$ 405,0.00000055,0.00000309,0.00000175,0.00000195,-0.00000436,0.00000061 $, 0.00000054,0.00000392,0.00000532,-0.00000011,0.00000274,-0.00000019,-$ $0.00000133,0.00000321,-0.00000162,-0.00000422,0.00000708,0.00000690,0$. $00000334,-0.00000242,-0.00000148,0.00000124,-0.00000233,0.00000262,0.0$ $0000463,0.00000059,0.00000253,-0.00000100,0.00000434,0.00000152,-0.000$ $00329,0.00000418,0.00000056,0.00000260,-0.00000217,0.00000134,0.000004$ $24,-0.00000204,0.00000302,-0.00000093,0.00000034,-0.00000479,-0.000008$ 44,0.00000310,-0.00000671III@

meta-Addition Product

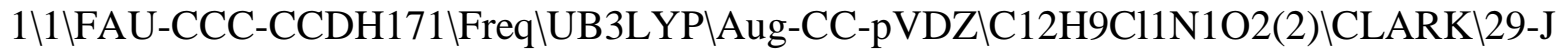
an-2015\0\\N Geom=AllCheck Guess=TCheck SCRF=Check GenChk UB3LYP/AugCC-pVDZ Freq \\Addition product p-Cl-Ph-radical + nitrobenzene (meta) \ $0,2 \backslash \mathrm{C},-6.4458406758,1.7087752645,1.4968561475 \backslash \mathrm{C},-5.1711585053,0.948941$ 5352,1.7634178036\C,-3.9495406948,1.5358049601,1.5999234091\C,-3.76944 $15848,2.873170357,1.1627063716 \backslash \mathrm{C},-4.9296098326,3.6370938879,0.87783710$ 56\C,-6.1894646946,3.1179693375,1.0185248891\H,-6.9968664188,1.1664374 $212,0.7020116214 \backslash \mathrm{H},-5.2350404821,-0.0818602055,2.1038006702 \backslash \mathrm{N},-2.73708$ $80734,0.7277769172,1.8948663067 \backslash \mathrm{H},-4.8079342802,4.6656942001,0.5360643$ $014 \backslash \mathrm{H},-7.0640581481,3.7286218922,0.7933993214 \backslash \mathrm{H},-2.769074813,3.2793377$ $069,1.0535851618 \backslash \mathrm{C},-9.0395441405,1.6329515191,4.968146329 \backslash \mathrm{C},-9.4424387$ $652,0.9830607572,3.8021081717 \backslash \mathrm{C},-8.6003131842,1.0120393942,2.685862588$ 5\C,-7.3705628924,1.6794352186,2.7244379521\C,-6.9907546702,2.32344113 79,3.9110986019\C,-7.8173063762,2.3055091733,5.035755134\Cl,-10.090179 $3579,1.6068403994,6.3814911786 \backslash \mathrm{H},-10.3982299434,0.4634965632,3.7665441$ $631 \backslash \mathrm{H},-8.9146513239,0.5039515258,1.7729689836 \backslash \mathrm{H},-6.0370813788,2.849104$ $3848,3.9621405888 \backslash \mathrm{H},-7.5191072009,2.8061129513,5.9552007942 \backslash \mathrm{O},-2.88979$ $24002,-0.4329750645,2.2630080333 \backslash 0,-1.6468206829,1.2741351986,1.750004$ $2539 \backslash$ Version=EM64L-G09RevC.01 $\backslash$ State $=2-A \backslash H F=-1128.0522713 \backslash S 2=0.789584 \backslash$ $\mathrm{S} 2-1=0 . \mathrm{IS} 2 \mathrm{~A}=0.750876 \backslash \mathrm{RMSD}=7.360 \mathrm{e}-09 \backslash \mathrm{RMSF}=2.599 \mathrm{e}-06 \backslash$ ZeroPoint $=0.1821618$ $\backslash$ Thermal $=0.1955152 \mathrm{ZPE}=114.3082423 \backslash$ Dipole $=-1.2885688,0.873976,-0.75435$ 
78|DipoleDeriv $=0.0039299,0.0238546,0.0286361,-0.0173793,0.0297786,0.09$ 41462,-0.0113275,0.0550318,0.2078081,0.1942653,-0.1798666,0.0090613,0. $0679187,-0.1861148,0.0798875,0.0617298,0.0337723,-0.2009894,-0.3169392$ $, 0.21271,0.0005683,0.2261478,0.2650502,-0.1490235,-0.0884513,-0.070375$ $4,0.105392,0.0146991,0.0683704,-0.0136585,-0.2109869,-0.201991,0.08012$ $65,0.0807233,0.0378488,-0.1308907,-0.1807499,-0.0451432,0.0504567,-0.0$ $335907,0.1364662,-0.0384511,0.0008435,-0.0762324,-0.0990921,0.0960619$, $0.0651206,0.0092325,0.1368747,0.0083808,-0.17224,0.0517587,-0.0351856$, $-0.19373,-0.0420847,-0.0781517,-0.0659438,-0.0515874,0.020668,-0.05528$ $33,-0.0455795,-0.0444999,0.0103685,0.1098354,-0.012909,-0.0157985,-0.0$ $288401,0.0663024,0.0277275,-0.0018221,0.025949,0.1171344,1.8157388,0.0$ $406049,-0.1369546,-0.2163972,1.3716811,-0.2511419,0.0643437,-0.3675368$ $, 0.3317287,0.044897,-0.009511,0.0177954,-0.0228,-0.0617493,0.0809609,-$ $0.015632,0.059154,0.1193519,-0.0175434,0.0508999,-0.0329591,0.0590031$, $0.0197893,0.0308371,-0.0278239,0.0222848,0.121816,0.0724409,-0.0312877$ $, 0.0060007,-0.017549,0.0945929,0.0161444,0.002509,0.0123001,0.1342384$, $0.4669517,0.045648,-0.5811496,0.0069085,-0.0160382,0.0257425,-0.697073$ $7,-0.0059007,0.8550642,-0.0698809,0.060999,0.1139281,0.0788582,-0.0560$ $588,-0.0178203,0.1881905,-0.0023926,-0.2967741,0.0327711,0.0452532,-0$. $0309574,0.1144275,-0.0443968,0.0232823,0.0920507,0.0849508,0.0150241,0$ $.0773241,0.0191439,-0.1155902,0.0081491,-0.0548036,-0.0104578,-0.22234$ $21,-0.0398657,0.0881297,-0.0884997,0.0388891,0.0650469,0.0138534,-0.05$ $62565,0.0402429,0.0161579,0.0128118,-0.0531338,-0.1532909,0.0057674,0$. $1264877,0.0108422,-0.0497561,0.0625882,0.1409287,0.0739366,-0.089685,-$ $0.4152186,-0.0231029,0.3937453,-0.0152922,-0.0703585,0.0028627,0.45330$ $61,0.0142893,-0.6086046,-0.00535,-0.0631969,0.0149506,-0.0623459,0.092$ $9886,-0.009083,0.0274289,-0.0057534,0.0800314,0.0850665,-0.0215158,-0$. $0046843,-0.0281981,0.0776549,-0.0654655,-0.0296124,-0.0764235,-0.05225$ $35,-0.0254305,-0.0629474,0.0092436,-0.0706965,0.0671816,-0.0009669,-0$. $0069887,-0.0007684,0.0895957,0.0789286,-0.0266434,-0.0062235,-0.024514$ $9,0.0932854,-0.0509719,0.0029091,-0.0499544,-0.0041914,-0.6181408,0.10$ $92186,0.0110854,0.1217195,-0.9914555,0.170501,-0.0392016,0.2456345,-0$. $283802,-1.1597819,-0.2322036,0.147681,-0.0445246,-0.5548408,0.0858556$, $0.0029749,0.0969249,-0.2625365 \backslash$ Polar $=227.2281444,12.5426597,147.650807$ $6,-42.5515733,-8.8762364,166.4974548 \backslash \mathrm{PG}=\mathrm{C} 01$ [X(C12H9Cl1N1O2)]\NImag=0 $10.47522638,0.02098120,0.48553101,0.04795080,0.01410968,0.44127210,-0$. $15983900,0.05464923,-0.02385344,0.64306285,0.04572246,-0.12039049,0.02$ $014811,0.08353896,0.62666607,-0.02101278,0.01959420,-0.07036644,-0.017$ $37691,-0.16142467,0.18550732,-0.02953534,-0.00257903,0.00004790,-0.322$ $86160,-0.12028195,0.03361544,0.60530452,0.00668497,0.01452571,-0.00403$ $666,-0.08153258,-0.15033116,0.03017369,0.09238628,0.55559937,-0.002917$ $63,-0.00324403,0.00777181,0.02008282,0.02815094,-0.06096234,-0.0189779$ $1,-0.13752485,0.17121768,0.00145005,0.00400016,-0.00110725,0.00902917$, $-0.03021751,0.01008312,-0.11825554,-0.05984101,0.01821207,0.69851311,0$ $.00275353,-0.00694089,-0.00030481,-0.06819757,-0.04394765,0.01572398,0$ $.01983430,-0.21593877,0.05412375,0.05707987,0.51687850,-0.00071270,-0$. $00031026,-0.00759364,0.02263269,0.01678278,0.00428739,-0.00821284,0.05$ $244934,-0.07026837,-0.00351517,-0.13555028,0.14853194,-0.00343683,-0.0$ $1538070,0.00354201,-0.03294937,-0.01526220,0.00409369,0.03817227,0.065$ $54727,-0.02088621,-0.19974805,0.01622189,-0.00930881,0.60204799,-0.027$ $36179,-0.01009147,0.00591206,0.00455970,0.00447720,-0.00170085,0.02232$ 
$205,-0.03454672,0.01301752,0.10271161,-0.16188805,0.03880895,0.0555036$ $9,0.65828130,0.00865050,0.00569463,0.00566956,-0.00230543,-0.00225773$, $0.00002327,-0.00693824,0.01454170,-0.00117762,-0.03821099,0.03698701,-$ $0.06636820,-0.00544207,-0.17911531,0.17976877,-0.08821967,-0.01488083$, $0.00266854,0.02189810,0.03549308,-0.01074472,-0.01812715,-0.02807889,0$ $.00867439,-0.07604011,0.04413116,-0.01688945,-0.32209604,-0.13202029,0$ $.03622032,0.74298444,-0.00615167,-0.18519772,0.03755089,0.01242284,-0$. $02016163,0.00622481,-0.00733760,-0.00971675,0.00249102,0.00093650,0.03$ $359548,-0.00796317,-0.08530203,-0.15925315,0.03407453,0.00165851,0.512$ $71591,-0.00128013,0.03778820,-0.07649026,-0.00391417,0.00805463,-0.002$ $93192,0.00178999,0.00197979,-0.00264520,-0.00228757,-0.00716998,0.0105$ $5164,0.02199614,0.03323517,-0.06163020,0.01295487,-0.12914798,0.173933$ $96,-0.09396485,-0.05527487,-0.09188412,-0.02358833,-0.00996982,-0.0179$ $9921,0.00102825,0.00541210,-0.00263090,-0.00127731,-0.00192774,0.00056$ $258,0.00199261,-0.00057201,-0.00003604,-0.00366817,-0.00906383,-0.0004$ $0630,0.11594857,-0.05170689,-0.09910653,-0.08316981,0.00709467,0.01112$ $582,0.01038129,0.00142372,-0.00242354,0.00099379,-0.00131205,0.0018005$ $8,-0.00094530,0.00404191,-0.00414483,0.00053530,-0.01939691,-0.0186824$ $0,-0.01639586,0.06267941,0.10999990,-0.08273141,-0.07872068,-0.1580974$ $5,-0.00571778,-0.00114642,-0.00173180,0.00084683,0.00205702,-0.0019288$ $6,-0.00017307,-0.00085646,0.00019719,0.00082218,0.00090132,-0.00107043$ $, 0.00605432,0.00398455,0.00969154,0.08912177,0.08846783,0.18151263,0.0$ $0433348,0.02334055,-0.00641406,-0.05554976,-0.01830040,0.00568516,0.00$ $411610,-0.02651017,0.00866956,-0.00557236,-0.00129444,0.00025548,-0.00$ $058549,0.00041052,-0.00018835,-0.00493583,0.00127451,-0.00052569,0.000$ $63871,-0.00064069,-0.00037974,0.05516660,-0.00221383,-0.00696073,0.002$ $89305,-0.02308474,-0.31580308,0.09247089,0.00010120,-0.00942085,0.0058$ $7424,-0.00193658,0.00261431,0.00111278,0.00040494,0.00011831,-0.000309$ $43,0.00184250,0.00098714,0.00053882,0.00044295,0.00012063,0.00036604,0$ $.02093523,0.33113238,0.00125511,0.00416061,-0.00008060,0.00691088,0.09$ $379880,-0.06314354,-0.00029220,0.00480376,0.00631776,0.00019612,0.0010$ $0704,0.00554651,-0.00007307,-0.00014819,-0.00079410,-0.00075595,0.0001$ $7365,0.00190632,0.00077226,-0.00030510,0.00068369,-0.00628669,-0.10244$ $627,0.05383833,-0.00013848,-0.00464096,0.00035861,-0.04322354,0.014822$ $89,-0.00574414,-0.10087414,0.00393179,-0.00123707,0.00305163,0.0244136$ $7,-0.00809779,0.00652007,-0.00646923,0.00242279,-0.00265175,-0.0007384$ $7,0.00014332,-0.00005770,-0.00027886,0.00026221,0.00081065,0.00016197$, $0.00014185,0.73257657,-0.00463378,-0.00227772,0.00513969,0.01212819,0$. $02030603,-0.00677861,-0.00419002,-0.11164436,0.00888743,0.03043745,-0$. $01507842,0.00525256,-0.00336878,-0.00503279,0.00328522,-0.00117732,-0$. $00006012,-0.00023729,-0.00027665,0.00028798,-0.00017542,-0.00330242,-0$ $.00337483,-0.00100168,0.14222092,0.78769793,0.00258807,0.00448112,0.01$ $141770,-0.00483329,-0.00567138,0.00072461,0.00052771,0.00878184,-0.086$ $16158,-0.00993202,0.00542499,-0.00373475,0.00114171,0.00357147,0.00468$ $856,0.00027723,-0.00021245,-0.00068261,0.00167466,-0.00172276,0.000133$ $57,0.00117702,-0.00112740,-0.00601610,-0.03164591,-0.17590881,0.284316$ $73,-0.00401897,-0.00038218,-0.00012494,-0.00085361,0.00003885,0.000046$ $13,-0.00364534,0.00203456,-0.00094593,0.00610003,0.02408509,-0.0080076$ $1,-0.06060772,-0.02903711,0.00913332,0.00015779,-0.02859779,0.00953444$ ,-0.00011651,-0.00063534,0.00020513,0.00010709,0.00003082,0.00001665,0 $.00099233,0.00128059,-0.00039406,0.06092178,-0.00124973,0.00280310,0.0$ 
$0214893,0.00015920,0.00045467,-0.00036299,0.00281038,0.00101253,0.0015$ $0240,-0.00380965,-0.01156389,0.00465711,-0.02954627,-0.30490457,0.0890$ $0069,0.00065465,-0.00806371,0.00378424,-0.00007836,0.00016834,-0.00005$ $511,-0.00001427,-0.00010033,-0.00015591,-0.00089948,-0.00027890,0.0003$ $3711,0.03161370,0.32072979,0.00038920,0.00283609,0.00833605,-0.0000736$ $0,-0.00038683,-0.00046390,-0.00107899,0.00140134,0.00469413,0.00155206$ $, 0.00531623,0.00115760,0.00867721,0.08877438,-0.06737352,0.00023205,0$. $00285191,0.00266131,0.00010181,0.00131250,-0.00063503,0.00002238,-0.00$ $018933,-0.00051485,0.00037151,0.00030830,0.00056000,-0.00973592,-0.098$ $10192,0.05836858,0.00620137,-0.00517347,0.00276213,0.00026923,-0.00165$ $955,0.00067406,0.00003628,-0.00089156,0.00028438,-0.00323046,-0.003426$ $02,0.00105875,-0.01749007,0.01821562,-0.00660804,-0.23832816,0.1265832$ $7,-0.04800063,0.00021300,0.00051144,-0.00031299,0.00047262,-0.00020047$ $, 0.00001337,-0.00027612,-0.00019852,0.00004486,0.00090322,-0.00002889$, $0.00008085,0.25102528,0.02238727,-0.01154285,0.00565703,-0.00257609,-0$ $.00422707,0.00192841,-0.00088894,-0.00104165,0.00024026,-0.00306511,0$. $00101447,0.00210217,-0.01154060,0.00960417,-0.00159134,0.12919363,-0.1$ $4261385,0.04121939,0.00000289,0.00076326,-0.00061414,0.00138658,-0.000$ $39650,0.00018782,-0.00044897,-0.00036463,-0.00020566,0.00020903,0.0004$ $0235,-0.00189299,-0.13501152,0.14690835,-0.00648904,0.00392920,-0.0004$ $0453,0.00071680,0.00201924,0.00113341,0.00046227,0.00018560,-0.0001719$ $1,0.00057494,0.00206588,0.00699303,0.00303922,-0.00077716,0.00603034,-$ $0.04812608,0.04023220,-0.04552461,0.00026953,0.00088046,0.00037445,-0$. $00048061,0.00010490,-0.00001000,0.00009793,-0.00015920,-0.00094365,0.0$ $0013838,-0.00186038,-0.00465078,0.05071265,-0.04617659,0.03604418,0.00$ $052912,0.00023764,0.00002153,0.00115755,0.00113409,-0.00040191,0.00054$ $677,-0.00108506,0.00025736,-0.30647991,-0.10690551,0.02838241,-0.01308$ $512,-0.01046783,0.00299631,-0.00166199,0.00304998,-0.00118998,0.000076$ $35,0.00000512,-0.00002175,0.00024517,0.00008813,-0.00004630,-0.0016179$ $8,-0.00073402,0.00025309,0.00089421,0.00002129,0.00009612,-0.00071870$, $-0.00048896,0.00014405,0.32188211,0.00038900,-0.00022491,-0.00029087,0$ $.00100902,-0.00513963,0.00257360,-0.02593062,-0.00514501,0.00216879,-0$ $.10469006,-0.09667531,0.02073408,0.01584591,0.01080687,-0.00187443,0.0$ $0331519,-0.00172066,0.00203013,-0.00004294,0.00001262,0.00002636,-0.00$ $147007,-0.00026585,0.00025029,-0.00440373,-0.00067477,-0.00066377,-0.0$ $0045397,0.00042579,-0.00108103,0.00089118,0.00038281,-0.00009740,0.111$ $76904,0.09607540,-0.00017137,-0.00036312,-0.00109371,-0.00024601,0.002$ $63789,0.00188894,0.00854063,0.00269910,0.00283660,0.02763396,0.0206764$ $4,-0.03225864,-0.00571000,-0.00240961,0.00455755,-0.00122521,0.0019697$ 2,0.00333115,-0.00012183,-0.00006013,0.00011995,0.00046102,0.00030689, $0.00049215,0.00138375,-0.00052519,-0.00214372,0.00024335,-0.00107766,-$ $0.00231593,-0.00032802,-0.00014860,0.00002516,-0.02933231,-0.02321495$, $0.02431602,-0.00124999,-0.00034423,0.00019029,0.00020632,0.00022276,0$. $00018696,-0.00017915,-0.00000305,0.00021853,0.00021253,0.00012775,-0.0$ $0001108,0.00000034,0.00011144,0.00010596,0.00001512,-0.00002098,0.0001$ $9733,-0.00002140,-0.00009229,0.00020917,-0.00013409,0.00005557,-0.0000$ $0002,-0.00020576,-0.00004341,-0.00012790,0.00006894,0.00001569,-0.0000$ $7750,-0.00003568,-0.00002544,-0.00001133,-0.00001107,0.00001061,0.0000$ $1521,0.49512579,-0.00047708,-0.00075580,0.00018791,0.00020444,0.000120$ $91,0.00015704,-0.00024293,0.00014385,0.00009295,0.00014982,-0.00000917$ $, 0.00004147,-0.00013159,-0.00001044,0.00008338,0.00009159,0.00021351,0$ 
$.00024801,-0.00011469,0.00010353,0.00015713,-0.00001149,-0.00002942,-0$ $.00007263,0.00037047,-0.00027535,-0.00003220,0.00001412,-0.00001882,-0$ $.00010760,-0.00003328,-0.00004327,0.00000408,-0.00000803,0.00000459,0$. $00001015,0.19495624,0.28322490,-0.00029059,-0.00019653,-0.00034142,0.0$ $0053077,0.00015108,0.00030661,-0.00061472,0.00035717,-0.00008964,0.000$ $13664,-0.00024567,0.00015442,-0.00033283,-0.00001680,-0.00002334,0.000$ $14478,0.00036111,0.00030667,0.00005382,0.00004740,0.00029393,0.0000389$ $1,-0.00010407,-0.00003339,0.00124843,-0.00078345,0.00002603,-0.0000164$ $4,-0.00007514,-0.00008093,-0.00002746,0.00006533,-0.00013250,0.0000186$ $6,-0.00000435,0.00000545,-0.00026306,0.14217337,0.50666921,-0.00092235$ $,-0.00469178,-0.00057075,0.00051076,0.00017741,0.00002832,-0.00011932$, $-0.00006694,-0.00000181,0.00006944,0.00006164,-0.00000044,0.00009842,-$ $0.00033699,0.00011728,-0.00100284,0.00047700,0.00088504,0.00012784,0.0$ $0016602,0.00025300,-0.00011825,0.00000625,-0.00002800,0.00026424,-0.00$ $022286,0.00005942,-0.00006485,-0.00004965,-0.00011220,-0.00006642,-0.0$ $0008824,-0.00000441,0.00001201,0.00001664,0.00000407,-0.12745173,-0.06$ $404056,-0.08256293,0.62599929,-0.00448482,0.00406845,-0.00209945,-0.00$ $108718,-0.00069098,0.00104955,0.00012241,0.00041161,-0.00021607,-0.000$ $04207,-0.00017463,0.00005796,-0.00006937,0.00028008,-0.00023133,0.0009$ $4596,-0.00012857,-0.00048768,0.00001442,-0.00002304,0.00000954,-0.0000$ $5503,-0.00000019,0.00005103,-0.00017601,-0.00004647,-0.00006628,0.0000$ $6280,0.00005855,0.00012345,0.00001715,-0.00005954,0.00002990,0.0000125$ $0,-0.00000020,0.00000408,-0.04394499,-0.13045977,-0.11230433,0.2356282$ $4,0.30794362,-0.00061451,-0.00213333,0.00182804,-0.00018794,-0.0000926$ $1,-0.00077931,0.00080804,-0.00049864,0.00001954,-0.00035993,0.00014784$ ,-0.00013407,0.00043073,-0.00007452,-0.00003199,-0.00051799,-0.0002796 $1,-0.00013482,-0.00004171,-0.00003584,-0.00023059,0.00005232,0.0001070$ $2,-0.00001345,-0.00094250,0.00083231,0.00015788,-0.00005017,0.00002326$ $, 0.00011069,0.00005560,-0.00004087,0.00007410,-0.00000175,0.00000443,-$ $0.00000385,-0.02878966,-0.08381546,-0.24823274,-0.07199687,0.13875898$, $0.60385604,-0.01749814,-0.00930557,0.00580225,-0.00054384,-0.00173479$, $0.00026746,0.00056848,0.00038252,-0.00015975,-0.00019444,0.00007050,-0$ $.00001617,0.00071989,-0.00053154,0.00022945,-0.00145632,0.00096317,-0$. $00097028,0.00026270,-0.00073032,-0.00273260,0.00001495,0.00031792,0.00$ $004979,-0.00073677,0.00041184,0.00006755,-0.00020962,0.00000902,0.0001$ $7577,0.00012638,-0.00038931,0.00026420,0.00000253,-0.00000481,0.000012$ $82,0.04880842,0.03618361,0.03783461,-0.19018939,-0.03670289,0.08308388$ $, 0.56131351,-0.00202616,0.00323893,0.00054398,0.00026563,-0.00063690,-$ $0.00095734,0.00050545,-0.00011994,0.00026957,-0.00003274,0.00031915,-0$ $.00011948,0.00012300,0.00009032,-0.00000718,0.00006505,-0.00004462,-0$. $00046679,0.00112844,0.00021554,-0.00088440,-0.00025185,0.00013187,-0.0$ $0007213,-0.00059394,0.00044404,0.00032198,0.00003534,0.00002210,-0.000$ $01663,0.00012779,0.00007264,0.00003355,-0.00000266,-0.00000654,-0.0000$ $1080,0.01888333,0.01604357,-0.00266717,-0.01577360,-0.08110849,-0.0270$ $0139,0.20343907,0.30480263,0.02442878,0.01106159,-0.00190501,0.0014915$ $8,0.00150045,-0.00185146,-0.00001102,-0.00070676,0.00049164,0.00035512$ $, 0.00018508,-0.00008645,-0.00017762,0.00100740,-0.00005724,0.00250263$, $-0.00085812,-0.00169090,0.00249254,0.00185467,0.00163826,0.00006965,-0$ $.00019598,0.00006503,-0.00008332,0.00034245,-0.00012851,0.00026170,0.0$ $0021636,0.00011597,-0.00020508,0.00014301,-0.00006682,-0.00003031,-0.0$ $0002944,-0.00007498,-0.00922460,-0.02750679,-0.05381305,0.13914288,0.0$ 
$0265619,-0.25193710,-0.06073324,0.16520053,0.66231453,-0.09786045,-0.0$ $0065560,0.04686972,-0.03083446,0.00398440,0.02087012,0.00038917,0.0051$ $5748,-0.00054380,-0.00071949,-0.00134089,0.00031746,0.00207865,0.00045$ $826,0.00099694,-0.00795557,-0.01248091,0.01022263,0.00460382,-0.001584$ $42,-0.00994737,0.00141912,-0.00068237,-0.00065184,0.00000042,-0.000979$ $47,-0.00176263,0.00019799,-0.00010432,-0.00029795,0.00070591,0.0007638$ $0,-0.00112466,0.00001856,0.00003794,0.00005986,-0.03372598,-0.02411361$ ,-0.02701597,-0.03369061,-0.00029953,0.05730226,-0.26533800,-0.1166015 $5,-0.02876815,0.54827091,-0.00415675,-0.06948151,-0.00010412,0.0076119$ $1,0.00250789,-0.01193157,0.00058364,-0.00252750,0.00080153,-0.00063704$ $, 0.00187830,-0.00038433,0.00270643,-0.00424457,0.00130889,-0.02138546$, $-0.00139946,0.02378835,0.00737563,-0.00116403,-0.01441403,0.00014060,0$ $.00047872,-0.00020714,-0.00056756,0.00069911,0.00078138,-0.00072344,-0$ $.00073889,-0.00119981,-0.00014871,0.00058860,-0.00029545,-0.00002956,0$ $.00012147,0.00009681,-0.02441697,-0.02692790,-0.01868115,-0.01507636,0$ $.01408746,0.04018959,-0.09577973,-0.12929801,-0.03484277,0.18654531,0$. $31984514,0.03394454,-0.00654521,-0.13104389,0.00739347,-0.00658577,0.0$ $0631683,-0.00135978,0.00130348,-0.00217449,0.00039076,-0.00057052,0.00$ $054602,-0.00171842,-0.00116700,-0.00113936,0.00887757,0.01201515,-0.01$ $122862,0.01595851,-0.00220687,-0.02767465,-0.00098672,0.00061397,0.000$ $87194,0.00061123,-0.00113305,0.00007681,-0.00036231,-0.00009804,0.0004$ $4712,-0.00005118,-0.00109817,0.00131889,0.00003486,-0.00002045,0.00015$ $307,-0.02749099,-0.01916868,-0.01910354,0.01706091,0.01891367,0.033482$ $52,0.02736063,-0.00460505,-0.11723320,-0.03481051,0.13098389,0.5678127$ $7,0.00655904,0.00961681,0.01829919,0.00207228,0.00014641,0.00110608,-0$ $.00088736,0.00046358,0.00014554,-0.00007579,-0.00037032,0.00003627,-0$. $00063762,-0.00038081,-0.00017166,0.00132422,0.00120829,0.00038716,-0.0$ $0173453,0.00021008,0.00432144,0.00003011,-0.00015838,0.00008849,0.0009$ $0395,-0.00050939,-0.00001310,-0.00002504,-0.00006801,-0.00007700,0.000$ $01314,-0.00009858,0.00007633,-0.00000173,0.00000540,0.00004738,-0.0362$ $5869,-0.01707503,0.01618626,-0.00348147,0.00343991,0.00927333,-0.00280$ $844,-0.01612651,-0.02800551,-0.12866995,-0.05753108,-0.06653608,0.6156$ $7977,0.00279710,0.00303505,-0.00048148,-0.00026387,0.00058311,-0.00005$ $702,-0.00029685,-0.00009164,-0.00045562,-0.00007335,-0.00034188,0.0000$ $8182,-0.00046299,-0.00003588,0.00030762,0.00011578,0.00026789,0.000534$ $90,-0.00052441,0.00106048,0.00068004,0.00000596,0.00006914,-0.00007946$ $, 0.00008696,-0.00010616,0.00002110,-0.00003049,0.00001285,-0.00006608$, $-0.00006385,0.00032941,-0.00011363,-0.00000829,0.00003076,0.00000484,0$ $.00047153,0.01437897,0.01848402,0.00350348,-0.00732881,-0.01603647,-0$. $03027110,-0.01124934,-0.00843047,-0.03828497,-0.12618390,-0.10448906,0$ $.22486485,0.30564081,0.00049849,-0.00991840,-0.02553757,-0.00233061,0$. $00067191,-0.00085410,-0.00020732,-0.00018651,0.00006159,-0.00041646,-0$ $.00015427,-0.00024225,-0.00034344,-0.00018934,-0.00024148,-0.00044289$, $-0.00260715,-0.00002677,0.00246390,-0.00027655,-0.00333948,0.00037388$, $-0.00025713,0.00014217,-0.00019361,0.00026428,-0.00010285,-0.00000402$, $-0.00001844,0.00007309,0.00004757,0.00025214,-0.00012696,0.00000819,0$. $00001511,0.00001967,0.06298720,0.04342994,0.03409953,0.00997820,-0.015$ $83051,-0.05983350,-0.06562042,-0.02840940,0.02510475,-0.01488058,-0.07$ $697040,-0.25123210,-0.08022297,0.13493705,0.61061419,0.00220095,-0.002$ $92600,-0.00273422,-0.00055663,-0.00014948,-0.00026617,0.00025520,-0.00$ $013888,-0.00018284,-0.00008575,0.00006092,-0.00004628,0.00008562,-0.00$ 
$021246,-0.00013375,-0.00050410,-0.00011807,0.00016176,0.00031873,-0.00$ 021946,-0.00101959,0.00009723,0.00001660,0.00001709,-0.00016560,0.0001 $3991,0.00006794,-0.00009131,-0.00002510,0.00004588,0.00007033,0.000085$ $81,0.00009645,-0.00000243,-0.00001286,-0.00001068,-0.26122571,-0.09933$ $391,0.01145576,-0.00099298,-0.02922911,-0.06398727,-0.04904390,-0.0154$ $9369,0.02322808,0.04709986,0.03376799,0.03479451,-0.18817648,-0.035283$ $95,0.08500281,0.55652822,-0.00290855,0.00263823,-0.00381391,-0.0005335$ $8,0.00002883,0.00047721,-0.00014976,-0.00001402,-0.00010265,0.00005270$ ,-0.00001738,-0.00006909,0.00008458,0.00025177,0.00009325,0.00003241,-$0.00035542,0.00000333,0.00036646,0.00001227,-0.00082259,0.00010435,-0$. $00002617,0.00011785,0.00002798,-0.00016731,-0.00008223,0.00003035,0.00$ $000357,0.00003971,0.00001530,-0.00000863,-0.00009267,0.00000578,-0.000$ $01338,0.00001230,-0.11954749,-0.13235882,-0.00970440,-0.01524852,-0.00$ $958890,-0.02792022,-0.01544101,-0.00864964,0.00850505,0.01887132,0.016$ $53423,-0.00282790,-0.01428274,-0.08046709,-0.02961489,0.20479948,0.305$ $03250,-0.00235559,-0.00363164,-0.00073902,-0.00021497,0.00040142,0.000$ $13925,0.00003205,0.00011465,0.00026307,0.00019667,0.00008025,-0.000026$ $50,0.00008191,0.00017352,0.00017346,-0.00025443,-0.00020590,0.00069945$ $, 0.00046462,-0.00006314,-0.00043909,-0.00002304,-0.00005586,-0.0001440$ $2,0.00024810,-0.00009070,-0.00008321,0.00005397,0.00000905,-0.00013297$ $,-0.00000282,0.00005636,-0.00009618,-0.00001454,-0.00000122,-0.0000101$ $8,-0.04268255,-0.03843084,-0.10801204,-0.02687245,-0.00819329,0.025355$ $46,0.02294781,0.00856905,-0.01365185,-0.00539546,-0.02418447,-0.049239$ $09,0.14133779,0.00019600,-0.26162798,-0.05487169,0.17463456,0.67737235$ ,-0.00000342,0.00026855,-0.00029073,0.00012567,-0.00016789,0.00033996, $-0.00023239,0.00024980,-0.00014004,0.00000423,-0.00016080,0.00006967,-$ $0.00008235,-0.00007374,0.00002706,-0.00006562,0.00035108,0.00015863,-0$ $.00001972,0.00002321,0.00005409,0.00006054,-0.00005747,-0.00000187,0.0$ $0063009,-0.00043386,0.00003889,-0.00004335,-0.00004595,-0.00003376,0.0$ $0002476,0.00001524,-0.00003984,0.00001466,-0.00000409,-0.00000145,-0.0$ $7755660,-0.00677555,0.05692018,-0.00028135,-0.00054078,0.00554275,0.00$ $161858,-0.00162764,0.00024693,0.00022033,0.00104094,0.00161460,-0.0012$ $4475,-0.00470358,-0.00448703,-0.02180027,-0.00124446,0.03428268,0.0994$ $1083,0.00037510,-0.00046249,0.00019636,0.00005803,0.00004997,-0.000050$ $44,0.00003039,-0.00001067,0.00003505,0.00000044,0.00000410,-0.00000318$ ,-0.00000076,-0.00002552,-0.00002400,-0.00005460,0.00001921,0.00001546 ,-0.00000558,0.00002165,0.00006525,-0.00000783,-0.00000608,-0.00000636 $, 0.00001103,-0.00001679,0.00002048,-0.00000530,-0.00000173,-0.00000396$ $,-0.00000202,0.00000737,-0.00000260,0.00000223,-0.00000064,-0.00000275$ $,-0.00676830,-0.02890806,-0.00273423,0.00787563,0.00444726,-0.01162938$ ,-0.00366461,0.00230615,-0.00165472,0.00098097,0.00014566,0.00078672,-$0.00266764,0.00204268,-0.00307672,-0.00969331,0.00405804,0.01186294,0$. $01077441,0.01933042,-0.00004406,0.00039018,0.00025942,-0.00022378,0.00$ $019564,-0.00040841,0.00033704,-0.00037908,0.00014267,0.00000303,0.0002$ $2943,-0.00009980,0.00014293,0.00019055,-0.00001083,0.00018314,-0.00050$ $563,-0.00023099,-0.00001789,-0.00000150,0.00006586,-0.00008289,0.00006$ $388,0.00000362,-0.00088621,0.00060037,-0.00007238,0.00007342,0.0000760$ $1,0.00004663,-0.00003296,-0.00003529,0.00004777,-0.00001779,0.00000527$ $,-0.00000465,0.05703846,-0.00277332,-0.11189858,0.02793562,0.00022464$, $-0.03423052,-0.00520221,-0.00457584,0.00011594,0.00140917,0.00067729,-$ $0.00091757,0.00107262,-0.00013715,0.00278855,0.01171984,-0.00004971,-0$ 
$.01203455,-0.09457046,0.00424159,0.15663493,-0.00024217,-0.00034487,-0$ $.00034141,-0.00006615,-0.00009191,0.00008247,-0.00001318,0.00005311,-0$ $.00000379,-0.00002793,-0.00000678,0.00000041,0.00003346,-0.00005228,0$. $00002205,-0.00012335,0.00007798,0.00012174,0.00001404,0.00000675,-0.00$ $004404,-0.00001029,-0.00000713,-0.00000251,0.00005163,-0.00008843,0.00$ $003175,-0.00001142,-0.00001731,-0.00001931,0.00000357,-0.00001036,0.00$ $000495,0.00000248,0.00000151,0.00000486,-0.00468612,-0.00304550,0.0033$ $8864,-0.27358302,-0.12902112,-0.00784827,-0.01064137,-0.00871631,-0.00$ $294638,0.00132897,-0.00149983,0.00390802,0.00021357,0.00076709,0.00002$ $861,0.00227468,-0.00203959,-0.00076998,0.00044215,0.00162501,-0.000092$ $53,0.28550872,0.00007108,0.00013480,-0.00014937,0.00000010,0.00000573$, $0.00007722,-0.00000192,0.00007847,-0.00003166,0.00000085,-0.00006609,0$ $.00000714,0.00000471,0.00001839,0.00003370,-0.00001930,-0.00002408,-0$. $00010759,0.00001226,-0.00001009,-0.00000294,0.00000963,0.00000616,-0.0$ $0000293,0.00005518,0.00000101,-0.00003433,-0.00000898,0.00000271,0.000$ $01884,-0.00000430,-0.00000752,0.00000304,0.00000101,-0.00000207,-0.000$ $00031,-0.01291332,-0.00284823,0.00115944,-0.12816818,-0.10761555,-0.01$ $096464,0.00058394,0.00364824,-0.00103436,-0.00164619,0.00548885,-0.001$ $58908,0.00079257,-0.00074575,-0.00000006,-0.00182142,0.00307153,-0.003$ $89054,0.00128976,-0.00157544,0.00124148,0.14038129,0.10374168,0.000826$ $91,0.00045865,0.00016057,0.00002172,0.00007089,-0.00017875,0.00002114$, $-0.00008381,0.00004954,0.00002083,0.00005555,-0.00001653,-0.00000058,0$ $.00004954,-0.00001494,0.00012848,-0.00009011,-0.00007776,-0.00002889,0$ $.00000311,0.00004058,0.00001139,-0.00000947,-0.00000252,-0.00007671,0$. $00007099,0.00000723,0.00001872,0.00001628,0.00000480,-0.00000313,0.000$ $02249,-0.00000854,-0.00000300,-0.00000013,-0.00000540,-0.02284551,-0.0$ $1272211,0.00197816,-0.00556851,-0.00969352,-0.05381436,0.02191353,0.01$ $203493,0.00499489,0.00352069,-0.00179610,-0.00279559,0.00022117,0.0000$ $9899,-0.00114623,-0.00034288,-0.00362836,-0.00381844,-0.00100511,0.000$ $72148,0.00069164,0.00527743,0.01368102,0.05333565,0.00003877,0.0012989$ $1,-0.00253720,-0.00015450,-0.00013090,0.00032403,-0.00008715,-0.000003$ $11,-0.00002250,0.00007640,0.00006445,-0.00000556,-0.00005611,0.0001605$ $2,-0.00006012,0.00042469,0.00006391,0.00012899,-0.00004729,0.00016851$, $0.00099139,-0.00007361,0.00000974,-0.00004339,-0.00011321,-0.00003651$, $-0.00007511,0.00004254,0.00001813,-0.00002003,-0.00000989,0.00002348$,$0.00004432,-0.00000167,0.00000209,-0.00000504,0.00010998,-0.00228225,0$ $.00362564,0.00827558,0.00882433,0.01691100,-0.07637595,-0.04548897,-0$. $06433482,-0.00327756,-0.01222998,-0.02250394,-0.00359721,-0.00466256,0$ $.00123094,-0.00094626,0.00024373,0.00056021,-0.00020959,0.00024567,0.0$ $0111843,-0.00008911,0.00178738,-0.00097127,0.07620919,0.00232447,-0.00$ $267616,0.00014072,0.00046056,0.00048688,-0.00016933,0.00011481,-0.0002$ $3880,0.00005904,0.00001864,0.00005732,-0.00000740,0.00007971,-0.000137$ $86,0.00007313,-0.00055867,0.00004262,0.00029909,-0.00016136,0.00036041$ $, 0.00067161,0.00005714,-0.00002588,-0.00002156,-0.00001140,0.00013775$, $-0.00002953,-0.00002423,-0.00002568,-0.00006453,-0.00005698,-0.0000875$ $4,0.00001067,0.00000162,0.00000222,0.00000145,-0.00218447,0.00548066,-$ $0.00091932,-0.00077843,0.00378197,0.00143991,-0.04558905,-0.10289467,-$ $0.11351216,-0.00318777,-0.00273226,-0.01307898,-0.00500196,0.00313065$, $-0.00079834,0.00020597,-0.00065129,0.00074093,-0.00010170,0.00010013,0$ $.00011005,0.00188766,-0.00272321,0.00119958,0.05227879,0.09937779,0.00$ $055179,0.00195049,0.00038944,-0.00019077,-0.00000400,0.00017485,-0.000$ 
$00387,0.00012415,-0.00004294,-0.00005129,-0.00006688,0.00000954,0.0000$ $2538,0.00010616,-0.00003664,0.00028804,-0.00010705,0.00013052,-0.00020$ $389,-0.00003476,0.00050240,-0.00008018,-0.00000799,-0.00001494,0.00006$ $999,-0.00013715,-0.00001762,0.00002689,0.00001203,-0.00001107,-0.00000$ $433,-0.00000703,-0.00002989,0.00000847,0.00000449,0.00000240,0.0039446$ $6,-0.00082547,-0.00064596,-0.00873506,-0.01208661,-0.01643762,-0.06475$ $191,-0.11375526,-0.24863551,0.00175918,-0.00029712,0.00040042,0.000417$ $90,-0.00119604,0.00227217,0.00042659,0.00068568,0.00013384,0.00018070$, $-0.00038372,-0.00118770,-0.00072534,0.00133366,0.00040168,0.06705340,0$ $.12439958,0.26276571,-0.00075894,0.00126183,0.00028823,0.00011540,0.00$ $009827,0.00034457,-0.00002707,-0.00007013,-0.00012916,0.00004393,-0.00$ $007496,0.00001715,0.00001352,0.00015034,0.00029071,0.00013274,0.000207$ $83,0.00000086,-0.00002833,0.00001044,-0.00009507,-0.00002079,-0.000004$ $10,-0.00001319,-0.00003871,0.00003212,0.00008083,0.00002703,0.00002864$ $, 0.00000391,-0.00006635,-0.00000422,-0.00013682,-0.00000469,0.00000291$ $, 0.00000963,0.00149763,-0.00171820,0.00357352,0.00023696,0.00075665,0$. $00004479,0.00232438,-0.00247803,-0.00083503,-0.00387974,-0.00345418,0$. $00121268,-0.26739166,-0.12614960,-0.00982117,-0.01149244,-0.00916262,-$ $0.00269163,-0.00071475,-0.00030556,0.00029739,-0.00021052,0.00024173$,$0.00010493,-0.00018506,-0.00012950,0.00014292,0.28068460,0.00099889,-0$ $.00209820,0.00148736,0.00002438,-0.00018343,-0.00032376,0.00020453,0.0$ $0026725,0.00034070,0.00000396,0.00005991,-0.00011839,0.00001034,-0.000$ $12382,-0.00022089,-0.00011621,0.00026900,0.00029555,-0.00002174,-0.000$ $10224,0.00011969,-0.00001578,-0.00004257,-0.00009865,0.00033735,0.0000$ $2836,-0.00004130,-0.00001021,-0.00002467,0.00008176,0.00005632,-0.0000$ $3036,0.00011580,-0.00000784,-0.00000567,0.00001267,-0.00184900,0.00565$ $857,-0.00163191,0.00079155,-0.00073427,0.00007311,-0.00214526,0.003584$ $59,-0.00429443,-0.01265438,-0.00299294,0.00064543,-0.12622587,-0.10838$ $588,-0.01255637,0.00043962,0.00375966,-0.00135777,0.00005780,0.0000399$ $7,-0.00009325,0.00022843,-0.00052029,0.00022634,-0.00064621,-0.0001448$ $4,0.00000540,0.13883111,0.10462119,-0.00083728,0.00087964,0.00026078,-$ $0.00006799,-0.00014198,0.00007559,0.00008011,-0.00010454,-0.00006011,0$ $.00023675,0.00001179,-0.00005157,0.00016654,0.00033241,-0.00005332,-0$. $00017300,-0.00013373,-0.00011957,-0.00008063,0.00011047,0.00024954,-0$. $00001303,0.00007369,0.00002095,0.00032088,-0.00025557,0.00015915,0.000$ $03844,0.00001212,0.00002526,-0.00000567,-0.00002878,-0.00004991,0.0000$ $0700,-0.00000171,0.00000673,0.00326442,-0.00175082,-0.00215889,0.00017$ $105,0.00008297,-0.00109081,-0.00022726,-0.00394054,-0.00395770,-0.0232$ $9031,-0.01235014,0.00374253,-0.00954216,-0.01235733,-0.05613507,0.0228$ $6402,0.01216103,0.00417695,0.00124640,0.00040958,-0.00061737,-0.000120$ $83,0.00022001,0.00001026,-0.00121517,-0.00072055,0.00002247,0.00830476$ $, 0.01589445,0.05518163,0.00030141,0.00033078,0.00068705,0.00007517,-0$. $00006133,-0.00000534,-0.00001824,0.00005943,0.00000867,-0.00001258,-0$. $00005987,-0.00001318,-0.00006530,-0.00001615,-0.00000991,0.00012621,0$. $00013039,-0.00010118,-0.00014000,0.00008515,0.00022888,-0.00000760,0.0$ $0000734,0.00001236,0.00006275,-0.00004977,0.00003263,-0.00000720,-0.00$ $000920,0.00001099,-0.00000394,-0.00000758,0.00000575,0.00000230,0.0000$ $0103,0.00000917,-0.00369660,-0.01241812,-0.02308923,-0.00349367,-0.004$ $19982,0.00126388,-0.00094678,0.00024754,0.00061944,-0.00038343,-0.0025$ $0638,0.00402947,0.00881755,0.00866030,0.01623041,-0.07379526,-0.043990$ $84,-0.06403374,0.00028127,0.00119461,-0.00095631,-0.00009804,-0.000616$ 
$72,-0.00110747,-0.00007347,0.00029554,-0.00012088,-0.00023408,0.001843$ $00,-0.00085455,0.07336810,-0.00012272,0.00017797,0.00009024,0.00012823$ $, 0.00001516,0.00000388,-0.00001291,-0.00008114,-0.00003586,-0.00000343$ $, 0.00004126,-0.00001060,0.00004302,0.00000503,0.00004832,-0.00008495,-$ $0.00006851,0.00000290,0.00005593,-0.00008049,0.00004387,-0.00000325,-0$ $.00000667,-0.00001244,-0.00004032,0.00009320,-0.00003317,-0.00000157,-$ $0.00000073,0.00001058,0.00000508,-0.00000828,0.00001414,-0.00000440,0$. $00000093,-0.00000407,-0.00266707,-0.00287297,-0.01328550,-0.00435896,0$ $.00282434,-0.00053692,0.00020765,-0.00062173,0.00073092,-0.00240050,0$. $00559844,-0.00095926,-0.00054492,0.00423073,0.00155698,-0.04479016,-0$. $10318846,-0.11672806,0.00152453,-0.00159291,0.00077490,-0.00017357,-0$. $00004661,-0.00062496,0.00028078,-0.00050119,0.00015080,0.00191452,-0.0$ $0282691,0.00134041,0.05103052,0.09899830,-0.00057981,-0.00056703,-0.00$ $049721,-0.00017288,0.00005892,-0.00000275,0.00003760,-0.00004537,0.000$ $04406,0.00002404,0.00003968,-0.00000601,-0.00000033,0.00003841,-0.0000$ $0659,-0.00000536,-0.00009038,0.00005709,0.00014977,-0.00000246,-0.0003$ $2509,0.00000029,-0.00000196,-0.00001261,-0.00010168,0.00006253,-0.0000$ $2778,0.00001649,0.00001029,-0.00001182,-0.00001071,0.00000957,-0.00002$ $097,-0.00000072,-0.00000010,-0.00000427,0.00313970,0.00064322,0.000825$ $07,0.00086766,-0.00076065,0.00199747,0.00041408,0.00061071,0.00010185$, $0.00450835,-0.00073094,-0.00108759,-0.00837040,-0.01139419,-0.01487584$ ,-0.06630109,-0.11801930,-0.25830237,-0.00007930,0.00122948,0.00079161 $, 0.00007669,0.00001052,0.00019524,-0.00014924,0.00013454,-0.00013186,-$ $0.00075888,0.00139646,0.00051487,0.06719193,0.12741714,0.27075499,0.00$ $152682,0.00136583,-0.00027407,-0.00788709,0.00242892,-0.00083261,0.011$ $11152,0.03165521,-0.01040353,-0.00532836,-0.00248375,0.00066366,-0.000$ $23328,0.00226103,-0.00075591,0.00006544,0.00044920,-0.00006680,-0.0001$ $9468,-0.00001796,-0.00010043,-0.00193178,0.00417046,-0.00136864,-0.130$ $41499,-0.02381758,0.00611941,-0.00053104,0.00035472,-0.00014380,0.0000$ $7281,0.00032638,-0.00009765,0.00006901,0.00095089,-0.00032424,0.000176$ $09,-0.00002450,-0.00031959,-0.00005863,-0.00000295,0.00014901,0.000065$ $36,0.00012345,0.00020808,0.00020717,0.00009734,-0.00056160,-0.00018523$ ,-0.00006535,0.00008873,-0.00002726,-0.00002966,0.00001358,-0.00021720 ,-0.00000577,0.00029752,-0.00003183,-0.00000909,0.00002991,0.00005392, $0.00002137,-0.00002077,-0.00000631,-0.00005619,-0.00011911,-0.00002649$ $, 0.00001536,0.00003859,0.14193856,0.00118941,0.00118071,-0.00082342,-0$ $.00745226,-0.00298976,0.00139967,0.01679966,-0.04821567,0.02214197,0.0$ $0629919,0.00338468,-0.00009606,0.00167409,-0.00202095,0.00057445,-0.00$ $136018,-0.00040746,0.00011529,0.00046957,-0.00041360,0.00013258,0.0028$ $7276,0.00047760,-0.00004572,-0.02509563,-0.48623283,0.13112982,0.00060$ $611,-0.00039613,0.00003604,0.00019121,0.00012607,-0.00000986,-0.000614$ $31,0.00132133,-0.00041794,-0.00001622,0.00014574,0.00048321,0.00014437$ $, 0.00001276,-0.00044942,-0.00027388,-0.00026257,-0.00020411,0.00018945$ ,-0.00031921,0.00057946,0.00033019,0.00010695,-0.00014572,-0.00007420, $0.00007111,0.00001539,0.00027276,0.00001748,-0.00038168,0.00005815,0.0$ $0000061,-0.00004914,-0.00000238,-0.00008013,0.00009405,0.00000830,-0.0$ $0001784,0.00018240,0.00004222,-0.00006286,-0.00004320,0.07032580,0.594$ $44537,-0.00048990,-0.00076404,-0.00099878,0.00220356,0.00144633,0.0012$ $0532,-0.00563534,0.02257451,0.01147816,-0.00221487,-0.00030620,0.00273$ $800,-0.00051167,0.00042960,-0.00086781,0.00042045,0.00022827,0.0001173$ $0,-0.00029428,0.00029156,-0.00007652,-0.00097265,0.00004960,0.00020114$ 
$, 0.00658190,0.13103729,-0.11776422,-0.00020505,0.00000755,-0.00037595$, $-0.00005515,0.00000766,0.00012076,0.00019424,-0.00032828,0.00033885,-0$ $.00000496,-0.00004200,-0.00012299,-0.00004212,0.00000476,0.00010010,0$. $00004283,0.00003279,0.00007773,0.00022222,-0.00002025,-0.00026174,-0.0$ $0011213,-0.00005927,0.00006686,0.00002546,-0.00000461,0.00003073,-0.00$ $007261,-0.00000679,0.00011560,-0.00001994,0.00000463,0.00001276,0.0000$ $2258,0.00003046,-0.00002004,-0.00000373,0.00004604,-0.00009390,-0.0000$ $2559,0.00003453,0.00002334,-0.01946344,-0.17991976,0.08506227,-0.00067$ $881,-0.00077139,0.00064394,0.00038539,0.01021923,-0.00336377,-0.066665$ $63,-0.01579670,0.00262955,-0.00150242,-0.00265614,0.00084053,-0.000689$ $51,0.00066743,-0.00035220,-0.00029209,-0.00004309,0.00005983,-0.000365$ $80,0.00018879,-0.00009455,0.00142748,-0.00024654,0.00008970,-0.4653495$ $8,-0.14258825,0.03460127,-0.00018698,0.00026078,-0.00008967,0.00008788$ $, 0.00024982,-0.00004869,-0.00186757,0.00276480,-0.00094954,0.00021740$, $-0.00012397,-0.00062379,-0.00007766,0.00003130,0.00042098,0.00015778,0$ $.00026957,0.00030766,-0.00020565,0.00027326,-0.00081024,-0.00043769,-0$ $.00012783,0.00003158,-0.00002443,-0.00005560,-0.00003651,-0.00036176,0$ $.00000779,0.00049850,-0.00003878,-0.00002204,0.00004607,0.00006656,0.0$ $0003926,-0.00000764,-0.00001675,-0.00007861,-0.00015337,-0.00003209,0$. $00000312,0.00006519,-0.00821253,-0.06658418,0.02041019,0.54466324,0.00$ $081929,0.00087351,-0.00093211,0.00041282,-0.00232647,0.00152112,0.0046$ $7371,0.01946938,0.00041981,0.00396940,-0.00900592,0.00348937,-0.001226$ $19,0.00252719,-0.00101019,0.00159411,-0.00021263,0.00007991,0.00007393$ $, 0.00009780,0.00000935,0.00133987,0.00032288,0.00012533,-0.14207566,-0$ $.18406397,0.03096000,-0.00013076,0.00002171,-0.00000043,0.00028478,0.0$ $0023219,-0.00004371,0.00402805,0.00068985,-0.00019721,-0.00001770,-0.0$ $0000077,0.00007188,0.00005286,0.00004329,-0.00006713,-0.00000536,-0.00$ $002733,-0.00013357,0.00002756,0.00003630,0.00030132,-0.00003684,0.0000$ $1685,-0.00011721,0.00000578,0.00004477,-0.00002068,0.00006944,0.000010$ $23,-0.00008884,0.00002800,-0.00001325,-0.00001212,0.00001247,-0.000038$ $89,0.00004259,0.00000646,-0.00005457,0.00007474,0.00000794,-0.00002591$ ,-0.00000589,-0.08803314,-0.05987140,0.02522562,0.21411920,0.23125516, $-0.00055739,-0.00094001,-0.00217592,-0.00027472,0.00162237,0.00189135$, $-0.00381452,0.00001906,0.02029485,-0.00134751,0.00315012,0.00017351,0$. $00034540,-0.00094965,-0.00001389,-0.00049173,0.00004347,0.00002727,-0$. $00034006,0.00034172,0.00004539,-0.00038341,0.00012913,0.00069049,0.034$ $74644,0.03110268,-0.08438931,0.00003944,-0.00000783,-0.00003446,-0.000$ 08606,-0.00005216,0.00007514,-0.00136194,-0.00020442,-0.00017836,0.000 $03126,0.00001927,0.00002391,-0.00000791,0.00000300,-0.00007711,-0.0000$ $5279,-0.00012770,0.00006918,0.00043063,-0.00021793,-0.00003750,0.00003$ $407,0.00000688,0.00007960,-0.00000972,0.00002255,0.00001294,-0.0000003$ $3,-0.00000557,0.00000910,-0.00001329,0.00001712,-0.00000395,0.00002111$ $, 0.00001096,0.00000638,-0.00002238,-0.00000404,-0.00005850,-0.00001325$ $, 0.00001122,0.00000720,0.02724370,0.02570170,0.01889303,-0.05411504,-0$ $.05969194,0.04466965 \backslash \backslash-0.00000668,0.00000090,0.00000736,0.00000150,-0$. $00000120,0.00000070,0.00000283,0.00000292,-0.00000117,0.00000211,-0.00$ $000358,0.00000089,-0.00000464,-0.00000044,-0.00000239,0.00000262,0.000$ 00034,-0.00000198,0.00000276,-0.00000122,-0.00000312,0.00000093,0.0000 $0107,0.00000432,0.00000388,0.00000659,-0.00000110,-0.00000054,-0.00000$ 095,-0.00000167,-0.00000072,-0.00000141,0.00000004,-0.00000086, 0.00000 $108,-0.00000087,0.00000081,0.00000131,0.00000309,0.00000077,-0.0000012$ 
9,-0.00000282,-0.00000359,-0.00000102,0.00000242,0.00000579,-0.0000026 $0,-0.00000516,-0.00000217,0.00000104,-0.00000316,-0.00000262,0.0000009$ $4,0.00000004,0.00000009,0.00000178,-0.00000146,0.00000058,-0.00000089$, $0.00000023,0.00000130,-0.00000196,0.00000074,-0.00000030,0.00000078,-0$ $.00000029,-0.00000109,0.00000237,-0.00000110,0.00000239,-0.00000300,0$. 00000425,-0.00000515,-0.00000157,0.0000021911।@

para-Addition Product

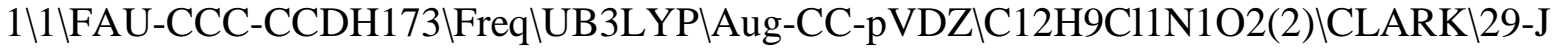
an-2015 \0\\NN Geom=AllCheck Guess=TCheck SCRF=Check GenChk UB3LYP/AugCC-pVDZ Freq \\Addition product p-Cl-Ph-radical + nitrobenzene (para)\ 0,2\C,-6.0363007264,1.5011677077,1.5652845511\C,-4.7640598917,0.806342 9646,1.9734986232\C,-3.5254612606,1.3260189788,1.7426110747\C,-3.39718 $08737,2.5843466047,1.0902832402 \backslash \mathrm{C},-4.538559464,3.3269352682,0.67653610$ $54 \backslash \mathrm{C},-5.787386207,2.8274547306,0.8966460258 \backslash \mathrm{H},-6.5444482091,0.84955068$ $53,0.8251459289 \backslash \mathrm{H},-4.8646312799,-0.1550663556,2.4779130048 \backslash \mathrm{H},-2.622590$ $321,0.8024664143,2.0473561133 \backslash \mathrm{H},-4.3872143449,4.2876586276,0.190474062$ $7 \backslash \mathrm{H},-6.6632819413,3.3972428896,0.5851876931 \mathrm{~N},-2.0810773951,3.12263848$ $15,0.8498990406 \backslash \mathrm{C},-8.7993663135,1.8699356298,4.8823298594 \backslash \mathrm{C},-9.1591106$ 637,1.0995939441,3.7774314361\C,-8.2619260435,0.9851999882,2.710494380 $7 \backslash \mathrm{C},-7.0192599524,1.6285024315,2.7382778405 \backslash \mathrm{C},-6.683685676,2.397011268$ ,3.8626562383\C,-7.5657424338,2.5230643477,4.9371014535\Cl,-9.91908435 $37,2.0226149912,6.2326419157 \backslash \mathrm{H},-10.124186217,0.5971152671,3.7505202888$ $\backslash \mathrm{H},-8.5436559523,0.382321779,1.8459836073 \backslash \mathrm{H},-5.7207120995,2.9065688274$ ,3.9048313622\H,-7.3013878541,3.1204915747,5.80787159\O,-1.1046557467, $2.4582844049,1.2225578052 \backslash \mathrm{O},-1.9970141761,4.2206401051,0.2833493965 ॥ \mathrm{~V}$ ersion=EM64L-G09RevC.01 \State $=2-A \backslash H F=-1128.059857 \backslash \mathrm{S} 2=0.775613 \backslash \mathrm{S} 2-1=0 . \backslash$ $\mathrm{S} 2 \mathrm{~A}=0.750413 \backslash \mathrm{RMSD}=7.997 \mathrm{e}-09 \backslash \mathrm{RMSF}=3.902 \mathrm{e}-06 \mathrm{ZeroPoint}=0.1828112 \backslash \mathrm{Thermal}$ $=0.1960303 \backslash \mathrm{ZPE}=114.7157509 \backslash \mathrm{Dipole}=-1.8281455,-0.9266106,-0.0017827 \mathrm{Dip}$ oleDeriv $=-0.2341194,-0.0631684,0.092854,-0.1429122,-0.0018873,0.154052$ $9,-0.0565839,0.0782949,0.1890482,0.1292397,0.1673646,-0.1283678,-0.125$ $9313,0.051198,-0.0484735,0.2213027,-0.0812322,-0.2272876,-0.015095,-0$. $1979905,0.1663808,0.2500812,-0.1464645,-0.0009096,-0.1248518,0.0265952$ $,-0.0639055,-0.5575543,-0.3538784,0.2427966,-0.3839992,0.1828804,-0.03$ 67195,0.1862686,-0.0653563,0.0971904,-0.0706461,0.2930643,-0.1499946,$0.1817264,-0.0467904,0.0541456,0.0912047,0.0012837,-0.1080497,0.078020$ $1,-0.1267957,0.1223071,0.2888855,0.0125372,-0.2133394,0.002185,-0.0643$ $366,-0.1374779,-0.0334575,-0.0834811,-0.0507483,-0.0644173,0.0173748,-$ $0.0526165,-0.0150164,-0.0345257,0.0274977,0.0581562,-0.0121556,-0.0126$ $633,-0.018423,-0.0113656,0.0653677,-0.0090176,0.0736486,0.0815163,0.06$ $6777,0.0405457,-0.0159656,-0.0078267,0.0858622,0.042301,-0.024502,0.02$ $40257,0.1326934,0.091639,-0.0111523,0.0166301,0.0070761,0.0615054,0.04$ $53131,-0.031425,0.034954,0.1321957,-0.0093927,0.0361053,-0.0353198,0.0$ $454744,0.0305752,0.0369525,-0.0331197,0.0316454,0.1071248,2.5268633,0$. $3643808,-0.3620624,0.4626343,1.3095347,-0.6583411,-0.1777064,-0.564986$ $5,0.5348349,0.5491077,-0.0265236,-0.6070574,-0.0783188,-0.0082402,0.12$ $9415,-0.7043249,0.0801955,0.7821513,-0.0842237,0.0660345,0.1197596,0.0$ $935775,-0.0626026,-0.0398354,0.1714444,-0.0136805,-0.2773111,0.0144245$ $, 0.0423983,-0.0317755,0.1130956,-0.0272356,0.0345731,0.1010038,0.10175$ $57,-0.0078013,0.1950271,0.0350299,-0.1709285,-0.0252029,-0.060864,0.01$ 
$11353,-0.2841387,-0.0460902,0.1294849,-0.089946,0.0309775,0.0665506,0$. $0121851,-0.0532273,0.0408976,0.0312428,0.0230472,-0.067631,-0.1708126$, $0.0201724,0.1235255,0.0314514,-0.0353306,0.0552366,0.1447101,0.0659506$ ,-0.0942763,-0.4604358,0.0153185,0.4069868,0.0446144,-0.0729589,-0.062 $3967,0.4619701,-0.0345644,-0.5660738,-0.0099065,-0.0603778,0.0185872,-$ $0.0542162,0.0937523,-0.0147374,0.0301587,-0.0088757,0.0837008,0.089332$ $7,-0.0146594,0.0007507,-0.0258264,0.0624745,-0.0818502,-0.0202127,-0.0$ $924515,-0.0352713,-0.0291021,-0.0596564,0.0114705,-0.070065,0.0663761$, $0.0017189,-0.0080339,-0.0081721,0.0932235,0.0761721,-0.0249489,0.00144$ $18,-0.0205099,0.0838097,-0.0616453,0.009756,-0.057431,0.0098887,-1.471$ $8078,0.1194888,0.026486,0.4338273,-0.4560961,0.0892825,-0.2568056,0.10$ 93394,-0.3003238,-0.6382599,-0.1960925,0.148356,-0.5835275,-1.0748173, $0.5104727,0.2944918,0.4209671,-0.5151417 \backslash$ Polar $=253.2438565,29.3789937$, 146.3147136,-55.037696,-10.9231397,169.0726174य $\mathrm{PG}=\mathrm{C} 01$ [X(C12H9Cl1N1O2) ]NImag=0\\0.47778787,0.02149836,0.48977202,0.03965739,0.00359680,0.44 $604825,-0.15957352,0.05301825,-0.03324809,0.66057497,0.04205829,-0.112$ $87523,0.02709527,0.09427806,0.55683117,-0.02819907,0.02717688,-0.08262$ $026,-0.03262024,-0.21739829,0.25439234,-0.02959454,-0.00608025,0.00176$ $899,-0.37635747,-0.11355336,0.04902321,0.76996532,0.00666917,0.0116744$ $7,-0.00274893,-0.07471825,-0.13396079,0.03865034,-0.02204122,0.5014252$ $7,-0.00530219,-0.00160437,0.01179903,0.02791459,0.03715178,-0.07289003$ $, 0.03320761,-0.19870033,0.22604071,-0.00103613,0.00112113,-0.00143023$, $0.00609319,-0.03313279,0.01803942,-0.11074521,-0.01524245,0.00634425,0$ $.55849867,0.00144233,-0.00564846,-0.00304438,-0.06036599,-0.03287738,0$ $.01929833,0.04440052,-0.20826219,0.08226161,-0.03261628,0.52407088,-0$. $00082846,-0.00274107,-0.00911385,0.03177858,0.01932490,-0.00255339,-0$. $02501301,0.08016259,-0.09643904,0.02873458,-0.21125827,0.22933515,-0.0$ $0458901,-0.01341482,0.00461756,-0.03110031,-0.00713366,0.00288928,0.03$ $798948,0.04659505,-0.02294332,-0.17139729,0.03230505,-0.01993031,0.643$ $80713,-0.02595754,-0.00692799,0.00926059,0.00203571,0.00219679,-0.0017$ $4273,0.02199767,-0.02198954,0.01434310,0.09239279,-0.16034589,0.057336$ $01,0.07590093,0.60663273,0.01207920,0.00831437,0.00539700,-0.00213791$, $-0.00212423,0.00022748,-0.01055244,0.01575489,-0.00321160,-0.05045684$, $0.05565861,-0.08370435,-0.02320741,-0.24638752,0.24699165,-0.08638483$, $-0.01387662,0.00619560,0.01959557,0.03106541,-0.01490854,-0.01438658,-$ $0.02041492,0.00979398,-0.07336117,0.02876226,-0.01710007,-0.35235433,-$ $0.13279097,0.05863338,0.76398355,-0.00342958,-0.17637558,0.05340369,0$. $01388396,-0.01930424,0.00935425,-0.01101357,-0.01099692,0.00496722,0.0$ $0205125,0.03151560,-0.01223574,-0.09314268,-0.15322145,0.04784637,0.01$ $162600,0.48000089,0.00017649,0.05283705,-0.09227808,-0.00744044,0.0126$ $8760,-0.00525097,0.00520141,0.00480598,-0.00329430,-0.00237709,-0.0117$ $1255,0.01250606,0.03904483,0.04711968,-0.07763628,0.00916105,-0.171969$ $75,0.22779374,-0.08488922,-0.06437679,-0.08121028,-0.02060142,-0.01332$ $172,-0.01737326,-0.00068252,0.00384617,-0.00203355,-0.00233877,-0.0006$ $8558,-0.00013560,0.00284165,-0.00066541,0.00043317,-0.00450392,-0.0082$ $9574,-0.00006105,0.10611116,-0.05946030,-0.12265367,-0.09263178,0.0044$ $2865,0.01060915,0.00875399,0.00268836,0.00043525,-0.00066835,-0.000802$ $86,-0.00132629,0.00039012,0.00220237,-0.00344316,0.00127355,-0.0166641$ $9,-0.02045213,-0.01417875,0.07047375,0.13687924,-0.07198512,-0.0879605$ $2,-0.13988153,-0.00683501,-0.00395363,-0.00466697,0.00001571,0.0013855$ $1,-0.00175242,-0.00035617,0.00027825,-0.00026642,0.00159395,0.00084503$ 
,-0.00139811,0.00701074,0.00744492,0.01029521,0.07697122,0.10000784,0. $16247493,0.00410569,0.02314742,-0.01055903,-0.05735806,-0.02161314,0.0$ $1047442,0.00016110,-0.02644532,0.01375371,-0.00476194,-0.00062349,-0.0$ $0000356,-0.00019013,0.00004273,-0.00005313,-0.00546502,0.00115282,-0.0$ $0090658,0.00073251,-0.00071961,-0.00019481,0.05964633,-0.00299314,-0.0$ $0792805,0.00450986,-0.02426194,-0.27276560,0.12481380,0.00141870,-0.00$ $569030,0.00650130,-0.00173912,0.00303285,0.00297053,-0.00025997,0.0001$ $4619,-0.00052652,0.00270262,0.00083127,0.00067913,0.00059606,0.0000700$ $1,0.00055766,0.02547669,0.28315850,0.00213980,0.00665756,-0.00246920,0$ $.01190087,0.12572102,-0.09911339,-0.00112423,0.00543174,0.00383545,0.0$ $0028968,0.00275587,0.00679694,0.00021057,-0.00042721,-0.00060231,-0.00$ $173621,0.00020555,0.00133022,0.00078492,-0.00043766,0.00071838,-0.0122$ $1971,-0.13747381,0.09309473,-0.00099238,-0.00294524,0.00124548,-0.0172$ $7957,0.01357068,-0.00797315,-0.25234070,0.11921905,-0.06951752,0.00583$ $550,-0.00093930,0.00053020,0.00090433,-0.00178100,0.00080287,-0.000048$ $50,-0.00029997,0.00021179,-0.00046608,0.00019581,-0.00010847,0.0011579$ $2,0.00006137,0.00023625,0.26684207,-0.00221040,0.00134233,0.00459528,-$ $0.01222987,0.01029457,-0.00365474,0.11770012,-0.12284043,0.04814646,0$. $02272349,-0.00912463,0.00730147,-0.00220393,-0.00301323,0.00346986,-0$. $00063418,-0.00025818,-0.00012503,0.00032236,-0.00031408,0.00003636,0.0$ $0019253,-0.00058419,-0.00280466,-0.12562389,0.12299947,0.00152285,0.00$ $416772,0.00787360,0.00559336,-0.00232245,0.00539224,-0.06923418,0.0481$ $6405,-0.06600885,-0.01174609,0.00639180,-0.00035081,0.00096057,0.00368$ $738,0.00188121,0.00042417,-0.00007732,-0.00052761,0.00127108,-0.000908$ $31,0.00038614,0.00007342,-0.00286855,-0.00440576,0.07391952,-0.0548069$ $4,0.05759798,-0.00382151,-0.00022575,-0.00014023,-0.00070968,-0.000122$ $00,0.00012631,-0.00447739,0.00204724,-0.00130971,0.00763112,0.02138414$ ,-0.01083929,-0.05934201,-0.03263947,0.01614561,-0.00005794,-0.0257684 $7,0.01298171,-0.00013940,-0.00055728,0.00024228,0.00011022,-0.00000447$ $, 0.00003558,0.00044785,0.00121739,-0.00064506,0.05948787,-0.00064759,0$ $.00392065,0.00309609,0.00025257,0.00021797,-0.00039714,0.00249973,0.00$ $112894,0.00140652,-0.00236846,-0.01066326,0.00708608,-0.03147854,-0.28$ $111756,0.12308378,0.00037452,-0.00408256,0.00401532,-0.00001360,0.0005$ $6249,-0.00033101,-0.00007626,-0.00016585,-0.00022004,-0.00020722,-0.00$ $038540,0.00035293,0.03608606,0.29306659,0.00016954,0.00382170,0.008124$ $20,-0.00001020,-0.00040631,-0.00034261,-0.00141189,0.00121729,0.003120$ $88,0.00126804,0.00791034,-0.00060811,0.01519033,0.12272624,-0.10073089$ $, 0.00005806,0.00300764,0.00254750,0.00012057,0.00125051,-0.00081712,0$. $00006700,-0.00026960,-0.00046558,0.00012476,0.00033145,0.00006603,-0.0$ $1745878,-0.13510503,0.09488503,0.00633165,-0.00456647,0.00342736,0.000$ $19517,-0.00163198,0.00090788,-0.00000774,-0.00044408,0.00021223,-0.002$ $39597,-0.00338779,0.00170078,-0.01859215,0.01626813,-0.00896946,-0.238$ $65026,0.11821534,-0.06592442,0.00022484,0.00047139,-0.00041572,0.00050$ $559,-0.00020938,0.00008292,-0.00002637,0.00008407,-0.00005838,0.001067$ $91,0.00006001,0.00006192,0.25175308,0.02147543,-0.01020476,0.00726461$, $-0.00335002,-0.00379293,0.00248992,-0.00003604,-0.00004643,-0.00043545$ $,-0.00255569,0.00087637,0.00366154,-0.01199128,0.00959007,-0.00171128$, $0.12119062,-0.12908397,0.05199279,-0.00002978,0.00093440,-0.00072003,0$ $.00137974,-0.00035937,0.00066208,0.00003434,-0.00002637,-0.00026498,0$. $00027792,-0.00039771,-0.00280519,-0.12575528,0.13122513,-0.00945590,0$. $00502315,-0.00241944,0.00142143,0.00280319,0.00029404,0.00014637,-0.00$ 
043486,-0.00059197,0.00087522,0.00360683,0.00658715,0.00516577,-0.0010 $1807,0.00730931,-0.06673586,0.05139722,-0.06150392,0.00013245,0.001049$ $06,0.00036176,-0.00071880,0.00057341,0.00047678,0.00001446,-0.00029351$ ,-0.00046839,0.00006151,-0.00278656,-0.00450202,0.06977022,-0.05929790 $, 0.05292201,0.00402673,0.00231721,0.00079340,0.00561744,0.00984284,-0$. $00620893,-0.01204587,-0.03079865,0.01552696,-0.13279923,-0.01065904,0$. $00401314,-0.04538891,-0.00483990,0.00102970,0.00513173,0.00054153,-0.0$ $0137951,0.00182868,0.00100403,-0.00033170,0.00066942,0.00048608,-0.000$ $17833,-0.00002794,0.00459672,-0.00232163,0.00231434,-0.00100109,0.0006$ $5472,-0.00048064,-0.00042498,0.00027744,0.66332045,0.00154318,0.000916$ $96,-0.00028263,0.00066482,-0.00411280,0.00378699,-0.03085537,-0.009158$ $32,0.00319184,-0.01068334,-0.10412393,0.01653294,-0.00468160,0.0179451$ $3,-0.00993579,0.01075510,-0.00377744,0.00385803,0.00089672,0.00002377$, $0.00013823,-0.00137081,-0.00022521,0.00049538,0.00083194,-0.00133641,-$ $0.00035204,0.00276687,-0.00323782,0.00067450,0.00134969,0.00068744,0.0$ $0006850,-0.07651041,0.69627417,-0.00065837,-0.00101788,-0.00099838,-0$. $00022348,0.00458055,0.00183202,0.01570258,0.00318804,-0.00212864,0.003$ $96837,0.01650821,-0.08030196,0.00104248,-0.00983642,0.00411119,-0.0054$ $2155,0.00404833,0.00198262,-0.00053361,0.00003622,0.00007083,0.0006924$ $3,0.00049534,0.00044930,-0.00036477,-0.00024299,-0.00196928,-0.0012951$ $6,0.00057019,-0.00240969,-0.00074751,-0.00000963,0.00068677,0.05246975$ $,-0.25259269,0.34578334,-0.00108411,-0.00023317,0.00013875,-0.00010341$ $, 0.00026293,0.00018111,0.00006321,0.00002266,0.00014758,0.00043967,0.0$ $0021742,0.00003562,0.00000736,0.00017516,0.00003611,-0.00002225,-0.000$ $05855,0.00033714,-0.00009194,-0.00002540,0.00018111,-0.00010641,0.0000$ $2137,-0.00000461,0.00003964,-0.00002435,-0.00010302,0.00006295,-0.0000$ $2022,-0.00007035,-0.00002773,-0.00007641,0.00000171,-0.00086690,-0.000$ $35256,0.00017880,0.50412693,-0.00052092,-0.00068886,0.00009609,-0.0000$ $1693,0.00012581,0.00036499,-0.00000428,-0.00000142,0.00004236,0.000036$ $98,0.00010317,0.00006887,-0.00002203,-0.00004857,0.00012774,0.00006931$ $, 0.00027886,0.00031555,-0.00011054,0.00017196,0.00014633,0.00001522,-0$ $.00006191,-0.00007081,0.00004996,-0.00002115,-0.00007743,0.00001412,-0$ $.00004964,-0.00013166,-0.00006039,-0.00002768,0.00000160,0.00022842,0$. $00010884,-0.00004563,0.18140815,0.31104603,-0.00040135,-0.00017653,-0$. $00042781,0.00026217,0.00006471,0.00051741,-0.00019417,0.00010550,-0.00$ $004259,-0.00030257,-0.00010262,0.00014301,-0.00004343,-0.00014828,0.00$ $006091,-0.00002977,0.00047142,0.00028423,0.00002154,0.00006594,0.00025$ $250,0.00002267,-0.00009114,-0.00002192,0.00001702,-0.00008279,-0.00011$ $247,0.00001362,-0.00002322,-0.00010744,-0.00000803,0.00003035,-0.00013$ 492,0.00126910,0.00050641,-0.00023800,-0.01790471,0.17402017,0.4704126 $4,-0.00119652,-0.00428817,-0.00004526,0.00027839,0.00008361,0.00028990$ $, 0.00006389,0.00000664,-0.00004420,-0.00005080,-0.00006807,0.00003237$, $0.00007366,-0.00030416,0.00015979,-0.00090613,0.00061555,0.00068872,0$. $00007336,0.00015271,0.00018505,-0.00010692,0.00002230,-0.00003999,0.00$ $002415,-0.00001084,-0.00001926,-0.00006337,-0.00005128,-0.00012625,-0$. $00008432,-0.00007506,0.00002874,0.00041797,0.00015972,-0.00006377,-0.1$ $2462774,-0.06853585,-0.07192516,0.64417829,-0.00443782,0.00369324,-0.0$ $0244116,-0.00075608,-0.00056312,0.00094399,-0.00020827,0.00026519,-0.0$ $0017309,-0.00012994,0.00000699,-0.00008537,0.00007433,0.00028490,-0.00$ $025906,0.00086356,-0.00018490,-0.00060909,0.00004864,-0.00001030,-0.00$ $000300,-0.00005280,0.00002522,0.00003196,-0.00008214,-0.00008993,-0.00$ 
$001887,0.00004921,0.00007268,0.00014889,0.00005253,-0.00007908,0.00005$ $434,-0.00004316,0.00001454,0.00000139,-0.04119266,-0.15184666,-0.12423$ $704,0.21149730,0.33444296,-0.00032198,-0.00258058,0.00269777,0.0003733$ $7,-0.00003116,-0.00104332,0.00032267,-0.00020294,-0.00002278,-0.000083$ $65,-0.00014372,-0.00007240,0.00010136,-0.00007326,-0.00005160,-0.00050$ $835,-0.00019675,-0.00023761,-0.00001071,-0.00010269,-0.00021540,0.0000$ $2663,0.00012831,-0.00000411,-0.00001731,0.00013550,0.00019359,-0.00007$ $362,0.00002211,0.00011892,0.00005976,0.00000829,0.00007732,-0.00044413$ ,-0.00019118,0.00009876,-0.02057861,-0.09826528,-0.22983518,-0.0907088 $1,0.18202535,0.55850034,-0.01926163,-0.00854002,0.00619683,-0.00002613$ $,-0.00174922,0.00044590,-0.00020361,0.00045251,-0.00037013,-0.00013077$ $, 0.00008467,-0.00000018,0.00067684,-0.00052700,0.00023603,-0.00149624$, $0.00088126,-0.00128909,0.00029768,-0.00126608,-0.00274903,-0.00003153$, $0.00039246,-0.00004275,-0.00014849,-0.00005521,0.00010826,-0.00018877$, $0.00005285,0.00018106,0.00015002,-0.00035997,0.00030555,-0.00016099,-0$ $.00003545,0.00004533,0.04861526,0.04149323,0.03816058,-0.20258901,-0.0$ $2412754,0.08816365,0.57793304,0.00125168,0.00465268,-0.00024061,0.0006$ $1796,-0.00059957,-0.00115176,0.00049337,0.00026568,0.00006880,0.000389$ $09,-0.00007024,0.00005821,-0.00028267,0.00029746,-0.00011505,0.0007635$ $1,-0.00034578,-0.00063189,0.00141430,0.00034798,-0.00051124,-0.0002705$ $9,0.00008519,-0.00005153,0.00002430,0.00011405,0.00020967,0.00004847,0$ $.00001039,0.00000759,0.00009556,0.00014277,0.00002222,-0.00064928,-0.0$ $0031165,0.00011335,0.01766141,0.01038289,-0.00996968,0.00438235,-0.085$ $04454,-0.04731873,0.18088659,0.33912222,0.02459050,0.00906037,-0.00219$ $024,0.00101897,0.00164590,-0.00223164,0.00047523,-0.00082677,0.0007171$ $7,0.00057172,0.00034735,-0.00018919,-0.00007185,0.00105175,-0.00019541$ $, 0.00235945,-0.00129275,-0.00101099,0.00228266,0.00203369,0.00155498,0$ $.00012880,-0.00028456,0.00013252,0.00020430,0.00015101,-0.00001934,0.0$ $0022584,0.00013780,0.00012437,-0.00025512,0.00005814,-0.00010535,-0.00$ $110766,-0.00047040,0.00012661,-0.00658848,-0.03260298,-0.04820593,0.14$ $169977,-0.02024139,-0.23465635,-0.08170889,0.21287352,0.61161724,-0.10$ $461674,0.00731519,0.04919455,-0.03046618,0.00577228,0.01954083,-0.0004$ $3875,0.00365611,-0.00116049,-0.00079886,0.00057465,0.00013167,0.003145$ $72,0.00058237,0.00106647,-0.00942806,-0.01137580,0.01149899,0.00430931$ ,-0.00230240,-0.00797509,0.00153837,-0.00124536,-0.00056122,-0.0002548 $9,-0.00078828,-0.00110538,0.00015173,-0.00019033,-0.00025859,0.0007532$ $1,0.00061056,-0.00136034,-0.00119647,-0.00027068,0.00003303,-0.0322726$ $5,-0.02617455,-0.02494462,-0.03762381,0.00633675,0.05408999,-0.2707407$ $4,-0.11719127,-0.02455576,0.56259193,0.00090578,-0.07026785,-0.0064900$ $0,0.00682278,-0.00081237,-0.01053917,0.00187838,0.00048513,-0.00004945$ $, 0.00026295,-0.00118574,0.00089496,0.00034733,-0.00440380,0.00139097,-$ $0.01983263,0.00363879,0.02102176,0.01049400,-0.00340407,-0.01723585,-0$ $.00000426,0.00072426,-0.00017657,0.00009229,0.00045621,0.00048012,-0.0$ $0070079,-0.00074398,-0.00118223,-0.00015435,0.00041824,-0.00038732,0.0$ $0059125,-0.00020230,0.00023471,-0.02659477,-0.03007021,-0.01846164,-0$. $01418160,0.02095095,0.04004574,-0.08791622,-0.12930037,-0.03745334,0.1$ $6949928,0.34484296,0.03716573,-0.01257944,-0.12620646,0.00558090,-0.00$ $523179,0.00975108,-0.00332604,0.00035755,-0.00222989,-0.00045440,0.000$ $59821,-0.00023967,-0.00054178,-0.00092663,-0.00092419,0.01154636,0.008$ $67510,-0.01574109,0.01604075,-0.00507458,-0.02430709,-0.00101799,0.000$ $78079,0.00059990,-0.00050248,-0.00058138,-0.00021076,-0.00016712,0.000$ 
$23083,0.00058307,-0.00000861,-0.00089233,0.00169019,0.00165150,0.00105$ $376,-0.00021811,-0.02573433,-0.01886058,-0.01776219,0.01556328,0.02056$ $041,0.03062543,0.03042046,-0.00964951,-0.11190228,-0.05322015,0.164621$ $32,0.53115465,0.00545487,0.01187209,0.01869942,0.00209444,0.00037080,0$ $.00151178,-0.00065007,-0.00014622,0.00022961,-0.00054938,0.00007369,-0$ $.00001011,-0.00025122,-0.00043895,0.00009508,0.00098577,0.00176285,0.0$ $0038701,-0.00200468,0.00073924,0.00426338,0.00003931,-0.00008884,0.000$ 07005,0.00000606,-0.00013096,-0.00011086,-0.00002093,-0.00005307,-0.00 $011138,0.00004839,-0.00011966,0.00007152,0.00088256,0.00045934,-0.0001$ $1346,-0.04045599,-0.01611043,0.01480763,-0.00415700,0.00528133,0.01109$ $743,0.00058785,-0.01810672,-0.02742744,-0.12679272,-0.06094505,-0.0570$ $7242,0.63479436,0.00269628,0.00109144,-0.00364492,-0.00060358,0.000466$ $89,-0.00038593,-0.00027612,-0.00001663,-0.00035115,-0.00014269,-0.0006$ $5220,0.00024030,-0.00078323,0.00000405,0.00021368,0.00028700,0.0002839$ $1,0.00046763,-0.00013861,0.00098211,0.00005701,0.00003477,0.00010506,-$ $0.00005972,-0.00003499,0.00003380,-0.00000302,-0.00004406,0.00000310,-$ $0.00006805,-0.00009482,0.00038546,-0.00018032,0.00036680,0.00005671,0$. $00003004,0.00769480,0.02172706,0.02036989,0.00569480,-0.01170350,-0.02$ $191306,-0.03761351,-0.01412714,-0.00490337,-0.03397070,-0.14600956,-0$. $11724048,0.20005527,0.33150068,0.00146685,-0.01236371,-0.02294159,-0.0$ $0194160,0.00046749,-0.00103701,-0.00036078,-0.00040005,0.00022567,-0.0$ $0041824,0.00003295,-0.00024738,-0.00020395,-0.00018667,-0.00019452,-0$. $00075748,-0.00271299,0.00025553,0.00261498,-0.00077642,-0.00291954,0.0$ $0034378,-0.00030639,0.00017646,-0.00002589,0.00002788,0.00006737,0.000$ $00087,0.00000104,0.00012548,0.00007665,0.00020841,-0.00011312,-0.00028$ $731,-0.00005807,0.00001930,0.05950868,0.04297752,0.03117147,0.01187200$ ,-0.02151982,-0.05460116,-0.06405687,-0.02342953,0.02435069,-0.0064220 $2,-0.09161963,-0.23234081,-0.09881471,0.17851087,0.56430504,0.00225385$ $,-0.00294651,-0.00240623,-0.00033197,-0.00023486,-0.00034618,0.0000252$ $6,-0.00000040,-0.00009993,-0.00006294,-0.00008224,-0.00008175,-0.00003$ $613,-0.00026651,-0.00010229,-0.00047740,-0.00023229,-0.00002136,0.0003$ $9716,-0.00036569,-0.00092958,0.00007135,0.00003820,0.00002265,-0.00005$ $560,0.00004299,0.00010203,-0.00009301,-0.00001112,0.00006523,0.0000562$ $4,0.00013774,0.00007695,0.00012495,0.00005479,-0.00005877,-0.26397361$, $-0.09296070,0.01415629,0.00258898,-0.03614374,-0.06204651,-0.05216168$, $-0.01249455,0.02295149,0.04676659,0.03879145,0.03547368,-0.20098203,-0$ $.02227081,0.09063535,0.57137150,-0.00265016,0.00194780,-0.00423792,-0$. $00061097,0.00007919,0.00038616,-0.00011814,0.00005078,-0.00010674,0.00$ $010439,0.00003350,-0.00006336,0.00016522,0.00025558,0.00006770,-0.0000$ $5212,-0.00046714,0.00002968,0.00034992,-0.00008480,-0.00075563,0.00008$ $847,-0.00002399,0.00009963,-0.00003476,-0.00006232,-0.00004925,0.00004$ $871,0.00002567,0.00003402,0.00002530,-0.00003596,-0.00010603,-0.000013$ $01,-0.00005281,-0.00000249,-0.12050523,-0.13331655,-0.01330418,-0.0172$ $3358,-0.01222231,-0.02296211,-0.01249082,-0.00619630,0.00640878,0.0180$ $8977,0.01151393,-0.00950675,0.00645301,-0.08542950,-0.05101077,0.18146$ $362,0.34217164,-0.00185066,-0.00395842,-0.00012822,-0.00045822,0.00044$ $007,0.00008320,0.00029265,0.00013675,0.00028246,0.00026917,0.00011466$, $-0.00002660,0.00009403,0.00012185,0.00013937,-0.00027882,-0.00021011,0$ $.00077443,0.00041454,-0.00007586,-0.00039662,0.00001449,-0.00011338,-0$ $.00012389,0.00009192,0.00000136,-0.00008627,0.00004151,-0.00003375,-0$. $00013697,-0.00003056,0.00004802,-0.00009688,-0.00018582,-0.00006683,0$. 
$00000622,-0.03757026,-0.03946617,-0.10370737,-0.02653660,-0.00500159,0$ $.02453463,0.02296099,0.00641236,-0.01305646,-0.00339699,-0.02916595,-0$ $.04411773,0.14456941,-0.02373090,-0.24339377,-0.07719653,0.22357695,0$. $62447537,-0.00009148,0.00014445,-0.00035077,0.00012263,-0.00022637,0.0$ $0047301,-0.00015184,0.00014510,-0.00011828,-0.00029033,-0.00011293,0.0$ $0005494,0.00004856,-0.00018075,0.00009771,-0.00015806,0.00046161,0.000$ $08285,-0.00000615,0.00002267,0.00004938,0.00005086,-0.00002534,-0.0000$ $1663,-0.00001439,-0.00005986,-0.00003232,-0.00003168,-0.00001149,-0.00$ $005458,0.00002176,0.00002396,-0.00004522,0.00095937,0.00039439,-0.0001$ $8458,-0.08389549,0.00126980,0.05890370,-0.00179620,0.00038539,0.006845$ $23,0.00171163,-0.00138591,0.00045374,0.00021163,0.00125113,0.00149964$, $-0.00118760,-0.00515408,-0.00416781,-0.02429140,0.00326893,0.03369555$, $0.10963978,0.00037004,-0.00038744,0.00032455,0.00003106,0.00008452,-0$. $00012536,0.00007208,-0.00001928,0.00006226,0.00004686,0.00000499,-0.00$ $001748,-0.00001222,0.00000854,-0.00003646,-0.00002786,-0.00006407,0.00$ $000059,-0.00002290,0.00002075,0.00005875,-0.00001389,-0.00000005,-0.00$ $000216,0.00000989,0.00001276,0.00000644,0.00000093,0.00000226,0.000005$ $05,-0.00000592,0.00000038,0.00000222,-0.00013228,-0.00006615,0.0000273$ $7,0.00130776,-0.03064607,-0.01298355,0.01185501,0.00244667,-0.01539449$ ,-0.00415442,0.00172540,-0.00207058,0.00110061,0.00024203,0.00068894,-$0.00233177,0.00183538,-0.00300139,-0.00821284,0.00527793,0.00884766,-0$ $.00257618,0.02205614,0.00007185,0.00053876,0.00019501,-0.00019096,0.00$ $025338,-0.00055310,0.00019024,-0.00019790,0.00012079,0.00035478,0.0001$ $3431,-0.00009450,-0.00003174,0.00031447,-0.00010758,0.00031192,-0.0005$ $9855,-0.00012419,-0.00003606,0.00001557,0.00006460,-0.00006078,0.00002$ 047,0.00002029,0.00001843,0.00006735,0.00003989,0.00004890,0.00002225, $0.00006770,-0.00003561,-0.00003793,0.00004892,-0.00117279,-0.00047300$, $0.00021298,0.05897607,-0.01294729,-0.10472652,0.02838354,-0.00449989,-$ $0.03076293,-0.00474527,-0.00469996,0.00061017,0.00121853,0.00054595,-0$ $.00081201,0.00113120,-0.00032114,0.00293513,0.01213289,-0.00205842,-0$. $01067001,-0.09750872,0.02118543,0.14448205,-0.00035263,-0.00040096,-0$. $00028620,-0.00005647,-0.00012324,0.00014558,0.00000068,0.00009454,-0.0$ $0004576,-0.00006193,-0.00005923,0.00004003,-0.00000127,-0.00006222,0.0$ $0002363,-0.00010372,0.00013839,0.00007934,0.00002829,0.00000232,-0.000$ $05442,-0.00001581,0.00001040,-0.00000584,-0.00001175,-0.00001240,0.000$ $00064,-0.00001035,-0.00001138,-0.00002087,0.00000466,-0.00000886,0.000$ $00805,0.00017110,0.00005895,-0.00002091,-0.00410035,-0.00206301,0.0033$ $7068,-0.27866355,-0.12567675,-0.00509453,-0.01177626,-0.00927792,-0.00$ $273710,0.00094080,-0.00082977,0.00411521,0.00023148,0.00071902,0.00001$ $802,0.00222536,-0.00187144,-0.00036327,0.00049401,0.00147102,-0.000296$ $75,0.29134777,0.00020248,0.00015727,-0.00015824,0.00004138,0.00006847$, $0.00001657,-0.00004006,-0.00005101,0.00002498,-0.00003689,0.00006104,-$ $0.00003290,0.00008882,0.00001176,0.00001948,-0.00006344,-0.00005705,-0$ $.00006146,0.00000958,-0.00002212,0.00001255,0.00001129,-0.00000187,0.0$ $0000392,0.00000654,-0.00000443,-0.00001661,-0.00000431,0.00000956,0.00$ $001743,-0.00000761,-0.00000647,-0.00000080,-0.00000711,0.00002197,-0.0$ $0001332,-0.01549701,-0.00376374,0.00102893,-0.12455124,-0.10457302,-0$. $01129022,0.00341731,0.00497110,-0.00083069,-0.00101830,0.00501286,-0.0$ $0260155,0.00080708,-0.00076032,0.00000499,-0.00162536,0.00223543,-0.00$ 466703,0.00103193,-0.00141575,0.00148146,0.13620830,0.10115609,0.00084 $704,0.00041469,0.00011371,-0.00001243,0.00004948,-0.00021233,0.0000690$ 
$2,-0.00001294,0.00003891,0.00008196,-0.00001175,-0.00000263,-0.0000602$ $3,0.00007315,-0.00002186,0.00016781,-0.00012973,-0.00003947,-0.0000407$ $5,0.00001946,0.00004165,0.00000680,-0.00001588,0.00000383,0.00001009,0$ $.00001908,0.00001988,0.00001847,0.00000645,0.00000448,-0.00000055,0.00$ $002104,-0.00001201,-0.00014482,-0.00007607,0.00002755,-0.02185647,-0.0$ $1173094,0.00225772,-0.00297904,-0.01022081,-0.05192437,0.02110058,0.01$ $122722,0.00472589,0.00376085,-0.00278027,-0.00187980,0.00018349,0.0000$ $8843,-0.00117948,0.00009868,-0.00443360,-0.00287631,-0.00112141,0.0010$ $6444,0.00033731,0.00209283,0.01501592,0.05047332,0.00037030,0.00083845$ ,-0.00259627,-0.00021054,-0.00000969,0.00029900,-0.00004686,-0.0000105 $9,-0.00000884,0.00017366,0.00008440,-0.00003159,-0.00008657,0.00013689$ $,-0.00011706,0.00037975,0.00002324,0.00017017,-0.00014873,0.00030659,0$ $.00098665,-0.00006964,-0.00000686,-0.00002620,-0.00000254,-0.00003011$, $-0.00004502,0.00003130,-0.00000149,-0.00001760,-0.00000858,0.00001545$, $-0.00003518,-0.00028053,-0.00011930,0.00005100,-0.00037433,-0.00168739$ $, 0.00387057,0.00804798,0.01128084,0.01695165,-0.07251443,-0.04684142,-$ $0.05344968,-0.00272516,-0.01466059,-0.02137834,-0.00389984,-0.00432535$ $, 0.00141750,-0.00099705,0.00029912,0.00047098,-0.00027214,0.00040688,0$ $.00112150,0.00008760,0.00155086,-0.00111634,0.07253515,0.00230123,-0.0$ $0240465,0.00085095,0.00040383,0.00051521,-0.00017588,0.00015850,-0.000$ $19738,0.00006275,0.00003704,-0.00000526,0.00000402,0.00005581,-0.00013$ $132,0.00008621,-0.00057948,0.00008504,0.00027281,-0.00023959,0.0004421$ $7,0.00052352,0.00005848,-0.00004249,-0.00000257,0.00003940,0.00002742$, $-0.00000785,-0.00002619,-0.00003184,-0.00006054,-0.00005360,-0.0001038$ $5,0.00002424,-0.00005687,-0.00001667,0.00000613,-0.00155564,0.00530267$ $,-0.00177949,-0.00181020,0.00209940,-0.00108381,-0.04712352,-0.1285640$ $2,-0.12872483,-0.00211560,-0.00395301,-0.01245839,-0.00475246,0.003139$ $94,-0.00112757,0.00024522,-0.00052735,0.00082865,-0.00006853,0.0000378$ $6,-0.00005943,0.00166119,-0.00244690,0.00162784,0.05320525,0.12743791$, $0.00014940,0.00223905,0.00004095,-0.00024141,-0.00008167,0.00026701,-0$ $.00000139,0.00012011,-0.00004514,-0.00012029,-0.00004093,0.00001131,0$. $00003983,0.00011188,-0.00007202,0.00035660,-0.00000027,0.00010656,-0.0$ $0003743,0.00000543,0.00033136,-0.00007085,0.00000609,-0.00003059,-0.00$ $000536,-0.00003118,-0.00003448,0.00002700,0.00000543,-0.00000909,0.000$ $00230,0.00001184,-0.00003057,0.00016806,0.00006545,-0.00002810,0.00411$ $781,-0.00165437,0.00001888,-0.00763134,-0.01351734,-0.01454289,-0.0539$ $7719,-0.12899118,-0.22713429,0.00217685,-0.00054447,0.00066098,0.00061$ $477,-0.00153343,0.00268107,0.00037021,0.00077755,0.00001606,0.00022876$ ,-0.00051099,-0.00107253,-0.00090952,0.00173247,-0.00003678,0.05484328 $, 0.14166494,0.23901717,-0.00051686,0.00132534,0.00003701,0.00001497,0$. $00009821,0.00030746,0.00003847,0.00006554,-0.00009588,0.00012641,-0.00$ $020342,0.00009285,-0.00011305,0.00022113,0.00016655,0.00024282,0.00010$ $812,-0.00008419,-0.00006507,-0.00000319,-0.00008943,-0.00002716,0.0000$ $0541,-0.00000943,-0.00002823,0.00004458,0.00004679,0.00002696,0.000025$ $14,0.00000348,-0.00006016,-0.00002218,-0.00011262,0.00005154,-0.000064$ $75,0.00004855,0.00107581,-0.00108707,0.00385405,0.00022697,0.00075112$, $0.00004392,0.00230789,-0.00227096,-0.00037760,-0.00330198,-0.00264770$, $0.00138566,-0.27207645,-0.12280383,-0.00706818,-0.01263115,-0.00970950$ $,-0.00244613,-0.00080940,-0.00026708,0.00030784,-0.00018322,0.00020440$ ,-0.00013478,-0.00012957,-0.00009476,0.00013295,0.28597601,0.00084888, $-0.00176653,0.00169412,0.00025538,-0.00010616,-0.00031846,0.00012819$,- 
$0.00004744,0.00040979,-0.00019629,0.00040034,-0.00023817,0.00033702,-0$ $.00017561,-0.00020647,-0.00033503,0.00014698,0.00030827,-0.00001530,-0$ $.00007583,0.00015928,-0.00000256,-0.00011263,-0.00008380,0.00007956,-0$ $.00000875,-0.00001305,-0.00001309,-0.00000282,0.00008961,0.00005028,-0$ $.00003371,0.00010734,0.00017475,0.00025669,-0.00011723,-0.00124611,0.0$ 0525663,-0.00262078,0.00079510,-0.00076746,0.00007940,-0.00192053,0.00 $267771,-0.00516556,-0.01533553,-0.00389230,0.00088629,-0.12269803,-0.1$ $0568130,-0.01291660,0.00334687,0.00502998,-0.00122384,0.00022222,0.000$ $06799,-0.00016383,0.00019654,-0.00047374,0.00029306,-0.00078382,-0.000$ $20381,-0.00000938,0.13458983,0.10233889,-0.00085849,0.00124171,0.00004$ $801,-0.00024302,-0.00021266,0.00016872,0.00028158,0.00010646,-0.000266$ $01,0.00010656,-0.00023064,0.00009116,0.00012445,0.00026602,0.00001385$, $-0.00006989,-0.00006910,-0.00031371,-0.00011263,0.00014762,0.00023154$, $-0.00001324,0.00011618,0.00001155,0.00000053,0.00003228,0.00003559,0.0$ $0004368,0.00004121,-0.00002558,0.00001222,-0.00003141,-0.00003354,0.00$ $049810,0.00011048,-0.00003559,0.00355579,-0.00277161,-0.00129859,0.000$ $12642,0.00012119,-0.00110479,0.00028053,-0.00483306,-0.00294171,-0.022$ $43948,-0.01116413,0.00374151,-0.00686744,-0.01281669,-0.05428207,0.022$ $06882,0.01117700,0.00401467,0.00122655,0.00030083,-0.00055718,-0.00014$ $953,0.00028557,-0.00005088,-0.00116113,-0.00066391,0.00005359,0.005008$ $05,0.01707713,0.05247630,0.00026333,0.00039764,0.00069425,0.00011313,-$ $0.00006860,0.00000613,-0.00002725,0.00002169,0.00000377,-0.00006754,-0$ $.00004488,-0.00000092,-0.00003956,-0.00003123,-0.00001795,0.00011349,0$ $.00016690,-0.00009348,-0.00014121,0.00010216,0.00022001,-0.00000978,0$. $00001933,0.00000820,-0.00001143,0.00000329,0.00001215,-0.00000625,-0.0$ $0000210,0.00001093,-0.00000512,-0.00000514,0.00000699,0.00013868,0.000$ $04621,-0.00000196,-0.00304587,-0.01503659,-0.02200595,-0.00380862,-0.0$ $0386929,0.00142314,-0.00098502,0.00031315,0.00055786,-0.00088199,-0.00$ $185930,0.00421752,0.00853801,0.01097932,0.01629092,-0.06969352,-0.0450$ $3850,-0.05268687,0.00045597,0.00098880,-0.00107202,-0.00007310,-0.0007$ $3941,-0.00106467,-0.00004284,0.00025676,-0.00014054,-0.00007285,0.0016$ $2454,-0.00102139,0.06938476,-0.00020826,0.00010571,0.00000558,0.000107$ $93,0.00004299,-0.00000883,-0.00001181,-0.00006136,-0.00000341,0.000005$ $88,0.00000944,-0.00002560,0.00002079,0.00002685,0.00004816,-0.00006528$ ,-0.00008193,0.00000679,0.00006292,-0.00007287,0.00001284,-0.00000262, $-0.00002061,-0.00000764,0.00000146,0.00000498,-0.00000390,-0.00000124$, $0.00000076,0.00000645,0.00000404,-0.00000359,0.00001138,-0.00005398,0$. $00001496,-0.00000571,-0.00163916,-0.00386734,-0.01230335,-0.00407085,0$ $.00280972,-0.00088369,0.00020625,-0.00048031,0.00081127,-0.00164115,0$. $00540529,-0.00187532,-0.00157924,0.00273753,-0.00083971,-0.04622801,-0$ $.13024254,-0.13283501,0.00147689,-0.00128130,0.00112522,-0.00013121,-0$ $.00009995,-0.00056636,0.00024060,-0.00046898,0.00020255,0.00165656,-0$. $00255110,0.00182341,0.05180265,0.12809849,-0.00044308,-0.00056976,-0.0$ $0044079,-0.00020506,0.00006380,-0.00005237,0.00004196,0.00000924,0.000$ $05530,0.00009431,0.00002260,-0.00002717,-0.00002138,0.00005314,-0.0000$ $2060,0.00001330,-0.00011878,0.00007202,0.00014636,-0.00002438,-0.00029$ $640,0.00000284,-0.00001049,-0.00000631,0.00000775,0.00000395,-0.000005$ $96,0.00001351,-0.00000233,-0.00000692,-0.00001166,0.00000207,-0.000027$ $96,-0.00019002,-0.00010090,0.00003646,0.00315097,0.00042059,0.00131046$ $, 0.00104452,-0.00107489,0.00232919,0.00035791,0.00070994,-0.00002358,0$ $.00462585,-0.00166875,-0.00029347,-0.00729203,-0.01276693,-0.01306451$, 
$-0.05491895,-0.13396473,-0.23551872,-0.00015554,0.00158895,0.00050387$, $0.00007758,0.00001137,0.00021568,-0.00017093,0.00018718,-0.00018472,-0$ $.00096143,0.00185369,0.00003406,0.05466660,0.14529336,0.24543337,-0.00$ $205421,-0.00072846,-0.00045282,-0.00021016,-0.00152784,0.00127733,-0.0$ $0083709,0.00428233,-0.00219479,-0.07739583,0.02188417,-0.01411738,0.00$ $266449,-0.00823740,0.00431223,-0.00498856,0.00256438,-0.00094299,-0.00$ $065859,-0.00052631,0.00023751,-0.00001183,-0.00014597,0.00005953,-0.00$ $354053,-0.00228178,0.00108710,0.00046523,0.00010642,-0.00007026,-0.000$ $68819,-0.00039567,0.00014416,-0.35975691,0.15254657,-0.08742318,0.0004$ $2875,-0.00012188,-0.00064850,-0.00020424,0.00005198,0.00024110,0.00008$ $988,0.00029625,0.00055398,0.00077322,-0.00043845,-0.00082177,-0.000449$ $09,-0.00020988,0.00017373,-0.00005949,-0.00001412,0.00008390,-0.000486$ 40,0.00006573,0.00059813,-0.00008711,0.00001109,0.00006580,0.00012896, $0.00004283,-0.00007525,-0.00005854,-0.00005438,-0.00027261,-0.00007225$ $, 0.00003563,0.00008936,0.42805184,0.00085568,0.00030611,0.00028838,0.0$ $0138211,0.00363759,-0.00209176,-0.00717042,-0.00927120,0.00518353,0.01$ $877116,0.01813308,0.00012823,-0.00310355,0.00035160,0.00146731,0.00063$ $625,0.00008418,-0.00050738,0.00031922,0.00046841,-0.00018675,0.0000061$ $9,0.00000326,0.00003067,-0.00276110,0.00198474,-0.00134758,-0.00098928$ $, 0.00042302,-0.00016862,-0.00022230,-0.00011579,-0.00023005,0.13028840$ ,-0.20316533,0.07176238,-0.00011303,0.00000409,0.00013221,0.00005950,$0.00000682,-0.00000335,-0.00001843,-0.00007728,-0.00017998,-0.00025013$ $, 0.00030403,0.00004061,0.00009485,0.00011639,-0.00002523,0.00001422,-0$ $.00000340,-0.00005846,0.00010287,-0.00000980,-0.00013111,0.00002892,-0$ $.00001165,-0.00001575,-0.00003941,-0.00000670,0.00003292,0.00004231,-0$ $.00005109,0.00007625,0.00002552,-0.00001837,-0.00002008,-0.20187340,0$. 24402580,-0.00052242,-0.00023440,-0.00023206,-0.00072037,-0.00198891,0 $.00110677,0.00370478,0.00557675,-0.00162436,-0.01252632,0.00013574,0.0$ $1675641,0.00158975,0.00135274,0.00286111,-0.00046989,-0.00022480,-0.00$ $027584,-0.00020243,-0.00013845,0.00006428,-0.00000627,0.00002788,0.000$ $04751,0.00132581,-0.00134941,0.00029991,0.00052910,-0.00011715,0.00024$ $935,0.00011238,-0.00025572,-0.00048096,-0.07579174,0.07243758,-0.11169$ $613,0.00005903,-0.00000680,-0.00007506,-0.00003584,0.00001262,-0.00000$ $311,0.00000553,0.00004279,0.00012888,0.00023046,-0.00025936,-0.0000456$ $7,-0.00010144,-0.00009322,0.00002932,0.00000682,0.00001570,0.00004081$, $-0.00006099,0.00000866,0.00008536,-0.00001779,0.00000772,0.00001151,0$. $00002984,0.00000982,-0.00001734,-0.00003849,0.00003153,-0.00007016,-0$. $00002926,0.00001286,0.00002001,0.11620326,-0.11843545,0.09156889,-0.00$ $053896,-0.00037227,0.00005882,-0.00031499,-0.00295463,0.00169084,-0.00$ $531389,0.00313829,-0.00188969,-0.00055413,-0.04136506,0.02094746,-0.00$ $867726,-0.00103100,0.00029697,0.00236026,-0.00057652,0.00049868,-0.000$ $10694,-0.00009676,0.00006756,-0.00063445,-0.00027057,0.00012415,-0.000$ $00812,-0.00063146,0.00029925,-0.00283519,-0.00331206,0.00163510,-0.000$ $08528,0.00006026,-0.00004462,-0.13265056,-0.04758402,0.02287879,0.0001$ $8919,-0.00007261,-0.00032135,-0.00009539,0.00004723,0.00014831,0.00004$ $518,0.00013327,0.00020528,0.00035651,-0.00017114,-0.00041527,-0.000186$ $10,-0.00006178,0.00010551,-0.00003426,-0.00001410,0.00000162,-0.000233$ $62,0.00003069,0.00028488,-0.00003422,-0.00000030,0.00002809,0.00005464$ $, 0.00001090,-0.00001724,-0.00001415,-0.00004849,-0.00012441,-0.0000232$ $0,0.00001120,0.00003727,0.01895665,0.06391384,-0.03327348,0.13037830,-$ $0.00160311,-0.00145162,-0.00016127,-0.00131081,-0.00340012,0.00195385$, 
$-0.00202671,0.00687996,-0.00173197,-0.03829619,-0.04396278,0.03053719$, $0.01041745,-0.00293355,0.00247970,-0.00375944,0.00188782,-0.00062277,-$ $0.00091807,-0.00008835,-0.00000642,-0.00041099,-0.00019969,-0.00027720$ $, 0.00049303,0.00079915,-0.00032193,-0.00284464,0.00143435,-0.00105149$, $-0.00008237,0.00005740,-0.00000928,-0.02524826,-0.38653434,0.16228176$, $0.00036260,-0.00009818,-0.00051125,-0.00014371,-0.00002081,0.00019233$, $0.00003083,0.00032168,0.00046765,0.00033252,0.00025090,-0.00101506,-0$. $00046813,-0.00008936,0.00006342,-0.00005947,0.00004354,0.00007897,-0.0$ $0039987,0.00006632,0.00048385,-0.00006120,-0.00001751,0.00007384,0.000$ $12744,0.00001662,-0.00006051,0.00003676,-0.00022495,-0.00013014,-0.000$ $05293,-0.00000646,0.00010214,0.03466817,-0.05710487,0.04343128,0.03121$ $710,0.48437487,0.00079047,0.00069689,0.00001038,0.00068422,0.00140197$, $-0.00146491,0.00077411,-0.00164805,0.00431128,0.01927382,0.03048921,0$. $00201028,-0.00560936,0.00208374,-0.00012175,0.00198405,-0.00098507,0.0$ $0028596,0.00055311,-0.00003177,0.00006944,0.00021777,-0.00022042,-0.00$ 045080,-0.00024621,-0.00034665,0.00034656,0.00137348,-0.00106180,0.000 $14500,0.00003901,0.00000276,0.00006679,0.01139252,0.16167949,-0.155420$ $08,-0.00019543,0.00004975,0.00026677,0.00008142,0.00000228,-0.00009794$ ,-0.00002978,-0.00015193,-0.00022575,-0.00016794,-0.00005739,0.0004244 $0,0.00018951,0.00003449,-0.00002757,0.00004701,-0.00001121,-0.00003141$ $, 0.00020581,-0.00002971,-0.00024352,0.00003092,0.00000948,-0.00003551$, $-0.00005797,-0.00000479,0.00003360,-0.00001701,0.00008768,0.00005926,0$ $.00001653,-0.00000133,-0.00004094,-0.01810757,0.04425906,0.00125058,-0$ $.01322249,-0.23624668,0.14887988 \backslash 1-0.00000593,0.00000248,0.00001951,-0$ $.00000564,0.00000071,-0.00000351,-0.00000201,-0.00000196,-0.00000184,0$ $.00000408,0.00000047,-0.00000347,-0.00000066,-0.00000053,-0.00000135,0$ $.00000223,0.00000057,-0.00000245,-0.00000114,-0.00000197,-0.00000466,0$ $.00000188,0.00000054,0.00000149,-0.00000074,-0.00000169,-0.00000086,-0$ $.00000195,-0.00000051,-0.00000076,-0.00000114,-0.00000057,-0.00000044$, $-0.00000967,-0.00000867,0.00000277,0.00000040,0.00000429,0.00000495,0$. $00000229,0.00000131,-0.00000292,-0.00000436,0.00000212,0.00000351,0.00$ $000638,-0.00000609,-0.00000583,0.00000297,-0.00000083,0.00000011,0.000$ $00030,0.00000154,-0.00000043,0.00000300,0.00000099,0.00000123,-0.00000$ $022,0.00000162,0.00000470,-0.00000044,0.00000092,0.00000237,0.00000118$ $, 0.00000119,-0.00000081,0.00000278,0.00000163,0.00000027,0.00000387,-0$ .00000225,-0.00000352,0.00000254,0.00000471,-0.00000807III@ 


\section{Phenolate addition}

Chlorophenyl + Phenolate

ortho-Complex

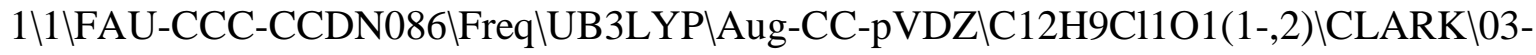
Mar-2015\0\\#N Geom=AllCheck Guess=TCheck SCRF=Check GenChk UB3LYP/Aug -CC-pVDZ Freq IPre-addition complex p-Cl-Ph-radical + phenolate (ortho )\-1,2\C,-2.1241701289,0.0085073485,-1.0536041687\C,-2.4062199482,1.1 003079531,-0.1283896585\C,-3.1049049995,0.7076393944,1.0780285265\C,-3 $.5495783905,-0.5894214561,1.2832111844 \backslash \mathrm{C},-3.3369162778,-1.60423051,0.3$ $200717199 \backslash \mathrm{C},-2.6446875425,-1.2826425008,-0.846959666 \backslash \mathrm{H},-1.6997427707,0$ $.2816586979,-2.0187215226 \backslash \mathrm{O},-2.055788632,2.2969663557,-0.3626083688 \mathrm{lH}$, $-3.2985086077,1.4894746597,1.8159780362 \backslash \mathrm{H},-3.7151920135,-2.6142528112$, $0.4871905421 \backslash \mathrm{H},-2.4779652472,-2.0510254073,-1.6073910183 \backslash \mathrm{H},-4.08939938$ $05,-0.8322781027,2.2038729975 \backslash C, 2.9925443574,-0.0710570929,0.095955954$ IC,2.272418005,1.1235262918,0.0301167081\C,0.8926557955,1.0636339295,$0.232738214 \backslash \mathrm{C}, 0.281175258,-0.1697243615,-0.4227225132 \backslash \mathrm{C}, 1.0059309245,-$ $1.3527149972,-0.3489260574 \backslash \mathrm{C}, 2.3879150991,-1.3146813803,-0.085493535 \backslash \mathrm{C}$ $1,4.7472239538,-0.007386134,0.4274258363 \backslash \mathrm{H}, 2.7789646162,2.0772662124,0$ $.1841645433 \backslash \mathrm{H}, 0.2906355341,1.9770270988,-0.2779509001 \backslash \mathrm{H}, 0.517860214,-2$ $.3221963122,-0.4862301881 \backslash \mathrm{H}, 2.9805381773,-2.2282754447,-0.0206041138 \backslash \backslash$ Version=EM64L-G09RevA.02 IState $=2-A \backslash H F=-998.1698846 \backslash S 2=0.763029 \backslash S 2-1=0$. $\backslash \mathrm{S} 2 \mathrm{~A}=0.750107 \backslash \mathrm{RMSD}=4.175 \mathrm{e}-09 \backslash \mathrm{RMSF}=9.069 \mathrm{e}-06 \backslash$ ZeroPoint $=0.1684022 \backslash$ Therma $\mathrm{l}=0.1803291 \backslash \mathrm{ZPE}=105.6739588 \backslash \mathrm{Dipole}=1.8246389,-1.4879086,0.1513893 \backslash \mathrm{Dipo}$ leDeriv $=-0.5745947,0.2524987,-0.0742152,-0.2105005,-0.4264787,0.094189$ $6,0.0729387,-0.2882765,0.0154824,0.0898546,0.4361631,-0.0977488,0.0342$ $888,1.5336211,-0.3166781,-0.0781753,-0.3266523,0.1123917,-0.0545683,-0$ $.26242,-0.0675099,0.2237905,-0.2436194,-0.0405176,-0.234954,0.4989485$, $-0.091282,-0.1552741,0.0774426,-0.0877296,-0.5231114,0.3554823,0.23246$ $75,-0.3554414,0.108479,0.1363401,-0.5762862,0.0358403,0.0283721,-0.059$ $0018,-0.1476677,-0.0173323,0.6778593,-0.0333282,-0.3180727,0.2819112,0$ $.0553358,-0.2397847,0.6446267,0.1479193,-0.0595478,-0.4609817,-0.09687$ $21,0.1000875,0.1737613,-0.044691,0.0766069,-0.0384125,0.0249617,0.0399$ $738,0.0409577,0.0291428,-0.0673512,-0.4022959,-0.3304139,0.0352876,0.1$ $687168,-1.7241185,0.2566446,-0.0327264,0.2892076,-0.4407129,0.0925379$, $0.007221,0.0587329,0.0536752,-0.0690119,-0.1242101,0.0868918,-0.102162$ $8,-0.0391437,0.0877123,-0.0935417,0.059018,-0.1450057,-0.1649844,0.019$ $1387,0.0818786,0.0216512,0.0544549,0.0940691,0.0251971,0.0660832,-0.03$ $8108,-0.0787464,-0.140515,0.0008908,-0.1251899,-0.0510949,-0.0013859,-$ $0.0467181,0.1444787,-0.0791734,0.0422966,0.0521255,0.1849452,0.0520758$ ,-0.131807,1.2267934,0.0303237,0.198176,0.0630411,0.031823,0.0105401,0 $.2464837,0.0113934,-0.0436343,-0.210294,0.0917842,-0.0043738,-0.249544$ $3,0.0895479,0.02159,-0.0397543,0.034424,-0.1011681,-0.6754837,-0.00059$ $23,0.0538661,0.3707,-0.0324001,-0.0089681,-0.0959727,0.0203022,-0.0739$ $554,1.5561553,-0.1793538,-0.0162352,0.1043432,-0.1692998,0.0023476,0.3$ $236238,-0.0420988,-0.1237776,-0.5811817,0.0871787,0.0544833,-0.4546402$ $, 0.1944466,0.0520315,-0.1202393,0.0148347,-0.0993542,-0.2567774,-0.093$ $4673,-0.00636,0.1989383,-0.0012688,-0.0101615,-0.0252878,-0.0136803,-0$ $.1174935,-1.0978487,-0.0248351,-0.1163473,-0.0331333,-0.1381424,-0.004$ 
$5241,-0.1801958,-0.0047343,-0.1507454,-0.0996983,-0.0785021,-0.0147665$ ,-0.1293246,-0.0598352,-0.0205211,-0.0478662,-0.0282049,0.1174682,0.15 $47167,0.0488014,-0.0083919,-0.1046291,0.0295324,-0.0023037,-0.0039059$, $-0.005777,0.0960214,0.0513437,-0.1038123,-0.0340748,0.0647328,-0.12644$ $09,-0.037243,-0.0021323,-0.0264177,0.0950647,-0.1231668,0.110561,-0.00$ $75671,0.1377314,-0.0676167,0.0014733,-0.0388364,0.0129356,0.1222818 \backslash \mathrm{Po}$ lar $=304.637464,3.6232819,189.3844144,-11.2944706,4.0836161,136.00537 \backslash \mathrm{P}$ $\mathrm{G}=\mathrm{C} 01[\mathrm{X}(\mathrm{C} 12 \mathrm{H} 9 \mathrm{Cl1O} 1)] \mathrm{NImag}=1 \backslash 0.19000077,0.12390995,0.60110796,-0.209$ $62300,-0.01674354,0.56895796,-0.07611381,0.01714917,0.04232981,0.29161$ $928,0.00967643,-0.14023775,-0.06429153,0.11088888,0.71064060,0.0354725$ $6,-0.04443731,-0.16982286,-0.15252307,-0.01462645,0.47532711,0.0029565$ $9,0.01369619,0.01254784,-0.10722686,-0.01419761,0.06192033,0.23021879$, $0.00841705,0.03137374,-0.01366964,-0.00203141,-0.10568610,-0.01391663$, $0.10346918,0.70774147,0.01296261,-0.01313393,-0.02133367,0.06859598,0$. $01034559,-0.19978155,-0.18482317,0.08519382,0.51001789,-0.00170620,-0$. $00022356,0.00124352,-0.00612535,-0.01549937,0.00560378,-0.09639051,-0$. $10078736,0.03343962,0.27280749,-0.00649602,-0.03444651,-0.00324429,-0$. $02831978,-0.00340214,0.05692019,-0.07577688,-0.33748504,0.00791090,0.1$ $2091832,0.63850265,-0.00786169,-0.01722687,-0.00648114,0.00094023,0.03$ $048622,0.01509517,0.04435092,0.05050168,-0.11097402,-0.21968273,0.0304$ $9099,0.57341591,-0.01139149,-0.03112325,0.01118126,-0.01000342,0.00682$ $939,0.01404024,0.01732367,0.00532109,-0.01168574,-0.07673460,-0.002084$ $40,0.03289960,0.25968159,-0.01918180,-0.00316629,0.02723644,0.00864196$ $, 0.00339360,-0.01337381,-0.01052197,-0.04869495,-0.01176895,0.02223693$ ,-0.20504964,-0.12386657,0.14122085,0.66129019,0.02498510,0.05577982,0 $.00529151,0.01110346,-0.01164419,-0.04025846,-0.02048833,-0.04012694,0$ $.02488005,0.04658676,-0.07592699,-0.18934258,-0.21217283,0.03379725,0$. $53468557,-0.08226688,-0.07676787,0.03996450,0.00900101,-0.00239641,-0$. $02464978,-0.00996674,-0.01484710,0.00774834,0.00213608,0.02608061,0.02$ $597651,-0.12823376,-0.04897359,0.11285292,0.27181859,-0.07740988,-0.29$ $242904,0.04259076,-0.00350704,-0.03449708,-0.02256088,-0.00665038,-0.0$ $1610957,0.00319167,0.00918750,0.03004397,-0.00483797,-0.02580242,-0.11$ $905652,0.01476177,0.10664342,0.62376556,0.02580677,-0.00367183,-0.1136$ $4486,-0.01682883,-0.00375093,0.03424177,0.01219272,0.02128698,-0.01223$ $955,0.01579510,-0.03802483,-0.04374079,0.12509533,0.06014618,-0.264623$ $63,-0.21392548,0.08407419,0.58585184,-0.06817218,-0.02966227,0.1051092$ $5,-0.00062307,-0.00287481,0.00703381,0.00264743,0.00116556,0.00231696$, $0.00000001,0.00137748,0.00036038,0.00556112,-0.00338661,-0.00106188,-0$ $.00643972,-0.00388094,0.01109922,0.07910704,-0.03330209,-0.07002005,0$. $05976982,0.00797445,0.00831712,-0.02041396,0.00030913,-0.00315969,-0.0$ $0301006,0.00022640,-0.00093787,-0.00035658,-0.00237293,-0.00440771,-0$. $00072379,-0.01016195,-0.00221598,0.02599188,0.03536358,0.06946776,0.10$ $947949,0.05905043,-0.27552810,0.00905447,0.01205132,-0.01408386,0.0017$ $8984,-0.00405841,-0.00133283,-0.00058911,-0.00119532,0.00000371,0.0011$ $3863,0.00023180,0.00291110,-0.00003016,-0.00307356,0.00023374,-0.11581$ $652,-0.06438152,0.28717499,0.01828544,-0.00518465,-0.00462414,-0.09866$ $662,-0.10431907,0.02969477,0.00021792,-0.03580317,0.02024278,0.0030066$ $2,0.00699648,0.00629644,0.00065499,-0.00318841,-0.00367509,0.00125344$, $0.00024968,0.00323269,-0.00214835,0.00033826,-0.00220014,0.08240974,-0$ $.00503411,-0.07516360,-0.01219898,-0.10178899,-0.43233816,0.06574267,-$ $0.02779486,-0.03328661,0.03576533,0.00009942,0.01000575,0.00128448,-0$. 
$00218306,-0.00734109,0.00129015,0.00282461,0.00413788,-0.00926274,-0.0$ $0075420,0.00232237,-0.00033045,0.13586235,0.53151231,-0.00656866,-0.02$ $614966,0.01855142,0.02993372,0.06530027,-0.09840715,0.02311942,0.05117$ $150,-0.00626994,0.00345530,-0.01131199,-0.00707859,-0.00323419,0.00372$ $077,0.00748223,0.00715560,0.00062128,-0.00577452,-0.00194742,0.0024874$ $2,0.00277111,-0.04588772,-0.08567636,0.08780478,0.00173253,-0.00251922$ $, 0.00391292,0.00175167,0.01270778,0.01099869,-0.04440842,0.03184354,0$. $04331828,0.00319762,-0.00825761,-0.00755852,0.00500845,-0.00299813,0.0$ $0325457,-0.00044595,-0.00006433,-0.00026781,-0.00066584,0.00075302,0.0$ $0044221,-0.00154693,0.00004508,-0.00347368,0.03858308,-0.00120373,0.00$ $097219,0.00079807,-0.00300087,0.00410530,0.00579777,0.03131805,-0.1915$ $6864,-0.13556464,0.00700241,-0.01517811,-0.02115763,-0.00371454,-0.003$ $06265,0.00216198,-0.00018352,-0.00001877,0.00057966,0.00003182,0.00023$ $995,0.00029893,0.00119782,0.00182687,-0.00029824,-0.03249136,0.2030539$ $3,0.00442170,0.00203085,-0.00354092,0.00327635,-0.02431701,-0.01152290$ $, 0.04321869,-0.13425572,-0.17781798,-0.00045142,0.00551439,0.00502036$, $0.00258274,0.00091481,0.00319150,0.00029217,0.00108408,-0.00047000,0.0$ $0010469,-0.00110392,-0.00112211,-0.00254080,0.00209018,0.00253314,-0.0$ $4798872,0.14760344,0.18362086,0.00316697,-0.00048301,0.00536254,-0.000$ $38197,0.00015598,0.00011706,0.00344974,-0.00011048,0.00187917,0.001834$ $80,0.00589139,-0.00066142,-0.06505945,-0.09505805,0.02610047,-0.003806$ $99,-0.01444170,-0.00027475,0.00032016,-0.00113985,0.00022812,-0.000248$ $66,0.00007755,-0.00046744,0.00067429,0.00000361,0.00032211,0.06469546$, $-0.00142203,0.00209246,0.00100021,0.00043883,0.00061488,-0.00044810,0$. $00096645,-0.00041683,-0.00468983,-0.00860133,-0.01651925,0.00771116,-0$ $.09513709,-0.28866974,0.03563774,-0.00013654,-0.00443399,-0.00215351,-$ $0.00031582,-0.00025599,-0.00015318,-0.00005017,-0.00023167,0.00032027$, $-0.00056640,-0.00095256,0.00019557,0.10294281,0.30747628,0.00416292,-0$ $.00047129,-0.00272582,0.00017884,-0.00058958,-0.00112329,0.00261244,-0$ $.00264344,-0.00207819,-0.00887859,-0.01978643,0.00609778,0.02634866,0$. $03529896,-0.05588064,0.00776266,0.02533654,0.00029740,0.00074482,0.000$ $98097,-0.00005010,-0.00035331,0.00037028,0.00034891,0.00001943,-0.0011$ $2854,0.00033761,-0.02914994,-0.03841337,0.05456939,0.00487425,-0.00917$ 266,-0.00826692,0.00431598,-0.00222107,0.00352793,-0.00046562,-0.00024 $447,-0.00019922,0.00338692,-0.00267926,0.00465098,0.00115892,0.0118480$ $8,0.00997068,-0.04519165,0.02420870,0.03746820,-0.00243285,0.00088128$, $-0.00126788,-0.00054007,-0.00064421,-0.00072893,-0.00065796,0.00021647$ $,-0.00041301,-0.00276678,0.00076081,-0.00198490,0.03822412,0.00572188$, $-0.01661687,-0.02251237,-0.00480674,-0.00099819,0.00318744,0.00000891$, $-0.00004869,0.00026752,-0.00172092,0.00152282,0.00045112,-0.00212339,0$ $.00661632,0.00601872,0.02345343,-0.18608301,-0.13356870,0.00096736,0.0$ $0083839,0.00099204,0.00054469,-0.00239828,-0.00104356,0.00025361,-0.00$ $010243,0.00002092,0.00138883,0.00063380,0.00008413,-0.02387511,0.19635$ $532,-0.00010669,0.00340280,0.00497327,0.00272628,0.00137545,0.00268139$ ,-0.00018596,0.00083232,0.00037096,0.00521219,0.00231235,-0.00267965,0 $.00182184,-0.02154987,-0.01284614,0.03778676,-0.13203274,-0.18096652,-$ $0.00178134,0.00003931,0.00014095,-0.00044667,-0.00029893,-0.00022924,-$ $0.00038871,-0.00003464,-0.00021877,-0.00179259,0.00092582,0.00008367,-$ $0.04348123,0.14585409,0.18960880,-0.00047991,0.00007739,-0.00079771,0$. $00746380,-0.00285834,0.00139269,-0.00106939,-0.00082499,0.01077101,-0$. $10454985,-0.03342238,0.11868931,-0.00005024,-0.00291633,0.00384624,0.0$ 
$0429177,0.00074926,0.00191978,-0.00048892,0.00006003,-0.00022363,-0.00$ $159837,-0.00063688,0.00045822,-0.00331722,0.00083280,-0.00252647,-0.00$ $198636,0.00108372,-0.00138792,0.00002104,-0.00012302,0.00040430,0.1018$ $0224,-0.00017461,-0.00043827,0.00026980,-0.00201013,-0.00371441,-0.002$ $83733,-0.01515862,-0.00212395,0.02600537,-0.03322518,-0.06795336,0.046$ $75038,0.01104591,0.00842580,-0.01830445,-0.00037019,-0.00262524,-0.003$ $33509,0.00016823,0.00003812,0.00013248,-0.00064819,-0.00107897,0.00174$ $044,0.00133941,0.00065870,0.00016512,0.00054454,0.00070527,0.00093884$, $0.00055522,0.00021790,-0.00107251,0.03793797,0.06785462,-0.00050683,-0$ $.00024328,0.00045160,0.00233147,-0.00040210,0.00466987,0.00323316,-0.0$ $0110955,-0.00252502,0.11876652,0.04642187,-0.24040749,0.01092724,0.009$ $05921,-0.01388560,0.00141271,-0.00545822,-0.00083321,-0.00023886,0.000$ $13047,-0.00020614,-0.00002845,0.00083886,-0.00099077,-0.00226804,0.000$ $90841,-0.00000039,-0.00160822,0.00006398,0.00020161,0.00072581,0.00019$ $381,-0.00102281,-0.13255761,-0.05044369,0.25454769,0.00076155,0.000249$ $23,-0.00025633,0.00000822,0.00031417,-0.00002920,-0.00015775,-0.000227$ $52,0.00036969,0.00004366,0.00067385,0.00032349,0.00048788,0.00008398,-$ $0.00082429,-0.00038012,-0.00072433,0.00036443,-0.00023074,0.00000036,0$ $.00008743,0.00005464,-0.00056997,0.00014699,-0.00000828,-0.00006746,-0$ $.00005074,-0.00001132,0.00009107,-0.00004399,-0.00006822,0.00007927,0$. $00007657,0.00005821,0.00005851,-0.00009477,0.45402523,-0.00014373,0.00$ $000838,0.00032622,-0.00047084,-0.00064737,0.00008909,0.00014441,0.0001$ $1030,-0.00001274,-0.00000805,-0.00015448,-0.00003892,-0.00003037,0.000$ $26011,-0.00012327,-0.00004006,-0.00010510,0.00002131,0.00005570,-0.000$ $02865,-0.00007030,0.00052345,0.00030647,-0.00007687,-0.00002293,0.0000$ $0242,-0.00002305,0.00002751,0.00001720,-0.00001050,0.00008664,0.000063$ $59,0.00005654,0.00002292,0.00001573,-0.00001156,-0.01005340,0.65623083$ $, 0.00013284,-0.00000936,0.00002829,0.00007711,0.00001468,0.00004410,-0$ $.00009407,-0.00004421,0.00001374,0.00000590,0.00017865,0.00007552,0.00$ $017983,0.00005335,-0.00016485,-0.00017865,-0.00010383,0.00011485,-0.00$ $008700,-0.00001112,-0.00004233,-0.00000216,-0.00012539,-0.00007860,0.0$ $0000727,-0.00001057,-0.00000472,0.00000650,0.00001670,-0.00003145,0.00$ $001111,-0.00001121,-0.00003547,0.00001082,0.00000967,-0.00002290,0.062$ $14956,0.02829598,0.13217857,0.00007787,0.00009184,0.00057091,-0.000209$ $50,-0.00074153,0.00011798,0.00027078,0.00019556,-0.00016615,-0.0000254$ $1,-0.00045220,-0.00015582,-0.00016827,0.00027143,0.00017771,0.00006016$ $, 0.00006852,-0.00015885,0.00010374,-0.00001198,-0.00022237,0.00011598$, $0.00061556,-0.00013852,-0.00003603,0.00003812,0.00000287,0.00004511,0$. $00000712,0.00000535,0.00011476,0.00006390,0.00007544,-0.00000741,-0.00$ $000701,0.00001074,-0.14469656,0.05809550,-0.01228501,0.62585140,-0.002$ $09402,-0.00040692,0.00060512,-0.00095920,-0.00290135,0.00047479,0.0004$ $6944,0.00081736,-0.00108802,-0.00001227,-0.00138151,-0.00060393,-0.000$ $68480,-0.00028350,0.00137901,0.00049923,0.00118526,-0.00052755,0.00017$ $234,0.00000728,-0.00018921,0.00266626,0.00235442,-0.00028299,-0.000038$ $67,0.00006468,0.00003178,-0.00000995,-0.00004560,0.00004167,-0.0000370$ $6,-0.00010565,-0.00009420,-0.00003618,-0.00005076,0.00005795,0.1221618$ $0,-0.28047958,0.01035894,0.03183646,0.70826345,-0.00063359,-0.00025235$ $, 0.00030258,0.00011989,-0.00017917,-0.00005024,0.00020653,-0.00000417$, $0.00014262,0.00003080,-0.00031338,-0.00022139,-0.00020426,0.00003748,0$ $.00029307,0.00009927,0.00035084,-0.00026163,0.00028157,-0.00003337,0.0$ $0002885,0.00012485,0.00030494,-0.00008395,-0.00004918,0.00003713,-0.00$ 
$000211,0.00000669,-0.00000641,0.00003895,0.00000132,0.00001161,0.00004$ $854,-0.00002155,-0.00000879,0.00001405,-0.00846067,-0.00216947,-0.0645$ $8969,0.09628957,0.04121947,0.14587503,-0.00735172,-0.00144468,0.001147$ $73,-0.00338170,-0.00418480,-0.00023571,0.00186622,0.00118665,-0.002076$ $37,-0.00025385,-0.00344185,-0.00138464,-0.00177614,-0.00067356,0.00390$ $765,0.00146401,0.00362506,-0.00164495,0.00041657,-0.00018641,-0.000288$ $55,0.00015990,0.00422211,-0.00112752,-0.00015321,0.00022629,0.00011151$ ,-0.00006332,-0.00019605,0.00011864,-0.00015546,-0.00038686,-0.0004028 $9,-0.00020616,-0.00015323,0.00013036,-0.03485311,0.07630462,-0.0037691$ $4,-0.30436615,-0.04138151,-0.04751174,0.60778472,0.00137565,0.00112269$ ,-0.00067314,0.00170658,0.00122723,-0.00041309,-0.00014518,-0.00040578 $, 0.00104326,-0.00016306,0.00104278,0.00076543,0.00091474,0.00085621,-0$ $.00118924,-0.00062613,-0.00113857,0.00003999,0.00006501,-0.00000065,0$. $00042281,-0.00195816,-0.00194580,-0.00009797,0.00012646,-0.00010299,-0$ $.00005325,0.00001865,0.00008627,-0.00002657,-0.00001544,0.00018642,0.0$ $0015836,0.00001583,0.00008506,-0.00012435,0.01757587,0.03706978,0.0049$ $3825,0.03174324,-0.11670802,0.00275458,-0.04609989,0.68533453,-0.00176$ $870,-0.00073490,0.00048161,-0.00019921,-0.00141244,0.00033155,0.000294$ $18,0.00026828,-0.00044380,-0.00003269,-0.00062219,-0.00017426,-0.00014$ $086,-0.00003691,0.00071277,0.00017421,0.00099380,-0.00036350,0.0000430$ $2,-0.00012594,-0.00027164,0.00041450,0.00093023,0.00059171,-0.00002424$ $, 0.00004677,0.00002331,-0.00000646,-0.00000671,0.00000034,-0.00000231$, $-0.00004003,-0.00017308,-0.00006415,-0.00001191,0.00000826,-0.00684848$ $, 0.01630287,0.00798213,-0.04315343,-0.01117329,-0.07249140,0.08345609$, $0.02757533,0.15510635,0.03396438,0.00113528,-0.00279298,-0.00680550,0$. $02097755,0.00629109,-0.00243186,-0.00094789,0.00192616,0.00025199,0.00$ $680832,0.00552180,0.00695251,0.00271743,-0.01363305,-0.01862773,-0.019$ $00843,0.00473448,-0.00771560,0.00119535,-0.00092242,-0.00717925,-0.014$ $59369,0.00076608,0.00046393,-0.00052500,-0.00053226,0.00029784,0.00043$ $293,-0.00042128,0.00060773,0.00107168,0.00122668,0.00040102,0.00038109$ $,-0.00045151,-0.00390364,0.00327300,0.00088152,-0.01944982,-0.02569582$ ,-0.00840923,-0.12516801,-0.10500547,-0.01864550,0.30246237,-0.0019278 $1,-0.00424707,0.00052256,0.00070308,-0.00327423,0.00086379,-0.00004426$ ,-0.00090055,-0.00020602,0.00046936,0.00165041,-0.00005913,0.00020449, $-0.00065673,-0.00301676,-0.00477066,-0.00107771,0.00175902,0.00161075$, $-0.00016064,0.00048997,0.00069942,0.00111585,-0.00037177,-0.00001677,-$ $0.00002412,0.00004952,0.00026780,-0.00001500,-0.00002389,0.00019783,-0$ $.00000307,0.00025991,0.00007625,0.00003169,0.00002695,0.00239445,-0.07$ $274032,-0.00305016,-0.07683859,0.02338770,-0.01446651,-0.07747477,-0.2$ $8846092,-0.02868283,-0.01465116,0.63116581,0.01121766,0.00267430,-0.00$ $710635,-0.00078836,0.00277470,0.00285711,-0.00021628,-0.00019565,0.000$ $15104,-0.00052666,0.00177714,0.00198253,0.00188323,0.00025873,-0.00381$ $881,-0.00339189,-0.00604591,0.00296480,-0.00640832,0.00071270,-0.00003$ $159,-0.00090753,-0.00180753,0.00008163,0.00003795,-0.00007404,-0.00003$ $530,-0.00004526,0.00006958,-0.00011196,0.00006724,0.00027839,0.0004459$ $1,0.00013014,0.00005972,-0.00006429,0.00078453,-0.00327971,-0.00755823$ ,-0.01113156,-0.00453913,0.01408688,-0.01710636,-0.03394130,-0.0621571 $2,0.04114192,0.02774570,0.08631677,-0.00515342,0.00011099,0.00168572,-$ $0.00174857,-0.00368316,-0.00013967,0.00141528,0.00173196,-0.00185502,-$ $0.00041875,-0.00380400,-0.00125899,-0.00176903,-0.00023088,0.00459544$, $0.00291062,0.00390813,-0.00266762,0.00024606,0.00015666,-0.00055030,0$. 
00277304,0.00400103,-0.00050446,-0.00012636,0.00019665,0.00007228,-0.0 $0007945,-0.00015131,0.00013802,-0.00013713,-0.00030311,-0.00032249,-0$. $00014157,-0.00015086,0.00011550,-0.02593179,-0.08116403,-0.01116413,-0$ $.04451991,0.02880934,-0.00583808,0.04885181,0.01765807,0.00899134,-0.1$ $4513924,0.09134815,-0.01179011,0.58867041,-0.00333431,0.00071927,0.000$ $58471,-0.00084980,-0.00211588,0.00007886,0.00088949,0.00073093,-0.0014$ $2919,-0.00012974,-0.00220152,-0.00090158,-0.00109026,-0.00088800,0.002$ $67091,0.00082206,0.00249210,-0.00044920,0.00035187,0.00001568,-0.00018$ $210,0.00140604,0.00281411,-0.00052977,-0.00007062,0.00013412,0.0000623$ $4,-0.00021349,-0.00017220,0.00009665,-0.00012321,-0.00045338,-0.000689$ $41,-0.00011856,-0.00013183,0.00011280,-0.02203477,0.02705761,-0.003340$ $33,0.02124217,-0.01531772,0.00367165,-0.00694831,-0.05099232,-0.004761$ $01,0.11836103,-0.27506205,0.01109890,0.02485344,0.69172948,-0.00142143$ $, 0.00017532,0.00070922,-0.00040762,-0.00115017,-0.00016617,0.00026598$, $0.00054216,-0.00056402,0.00000686,-0.00087360,-0.00024602,-0.00020161$, $-0.00004729,0.00109240,0.00059944,0.00082658,-0.00056095,0.00021277,0$. $00001260,-0.00044047,0.00072104,0.00080376,0.00002772,-0.00002903,0.00$ $004235,0.00001930,-0.00009316,-0.00004917,0.00000232,0.00000394,-0.000$ $02765,-0.00024823,-0.00004865,-0.00003903,0.00003437,-0.00761565,-0.01$ $451130,0.00613633,-0.00658062,0.00488980,-0.00565323,0.00772470,0.0004$ $5025,0.00533626,-0.01079122,0.00632350,-0.05596460,0.08743220,0.032187$ $05,0.14931506,0.00079075,-0.00032728,-0.00023963,0.00053520,0.00111964$ ,-0.00006666,-0.00001040,0.00003705,-0.00002342,-0.00004199,-0.0002115 $6,-0.00006542,-0.00019652,-0.00028463,0.00059185,0.00044509,0.00014715$ ,-0.00017589,-0.00010062,0.00010229,-0.00001932,-0.00086376,-0.0005248 $6,0.00008779,0.00002188,0.00000990,-0.00000378,-0.00003140,-0.00003520$ $, 0.00000444,-0.00012396,-0.00011162,-0.00013951,-0.00003809,-0.0000255$ $4,0.00001433,-0.13028124,-0.04574595,-0.01517927,0.04971785,-0.0170610$ $7,0.00712049,-0.04005061,-0.02524578,-0.00846715,-0.02818344,0.0750506$ $0,-0.00422018,-0.30109449,-0.04333094,-0.04727497,0.63138619,0.0014787$ $0,0.00010315,-0.00056525,0.00062545,0.00067261,-0.00001077,-0.00050220$ ,-0.00051362,0.00067492,0.00007761,0.00130859,0.00045559,0.00065237,0. $00030914,-0.00157678,-0.00081338,-0.00119331,0.00062723,-0.00017552,-0$ $.00004460,0.00020023,-0.00063240,-0.00103202,0.00017967,0.00003638,-0$. $00006620,-0.00000548,0.00006057,0.00004932,-0.00003499,0.00012320,0.00$ $018099,0.00028355,0.00005546,0.00006280,-0.00003464,-0.10909230,-0.295$ $95483,-0.03307777,0.02698298,-0.05585287,0.00145989,-0.03101944,-0.019$ $65577,-0.00656595,0.02249210,0.03075894,0.00502816,0.02884121,-0.11440$ $592,0.00319146,-0.04529670,0.70651498,-0.00030656,-0.00026509,0.000565$ $70,0.00007480,0.00046589,-0.00020630,-0.00002069,-0.00003236,0.0001195$ $4,0.00005933,-0.00000674,-0.00008236,-0.00022609,-0.00008282,0.0001853$ $3,0.00023121,0.00023026,-0.00022542,0.00031285,-0.00001633,0.00005316$, $-0.00026657,-0.00025806,0.00000755,0.00000018,-0.00000423,-0.00000535$, $-0.00000799,-0.00001084,0.00000412,-0.00001889,-0.00000984,0.00006675$, $-0.00002391,-0.00000318,0.00001351,-0.01865286,-0.02069590,-0.06752062$ $, 0.00961712,-0.00712259,0.01049231,-0.00842939,-0.00567021,-0.00686393$ ,-0.00728141,0.01493647,0.01655078,-0.04346274,-0.01055383,-0.07171081 $, 0.09361161,0.02173292,0.14237919,-0.00102558,-0.00024649,0.00024055,-$ $0.00003181,-0.00050932,-0.00004557,0.00028349,0.00030574,-0.00041957,-$ $0.00007657,-0.00089588,-0.00038393,-0.00056582,-0.00010460,0.00108789$, $0.00053856,0.00104081,-0.00055628,0.00020904,-0.00003387,0.00003605,-0$ 
$.00028327,0.00085951,-0.00017525,-0.00001514,0.00008935,0.00007182,-0$. $00001869,-0.00011386,0.00005960,0.00005299,-0.00008690,-0.00005242,-0$. $00008864,-0.00006873,0.00011832,-0.12269168,-0.00318287,-0.01934625,-0$ $.03805734,-0.00451849,-0.00811756,0.00182037,0.00762883,0.00001829,0.0$ $0189084,-0.00028789,0.00058436,0.00211917,-0.00620578,-0.00077600,-0.0$ $3598246,0.00130674,-0.00735662,0.19223182,-0.00005480,0.00003512,0.000$ $04331,-0.00006589,-0.00024499,0.00004226,0.00003491,0.00005057,-0.0000$ $5690,-0.00001521,-0.00006413,-0.00003707,-0.00001701,0.00002247,0.0000$ $4456,0.00001733,0.00004109,-0.00003179,0.00000254,-0.00000733,-0.00000$ $893,0.00018951,0.00022360,-0.00001932,-0.00000536,0.00000763,0.0000086$ $2,0.00000841,0.00000241,0.00000265,0.00001229,0.00000715,0.00002122,0$. $00000058,-0.00000260,0.00000473,-0.00306804,-0.03866642,-0.00156511,0$. $02383759,0.00415890,0.00450858,-0.00105651,-0.00360656,-0.00070493,-0$. $00003887,0.00189835,0.00020188,0.00173666,-0.00383712,-0.00009888,-0.0$ $2702133,0.00227080,-0.00514001,0.00587164,0.03525604,-0.00011563,-0.00$ $001838,0.00001514,-0.00003902,-0.00008778,-0.00000892,0.00007760,0.000$ $08297,-0.00009744,-0.00002179,-0.00019553,-0.00006925,-0.00010528,-0.0$ $0001966,0.00020120,0.00010933,0.00016435,-0.00010399,0.00002164,0.0000$ $0202,-0.00000112,-0.00004486,0.00015640,-0.00000964,-0.00000718,0.0000$ $1751,0.00001185,-0.00000472,-0.00001974,0.00001213,0.00000685,-0.00000$ $755,-0.00000385,-0.00001724,-0.00001378,0.00002579,-0.01942535,-0.0015$ $7532,-0.02403937,-0.00634883,-0.00086806,0.00222483,-0.00061799,0.0009$ $5890,0.00454106,0.00064438,0.00014442,-0.00160310,-0.00039785,-0.00161$ $635,0.00452122,-0.00881581,0.00022285,0.00171983,0.03424888,0.00241415$ $, 0.01753574,-0.00085936,-0.00015373,0.00029535,-0.00033313,-0.00083738$ $, 0.00007952,0.00028523,0.00028900,-0.00040816,-0.00003216,-0.00063863$, $-0.00024457,-0.00031940,-0.00008424,0.00064702,0.00025342,0.00056658,-$ $0.00028451,0.00011182,-0.00000100,-0.00004828,0.00049906,0.00092949,-0$ $.00012727,-0.00003286,0.00004272,0.00002268,-0.00000919,-0.00003488,0$. $00002328,-0.00000333,-0.00004724,-0.00004481,-0.00003088,-0.00002977,0$ $.00003577,0.00708878,0.01546567,0.00188471,-0.11163085,-0.11518854,-0$. $02176695,-0.00846049,-0.02625080,-0.00393195,-0.00397402,-0.00084625,-$ $0.00217240,-0.00137695,0.00085811,0.00002387,-0.00054027,0.00350544,-0$ $.00101365,0.00141473,0.00071882,0.00081856,0.11634991,-0.00012714,-0.0$ $0001221,0.00010524,-0.00006864,-0.00009100,-0.00003049,0.00010479,0.00$ $004901,-0.00011415,-0.00000886,-0.00018321,-0.00007082,-0.00011375,-0$. $00003354,0.00018504,0.00006386,0.00014484,-0.00007114,0.00002011,0.000$ $00124,-0.00001782,0.00005712,0.00021843,-0.00003786,-0.00001603,0.0000$ $2534,0.00001619,0.00000136,-0.00001850,0.00001050,0.00000367,-0.000016$ $70,-0.00001132,-0.00001739,-0.00001647,0.00001585,-0.01610146,-0.01844$ $767,-0.00433603,-0.10977042,-0.27011312,-0.03520640,0.00156598,0.00222$ $882,0.00011657,-0.00021306,0.00166337,-0.00030307,0.00032527,0.0004750$ $7,0.00008632,0.00441227,-0.00221503,0.00040741,-0.00011800,0.00119732$, $0.00022545,0.12083160,0.28561203,-0.00009229,0.00004230,0.00006258,-0$. $00003906,-0.00025972,0.00007153,0.00001912,0.00005013,-0.00010326,-0.0$ $0000380,-0.00007924,-0.00002791,-0.00003148,-0.00001323,0.00004564,-0$. $00002616,0.00000742,-0.00000740,-0.00004417,-0.00001074,-0.00000196,0$. $00014869,0.00023744,-0.00003471,0.00000864,-0.00000495,0.00000488,0.00$ $000051,-0.00000887,0.00000209,-0.00001007,-0.00001598,-0.00001772,-0.0$ $0000245,-0.00000346,0.00000369,-0.00006021,0.00177884,0.00256189,-0.02$ $133897,-0.03636578,-0.04015822,-0.00251879,-0.00515200,0.00359861,-0.0$ 
$0190414,-0.00025287,0.00596445,-0.00001818,0.00020174,-0.00072314,-0.0$ $0095445,0.00014657,0.00530553,0.00076120,0.00037740,-0.00258689,0.0250$ $6827,0.03888020,0.03019738,-0.00200451,0.00125736,0.00081903,-0.002963$ $51,-0.00834727,0.00138011,0.00104331,0.00159376,-0.00159138,-0.0002239$ $2,-0.00159349,-0.00055401,-0.00024880,0.00082710,0.00097231,0.00037504$ $, 0.00044405,-0.00028949,0.00025543,-0.00018646,0.00000460,0.00357409,0$ $.00482224,-0.00075238,-0.00001859,-0.00003683,-0.00000560,0.00003210,0$ $.00003428,0.00002198,-0.00000114,0.00010441,0.00002859,0.00004405,0.00$ $002436,-0.00006438,-0.00291840,-0.00140278,-0.00174983,-0.01252000,0.0$ $2364317,-0.00196109,-0.13472182,0.12339929,-0.00897292,0.01439919,-0.0$ $0921128,0.00197454,-0.00111575,-0.00467601,-0.00158383,-0.00034632,-0$. $00038785,-0.00002625,-0.00135824,0.00066429,-0.00025338,0.00118235,-0$. $00008638,0.00093715,0.13717595,0.00062189,-0.00047678,0.00018447,-0.00$ $046754,0.00109988,-0.00007857,-0.00032222,-0.00025479,0.00016427,0.000$ $16636,0.00013167,-0.00004859,-0.00008225,-0.00036576,-0.00012784,-0.00$ $003588,-0.00006661,0.00019795,-0.00020788,0.00001740,-0.00021639,0.001$ $17005,-0.00157737,0.00019341,-0.00005032,0.00002124,-0.00002587,0.0000$ $0065,-0.00000586,-0.00001695,0.00000527,-0.00004717,-0.00001295,-0.000$ $00778,-0.00001478,0.00002972,-0.00061391,0.00183224,-0.00052465,-0.004$ $05326,0.00601698,-0.00071025,0.12545572,-0.24259137,0.00881286,0.01136$ $754,-0.02344897,0.00054417,-0.00150807,-0.00411380,-0.00101252,-0.0011$ $8806,-0.00001476,-0.00017955,-0.00088866,0.00019398,-0.00018440,0.0001$ $5820,0.00036133,0.00035404,-0.13018438,0.26373910,-0.00007000,0.000131$ $77,0.00014380,0.00002761,0.00000810,-0.00005461,-0.00005683,0.00012469$ ,-0.00007304,0.00003357,0.00010844,-0.00006121,-0.00006703,-0.00003826 $,-0.00004925,0.00020046,-0.00008110,0.00002220,-0.00001062,-0.00003056$ ,-0.00012488,0.00006207,0.00001307,-0.00119097,0.00004329,-0.00001576, $-0.00002171,0.00001269,-0.00000536,-0.00001594,0.00000506,-0.00001123$, $0.00004155,0.00002495,0.00000838,-0.00000145,-0.00189870,-0.00051815,0$ $.00626668,-0.00386340,0.00454635,0.00554645,-0.00827759,0.00820304,-0$. $04205566,0.00288358,-0.00377607,0.00126431,-0.00129634,-0.00140707,0.0$ $0651345,-0.00007780,-0.00010492,-0.00071020,-0.00038742,0.00015124,0.0$ $0014542,0.00096110,0.00030960,-0.00382264,0.01141056,-0.00754296,0.029$ $38153,0.00068999,-0.00029755,-0.00001118,0.00016471,0.00052538,-0.0000$ $1799,-0.00008632,-0.00000753,0.00004232,-0.00005667,-0.00003149,0.0000$ $2788,0.00000843,-0.00012412,0.00042638,0.00045269,0.00002726,-0.000301$ $76,-0.00003351,0.00002874,-0.00003158,-0.00027238,-0.00049040,0.000105$ $75,0.00001867,-0.00002284,-0.00000883,-0.00001813,0.00004727,-0.000002$ $66,-0.00019126,-0.00000352,-0.00012565,0.00001335,0.00000066,-0.000042$ $00,-0.00326631,0.00121742,-0.00170472,-0.00078744,0.00069334,-0.000069$ $88,-0.00033170,0.00419309,-0.00109410,0.01117086,0.01302973,0.00293703$ ,-0.10810798,-0.10522700,-0.01880999,-0.01133554,-0.02780979,-0.004440 $89,-0.00180839,-0.00096746,-0.00045488,0.00000323,-0.00006129,0.000221$ $57,0.00047660,0.00060074,0.00014781,0.11217316,0.00049361,0.00006681,-$ $0.00007640,0.00016657,-0.00004526,-0.00004326,-0.00021569,-0.00014126$, $0.00039703,-0.00000604,0.00054889,0.00023361,0.00027207,0.00046188,-0$. $00074499,-0.00020292,-0.00056541,0.00001162,-0.00007376,-0.00002454,0$. $00001005,-0.00006962,-0.00026395,0.00012145,0.00000596,-0.00001789,0.0$ $0000761,0.00006067,0.00003782,-0.00000673,0.00012278,0.00022924,0.0002$ $6090,0.00003191,0.00004114,-0.00001701,0.00011028,0.00239723,-0.000084$ $17,0.00100786,0.00019773,0.00019415,0.00258919,-0.00398057,-0.00011023$ 
,-0.00953980,-0.02325729,-0.00287925,-0.10988517,-0.26027392,-0.031096 $42,0.00153586,0.00089419,0.00003581,0.00016216,-0.00000795,0.00001634$, $-0.00009970,0.00000133,0.00003196,-0.00073299,-0.00047376,-0.00012961$, $0.11453276,0.28368992,0.00064009,0.00011432,-0.00041415,0.00009596,-0$. $00005893,0.00016792,-0.00000336,-0.00003055,-0.00000664,-0.00002741,0$. $00005832,-0.00005967,0.00006232,0.00004996,-0.00025660,-0.00026699,-0$. $00017028,0.00013646,-0.00027017,0.00008382,-0.00008437,0.00000550,0.00$ $002713,-0.00001027,0.00001210,-0.00000214,-0.00000302,0.00006895,0.000$ $02814,0.00000896,-0.00006252,-0.00009972,-0.00000110,0.00002409,-0.000$ $00105,-0.00001192,-0.00187792,-0.00006556,0.00606936,-0.00005556,0.000$ $25681,-0.00065904,-0.00125758,0.00013397,0.00715565,0.00169528,0.00116$ $261,0.00004258,-0.01890736,-0.02980083,-0.04102445,-0.00301328,-0.0056$ $1264,0.00423367,-0.00041641,-0.00022000,0.00020843,0.00021712,0.000064$ $13,-0.00113660,0.00009493,0.00010109,-0.00015262,0.02229789,0.03373223$ $, 0.02943425,-0.00077601,-0.00007120,0.00020093,-0.00024503,-0.00052508$ $, 0.00003710,0.00023542,0.00027579,-0.00036472,-0.00002935,-0.00064101$, $-0.00022579,-0.00032061,-0.00012082,0.00074901,0.00035906,0.00062334,-$ $0.00033252,0.00007297,0.00000699,-0.00005056,0.00034281,0.00065627,-0$. $00009542,-0.00001932,0.00004013,0.00002201,-0.00003474,-0.00004462,0.0$ $0002153,-0.00002127,-0.00006954,-0.00008770,-0.00004250,-0.00003385,0$. $00003873,0.00687101,-0.01295183,0.00021308,0.00011704,-0.00323379,-0.0$ $0123720,-0.00107013,-0.00091138,-0.00003948,-0.00428456,0.00001753,-0$. $00205369,-0.01012601,0.02556860,-0.00135699,-0.13367584,0.12949136,-0$. $01237258,0.00144261,-0.00078408,0.00074255,0.00041434,-0.00068360,-0.0$ $0012094,-0.00011710,0.00006380,0.00019297,0.00112392,-0.00026600,0.000$ $94431,0.13978330,0.00021092,0.00005958,-0.00006158,0.00005190,0.000122$ $68,-0.00000620,-0.00008106,-0.00007730,0.00011474,0.00001663,0.0001992$ $3,0.00007502,0.00009861,0.00004039,-0.00022169,-0.00007834,-0.00019481$ $, 0.00011129,-0.00002056,-0.00000160,-0.00000287,-0.00004729,-0.0001805$ $2,0.00003988,0.00000652,-0.00001802,-0.00001113,0.00000621,0.00002148$, $-0.00000895,-0.00000975,0.00002149,0.00001395,0.00001773,0.00001379,-0$ $.00002021,0.01876031,-0.01814745,0.00242768,-0.00422288,-0.00280715,-0$ $.00120006,-0.00052775,0.00034952,-0.00005744,0.00000590,0.00165656,-0$. $00015847,-0.00279697,0.00379729,-0.00062512,0.12399578,-0.25217658,0.0$ $1224284,-0.00000346,0.00107167,0.00020119,0.00084654,-0.00083069,0.000$ $05880,0.00003141,0.00003795,0.00005636,0.00014660,0.00048562,0.0002491$ $5,-0.13640701,0.26655686,-0.00020665,-0.00004409,0.00006320,-0.0000407$ $9,-0.00005223,-0.00000122,0.00003990,0.00003273,-0.00005488,-0.0000250$ $6,-0.00011271,-0.00004464,-0.00009156,-0.00000759,0.00015418,0.0001030$ $2,0.00013096,-0.00004708,0.00002531,0.00000688,0.00000389,0.00003411,0$ $.00010353,-0.00004187,-0.00000041,0.00000334,0.00000248,0.00000716,-0$. $00000407,0.00001213,-0.00000324,-0.00001194,-0.00001919,-0.00000026,-0$ $.00000591,0.00000157,0.00183649,-0.00368823,0.00261675,-0.00126860,-0$. $00100694,0.00507675,0.00005253,-0.00010304,-0.00087521,-0.00192799,-0$. $00037842,0.00574256,-0.00285717,0.00489504,0.00359445,-0.01269399,0.01$ $338615,-0.03437197,0.00080633,0.00006233,-0.00263908,-0.00003470,-0.00$ $022337,0.00080218,0.00016073,0.00009949,-0.00099121,0.00090945,0.00013$ $529,-0.00363683,0.01517539,-0.01321719,0.02465306 \backslash 1-0.00002157,0.00001$ $651,0.00000600,0.00000165,-0.00000842,-0.00001036,-0.00000760,0.000000$ $60,0.00000628,0.00000219,0.00001292,0.00000284,-0.00000461,-0.00000081$ $,-0.00001305,0.00000515,0.00000074,0.00001107,-0.00000042,0.00000037,0$ 
$.00000181,0.00001801,0.00000304,0.00000526,0.00000086,0.00000258,-0.00$ $000069,-0.00000104,0.00000276,0.00000467,-0.00000162,0.00000381,-0.000$ $00110,-0.00000126,0.00000088,-0.00000063,-0.00000665,-0.00000086,-0.00$ $000217,0.00000003,0.00000194,0.00001474,0.00002110,0.00001313,-0.00001$ $642,0.00002292,0.00000135,-0.00001309,-0.00000002,-0.00000546,0.000004$ $54,-0.00000452,-0.00000571,0.00000483,0.00000342,-0.00000483,0.0000009$ $0,0.00000323,-0.00000383,-0.00000350,-0.00002794,-0.00002875,0.0000006$ $1,-0.00000111,-0.00000022,-0.00000293,-0.00000022,-0.00000175,0.000000$ 3911!@

meta-Complex

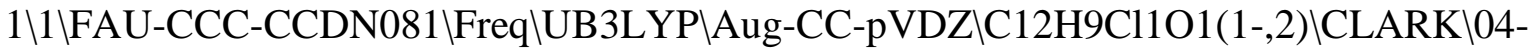
Mar-2015\0\\\# Geom=AllCheck Guess=TCheck SCRF=Check GenChk UB3LYP/Aug -CC-pVDZ Freq IIPre-addition complex p-Cl-Ph-radical + phenolate (meta) II-1,2\C,2.5961108398,0.9761094166,-1.2632941385\C,3.2390867951,-0.247 710267,-1.3197931702\C,3.6432067723,-0.967269945,-0.1227724013\C,3.306 $826606,-0.3022864734,1.1256587398 \backslash \mathrm{C}, 2.663257848,0.9220645866,1.1594668$ $212 \backslash \mathrm{C}, 2.2729409981,1.5859776209,-0.0278165516 \backslash \mathrm{H}, 2.3144131682,1.4790577$ $548,-2.1925079057 \backslash \mathrm{H}, 3.4745870169,-0.713466593,-2.2795332919 \backslash \mathrm{O}, 4.242410$ $7261,-2.0817513896,-0.1642503946 \backslash \mathrm{H}, 2.4341132067,1.3828052622,2.1243247$ $555 \backslash \mathrm{H}, 1.8472809535,2.5852745912,0.0063152307 \backslash \mathrm{H}, 3.5946416736,-0.8099974$ 716,2.0491761436\C,-2.8780948739,-0.2387837671,0.0723693231\C,-2.84298 $72733,1.1543116493,0.1031240188 \mathrm{IC},-1.5879715746,1.7925255114,0.0835217$ $563 \backslash \mathrm{C},-0.4165561972,1.041174172,0.0344907633 \backslash \mathrm{C},-0.4731509097,-0.348280$ $1486,0.0043983582 \backslash \mathrm{C},-1.7138390862,-1.0076950933,0.023158959 \backslash \mathrm{Cl},-4.4610$ $545421,-1.062465249,0.0966391352 \backslash \mathrm{H},-3.7703991085,1.7273059546,0.141430$ $043 \backslash \mathrm{H},-1.5611154242,2.8863087502,0.1077575773 \backslash \mathrm{H}, 0.4461616194,-0.939529$ $1865,-0.0341031286 \mathrm{LH},-1.775430086,-2.0962099937,0.0000401652 \mathrm{llVersion}=$ EM64L-G09RevC.01 \State=2-A $\backslash H F=-998.1700037 \backslash S 2=0.761846 \backslash S 2-1=0 . \backslash S 2 A=0.7$ $50098 \backslash \mathrm{RMSD}=6.684 \mathrm{e}-09 \backslash \mathrm{RMSF}=4.080 \mathrm{e}-06 \backslash \mathrm{ZeroPoint}=0.1685158 \backslash \mathrm{Thermal}=0.1814$ 37\ZPE=105.7452708\Dipole=-3.1576246,1.5136167,0.1205478\DipoleDeriv $=0$ $.2160405,-0.2657848,-0.087505,-0.6462612,0.1817495,0.2349218,-0.489804$ $3,0.0669119,0.2478574,-0.4390082,0.0828152,0.055722,0.4353842,-0.35402$ $24,-0.0787294,-0.5132393,0.4257143,0.0202614,0.6243871,-0.6883284,-0.0$ $296437,-1.0459226,1.3585469,0.056764,-0.0360725,0.0465979,0.1231891,-0$ $.4126469,0.0618283,-0.0296435,0.4105745,-0.3359908,0.0393972,0.5542877$ ,-0.4473434,-0.0254563,0.247894,-0.2748178,0.0767309,-0.6794998,0.1953 $992,-0.2001357,0.4624227,-0.0523547,0.2054272,-0.5279807,0.0263243,0.0$ $049989,0.004253,-0.1458652,0.0042637,0.0028738,0.0040195,-0.3475927,0$. $0843877,0.0707512,-0.0698384,-0.0101845,0.0171572,0.0977538,0.0378573$, $0.0931086,-0.1246132,0.0907209,0.056544,0.0385682,0.0882056,0.0259615$, $-0.0918585,0.0774258,-0.0688628,-0.127984,-1.11036,0.6119989,0.033331$, $1.2714406,-1.5716536,-0.0611255,0.0480315,-0.0436967,-0.4129357,0.0851$ $7,0.0616848,0.0604453,-0.0127681,0.0250916,-0.0900628,-0.0492118,-0.09$ $06601,-0.1341997,0.2256764,0.1017387,-0.002967,-0.0340959,-0.0574364,-$ $0.0008929,-0.0067299,-0.0045193,0.0277602,0.0850425,0.0628341,-0.04761$ $12,0.1002241,0.0172746,0.0934455,-0.0855902,0.0737707,-0.1132081,1.088$ $4699,0.412028,-0.0227101,0.5858,0.2178911,-0.0100503,-0.017226,-0.0039$ $141,-0.0785557,-0.3685587,-0.0914895,0.0048183,0.1511204,-0.0940332,-0$ $.0045073,0.0118038,0.0014644,-0.1138104,-0.5686,0.0653273,0.0134597,-1$ 
$.0682964,0.3504069,0.040516,-0.0135618,0.0123907,-0.120495,2.1842557,-$ $0.2884066,-0.0712102,1.3166511,-0.4068901,-0.0407435,-0.0343983,0.0008$ $542,-0.1735335,-1.1500863,0.1316286,0.0318394,0.0909052,-0.0124432,-0$. $0022755,0.0316072,-0.0018812,-0.0757849,-0.069386,-0.0590808,-0.000928$ $4,-0.4698047,0.0852467,0.0179316,-0.0133504,0.0065532,-0.0867227,-1.02$ $74338,-0.2650555,0.0187534,-0.4677897,-0.269551,0.0095033,0.0139081,0$. $0034476,-0.1242205,-0.2739056,0.123335,0.0137151,0.0570523,0.0273401,-$ $0.0032388,0.0119564,-0.0056193,0.1239145,-0.0157592,0.0013467,0.003685$ $7,0.1654869,-0.2111337,-0.01214,0.0083647,-0.0088219,0.1094852,0.09966$ $07,0.066467,0.0011763,-0.085744,0.0346083,0.0013064,-0.0027883,-0.0030$ $794,0.0817668,-0.0679799,-0.0036889,0.0048133,-0.1567309,-0.077654,-0$. 0000432,0.0014337,-0.0040802,0.1194506\Polar $=388.0384022,-41.8425786,1$ $89.8983918,-7.7905548,2.3640829,139.6535729 \backslash \mathrm{PG}=\mathrm{C} 01[\mathrm{X}(\mathrm{C} 12 \mathrm{H} 9 \mathrm{Cl1O} 1)] \mathrm{NIm}$ $\mathrm{ag}=0 \backslash 0.25560062,-0.23424879,0.57223659,0.01987041,-0.02724654,0.67228$ $906,-0.12479053,0.13501229,-0.00766097,0.24331490,0.13573754,-0.308895$ $72,0.01509903,-0.24800939,0.57895971,0.01597009,-0.03028795,-0.1218324$ $6,-0.02508455,0.06167863,0.62978080,-0.00675725,0.01387473,-0.00887638$ $,-0.08260828,0.01887800,-0.02583256,0.31328329,0.01337603,-0.02433934$, $0.01842312,0.01864725,-0.10721421,0.04371469,-0.23595949,0.62884965,-0$ $.02284470,0.04259918,0.03647307,-0.01400799,0.02084457,-0.21576948,0.0$ $0102872,0.00850921,0.54070788,-0.00610557,0.01012598,0.00961724,0.0138$ $5723,-0.01219975,-0.00112292,-0.08076042,0.01584784,0.00720137,0.24668$ $830,0.00968077,-0.02243600,-0.02023146,-0.01227864,0.03166158,0.000528$ $04,0.01681599,-0.10449242,-0.01682067,-0.25245314,0.58392441,0.0028409$ $2,-0.00630201,-0.01182067,-0.00193717,0.00293905,-0.03111854,0.0190660$ $2,-0.03964542,-0.22031795,0.03507586,-0.04978174,0.62143683,0.01191208$ ,-0.01253645,-0.00709657,-0.00676426,0.01084625,-0.00260859,-0.0048471 $8,0.01118708,0.02562395,-0.12492691,0.13504088,-0.00976356,0.25411603$, $-0.01202724,0.03428025,0.01581127,0.01087817,-0.02355672,0.00514636,0$. $01097185,-0.02148030,-0.04582638,0.13577048,-0.30886169,0.01441936,-0$. $23191926,0.56946376,0.00287645,-0.01117111,-0.05375461,-0.00938405,0.0$ $1911778,-0.00999583,0.01165280,-0.02166106,0.03167950,0.01387625,-0.03$ $094607,-0.12171337,-0.00699581,0.03555192,0.67655482,-0.07177387,0.035$ $69693,0.04410954,-0.00096982,0.01799974,0.01195390,-0.00265637,-0.0078$ $8743,-0.00140087,-0.00300425,0.02143339,-0.01000416,-0.07617870,0.0404$ $4893,-0.05243219,0.20260015,0.03806871,-0.13653836,-0.09324523,0.01568$ $620,-0.02114960,-0.02527810,-0.00712016,0.00559040,0.00135545,0.018179$ $78,-0.02461894,0.02256663,0.04470814,-0.14284971,0.09609532,-0.2309735$ $9,0.61329427,0.02685660,-0.04500667,-0.26018797,0.02678713,-0.05491169$ $, 0.01452487,-0.00138553,0.00138612,-0.04660954,-0.02496837,0.05203064$, $0.02002205,-0.03502796,0.04803826,-0.24947051,0.00587626,0.00667750,0$. $60094528,-0.05769343,0.04024186,-0.06028543,0.00084212,0.00697051,-0.0$ $1351864,0.00671391,0.00515254,0.00205248,-0.00071817,-0.00032396,-0.00$ $000369,0.00390407,0.00234426,0.00112785,0.00434861,-0.00303737,0.00691$ $179,0.05016694,0.04023268,-0.10827193,0.10395573,0.00745142,-0.0082818$ $7,0.02615191,0.00504981,-0.00048651,-0.00367927,-0.00023976,-0.0004035$ $5,-0.00007476,0.00241666,0.00068179,-0.00245915,-0.00338701,0.00876480$ $,-0.01131085,-0.04763441,0.11002187,-0.05855980,0.10464703,-0.25090474$ $, 0.00099363,-0.00207708,0.00238145,0.00053981,-0.00132971,0.00241868,0$ $.00039750,-0.00074994,0.00067104,0.00195685,-0.00379633,-0.00405146,-0$ $.00882116,0.01574193,-0.01837171,0.06427658,-0.11363886,0.26689651,-0$. 
$00047372,0.00465846,0.01288277,-0.04879665,0.03389558,0.05142558,0.005$ $66872,-0.00180419,-0.00730954,0.00276140,0.00225729,-0.00134977,-0.000$ $44919,-0.00010122,-0.00011561,0.00456959,0.00563341,-0.00081967,-0.003$ $66635,-0.00250305,-0.00002485,0.04259280,0.00461925,-0.00734982,-0.025$ $28874,0.03396056,-0.09714429,-0.10222905,-0.00144269,0.00772902,0.0129$ $1806,0.00226205,-0.00006849,0.00238596,-0.00006979,-0.00042431,0.00037$ $355,0.00506773,-0.00219674,0.00148281,-0.00237765,-0.00029588,0.000343$ $67,-0.03966338,0.09921062,-0.00159972,0.00258086,0.00149218,0.05121426$ $,-0.10164235,-0.26959717,0.00926209,-0.01792870,-0.01890275,-0.0020884$ $6,0.00381374,-0.00375242,-0.00070868,0.00118722,-0.00000611,-0.0001283$ $0,0.00015816,0.00264223,0.00036601,-0.00046767,0.00116909,-0.05611690$, $0.11187951,0.28422224,0.00590039,-0.00283196,-0.00413234,-0.00108839,0$ $.03542746,-0.01391013,-0.15842344,0.17621075,0.00579081,0.00102575,0.0$ $3233328,0.01581859,0.00591520,-0.00267841,0.00309427,-0.00306496,0.003$ $81279,0.00041491,-0.00158589,0.00034676,-0.00024859,-0.00204144,-0.002$ $21252,-0.00049567,0.15675563,-0.00266001,0.00949037,0.00717214,0.03520$ $794,-0.04631369,0.02574569,0.17595613,-0.39154428,-0.01157411,0.032623$ $39,-0.04311037,-0.02993634,-0.00287590,0.00953086,-0.00612434,0.004724$ $58,-0.00748292,-0.00049144,0.00029147,-0.00186504,0.00047956,-0.002108$ $31,0.00087423,0.00108658,-0.23842864,0.47157942,0.00293341,-0.00533880$ ,-0.01044609,-0.02248962,0.04201919,0.00973719,0.00578462,-0.01157709, $-0.09187218,0.02440646,-0.04622505,0.00438672,-0.00397033,0.00637903,-$ $0.01048183,0.00043085,-0.00045833,0.00858742,-0.00081827,0.00153778,-0$ $.00029247,0.00093280,-0.00150800,0.00422804,-0.00743348,0.01533249,0.0$ $8115522,0.00372012,0.00260215,-0.00144661,-0.00073923,-0.00028961,0.00$ $007196,0.00657027,0.00531203,-0.00204715,0.00156698,0.00643602,0.01382$ $518,-0.05162950,0.03209163,0.05059163,0.00437611,-0.00352273,-0.008327$ $84,0.00035008,-0.00003969,-0.00037757,-0.00059659,-0.00032930,0.000065$ $59,-0.00152337,0.00022525,0.00089509,0.04361316,0.00264671,0.00040559$, $0.00250752,-0.00021757,-0.00044084,0.00005155,0.00526324,-0.00069118,0$ $.00325195,0.00600645,-0.00717204,-0.02693335,0.03220837,-0.09918989,-0$ $.09907156,-0.00308444,0.00889767,0.01271054,-0.00002360,0.00034695,0.0$ $0069206,-0.00032762,-0.00016368,-0.00008034,0.00024859,-0.00177180,-0$. $00161413,-0.03883442,0.10011687,-0.00226092,0.00385282,-0.00357384,-0$. $00032953,0.00071319,0.00072084,-0.00053479,0.00089726,0.00277267,-0.00$ $068035,0.00129193,0.00054142,0.04885578,-0.09974798,-0.26606091,0.0074$ $0870,-0.01434231,-0.01853836,0.00028678,-0.00061532,-0.00131999,0.0000$ $7080,-0.00008465,-0.00006262,0.00032800,-0.00055790,-0.00045488,-0.053$ $53665,0.10845374,0.28336265,-0.00373372,0.00634218,0.00004541,0.004134$ $36,0.00038989,0.00170413,-0.00048789,0.00021122,-0.00002466,0.00399276$ $, 0.00055053,-0.00215863,-0.00429602,0.00775695,0.00072053,-0.06766076$, $0.11564441,0.00292671,-0.00219425,-0.00152957,0.00007753,0.00056998,0$. $00060199,-0.00002431,-0.00002744,-0.00080790,0.00000148,-0.00218132,-0$ $.00156143,0.00003534,0.07579838,0.00407649,-0.00799221,0.00355903,0.00$ $111557,0.00168138,-0.00303807,-0.00075458,0.00055904,0.00007002,0.0013$ $1095,0.00149499,0.00323947,0.00432881,-0.00897441,-0.00435758,0.117070$ $69,-0.29416431,-0.00857635,-0.00128475,-0.00006061,-0.00026312,0.00058$ $528,-0.00038831,0.00013142,-0.00008806,-0.00002151,-0.00001559,-0.0012$ $8282,-0.00004445,0.00028411,-0.12505754,0.30802446,0.01082799,-0.02633$ $955,0.00343298,0.00031493,-0.00095293,-0.00464085,-0.00004468,0.000043$ $05,-0.00155843,-0.00072966,0.00119711,-0.00430546,-0.01019063,0.025380$ 
$81,0.00497541,0.00295300,-0.00853668,-0.05584841,-0.00020348,0.0006322$ $5,0.00109753,0.00090163,-0.00106555,-0.00005247,0.00001771,0.00000509$, $0.00075328,0.00033122,-0.00059696,0.00106743,-0.00337584,0.00911942,0$. $05524543,-0.00039547,-0.00016758,0.00014264,0.00292984,0.00200288,0.00$ $106558,0.00547979,-0.00107025,0.00590390,-0.05507290,0.04220689,-0.061$ 40848,-0.00107362,0.00506901,-0.01249822,0.00462788,0.00555975,0.00095 $077,-0.00060325,-0.00032199,-0.00005900,-0.00032241,-0.00039093,0.0003$ $0917,-0.00204851,-0.00213724,-0.00067915,-0.00367925,-0.00248063,0.000$ $18837,0.00052198,0.00061881,-0.00090623,0.04944529,-0.00013797,-0.0003$ $4338,-0.00038566,0.00205554,0.00019242,-0.00230398,-0.00167496,0.00744$ $123,-0.01165824,0.04228999,-0.10645875,0.10724565,0.00592273,-0.008333$ $74,0.02453085,0.00497254,-0.00209613,-0.00196725,-0.00032632,-0.000170$ $60,0.00009817,-0.00041059,0.00023028,-0.00068787,-0.00216258,0.0008622$ $6,0.00148319,-0.00237813,-0.00030244,-0.00027645,0.00065356,-0.0004276$ $6,0.00101299,-0.04877427,0.10941834,0.00071566,-0.00118442,-0.00011373$ $, 0.00180866,-0.00372672,-0.00420763,-0.01067960,0.01917583,-0.01843089$ ,-0.06119281,0.10667135,-0.25399286,0.00201298,-0.00330119,0.00306720, $0.00023887,-0.00069006,0.00247983,-0.00005113,0.00008542,-0.00004906,-$ $0.00038915,0.00076945,-0.00143697,0.00074918,-0.00112262,0.00426198,-0$ $.00019823,0.00053729,0.00119624,0.00002449,-0.00018174,0.00003214,0.06$ $691468,-0.11706540,0.26714642,-0.00041919,0.00061108,0.00059252,0.0002$ $3078,-0.00053810,0.00043388,-0.00022717,0.00053062,0.00001150,0.000214$ $76,-0.00053202,-0.00044946,-0.00044428,0.00064036,-0.00054722,0.000552$ $87,0.00012067,-0.00001738,-0.00003283,0.00009030,-0.00007492,-0.000009$ $78,-0.00005402,-0.00006075,0.00054842,-0.00088039,-0.00002757,-0.00001$ $837,0.00008222,0.00007858,-0.00021294,0.00008166,0.00001285,-0.0000166$ $9,-0.00005294,0.00004722,0.50812547,0.00010813,0.00008549,0.00025491,0$ $.00022891,0.00001416,0.00019300,-0.00030666,0.00058254,0.00000753,0.00$ $024231,0.00002152,-0.00019094,0.00007631,0.00011262,-0.00025979,0.0002$ $1927,-0.00015805,-0.00001167,-0.00005896,-0.00000817,-0.00005164,-0.00$ $000055,-0.00002129,-0.00004062,0.00035434,-0.00067692,-0.00002315,-0.0$ $0004931,-0.00001901,0.00004583,-0.00001183,0.00013887,0.00001171,-0.00$ $001219,-0.00003839,0.00004114,-0.07520605,0.62080053,-0.00006197,0.000$ $02704,-0.00000287,0.00001059,-0.00003900,-0.00004611,0.00000291,-0.000$ $00984,0.00005130,-0.00001573,0.00008322,-0.00002355,0.00008082,-0.0000$ $4466,-0.00000751,-0.00000405,-0.00001307,0.00007613,0.00001633,0.00000$ $467,-0.00001114,-0.00001289,-0.00000671,0.00000557,-0.00000860,0.00001$ $005,-0.00000749,-0.00001585,-0.00001530,-0.00000541,0.00000577,0.00000$ $135,0.00000985,0.00001752,-0.00000054,0.00000407,-0.01229450,0.0134567$ $4,0.12013518,0.00002937,-0.00034802,-0.00054600,-0.00022176,0.00013218$ $,-0.00033268,0.00021446,-0.00043464,-0.00000694,-0.00020533,0.00014883$ $, 0.00033246,0.00006994,-0.00039106,0.00054278,-0.00023988,0.00031772,0$ $.00001194,0.00002875,-0.00006047,0.00015320,0.00000276,0.00003807,0.00$ $005772,-0.00039779,0.00075793,0.00001810,0.00000839,-0.00005366,-0.000$ $16024,-0.00002193,-0.00037797,-0.00000806,0.00000572,0.00002907,-0.000$ $04301,-0.10187347,0.04278622,0.00203826,0.68882217,-0.00041655,0.00079$ $465,0.00068095,0.00007573,-0.00063561,0.00037465,0.00002179,0.00009472$ $, 0.00000024,0.00007539,-0.00067829,-0.00043883,-0.00048606,0.00086513$, $-0.00058655,0.00087625,0.00005993,-0.00004034,0.00005289,0.00010608,-0$ $.00015680,0.00001704,-0.00004666,-0.00002153,0.00031773,-0.00063533,-0$ $.00002350,0.00005190,0.00009908,0.00016141,-0.00001128,0.00008856,0.00$ 
$000158,0.00002847,-0.00002460,0.00002747,-0.02159536,-0.33134583,-0.00$ $550668,-0.04875438,0.66801103,-0.00033964,0.00038977,0.00022719,0.0000$ $2708,-0.00024583,0.00004411,-0.00000493,0.00002308,0.00019006,-0.00002$ $297,0.00017715,-0.00001055,0.00036001,-0.00034142,0.00013951,0.0000131$ $8,-0.00000602,-0.00024985,0.00004370,0.00001004,-0.00002250,0.00001404$ $, 0.00000651,-0.00002113,0.00002039,-0.00003767,-0.00009295,-0.00005321$ $, 0.00001072,-0.00000510,-0.00000275,0.00001401,0.00001084,-0.00001417$, $0.00000360,-0.00000127,0.00057037,-0.00728909,-0.06306529,-0.01650971$, $0.01372750,0.12389029,0.00098259,-0.00206643,-0.00182346,-0.00035844,0$ $.00172980,-0.00093686,0.00000342,-0.00059060,-0.00000882,-0.00030204,0$ $.00165858,0.00098349,0.00104895,-0.00211976,0.00171196,-0.00172729,0.0$ $0073062,0.00004987,-0.00011173,-0.00030837,0.00039359,-0.00005234,0.00$ $006557,0.00011287,-0.00080609,0.00178523,0.00005578,-0.00015259,-0.000$ $28731,-0.00039581,0.00068109,-0.00072700,-0.00003608,-0.00003578,0.000$ $08995,-0.00008884,0.02268488,-0.08452761,-0.00229903,-0.26530722,-0.04$ $259070,0.00454375,0.58842319,0.00117041,-0.00271583,-0.00260957,-0.000$ $60219,0.00208495,-0.00150687,0.00043030,-0.00119362,-0.00003395,-0.000$ $51682,0.00197432,0.00166551,0.00134232,-0.00283980,0.00240921,-0.00244$ $858,0.00038009,0.00006017,-0.00000836,-0.00030714,0.00054762,-0.000104$ $59,0.00015119,0.00020059,-0.00153866,0.00324607,0.00012783,-0.00002142$ ,-0.00028073,-0.00055249,0.00013337,-0.00080505,-0.00002285,-0.0001279 $1,0.00018209,-0.00021728,-0.02410838,-0.02428535,-0.00009303,-0.116467$ $93,-0.15494852,0.00107936,-0.01581268,0.69354440,0.00021182,-0.0001226$ $6,-0.00015534,0.00006679,-0.00008084,0.00003042,0.00000094,-0.00001560$ ,-0.00034194,-0.00008425,0.00013570,0.00010267,-0.00022591,0.00013680, $-0.00015355,-0.00000303,-0.00003459,0.00021932,-0.00003644,0.00001064$, $-0.00002393,-0.00000982,-0.00000999,0.00001690,-0.00000849,0.00002022$, $0.00010071,0.00004896,-0.00002807,-0.00001327,-0.00000534,0.00000045,0$ $.00012221,0.00001079,-0.00000930,-0.00001413,-0.00096703,0.00156602,0$. $00819994,0.00288981,-0.00093325,-0.06251280,-0.01281157,0.01323607,0.1$ $3049494,-0.00870333,0.00944960,0.01033555,0.00217981,-0.00766485,0.003$ 22993,-0.00170170,0.00381820,0.00009445,0.00201593,-0.00754741,-0.0036 $2941,-0.00937152,0.00998842,-0.00953685,0.01422607,-0.00245746,-0.0003$ $9192,0.00026423,0.00103107,-0.00142722,0.00039598,-0.00033189,-0.00043$ $824,0.00357510,-0.00735624,-0.00024099,0.00037759,0.00096756,0.0014681$ $6,-0.00568763,0.00158900,0.00018588,0.00039644,-0.00038571,0.00038963$, $-0.01768323,0.02840225,0.00090478,-0.05570313,-0.01844453,0.00155612,-$ $0.24465511,0.12713469,0.00807943,0.36743735,-0.00149999,0.00418527,0.0$ $0394222,0.00052502,-0.00301348,0.00176205,0.00013810,0.00048588,0.0000$ $2716,0.00037689,-0.00279883,-0.00192609,-0.00159622,0.00423713,-0.0036$ $2689,0.00265189,-0.00476468,-0.00017342,0.00011536,0.00047779,-0.00079$ $901,0.00000046,-0.00022432,-0.00022108,0.00170447,-0.00308301,-0.00013$ $646,0.00014394,0.00044016,0.00079092,-0.00373648,0.00115286,0.00013460$ $, 0.00001436,-0.00025613,0.00021980,0.02764605,-0.05810376,-0.00191277$, $0.03523097,0.05831585,-0.00002080,0.10141361,-0.16462125,-0.00527481,-$ $0.12375050,0.55203842,0.00253278,-0.00219647,-0.00172600,-0.00053983,0$ $.00170914,0.00003574,0.00003345,-0.00008123,-0.00067711,0.00046618,-0$. $00144618,0.00002349,-0.00220576,0.00190400,-0.00110542,-0.00030162,-0$. $00003857,0.00090414,-0.00019893,-0.00016830,0.00046307,-0.00010068,0.0$ $0004247,0.00005940,-0.00004833,0.00012466,0.00054390,0.00021312,0.0001$ $2166,0.00040175,0.00006069,-0.00001042,-0.00046708,0.00007978,-0.00002$ 
$920,0.00005015,0.00092768,-0.00192740,-0.00735126,0.00276814,0.0014374$ $1,0.01709091,0.00749249,-0.00596871,-0.05392187,-0.01073996,0.01420730$ $, 0.07626107,0.00149383,-0.00373802,-0.00362231,-0.00108835,0.00279796$, $-0.00176025,0.00086335,-0.00243260,-0.00007626,-0.00099307,0.00262093$, $0.00189449,0.00165030,-0.00387006,0.00343298,-0.00566798,-0.00118649,0$ $.00008263,-0.00001388,-0.00043681,0.00060968,-0.00020009,0.00013358,0$. $00026584,-0.00174694,0.00448902,0.00014728,-0.00006624,-0.00040513,-0$. $00061607,0.00045797,-0.00096581,-0.00003231,-0.00018937,0.00018678,-0$. $00023670,-0.05943942,0.03234875,0.00259599,-0.01609187,-0.02958709,-0$. $00036794,0.02641868,0.02876743,0.00004189,-0.08053965,0.01264755,0.000$ $93101,0.65846944,0.00006273,-0.00070478,-0.00094202,0.00091908,0.00010$ $881,0.00021265,-0.00014951,0.00070899,0.00003465,0.00095043,0.00010952$ ,-0.00031313,0.00009742,-0.00070827,0.00095141,-0.00239778,0.00012945, $0.00005420,-0.00001952,0.00002079,0.00008332,0.00000008,0.00001403,-0$. $00002967,0.00007657,-0.00103315,-0.00003783,-0.00004435,0.00002013,-0$. $00008585,0.00005663,0.00004365,-0.00000080,0.00001420,0.00001441,0.000$ $05367,-0.02754852,0.06146075,0.00198823,-0.02436471,-0.04622952,-0.000$ $29377,0.05557740,-0.02947515,-0.00221122,-0.01529836,-0.33241548,-0.00$ $589686,-0.05160915,0.64811781,0.00035047,0.00019218,0.00013523,0.00048$ $380,0.00027220,0.00011307,-0.00004401,0.00010643,-0.00040643,-0.000400$ $24,-0.00042551,0.00000720,-0.00039055,-0.00001520,-0.00000610,0.000085$ $83,0.00002670,-0.00025582,0.00000724,-0.00000481,0.00002471,-0.0000642$ $8,-0.00003882,0.00003639,0.00005831,-0.00015667,0.00007168,-0.00001208$ $, 0.00003150,0.00006178,-0.00000920,0.00002746,0.00013741,0.00007773,0$. $00003466,0.00005016,0.00120119,0.00033410,0.00790200,-0.00024027,-0.00$ $014994,-0.00482868,0.00063996,-0.00153587,0.00317923,0.00029951,-0.006$ $61649,-0.05646821,-0.01538315,0.01305177,0.13758827,0.00026815,0.00014$ $905,0.00013296,0.00022116,0.00005470,0.00014231,-0.00021628,0.00045324$ $, 0.00003136,0.00018860,0.00008345,-0.00016391,0.00026285,0.00012393,-0$ $.00013830,0.00071079,-0.00010671,-0.00001522,-0.00004707,-0.00004766,-$ $0.00004131,-0.00000185,-0.00000852,-0.00003672,0.00017297,-0.00061712$, $-0.00002460,-0.00005093,-0.00003881,0.00004649,0.00001792,0.00010235$,$0.00000472,0.00001145,-0.00001097,0.00004028,-0.24137183,0.07619583,0$. $00659588,0.02861476,0.06729279,0.00100591,-0.05988561,0.00003096,0.001$ $52985,0.02659319,-0.07615102,-0.00204228,-0.27256828,-0.04237558,0.004$ $74887,0.62961438,0.00077726,-0.00105630,-0.00103834,-0.00021994,0.0010$ $1921,-0.00045382,-0.00012855,-0.00019380,-0.00000823,-0.00022059,0.001$ $03796,0.00055928,0.00087019,-0.00112181,0.00088656,-0.00102248,-0.0004$ $7678,0.00004524,-0.00005245,-0.00015370,0.00017854,-0.00005881,0.00005$ $424,0.00004418,-0.00049084,0.00097127,0.00004486,-0.00004381,-0.000145$ $63,-0.00018035,0.00005361,-0.00020163,-0.00000893,-0.00007465,0.000044$ $95,-0.00005952,0.14117900,-0.18713462,-0.00669295,0.02264648,-0.032792$ $57,-0.00154855,0.00752217,-0.00126682,-0.00018346,-0.02371852,-0.02467$ $415,-0.00025454,-0.11831925,-0.15861357,0.00104953,-0.00135826,0.73109$ $310,0.00016509,0.00017842,0.00014075,-0.00005175,0.00010517,-0.0000162$ $1,0.00001211,-0.00004184,0.00010351,0.00005376,-0.00002816,0.00003233$, $-0.00018479,-0.00024837,0.00020145,-0.00003925,0.00001133,-0.00019236$, $-0.00001179,-0.00005314,-0.00001216,0.00002303,0.00000261,0.00000230,-$ $0.00002429,0.00005061,-0.00002953,0.00002488,0.00003522,-0.00002782,0$. $00000987,-0.00001258,0.00006230,-0.00002819,-0.00001341,-0.00001603,0$. $00807446,-0.00488962,-0.06321624,-0.00002521,-0.00276759,0.00878909,0$. 
$00168752,0.00004909,-0.00400776,-0.00080909,0.00115111,0.01579209,0.00$ $299240,-0.00102501,-0.06330642,-0.01377578,0.01383209,0.12588728,0.000$ $30989,-0.00072085,-0.00081468,-0.00031433,0.00050915,-0.00052780,0.000$ $46778,-0.00084375,-0.00003346,-0.00028535,0.00048518,0.00057439,0.0003$ $5800,-0.00075738,0.00075634,-0.00073784,0.00001553,0.00002310,0.000049$ $95,-0.00007821,0.00010446,-0.00000728,0.00007539,0.00008093,-0.0006982$ $1,0.00128072,0.00005038,0.00004715,-0.00007308,-0.00010973,0.00025428$, $-0.00008042,-0.00000929,-0.00001409,0.00007983,-0.00008093,-0.10956918$ $,-0.03722060,0.00156803,-0.02011983,-0.00947506,0.00042699,0.00258674$, $-0.00355642,-0.00003136,0.00284932,0.00013082,-0.00011676,-0.00179316$, $0.00865482,0.00036818,-0.03916446,-0.02505281,0.00059393,0.16543211,0$. $00016752,-0.00040037,-0.00042945,-0.00016438,0.00030732,-0.00028807,0$. $00024258,-0.00047915,-0.00001800,-0.00014912,0.00029530,0.00031501,0.0$ $0019297,-0.00042234,0.00039850,-0.00034726,0.00002734,0.00001043,0.000$ $02503,-0.00004225,0.00005463,-0.00001149,0.00004026,0.00004679,-0.0003$ $7028,0.00074481,0.00002770,0.00002408,-0.00004007,-0.00005810,0.000151$ $88,-0.00003799,-0.00000447,-0.00001607,0.00004171,-0.00004674,-0.03717$ $713,-0.05826750,0.00015346,-0.03818828,-0.01534438,0.00061095,0.004725$ $63,-0.00468061,-0.00034640,0.00057213,0.00206221,0.00007273,-0.0004096$ $2,-0.00011579,-0.00009560,0.00373752,0.00389928,-0.00009631,0.06845732$ $, 0.07061071,-0.00000116,-0.00002835,-0.00001717,-0.00000398,-0.0000118$ $3,0.00001700,-0.00000860,0.00001546,-0.00001612,0.00001348,-0.00000993$ ,-0.00000612,-0.00000521,0.00004734,-0.00003294,0.00001138,0.00000122, $0.00002574,-0.00000290,0.00000112,0.00000489,-0.00000388,0.00000136,-0$ $.00000032,0.00001169,-0.00001866,0.00001717,-0.00000007,0.00000424,0.0$ $0000570,-0.00000415,0.00000150,-0.00000808,0.00000299,0.00000022,0.000$ $00478,0.00157194,0.00015519,-0.02071420,-0.00023013,-0.00017602,0.0034$ $7985,0.00015793,-0.00010987,0.00467108,-0.00010571,0.00008266,-0.00172$ $139,0.00015801,-0.00035008,0.00456544,0.00124956,0.00069560,0.00359422$ - $-0.00263675,-0.00050546,0.01115547,0.00020003,-0.00046346,-0.00048807$ $,-0.00013437,0.00034211,-0.00027940,0.00012693,-0.00027439,-0.00000819$ ,-0.00011772,0.00032301,0.00030705,0.00023275,-0.00048909,0.00045766,$0.00044685,0.00001441,0.00000618,-0.00000231,-0.00005766,0.00010148,-0$ $.00001312,0.00003657,0.00003694,-0.00031286,0.00063301,0.00002478,-0.0$ $0000440,-0.00005348,-0.00010277,0.00007532,-0.00007867,-0.00000301,-0$. $00001720,0.00004292,-0.00003984,0.00050651,-0.00464679,-0.00005186,-0$. $25450271,0.12901089,0.00898227,-0.01680455,0.01631774,0.00092532,-0.00$ $297512,-0.00243281,0.00019373,-0.00049718,-0.00121987,-0.00003589,0.00$ $229215,-0.00100696,0.00005509,0.00181008,-0.00060724,-0.00013715,0.269$ $50187,0.00002054,-0.00008002,-0.00006970,-0.00000316,0.00006723,-0.000$ $03452,0.00000183,-0.00003365,-0.00000323,-0.00001391,0.00009033,0.0000$ $5102,0.00003152,-0.00009361,0.00004094,-0.00008423,0.00002962,0.000019$ $98,-0.00000990,-0.00001200,0.00001248,-0.00000152,0.00000074,0.0000036$ $9,-0.00002531,0.00007281,0.00000332,-0.00000687,-0.00000969,-0.0000127$ $1,0.00000033,-0.00001063,-0.00000231,-0.00000482,-0.00000905,-0.000006$ $39,0.02766885,-0.01134099,-0.00107604,0.12343988,-0.13301220,-0.005643$ $29,-0.01269707,0.00994369,0.00047835,-0.00244158,0.00042911,-0.0000579$ $5,-0.00081693,-0.00043215,0.00003165,-0.00190817,-0.00541246,-0.000190$ $71,0.00023799,0.00109926,0.00008349,-0.13513821,0.13843534,-0.00006707$ $, 0.00010208,0.00007510,-0.00000384,-0.00005972,-0.00000256,-0.00000102$ $, 0.00000719,0.00006673,0.00001224,0.00004216,-0.00001181,0.00005835,-0$ 
$.00008488,0.00004562,0.00000852,0.00000354,-0.00008918,-0.00000393,0.0$ $0000634,-0.00000583,0.00000537,-0.00000156,-0.00000397,0.00000647,-0.0$ $0001517,-0.00002919,0.00000392,-0.00000250,-0.00000271,-0.00000333,0.0$ $0000163,0.00000149,-0.00000545,0.00000067,-0.00000228,0.00068797,-0.00$ $018465,0.00251128,0.00885076,-0.00580141,-0.03306500,0.00027091,-0.000$ $30270,0.00387219,0.00019288,-0.00006477,0.00604640,-0.00002904,0.00004$ $049,-0.00079762,0.00003650,-0.00021614,0.00533438,-0.00011887,0.000104$ $93,-0.00275741,-0.00980536,0.00632188,0.02282409,0.00004296,-0.0001424$ $1,-0.00008175,-0.00004447,0.00000767,-0.00008925,-0.00000078,0.0000195$ $1,-0.00000784,-0.00004321,0.00000946,0.00010964,0.00004549,-0.00014013$ $, 0.00005121,-0.00000961,0.00016805,0.00001520,-0.00000209,0.00000317,0$ $.00004681,0.00001539,0.00000061,0.00000160,-0.00004327,0.00007570,0.00$ $000763,0.00000209,0.00000453,-0.00004993,-0.00014436,-0.00019197,0.000$ $00124,0.00000719,-0.00000644,-0.00000931,-0.00290177,-0.00146683,0.000$ $21441,0.00070477,-0.02861274,-0.00052979,-0.05220083,-0.00805801,0.000$ $25337,0.00318725,0.02580122,0.00048216,-0.00344552,0.00451094,0.000397$ $57,-0.00141824,-0.00004874,0.00002063,-0.00124555,-0.00158565,0.000007$ $91,0.00105637,0.00044605,-0.00012066,0.05604520,-0.00013536,0.00029496$ $, 0.00038040,0.00008618,-0.00017932,0.00021887,-0.00011053,0.00015558,0$ $.00000931,0.00007975,-0.00015932,-0.00024070,-0.00015662,0.00030555,-0$ $.00034889,0.00034431,0.00016940,-0.00000816,-0.00001058,0.00003318,-0$. $00008438,0.00001708,-0.00001923,-0.00002219,0.00023961,-0.00043725,-0$. $00002116,-0.00001008,0.00002771,0.00008546,0.00018575,0.00004144,-0.00$ $000373,0.00002068,-0.00002495,0.00002828,-0.00254554,0.00178524,-0.000$ $03590,0.00161259,-0.01321547,-0.00046861,-0.01325639,-0.31840342,-0.00$ $607766,0.00272138,-0.01454829,-0.00038856,0.00319802,-0.00067671,-0.00$ $027198,0.00019663,0.00077544,0.00003369,-0.00045996,-0.00075010,-0.000$ $01302,0.00004915,0.00076004,0.00010225,0.00830763,0.34443610,0.0001672$ $5,-0.00024431,-0.00006077,0.00000987,0.00013304,-0.00001814,-0.0000048$ $5,0.00000838,-0.00012337,0.00000084,-0.00016236,-0.00005917,-0.0001802$ $5,0.00026269,-0.00005026,0.00001232,0.00000464,0.00009180,0.00001811,-$ $0.00000250,-0.00003216,-0.00000369,0.00000067,-0.00000369,0.00000600$,$0.00001084,0.00004738,-0.00002282,0.00001302,-0.00003016,0.00000520,0$. $00000570,0.00001206,0.00000390,0.00000587,0.00001065,0.00020709,-0.000$ $07155,0.00650793,0.00014673,0.00035897,0.00514020,0.00013977,-0.006210$ $87,-0.03581213,-0.00005169,-0.00102238,-0.00107536,0.00037161,-0.00032$ $375,0.00744401,0.00001973,0.00004801,-0.00075286,0.00003381,0.00002133$ $, 0.00027955,-0.00012627,0.00009118,-0.00378477,-0.00069799,0.00709626$, $0.02351451,0.00014462,-0.00107174,-0.00045129,0.00031325,0.00063020,0$. $00023264,-0.00081610,0.00083993,0.00005564,0.00030572,0.00071786,-0.00$ $021800,0.00020947,-0.00115477,0.00039369,-0.00136515,-0.00017912,0.000$ $02524,0.00001269,0.00007071,-0.00008014,0.00008299,0.00000066,-0.00011$ $580,0.00012668,-0.00076902,-0.00002622,0.00001853,0.00006228,0.0000779$ $0,0.00008557,0.00006176,-0.00000270,0.00008220,-0.00001898,0.00010968$, $-0.00159989,-0.00319567,0.00014363,0.00025249,-0.00048428,-0.00003514$, $0.00117058,-0.00175752,0.00011221,0.00599570,0.00342559,-0.00007510,-0$ $.24754662,0.12072873,0.00842771,-0.01978638,0.01503100,0.00104370,-0.0$ $0146613,0.00004292,0.00004710,-0.00006999,0.00000044,-0.00002536,0.000$ $29672,-0.00035934,-0.00002111,0.26242798,0.00021707,-0.00035213,0.0000$ $3388,-0.00023824,0.00046975,-0.00013159,-0.00017458,0.00027073,-0.0000$ $0395,-0.00023882,0.00040688,0.00017655,0.00018689,-0.00030501,-0.00007$ 
$378,0.00037758,-0.00074214,-0.00001386,-0.00003312,-0.00003952,0.00000$ $713,0.00006172,0.00002395,-0.00003311,-0.00002669,0.00003446,0.0000092$ $7,-0.00002795,-0.00003590,-0.00000411,-0.00007076,-0.00005186,0.000002$ $98,0.00006337,0.00003108,0.00002492,-0.00220192,0.00022999,-0.00008576$ ,-0.00113847,-0.00068007,0.00003307,0.00041061,-0.00613138,-0.00031719 $, 0.02572582,-0.01569128,-0.00105833,0.12484021,-0.13051067,-0.00548449$ ,-0.01463038,0.00947712,0.00047965,-0.00141855,-0.00019060,0.00003118, $-0.00000450,0.00005665,0.00002506,0.00090909,-0.00034747,-0.00002513,-$ $0.13296794,0.14331690,-0.00067139,0.00100968,0.00038457,-0.00021725,-0$ $.00095614,-0.00014178,0.00003554,-0.00002772,0.00061555,0.00018512,0.0$ $0093794,-0.00009027,0.00068927,-0.00098285,0.00028139,0.00002346,0.000$ $00895,-0.00080550,0.00002925,0.00007021,-0.00002400,0.00005074,0.00002$ $734,-0.00002293,-0.00000943,0.00002567,-0.00018282,-0.00003312,-0.0000$ $7193,-0.00003278,-0.00000705,-0.00000147,-0.00007687,-0.00005490,-0.00$ $002142,-0.00002616,0.00017686,-0.00005395,0.00657664,-0.00005155,0.000$ $00763,-0.00063628,0.00015383,-0.00024044,0.00674863,0.00043996,-0.0004$ $6232,0.00033299,0.00851448,-0.00537226,-0.03946029,0.00036116,-0.00032$ $206,0.00586244,0.00001328,-0.00000889,0.00018312,-0.00002472,0.0000252$ $3,-0.00101266,0.00001301,0.00000261,-0.00007682,-0.00949006,0.00631345$ $, 0.02550594,0.00024143,-0.00042859,-0.00040318,-0.00010455,0.00035008$, $-0.00022637,0.00011095,-0.00030890,-0.00001777,-0.00008249,0.00033283$, $0.00025815,0.00025604,-0.00043559,0.00036187,-0.00050874,-0.00007658,0$ $.00001555,-0.00001010,-0.00004792,0.00006344,-0.00002880,0.00001867,0$. $00004052,-0.00021152,0.00059040,0.00002161,-0.00000870,-0.00004879,-0$. $00006809,0.00005864,-0.00005178,0.00000023,-0.00003926,0.00001693,-0.0$ $0004177,0.00295635,0.02574072,0.00056952,-0.00406867,0.00263718,0.0003$ $1644,-0.00160049,-0.00019774,0.00001207,-0.00247354,-0.00279928,0.0001$ $8804,0.00253405,-0.02662168,-0.00056669,-0.05445743,-0.01626308,0.0001$ $9367,0.00125008,0.00068692,-0.00009231,0.00009636,0.00130424,0.0000500$ 4,0.00009626,-0.00001435,-0.00003355,0.00112506,0.00037954,-0.00012650 $, 0.05486905,0.00016408,-0.00029698,-0.00033347,-0.00011635,0.00025881$, $-0.00022607,0.00017736,-0.00031932,-0.00000130,-0.00010975,0.00024189$, $0.00021983,0.00018375,-0.00031551,0.00033075,-0.00025104,-0.00010463$,$0.00000419,0.00000703,-0.00005449,0.00006075,-0.00003108,0.00001447,0$. $00004238,-0.00021840,0.00056872,0.00001441,-0.00000652,-0.00004682,-0$. $00005844,0.00005447,-0.00004464,-0.00000682,-0.00001989,0.00002706,-0$. $00003256,-0.00621044,-0.01383184,-0.00019249,0.00343964,0.00115404,-0$. $00019071,-0.00072524,0.00044789,0.00006081,-0.00200307,0.00036996,-0.0$ $0009664,0.00174674,-0.00983311,-0.00036278,-0.01116726,-0.33512871,-0$. $00656223,-0.00018966,0.00164294,0.00010265,-0.00029669,-0.00053837,-0$. $00002472,-0.00001072,-0.00002309,0.00002394,0.00034629,0.00076052,0.00$ $009275,0.01523677,0.35505119,0.00008741,-0.00011021,-0.00005386,-0.000$ $00620,0.00007314,0.00000129,-0.00000208,0.00000840,-0.00005275,-0.0000$ $0052,-0.00008190,-0.00000101,-0.00008342,0.00012420,-0.00005449,0.0000$ $0882,-0.00000843,0.00010042,-0.00000149,0.00000853,-0.00000582,0.00001$ $398,-0.00000087,-0.00001177,0.00000299,-0.00000699,-0.00000128,-0.0000$ $0034,-0.00000688,-0.00000263,-0.00000190,0.00000138,-0.00000271,-0.000$ $01403,0.00000285,-0.00001260,-0.00015529,-0.00107211,0.00247119,0.0003$ $4390,-0.00017537,0.00550934,0.00000677,0.00002907,-0.00080153,0.000194$ $81,-0.00006053,0.00650861,0.00008576,0.00041186,0.00421300,0.00030198$, $-0.00641699,-0.03422476,-0.00011420,0.00008104,-0.00273025,0.00001205$, 
$-0.00006634,0.00078770,-0.00003587,0.00002783,-0.00116845,-0.00012820$, $0.00009417,-0.00390213,-0.00051493,0.00714415,0.02343450 \backslash 10.00000237,-$ $0.00000582,0.00000272,0.00001386,0.00000922,-0.00000959,-0.00000126,0$. $00000527,0.00000137,0.00000260,-0.00000015,-0.00000024,-0.00000316,0.0$ $0000702,0.00000506,-0.00000675,-0.00000052,-0.00000014,-0.00000157,0.0$ $0000029,-0.00000398,-0.00000406,0.00000034,0.00000022,0.00000427,0.000$ $00131,-0.00000122,-0.00000085,0.00000293,0.00000042,-0.00000560,0.0000$ $0778,0.00000477,0.00000084,0.00000405,0.00000051,0.00000381,-0.0000031$ $7,0.00000562,-0.00000205,-0.00000631,-0.00000567,-0.00000189,-0.000004$ $56,0.00000127,0.00000061,0.00000379,-0.00000111,0.00000345,-0.00000429$ ,-0.00000016,-0.00000160,-0.00000209,-0.00000258,0.00000060,-0.0000068 $9,-0.00000131,-0.00000206,-0.00000569,0.00000035,-0.00000315,-0.000003$ $25,0.00000229,-0.00000090,0.00000387,0.00000186,0.00000249,-0.00000315$ ,-0.00000043III@

para-Complex

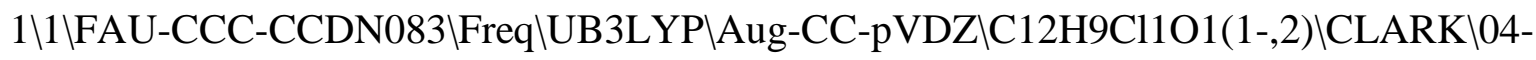
Mar-2015\0\॥N Geom=AllCheck Guess=TCheck SCRF=Check GenChk UB3LYP/Aug -CC-pVDZ Freq \Pre-addition complex p-Cl-Ph-radical + phenolate (para) II-1,2\C,-2.0885458242,1.5709989273,0.1053066808\C,-2.4633673655,0.880 0863375,1.2821308155\C,-3.1186147793,-0.3373463006,1.2287347471\C,-3.4 $836368035,-0.9669283306,-0.0299606735 \backslash \mathrm{C},-3.0946953799,-0.2202459948,-1$ $.2153296378 \backslash \mathrm{C},-2.4396499323,0.9960970695,-1.1392699092 \backslash \mathrm{H},-1.6531654754$ ,2.5653266646,0.1571910552\H,-2.2125856201,1.3137528989,2.254129782\H, $-3.3943273465,-0.8662170631,2.1440327025 \mathrm{H},-3.3519720552,-0.6588978013$ $,-2.182224097 \mathrm{H},-2.170299197,1.5204649133,-2.0602629184 \backslash \mathrm{O},-4.093699642$ $8,-2.0747106428,-0.0889998932 \backslash C, 3.0462374911,-0.3012197386,0.064450048$ 6\C,3.0245584245,1.0908602733,0.1318503639\C,1.7753283691,1.7403316896 ,0.1514170507\C,0.5963955546,1.0007462643,0.1041978966\C,0.6395502205, $-0.387889232,0.0372003137 \backslash \mathrm{C}, 1.8742521475,-1.0583894081,0.0164702213 \backslash \mathrm{Cl}$ ,4.6217144457,-1.1390995705,0.0388575511\H,3.9577615252,1.6545038375,0 $.1681746035 \backslash \mathrm{H}, 1.7588917138,2.8333081143,0.2043302013 \backslash \mathrm{H},-0.2857276149,-$ $0.9698554956,0.0000983559 \backslash \mathrm{H}, 1.9254151057,-2.1464321739,-0.0358641275 \backslash \backslash$ Version=EM64L-G09RevA.02 $\backslash$ State $=2-A \backslash H F=-998.1700038 \backslash \mathrm{S} 2=0.761844 \backslash \mathrm{S} 2-1=0$. $\backslash \mathrm{S} 2 \mathrm{~A}=0.750098 \backslash \mathrm{RMSD}=5.282 \mathrm{e}-09 \backslash \mathrm{RMSF}=4.352 \mathrm{e}-06 \backslash$ ZeroPoint $=0.1685178 \backslash$ Therma $\mathrm{l}=0.1814398 \backslash \mathrm{ZPE}=105.7464867 \backslash \mathrm{Dipole}=3.1732909,1.4823646,0.1015194 \backslash \mathrm{Dipol}$ eDeriv $=-0.5233771,-0.022922,-0.0029966,-0.0028573,-0.1498087,0.0101349$ ,-0.0082441,0.0113968,-0.3461306,0.2376192,0.2710901,-0.0708477,0.6773 $779,0.1953972,-0.2112257,-0.4462127,-0.0554059,0.2081127,-0.4352516,-0$ $.0666218,0.0364299,-0.3999934,-0.3254034,0.0393552,-0.557204,-0.448823$ $9,-0.0260076,0.6558887,0.694402,0.0366674,1.0540069,1.3437384,0.068997$ $2,0.0587876,0.065573,0.1238598,-0.4445813,-0.0716169,-0.0487887,-0.453$ $5723,-0.359441,-0.079617,0.5098677,0.4146797,0.0209133,0.2321029,0.260$ $3314,0.0975627,0.6361679,0.1684857,0.2204581,0.5136282,0.0549626,0.243$ $6399,0.2267678,-0.1017612,-0.0034753,0.0392711,-0.056925,-0.0047195,0$. $0042952,-0.0052387,0.0275675,0.0910155,-0.06227,-0.067993,0.0151929,0$. $030917,-0.0863011,0.0512823,-0.0858381,-0.1393266,0.0863682,-0.0631156$ $, 0.0433026,-0.0965186,0.0165475,0.0974254,0.0783717,0.0770314,-0.11118$ $5,0.0879099,-0.0571132,-0.0452249,-0.0890585,0.0309448,-0.0854735,-0.0$ $866803,-0.0645837,-0.1293755,0.0861121,-0.0687556,0.0647306,0.0154678$, 
$0.0130388,0.1001857,-0.0441243,0.0990491,-0.1196865,-1.1318087,-0.6177$ 487,-0.0361984,-1.273566,-1.553754,-0.0674205,-0.0700374,-0.06094,-0.4 $138273,1.0728085,-0.4169864,-0.0088359,-0.5895173,0.2237745,0.0087775$, $-0.0148429,0.0098057,-0.0792412,-0.3684101,0.095912,0.0016269,-0.15238$ $13,-0.0914996,-0.0013725,-0.007372,0.000697,-0.1140071,-0.5586263,-0.0$ $582334,-0.0062041,1.0726354,0.3435896,0.0332546,0.0529131,0.0199211,-0$ $.1201241,2.1705961,0.2680793,0.0353133,-1.3266461,-0.4025414,-0.024432$ $6,-0.0377092,-0.0101512,-0.1753983,-1.146909,-0.1230094,-0.0159982,-0$. $0883219,-0.0058608,0.002515,-0.0275738,0.0070766,-0.0739149,-0.0480721$ $, 0.0531558,0.0016069,0.4725965,0.0786948,0.0134535,0.0271792,0.0085402$ $,-0.0866582,-1.0195312,0.2723389,0.0043473,0.4740642,-0.2751342,-0.002$ $8537,0.0134832,-0.0045899,-0.1238612,-0.2729291,-0.1232942,-0.0098696$, $-0.0526337,0.0281893,-0.0049819,-0.0087784,-0.0049173,0.1243369,-0.021$ $3658,-0.0016942,-0.001207,-0.1655063,-0.2099011,-0.017389,-0.0123129,-$ $0.0140251,0.1091979,0.0937091,-0.0659124,-0.0026052,0.084579,0.033852$, $-0.0013001,0.0066901,-0.0038635,0.0816512,-0.0700354,0.0057455,-0.0013$ $431,0.149213,-0.0769007,-0.0074699,0.0045937,-0.0103557,0.1194648 \backslash$ Pola $\mathrm{r}=389.0371964,40.1737828,189.0863829,4.2737771,2.7961425,139.5820841 \mathrm{PP}$ $\mathrm{G}=\mathrm{C} 01[\mathrm{X}(\mathrm{C} 12 \mathrm{H} 9 \mathrm{Cl1O} 1)] \backslash \mathrm{NImag}=0 \backslash 10.20682721,0.23470988,0.60890446,0.0073$ $5600,0.00264819,0.60119818,-0.07542128,-0.04440866,0.03118585,0.258760$ $85,-0.04150296,-0.14592629,0.05118183,0.23489488,0.56369062,0.04912241$ $, 0.09914758,-0.24725993,0.02094559,0.02828777,0.67772937,-0.00275019,-$ $0.01867852,0.02537600,-0.12751629,-0.13674982,-0.01764226,0.25011416$,$0.02097107,-0.02615765,0.05104417,-0.13746647,-0.30595241,-0.03310883$, $0.25525796,0.58208361,0.01011838,0.02160534,0.02127308,0.00640951,0.01$ 203450,-0.12208257,-0.02219999,-0.05514787,0.61983366,-0.00244062,0.00 $724656,0.00077823,-0.00569992,-0.01165136,-0.01143103,-0.08154730,-0.0$ $1679461,-0.02235198,0.31764702,0.00796721,0.00533943,0.00256455,-0.011$ $05934,-0.01950747,-0.02269204,-0.01651883,-0.10280477,-0.03614372,0.23$ $877524,0.62420813,0.00082316,0.00257189,-0.04655160,-0.02564160,-0.046$ $71648,0.03060912,-0.01029135,-0.01342872,-0.22112979,0.00924459,0.0063$ $3647,0.54094440,-0.00201461,-0.01566396,-0.02741406,-0.00677418,-0.011$ $17066,0.00919249,0.01409012,0.01242215,0.00143714,-0.08223624,-0.01836$ $701,0.01131537,0.24895276,-0.01885755,-0.01880402,-0.05521658,-0.01076$ $653,-0.02397268,0.01887540,0.01240954,0.03126389,0.00429067,-0.0193273$ $1,-0.10857936,0.02385996,0.25121160,0.57145568,-0.01240327,-0.02575023$ $, 0.01317565,0.00235390,0.00498401,-0.00959359,0.00061501,0.00195307,-0$ $.03107431,0.02329962,0.04658542,-0.21472960,0.03911378,0.05539395,0.63$ $165007,-0.07382454,-0.03952560,-0.03167811,0.01218015,0.01258718,-0.00$ $385634,-0.00652947,-0.01011698,-0.00326498,-0.00639825,-0.01381186,0.0$ $2367269,-0.12745522,-0.13668064,-0.01966832,0.25932124,-0.03585450,-0$. $13243580,-0.04079497,0.01239137,0.03392620,-0.00913564,-0.01009785,-0$. $02156145,-0.00633000,-0.01354856,-0.02562649,0.04103563,-0.13733408,-0$ $.30600048,-0.03241370,0.23682769,0.56963926,-0.04928345,-0.08885179,-0$ $.26224025,0.00637668,0.01781978,-0.05362690,-0.01010368,-0.02025423,-0$ $.01226371,0.00942382,0.01701912,0.03739921,0.00439426,0.01276446,-0.12$ $205475,-0.00650267,-0.03404951,0.67115801,-0.06993977,-0.11773891,-0.0$ $0576441,-0.00420565,-0.00728568,-0.00079319,0.00406681,-0.00057933,0.0$ $0202511,-0.00052071,-0.00021263,-0.00000842,0.00410593,-0.00035509,-0$. $00190622,-0.00401671,-0.00684542,-0.00006083,0.07822554,-0.11914032,-0$ $.29149792,-0.01246791,-0.00470394,-0.00939947,-0.00461732,-0.00126373$, 
$0.00137889,0.00341500,0.00074614,0.00055114,0.00010231,-0.00116783,0.0$ $0179667,-0.00285164,-0.00375657,-0.00733005,0.00332092,0.12717871,0.30$ $518666,-0.00584271,-0.01245168,-0.05623094,0.01046583,0.02505073,0.005$ $33773,0.00055976,0.00134627,-0.00424036,0.00005197,0.00009874,-0.00155$ $018,-0.00051691,-0.00077908,-0.00470040,-0.01103874,-0.02640285,0.0030$ $4342,0.00632189,0.01323468,0.05564102,0.00445693,0.00312328,0.00811475$ ,-0.05410057,-0.03286197,-0.05617426,0.00123735,-0.00613589,-0.0142409 $5,0.00648811,-0.00541015,0.00185658,-0.00070416,0.00031839,-0.00004125$ $, 0.00374415,-0.00261619,0.00123779,-0.00218036,0.00129544,-0.00024087$, $0.04635986,0.00356989,0.00888982,0.01327769,-0.03299716,-0.09362968,-0$ $.09357474,-0.00657775,-0.00639078,-0.02686278,-0.00529356,-0.00071473$, $0.00323225,0.00022061,-0.00044913,0.00000131,-0.00266717,0.00026722,0$. $00263093,0.00154587,-0.00006825,-0.00064884,0.03974803,0.09397419,-0.0$ $0785235,-0.01361240,-0.01856715,-0.05446387,-0.09427998,-0.26911143,0$. $00053587,0.00119822,0.00014991,0.00031737,0.00091027,0.00282543,0.0003$ $8506,0.00067641,0.00072524,0.00209856,0.00396669,-0.00350064,0.0000553$ $9,0.00022066,0.00106670,0.05984545,0.10238301,0.28671119,0.00450928,-0$ $.00559970,-0.00114445,-0.00097630,-0.00550927,0.01266306,-0.05364077,-$ $0.04224623,0.05767918,0.00556638,0.00159818,-0.00640813,0.00285466,-0$. $00205323,-0.00126008,-0.00040826,0.00015029,-0.00014455,0.00051689,-0$. $00066476,0.00088629,-0.00363998,0.00251562,-0.00004474,0.04795141,-0.0$ $0504472,-0.00192852,-0.00200578,-0.00548611,-0.00878053,0.02422988,-0$. $04232986,-0.11130946,0.11120191,0.00122952,0.00719629,-0.01096874,-0.0$ $0207275,0.00040880,-0.00216837,0.00015369,-0.00031951,-0.00037946,-0.0$ $0065736,-0.00043002,0.00100901,0.00239778,-0.00035615,-0.00035030,0.04$ $883776,0.11468625,-0.00042801,-0.00068383,0.00244554,-0.00208524,-0.00$ $348265,0.00342478,0.05746455,0.11062348,-0.25057186,0.01043359,0.01970$ $796,-0.01831249,-0.00201376,-0.00355206,-0.00428652,-0.00070457,-0.001$ $19244,-0.00014678,-0.00005682,-0.00019525,0.00003788,0.00034590,0.0004$ $4207,0.00118446,-0.06277587,-0.12133657,0.26338323,0.00448658,-0.00570$ $456,0.00065063,-0.00042565,0.00012541,0.00013829,0.00278239,-0.0022484$ $1,0.00119231,0.00564746,0.00131526,0.00701608,-0.05140276,-0.03470088$, $-0.05727293,-0.00076903,-0.00433573,-0.01319272,0.00054060,-0.00057260$ $,-0.00093542,-0.00059599,0.00032777,-0.00005921,-0.00031328,0.00039345$ $, 0.00038618,0.04549638,-0.00510227,-0.00213447,0.00144219,0.00006586,-$ $0.00047984,0.00035875,-0.00231191,-0.00017022,0.00250278,0.00196095,0$. $00779634,0.01351389,-0.03479844,-0.09140840,-0.09653661,-0.00516868,-0$ $.00668110,-0.02529097,-0.00063616,-0.00034748,-0.00105561,0.00033904,-$ $0.00016249,-0.00008665,0.00041783,0.00021141,0.00080693,0.04059724,0.0$ $9286888,-0.00003205,0.00012403,0.00266233,0.00069966,0.00115574,0.0000$ $1410,0.00193939,0.00390440,-0.00365284,-0.00980589,-0.01716680,-0.0189$ $6129,-0.05706622,-0.09597327,-0.27271389,0.00151336,0.00244032,0.00111$ $966,0.00001284,0.00014565,-0.00006054,-0.00003565,-0.00007342,-0.00005$ $623,-0.00032245,-0.00063472,-0.00143335,0.06257923,0.10570239,0.287646$ $94,0.00442985,0.00355159,-0.00732934,0.00381903,-0.00237925,-0.0013586$ $8,-0.00069297,0.00032850,0.00004069,0.00654338,-0.00519253,-0.00230287$ $, 0.00096311,-0.00741206,0.01358599,-0.05629590,-0.04027830,0.05667263$, $-0.00217485,0.00127682,0.00030126,0.00033834,0.00002519,-0.00032932,-0$ $.00060121,0.00032002,0.00006388,-0.00362650,0.00241418,-0.00020673,0.0$ $4873049,0.00300937,0.00859105,-0.01061853,-0.00242145,0.00087520,-0.00$ $226634,0.00025296,-0.00037603,-0.00010306,-0.00516302,-0.00027804,-0.0$ 
$0363941,-0.00702979,-0.00875851,0.02586594,-0.04022828,-0.11304769,0.1$ $0775128,0.00154360,-0.00009462,0.00057149,0.00001618,0.00033859,-0.000$ $57009,0.00032002,-0.00018426,0.00007287,0.00251966,-0.00033451,-0.0005$ $4098,0.04767111,0.11514478,0.00862305,0.01629035,-0.01828469,-0.002202$ $88,-0.00361791,-0.00415582,-0.00040435,-0.00076285,0.00064995,-0.00073$ $640,-0.00131157,0.00234916,-0.00118671,-0.00221764,0.00276474,0.054956$ $37,0.10846340,-0.24750878,0.00002590,-0.00030407,0.00110116,0.00037085$ $, 0.00073571,-0.00130497,0.00008455,0.00008169,-0.00004267,0.00018411,0$ $.00025424,0.00116495,-0.06013249,-0.11789472,0.26319023,-0.00315954,-0$ $.00385269,-0.00029953,0.00597238,0.00290139,-0.00337842,-0.00032183,-0$ $.03313114,-0.01688382,-0.16186085,-0.17831862,-0.00923154,-0.00107675$, $-0.03544967,0.01344497,0.00597033,0.00267005,0.00396965,-0.00001465,0$. $00009186,-0.00000432,-0.00154423,-0.00026296,-0.00031847,-0.00200574,0$ $.00222906,-0.00057694,-0.00200649,0.00219903,0.00066705,-0.00156789,-0$ $.00032428,0.00026629,0.16140783,-0.00475377,-0.00735400,-0.00082213,0$. $00270071,0.00944039,-0.00562280,-0.03291844,-0.04056374,-0.03035896,-0$ $.17804828,-0.38766450,-0.01592975,-0.03575443,-0.04741938,0.02479409,0$ $.00291475,0.00937225,0.00758799,0.00080317,-0.00001969,-0.00002698,-0$. $00021005,-0.00170211,-0.00058090,0.00211794,0.00082300,-0.00124268,0.0$ $0217584,0.00085718,0.00095192,-0.00031128,-0.00190300,0.00044204,0.241$ $27439,0.46636205,-0.00034776,-0.00081042,0.00856824,0.00379349,0.00680$ $673,-0.01048369,-0.02561033,-0.04657248,0.00316815,-0.00921083,-0.0159$ $3411,-0.09230357,0.02215087,0.04100154,0.01079807,-0.00321173,-0.00484$ $440,-0.01039285,0.00003742,-0.00003173,0.00075015,-0.00088891,-0.00163$ $135,-0.00048214,0.00086374,0.00134726,0.00426649,-0.00077547,-0.001632$ $99,0.00421744,0.00085226,0.00149434,-0.00026761,0.01234807,0.02083956$, $0.08172447,0.00052273,-0.00017489,0.00000314,-0.00046808,-0.00062522,0$ $.00049696,0.00021044,0.00050938,0.00047935,-0.00021142,-0.00050702,-0$. $00001611,0.00021199,0.00055357,-0.00042806,-0.00045719,-0.00055996,-0$. $00056272,-0.00017421,-0.00006935,-0.00000726,-0.00000708,-0.00008109,-$ $0.00006840,-0.00000535,0.00005548,-0.00005521,-0.00000443,0.00003906,0$ $.00005427,-0.00000480,-0.00009350,0.00005948,0.00054108,0.00084988,0.0$ $0004218,0.50983626,-0.00018683,-0.00014388,-0.00002741,-0.00010388,0.0$ $0008907,-0.00024186,-0.00023558,-0.00000078,-0.00019194,0.00033869,0.0$ $0059189,0.00003050,-0.00023746,-0.00001585,0.00019992,-0.00010709,0.00$ $007179,0.00025325,-0.00000161,0.00015475,0.00000963,0.00005772,0.00000$ $076,0.00005788,0.00000149,-0.00001712,0.00003973,-0.00000062,-0.000015$ $43,-0.00004905,0.00005181,0.00000310,-0.00005072,-0.00037282,-0.000670$ $58,-0.00003825,0.07627889,0.61843718,0.00000547,-0.00000021,0.00006532$ ,-0.00007554,-0.00003846,0.00000150,0.00000514,0.00004987,-0.00003405, $0.00001040,0.00002373,0.00004857,-0.00001464,-0.00005062,-0.00003267,0$ $.00006173,0.00001864,-0.00000525,-0.00000946,0.00000457,0.00001398,0.0$ $0001319,-0.00001010,-0.00001074,-0.00001687,0.00001026,0.00000377,0.00$ $001227,-0.00000187,0.00000332,-0.00001734,0.00001361,-0.00000461,-0.00$ $001163,-0.00001974,-0.00000637,0.00743876,0.02496464,0.12078150,-0.000$ $26213,-0.00029898,-0.00001053,0.00009768,0.00043090,-0.00053313,-0.000$ $19299,-0.00010958,-0.00034534,0.00019292,0.00040282,0.00001455,-0.0002$ $1313,-0.00012916,0.00032658,0.00007444,0.00035239,0.00056307,-0.000033$ $84,0.00036388,0.00001817,0.00000231,0.00002892,0.00015408,-0.00000521$, $-0.00004958,0.00004423,0.00000326,-0.00004098,-0.00004390,0.00000943,0$ $.00004968,-0.00015054,-0.00039947,-0.00075113,-0.00003338,-0.10209833$, 
$-0.04499292,-0.00256369,0.69013466,-0.00092487,0.00005535,0.00007109,0$ $.00052685,0.00098202,-0.00060532,0.00002471,-0.00066180,-0.00047457,-0$ $.00013855,0.00010283,-0.00002487,-0.00000978,-0.00063219,0.00039476,0$. $00042626,0.00080733,0.00063488,0.00003096,0.00002275,-0.00000260,-0.00$ $009912,0.00005330,0.00011641,-0.00004138,-0.00004596,0.00003490,-0.000$ $02040,-0.00004264,-0.00001860,-0.00004400,0.00008406,-0.00011840,-0.00$ 031207,-0.00064826,-0.00001411,0.01945620,-0.33069862,-0.01284961,0.04 $863387,0.66615495,-0.00002939,0.00001080,-0.00024692,-0.00033732,-0.00$ $033814,0.00013110,0.00000656,0.00016486,0.00001929,0.00001632,0.000001$ $18,0.00018808,-0.00003923,-0.00026206,0.00002051,0.00036484,0.00038949$ $, 0.00023351,0.00000192,0.00001746,0.00001529,0.00005043,0.00001402,-0$. $00000250,0.00001506,0.00000330,-0.00001396,-0.00001883,0.00000652,-0.0$ $0001247,-0.00004605,0.00001541,-0.00002582,-0.00002521,-0.00002474,-0$. $00009834,0.00056554,-0.01343130,-0.06353284,0.00780777,0.02682363,0.12$ $449529,-0.00167338,-0.00069309,-0.00011287,0.00100274,0.00204595,-0.00$ $162535,-0.00042225,-0.00159937,-0.00101007,0.00010999,0.00054877,0.000$ $04797,-0.00041806,-0.00173478,0.00085303,0.00102456,0.00195146,0.00188$ $458,0.00066531,0.00076668,0.00004526,-0.00009057,0.00030861,0.00044639$ ,-0.00003093,-0.00007883,0.00008944,-0.00004447,-0.00006798,-0.0001142 $1,-0.00012889,0.00033233,-0.00041036,-0.00081438,-0.00171910,-0.000103$ $84,0.02366636,0.08406044,0.00425804,-0.26391565,0.04353208,0.00020195$, $0.58906715,0.00242948,0.00036894,0.00005363,-0.00126879,-0.00286195,0$. $00237594,0.00057924,0.00202760,0.00166091,-0.00049371,-0.00125610,-0.0$ $0005838,0.00062727,0.00218998,-0.00146726,-0.00121498,-0.00263477,-0.0$ $0265811,-0.00017077,-0.00083917,-0.00005160,-0.00000193,-0.00030612,-0$ $.00058031,0.00012157,0.00013704,-0.00019569,0.00011503,0.00013144,0.00$ $022822,-0.00000451,-0.00035491,0.00054180,0.00159301,0.00324757,0.0001$ $7319,0.02367973,-0.02528400,-0.00140443,0.11740667,-0.15620519,-0.0034$ $4275,0.01681376,0.69219393,0.00011942,0.00002610,0.00021675,0.00017758$ $, 0.00000229,-0.00002185,0.00011223,0.00017419,0.00014258,-0.00002942,-$ $0.00003442,-0.00034710,-0.00004369,-0.00003330,-0.00000085,-0.00026371$ $,-0.00025132,-0.00030179,-0.00001904,-0.00004366,0.00012334,-0.0000543$ $6,-0.00002713,-0.00005334,-0.00001034,0.00001295,-0.00000744,0.0000129$ $7,0.00001748,0.00001349,0.00003237,0.00001203,0.00001674,0.00007277,0$. $00014430,0.00010972,0.00128866,-0.00080547,0.00810942,0.00376204,-0.00$ $419993,-0.06256826,0.00527735,0.02745583,0.13122022,0.01414838,0.00224$ $308,0.00033889,-0.00916404,-0.00992752,0.00934636,0.00226790,0.0074224$ $8,0.00373269,-0.00192124,-0.00377702,-0.00022743,0.00231885,0.00777047$ ,-0.00298951,-0.00900534,-0.00905212,-0.01047133,-0.00564461,-0.001620 $86,-0.00013767,0.00025598,-0.00099201,-0.00155966,0.00039438,0.0003413$ $2,-0.00037169,0.00039749,0.00031766,0.00045152,0.00029406,-0.00110545$, $0.00143726,0.00365062,0.00724253,0.00039936,-0.01813054,-0.02884329,-0$ $.00154682,-0.05589950,0.01963238,0.00020695,-0.24705153,-0.12624439,-0$ $.00806793,0.37021696,-0.00276449,-0.00470576,-0.00033260,0.00155728,0$. $00427260,-0.00353062,-0.00048801,-0.00287181,-0.00198200,-0.00005278,0$ $.00056131,0.00006721,-0.00057738,-0.00320486,0.00169929,0.00173470,0.0$ $0413448,0.00411679,0.00378270,0.00116026,0.00012180,-0.00011169,0.0004$ $6734,0.00081322,-0.00001123,-0.00020492,0.00019517,-0.00001723,-0.0002$ $0105,-0.00024099,-0.00013476,0.00055169,-0.00079707,-0.00178072,-0.003$ $10858,-0.00020071,-0.02807754,-0.05741094,-0.00268951,-0.03412508,0.05$ $821265,0.00168413,-0.10045075,-0.16231351,-0.00621920,0.12526753,0.548$ 
$96789,0.00000569,-0.00021729,0.00089013,0.00241112,0.00220904,-0.00134$ $535,-0.00049688,-0.00165565,-0.00012488,-0.00003901,0.00002961,-0.0006$ $8034,0.00052944,0.00149186,0.00017155,-0.00237709,-0.00187201,-0.00146$ $095,0.00011316,0.00004830,-0.00046376,-0.00022816,0.00013698,0.0004490$ $1,-0.00009348,-0.00003717,0.00005955,0.00009051,0.00003195,0.00004970$, $0.00018553,-0.00015112,0.00041905,-0.00004868,-0.00010531,0.00054080,-$ $0.00147454,-0.00270187,-0.00742713,-0.00237032,0.00212432,0.01721767,-$ $0.00668156,-0.00649758,-0.05396651,0.00884366,0.02419409,0.07690271,-0$ $.00555152,0.00125969,-0.00009703,0.00155672,0.00379612,-0.00331103,-0$. $00119546,-0.00261065,-0.00189108,0.00107705,0.00241796,0.00016542,-0.0$ $0121881,-0.00284724,0.00163356,0.00158054,0.00357213,0.00376180,0.0004$ $4787,0.00106137,0.00006306,0.00003086,0.00045083,0.00070756,-0.0001853$ $7,-0.00014328,0.00021296,-0.00019485,-0.00013374,-0.00027224,-0.000031$ $55,0.00048806,-0.00064430,-0.00181282,-0.00444119,-0.00025303,-0.05962$ $841,-0.03108227,-0.00211156,-0.01559964,0.02915997,0.00136371,0.025675$ $18,-0.02937036,-0.00112573,-0.08071584,-0.01481654,-0.00088652,0.65996$ $193,0.00240378,0.00001804,0.00008254,-0.00013950,-0.00069324,0.0008782$ $9,-0.00088767,0.00016313,-0.00024623,0.00010388,0.00071537,0.00005311$, $-0.00086417,0.00013442,0.00022718,-0.00011494,-0.00064563,-0.00097996$, $-0.00001175,0.00001635,-0.00001043,0.00002533,0.00000055,-0.00009516,-$ $0.00000785,0.00000584,0.00003750,-0.00000513,0.00000169,-0.00003638,0$. $00005494,-0.00000235,0.00008694,-0.00008146,-0.00102664,-0.00005030,0$. $02875787,0.06134350,0.00285072,0.02406499,-0.04653628,-0.00181326,-0.0$ $5618731,-0.02856740,-0.00212319,0.01304183,-0.33207881,-0.01329334,0.0$ $5138034,0.64639066,0.00001893,-0.00004284,-0.00024630,0.00035595,-0.00$ $007943,0.00000885,0.00034743,-0.00032879,0.00002039,0.00005164,0.00006$ $523,-0.00039498,-0.00051733,0.00034501,0.00009409,-0.00037869,0.000161$ $26,0.00012368,0.00004414,0.00002144,0.00013624,0.00004128,0.00001522,0$ $.00007050,-0.00005900,0.00000851,0.00005247,0.00007581,-0.00006076,0.0$ $0003526,0.00001881,-0.00002621,0.00000719,-0.00003439,-0.00011432,0.00$ $006169,0.00077011,0.00226438,0.00804238,0.00108910,-0.00158663,-0.0049$ $1314,-0.00265615,-0.00179396,0.00312729,0.00060193,-0.01358725,-0.0569$ $6256,0.00729069,0.02532194,0.13817309,0.00081274,0.00017171,-0.0000350$ $1,0.00024788,-0.00025198,0.00019618,0.00018063,-0.00012174,0.00020764$, $-0.00017003,-0.00045668,-0.00002290,0.00017460,-0.00010850,-0.00018623$ $, 0.00028425,-0.00021434,-0.00019702,-0.00003424,-0.00007030,0.00000594$ $,-0.00004947,0.00007765,-0.00003161,-0.00001127,0.00004804,-0.00005409$ $,-0.00001167,0.00003750,0.00003687,-0.00008049,0.00007264,0.00005281,0$ $.00018982,0.00067407,0.00002918,-0.24362516,-0.07556651,-0.00538070,0$. $02772556,-0.06803318,-0.00308902,-0.05983418,0.00042485,-0.00047602,0$. $02728184,0.07578324,0.00380978,-0.27086624,0.04324057,0.00002086,0.630$ $22042,0.00104409,-0.00045000,-0.00006766,-0.00087033,-0.00121310,0.000$ $90904,0.00012484,0.00104530,0.00057525,0.00022326,-0.00022665,0.000000$ $07,0.00015688,0.00103388,-0.00046420,-0.00078170,-0.00107472,-0.001002$ $21,-0.00008382,-0.00015160,-0.00000784,0.00008608,-0.00011770,-0.00014$ $670,0.00008317,0.00004508,-0.00005335,0.00006729,0.00005054,0.00004891$ $, 0.00003111,-0.00014198,0.00014230,0.00050416,0.00098769,0.00004343,-0$ $.14066718,-0.18478422,-0.00723527,-0.02323481,-0.03192066,-0.00218058$, $-0.00692579,-0.00127033,0.00012868,0.02311800,-0.02537117,-0.00171836$, $0.11936364,-0.16009791,-0.00371808,0.00257664,0.72997771,0.00005603,0$. $00003711,-0.00018463,0.00016839,-0.00025708,0.00023763,0.00002492,-0.0$ 
$0000851,-0.00000012,-0.00007228,-0.00001520,0.00008697,0.00011054,0.00$ $013863,0.00001467,-0.00017828,0.00013496,0.00008722,-0.00003709,-0.000$ $05626,0.00005539,-0.00005351,0.00001405,-0.00005716,0.00001592,-0.0000$ $1396,-0.00000121,-0.00002382,0.00001814,0.00000282,-0.00001055,-0.0000$ $6546,0.00001588,0.00005192,0.00004615,-0.00000899,-0.00855979,-0.00663$ $616,-0.06329474,-0.00098849,-0.00270605,0.00873386,-0.00081797,0.00014$ $292,-0.00398740,0.00119885,-0.00126892,0.01569042,0.00383796,-0.004310$ $10,-0.06334960,0.00493945,0.02944163,0.12661527,-0.00073885,-0.0000119$ $5,0.00001007,0.00035429,0.00079344,-0.00073930,-0.00026563,-0.00047236$ ,-0.00058216,0.00044435,0.00084327,0.00003870,-0.00028245,-0.00050984, $0.00052693,0.00032276,0.00069726,0.00080374,0.00025302,0.00006121,0.00$ $000480,0.00003454,0.00006049,0.00009681,-0.00001667,-0.00007986,0.0000$ $7707,-0.00001076,-0.00007254,-0.00007968,0.00005033,0.00007506,-0.0000$ $9139,-0.00070833,-0.00127346,-0.00006420,-0.10887580,0.03765468,0.0009$ $7570,-0.01967546,0.00950563,0.00023367,0.00257819,0.00349087,0.0001328$ $8,0.00284617,-0.00016899,0.00002058,-0.00186921,-0.00860873,-0.0004376$ 6,-0.03903097,0.02544784,0.00081141,0.16419745,0.00035156,0.00002200,$0.00000061,-0.00018927,-0.00043447,0.00039442,0.00015871,0.00030064,0$. $00032683,-0.00024931,-0.00049162,-0.00002459,0.00016163,0.00032783,-0$. $00029507,-0.00017761,-0.00038989,-0.00043227,-0.00015664,-0.00003633,-$ $0.00000250,-0.00002379,-0.00004365,-0.00005601,0.00001441,0.00003835,-$ $0.00004838,0.00001391,0.00003518,0.00005227,-0.00002816,-0.00004844,0$. $00005053,0.00038488,0.00075208,0.00003954,0.03762980,-0.05890513,-0.00$ $149458,0.03819791,-0.01574967,-0.00056323,-0.00480772,-0.00466021,-0.0$ $0049716,-0.00056382,0.00207207,0.00017924,0.00040716,-0.00002143,-0.00$ $022399,-0.00333197,0.00367415,-0.00003645,-0.06928625,0.07180077,0.000$ $00650,-0.00000419,0.00002915,-0.00000498,0.00002612,-0.00001552,-0.000$ $00593,0.00001465,0.00001402,-0.00000645,-0.00001515,-0.00001724,0.0000$ $1310,0.00000872,-0.00000163,-0.00000850,-0.00004509,-0.00003792,-0.000$ $00243,-0.00000022,-0.00000905,-0.00000047,0.00000009,0.00000430,-0.000$ $00135,-0.00000154,0.00000201,0.00000431,0.00000109,0.00000330,0.000002$ $39,-0.00000386,0.00000509,0.00001116,0.00002197,0.00001837,0.00098228$, $-0.00149798,-0.02073932,0.00163501,-0.00083373,0.00345012,-0.00026140$, $-0.00041655,0.00465595,0.00002938,0.00017737,-0.00171576,-0.00005532,-$ $0.00030091,0.00456121,-0.00057639,0.00023904,0.00362538,-0.00189581,0$. $00228410,0.01118856,-0.00042483,0.00000441,-0.00001394,0.00021139,0.00$ $046559,-0.00043080,-0.00013970,-0.00034466,-0.00030468,0.00014929,0.00$ 028913,0.00001708,-0.00014344,-0.00037418,0.00027439,0.00020826,0.0004 $3104,0.00048180,0.00007481,0.00008915,0.00000650,0.00000234,0.00006821$ $, 0.00010636,-0.00001523,-0.00002523,0.00003734,-0.00001633,-0.00002491$ $,-0.00004617,-0.00000200,0.00007512,-0.00009607,-0.00032150,-0.0006226$ $9,-0.00003574,0.00025467,0.00455103,0.00020357,-0.25707215,-0.12783123$ ,-0.00835714,-0.01679753,-0.01605140,-0.00096880,-0.00300465,0.0024886 $0,0.00004154,-0.00040275,0.00117021,0.00003745,0.00237705,0.00098966,0$ $.00002830,0.00180566,0.00060457,0.00007135,0.27226794,0.00011353,0.000$ $04286,-0.00003302,-0.00005064,-0.00014832,0.00005824,-0.00004509,0.000$ $07730,0.00007594,0.00006137,-0.00004018,0.00000731,-0.00004042,0.00006$ $243,-0.00006035,-0.00002224,-0.00010935,-0.00004966,-0.00000575,0.0000$ $2290,0.00000416,0.00003458,0.00001097,0.00001157,0.00000983,0.00000391$ ,-0.00001312,0.00000436,0.00000191,0.00000101,0.00000484,0.00000159,-0 $.00000432,0.00002662,0.00009240,-0.00000110,-0.02781914,-0.01105220,-0$ 
$.00090945,-0.12221173,-0.13048728,-0.00590284,0.01297088,0.00994214,0$. $00045227,0.00241202,0.00048366,-0.00022095,0.00086615,-0.00052342,-0.0$ $0006180,0.00194749,-0.00539431,-0.00048888,-0.00024035,0.00108097,0.00$ $017966,0.13383518,0.13568347,-0.00000567,-0.00000788,-0.00009200,-0.00$ $007121,-0.00009535,0.00004201,-0.00001843,0.00005780,-0.00000275,0.000$ $01330,-0.00000525,0.00007261,-0.00000440,-0.00004760,-0.00001458,0.000$ $06100,0.00010035,0.00008358,0.00000926,0.00000708,0.00000028,0.0000055$ $0,-0.00000211,0.00000416,0.00000731,-0.00000074,-0.00000378,-0.0000025$ $0,-0.00000743,-0.00000455,0.00000953,0.00000304,-0.00001155,-0.0000049$ $5,-0.00000274,-0.00003185,-0.00136852,-0.00062165,0.00249354,-0.008081$ $40,-0.00594407,-0.03305802,0.00040305,0.00014503,0.00390057,0.00006787$ ,-0.00025685,0.00604233,0.00000685,0.00004365,-0.00080167,0.00005113,-$0.00054318,0.00531996,0.00004046,0.00019188,-0.00275414,0.00889157,0.0$ $0676917,0.02282529,-0.00004182,-0.00018826,0.00001396,0.00005437,0.000$ $18413,-0.00007933,-0.00002049,-0.00000275,-0.00011475,-0.00003269,-0.0$ $0000835,-0.00000359,-0.00002375,-0.00000470,0.00010718,0.00003870,0.00$ $016240,0.00008387,-0.00012611,0.00017690,0.00000418,-0.00000917,-0.000$ $01711,0.00003899,0.00001076,-0.00000420,0.00001163,0.00001335,-0.00000$ $335,-0.00000457,0.00000851,-0.00000948,-0.00004389,-0.00004579,-0.0000$ $9497,-0.00000235,-0.00284306,0.00148612,-0.00002734,0.00097140,0.02851$ $047,0.00133183,-0.05207203,0.00554758,0.00009190,0.00299311,-0.0260323$ $6,-0.00123891,-0.00360461,-0.00441680,-0.00027278,-0.00149742,0.000021$ $79,-0.00000833,-0.00121902,0.00159330,0.00006583,0.00103067,-0.0004789$ $5,0.00003172,0.05595925,-0.00032883,0.00017695,-0.00000743,0.00013108$, $0.00028983,-0.00034284,-0.00008255,-0.00016957,-0.00022276,0.00012274$, $0.00016947,0.00001677,-0.00009161,-0.00020475,0.00021148,0.00014631,0$. $00028391,0.00038099,-0.00018158,0.00004899,0.00000181,0.00001199,0.000$ $03416,0.00009120,-0.00002221,-0.00001344,0.00002128,-0.00002067,-0.000$ $01317,-0.00002770,0.00001103,0.00004263,-0.00008146,-0.00024534,-0.000$ $43289,-0.00002640,0.00257430,0.00176839,-0.00020309,-0.00175291,-0.013$ $46294,-0.00091257,0.01072829,-0.31806552,-0.01360484,-0.00291771,-0.01$ 423940,-0.00066752,-0.00313603,-0.00060061,-0.00043182,-0.00013839,0.0 $0079419,0.00006397,0.00046012,-0.00076666,-0.00004738,-0.00004773,0.00$ $076531,0.00021938,-0.00555433,0.34398028,-0.00002930,-0.00001277,0.000$ $08942,0.00018107,0.00028146,-0.00007312,-0.00000886,-0.00014825,-0.000$ $05598,0.00001233,0.00001004,-0.00012154,-0.00001036,0.00015243,-0.0000$ $2318,-0.00017094,-0.00022245,-0.00003020,-0.00000128,0.00000638,0.0000$ $1134,0.00002759,0.00000728,-0.00001965,-0.00000194,-0.00000200,0.00000$ $383,0.00000430,-0.00001250,0.00000140,-0.00001485,-0.00000839,-0.00004$ $360,-0.00001482,-0.00002894,0.00004605,0.00004165,-0.00022280,0.006506$ $67,-0.00012126,-0.00059107,0.00509977,0.00032618,-0.01365256,-0.036323$ $77,-0.00005133,-0.00090283,-0.00109318,-0.00031244,-0.00040717,0.00743$ $199,-0.00003694,0.00003329,-0.00074156,0.00001874,-0.00003769,0.000277$ $76,0.00003919,0.00019464,-0.00378031,0.00005576,0.01551340,0.02408686$, $-0.00140859,0.00016890,0.00003470,0.00022053,0.00118825,-0.00038440,0$. $00033452,-0.00067593,0.00015847,-0.00087965,-0.00083035,-0.00006372,0$. $00034435,-0.00062989,-0.00022441,0.00017304,0.00110016,0.00047411,0.00$ $009903,-0.00009355,-0.00000782,-0.00000011,-0.00008113,-0.00010245,0.0$ $0008508,-0.00000221,-0.00009375,0.00008363,-0.00000474,0.00011181,0.00$ $002139,-0.00008521,0.00008671,0.00014175,0.00075066,0.00004490,-0.0015$ $2165,0.00317085,0.00006276,0.00027993,0.00054449,0.00001233,0.00113003$ 
$, 0.00173336,0.00000567,0.00582364,-0.00373131,-0.00014207,-0.25014701$, $-0.11947136,-0.00775967,-0.01991772,-0.01479182,-0.00095226,-0.0014471$ $6,-0.00002731,-0.00001266,-0.00009744,-0.00003436,0.00001677,0.0003266$ $5,0.00033717,0.00003552,0.26518795,-0.00037335,-0.00072773,-0.00000299$ ,-0.00016092,-0.00029368,-0.00007611,0.00023764,0.00039854,0.00016951, $0.00018144,0.00026600,-0.00001722,0.00023012,0.00049838,-0.00011184,-0$ $.00022787,-0.00039091,0.00001148,0.00006233,-0.00005342,0.00000135,0.0$ 0002636,-0.00003380,-0.00000625,-0.00006389,0.00002236,0.00003703,-0.0 $0006192,0.00002817,-0.00003084,0.00002711,-0.00004137,0.00000485,0.000$ $03251,0.00003219,0.00001099,0.00220582,0.00019292,-0.00028750,0.001126$ $62,-0.00069780,0.00000528,-0.00047801,-0.00612167,-0.00062460,-0.02591$ $919,-0.01537666,-0.00100385,-0.12364850,-0.12804492,-0.00551448,0.0149$ $1348,0.00948155,0.00033236,0.00142678,-0.00020523,-0.00000544,0.000011$ $84,0.00005807,0.00004877,-0.00091702,-0.00034005,-0.00002524,0.1317548$ $9,0.14059378,-0.00001026,0.00000865,-0.00080827,-0.00070737,-0.0010264$ $1,0.00027614,-0.00018215,0.00095880,-0.00004999,0.00000942,-0.00002475$ $, 0.00062129,0.00019538,-0.00093851,-0.00017828,0.00067833,0.00096225,0$ $.00038701,0.00000254,0.00000930,-0.00007669,0.00003570,-0.00006392,-0$. $00002792,0.00004812,-0.00001408,-0.00002989,-0.00005474,0.00002966,-0$. $00002402,-0.00002903,0.00007501,-0.00002438,0.00000096,0.00002585,-0.0$ $0018634,0.00002551,-0.00027262,0.00657216,0.00006733,-0.00001556,-0.00$ $063453,-0.00005818,-0.00062145,0.00673286,-0.00124819,-0.00078267,0.00$ $030340,-0.00797769,-0.00547641,-0.03945382,0.00050791,0.00005312,0.005$ $88661,0.00004835,-0.00001666,0.00018195,0.00001376,0.00006775,-0.00101$ $246,-0.00005147,-0.00000370,-0.00007904,0.00867452,0.00686526,0.025522$ $59,-0.00054201,0.00006068,0.00003706,0.00027395,0.00050244,-0.00036382$ ,-0.00004897,-0.00031330,-0.00029067,0.00005295,0.00031252,0.00000549, $-0.00005281,-0.00032234,0.00024536,0.00023747,0.00043079,0.00039116,0$. $00006991,0.00001566,-0.00000319,-0.00002907,0.00002226,0.00004218,-0.0$ $0003328,-0.00003213,0.00004916,-0.00002891,-0.00002264,-0.00003626,-0$. $00000088,0.00003467,-0.00004800,-0.00021354,-0.00060514,-0.00002392,0$. $00281198,-0.02592083,-0.00127145,-0.00412411,-0.00252929,-0.00023667,-$ $0.00162569,0.00025843,-0.00002681,-0.00235212,0.00272677,0.00002619,0$. $00269393,0.02655294,0.00135156,-0.05436593,0.01351701,0.00045870,0.001$ $25354,-0.00067600,0.00001038,0.00005545,-0.00133820,-0.00005824,0.0001$ $2918,-0.00000315,0.00003197,0.00116780,-0.00038629,0.00001124,0.054671$ $16,0.00020670,-0.00014285,0.00003176,-0.00017504,-0.00027280,0.0002991$ $4,0.00014001,0.00025154,0.00020526,-0.00022875,-0.00030830,-0.00001920$ $, 0.00014953,0.00027764,-0.00019114,-0.00018837,-0.00025458,-0.00034370$ ,-0.00002482,-0.00007134,-0.00000966,-0.00000942,-0.00006231,-0.000080 $81,0.00003163,0.00001156,-0.00002290,0.00003292,0.00000806,0.00003789$, $0.00001660,-0.00006696,0.00006690,0.00022480,0.00054656,0.00003176,0.0$ $0608491,-0.01365461,-0.00074161,-0.00336180,0.00131404,-0.00026462,0.0$ $0068345,0.00050221,0.00003811,0.00215408,0.00020352,-0.00030336,-0.001$ $99362,-0.00995090,-0.00063262,0.00832569,-0.33487575,-0.01450688,0.000$ $20769,0.00164871,0.00021925,0.00024829,-0.00057481,-0.00005128,0.00004$ $993,-0.00004510,0.00007575,-0.00028956,0.00075528,0.00020110,-0.012284$ $85,0.35476118,0.00000917,-0.00002450,0.00009889,0.00006539,0.00009192$, $-0.00004253,-0.00002266,-0.00007359,0.00001218,0.00002069,-0.00000932$, $-0.00004511,-0.00001013,0.00007168,-0.00001033,-0.00010421,-0.00011860$ $,-0.00006454,0.00000964,0.00001430,-0.00000202,0.00001337,0.00000381,0$ 
$.00000296,0.00002000,0.00000866,-0.00001404,-0.00001139,-0.00000062,-0$ $.00001553,0.00001191,0.00001318,-0.00000963,-0.00000013,0.00002615,-0$. $00000791,0.00029271,-0.00103084,0.00244561,-0.00023995,-0.00021487,0.0$ $0550232,0.00001870,0.00006316,-0.00079059,0.00001685,-0.00027784,0.006$ $50410,-0.00010557,-0.00043904,0.00417573,0.00022819,-0.01447733,-0.034$ $74664,0.00005029,0.00021036,-0.00272656,0.00000313,-0.00007622,0.00078$ $333,0.00001046,0.00005922,-0.00116997,0.00002494,0.00021948,-0.0038983$ $9,-0.00030141,0.01596086,0.02401865 \backslash 0.00000378,0.00000089,0.00000262$, $-0.00000030,0.00000509,0.00000213,0.00000244,0.00000383,0.00000088,0.0$ $0000111,0.00000911,-0.00000248,-0.00000260,-0.00000067,0.00000225,0.00$ $000012,0.00000244,-0.00000334,0.00000316,0.00000308,-0.00000170,-0.000$ $00002,0.00000198,0.00000075,-0.00000238,0.00000239,-0.00000107,-0.0000$ $0154,0.00000496,-0.00000017,0.00000133,0.00000272,0.00000028,-0.000005$ $12,-0.00000013,0.00000099,-0.00000560,0.00000034,-0.00000190,0.0000041$ $3,-0.00000702,-0.00000028,-0.00000077,0.00000130,0.00000135,0.00002234$ ,-0.00000493,0.00000091,-0.00001447,-0.00000260,-0.00000237,-0.0000013 $5,-0.00000311,-0.00000016,0.00000030,-0.00000798,-0.00000006,0.0000019$ $0,-0.00000604,0.00000014,-0.00000435,-0.00000299,-0.00000024,-0.000000$ 47,0.00000046,0.00000176,-0.00000166,-0.00000312,-0.00000030॥!@

ortho Transition State

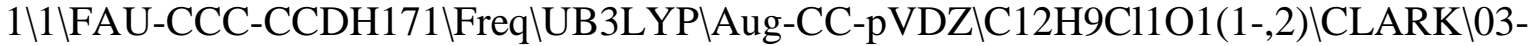
Mar-2015\0\\N Geom=AllCheck Guess=TCheck SCRF=Check GenChk UB3LYP/Aug -CC-pVDZ Freq I\Addition TS p-Cl-Ph-radical + phenolate anion (ortho)\\ $-1,2 \backslash \mathrm{C}, 2.220042389,0.0456740471,1.0851462771 \backslash \mathrm{C}, 2.5143927344,1.08982215$ $96,0.11019642 \backslash \mathrm{C}, 3.2015008252,0.6317736206,-1.0797899391 \backslash \mathrm{C}, 3.6250260678$ ,-0.680054764,-1.2274957831\C,3.4008179377,-1.6467365604,-0.2186281615 \C,2.7189693028,-1.2615281781,0.9352818397\Н,1.8022784761,0.3684299468 ,2.0378048024\O,2.1835702023,2.3012252519,0.290871534\H,3.4037907512,1 $.3761068706,-1.8533338785 \backslash \mathrm{H}, 3.762304146,-2.6692106,-0.3412129385 \backslash \mathrm{H}, 2.5$ $429183553,-1.9921325762,1.7300856046 \mathrm{H}, 4.1568730905,-0.9728107852,-2.1$ 382388554\C,-2.9063283394,-0.0030328227,-0.0368701482\C,-2.1673246764, $1.1817696894,-0.0276561934 \backslash \mathrm{C},-0.7866003266,1.1115145125,0.227572486 \backslash \mathrm{C}$, $-0.1929522434,-0.1221238733,0.465881026 \backslash \mathrm{C},-0.9363371319,-1.2956452735$, $0.4485274011 \backslash \mathrm{C},-2.3196169723,-1.2471120317,0.1936936196 \backslash \mathrm{Cl},-4.66233996$ $75,0.074060568,-0.3581353269 \backslash \mathrm{H},-2.6602942138,2.1358959389,-0.218885993$ $3 \backslash \mathrm{H},-0.1704417645,2.0165738435,0.2297970866 \backslash \mathrm{H},-0.4622477131,-2.2658796$ $978,0.6240609611 \mathrm{LH},-2.9267919292,-2.153178286,0.1725601597 \backslash \backslash V e r s i o n=E M$ 64L-G09RevC.01 $\backslash$ State $=2-A \backslash H F=-998.1698845 \backslash S 2=0.762963 \backslash S 2-1=0 . \mid S 2 A=0.750$ 106\RMSD $=8.360 \mathrm{e}-09 \backslash \mathrm{RMSF}=1.380 \mathrm{e}-05 \backslash$ ZeroPoint $=0.168406 \backslash$ Thermal $=0.1803323$ $\backslash \mathrm{ZPE}=105.676354 \backslash \mathrm{Dipole}=-1.853023,-1.4641797,-0.076241 \backslash \mathrm{DipoleDeriv}=-0.5$ $805537,-0.253789,-0.0617269,0.2189209,-0.4302963,-0.0763314,0.0689363$, $0.3107714,0.0054062,0.0805989,-0.4152297,-0.0734903,-0.0133797,1.57772$ $92,0.2528991,-0.0739424,0.2665927,0.0874616,-0.0570536,0.2596449,-0.07$ $77852,-0.2354011,-0.2666053,0.0496944,-0.2301515,-0.4998403,-0.0708514$ $,-0.1508348,-0.0699256,-0.0866485,0.5098461,0.3397422,-0.241123,-0.381$ $248,-0.1085254,0.1546803,-0.5735777,-0.0261184,0.0314732,0.0975639,-0$. $1467805,0.0088897,0.6790308,0.006808,-0.3241548,0.2732124,-0.079697,-0$ $.2383129,-0.6688317,0.1685551,0.0643518,-0.4320963,0.1089777,0.0969005$ $, 0.1741367,0.049168,0.073893,0.0384843,0.0186848,-0.0453975,0.0386157$, 
$-0.0358087,-0.0647041,-0.3975991,0.3080042,0.0170019,-0.1958469,-1.745$ $1321,-0.1965611,-0.0286564,-0.230874,-0.4187451,0.0925205,-0.0082451,0$ $.0602944,-0.0517831,-0.0578713,0.1243752,0.0893973,0.1066897,-0.049738$ $9,0.0914431,0.0937094,0.0546064,0.147389,-0.1785968,-0.0113509,0.07432$ $19,-0.0113709,0.0555164,0.094012,-0.0232054,0.0690387,0.0353397,-0.063$ $3971,0.1403917,0.000073,0.1270808,-0.0632981,0.0019445,0.0528446,0.140$ $7294,0.0880206,0.0357266,-0.0623825,0.1811497,-0.0662534,-0.1284019,1$. $2275195,-0.037832,0.1909659,-0.0774363,0.0485385,-0.0148743,0.2427854$, $-0.0276263,-0.0465027,-0.2080535,-0.0875093,-0.0003637,0.2609846,0.065$ $1755,-0.0325278,-0.0478648,-0.0471485,-0.0989581,-0.6821294,0.0136887$, $0.0553729,-0.3729632,-0.0060941,0.0098019,-0.0789212,-0.0116908,-0.072$ $6334,1.5664518,0.1541797,-0.0299879,-0.1108493,-0.189,-0.0016633,0.314$ $5821,0.0477758,-0.1247784,-0.5692952,-0.0963328,0.0556685,0.4634464,0$. $1822072,-0.0671924,-0.1358091,-0.0286371,-0.0962643,-0.2565995,0.09033$ $97,-0.0102477,-0.2025682,0.0178109,0.0074186,-0.0140601,0.0055724,-0.1$ $184846,-1.1011096,0.0395475,-0.1108892,0.0414646,-0.141695,0.0053037,-$ $0.175097,0.0076282,-0.1490508,-0.098925,0.0825065,-0.0161983,0.1294568$ $,-0.0672359,0.0270446,-0.0520945,0.0382243,0.1156767,0.1550046,-0.0509$ $724,-0.0062343,0.1026926,0.0288441,0.0048449,-0.0073667,0.0044727,0.09$ $53651,0.0498942,0.104847,-0.0367099,-0.0646711,-0.1333022,0.0462448,0$. $0008205,0.0368658,0.0924589,-0.1310071,-0.0996233,-0.0004496,-0.139878$ $7,-0.0570075,0.0081439,-0.0324048,0.000316,0.1231009 \backslash$ Polar $=304.8268282$ ,-5.9723141,189.0751193,-11.9670105,-6.2087183,136.4915155\PG=C01 [X(C 12H9Cl1O1)] $\backslash N I m a g=1 \backslash 0.18560886,-0.12688161,0.60672074,-0.20261980,0.0$ $1884667,0.56853572,-0.07633946,-0.01591429,0.04370982,0.28685689,-0.00$ $878301,-0.13503656,0.06203947,-0.11108352,0.71525508,0.03642580,0.0423$ $7502,-0.17507347,-0.14648253,0.00650410,0.47562377,0.00282971,-0.01252$ $487,0.01316475,-0.10635459,0.01689704,0.06094358,0.22536155,-0.0073713$ $4,0.03298888,0.01103013,0.00516929,-0.10615738,0.00860729,-0.10478239$, $0.70313829,0.01328180,0.01035711,-0.02241537,0.06776734,-0.01565633,-0$ $.20019146,-0.18009946,-0.09044137,0.51931216,-0.00166307,-0.00008440,0$ $.00124807,-0.00540911,0.01557479,0.00407341,-0.09342179,0.09816138,0.0$ $2888390,0.26718688,0.00571163,-0.03375350,0.00440281,0.02813495,-0.007$ $90192,-0.05601398,0.07391135,-0.34262157,0.00164697,-0.12503328,0.6399$ $0662,-0.00781274,0.01859547,-0.00746927,-0.00074828,-0.02948220,0.0190$ $4564,0.04016958,-0.04119908,-0.10906410,-0.21332711,-0.02946545,0.5779$ $9316,-0.01039870,0.03153914,0.00937835,-0.01016282,-0.00594332,0.01441$ $178,0.01724121,-0.00671089,-0.01114444,-0.07673599,0.00196896,0.034308$ $16,0.25334739,0.02021118,-0.00772855,-0.02689237,-0.00785518,0.0046703$ $8,0.01113724,0.00864121,-0.04625759,0.01513264,-0.02153329,-0.19570731$ $, 0.12375005,-0.14437063,0.66276173,0.02323192,-0.05589538,0.00895912,0$ $.01156048,0.00945534,-0.04143847,-0.02018943,0.04384584,0.02241163,0.0$ $4837177,0.07537233,-0.19880405,-0.20502262,-0.03562329,0.53941015,-0.0$ $7982901,0.07509495,0.03595484,0.00893053,0.00064203,-0.02428967,-0.009$ $49706,0.01515898,0.00692038,0.00181080,-0.02445417,0.02695217,-0.12591$ $563,0.05379744,0.10952413,0.26666649,0.07530255,-0.29663666,-0.0353388$ $8,0.00209764,-0.03318372,0.02615580,0.00714845,-0.01765806,-0.00321331$ $,-0.00797918,0.03249836,0.00104369,0.03136027,-0.12414230,-0.02304299$, $-0.11097744,0.62014145,0.02242106,0.01128868,-0.11248704,-0.01677807,0$ $.00708679,0.03309242,0.01149727,-0.02141367,-0.01113479,0.01650395,0.0$ $3454549,-0.04566813,0.12209793,-0.06872834,-0.26160455,-0.20899393,-0$. 
$08165873,0.59475823,-0.06680305,0.03412254,0.10219355,-0.00060113,0.00$ $332939,0.00709929,0.00261882,-0.00116275,0.00244062,-0.00003262,-0.001$ $32040,0.00041942,0.00563825,0.00318311,-0.00117091,-0.00613612,0.00439$ $974,0.01049179,0.07736662,0.03789328,-0.07688682,-0.07038766,-0.007376$ $78,0.00871181,0.01927298,-0.00029608,-0.00278902,0.00304570,-0.0002624$ $1,-0.00087237,0.00038461,0.00227622,-0.00445790,0.00106894,0.01016439$, $-0.00348659,-0.02603386,-0.04044455,0.07694699,0.10632395,-0.06982738$, $-0.27010090,0.00919071,-0.01318354,-0.01445481,0.00189121,0.00412400,-$ $0.00174189,-0.00052937,0.00124862,-0.00005617,0.00104373,0.00007663,0$. $00283085,-0.00046532,0.00320490,0.00126108,-0.11254826,0.07571178,0.28$ $134529,0.01839378,0.00361100,-0.00459279,-0.09533695,0.10006059,0.0241$ $4623,0.00141131,0.03591980,0.01800247,0.00295221,-0.00656721,0.0065484$ $1,0.00072037,0.00287332,-0.00383936,0.00122826,-0.00004703,0.00337523$, $-0.00215026,-0.00037375,-0.00217875,0.07791443,0.00335813,-0.07327311$, $0.01641233,0.09754110,-0.44087285,-0.05104656,0.02805775,-0.03826543,-$ $0.03470690,0.00014775,0.01060260,-0.00221031,0.00187682,-0.00761780,-0$ $.00047934,-0.00238626,0.00455096,0.00869784,0.00071041,0.00221536,0.00$ $040881,-0.13039001,0.54265161,-0.00636680,0.03045079,0.01667785,0.0244$ $8687,-0.05060456,-0.09334429,0.02103269,-0.05022399,-0.00251540,0.0035$ $8260,0.01047874,-0.00754903,-0.00335949,-0.00299946,0.00773219,0.00724$ $745,-0.00124149,-0.00620915,-0.00200210,-0.00243068,0.00287633,-0.0386$ $0624,0.06624663,0.08122787,0.00181156,0.00261623,0.00377969,0.00167571$ -0.01202355,0.01141446,-0.04498964,-0.03139928,0.04622571,0.00319930, $0.00760360,-0.00756474,0.00514599,0.00301201,0.00304912,-0.00047729,0$. $00004434,-0.00025171,-0.00066428,-0.00071535,0.00045225,-0.00160049,-0$ $.00014474,-0.00344509,0.03916147,0.00137447,0.00074132,-0.00105321,0.0$ $0317392,0.00507610,-0.00673413,-0.03088215,-0.17831705,0.13500374,-0.0$ $0719459,-0.01442782,0.02219988,0.00369255,-0.00329369,-0.00195078,0.00$ $017657,-0.00009992,-0.00057735,-0.00000560,0.00028282,-0.00037446,-0.0$ $0126318,0.00177668,0.00039329,0.03205151,0.18861460,0.00433742,-0.0021$ $8635,-0.00349320,0.00344906,0.02350849,-0.01241385,0.04606516,0.133682$ $43,-0.19034043,-0.00024341,-0.00463574,0.00427968,0.00237030,-0.000675$ $05,0.00337797,0.00034718,-0.00108686,-0.00045933,0.00009463,0.00104758$ ,-0.00112097,-0.00247789,-0.00205017,0.00263831,-0.05108800,-0.1471933 $8,0.19740233,0.00323236,0.00072959,0.00527100,-0.00038756,-0.00013911$, $0.00012600,0.00344321,0.00012657,0.00194816,0.00182163,-0.00609750,-0$. $00049443,-0.06186691,0.09238429,0.02143344,-0.00353221,0.01429007,-0.0$ $0089124,0.00034739,0.00113218,0.00018995,-0.00024888,-0.00010449,-0.00$ $046585,0.00069116,-0.00000660,0.00030327,0.06122852,0.00158677,0.00201$ $553,-0.00126186,-0.00042566,0.00062430,0.00036080,-0.00085514,0.000032$ $97,0.00453401,0.00789035,-0.01609356,-0.00675477,0.09248747,-0.2944471$ $4,-0.02566607,0.00050083,-0.00576162,0.00242550,0.00032658,-0.00030315$ $, 0.00012524,0.00002467,-0.00025939,-0.00027938,0.00052160,-0.00093387$, $-0.00010593,-0.10007173,0.31377344,0.00407839,0.00021163,-0.00274501,0$ $.00020663,0.00049895,-0.00117193,0.00265556,0.00248955,-0.00239661,-0$. $00892078,0.02097012,0.00553661,0.02165132,-0.02532152,-0.05328637,0.00$ $737004,-0.02524522,0.00129585,0.00071114,-0.00098805,-0.00000508,-0.00$ $035416,-0.00034018,0.00038245,0.00001700,0.00118833,0.00027511,-0.0240$ $6704,0.02758660,0.05178920,0.00488910,0.00842704,-0.00829833,0.0044647$ $8,0.00230987,0.00337340,-0.00046612,0.00022119,-0.00021420,0.00349985$, $0.00286682,0.00449937,0.00105790,-0.01120857,0.01030692,-0.04571955,-0$ 
$.02414777,0.04022027,-0.00247534,-0.00089506,-0.00123886,-0.00054483,0$ $.00058381,-0.00073937,-0.00066726,-0.00022365,-0.00040289,-0.00281149$, $-0.00081383,-0.00193672,0.03868286,-0.00595220,-0.01567052,0.02356578$, $0.00482069,-0.00135320,-0.00310222,-0.00006265,-0.00013632,-0.00015392$ $, 0.00188471,0.00132863,-0.00072820,0.00226335,0.00742370,-0.00704801,-$ $0.02332383,-0.17335738,0.13263087,-0.00101483,0.00082266,-0.00097155$,$0.00057096,-0.00235708,0.00115390,-0.00024222,-0.00008620,-0.00006806$, $-0.00142280,0.00065227,-0.00008063,0.02376350,0.18243518,0.00011097,-0$ $.00250456,0.00408731,0.00248619,-0.00123421,0.00286550,-0.00019837,-0$. $00082453,0.00042423,0.00506910,-0.00256833,-0.00257897,0.00200972,0.02$ $065349,-0.01355554,0.04043421,0.13114526,-0.19316027,-0.00172999,-0.00$ $004401,0.00020061,-0.00041240,0.00039797,-0.00029193,-0.00037243,0.000$ $03807,-0.00022048,-0.00173253,-0.00092766,0.00014392,-0.04631915,-0.14$ $488901,0.20303795,-0.00049215,-0.00015620,-0.00077664,0.00755739,0.002$ $77444,0.00131383,-0.00073550,0.00128343,0.01027107,-0.10244301,0.03903$ $455,0.11566682,-0.00009831,0.00319385,0.00390018,0.00426555,-0.0007423$ $2,0.00201336,-0.00048559,-0.00006706,-0.00023181,-0.00158418,0.0006714$ $1,0.00040194,-0.00338121,-0.00089059,-0.00245626,-0.00202104,-0.001124$ $84,-0.00133863,0.00002225,0.00015781,0.00041196,0.09943494,0.00016993$, $-0.00037007,-0.00023964,0.00195002,-0.00367156,0.00317206,0.01512307,-$ $0.00351952,-0.02613865,0.03880904,-0.07376614,-0.05634964,-0.01033862$, $0.00891314,0.01729405,0.00039837,-0.00221578,0.00333060,-0.00018957,0$. $00003601,-0.00012635,0.00065580,-0.00123481,-0.00173826,-0.00136869,0$. $00064651,-0.00017842,-0.00058695,0.00072372,-0.00094504,-0.00051992,0$. $00023999,0.00100458,-0.04409516,0.07418430,-0.00050586,0.00030069,0.00$ $047423,0.00223394,0.00070419,0.00449018,0.00256875,0.00114152,-0.00145$ $493,0.11575334,-0.05607239,-0.23671613,0.01120657,-0.01028446,-0.01431$ $471,0.00149116,0.00548312,-0.00125383,-0.00024050,-0.00013028,-0.00020$ $508,-0.00007025,-0.00083432,-0.00087354,-0.00221424,-0.00090499,0.0000$ $8036,-0.00157723,-0.00002483,0.00025739,0.00073415,-0.00025617,-0.0010$ $7038,-0.12918968,0.06090921,0.25058760,0.00075387,-0.00032067,-0.00025$ $033,0.00002204,-0.00027678,0.00000025,-0.00014223,0.00020167,0.0003296$ $4,0.00004744,-0.00058789,0.00037304,0.00048425,-0.00011945,-0.00085172$ ,-0.00043070,0.00076712,0.00036365,-0.00021216,-0.00000484,0.00009309, $0.00004705,0.00059922,0.00011583,-0.00000694,0.00007175,-0.00004811,-0$ $.00000620,-0.00014685,-0.00003293,-0.00005941,-0.00006953,0.00008686,0$ $.00006154,-0.00007390,-0.00011691,0.45540130,0.00012281,-0.00006344,-0$ $.00039771,0.00049747,-0.00051790,-0.00002465,-0.00016907,-0.00011457,0$ $.00001209,0.00001152,0.00015686,0.00009527,0.00001274,0.00028838,0.000$ $07908,-0.00011390,-0.00001102,0.00003335,-0.00001045,-0.00006183,0.000$ $12621,-0.00051886,0.00033496,0.00004081,0.00005162,0.00003572,-0.00002$ $448,-0.00001526,-0.00014270,0.00001954,-0.00004510,0.00002996,-0.00003$ $730,0.00001507,-0.00005209,-0.00006867,0.01559769,0.65276940,0.0001102$ $7,-0.00003470,0.00010534,0.00004379,-0.00000643,0.00004864,-0.00003652$ $, 0.00015943,-0.00006341,0.00002602,-0.00029922,0.00006914,0.00019532,-$ $0.00008603,-0.00019445,-0.00016385,0.00006284,0.00012799,-0.00008556,0$ $.00002335,-0.00009120,0.00000913,0.00011555,-0.00008155,-0.00001984,-0$ $.00001005,0.00005217,0.00001175,0.00002323,-0.00002526,-0.00000003,0.0$ $0004775,-0.00004213,-0.00001533,0.00003241,0.00000266,0.05850583,-0.05$ $190226,0.13477234,0.00009893,-0.00008214,0.00056531,-0.00018458,0.0007$ $1196,0.00007944,0.00026079,-0.00019425,-0.00014976,-0.00001532,0.00043$ 
$023,-0.00016423,-0.00016207,-0.00026735,0.00016248,0.00004392,-0.00005$ $910,-0.00014137,0.00010292,-0.00000073,-0.00022174,0.00006998,-0.00058$ $409,-0.00010621,-0.00003671,-0.00003757,0.00000831,0.00004710,-0.00001$ $339,0.00000689,0.00011569,-0.00006061,0.00008002,-0.00000731,0.0000068$ $3,0.00000739,-0.14767568,-0.06056220,-0.00919565,0.62634268,0.00210715$ ,-0.00023517,-0.00053926,0.00088290,-0.00320100,-0.00041778,-0.0004755 $2,0.00115661,0.00116964,-0.00005896,-0.00176686,0.00056999,0.00068258$, $-0.00039028,-0.00122815,-0.00018549,0.00107556,0.00032770,-0.00025265$, $0.00007811,0.00014692,-0.00260514,0.00234478,0.00020709,0.00002293,0.0$ $0002329,-0.00003179,-0.00002125,0.00022550,-0.00006805,-0.00002473,-0$. $00010044,0.00005962,-0.00000210,0.00001817,0.00007158,-0.12468466,-0.2$ $7816999,0.00021706,-0.02692173,0.70547153,-0.00073792,0.00023365,0.000$ $35031,0.00008313,0.00030329,-0.00004412,0.00026484,0.00004877,0.000029$ $11,0.00005951,0.00027867,-0.00026129,-0.00021188,-0.00000756,0.0003073$ $6,0.00010138,-0.00045091,-0.00022949,0.00029049,0.00003414,-0.00001165$ $, 0.00021863,-0.00038888,-0.00007189,-0.00007075,-0.00005143,0.00004962$ $, 0.00001337,0.00001905,0.00004790,-0.00000783,0.00003140,0.00004107,-0$ $.00004140,0.00004097,0.00002438,-0.00244769,0.01185734,-0.06457326,0.0$ $9308048,-0.06634619,0.14909266,-0.00727523,0.00184602,0.00106910,-0.00$ $336504,0.00410527,-0.00045625,0.00174331,-0.00129772,-0.00185414,-0.00$ $028036,0.00329693,-0.00160858,-0.00178520,0.00088183,0.00405825,0.0016$ $2342,-0.00375358,-0.00162305,0.00035227,0.00019504,-0.00027105,0.00018$ $911,-0.00437875,-0.00092068,-0.00012819,-0.00022012,0.00003459,-0.0000$ $9166,0.00034794,0.00007786,-0.00017297,0.00030817,-0.00044178,-0.00019$ $054,0.00015013,0.00018501,-0.03641811,-0.07564927,-0.00009850,-0.30482$ $475,0.04269167,-0.04745742,0.61029293,-0.00130432,0.00128286,0.0004864$ $5,-0.00162053,0.00133424,0.00037347,0.00000396,-0.00071159,-0.00084486$ $, 0.00012087,0.00127633,-0.00077989,-0.00092642,0.00093192,0.00121162,0$ $.00058598,-0.00102212,-0.00003421,-0.00006389,-0.00004058,-0.00032764$, $0.00199113,-0.00199028,0.00020551,-0.00006676,-0.00005549,-0.00007463$, $-0.00002856,0.00000385,0.00000725,0.00005110,0.00007851,-0.00015168,0$. $00004523,-0.00000780,0.00006835,-0.01663248,0.03785628,-0.00604714,-0$. $03060826,-0.11692794,0.00011628,0.05001288,0.68057885,-0.00161342,0.00$ $078135,0.00034068,-0.00007783,0.00132586,0.00025067,0.00020948,-0.0003$ $6670,-0.00027715,-0.00005977,0.00065172,-0.00015745,-0.00011248,0.0000$ $3784,0.00068809,0.00013560,-0.00087709,-0.00034219,0.00003420,0.000107$ $68,-0.00020813,0.00032400,-0.00080832,0.00062268,0.00001005,-0.0000210$ $5,-0.00004374,-0.00001437,-0.00001709,-0.00001151,0.00001016,-0.000026$ $26,-0.00015977,-0.00003539,-0.00003380,-0.00001271,-0.00599899,-0.0167$ $6189,0.00900724,-0.03988591,0.01318302,-0.07230471,0.07730193,-0.05168$ $991,0.15733293,0.03358105,-0.00199516,-0.00285930,-0.00694880,-0.02051$ $833,0.00714783,-0.00240726,0.00107030,0.00188308,0.00022765,-0.0064941$ $1,0.00584203,0.00684307,-0.00341638,-0.01356734,-0.01825867,0.01939308$ $, 0.00399228,-0.00763006,-0.00112148,-0.00081845,-0.00703984,0.01484591$ $, 0.00016090,0.00045848,0.00050273,-0.00052122,0.00030691,-0.00057052,-$ $0.00037971,0.00061182,-0.00100085,0.00128661,0.00039420,-0.00041799,-0$ $.00048598,-0.00395528,-0.00402598,0.00096896,-0.01800226,0.02568476,-0$ $.00912083,-0.12267353,0.10211219,-0.02203092,0.30370665,0.00168327,-0$. $00447598,-0.00045462,-0.00065228,-0.00307105,-0.00069051,0.00018433,-0$ $.00062712,0.00001808,-0.00039241,0.00139648,-0.00001905,-0.00017100,-0$ $.00048761,0.00295922,0.00481705,-0.00140071,-0.00158668,-0.00173624,-0$ 
$.00014661,-0.00055947,-0.00066068,0.00100283,0.00032754,-0.00005609,-0$ $.00007377,0.00010858,-0.00025397,0.00004461,0.00004643,-0.00022554,0.0$ $0010248,-0.00026790,-0.00013757,0.00013419,0.00001457,-0.00350016,-0.0$ $7247246,0.00584594,0.07706777,0.02302277,0.01358761,0.07474221,-0.2882$ $2401,0.03811135,0.02100427,0.62718333,0.01088426,-0.00288759,-0.006900$ $72,-0.00082012,-0.00234530,0.00297067,-0.00023558,0.00008070,0.0001667$ $3,-0.00054472,-0.00153309,0.00203396,0.00180957,-0.00041602,-0.0038186$ $1,-0.00344856,0.00620667,0.00269191,-0.00623122,-0.00061151,0.00008677$ $,-0.00077983,0.00168130,-0.00002421,0.00006301,0.00008989,-0.00009160$, $-0.00003885,-0.00011692,-0.00011130,0.00008392,-0.00029304,0.00047035$, $0.00015474,-0.00010784,-0.00008594,0.00099233,0.00623013,-0.00800703,-$ $0.01410702,0.00386999,0.01348681,-0.01949188,0.04317058,-0.06497468,0$. $03822688,-0.05184477,0.08910375,-0.00514445,0.00004805,0.00183307,-0.0$ $0172025,0.00357067,-0.00032814,0.00144567,-0.00151062,-0.00187011,-0.0$ $0033024,0.00333107,-0.00146200,-0.00168386,0.00042756,0.00452660,0.002$ $94628,-0.00418529,-0.00249038,0.00020869,-0.00014007,-0.00063678,0.002$ $67585,-0.00403679,-0.00030765,-0.00018116,-0.00023827,0.00019255,-0.00$ $007522,0.00031785,0.00013031,-0.00017820,0.00036416,-0.00035511,-0.000$ $19887,0.00026044,0.00019425,-0.02461379,0.08106352,-0.01426288,-0.0453$ $9949,-0.02782841,-0.00433403,0.04919623,-0.01903675,0.00949971,-0.1488$ $3012,-0.09364700,-0.00734718,0.58964871,0.00338716,0.00070570,-0.00062$ $117,0.00082311,-0.00221338,-0.00000556,-0.00090641,0.00084824,0.001460$ $48,0.00008103,-0.00224267,0.00098890,0.00107784,-0.00104084,-0.0026081$ $0,-0.00072801,0.00252379,0.00031241,-0.00036963,0.00004263,0.00017326$, $-0.00136536,0.00286938,0.00041942,0.00008003,0.00012848,-0.00009012,0$. $00019695,-0.00011552,-0.00010002,0.00010834,-0.00043992,0.00069005,0.0$ $0011622,-0.00013552,-0.00008026,0.02249076,0.02602415,0.00253974,-0.02$ $107498,-0.01469778,-0.00297545,0.00579908,-0.05066649,0.00692033,-0.12$ $082751,-0.27198917,-0.00074248,-0.01972107,0.68874648,-0.00152373,-0.0$ $0015517,0.00075049,-0.00041649,0.00118407,-0.00022119,0.00029075,-0.00$ $053462,-0.00059599,0.00002289,0.00085019,-0.00032787,-0.00022380,0.000$ $13942,0.00117153,0.00062156,-0.00095641,-0.00052896,0.00021487,-0.0000$ $2542,-0.00045674,0.00074538,-0.00090878,0.00005392,-0.00004147,-0.0000$ $5348,0.00004061,-0.00010213,0.00008788,0.00000283,-0.00000748,0.000041$ $68,-0.00027977,-0.00006202,0.00006383,0.00005313,-0.00814770,0.0130383$ $4,0.00560954,-0.00544404,-0.00402985,-0.00523173,0.00721543,0.00192213$ $, 0.00493428,-0.00502770,0.00396121,-0.05557905,0.08447248,-0.05661812$, $0.15188631,0.00075983,0.00031199,-0.00020994,0.00049550,-0.00116009,-0$ $.00002511,0.00001953,0.00004374,-0.00005466,-0.00003318,0.00011328,-0$. $00009963,-0.00018899,0.00031496,0.00060717,0.00049573,-0.00022760,-0.0$ $0019278,-0.00010961,-0.00008823,-0.00004344,-0.00085031,0.00052081,0.0$ $0006679,0.00000580,-0.00002506,0.00002510,-0.00003402,0.00008887,0.000$ $00178,-0.00013840,0.00013016,-0.00015562,-0.00005592,0.00005832,0.0000$ $4132,-0.12822902,0.04250493,-0.01599834,0.04966897,0.01599990,0.006116$ $60,-0.03919035,0.02513810,-0.00920740,-0.02986676,-0.07429227,-0.00077$ $484,-0.30135375,0.04438326,-0.04742487,0.63428278,-0.00151419,0.000147$ $22,0.00050392,-0.00059519,0.00079059,-0.00000802,0.00043552,-0.0007711$ $0,-0.00059498,-0.00006495,0.00156406,-0.00047955,-0.00067826,0.0004158$ $7,0.00156051,0.00069605,-0.00114555,-0.00056183,0.00022272,-0.00008440$ ,-0.00012876,0.00063321,-0.00104493,-0.00014646,-0.00000191,-0.0000300 $8,-0.00006038,-0.00005680,-0.00007778,0.00003525,-0.00008393,0.0001198$ 
$6,-0.00026259,-0.00001256,-0.00000781,-0.00003398,0.10599290,-0.295237$ $61,0.04219882,-0.02818700,-0.05589604,0.00144989,0.03071069,-0.0197869$ $3,0.00707958,-0.02164119,0.03126674,-0.00531127,-0.02809906,-0.1143545$ $9,-0.00046747,0.04994573,0.70231128,-0.00026369,0.00023055,0.00054465$, $0.00010036,-0.00047390,-0.00018183,-0.00001224,0.00009508,0.00010013,0$ $.00008051,-0.00007814,-0.00005149,-0.00017923,0.00007550,0.00006415,0$. 00015430,-0.00020614,-0.00014800,0.00031298,0.00001545,0.00004406,-0.0 $0029638,0.00031877,0.00000239,-0.00000955,0.00000177,0.00002424,0.0000$ 0206,-0.00000556,0.00000927,-0.00001195,0.00002890,0.00008018,-0.00003 $015,0.00001401,0.00000789,-0.02242526,0.03048583,-0.07001586,0.0106326$ $3,0.00968623,0.00998731,-0.00938736,0.00622371,-0.00725161,-0.00622543$ ,-0.01493158,0.01752371,-0.04035365,0.01273633,-0.07148219,0.08728835, $-0.04768069,0.14401523,-0.00103268,0.00025683,0.00021042,-0.00001744,0$ $.00054623,-0.00006090,0.00026788,-0.00038965,-0.00040418,-0.00006303,0$ $.00096073,-0.00041155,-0.00056694,0.00017187,0.00107690,0.00048292,-0$. $00104835,-0.00049630,0.00022162,0.00002219,0.00005042,-0.00029466,-0.0$ $0085828,-0.00013916,-0.00000801,-0.00007648,0.00006202,-0.00001423,0.0$ $0007394,0.00005679,0.00006470,0.00007437,-0.00005027,-0.00007633,0.000$ $05662,0.00009433,-0.12287561,0.00380680,-0.01875777,-0.03845413,0.0046$ $6570,-0.00809210,0.00163312,-0.00763352,0.00040045,0.00198348,0.000253$ $22,0.00058520,0.00205283,0.00605124,-0.00104280,-0.03573616,-0.0009261$ $1,-0.00693832,0.19260520,0.00005948,-0.00000248,-0.00002338,0.00005652$ ,-0.00024687,-0.00002701,-0.00001152,0.00009074,0.00001971,0.00002725, $-0.00010392,0.00004356,0.00003020,0.00001680,-0.00007438,-0.00003033,0$ $.00003679,0.00004877,-0.00000167,-0.00000548,-0.00000800,-0.00018932,0$ $.00024201,0.00001317,-0.00000531,0.00000145,0.00001511,-0.00000482,0.0$ $0000437,0.00000070,-0.00001582,0.00002305,-0.00002166,-0.00000940,0.00$ $001173,-0.00000149,0.00356546,-0.03862300,0.00246550,-0.02345443,0.004$ $35957,-0.00429319,0.00100448,-0.00348234,0.00105248,0.00002968,0.00184$ $660,-0.00035860,-0.00183233,-0.00379776,0.00048847,0.02726170,0.002064$ $87,0.00501485,-0.00690878,0.03519307,-0.00011135,0.00001297,0.00001289$ ,-0.00003994,0.00010422,-0.00000783,0.00007486,-0.00010042,-0.00009550 ,-0.00001821,0.00020536,-0.00007436,-0.00010234,0.00002989,0.00019280, $0.00009457,-0.00015999,-0.00009142,0.00002286,-0.00000431,0.00000074,-$ $0.00003657,-0.00015945,-0.00000308,-0.00000626,-0.00001486,0.00001091$, $-0.00000319,0.00001039,0.00001159,0.00000928,0.00000541,-0.00000163,-0$ $.00001441,0.00001161,0.00002029,-0.01878873,0.00250186,-0.02393674,-0$. $00501348,0.00080481,0.00247990,-0.00066490,-0.00052585,0.00454243,0.00$ $062592,-0.00031977,-0.00157898,-0.00030011,0.00192539,0.00443514,-0.00$ $971336,-0.00006938,0.00163395,0.03316819,-0.00366284,0.01729017,-0.000$ $86143,0.00015535,0.00027489,-0.00031397,0.00088659,0.00005208,0.000274$ $04,-0.00036474,-0.00039966,-0.00001939,0.00069735,-0.00026076,-0.00031$ $892,0.00012648,0.00063137,0.00020443,-0.00055785,-0.00024417,0.0001236$ $8,-0.00001190,-0.00003638,0.00048624,-0.00093608,-0.00009039,-0.000027$ $68,-0.00003261,0.00001579,-0.00000579,-0.00000561,0.00002425,0.0000069$ $8,0.00003910,-0.00004016,-0.00002142,0.00001657,0.00001452,0.00713975$, $-0.01567334,0.00255673,-0.10852629,0.11182625,-0.02551800,-0.00819921$, $0.02633173,-0.00494869,-0.00392652,0.00078583,-0.00208053,-0.00150962$, $-0.00085816,0.00004010,-0.00071551,-0.00347743,-0.00081770,0.00144379$, $-0.00069789,0.00081964,0.11307392,0.00009713,-0.00019635,-0.00002793,0$ $.00006874,-0.00000876,0.00006901,-0.00001198,0.00012288,-0.00005556,0$. 
$00006937,-0.00021271,0.00010739,0.00014573,-0.00004530,-0.00030607,-0$. $00018257,0.00013032,0.00016439,0.00001166,-0.00000487,-0.00003316,-0.0$ $0008561,0.00027237,0.00002714,-0.00001858,0.00001127,0.00007563,0.0000$ $1961,-0.00007791,0.00001281,-0.00000098,0.00005168,0.00001782,-0.00000$ $895,0.00002028,-0.00003390,0.01571681,-0.01823036,0.00499254,0.1065762$ $3,-0.27011732,0.04449420,-0.00139071,0.00224876,0.00012101,0.00017982$, $0.00146982,0.00059938,-0.00046360,0.00050212,-0.00015896,-0.00451409,-$ $0.00199452,-0.00008190,0.00016624,0.00113723,-0.00039775,-0.11732346,0$ $.28541527,-0.00009052,-0.00001841,0.00005082,-0.00003436,0.00026007,0$. $00005555,0.00000568,-0.00008330,-0.00007929,-0.00001169,0.00011108,-0$. $00003274,-0.00004015,0.00001822,0.00006352,-0.00001911,0.00000114,-0.0$ $0001864,-0.00004445,0.00000908,0.00000871,0.00014836,-0.00024870,-0.00$ $002569,0.00001534,0.00000865,-0.00001166,-0.00000133,0.00000484,-0.000$ $00041,-0.00000704,0.00000346,-0.00001748,0.00000385,-0.00000660,0.0000$ $0019,-0.00078892,-0.00073341,0.00240435,-0.02482823,0.04562326,-0.0432$ $8290,-0.00231427,0.00504371,0.00343454,-0.00180745,0.00048011,0.005960$ $23,-0.00002042,-0.00026477,-0.00071253,-0.00072193,0.00022559,0.005332$ $67,0.00072453,-0.00054267,-0.00256356,0.02887084,-0.04927127,0.0335771$ $1,-0.00203245,-0.00124117,0.00088221,-0.00280077,0.00846796,0.00101603$ $, 0.00102994,-0.00168757,-0.00155774,-0.00018724,0.00159105,-0.00061106$ $,-0.00024964,-0.00077922,0.00097447,0.00032302,-0.00045767,-0.00024108$ $, 0.00027134,0.00017642,-0.00000680,0.00346883,-0.00490105,-0.00055241$, $-0.00002212,0.00003771,0.00001060,0.00003793,-0.00006464,0.00003028,0$. $00000210,-0.00009054,0.00003733,0.00004125,-0.00002671,-0.00007646,-0$. $00290114,0.00146818,-0.00176944,-0.01286982,-0.02352652,-0.00083721,-0$ $.13866329,-0.12518508,-0.00301392,0.01439704,0.00864902,0.00154223,-0$. $00112649,0.00455617,-0.00172687,-0.00034814,0.00045388,-0.00005287,-0$. $00134403,-0.00066245,-0.00020757,0.00121102,0.00008539,0.00089940,0.14$ $134124,-0.00058923,-0.00048083,-0.00016569,0.00053123,0.00095362,0.000$ $01341,0.00030645,-0.00024282,-0.00014749,-0.00015116,0.00011016,0.0000$ $5770,0.00008358,-0.00034473,0.00010362,0.00001188,-0.00006028,-0.00017$ $286,0.00020750,0.00001981,0.00020461,-0.00125350,-0.00146853,-0.000161$ $91,0.00004992,0.00002159,0.00003308,0.00000251,-0.00001786,0.00001943$, $-0.00000375,-0.00003703,0.00001795,0.00000617,-0.00001454,-0.00003349$, $0.00061726,0.00187872,0.00071425,0.00417877,0.00612594,0.00067285,-0.1$ $2730967,-0.23900296,0.00067021,-0.01183373,-0.02325531,0.00057782,0.00$ $138000,-0.00408872,0.00146987,0.00117972,-0.00000067,0.00013650,0.0009$ $0281,0.00018617,0.00017968,-0.00012781,0.00031187,-0.00053927,0.132447$ $87,0.25985003,-0.00003868,-0.00012455,0.00018704,0.00001114,-0.0000989$ $6,-0.00005782,-0.00004864,-0.00005464,-0.00009503,0.00005265,-0.000183$ $25,-0.00005779,-0.00005693,0.00005132,-0.00008148,0.00019396,0.0000608$ $8,0.00003884,-0.00002004,0.00002807,-0.00015611,0.00008267,0.00002750$, $-0.00118036,0.00002724,0.00000392,0.00000614,0.00001564,0.00001985,-0$. $00001379,-0.00000092,0.00003674,0.00003727,0.00001117,0.00001343,0.000$ $01023,-0.00186896,0.00067041,0.00624201,-0.00396010,-0.00421453,0.0057$ $4928,-0.00220675,0.00122436,-0.04165516,0.00330060,0.00468527,0.001044$ $83,-0.00123641,0.00187952,0.00641101,-0.00011456,0.00003338,-0.0007276$ $5,-0.00043031,-0.00014505,0.00014516,0.00090970,-0.00054573,-0.0037994$ 7,0.00502981,-0.00343158,0.02908869,0.00068161,0.00028915,-0.00005873, $0.00016393,-0.00050030,0.00000873,-0.00010399,-0.00006039,0.00006186,-$ $0.00006310,0.00012680,0.00003468,-0.00000146,0.00015794,0.00043562,0.0$ 
$0042804,-0.00002737,-0.00029927,-0.00002672,-0.00003913,-0.00000756,-0$ $.00025771,0.00049263,0.00007911,0.00003158,0.00003298,-0.00003565,-0.0$ $0002064,-0.00008238,-0.00000082,-0.00017943,-0.00001351,-0.00012204,0$. $00002700,-0.00002619,-0.00006099,-0.00327831,-0.00109485,-0.00160666,-$ $0.00081133,-0.00085444,-0.00004699,-0.00054767,-0.00423978,-0.00083850$ $, 0.01121534,-0.01346679,0.00350189,-0.10520849,0.10218236,-0.02226409$, $-0.01101421,0.02790214,-0.00551247,-0.00177380,0.00097511,-0.00047595$, $0.00003534,0.00010714,0.00021158,0.00049956,-0.00060058,0.00016244,0.1$ $0903963,-0.00049844,-0.00001445,0.00013271,-0.00017958,-0.00002571,0.0$ $0005806,0.00028018,-0.00003937,-0.00049577,0.00004843,0.00040206,-0.00$ $026545,-0.00023331,0.00048729,0.00065385,0.00016250,-0.00060968,0.0000$ $6066,0.00006842,-0.00002013,-0.00006456,0.00005245,-0.00026035,-0.0001$ $0613,-0.00003901,-0.00003938,0.00006696,-0.00004794,0.00006165,0.00001$ $447,-0.00013179,0.00027433,-0.00027292,-0.00006064,0.00009115,0.000033$ $63,-0.00014761,0.00232788,0.00027100,-0.00099958,0.00042605,-0.0002435$ $2,-0.00252147,-0.00384093,0.00064412,0.00897910,-0.02311878,0.00376183$ $, 0.10694372,-0.26055302,0.04003688,-0.00145102,0.00066641,0.00016662,-$ $0.00018005,-0.00002349,-0.00000466,0.00007795,-0.00010990,-0.00007907$, $0.00068703,-0.00048811,0.00013004,-0.11111045,0.28385873,0.00066462,-0$ $.00013190,-0.00041971,0.00009934,0.00006856,0.00016530,-0.00002115,0.0$ $0001258,0.00002093,-0.00003541,-0.00005565,-0.00004574,0.00006482,-0.0$ $0008459,-0.00027377,-0.00026603,0.00021602,0.00012274,-0.00027401,-0.0$ $0008394,-0.00006993,0.00000815,-0.00002161,-0.00000606,0.00001788,0.00$ $000604,-0.00001494,0.00006988,-0.00003450,0.00000818,-0.00005371,0.000$ $08247,0.00000640,0.00003051,-0.00000942,-0.00001583,-0.00179368,0.0002$ $7770,0.00607868,-0.00001442,-0.00032463,-0.00063017,-0.00110868,0.0003$ $8902,0.00713712,0.00120996,-0.00007225,-0.00011061,-0.02258140,0.03874$ $589,-0.04373189,-0.00274563,0.00560665,0.00405943,-0.00038654,0.000230$ $12,0.00020603,0.00020777,-0.00009935,-0.00113314,0.00006340,-0.0000811$ $2,-0.00015540,0.02595390,-0.04418144,0.03243015,-0.00076897,0.00015263$ $, 0.00013078,-0.00022431,0.00055119,0.00001045,0.00017120,-0.00042475,-$ $0.00026768,-0.00004831,0.00077527,-0.00024858,-0.00034301,0.00017302,0$ $.00079055,0.00034685,-0.00059270,-0.00033345,0.00007536,-0.00002230,-0$ $.00000074,0.00035069,-0.00068520,-0.00007276,0.00000993,-0.00001772,-0$ $.00004256,-0.00004036,0.00000774,0.00001257,-0.00000503,0.00001851,-0$. $00008312,-0.00001273,-0.00001143,0.00001337,0.00684734,0.01272592,-0.0$ $0041743,0.00021877,0.00287777,-0.00130438,-0.00122430,0.00095314,-0.00$ $008800,-0.00418659,0.00000504,-0.00194369,-0.01062025,-0.02548243,-0.0$ $0016828,-0.13786379,-0.13165426,-0.00605491,0.00150151,0.00081420,0.00$ $068107,0.00044658,0.00075668,-0.00014971,-0.00007814,-0.00003623,0.000$ $18529,0.00116481,0.00030112,0.00089044,0.14428272,-0.00017057,0.000222$ $24,-0.00001688,-0.00004454,0.00004574,-0.00002882,-0.00001152,-0.00015$ $010,0.00004896,-0.00007183,0.00022820,-0.00010293,-0.00012500,0.000051$ $82,0.00031818,0.00017499,-0.00016583,-0.00018552,-0.00000982,0.0000035$ $6,0.00005386,0.00006864,-0.00021898,-0.00003172,0.00002770,-0.00000255$ $,-0.00007650,-0.00002400,0.00006831,-0.00001236,0.00000875,-0.00004422$ ,-0.00001844,0.00000922,-0.00002354,0.00003237,-0.01905582,-0.01800531 ,-0.00132357,0.00413561,-0.00282507,0.00158902,0.00042895,0.00016785,$0.00008445,0.00006140,0.00187274,0.00030016,0.00303224,0.00394439,0.00$ $062432,-0.12623711,-0.24891491,-0.00177219,0.00001243,0.00107088,-0.00$ $037230,-0.00087631,-0.00069586,-0.00000175,0.00000194,0.00004449,-0.00$ 
$006641,-0.00015012,0.00054774,-0.00045466,0.13881477,0.26277841,-0.000$ $18595,0.00008141,0.00002823,-0.00003118,0.00004202,-0.00000726,0.00000$ $722,-0.00007500,-0.00000360,-0.00003725,0.00014318,-0.00004362,-0.0000$ $9421,0.00001647,0.00016267,0.00010354,-0.00010228,-0.00005326,0.000021$ $21,-0.00000964,0.00002424,0.00003598,-0.00010316,-0.00003780,0.0000123$ $3,0.00000557,-0.00002724,0.00000366,-0.00000284,0.00000698,0.00000102$, $-0.00001131,-0.00001645,0.00001226,-0.00001312,-0.00000545,0.00269940$, $0.00448242,0.00251079,-0.00139077,0.00126331,0.00499547,-0.00003687,0$. $00003337,-0.00089186,-0.00180071,0.00060068,0.00574301,-0.00296271,-0$. $00468567,0.00379911,-0.00666502,-0.00288822,-0.03357061,0.00078095,-0$. $00023417,-0.00262890,0.00001877,0.00033025,0.00078984,0.00016767,-0.00$ $013760,-0.00098045,0.00087990,-0.00031733,-0.00362777,0.00846076,0.001$ $58166,0.02383394 \backslash 10.00001408,-0.00003510,0.00002080,-0.00001649,0.0000$ $1205,0.00000579,0.00000995,-0.00002281,-0.00000607,-0.00001291,0.00002$ $405,0.00001488,-0.00000957,-0.00001022,-0.00002599,0.00004254,0.000041$ 42,0.00003051,-0.00000004,-0.00000446,0.00000083,-0.00000060,0.0000006 $4,-0.00002083,-0.00000207,0.00000101,-0.00000118,0.00000007,0.00000262$ $,-0.00000360,-0.00001829,-0.00000517,-0.00000748,0.00000411,0.00000515$ $, 0.00000169,-0.00000230,-0.00000322,-0.00000104,0.00000107,0.00000091$, $-0.00000119,-0.00001539,0.00000266,-0.00000346,-0.00001937,0.00001946$, $-0.00002704,0.00002533,-0.00000978,0.00000805,0.00000073,-0.00000448,0$ $.00000707,0.00000017,-0.00000492,-0.00000023,-0.00000280,-0.00000252,0$ $.00000074,0.00000203,-0.00000137,0.00001375,-0.00000256,-0.00000266,-0$ $.00000627,0.00000229,-0.00000327,0.00000028111 @$

meta-Addition Transition State

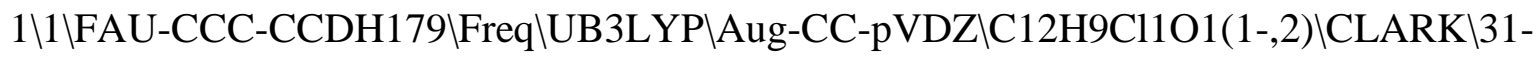
Jul-201610\\N Geom=AllCheck Guess=TCheck SCRF=Check GenChk UB3LYP/Aug -CC-pVDZ Freq \\Addition TS p-Cl-Ph-radical + phenolate anion (meta) \$1,2 \backslash \mathrm{C}, 1.871286,1.094462,-0.770915 \backslash \mathrm{C}, 2.549217,-0.080507,-1.159448 \backslash \mathrm{C}, 3.3$ $13058,-0.878905,-0.237879 \backslash \mathrm{C}, 3.404055,-0.321355,1.109161 \backslash \mathrm{C}, 2.810023,0.8$ $90303,1.46697 \backslash \mathrm{C}, 2.06286,1.631802,0.549999 \backslash \mathrm{H}, 1.516546,1.770772,-1.55209$ $6 \backslash \mathrm{H}, 2.469987,-0.438906,-2.188042 \backslash \mathrm{O}, 3.889336,-1.967093,-0.548112 \backslash \mathrm{H}, 2.93$ $3685,1.267738,2.487053 \backslash \mathrm{H}, 1.591264,2.572421,0.834216 \backslash \mathrm{H}, 3.986805,-0.8901$ $51,1.837972 \backslash \mathrm{C},-2.863395,-0.165915,0.024919 \backslash \mathrm{C},-2.606198,1.14873,-0.3628$ 54\C,-1.271539,1.530332,-0.585671\C,-0.243316,0.606616,-0.429604\C,-0. $517018,-0.702234,-0.049593 \backslash \mathrm{C},-1.840327,-1.102868,0.188518 \backslash \mathrm{Cl},-4.550597$ ,-0.666069,0.313191\H,-3.427594,1.855802,-0.486155\H,-1.061757,2.56316 $3,-0.881146 \backslash \mathrm{H}, 0.300614,-1.419945,0.056523 \backslash \mathrm{H},-2.076325,-2.123746,0.4907$ $08 \mathrm{IV}$ ersion=ES64L-G09RevD.01 $\backslash$ State $=2-\mathrm{A} \backslash \mathrm{HF}=-998.157143 \backslash \mathrm{S} 2=0.778448 \backslash \mathrm{S} 2-1$ $=0 . \backslash \mathrm{S} 2 \mathrm{~A}=0.750521 \backslash \mathrm{RMSD}=5.582 \mathrm{e}-09 \backslash \mathrm{RMSF}=3.205 \mathrm{e}-06 \backslash \mathrm{ZeroPoint}=0.1676181 \backslash \mathrm{The}$ $\mathrm{rmal}=0.1796061 \mathrm{ZPE}=105.1819205 \backslash \mathrm{Dipole}=-3.5212652,1.7108002,0.4575857 \mathrm{DD}$ ipoleDeriv $=0.2319897,-0.255061,-0.2636855,-0.7078769,0.2715423,-0.0165$ $843,-0.7412478,0.105164,0.0837325,-0.3728636,0.2659302,0.3514351,0.261$ $9295,-0.5846179,-0.0626987,-0.0475785,0.3525424,0.0822086,0.4425758,-0$ $.789576,-0.3085153,-1.1467204,1.4970163,0.4026904,-0.9736474,0.2882416$ ,-0.1767835,-0.096575,-0.1028209,0.0655493,0.1945859,-0.2485487,0.0337 $879,0.5776277,-0.4054269,0.0343722,0.0093222,-0.2100061,0.165921,-0.16$ $48954,0.1802264,-0.0827274,0.2626798,0.0554408,0.2154344,-0.3010682,-0$ $.0386705,0.0226598,-0.1405365,-0.2525265,-0.0523409,0.3463326,0.070328$ 
,-0.2421706,0.125208,0.089093,-0.0232959,0.0823051,-0.0409863,0.115625 $2,-0.01055,0.0953721,-0.025919,0.1034734,0.0370276,-0.0557029,0.064895$ $3,0.0374761,-0.0970689,0.0075529,-0.0612552,-0.1327733,-0.9662673,0.61$ $75886,0.1847978,1.1068819,-1.5946492,-0.2466144,0.5488594,-0.3360937,-$ $0.3876218,0.0887004,0.0206441,-0.0372855,-0.0069665,0.0279123,-0.11523$ $79,-0.0913343,-0.1220091,-0.1921259,0.087599,0.1562707,0.0048875,0.092$ $6828,-0.0688891,-0.0583717,-0.0012104,-0.0673161,0.0272165,0.0142924,0$ $.0872955,-0.1064203,0.1138444,0.008023,0.0750228,-0.0946198,0.0520293$, $-0.0473719,1.091768,0.281025,-0.1481423,0.3612463,0.0904632,-0.0524273$ $,-0.1904453,-0.0725653,-0.0552721,-0.2139396,-0.1309441,-0.0013669,0.1$ $062687,-0.0211902,0.0108302,-0.0550221,-0.0080606,-0.1201226,-0.483175$ $4,-0.0355778,-0.0775571,-0.5322291,0.1828011,-0.1974612,0.1374784,-0.0$ $498672,-0.0474384,0.9451304,0.1863715,0.2178243,0.6463746,-0.147026,0$. $1660344,0.0928827,-0.0407096,-0.1069999,-0.6195367,-0.0759615,-0.10396$ $25,0.1010971,0.0188442,0.0489354,0.00054,-0.0131433,-0.0750691,-0.0741$ $582,0.012231,0.0175789,-0.2512446,0.0486908,-0.0764042,0.0628344,-0.03$ $65924,-0.080581,-0.9995975,-0.1937251,0.0842923,-0.2585112,-0.18895,0$. $0270757,0.1421166,0.0359339,-0.1335363,-0.1746893,0.104399,-0.0388263$, $0.1057757,-0.0038953,0.0280373,-0.0098169,0.0243334,0.1160304,0.043246$ $4,-0.0600783,0.0248229,0.1006104,-0.1579135,0.1056299,-0.0000523,0.068$ $1837,0.0763715,0.1437086,0.0847416,0.0232169,-0.0228431,0.0181062,0.00$ $01717,-0.023932,0.0082557,0.0786922,-0.0251434,-0.0501963,0.0017748,-0$ $.1066739,-0.0719093,0.0440962,0.0605523,0.0572144,0.1097273 \backslash$ Polar=297. $1216675,-15.0901263,178.9189191,18.2605148,-1.6981271,147.2109417 \mathrm{PG}=\mathrm{C}$ $01[\mathrm{X}(\mathrm{C} 12 \mathrm{H} 9 \mathrm{Cl1O} 1)] \mathrm{NImag}=1 \backslash 0.12839506,-0.21127171,0.55124920,0.091582$ $13,-0.00515392,0.60024659,-0.08841192,0.11282111,0.01259157,0.27361888$ $, 0.08001956,-0.25003755,-0.03859706,-0.22229475,0.49533563,0.02262210$, $-0.07011809,-0.12866955,0.08575186,0.10620426,0.63127727,-0.02296666,0$ $.01811483,0.00221816,-0.10984923,0.04418180,-0.06643257,0.34483954,0.0$ $2310076,-0.02808435,0.00681672,0.05189629,-0.12116485,0.05005855,-0.23$ $839426,0.61054623,-0.02370784,0.01004485,0.02771232,-0.04547404,0.0281$ $5254,-0.18521637,0.04886203,0.08000487,0.49951905,0.00258188,0.0066650$ $1,0.00791891,0.00508272,-0.00836046,-0.01513779,-0.08529320,0.00634182$ ,-0.03508663,0.29818121,-0.00819872,-0.01384449,-0.01650882,-0.0094362 $1,0.02428172,0.00761826,0.01529640,-0.10114183,-0.01118668,-0.25204049$ $, 0.54620642,0.00353570,0.00034565,-0.00657892,-0.02023175,0.01058805,-$ $0.02962237,-0.01698108,-0.03707569,-0.19727493,0.12484342,0.03046560,0$ $.54582335,-0.00924351,-0.00834239,-0.02582139,-0.00714014,0.01135155,-$ $0.00162945,0.01198241,-0.00223518,0.03091121,-0.10965526,0.09579964,-0$ $.00448796,0.28698957,0.01576088,0.02819532,0.01697802,0.00691576,-0.01$ $500968,0.00014454,-0.00841071,-0.00977232,-0.04312534,0.11222298,-0.26$ $199518,-0.03878607,-0.20090385,0.50967171,-0.02216891,0.00529620,-0.03$ $770854,-0.00819681,0.00879991,-0.00504171,0.01969638,-0.02707946,0.014$ $08004,0.02521977,-0.09002441,-0.13357566,0.12613470,0.09477304,0.65389$ $920,-0.04030176,0.01385836,-0.02910484,0.00589998,0.00914256,0.0138658$ $9,-0.00559096,-0.00551472,-0.01000987,-0.01357447,0.04163017,0.0043065$ $7,-0.13173300,0.06891372,-0.10653847,0.29343667,0.01816434,-0.12246312$ ,-0.08097474,-0.00021748,-0.00777699,-0.02718348,-0.00193326,0.0030396 $5,0.00640994,0.02989005,-0.03712384,0.00910493,0.08887724,-0.15054793$, $0.09026761,-0.24881815,0.56156294,0.00168299,-0.04605767,-0.24547273,0$ $.01093555,-0.03301356,-0.00600995,-0.01080631,0.00779882,-0.02867679,-$ 
$0.01756608,0.04251384,0.03068371,-0.07656951,0.03780879,-0.20680454,0$. $11414955,0.06919187,0.54677280,-0.04732240,0.06273255,-0.06354821,-0.0$ $0766637,0.01384105,-0.01230040,0.00606121,0.00393795,0.00448913,-0.000$ $99428,0.00089347,-0.00005428,0.00487459,-0.00285639,-0.00090931,-0.005$ $02712,0.00247874,-0.00914234,0.06989279,0.07316055,-0.15285532,0.11902$ $722,0.00788050,-0.00926344,0.02024145,0.00177794,-0.00130050,-0.002858$ $08,-0.00047395,0.00039091,-0.00074989,0.00094201,-0.00092651,-0.001991$ $67,-0.00572663,0.00963523,-0.01212154,-0.06865279,0.15868525,-0.069981$ $12,0.11801442,-0.19141721,0.00270803,-0.00676786,0.00800737,0.00077794$ ,-0.00223852,0.00213662,0.00088935,-0.00085502,-0.00020441,-0.00001031 ,-0.00343700,-0.00502456,-0.00940468,0.01986050,-0.01437426,0.07170255 ,-0.12674023,0.20181910,0.00047249,0.00547719,0.01622393,-0.03641395,0 $.00346476,-0.02645150,0.00409281,-0.00581247,-0.01615513,0.00155539,0$. $00430615,-0.00254947,-0.00031538,-0.00004431,0.00009464,0.00212840,0.0$ $0387903,-0.00133388,-0.00211841,-0.00122336,0.00026147,0.03193748,-0.0$ $0454563,-0.00531530,-0.02381436,0.00561603,-0.07475194,-0.08734013,0.0$ $0273040,0.00807631,0.01494813,0.00377840,-0.00008047,0.00150252,0.0001$ $2900,-0.00061197,0.00022921,0.00507200,-0.00228543,-0.00081952,-0.0021$ $1113,0.00031325,0.00067228,-0.00740435,0.07464618,-0.00120036,0.000691$ $58,-0.00465698,-0.02579172,-0.08543020,-0.29790916,0.00223450,-0.01423$ $614,-0.01642040,-0.00371445,0.00298197,-0.00189731,-0.00040654,0.00086$ $946,0.00022886,0.00021295,-0.00147196,0.00232738,0.00105684,-0.0002584$ $0,0.00072207,0.02719314,0.09589066,0.31613424,0.00802898,-0.00277016,-$ $0.00929620,-0.01237266,0.04765856,-0.00315840,-0.15288779,0.16127025,0$ $.04007098,0.01311093,0.01415848,0.01109542,0.00236391,0.00119253,-0.00$ $205251,-0.00223215,0.00200329,0.00397933,-0.00187113,0.00115573,0.0002$ $5552,-0.00174311,-0.00306810,0.00111884,0.15069471,-0.00290266,0.01213$ $891,0.01145703,0.04046287,-0.04705290,0.00596727,0.16246153,-0.3688461$ $9,-0.08195261,0.01961981,-0.03353951,-0.03882880,-0.00238184,0.0056863$ $8,-0.00333034,0.00474093,-0.00487327,-0.00403440,0.00049812,-0.0018007$ $5,-0.00005666,-0.00195440,0.00052901,0.00154214,-0.21995139,0.44243850$ $, 0.00213303,0.00236172,-0.00309943,-0.01637944,0.02246331,0.02097724,0$ $.04123303,-0.08626317,-0.10598564,0.02206661,-0.05475281,-0.01853275,-$ $0.00833805,0.00671965,-0.00911647,0.00340820,-0.00177197,0.00514427,-0$ $.00114231,0.00054744,-0.00041748,0.00278437,-0.00081477,0.00315755,-0$. $04703806,0.11405942,0.10579918,0.00193521,0.00525020,-0.00315929,-0.00$ $024256,-0.00048837,0.00045521,0.00455920,0.00484601,-0.00219536,0.0051$ $8514,0.00480290,0.01249454,-0.04496474,-0.00141791,-0.03294121,0.00111$ $661,-0.00694227,-0.01533760,0.00017304,-0.00039199,-0.00124252,-0.0004$ $6003,-0.00041484,0.00017519,-0.00088512,-0.00002791,0.00093117,0.03785$ $626,0.00408912,0.00017837,0.00104913,-0.00012452,-0.00020198,0.0001145$ $3,0.00550053,-0.00076377,0.00151140,-0.00398529,-0.00485896,-0.0273564$ $2,-0.00159090,-0.08289656,-0.08780925,0.00174890,0.00885397,0.01515214$ $, 0.00021064,0.00020685,0.00049885,-0.00038138,-0.00018413,0.00001194,-$ $0.00007330,-0.00170242,-0.00169268,-0.00137717,0.08175521,-0.00378216$, $0.00295262,-0.00087600,0.00034230,0.00048702,0.00076609,-0.00105592,-0$ $.00126559,0.00398519,-0.00262286,-0.00123379,-0.00659311,-0.03384464,--$ $0.08717883,-0.28490196,0.00001908,-0.01074572,-0.01590754,-0.00040492$, $-0.00039908,-0.00093251,0.00021338,0.00005079,-0.00015740,0.00065065$,$0.00089094,-0.00118443,0.03813865,0.09675541,0.30425967,0.00096797,-0$. $00669693,-0.00220741,0.00174453,0.00100254,-0.00118568,-0.00009861,-0$. 
$00060299,-0.00021173,0.00244065,0.00241992,-0.00406264,-0.00423469,0.0$ $1300119,0.00702780,-0.08532868,0.12017561,0.02469340,-0.00122722,-0.00$ $060809,0.00023505,0.00096664,0.00040017,-0.00031832,-0.00037917,0.0000$ $6850,0.00034878,-0.00224723,-0.00200476,0.00074400,0.08687469,0.004089$ $07,-0.00794325,0.00047469,-0.00043987,0.00196946,-0.00244176,-0.000326$ $47,0.00031554,0.00019410,0.00315381,0.00158582,0.00288071,0.00455577,-$ $0.01077895,-0.00796530,0.11758794,-0.25439697,-0.06631172,-0.00115459$, $0.00021465,-0.00034786,0.00075839,-0.00018730,-0.00016638,-0.00017295$, $-0.00014950,-0.00000059,-0.00183398,-0.00022478,0.00092598,-0.12755435$ $, 0.26981266,0.01527341,-0.02404200,-0.00285644,-0.00274527,-0.00076139$ ,-0.00461669,-0.00039552,0.00058501,-0.00069104,-0.00305077,0.00078737 ,-0.00250118,-0.00880736,0.01908908,0.00849045,0.02237781,-0.06925059, $-0.07160958,0.00056292,0.00105671,0.00084576,0.00039245,-0.00146829,-0$ $.00027292,0.00045957,-0.00028907,0.00037229,0.00146698,0.00020572,0.00$ $068906,-0.02464190,0.07307063,0.07155511,0.00004192,-0.00076061,0.0003$ $5860,0.00289947,0.00046312,-0.00149108,0.00197115,0.00654494,-0.000968$ $63,-0.11559651,0.08508990,-0.10472425,-0.00375895,0.00479526,-0.009835$ $18,0.00419031,0.00392473,0.00091376,-0.00037456,-0.00012870,0.00001767$ ,-0.00088234,-0.00033061,0.00001964,-0.00102881,-0.00273765,0.00117120 ,-0.00256094,-0.00184961,0.00151186,0.00010366,0.00055363,-0.00064399, $0.11496109,-0.00025456,-0.00016920,-0.00006255,0.00123949,0.00060045,-$ $0.00228139,-0.00332342,0.00694884,-0.00852613,0.08501759,-0.12064781,0$ $.09205206,0.01311794,-0.00884811,0.01948749,0.00388549,-0.00216757,-0$. $00375543,-0.00019384,-0.00011799,0.00011595,-0.00078464,0.00005476,-0$. $00033185,-0.00173799,0.00063144,0.00175520,-0.00215998,-0.00029050,0.0$ $0042229,0.00095836,-0.00054569,0.00079881,-0.09565636,0.12455720,0.000$ $95121,-0.00073735,-0.00031731,-0.00054657,-0.00395510,-0.00498768,-0.0$ $1848054,0.02038226,-0.01233418,-0.10412072,0.09182166,-0.17778700,0.00$ $550612,-0.00642283,0.00698830,-0.00026279,-0.00257603,0.00166347,0.000$ $05337,0.00013078,-0.00004864,-0.00067412,0.00106738,-0.00132187,0.0027$ $2032,-0.00089112,0.00355157,0.00099541,0.00124343,0.00083848,0.0001429$ $2,-0.00040751,0.00031205,0.11374967,-0.09956992,0.18340499,-0.00009671$ $, 0.00095908,0.00089827,-0.00038418,-0.00013354,-0.00030322,0.00011481$, $0.00045132,0.00123234,0.00007742,-0.00004608,-0.00029254,0.00018256,-0$ $.00063035,-0.00021906,-0.00065098,0.00000707,-0.00079769,-0.00020971,-$ $0.00003532,-0.00004875,0.00000152,-0.00002147,-0.00007339,0.00033286,-$ $0.00059694,-0.00046968,0.00000223,0.00003011,0.00010424,-0.00001372,-0$ $.00007146,-0.00005737,0.00001503,-0.00005028,0.00002003,0.48486738,-0$. $00001651,-0.00044205,0.00071022,0.00042369,0.00024597,0.00023440,-0.00$ $014096,0.00063149,0.00047490,-0.00027933,0.00003715,-0.00030926,-0.000$ $10715,0.00029706,-0.00019804,0.00026010,0.00033140,-0.00013958,-0.0000$ $0760,0.00017716,-0.00029923,-0.00015151,-0.00007337,0.00004531,0.00018$ $179,-0.00055297,-0.00029003,-0.00003703,0.00001616,0.00003750,0.000007$ $94,0.00026645,0.00008376,0.00004799,-0.00000583,0.00001550,-0.05933894$ $, 0.60522693,-0.00002194,0.00012867,-0.00020380,0.00001417,-0.00033927$, $0.00000657,-0.00002168,-0.00011118,-0.00032228,0.00007154,-0.00000079$, $0.00007414,-0.00000469,0.00005405,-0.00002715,0.00012148,-0.00008095,0$ $.00009749,0.00005844,-0.00000995,0.00008193,0.00006871,0.00004621,-0.0$ $0005481,-0.00003501,0.00010416,0.00013512,0.00001638,0.00001208,0 .,-0$. $00005101,-0.00013202,-0.00000079,-0.00001788,-0.00000116,0.00000169,-0$ $.01731730,-0.12923234,0.15554236,0.00124701,0.00052147,-0.00163779,-0$. 
$00019089,-0.00034023,0.00031638,-0.00043585,-0.00037146,-0.00129878,0$. $00025463,-0.00019279,0.00038228,-0.00015644,0.00048694,0.00014851,0.00$ $076581,-0.00023023,0.00076539,0.00021278,-0.00047501,0.00056498,0.0001$ $6752,0.00009959,-0.00004694,0.00000419,0.00033994,0.00051378,0.0000086$ $8,-0.00002094,-0.00004352,-0.00014334,-0.00039209,-0.00008794,-0.00002$ $671,-0.00001744,0.00001877,-0.10432217,0.00741847,0.00173208,0.6746322$ $5,0.00066220,0.00089198,0.00174688,-0.00040594,-0.00014157,-0.00067559$ $, 0.00045236,0.00008019,0.00164410,0.00010663,-0.00033470,-0.00031613,0$ $.00000531,-0.00048590,-0.00036138,0.00040114,0.00019728,-0.00087124,-0$ $.00003727,-0.00021735,-0.00009584,0.00016288,0.00000565,-0.00017054,0$. $00004309,-0.00039976,-0.00055932,0.00003434,0.00003547,0.00008516,-0.0$ $0004012,0.00001717,0.00008839,0.00001310,-0.00000390,-0.00002360,-0.05$ $529982,-0.30733426,0.07269565,-0.05954627,0.64577518,0.00043999,-0.000$ $63763,-0.00020370,-0.00053695,0.00084964,-0.00005481,-0.00026813,-0.00$ $042366,-0.00067520,0.00007153,0.00036253,0.00059603,0.00018945,-0.0001$ $9972,0.00035984,0.00025551,0.00001034,0.00022699,-0.00008970,-0.000194$ $28,0.00016033,0.00002397,0.00003422,0.00005495,-0.00016425,0.00062021$, $0.00035217,-0.00003750,-0.00007195,-0.00001406,0.00007274,0.00022266,-$ $0.00006569,0.00001527,0.00001910,0.00000337,0.01930631,0.06769529,-0.0$ $8300431,-0.03279752,-0.13906455,0.16453321,-0.00245784,0.00009883,-0.0$ $0461153,-0.00047892,0.00033117,0.00042366,-0.00093100,-0.00107564,-0.0$ $0370369,0.00011569,0.00096281,0.00141511,0.00036105,0.00043508,0.00095$ $937,-0.00161432,-0.00048032,0.00219346,0.00079380,0.00025235,0.0002630$ $0,-0.00022088,0.00012391,0.00044540,-0.00047564,0.00204011,0.00146991$, $-0.00006208,-0.00009690,-0.00022084,0.00018395,0.00004322,-0.00014541$, $-0.00008699,0.00003876,0.00003849,0.00465777,-0.08528737,0.02376458,-0$ $.29309395,-0.01687682,0.02501876,0.60079583,-0.00034554,-0.00238227,-0$ $.00528727,0.00008662,0.00073248,0.00130210,-0.00089269,-0.00154965,-0$. $00547358,0.00047693,0.00064249,0.00147136,-0.00031177,0.00126797,0.001$ $05967,-0.00034277,-0.00091079,0.00282028,-0.00079573,0.00061035,0.0005$ $3487,-0.00011085,0.00008971,0.00030949,-0.00049170,0.00239583,0.002179$ $20,-0.00005905,-0.00012931,-0.00024030,-0.00007167,-0.00028518,-0.0003$ $2443,-0.00010467,0.00003407,0.00002784,-0.02784816,-0.00174497,0.00517$ $384,-0.08723058,-0.12633128,0.02522699,-0.00607906,0.65984379,0.000886$ $33,0.00040545,0.00229011,-0.00043337,0.00028870,-0.00066007,0.00071373$ $, 0.00026980,0.00253630,0.00007811,-0.00063421,-0.00049690,-0.00033636$, $-0.00010246,-0.00053497,0.00052452,0.00038211,-0.00093570,-0.00025647$, $-0.00023812,-0.00028004,-0.00008013,-0.00000846,-0.00018400,-0.0000879$ $2,-0.00051277,-0.00082446,0.00000181,0.00000846,0.00009702,-0.00002542$ $, 0.00024828,0.00004801,0.00005144,0.00002165,-0.00004743,0.00743654,0$. $01157646,0.00400602,0.04380996,0.01840949,-0.06927876,-0.03939420,-0.1$ $4295591,0.16931049,0.07125001,0.01392669,0.01154536,-0.02835450,0.0213$ $2748,0.00441933,0.00531967,-0.00145475,0.01908241,-0.00337106,0.003226$ $19,-0.00568545,0.00453326,-0.01101689,0.00289434,-0.02147233,-0.010746$ $55,-0.03350041,-0.01501068,-0.00651466,0.00171063,0.00114576,-0.000243$ $79,-0.00152342,0.00025590,-0.00333832,-0.00502277,0.00068210,0.0000192$ $2,0.00071276,0.00039442,0.00159424,0.00022656,0.00034815,-0.00013804,--$ $0.00012822,-0.00871179,0.02011261,-0.00551764,-0.05081157,0.00091986,0$ $.00598091,-0.21202100,0.13129438,-0.02147137,0.33343106,0.00958809,-0$. $00910696,0.01004477,0.00380755,0.00231300,-0.00026735,0.00263016,0.001$ $36174,0.00784395,-0.00170826,0.00028891,-0.00385103,0.00082645,-0.0027$ 
$0810,-0.00093212,-0.00599559,-0.00132542,-0.00823002,-0.00893646,-0.00$ $355192,0.00141574,0.00004522,-0.00023016,-0.00037095,0.00101702,-0.003$ $73789,-0.00322472,0.00022288,0.00021132,0.00032187,-0.00063573,0.00042$ $632,0.00038481,0.00012210,-0.00005283,-0.00009194,0.01962916,-0.064084$ $99,0.01399408,0.05240620,0.04969047,-0.01458100,0.10444768,-0.20283070$ $, 0.03120503,-0.09465820,0.57686462,-0.01875794,0.00718572,-0.00825304$, $0.00816391,-0.00988974,0.00141016,-0.00136606,0.00265458,-0.00207267,0$ $.00009907,-0.00126760,-0.00236313,-0.00253286,0.00276501,-0.00132438,-$ $0.00420900,-0.00099372,0.00267624,0.01025426,0.00391504,-0.00078372,-0$ $.00008607,-0.00007613,0.00038166,0.00139173,-0.00286975,-0.00040033,0$. $00021219,0.00036890,-0.00007220,-0.00033831,0.00000186,0.00037446,-0.0$ $0011485,-0.00010869,0.00007812,-0.00497839,0.01256978,-0.00982367,-0.0$ $0744651,-0.00935627,0.01845815,-0.01305462,0.03088041,-0.06145917,0.00$ $300648,-0.12650092,0.12124498,-0.00681633,-0.00963537,-0.00322948,0.00$ $076672,-0.00126010,0.00019730,-0.00018454,-0.00291653,-0.00500816,0.00$ $055884,0.00117010,0.00148584,0.00014982,0.00055610,0.00084915,-0.00082$ $821,-0.00060764,0.00457642,-0.00042787,-0.00074154,0.00101200,-0.00010$ $522,0.00018178,0.00049921,-0.00038385,0.00335711,0.00206750,-0.0000537$ $8,-0.00009741,-0.00029874,0.00005912,-0.00051967,-0.00013848,-0.000209$ $68,0.00001632,0.00001452,-0.05458516,0.05085875,-0.00779058,-0.0254082$ $3,-0.03095113,0.01006068,0.03983573,0.01585520,-0.00841522,-0.09588784$ ,-0.02001065,0.01163471,0.65472522,-0.00179825,0.00348466,0.00475136,0 $.00011150,-0.00178702,-0.00150340,0.00160292,0.00182296,0.00456124,-0$. $00094980,-0.00066883,-0.00142194,0.00033069,-0.00096440,-0.00078303,-0$ $.00098179,0.00086862,-0.00164942,-0.00045620,-0.00019441,-0.00024386,-$ $0.00008794,0.00000850,-0.00022669,-0.00043021,-0.00201674,-0.00164865$, $0.00005590,0.00004502,0.00005584,0.00011267,-0.00017818,-0.00024103,0$. $00014220,0.00001294,-0.00009202,-0.00628240,0.05294880,-0.01171645,-0$. $02536340,-0.03347818,0.01078133,0.04118185,-0.03772336,0.00657530,-0.0$ $5022756,-0.30910016,0.07210188,-0.05752505,0.63351641,0.00222025,-0.00$ $111550,-0.00081596,-0.00006742,0.00187365,0.00017030,-0.00014181,-0.00$ $039547,-0.00060492,0.00007891,-0.00038601,0.00019100,-0.00057698,0.000$ $70084,0.00014824,0.00078842,-0.00030721,0.00079323,-0.00025798,0.00018$ $993,-0.00021290,-0.00016718,-0.00005200,0.00013243,-0.00001032,0.00052$ $691,0.00032861,0.00003782,0.00000551,0.00001554,-0.00014182,0.00000326$ $, 0.00008083,0.00003177,0.00001985,0.00004326,0.00790092,-0.01779733,0$. $01160882,0.00941695,0.01124653,-0.00838162,-0.01553188,0.00955831,0.00$ $205255,0.01810022,0.07054650,-0.07688450,-0.03711570,-0.12876849,0.171$ $69869,0.00144835,0.00015612,0.00117396,0.00097787,0.00014826,0.0001975$ $3,-0.00031853,0.00094776,0.00039005,-0.00042482,-0.00017722,-0.0003844$ $9,-0.00021719,0.00076262,-0.00035872,0.00140862,0.00048631,-0.00027283$ ,-0.00008006,0.00011299,-0.00029047,-0.00014646,-0.00008249,0.00005106 $, 0.00018010,-0.00086919,-0.00032651,-0.00005163,-0.00000400,0.00001333$ - $0.00009634,0.00024035,0.00016821,0.00007157,0.00000732,0.00001021,-0$ $.20418545,0.07828221,-0.00901756,0.04119980,0.05244162,-0.01721169,-0$. $05665012,0.01040623,0.00287123,0.00832760,-0.07947966,0.02120203,-0.29$ $978674,-0.01659707,0.02735987,0.63237052,0.00024241,-0.00260340,-0.001$ $61065,0.00126085,0.00084452,0.00017316,-0.00081702,-0.00022631,-0.0021$ $6029,0.00030550,0.00036952,0.00039671,0.00007258,0.00037756,0.00044574$ $, 0.00018983,-0.00047173,0.00136267,-0.00019354,-0.00017420,0.00024830$, $-0.00012069,0.00000668,0.00021120,-0.00002972,0.00068772,0.00076209,-0$ 
$.00005317,-0.00004910,-0.00007999,-0.00002191,-0.00013521,0.00004156,-$ $0.00006315,0.00000095,0.00005061,0.14145236,-0.21560515,0.02947904,0.0$ $0860001,-0.04289799,0.01302617,0.01764249,-0.00536688,-0.00135794,-0.0$ $3059282,-0.00192373,0.00814193,-0.08718341,-0.12906515,0.02704359,0.00$ $487984,0.68528805,0.00063327,-0.00038321,0.00084743,-0.00013409,0.0007$ $5615,-0.00009944,0.00003205,0.00010179,0.00001701,0.00029069,-0.000068$ 40,0.00036332,0.00019565,-0.00029604,0.00018152,0.00028367,0.00010069, $0.00007851,-0.00033718,-0.00009514,0.00001142,0.00000285,-0.00004656,-$ $0.00000828,-0.00006740,0.00007624,0.00004636,-0.00000862,-0.00000846,0$ $.00002567,0.00001183,0.00001346,-0.00003749,0.00002416,-0.00000488,-0$. $00000467,-0.02585627,0.03550002,-0.06953530,-0.00566305,0.00923957,0.0$ $0665936,0.00001574,-0.00113304,-0.00360210,0.00824126,0.01209298,0.009$ $14655,0.04683404,0.02094615,-0.07141068,-0.04920885,-0.15508837,0.1723$ $2556,0.00019643,-0.00072169,-0.00088394,-0.00028022,-0.00001226,-0.000$ $19655,0.00036191,-0.00107389,-0.00104831,-0.00002311,0.00028814,0.0005$ $0769,0.00008024,-0.00005697,0.00032415,-0.00038608,-0.00008322,0.00053$ $288,0.00018028,0.00003424,0.00001768,0.00005255,0.00007506,0.00006853$, $-0.00058765,0.00119054,0.00056632,-0.00000375,-0.00004049,-0.00015123$, $0.00010138,0.00001618,0.00000979,-0.00005674,0.00006237,-0.00004240,-0$ $.12094428,-0.02424456,0.01587209,-0.02664251,-0.00618655,0.00428860,0$. $00214543,-0.00383552,0.00118243,0.00118732,-0.00023459,0.00038556,-0.0$ $0022883,0.00765710,-0.00176709,-0.04037904,-0.01579761,0.00820148,0.18$ $520821,0.00010941,-0.00020798,-0.00036317,-0.00012106,0.00007471,-0.00$ $008686,0.00011311,-0.00039886,-0.00035032,0.00000885,0.00010683,0.0001$ $6861,0.00003620,-0.00003074,0.00010379,-0.00015267,-0.00005549,0.00014$ $781,0.00009640,0.00003438,-0.00002140,0.00001110,0.00001886,0.00003312$ ,-0.00018125,0.00045090,0.00019252,-0.00000013,-0.00001308,-0.00004149 $, 0.00003803,0.00001331,0.00001735,-0.00002675,0.00001851,-0.00001401,-$ $0.02425431,-0.04483502,0.00893867,-0.03391697,-0.00612212,0.00560039,0$ $.00333254,-0.00379803,0.00192558,-0.00027744,0.00167692,-0.00060238,-0$ $.00012019,-0.00149885,0.00169510,0.01202074,0.00608938,-0.00186398,0.0$ 4432013,0.04698058,-0.00015782,0.00021984,0.00014197,-0.00005550,-0.00 $010475,-0.00000002,0.00003829,0.00011520,0.00030511,-0.00005669,-0.000$ $07876,-0.00010939,-0.00000630,0.00000900,-0.00010604,-0.00001731,0.000$ $00414,-0.00014613,-0.00002629,0.00001280,-0.00002350,-0.00000905,-0.00$ $000942,-0.00000550,0.00008700,-0.00018265,-0.00012616,0.00000948,0.000$ $01367,0.00002750,-0.00001337,-0.00001345,-0.00000876,0.00001299,-0.000$ $01079,0.00000257,0.01557390,0.00890352,-0.02423998,0.01214968,0.003184$ $25,0.00138499,-0.00072971,0.00277153,0.00386668,-0.00011372,-0.0007997$ $0,-0.00128078,0.00045988,0.00086847,0.00423119,0.00077580,0.00062172,0$ $.00315475,-0.02785835,-0.01382605,0.01735733,0.00009322,-0.00051315,-0$ $.00098697,-0.00007688,0.00013562,0.00014951,-0.00012340,-0.00040392,-0$ $.00100558,0.00005287,0.00020522,0.00030706,0.00003941,0.00013923,0.000$ $24509,-0.00012579,-0.00018899,0.00048231,0.00008006,0.00000878,0.00010$ $378,-0.00000547,0.00003558,0.00007529,-0.00015339,0.00055266,0.0004254$ $9,-0.00001746,-0.00002777,-0.00007053,0.00002140,-0.00006976,-0.000015$ $12,-0.00002909,0.00001225,0.00000172,0.00406618,-0.00676947,0.00171053$ $,-0.21074033,0.14019191,-0.02303330,-0.01535411,0.01992441,-0.00386078$ ,-0.00438317,-0.00139670,0.00173495,-0.00048744,-0.00107251,0.00022951 $, 0.00152921,-0.00187465,0.00076312,0.00159429,-0.00070959,-0.00018583$, $0.22258118,-0.00021365,0.00010306,-0.00001455,0.00004832,-0.00008343,0$ 
$.00002438,-0.00006364,0.00006314,-0.00003309,-0.00000297,0.00000926,-0$ $.00001730,0.00000288,-0.00000413,-0.00001317,0.00002661,0.00004204,0.0$ $0002894,0.00004544,0.00000934,0.00003519,-0.00000022,0.00000018,-0.000$ $00589,0.00005759,-0.00006728,-0.00000972,0.00000005,0.00000320,0.00000$ $770,-0.00001202,-0.00000403,-0.00000256,0.00000095,-0.00000746,0.00000$ $663,0.02408578,-0.01384328,0.00240614,0.13496303,-0.17231737,0.0266796$ 6,-0.00771181,0.00833076,-0.00060054,-0.00142116,0.00150985,0.00144143 ,-0.00081486,-0.00018491,-0.00010697,-0.00276216,-0.00394034,0.0028508 $0,0.00013609,0.00074532,-0.00098121,-0.14778154,0.17993427,-0.00009178$ $, 0.00005904,0.00006542,-0.00005792,-0.00014219,-0.00004530,-0.00000815$ ,-0.00001984,-0.00003160,-0.00005909,0.00008035,-0.00000307,0.00008783 $,-0.00011502,0.00005336,-0.00018048,0.00003470,-0.00008848,0.00005965$, $-0.00000282,0.00004064,0.00003488,0.00000943,-0.00001264,0.00001541,-0$ $.00000633,0.00001107,-0.00000772,-0.00000456,-0.00000879,0.00000607,-0$ $.00000592,0.00001625,0.00000188,0.00000297,0.00000088,-0.00679274,0.00$ $511609,0.00171217,-0.02172113,0.02620228,-0.03831220,0.00402297,-0.002$ $96589,0.00423733,0.00127871,0.00156038,0.00574542,0.00037405,-0.000216$ $05,-0.00082410,0.00111732,0.00276836,0.00459566,-0.00040840,-0.0008853$ $9,-0.00249099,0.02318762,-0.03054230,0.02902117,0.00049477,0.00040137$, $-0.00076781,0.00013147,-0.00016908,0.00021621,-0.00019238,0.00006455,-$ $0.00006333,0.00003597,-0.00008980,0.00000300,-0.00006695,0.00005239,-0$ $.00007222,0.00006590,0.00001898,0.00026317,0.00021045,-0.00028976,0.00$ $048906,0.00003260,0.00002261,-0.00004292,0.00012461,-0.00016667,-0.000$ $00802,0.00002471,0.00002299,0.00001485,-0.00007539,-0.00008325,-0.0001$ $8257,0.00000267,-0.00002100,0.00001035,-0.00347989,-0.00032901,0.00100$ $536,-0.00370515,-0.02878350,0.00838086,-0.06448748,-0.04770539,0.01566$ $750,0.00815213,0.02101206,-0.00635461,-0.00247009,0.00448526,-0.000267$ $38,-0.00126358,0.00029697,-0.00011735,-0.00144413,-0.00131923,0.000537$ $54,0.00115300,0.00028112,-0.00048904,0.06628507,-0.00066535,0.00082664$ $, 0.00110855,0.00006206,-0.00018411,-0.00044434,0.00015110,0.00031674,0$ $.00124128,-0.00004640,-0.00017004,-0.00040315,-0.00010917,-0.00016536$, $-0.00031878,0.00035098,0.00040158,-0.00045739,0.00056633,0.00011378,-0$ $.00032858,0.00001343,-0.00004127,-0.00004296,0.00008555,-0.00042551,-0$ $.00050416,0.00000680,0.00003900,0.00003127,0.00001838,0.00017816,0.000$ $11627,0.00000230,-0.00000271,-0.00000704,-0.00135908,0.00255525,0.0012$ $0575,0.00027153,-0.00636016,0.00299580,-0.05281289,-0.28673199,0.07229$ $033,-0.00074583,-0.01893084,0.00430228,0.00320174,-0.00166478,0.002208$ $91,0.00060495,0.00054324,-0.00041450,-0.00026757,-0.00032590,0.0001788$ $2,-0.00007833,0.00031662,-0.00106474,0.05147542,0.31031994,-0.00033180$ ,-0.00044298,-0.00019572,0.00047231,-0.00014462,0.00024515,0.00002696, $0.00024700,-0.00020298,-0.00003537,-0.00009841,-0.00014956,-0.00006029$ $, 0.00017596,-0.00013925,-0.00020643,-0.00003588,0.00021333,-0.00017621$ $, 0.00033109,-0.00013879,-0.00005964,-0.00007303,0.00002684,0.00005152$, $-0.00023188,-0.00001563,0.00002192,0.00003467,-0.00003240,-0.00009015$, $-0.00025185,-0.00010315,-0.00001152,-0.00002452,-0.00000519,0.00125498$ $, 0.00089640,0.00624825,0.00106612,0.00566424,0.00282462,0.01632859,0.0$ $7200111,-0.05549252,-0.00016320,0.00288683,-0.00107681,0.00011614,0.00$ $199184,0.00693817,-0.00020950,-0.00025950,-0.00083714,0.00024664,0.000$ $31254,0.00023175,-0.00034911,-0.00113868,-0.00340683,-0.01783563,-0.08$ $159813,0.04629520,0.00067053,0.00061616,0.00138046,0.00051781,-0.00011$ $130,-0.00041698,-0.00035652,0.00083175,0.00172575,-0.00036577,-0.00025$ 
$328,-0.00113963,-0.00008014,-0.00004529,-0.00045602,-0.00015982,0.0004$ $3158,-0.00035396,-0.00013068,-0.00004527,-0.00009273,-0.00011524,-0.00$ $005779,-0.00010230,-0.00001627,-0.00090314,-0.00071301,0.00002419,0.00$ $007366,0.00007469,-0.00001983,0.00006056,-0.00002904,0.00004953,-0.000$ $02378,0.00002175,-0.00244582,-0.00246803,0.00160828,0.00014361,-0.0006$ $3364,0.00020226,0.00049851,-0.00203897,0.00112819,0.00905846,-0.001000$ $98,-0.00062825,-0.20687534,0.13277970,-0.01793059,-0.01784621,0.018810$ $60,-0.00282368,-0.00141083,0.00034721,0.00011299,-0.00001009,0.0000152$ $4,-0.00010182,0.00038447,-0.00061948,0.00002383,0.21729296,0.00108038$, $-0.00116907,-0.00138005,0.00037791,0.00094549,0.00022617,-0.00074638,0$ $.00033610,-0.00086243,0.00021375,0.00035726,-0.00002620,-0.00007884,0$. $00020714,0.00020974,-0.00018536,-0.00044675,0.00042188,0.00012191,0.00$ $001849,0.00001577,-0.00003348,-0.00000103,0.00008515,0.00017464,-0.000$ $16241,0.00027341,-0.00000522,-0.00001203,-0.00001671,0.00002127,0.0000$ $4312,0.00005266,-0.00001742,-0.00001243,0.00004677,-0.00165494,0.00133$ $282,0.00164019,-0.00113514,-0.00040320,-0.00012805,-0.00035099,-0.0046$ $9781,0.00322069,0.02053911,-0.01822447,0.00349794,0.13666685,-0.170236$ $83,0.02117478,-0.00927705,0.00879522,-0.00025129,-0.00102524,0.0000633$ $0,0.00012723,0.00004772,-0.00001672,-0.00025796,0.00087633,-0.00049701$ $, 0.00003631,-0.14586176,0.18348482,-0.00176703,0.00066997,-0.00029236$, $-0.00051735,-0.00126482,-0.00003035,0.00017153,-0.00020476,-0.00006111$ ,-0.00017630,0.00053469,0.00011348,0.00045872,-0.00040263,-0.00006711, $-0.00028967,0.00000525,-0.00048108,0.00044132,0.00018470,0.00008717,0$. $00028889,0.00009367,-0.00023372,-0.00005037,0.00003716,-0.00000353,-0$. $00002683,-0.00004693,-0.00003341,-0.00001094,-0.00000583,-0.00000410,-$ $0.00007219,-0.00001626,-0.00003444,0.00137992,0.00142343,0.00614121,0$. $00023317,0.00011579,-0.00091174,0.00092979,0.00351756,0.00604144,-0.00$ $567510,0.00500801,0.00049522,-0.01844690,0.02120865,-0.04220661,0.0044$ $8825,-0.00261276,0.00534051,0.00042810,0.00001941,0.00021975,-0.000058$ $31,-0.00027758,-0.00095120,-0.00030019,0.00018153,-0.00012765,0.019162$ $68,-0.02696890,0.03059655,-0.00020669,-0.00088695,-0.00063651,0.000222$ $98,0.00010206,0.00008866,-0.00007404,-0.00037758,-0.00082683,0.0000651$ $5,0.00018510,0.00021692,0.00000958,0.00014801,0.00015452,0.00001335,-0$ $.00008887,0.00051240,-0.00001851,-0.00001801,0.00006184,-0.00002667,0$. $00001565,0.00006977,-0.00007946,0.00049797,0.00031846,-0.00001804,-0.0$ $0002072,-0.00004444,0.00000581,-0.00003471,0.00000302,-0.00004323,0.00$ $000543,0.00000050,0.00571207,0.02158392,-0.00624836,-0.00295912,0.0033$ $2662,-0.00016779,-0.00145342,0.00022036,0.00000945,-0.00406189,-0.0016$ $9774,0.00178259,-0.00135628,-0.02668759,0.00781150,-0.06606747,-0.0570$ $0572,0.01868668,0.00127913,0.00064585,-0.00052759,0.00029580,0.0011209$ $3,-0.00026811,0.00008724,-0.00010647,-0.00008017,0.00119250,0.00025195$ ,-0.00059119,0.06748121,0.00014580,-0.00044384,-0.00039744,0.00002927, $0.00011203,0.00003331,0.00003573,-0.00029029,-0.00050274,0.00004581,0$. $00012938,0.00016568,0.00001383,0.00004272,0.00013457,-0.00009105,-0.00$ $008740,0.00021445,-0.00002705,-0.00000727,0.00002017,-0.00000854,0.000$ $01331,0.00003992,-0.00009172,0.00036839,0.00021865,-0.00001004,-0.0000$ $1793,-0.00004097,0.00002400,-0.00001365,0.00000124,-0.00002468,0.00001$ 286,-0.00000440,-0.00899179,-0.01554473,0.00574560,0.00412536,0.000134 $37,0.00117652,-0.00015899,0.00053476,-0.00041692,-0.00158378,0.0014650$ $3,0.00183845,0.00051959,-0.00394608,0.00213531,-0.05224848,-0.30054365$ $, 0.07797601,-0.00019966,0.00101306,-0.00102339,-0.00036698,-0.00059808$ 
$, 0.00039539,-0.00005335,-0.00011125,-0.00027849,0.00009625,0.00029600$, $-0.00119933,0.05882049,0.31748225,0.00000828,0.00031263,0.00031383,0.0$ $0018852,-0.00005267,0.00010332,-0.00004682,0.00027890,0.00027847,-0.00$ $000115,-0.00007057,-0.00015535,-0.00005098,-0.00002150,-0.00005746,-0$. $00009011,0.00002018,-0.00017063,-0.00003286,0.00002204,-0.00005440,-0$. $00001651,-0.00002182,-0.00003099,0.00006413,-0.00031323,-0.00016912,-0$ $.00000010,0.00000442,0.00000957,0.00001612,0.00001456,-0.00001644,-0.0$ $0000034,-0.00000178,-0.00000033,0.00209893,0.00298325,0.00155631,-0.00$ $037489,0.00117214,0.00528807,0.00010306,-0.00045412,-0.00068982,0.0016$ $0234,0.00154480,0.00578345,0.00026179,0.00466898,0.00290842,0.01746176$ $, 0.07839666,-0.05725852,-0.00027568,-0.00109983,-0.00238635,0.00014955$ $, 0.00026268,0.00067584,-0.00008685,-0.00027703,-0.00109972,-0.00065288$ $,-0.00113709,-0.00359694,-0.02032532,-0.08623158,0.04876876110 .0000112$ $0,-0.00000066,-0.00000875,-0.00000885,0.00000306,0.00000746,-0.0000013$ $3,0.00000021,-0.00000656,-0.00000014,-0.00000044,0.00000376,0.00000203$ ,-0.00000071,0.00000164,-0.00000383,-0.00000476,-0.00000085,0.00000521 $, 0.00000183,0.00000431,-0.00000031,-0.00000016,0.00000062,-0.00000088$, $-0.00000077,0.00000185,-0.00000087,-0.00000223,0.00000074,0.00000047$, $0.00000110,0.00000140,-0.00000148,-0.00000264,0.00000118,-0.00000164,0$ $.00000283,-0.00000188,0.00000029,0.00000088,-0.00000160,0.00000565,0.0$ $0000080,-0.00000290,-0.00000479,-0.00000313,0.00000649,-0.00000213,-0$. $00000029,-0.00000149,0.00000084,-0.00000101,-0.00000157,-0.00000065,0$. $00000218,-0.00000259,0.00000119,0.00000219,-0.00000079,0.00000194,0.00$ $000253,0.00000157,-0.00000046,0.00000031,0 .,-0.00000145,0.00000109,-0$. 00000204III@

para-Transition State

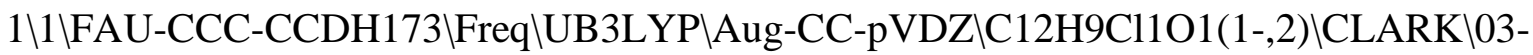
Mar-2015\0\\#N Geom=AllCheck Guess=TCheck SCRF=Check GenChk UB3LYP/Aug -CC-pVDZ Freq \\Addition TS p-Cl-Ph-radical + phenolate anion (para) \$1,2 \backslash \mathrm{C},-1.8142462983,1.4043419831,0.4258162626 \backslash \mathrm{C},-2.4024839837,0.569674$ 2096,1.4264253138\C,-3.2202484052,-0.4913342747,1.0981655961\C,-3.6014 995638,-0.7884722718,-0.2737413638IC,-3.073550998,0.1389841412,-1.2600 516676\C,-2.2553339128,1.1960353939,-0.918981464\H,-1.4157809314,2.376 $2343454,0.7099153062 \backslash \mathrm{H},-2.1589991273,0.7584364416,2.4756252085 \backslash \mathrm{H},-3.63$ $19801498,-1.1400138676,1.8752327194 \backslash \mathrm{H},-3.3699336664,-0.0244612218,-2.2$ 990209274\H,-1.8980437005,1.8733517679,-1.6997134017\O,-4.3493016979,-$1.7647085377,-0.5794598358 \backslash \mathrm{C}, 2.96549633,-0.1994159044,-0.0401154843 \backslash \mathrm{C}$, $2.7341395987,1.1753789899,-0.0617968342 \backslash \mathrm{C}, 1.4224893328,1.6336364715,0$. $156243835 \backslash \mathrm{C}, 0.3768736878,0.7423520994,0.3963858327 \backslash \mathrm{C}, 0.6397927738,-0.6$ $274330095,0.4059837718 \backslash \mathrm{C}, 1.9381184033,-1.1166102511,0.1882721334 \backslash \mathrm{Cl}, 4$. 6237884977,-0.8021122994,-0.3117639483\Н,3.5580192934,1.8677019975,-0. $2427141885 \backslash \mathrm{H}, 1.2432819191,2.714817323,0.134542469 \backslash \mathrm{H},-0.172483768,-1.33$ $87109584,0.5828457275 \backslash \mathrm{H}, 2.1508643665,-2.1865795678,0.1946069397 \backslash \mathrm{Versi}$ on=EM64L-G09RevC.01 \State=2-A $\backslash H F=-998.1688093 \backslash S 2=0.765452 \backslash \mathrm{S} 2-1=0 . \backslash \mathrm{S} 2 \mathrm{~A}=$ $0.750114 \backslash \mathrm{RMSD}=8.871 \mathrm{e}-09 \backslash \mathrm{RMSF}=3.803 \mathrm{e}-06 \backslash$ ZeroPoint $=0.1682297 \backslash \mathrm{Thermal}=0.1$ 802455ไZPE=105.565716 Dipole=3.1796188,1.3650042,0.4614952 \DipoleDeriv $=-0.0381644,-0.127483,0.0147404,-0.049421,-0.000812,0.0942631,0.066431$ $8,0.088903,-0.3447861,0.2354163,0.3142244,-0.0662548,0.5883346,0.09228$ $3,-0.3014442,-0.0932455,0.0009248,0.1315917,-0.3927959,-0.0354909,0.07$ 
49425,-0.184941,-0.1944549,0.0707568,-0.6010241,-0.4152644,-0.0221924, $0.8563429,0.7085778,0.2302884,1.0391113,1.0207758,0.3164776,0.3618646$, $0.3091416,0.0935726,-0.4323263,-0.0354107,-0.134654,-0.3840727,-0.2967$ $705,-0.2618664,0.3735711,0.2505991,0.0971902,0.1681976,0.2468769,0.205$ $5038,0.3887525,-0.0520372,0.2420824,0.3345669,-0.0613662,0.2934274,0.1$ $553148,-0.0845401,-0.0140692,-0.0521448,-0.1102225,-0.0340788,0.001032$ $4,-0.0307913,0.0244384,0.0794161,-0.0518821,-0.0786771,-0.0129434,0.06$ $95044,-0.0296544,-0.0041521,-0.0450248,-0.1727269,0.0591302,-0.0859469$ $, 0.0513974,-0.1223934,0.0034779,0.1133289,0.0656548,0.0851509,-0.06757$ $3,0.0779055,-0.0475823,-0.0640223,-0.0680583,0.075663,-0.0363713,-0.09$ $5754,-0.0109211,-0.1544876,0.0767905,-0.1003968,0.0615057,-0.0361227,-$ $0.0192844,0.1111111,0.0197003,0.1145543,-0.0688445,-1.2980679,-0.62830$ $53,-0.2051824,-1.1075525,-1.2559163,-0.2746682,-0.3573602,-0.2669532,-$ $0.4825155,1.0844108,-0.3224771,-0.1597373,-0.4037806,0.12136,0.0408897$ ,-0.1878885,0.0523785,-0.0535619,-0.2207079,0.1302402,-0.0188525,-0.10 $98917,-0.0445388,-0.0300737,0.0757747,-0.0272854,-0.1089266,-0.4189081$ $, 0.011885,-0.0639574,0.6599535,0.3175075,0.0313684,0.0632288,-0.031104$ $3,-0.1121701,0.935324,-0.0150684,0.0542906,-0.5797857,-0.2717135,-0.04$ $72459,-0.3779314,-0.0302421,-0.1472108,-0.6428194,0.0023599,-0.0701553$ ,-0.0768402,0.033072,-0.0197297,0.1464044,0.0052436,-0.0800723,-0.1269 $814,0.0139387,0.017773,0.2883662,0.0822175,-0.0007545,0.0509658,-0.005$ $2149,-0.0990842,-1.0026816,0.2184269,0.0931237,0.3144272,-0.215143,-0$. $0316309,0.1332624,-0.0338026,-0.1414554,-0.200392,-0.1205178,0.0267635$ ,-0.1180471,-0.0135469,0.0218898,0.04766,0.0281844,0.1156327,0.0233418 $, 0.0561862,0.0110225,-0.1385529,-0.2418899,-0.0043651,-0.0001499,0.009$ $3963,0.0998452,0.0577767,-0.0881547,0.0329295,0.0280848,-0.0007206,0.0$ $19021,-0.0375228,0.01337,0.0811136,-0.0355226,0.0405401,0.0012813,0.13$ $7518,-0.0988105,0.0106945,0.0149106,0.000124,0.1187957 \backslash$ Polar $=323.59836$ $08,28.9996684,179.3755199,3.55939,1.4487685,142.4774959 \backslash \mathrm{PG}=\mathrm{C} 01[\mathrm{X}(\mathrm{C} 12 \mathrm{H}$ 9C11O1)]\NImag=1\0.17793621,0.24604974,0.56264083,0.03912962,0.012289 $94,0.57356907,-0.08250643,-0.06622685,0.04068003,0.33993233,-0.0525990$ $2,-0.15526442,0.04794859,0.26690883,0.47583233,0.04398437,0.09305772,-$ $0.20082760,0.05853609,0.01520253,0.67035827,-0.01296023,-0.02619288,0$. $02335422,-0.17351531,-0.15501111,-0.05627385,0.33881378,-0.02760511,-0$ $.02831014,0.03270685,-0.16088455,-0.25324256,-0.06656535,0.30084448,0$. $51937346,0.00820755,0.00847429,0.03649161,-0.02936107,-0.02773505,-0.1$ $3459746,0.01267913,-0.05081629,0.59609745,-0.00696737,0.00644337,0.003$ $68045,-0.00426791,-0.00542425,-0.02010867,-0.08590088,-0.01538063,-0.0$ $2418494,0.38106197,0.00123225,-0.00111457,0.01192730,-0.00171621,0.003$ $86283,-0.02930205,-0.01764345,-0.09567481,-0.00959239,0.26551972,0.547$ $28799,0.00418244,0.01297852,-0.04501627,-0.03533745,-0.05279121,0.0096$ $9737,-0.00380021,0.01770008,-0.22162302,0.06067640,0.01950249,0.539276$ $09,-0.00706409,-0.00730524,-0.03573035,-0.00982654,-0.01656510,0.00651$ $262,0.01908482,0.01397832,0.00946705,-0.09013234,-0.02658237,0.0088577$ $2,0.32670518,-0.01172678,0.00641341,-0.04983626,-0.01346307,-0.0269171$ $8,0.01111369,0.01520474,0.02565332,0.02073740,-0.03140835,-0.12282824$, $0.03633019,0.27114715,0.47722658,-0.02315689,-0.02638388,-0.00486422,0$ $.00130983,0.00372084,-0.00590764,0.00474101,0.01309476,-0.02900911,0.0$ $2796911,0.06376657,-0.19248139,0.10284804,0.01912983,0.65089306,-0.071$ $91059,-0.03435781,-0.04644113,0.01165101,0.01229346,0.00615551,-0.0081$ $2312,-0.00937957,-0.00674385,-0.01015456,-0.02076466,0.02430001,-0.172$ 
$26790,-0.15413605,-0.06190923,0.33856735,-0.02751366,-0.10410261,0.005$ $37224,0.01333746,0.02626937,0.00962194,-0.01069945,-0.01486021,-0.0106$ $0694,-0.01876775,-0.02922513,0.02728036,-0.15808779,-0.25364195,-0.063$ $77843,0.27575074,0.51949305,-0.04419147,-0.04102116,-0.26206551,0.0068$ $6617,0.02932026,-0.03890192,-0.01310809,-0.01705907,-0.01995620,0.0087$ $9630,0.00406437,0.04782679,-0.03381677,-0.02609589,-0.13352772,0.04707$ $504,-0.06187906,0.62325696,-0.05625727,-0.10275884,-0.02723382,-0.0090$ $4299,-0.01345995,-0.00231445,0.00365956,-0.00091835,0.00325139,0.00005$ $548,0.00008106,-0.00003452,0.00392931,0.00046693,-0.00216832,-0.007071$ $20,-0.00898567,-0.00506907,0.07843006,-0.11383221,-0.27258893,-0.06571$ $995,-0.00402644,-0.01061027,-0.00843447,-0.00117892,-0.00022792,0.0035$ $9809,0.00053556,0.00017188,0.00040095,-0.00063258,0.00135826,-0.000935$ $90,0.00067944,0.00001376,0.00339852,0.11116326,0.28558583,-0.03006214$, $-0.06491817,-0.07311224,0.00878676,0.02110086,0.00895787,0.00060241,0$. $00175583,-0.00321172,0.00024338,0.00035829,-0.00105848,-0.00014320,0.0$ $0111481,-0.00515791,-0.01149736,-0.02779701,-0.00338507,0.03140712,0.0$ $6907601,0.07394426,0.00493111,0.00202060,0.01352213,-0.05496186,-0.020$ $34932,-0.05990517,0.00027440,-0.00337744,-0.01910035,0.00473750,-0.006$ $68236,0.00110929,-0.00069215,0.00031847,-0.00008340,0.00168989,-0.0032$ $5067,0.00112877,-0.00139953,0.00087685,-0.00018584,0.04928998,0.004994$ $00,0.00646513,0.01645555,-0.02130694,-0.05618221,-0.04178411,-0.005968$ $76,0.00039768,-0.02493802,-0.00610110,0.00065848,0.00208572,0.00010388$ $,-0.00075026,-0.00010766,-0.00362103,-0.00018899,0.00285146,0.00169313$ $, 0.00007166,-0.00074978,0.02710458,0.05217609,-0.00731507,-0.00658737$, $-0.01732260,-0.05764142,-0.04345054,-0.30335683,-0.00047484,0.00093001$ ,-0.00617595,-0.00031329,-0.00026071,0.00367462,0.00041606,0.00038127, $0.00085034,0.00256391,0.00391046,-0.00187908,0.00019666,0.00015082,0.0$ $0088284,0.06381761,0.04659921,0.32413207,0.00367579,-0.00620507,-0.003$ $12024,-0.00420936,-0.00963272,0.01410504,-0.07798090,-0.07050515,0.072$ $83663,0.00549282,0.00184102,-0.00681771,0.00218795,-0.00183628,-0.0014$ $6331,-0.00008563,0.00042333,0.00004749,-0.00007349,-0.00093957,0.00109$ $942,-0.00303110,0.00303970,0.00011261,0.07569995,-0.00553934,0.0005474$ $8,-0.00197788,-0.00715400,-0.00979760,0.01882601,-0.07086703,-0.140948$ $27,0.11677008,-0.00083871,0.00395707,-0.00462428,-0.00164406,0.0021230$ $6,-0.00084335,0.00017899,-0.00033274,-0.00057672,-0.00072802,-0.000226$ $11,0.00048732,0.00278276,-0.00121344,-0.00061658,0.08042873,0.14601274$ $,-0.00181085,-0.00134405,0.00168114,-0.00457746,-0.00618876,0.00730012$ $, 0.07192874,0.11658879,-0.19630365,0.01484594,0.02240944,-0.01502793,-$ $0.00249151,-0.00194657,-0.00545380,-0.00081596,-0.00118567,-0.00041717$ ,-0.00013443,-0.00028638,0.00015033,0.00069288,0.00011345,0.00122108,$0.07793019,-0.12762067,0.20402263,0.00337074,-0.00699616,-0.00019507,-$ $0.00019196,0.00033059,0.00013031,0.00182003,-0.00293749,0.00092270,0.0$ $0609071,-0.00068761,0.01018731,-0.05894541,-0.02018414,-0.07264435,-0$. $00242818,-0.00150904,-0.01789178,0.00007589,-0.00028098,-0.00140173,-0$ $.00041345,0.00039880,-0.00004380,-0.00019401,0.00033502,0.00054637,0.0$ $5499935,-0.00579717,-0.00023680,-0.00019120,-0.00029005,-0.00114532,0$. $00014434,-0.00317058,-0.00076318,0.00310773,0.00455318,0.00680534,0.01$ $768381,-0.02099867,-0.05236114,-0.03503028,-0.00571821,0.00018576,-0.0$ $2309943,-0.00060270,-0.00000031,-0.00063464,0.00037075,-0.00024099,-0$. $00007443,0.00044472,0.00003573,0.00116324,0.02583811,0.04824369,-0.001$ $03284,-0.00068664,0.00268943,0.00074899,0.00077239,0.00043967,0.001903$ 
46,0.00411083,-0.00210404,-0.01068749,-0.00939623,-0.01836746,-0.07223 $016,-0.03467749,-0.30399080,0.00037375,0.00171732,-0.00437938,-0.00021$ $958,0.00013520,-0.00023092,0.00002776,-0.00014898,-0.00011056,-0.00018$ $312,-0.00031246,-0.00131102,0.07985097,0.03941955,0.32245754,0.0050433$ $9,0.00606950,-0.00857122,0.00218262,-0.00207400,-0.00181605,-0.0005773$ $9,0.00037220,0.00014582,0.00508375,-0.00520253,-0.00403829,-0.00167401$ $,-0.01219811,0.01471461,-0.07030068,-0.06496064,0.06159858,-0.00139268$ $, 0.00076556,0.00035989,0.00049448,0.00015725,-0.00033312,-0.00042149,0$ $.00033016,0.00011309,-0.00305608,0.00285775,0.00031127,0.06590500,0.00$ $106233,0.00462257,-0.00326198,-0.00172996,0.00253239,-0.00063135,0.000$ $37951,-0.00030574,-0.00055233,-0.00525796,0.00250214,-0.00336009,-0.00$ $812986,-0.01115977,0.02057949,-0.06461476,-0.15070050,0.11916177,0.001$ $56988,-0.00004353,0.00044689,-0.00003365,0.00015945,-0.00018851,0.0003$ $1941,-0.00031710,0.00006785,0.00285354,-0.00095247,-0.00094598,0.07444$ $837,0.15576254,0.01166036,0.01976402,-0.01547322,-0.00319483,-0.001575$ $27,-0.00508920,-0.00054320,-0.00096020,0.00025759,-0.00237602,-0.00118$ $998,0.00143476,-0.00349993,-0.00527299,0.00712524,0.05908738,0.1210668$ $0,-0.19321844,0.00029664,-0.00066533,0.00104024,0.00061041,0.00100480$, $-0.00126536,0.00019689,-0.00000141,0.00000185,0.00093118,-0.00030518,0$ $.00105887,-0.06372387,-0.13177639,0.20390245,0.00231652,-0.00327867,-0$ $.00060402,0.00459424,0.00076874,-0.00062859,-0.00643984,-0.02807554,-0$ $.02665180,-0.20838116,-0.19338511,-0.05871646,-0.01194703,-0.04174708$, $0.00611290,0.00514590,0.00040380,0.00263441,-0.00022572,0.00014399,0.0$ $0000759,-0.00148519,-0.00021910,-0.00068329,-0.00121385,0.00263534,-0$. $00053951,-0.00127025,0.00239713,0.00119047,-0.00165036,-0.00064709,0.0$ $0028944,0.22509559,0.00355965,-0.00443852,-0.00245405,-0.00049697,0.00$ $284387,0.00130186,-0.02609809,-0.01246000,-0.03054359,-0.19290669,-0.3$ $1489904,-0.07188456,-0.04417419,-0.04449898,0.00759160,0.00182237,0.00$ $462046,0.00600920,0.00025295,0.00001782,-0.00012380,0.00004024,-0.0012$ $1803,-0.00076023,0.00236557,0.00029997,-0.00172462,0.00274024,0.000559$ $91,-0.00001985,-0.00061044,-0.00219045,0.00004523,0.25952399,0.3692992$ $5,0.00016277,-0.00370877,0.00815326,0.00558098,0.00978736,-0.00799078$, $-0.03900765,-0.04785119,-0.01651194,-0.05872327,-0.07208684,-0.1114214$ $0,0.01824528,0.02488773,0.02164352,-0.00327057,-0.00220457,-0.01029244$ $, 0.00003864,-0.00018446,0.00065395,-0.00124674,-0.00160180,-0.00108341$ $, 0.00128374,0.00045036,0.00415678,-0.00062576,-0.00215115,0.00386511,0$ $.00095869,0.00085142,0.00005323,0.07808067,0.09320106,0.10915765,-0.00$ $021581,-0.00001604,0.00014143,-0.00031756,-0.00039434,0.00023015,0.000$ $25112,0.00014040,0.00032873,-0.00036667,-0.00054152,0.00003882,0.00042$ $892,0.00054196,-0.00030935,-0.00050711,-0.00024337,-0.00040717,-0.0002$ $3253,-0.00004553,0.00001417,-0.00004080,-0.00001489,-0.00007856,-0.000$ $00122,0.00008343,-0.00004850,-0.00000254,0.00004777,0.00006531,-0.0000$ $7235,-0.00002065,-0.00001993,0.00058779,0.00070740,0.00018228,0.476258$ $29,0.00038956,-0.00029566,-0.00042997,0.00005062,-0.00016367,0.0001022$ $9,-0.00006946,0.00018774,-0.00014600,0.00029997,0.00019560,0.00001011$, $-0.00005157,0.00017420,0.00006661,-0.00032682,0.00015873,0.00009745,-0$ $.00016231,0.00025442,0.00003285,0.00003667,-0.00003495,-0.00005289,0.0$ $0000492,-0.00001761,0.00003685,0.00005256,-0.00001133,-0.00003476,-0.0$ $0001548,0.00008081,-0.00011921,-0.00022483,-0.00022079,-0.00007122,0.0$ $5345235,0.63639807,-0.00000858,-0.00009073,0.00004454,0.00006703,0.000$ $18251,-0.00004331,0.00003312,-0.00001940,-0.00006813,-0.00000509,0.000$ 
$06146,-0.00001142,-0.00004839,-0.00009038,-0.00001744,0.00020122,0.000$ $08168,0.00008268,0.00005860,0.00002208,0.00005476,0.00000814,-0.000016$ $07,0.00000385,-0.00001326,-0.00002522,0.00000668,-0.00001223,-0.000042$ $79,0.00000776,-0.00004956,0.00001072,-0.00001204,-0.00008910,-0.000103$ $24,-0.00002786,-0.06760603,-0.03318822,0.13227861,0.00157324,-0.001022$ $62,-0.00047218,-0.00028211,-0.00031242,-0.00002231,0.00001071,0.000492$ $92,0.00010822,0.00007416,0.00009008,-0.00033760,-0.00030734,-0.0002143$ $6,0.00003879,0.00029291,0.00026738,0.00032087,0.00006084,0.00072307,0$. $00009009,0.00007715,-0.00007530,-0.00001161,0.00004977,-0.00000469,0.0$ $0002123,0.00002281,-0.00002770,-0.00000856,-0.00008247,0.00015489,-0.0$ $0019779,-0.00010767,-0.00009856,-0.00000361,-0.10173680,-0.00998810,0$. $00764793,0.65891882,-0.00130948,-0.00034243,0.00013843,-0.00000708,0.0$ $0098321,-0.00032074,-0.00013764,-0.00039549,-0.00033619,0.00010901,0.0$ $0031500,0.00000440,-0.00037460,-0.00086391,0.00026730,0.00064252,0.000$ $69232,0.00083020,-0.00004008,-0.00005961,0.00013141,-0.00003414,0.0000$ $3930,0.00019367,0.00000900,-0.00002409,-0.00000446,-0.00003190,-0.0000$ $1459,-0.00002682,0.00002905,0.00005562,-0.00008495,-0.00043836,-0.0006$ $3648,-0.00020169,0.05364638,-0.32836748,0.00234461,0.04375511,0.679409$ $85,-0.00061123,0.00065260,0.00044163,-0.00057615,-0.00082400,-0.000110$ $69,0.00025970,0.00050853,0.00063048,-0.00018876,-0.00022450,-0.0004974$ $1,-0.00028860,-0.00040227,-0.00004094,0.00112447,0.00001998,0.00021777$ ,-0.00005303,-0.00018259,-0.00005151,0.00006498,-0.00002180,-0.0000639 $7,0.00003269,-0.00001225,-0.00001299,0.00000474,0.00002155,0.00001913$, $0.00000329,-0.00004336,0.00007027,0.00029965,0.00038789,0.00016050,0.0$ $0499743,0.01429577,-0.06459076,-0.09930784,-0.03388798,0.14383657,-0.0$ $0296686,-0.00338709,0.00052464,-0.00032892,0.00201748,-0.00117660,-0.0$ $0048885,-0.00080272,-0.00066940,0.00031169,0.00072773,0.00012399,-0.00$ $069197,-0.00175831,0.00037056,0.00094140,0.00176676,0.00229971,0.00132$ $159,0.00049896,0.00014306,-0.00005809,0.00012031,0.00044471,0.00002996$ -0.00019860,0.00005973,-0.00003844,-0.00009941,-0.00010446,0.00003580 $, 0.00021492,-0.00016604,-0.00092932,-0.00136039,-0.00041793,0.00642664$ $, 0.08789605,-0.00374727,-0.28324818,0.02572298,0.03857407,0.58408670,0$ $.00206148,0.00178004,0.00067659,-0.00034377,-0.00249185,0.00058920,0.0$ $0043446,0.00094254,0.00138408,-0.00088725,-0.00148628,0.00017195,0.001$ $33487,0.00224154,-0.00084860,-0.00078657,-0.00222313,-0.00226979,0.000$ $91452,0.00034326,-0.00038215,-0.00000129,-0.00011336,-0.00036258,0.000$ $02851,0.00014686,-0.00007209,-0.00003959,0.00009291,0.00015579,-0.0000$ $5540,-0.00029520,0.00045173,0.00168738,0.00239417,0.00065029,0.0289683$ $4,-0.00942230,-0.00452106,0.09673880,-0.13686873,-0.01377179,-0.008331$ $13,0.68772685,0.00120932,0.00003796,-0.00037510,-0.00028636,-0.0008290$ 4,0.00010387,0.00047506,0.00055953,0.00074544,-0.00025443,-0.00012677, $-0.00060816,0.00005007,0.00015676,-0.00018867,-0.00025215,-0.00010920$, $-0.00040696,-0.00037566,-0.00018692,-0.00000831,0.00000090,-0.00009100$ $,-0.00007377,-0.00000320,0.00004143,-0.00000972,0.00002624,-0.00002727$ ,0.00002099,0.00001793,-0.00010720,0.00010639,0.00033054,0.00040057,0. $00026257,-0.00075617,-0.01604776,0.00961368,0.03419446,-0.00054757,-0$. $06971159,-0.08118164,-0.02587247,0.14819797,0.04381031,-0.00650314,0.0$ $0070751,-0.02288896,-0.01224246,0.01493355,0.00390958,0.00719169,0.001$ $88720,0.00213403,0.00099314,-0.00111017,0.00260388,0.00416891,0.002707$ $08,-0.01753489,0.00014691,-0.02010618,-0.01134558,0.00362223,0.0000690$ $6,0.00067794,-0.00056590,-0.00133842,0.00031333,0.00046936,-0.00001511$ 
$, 0.00023613,0.00039762,0.00026761,0.00045202,-0.00103220,0.00110749,-0$ $.00127877,-0.00116248,-0.00013251,-0.00869139,-0.02169042,0.00118349,--$ $0.04818691,0.00288103,0.01202702,-0.21182387,-0.12308984,0.03439819,0$. $34135914,-0.01436961,-0.01506527,-0.00160278,-0.00048312,0.00470960,-0$ $.00416524,-0.00052768,-0.00086412,0.00031787,-0.00062659,-0.00007424,-$ $0.00102532,-0.00192611,-0.00248193,-0.00020180,0.00281953,0.00355231,0$ $.00665238,0.01185517,-0.00381855,-0.00006384,-0.00001688,0.00015123,0$. $00042398,0.00027581,-0.00034199,-0.00002741,0.00006241,-0.00021447,-0$. $00012978,0.00005133,0.00040029,-0.00040526,0.00004337,0.00001858,0.000$ $05771,-0.02121372,-0.06411660,0.00654909,-0.04728797,0.05433954,0.0073$ $2047,-0.09924045,-0.19534588,0.02654235,0.08600117,0.58279645,-0.00027$ $960,-0.00308879,-0.00276647,0.00887929,0.00693247,-0.00038767,-0.00328$ $585,-0.00542212,-0.00535928,0.00116492,0.00051728,0.00462473,0.0017909$ $1,0.00443110,0.00047215,-0.00978980,-0.00154644,-0.00330651,0.00229753$ $, 0.00020926,0.00048459,-0.00055964,0.00043346,0.00075990,-0.00026585,--$ $0.00003226,0.00002365,0.00019699,0.00002437,-0.00011141,0.00040516,-0$. $00026895,0.00002721,-0.00163907,-0.00211924,-0.00100561,0.00042613,0.0$ $0741481,-0.00807596,0.01561049,-0.00282016,0.01510128,0.03188800,0.027$ $82653,-0.06227515,-0.05169556,-0.03815742,0.09239117,-0.00340933,0.004$ $90273,0.00013567,0.00201519,0.00337558,-0.00141466,-0.00064812,-0.0017$ $6555,-0.00172963,0.00082884,0.00132906,0.00055316,-0.00061375,-0.00168$ $628,0.00040415,0.00108773,0.00148664,0.00288053,-0.00115549,0.00135844$ $, 0.00022199,-0.00007403,0.00018578,0.00051414,-0.00023087,-0.00013524$, $0.00014788,-0.00005049,-0.00011524,-0.00015533,-0.00005947,0.00037069$, $-0.00043063,-0.00162358,-0.00246839,-0.00070363,-0.05475913,-0.0485994$ $5,0.01379795,-0.02394905,0.03225402,0.00181871,0.03648799,-0.02065806$, $-0.00514177,-0.09460237,0.02251304,0.00555538,0.63477179,0.00247855,0$. $00003375,-0.00196080,-0.00090813,-0.00020692,0.00099244,0.00002806,-0$. $00025256,0.00013993,0.00041756,-0.00028309,-0.00050841,-0.00048434,-0$. $00049828,0.00019830,0.00009246,0.00072886,0.00032375,0.00028928,0.0000$ $3384,0.00000516,0.00005351,0.00009099,0.00005012,-0.00016598,0.0000222$ $9,0.00006648,0.00001465,-0.00000081,0.00001529,0.00007256,-0.00002874$, $0.00016478,-0.00032863,-0.00049618,-0.00007610,0.01008968,0.05808350,-$ $0.00402914,0.02597848,-0.03750748,-0.00371648,-0.04317986,-0.03869626$, $0.00910163,0.04739152,-0.31958066,0.00477470,0.04337789,0.65375877,0.0$ $0066681,-0.00005862,-0.00068167,-0.00022612,-0.00146666,0.00019199,0.0$ $0031578,0.00061728,0.00088421,-0.00048006,-0.00037736,-0.00087095,-0.0$ $0014963,0.00017699,-0.00011923,0.00004051,-0.00004144,0.00000093,0.000$ $01457,-0.00023495,0.00011423,0.00002359,-0.00002915,-0.00002844,0.0000$ $3401,0.00003161,0.00001216,0.00005383,-0.00004941,0.00008369,-0.000047$ $63,-0.00009678,0.00011240,0.00055691,0.00083087,0.00041750,0.01154953$, $0.00730172,0.00583300,0.00245669,-0.00462669,-0.00590143,-0.00392103,0$ $.00623362,0.00379007,0.00448171,0.00637679,-0.05732814,-0.09234288,-0$. $03083351,0.15325124,0.00136657,-0.00012454,0.00029209,0.00024732,-0.00$ $044770,-0.00020172,-0.00012240,0.00016605,0.00019845,-0.00028744,-0.00$ $004175,-0.00021344,-0.00019411,-0.00042218,0.00000588,0.00073947,-0.00$ $029494,-0.00022737,0.00020727,-0.00022349,-0.00006793,-0.00003567,0.00$ 005490,0.00005270,0.00002403,-0.00004731,-0.00002721,-0.00002805,-0.00 $000981,-0.00000362,-0.00000492,-0.00001637,0.00013510,0.00017713,0.000$ $19520,0.00011459,-0.20528565,-0.07712022,0.02960015,0.03861139,-0.0555$ $4660,-0.00322972,-0.05540716,-0.00719820,0.00951118,0.01162950,0.07924$ 
$855,-0.00202777,-0.28731822,0.02625624,0.03886536,0.61635866,-0.000207$ $77,-0.00095553,0.00014944,-0.00106710,-0.00076655,0.00022695,0.0004302$ $3,0.00090524,0.00062069,-0.00014382,-0.00033882,0.00001430,0.00031049$, $0.00068286,-0.00021208,-0.00046061,-0.00047684,-0.00066331,0.00028739$, $-0.00021021,-0.00000576,0.00007342,-0.00010305,-0.00017896,0.00009636$, $0.00002462,-0.00004948,0.00001442,0.00000579,0.00003208,0.00000341,-0$. $00014479,0.00006406,0.00048884,0.00073540,0.00017935,-0.14118300,-0.21$ $656726,0.03251895,-0.01201152,-0.04352458,0.00465160,-0.01520135,-0.00$ $377369,0.00278970,0.03085274,-0.00909108,-0.00492968,0.09847894,-0.140$ $31034,-0.01456686,-0.01621479,0.72760794,-0.00035717,0.00076474,0.0000$ $9144,-0.00024511,-0.00056707,-0.00004925,0.00049053,0.00034140,0.00051$ $664,-0.00017064,-0.00032014,-0.00056487,-0.00000553,-0.00022048,-0.000$ $00852,0.00045801,0.00023487,0.00046429,-0.00016212,-0.00002914,-0.0000$ $3935,0.00000653,0.00002480,-0.00002662,0.00000432,0.00001350,0.0000032$ $2,0.00001030,0.00000820,0.00000250,-0.00000793,0.00004847,-0.00004253$, $0.00027260,0.00042498,0.00018999,0.03234508,0.01995258,-0.06961314,-0$. $00523610,0.01311078,0.00962280,0.00998350,0.00177495,-0.00736874,-0.00$ 080806,-0.01416458,0.01745280,0.03672757,-0.00162845,-0.06942277,-0.08 $932922,-0.02259050,0.14200478,0.00017935,-0.00016604,0.00008640,0.0003$ $0057,0.00065667,-0.00035530,-0.00033748,-0.00024122,-0.00056187,0.0006$ $0058,0.00079736,0.00034906,-0.00031111,-0.00040213,0.00029118,0.000123$ $42,0.00032198,0.00054751,0.00023528,0.00006012,0.00000741,0.00006154,0$ $.00001903,0.00006356,-0.00001052,-0.00008884,0.00003938,-0.00001431,-0$ $.00005242,-0.00009947,0.00007955,0.00004695,-0.00002817,-0.00081629,-0$ $.00104134,-0.00035138,-0.11517167,0.02836104,0.01595963,-0.02582638,0$. $00777474,0.00499057,0.00303718,0.00414859,0.00013199,0.00103138,0.0003$ $9815,-0.00069130,0.00011923,-0.00824758,0.00111670,-0.03972238,0.01864$ $426,0.00707692,0.17673108,-0.00005916,0.00008796,-0.00003431,-0.000122$ $43,-0.00023770,0.00011968,0.00012609,0.00012879,0.00021234,-0.00023887$ ,-0.00030756,-0.00011068,0.00012505,0.00016437,-0.00011525,-0.00005868 $,-0.00013570,-0.00020760,-0.00014030,0.00000564,0.00000879,-0.00001836$ ,-0.00001672,-0.00002357,0.00001489,0.00003448,-0.00002274,0.00000978, $0.00001717,0.00003764,-0.00002912,-0.00002017,-0.00000099,0.00030706,0$ $.00042334,0.00013401,0.02833225,-0.04897910,-0.00388263,0.03622370,-0$. $00892703,-0.00603998,-0.00387656,-0.00456080,0.00118686,0.00029536,0.0$ $0187572,-0.00016804,0.00029672,-0.00145539,0.00017258,-0.00984587,0.00$ $601194,0.00163839,-0.05226767,0.05395815,0.00006396,-0.00003951,-0.000$ $09089,-0.00002622,-0.00006161,0.00006683,-0.00001245,-0.00000199,0.000$ $02411,-0.00007816,-0.00008446,0.00002728,0.00008054,0.00010878,-0.0000$ $4096,-0.00016882,-0.00008172,-0.00015327,-0.00002675,0.00000380,-0.000$ $00528,-0.00000935,-0.00000239,0.00000121,-0.00000280,0.00001471,-0.000$ $00535,0.00000994,0.00001280,0.00001657,-0.00000232,-0.00001406,0.00000$ $751,0.00009761,0.00011775,0.00004294,0.01584960,-0.00389952,-0.0229066$ $6,0.00350167,-0.00068858,0.00273707,0.00066789,-0.00040046,0.00461532$, $-0.00049327,-0.00027861,-0.00166061,0.00089336,0.00173047,0.00442126,0$ $.00818401,-0.00348960,0.00207373,-0.02765290,0.00759661,0.01558166,-0$. $00026352,-0.00018290,-0.00009288,0.00003665,0.00047939,-0.00019785,-0$. $00009800,-0.00016416,-0.00029451,0.00023621,0.00036420,0.00003344,-0.0$ $0023549,-0.00045375,0.00017184,0.00017229,0.00042967,0.00051666,0.0000$ $5774,0.00004522,0.00003510,0.00000708,0.00001463,0.00007975,-0.0000175$ $0,-0.00004122,0.00001519,-0.00000989,-0.00002888,-0.00004415,0.0000098$ 
$9,0.00006992,-0.00007703,-0.00040455,-0.00055124,-0.00016375,0.0033856$ $2,0.00667329,-0.00057572,-0.20984055,-0.13645010,0.03843336,-0.0150044$ $4,-0.02001079,0.00417337,-0.00398480,0.00213085,0.00207545,-0.00057584$ $, 0.00139043,-0.00018928,0.00193143,0.00192833,0.00053914,0.00165990,0$. $00048356,-0.00080016,0.22166156,-0.00002867,0.00009841,0.00001262,0.00$ $001388,0.00006865,-0.00003356,-0.00002278,-0.00001186,-0.00006560,0.00$ $007967,0.00009328,0.00004597,-0.00002427,-0.00003577,0.00004075,-0.000$ $00535,0.00003164,0.00005806,0.00000165,-0.00002018,-0.00001486,0.00000$ $967,-0.00000184,0.00000532,-0.00000509,-0.00001224,-0.00000212,-0.0000$ $0529,-0.00000718,-0.00001104,0.00001444,0.00000337,-0.00000708,-0.0000$ $9670,-0.00011526,-0.00004123,-0.02513914,-0.01431440,0.00534143,-0.130$ $84718,-0.16845331,0.02998523,0.00883924,0.00931327,-0.00184345,0.00168$ $372,0.00095455,-0.00004585,0.00093340,-0.00018453,-0.00019700,0.002939$ $62,-0.00483758,-0.00006805,-0.00025576,0.00093029,-0.00015806,0.143600$ $20,0.17651126,-0.00030586,0.00007950,0.00007209,-0.00001965,0.00005027$ -0.00003038,-0.00000981,-0.00003293,-0.00002052,-0.00002232,0.0000268 $6,0.00005608,-0.00003745,-0.00000858,-0.00000455,0.00003919,-0.0000615$ $2,0.00007217,0.00002895,-0.00000809,0.00003780,0.00000576,0.00000113,-$ $0.00002323,0.00000286,0.00000871,0.00000055,-0.00000624,0.00000644,-0$. $00000120,0.00001871,-0.00001460,0.00000437,0.00002118,0.00000853,-0.00$ $002124,0.00100362,-0.00020320,0.00238845,0.03808221,0.03082803,-0.0414$ $5208,0.00328326,0.00329929,0.00334052,0.00178151,-0.00045181,0.0056180$ $9,-0.00003079,0.00004380,-0.00077113,0.00056127,0.00010801,0.00563598$, $-0.00080844,-0.00032334,-0.00264585,-0.04269802,-0.03308756,0.03179800$ $, 0.00076182,-0.00096140,-0.00042145,0.00007518,0.00024485,0.00006961,-$ $0.00007070,0.00005173,-0.00008587,-0.00000624,-0.00011050,0.00005048,0$ $.00000912,0.00008809,-0.00000098,-0.00017564,0.00009302,0.00003589,0.0$ $0018470,0.00066027,0.00022259,0.00000782,-0.00004394,-0.00001665,0.000$ $02354,0.00001584,-0.00000699,0.00003017,0.00000567,0.00001259,-0.00000$ $676,-0.00000069,-0.00005685,0.00002595,0.00007658,0.00000555,-0.003122$ $03,0.00068565,0.00176882,-0.00333259,0.03005117,0.00009625,-0.06128576$ $, 0.04298556,0.00259149,0.00696676,-0.02238954,-0.00031958,-0.00208872$, $-0.00447348,0.00206529,-0.00126378,-0.00033929,0.00005824,-0.00149680$, $0.00149407,0.00024366,0.00107503,-0.00046805,-0.00083584,0.06331708,-0$ $.00011190,0.00032326,-0.00019000,0.00015454,0.00066015,0.00005951,-0.0$ $0006571,-0.00016776,-0.00047505,0.00025185,0.00035061,0.00007134,-0.00$ $020392,-0.00042417,0.00022243,-0.00000701,0.00047760,0.00043571,-0.000$ $79989,-0.00004565,0.00013286,0.00000463,-0.00000089,0.00006656,-0.0000$ $0462,-0.00000589,0.00000776,0.00002089,-0.00000976,-0.00003347,0.00000$ $407,0.00009106,-0.00018451,-0.00043986,-0.00057073,-0.00018312,0.00187$ $397,0.00224069,-0.00006550,0.00038176,-0.00943977,0.00048211,0.0491742$ $9,-0.30447714,0.00454131,-0.00108382,-0.01892733,0.00155978,-0.0027923$ $4,-0.00207041,0.00097877,-0.00053675,0.00068065,-0.00003252,0.00047015$ ,-0.00051812,0.00001212,-0.00004538,0.00057116,-0.00023214,-0.04675460 $, 0.33176318,0.00024939,0.00013768,-0.00025913,0.00038749,0.00026348,0$. $00016272,-0.00015769,-0.00027046,-0.00034939,0.00013213,-0.00000217,0$. $00031434,0.00022268,0.00029998,-0.00007292,-0.00059555,-0.00020004,-0$. $00019868,0.00001778,-0.00012769,-0.00006019,-0.00002125,0.00003672,-0$. $00000038,-0.00002352,0.00000633,-0.00000102,-0.00000524,-0.00001708,-0$ $.00000668,-0.00007032,0.00007626,-0.00008857,-0.00010690,-0.00009784,-$ $0.00007837,0.00147645,-0.00017486,0.00618408,0.00181557,-0.00462103,0$. 
$00506793,0.00178311,0.00526260,-0.03655470,-0.00132593,0.00529911,-0.0$ $0069681,0.00193660,0.00130803,0.00757146,0.00040454,0.00001871,-0.0009$ $7442,0.00027112,-0.00021207,0.00030567,-0.00083862,-0.00012393,-0.0035$ $6355,-0.00519610,-0.00702191,0.02458269,-0.00026150,-0.00005528,0.0006$ $1556,0.00103445,0.00105750,-0.00012111,-0.00042903,-0.00097696,-0.0003$ $6708,-0.00045253,0.00010476,-0.00013905,0.00009210,0.00000731,-0.00006$ $015,-0.00003402,-0.00034193,-0.00023722,0.00003915,-0.00002366,0.00004$ $166,-0.00004700,0.00000005,-0.00006884,0.00000608,0.00005102,-0.000045$ $31,0.00001552,0.00000267,0.00004731,0.00000805,-0.00005768,0.00002789$, $0.00011288,0.00014962,0.00012453,-0.00204575,0.00298786,0.00146637,-0$. $00000305,0.00064369,-0.00014816,0.00119615,0.00234645,0.00099942,0.008$ $90986,0.00040679,-0.00161390,-0.20395803,-0.12766545,0.03556399,-0.018$ $56015,-0.01902759,0.00519506,-0.00170161,-0.00022540,0.00035483,-0.000$ $06724,-0.00005679,-0.00022556,0.00031107,0.00043693,-0.00009924,0.2148$ 4746,-0.00110996,-0.00019362,-0.00001005,-0.00015874,-0.00045083,-0.00 $022831,0.00015692,0.00073657,0.00021571,-0.00002179,0.00029744,0.00010$ $785,0.00007193,0.00016350,-0.00010697,0.00005154,-0.00015373,-0.000090$ $82,-0.00000088,0.00001260,0.00003686,0.00008172,-0.00011990,-0.0001164$ $7,-0.00001701,0.00001355,0.00004479,-0.00001158,0.00000718,-0.00000342$ ,-0.00001251,-0.00001296,-0.00002750,0.00018612,0.00010598,0.00003041, $0.00196782,0.00100791,-0.00025522,0.00113364,-0.00059110,-0.00016015,0$ $.00020210,-0.00593395,0.00046890,-0.02098348,-0.01952836,0.00485553,-0$ $.13181535,-0.16498545,0.02917586,0.01021520,0.00879656,-0.00198561,0.0$ $0106876,0.00000616,-0.00017717,-0.00009417,0.00006429,-0.00004426,-0.0$ $0092523,-0.00047178,0.00022779,0.14044615,0.18059789,-0.00037685,-0.00$ $022394,-0.00055050,-0.00046830,0.00013134,-0.00005412,-0.00004484,0.00$ $018222,-0.00028886,0.00002235,0.00035016,0.00049370,-0.00016629,-0.000$ $45814,-0.00008794,0.00026390,0.00021598,0.00031839,0.00017175,0.000020$ $45,0.00003035,0.00010599,-0.00012156,-0.00010609,0.00005896,-0.0000160$ $3,-0.00002711,-0.00003344,0.00002084,-0.00003990,0.00002031,0.00001278$ $,-0.00001170,-0.00022354,-0.00048483,-0.00028963,0.00163004,0.00008068$ $, 0.00636724,-0.00012751,-0.00044675,-0.00087356,0.00131347,0.00015861$, $0.00742313,-0.00041924,0.00104426,0.00022744,0.03549271,0.02850206,-0$. $04587824,0.00398626,0.00309734,0.00458383,0.00030870,0.00006513,0.0002$ $2522,-0.00017720,-0.00006307,-0.00127133,-0.00000651,-0.00006331,0.000$ $04070,-0.04044678,-0.03187560,0.03345092,-0.00018207,0.00025464,0.0000$ $1258,0.00027035,0.00037929,-0.00012091,-0.00013175,-0.00022335,-0.0002$ $8250,0.00020937,0.00027769,0.00008277,-0.00013804,-0.00030672,0.000110$ $99,0.00018217,0.00022722,0.00030720,-0.00006042,0.00005042,0.00000652$, $-0.00001003,0.00003093,0.00005718,-0.00003331,-0.00002358,0.00003259$,$0.00001828,-0.00000453,-0.00003071,0.00000409,0.00005044,-0.00002849$,$0.00028243,-0.00046504,-0.00013093,0.00523062,-0.02264188,0.00036277,-$ $0.00278869,-0.00314872,0.00167659,-0.00160323,-0.00024706,0.00014123$,$0.00369632,0.00227549,0.00187766,-0.00019429,0.02752258,-0.00044865,-0$ $.06306282,0.05424800,0.00315409,0.00124947,-0.00082613,-0.00067387,0.0$ $0026845,-0.00116042,0.00017143,0.00006078,0.00007273,-0.00025651,0.000$ $98712,-0.00043122,-0.00088396,0.06373928,-0.00001775,-0.00010434,-0.00$ $000834,-0.00007992,-0.00028531,0.00010703,0.00004907,0.00015930,0.0002$ $2924,-0.00020614,-0.00026588,-0.00008350,0.00011299,0.00026049,-0.0001$ $1428,-0.00009393,-0.00018232,-0.00024030,0.00002177,-0.00001770,0.0000$ $0209,-0.00000975,-0.00002537,-0.00003858,0.00001952,0.00001924,-0.0000$ 
$2364,0.00001276,0.00000486,0.00003081,-0.00001741,-0.00003882,0.000014$ $82,0.00029520,0.00042571,0.00013242,0.00896044,-0.01627051,-0.00078294$ $,-0.00404941,0.00018127,0.00096142,0.00025504,0.00070714,-0.00003834,0$ $.00225833,0.00065221,-0.00038034,-0.00082704,-0.00619491,0.00068236,0$. $04901478,-0.32435066,0.00326888,-0.00000482,0.00151426,-0.00021932,0.0$ $0041292,-0.00064599,0.00000746,0.00001421,-0.00002894,-0.00006761,-0.0$ $0021007,0.00063208,-0.00012861,-0.05591078,0.34385420,-0.00000807,-0.0$ $0008534,0.00017122,0.00011748,0.00007631,0.00006814,-0.00006468,-0.000$ 09712,-0.00006827,0.00006215,-0.00002062,0.00012297,0.00011685,0.00014 $053,-0.00001055,-0.00019847,-0.00002959,-0.00015163,0.00001208,-0.0000$ $1931,-0.00003023,-0.00001299,0.00000602,-0.00000962,0.00000854,0.00000$ $907,-0.00001043,-0.00001123,0.00000997,-0.00000789,-0.00000703,0.00000$ $590,-0.00000131,-0.00001168,-0.00000371,-0.00003433,-0.00105306,0.0049$ $7585,0.00250137,0.00183688,0.00076481,0.00540149,-0.00007535,-0.000134$ $24,-0.00063907,0.00189302,0.00008983,0.00579061,0.00086951,-0.00466403$ $, 0.00431786,0.00341641,0.00221504,-0.03453178,-0.00069131,-0.00003082$, $-0.00263751,0.00007588,0.00027309,0.00078095,-0.00027364,-0.00008874,-$ $0.00132476,-0.00086423,-0.00008215,-0.00368195,-0.00513707,-0.00331075$ $, 0.02398470 \backslash 10.00000540,0.00000424,-0.00000118,0.00000096,0.00000260,-$ $0.00000016,-0.00000217,0.00000702,-0.00000034,-0.00000248,0.00000494,0$ $.00000351,0.00000146,0.00000401,-0.00000056,0.00000224,-0.00000223,-0$. $00000417,0.00000383,0.00000459,-0.00000369,-0.00000172,0.00000921,-0.0$ $0000155,-0.00000281,0.00000880,0.00000189,0.00000045,-0.00000022,-0.00$ $000041,0.00000204,-0.00000065,-0.00000343,-0.00000361,0.00000375,0.000$ $00212,-0.00000040,-0.00000651,0.00000213,0.00000113,-0.00000538,0.0000$ $0058,0.00000081,-0.00000179,-0.00000112,-0.00000094,-0.00000311,-0.000$ $00693,0.00000078,-0.00000270,0.00000435,-0.00000275,-0.00000375,0.0000$ $0430,-0.00000159,-0.00000929,0.00000375,0.00000259,-0.00000739,-0.0000$ $0199,0.00000387,-0.00000276,-0.00000583,-0.00000299,0.00000091,0.00000$ 272,-0.00000411,-0.00000429,0.00000600II! 


\section{Addition to disubstituted benzenes}

Addition Products: p-Chlorophenyl + disubstituted benzenes

ortho-Addition Product to 4-fluoroaniline

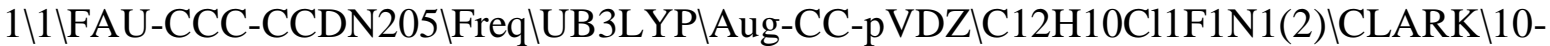
Dec-2014\0\\ ub3lyp/aug-cc-pvdz geom=check guess=read freq name=clark IIAddition product p-Cl-Ph-radical + 4-fluoro-aniline (ortho) $\backslash 0,2 \backslash \mathrm{C}, 1$ $.5236261652,0.1704525647,0.8163740911 \backslash \mathrm{C}, 2.2658111417,0.9644786043,-0.2$ 492032262\C,3.3649931862,0.4546152028,-0.9109460893\C,3.8754489448,-0. 8309559708,-0.6294096624\C,3.2222466209,-1.6046702223,0.3600833718\C,2 $.1332442148,-1.1907882231,1.0561616865 \backslash \mathrm{H}, 1.5920231581,0.7594011326,1.7$ $533619203 \backslash \mathrm{N}, 1.8208866384,2.2642498796,-0.4346653367 \backslash \mathrm{H}, 3.8593076977,1.0$ $670510425,-1.6677019324 \mathrm{~W}, 3.7387055825,-2.8463039728,0.6112594205 \backslash \mathrm{H}, 1$. $6793062493,-1.8370841263,1.8050704769 \backslash \mathrm{H}, 4.739154955,-1.2333285599,-1.1$ $53090241 \backslash \mathrm{C},-2.7093195641,-0.1136469809,-0.0849151788 \backslash \mathrm{C},-2.2975522025,0$ $.6243226624,1.022816035 \backslash \mathrm{C},-0.929095957,0.7099781429,1.3069310176 \backslash \mathrm{C}, 0.0$ $217423668,0.0703893897,0.502603735 \backslash \mathrm{C},-0.4251196005,-0.669093905,-0.603$ $5106062 \backslash \mathrm{C},-1.7840262993,-0.7653760827,-0.9050855362 \mathrm{lCl},-4.4284591018,-$ $0.2319499965,-0.457340627 \backslash \mathrm{H},-3.0314636211,1.1209995751,1.6549141138 \backslash \mathrm{H}$, $-0.6043229336,1.28111214,2.1783617837 \backslash \mathrm{H}, 0.2998928812,-1.1819738247,-1$. $2357450737 \backslash \mathrm{H},-2.1261661134,-1.3408379857,-1.7635946833 \backslash \mathrm{H}, 2.1861913971$, $2.7575400814,-1.2389295506 \backslash \mathrm{H}, 0.8343196847,2.4326568887,-0.2792913683 \backslash \backslash$ Version=EM64L-G09RevC.01 $\backslash$ State $=2-A \backslash H F=-1078.1402412 \backslash \mathrm{S} 2=0.777331 \backslash \mathrm{S} 2-1=0$ $. \mathrm{S} 2 \mathrm{~A}=0.750437 \backslash \mathrm{RMSD}=8.715 \mathrm{e}-09 \backslash \mathrm{RMSF}=6.518 \mathrm{e}-06 \backslash \mathrm{ZeroPoint}=0.1885567 \backslash \mathrm{Therm}$ $\mathrm{al}=0.2015807 \mathrm{ZPE}=118.3211081 \backslash \mathrm{Dipole}=-0.045194,1.1911448,-0.3313271 \mathrm{Dip}$ oleDeriv $=0.2848418,-0.1052954,0.0654662,0.0594103,0.0626738,0.0944097$, $0.1174467,0.1760605,0.155253,0.0983856,-0.4593918,0.0338409,-0.4370663$ $, 1.134268,-0.1002858,-0.1382679,-0.023135,-0.0118606,-0.0652615,0.3139$ $925,-0.2081482,-0.2409955,-0.1614345,0.055786,0.0264112,-0.2284091,-0$. $0352274,-0.0858133,-0.1227824,-0.0075457,0.1451378,-0.0591705,-0.10951$ $71,-0.1635305,0.0545862,-0.0818887,0.4574961,-0.3883637,-0.0471297,-0$. $6176493,1.1567095,-0.224503,-0.008259,-0.2799815,0.2191751,-0.3511698$, $0.0361586,-0.0423873,-0.1014223,-0.17063,-0.0478752,-0.0387064,-0.0395$ $883,-0.1495039,-0.0119364,0.0263355,0.0484703,0.0498795,-0.0317557,-0$. $1223458,0.0406685,-0.1120116,-0.075837,-0.3490051,0.1377075,-0.0768929$ $, 0.3397387,-1.4112573,0.0739389,0.0208479,-0.2093638,-0.2500895,0.0557$ $78,-0.0226826,0.0661737,-0.0254484,-0.0107938,0.1054191,0.1077586,0.09$ $45254,0.0185362,-0.4528606,0.3671602,-0.0278296,0.6846959,-1.0697473,0$ $.1528201,-0.0782784,0.2104933,-0.1918353,0.0967426,-0.0050603,0.071438$ $1,0.0055604,0.031898,0.0622595,0.072001,0.0610209,0.0608094,0.0316435$, $0.0496129,0.0714983,0.0536833,0.0642706,0.0081413,0.0844909,0.0014292$, $0.0863684,1.2360913,0.0668639,0.2266504,0.1055931,-0.0121479,0.0529842$ $, 0.2962146,0.0513637,0.0592169,-0.2606354,-0.0648579,-0.1080402,-0.089$ $4155,-0.0637042,0.0290596,-0.1323698,0.0329743,-0.0598043,-0.0435994,0$ $.0175444,0.0484639,-0.0687137,-0.0621998,0.0889075,-0.0533056,0.089567$ $4,0.0396083,0.2073906,0.0133716,-0.0107182,0.0330385,-0.0649694,-0.018$ $7897,0.0566272,-0.0138638,-0.084998,-0.0969761,-0.037098,-0.0258711,-0$ $.0065086,-0.0484362,0.0430007,-0.0020195,0.0378873,-0.0294214,-0.25946$ 
49,0.0309188,0.0208959,0.0104849,-0.0361386,0.0648535,-0.0058269,0.065 $9778,0.0091535,-0.9009538,-0.045453,-0.1363141,-0.0549836,-0.0778516$,$0.0188262,-0.1751592,-0.0192805,-0.1115009,0.0017418,0.0392717,0.04799$ $54,0.0379849,0.0918069,-0.0461425,0.0391118,-0.0462754,0.0654282,0.051$ $9311,-0.0394894,-0.0711283,-0.0172828,0.062451,-0.076813,-0.0503357,-0$ $.0756201,-0.0029982,0.0322408,0.0435978,0.0509339,0.0447371,0.0700772$, $-0.0409943,0.0621866,-0.0341051,0.0488666,0.0482179,-0.0203265,-0.0346$ $404,-0.0193263,0.0823009,-0.0632592,-0.0440225,-0.0628948,0.032096,0.1$ $352756,0.0991301,0.0407113,0.0602518,0.2598105,0.0062227,0.0467176,0.0$ $971822,0.1312431,0.1398996,0.0691356,0.0041072,0.0486161,0.2639703,0.0$ $315488,-0.0804013,0.1714609,0.1592105 \backslash$ Polar $=229.9251437,-16.2409696,15$ 3.4583888,-9.504556,5.0595217,136.8985689\PG=C01 [X(C12H10Cl1F1N1)]\NI $\mathrm{mag}=0 \backslash 0.41793207,-0.01729745,0.49684742,-0.01487254,0.02996189,0.4958$ $1710,-0.09892715,-0.02008807,0.04099995,0.52112885,-0.01290680,-0.1059$ $7616,0.03776264,-0.17450938,0.57505749,0.03777642,0.03643619,-0.124944$ $99,-0.21948157,-0.07335649,0.36901180,-0.00876677,0.00034882,0.0131898$ $4,-0.24794167,0.08659809,0.11918005,0.46385093,-0.01027786,0.01325922$, $0.00459543,0.04570622,-0.12518716,-0.00595203,-0.09827859,0.56890447,0$ $.01982105,-0.00270970,-0.00798964,0.13927815,-0.03428549,-0.15343251,-$ $0.25509474,-0.16512339,0.41626059,0.00046284,-0.00295631,-0.00331337,-$ $0.01465120,0.03177106,-0.00143398,-0.11086989,0.09742197,0.00460804,0$. $48722316,-0.00156753,-0.00611627,0.00060635,0.05772223,-0.02425936,-0$. $02795181,0.04317025,-0.24797534,0.07367672,-0.16684663,0.53390667,-0.0$ $0416730,0.00373500,-0.00215806,-0.01478247,-0.01089886,0.02141685,0.03$ $387817,0.03059549,-0.08835982,-0.24840953,-0.10318180,0.36262814,-0.01$ $236498,0.01667189,0.00303686,-0.01939190,0.00336094,0.01107742,0.03867$ $897,-0.03620741,-0.00904536,-0.11930825,0.00940715,0.04935586,0.486212$ $42,0.02285443,-0.01099044,-0.01282502,-0.00362733,-0.00329485,0.002580$ $49,-0.00475573,-0.03191502,0.02337113,-0.05412929,-0.14069746,0.093076$ $96,-0.13651631,0.52658573,-0.00198067,-0.00910289,0.00298204,0.0176765$ $7,-0.00199599,-0.01175554,-0.02739251,0.05066238,-0.00257647,0.0847217$ $1,0.03959459,-0.15086967,-0.22874049,-0.10481675,0.38555340,-0.0880155$ $6,0.04633906,0.00157492,0.01867844,-0.01445265,-0.00938501,-0.01682467$ $, 0.02173256,0.00209093,-0.02600463,-0.03381325,0.05071896,-0.27681226$, $0.10415098,0.13630164,0.52769511,0.03957601,-0.17891964,0.02891496,-0$. $00226873,-0.01677993,0.00897029,0.00691599,-0.00725010,-0.00137955,-0$. $00797450,0.02650885,-0.00197934,0.06856193,-0.12162094,-0.01815718,-0$. $10715271,0.50126100,0.00685642,0.02250612,-0.07876069,-0.01727017,0.02$ $293771,-0.00002558,0.01146716,-0.01505069,-0.00057235,0.03554160,0.020$ $70880,-0.03033818,0.15539082,-0.04820850,-0.15684539,-0.29254981,-0.12$ $567148,0.43044629,-0.03848349,-0.00675460,-0.00941474,-0.00246664,0.00$ $710993,0.01827741,0.00133173,-0.00315740,0.00034615,-0.00093615,0.0016$ $3172,-0.00033213,0.00059768,0.00079542,-0.00022151,-0.00113313,0.01119$ $825,0.01088280,0.05736693,-0.01227873,-0.10639302,-0.10237504,0.003300$ $09,0.00808670,0.01222397,-0.00195853,-0.00043967,0.00151144,0.00147664$ ,-0.00031412,-0.00092816,-0.00454340,-0.00257670,0.00169612,0.00701343 ,-0.01996261,-0.02153048,0.00755068,0.12069929,-0.01877523,-0.09957222 $,-0.20991314,0.00726513,-0.00871706,-0.02470668,-0.00321109,0.00494032$ $,-0.00144356,0.00112448,-0.00257741,-0.00001658,-0.00056485,0.00049642$ $, 0.00046172,0.00242088,-0.00134833,0.00891307,0.01371664,0.11242751,0$. $23227710,0.00787062,-0.01494438,0.00124685,-0.11914470,0.08992373,0.02$ 
$996184,-0.01269668,0.04691716,-0.00203530,-0.00128047,-0.00294371,0.00$ $688123,0.00035839,-0.00082912,-0.00031864,-0.00269817,0.00000779,0.003$ $24492,0.00052031,-0.00119692,-0.00038407,0.64928295,0.00068683,-0.0270$ $1116,0.01067148,0.07006679,-0.28063388,0.03934606,0.02122836,-0.025601$ $71,-0.00337172,0.00132692,0.00209629,0.00212165,0.00078056,0.00382486$, $-0.00302559,-0.00227447,-0.00220502,0.00226437,-0.00000295,0.00171859$, $-0.00046861,-0.12022280,0.51293780,-0.00764531,0.02815508,-0.00463267$, $0.02169745,0.06768376,-0.07203706,0.01088547,-0.02061879,0.00886080,0$. $00396431,0.00299802,-0.00080179,-0.00006212,0.00052654,-0.00060592,0.0$ $0464246,0.00100929,-0.00258645,-0.00141291,0.00052246,0.00247740,-0.23$ $071927,-0.25367429,0.39173619,0.00006332,0.00398552,0.00362339,-0.0079$ $2365,-0.01535437,0.01832212,-0.10551369,-0.06365624,0.09710058,0.00770$ $256,0.00647118,-0.00845478,0.00164996,0.00323082,0.00128227,-0.0004677$ $6,0.00039532,-0.00045302,-0.00064397,-0.00071370,0.00087927,-0.0002107$ $6,-0.00234932,-0.00170651,0.10589644,0.00319820,0.00057502,0.00083305$, $0.00624761,0.00680861,-0.00703369,-0.06387331,-0.13865981,0.11264972,-$ $0.01456382,-0.01121689,0.01959005,0.00394886,-0.00181500,0.00037890,0$. $00016596,-0.00010952,-0.00041393,-0.00020854,-0.00003936,0.00024594,-0$ $.00067613,0.00116251,-0.00213486,0.06711572,0.14389853,0.00362534,-0.0$ $0032795,0.00410643,0.00612604,0.01129459,-0.00751607,0.09799368,0.1114$ $8716,-0.17722677,0.00298217,0.00251488,-0.00200383,0.00072050,0.001458$ $25,0.00391196,-0.00025053,-0.00045251,-0.00017549,-0.00011928,-0.00032$ $419,0.00025351,-0.00298327,-0.00101159,-0.00080377,-0.10788295,-0.1239$ $2456,0.18214547,0.00110044,-0.00011975,0.00756872,0.00034758,-0.001103$ $48,0.00004091,-0.00100202,0.00259984,0.00299731,0.00885941,-0.01142766$ $, 0.00328335,-0.08801026,0.07717491,-0.00124350,-0.01483482,0.04535067$, $-0.00751563,-0.00092214,0.00221862,-0.00077610,0.00025778,0.00087308,-$ $0.00069366,0.00025724,-0.00032444,0.00020514,0.09411285,0.00266561,0.0$ $0548033,-0.00067849,0.00071410,0.00228100,-0.00128057,-0.00459286,0.00$ $159416,0.00546783,0.01675658,-0.04178736,0.01194403,0.08132167,-0.2328$ $1582,0.03878974,0.01654543,-0.03071284,0.00706906,0.00028908,-0.001033$ $97,0.00044192,0.00028235,-0.00331780,0.00003627,0.00085720,-0.00187497$ $, 0.00046398,-0.11296234,0.30240889,0.00547696,0.00306850,0.00395027,-0$ $.00096881,-0.00066021,0.00044599,0.00695656,-0.00055426,-0.00124501,-0$ $.01256569,0.03564460,-0.00401008,-0.00307343,0.04237259,-0.05997897,0$. $00834365,-0.01816068,0.00935883,-0.00042354,0.00076707,-0.00053680,-0$. $00051237,0.00100347,0.00010988,-0.00048672,0.00147461,-0.00015474,0.00$ $219268,-0.05989307,0.05411081,0.00544234,0.00736859,-0.00987424,-0.000$ $94059,0.00294167,0.00058077,-0.00052730,0.00048199,-0.00057788,-0.0014$ $8938,0.00387418,0.00343697,-0.00360857,-0.01682002,0.01857965,-0.09618$ $503,-0.06426707,0.09657139,0.00033933,0.00008364,0.00038852,0.00057237$ $, 0.00023549,-0.00083951,-0.00011105,-0.00015929,-0.00015130,-0.0003237$ $6,-0.00181003,-0.00369537,0.09528068,-0.01139009,-0.01137634,0.0174763$ $4,0.00434166,-0.00341530,-0.00134422,-0.00031772,-0.00045528,-0.000175$ $65,0.00332891,0.00051025,-0.00014717,0.00635873,0.00766288,-0.00569832$ ,-0.06642919,-0.14876563,0.12639055,-0.00049835,0.00084328,-0.00040868 ,-0.00122492,-0.00009631,0.00160665,-0.00015047,-0.00004122,-0.0000444 $5,-0.00233539,0.00042677,-0.00158198,0.06862170,0.15386275,0.00072031$, $-0.00024847,-0.00010875,0.00027557,0.00019219,0.00154830,-0.00072354,-$ $0.00047755,-0.00011048,0.00415224,-0.00106276,0.00227598,0.00599041,0$. $01390352,-0.00568390,0.09773625,0.12379742,-0.17852500,-0.00033044,0.0$ 
$0084823,0.00025973,-0.00001155,-0.00003430,-0.00009633,-0.00016276,-0$. $00004893,-0.00023806,-0.00358911,-0.00185543,-0.00189416,-0.10518508,-$ $0.13543724,0.18234717,-0.00014449,-0.00063345,-0.00066823,0.00211759,0$ $.00183246,0.00047596,-0.00345957,0.00037838,0.00472710,-0.22813323,0.0$ $9191566,0.12785038,-0.00811351,0.00901513,0.00492912,0.00112252,-0.001$ $63700,0.00191971,0.00016840,-0.00008090,-0.00007067,-0.00022150,0.0000$ $0577,0.00032325,-0.00059199,-0.00072267,-0.00158324,0.00020982,-0.0010$ $8802,-0.00199730,-0.00013310,0.00039724,0.00049773,0.23710919,-0.00019$ $273,-0.00046149,-0.00022185,0.00158673,-0.00384332,0.00265794,0.020095$ $09,-0.00376733,-0.01433160,0.09296599,-0.08899751,-0.04217839,-0.01324$ $782,0.00871876,0.00843454,-0.00115668,-0.00198681,0.00325919,0.0001093$ $1,-0.00000665,-0.00006720,0.00135154,-0.00001471,-0.00053370,-0.001180$ $84,0.00056097,-0.00090858,-0.00028233,0.00124883,-0.00198520,-0.000627$ $00,0.00048093,0.00065773,-0.09952909,0.08834609,-0.00078917,-0.0000372$ $6,-0.00046006,0.00047574,0.00259922,0.00181140,-0.00643728,0.00278050$, $0.00622720,0.12775225,-0.04129119,-0.10835693,0.01690813,-0.01074270,-$ $0.00525896,0.00157441,0.00375263,-0.00143639,0.00012822,-0.00005360,-0$ $.00000077,-0.00027204,0.00035063,0.00049394,-0.00131806,-0.00122384,-0$ $.00112899,-0.00241236,-0.00131405,0.00002444,0.00107809,-0.00021521,-0$ $.00001213,-0.13652994,0.04525459,0.10798210,-0.00124092,0.00026477,0.0$ $0026560,0.00002519,0.00005803,-0.00005153,-0.00013126,0.00029906,-0.00$ $007459,0.00006307,-0.00030812,0.00013066,-0.00028714,0.00051197,-0.000$ $28759,0.00004910,0.00006758,0.00000141,0.00004401,0.00004537,0.0000434$ $7,0.00026433,0.00015748,-0.00016288,0.00006040,0.00002897,-0.00005720$, $0.00037582,-0.00066424,0.00010909,-0.00006524,0.00004881,-0.00005420,0$ $.00002253,-0.00001792,-0.00002631,0.50405848,0.00057530,-0.00074976,-0$ $.00042874,0.00016057,-0.00015828,0.00039670,0.00002573,0.00002425,0.00$ $004078,0.00000822,0.00008942,0.00002269,-0.00013323,0.00031274,0.00018$ $723,0.00052911,0.00018909,0.00019203,0.00019674,0.00013208,0.00012668$, $0.00000678,0.00017586,0.00027073,-0.00007246,-0.00000090,-0.00005918,0$ $.00001910,-0.00030886,-0.00004267,0.00001058,-0.00004063,-0.00004686,0$ $.00001006,0.00001864,-0.00000718,-0.03740355,0.30100894,0.00070682,-0$. $00027647,-0.00113334,0.00056670,-0.00046159,0.00058916,-0.00005437,0.0$ $0019577,0.00006165,-0.00008266,0.00007213,0.00008698,-0.00021322,0.000$ $58442,0.00022379,0.00084026,0.00026893,0.00023588,0.00020906,0.0000069$ $4,0.00035930,-0.00017372,0.00037305,0.00045167,-0.00009692,-0.00002792$ $,-0.00012523,0.00007767,-0.00054371,-0.00001065,-0.00009041,0.00000570$ $,-0.00013054,0.00003369,-0.00000821,-0.00000421,-0.00834268,0.24596793$ $, 0.47725754,0.00060586,0.00180506,0.00227101,-0.00103220,0.00103256,-0$ $.00074399,0.00014973,-0.00041943,-0.00004649,0.00014854,-0.00001965,-0$ $.00016322,0.00018328,-0.00042843,-0.00020793,-0.00127763,-0.00054844,-$ $0.00025318,-0.00011566,-0.00016140,-0.00043321,0.00024107,-0.00065713$, $-0.00049617,0.00007299,0.00002498,0.00015183,-0.00013159,0.00061862,0$. $00004319,-0.00000236,0.00004460,0.00013414,-0.00001601,-0.00000665,0.0$ $0000013,-0.12092906,-0.00742210,-0.01704202,0.69486194,0.00167714,0.00$ $404354,-0.00460998,0.00122534,-0.00054691,0.00007895,-0.00002258,0.000$ $46623,-0.00011798,-0.00004589,-0.00017196,0.00004977,0.00017858,-0.000$ $05881,-0.00038482,-0.00138253,-0.00012246,0.00033635,0.00004529,-0.000$ $03267,0.00000946,0.00011503,-0.00008176,0.00000913,0.00005208,-0.00006$ $394,-0.00006738,-0.00010808,0.00034821,0.00010464,0.00000696,-0.000049$ $53,0.00006035,-0.00000146,-0.00001233,0.00002360,-0.04380805,-0.145778$ 
$84,-0.12106829,-0.05807007,0.32263180,0.00108069,-0.00480805,0.0004711$ $8,-0.00080003,0.00022156,-0.00028677,-0.00014179,-0.00023713,0.0001055$ $5,0.00006661,-0.00014976,-0.00009607,0.00003711,-0.00040668,0.00013257$ $, 0.00081015,0.00005686,-0.00018570,-0.00001479,0.00015764,0.00007424,-$ $0.00017755,0.00006002,-0.00020356,0.00003632,0.00007178,0.00012104,0.0$ $0003720,0.00014791,-0.00006993,0.00004904,-0.00007188,0.00003684,-0.00$ $001065,0.00002215,0.00002282,-0.06949320,-0.11743512,-0.24158219,-0.01$ $774876,0.27022570,0.51778447,-0.02545407,0.01277579,0.01450201,-0.0018$ $5121,0.00230628,-0.00052260,0.00030094,-0.00093824,0.00068689,-0.00021$ $849,0.00040638,-0.00000066,0.00023786,0.00074968,-0.00051808,-0.001795$ $41,-0.00111999,0.00138497,0.00087529,0.00187808,0.00313684,0.00063247$, $-0.00026184,0.00055230,-0.00030146,0.00002216,0.00008509,-0.00043156,0$ $.00030162,0.00018010,0.00000780,0.00032268,-0.00035747,-0.00004726,-0$. $00000819,-0.00005171,-0.00502886,-0.05084573,-0.07414986,-0.32392573,0$ $.01054705,-0.01577130,0.66706540,0.00116362,0.00477028,0.00056695,-0.0$ $0088494,-0.00185860,0.00026115,0.00000299,0.00002703,-0.00005402,-0.00$ $013875,0.00028713,0.00003244,0.00000126,-0.00026350,-0.00001256,-0.000$ $58920,0.00016216,-0.00004921,-0.00156826,0.00017714,0.00028024,-0.0009$ $0488,-0.00004472,0.00030437,0.00012699,0.00004463,0.00015830,-0.000127$ $32,0.00030941,-0.00004829,-0.00004247,0.00007716,0.00008103,-0.0000402$ $1,0.00000424,-0.00003476,-0.01872928,0.01127839,0.00376311,-0.02657702$ $,-0.08304225,-0.03293159,-0.01732815,0.32279034,-0.00289158,0.00166387$ $, 0.00690407,-0.00208115,0.00114424,-0.00009961,0.00052007,-0.00106786$, $0.00009614,-0.00006189,0.00070106,-0.00026380,-0.00023065,-0.00032949$, $0.00030123,-0.00205559,0.00008220,-0.00089158,-0.00205030,0.00074146,0$ $.00076982,0.00001707,-0.00089714,-0.00042711,-0.00001654,0.00001587,0$. $00019236,-0.00018812,0.00078003,0.00011518,0.00016729,-0.00012516,0.00$ $008123,-0.00003447,-0.00001610,-0.00002366,-0.02840740,0.00023793,0.00$ $423592,-0.06985849,-0.02900394,-0.11212415,0.03921761,0.27116966,0.531$ $97432,-0.15183123,-0.01184503,-0.02665354,-0.02724704,-0.01136880,0.01$ $196869,0.00165394,-0.00229287,-0.00217819,-0.00124950,0.00086670,0.000$ $79952,0.00037056,-0.00023517,-0.00317872,-0.02227134,0.01583121,-0.004$ $52213,-0.01010386,-0.00158435,-0.00251775,-0.00043473,0.00206354,-0.00$ $160868,0.00058595,0.00023812,0.00026146,0.00100694,-0.00099205,0.00050$ $710,0.00169874,-0.00125526,0.00102057,0.00006876,-0.00001166,-0.000116$ $13,-0.00131465,0.00778778,0.01208168,-0.04394343,0.00900381,0.00737529$ $,-0.21258925,0.08306561,0.10290782,0.59900652,-0.00533849,-0.07184393$, $-0.00727887,-0.01270652,-0.00253284,-0.00362640,-0.00065760,-0.0013658$ $3,0.00104763,0.00053905,0.00022377,-0.00086140,-0.00090687,-0.00160987$ $, 0.00391869,0.03094370,0.00025553,0.00354293,-0.01687763,-0.00257652,-$ $0.00618520,-0.00292011,0.00079291,0.00127405,0.00010419,0.00042305,0.0$ $0038999,0.00060911,-0.00223595,-0.00144014,-0.00012865,0.00056815,-0.0$ $0026652,0.00010693,0.00011112,0.00007115,0.00869631,-0.02899377,-0.030$ $64120,0.03566950,0.01951410,0.02174573,0.04677151,-0.12620860,-0.08364$ $984,-0.03281515,0.33971981,-0.01457622,-0.00735511,-0.08047153,0.02380$ $040,-0.00050219,0.00610050,-0.00016911,0.00382924,-0.00244958,0.000410$ $16,-0.00048154,0.00044360,-0.00049543,-0.00044736,-0.00031255,-0.00341$ $626,0.00162948,0.00044221,-0.02851360,-0.00544040,-0.00985177,0.001073$ $37,0.00006929,0.00016735,0.00016721,-0.00054457,-0.00111228,0.00022533$ ,-0.00003250,0.00002994,-0.00022725,-0.00032086,0.00045036,0.00008320, $0.00000505,0.00014214,0.01295258,-0.03057253,-0.04908065,0.04496983,0$. 
$01884739,0.03912649,0.04898294,-0.07940311,-0.17327136,-0.00187850,0.2$ $2940750,0.50109334,-0.01409478,-0.01695931,-0.02555647,0.00107931,0.00$ $088353,-0.00303062,0.00020375,-0.00057397,0.00019278,0.00003229,0.0003$ $6459,-0.00015873,0.00009196,-0.00075964,-0.00020044,0.00041597,-0.0022$ $3221,-0.00079975,-0.00607174,-0.00087709,-0.00215393,-0.00000772,-0.00$ $057793,-0.00023976,0.00007587,0.00002178,0.00016778,-0.00019157,0.0008$ $8827,0.00002280,-0.00008511,0.00019624,-0.00009020,-0.00003931,-0.0000$ $0177,-0.00000478,-0.04864060,0.03932699,0.04933661,-0.03503409,-0.0166$ $3773,-0.02736670,0.05421188,-0.00139727,0.00416235,-0.13791609,-0.0086$ $4250,-0.02142323,0.70451377,-0.00502740,0.00169396,-0.00101453,0.00049$ $495,0.00065702,-0.00067387,-0.00033861,0.00041612,-0.00044865,0.000083$ $20,-0.00076658,0.00012784,0.00063923,0.00025981,-0.00008451,0.00058781$ $, 0.00004439,0.00037377,0.00038165,0.00112511,-0.00001476,0.00000543,-0$ $.00024410,-0.00033744,0.00001988,0.00001388,-0.00008616,-0.00001384,-0$ $.00019016,-0.00006775,-0.00010406,0.00030555,-0.00013249,0.00004121,0$. $00002589,0.00001052,0.00741280,0.02006481,0.01965201,-0.01696475,-0.01$ $062041,-0.01268512,0.02377961,-0.01312584,-0.02696266,-0.04325662,-0.1$ $4030471,-0.10988363,-0.05352069,0.32366518,-0.00900385,-0.00181491,-0$. $00211960,-0.00001518,0.00170750,-0.00093936,0.00021948,0.00020966,0.00$ 005207,0.00014999,-0.00043144,-0.00020796,0.00045281,-0.00010676,-0.00 $066016,-0.00044194,-0.00174708,0.00029486,-0.00025615,-0.00015328,0.00$ $072621,0.00085399,-0.00026642,-0.00011636,-0.00000174,-0.00004239,-0.0$ $0001930,0.00003870,-0.00017290,0.00000146,-0.00005235,0.00012807,0.000$ $03404,0.00002537,0.00000099,0.00005234,0.00392136,0.02348959,0.0409526$ $7,-0.02794623,-0.01302354,-0.02480583,0.04020184,-0.02967636,-0.030126$ $01,-0.07050949,-0.10542011,-0.22506841,-0.00632627,0.25963430,0.502777$ $68,0.00362696,-0.00014856,-0.00090119,0.00008220,0.00000789,0.00065183$ $, 0.00005982,-0.00000902,0.00017119,-0.00002097,0.00018089,-0.00008380$, $-0.00008396,0.00041895,0.00049279,0.00063347,0.00008478,0.00035998,0.0$ $0032604,0.00022397,0.00051492,-0.00002817,0.00019713,0.00073177,-0.000$ $15770,-0.00002164,-0.00005447,-0.00014251,-0.00014560,-0.00005504,-0.0$ $0008557,-0.00005603,-0.00016889,-0.00000522,0.00000784,0.00000011,-0.1$ $8792031,0.04798243,0.05379025,0.05387518,0.02367654,0.03890691,-0.0587$ $4893,0.01374909,0.01320270,-0.00494211,-0.04650738,-0.06758422,-0.3334$ $5983,0.00763600,-0.02215148,0.66656975,0.00003822,0.00174231,-0.005056$ $25,0.00092919,0.00009825,-0.00007970,0.00004500,-0.00000775,-0.0000462$ $1,-0.00009028,0.00009197,-0.00003090,-0.00010947,0.00015613,0.00011906$ $, 0.00003653,-0.00008447,0.00025662,-0.00096925,-0.00010549,-0.00043019$ $, 0.00003513,0.00014788,-0.00006502,0.00002517,-0.00003425,-0.00002365$, $-0.00001780,0.00005460,0.00002934,-0.00007474,-0.00000038,-0.00007857$, $-0.00000591,-0.00001877,-0.00000013,0.08518057,-0.13120196,-0.08456836$ ,-0.00187737,-0.01147107,-0.02923246,0.01456167,-0.00664508,-0.0025455 $9,-0.01970945,0.01240628,0.00497542,-0.03085082,-0.08323234,-0.0355077$ $3,-0.01042583,0.32980631,-0.00056677,-0.00514373,-0.00168825,0.0000805$ $7,0.00036019,-0.00037328,0.00004186,-0.00013724,0.00019306,-0.00005720$ $, 0.00012008,-0.00000555,-0.00009299,-0.00003469,0.00003924,0.00074089$, $-0.00008729,0.00020648,-0.00113783,-0.00019804,-0.00033612,0.00011570$, $-0.00011661,0.00018524,-0.00002737,0.00000028,0.00002615,-0.00000013,0$ $.00000915,-0.00003877,-0.00002194,0.00008770,0.00000692,-0.00000561,0$. $00000678,-0.00002127,0.10646328,-0.08857186,-0.17910533,0.00257080,-0$. $02639058,-0.02831999,0.01420953,-0.00285523,-0.00564493,-0.02911427,0$. 
$00219964,0.00663576,-0.07668970,-0.03106367,-0.11484161,0.04958900,0.2$ $8257431,0.53943508,0.00053348,0.00011224,0.00035142,-0.00036137,0.0003$ $7425,-0.00044693,0.00018656,-0.00052021,0.00012742,-0.00005590,0.00031$ $034,-0.00014574,0.00035630,-0.00067919,0.00000262,-0.00057312,-0.00041$ $201,0.00003191,-0.00007041,0.00000868,0.00000552,-0.00026878,-0.000442$ $80,0.00014141,-0.00004602,0.00000087,0.00012274,-0.00040433,0.00089874$ ,-0.00005462,0.00007578,-0.00001598,0.00004041,-0.00002665,0.00001297, $0.00002239,-0.14671582,-0.00659175,-0.02387139,-0.03025838,-0.00230173$ ,-0.00724263,0.00313874,-0.00177800,-0.00279401,-0.00147964,-0.0007421 $6,-0.00105461,0.00181979,0.00380773,0.00509982,-0.04104182,-0.00319586$ ,-0.00983048,0.21543822,-0.00009832,-0.00042305,0.00043932,-0.00009253 $, 0.00005797,-0.00004774,-0.00000628,-0.00004518,0.00006216,-0.00001287$ $, 0.00002605,0.00000145,0.00002670,-0.00009836,-0.00003688,-0.00001834$, $-0.00000262,-0.00001677,0.00003979,0.00003443,0.00005332,-0.00001947$, $0.00000486,0.00002164,-0.00000864,0.00000283,0.00001533,-0.00002480,0$. $00008333,-0.00000619,0.00000313,0.00000561,-0.00000171,-0.00000414,-0$. $00000201,0.00000033,-0.00679671,-0.02971266,-0.00901838,-0.01758199,0$. $00291442,-0.00438764,0.00188943,0.00197628,-0.00406629,-0.00035837,0.0$ $0018296,0.00119466,-0.00005447,0.00186722,-0.00383709,0.01244141,0.004$ $92990,0.00201149,0.01125576,0.02081626,0.00008843,0.00048043,-0.000025$ $87,-0.00006777,0.00003246,-0.00010777,0.00004560,-0.00012567,0.0000281$ $5,-0.00001342,0.00007589,-0.00003481,0.00006333,-0.00011097,0.00000920$ ,-0.00019060,-0.00009506,0.00006526,0.00005828,0.00003201,0.00004187,-$0.00004894,-0.00006077,0.00004566,-0.00000807,-0.00000143,0.00002671,-$ $0.00009644,0.00021272,-0.00000126,0.00001777,-0.00001229,-0.00000035,-$ $0.00001143,0.00000259,0.00000119,-0.02378515,-0.00898088,-0.03966508$, $0.02940159,-0.00266062,-0.00262811,0.00250857,-0.00449712,-0.00127779$, $-0.00067987,0.00113415,0.00091737,-0.00033759,-0.00332965,-0.00029038$, $0.01223568,0.00019331,0.00634685,0.03997618,0.01407764,0.03677912,-0.0$ $0013856,0.00054924,0.00083270,-0.00026847,0.00025272,-0.00008289,0.000$ $11474,-0.00019615,0.00002444,-0.00004018,0.00011690,-0.00003524,0.0000$ $3197,-0.00006251,-0.00003874,-0.00026008,-0.00007030,0.00000670,0.0000$ $3520,-0.00000187,0.00000980,0.00000184,-0.00019486,-0.00000731,-0.0000$ $1356,0.00000136,0.00003103,-0.00006231,0.00014425,0.00000751,0.0000010$ $1,0.00002227,-0.00000247,-0.00000760,-0.00000073,-0.00000280,0.0073255$ $5,-0.00558351,-0.00766759,-0.18481261,0.09038693,0.11330272,-0.0141571$ $2,0.01412152,0.01823404,-0.00421347,-0.00099388,-0.00260031,-0.0004152$ $7,-0.00050186,-0.00060923,0.00037698,-0.00121037,-0.00232240,0.0012027$ $3,-0.00057163,-0.00038230,0.19375350,-0.00022433,0.00011889,0.00000672$ $, 0.00009750,0.00015207,0.00004290,-0.00001554,0.00001263,0.00001057,0$. $00000333,-0.00002612,0.00001723,0.00003163,0.00000500,-0.00002536,-0.0$ $0014056,-0.00001636,-0.00009438,-0.00000251,0.00001148,-0.00001174,-0$. $00002721,-0.00008368,-0.00004806,-0.00001260,-0.00000301,-0.00000792,0$ $.00001038,0.00000979,0.00001762,0.00000498,-0.00000580,-0.00000356,-0$. $00000169,-0.00000385,0.00000009,0.01224585,-0.00348378,-0.00857000,0.0$ $8879391,-0.10211781,-0.08642310,-0.00278671,0.00458211,0.00126160,-0.0$ $0057030,0.00507651,-0.00260359,-0.00054684,-0.00078480,0.00056775,-0.0$ $0156138,0.00253159,-0.00456493,0.00001544,-0.00144246,0.00187469,-0.09$ $569493,0.09850866,-0.00034031,0.00011429,0.00026380,-0.00009939,-0.000$ $06881,-0.00008617,0.00000826,-0.00001036,0.00002113,0.00000184,0.00002$ $140,-0.00002486,-0.00003853,0.00000659,0.00004452,0.00005636,-0.000025$ 
$54,0.00003227,0.00000571,-0.00001278,-0.00000311,0.00003273,0.00004865$ $, 0.00000705,0.00000738,0.00000213,0.00001020,-0.00000604,-0.00000329,-$ $0.00000604,-0.00000554,0.00001036,-0.00000367,0.00000003,0.00000142,-0$ $.00000186,0.01801503,-0.01055028,-0.00968344,0.11093885,-0.08633943,-0$ $.14706383,-0.00592898,0.00315482,0.00736132,-0.00219235,-0.00253029,0$. $00288865,-0.00083707,0.00053201,-0.00054120,-0.00278319,-0.00451779,-0$ $.00127489,0.00049494,0.00181455,-0.00006152,-0.11858744,0.09640807,0.1$ $4955537,0.00101082,0.00087889,0.00186176,0.00008966,-0.00011070,-0.000$ $19415,-0.00024152,0.00030031,0.00002636,0.00009930,-0.00027079,0.00002$ $729,-0.00004510,0.00012464,-0.00002421,0.00006411,-0.00025415,-0.00006$ $342,-0.00014472,-0.00032595,-0.00060147,-0.00004203,0.00019770,-0.0000$ $3160,0.00001002,0.00002105,0.00001295,0.00006816,-0.00015262,0.0000331$ $3,0.00001260,-0.00001206,0.00001571,0.00000341,-0.00000240,0.00000531$, $-0.00406383,0.00046956,-0.00059354,-0.00544973,-0.01639238,-0.02473072$ ,-0.08078994,-0.04146680,-0.06612613,0.00877745,0.01031594,0.01581212, $-0.00230914,0.00253012,0.00251176,-0.00102954,0.00024286,0.00037154,-0$ $.00125683,-0.00058659,-0.00101662,0.00103643,-0.00017408,0.00034100,0$. $08365774,-0.00179253,-0.00272207,0.00182112,-0.00057933,0.00073869,0.0$ $0008688,-0.00003870,-0.00027511,0.00007670,0.00003140,0.00000974,-0.00$ $001047,0.00000950,-0.00002800,0.00013105,0.00061960,-0.00002129,-0.000$ $11535,0.00050833,0.00052531,0.00052930,0.00008559,-0.00016271,-0.00002$ $181,-0.00005191,0.00001612,0.00005132,-0.00000858,-0.00006831,-0.00004$ $083,0.00005469,-0.00007800,-0.00004717,-0.00000560,-0.00000124,0.00000$ $760,0.00024479,0.00544475,-0.00227432,0.00079090,0.00221071,-0.0012821$ $9,-0.04116800,-0.11810122,-0.12533795,-0.00533628,-0.00373364,-0.01139$ $862,0.00303269,0.00326131,-0.00404992,0.00032652,-0.00058552,0.0007793$ $1,0.00005544,0.00007727,-0.00008935,-0.00036524,-0.00252832,0.00220536$ $, 0.04416922,0.11672265,-0.00144772,0.00233688,-0.00103143,0.00067432,-$ $0.00058154,0.00004188,-0.00017529,0.00032161,0.00006848,-0.00001304,-0$ $.00010370,0.00004799,0.00008501,0.00005362,-0.00009021,-0.00032218,0.0$ $0001319,0.00048666,0.00081851,0.00024872,0.00065460,-0.00000363,0.0003$ $5457,0.00027773,-0.00001786,-0.00003380,-0.00005475,-0.00009238,0.0001$ $1492,0.00000345,-0.00003557,-0.00000871,-0.00005836,-0.00000264,0.0000$ $0482,0.00000250,-0.00086911,-0.00220442,0.00359191,0.00025487,-0.00332$ $498,-0.00138760,-0.06565978,-0.12556372,-0.22721360,-0.00789273,-0.009$ $26801,-0.01068410,0.00329375,-0.00392508,0.00094530,0.00053653,0.00073$ $071,0.00004265,-0.00008474,-0.00014692,-0.00009105,0.00006964,0.002214$ $55,-0.00083170,0.07149482,0.13902720,0.23671983,-0.00019664,-0.0002236$ $4,-0.00016008,0.00025314,-0.00051601,-0.00033462,-0.00008474,0.0001351$ $2,0.00022261,-0.00015516,-0.00003536,0.00006083,-0.00037541,0.00029353$ ,-0.00012786,0.00010340,-0.00030664,-0.00023724,0.00016860,0.00010161, $0.00017821,-0.00016304,0.00034156,0.00004906,-0.00001237,0.00001241,-0$ $.00001669,0.00005569,-0.00022244,0.00003049,-0.00000031,-0.00001058,0$. $00006833,0.00000431,-0.00000855,-0.00000420,-0.00338866,-0.00061823,-0$ $.00224429,-0.00033663,-0.00050831,-0.00061853,0.00021228,-0.00102580,-$ $0.00233378,0.01027568,-0.00458257,-0.00581745,-0.17980094,0.08848581,0$ $.10751557,-0.01549732,0.01439367,0.01804256,-0.00141755,0.00041370,0.0$ $0038388,-0.00000235,0.00000111,0.00009608,0.00041273,-0.00039743,-0.00$ $049075,0.18896153,0.00056559,-0.00190892,0.00179752,-0.00030201,0.0002$ $1089,-0.00009383,0.00011108,-0.00006703,0.00040974,-0.00012562,0.00019$ $961,-0.00008566,-0.00018127,0.00012955,-0.00021752,0.00015043,0.000215$ 
$64,0.00008628,0.00006238,-0.00007143,0.00010569,0.00009326,-0.00020847$ $, 0.00019715,-0.00006808,-0.00001580,0.00001076,0.00011596,-0.00019576$, $0.00008002,0.00006929,-0.00000253,0.00015400,0.00000850,-0.00000486,0$. $00000115,-0.00046862,0.00540334,-0.00281013,-0.00056878,-0.00079488,0$. 00055971,-0.00157071,0.00291965,-0.00510998,0.01187928,-0.00368390,-0. $00868671,0.08862953,-0.10680198,-0.08735363,-0.00308805,0.00476857,0.0$ $0092532,-0.00028216,0.00007745,-0.00000160,0.00002841,-0.00048335,0.00$ $034284,0.00059821,-0.00022704,-0.00046302,-0.09545331,0.10330272,0.001$ $19308,0.00160747,-0.00058278,0.00034787,-0.00056749,0.00018752,-0.0002$ $3277,0.00012335,-0.00004715,-0.00012058,-0.00018783,0.00011224,-0.0003$ $4948,0.00052067,0.00018808,0.00027238,0.00024028,0.00012177,0.00009144$ $, 0.00009928,0.00004932,-0.00017300,0.00039735,-0.00000519,0.00005629,0$ $.00007052,0.00002571,0.00006006,-0.00019959,0.00005719,-0.00009277,-0$. $00005182,-0.00017029,0.00003485,0.00000249,-0.00001035,-0.00190701,-0$. $00288327,0.00311544,-0.00071372,0.00055718,-0.00044986,-0.00289759,-0$. $00514167,-0.00124964,0.01750584,-0.01096434,-0.00966062,0.10728684,-0$. $08687665,-0.14597042,-0.00665171,0.00296186,0.00729632,-0.00054766,0.0$ $0007031,0.00010619,0.00010643,0.00035885,-0.00022957,0.00087642,-0.000$ $55205,-0.00058318,-0.11455417,0.09792953,0.14871600,0.00013812,-0.0007$ $8213,-0.00104300,0.00006586,0.00013043,-0.00009690,0.00007465,-0.00013$ $943,0.00003842,-0.00000674,0.00013340,-0.00002648,-0.00002284,-0.00008$ $589,0.00004524,0.00015683,-0.00007269,0.00005213,-0.00044789,-0.000095$ $15,-0.00015103,0.00000076,-0.00004957,0.00006629,-0.00002424,-0.000011$ $35,0.00000671,-0.00005615,0.00013001,-0.00000791,-0.00001151,0.0000103$ $4,-0.00001766,-0.00001229,0.00000047,-0.00000366,0.00775295,0.01153699$ $, 0.01694173,-0.00254357,0.00242024,0.00248561,-0.00107741,0.00018452,0$ $.00030000,-0.00518376,0.00062259,-0.00061544,-0.00427055,-0.01595339,-$ $0.02369502,-0.08248669,-0.04695254,-0.07227505,0.00109897,0.00015482,0$ $.00064449,0.00051473,0.00055310,0.00083186,0.00005277,-0.00009345,-0.0$ $0004968,0.00095346,-0.00020091,0.00039818,0.08535531,0.00013147,0.0001$ $1011,-0.00006419,-0.00017180,0.00003567,0.00004225,0.00005790,-0.00009$ $519,-0.00002814,-0.00000372,0.00004974,-0.00003683,-0.00004176,0.00004$ $269,0.00007542,0.00014628,-0.00009621,-0.00003799,-0.00003992,-0.00008$ $799,0.00008150,0.00002552,-0.00002902,0.00004056,-0.00000941,-0.000005$ $28,0.00000313,0.00000635,-0.00002978,0.00000557,0.00000678,-0.00000600$ $, 0.00001154,0.00000063,0.00000368,0.00000102,-0.00645712,-0.00376938,-$ $0.01083312,0.00270488,0.00286322,-0.00321108,0.00035956,-0.00053108,0$. $00073486,0.00036124,0.00564112,-0.00243658,0.00077161,0.00296383,-0.00$ $122720,-0.04529010,-0.12321595,-0.12972396,-0.00044950,-0.00133969,0.0$ $0175915,-0.00025757,-0.00006390,-0.00056386,-0.00007020,-0.00049500,0$. $00030661,-0.00031307,-0.00261832,0.00244448,0.04854526,0.12067077,0.00$ $030914,-0.00023926,-0.00011220,0.00006200,0.00005783,-0.00004681,0.000$ $02479,-0.00001236,0.00003081,-0.00002156,0.00001246,-0.00000187,0.0000$ $8191,-0.00009128,-0.00005197,-0.00015149,0.00005876,0.00004068,-0.0000$ 9833,0.00005162,-0.00008026,-0.00001186,-0.00003818,-0.00000824,0.0000 $0094,0.00000437,0.00000513,-0.00003981,0.00010461,-0.00001039,0.000002$ $82,0.00000415,-0.00001339,-0.00000241,-0.00000183,0.00000224,-0.008632$ $18,-0.00876635,-0.01008072,0.00291518,-0.00322297,0.00102515,0.0004299$ $1,0.00074961,0.00013407,-0.00112293,-0.00251209,0.00365825,0.00016192$, $-0.00320466,-0.00052178,-0.06986433,-0.12975370,-0.22862401,-0.0002208$ $0,0.00181338,-0.00012058,-0.00032544,-0.00045828,-0.00038003,-0.000015$ 
$70,0.00031549,-0.00027255,0.00030855,0.00241225,-0.00091810,0.07620295$ $, 0.14269617,0.23633784,0.00075388,0.00265147,0.00030087,0.00696215,-0$. $00061603,-0.01097988,-0.00018530,0.00149888,-0.00168943,-0.00087617,-0$ $.00015207,-0.00027928,-0.00002337,-0.00036849,0.00018866,0.00043914,0$. $00049781,-0.00014322,-0.00025817,-0.00012394,0.00047221,-0.10415485,-0$ $.04315500,0.11865311,-0.00008644,-0.00012861,0.00108113,-0.00012177,0$. $00022520,0.00002725,0.00001796,0.00012137,-0.00000157,0.00007138,0.000$ $06307,0.00002749,0.00004194,-0.00010111,-0.00013955,0.00020921,0.00001$ $215,0.00008268,0.00000481,-0.00022542,0.00004485,-0.00005038,-0.000209$ $74,-0.00004986,0.00026484,0.00015941,0.00025431,-0.00022891,0.00004423$ ,-0.00003832,-0.00000752,0.00000434,-0.00001195,0.00000886,-0.00000257 $, 0.00000192,-0.00001953,0.00003126,-0.00008996,0.00001033,-0.00005605$, $-0.00002830,-0.00000998,-0.00001609,0.00000804,0.10388423,0.00462211$, $0.00393950,-0.00047902,-0.01435122,-0.01885697,0.02872462,-0.00363256$, $0.00263137,-0.00034544,-0.00014843,-0.00101367,-0.00018804,0.00008039$, $-0.00002474,-0.00007095,-0.00131562,-0.00002259,0.00146478,0.00007961$, $0.00025974,0.00022436,-0.04773557,-0.12792732,0.15352278,0.00127268,0$. $00027348,-0.00050981,0.00005182,0.00019956,-0.00015483,0.00033324,-0.0$ $0045095,0.00003445,0.00001216,0.00010825,0.00003909,-0.00016940,-0.000$ $01460,-0.00000416,-0.00003370,0.00001889,0.00000267,-0.00003098,0.0000$ $8860,-0.00003441,0.00069295,0.00021030,-0.00013417,-0.00006197,0.00005$ $225,-0.00006685,0.00000488,-0.00001962,-0.00003609,0.00011957,0.000003$ $40,0.00001934,0.00000987,0.00000300,-0.00000756,-0.00001618,-0.0000038$ $1,0.00005476,0.00000742,-0.00008060,0.00000167,-0.00001309,-0.00000290$ $, 0.00000358,0.05830283,0.14510366,-0.00010658,0.00061510,0.00193392,-0$ $.00042288,-0.00428197,-0.00311340,-0.00052128,-0.00227324,0.00111491,0$ $.00002147,0.00062369,-0.00039668,-0.00019346,-0.00018481,0.00032732,0$. $00071391,-0.00000827,0.00016893,-0.00023274,-0.00033912,0.00062542,0.1$ 4090923,0.18012953,-0.28711514,-0.00038235,0.00026927,-0.00020953,-0.0 $0011008,0.00009416,-0.00011280,-0.00000026,0.00009395,0.00005376,0.000$ $03024,-0.00001062,0.00003639,-0.00001034,-0.00000747,-0.00004631,0.000$ $05748,-0.00000714,0.00003552,0.00003427,-0.00012725,0.00000116,-0.0000$ $8743,-0.00004820,-0.00026571,0.00009501,0.00003360,0.00015383,-0.00004$ $761,0.00002625,0.00000441,0.00001150,-0.00000110,-0.00000173,0.0000082$ $5,0.00000714,-0.00000339,-0.00001955,0.00002095,0.00001659,0.00002084$, $-0.00003061,0.00000509,-0.00000843,-0.00001024,0.00000242,-0.13484050$, $-0.17379225,0.29029540,0.00011789,-0.00099869,0.00083151,-0.00868219,0$ $.00235463,-0.00152874,0.00163930,-0.00080467,-0.00171746,0.00070513,0$. $00081320,0.00061612,-0.00032182,-0.00002332,0.00026065,-0.00020448,-0$. $00017090,0.00009567,0.00002446,0.00004113,-0.00051648,-0.41865132,0.07$ $202745,0.08391881,-0.00027010,-0.00035826,-0.00047468,-0.00001694,-0.0$ $0000852,-0.00008970,0.00012026,-0.00011852,0.00004328,0.00005380,0.000$ $08136,-0.00000382,-0.00035700,0.00012575,0.00017776,-0.00053911,-0.000$ $06833,-0.00015368,-0.00027079,0.00040816,0.00035629,0.00132594,-0.0003$ 4957,-0.00005564,-0.00037270,-0.00038363,-0.00023606,0.00032913,-0.000 04253,-0.00004564,0.00013357,0.00000607,0.00002954,-0.00001196,0.00000 403,-0.00001314,0.00009671,-0.00012290,0.00001960,0.00001997,0.0000536 $6,0.00003907,-0.00001079,0.00000278,-0.00000132,-0.00664636,0.00191916$ ,-0.00491872,0.43178940,0.00063771,0.00019067,-0.00042179,0.03698889,-$0.00684080,-0.01040802,-0.00011067,-0.00422798,0.00409312,0.00132284,-$ $0.00065682,-0.00078758,0.00050851,0.00011138,-0.00035841,-0.00023568,0$ 
$.00004238,-0.00019976,0.00008125,0.00002707,-0.00016124,0.05479592,-0$. $05518950,0.01925549,-0.00002872,0.00010032,-0.00009976,-0.00017402,0.0$ $0012669,0.00006809,-0.00006358,0.00004055,0.00000111,0.00002604,-0.000$ $50652,0.00002780,-0.00043935,0.00007111,0.00021587,-0.00014811,-0.0000$ $4547,-0.00000993,-0.00061297,0.00032631,0.00061364,-0.00015889,-0.0000$ $3603,0.00028545,-0.00042102,-0.00034539,-0.00084026,0.00031525,-0.0001$ $1115,-0.00015034,0.00043934,0.00001553,0.00007105,0.00007195,0.0000315$ $2,-0.00003250,-0.00001359,0.00002475,-0.00015827,0.00003598,-0.0000625$ $7,0.00003942,0.00002740,0.00000487,0.00001668,-0.01845691,0.00340479,-$ $0.00079132,-0.07438756,0.06350428,-0.00049140,0.00095521,-0.00029580$,$0.00333705,-0.00961619,-0.00168182,-0.00025722,0.00189967,0.00233277,0$ $.00059606,0.00004859,0.00093477,-0.00010013,-0.00064620,0.00051463,-0$. $00007465,0.00031803,-0.00047039,0.00002406,0.00002063,-0.00105454,0.05$ $348829,0.02577510,-0.03537332,-0.00046012,-0.00017655,-0.00005585,0.00$ $003630,0.00029728,-0.00013784,-0.00002282,-0.00015982,-0.00004892,-0.0$ $0001401,-0.00004700,-0.00008311,0.00025889,-0.00037546,-0.00051421,0.0$ $0074767,-0.00008087,0.00026773,-0.00025483,-0.00012680,0.00044641,-0.0$ $0017526,-0.00003886,0.00013260,0.00049541,0.00042723,0.00023791,-0.000$ $83775,0.00006939,-0.00014412,-0.00013467,-0.00000885,-0.00006129,0.000$ $03091,0.00002314,0.00001096,0.00002696,0.00016775,-0.00060107,-0.00003$ $736,-0.00015928,-0.00010373,-0.00002336,-0.00002909,0.00000632,0.02714$ $929,-0.00826851,-0.00351035,-0.07663323,-0.01026854,0.03925225 \backslash \backslash 0.0000$ $0698,-0.00000922,0.00000127,-0.00000304,-0.00001691,-0.00000554,0.0000$ 0396,-0.00000938,-0.00000215,-0.00000915,0.00002003,-0.00000428,0.0000 $0279,-0.00001018,0.00000990,0.00000045,0.00001992,-0.00000779,-0.00000$ 010,0.00000395,0.00000171,0.00000877,0.00001949,0.00001018,0.00000178, $-0.00000765,-0.00000353,-0.00000072,-0.00000329,0.00000632,-0.00000237$ $, 0.00000206,0.00000753,0.00000202,-0.00000611,0.00000096,-0.00000022,-$ $0.00000080,0.00000155,0.00000087,0.00000399,-0.00000195,0.00000244,-0$. $00000171,0.00000146,-0.00000584,0.00000326,0.00000355,-0.00000243,-0.0$ $0000132,-0.00000023,-0.00000013,-0.00000304,0.00000297,0.00000066,-0.0$ $0000001,0.00000183,0.00000149,0.00000479,-0.00000120,0.00000092,0.0000$ $0623,-0.00000326,-0.00000061,-0.00000307,0.00000257,-0.00000112,-0.000$ $00352,0.00000347,-0.00000101,-0.00000332,-0.00001350,-0.00000638,-0.00$ 000419,-0.00001185॥I@

meta-Addition Product to 4-fluoroaniline

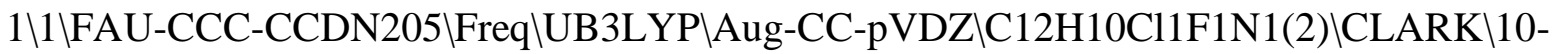
Dec-201410\\\# ub3lyp/aug-cc-pvdz geom=check guess=read freq name=clark \\Addition product p-Cl-Ph-radical + aniline (meta) \\0,2\C,-1.28378636 $17,0.8483465351,0.6580918066 \backslash \mathrm{C},-2.1302245586,-0.3660878682,0.958205412$ 4IC,-3.2248195647,-0.7305197106,0.2137674819\C,-3.6538363143,0.0810995 $615,-0.8855119918 \mathrm{IC},-2.9614014461,1.2745125093,-1.2005236551 \backslash \mathrm{C},-1.8657$ $766595,1.6332130685,-0.4753816735 \backslash \mathrm{H},-1.2771067528,1.5127158192,1.54566$ $82871 \backslash \mathrm{H},-1.8074365649,-0.9852371027,1.7964112479 \backslash \mathrm{N},-3.9205276638,-1.93$ 04785695,0.4517344954\H,-3.2948436781,1.9135837168,-2.017785064\F,-1.2 $182356698,2.7962297241,-0.7563790425 \backslash \mathrm{H},-4.5104443474,-0.2245914362,-1$. 4848225047\C, $2.8653355279,-0.1702746813,-0.0788457143 \backslash \mathrm{C}, 2.5577900201,0$ $.7647884976,0.907507157 \backslash \mathrm{C}, 1.2159232495,1.0878293786,1.1372122408 \backslash \mathrm{C}, 0.1$ $903823512,0.487966776,0.39928493 \backslash \mathrm{C}, 0.5298497842,-0.4496039414,-0.58636$ 
50827\C, $1.8624302244,-0.7834070214,-0.833449553 \backslash \mathrm{Cl}, 4.5521617817,-0.590$ 946298,-0.3786459176\H,3.3519331186,1.2338477099,1.4855215702\H,0.9723 $4558,1.823302523,1.9051848411 \backslash \mathrm{H},-0.2569653299,-0.9304003375,-1.1678364$ 991\H,2.1228665431,-1.5111600529,-1.6000959164\H,-3.7544357197,-2.3391 $186851,1.3632950445 \backslash \mathrm{H},-4.9115825434,-1.8889610437,0.2467240529 \mid$ Versio $\mathrm{n}=\mathrm{EM} 64 \mathrm{~L}-\mathrm{G} 09 \mathrm{RevC} .01 \backslash \mathrm{State}=2-\mathrm{A} \backslash \mathrm{HF}=-1078.1344546 \backslash \mathrm{S} 2=0.784048 \backslash \mathrm{S} 2-1=0 . \mathrm{S} 2 \mathrm{~A}=$ $0.750671 \backslash \mathrm{RMSD}=7.040 \mathrm{e}-09 \backslash \mathrm{RMSF}=1.277 \mathrm{e}-05 \backslash \mathrm{Z}$ eroPoint $=0.1881425 \backslash \mathrm{Thermal}=0.2$ 01343 \ZPE=118.0611697\Dipole=-1.3579373,-0.3470025,0.5385732\DipoleDer iv $=0.1674118,0.0329656,-0.1214428,-0.0776454,0.100363,0.0984492,-0.141$ $2071,0.205329,0.0898167,-0.3384611,-0.1911511,0.1786015,0.125314,-0.22$ $40248,-0.0628828,0.0272873,-0.184121,-0.0456723,0.3812234,0.627378,-0$. $1915411,0.7907647,0.8564965,-0.0921592,-0.2270515,-0.0858196,-0.076789$ $1,-0.0190754,0.1128095,0.0878827,-0.1814108,-0.0838975,-0.1391292,0.34$ $03526,0.0345103,0.0532043,0.0022868,-0.1305467,0.1616039,0.2424272,-0$. $0768084,0.0459815,-0.0795969,-0.1613613,0.001013,0.3084704,0.4701785,0$ $.0048292,0.4513155,0.9635445,-0.2118073,0.1305871,-0.2726736,0.1315823$ $, 0.0005157,-0.0364608,-0.0568793,-0.0408451,-0.0238349,-0.0957433,-0.0$ $342079,-0.1072148,-0.0445319,0.0822552,-0.015893,-0.0557093,-0.035139$, $-0.0043676,0.1027712,-0.0527186,0.0862527,0.0009951,-0.449806,-0.24329$ $79,0.106498,-0.7964527,-1.0509413,-0.0361246,0.0659504,-0.0941254,-0.2$ $218329,0.095168,0.0023746,-0.0439877,-0.0142684,0.0432975,0.0845547,-0$ $.0741958,0.0898823,0.0409438,-0.4442922,-0.4416112,0.0663287,-0.453014$ $1,-1.0250556,0.2361554,0.0311523,0.2569462,-0.2149383,-0.0097429,-0.05$ $80962,-0.0767569,-0.0803083,0.0531748,0.000892,-0.0883011,0.0080607,0$. $0511507,1.179179,-0.2597679,-0.1700635,-0.3279535,0.0577762,0.0832676$, $-0.2443247,0.0876124,0.0317172,-0.2561413,0.1044549,0.0930241,0.134071$ $2,-0.0816279,0.0188726,0.1167054,0.0156021,-0.0646261,-0.0417012,-0.03$ $15235,-0.0289601,0.0582144,0.0045926,0.1104575,0.0678134,0.0938168,0.0$ $086866,0.2837897,-0.040903,0.023233,-0.1298779,-0.0596244,-0.0278615,-$ $0.017462,-0.0208772,-0.075784,-0.1146353,0.0243711,0.0217814,0.0104736$ $,-0.0463664,0.038955,0.0109138,0.0394008,-0.0367532,-0.2495444,0.00601$ $12,-0.0124496,0.0282567,-0.0261169,0.069047,0.0160173,0.067606,-0.0111$ $591,-0.8845293,0.161394,0.1035773,0.2003629,-0.1212043,-0.0365006,0.13$ $78573,-0.039045,-0.1019411,-0.0113612,-0.0363104,-0.0523602,-0.029008$, $0.0906073,-0.0439648,-0.0436039,-0.0453602,0.0754155,0.058965,0.050501$ $1,0.0527792,0.0286544,0.0283844,-0.0950169,0.0315155,-0.0883911,0.0200$ $727,0.020482,-0.0390706,-0.0551069,-0.0467338,0.0692998,-0.0384688,-0$. $0729194,-0.0365623,0.0544377,0.0513228,0.0262861,0.021737,0.0300549,0$. $0558075,-0.0735686,0.0318399,-0.0733743,0.0492795,0.1080955,-0.0654973$ ,-0.0537971,0.0068129,0.2670572,-0.0045665,-0.0047675,0.0887975,0.0845 $315,0.0801251,-0.028595,-0.0028216,0.1059345,0.2334685,0.0683903,0.072$ $3644,0.1351091,0.2011814 \backslash$ Polar $=237.3165123,8.9290862,146.052941,12.366$ $6614,5.8810763,136.1015199 \backslash \mathrm{PG}=\mathrm{C} 01[\mathrm{X}(\mathrm{C} 12 \mathrm{H} 10 \mathrm{Cl1F} 1 \mathrm{~N} 1)] \mathrm{NImag}=0 \backslash 10.419511$ $63,0.02411785,0.49153644,0.03079272,0.02145197,0.50623660,-0.10051551$, $-0.05955283,0.00733825,0.49651052,-0.05435758,-0.15731087,0.03487434,0$ $.13993424,0.44232578,0.00022132,0.02845887,-0.08160028,0.24300608,-0.1$ $5620539,0.47552832,-0.01850973,-0.01142008,-0.00842674,-0.25323722,-0$. $06201045,-0.13675820,0.50134487,-0.02008268,-0.00675822,-0.01309952,-0$ $.09338468,-0.11525769,-0.03144395,0.18820979,0.50906039,-0.00048279,-0$ $.00647119,0.00212830,-0.11290382,-0.00478062,-0.15488669,0.17131174,-0$ $.13275573,0.41673648,-0.00018007,0.00216329,0.00344639,-0.01261286,0.0$ 
$1234059,-0.03051906,-0.10108297,0.02362633,-0.06548240,0.47540847,0.00$ $150626,-0.00439262,0.00005837,0.03326714,0.02116856,0.02094630,-0.0295$ $2530,-0.13711735,0.04034509,0.19129138,0.46512170,0.00449791,0.0012086$ $4,-0.00215322,-0.04857273,0.00102228,-0.03949845,-0.01890906,0.0921285$ $0,-0.15216776,0.21940291,-0.12087172,0.40750847,-0.00465281,-0.0004914$ $9,-0.01227447,-0.02019884,-0.00658696,-0.01384151,0.04129897,-0.004464$ $49,0.04257998,-0.12191833,-0.05261266,-0.03275158,0.46885533,0.0093266$ $3,0.01156078,0.00420590,-0.01669810,-0.00495273,-0.01159593,0.02075892$ $,-0.02997684,0.04538255,-0.10702898,-0.21168253,0.03468302,0.11969852$, $0.50388403,-0.01849244,-0.00242998,-0.00690581,-0.00617140,-0.00219356$ $,-0.00330143,0.02139978,0.02256426,0.00325083,0.01203961,0.08505437,-0$ $.10209563,0.24019981,-0.20281506,0.49294513,-0.09379597,0.02475923,-0$. $05082072,0.01954181,-0.00265316,0.02131754,-0.01957430,0.00714210,-0.0$ $2363001,-0.03149340,-0.06540047,0.01879410,-0.25978442,-0.02668670,-0$. $17340870,0.52491647,0.01930584,-0.10074104,0.05176969,0.00646723,-0.01$ $882735,0.02151025,-0.00409941,-0.00107136,-0.00307045,-0.03145763,-0.0$ $1992863,-0.00906498,-0.07111565,-0.09631754,-0.02830872,0.16726573,0.4$ $4735910,-0.04788044,0.05112277,-0.15198455,0.01379279,0.01149366,-0.00$ $011514,-0.01444369,0.00594605,-0.01912978,-0.00784930,-0.03907820,0.02$ $950441,-0.13901387,0.01143829,-0.18622297,0.23771157,-0.09518443,0.416$ $68159,-0.03470150,-0.00204338,-0.00389236,-0.00445485,-0.01373719,-0.0$ $1308074,0.00048206,-0.00196726,0.00065559,-0.00098311,-0.00104136,-0.0$ $0013876,0.00140009,0.00221535,-0.00017221,-0.00338639,-0.00683091,-0.0$ $1475961,0.05540122,0.00352343,-0.12395254,-0.10929213,-0.00821890,-0.0$ $1658072,-0.01726476,0.00229259,-0.00290857,0.00084032,-0.00066741,-0.0$ $0070439,-0.00000957,0.00203963,-0.00102843,0.00148881,-0.00196473,0.01$ $088910,0.01252898,0.00036005,0.13812366,0.00532643,-0.10923665,-0.1936$ $0878,-0.00270399,0.00103957,0.00905055,0.00150415,-0.00001513,0.001514$ $61,-0.00126046,-0.00099922,-0.00068480,0.00281060,0.00395540,-0.001600$ $75,-0.00841429,-0.01168091,-0.02693911,0.00190471,0.12196237,0.2153856$ $0,0.00483578,-0.00974611,0.01450977,-0.07036645,0.03741401,-0.07520478$ $,-0.00144173,0.01500417,-0.01956448,-0.00153601,-0.00392527,-0.0037873$ $6,-0.00049452,-0.00035691,0.00032768,-0.00203403,-0.00219513,-0.001237$ $58,0.00037618,-0.00007595,-0.00042543,0.06971195,0.00835450,-0.0116839$ $5,0.01834891,0.03856088,-0.13481860,0.13277274,-0.00561305,0.00621729$, $-0.00584976,-0.00324124,0.00166070,0.00071243,0.00035062,-0.00039474,-$ $0.00002900,-0.00457677,-0.00119369,-0.00159297,0.00065021,0.00086161,-$ $0.00031381,-0.03749076,0.13979279,-0.00184308,0.00115870,-0.00244391,-$ $0.07515535,0.12976519,-0.20931974,-0.00377836,0.01285282,-0.00902017,-$ $0.00471096,-0.00036488,0.00185887,0.00035259,-0.00056794,0.00008956,0$. $00029592,0.00039701,0.00195142,0.00044240,0.00071556,0.00032699,0.0828$ $7871,-0.14263706,0.21777791,0.00182755,-0.00080306,-0.00482467,-0.0226$ $5858,-0.04069586,0.00136097,-0.13901056,-0.10945037,-0.01254372,0.0078$ $7541,0.00729428,-0.00281247,-0.00226594,-0.00087381,-0.00355588,-0.000$ $37096,0.00076130,-0.00034944,-0.00084058,-0.00160079,0.00067120,-0.000$ $32946,0.00164040,0.00306409,0.64509637,0.00053864,0.00710933,0.0026801$ $1,-0.02090760,-0.01621450,-0.00116752,-0.07913255,-0.20983988,0.033378$ $64,-0.01373948,-0.03443863,0.01793715,0.00368912,0.00403246,0.00206833$ $,-0.00308418,0.00054436,-0.00287950,0.00026442,-0.00060950,0.00047475$, $0.00275352,-0.00026662,-0.00130742,0.10825318,0.37408648,-0.00240491,0$ $.00278247,0.00101289,-0.01818950,-0.01968084,0.00697714,0.00275951,0.0$ 
$6950802,-0.08206527,0.01567993,0.03826348,-0.00906427,-0.00757785,-0.0$ $0059340,-0.00329587,0.00144341,0.00021079,0.00096455,0.00006350,-0.000$ $10528,-0.00027719,0.00107985,-0.00229168,-0.00032025,0.16108798,-0.259$ $77121,0.49767609,-0.00030109,-0.00421316,-0.00371455,-0.00054582,-0.00$ $033393,0.00049014,0.00045529,-0.00423496,-0.00113314,0.00782007,-0.008$ $48763,0.01246679,-0.07643577,0.04484536,-0.07467390,-0.00331346,0.0157$ $8501,-0.02074886,-0.00056147,0.00083564,-0.00095805,-0.00004808,0.0001$ $4437,0.00012019,0.00048072,-0.00030895,0.00067923,0.07289087,-0.003419$ $20,0.00158356,0.00145515,-0.00033066,-0.00029429,-0.00062733,-0.004090$ $69,-0.00145834,0.00114104,0.01003032,-0.00876482,0.01805247,0.04495353$ ,-0.14709214,0.13087707,-0.00399420,0.00723162,-0.00469777,0.00022385, $-0.00026678,0.00031901,0.00016719,-0.00005234,-0.00003311,0.00043815$,$0.00040200,0.00109549,-0.04704368,0.14928231,-0.00379618,0.00032821,0$. $00334964,0.00016695,-0.00063034,-0.00008129,-0.00065441,0.00150925,0.0$ $0361175,-0.00178775,0.00224946,-0.00181565,-0.07706568,0.12922078,-0.2$ 0848504,-0.00365387,0.01370020,-0.00845580,0.00008719,-0.00032661,0.00 $025909,0.00010338,-0.00004939,-0.00015601,-0.00024006,0.00020903,-0.00$ $020411,0.08272851,-0.14360780,0.21455821,0.00547961,0.00223852,0.00176$ $562,-0.00202293,-0.00133707,-0.00224596,0.00291258,0.00121348,0.002259$ $72,-0.00126565,0.00221867,-0.00939261,-0.02121033,-0.04571504,0.010425$ $73,-0.10695103,-0.10174916,0.00329950,0.00040454,0.00113705,0.00026550$ $, 0.00035040,0.00032376,-0.00004326,-0.00027927,-0.00103366,0.00021247$, $-0.00004972,0.00251152,0.00321558,0.12186606,-0.01691005,-0.03155570,0$ $.00816610,0.00264838,-0.00225800,0.00534639,0.00325952,0.00309748,0.00$ $024906,-0.00555685,0.00186773,-0.00138171,-0.01689732,-0.02359901,0.00$ $587013,-0.10444614,-0.22012448,0.04945040,0.00023943,0.00196719,0.0002$ $4662,-0.00140699,-0.00164107,0.00025165,-0.00099808,-0.00292842,-0.000$ $23751,0.00208177,-0.00016264,-0.00200404,0.13991150,0.27427768,0.01709$ $600,0.02769106,-0.00310559,-0.00510883,0.00234809,-0.00401487,0.000173$ $34,-0.00158533,0.00079908,-0.00403629,0.00448948,-0.00049679,-0.009636$ $14,-0.01895111,0.01103313,0.00410562,0.05309941,-0.06247730,0.00142190$ ,-0.00117018,0.00261036,0.00122274,0.00177371,-0.00025325,0.00087231,0 $.00115196,0.00015422,0.00354655,-0.00198375,-0.00094910,-0.00999923,-0$ $.06640344,0.05608304,0.00010248,0.00055161,0.00054967,0.00175201,0.000$ $70150,-0.00184541,-0.00471297,-0.00599381,-0.00255916,-0.21906014,-0.0$ $7194175,-0.13669247,-0.00793140,-0.00175776,-0.00885040,0.00200712,-0$. $00241659,0.00078853,0.00012618,-0.00003918,0.00006991,-0.00025988,-0.0$ $0003481,-0.00021239,0.00061281,0.00058467,0.00193486,-0.00049787,0.001$ $03913,0.00140945,-0.00053359,-0.00069536,-0.00069257,0.22831422,0.0000$ $9858,-0.00041957,-0.00031286,0.00014621,-0.00145414,0.00375299,0.01299$ $859,0.00786310,0.00895192,-0.07310993,-0.07023313,-0.03201650,-0.01917$ $932,-0.00236046,-0.01473056,-0.00172933,-0.00253109,0.00263372,-0.0001$ $2234,0.00003776,-0.00009306,0.00081902,0.00032676,0.00052734,0.0011730$ $5,0.00055759,-0.00129361,0.00132896,0.00024709,-0.00106353,-0.00192952$ ,-0.00090502,-0.00035767,0.07962047,0.06849446,0.00076891,-0.00012078, $-0.00016961,-0.00110797,0.00430477,-0.00208152,-0.01789994,-0.00938025$ $,-0.00669658,-0.13726960,-0.03224489,-0.13152019,0.00698757,0.00319508$ $, 0.00682499,-0.00010823,0.00222320,0.00110835,-0.00010347,0.00001650,-$ $0.00005627,-0.00102549,-0.00031107,-0.00049317,0.00031441,-0.00155641$, $0.00025822,0.00102091,-0.00132346,-0.00065694,0.00040594,0.00073255,0$. $00058194,0.14679274,0.03355453,0.13338113,-0.00106536,-0.00026157,-0.0$ 
0020042,-0.00008358,-0.00007220,-0.00004607,-0.00020319,-0.00034703,0. $00039447,0.00009818,0.00024325,-0.00017949,-0.00010597,-0.00015720,0.0$ $0015535,-0.00034532,0.00011456,-0.00012413,-0.00002188,-0.00004690,-0$. $00003723,-0.00006459,0.00000172,0.00003235,0.00029715,0.00048291,-0.00$ $001775,0.00004754,-0.00000605,0.00004249,0.00034081,-0.00003373,0.0001$ $5328,0.00003295,0.00003322,0.00003105,0.50876670,-0.00060587,-0.000811$ $60,-0.00028690,-0.00068725,0.00026141,0.00001915,0.00019923,0.00064921$ $, 0.00006311,0.00011675,-0.00004354,0.00018255,0.00001619,0.00015369,0$. $00013281,-0.00039124,0.00000047,0.00032699,-0.00019372,0.00015794,0.00$ $015527,0.00001345,-0.00003585,-0.00005901,-0.00011028,-0.00069492,-0.0$ $0007345,0.00008784,-0.00002860,-0.00008286,0.00015618,0.00007350,-0.00$ $001349,-0.00002110,0.00000774,-0.00000100,0.01022608,0.36281338,-0.000$ $62608,-0.00043387,-0.00093625,-0.00082539,0.00031544,-0.00002048,0.000$ $30949,0.00072358,0.00006436,0.00005965,-0.00007422,0.00026448,0.000114$ $46,0.00027116,0.00002486,-0.00076375,-0.00014785,0.00049390,-0.0001448$ $4,0.00004947,0.00035474,0.00008880,-0.00001312,-0.00012311,-0.00010667$ ,-0.00070919,-0.00007370,0.00009151,-0.00006513,-0.00005577,0.00038594 $, 0.00026679,0.00002513,-0.00002900,-0.00001731,-0.00001789,0.04561883$, $0.25796119,0.40964862,0.00009857,-0.00175881,-0.00248525,-0.00109933,0$ $.00061677,0.00008565,0.00024463,0.00061834,-0.00000982,0.00010992,-0.0$ $0009611,0.00029975,0.00016113,0.00033826,0.00003533,-0.00143938,-0.000$ $61209,0.00071883,-0.00008892,0.00020635,0.00041471,0.00001347,-0.00006$ $322,-0.00012202,-0.00013575,-0.00073394,-0.00006927,0.00008518,-0.0000$ $7413,-0.00011099,0.00067471,0.00057478,-0.00006815,-0.00001418,-0.0000$ $0554,0.00000082,-0.11390962,0.00358135,-0.00067017,0.70418923,-0.00165$ $219,0.00336899,-0.00450868,0.00125654,-0.00027855,0.00042585,-0.000062$ $57,-0.00040082,-0.00029011,-0.00014180,-0.00000090,-0.00012107,0.00010$ $816,0.00022993,-0.00019299,-0.00112645,-0.00058747,0.00012121,-0.00005$ $306,-0.00012074,-0.00012314,-0.00003834,-0.00003535,0.00009518,0.00012$ $801,0.00059939,0.00013562,-0.00007219,-0.00004726,-0.00003967,0.000078$ $39,0.00007723,0.00001461,0.00001506,-0.00000669,0.00001628,0.04686774$, $-0.18814320,-0.12938793,0.00682596,0.39083432,-0.00176541,-0.00472929$, $0.00162153,-0.00078821,0.00027817,-0.00012402,-0.00031367,-0.00043416$, $0.00011254,-0.00002696,-0.00008823,-0.00013132,0.00002129,-0.00039834$, $0.00020433,0.00122735,0.00003645,-0.00025680,0.00002480,0.00011262,0.0$ $0008906,-0.00004898,-0.00004505,0.00002572,0.00000582,0.00023711,-0.00$ $003031,-0.00004461,0.00011005,0.00009844,-0.00014887,0.00013443,-0.000$ $06551,0.00001536,0.00002256,0.00001882,0.04645337,-0.13318909,-0.20502$ $676,0.05842218,0.28247793,0.44129009,-0.02748945,-0.00942667,-0.013508$ $20,-0.00114164,0.00149585,-0.00134819,-0.00033631,-0.00116619,0.000410$ $86,-0.00021804,-0.00018126,0.00000956,0.00029694,0.00072576,-0.0007052$ $6,-0.00204542,-0.00289875,0.00094153,0.00083072,-0.00233226,-0.0026870$ $3,-0.00012993,-0.00027397,0.00043949,-0.00024947,0.00014498,-0.0001712$ $3,-0.00027918,-0.00002035,-0.00008786,0.00045693,0.00083185,-0.0003515$ $9,-0.00002748,0.00000884,0.00003874,0.00457709,0.06157608,0.06669557,-$ $0.31862181,0.02606410,0.00529397,0.66678034,0.00515411,0.00650869,0.00$ $291113,0.00123923,-0.00055238,0.00008607,-0.00006332,-0.00008605,-0.00$ $007253,0.00018904,0.00019074,-0.00006411,-0.00000627,-0.00022692,0.000$ $26379,0.00101434,-0.00048088,-0.00051442,0.00186015,0.00041571,0.00064$ $114,0.00000813,0.00011963,-0.00002636,0.00015319,0.00039899,0.00007610$ $,-0.00006948,0.00011165,0.00021868,0.00039745,-0.00028672,0.00008140,0$ 
$.00004805,0.00000070,-0.00001734,0.02372061,0.00001667,-0.00575147,0.0$ $7147918,-0.10212305,-0.03645849,-0.03618020,0.41022253,0.00261265,0.00$ $085838,0.00592741,0.00194448,-0.00009878,-0.00075532,0.00029970,-0.000$ $47657,0.00052834,0.00012986,0.00050930,-0.00025851,-0.00045854,-0.0005$ $3692,0.00006760,0.00181854,0.00082233,-0.00007279,0.00183447,0.0006365$ 2,0.00066395,-0.00021799,-0.00009967,0.00008041,0.00009401,0.00072998, $0.00007231,0.00003456,0.00002133,0.00011724,-0.00042891,-0.00085643,-0$ $.00001751,0.00002687,-0.00001156,-0.00002109,0.02503934,-0.00266334,0$. $00639174,0.05393179,-0.04002038,-0.10074232,0.00868016,0.29347491,0.45$ $144253,-0.14384823,0.02562683,0.02238705,-0.02897316,-0.01071460,0.004$ $51999,0.00077517,0.00010296,0.00435668,-0.00124596,-0.00023557,-0.0011$ $5861,0.00192825,0.00177028,0.00242102,-0.02287482,0.01484531,-0.014757$ $47,-0.00810896,0.00263778,0.00135206,0.00212229,0.00093199,-0.00127737$ $, 0.00069815,0.00035559,-0.00038201,0.00049760,-0.00020195,-0.00018241$, $0.00002022,-0.00331835,0.00186091,0.00010036,0.00002277,0.00005745,-0$. $00430517,-0.01336044,-0.01451058,-0.04902649,0.00073016,-0.00433811,-0$ $.22797965,-0.08426449,-0.10575248,0.59909350,0.01750601,-0.07712944,-0$ $.01117361,-0.02420260,0.00762800,0.00117897,-0.00097871,-0.00113125,0$. $00195215,0.00005681,-0.00034056,0.00029132,-0.00008362,-0.00115995,0.0$ $0059572,0.01541529,-0.00616707,0.00044249,0.01939357,-0.00727393,-0.00$ $654074,-0.00006062,0.00023596,-0.00011082,-0.00068663,-0.00243056,-0.0$ $0106381,-0.00022628,0.00046167,0.00038472,0.00173308,0.00201874,-0.000$ $25104,-0.00008852,0.00007365,0.00000882,-0.01434123,-0.03415076,-0.030$ $59050,-0.03119410,0.03159176,0.02390847,-0.03895143,-0.13067288,-0.074$ 66836,-0.00228843,0.39885023,0.00924994,-0.00892210,-0.07484891,0.0044 $4294,0.00020475,0.00014459,0.00083685,-0.00119797,0.00022272,-0.000694$ $00,0.00009040,-0.00006089,0.00097787,0.00340726,-0.00258821,-0.0258539$ $1,0.00352104,0.00509658,0.02556568,-0.01040788,-0.00768387,0.00001346$, $-0.00034246,0.00056628,-0.00000854,0.00078642,0.00006116,0.00001327,-0$ $.00077501,-0.00112659,0.00018728,-0.00036686,0.00021498,-0.00009414,0$. $00004316,0.00014679,-0.01536028,-0.03088290,-0.04180940,-0.03898984,0$. $02633579,0.03249227,-0.05644369,-0.07861650,-0.15544233,0.03887447,0.2$ $4425141,0.43652094,-0.01179222,0.02152692,0.02185632,0.00028270,0.0021$ $7977,0.00065153,0.00007192,0.00139414,-0.00000223,0.00006778,-0.000552$ $53,0.00043367,0.00012794,0.00051593,-0.00021923,0.00029598,-0.00075412$ $, 0.00317928,-0.00559389,0.00219491,0.00188923,-0.00012493,-0.00008680$, $0.00009698,-0.00016630,-0.00134033,-0.00017717,0.00011117,-0.00007901$, $-0.00011424,0.00054174,0.00060000,-0.00026799,-0.00004782,-0.00000853$, $-0.00000210,-0.05368884,-0.03453776,-0.04260065,-0.03010401,0.02297040$ $, 0.02251980,0.05058241,-0.00864846,-0.00605546,-0.13012196,0.00969362$, $0.00417154,0.70968332,0.00758538,-0.00268555,-0.00472659,-0.00052730$,$0.00044661,0.00040227,-0.00082002,0.00029934,-0.00043752,-0.00000334,-$ $0.00064858,-0.00004226,0.00015006,0.00017783,-0.00015045,-0.00010604,0$ $.00092668,-0.00134124,0.00061993,0.00080502,-0.00026312,0.00016685,0.0$ $0031814,-0.00015724,0.00009657,-0.00007422,0.00004070,-0.00005348,0.00$ $006043,-0.00008064,-0.00040045,-0.00029818,0.00004454,-0.00003142,0.00$ $005120,0.00002802,0.00334729,0.03248452,0.02743894,0.02285940,-0.02060$ $989,-0.01716383,-0.03872980,-0.01561086,-0.02811835,0.05040255,-0.1810$ $2746,-0.11883434,-0.00080102,0.38815851,0.00716531,-0.00258817,-0.0008$ $9764,0.00041365,-0.00163377,0.00039382,-0.00027170,-0.00020611,-0.0004$ $9881,-0.00023512,-0.00006438,-0.00034764,-0.00039396,0.00011663,-0.000$ 
$12525,0.00069133,0.00124648,-0.00013726,0.00011496,-0.00014120,0.00069$ $318,0.00007057,0.00000542,0.00002927,0.00000166,-0.00017902,-0.0000413$ $5,0.00002878,-0.00009186,0.00000223,-0.00095804,-0.00047390,0.00008796$ ,-0.00003552,-0.00002651,0.00003978,-0.00154235,0.02461362,0.03366215, $0.02261377,-0.01733554,-0.02003415,-0.03898394,-0.02589068,-0.02348295$ ,0.04938596,-0.12257906,-0.19429688,0.04706247,0.27284960,0.43382043,0 $.00347160,0.00003531,0.00005264,0.00077172,-0.00010247,-0.00008932,-0$. $00019795,-0.00070874,-0.00033561,-0.00011449,-0.00002347,-0.00006241,0$ $.00001718,-0.00013483,-0.00032807,0.00044776,-0.00025463,-0.00040649,0$ $.00041694,-0.00027262,-0.00053977,-0.00006343,0.00003063,0.00016673,-0$ $.00005515,0.00050878,0.00006623,-0.00017425,0.00004865,0.00001702,-0.0$ $0039438,-0.00002185,-0.00018213,0.00000122,-0.00000744,-0.00000454,-0$. $20509294,-0.04508559,-0.06153269,0.04962594,-0.03787410,-0.03754346,-0$ $.06195672,-0.00800041,-0.01291773,0.00444327,0.05624043,0.06119743,-0$. $32410580,0.03068246,0.01037883,0.66176791,-0.00010129,0.00035994,-0.00$ $543802,-0.00043304,0.00006273,0.00038173,0.00022041,0.00021671,0.00020$ 613,-0.00003716,0.00009910,0.00012848,0.00002435,0.00001987,-0.0002102 $7,-0.00085124,0.00022517,-0.00009079,0.00103045,-0.00032217,-0.0003026$ $9,0.00011207,-0.00002741,-0.00010761,0.00003736,0.00001602,-0.00003312$ $, 0.00000980,-0.00005943,-0.00000061,0.00001941,0.00005303,0.00009247,0$ $.00000320,-0.00002827,-0.00003042,-0.08871508,-0.13392575,-0.08275671$, $-0.00758235,-0.01388098,-0.02548104,-0.00847888,-0.00380319,-0.0011908$ $2,0.02447971,0.00149308,-0.00405930,0.07577684,-0.10311549,-0.03895194$ $,-0.04184916,0.41353324,0.00019778,-0.00525171,-0.00010478,-0.00070679$ $,-0.00003371,0.00018176,0.00004805,-0.00011288,0.00003726,0.00016635,0$ $.00006854,-0.00005327,-0.00008841,-0.00012993,0.00030576,0.00013468,0$. $00023321,-0.00056901,0.00080012,-0.00038268,-0.00026241,0.00003461,0.0$ $0008794,0.00001539,0.00000215,0.00009483,0.00000525,0.00002337,0.00002$ 408,-0.00001520,-0.00004280,-0.00009869,0.00009743,-0.00000235,0.00002 $660,-0.00000427,-0.10885070,-0.07906896,-0.16058539,-0.00456930,-0.028$ $15709,-0.02182504,-0.01420853,-0.00090172,-0.00585070,0.02586435,-0.00$ $152275,0.00839141,0.05948337,-0.04281726,-0.10284756,0.00096577,0.3037$ $5035,0.46035366,0.00034603,-0.00021268,-0.00031835,-0.00049453,0.00049$ $398,-0.00010804,0.00029061,0.00070326,-0.00016772,-0.00003599,-0.00032$ $004,0.00032642,0.00020993,0.00044430,-0.00016446,-0.00032611,-0.000468$ $08,0.00059665,-0.00004231,0.00001295,0.00002486,0.00007530,-0.00004413$ ,-0.00000790,-0.00034378,-0.00092680,-0.00007982,-0.00001525,-0.000041 $31,-0.00010421,-0.00005878,0.00029759,-0.00021677,-0.00004135,-0.00003$ $190,-0.00003017,-0.14116870,0.02612792,0.01744768,-0.02701952,0.007464$ $07,0.00520104,0.00314784,0.00201167,0.00197424,-0.00125440,0.00074581$, $0.00112901,0.00119623,-0.00430767,-0.00503434,-0.04126344,0.01143547,0$ $.00817393,0.20710265,0.00001526,-0.00027350,0.00052151,0.00013981,-0.0$ $0011090,0.00001694,-0.00007907,-0.00023983,0.00002049,0.00000644,0.000$ $08264,-0.00009604,-0.00004665,-0.00012639,0.00009677,0.00015013,0.0001$ $1680,-0.00015873,-0.00004280,0.00004943,0.00005337,-0.00001616,0.00001$ $784,-0.00000667,0.00008545,0.00028877,0.00003000,0.00000819,0.00001206$ $, 0.00003773,0.00003839,-0.00003286,0.00004765,0.00001271,0.00000637,0$. $00000750,0.02615137,-0.03745300,-0.01244290,0.02574309,-0.00242848,-0$. $00509328,-0.00239464,0.00071230,-0.00471203,0.00063253,0.00034652,0.00$ $111157,0.00019302,0.00154623,-0.00328824,-0.00702695,0.00557655,0.0005$ $4824,-0.04390427,0.03374582,0.00001714,0.00051389,-0.00014559,0.000134$ 
$54,-0.00009941,0.00009705,-0.00003326,-0.00007402,0.00003760,0.0000071$ $9,0.00006892,-0.00004876,-0.00004044,-0.00010321,0.00001247,0.00001830$ $, 0.00003945,-0.00011531,-0.00006094,0.00002565,0.00001744,-0.00001472$, $0.00000058,-0.00000252,0.00006525,0.00017308,0.00002228,0.00000396,0.0$ $0000302,0.00001374,0.00001578,-0.00003830,0.00004551,0.00001267,0.0000$ $0377,0.00000378,0.01752631,-0.01245154,-0.03571128,0.02508753,-0.00686$ $762,-0.00062508,-0.00282786,-0.00434442,-0.00005730,0.00073425,0.00114$ $725,0.00061547,-0.00014952,-0.00361297,0.00054820,-0.01169876,0.002240$ $74,0.00592974,-0.02961851,0.01971036,0.03048346,-0.00030804,-0.0005860$ $7,-0.00073957,-0.00021625,0.00010770,-0.00004194,0.00000100,0.00008386$ $, 0.00001159,-0.00004481,-0.00009938,0.00004372,0.00009126,0.00013032,-$ $0.00001894,-0.00027317,-0.00023406,0.00014515,0.00004129,-0.00001059,-$ $0.00000672,-0.00000280,-0.00002986,0.00001211,-0.00004883,-0.00013964$, $-0.00002287,-0.00000708,-0.00000717,-0.00002489,0.00007654,0.00017405$, $-0.00005198,-0.00000764,-0.00000190,-0.00000014,0.00610790,0.00510432$, $0.00576444,-0.20717794,-0.09087998,-0.11354716,-0.01533955,-0.01356600$ ,-0.01634038,-0.00374478,0.00256673,0.00173710,-0.00031833,0.00050928, $0.00065755,0.00084201,0.00169136,0.00140699,0.00123209,0.00024608,0.00$ $060120,0.21761924,0.00033001,0.00015624,0.00015434,0.00012557,-0.00006$ $366,-0.00008012,0.00002185,-0.00001969,0.00005781,0.00000694,0.0000280$ $1,-0.00004363,-0.00004931,-0.00003527,0.00001325,0.00008342,0.00018920$ ,-0.00002468,-0.00000316,0.00001063,-0.00000304,-0.00000619,-0.0000026 $0,0.00000054,-0.00000182,0.00005403,0.00000511,0.00001367,-0.00000430$, $-0.00000183,-0.00006109,-0.00009637,0.00000547,0 .,-0.00000230,0.000000$ $23,-0.01611468,-0.00477380,-0.01026372,-0.08897150,-0.09871223,-0.0766$ $7544,0.00646111,0.00672085,0.00390816,0.00216433,0.00389864,-0.0029939$ $5,0.00062902,-0.00082437,0.00046285,0.00210105,0.00063522,-0.00508839$, $-0.00048083,-0.00094420,0.00189964,0.09497018,0.09613605,0.00034564,0$. $00006446,0.00017827,-0.00005073,-0.00001382,0.00005704,-0.00000240,0.0$ $0003748,-0.00001431,-0.00000377,-0.00001878,0.00001942,0.00003210,0.00$ $001983,0.00002627,-0.00000220,-0.00009648,-0.00007128,-0.00000647,-0.0$ $0000304,-0.00001446,0.00000880,0.00000819,-0.00000476,0.00000303,-0.00$ $003112,-0.00000081,-0.00001156,0.00000678,0.00001095,0.00003483,0.0000$ 4792,-0.00000984,0.00000161,0.00000094,-0.00000194,-0.01727864,-0.0084 $7998,-0.00733721,-0.11146155,-0.07666775,-0.12806460,0.00536241,0.0020$ $9547,0.00645606,0.00136275,-0.00302173,0.00375180,0.00079630,0.0005150$ $4,-0.00061183,0.00186886,-0.00512567,0.00013021,-0.00014422,0.00194057$ ,-0.00061822,0.11989358,0.08643926,0.12822890,0.00111724,-0.00158970,$0.00132769,0.00004217,0.00020297,0.00007475,-0.00004222,-0.00009538,0$. $00004937,0.00006423,0.00016410,-0.00002866,-0.00015935,-0.00019894,-0$. $00000691,0.00010463,0.00013843,0.00009489,-0.00022426,0.00041458,0.000$ $52755,0.00000694,-0.00000235,-0.00004047,0.00005961,0.00016033,-0.0000$ $0150,0.00001566,-0.00000913,-0.00001012,-0.00006147,-0.00010322,0.0000$ $6465,0.00000569,0.00000780,-0.00000077,-0.00395271,0.00095853,0.000041$ $70,-0.00321940,0.02067959,0.02148259,-0.07035736,0.04206115,0.04257806$ $, 0.00771006,-0.01438919,-0.01511787,-0.00291582,-0.00192145,-0.0028818$ $8,-0.00107800,-0.00020083,-0.00023662,-0.00118154,0.00089917,0.0008812$ $2,0.00104036,-0.00039394,0.00007405,0.07257575,0.00142762,-0.00212036$, $0.00203583,-0.00054159,0.00006631,-0.00006425,-0.00002083,0.00000558,0$ $.00013703,-0.00002676,-0.00000750,0.00000288,0.00003305,-0.00018264,0$. $00015796,0.00059718,0.00059895,0.00001694,-0.00057954,0.00065769,0.000$ 
43994,-0.00003720,-0.00007100,-0.00004279,0.00001578,-0.00006694,-0.00 $002992,0.00004775,0.00001378,0.00003225,-0.00011728,-0.00012669,0.0000$ $3612,0.00000532,-0.00000077,0.00000852,0.00116270,0.00456152,-0.002423$ $38,-0.00016654,-0.00115079,-0.00501149,0.04179832,-0.17227970,-0.14253$ $971,0.00487993,-0.00537963,-0.00940864,-0.00256570,0.00287447,-0.00385$ $474,-0.00030550,-0.00027310,0.00086003,0.00012944,-0.00006962,-0.00022$ $407,-0.00016454,-0.00186409,0.00240437,-0.04596090,0.17554652,0.001495$ $68,0.00240143,-0.00157014,0.00035778,-0.00003984,0.00053998,-0.0000150$ $6,0.00010810,-0.00015414,-0.00000817,-0.00003222,0.00003329,0.00011822$ $, 0.00020632,-0.00000576,-0.00098784,-0.00063985,0.00003967,-0.00070586$ $, 0.00021579,0.00048705,0.00004354,0.00000686,-0.00003752,0.00006663,0$. $00008770,0.00004546,0.00001740,-0.00004926,-0.00005298,0.00040651,0.00$ $041538,0.00007761,0.00000628,0.00000231,0.00000432,0.00034719,-0.00247$ $086,0.00432050,-0.00073748,-0.00336456,-0.00009804,0.04196603,-0.14218$ $560,-0.18517695,0.00615061,-0.01146341,-0.00782867,-0.00359568,-0.0036$ $7943,0.00190094,-0.00035328,0.00083720,-0.00015460,0.00005448,-0.00017$ $527,-0.00004140,0.00031044,0.00243284,-0.00154346,-0.04539161,0.157623$ $77,0.19005041,-0.00027517,0.00000880,0.00046901,-0.00004644,0.00045882$ $, 0.00030160,-0.00041791,-0.00025363,0.00018705,-0.00009384,0.00012446$, $-0.00017309,-0.00017667,-0.00003677,-0.00008971,0.00031664,0.00035885$, $0.00014080,0.00013112,-0.00015558,-0.00012227,0.00000136,0.00000407,-0$ $.00003439,0.00003095,0.00025804,0.00003799,0.00001261,-0.00002311,-0.0$ $0000221,-0.00020659,-0.00033140,0.00005089,-0.00000232,0.00001567,0.00$ $001094,-0.00305255,0.00210306,0.00150713,-0.00021155,0.00053936,0.0006$ $2168,0.00063696,0.00157913,0.00133240,0.00899397,0.00319729,0.00410864$ ,-0.20176897,-0.08840381,-0.10905586,-0.01664894,-0.01343962,-0.016372 $50,-0.00143674,-0.00024133,-0.00036362,-0.00001479,-0.00008191,-0.0000$ $2794,0.00037225,0.00036890,0.00034288,0.21287833,-0.00078948,-0.001411$ $94,0.00192486,-0.00030988,0.00023742,-0.00007736,0.00046421,0.00017826$ $, 0.00001303,0.00016413,0.00024228,-0.00006511,-0.00006250,-0.00009426$, $0.00032986,0.00014673,-0.00017135,-0.00014040,-0.00016730,-0.00000585$, $0.00010231,-0.00004196,-0.00002573,0.00012190,-0.00009034,-0.00024646$, $-0.00003367,0.00003754,-0.00001267,0.00003383,0.00006608,-0.00005594,0$ $.00000978,-0.00001621,-0.00001434,-0.00000607,0.00193762,0.00413138,-0$ $.00322156,0.00051172,-0.00079649,0.00052901,0.00223126,0.00101406,-0.0$ $0570574,-0.01638704,-0.00467148,-0.01020192,-0.08838524,-0.10181060,-0$ $.07731929,0.00714822,0.00670126,0.00372935,0.00057288,0.00007552,0.000$ $05289,-0.00009537,-0.00036289,0.00036006,-0.00077379,-0.00032407,-0.00$ $054419,0.09425869,0.09930122,-0.00079699,0.00177925,-0.00112820,-0.000$ $01099,0.00021162,0.00018887,0.00012934,0.00063518,-0.00006995,0.000127$ $59,-0.00036226,0.00035654,0.00032327,0.00007176,-0.00007829,-0.0004912$ $4,-0.00047771,0.00013283,-0.00004648,0.00009013,0.00001750,0.00009913$, $-0.00005123,-0.00022841,-0.00003757,-0.00029278,0.00000980,-0.00004447$ $, 0.00006777,0.00001096,0.00026639,0.00039257,-0.00007862,-0.00002749,0$ $.00000951,-0.00001143,0.00116665,-0.00314914,0.00400677,0.00078733,0.0$ $0048893,-0.00061504,0.00186384,-0.00584137,0.00029872,-0.01717660,-0.0$ $0849840,-0.00684654,-0.10854650,-0.07693249,-0.12851263,0.00575852,0.0$ $0183301,0.00638686,0.00045175,-0.00001805,0.00010835,-0.00004162,0.000$ $37199,-0.00031910,-0.00086567,-0.00044857,-0.00047268,0.11708217,0.087$ $46427,0.12837367,0.00010563,0.00088787,0.00085692,0.00014521,0.0000426$ $7,-0.00005131,-0.00001181,0.00011113,-0.00006764,-0.00000699,-0.000105$ 
$94,0.00003708,0.00004614,0.00007548,-0.00004410,0.00010355,-0.00015888$ $, 0.00017603,-0.00039565,0.00016602,0.00014401,-0.00001585,-0.00001465$, $0.00002557,-0.00003586,-0.00015604,-0.00002156,-0.00001850,0.00000985$, $-0.00000456,0.00000405,0.00006520,-0.00004236,-0.00001427,0.00000055,0$ $.00000251,0.00682213,-0.01524714,-0.01628109,-0.00309623,-0.00185837,-$ $0.00274564,-0.00114289,-0.00013561,-0.00016837,-0.00502457,0.00100214$, $0.00002246,-0.00210023,0.01967177,0.02080316,-0.07068774,0.04682075,0$. $04776727,0.00110084,-0.00058331,-0.00030413,0.00045753,-0.00074452,-0$. $00079518,0.00006188,0.00002456,0.00008969,0.00095809,-0.00037521,0.000$ $02622,0.07276531,-0.00014541,-0.00001203,-0.00025052,-0.00013349,-0.00$ $007670,-0.00001212,0.00000972,0.00003239,0.00005242,0.00002791,0.00002$ $877,-0.00002585,-0.00005482,-0.00007159,0.00000054,0.00012165,0.000077$ $33,0.00000375,0.00011057,-0.00009949,0.00004682,-0.00000545,-0.0000008$ $8,0.00000663,0.00000887,-0.00000841,0.00000189,0.00001206,-0.00000511$, $-0.00000145,-0.00003466,-0.00005925,0.00003550,0.00000253,0.00000555,0$ $.00000467,0.00592758,-0.00536799,-0.00884512,-0.00221052,0.00270876,-0$ $.00302684,-0.00027031,-0.00026694,0.00083031,0.00129568,0.00472943,-0$. $00264891,-0.00012260,-0.00054302,-0.00471937,0.04494710,-0.17355372,-0$ $.14584290,0.00010634,-0.00083746,0.00197124,0.00020536,-0.00011950,-0$. $00048544,-0.00001137,-0.00040436,0.00031609,-0.00024213,-0.00192737,0$. $00261071,-0.04953167,0.17577059,-0.00032136,-0.00019923,-0.00002610,0$. $00015894,0.00004910,0.00004625,-0.00006484,-0.00012046,-0.00002331,-0$. $00000166,0.00003321,-0.00003201,-0.00001812,-0.00001207,0.00002531,-0$. $00006085,0.00003725,-0.00007439,0.00007387,0.00002963,-0.00007788,0.00$ $000380,0.00000731,-0.00002148,0.00002869,0.00013051,0.00001259,-0.0000$ $0639,0.00000754,0.00000927,0.00000922,-0.00001217,-0.00000126,-0.00000$ $015,0.00000012,0.00000152,0.00673910,-0.01065624,-0.00765187,-0.003183$ $06,-0.00298240,0.00174813,-0.00031203,0.00085601,-0.00011306,0.0005105$ $6,-0.00267162,0.00436581,-0.00059661,-0.00297346,0.00063428,0.04557273$ $,-0.14568157,-0.18953111,0.00050080,0.00188827,-0.00054483,0.00024235$, $-0.00056926,-0.00025203,0.00007089,0.00031337,-0.00035350,0.00009366,0$ $.00257924,-0.00157655,-0.04945980,0.15997587,0.19345807,-0.00060041,0$. $00073112,-0.00013683,0.00053035,-0.00134813,0.00160519,0.00505174,-0.0$ $0113959,0.01714159,-0.00105782,-0.00399560,0.00012526,0.00149722,-0.00$ $008047,0.00031129,-0.00024313,0.00006296,-0.00021480,0.00003562,-0.000$ $07764,0.00009708,-0.00025847,-0.00000914,-0.00123334,-0.06709200,0.001$ $92877,-0.05876726,-0.00016139,-0.00015519,0.00007183,-0.00005614,-0.00$ $003914,-0.00004240,0.00001981,0.00005920,0.00037486,-0.00003566,-0.000$ $04869,-0.00005422,-0.00005368,0.00005197,0.00001935,0.00001806,0.00001$ $418,0.00003180,-0.00000376,-0.00022918,0.00009505,-0.00013077,0.000020$ $47,-0.00000428,0.00005242,0.00001748,0.00001345,-0.00002624,0.00001481$ $, 0.00000635,-0.00000817,0.00000481,-0.00000192,0.00001019,-0.00001532$, $0.00001466,0.00002862,-0.00004102,0.00001342,-0.00001572,-0.00000828,0$ $.00001641,0.06509646,0.00042710,-0.00074089,-0.00080043,0.00311951,0.0$ $0172952,-0.00023160,0.00635280,-0.01797347,0.03187690,-0.00516966,-0.0$ $0292588,-0.00041839,0.00034720,-0.00036439,0.00158865,0.00044161,-0.00$ $007037,0.00031739,-0.00010005,-0.00003805,0.00007146,-0.00110147,0.000$ $66652,-0.00028519,0.00607523,-0.08376789,0.13442111,-0.00019370,-0.000$ $38891,-0.00002708,0.00004926,0.00033245,-0.00021435,0.00016095,-0.0000$ $0171,-0.00010620,-0.00006569,0.00006534,0.00006387,0.00006283,-0.00007$ $107,-0.00000558,0.00006078,-0.00003153,-0.00006938,-0.00009845,0.00036$ 
$364,-0.00006259,0.00014912,-0.00001088,0.00003854,-0.00002972,-0.00001$ $321,-0.00000104,0.00012280,-0.00003894,-0.00002463,0.00002018,-0.00000$ $125,0.00000056,-0.00002141,-0.00000159,-0.00001676,-0.00003902,0.00004$ $376,-0.00000700,0.00002411,0.00000231,-0.00002274,-0.01127223,0.104945$ $92,-0.00009056,0.00027384,0.00010845,-0.00050427,-0.00199612,0.0015721$ $6,-0.00046743,-0.00504915,-0.00402558,0.00056567,0.00080663,0.00069541$ ,-0.00109017,0.00002026,0.00047512,0.00007934,-0.00004718,0.00020359,$0.00003386,-0.00005570,0.00000368,0.00064044,0.00006859,-0.00026834,-0$ $.07877562,0.17284614,-0.36254179,0.00008503,0.00002560,-0.00002030,0.0$ $0014160,0.00002166,-0.00010563,0.00022755,-0.00036294,-0.00000272,-0.0$ $0000425,0.00000580,0.00000380,-0.00000145,-0.00000121,-0.00000359,0.00$ $000629,0.00000057,-0.00003187,-0.00008927,-0.00000699,0.00002765,0.000$ $05185,0.00001809,0.00005183,0.00000264,-0.00002036,-0.00001239,0.00001$ $213,-0.00000706,-0.00000595,0.00000178,-0.00000286,0.00000051,-0.00000$ $597,-0.00000824,-0.00000401,-0.00001535,0.00003115,-0.00002805,0.00001$ $433,0.00000329,-0.00000830,0.07412490,-0.16505531,0.36929819,0.0011394$ $4,-0.00054175,-0.00135454,-0.00090848,-0.00203925,0.00154578,-0.014149$ $96,0.00507923,-0.00047609,0.00150643,0.00243481,-0.00177787,-0.0005960$ $1,-0.00111963,-0.00041399,0.00051736,0.00013987,0.00020011,-0.00024219$ ,-0.00035843,0.00015323,-0.00032351,0.00048763,0.00053175,-0.42309622, $0.00158020,-0.09716226,-0.00000778,0.00007364,-0.00000294,-0.00003829$, $0.00007442,0.00012822,0.00007606,-0.00020720,0.00074557,0.00000564,0.0$ $0002211,0.00001994,0.00001504,-0.00002046,0.00000249,-0.00001243,0.000$ $00464,-0.00002303,0.00012908,0.00016976,-0.00006073,0.00001870,-0.0000$ $0621,0.00000899,-0.00002473,0.00000999,-0.00000425,0.00000717,-0.00000$ $128,0.00000006,0.00000296,-0.00000301,0.00000263,0.00000546,0.00001114$ $, 0.00000694,-0.00000844,-0.00000373,-0.00001000,-0.00000407,-0.0000046$ $7,0.00000385,-0.00259713,0.00067793,0.00512463,0.43858590,-0.00115346$, $-0.00018412,-0.00066894,-0.00097682,-0.00398205,0.00347182,-0.03687158$ $,-0.00338037,-0.01093977,0.00016033,0.00073884,-0.00212881,0.00024064$, $-0.00039274,-0.00004174,-0.00012675,0.00005356,-0.00013955,-0.00008591$ $,-0.00006462,0.00006349,0.00004608,0.00003257,-0.00018642,0.02915024,-$ $0.03569848,0.03863672,-0.00000053,0.00003545,-0.00000830,0.00028519,0$. $00036530,0.00000910,0.00029128,0.00028552,0.00099723,-0.00005531,0.000$ $05223,0.00005353,0.00005083,-0.00001642,-0.00004665,-0.00009423,-0.000$ $01143,-0.00002319,-0.00000907,-0.00015637,-0.00003049,0.00005842,0.000$ $00335,0.00001063,-0.00004516,0.00001077,-0.00001347,0.00006920,-0.0000$ $2168,-0.00001028,0.00000512,-0.00000236,0.00000288,-0.00001505,-0.0000$ $0196,0.00000745,-0.00002684,0.00000628,0.00005230,0.00000925,-0.000001$ $32,-0.00000621,0.01555384,-0.00170943,-0.00150851,-0.00645973,0.044039$ 48,-0.00128719,0.00028904,0.00106629,0.00104357,0.00149920,0.00102170, $0.00326681,-0.01006201,-0.00029152,0.00226037,0.00121544,0.00018829,-0$ $.00026702,-0.00049035,-0.00058035,0.00055792,-0.00012821,0.00044460,0$. $00015642,0.00044669,-0.00026827,0.00040153,-0.00016641,-0.00002919,-0$. $06438357,0.03490843,-0.04935703,-0.00001468,0.00004806,0.00003324,-0.0$ $0003792,0.00028140,-0.00023514,-0.00079447,0.00075662,-0.00063601,-0.0$ $0005845,0.00006826,0.00008805,0.00008068,-0.00005126,-0.00005835,-0.00$ $007303,-0.00002023,-0.00002363,-0.00021321,0.00000151,-0.00008617,0.00$ $013503,-0.00000600,-0.00003011,-0.00005313,0.00001080,-0.00001898,0.00$ $010151,-0.00003270,-0.00001884,0.00000819,-0.00000455,0.00000444,-0.00$ $002398,-0.00001324,-0.00000537,-0.00002992,0.00003379,0.00007464,0.000$ 
$02047,-0.00000120,-0.00001354,-0.03360743,-0.00103022,-0.00538189,0.09$ $281150,-0.02755285,0.05411312 \backslash \backslash-0.00004383,0.00004442,-0.00001909,0.00$ $000713,-0.00000147,0.00000611,-0.00000840,-0.00001008,-0.00000347,-0.0$ $0000788,-0.00001005,0.00001063,0.00002499,0.00004075,-0.00001382,0.000$ $00276,-0.00003570,0.00002997,0.00001590,-0.00001230,-0.00000213,-0.000$ $00304,0.00000138,0.00000419,0.00000541,0.00000531,0.00000545,-0.000004$ $15,-0.00000770,0.00000412,-0.00000789,-0.00000553,-0.00000432,-0.00000$ $255,-0.00000155,0.00000436,0.00000211,-0.00000392,0.00000567,0.0000058$ $7,-0.00000244,-0.00000842,-0.00001308,0.00000460,-0.00000388,0.0000365$ $6,-0.00000755,-0.00000422,-0.00000601,0.00000178,-0.00000156,-0.000004$ $68,0.00000206,-0.00000526,-0.00000124,0.00000020,-0.00000669,0.0000011$ $0,-0.00000003,-0.00000399,0.00000354,-0.00000092,-0.00000106,-0.000000$ $55,-0.00000113,0.00000036,-0.00000154,-0.00000171,-0.00000147,-0.00000$ 088,0.00000111,0.00000435,0.00000035,0.00000046,0.00000417II!@

ortho-Addition Product to 4-fluoroanisole

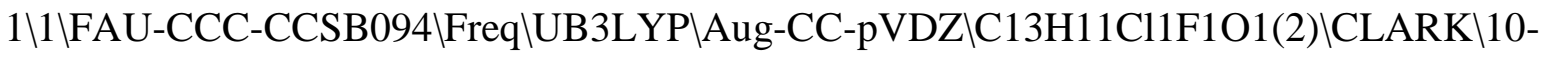
Dec-2014I0I\\#N Geom=AllCheck Guess=TCheck SCRF=Check GenChk UB3LYP/Aug -CC-pVDZ Freq \Addition product p-Cl-Ph-radical + 4-fluoro-anisol (ort ho) $\backslash 10,2 \backslash \mathrm{C}, 1.3158144155,-0.4023748212,0.9182686041 \backslash \mathrm{C}, 2.1631965713,0.55$ 45014148,0.1035887521\C,3.1774094569,0.1420213724,-0.7273222184\C,3.52 $1895946,-1.2317633897,-0.8272074194 \backslash \mathrm{C}, 2.812870887,-2.1616081611,-0.034$ $5697665 \backslash \mathrm{C}, 1.7967329586,-1.8290004891,0.8045009218 \backslash \mathrm{H}, 1.3957191425,-0.07$ $87695051,1.9735634549 \backslash \mathrm{O}, 1.7898989355,1.842233257,0.3124709081 \backslash \mathrm{H}, 3.7447$ $103297,0.8632943287,-1.3111428478 \backslash \mathrm{F}, 3.1943169678,-3.4710045205,-0.1300$ 67399\Н,1.2911768368,-2.5951903946,1.3892831541\Н,4.3222337251,-1.5712 498144,-1.480174342\C,-2.8808123149,-0.0562797831,-0.1121465344\C,-2.4 $480193322,0.3769151274,1.1399160246 \backslash \mathrm{C},-1.0918616034,0.2621473122,1.463$ 0519894\C, $-0.1743595048,-0.2786350947,0.5563124699 \backslash \mathrm{C},-0.6396009379,-0$. $7103435448,-0.6936974766 \backslash \mathrm{C},-1.9881868729,-0.6010581313,-1.0377610245 \backslash \mathrm{C}$ $1,-4.5880465723,0.0816298654,-0.5346699475 \backslash \mathrm{H},-3.1577362432,0.797276025$ $8,1.8501704062 \backslash \mathrm{H},-0.7491137792,0.6051735784,2.4400624677 \backslash \mathrm{H}, 0.058615873$ -1.14289922,-1.4103957506ไH,-2.3457336603,-0.9370667888,-2.0095700058 IC ,2.4716289464,2.8706829019,-0.4023519375\H,2.351478643,2.7383163026, $-1.4877955699 \backslash \mathrm{H}, 3.541685363,2.8845175293,-0.1464509352 \backslash \mathrm{H}, 2.0048008419$, 3.8096046474,-0.0910521258॥Version=EM64L-G09RevC.01 $\backslash$ State=2-A $\backslash H F=-113$

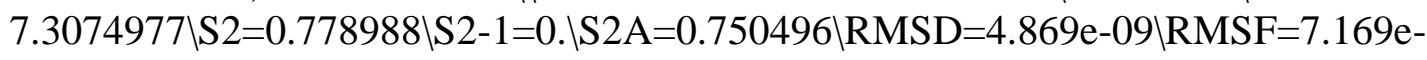
06\ZeroPoint $=0.2041776 \backslash$ Thermal $=0.218394 \mathrm{ZPE}=128.123395 \mathrm{\backslash Dipole}=0.745074$ 9,1.1642227,-0.0171626\DipoleDeriv=0.1837667,-0.0993456,0.0740503,-0.0 $31796,-0.0029253,0.0471971,0.1380626,0.1009883,0.1821979,0.1424967,-0$. 4460996,-0.093927,-0.0634595,1.3339799,0.0885081,-0.2344738,0.3277073, $0.0568133,-0.1124146,0.2875267,-0.0884884,-0.253851,-0.1884573,0.07893$ $65,-0.0243326,-0.2658811,-0.099786,-0.039396,-0.104745,-0.0836678,0.17$ $21198,0.014398,-0.0927904,-0.1398488,0.0717077,-0.0606076,0.2889392,-0$ $.3473616,-0.1006885,-0.4996903,1.2860717,0.0677422,-0.1215477,0.079607$ $5,0.1351135,-0.272064,0.0910655,-0.0736054,-0.1018421,-0.1325837,-0.03$ $01734,-0.0747814,-0.0529797,-0.1520935,0.0152742,0.015638,0.0440897,0$. $031023,0.0314785,-0.0862808,0.0208417,-0.076798,-0.1005566,-0.4552611$, $0.0983456,0.1118679,-0.0591535,-1.8639029,0.0821581,0.1879163,0.035892$ $8,-0.3230546,0.066903,-0.0591633,0.0589175,-0.0450873,-0.0037854,0.059$ 
$2036,0.0934909,0.0604209,0.080908,-0.3305772,0.2933603,0.0559034,0.571$ $8367,-1.2096249,-0.0973034,0.1015743,-0.0605365,-0.1596368,0.0984919,-$ $0.0275101,0.0700414,-0.021083,0.0049217,0.0402579,0.0668625,0.0336445$, $0.0876923,0.0464995,0.0362047,0.0807556,0.0267662,0.0489153,-0.006894$, $0.0895181,-0.0136575,0.0796101,1.2089738,-0.1021711,0.2548732,-0.09051$ $79,-0.0239375,0.007864,0.332864,0.0049456,0.0905665,-0.2714252,-0.0327$ $214,-0.1206183,-0.0339352,-0.0677592,0.0385092,-0.1428779,0.0362404,-0$ $.0526793,-0.0461379,0.0095142,0.0591582,-0.0319118,-0.0614054,0.072638$ $3,-0.0637807,0.0704225,0.07521,0.2707397,0.0121611,-0.013057,0.045998$, $-0.0500622,-0.0123264,0.0561974,-0.0141048,-0.0924999,-0.1033761,-0.02$ $70431,-0.0205514,0.0033929,-0.0675956,0.0335712,-0.0001628,0.0253543,-$ $0.014154,-0.2563992,0.041248,0.0225529,0.0269387,-0.0722931,0.0445804$, $-0.0033343,0.0506621,0.0295585,-0.8929601,0.0608973,-0.1522463,0.06695$ $57,-0.0789618,0.005298,-0.198507,0.0073196,-0.127193,0.0048708,0.03991$ $09,0.0532825,0.0412172,0.1031724,-0.0422886,0.0427712,-0.0403489,0.046$ $9613,0.0485574,-0.0141862,-0.0824059,-0.0047112,0.0935762,-0.052055,-0$ $.0558086,-0.050815,-0.0359337,0.0284746,0.0405422,0.0577582,0.0384827$, $0.0793394,-0.038201,0.073551,-0.033582,0.0297561,0.0470031,-0.0054743$, $-0.0413223,-0.0029289,0.1082031,-0.043654,-0.0515119,-0.0434932,0.0020$ $996,0.3869121,0.3530341,-0.0461835,0.1811207,0.7940862,-0.1059305,-0.0$ $781436,-0.3561519,0.3534485,0.0286538,-0.0674357,-0.0279997,-0.0195193$ $, 0.0090045,-0.0713185,-0.0008121,0.095711,-0.120425,-0.1378061,-0.1265$ $068,-0.0258526,0.0379793,0.0123544,0.0213688,-0.043126,0.0552944,0.025$ $46,0.0512611,0.0803152,0.0273632,0.015656,-0.0962069,-0.0086174,0.0293$ $99,-0.0475707,0.0632244 \backslash$ Polar $=228.9219659,-14.4027381,163.8220839,-11$. $2702638,6.9877262,146.635381 \backslash \mathrm{PG}=\mathrm{C} 01$ [X(C13H11Cl1F1O1)]\NImag=0\0.4113 $4051,-0.00273450,0.48866995,-0.02356452,0.02977484,0.52252769,-0.10807$ $912,-0.03813159,0.04364197,0.47586611,-0.03127473,-0.11363794,0.036832$ $73,-0.07383656,0.58658573,0.04181280,0.03578390,-0.11017663,-0.2750533$ $6,0.01850780,0.38201650,-0.00872687,0.00069242,0.01426956,-0.23206936$, $0.05539047,0.14926340,0.44582706,-0.01122528,0.01254089,0.00780432,0.0$ $1762880,-0.12626860,-0.00858440,0.00505166,0.62942981,0.01480705,-0.00$ $051158,-0.00570040,0.15441010,-0.03960376,-0.18152478,-0.29670455,-0.0$ $7238080,0.37364853,-0.00044261,-0.00258496,-0.00345573,-0.00382727,0.0$ $2927690,0.00290392,-0.09370272,0.06737718,0.03301168,0.43534298,-0.000$ $47354,-0.00502412,0.00047761,0.05502831,-0.02057079,-0.04286372,0.0086$ $0734,-0.26126411,0.02177541,-0.07639558,0.58273091,-0.00378270,0.00284$ $701,-0.00175611,-0.00041009,-0.02218713,0.00712605,0.04083675,-0.02584$ $366,-0.07576176,-0.29308098,-0.01163834,0.35444479,-0.00921491,0.01374$ $395,0.00622802,-0.01790143,0.00305182,0.01394403,0.03263861,-0.0391037$ $0,-0.01957377,-0.11964655,-0.00512372,0.05844851,0.43371023,0.02091600$ ,-0.00837242,-0.01642060,-0.00543500,-0.00382865,0.00425657,-0.0052011 $8,-0.04368639,0.01234261,-0.07588530,-0.16804006,0.08634743,-0.0652171$ $1,0.58689478,0.00410883,-0.01108784,-0.00309898,0.01545159,-0.00196298$ ,-0.01353009,-0.02484823,0.04143000,0.01770496,0.07116412,0.02607170,$0.12304704,-0.25736822,-0.02630200,0.36270378,-0.07919645,0.03554089,0$ $.01373133,0.01711652,-0.01409904,-0.01373827,-0.01369796,0.02058310,0$. $00879810,-0.02913294,-0.04055922,0.04225669,-0.24140002,0.08022454,0.1$ $5533560,0.48658077,0.02722320,-0.19354717,0.00017170,-0.00133247,-0.02$ $260104,0.00428380,0.00506618,-0.00514006,-0.00357902,-0.01122179,0.024$ $98217,0.00724608,0.03976772,-0.11942491,-0.02128441,-0.02933970,0.5655$ 
$8849,0.01629893,-0.00618986,-0.07384586,-0.01573003,0.01610137,0.00809$ $340,0.01119190,-0.01764105,-0.00698589,0.03754302,0.03373814,-0.027968$ $02,0.16010484,-0.05686491,-0.18269326,-0.31730029,-0.06395986,0.402479$ $81,-0.03772155,-0.00607363,-0.01274844,-0.00239225,0.00307038,0.021541$ $23,0.00032008,-0.00325381,-0.00017708,-0.00056269,0.00116933,0.0003733$ 4,0.00063525,0.00084046,-0.00031711,-0.00091364,0.00678901,0.01072316, $0.05707682,-0.00862141,-0.06336372,-0.06506023,0.00187895,0.00526389,0$ $.01857233,-0.00151701,-0.00158943,0.00131270,0.00090937,-0.00014815,-0$ $.00048697,-0.00361800,-0.00278585,0.00050808,0.00454918,-0.01252059,-0$ $.02828258,0.00455289,0.07167681,-0.02191531,-0.06110399,-0.25926123,0$. $00682205,-0.00204612,-0.02103104,-0.00180727,0.00451004,-0.00000257,0$. $00068810,-0.00150230,-0.00087240,-0.00206241,-0.00046073,0.00110347,0$. $00343987,-0.00617617,0.00128717,0.01685782,0.07122750,0.28657969,0.007$ $18747,-0.01822688,-0.00454630,-0.09430822,0.04542843,0.04673935,-0.004$ $56489,0.03698038,0.00858198,-0.00064064,-0.00296695,0.00673371,-0.0000$ $3522,-0.00084460,0.00000243,-0.00284958,-0.00067621,0.00311828,0.00055$ $113,-0.00073605,-0.00067523,0.21337056,0.00152623,-0.04472098,0.001250$ $34,0.07230396,-0.27157966,-0.04152186,0.01562467,-0.02617266,-0.011307$ $79,0.00201066,-0.00150615,-0.00063635,0.00033074,0.00559499,-0.0017747$ $6,-0.00412864,-0.00424184,0.00359406,0.00114033,0.00223506,-0.00103173$ $, 0.00222609,0.53363997,-0.00674337,0.02036255,0.00396946,0.04581513,-0$ $.01648654,-0.07377739,0.00951020,-0.02738095,0.00400163,0.00617099,0.0$ $0219806,0.00078600,0.00038378,0.00041669,-0.00069174,0.00383342,0.0013$ $9281,-0.00174381,-0.00107122,-0.00008830,0.00165139,-0.12799837,-0.047$ $10546,0.18609097,0.00125342,0.00291782,0.00491894,-0.00829182,-0.01778$ $354,0.01233024,-0.12442015,-0.09690394,0.09298072,0.00641922,0.0062569$ $0,-0.00477764,0.00209424,0.00213680,0.00183496,-0.00022262,0.00046914$, $-0.00043056,-0.00085640,-0.00090357,0.00070204,-0.00042648,-0.00056524$ ,-0.00285891,0.12479769,0.00199661,-0.00019229,-0.00063542,0.00449508, $0.00992478,-0.00448708,-0.09758719,-0.17955680,0.10314526,-0.01636666$, $-0.01523024,0.01659249,0.00256837,-0.00269048,-0.00144732,0.00043282$,$0.00001957,-0.00040439,-0.00004029,0.00012427,-0.00004338,-0.00128565$, $-0.00067581,0.00074646,0.10369188,0.19382534,0.00425705,-0.00188986,0$. $00349796,0.00852363,0.01485739,-0.00572996,0.09446409,0.10095233,-0.12$ $614373,-0.00195237,-0.00154738,0.00353363,0.00146999,-0.00063346,0.003$ $56411,-0.00008416,-0.00047278,-0.00043222,-0.00017291,-0.00027727,0.00$ $017294,-0.00268021,0.00033425,-0.00132562,-0.10210000,-0.11188944,0.12$ $504025,0.00202046,-0.00136805,0.00742935,0.00024349,-0.00110555,-0.000$ $33346,-0.00078622,0.00194538,0.00441848,0.00809905,-0.01736730,0.00043$ $572,-0.07083092,0.05829584,0.01733026,-0.00889892,0.04255611,0.0051330$ $6,-0.00068794,0.00229217,-0.00012606,0.00022228,0.00103722,-0.00066851$ $, 0.00028420,-0.00051148,0.00013164,0.07136948,0.00127122,0.00501242,-0$ $.00112121,0.00056031,0.00306732,-0.00044633,-0.00535360,0.00012369,0.0$ $0556149,0.01359521,-0.05100068,-0.00157612,0.06141688,-0.25735398,-0.0$ $1372269,0.01193054,-0.03150020,-0.00354029,0.00030624,-0.00155708,0.00$ $021927,0.00035321,-0.00471125,0.00007357,0.00063993,-0.00208493,-0.000$ $26026,-0.08478096,0.33786207,0.00582630,0.00289220,0.00377181,-0.00033$ $385,0.00024347,0.00016277,0.00554840,-0.00075518,-0.00033451,-0.005543$ $38,0.02482875,0.00564559,0.01762688,-0.01058937,-0.05322015,0.01043291$ $,-0.03099530,0.00313958,-0.00013890,0.00037240,-0.00027971,-0.00052617$ $, 0.00000233,0.00015519,-0.00012375,0.00090250,0.00030997,-0.02711236,0$ 
$.01646506,0.04371377,0.00518638,0.00763033,-0.00678201,-0.00041378,0.0$ $0187591,0.00132066,-0.00040153,0.00052094,-0.00042009,-0.00053912,0.00$ $289679,0.00433508,-0.00374676,-0.01898303,0.01312452,-0.10684108,-0.09$ $152758,0.08825339,0.00029743,0.00003652,0.00041922,0.00046953,0.000678$ $64,-0.00061124,-0.00012901,-0.00007434,-0.00016016,-0.00091749,-0.0005$ $3370,-0.00408430,0.10570226,-0.01306418,-0.01459167,0.01480076,0.00418$ $595,-0.00242582,-0.00263877,-0.00017601,-0.00035033,-0.00004566,0.0022$ $6588,0.00028005,-0.00101162,0.00562726,0.00654734,-0.00478721,-0.09364$ $898,-0.19396901,0.11440514,-0.00036736,0.00086495,-0.00032068,-0.00127$ $795,-0.00122354,0.00133645,-0.00008611,0.00000247,0.00002566,-0.001224$ $49,0.00129951,-0.00024294,0.09829023,0.20259312,-0.00243879,-0.0040319$ $0,0.00357348,0.00135623,-0.00111918,0.00107890,-0.00076021,-0.00046943$ ,-0.00012925,0.00477076,-0.00212926,0.00119907,0.00948740,0.01680726,-$0.00380475,0.08788231,0.11108498,-0.12388384,-0.00039491,0.00098375,0$. $00049112,-0.00035176,-0.00014361,0.00014476,-0.00016516,0.00002997,-0$. $00019378,-0.00423678,-0.00042814,-0.00208445,-0.09605230,-0.12100895,0$ $.12344854,-0.00013693,-0.00045698,-0.00086820,0.00222339,0.00082245,0$. $00063964,-0.00083411,-0.00072022,0.00307007,-0.20040292,0.06763482,0.1$ 4733560,-0.00734603,0.00893510,0.00845645,0.00073943,-0.00232293,0.001 $89150,0.00016998,-0.00003354,-0.00008226,0.00002929,-0.00001203,0.0003$ $1669,-0.00072878,-0.00012943,-0.00163466,-0.00009049,-0.00043627,-0.00$ $238371,-0.00017569,0.00027638,0.00059474,0.20655102,0.00007803,-0.0003$ $2451,-0.00014364,0.00015570,-0.00492091,0.00079760,0.02057853,-0.00287$ $968,-0.01716372,0.06846888,-0.08262860,-0.05045675,-0.01458065,0.00945$ $035,0.01190544,-0.00189103,-0.00328437,0.00269160,0.00005339,0.0000325$ $4,-0.00003022,0.00138130,-0.00091658,-0.00105998,-0.00048794,0.0011885$ $5,-0.00007529,0.00044760,0.00194005,-0.00123351,-0.00080666,0.00040533$ $, 0.00071942,-0.07353082,0.08148247,-0.00085724,0.00008440,-0.00043088$, $0.00076963,0.00054541,0.00225896,-0.00027780,0.00112063,0.00251867,0.1$ 4701893,-0.04951061,-0.14323298,0.01238874,-0.00873225,-0.00553428,0.0 $0169837,0.00318170,0.00016300,0.00014498,-0.00002300,-0.00005097,0.000$ $04523,0.00025387,0.00037013,-0.00151125,-0.00032814,-0.00124834,-0.002$ $50057,-0.00060573,-0.00053245,0.00079312,-0.00024323,-0.00005193,-0.15$ $756270,0.05419139,0.14548366,-0.00097280,0.00027323,0.00028900,-0.0001$ $5030,-0.00020408,0.00000629,-0.00002204,0.00031463,-0.00004813,0.00002$ $481,-0.00027526,0.00006814,-0.00019452,0.00055389,-0.00014361,0.000026$ $24,0.00012834,0.00005858,0.00002225,0.00004410,0.00006841,0.00043690,0$ $.00047824,-0.00025439,0.00005408,0.00001839,-0.00005280,0.00027707,-0$. $00066002,-0.00008684,-0.00006246,0.00005427,-0.00004133,0.00001421,-0$. $00000433,-0.00002777,0.49344411,0.00046258,-0.00073052,-0.00025058,0.0$ $0006496,-0.00004092,0.00013298,0.00005998,-0.00006724,0.00006440,-0.00$ $001581,0.00010797,0.00006721,0.00000839,0.00009978,0.00013192,0.000260$ $10,0.00005260,0.00012271,0.00015760,0.00007133,0.00005624,-0.00000303$, $-0.00004917,0.00011738,-0.00005054,-0.00000228,-0.00001518,-0.00005609$ $,-0.00010347,-0.00006425,0.00003355,-0.00004521,-0.00001608,0.00000187$ $, 0.00001357,-0.00000082,-0.06835110,0.21387640,0.00083652,-0.00044779$, $-0.00114552,0.00044085,-0.00051192,0.00047406,0.00005374,0.00011245,0$. $00012595,-0.00008087,0.00005322,0.00016294,-0.00006865,0.00059831,0.00$ $029381,0.00071549,0.00011829,0.00021813,0.00021215,-0.00005673,0.00036$ $538,-0.00020858,0.00035107,0.00024417,-0.00012796,-0.00000388,-0.00010$ $831,0.00000849,-0.00057075,-0.00014454,-0.00006688,0.00005595,-0.00012$ 
$158,0.00002635,-0.00000903,-0.00001027,-0.00117243,0.18423322,0.574661$ $34,0.00076879,0.00204712,0.00216720,-0.00070302,0.00098931,-0.00038576$ $, 0.00000016,-0.00021730,-0.00015502,0.00013770,0.00000448,-0.00018735$, $0.00004642,-0.00038876,-0.00025413,-0.00124864,-0.00033162,-0.00025273$ ,-0.00010179,-0.00005753,-0.00042928,0.00046605,-0.00065371,-0.0002778 4,0.00009713,0.00000882,0.00010723,-0.00005013,0.00060319,0.00019720,-$0.00000979,-0.00000477,0.00012937,-0.00001276,-0.00000613,-0.00000278$, $-0.12102246,0.00075278,-0.02336383,0.68190102,0.00216979,0.00542393,-0$ $.00350625,0.00149194,-0.00107963,0.00012089,-0.00002302,0.00051355,-0$. $00006522,0.00000348,-0.00011091,0.00010721,0.00003788,0.00011402,-0.00$ $039488,-0.00120752,-0.00001089,0.00037516,0.00005208,-0.00006613,-0.00$ $003494,-0.00020541,0.00007515,0.00004568,0.00001035,-0.00003976,-0.000$ $13203,-0.00005164,0.00020058,0.00017275,-0.00001410,-0.00004352,0.0000$ $1572,-0.00000041,-0.00001703,0.00000923,-0.02319542,-0.09630794,-0.087$ $41611,-0.10398627,0.23235911,0.00142090,-0.00357245,-0.00120627,-0.000$ $54224,0.00011980,-0.00023725,-0.00018389,-0.00011145,0.00002720,0.0000$ $6211,-0.00016113,-0.00016561,-0.00001335,-0.00040378,-0.00005223,0.000$ $58314,0.00002796,0.00002630,0.00000613,0.00005276,0.00006900,0.0000529$ $8,0.00009916,-0.00005813,0.00006970,0.00002119,0.00012496,0.00003119,0$ $.00022909,0.00000092,0.00003326,-0.00008970,0.00002171,-0.00001359,0.0$ $0001384,0.00002848,-0.08220264,-0.07695976,-0.28909156,-0.00766965,0.2$ $0308953,0.62170207,-0.02512911,0.01180108,0.01621488,-0.00146092,0.002$ $68714,-0.00004193,0.00006599,-0.00104122,0.00048070,-0.00018587,0.0003$ $3639,0.00013963,0.00039948,0.00077509,-0.00029748,-0.00217722,-0.00118$ $290,0.00080632,0.00084055,0.00102660,0.00337540,-0.00013047,-0.0006506$ $8,0.00037316,-0.00026188,0.00004763,0.00012142,-0.00038355,0.00027939$, $0.00027420,0.00000962,0.00039166,-0.00024701,-0.00004719,0.00000987,-0$ $.00004976,-0.00724519,-0.03154409,-0.08332032,-0.32196927,0.03924633,-$ $0.01975477,0.65900044,0.00423774,0.00308497,-0.00143414,-0.00037013,-0$ $.00143087,-0.00039514,-0.00008799,0.00027881,0.00006277,-0.00010627,-0$ $.00002880,-0.00002530,0.00013537,-0.00017668,-0.00006670,-0.00013941,0$ $.00005908,-0.00007872,-0.00126545,-0.00003760,-0.00038600,-0.00093274$, $0.00008014,0.00017065,0.00016002,-0.00004345,0.00013205,-0.00003579,0$. $00013410,-0.00003396,-0.00003698,0.00003421,0.00012999,-0.00002949,0.0$ $0001184,-0.00000791,-0.01082215,0.01494095,0.01432790,0.01393470,-0.07$ $454618,-0.02353880,-0.07204886,0.23077743,-0.00394779,0.00172667,0.007$ $31240,-0.00190931,0.00110955,0.00001423,0.00027359,-0.00089062,-0.0001$ $0369,-0.00002727,0.00064262,-0.00001009,-0.00022860,-0.00047343,0.0002$ $0888,-0.00210307,0.00046013,-0.00086719,-0.00216512,0.00087886,0.00108$ $257,0.00005569,-0.00098922,-0.00039108,0.00001125,0.00001367,0.0001906$ 7,-0.00010215,0.00081043,0.00032577,0.00015095,-0.00016167,0.00004329, $-0.00004015,-0.00000921,-0.00003296,-0.03151064,0.00446194,0.00317317$, $-0.08072796,-0.01191948,-0.12577701,0.05548845,0.19897656,0.64059569,-$ $0.14869476,0.00254532,-0.03043538,-0.03127876,-0.01208382,0.00770158,0$ $.00156729,-0.00205346,-0.00273951,-0.00104098,0.00050178,0.00107885,0$. $00068809,0.00056578,-0.00338569,-0.01813357,0.01839352,-0.00002909,-0$. $01039026,0.00019474,-0.00321098,-0.00016244,0.00395800,-0.00122133,0.0$ $0066445,0.00003266,0.00045862,0.00090149,-0.00129620,0.00009876,0.0015$ $9257,-0.00149471,0.00057123,0.00004902,0.00000413,-0.00010286,-0.00121$ $001,0.00396011,0.01269559,-0.04179419,0.01234645,0.00814644,-0.2048505$ $0,0.07178662,0.11337598,0.58816876,0.00639883,-0.06825783,-0.00155044$, 
$-0.01589431,0.00305127,-0.00610868,-0.00054245,-0.00228237,0.00096722$, $0.00050493,0.00044531,-0.00094618,-0.00095577,-0.00202971,0.00346645,0$ $.03204430,-0.00558016,0.00364630,-0.00813585,0.00084003,-0.00379433,-0$ $.00190267,0.00077080,0.00087270,0.00013371,0.00030310,0.00076843,0.000$ $12156,-0.00175307,-0.00189249,-0.00024240,0.00083790,-0.00023291,0.000$ $05540,0.00007889,0.00003909,0.00458876,-0.01779105,-0.02436178,0.02979$ $785,0.00811486,0.01591224,0.04673235,-0.10568433,-0.07715342,-0.070174$ $87,0.25818373,-0.01637224,-0.00327486,-0.08301195,0.01823340,-0.004689$ $03,0.00508639,0.00008845,0.00351356,-0.00115871,0.00044925,-0.00044380$ $, 0.00017226,-0.00082949,-0.00083678,0.00020385,0.00429373,0.00096434,0$ $.00170594,-0.03183717,0.00030635,-0.01276343,0.00010349,0.00021228,0.0$ $0072299,-0.00001021,-0.00023017,-0.00102889,0.00028319,-0.00055324,-0$. $00047073,-0.00011791,-0.00027939,0.00037607,0.00013084,-0.00001732,0.0$ $0016240,0.01354928,-0.02492803,-0.06136117,0.05142721,0.00748270,0.048$ $76810,0.05160517,-0.06606645,-0.20374974,0.00814147,0.17388582,0.59414$ $431,-0.01527652,-0.00868885,-0.02873859,0.00123235,0.00159528,-0.00280$ $791,-0.00016423,-0.00045101,0.00005546,0.00016920,0.00027232,-0.000089$ $84,-0.00002863,-0.00080777,-0.00039984,0.00019899,-0.00194288,-0.00131$ $897,-0.00580582,0.00038839,-0.00233901,0.00018103,-0.00060615,-0.00001$ $462,0.00010458,0.00001811,0.00013582,-0.00009801,0.00089776,0.00022757$ $,-0.00007784,0.00020770,-0.00004128,-0.00002996,0.00000413,-0.00000339$ $,-0.04606947,0.03305883,0.05605679,-0.03560017,-0.00707081,-0.03157868$ $, 0.05471203,-0.00761562,0.00332534,-0.13872640,0.00086898,-0.02809185$, $0.68738247,-0.00072434,0.00332986,0.00281799,0.00027984,0.00009355,-0$. $00008859,-0.00031819,0.00053522,-0.00030194,-0.00011452,-0.00068624,-0$ $.00000566,0.00064752,0.00031899,0.00011383,0.00063936,0.00046688,0.000$ $61736,0.00115903,0.00095738,0.00034157,-0.00019235,0.00005118,-0.00016$ $267,0.00003836,0.00002415,-0.00005474,-0.00004019,-0.00020608,-0.00010$ $723,-0.00004026,0.00028506,-0.00004372,0.00004060,0.00001463,0.0000018$ $3,0.01185308,0.00790112,0.00696083,-0.00666460,-0.00414360,-0.00532999$ $, 0.00888814,-0.00228471,-0.02037077,-0.02160687,-0.09608494,-0.0802480$ $4,-0.10169136,0.23615791,-0.00978460,0.00016716,-0.00332085,0.00014511$ $, 0.00170896,-0.00032517,0.00032867,0.00027010,-0.00002072,0.00003407,-$ $0.00036684,-0.00042557,0.00057451,0.00002999,-0.00061100,-0.00057469,-$ $0.00161314,-0.00001687,-0.00030069,-0.00000127,0.00062768,0.00086483,-$ $0.00044615,-0.00017483,-0.00001926,-0.00001821,-0.00006450,0.00000600$, $-0.00023623,-0.00009804,-0.00007780,0.00018099,0.00003100,0.00004258,-$ $0.00001030,0.00005529,0.00504489,0.01673505,0.05039724,-0.03158989,-0$. $00542473,-0.03126431,0.04419697,-0.02864059,-0.04048204,-0.08456207,-0$ $.06967402,-0.27188301,0.00431254,0.19594241,0.60777349,0.00365540,0.00$ $000218,-0.00102700,0.00024188,0.00000874,0.00053214,0.00011229,-0.0001$ $1635,0.00023118,-0.00005573,0.00019491,0.00000085,0.00001200,0.0003099$ $1,0.00051022,0.00050581,-0.00009323,0.00026084,0.00028630,0.00003199,0$ $.00048887,-0.00016180,-0.00012090,0.00041802,-0.00018019,-0.00000960,-$ $0.00004968,-0.00015772,-0.00010601,-0.00005224,-0.00007558,0.00000891$, $-0.00016615,0.00000082,0.00000134,0.00000675,-0.18189258,0.04528514,0$. $05795598,0.05348545,0.00928327,0.04277468,-0.05700072,0.01664259,0.014$ $67728,-0.00641264,-0.02836275,-0.07638687,-0.32684787,0.03855143,-0.02$ $617867,0.65433851,0.00011146,0.00382500,-0.00379833,0.00078281,-0.0000$ $7830,-0.00000955,0.00004179,0.00008004,-0.00012011,-0.00006923,-0.0000$ $3635,-0.00002067,0.00000173,0.00012390,0.00015872,-0.00011797,-0.00014$ 
$707,0.00010171,-0.00074528,0.00010582,-0.00035635,0.00008108,0.0002427$ $4,-0.00008251,0.00001463,-0.00000753,-0.00005405,0.00000051,0.00005638$ $, 0.00003900,-0.00005919,0.00000975,-0.00006225,-0.00000322,-0.00001036$ $, 0.00000631,0.06989173,-0.10696123,-0.07099375,-0.00756946,-0.00089102$ $,-0.02744701,0.01650968,-0.00831274,-0.00395962,-0.01099404,0.01483530$ $, 0.01363960,0.01330855,-0.07303160,-0.02306143,-0.07026020,0.22725444$, $-0.00107281,-0.00361625,-0.00363463,0.00036278,0.00036824,-0.00039411$, $-0.00001266,-0.00017761,0.00016345,-0.00006668,0.00013579,0.00008145$,$0.00016113,0.00003404,0.00009644,0.00078798,-0.00027657,0.00020217,-0$. $00123573,-0.00001158,-0.00048225,0.00005283,-0.00008187,0.00011197,-0$. $00001564,0.00000117,-0.00000095,-0.00000705,0.00002082,-0.00003114,-0$. $00003699,0.00007497,0.00001608,-0.00000356,0.00001343,-0.00002534,0.11$ $674187,-0.08146013,-0.21180149,0.00174615,-0.02016231,-0.03869301,0.01$ $606881,-0.00386129,-0.00643292,-0.03257714,0.00498974,0.00600843,-0.08$ $713224,-0.01175447,-0.12913887,0.06553875,0.20388247,0.65441211,0.0004$ $0085,-0.00016696,0.00040506,-0.00019621,0.00075204,-0.00035279,0.00004$ $035,-0.00054034,0.00002662,0.00001373,0.00030628,-0.00009828,0.0002248$ $6,-0.00071417,-0.00013918,-0.00050459,-0.00037890,-0.00008548,-0.00003$ $915,0.00000917,-0.00001694,-0.00033122,-0.00077466,0.00022806,-0.00003$ $597,-0.00000009,0.00012372,-0.00028368,0.00089296,0.00018848,0.0000714$ $0,-0.00003701,0.00003124,-0.00002141,0.00000358,0.00002305,-0.14344044$ $, 0.01098173,-0.02680036,-0.03027260,0.00297699,-0.00817903,0.00320518$, $-0.00109687,-0.00333035,-0.00141060,0.00003390,-0.00104258,0.00185561$, $0.00273173,0.00546780,-0.03976532,0.00348614,-0.01112667,0.21088249,-0$ $.00022705,-0.00056357,0.00025721,-0.00005073,-0.00001927,0.00002466,-0$ $.00002299,0.00002135,0.00005530,-0.00000024,-0.00002048,0.00000239,-0$. $00002359,0.00000766,-0.00002636,0.00006402,0.00005171,-0.00002361,0.00$ $003147,0.00001315,0.00005010,0.00001462,0.00009760,-0.00002120,0.00000$ $164,0.00000220,-0.00000232,0.00002869,-0.00004953,-0.00001512,-0.00000$ $567,0.00001332,-0.00000214,-0.00000022,-0.00000202,-0.00000254,0.01089$ $651,-0.02711452,-0.00329882,-0.00709557,0.00486426,-0.00219009,0.00135$ $017,0.00357585,-0.00258058,-0.00010860,-0.00028214,0.00104167,0.000246$ $13,0.00307559,-0.00357951,0.01378121,0.00318909,0.00292408,-0.01796323$ $, 0.01712896,0.00007296,0.00032680,0.00016416,-0.00003294,0.00013978,-0$ $.00011861,0.00000347,-0.00014858,0.00001112,-0.00000244,0.00008031,-0$. $00001820,0.00005182,-0.00014737,-0.00002306,-0.00017560,-0.00010248,0$. $00002510,0.00007674,0.00000766,0.00004693,-0.00008870,-0.00016343,0.00$ $007297,-0.00000982,-0.00000303,0.00002984,-0.00007712,0.00024129,0.000$ $06139,0.00001658,-0.00001384,-0.00000097,-0.00001020,0.00000129,0.0000$ $0309,-0.02683629,-0.00326324,-0.04389878,-0.03293747,0.00223972,-0.004$ $60510,0.00264481,-0.00371941,-0.00299568,-0.00068957,0.00100975,0.0013$ $7089,-0.00054824,-0.00238854,-0.00162000,0.01367512,-0.00160279,0.0068$ $1656,0.04512381,0.00470791,0.04355716,-0.00026146,0.00037960,0.0008625$ $7,-0.00018981,0.00034631,-0.00001367,0.00006249,-0.00018969,-0.0000286$ $2,-0.00002358,0.00011750,0.00000147,0.00001999,-0.00007033,-0.00004769$ $,-0.00025462,-0.00005288,-0.00001812,0.00004121,-0.00000246,0.00001413$ $, 0.00002769,-0.00024597,-0.00000605,-0.00001246,0.00000037,0.00002857$, $-0.00004107,0.00014316,0.00004730,0.00000136,0.00001874,-0.00000093,-0$ $.00000585,-0.00000123,-0.00000248,0.00734961,-0.00434571,-0.00881629,-$ $0.17576190,0.07583831,0.12255295,-0.01339777,0.01152432,0.02041467,-0$. $00400172,0.00071052,-0.00294170,-0.00049868,-0.00042482,-0.00069800,0$. 
$00044201,-0.00019762,-0.00278349,0.00111898,-0.00082877,-0.00035954,0$. $18396993,-0.00011314,0.00005409,-0.00011157,0.00004472,0.00011638,0.00$ $009187,0.00002389,0.00000036,-0.00000569,-0.00000492,-0.00000362,-0.00$ $001069,0.00000082,0.00000717,0.00002498,-0.00007967,0.00001984,-0.0000$ $9795,-0.00000550,0.00000358,0.00000226,0.00003302,-0.00006228,-0.00002$ $132,-0.00001429,-0.00000085,-0.00001132,0.00001977,-0.00000948,0.00001$ $012,0.00000719,-0.00001021,-0.00000495,0.00000102,-0.00000356,0.000000$ $03,0.00735188,-0.00009797,-0.00518286,0.07476060,-0.08159391,-0.080169$ $65,0.00044312,0.00281879,-0.00177129,0.00095003,0.00611918,-0.00155998$ $,-0.00044471,-0.00093860,0.00047901,-0.00041392,0.00432629,-0.00304057$ $,-0.00044031,-0.00211031,0.00142213,-0.08165634,0.07513896,-0.00040905$ $, 0.00013679,0.00025767,-0.00002754,-0.00000601,-0.00007506,-0.00001321$ ,-0.00000258,0.00002702,0.00000302,0.00000781,-0.00000394,-0.00000938, $0.00000153,0.00002350,-0.00000258,-0.00002649,0.00000101,0.00000955,0$. $00000283,-0.00001185,-0.00001344,0.00003295,-0.00001329,0.00000834,-0$. $00000086,0.00000717,-0.00000434,0.00000148,-0.00000053,-0.00000335,0.0$ 0000953,-0.00000306,-0.00000170,-0.00000019,-0.00000171,0.01984651,-0. $01045889,-0.01325970,0.11998847,-0.07960666,-0.17633351,-0.00663143,0$. $00312241,0.00843497,-0.00248997,-0.00164990,0.00181679,-0.00088648,0.0$ 0054200,-0.00029367,-0.00333987,-0.00292793,-0.00319122,0.00059707,0.0 $0123943,0.00063306,-0.12812134,0.08829992,0.18263783,0.00090636,-0.000$ $07064,0.00200483,-0.00007421,0.00008968,-0.00007680,-0.00012535,0.0001$ $7008,0.00005918,0.00004038,-0.00018561,-0.00004633,-0.00004149,0.00010$ $767,0.00002543,0.00014293,-0.00018851,-0.00014224,-0.00026588,-0.00010$ $694,-0.00048154,0.00003910,0.00017653,-0.00009100,0.00001662,0.0000153$ $8,0.00002389,0.00005507,-0.00016155,-0.00001940,0.00000717,-0.00001280$ $, 0.00002363,0.00000257,-0.00000152,0.00000287,-0.00378339,0.00163352,-$ $0.00076623,-0.00586630,-0.00980374,-0.02762346,-0.08324715,-0.02399857$ $,-0.07933090,0.00866186,0.00620954,0.01711093,-0.00194274,0.00258911,0$ $.00269462,-0.00100618,0.00014180,0.00041758,-0.00126168,-0.00020103,-0$ $.00114490,0.00091276,-0.00069884,0.00047808,0.08640936,-0.00188138,-0$. $00326227,0.00095240,-0.00066113,0.00086003,0.00026803,-0.00003260,-0.0$ $0032870,0.00002951,0.00000671,0.00005062,-0.00001992,0.00001114,-0.000$ $07415,0.00013533,0.00061872,-0.00002704,-0.00017949,0.00040430,0.00036$ $602,0.00054584,0.00020857,-0.00023708,-0.00012283,-0.00004106,0.000021$ $98,0.00005340,-0.00001576,-0.00005661,-0.00006297,0.00006126,-0.000051$ $32,-0.00004958,-0.00000172,-0.00000177,0.00000685,0.00157726,0.0060131$ $7,-0.00165514,0.00183674,0.00433306,0.00262009,-0.02402771,-0.06752497$ ,-0.08598556,-0.00420301,0.00029386,-0.01057383,0.00288897,0.00429254, $-0.00327291,0.00015424,-0.00090632,0.00050037,0.00023417,0.00013008,0$. $00010326,-0.00085171,-0.00333396,0.00162022,0.02418302,0.06010369,-0.0$ $0139335,0.00197187,-0.00017015,0.00052110,-0.00048073,-0.00005760,-0.0$ $0012432,0.00016479,0.00015407,-0.00000443,-0.00007701,0.00001686,0.000$ $06929,0.00008103,-0.00006857,-0.00019810,-0.00010167,0.00045008,0.0008$ $5252,0.00003291,0.00064945,-0.00026575,0.00022025,0.00026762,-0.000037$ $89,-0.00001317,-0.00004455,-0.00007758,0.00009775,0.00003070,-0.000023$ $64,-0.00000210,-0.00006342,-0.00000547,0.00000494,0.00000392,-0.001143$ $52,-0.00156454,0.00275652,0.00011674,-0.00266956,-0.00286537,-0.078447$ $87,-0.08565120,-0.27750290,-0.00933308,-0.00612788,-0.01454916,0.00350$ $635,-0.00337077,-0.00049462,0.00060590,0.00045374,0.00037607,-0.000124$ $83,-0.00010747,-0.00016833,0.00018359,0.00172270,0.00000976,0.08590372$ 
$, 0.09533652,0.29224053,-0.00026627,-0.00051787,-0.00017534,0.00018358$, $-0.00026344,-0.00034989,-0.00009988,0.00004630,0.00020633,-0.00011875$, $-0.00001639,0.00004009,-0.00034075,0.00041499,-0.00009777,-0.00010057$, $-0.00026280,-0.00034320,0.00016970,0.00003226,0.00017595,-0.00008592,0$ $.00036162,0.00004235,-0.00001492,0.00000600,0.00000317,0.00003990,-0.0$ $0024359,-0.00003343,0.00001511,-0.00001955,0.00009136,-0.00000295,-0.0$ $0000554,-0.00000790,-0.00320872,0.00091428,-0.00260867,-0.00038530,-0$. $00043329,-0.00068082,0.00029984,0.00003415,-0.00280568,0.01024684,-0.0$ 0395176,-0.00677096,-0.17023903,0.07382967,0.11658248,-0.01485228,0.01 $192938,0.02039439,-0.00142627,0.00046890,0.00042109,-0.00001645,-0.000$ $08380,0.00011920,0.00040221,-0.00030708,-0.00052737,0.17899224,-0.0000$ $4268,-0.00263275,0.00141269,-0.00033004,0.00034723,-0.00011609,0.00017$ $133,-0.00028869,0.00034766,0.00000302,0.00039922,-0.00006153,-0.000188$ 23,0.00003932,-0.00012971, $0.00025933,0.00020524,0.00013129,0.00005308$, $-0.00012270,0.00004693,0.00007269,-0.00033795,0.00002286,-0.00007456,-$ $0.00002534,-0.00000042,0.00009157,-0.00017134,0.00001748,0.00008715,-0$ $.00006455,0.00017559,0.00000019,-0.00000761,0.00000337,0.00098281,0.00$ $631085,-0.00174326,-0.00044026,-0.00089541,0.00049104,-0.00030905,0.00$ $499269,-0.00345416,0.00680561,-0.00009200,-0.00569972,0.07398800,-0.08$ $474460,-0.08136101,0.00040019,0.00298793,-0.00192046,0.00000780,0.0000$ $4802,-0.00006985,-0.00006332,-0.00060209,0.00023489,0.00032585,-0.0000$ $3391,-0.00025545,-0.08098928,0.07818567,0.00128012,0.00088693,-0.00004$ $003,0.00024911,-0.00059851,-0.00012804,-0.00020698,0.00011721,0.000100$ $33,-0.00023972,-0.00019466,0.00011975,-0.00016254,0.00054935,0.0002532$ $1,0.00013446,0.00021630,0.00022510,0.00012177,0.00005374,0.00009160,-0$ $.00016146,0.00050677,0.00000592,0.00004809,0.00003749,0.00004296,0.000$ $04222,-0.00024416,0.00001042,-0.00007248,0.00001302,-0.00013830,0.0000$ $3732,0.00000043,-0.00000427,-0.00225596,-0.00178626,0.00192109,-0.0008$ $3771,0.00046304,-0.00026833,-0.00344825,-0.00337676,-0.00339791,0.0195$ $0699,-0.01076151,-0.01293707,0.11633404,-0.08071243,-0.17683797,-0.007$ $25734,0.00308947,0.00818924,-0.00059989,0.00011838,0.00011720,0.000140$ $40,0.00024868,-0.00008807,0.00096908,-0.00053209,-0.00078602,-0.124134$ $72,0.09021237,0.18340190,0.00000155,-0.00048763,-0.00116765,0.00011494$ ,0.00018402,-0.00008669,0.00002363,-0.00014494,0.00001019,0.00001775,0 $.00011580,0.00001335,-0.00002629,-0.00009375,0.00002878,0.00015816,-0$. $00012745,0.00001513,-0.00043135,-0.00001726,-0.00016574,-0.00002429,-0$ $.00011717,0.00005730,-0.00002354,-0.00000475,0.00000304,-0.00004148,0$. $00012988,0.00002838,-0.00001055,0.00001342,-0.00001448,-0.00001360,0.0$ $0000276,-0.00000424,0.00780873,0.00679610,0.01854602,-0.00220982,0.002$ $50158,0.00263919,-0.00105996,0.00011457,0.00036135,-0.00481058,0.00193$ $255,-0.00078326,-0.00460308,-0.00941890,-0.02657199,-0.08453821,-0.026$ $34780,-0.08582980,0.00103049,-0.00034919,0.00078973,0.00052030,0.00033$ $215,0.00090020,0.00003383,-0.00014467,-0.00003908,0.00083182,-0.000763$ $15,0.00054807,0.08724832,0.00006119,0.00024157,0.00009876,-0.00014017$, $0.00002155,0.00007211,0.00001457,-0.00004506,-0.00004987,-0.00000579,0$ $.00001325,-0.00000939,-0.00001862,0.00005848,0.00007903,0.00011388,-0$. $00008259,-0.00006634,0.00000291,-0.00009511,0.00007878,0.00000334,-0.0$ 0005466,0.00001947,-0.00000913,-0.00000143,-0.00000147,0.00001086,-0.0 $0006747,-0.00000861,0.00000494,-0.00001242,0.00001013,0.00000379,0.000$ $00319,0.00000163,-0.00504035,-0.00009418,-0.01048332,0.00265355,0.0036$ $9537,-0.00273192,0.00027149,-0.00087836,0.00049945,0.00178202,0.006191$ 
$04,-0.00168801,0.00159654,0.00454419,0.00243105,-0.02526561,-0.0665778$ $6,-0.08653479,-0.00072371,-0.00201605,0.00123929,-0.00019309,0.0001562$ $0,-0.00055360,-0.00012437,-0.00057997,0.00023418,-0.00086032,-0.003460$ $05,0.00178365,0.02586548,0.05902083,0.00030651,-0.00021336,-0.00017749$ $, 0.00003449,0.00006648,-0.00003449,0.00003389,-0.00004582,0.00002592,-$ $0.00001445,0.00003191,-0.00002495,0.00005419,-0.00008870,-0.00003251,-$ $0.00010232,0.00002507,0.00002986,-0.00009645,0.00004818,-0.00005469,-0$ $.00001200,-0.00006201,0.00001473,-0.00000219,0.00000304,0.00000513,-0$. $00002743,0.00010404,0.00001760,0.00000383,0.00000330,-0.00001000,0.000$ $00050,0 ., 0.00000755,-0.01007104,-0.00533383,-0.01388660,0.00318009,-0$. $00277867,-0.00014606,0.00050484,0.00046443,0.00044050,-0.00133518,-0.0$ $0160174,0.00266319,0.00005991,-0.00242589,-0.00193862,-0.08314674,-0.0$ $8691914,-0.28264047,-0.00014306,0.00141103,0.00059076,-0.00038928,-0.0$ $0030923,-0.00058260,-0.00000748,0.00023072,-0.00015410,0.00040568,0.00$ $176165,0.00008486,0.09075315,0.09560151,0.29581586,0.00116319,0.003152$ $60,0.00141247,0.00618587,-0.00005726,-0.00817513,-0.00115682,0.0063707$ $3,-0.00134655,-0.00054579,-0.00014748,0.00077982,0.00058620,-0.0008445$ $4,-0.00021533,0.00026662,0.00090948,0.00016631,-0.00026215,-0.00048551$ $, 0.00043828,-0.10939871,-0.05312990,0.04961762,-0.00059924,0.00134691$, $0.00006564,-0.00040450,0.00089620,-0.00000477,-0.00000979,0.00019431$,$0.00001975,0.00006944,0.00021646,0.00004183,-0.00027119,-0.00003220,-0$ $.00014227,0.00009148,-0.00000142,0.00007370,0.00010579,0.00003759,0.00$ $013163,-0.00013275,-0.00017411,-0.00025947,0.00015848,-0.00002679,0.00$ $001041,-0.00005081,0.00000387,-0.00001988,0.00026791,-0.00002182,0.000$ $06671,0.00003223,0.00000106,-0.00000354,-0.00004964,-0.00000998,0.0000$ $2002,-0.00004635,-0.00001560,-0.00005735,0.00002416,0.00001184,0.00001$ $460,0.54824400,0.00365627,-0.00131717,-0.00252657,-0.01447911,-0.05314$ $882,0.01772561,-0.00165694,0.00472451,0.00089070,-0.00182824,-0.001039$ $27,0.00162767,0.00083920,-0.00132100,-0.00035017,-0.00121824,0.0007136$ $2,0.00115148,0.00001809,-0.00023631,0.00048486,-0.03108066,-0.13534322$ $, 0.03478457,-0.00025880,-0.00231187,0.00053061,-0.00036888,0.00116170$, $0.00005902,0.00010757,-0.00019724,-0.00018857,-0.00008871,0.00043541,0$ $.00001164,-0.00051530,0.00001971,-0.00003973,0.00005079,0.00005763,0.0$ $0003761,0.00002471,0.00017980,0.00017790,-0.00037147,-0.00010682,0.000$ $00906,0.00001541,0.00001050,-0.00027050,0.00015512,-0.00009711,-0.0000$ $5195,0.00053300,-0.00005412,0.00012923,0.00007194,0.00001524,-0.000027$ $97,-0.00011701,-0.00001343,0.00011222,-0.00008425,0.00001317,-0.000108$ $22,0.00004683,0.00002431,0.00003445,-0.06661866,0.53197130,0.00144101$, $-0.00219956,0.00176113,-0.00679458,0.00476437,0.00225602,-0.00076288,-$ $0.00628285,-0.00090824,0.00112151,0.00022701,-0.00046310,-0.00049423,0$ $.00094164,0.00031592,0.00013821,-0.00088685,0.00049149,-0.00024503,0.0$ $0016875,0.00040024,0.04788278,0.05568381,-0.10827212,0.00030407,-0.001$ $13466,-0.00049487,0.00015189,-0.00084527,-0.00020351,0.00012399,-0.000$ $18190,0.00005816,0.00009279,-0.00020996,0.00012493,0.00024344,-0.00001$ $638,0.00004303,0.00000898,-0.00002972,-0.00001482,-0.00012412,-0.00013$ $349,-0.00015332,0.00080521,0.00035675,-0.00023432,-0.00007641,0.000093$ $06,0.00020272,-0.00013927,0.00007059,-0.00004611,-0.00028153,0.0000290$ $4,-0.00008146,-0.00004204,-0.00000769,0.00000982,0.00005284,0.00003633$ $,-0.00005989,0.00009695,-0.00011719,0.00008817,-0.00006324,-0.00003883$ ,-0.00002209,0.02300837,0.06344068,0.53998646,-0.00016561,-0.00015766, $-0.00009163,-0.00010413,0.00242201,0.00005671,0.00072760,-0.00043369$, 
$0.00094521,-0.00014122,0.00006819,0.00021125,-0.00005182,0.00006849,0$. $00003275,-0.00004818,-0.00008047,-0.00006558,0.00000192,0.00003125,-0$. $00009755,0.00012333,-0.00431689,0.02295237,-0.00041969,0.00043632,-0.0$ $0012856,0.00002866,-0.00018476,0.00002636,0.00000927,-0.00002258,0.000$ $01666,0.00000036,0.00000079,-0.00004440,0.00000852,-0.00001349,0.00000$ $324,-0.00000150,0.00000622,-0.00000602,-0.00004669,-0.00000127,0.00001$ $234,0.00015033,0.00003717,0.00007604,0.00000405,0.00002736,-0.00001586$ ,-0.00004325,0.00002750,-0.00003078,-0.00004631,0.00000809,-0.00001350 ,-0.00000999,-0.00000243,0.00000094,0.00001607,-0.00000239,-0.00002343 $, 0.00002027,-0.00003683,0.00001692,-0.00001173,-0.00001414,-0.00000176$ ,-0.05352091,-0.00444260,-0.03785215,0.05323050,-0.00052872,-0.0003963 $2,-0.00021061,-0.00163252,0.00579912,0.00301430,0.00097671,0.00077763$, $-0.00088236,-0.00035330,-0.00082088,-0.00024893,0.00031947,0.00013022$, $-0.00037680,-0.00028524,0.00007493,0.00009234,0.00009886,-0.00001739,-$ $0.00025432,-0.00150788,-0.00678948,0.03259814,0.00121665,-0.00122526,0$ $.00098659,0.00006176,-0.00002618,0.00007184,0.00001507,-0.00002592,0.0$ $0001087,0.00001088,-0.00000490,0.00000943,-0.00000677,-0.00000541,-0.0$ $0000913,0.00000748,-0.00000903,-0.00001478,0.00000559,0.00000016,0.000$ $02568,0.00012116,-0.00007657,0.00005040,-0.00001733,0.00003123,0.00001$ $061,-0.00001125,0.00001077,-0.00001358,-0.00000447,-0.00000250,-0.0000$ $0191,-0.00000158,0.00000441,-0.00000194,0.00000696,-0.00001322,-0.0000$ $0800,-0.00000711,-0.00004442,-0.00000617,-0.00000554,0.00000097,0.0000$ $0099,-0.00448204,-0.05530370,-0.04536398,0.00889485,0.05824275,0.00011$ $308,0.00042281,-0.00000336,0.00143718,-0.00487943,-0.00015769,0.000182$ $03,0.00056587,-0.00017741,-0.00050808,-0.00010354,0.00037044,0.0001646$ $9,-0.00029907,-0.00013916,0.00001264,0.00018082,0.00013122,-0.00007046$ $,-0.00017870,0.00022395,0.00633506,0.01232119,-0.01861945,-0.00062711$, $-0.00010585,0.00063631,-0.00009679,0.00020046,0.00005561,-0.00002879,0$ $.00002906,-0.00000991,0.00000642,0.00006277,-0.00001386,-0.00005892,-0$ $.00000059,-0.00000953,-0.00000758,0.00000322,0.00002042,0.00003544,0.0$ $0000717,-0.00000519,-0.00022595,-0.00008479,-0.00000347,0.00003545,-0$. $00002417,-0.00003071,0.00003368,-0.00002035,0.00002857,0.00007481,-0.0$ $0001121,0.00002420,0.00000996,0.00000610,-0.00000529,-0.00003327,-0.00$ $000730,0.00004638,-0.00003271,0.00003551,-0.00001940,0.00002042,0.0000$ $1225,0.00000733,-0.03450355,-0.03855157,-0.29358088,0.03104094,0.03489$ $417,0.32356631,0.00001297,-0.00036297,0.00005353,-0.00049065,0.0045206$ $9,0.00071751,-0.00019315,-0.00067889,0.00024214,0.00040033,0.00017600$, $-0.00045773,-0.00028379,0.00025411,0.00011107,0.00008910,-0.00016042,-$ $0.00001793,-0.00000404,0.00011470,-0.00005404,-0.01684628,-0.01359656$, $0.00566663,0.00070268,0.00046713,-0.00032072,0.00012039,-0.00020875,-0$ $.00004030,0.00001925,-0.00002789,0.00000105,-0.00000172,-0.00006411,0$. $00001072,0.00012186,-0.00001688,-0.00000494,0.00003051,0.00000765,0.00$ $000754,-0.00003083,-0.00005104,-0.00006861,0.00021671,0.00011890,-0.00$ $002533,0.00001893,0.00001102,0.00008854,-0.00004554,0.00002290,0.00000$ $950,-0.00009348,0.00000794,-0.00001924,-0.00001735,-0.00000585,0.00000$ $055,0.00001602,0.00001722,-0.00002652,0.00000224,-0.00000120,0.0000057$ $3,-0.00000705,0.00000310,-0.00000375,-0.28619021,0.00498700,-0.0651844$ $8,-0.00227724,0.00183914,-0.00092597,0.31530241,0.00021691,-0.00027610$ $, 0.00052922,-0.00340116,0.00576816,0.00068855,0.00059272,0.00077074,-0$ $.00083851,0.00036891,-0.00078864,0.00044859,0.00039157,0.00016907,-0.0$ 0033846,-0.00020667,0.00004408,0.00028324,-0.00002316,-0.00005270,-0.0 
$0004586,-0.03101895,-0.01128735,-0.00062171,-0.00055723,-0.00149784,-0$ $.00119513,-0.00004762,-0.00003329,-0.00004702,0.00001927,-0.00004283,-$ $0.00001632,-0.00002037,-0.00001737,-0.00001695,0.00003300,-0.00000500$, $-0.00002898,0.00001267,-0.00001061,0.00000691,0.00002423,-0.00003338$,$0.00000226,0.00019350,-0.00004784,-0.00006991,0.00002077,-0.00000950,0$ $.00005906,-0.00004343,0.00001471,0.00000037,-0.00003838,0.00000408,-0$. $00000944,-0.00000425,-0.00000255,0.00000507,0.00001036,0.00000175,-0.0$ $0001698,-0.00000519,-0.00000262,-0.00001006,-0.00000361,-0.00000094,-0$ $.00000252,0.01096028,-0.04900209,0.00115142,-0.00440681,0.00097691,-0$. $00284743,0.00226757,0.05305522,-0.00013577,0.00003249,-0.00020284,0.00$ $056438,-0.00241462,0.00020328,-0.00117108,0.00020056,0.00067032,0.0002$ $5267,0.00011089,-0.00018296,-0.00003700,-0.00006934,0.00004268,0.00001$ $983,0.00006058,-0.00008550,0.00007757,0.00000024,-0.00004513,0.0235606$ $2,0.00661378,0.00296934,0.00015976,-0.00030360,-0.00038194,0.00000584$, $0.00014862,-0.00002177,-0.00000403,0.00002347,0.00000436,-0.00003614,-$ $0.00001671,-0.00000539,-0.00003050,-0.00000535,0.00000219,0.00001029,0$ $.00001910,-0.00000734,0.00001466,0.00003411,0.00004287,-0.00014390,-0$. $00000463,-0.00002052,-0.00000070,-0.00002015,-0.00004556,0.00003862,-0$ $.00000956,0.00001030,0.00005037,-0.00000518,0.00001425,0.00000722,-0.0$ $0000350,-0.00000091,-0.00000756,0.00000691,0.00000683,-0.00002053,0.00$ $002401,-0.00001098,0.00001092,0.00001072,0.00000318,-0.06876023,-0.000$ $17335,-0.06677788,-0.02923540,0.00070493,-0.00588729,0.06487545,-0.004$ $67385,0.06669168,0.00038760,-0.00069737,-0.00002794,-0.00147684,-0.001$ $80357,0.00190615,-0.00042027,0.00008465,0.00074857,0.00034424,-0.00017$ $610,-0.00015789,0.00003377,0.00008686,-0.00000274,-0.00020397,-0.00007$ $996,0.00016894,0.00002230,0.00000699,-0.00002650,0.00686183,-0.0229344$ $0,-0.00356887,0.00013484,0.00042700,-0.00058132,0.00001057,0.00004933$, $-0.00003936,0.00002913,-0.00007028,-0.00002515,0.00000085,-0.00002655$, $0.00001093,-0.00004283,-0.00000257,-0.00000291,-0.00001507,0.00002072$, $-0.00000577,-0.00001466,0.00003094,0.00001909,0.00014274,0.00004768,-0$ $.00002341,-0.00000928,-0.00002307,-0.00002047,0.00000594,0.00000847,-0$ $.00000840,0.00001709,-0.00000068,0.00000273,-0.00000585,-0.00000553,0$. $00000530,0.00000168,-0.00002214,0.00001700,0.00000065,0.00000559,0.000$ $00011,-0.00000215,-0.00000418,0.00000145,-0.10455653,0.11262293,0.0365$ $8792,0.00256735,-0.00473082,-0.00238262,-0.01057206,0.02466508,0.00993$ $465,0.10675895,0.00058492,-0.00097134,-0.00041098,-0.00320640,0.000732$ $20,0.00291919,-0.00037307,0.00012354,0.00030099,0.00016150,-0.00006141$ $,-0.00010087,-0.00007043,0.00022820,0.00002679,-0.00033942,-0.00016213$ $, 0.00027716,0.00001088,0.00003792,0.00002373,0.00607522,-0.03287796,-0$ $.00200459,0.00004811,0.00018888,-0.00012833,0.00006474,-0.00017319,-0$. $00002897,0.00005854,-0.00011942,-0.00002068,0.00000388,-0.00003756,0.0$ $0000438,0.00003467,0.00000866,0.00000175,-0.00004408,0.00001406,-0.000$ $00069,-0.00004618,0.00001327,-0.00006215,0.00024561,0.00000355,0.00005$ $279,-0.00003938,0.00003988,-0.00003755,-0.00000791,-0.00000468,0.00001$ $794,-0.00007038,0.00001160,-0.00001450,-0.00002100,-0.00001109,0.00000$ $616,0.00001161,-0.00002207,0.00001362,0.00001370,-0.00000331,0.0000340$ $3,-0.00000934,-0.00000482,-0.00000590,0.10194599,-0.23983476,-0.069515$ $15,0.00208777,-0.00128791,-0.00162740,0.00035952,0.00230592,-0.0002905$ $3,-0.10747903,0.27186399,-0.00016452,0.00072289,0.00034742,0.00205519$, $0.00162060,-0.00147266,0.00082493,-0.00004688,-0.00022753,-0.00021775$, $0.00015938,0.00027391,0.00001210,-0.00008467,0.00003221,0.00018500,0.0$ 
$0006017,-0.00014692,-0.00002576,-0.00006241,0.00001269,-0.00660130,0.0$ $2378813,0.00348820,-0.00055074,-0.00044465,0.00004176,-0.00003712,-0.0$ $0002293,0.00002043,-0.00003260,0.00006936,0.00000868,0.00000087,0.0000$ $3367,-0.00000446,0.00002041,0.00000087,0 ., 0.00000006,-0.00000339,-0.00$ $000325,0.00001805,-0.00001217,0.00003673,-0.00009900,0.00003020,-0.000$ $02947,0.00001877,-0.00002110,-0.00000049,-0.00000505,0.00000380,-0.000$ $00032,-0.00001912,0.00000497,-0.00000849,0.00000642,0.00000046,0.00000$ $578,0.00000444,0.00001584,-0.00002410,0.00000993,0.00001151,0.00000464$ $, 0.00000094,-0.00000586,-0.00000180,0.03766041,-0.07891871,-0.07442551$ $, 0.01410533,-0.02507779,-0.00644811,-0.00464039,0.00760897,0.00301471$, $-0.04252950,0.07056974,0.07550594 \backslash 1-0.00000098,-0.00000972,-0.00000782$ $,-0.00000436,-0.00001334,0.00000328,0.00000906,-0.00001552,-0.00000634$ ,-0.00001091,0.00003363,-0.00000147,0.00000001,-0.00001605,0.00000385, $0.00000371,0.00002011,-0.00001354,0.00000191,-0.00000313,0.00000666,-0$ $.00000632,0.00001415,0.00000124,0.00000307,-0.00000216,-0.00000001,-0$. 00000296,-0.00000704,-0.00000664,-0.00000641,-0.00000474,-0.00000142,0 $.00000254,-0.00000356,-0.00000408,-0.00000350,0.00000222,0.00000810,-0$ $.00000402,0.00000039,-0.00000433,0.00000800,-0.00000521,-0.00000250,-0$ $.00000204,0.00000178,0.00001247,-0.00000597,0.00000480,-0.00000612,0.0$ $0000622,-0.00000130,-0.00000109,0.00000146,0.00000505,-0.00000001,0.00$ $000214,-0.00000002,0.00000300,0.00000031,-0.00000141,0.00000190,0 ., 0.0$ $0000193,-0.00000222,-0.00000156,0.00000555,-0.00000103,0.00000453,-0.0$ $0000680,0.00000806,0.00000133,0.00000148,0.00000233,0.00000242,-0.0000$ 0048,0.00000252,0.00000235,-0.00000061,0.00000523II!@

meta-Addition Product to 4-fluoroanisole

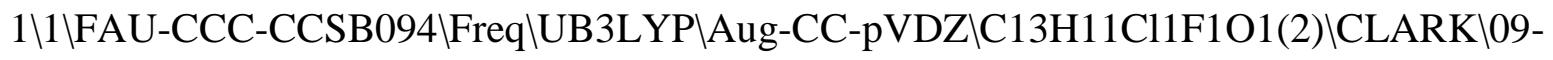
Dec-2014\0\\N Geom=AllCheck Guess=TCheck SCRF=Check GenChk UB3LYP/Aug -CC-pVDZ Freq \\Addition product p-Cl-Ph-radical + 4-fluoro-anisol (met a) $\backslash \backslash 0,2 \backslash \mathrm{C},-0.8292450342,1.0855548213,0.6498529078 \backslash \mathrm{C},-1.7633461526,-0.0$ $550755569,0.9662906095 \backslash \mathrm{C},-2.9106700632,-0.3121964289,0.2492849401 \backslash \mathrm{C},-3$ $.3009689625,0.5156522498,-0.8369998991 \backslash \mathrm{C},-2.4956896369,1.6392713932,-1$ $.1799256033 \backslash \mathrm{C},-1.3596116548,1.9037494205,-0.485848109 \backslash \mathrm{H},-0.7630895603$, $1.7532542589,1.5331255004 \backslash \mathrm{H},-1.4960624937,-0.7138523117,1.7910289065 \backslash \mathrm{O}$ ,-3.6367925126,-1.4065608607,0.6526143874\H,-2.7893127075,2.2993108148 ,-1.9955775395\F,-0.6136613846,2.9983258473,-0.7947672988\H,-4.2019726 $197,0.319078051,-1.4095042794 \backslash \mathrm{C}, 3.2146954977,-0.2905244795,-0.08759630$ 06\C,2.9816079066,0.6219615415,0.9395271495\C,1.6736160454,1.062921117 2,1.167891844\C,0.6083309441,0.601486163,0.3882931079\C,0.8721560489,-$0.317772233,-0.6365397415 \backslash \mathrm{C}, 2.1702389243,-0.7670536809,-0.8833712447 \backslash \mathrm{C}$ $1,4.8576536027,-0.8571682615,-0.3875964878 \backslash \mathrm{H}, 3.8070475491,0.983374646$, $1.5500775591 \backslash \mathrm{H}, 1.4890328268,1.7805053851,1.968502438 \backslash \mathrm{H}, 0.0529973887,-0$ $.692097091,-1.2507854846 \backslash \mathrm{H}, 2.3722377529,-1.4796958482,-1.6811524469 \backslash \mathrm{C}$, $-4.8525353086,-1.7045464922,-0.02349153 \backslash \mathrm{H},-5.2565510431,-2.5926678704$, $0.4724033904 \mathrm{UH},-4.6790177828,-1.9312929242,-1.0870359637 \backslash \mathrm{H},-5.57584151$ 71,-0.8789240143,0.0642042034॥Version=EM64L-G09RevC.01 \State=2-A $\backslash H F=-$ $1137.301452 \backslash \mathrm{S} 2=0.78654 \backslash \mathrm{S} 2-1=0 . \mathrm{S} 2 \mathrm{~A}=0.750728 \backslash \mathrm{RMSD}=9.250 \mathrm{e}-09 \backslash \mathrm{RMSF}=1.388 \mathrm{e}$ $-05 \mathrm{VZeroPoint}=0.2037139 \backslash \mathrm{Thermal}=0.2180558 \mathrm{ZPE}=127.8324307 \mathrm{Dipole}=-1.30$ $72529,-0.1634936,-0.0419092 \backslash$ DipoleDeriv $=0.1628568,0.0183665,-0.0853384$ $,-0.1033215,0.0862854,0.1097549,-0.113022,0.1943696,0.0924844,-0.29912$ 
$56,-0.1578552,0.1755381,0.1139158,-0.1934346,-0.0835297,0.0404511,-0.1$ $737113,-0.0083283,0.664569,0.702832,-0.2397978,0.9755509,0.8192859,-0$. $0780146,-0.377028,-0.1067259,-0.0665385,-0.0802442,0.112857,0.008981,-$ $0.206957,-0.0617424,-0.1802169,0.2846783,-0.0120944,0.007551,-0.014285$ -0.1718379,0.1926807,0.2212641,-0.088144,0.0247214,-0.1129271,-0.1828 $501,0.0159221,0.4299574,0.5591537,-0.0481113,0.5193363,0.8755104,-0.22$ $17384,0.112127,-0.2668547,0.1177306,-0.0002407,-0.0412174,-0.0646022,-$ $0.0420339,-0.0109032,-0.0931839,-0.0453688,-0.1056337,-0.0353112,0.087$ $3322,-0.0157722,-0.0472802,-0.0229529,0.0400115,0.0746836,-0.0443734,0$ $.0651588,0.0406865,-1.3021649,-0.6236227,-0.0631873,-1.0203455,-0.9305$ $942,0.0379787,0.0533454,0.1206827,-0.3229043,0.0865231,-0.0035392,-0.0$ $393561,-0.0272514,0.0416994,0.0864907,-0.0652009,0.0967079,0.0427525,-$ $0.5500494,-0.4967509,0.0961804,-0.5192719,-0.9432063,0.2346645,0.07222$ $41,0.2655985,-0.2148892,-0.0013426,-0.0443965,-0.0634321,-0.0369651,0$. $092015,0.0080516,-0.0826215,0.0013712,0.0724326,1.1294403,-0.352381,-0$ $.1611072,-0.4225206,0.1164361,0.0964088,-0.236593,0.1092141,0.0363678$, $-0.2383822,0.1141785,0.0987536,0.143953,-0.1088195,0.0097621,0.1251974$ $, 0.0028365,-0.0598446,-0.0339511,-0.0318184,-0.019731,0.0595527,-0.007$ $6658,0.1093589,0.0836044,0.086464,0.0188234,0.266441,-0.061523,0.02221$ $72,-0.1431482,-0.0343988,-0.0324994,-0.0319002,-0.0232254,-0.0712544,-$ $0.1070208,0.0301758,0.0261086,0.0104912,-0.0515149,0.0367776,0.0126378$ $, 0.0384874,-0.0312476,-0.2517258,0.0238729,-0.0076505,0.045754,-0.0367$ $902,0.0683638,0.0169082,0.063618,-0.0055759,-0.8489571,0.2243826,0.098$ $4845,0.2652518,-0.1559901,-0.0449387,0.1350126,-0.0508913,-0.1014529,--$ $0.0133163,-0.0226099,-0.0578084,-0.0144579,0.0996898,-0.0374165,-0.049$ $7788,-0.0394921,0.0706112,0.065777,0.0474226,0.0456044,0.0262196,0.030$ $966,-0.0973284,0.0252329,-0.0898136,0.014215,0.0084436,-0.0307008,-0.0$ $617264,-0.0401366,0.0784176,-0.0330685,-0.0797055,-0.0298637,0.0471964$ $, 0.0563988,0.0261642,0.0156664,0.03038,0.0581208,-0.0742629,0.025242,-$ $0.0748779,0.0430823,0.9097905,0.2691194,0.1077916,0.1227303,0.3555954$, $0.0524422,0.3337407,0.1878836,0.3606783,0.0034131,-0.0608448,0.002666$, $-0.117161,-0.0565617,0.0503217,0.0420136,0.0818677,0.0416225,-0.030907$ $2,-0.0191787,0.0711377,0.0163648,0.0461596,-0.013237,-0.07528,-0.09759$ $17,-0.1398371,-0.0992302,0.0055235,-0.0026815,0.1657591,-0.0604271,-0$. $0103455,-0.0486165,-0.0606344,0.0350274 \backslash$ Polar $=251.2883457,3.1621842,15$ $0.7024963,14.1394929,5.4623011,142.8102748 \backslash \mathrm{PG}=\mathrm{C} 01[\mathrm{X}(\mathrm{C} 13 \mathrm{H} 11 \mathrm{Cl1F} 1 \mathrm{O} 1)] \mathrm{N}$ Imag=0\10.42603978,0.02874933,0.48561385,0.03267186,0.01522944,0.50518 $843,-0.11239627,-0.06524239,0.01057262,0.52056134,-0.05848077,-0.14897$ $505,0.03692796,0.12786738,0.42657685,0.00226732,0.03023565,-0.08254568$ $, 0.21649252,-0.18886550,0.46076027,-0.01768025,-0.00995902,-0.00769137$ $,-0.25152050,-0.03987978,-0.12675980,0.50417831,-0.01887239,-0.0035168$ $3,-0.01118119,-0.07110729,-0.09491752,-0.01943989,0.16398099,0.4529723$ 9,0.00203096,-0.00462091,0.00421770,-0.10051311,0.00612314,-0.14198167 $, 0.15115824,-0.15316201,0.40618302,0.00054712,0.00181917,0.00297414,-0$ $.00774393,0.01579799,-0.02847089,-0.11472582,0.02423241,-0.07214400,0$. $53372916,0.00184495,-0.00427068,0.00067577,0.04325357,0.01777860,0.028$ $46578,-0.04109446,-0.13817307,0.04359125,0.16813948,0.40841235,0.00315$ $915,0.00116445,-0.00309583,-0.05361815,0.00523657,-0.04023805,-0.01310$ $419,0.09653498,-0.15495591,0.21486882,-0.14733343,0.40657182,-0.005461$ $07,0.00042910,-0.01272736,-0.02663196,-0.00531311,-0.01781384,0.051332$ $20,-0.00979564,0.05054868,-0.13668600,-0.05128911,-0.02869610,0.515686$ 
$87,0.00994816,0.01053307,0.00469241,-0.01735272,-0.00266637,-0.0118620$ $3,0.01944443,-0.03073206,0.04491616,-0.10829998,-0.18483601,0.03640055$ $, 0.11983063,0.47194341,-0.01971694,-0.00073795,-0.00650295,-0.00672549$ ,-0.00169778,-0.00377615,0.02392813,0.02080845,0.00145327,0.02072667,0 $.08067245,-0.10020879,0.22759954,-0.21924955,0.48771668,-0.09184790,0$. $02516989,-0.04629827,0.02270204,-0.00564184,0.02539932,-0.02484307,0.0$ $0726409,-0.02713489,-0.03804600,-0.06166284,0.01995075,-0.28827411,-0$. $01889148,-0.17815756,0.57765829,0.01929860,-0.10342876,0.05433442,0.00$ $428359,-0.01883585,0.02008326,-0.00395193,-0.00235040,-0.00127509,-0.0$ $2913116,-0.01101728,-0.00980924,-0.05917985,-0.09101737,-0.01357286,0$. $15842397,0.41705844,-0.04325019,0.05416968,-0.15261799,0.01596889,0.00$ $983069,0.00076777,-0.01677180,0.00692812,-0.02045673,-0.00782389,-0.03$ $515796,0.02894104,-0.14389505,0.01809350,-0.18363029,0.22820786,-0.116$ $51922,0.41804702,-0.03458134,-0.01043960,-0.01410006,-0.00665825,-0.01$ $473246,-0.01395428,0.00054051,-0.00173706,0.00083905,-0.00123069,-0.00$ $103189,-0.00011622,0.00180059,0.00208329,-0.00006547,-0.00425107,-0.00$ $580511,-0.01403779,0.05596150,-0.00415113,-0.12460197,-0.10862913,-0.0$ $0942056,-0.01517021,-0.01579026,0.00212322,-0.00263769,0.00052712,-0.0$ $0104168,-0.00061167,-0.00004514,0.00207439,-0.00123862,0.00157393,-0.0$ $0107167,0.01170560,0.01320918,0.00798377,0.13883105,-0.00454287,-0.108$ $62307,-0.19083624,-0.00273772,0.00107827,0.00931036,0.00174966,-0.0000$ $5431,0.00140759,-0.00115923,-0.00106939,-0.00055403,0.00312045,0.00383$ $549,-0.00171066,-0.00930023,-0.01116764,-0.02665839,0.01309971,0.12145$ $309,0.21274872,0.00489398,-0.01074118,0.01557856,-0.06553660,0.0342038$ $3,-0.06421969,-0.00009567,0.01600584,-0.02063480,-0.00278239,-0.003251$ $80,-0.00325184,-0.00036432,-0.00033516,0.00045757,-0.00264959,-0.00220$ $709,-0.00134371,0.00043701,-0.00011398,-0.00046015,0.06358741,0.006935$ $97,-0.01065902,0.01592795,0.03609955,-0.14810978,0.14339343,-0.0044961$ $7,0.00696273,-0.00350482,-0.00255771,0.00160900,0.00096979,0.00031525$, $-0.00038310,-0.00008023,-0.00427443,-0.00062819,-0.00129034,0.00065432$ $, 0.00083739,-0.00023567,-0.03497206,0.15201055,-0.00131885,0.00056141$, $-0.00147026,-0.06569348,0.14037628,-0.20936202,-0.00263296,0.01330721$, $-0.00718938,-0.00439812,0.00011531,0.00125690,0.00024016,-0.00065213,0$ $.00009775,0.00020897,0.00040549,0.00182917,0.00048002,0.00078080,0.000$ $28053,0.07079675,-0.15385817,0.21599303,0.00083035,0.00056938,-0.00410$ $359,-0.03756725,-0.04794134,0.00592552,-0.12322791,-0.09649024,0.01968$ $214,0.00594585,0.00146600,-0.00129181,-0.00278910,-0.00284978,-0.00369$ $694,0.00133214,0.00211782,-0.00013126,-0.00101538,-0.00172470,0.000627$ $82,0.00055514,0.00140534,0.00269563,0.39603701,0.00141048,0.00459084,0$ $.00158298,-0.02820988,-0.01705320,0.00019445,-0.06895615,-0.18657151,0$ $.07658487,-0.01998067,-0.02709824,0.01428499,0.00130137,0.00122688,0.0$ $0409130,0.00008791,0.00156256,-0.00112736,-0.00011187,-0.00081610,0.00$ $031632,0.00183690,-0.00098455,-0.00095462,0.17974773,0.30538020,-0.003$ $99965,0.00172485,0.00038291,-0.01255676,-0.01620235,0.01362456,-0.0041$ $5052,0.05541356,-0.09267660,0.01833925,0.03237295,-0.00798091,-0.00771$ $218,0.00056975,-0.00224926,0.00139489,-0.00010088,0.00088022,-0.000001$ $73,0.00011853,-0.00048402,0.00199614,-0.00170030,-0.00094002,0.1014788$ $4,-0.07625083,0.20892574,-0.00109469,-0.00400862,-0.00354468,-0.000604$ $69,-0.00030666,0.00045486,-0.00060917,-0.00390380,-0.00066872,0.007759$ $83,-0.01018364,0.01380149,-0.07201518,0.04063183,-0.06625284,-0.002503$ $86,0.01666373,-0.02144400,-0.00050137,0.00083994,-0.00093062,-0.000000$ 
$92,0.00010845,0.00006544,0.00012825,-0.00091379,0.00087258,0.06866205$, $-0.00328840,0.00231113,0.00203313,-0.00029682,-0.00024141,-0.00066974$, $-0.00395253,-0.00062263,0.00106103,0.00843598,-0.00906307,0.01654327,0$ $.04095835,-0.15206181,0.13605989,-0.00344283,0.00634137,-0.00291707,0$. $00030029,-0.00037814,0.00043291,0.00011756,-0.00003845,-0.00003110,-0$. $00000527,-0.00104140,0.00113543,-0.04224456,0.15468439,-0.00384218,0.0$ 0089995,0.00363545,0.00011991,-0.00065021,-0.00010603,0.00002291,0.001 $44509,0.00331111,-0.00196002,0.00253308,-0.00217405,-0.06841975,0.1346$ $8949,-0.20740485,-0.00278089,0.01354878,-0.00802286,0.00010913,-0.0003$ $8687,0.00033004,0.00005296,-0.00002432,-0.00010782,-0.00012415,0.00025$ $643,-0.00015430,0.07340420,-0.14915366,0.21354192,0.00392451,-0.000727$ $86,0.00229465,-0.00206325,-0.00122278,-0.00222165,0.00386169,0.0015116$ $3,0.00199676,-0.00112008,0.00235933,-0.00917929,-0.02719523,-0.0457597$ $1,0.01173530,-0.12716300,-0.10861855,0.01126747,0.00046193,0.00134316$, $0.00018483,0.00029103,0.00025840,-0.00007707,-0.00183192,-0.00199777,0$ $.00061336,0.00040948,0.00248174,0.00308647,0.14918881,-0.01994418,-0.0$ $2945189,0.00869689,0.00257019,-0.00236527,0.00544065,0.00351366,0.0030$ $7896,-0.00038380,-0.00461821,0.00277142,0.00006459,-0.01777630,-0.0181$ $6583,0.00508133,-0.11081134,-0.19973120,0.05267787,0.00029680,0.001740$ $76,0.00019303,-0.00148674,-0.00128028,0.00006465,-0.00293156,-0.003374$ $51,0.00036621,0.00209846,-0.00078803,-0.00242768,0.14999636,0.24657015$ $, 0.01961751,0.02658585,-0.00376361,-0.00518440,0.00290293,-0.00420480$, $0.00004692,-0.00148132,0.00108732,-0.00368963,0.00491374,0.00000868,-0$ $.01015244,-0.01638720,0.01151076,0.01203315,0.05549598,-0.06549592,0.0$ $0125848,-0.00129145,0.00253496,0.00138779,0.00148649,-0.00013116,0.001$ $44978,0.00102290,-0.00018454,0.00336385,-0.00242350,-0.00118451,-0.020$ 09752,-0.07020673,0.05912645,0.00019885,0.00047964,0.00057911,0.001572 $52,0.00030827,-0.00165430,-0.00325048,-0.00319803,-0.00240216,-0.24836$ $692,-0.05251509,-0.14120518,-0.00972945,-0.00090935,-0.00929539,0.0011$ $6018,-0.00256726,0.00095460,0.00013025,-0.00001755,0.00004396,-0.00019$ $936,-0.00015823,-0.00004953,-0.00105634,0.00037196,0.00050703,-0.00004$ $212,0.00101860,0.00104269,-0.00070525,-0.00068407,-0.00063236,0.261541$ $16,0.00015299,-0.00047471,-0.00031794,-0.00003586,-0.00148803,0.004298$ $86,0.01440234,0.00881449,0.00669983,-0.05280526,-0.05461012,-0.0137253$ $0,-0.01942939,-0.00079610,-0.01313724,-0.00208084,-0.00215627,0.002472$ $65,-0.00012813,0.00004557,-0.00006157,0.00084304,0.00021813,0.00052307$ ,-0.00073202,-0.00041378,-0.00200289,0.00127128,0.00014096,-0.00118295 ,-0.00179293,-0.00055911,-0.00024522,0.06123685,0.05469012,0.00070596, $-0.00020981,-0.00016303,-0.00116518,0.00463588,-0.00215936,-0.01834003$ ,-0.00769293,-0.00515152,-0.14086729,-0.01357761,-0.12655551,0.0075364 $0,0.00205995,0.00682556,0.00050626,0.00224386,0.00112055,-0.00009605,0$ $.00003061,-0.00003487,-0.00100197,-0.00031439,-0.00047199,0.00147833,-$ $0.00120514,0.00026780,0.00079276,-0.00135481,-0.00058995,0.00043249,0$. $00070762,0.00057352,0.14967909,0.01464139,0.12654813,-0.00114027,-0.00$ $022130,-0.00025660,-0.00012293,-0.00002341,0.00000416,-0.00034963,-0.0$ $0037726,0.00040713,0.00016519,0.00023739,-0.00011611,-0.00009653,-0.00$ $013831,0.00018204,-0.00031198,0.00013628,-0.00013799,-0.00004263,-0.00$ $000616,-0.00004119,-0.00007553,-0.00003423,0.00005454,0.00065319,0.000$ $51025,-0.00003530,0.00005287,-0.00000580,0.00003706,0.00032836,-0.0000$ $5449,0.00015199,0.00002495,0.00000655,0.00002643,0.50807553,-0.0005908$ $7,-0.00067066,-0.00030430,-0.00045770,0.00032775,0.00014518,0.00021078$ 
$, 0.00058688,-0.00014048,0.00005216,-0.00004353,0.00021909,0.00005456,0$ $.00013836,0.00004426,-0.00024282,-0.00000170,0.00030831,-0.00015052,0$. $00019260,0.00011666,0.00000114,-0.00002479,-0.00004715,-0.00041368,-0$. $00062717,0.00007797,0.00006539,-0.00004086,-0.00006978,0.00005475,0.00$ $004509,-0.00001203,-0.00002186,0.00000139,-0.00001640,-0.01144089,0.33$ $859424,-0.00079061,-0.00026012,-0.00099976,-0.00062012,0.00036398,0.00$ $013834,0.00023473,0.00072984,-0.00010844,0.00005556,-0.00013214,0.0002$ $3252,0.00011373,0.00023889,0.00005065,-0.00068498,-0.00009992,0.000472$ $54,-0.00018435,0.00007468,0.00030338,0.00007229,-0.00003972,-0.0000969$ $8,-0.00035242,-0.00063296,0.00009278,0.00008323,-0.00007093,-0.0000772$ $4,0.00035311,0.00021598,0.00004261,-0.00002425,0.00000275,-0.00000448$, $0.07138300,0.24902873,0.43647061,-0.00020679,-0.00101581,-0.00309750,-$ $0.00066033,0.00060026,0.00026491,0.00021899,0.00056341,-0.00018359,0.0$ $0007593,-0.00011792,0.00026223,0.00018144,0.00035924,-0.00002891,-0.00$ $153758,-0.00052687,0.00075678,-0.00010002,0.00020273,0.00035881,-0.000$ $03426,-0.00006101,-0.00010356,-0.00037499,-0.00061145,0.00007831,0.000$ $06339,-0.00009711,-0.00012433,0.00062062,0.00045340,-0.00004631,-0.000$ $01190,0.00000220,0.00001094,-0.11039138,-0.00131067,-0.01171995,0.6998$ $5006,-0.00109884,0.00398009,-0.00422373,0.00131587,-0.00050579,0.00025$ $772,-0.00003815,-0.00035604,-0.00009887,-0.00008511,0.00000923,-0.0001$ $7527,0.00003737,0.00022497,-0.00018875,-0.00110558,-0.00036014,0.00011$ $016,-0.00003585,-0.00008201,-0.00011628,-0.00001671,-0.00003967,0.0000$ $9625,0.00034913,0.00054835,-0.00000994,-0.00007174,-0.00003930,-0.0000$ $5541,0.00002427,-0.00001934,0.00001022,0.00001233,0.00000210,0.0000253$ $4,0.03982981,-0.17956757,-0.12673881,-0.03264415,0.36629484,-0.0023632$ $4,-0.00432793,0.00126895,-0.00077861,0.00031425,-0.00022588,-0.0001840$ $8,-0.00046099,0.00017855,-0.00007195,-0.00003204,-0.00013091,0.0000282$ $9,-0.00036740,0.00014866,0.00095905,-0.00008173,-0.00019176,0.00003347$ $, 0.00007069,0.00005174,-0.00006142,-0.00001792,0.00001109,0.00009123,0$ $.00026785,-0.00009135,-0.00003477,0.00010706,0.00009983,-0.00007534,0$. $00014566,-0.00008287,0.00001593,0.00001400,0.00001552,0.03683672,-0.13$ $599316,-0.21740461,0.08864449,0.27132493,0.47049163,-0.02742051,-0.005$ $38025,-0.01400761,-0.00098251,0.00135415,-0.00153171,-0.00004762,-0.00$ $096998,0.00061782,-0.00021597,-0.00013292,-0.00011355,0.00032403,0.000$ $70455,-0.00075090,-0.00251462,-0.00266726,0.00107925,0.00074316,-0.002$ $35528,-0.00277315,-0.00009667,-0.00020612,0.00040118,-0.00028957,0.000$ $17340,-0.00026523,-0.00028716,-0.00000151,-0.00008271,0.00065547,0.000$ $72751,-0.00035371,-0.00002431,0.00002935,0.00003844,0.01131191,0.05735$ $306,0.06876651,-0.30823332,0.04690073,0.00023991,0.65644548,0.00826203$ $, 0.00619684,0.00426683,0.00119911,-0.00066106,0.00034797,-0.00024045,-$ $0.00001908,-0.00016804,0.00022410,0.00018016,0.00005025,0.00004255,-0$. $00031694,0.00034628,0.00130536,-0.00043143,-0.00060986,0.00157262,0.00$ $038987,0.00108510,0.00002989,0.00015223,-0.00004755,0.00042723,0.00035$ $852,0.00005559,-0.00003440,0.00013351,0.00025190,0.00034086,-0.0002653$ $8,0.00012217,0.00004190,-0.00003311,-0.00003902,0.02131851,-0.00755473$ $,-0.01253569,0.08995157,-0.10962881,-0.03576287,-0.06695274,0.39212510$ $, 0.00289907,0.00044234,0.00601407,0.00165119,-0.00032498,-0.00079240,0$ $.00019660,-0.00044876,0.00058013,0.00021734,0.00048719,-0.00023088,-0$. $00045100,-0.00049687,0.00017475,0.00193730,0.00057825,-0.00023449,0.00$ $196220,0.00038533,0.00075686,-0.00018611,-0.00010664,0.00007948,0.0003$ $9265,0.00063308,0.00000520,0.00003661,0.00003015,0.00012973,-0.0003850$ 
$9,-0.00073495,0.00000584,0.00002100,-0.00002636,-0.00002264,0.02577018$ ,-0.00479329,0.00704215,0.05074855,-0.04484565,-0.10363499,0.03741249, $0.28755872,0.48111675,-0.13974548,0.03192144,0.02116345,-0.03164402,-0$ $.00746950,0.00521710,0.00009393,-0.00014547,0.00381996,-0.00119450,-0$. $00056236,-0.00073019,0.00247035,0.00197810,0.00250409,-0.02076064,0.01$ $543969,-0.01461071,-0.00622267,0.00252239,0.00092862,0.00220827,0.0006$ $2524,-0.00116048,0.00092773,0.00042035,-0.00041528,0.00047400,-0.00021$ $505,-0.00020318,-0.00007003,-0.00311155,0.00186994,0.00006913,0.000007$ $89,0.00007608,-0.00682200,-0.01466276,-0.01738737,-0.05061963,0.008439$ $42,-0.00296338,-0.23607905,-0.06758083,-0.11768573,0.59664097,0.023231$ $19,-0.08007279,-0.01217042,-0.02091738,0.01008645,0.00091100,-0.000553$ $81,-0.00117388,0.00128183,-0.00012596,-0.00038496,-0.00001241,-0.00033$ $531,-0.00127455,0.00054331,0.01625227,-0.00872230,0.00123445,0.0197159$ $5,-0.00901151,-0.00692701,-0.00023868,0.00029249,0.00005930,-0.0016056$ $8,-0.00175360,-0.00064117,-0.00021687,0.00051269,0.00039632,0.00196262$ $, 0.00215555,-0.00050508,-0.00006819,0.00009251,0.00003862,-0.01552426$, $-0.02849399,-0.02882743,-0.02202143,0.03112401,0.02370754,-0.02422937$, $-0.11337520,-0.06244098,-0.02818473,0.37842152,0.00873602,-0.00887482$, $-0.07600073,0.00473902,-0.00041980,-0.00004203,0.00107534,-0.00092651$, $0.00044761,-0.00047832,0.00018402,0.00017620,0.00119765,0.00338897,-0$. $00290299,-0.02571298,0.00552649,0.00565330,0.02434164,-0.01239530,-0.0$ $0821746,0.00002502,-0.00031909,0.00055795,-0.00004148,0.00024501,-0.00$ $012201,-0.00008152,-0.00081781,-0.00122022,0.00005307,-0.00050441,0.00$ $023890,-0.00008918,0.00006414,0.00013308,-0.01838674,-0.02882421,-0.04$ $505116,-0.03901744,0.03014492,0.03435881,-0.06624399,-0.07263180,-0.16$ $553703,0.06342938,0.23692995,0.46295618,-0.00963607,0.02115071,0.02221$ $190,0.00087268,0.00219258,0.00079440,-0.00003454,0.00125519,-0.0002300$ $9,-0.00001928,-0.00053751,0.00031377,0.00015698,0.00040423,-0.00031040$ $, 0.00037685,-0.00062282,0.00311849,-0.00530614,0.00270475,0.00183344,-$ $0.00011303,-0.00005112,0.00003353,-0.00062009,-0.00106961,0.00011141,0$ $.00007244,-0.00007616,-0.00009650,0.00050131,0.00056502,-0.00025574,-0$ $.00005031,0.00000950,0.00000560,-0.05522551,-0.02457126,-0.04275876,-0$ $.02622037,0.02275567,0.02178370,0.04619083,-0.01355025,-0.00789215,-0$. $12569857,0.00618309,-0.00613355,0.70411494,0.00793020,-0.00504111,-0.0$ $0672119,-0.00049884,-0.00052507,0.00029377,-0.00087000,0.00016926,-0.0$ $0035189,-0.00011275,-0.00059943,0.00003824,0.00015588,0.00006866,-0.00$ $029767,-0.00013732,0.00087650,-0.00153289,0.00127215,0.00045893,-0.000$ $44559,0.00017299,0.00034227,-0.00017701,0.00014534,0.00007430,-0.00003$ $505,-0.00005606,0.00007004,-0.00005238,-0.00034664,-0.00025500,0.00002$ $862,-0.00002466,0.00003943,0.00000500,0.01139162,0.03210742,0.03162522$ $, 0.02286210,-0.02351513,-0.01901698,-0.04252801,-0.00799174,-0.0275074$ $3,0.04555218,-0.17503408,-0.11719673,-0.04002741,0.36679081,0.00684460$ $,-0.00319448,-0.00095471,0.00006832,-0.00155611,0.00036888,-0.00018870$ ,-0.00016883,-0.00046018,-0.00023003,-0.00016260,-0.00040633,-0.000432 $36,0.00021801,-0.00008872,0.00076989,0.00123612,-0.00018332,0.00008137$ ,-0.00010155,0.00077392,0.00006169,0.00005015,0.00000443,-0.00005959,-$0.00009524,-0.00000941,0.00002979,-0.00009151,-0.00001934,-0.00102109$, $-0.00038059,0.00010167,-0.00003737,0.00001259,0.00005077,-0.00017424,0$ $.02402377,0.03547104,0.02189158,-0.01919581,-0.02108391,-0.04226920,-0$ $.02126643,-0.02666910,0.04091524,-0.12646551,-0.20592574,0.07604741,0$. $26342619,0.46176561,0.00363472,-0.00011468,-0.00043228,0.00051855,-0.0$ 
$0019845,-0.00025176,-0.00005454,-0.00057580,-0.00017098,-0.00011092,-0$ $.00000216,-0.00007605,-0.00000549,-0.00010654,-0.00030163,0.00018573,-$ $0.00026616,-0.00032342,0.00046912,-0.00038773,-0.00050611,-0.00002702$, $0.00005357,0.00013612,0.00002594,0.00033522,-0.00008877,-0.00015623,0$. $00005658,0.00001591,-0.00028135,0.00003212,-0.00020726,0.00000534,-0.0$ $0000120,-0.00000612,-0.21420611,-0.03243579,-0.07164633,0.04551687,-0$. $04122925,-0.04059125,-0.06259552,-0.00157608,-0.01433235,0.01076275,0$. $05267022,0.06329212,-0.31310247,0.05150043,0.00554332,0.65068561,-0.00$ $016829,0.00095353,-0.00524868,-0.00033029,0.00001869,0.00036224,0.0001$ $9321,0.00027700,0.00012806,0.00002035,0.00001985,0.00007158,0.00004125$ $, 0.00006525,-0.00007021,-0.00086314,0.00033153,-0.00005611,0.00082019$, $-0.00031440,-0.00026180,0.00010200,-0.00006756,-0.00010253,0.00000311$, $-0.00004962,-0.00000541,0.00002583,-0.00006358,-0.00002484,0.00004956$, $0.00004678,0.00009961,0.00000326,-0.00001099,0.00000310,-0.07384064,-0$ $.11522458,-0.07572435,-0.01251265,-0.00647941,-0.02105792,-0.00201329$, $-0.00279230,0.00034787,0.02222626,-0.00559009,-0.01052151,0.09447846$,$0.11155506,-0.03851744,-0.07175276,0.39605562,-0.00020356,-0.00530306$, $-0.00062289,-0.00074387,0.00004098,0.00014545,0.00005221,-0.00012764,0$ $.00003983,0.00012422,0.00011006,0.00002712,-0.00002073,-0.00009427,0.0$ $0026056,0.00002869,0.00031345,-0.00057251,0.00089205,-0.00048811,-0.00$ $029275,0.00005113,0.00008607,0.00001153,-0.00001287,0.00002475,-0.0000$ $3742,0.00001709,0.00001551,0.00000586,-0.00006266,-0.00011209,0.000103$ $58,0.00000172,-0.00000038,-0.00002183,-0.12047431,-0.06649767,-0.17074$ $686,-0.00661683,-0.02747954,-0.02488848,-0.01528123,0.00070897,-0.0062$ $5222,0.02652800,-0.00380039,0.00899838,0.05627942,-0.04798245,-0.10572$ $329,0.02999095,0.29808084,0.48987750,0.00033055,-0.00032698,-0.0002406$ $5,-0.00036943,0.00050058,-0.00007180,0.00040136,0.00071457,-0.00027490$ $,-0.00009524,-0.00029835,0.00024600,0.00019701,0.00038421,-0.00017780$, $-0.00030129,-0.00039783,0.00061506,-0.00002909,0.00001788,0.00002223,0$ $.00006946,-0.00000184,-0.00002869,-0.00082877,-0.00087346,0.00005582,-$ $0.00002232,-0.00004484,-0.00009673,-0.00005042,0.00028079,-0.00021413$, $-0.00003151,-0.00000487,-0.00002607,-0.13650851,0.03506379,0.01633277$, $-0.02408890,0.00948961,0.00479984,0.00315933,0.00174518,0.00164742,-0$. $00117898,0.00089873,0.00118524,0.00093514,-0.00393561,-0.00546806,-0.0$ $4039591,0.01541583,0.00811204,0.19877288,-0.00006845,-0.00027264,0.000$ $54727,0.00012095,-0.00016337,0.00000656,-0.00014485,-0.00030556,0.0000$ $7792,0.00003511,0.00010890,-0.00009641,-0.00006233,-0.00014963,0.00012$ 497,0.00016813,0.00014352,-0.00022456,-0.00003983,0.00004076,0.0000439 $0,-0.00002227,0.00000838,0.00000100,0.00029584,0.00034341,-0.00002463$, $0.00001317,0.00001832,0.00004524,0.00004516,-0.00006223,0.00006757,0.0$ $0001356,-0.00000015,0.00000863,0.03511614,-0.04210407,-0.01374230,0.02$ $672450,-0.00515020,-0.00539291,-0.00240556,0.00120733,-0.00488171,0.00$ $075196,0.00008439,0.00100089,0.00031589,0.00223083,-0.00269173,-0.0019$ $8447,0.00460783,-0.00033243,-0.05879204,0.04166966,0.00008548,0.000492$ $22,-0.00010928,0.00012649,-0.00010843,0.00008420,-0.00005568,-0.000092$ $69,0.00005239,0.00002086,0.00006674,-0.00003930,-0.00004004,-0.0000904$ $0,0.00002330,0.00002706,0.00004104,-0.00012318,-0.00006161,0.00003966$, $0.00002900,-0.00001604,-0.00000390,-0.00000313,0.00015975,0.00016780,-$ $0.00000271,0.00000640,0.00000194,0.00001623,0.00001363,-0.00004100,0.0$ $0004474,0.00000982,-0.00000105,0.00000312,0.01639856,-0.01377212,-0.03$ $657129,0.02521350,-0.00930723,-0.00082025,-0.00329252,-0.00395885,-0.0$ 
$0051345,0.00083534,0.00105765,0.00075854,-0.00047358,-0.00358039,0.000$ $16071,-0.01233438,0.00355045,0.00602675,-0.02777237,0.02201704,0.03170$ $639,-0.00034351,-0.00048344,-0.00075380,-0.00017338,0.00010729,-0.0000$ $4906,0.00004399,0.00008789,0.00000784,-0.00005790,-0.00007651,0.000029$ $11,0.00008202,0.00011025,-0.00003487,-0.00028607,-0.00016843,0.0001610$ $2,0.00004447,-0.00001548,-0.00001288,-0.00000627,-0.00001959,0.0000049$ $4,-0.00012161,-0.00011972,0.00000090,-0.00000622,-0.00001007,-0.000026$ $15,0.00007235,0.00013682,-0.00005253,-0.00000632,0.00000265,-0.0000006$ $0,0.00523490,0.00401225,0.00517905,-0.21919184,-0.07172481,-0.12563378$ ,-0.01563966,-0.01060258,-0.01677845,-0.00323972,0.00320838,0.00146271 - $0.00023709,0.00041035,0.00073630,0.00115750,0.00169820,0.00100220,0$. $00116381,-0.00004785,0.00085013,0.23015717,0.00036997,0.00015877,0.000$ $21764,0.00010070,-0.00008039,-0.00008801,0.00002728,-0.00002035,0.0000$ $6735,0.00001582,0.00004300,-0.00003918,-0.00005719,-0.00003880,0.00001$ $862,0.00012617,0.00020672,-0.00003233,-0.00001117,0.00000805,0.0000023$ $3,-0.00000332,0.00000019,-0.00000612,0.00001972,0.00004252,0.00000083$, $0.00001565,-0.00000619,-0.00000098,-0.00007668,-0.00011018,0.00000972$, $0.00000112,-0.00000304,-0.00000034,-0.01603506,-0.00275954,-0.01092444$ ,-0.06993802,-0.07612669,-0.06389810,0.00832733,0.00687906,0.00561501, $0.00286085,0.00367035,-0.00310429,0.00053109,-0.00095233,0.00039514,0$. $00205216,0.00076951,-0.00513112,-0.00072534,-0.00112286,0.00178372,0.0$ $7397641,0.07185383,0.00036198,0.00000932,0.00019345,-0.00002468,-0.000$ $01340,0.00005842,-0.00001479,0.00003239,-0.00001900,-0.00000980,-0.000$ $02716,0.00001127,0.00003302,0.00000937,0.00003619,0.00000944,-0.000086$ $97,-0.00008174,-0.00000420,0.00000058,-0.00000993,0.00000870,0.0000053$ $5,0.00000002,-0.00000547,-0.00001740,-0.00000061,-0.00000942,0.0000098$ $9,0.00001305,0.00003494,0.00004794,-0.00000874,0.00000146,0.00000030,-$ $0.00000118,-0.01855283,-0.00647983,-0.00836862,-0.12350419,-0.06426759$ $,-0.13864622,0.00557473,0.00142695,0.00662647,0.00107426,-0.00309322,0$ $.00348923,0.00087738,0.00041022,-0.00055266,0.00147800,-0.00522594,-0$. $00031934,0.00005224,0.00193312,-0.00039738,0.13314489,0.07299664,0.139$ $85968,0.00106189,-0.00188809,-0.00106508,-0.00002267,0.00020579,0.0000$ $3435,-0.00006616,-0.00011821,0.00006255,0.00008245,0.00015857,-0.00000$ $510,-0.00015852,-0.00021199,0.00002571,0.00021065,0.00021940,0.0000929$ $0,-0.00025207,0.00048632,0.00049429,0.00000931,-0.00001657,-0.00003073$ $, 0.00012310,0.00013379,-0.00002942,0.00002224,-0.00000616,-0.00000400$, $-0.00008451,-0.00011655,0.00006434,0.00000770,-0.00000089,-0.00000187$, $-0.00366698,0.00181827,-0.00023482,-0.00156205,0.01968174,0.02187097,-$ $0.06420022,0.03258815,0.03222664,0.00679405,-0.01463843,-0.01647826,-0$ $.00317567,-0.00110355,-0.00338630,-0.00110536,-0.00012375,-0.00017044$, $-0.00108090,0.00095795,0.00087887,0.00093344,-0.00069749,0.00031810,0$. $06577925,0.00092728,-0.00222313,0.00230932,-0.00055879,0.00008460,-0.0$ $0012733,-0.00000997,-0.00000382,0.00010067,-0.00001979,-0.00003278,0.0$ $0000221,0.00002699,-0.00017194,0.00015968,0.00065333,0.00055679,0.0000$ $0929,-0.00046080,0.00057962,0.00032127,-0.00004298,-0.00006341,-0.0000$ $3517,-0.00002353,-0.00006707,-0.00000961,0.00004853,0.00001179,0.00003$ $277,-0.00013038,-0.00011081,0.00001966,0.00000151,0.00000261,0.0000089$ $5,0.00199664,0.00449269,-0.00238770,0.00003706,-0.00265437,-0.00723735$ $, 0.03229763,-0.16539843,-0.14454266,0.00365630,-0.00340239,-0.00765828$ $,-0.00172081,0.00356461,-0.00343801,-0.00020781,-0.00029222,0.00085360$ $, 0.00023221,-0.00016083,-0.00031023,-0.00050659,-0.00200910,0.00235170$ 
,-0.03644844,0.16795825,0.00178538,0.00208403,-0.00137976,0.00035879,-$0.00005987,0.00052581,-0.00003263,0.00009256,-0.00012155,-0.00001601,-$ $0.00002546,0.00002319,0.00011885,0.00016277,0.00000947,-0.00091954,-0$. $00042442,0.00005696,-0.00067874,0.00037560,0.00046150,0.00003393,-0.00$ $000710,-0.00004220,0.00011221,0.00009015,0.00001844,0.00002021,-0.0000$ $5261,-0.00005366,0.00035969,0.00030999,0.00006812,0.00000528,0.0000030$ $2,0.00000458,0.00005201,-0.00246837,0.00408377,-0.00108607,-0.00303471$ ,-0.00023266,0.03162950,-0.14410633,-0.19833670,0.00545116,-0.01209155 ,-0.00891878,-0.00410146,-0.00325974,0.00144064,-0.00029811,0.00087157 $,-0.00007729,0.00002671,-0.00016061,-0.00004607,0.00055024,0.00234006$, $-0.00128693,-0.03381302,0.15964489,0.20464974,-0.00038179,-0.00014403$, $0.00072573,-0.00001755,0.00041063,0.00004687,-0.00023793,-0.00028972,0$ $.00023045,-0.00001514,0.00011276,-0.00014089,-0.00016622,-0.00001753,-$ $0.00005894,0.00040033,0.00036050,0.00019179,0.00010952,-0.00015511,-0$. $00011319,-0.00000999,0.00000115,-0.00003038,0.00013524,0.00022917,-0.0$ $0000643,0.00001110,-0.00001812,0.00000197,-0.00023236,-0.00032199,0.00$ $006191,-0.00000183,0.00000673,0.00001086,-0.00264923,0.00277036,0.0012$ $2166,-0.00014525,0.00043740,0.00069659,0.00101582,0.00163631,0.0008684$ $7,0.00773327,0.00214700,0.00358720,-0.21349814,-0.07043935,-0.12077169$ ,-0.01692380,-0.01044894,-0.01694024,-0.00140878,-0.00007397,-0.000395 $48,-0.00003428,-0.00012103,0.00000633,0.00033209,0.00028037,0.00031362$ $, 0.22522617,-0.00086658,-0.00155305,0.00175359,-0.00033669,0.00023154$, $-0.00001152,0.00057058,0.00011336,-0.00005048,0.00013443,0.00028573,-0$ $.00012219,-0.00004886,-0.00005303,0.00038427,0.00011850,-0.00005947,-0$ $.00021689,-0.00014902,0.00000161,0.00011542,-0.00004639,-0.00001038,0$. $00014629,-0.00022904,-0.00026172,0.00004555,0.00005129,-0.00002770,0.0$ 0002789,0.00002160,-0.00012674,0.00002199,-0.00001946,-0.00000729,-0.0 $0000696,0.00259157,0.00398441,-0.00327836,0.00042923,-0.00091641,0.000$ $45026,0.00226167,0.00116001,-0.00571850,-0.01644592,-0.00262836,-0.010$ $70495,-0.07041373,-0.07934041,-0.06518104,0.00905199,0.00678731,0.0053$ $7307,0.00067579,0.00002934,0.00009728,-0.00013562,-0.00038127,0.000360$ $32,-0.00079462,-0.00021738,-0.00058765,0.07425763,0.07503455,-0.000489$ $63,0.00181417,-0.00101559,0.00012319,0.00026382,0.00035206,0.00001464$, $0.00059034,-0.00019198,0.00007465,-0.00036960,0.00032756,0.00035345,0$. $00000492,-0.00002273,-0.00053082,-0.00043875,0.00012217,-0.00008071,0$. $00009591,0.00001327,0.00008587,-0.00006656,-0.00017209,-0.00015777,-0$. $00026037,0.00004134,-0.00004561,0.00008034,0.00001885,0.00030307,0.000$ $35225,-0.00008522,-0.00001626,0.00000448,-0.00001052,0.00086730,-0.003$ $20204,0.00371356,0.00084321,0.00040647,-0.00054605,0.00144466,-0.00591$ $385,-0.00021198,-0.01850914,-0.00659118,-0.00797947,-0.12030852,-0.064$ $96604,-0.13858363,0.00589529,0.00105834,0.00650707,0.00044696,-0.00006$ $472,0.00010801,-0.00000732,0.00037026,-0.00028398,-0.00092391,-0.00035$ $656,-0.00051628,0.13015774,0.07455216,0.13965165,0.00013939,0.00081694$ $, 0.00086524,0.00013604,0.00004264,-0.00004360,0.00001222,0.00010273,-0$ $.00006434,-0.00001938,-0.00009453,0.00001472,0.00003038,0.00005693,-0$. $00005396,0.00011078,-0.00016233,0.00018506,-0.00036719,0.00018526,0.00$ $015207,-0.00001672,-0.00000410,0.00001924,-0.00009599,-0.00012594,0.00$ $000576,-0.00001885,0.00001090,-0.00000501,0.00000408,0.00006185,-0.000$ $04237,-0.00001165,0.00000523,0.00000294,0.00603280,-0.01537166,-0.0175$ $6708,-0.00332746,-0.00107048,-0.00317706,-0.00116523,-0.00006938,-0.00$ $011582,-0.00469168,0.00195877,-0.00028705,-0.00052420,0.01870642,0.021$ 
$12912,-0.06392890,0.03693270,0.03741677,0.00102234,-0.00079900,-0.0001$ $2707,0.00041469,-0.00074639,-0.00086209,0.00005414,-0.00003230,0.00012$ $585,0.00084589,-0.00068286,0.00030309,0.06534763,-0.00015243,-0.000062$ $22,-0.00034025,-0.00014971,-0.00007790,-0.00000808,-0.00000320,0.00002$ $164,0.00005369,0.00002916,0.00003281,-0.00002006,-0.00005750,-0.000071$ $72,0.00000588,0.00012532,0.00008737,-0.00001899,0.00012345,-0.00012901$ $, 0.00003184,-0.00000407,0.00000165,0.00000351,0.00003081,0.00000628,0$. $00000962,0.00001296,-0.00000671,-0.00000027,-0.00004295,-0.00006127,0$. $00004472,0.00000432,0.00000193,0.00000002,0.00466899,-0.00342671,-0.00$ $694947,-0.00139328,0.00328162,-0.00270119,-0.00020043,-0.00030699,0.00$ $082006,0.00225709,0.00467129,-0.00258529,0.00001831,-0.00197786,-0.006$ $82737,0.03510763,-0.16751653,-0.14826077,-0.00012768,-0.00095784,0.001$ $96770,0.00015375,-0.00001959,-0.00038610,-0.00006424,-0.00042942,0.000$ $29979,-0.00058148,-0.00206808,0.00255479,-0.03973642,0.16900869,-0.000$ $32202,-0.00015838,-0.00002480,0.00012935,0.00003574,0.00003881,-0.0000$ $6043,-0.00011176,-0.00000717,0.00000900,0.00002927,-0.00002939,-0.0000$ $2190,-0.00000378,0.00003111,-0.00005603,0.00003596,-0.00007268,0.00008$ $183,0.00002472,-0.00007121,0.00000290,0.00000102,-0.00001741,0.0000711$ $4,0.00011156,-0.00001646,-0.00000284,0.00000649,0.00000758,0.00000899$, $-0.00001401,-0.00000159,0.00000008,-0.00000038,0.00000423,0.00613649,-$ $0.01140271,-0.00873956,-0.00360986,-0.00260771,0.00138950,-0.00024895$, $0.00087917,-0.00003813,0.00017791,-0.00266339,0.00410587,-0.00094556,-$ $0.00270324,0.00052398,0.03520034,-0.14777474,-0.20244167,0.00069051,0$. $00178924,-0.00035996,0.00020758,-0.00058846,-0.00031457,0.00010170,0.0$ $0030076,-0.00031711,0.00036488,0.00251194,-0.00131611,-0.03789855,0.16$ $231567,0.20767453,0.00012342,-0.00079464,-0.00126529,0.00237279,0.0006$ $1908,0.00061502,-0.03053534,-0.01908454,-0.00161150,0.00372902,0.00453$ $294,-0.00015762,-0.00174334,0.00013070,-0.00169741,0.00050775,-0.00108$ $435,0.00091236,-0.00003822,0.00014958,-0.00003703,-0.00023426,0.000365$ $92,0.00022175,-0.17201870,-0.03435804,-0.06018578,0.00029831,0.0004662$ $5,-0.00014884,0.00074091,0.00157306,-0.00058322,-0.00091855,-0.0000272$ $2,-0.00092941,-0.00036224,0.00027374,0.00024814,0.00024343,-0.00020183$ ,-0.00009713,-0.00003344,-0.00014332,-0.00021969,-0.00030962,0.0006630 $9,-0.00014657,0.00033453,-0.00002434,-0.00008453,-0.00008708,0.0000554$ $8,0.00003254,0.00045995,-0.00016169,-0.00008148,0.00005486,-0.00001759$ $, 0.00001395,-0.00005171,0.00001529,-0.00001744,-0.00010857,0.00003784$, $0.00019447,0.00003297,-0.00002090,-0.00002743,0.47648787,-0.00142049,0$ $.00090117,-0.00001488,-0.00000703,-0.00247509,0.00337337,-0.03361838,-$ $0.01658663,-0.01688300,-0.00079004,-0.00181442,-0.00010669,-0.00063812$ $, 0.00045929,-0.00062695,-0.00021193,-0.00017868,0.00014364,0.00001741$, $0.00015661,-0.00002179,0.00015212,-0.00015488,-0.00056343,-0.01259310$, $-0.07836533,0.01037180,0.00009576,0.00020414,-0.00007157,0.00033906,0$. $00033981,-0.00022158,-0.00177083,-0.00150444,0.00009468,-0.00009374,0$. $00006261,0.00004591,0.00005001,-0.00002103,-0.00004734,-0.00006859,-0$. $00000608,-0.00001128,-0.00025566,-0.00022707,0.00004731,0.00006252,-0$. $00001256,-0.00003771,-0.00001169,0.00001906,0.00001089,0.00009418,-0.0$ $0004004,-0.00001459,0.00000638,0.00000075,0.00000024,-0.00002051,-0.00$ $001570,0.00000234,-0.00003774,0.00003385,0.00007327,0.00001273,-0.0000$ $0123,-0.00001074,-0.01060566,0.59226886,-0.00048371,0.00075424,0.00125$ $601,0.00122034,0.00353593,-0.00106435,0.01043533,-0.00586942,0.0068339$ $0,0.00476042,0.00377306,-0.00087913,-0.00084250,0.00007884,-0.00043486$ 
$, 0.00027362,-0.00053269,0.00080667,0.00025106,0.00049121,-0.00020666,0$ $.00044262,-0.00039544,-0.00024343,-0.07880538,-0.00609777,-0.11120695$, $0.00013322,0.00017349,-0.00002413,0.00050380,0.00079388,-0.00050634,0$. $00073114,0.00161978,-0.00069064,-0.00019364,0.00010898,0.00011652,0.00$ $011529,-0.00007806,-0.00006105,0.00003138,-0.00011133,-0.00012835,-0.0$ $0047289,0.00027318,0.00004115,0.00020903,-0.00001813,0.00002750,-0.000$ $01400,0.00003670,-0.00001645,0.00023646,-0.00008881,-0.00005075,0.0000$ $2655,-0.00000925,0.00000518,-0.00004647,-0.00001658,-0.00002771,-0.000$ $05046,0.00007961,0.00012116,0.00004126,-0.00001855,-0.00001229,-0.0562$ $6302,-0.03513801,0.54933409,-0.00012928,0.00010863,-0.00024007,-0.0008$ $3012,-0.00138613,0.00051460,-0.00254548,0.00118990,-0.00308753,-0.0003$ $0224,0.00049258,-0.00102210,0.00010710,-0.00025300,0.00020676,0.000090$ $08,0.00019325,-0.00008484,-0.00006295,-0.00008566,0.00001620,-0.000019$ $99,0.00002388,-0.00002797,-0.02369996,-0.03147152,0.00940188,-0.000029$ $94,-0.00005564,0.00000108,-0.00004988,-0.00011515,0.00008752,0.0003586$ $9,0.00022780,0.00040631,0.00003542,-0.00001891,-0.00001952,-0.00002184$ $, 0.00001684,0.00000307,-0.00002481,0.00002873,0.00002504,0.00005474,-0$ $.00012495,-0.00001342,-0.00002907,0.00000670,0.00000209,-0.00000644,0$. $00000159,-0.00000875,-0.00004656,0.00001762,0.00000977,-0.00000697,0.0$ $0000235,0.00000025,0.00000590,-0.00000582,0.00001030,0.00001265,-0.000$ $01629,-0.00000954,-0.00000661,0.00000106,0.00000625,-0.07521494,-0.069$ $16249,0.04302075,0.09433908,-0.00002089,0.00006238,-0.00007748,-0.0001$ $5205,-0.00026229,0.00022493,0.00023995,0.00196779,-0.00112015,0.000031$ $14,0.00012165,0.00026237,-0.00006068,0.00014800,-0.00003427,0.00004156$ $, 0.00012013,-0.00003627,-0.00003950,-0.00003311,0.00000129,-0.00003351$ $, 0.00000796,-0.00000660,-0.00715980,-0.00621344,0.00053532,-0.00000532$ $,-0.00000600,0.00000989,-0.00011737,-0.00018894,0.00002749,-0.00008381$ $,-0.00018327,-0.00028420,0.00004757,-0.00003012,-0.00001097,-0.0000211$ $8,0.00002313,0.00000023,-0.00001062,0.00001064,0.00002814,0.00004797,-$ $0.00004985,-0.00002369,-0.00003137,-0.00001038,0.00002257,-0.00000239$, $0.00000071,-0.00001702,-0.00005259,0.00001906,0.00000824,-0.00000844,0$ $.00000196,0.00000034,0.00000664,-0.00000505,0.00000496,0.00001031,-0.0$ $0001300,-0.00001884,-0.00000393,0.00000225,0.00000329,-0.07803116,-0.2$ $2987925,0.10417853,0.08961725,0.24452831,-0.00009125,0.00001814,-0.000$ $10549,-0.00052208,-0.00076404,0.00031951,-0.00260194,-0.00054221,-0.00$ $107414,-0.00060213,0.00056426,-0.00065759,0.00006760,-0.00021237,0.000$ $34632,0.00000354,0.00007525,-0.00003666,-0.00001434,-0.00002086,0.0000$ $2287,-0.00001512,0.00004059,0.00000965,-0.01251525,-0.01830985,0.00758$ $109,-0.00003365,-0.00001844,0.00000656,0.00009966,0.00005161,0.0000105$ $0,0.00068223,-0.00003624,0.00019954,-0.00001784,0.00001305,0.00000150$, $0.00000714,-0.00000412,-0.00000504,-0.00001182,0.00000632,-0.00000505$, $-0.00001450,-0.00003654,0.00000594,0.00000355,0.00000483,-0.00000996,0$ $.00000347,-0.00000570,0.00001248,0.00001438,-0.00000635,-0.00000246,0$. $00000018,-0.00000055,-0.00000003,-0.00000317,-0.00000063,0.00000256,-0$ $.00000636,0.00000615,0.00000231,0.00000234,0.00000169,-0.00000227,0.05$ $108928,0.11111675,-0.11078060,-0.04369049,-0.11190112,0.11369414,-0.00$ $004704,0.00028959,0.00028674,-0.00163993,-0.00038200,-0.00031790,0.004$ $75937,0.00328469,-0.00174724,0.00056277,0.00002511,0.00068566,-0.00081$ $062,-0.00024840,-0.00009897,0.00030512,0.00009957,0.00027700,0.0000119$ $0,0.00002313,-0.00003826,0.00019573,-0.00014452,-0.00008778,-0.0060902$ $0,-0.01117103,-0.03794626,0.00001196,-0.00003850,-0.00000159,-0.000102$ 
$89,-0.00018620,-0.00002499,-0.00014820,-0.00085585,-0.00031577,0.00001$ $092,-0.00001793,-0.00002598,-0.00001692,0.00001689,0.00001200,-0.00000$ $493,0.00002477,0.00001695,-0.00001236,-0.00008015,0.00000650,-0.000053$ $14,0.00000189,-0.00002456,0.00001920,-0.00000266,0.00001331,-0.0000280$ $1,0.00001138,0.00000644,-0.00000493,0.00000120,0.00000029,0.00000528,-$ $0.00000325,0.00000605,0.00000120,-0.00001868,-0.00000421,-0.00000930,-$ $0.00000086,0.00000328,-0.05904025,0.01141601,0.05629128,-0.00103455,0$. $00356403,0.00928072,0.06490722,0.00002391,0.00011649,0.00010167,-0.001$ $03677,-0.00046975,-0.00032475,0.00256783,0.00166332,-0.00110332,-0.000$ $06046,0.00066245,-0.00073490,-0.00048724,-0.00032022,0.00033325,0.0002$ $3380,0.00002850,0.00019998,0.00003176,0.00002145,-0.00001076,0.0000906$ $7,-0.00002094,0.00000520,-0.00484253,-0.00106989,-0.00787002,-0.000014$ $91,-0.00004285,-0.00004168,0.00009568,0.00003786,-0.00002716,-0.000085$ $27,-0.00171309,-0.00013047,-0.00001837,0.00001087,0.00000223,0.0000080$ $5,-0.00000395,0.00000191,0.00000565,-0.00000370,-0.00002185,-0.0000504$ 3,-0.00000114,0.00001851,0.00001062,0.00001260,0.00000630,0.00000236,$0.00000818,0.00000485,0.00002224,-0.00000882,-0.00000328,0.00000363,-0$ $.00000094,-0.00000015,-0.00000448,0.00000117,-0.00000104,-0.00000296,0$ $.00001177,0.00000089,0.00000768,0.00000142,-0.00000494,0.01054657,-0.0$ $6003358,-0.04684306,-0.00465036,0.00694133,0.02436888,-0.00812376,0.05$ $832828,0.00011139,0.00021082,0.00013448,-0.00056157,-0.00074758,0.0002$ $3796,0.00228303,0.00412280,-0.00111151,-0.00065411,-0.00002510,-0.0001$ $8724,0.00042295,-0.00011146,0.00038130,-0.00013210,0.00014035,-0.00027$ $991,0.00001577,-0.00004573,0.00004117,0.00017978,-0.00005647,0.0000046$ $6,-0.01346661,-0.00681859,-0.01573248,-0.00005626,-0.00006745,0.000013$ $49,-0.00007258,-0.00021775,0.00015648,0.00061065,0.00014193,0.00077305$ $, 0.00004338,-0.00004168,-0.00004361,-0.00003654,0.00003935,0.00002216$, $-0.00000344,0.00002124,0.00004705,0.00008309,-0.00013664,0.00001563,-0$ $.00007793,0.00001238,-0.00000851,0.00002245,-0.00001052,-0.00000314,-0$ $.00006791,0.00002716,0.00001710,-0.00000786,0.00000433,-0.00000234,0.0$ $0001218,0.00000493,0.00000701,0.00002013,-0.00002719,-0.00002907,-0.00$ $001148,0.00000532,0.00000359,0.05034012,-0.04880403,-0.28013118,0.0040$ 4346,-0.00348379,-0.01 183056,-0.04491514,0.05495486,0.30920609,0.00038 $771,-0.00001593,-0.00009977,-0.00155039,-0.00123226,-0.00067067,0.0043$ $8153,0.00647210,-0.00053374,0.00013507,0.00022839,-0.00050729,-0.00024$ $183,-0.00069261,0.00005860,0.00025085,0.00012059,0.00006613,-0.0000508$ $9,-0.00010984,0.00002055,0.00001525,0.00015066,0.00028132,-0.03506615$, $0.01341235,-0.00746288,-0.00007319,-0.00004689,0.00002173,-0.00011036$, $-0.00014771,0.00014342,-0.00051424,-0.00030993,0.00104149,0.00004542,-$ $0.00002295,-0.00002724,-0.00002589,0.00001541,0.00001674,0.00000910,0$. $00001116,0.00001667,0.00005975,-0.00002613,-0.00002043,-0.00001204,-0$. $00000370,0.00002701,0.00000581,-0.00001087,-0.00000685,-0.00004720,0.0$ $0001494,0.00000815,-0.00000344,0.00000145,-0.00000247,0.00000531,-0.00$ $000187,0.00000324,0.00000759,0.00000164,-0.00002412,-0.00000021,0.0000$ $0229,0.00000132,-0.14468957,0.11905983,0.01844851,0.00905798,-0.007771$ $79,-0.00111413,-0.00174740,0.00572708,0.00187512,0.16977143,0.00003865$ ,-0.00014368,-0.00006807,-0.00070014,-0.00006107,-0.00084772,0.0004945 $7,0.00135923,0.00164995,0.00066150,0.00052010,0.00037730,-0.00073710,-$ $0.00051377,-0.00055441,0.00046775,-0.00009999,0.00035231,0.00000080,0$. $00001458,-0.00003201,-0.00004007,0.00002206,0.00003581,-0.00703519,0.0$ $1162729,0.00205511,0.00001812,0.00003981,0.00000577,-0.00005057,0.0001$ 
$2962,-0.00005838,-0.00136967,-0.00009574,0.00032845,-0.00003740,0.0000$ $2572,0.00002711,0.00002780,-0.00002247,-0.00000857,-0.00001311,-0.0000$ $0831,-0.00002424,-0.00002285,0.00008451,-0.00004172,0.00003440,0.00000$ $450,-0.00001888,-0.00000930,0.00001185,0.00000390,0.00004585,-0.000013$ $48,-0.00000733,0.00000486,-0.00000347,0.00000309,-0.00000265,0.0000057$ $5,-0.00000228,-0.00000838,-0.00000921,0.00002609,-0.00000143,-0.000003$ $89,-0.00000061,0.12512574,-0.20313014,-0.02068032,0.01541771,-0.017080$ $61,-0.00440850,0.00251552,-0.00414045,0.00090849,-0.13482540,0.2114812$ $6,0.00013802,-0.00008603,-0.00008287,-0.00012543,-0.00025638,-0.000233$ $99,0.00139120,0.00175510,-0.00028665,0.00040448,-0.00064244,0.00074757$ ,-0.00015553,0.00016839,-0.00013405,-0.00000150,0.00005074,-0.00003897 ,-0.00005024,-0.00005724,0.00001031,-0.00004286,0.00006024,0.00011167, $-0.01940046,0.01189568,0.00006897,0.00002320,-0.00002189,0.00000924,-0$ $.00014900,-0.00018714,0.00003517,0.00052018,0.00023872,-0.00064194,0.0$ $0003580,-0.00002076,-0.00001936,-0.00002067,0.00001211,0.00000961,-0.0$ $0000613,0.00002417,0.00002893,0.00007926,-0.00003023,-0.00001915,-0.00$ $003882,0.00000032,-0.00001032,0.00000281,-0.00000265,0.00000005,-0.000$ $04457,0.00001667,0.00000871,-0.00000632,0.00000149,0.00000028,0.000009$ $98,0.00000042,0.00000492,0.00000786,-0.00002006,-0.00001434,-0.0000079$ $7,0.00000209,0.00000315,0.01988577,-0.02270661,-0.05207732,-0.00955108$ $, 0.01170680,0.00229488,0.01866242,-0.02288114,-0.00170165,-0.01156038$, $0.02097965,0.05192714 \backslash \backslash-0.00003759,0.00004824,-0.00002350,0.00001120$,$0.00000340,0.00000721,-0.00001835,-0.00001533,-0.00001307,-0.00000857$, $-0.00001320,0.00000557,0.00002721,0.00004685,-0.00001641,0.00000856,-0$ $.00003289,0.00003852,0.00000828,-0.00001500,0.00000727,-0.00000882,-0$. $00000580,-0.00000150,0.00000618,0.00001291,-0.00000890,0.00000406,0.00$ 000095,0.00000790,-0.00000298, $0.00000027,0.00000673,0.00000323,0.00000$ $941,-0.00000137,-0.00000178,-0.00000476,0.00000621,0.00000103,-0.00000$ $868,0.00000684,-0.00001294,0.00000140,0.00000793,0.00003394,-0.0000207$ $0,-0.00000355,-0.00000731,0.00000861,0.00000117,0.00000379,-0.00000034$ $, 0.00000207,0.00000042,-0.00000181,0.00000330,-0.00000114,-0.00000881$, $0.00000901,-0.00000041,-0.00000853,0.00000841,0.00000169,0.00000342,-0$ $.00000134,0.00000181,0.00000264,-0.00000044,-0.00000820,-0.00000623,-0$ $.00001262,-0.00000270,0.00000169,-0.00001577,0.00000050,0.00000708,-0$. 00001066,-0.00000110,0.00000202,-0.00000900III@

ortho-Addition Product to 4-fluorophenolate

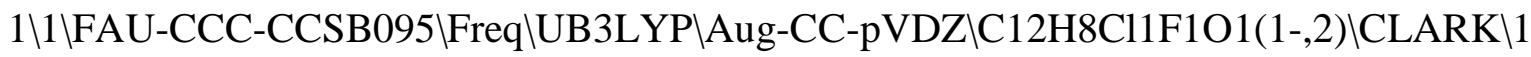
1-Dec-2014\0\\\#N Geom=AllCheck Guess=TCheck SCRF=Check GenChk UB3LYP/A ug-CC-pVDZ Freq I\Addition product p-Cl-Ph-radical + 4-fluoro-phenolate (ortho)\-1,2\C,1.3144473409,1.0463586621,-0.4253508205\C,2.078278480 $7,-0.0493487648,-1.2518563487 \backslash \mathrm{C}, 3.1758827434,-0.7018673362,-0.63610843$ 23\C,3.6861718401,-0.3367319205,0.6270475294\C,3.0828402551,0.74438784 $49,1.3045000753 \backslash \mathrm{C}, 2.0017515865,1.4430285232,0.8604390206 \backslash \mathrm{H}, 1.267248941$ $6,1.9179966151,-1.107507498 \backslash \mathrm{O}, 1.6637745718,-0.2793403229,-2.4222132619$ $\backslash \mathrm{H}, 3.6581337288,-1.492887473,-1.214335655 \mathrm{~F}, 3.6648974719,1.1274243586$, 2.5094117503\H,1.6284188456,2.2925314874,1.4341710962\Н,4.5407788375,$0.8430674692,1.0742647487 \backslash \mathrm{C},-2.8013171004,-0.2409964091,0.0492745975 \backslash \mathrm{C}$ ,-2.3413295531,0.1545328888,-1.206767963\C,-1.0161491946,0.5783745251, $-1.3378964499 \backslash \mathrm{C},-0.1420876357,0.6075617592,-0.2357674722 \backslash \mathrm{C},-0.64077291$ 
$94,0.1938921095,1.0068948499 \backslash \mathrm{C},-1.9668540449,-0.2286551167,1.163695024$ 9\Cl,-4.4948281349,-0.7665418956,0.2320785731\H,-3.0078868766,0.129009 $6222,-2.068138434 \backslash \mathrm{H},-0.6333307259,0.8645085051,-2.3161639541 \backslash \mathrm{H}, 0.02609$ $83929,0.2009932406,1.8694239069 \backslash \mathrm{H},-2.3426889434,-0.5437842618,2.136459$ $1546 \backslash \backslash$ Version=EM64L-G09RevC.01 $\backslash S t a t e=2-A \backslash H F=-1097.470245 \backslash S 2=0.760503 \backslash \mathrm{S}$ $2-1=0 . \backslash S 2 A=0.750069 \backslash \mathrm{RMSD}=8.876 \mathrm{e}-09 \backslash \mathrm{RMSF}=5.135 \mathrm{e}-06 \backslash$ ZeroPoint $=0.1624423 \backslash$ Thermal $=0.1750053 \backslash Z P E=101.9340663 \backslash$ Dipole $=-2.1451324,0.3405935,0.909595$ 4LDipoleDeriv $=0.0708069,0.0988473,0.2329437,0.2684511,0.0846877,-0.301$ $0138,0.3799726,-0.1928318,0.0230901,0.3754208,0.0253391,0.6357224,-0.3$ $636284,0.0565121,0.134324,0.2717668,0.2379003,1.4099483,-0.2085624,-0$. $2805154,-0.3242087,0.1514829,-0.1074421,0.3697482,0.4321638,-0.0831065$ ,-0.1537895,-0.2566816,0.0746044,0.1406634,-0.161929,-0.2302333,-0.124 $8171,-0.2628563,0.133804,-0.1821809,0.5867335,-0.2854463,0.2378771,-0$. $0543249,0.5092601,0.5637693,0.6083936,0.3461462,0.97807,-0.4570764,0.0$ $885225,0.1156342,-0.1352001,-0.3191048,-0.0805454,0.0321841,0.1771953$, $-0.1747818,-0.0825599,0.0398605,-0.0405978,0.0524912,-0.1053192,0.1541$ $367,-0.1474773,0.165528,-0.0903689,-0.7084985,-0.1049181,-0.4179058,0$. $0330926,-0.4270843,-0.2270434,-0.3106726,-0.2710888,-1.6752512,0.01273$ $98,0.1128849,0.0286454,0.1664623,-0.018211,-0.1044696,0.0318604,-0.127$ $8974,-0.0100779,-0.5206104,-0.0761348,-0.406506,-0.1720845,-0.272489,-$ $0.3084468,-0.6957637,-0.2688031,-1.0363788,0.0910975,0.0991934,-0.0069$ $406,0.0929256,-0.0291424,-0.1285295,-0.0312286,-0.1311042,0.0107128,-0$ $.0487099,0.1159287,-0.1043339,0.1315286,0.0654111,0.0120379,-0.1215029$ $, 0.0054496,0.0389367,1.0858245,0.3294902,-0.0859148,0.3572151,0.031791$ $5,-0.0584814,-0.1906287,-0.0525096,0.0590373,-0.2261122,-0.0234562,0.1$ $194517,-0.07622,-0.1132832,0.0254237,0.0572456,-0.0115458,0.0256191,-0$ $.1210925,0.0244597,-0.0417039,-0.0466098,-0.0872811,0.0137115,-0.17848$ $29,-0.0517207,0.0587946,0.5279075,0.073184,-0.1000266,0.1400039,-0.045$ $0718,0.0189338,0.0018726,0.003953,-0.0844911,-0.1892076,0.0085264,0.07$ $45609,-0.0744169,-0.0868796,-0.0062672,0.0264869,-0.011169,-0.0121426$, $-0.1659743,-0.0114384,-0.0640487,-0.025287,-0.1098599,-0.0499442,0.074$ $6497,-0.0186763,0.037207,-0.9858232,-0.2115361,0.0720782,-0.2623951,-0$ $.1852625,0.0233603,0.0945846,0.0263717,-0.14137,-0.0221031,-0.0132055$, $-0.0875975,-0.0325198,0.1177119,0.0008741,-0.1063436,0.0076436,-0.0197$ $502,0.1131013,-0.044232,0.0515967,0.0089163,0.0792332,0.0263229,0.0006$ $484,0.0500388,-0.0053561,0.1064009,-0.0052607,-0.0881268,0.0284813,0.0$ $870319,-0.0103355,-0.054804,0.0162174,-0.0022817,0.0229792,-0.0346977$, $0.0587375,-0.0264356,0.1050248,0.0572516,0.0879315,0.0502054,-0.053195$ Polar $=252.8183709,1.3682459,134.8310048,14.396761,5.6178493,180.94299$ 93\PG=C01 [X(C12H8Cl1F1O1)]\NImag=0\0.39139567,0.00368750,0.44530048, $0.05612508,-0.04881849,0.49333258,-0.08908233,0.03046459,0.01096063,0$. $45384639,0.01292371,-0.09402259,-0.00376202,-0.13380507,0.32815936,-0$. $01209760,-0.00299746,-0.05822694,0.20353965,0.06329395,0.63825702,-0.0$ $0838906,0.01371371,-0.00382962,-0.20499766,0.09651212,-0.06036464,0.42$ $587235,0.02229894,-0.00691291,0.00287255,0.09763100,-0.13918971,0.0180$ $0334,-0.22570267,0.40984837,0.01140379,-0.00733792,0.01003291,-0.05475$ $605,0.01617694,-0.12203651,0.13515992,0.18061270,0.57739313,0.00154677$ ,-0.00009901,0.00368551,-0.00909484,-0.00754863,-0.02810962,-0.1107381 $1,-0.00104806,-0.10461252,0.45956343,-0.00365742,-0.00298959,-0.005954$ $39,-0.00168906,0.01460488,0.02094072,0.01049723,-0.08577544,-0.0641069$ $6,-0.23418336,0.40498403,0.00084781,-0.00216550,-0.00360246,-0.0226921$ 
$8,0.01032776,-0.00978785,-0.08468105,-0.07710911,-0.27589172,0.1878605$ $0,0.14175051,0.54197699,-0.01552435,-0.00091669,-0.01684715,0.00304841$ ,-0.00027208,0.00882454,0.01261356,0.00337878,0.01403207,-0.11292791,0 $.06444421,0.01322159,0.49278706,-0.00325000,0.00766946,0.00975753,-0.0$ $0420299,-0.00061904,-0.00971948,-0.00238488,-0.01764656,-0.03261144,0$. $09221119,-0.19063949,-0.06943163,-0.24598558,0.44868871,-0.02039920,0$. $01181335,-0.01313594,-0.00049104,-0.00054222,-0.00309031,0.00746187,-0$ $.02700722,-0.02575136,0.04690425,-0.09809679,-0.12684051,0.14036722,0$. $11886010,0.45140700,-0.09460527,-0.00164025,-0.04800997,0.00814821,-0$. $00576089,0.00245958,-0.00373451,-0.00548636,-0.01133506,-0.02332431,0$. $04650061,0.01364918,-0.27817335,0.14210562,-0.10885541,0.51315175,-0.0$ $0173758,-0.08890576,-0.03348473,-0.01216191,-0.00350825,-0.01048930,0$. $00344506,0.00441959,0.01092491,0.03829971,-0.03052614,-0.01043306,0.15$ $931223,-0.16065013,0.04749154,-0.26706180,0.47126493,-0.05213744,-0.03$ $376937,-0.16294979,-0.00310390,-0.00637301,-0.01791123,0.00012748,-0.0$ $0142531,-0.00034782,0.00347208,0.00127121,0.01697463,-0.08250147,0.022$ $22278,-0.11207297,0.15425114,0.13656317,0.43825769,-0.03506812,0.00741$ $923,-0.00774032,-0.00488851,0.01859766,-0.00713765,0.00115074,0.000846$ $49,0.00388339,-0.00089082,-0.00059015,-0.00188476,0.00161291,-0.000761$ $46,-0.00111433,-0.00387144,0.01088339,-0.01349016,0.05497253,-0.000271$ $19,-0.18897246,0.11070224,0.00792455,-0.02262084,0.01018787,-0.0007529$ $9,-0.00200735,-0.00513262,-0.00030770,0.00114859,0.00293983,0.00144715$ ,-0.00131209,-0.00117202,0.00029243,0.01257641,-0.00403056,-0.00439448 $, 0.20595024,-0.00439177,0.11411836,-0.12740564,-0.00310794,-0.01133363$ $, 0.00488059,0.00299979,-0.00123066,0.00182858,-0.00111404,-0.00005689$, $-0.00213654,0.00358619,-0.00112547,-0.00265783,-0.00934709,0.01635599$, $-0.01919045,0.00743464,-0.12231234,0.14515578,0.01519583,-0.00057162,0$ $.00441925,-0.11898844,-0.01157333,-0.13275120,-0.01477308,0.00177037,-$ $0.05083089,-0.00013527,0.00259687,-0.00218039,-0.00613990,0.00671803,0$ $.00163272,0.00230393,0.00028032,0.00177882,0.00088230,-0.00036650,0.00$ $261486,0.12223713,-0.00344055,-0.00422445,-0.03415751,-0.00838089,-0.0$ $8817500,-0.07670226,0.00607553,0.02148872,0.01738148,0.00462275,-0.001$ $23870,-0.00275495,-0.00010000,0.00020241,0.00023840,0.00572899,-0.0022$ $4998,-0.00189344,-0.00317669,0.00391859,0.00000243,0.00509608,0.071989$ $52,0.00240880,-0.02434371,-0.04898886,-0.12885508,-0.07904759,-0.43232$ $077,-0.03928330,0.01087829,-0.03564446,-0.00094255,-0.00780745,-0.0055$ $5424,-0.01089346,0.01234268,0.00335214,0.01192480,-0.00977935,0.002028$ $51,-0.00102820,0.00075956,0.00359427,0.17033742,0.10023638,0.51762100$, $-0.00148851,0.00325662,-0.00356273,-0.00829610,0.01927051,0.01485529,-$ $0.09694677,0.10099359,0.05771960,0.00629837,-0.00965116,-0.00675321,0$. $00121529,0.00111672,-0.00296514,-0.00002399,-0.00055673,-0.00043886,-0$ $.00072362,0.00107841,0.00052692,0.00100224,-0.00416345,0.00131645,0.09$ $881881,0.00378000,0.00161823,0.00041010,0.00576747,-0.00775306,-0.0119$ $1463,0.10122321,-0.18648075,-0.11073059,0.00346747,-0.00372289,-0.0017$ $9653,0.00076734,0.00317156,-0.00179753,-0.00023244,0.00017633,0.000573$ $40,0.00004196,0.00006617,-0.00004671,-0.00335333,-0.00024897,0.0016162$ $6,-0.10981316,0.19504010,-0.00297913,-0.00017447,0.00042067,-0.0069468$ $9,0.00781973,0.00635663,0.05851054,-0.11361228,-0.12512769,0.01393939$, $-0.01922414,-0.00993992,-0.00414000,0.00043310,-0.00167497,0.00004627$, $0.00011851,0.00010452,0.00017888,-0.00018126,0.00015467,0.00207170,0.0$ $0122494,0.00133217,-0.06135338,0.12376998,0.12952867,0.00133460,0.0083$ 
$5870,-0.00048513,-0.00086903,0.00094261,0.00144064,0.00071984,0.001502$ $27,-0.00235609,0.00782544,0.00170259,0.00729801,-0.08789896,-0.0019062$ $4,-0.06903027,-0.01443738,-0.01503396,-0.04177422,-0.00132709,-0.00110$ $032,-0.00214169,0.00032670,-0.00117311,-0.00114235,0.00036049,0.000319$ $78,-0.00005710,0.09424912,0.00438405,0.00348620,-0.00257742,0.00094345$ $,-0.00059681,0.00160896,0.00548447,-0.00165191,-0.00031245,-0.01736890$ ,-0.00686971,-0.03532019,-0.00507091,-0.06849591,-0.04980561,0.0062905 $9,0.00978100,0.01360527,-0.00037949,-0.00054989,-0.00061724,-0.0012660$ $1,-0.00017104,-0.00196522,-0.00037826,-0.00002615,-0.00198018,0.011997$ $44,0.06527569,-0.00345150,0.00185613,0.00570584,0.00110032,0.00100761$, $0.00419679,0.00225254,-0.00521851,0.00122042,-0.01631572,-0.01459195,-$ $0.03451264,-0.07523599,-0.04260911,-0.18115093,-0.01343165,-0.01352182$ $,-0.02835494,-0.00067824,-0.00023110,-0.00114741,-0.00151384,-0.001017$ $67,-0.00493835,-0.00091691,-0.00063425,-0.00173066,0.10493850,0.071757$ $36,0.24154790,0.00571587,-0.01196312,-0.00586951,-0.00159057,0.0011391$ $8,-0.00232575,-0.00017324,-0.00092067,-0.00027290,-0.00186178,0.002345$ $12,-0.00395777,-0.00347181,0.02029850,0.01571424,-0.07432547,0.0882171$ $2,0.03738437,0.00026565,0.00031177,-0.00015457,0.00047096,-0.00100051$, $-0.00033907,-0.00012324,-0.00014565,0.00017609,-0.00038130,-0.00332847$ $, 0.00209186,0.07408798,0.00266322,-0.00312312,-0.00132502,-0.00072187$, $0.00266754,-0.00013707,-0.00089722,-0.00001922,0.00046742,0.00367845,0$ $.00135668,0.00197985,0.00298554,-0.00995965,-0.01351756,0.09113346,-0$. $21090404,-0.12186055,-0.00065776,0.00037091,-0.00084768,-0.00026333,-0$ $.00097193,-0.00068599,-0.00013826,-0.00023329,0.00001950,-0.00300127,-$ $0.00069398,0.00218217,-0.09661719,0.22087007,0.01085194,-0.01899737,-0$ $.00945159,-0.00663927,0.00225013,-0.00111006,0.00111399,-0.00021530,-0$ $.00032721,-0.00322150,0.00051712,0.00078545,-0.00510198,0.00596406,0.0$ $0525973,0.04110470,-0.12495444,-0.11787082,0.00053931,0.00064322,0.000$ $99366,0.00163558,-0.00163798,-0.00189897,0.00023053,0.00005032,-0.0000$ $8588,0.00295739,0.00224533,0.00064788,-0.04317329,0.13420589,0.1223093$ $9,-0.00032034,-0.00085626,0.00101874,0.00334811,-0.00022918,-0.0019884$ $9,-0.00512700,0.00589521,-0.00086770,-0.21457756,0.12033645,-0.0990890$ $0,-0.01015683,0.00382875,-0.01033639,0.00153804,0.00184101,0.00205552$, $0.00018468,-0.00008266,0.00007832,-0.00090947,0.00029123,-0.00067067,-$ $0.00053762,-0.00189465,0.00067863,0.00032922,-0.00198862,0.00204980,0$. $00011776,0.00109537,-0.00068974,0.22613506,-0.00124561,-0.00054310,0.0$ $0035644,0.00113566,0.00140856,-0.00296317,-0.00765856,0.00664952,-0.00$ $325344,0.11891643,-0.10113388,0.04142438,0.01900093,-0.00728111,0.0127$ $8994,0.00072187,-0.00237659,-0.00390226,0.00020674,-0.00006193,0.00010$ $690,-0.00049742,0.00023577,-0.00018073,-0.00126385,-0.00095039,0.00135$ $997,-0.00244858,0.00036874,0.00179504,0.00169561,0.00027168,-0.0002752$ $9,-0.12836339,0.10324630,0.00023790,0.00066338,-0.00049020,-0.00029124$ ,-0.00419311,-0.00221066,-0.02060677,0.01476216,-0.00520543,-0.1009446 $8,0.04365200,-0.09166303,0.01207579,-0.00804350,0.00643852,0.00029799$, $-0.00253854,-0.00123923,-0.00020364,0.00012114,-0.00012163,-0.00142472$ $, 0.00075416,-0.00141787,0.00147724,0.00083664,0.00036799,0.00107877,0$. $00219318,0.00107133,0.00018342,-0.00095139,0.00072781,0.10794225,-0.04$ $720403,0.09360666,-0.00170208,0.00048790,0.00052309,-0.00041677,-0.000$ $64885,-0.00125333,-0.00014723,-0.00003136,0.00011296,0.00020711,-0.000$ $06781,0.00024083,-0.00047424,0.00009685,-0.00035476,0.00057387,0.00007$ $202,0.00001972,0.00018838,-0.00004729,-0.00018520,0.00113921,0.0001161$ 
$5,0.00021485,0.00006715,0.00000963,-0.00009691,0.00031969,0.00010419,0$ $.00049195,-0.00001952,-0.00001164,0.00002177,0.00005345,-0.00003339,0$. $00003808,0.45214910,-0.00064635,-0.00043926,0.00075964,0.00018802,-0.0$ $0021119,0.00000405,-0.00006016,0.00002759,-0.00016566,0.00007581,0.000$ $03890,0.00015131,-0.00012689,-0.00006177,-0.00012277,0.00015127,0.0001$ $6048,-0.00017941,-0.00026930,0.00006026,-0.00011717,-0.00028289,0.0001$ $7383,-0.00021444,-0.00001766,-0.00001819,-0.00001477,0.00009823,0.0000$ $4171,0.00016333,-0.00005055,-0.00011513,-0.00001360,0.00001602,-0.0000$ $1567,0.00001259,0.08807604,0.16253433,0.00013955,0.00046958,-0.0015723$ $2,-0.00070607,-0.00038486,-0.00066281,0.00008508,-0.00038360,0.0003467$ $6,0.00010806,-0.00020594,-0.00045129,-0.00021123,0.00066562,0.00031912$ $, 0.00015347,-0.00019459,0.00038323,0.00050610,-0.00063942,0.00047556,0$ $.00078547,0.00002638,0.00116263,0.00021179,0.00011420,-0.00001214,-0.0$ $0003981,-0.00011404,-0.00028191,0.00027842,0.00010214,0.00012959,-0.00$ $002726,-0.00001002,-0.00000932,0.04983201,-0.09369055,0.63948330,0.003$ $58168,-0.00120880,-0.00476947,0.00002265,0.00054490,0.00076548,0.00004$ $107,-0.00034707,-0.00062137,0.00016085,0.00024857,0.00017007,0.0004414$ $3,-0.00021016,0.00008659,-0.00109215,0.00009062,0.00058148,0.00041371$, $-0.00058696,0.00109161,0.00020899,0.00014031,0.00095963,0.00012780,-0$. $00006301,0.00011989,-0.00013635,0.00000014,-0.00025676,0.00022205,0.00$ $020697,0.00017701,-0.00003829,0.00001434,0.00002085,-0.11586420,-0.020$ $20841,0.02246399,0.64377343,-0.00108718,0.00447357,0.00138115,-0.00158$ $112,-0.00036040,-0.00056210,0.00006910,0.00002049,0.00049172,0.0001332$ $9,-0.00027091,-0.00051027,-0.00028392,0.00070002,0.00006166,0.00083337$ ,-0.00019647,0.00015381,0.00049254,0.00018101,-0.00048071,0.00039797,0 $.00057503,0.00082851,0.00003608,0.00005241,0.00003079,-0.00000392,-0.0$ $0010726,0.00002604,0.00003714,0.00002883,0.00008121,-0.00002318,-0.000$ $01013,-0.00001855,-0.03350005,-0.08274955,0.05333830,0.13939157,0.1865$ $4184,-0.00191234,0.00162446,-0.00329183,-0.00021138,-0.00029184,-0.001$ $51796,0.00038891,0.00007218,0.00036804,-0.00016121,0.00009349,0.000066$ $81,0.00040529,-0.00084383,-0.00012624,-0.00164803,-0.00016691,-0.00009$ $360,-0.00002205,0.00011829,-0.00029571,0.00121303,-0.00039014,0.000719$ $23,-0.00013252,-0.00004820,0.00001788,-0.00008579,0.00013820,-0.000092$ $12,-0.00012273,0.00001445,-0.00014227,0.00000106,0.00001165,0.00001596$ $, 0.08342784,0.07239979,-0.29645443,0.07260936,-0.09170692,0.68884661,-$ $0.02439374,-0.00211301,-0.02099638,-0.00080620,-0.00186400,-0.00395741$ $, 0.00039437,0.00059553,0.00095232,-0.00036798,-0.00007077,-0.00000178$, $0.00060539,-0.00165157,-0.00024441,-0.00436142,0.00043716,0.00156850,0$ $.00034695,0.00033119,-0.00240932,-0.00156566,0.00170718,0.00061406,-0$. $00026789,0.00016211,-0.00010328,-0.00059571,0.00025920,-0.00106309,-0$. $00031019,-0.00039035,-0.00059275,-0.00008170,-0.00001653,-0.00000381,-$ $0.01159721,-0.02347948,0.08263684,-0.31233703,-0.07211214,-0.01512861$, $0.63338017,-0.00694006,0.00282242,-0.00574935,-0.00057107,0.00007058$,$0.00295664,0.00042895,0.00040410,0.00093673,-0.00029043,-0.00016391,-0$ $.00019373,-0.00004748,-0.00043393,-0.00011749,-0.00280590,0.00098181,-$ $0.00001803,0.00022444,0.00144888,-0.00164105,0.00268334,-0.00082679,0$. $00175933,-0.00006732,0.00015293,-0.00009326,-0.00023538,0.00008742,-0$. $00046490,0.00035653,-0.00010452,0.00010200,0.00001282,0.00000210,0.000$ $00083,-0.01073787,-0.00078328,0.02296264,-0.08461662,-0.08687602,0.007$ $25179,0.14806514,0.19896751,-0.00591520,-0.00104671,0.00823945,0.00112$ $150,-0.00124996,0.00026930,-0.00094829,0.00039248,-0.00001087,0.000382$ 
$84,-0.00048183,-0.00055958,-0.00135365,0.00170060,0.00003009,0.0033669$ $7,-0.00041178,0.00039655,0.00155805,0.00119243,-0.00014675,-0.00169557$ $, 0.00104725,-0.00112057,0.00005608,0.00008971,0.00000963,0.00021624,-0$ $.00020442,0.00026858,-0.00019100,-0.00029261,-0.00006174,-0.00000401,-$ $0.00000282,-0.00009042,0.02889092,0.00631585,0.02071830,0.04678208,0.0$ $2643532,-0.12362872,-0.00712746,-0.11032638,0.68570092,-0.13792455,-0$. $03900506,0.00678082,-0.02847059,0.01723591,0.02333501,0.00427763,-0.00$ $285154,0.00068008,-0.00168585,0.00116278,-0.00148077,0.00205319,-0.004$ $95600,0.00022045,-0.02385542,-0.00505234,-0.01211750,-0.00854895,-0.00$ $136239,0.00405899,-0.00085178,-0.00385638,-0.00799917,0.00077623,-0.00$ $056747,0.00008512,0.00103504,0.00065520,0.00154985,0.00158989,0.001278$ $49,0.00096151,0.00014377,-0.00018918,0.00018419,-0.00013256,0.00492246$ ,-0.01167079,-0.03808823,-0.00983380,-0.01681715,-0.18383091,-0.009860 $31,-0.11788386,0.55139556,-0.02105707,-0.07749028,0.00278129,0.0179893$ $9,0.01274881,0.00409012,0.00087678,-0.00402092,-0.00295335,-0.00085536$ $, 0.00122802,0.00071987,0.00176397,-0.00322113,0.00045552,-0.00983460,0$ $.00030608,-0.00305581,-0.02775442,-0.00933829,0.00775145,-0.00099378,-$ $0.00029787,-0.00259817,0.00043410,-0.00100759,0.00056507,0.00017407,0$. $00021965,-0.00010482,0.00013677,0.00072770,0.00005080,0.00013736,0.000$ $14406,0.00002168,0.00382714,-0.00770391,0.01121303,-0.00017113,0.00931$ $718,-0.01267932,-0.02142672,-0.07459383,0.00077461,0.10474920,0.222922$ $41,0.01025354,0.00541921,-0.08502452,0.01356792,0.00272367,-0.00701185$ $, 0.00060004,-0.00081105,-0.00071280,-0.00057633,0.00156979,0.00120747$, $0.00247950,-0.00618729,-0.00132272,-0.03059182,-0.00414445,0.00187548$, $0.02037879,0.00729115,-0.00508759,0.00235078,-0.00078552,0.00256279,-0$ $.00018837,-0.00027298,0.00016188,-0.00089366,0.00138227,-0.00206838,0$. $00019933,0.00027824,-0.00012121,-0.00012919,-0.00006031,0.00016075,-0$. $01131200,0.00932567,-0.06833492,-0.05599442,-0.02420195,0.04283188,-0$. $06726604,0.01579949,-0.23856628,0.02480807,-0.08810430,0.64751084,-0.0$ $1197189,-0.00899787,0.02812251,-0.00050684,-0.00476133,-0.00413447,-0$. $00025876,0.00059685,0.00092387,-0.00016676,0.00037424,0.00000530,0.001$ $48635,-0.00085133,0.00102803,-0.00325672,-0.00092878,0.00287240,-0.003$ $50014,-0.00248587,0.00127876,0.00214982,0.00101136,0.00221476,0.000060$ $08,0.00043928,-0.00016109,-0.00038472,-0.00024345,-0.00100380,0.000315$ $41,-0.00016346,-0.00026704,-0.00008291,-0.00000622,-0.00000880,-0.0430$ $1184,-0.00098888,-0.06726588,-0.03333000,-0.01530199,0.02970852,0.0526$ $2710,0.01304865,0.00335922,-0.14459330,-0.02662016,0.02978942,0.651767$ $47,-0.00849128,0.00232602,0.00819100,-0.00081562,-0.00270471,-0.003493$ $04,0.00011265,0.00047115,0.00058739,-0.00008938,0.00024293,-0.00001121$ $, 0.00048600,-0.00056743,0.00062237,-0.00138282,0.00002204,0.00163529,-$ $0.00093809,-0.00051104,0.00058707,0.00190275,0.00040768,0.00133286,-0$. $00009743,0.00009320,-0.00003240,-0.00017024,-0.00012729,-0.00021796,0$. $00020661,0.00049044,-0.00002004,0.00001267,0.00005753,-0.00004850,-0.0$ $1208153,0.01000808,-0.02791217,-0.01448534,-0.01080846,0.01313823,0.01$ $998614,0.01037142,0.01407518,-0.03936588,-0.08884543,0.05369873,0.1372$ $8141,0.19482105,0.01224473,0.00229914,-0.00026978,-0.00113915,0.002849$ $59,0.00403569,0.00074766,-0.00015269,0.00039605,-0.00035212,-0.0000888$ $6,-0.00109713,-0.00038687,0.00087638,0.00007645,-0.00058149,-0.0004942$ $8,-0.00116344,-0.00136390,-0.00005431,0.00114774,-0.00169993,-0.000499$ $84,-0.00124983,-0.00001720,-0.00017502,0.00012929,0.00006029,-0.000064$ $70,-0.00039646,0.00023325,-0.00035997,0.00020228,-0.00003814,-0.000052$ 
$14,-0.00000116,-0.01325593,-0.01072929,0.04002417,0.02203080,0.0111882$ $5,-0.02286068,-0.02900047,0.00226956,-0.04997937,0.07901696,0.06769034$ $,-0.27865984,0.06264731,-0.09698345,0.66932776,0.00333848,-0.00285245$, $-0.00055988,0.00089969,0.00169659,0.00271873,0.00003850,0.00026707,-0$. $00053098,0.00007729,0.00000683,-0.00001598,-0.00006898,-0.00019647,-0$. $00019169,0.00000717,0.00002832,0.00015826,-0.00095713,0.00080892,-0.00$ $021906,-0.00197442,-0.00009788,-0.00152136,-0.00020362,-0.00022380,0.0$ $0011485,-0.00003122,0.00004909,0.00005108,-0.00028604,-0.00014466,-0.0$ $0007246,0.00000969,-0.00000840,0.00000616,-0.16876436,-0.01532757,-0.0$ $7823738,0.04716063,0.01961393,-0.03550766,-0.04947093,-0.01091139,-0.0$ $2110672,-0.00688217,-0.02013132,0.07215150,-0.30552198,-0.06839603,-0$. $01112741,0.61780179,-0.00092197,0.00555518,0.00132495,-0.00084164,-0.0$ $0046401,0.00107748,-0.00011481,0.00040046,0.00011002,-0.00003703,-0.00$ $011909,-0.00010349,-0.00020760,0.00011775,0.00000285,0.00037807,0.0001$ $3587,-0.00005314,0.00083223,0.00039920,-0.00010910,-0.00059605,0.00004$ $110,-0.00049779,-0.00002053,0.00009231,-0.00003927,-0.00001496,0.00001$ $820,-0.00003795,-0.00005152,-0.00006053,-0.00002150,-0.00002645,-0.000$ $01383,0.00000452,-0.00267230,-0.06689262,0.01976561,0.00976034,0.01215$ $277,0.00260331,-0.00787450,-0.00499237,-0.00479234,-0.01111535,-0.0012$ $2226,0.01910246,-0.08223205,-0.08659111,0.01003967,0.14396915,0.193430$ $26,-0.00118881,0.00216725,-0.00365281,-0.00041065,0.00024318,-0.000640$ $28,-0.00002131,0.00001311,-0.00046760,-0.00008282,0.00063179,0.0007073$ $6,0.00111609,-0.00144191,0.00027005,-0.00220370,-0.00011428,0.00033734$ $, 0.00097313,0.00045677,-0.00043473,0.00047819,-0.00015768,0.00018357,-$ $0.00006498,-0.00000385,-0.00001138,-0.00002275,0.00006758,0.00009487,0$ $.00010412,0.00033400,-0.00004259,-0.00000189,0.00002310,0.00003404,-0$. $13837216,0.00188877,-0.26471091,0.00776176,0.01590415,-0.05271500,-0.0$ $2669305,-0.00560565,-0.01208993,0.03307506,0.00490057,0.02138250,0.049$ $38110,0.02876522,-0.12230732,0.00328370,-0.12178884,0.70647888,-0.0005$ $0803,0.00069569,0.00074910,-0.00005633,-0.00094804,-0.00088664,0.00005$ $268,0.00030301,0.00055396,-0.00020229,0.00000671,-0.00034272,0.0007457$ $5,-0.00020416,0.00064029,-0.00086662,-0.00009322,0.00045968,-0.0002061$ $2,-0.00013738,-0.00012760,-0.00035588,0.00041268,0.00067435,-0.0000999$ $9,0.00018364,0.00001615,-0.00047388,-0.00019381,-0.00084717,0.00011680$ $, 0.00004290,-0.00012689,-0.00008967,0.00004101,-0.00005797,-0.12014617$ ,-0.03064091,0.00779008,-0.02992271,-0.00979887,0.00073621,0.00268016, $-0.00139675,0.00432951,-0.00005885,-0.00018509,0.00000561,0.00127413,0$ $.00041107,-0.00626900,-0.03665309,-0.01297236,0.00714223,0.18478379,0$. $00031307,-0.00031031,-0.00016004,0.00001545,-0.00007435,-0.00005315,0$. $00009222,0.00002309,0.00027357,-0.00006996,-0.00006166,-0.00026041,0.0$ $0013707,0.00005698,0.00015650,-0.00019587,-0.00020186,0.00020696,-0.00$ $008788,-0.00002983,0.00002804,-0.00015982,0.00008497,0.00008314,-0.000$ $02257,0.00003785,0.00001407,-0.00013378,-0.00005316,-0.00024648,0.0000$ $3140,0.00002128,-0.00002604,-0.00002875,0.00001215,-0.00001874,-0.0306$ $0585,-0.03058006,0.00610326,-0.01541157,-0.00157682,0.00083016,0.00006$ $998,0.00396119,0.00299094,-0.00009460,-0.00079370,-0.00052489,-0.00108$ $193,0.00447197,-0.00026418,-0.00740337,0.00069486,0.00159988,0.0534643$ $1,0.02849254,0.00012620,-0.00015338,0.00010561,-0.00010713,0.00010560$, $-0.00012384,0.00005279,-0.00006510,-0.00001267,0.00000964,0.00001688,0$ $.00006403,0.00000561,-0.00007647,-0.00008848,-0.00001594,-0.00003625,0$ $.00004976,-0.00006260,-0.00002831,0.00005257,0.00020757,-0.00007391,0$. 
$00009865,-0.00000186,-0.00001843,-0.00000479,0.00005492,0.00003125,0.0$ $0012880,-0.00000052,0.00003402,-0.00000044,0.00001656,0.00000118,0.000$ $00651,0.00780864,0.00623118,-0.03944584,0.02796781,0.00857340,0.000791$ $96,-0.00256857,0.00086798,-0.00391918,0.00033314,-0.00038054,0.0016379$ $7,0.00051304,0.00182557,-0.00300336,-0.02004421,-0.00629006,0.00546886$ ,-0.01499281,-0.00942356,0.03560244,0.00015200,0.00031434,-0.00123054, $-0.00012983,-0.00011500,-0.00041857,0.00016600,0.00001273,0.00023637,-$ $0.00009877,-0.00000160,-0.00013190,0.00014329,-0.00022431,0.00007280,-$ $0.00062105,0.00007134,0.00005646,0.00003424,-0.00005338,0.00001691,0.0$ $0014497,0.00012644,0.00043224,-0.00002124,0.00003025,0.00000516,-0.000$ $09780,0.00000577,-0.00018420,0.00003670,0.00000347,-0.00004687,-0.0000$ $1595,0.00000123,-0.00000408,0.00616149,-0.00096118,0.01012170,-0.15692$ $827,-0.00871156,-0.13962323,-0.01210324,0.00002287,-0.02396904,-0.0039$ $1558,-0.00365111,0.00164790,-0.00080439,-0.00004997,0.00086705,0.00084$ $121,-0.00177959,0.00232047,0.00121050,0.00099028,0.00081498,0.16465402$ $, 0.00003442,-0.00005464,-0.00035967,0.00011258,0.00000276,-0.00013440$, $0.00008128,0.00002076,0.00009631,0.00001319,-0.00001066,-0.00009859,0$. $00004973,0.00003609,0.00001558,0.00000289,-0.00020186,-0.00005542,-0.0$ $0008802,-0.00003777,0.00002956,0.00007381,-0.00007999,0.00018065,-0.00$ $000514,-0.00000512,0.00000462,-0.00002433,-0.00000823,-0.00004737,-0.0$ $0006364,-0.00004619,-0.00000844,-0.00000897,-0.00000838,0.00000250,0.0$ $0530029,0.00263840,0.00740085,-0.00956129,-0.03609985,-0.00189503,-0.0$ $0555602,0.00388272,-0.00782920,-0.00346004,0.00595489,0.00177375,0.000$ $01017,-0.00105328,-0.00013974,-0.00205423,0.00387608,0.00234282,0.0011$ $2922,-0.00222902,-0.00051762,0.01245430,0.02715070,0.00036132,0.000082$ $90,0.00018575,-0.00006127,0.00002698,-0.00011917,0.00008952,0.00001405$ $, 0.00013836,-0.00005015,-0.00002524,-0.00013187,0.00003119,0.00001421$, $0.00007908,-0.00001523,-0.00005275,-0.00005275,-0.00005982,0.00001105$, $-0.00000663,-0.00000400,0.00001058,0.00011216,-0.00001070,0.00001012$, $0.00000089,-0.00003591,-0.00002521,-0.00005817,-0.00000402,-0.00001181$ ,-0.00001599,-0.00000680,-0.00000288,-0.00000283,-0.01988548,-0.001934 $39,-0.01722491,-0.13623428,-0.00032404,-0.23295606,0.00344287,0.000505$ $85,0.00555476,0.00089769,0.00135648,0.00194222,0.00085127,-0.00005112$, $0.00020468,0.00324317,0.00266576,-0.00337869,-0.00003398,-0.00075290,0$ $.00116797,0.14824548,-0.00052771,0.24485556,-0.00030925,0.00146943,-0$. $00007311,-0.00152147,-0.00053951,-0.00302028,0.00027477,-0.00007618,0$. $00048006,0.00012479,0.00005795,0.00013352,0.00009902,-0.00023199,-0.00$ $013576,0.00003082,0.00004928,0.00038850,0.00011629,-0.00033184,-0.0002$ $5114,0.00014321,-0.00001938,0.00225573,-0.00000441,0.00004326,-0.00003$ 496,0.00007527,0.00007266,0.00020949,0.00007087,0.00010554,0.00003622, $0.00002094,-0.00000033,0.00003523,-0.00298354,-0.00255292,-0.00093186$, $-0.00682634,-0.00748677,0.02572009,-0.08694334,-0.03430322,0.08876635$, $0.01066960,0.00530766,-0.01745616,-0.00203649,-0.00106040,-0.00367247$, $-0.00064918,0.00006045,-0.00041170,-0.00114908,-0.00067028,0.00086807$, $0.00075240,0.00126907,0.00013423,0.08969665,0.00126931,-0.00192984,-0$. $00215278,0.00130881,0.00050454,0.00236718,-0.00056621,-0.00005897,-0.0$ $0104387,0.00006762,0.00028179,0.00066395,0.00010738,-0.00031555,-0.000$ 03256,-0.00019261,0.00020507,0.00020201,0.00004627,-0.00055326,0.00080 $264,-0.00103346,-0.00053420,-0.00212302,0.00002066,-0.00001956,0.00000$ $201,0.00002592,0.00004889,0.00000360,-0.00002415,0.00002734,-0.0000229$ $9,-0.00001035,0.00001075,-0.00000151,-0.00332778,0.00586869,0.00095280$ 
$,-0.00248162,0.00118811,0.00924180,-0.03266605,-0.05846891,0.06915740$, $-0.00078739,0.00400648,-0.00031717,-0.00116666,0.00585576,0.00118158,0$ $.00014801,-0.00082376,-0.00083682,-0.00055018,0.00008454,0.00045582,0$. $00147290,-0.00378535,-0.00094096,0.03815517,0.04939855,0.00197565,0.00$ $068969,0.00037386,-0.00068013,0.00020778,-0.00077915,0.00020902,-0.000$ $16767,0.00026266,-0.00006438,-0.00005619,-0.00009532,-0.00001394,-0.00$ $004334,0.00007474,-0.00012697,-0.00038781,0.00002558,-0.00073315,-0.00$ $065775,0.00043001,0.00215866,-0.00081719,-0.00005517,0.00001380,0.0000$ $0496,-0.00002419,0.00007552,0.00000696,0.00005261,0.00003113,0.0001033$ $1,-0.00004654,0.00001649,-0.00000357,0.00000145,-0.00030926,0.00055999$ $, 0.00204094,-0.00212710,-0.00084797,0.00242550,0.08576118,0.07204923,-$ $0.28747363,0.00993284,0.00691878,-0.01808854,-0.00423591,0.00051615,-0$ $.00272749,-0.00066162,-0.00048963,0.00024052,-0.00047637,-0.00008732,0$ $.00016701,0.00041940,-0.00054352,0.00057623,-0.09206131,-0.07727064,0$. $30359504,-0.00143432,0.00055477,0.00036860,0.00044585,-0.00065052,0.00$ $060421,-0.00044331,0.00028912,-0.00020408,0.00031587,-0.00030107,0.000$ $02137,-0.00206265,0.00057079,-0.00011521,0.00069384,-0.00039508,0.0004$ $2463,0.00023667,0.00021905,-0.00035365,-0.00052768,0.00001331,-0.00076$ $135,0.00003756,0.00003806,-0.00008084,0.00014646,0.00001422,0.00035945$ $,-0.00045560,-0.00014528,0.00033100,0.00008036,0.00000093,-0.00002483$, $-0.00261859,-0.00295228,0.00133253,-0.00020733,-0.00005463,0.00084152$, $0.00013909,-0.00224905,0.00177479,0.01161989,0.00038089,0.00689491,-0$. $15552818,-0.00496500,-0.13542839,-0.01357086,0.00015304,-0.02253343,-0$ $.00117773,-0.00035409,-0.00061024,-0.00005205,0.00018502,-0.00002191,0$ $.00034974,0.00000167,0.00076265,0.16325090,0.00103239,-0.00328177,-0.0$ $0043654,0.00105460,0.00022209,0.00042204,-0.00031506,-0.00007531,-0.00$ $016280,0.00044011,-0.00049607,-0.00006324,-0.00107568,0.00082009,-0.00$ $035853,0.00068701,-0.00061404,0.00020532,-0.00050321,0.00004054,0.0000$ $3407,-0.00045254,-0.00012616,-0.00063009,0.00000231,-0.00003510,0.0000$ $0136,0.00016805,0.00003803,0.00022495,-0.00060598,-0.00061113,0.000127$ $33,0.00003774,-0.00001734,-0.00000689,-0.00292809,0.00557819,0.0013791$ $6,-0.00004777,-0.00099832,0.00004123,-0.00252176,0.00528096,0.00276581$ $, 0.00581765,0.00506408,0.00667225,-0.00452110,-0.03922217,0.00319014,--$ $0.00615601,0.00299171,-0.00730236,-0.00060424,0.00022899,-0.00013367,0$ $.00019911,-0.00059530,-0.00012613,0.00052888,-0.00031057,0.00037018,0$. $00827192,0.02972198,-0.00203389,-0.00137794,-0.00006096,-0.00023869,0$. $00012577,-0.00063632,0.00006798,-0.00022189,-0.00020470,0.00012346,0.0$ $0023398,0.00024252,0.00062561,-0.00069530,0.00072884,-0.00117688,-0.00$ $028341,0.00078188,-0.00008188,-0.00000010,-0.00004446,0.00047442,-0.00$ $000420,0.00058134,-0.00000563,-0.00001984,0.00003598,-0.00012039,-0.00$ $008891,-0.00034038,0.00003703,0.00027666,0.00010630,-0.00002463,0.0000$ $4401,0.00001368,0.00081935,0.00143497,0.00193019,0.00103543,0.00004635$ ,-0.00013221,0.00255814,0.00314959,-0.00403374,-0.01810176,-0.00039903 $,-0.01852313,-0.13468531,0.00361269,-0.23253791,0.00504745,0.00062004$, $0.00637081,0.00065346,0.00024473,0.00013762,-0.00007680,-0.00015427,-0$ $.00003036,-0.00106126,-0.00016245,-0.00094532,0.14618649,-0.00528967,0$ $.24602089,0.00046091,-0.00019832,0.00122047,-0.00004380,-0.00014677,-0$ $.00009749,0.00012722,0.00008295,0.00024924,-0.00004765,-0.00006774,-0$. $00023667,0.00004731,0.00007068,0.00010513,0.00010147,-0.00000619,-0.00$ $003550,-0.00042309,-0.00013250,0.00011608,0.00001632,0.00006682,0.0000$ $8347,-0.00002703,0.00002005,0.00001089,-0.00008845,-0.00004255,-0.0001$ 
$9599,-0.00001117,-0.00004111,-0.00002917,-0.00002372,-0.00000328,-0.00$ $000733,0.00701886,0.00503758,-0.01735480,-0.00138340,-0.00122583,-0.00$ $367914,-0.00114008,0.00019010,-0.00040975,-0.00472206,-0.00309659,-0.0$ $0071933,-0.00472542,-0.00801030,0.02648441,-0.08514046,-0.03367456,0.0$ $8637139,0.00110264,0.00120655,-0.00037511,0.00049136,0.00010573,-0.000$ $87319,-0.00000128,0.00018272,0.00013382,0.00076207,0.00149169,-0.00002$ $171,0.08764945,0.00035966,-0.00005150,0.00040107,0.00023567,-0.0000891$ $6,0.00012698,-0.00015974,0.00006520,-0.00024807,-0.00002820,0.00013230$ $, 0.00029443,0.00020652,-0.00025927,0.00006248,-0.00023265,0.00030552,-$ $0.00002291,0.00001000,-0.00016604,0.00010217,-0.00010949,0.00002744,-0$ $.00014411,-0.00001349,-0.00000003,-0.00001163,-0.00004354,-0.00001422$, $-0.00007231,-0.00000431,0.00001025,-0.00004443,0.00000782,0.00000517,-$ $0.00000001,-0.00139640,0.00244014,-0.00095837,-0.00099799,0.00482293,0$ $.00044316,0.00012928,-0.00119048,-0.00029954,-0.00346397,0.00589584,0$. $00037321,-0.00241088,0.00079164,0.00847381,-0.03268433,-0.05872618,0.0$ $7791322,0.00103477,-0.00225965,-0.00094557,-0.00015731,0.00065322,0.00$ $007717,0.00015349,-0.00068055,-0.00005442,0.00137423,-0.00360337,-0.00$ $109180,0.03819086,0.05189079,-0.00046944,-0.00002112,-0.00027303,0.000$ $14804,0.00001954,0.00018970,-0.00013214,-0.00000048,-0.00024609,0.0000$ $6190,0.00001913,0.00023886,-0.00008495,-0.00000832,-0.00012349,0.00008$ $515,0.00003364,0.00003186,0.00014839,0.00001625,-0.00003807,-0.0000579$ $4,-0.00002970,-0.00015937,0.00000379,-0.00001713,-0.00000749,0.0000648$ $6,0.00003921,0.00013730,-0.00002213,-0.00000800,0.00001321,0.00001652$, $-0.00000195,0.00000708,0.01258302,0.00793492,-0.01785300,-0.00462044,0$ $.00011183,-0.00177705,-0.00037747,-0.00048853,0.00049323,0.00015122,0$. $00098574,0.00224799,-0.00093323,0.00018216,0.00070966,0.08302149,0.076$ $90642,-0.28111754,0.00041054,-0.00071352,0.00111882,0.00042830,0.00046$ $434,-0.00083845,0.00008248,-0.00012603,-0.00003078,0.00023176,-0.00092$ $390,0.00053946,-0.09073972,-0.08437450,0.29673720 \backslash 1-0.00000281,0.00000$ $581,0.00000813,-0.00000243,0.00000861,0.00000221,0.00000690,-0.0000005$ $5,0.00000758,0.00000082,-0.00001071,-0.00001631,0.00000195,0.00001430$, $0.00000485,0.00000451,-0.00000721,-0.00001218,0.00000007,-0.00000100,-$ $0.00000038,-0.00000194,-0.00000013,-0.00000493,-0.00000306,0.00000145$, $-0.00000819,-0.00000121,-0.00000471,-0.00000301,0.00000083,-0.00000047$ $, 0.00000498,0.00000070,0.00000114,-0.00000089,0.00000170,-0.00000165,0$ $.00000990,0.00000406,0.00000345,-0.00000295,-0.00000902,-0.00000115,-0$ $.00000257,-0.00000116,-0.00000148,0.00001026,0.00000690,0.00000027,-0$. $00000131,-0.00000719,-0.00000254,-0.00000286,0.00000024,-0.00000124,0$. $00000200,-0.00000246,0.00000154,0.00000334,0.00000078,0.00000214,0.000$ $00211,-0.00000061,-0.00000162,-0.00000092,0.00000243,-0.00000423,0.000$ 00114III@

meta-Addition Product to 4-fluorophenolate

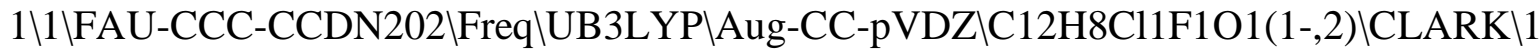
1-Dec-2014\0\\\#N Geom=AllCheck Guess=TCheck SCRF=Check GenChk UB3LYP/A ug-CC-pVDZ Freq IIAddition product p-Cl-Ph-radical + 4-fluoro-phenolate (meta)\-1,2\C,1.3424541,-0.8462015007,0.6130442825\C,2.1377936528,0. $3849273311,0.9828369146 \backslash \mathrm{C}, 3.2841616785,0.8649748448,0.2952797768 \backslash \mathrm{C}, 3.7$ 088556189,0.058229386,-0.8531814926\C,3.0345388133,-1.1207656189,-1.24 $56453925 \backslash \mathrm{C}, 1.9302947728,-1.5442421001,-0.5656029092 \backslash \mathrm{H}, 1.341587581,-1.5$ 
$740611221,1.4570354424 \backslash \mathrm{H}, 1.7755679471,0.956513853,1.8396213574 \backslash \mathrm{O}, 3.931$ $1185312,1.9163308903,0.619155824 \backslash \mathrm{H}, 3.389956275,-1.7141228501,-2.092302$ $3872 \mathrm{VF}, 1.3033490555,-2.7255741435,-0.932580177 \mathrm{~W}, 4.5872529569,0.395734$ $566,-1.4055483643 \backslash \mathrm{C},-2.8234705736,0.1565595061,-0.069844371 \backslash \mathrm{C},-2.50672$ $09038,-0.757843791,0.9279956627 \backslash \mathrm{C},-1.1584536779,-1.0865643574,1.134896$ $0773 \backslash \mathrm{C},-0.1386113138,-0.5086744661,0.3711658848 \backslash \mathrm{C},-0.4971496227,0.4174$ 782101,-0.6220823881\C,-1.831138683,0.7510013091,-0.8563893468\Cl,-4.5 $272468056,0.5865656762,-0.3499746822 \backslash \mathrm{H},-3.2932579079,-1.2079912598,1.5$ $32324503 \backslash \mathrm{H},-0.901305858,-1.8103966354,1.9104642936 \backslash \mathrm{H}, 0.292441098,0.889$ $5458754,-1.2071722113 \backslash \mathrm{H},-2.1020838118,1.4664040691,-1.6317474546 \mid \mathrm{Vers}$ ion $=E M 64 L-G 09 R e v C .01 \backslash$ State $=2-A \backslash H F=-1097.4443442 \backslash S 2=0.780592 \backslash S 2-1=0 . \mid S 2$ $\mathrm{A}=0.750542 \backslash \mathrm{RMSD}=8.438 \mathrm{e}-10 \backslash \mathrm{RMSF}=7.959 \mathrm{e}-06 \backslash$ ZeroPoint $=0.161253 \backslash$ Thermal $=0$. 1740215 ZPE $=101.187788$ Dipole $=-3.6321678,-1.7410374,-0.5368317 \mathrm{VipoleD}$ eriv $=0.2463442,0.0751343,0.1124099,-0.1046886,0.1498251,-0.2117077,0.2$ $299286,-0.3342482,0.0418702,-0.7427222,-0.4908271,-0.4796803,0.1134988$ ,-0.3797678,0.0675624,-0.0133419,0.4636528,0.1301785,0.4258604,1.22209 $61,0.831009,1.2288109,1.2823729,0.2916498,0.5811657,-0.054763,-0.49442$ $08,0.0504677,0.1401573,-0.1255305,-0.3870756,-0.1840847,0.2498189,-0.6$ $131566,-0.0759686,0.1083431,0.3957325,0.0067696,-0.3315486,0.4641663,0$ $.0741154,-0.0399584,-0.1153686,0.2051587,0.1501693,-0.2055007,0.318355$ $7,0.1105424,0.3746664,0.8575301,0.283636,0.1187659,0.3745116,-0.014795$ $1,-0.0534577,-0.0251549,0.0937574,-0.1201034,-0.0954525,0.2195226,0.03$ $6217,0.2050186,-0.0689839,0.0515891,-0.0170833,0.0968895,-0.0470697,0$. $0031548,-0.1124089,0.0864861,-0.1002255,-0.0400662,-1.0345115,-0.72278$ $04,-0.3413445,-1.1835142,-1.4910569,-0.2282322,-0.4588097,-0.2398417,-$ $0.3436312,0.0733328,0.0251175,0.0708352,0.0274725,-0.0060871,-0.143121$ $1,0.1069135,-0.1497801,-0.0511127,-0.2825175,-0.4727404,-0.1921621,-0$. $2787408,-1.1322749,-0.4475794,-0.0364916,-0.3359444,-0.2786659,-0.0722$ $542,-0.0688235,0.110571,-0.0962679,0.0525479,0.0148054,0.1100414,-0.00$ $39801,0.0292913,1.0146815,-0.2569823,0.1303645,-0.4651238,0.0219128,-0$ $.0943771,0.3875362,-0.0758559,0.0184602,-0.297054,0.0665806,-0.0765415$ $, 0.1817101,-0.073645,-0.0418974,-0.1255293,-0.0558476,-0.0667957,-0.12$ $87032,-0.0089778,0.0094301,-0.0710675,-0.0199986,-0.1332321,0.1180996$, $-0.0930746,0.0223712,0.6064723,-0.0035291,0.0246206,-0.0668016,-0.0268$ $364,0.1032992,-0.0782669,0.0677172,-0.0437168,-0.1061902,0.0386805,-0$. $0116595,0.0957101,-0.0317969,-0.0354142,-0.0592536,-0.0362131,-0.04600$ $44,-0.0707402,0.0023567,0.022196,0.1527575,-0.0289009,-0.0752788,-0.19$ $5307,-0.080847,-0.0218208,-0.9908504,0.1683198,-0.0948817,0.2315337,-0$ $.1607249,0.0333846,-0.1333981,0.0360756,-0.1384319,-0.0592428,-0.05010$ $99,0.0787683,-0.0421796,0.0788821,0.0549904,0.0846777,0.0598542,0.0472$ $924,0.0640862,0.0557447,-0.0620286,-0.0014812,0.0100913,0.1186504,-0.0$ $290112,0.1076133,-0.0053684,0.0723588,-0.0398409,0.0530949,-0.033087,0$ $.0620398,0.0300016,0.0664462,0.0227,0.0394752,0.0428191,0.0375367,-0.0$ $291115,0.0268745,0.0381543,0.0958859,-0.0683434,0.0942878,0.0263623 \backslash \mathrm{Po}$ lar $=282.641007,11.5182067,158.2143999,-19.5593775,-0.8381758,156.68237$ 69\PG $=\mathrm{C} 01[\mathrm{X}(\mathrm{C} 12 \mathrm{H} 8 \mathrm{Cl} 1 \mathrm{~F} 1 \mathrm{O} 1)] \mathrm{NImag}=0 \backslash 10.40511184,0.00962730,0.46750717$, $-0.03686430,-0.01892491,0.48925783,-0.09301469,-0.05653476,-0.00566834$ $, 0.42193092,-0.04786124,-0.14730114,-0.03920563,0.13120942,0.40847718$, $0.00500092,-0.02799908,-0.07964220,-0.20444665,0.16751913,0.42825442,--$ $0.01112755,-0.00312067,0.00814940,-0.17566885,-0.04717299,0.07799000,0$ $.42579191,-0.02079754,-0.01111440,0.01055230,-0.05592884,-0.09945378,0$ 
$.00982922,0.21539325,0.55149451,-0.01033737,-0.00557280,-0.00154083,0$. $07376784,0.00219793,-0.11858801,-0.06181211,0.14000452,0.35529422,0.00$ $108526,0.00051772,-0.00392724,-0.00991183,0.01160746,0.02650652,-0.104$ $47432,0.01522794,0.05379774,0.47290891,0.00575937,-0.00030854,-0.00252$ $665,0.02063468,0.01826380,-0.01072712,-0.02125534,-0.10636938,-0.01869$ $468,0.20339557,0.44089840,0.00045103,0.00219048,-0.00077762,0.03455687$ $,-0.00140620,-0.02842895,0.02186084,-0.05230902,-0.13408153,-0.1925495$ $7,0.11928596,0.38286116,-0.00993681,-0.00224788,0.01302047,-0.01536963$ $,-0.00698567,0.01079286,0.04293535,-0.00168493,-0.04146343,-0.11853083$ $,-0.05243078,0.02411379,0.46431228,0.00584508,0.00966250,-0.00023140,-$ $0.00756237,-0.00315970,0.00391442,0.01227399,-0.02081987,-0.03146689,-$ $0.10349971,-0.21328928,-0.05155682,0.13467720,0.48113867,0.01998985,0$. $00506871,-0.00772199,0.00953339,0.00327250,-0.00616812,-0.02954427,-0$. $01840423,0.01267070,-0.01785230,-0.09655741,-0.11110888,-0.22438581,0$. $19302915,0.48772103,-0.09101780,0.02350738,0.05561419,0.01685341,-0.00$ $331910,-0.01758920,-0.02069838,0.00551627,0.02302457,-0.03275905,-0.06$ $692783,-0.01829150,-0.25429658,-0.04094805,0.16664843,0.49825199,0.019$ $71596,-0.08847878,-0.05096583,0.00596611,-0.01732807,-0.02496606,-0.00$ $607015,-0.00109353,0.00596220,-0.03264687,-0.02068399,0.00513352,-0.08$ $763438,-0.09721741,0.03515641,0.17090910,0.40074202,0.05406104,-0.0507$ $6589,-0.15954753,-0.00986047,-0.01339672,-0.00219701,0.01682598,-0.003$ $18068,-0.02013929,0.00802638,0.03487861,0.03026169,0.13117327,-0.00418$ $613,-0.16872597,-0.24056539,0.09480867,0.40749373,-0.03439228,0.000218$ $70,0.00469858,-0.00296225,-0.01352217,0.00978341,-0.00103572,-0.001246$ $64,0.00116881,-0.00079278,-0.00106936,-0.00025368,0.00139974,0.0015049$ $1,-0.00022535,-0.00277059,-0.00848971,0.01404813,0.05490989,0.00886442$ $,-0.12449714,0.10772342,-0.00963568,-0.01911102,0.01145763,-0.00003614$ $,-0.00200176,0.00370935,-0.00068516,-0.00193320,-0.00140129,0.00298631$ ,-0.00071986,-0.00245967,-0.00332947,0.00852905,-0.00910853,-0.0028117 $4,0.14365243,-0.00846457,0.10424320,-0.16670581,0.00293795,-0.00133987$ $, 0.01018763,-0.00300087,-0.00018637,0.00302751,0.00173776,0.00116833,-$ $0.00142052,-0.00319339,-0.00417152,-0.00211381,0.00947721,0.01366613,-$ $0.02794399,0.00029361,-0.11865403,0.18902448,0.00268369,-0.00867190,-0$ $.01440467,-0.07253935,0.03858862,0.08698098,-0.00058993,0.01539828,0.0$ $2018572,-0.00248429,-0.00373665,0.00349187,-0.00040913,-0.00054579,-0$. $00012345,-0.00203590,-0.00174722,0.00057394,0.00035325,-0.00014463,0.0$ $0025419,0.07268340,0.00905348,-0.01173587,-0.01804140,0.03945848,-0.11$ $573391,-0.12631432,-0.00700412,0.00665401,0.00707484,-0.00268196,0.000$ $87342,-0.00091707,0.00024948,-0.00023005,-0.00031183,-0.00388010,-0.00$ $154064,0.00208833,0.00067263,0.00090332,0.00039518,-0.03957800,0.12174$ $908,0.00151039,-0.00007000,-0.00399297,0.08689071,-0.12389465,-0.21217$ $610,0.00194184,-0.01404138,-0.00829716,0.00498340,0.00042868,0.0003368$ $5,0.00030433,0.00077111,0.00031583,-0.00122087,-0.00000416,0.00171137$, $-0.00054305,-0.00115767,0.00025631,-0.09265175,0.13587715,0.22255878,0$ $.00079199,-0.00192592,0.00249804,-0.03884440,-0.05266919,0.00594626,-0$ $.15652688,-0.16117558,-0.05455511,0.01530032,-0.00089544,0.00026836,-0$ $.00590008,-0.00266493,0.00599982,0.00286631,0.00229415,-0.00236528,-0$. $00034759,-0.00125436,0.00001930,0.00030887,0.00302763,-0.00265516,0.18$ $296045,0.00049028,0.00568987,0.00038751,-0.04563797,-0.02598397,0.0053$ $1307,-0.15807479,-0.32968082,-0.08814259,-0.01849765,-0.04208190,-0.03$ $595356,0.00402831,0.00282582,-0.00375071,-0.00270539,0.00117642,0.0023$ 
$5658,-0.00031368,-0.00142427,-0.00053207,0.00091290,-0.00008546,0.0026$ $9213,0.21961338,0.39690478,0.00491655,0.00143044,0.00363555,0.01213254$ $, 0.01282603,0.01587079,-0.05166623,-0.08539579,-0.09068065,-0.01541593$ ,-0.05246157,-0.01558788,0.01183738,0.00268953,-0.00639238,-0.00522491 -0.00084901,0.00441462,-0.00071519,-0.00171070,-0.00110624,-0.0038292 $2,0.00123959,0.00030445,0.04976233,0.11999061,0.09013763,-0.00075950,-$ $0.00443751,0.00346422,-0.00009337,-0.00018476,-0.00091662,0.00056518,-$ $0.00359249,0.00200998,0.00870476,-0.00754625,-0.01186268,-0.07893511,0$ $.04093202,0.07733093,-0.00347054,0.01522284,0.02134108,-0.00071467,0.0$ $0067844,0.00107455,-0.00001512,0.00010738,-0.00006385,-0.00020815,-0.0$ $0027833,-0.00088609,0.07527536,-0.00349576,0.00185913,-0.00133306,0.00$ $015257,-0.00017322,0.00044213,-0.00504058,0.00087793,0.00062339,0.0113$ $5531,-0.00819245,-0.02063777,0.04202355,-0.12741399,-0.11969411,-0.004$ $33755,0.00586406,0.00460288,0.00040182,-0.00017174,-0.00047038,0.00014$ $072,-0.00005670,-0.00001687,0.00003391,-0.00218288,-0.00130702,-0.0443$ $0038,0.12887242,0.00415176,-0.00037670,0.00303160,-0.00027417,0.000870$ $89,0.00019651,0.00019362,-0.00147699,0.00474316,0.00378277,-0.00383960$ $,-0.00406999,0.08033506,-0.11725949,-0.21075853,0.00327666,-0.01403263$ ,-0.00987692,-0.00009106,0.00036581,0.00017616,-0.00003365,-0.00000434 ,-0.00012713,-0.00037803,-0.00072908,-0.00115046,-0.08713855,0.1330132 $8,0.21988015,0.00322899,0.00238088,-0.00305111,-0.00361788,-0.00161528$ $, 0.00380856,0.00865083,0.00150703,-0.00717698,-0.00213590,0.00384630,0$ $.01047085,-0.01977989,-0.04181928,-0.01408155,-0.08651020,-0.08409866$, $-0.00124154,0.00076134,0.00227197,-0.00012298,0.00059728,0.00011731,0$. $00041518,-0.00271138,-0.00149437,0.00090312,-0.00002467,0.00262546,-0$. $00272724,0.10044673,-0.01564991,-0.03104338,-0.00745548,0.00069616,-0$. $00237145,-0.00175781,0.01204814,0.00346960,-0.00732317,-0.00667041,0.0$ $0348273,0.00485694,-0.01360551,-0.02488823,-0.00747355,-0.08752695,-0$. $18293772,-0.05489832,0.00103726,0.00375918,-0.00046815,-0.00156661,-0$. $00119041,-0.00059452,-0.00464775,-0.00379972,0.00103561,0.00202430,0.0$ $0031653,0.00254249,0.11645514,0.23458065,-0.01860901,-0.02727146,-0.00$ $549234,0.00475005,-0.00166954,-0.00435014,0.00096155,0.00181652,0.0003$ $1469,0.00265702,-0.00392196,-0.00067114,0.00622075,0.01622480,0.008994$ $70,-0.00144219,-0.05663147,-0.06052238,-0.00120718,0.00117407,0.002673$ $43,-0.00096068,-0.00218371,0.00004641,-0.00155774,-0.00153052,-0.00029$ $517,-0.00316175,0.00217205,-0.00038254,0.01257462,0.07174980,0.0586354$ $7,0.00042106,0.00039138,-0.00050128,0.00052579,0.00120878,0.00302708,-$ $0.00491762,-0.00963881,0.00218294,-0.22561798,-0.07783456,0.12757730,-$ $0.00789195,-0.00111916,0.00835563,0.00221021,-0.00178896,-0.00134374,0$ $.00015469,0.00003676,-0.00000074,-0.00084943,0.00021585,0.00000184,0.0$ $0104901,0.00217378,-0.00177542,-0.00052999,0.00081708,-0.00171744,-0.0$ $0043553,-0.00123273,0.00083613,0.23586495,0.00000404,-0.00015865,0.000$ $15841,0.00010390,-0.00099507,-0.00327283,0.01067813,0.00843556,-0.0083$ $8503,-0.07798721,-0.06982242,0.03330419,-0.01974154,-0.00457129,0.0133$ $8865,-0.00088835,-0.00205202,-0.00286475,-0.00013408,-0.00002184,0.000$ $03735,0.00112452,-0.00000529,-0.00016955,0.00047485,0.00045896,0.00250$ $345,0.00153429,-0.00000583,0.00122275,-0.00205621,-0.00069917,-0.00003$ $444,0.08679425,0.06940226,-0.00060221,0.00001617,0.00026453,0.00170428$ ,-0.00453810,-0.00340208,0.02074210,0.01141925,-0.00375512,0.12742435, $0.03330982,-0.11666396,-0.00829184,-0.00458927,0.00575916,-0.00035294$, $-0.00212507,0.00106570,0.00004546,-0.00011862,-0.00012439,0.00090725,0$ 
$.00075320,-0.00120162,-0.00334510,0.00088787,0.00126921,-0.00112794,0$. $00183214,-0.00048169,-0.00005374,-0.00092209,0.00079541,-0.13678711,-0$ $.03580400,0.11638036,-0.00112783,-0.00104919,0.00057317,-0.00078053,-0$ $.00010105,0.00066173,-0.00045285,0.00012366,-0.00105608,0.00027152,0.0$ $0003852,0.00000564,-0.00005198,0.00019103,-0.00006600,-0.00085302,-0.0$ $0006310,0.00018112,0.00012064,0.00015140,-0.00002791,-0.00000740,-0.00$ $006440,-0.00009540,0.00027810,0.00002435,0.00033424,0.00006514,-0.0000$ $2212,-0.00002741,0.00044789,0.00006926,-0.00015019,0.00003479,0.000025$ $67,-0.00002411,0.48526588,-0.00020503,-0.00086379,0.00076149,-0.002260$ $88,0.00004561,0.00006512,0.00023695,0.00148331,0.00007301,0.00038329,-$ $0.00057141,-0.00081725,0.00067493,0.00076258,-0.00030434,-0.00179417,-$ $0.00016780,-0.00006087,-0.00014300,0.00025046,-0.00027808,0.00000647,-$ $0.00011920,-0.00005014,-0.00056854,-0.00149891,-0.00031026,0.00007659$, $0.00003498,0.00012519,0.00062329,0.00040106,0.00008053,-0.00005515,-0$. $00001460,0.00003925,0.00582592,0.34899316,0.00038774,0.00099386,-0.001$ $17324,0.00287859,-0.00040501,-0.00022213,-0.00038731,-0.00185373,-0.00$ $001738,-0.00042227,0.00068629,0.00094016,-0.00084920,-0.00095781,0.000$ $27616,0.00240494,0.00027419,-0.00006202,0.00005308,-0.00027938,0.00059$ $508,-0.00014638,0.00016292,-0.00005059,0.00064213,0.00177220,0.0003726$ $7,-0.00008781,-0.00002152,-0.00009693,-0.00089554,-0.00067843,-0.00001$ $562,0.00006036,0.00003662,-0.00001889,-0.04940852,-0.26234480,0.420097$ $06,0.00032919,-0.00128295,0.00271613,-0.00240941,0.00098656,-0.0005125$ $6,0.00080442,0.00107950,0.00058425,0.00031878,-0.00040657,-0.00082483$, $0.00066858,0.00081890,-0.00015470,-0.00254626,-0.00053876,-0.00072845$, $0.00004709,0.00035219,-0.00074403,0.00003265,-0.00016483,0.00000285,-0$ $.00056617,-0.00124002,-0.00044897,0.00006235,-0.00001230,0.00013006,0$. $00096915,0.00063930,0.00015487,-0.00002293,-0.00001691,-0.00000429,-0$. $10957811,0.00641480,-0.00123713,0.68603225,-0.00098077,0.00346479,0.00$ $444133,0.00255442,-0.00034823,-0.00049013,0.00017179,-0.00108891,0.000$ $03316,-0.00040805,0.00046341,0.00067186,-0.00044575,-0.00006531,0.0003$ $7008,-0.00038577,-0.00025914,-0.00041320,-0.00006742,-0.00001495,0.000$ $22205,-0.00008367,0.00009529,-0.00007002,0.00037390,0.00119210,0.00019$ $514,-0.00004721,-0.00013527,0.00007378,-0.00030333,-0.00032117,-0.0000$ $3503,0.00002461,0.00001039,-0.00003184,0.04879460,-0.18440132,0.132117$ $57,-0.00203571,0.37785379,0.00067658,0.00470289,0.00097044,-0.00024358$ ,-0.00023006,0.00003908,0.00079548,0.00072388,0.00000578,0.00011787,-0 $.00012333,-0.00038977,0.00031530,0.00084041,0.00013206,-0.00199980,-0$. $00008809,0.00009690,0.00001075,-0.00015470,0.00019049,-0.00001624,0.00$ $006069,-0.00005183,-0.00031603,-0.00058215,-0.00013338,0.00003516,-0.0$ $0006901,0.00008444,0.00031279,-0.00014561,-0.00009198,-0.00003878,-0.0$ $0003913,0.00004183,-0.04944678,0.13770604,-0.21217851,-0.06506274,-0.2$ $8389744,0.45523307,-0.02541144,-0.00998497,0.01516796,0.00041598,0.001$ $03817,0.00247769,-0.00086737,-0.00101682,-0.00148478,-0.00020279,0.000$ $05597,0.00052474,-0.00018222,0.00049162,0.00083196,-0.00171521,-0.0031$ $8320,-0.00112523,0.00070746,-0.00234964,0.00297193,-0.00001277,-0.0002$ $3050,-0.00053073,-0.00018068,0.00006741,0.00051928,-0.00027281,-0.0000$ $9088,0.00024878,0.00035590,0.00111807,0.00034335,-0.00003961,0.0000172$ $1,-0.00002988,0.00022105,0.06023539,-0.06732024,-0.30919959,0.02724071$ $,-0.00072934,0.65682578,0.00247324,0.00626015,-0.00242755,0.00030392,0$ $.00013364,0.00047071,0.00018478,0.00033625,-0.00045723,0.00038828,0.00$ $007599,-0.00018026,0.00007002,0.00019818,-0.00012902,0.00045851,-0.001$ 
$07093,0.00061147,0.00199649,0.00023521,-0.00091240,0.00013384,0.000057$ $84,0.00010935,-0.00001030,-0.00005734,0.00008796,-0.00005879,0.0001200$ $8,-0.00020763,0.00055847,0.00016401,-0.00010972,0.00001940,-0.00000604$ $, 0.00006132,0.02229188,-0.00135573,0.00797940,0.07025521,-0.09888682,0$ $.03454478,-0.04187433,0.39350670,0.00016466,0.00086568,0.00650214,-0.0$ $0072773,-0.00019938,-0.00186610,-0.00037324,0.00021586,0.00133879,-0.0$ $0036778,-0.00050482,0.00012665,0.00026539,0.00038882,0.00001601,-0.001$ $53062,-0.00042671,-0.00015189,-0.00209170,-0.00041897,0.00062438,0.000$ $36930,-0.00002515,0.00039385,0.00027628,0.00011111,-0.00032405,-0.0000$ $4461,-0.00004057,0.00013127,0.00006332,0.00052134,0.00000119,-0.000014$ $93,0.00005636,-0.00005669,-0.02355014,0.00387774,0.00615268,-0.0492110$ $1,0.03897768,-0.09839656,-0.01481149,-0.28857809,0.45466943,-0.1401454$ $5,0.03350661,-0.02061072,-0.02575260,-0.01449287,-0.00953947,-0.001732$ $45,-0.00038363,0.00082584,-0.00158127,-0.00083079,0.00063692,0.0018835$ $3,0.00080108,-0.00291186,-0.02262693,0.01271146,0.01441390,-0.00964105$ $, 0.00231296,-0.00113102,0.00239406,0.00088647,0.00186138,0.00229252,0$. $00238400,-0.00019490,0.00047635,-0.00017921,0.00002742,0.00020135,-0.0$ $0344016,-0.00193644,0.00013859,0.00010139,-0.00021560,-0.00182947,-0.0$ $1133903,0.01249102,-0.04743569,-0.00074678,0.00767441,-0.22610414,-0.0$ $7597960,0.10407173,0.58734724,0.01851242,-0.07355969,0.01179513,-0.022$ $45157,0.00522702,-0.00206944,-0.00323416,-0.00107516,0.00012703,0.0003$ $5791,-0.00095191,-0.00074348,0.00026335,-0.00126050,-0.00108992,0.0143$ $3422,-0.00557509,-0.00227610,0.02067472,-0.00826894,0.00724696,-0.0003$ $9731,0.00009627,-0.00007326,-0.00045768,-0.00194237,-0.00004508,-0.000$ $22703,0.00039632,-0.00016980,0.00182651,0.00183808,0.00046139,0.000010$ $08,0.00000604,-0.00015734,-0.01270004,-0.03178871,0.02994830,-0.029658$ $42,0.02987124,-0.02356996,-0.03696471,-0.12623902,0.07269930,-0.009824$ $36,0.38909985,-0.00719340,0.00958172,-0.07299047,-0.00543700,-0.002622$ $35,-0.00054323,-0.00377609,0.00121529,0.00207905,0.00126137,-0.0006088$ $1,-0.00088029,-0.00049698,-0.00382774,-0.00330669,0.02811173,-0.004056$ $60,0.00429801,-0.02485322,0.01083579,-0.00816508,-0.00049587,0.0003252$ $3,0.00030847,0.00066322,-0.00085428,-0.00062044,0.00004338,0.00084070$, $-0.00133562,0.00021841,0.00091106,0.00015768,0.00014316,-0.00010408,0$. $00005445,0.01382546,0.02982585,-0.04172639,0.03972184,-0.02645639,0.03$ $186101,0.05905856,0.07774475,-0.16045319,-0.04153451,-0.23835110,0.445$ $05354,-0.00982002,0.02013170,-0.02085060,-0.00037195,0.00159746,-0.000$ $07209,-0.00120693,0.00251220,-0.00012118,0.00037585,-0.00114237,-0.000$ $54434,0.00045995,0.00085280,-0.00001611,-0.00064872,-0.00020701,-0.002$ $29330,-0.00573822,0.00258244,-0.00193924,-0.00001775,-0.00026180,-0.00$ $009520,-0.00007494,-0.00184001,-0.00015089,0.00008921,-0.00007149,0.00$ $014631,0.00049761,0.00037584,0.00003514,-0.00003428,-0.00001747,-0.000$ $02339,-0.05415750,-0.03244094,0.04310234,-0.02624062,0.02244429,-0.022$ $53644,0.04661680,-0.01001395,0.00783048,-0.12704668,0.01383820,-0.0089$ $8388,0.71079807,0.00825658,-0.00164277,0.00487018,-0.00029982,-0.00061$ $942,-0.00104120,-0.00069908,-0.00001072,0.00079600,-0.00012570,-0.0005$ $3846,0.00001094,0.00008799,-0.00025482,0.00027109,0.00063417,0.0007714$ $7,0.00161512,0.00077194,0.00042293,0.00045569,0.00012731,0.00040117,0$. $00025699,0.00037558,0.00026412,-0.00008610,-0.00003056,0.00006579,0.00$ $003752,-0.00044105,-0.00012955,0.00001447,-0.00002909,0.00005416,0.000$ $00820,0.00434737,0.03160586,-0.02729570,0.02038524,-0.01969227,0.01682$ $927,-0.03431699,-0.01330785,0.02581911,0.04798916,-0.17635689,0.116351$ 
$90,-0.00494323,0.37819918,-0.00871099,0.00151448,-0.00079719,-0.001437$ $40,0.00278710,0.00056258,0.00057065,0.00062586,-0.00120626,0.00015050$, $0.00021479,-0.00050488,0.00031894,0.00011967,0.00014183,-0.00082819,-0$ $.00130579,0.00001096,0.00009255,0.00010859,0.00078666,0.00002388,-0.00$ $004895,-0.00005167,-0.00024828,-0.00038469,0.00016652,-0.00003990,0.00$ $007133,0.00003990,0.00085088,0.00039461,0.00003357,0.00000768,-0.00001$ $050,0.00008999,0.00169684,-0.02274922,0.03252181,-0.01939739,0.0164095$ $5,-0.01908694,0.03431620,0.02374326,-0.02305464,-0.04668861,0.12024007$ ,-0.19557411,-0.05080811,-0.26805679,0.43663757,0.00238187,0.00125516, $-0.00102186,0.00244106,0.00028089,-0.00057147,0.00053538,-0.00205432,0$ $.00072172,-0.00045052,0.00075572,0.00058219,-0.00057395,-0.00091805,0$. $00041362,0.00212622,-0.00029694,-0.00010842,0.00029511,-0.00071839,0.0$ $0063586,-0.00013892,0.00021088,-0.00012310,0.00023149,0.00148293,0.000$ $08768,-0.00018159,0.00002821,-0.00008664,-0.00064033,-0.00013950,0.000$ $27898,0.00001268,0.00000773,0.00000549,-0.19640249,-0.04169521,0.06341$ $936,0.04435617,-0.03669413,0.03692048,-0.05648406,-0.00476798,0.010318$ $67,0.00005951,0.05205686,-0.05816568,-0.32635441,0.03121294,-0.0077926$ $7,0.65522656,-0.00081086,0.00042186,0.00486861,0.00028508,0.00053622,-$ $0.00084170,-0.00014197,-0.00002659,0.00023730,-0.00012325,0.00018815,0$ $.00008947,-0.00018763,-0.00017577,0.00010237,-0.00029386,0.00022633,0$. $00025219,0.00095884,-0.00036692,0.00037566,0.00012290,-0.00004804,0.00$ $017440,0.00027650,0.00039196,0.00001789,-0.00000482,-0.00006803,-0.000$ $02691,-0.00022805,-0.00013848,-0.00017961,0.00002685,-0.00001535,-0.00$ $002469,-0.08456098,-0.12646485,0.08291334,-0.00615251,-0.01350584,0.02$ $631003,-0.00979708,-0.00335227,0.00093660,0.02467793,0.00136710,0.0033$ $7348,0.07533303,-0.10164885,0.03802030,-0.04871113,0.39716123,0.000424$ $38,0.00486138,-0.00019370,-0.00033681,0.00014622,0.00037684,-0.0001340$ $3,0.00069639,0.00020317,0.00011666,-0.00041949,-0.00042852,0.00039855$, $0.00045519,0.00009025,-0.00081068,-0.00059125,-0.00001732,-0.00094484$, $0.00048798,-0.00045390,0.00002413,-0.00018577,-0.00000047,-0.00024040$, $-0.00072576,-0.00021958,-0.00000679,0.00002549,0.00002835,0.00044922,0$ $.00058059,0.00015871,-0.00000186,-0.00001928,-0.00002439,0.11098726,0$. $07768920,-0.16278820,0.00269522,0.03012660,-0.02468076,0.01694045,0.00$ $022289,-0.00569536,-0.02618447,-0.00036501,0.00975970,-0.05626369,0.04$ $348687,-0.10244029,-0.00327336,-0.30033218,0.46563508,0.00022068,-0.00$ $030561,0.00037663,-0.00091839,0.00048433,0.00011842,0.00031872,0.00117$ $220,0.00048447,0.00003997,-0.00055299,-0.00052138,0.00054811,0.0005748$ $6,-0.00006061,-0.00073170,-0.00049022,-0.00035747,-0.00010746,-0.00005$ $918,-0.00000398,0.00007225,-0.00007910,-0.00001358,-0.00071848,-0.0013$ $5985,-0.00040855,-0.00003774,0.00002092,0.00014123,0.00012099,0.000509$ $28,0.00026670,-0.00007275,-0.00004018,0.00003750,-0.12631083,0.0228282$ $8,-0.01358959,-0.02769594,0.00648904,-0.00336990,0.00230399,0.00236557$ ,-0.00229033,0.00010745,0.00087636,-0.00118365,0.00036731,-0.00433990, $0.00552397,-0.03958758,0.01285880,-0.00917151,0.19200948,0.00001018,-0$ $.00031185,-0.00056747,0.00033998,-0.00005857,0.00002688,-0.00003050,-0$ $.00045189,-0.00018615,-0.00002988,0.00018786,0.00022217,-0.00016274,-0$ $.00017027,-0.00002187,0.00028548,0.00019516,0.00006392,-0.00001184,0.0$ $0008780,-0.00007898,-0.00001161,0.00002833,0.00002495,0.00019492,0.000$ $48145,0.00013594,0.00001358,-0.00000408,-0.00005210,-0.00004600,-0.000$ $12927,-0.00006412,0.00002185,0.00000951,-0.00001014,0.02331376,-0.0342$ $7063,0.01283685,0.02499179,-0.00259131,0.00355595,-0.00193556,0.000889$ 
$16,0.00464325,0.00011705,0.00009906,-0.00094972,0.00046198,0.00154398$, $0.00292456,-0.00646237,0.00433960,-0.00054165,-0.04127009,0.03177373$,$0.00003147,-0.00052416,-0.00014652,-0.00020517,0.00007403,0.00019725,0$ $.00009070,0.00012507,-0.00000177,0.00000251,-0.00011065,-0.00004030,0$. $00012202,0.00015354,-0.00001468,-0.00016877,-0.00001118,-0.00005331,0$. $00005464,-0.00004964,0.00003115,0.00001464,-0.00000622,0.00000245,-0.0$ $0013583,-0.00021175,-0.00003554,-0.00001070,0.00001154,0.00001926,0.00$ $001224,0.00006293,0.00005105,-0.00001866,-0.00000521,0.00000497,-0.013$ $48130,0.01281773,-0.03355629,-0.02481596,0.00599823,-0.00042038,0.0028$ $0366,0.00417076,-0.00020836,-0.00044696,-0.00101649,0.00048065,0.00011$ $333,0.00345039,0.00030819,0.01202201,-0.00287195,0.00510128,0.02503423$ ,-0.01848615,0.02897455,0.00018846,-0.00060792,0.00095577,-0.00027362, $-0.00000160,-0.00000907,-0.00001295,0.00021102,0.00008608,-0.00005076$, $-0.00015323,-0.00008518,0.00015895,0.00016896,-0.00002306,-0.00041461$, $-0.00023648,-0.00011037,0.00000739,0.00000747,-0.00001451,0.00000819$, $0.00004866,-0.00001896,-0.00011386,-0.00024875,-0.00008129,-0.00000989$ ,-0.00000083,0.00004572,0.00012426,0.00024444,0.00008200,-0.00001507,-$0.00000345,0.00000008,0.00511403,0.00497771,-0.00596812,-0.20102416,-0$ $.08490895,0.11625932,-0.01501563,-0.01349797,0.01739848,-0.00424305,0$. $00287158,-0.00173260,-0.00047024,0.00055341,-0.00075430,0.00111894,0.0$ $0164283,-0.00132367,0.00149422,0.00016683,-0.00067829,0.21193965,0.000$ $22261,-0.00007525,-0.00033561,0.00011450,-0.00004388,0.00025967,0.0001$ $7967,-0.00000670,-0.00026170,-0.00004224,0.00005316,0.00008994,-0.0000$ $2371,0.00000350,0.00002042,-0.00002028,0.00024236,0.00003031,0.0000217$ $3,0.00005712,0.00005845,-0.00000426,0.00000042,0.00000269,-0.00006022$, $0.00001316,0.00005920,0.00001636,-0.00000807,0.00000340,-0.00008453,-0$ $.00014004,0.00000530,-0.00000188,-0.00000187,0.00000497,-0.01615299,-0$ $.00451178,0.01041024,-0.08235633,-0.09201001,0.07651703,0.00587833,0.0$ $0639952,-0.00403669,0.00231057,0.00389464,0.00286005,0.00036230,-0.000$ $86098,-0.00064946,0.00242577,0.00071294,0.00493229,-0.00044648,-0.0010$ $8236,-0.00195328,0.08897151,0.08953816,-0.00045092,-0.00022910,-0.0000$ $6008,0.00012673,0.00005988,0.00022481,0.00014096,-0.00013644,-0.000229$ $28,-0.00000985,0.00010542,0.00009033,-0.00007305,-0.00002838,0.0000813$ $2,-0.00001420,0.00022580,-0.00007772,0.00003919,0.00006850,0.00001860$, $-0.00001431,0.00000696,-0.00000646,-0.00000605,0.00010214,0.00007962,0$ $.00001546,-0.00001386,0.00000392,-0.00009594,-0.00017375,-0.00001966,0$ $.00000045,0.00000274,0.00000015,0.01782317,0.00799336,-0.00832966,0.11$ $366736,0.07670187,-0.13365121,-0.00466280,-0.00171202,0.00593493,-0.00$ $097030,0.00273371,0.00370208,-0.00087905,-0.00062131,-0.00064509,-0.00$ 207850,0.00504186,-0.00008075,0.00003137,-0.00206549,-0.00053728,-0.12 $334982,-0.08608040,0.13532725,0.00075746,-0.00224772,0.00148078,0.0000$ $2701,0.00047607,0.00012818,0.00003071,-0.00017018,-0.00034044,0.000098$ $17,0.00027150,0.00005754,-0.00026823,-0.00021757,0.00010105,0.00023906$ $, 0.00024171,-0.00029867,0.00001814,0.00077483,-0.00079290,0.00000476,-$ $0.00000078,0.00005001,0.00006470,0.00020132,0.00015513,0.00002194,-0.0$ $0002181,-0.00000030,-0.00016675,-0.00025360,-0.00006343,0.00000944,0.0$ $0000392,0.00000032,-0.00377568,0.00088370,0.00038168,-0.00402854,0.020$ $30043,-0.02162638,-0.07073135,0.04336971,-0.04470191,0.00849749,-0.013$ $70909,0.01492430,-0.00287392,-0.00169488,0.00297416,-0.00090400,-0.000$ $23874,0.00027056,-0.00134503,0.00092770,-0.00090475,0.00109916,-0.0005$ $2800,-0.00009723,0.07281094,0.00218749,-0.00209207,-0.00185657,-0.0007$ 
$1605,-0.00015528,0.00003410,-0.00024469,0.00022964,0.00000950,0.000025$ $67,-0.00011770,-0.00010793,0.00012416,-0.00023088,-0.00021977,0.000570$ $55,0.00052329,-0.00002652,-0.00051205,0.00072706,-0.00059983,0.0000123$ $8,-0.00014378,0.00000929,0.00000073,-0.00023897,-0.00006484,0.00003965$ $, 0.00002709,-0.00004201,-0.00000651,0.00007582,-0.00000406,0.00000003$, $-0.00000550,-0.00001282,0.00130821,0.00420381,0.00207374,-0.00016466$, $0.00161210,0.00484540,0.04353993,-0.16485333,0.14023047,0.00372177,-0$. $00526425,0.00998401,-0.00247570,0.00274366,0.00421312,-0.00031465,-0.0$ $0013495,-0.00077848,0.00010385,0.00012370,0.00037041,-0.00020576,-0.00$ $185543,-0.00229978,-0.04754488,0.16890920,-0.00196879,-0.00217640,-0.0$ $0157515,-0.00038600,0.00004173,0.00065826,-0.00004864,-0.00000914,-0.0$ $0019074,0.00009641,0.00002782,-0.00002135,-0.00008836,-0.00024482,0.00$ $004293,0.00098532,0.00047039,0.00001341,0.00070854,-0.00043496,0.00047$ $163,-0.00003619,-0.00001898,-0.00005715,-0.00008232,-0.00015703,0.0000$ $3428,-0.00003458,0.00006172,-0.00006971,-0.00034109,-0.00033439,0.0000$ $4662,-0.00000421,-0.00001021,0.00000146,-0.00024361,0.00205688,0.00414$ $703,0.00084523,0.00271017,-0.00030652,-0.04403773,0.14002984,-0.185924$ $25,-0.00619523,0.01236549,-0.00866904,0.00380849,0.00397683,0.00155188$ $, 0.00053632,-0.00068599,-0.00015867,-0.00002879,0.00028978,0.00009046$, $-0.00048736,-0.00233547,-0.00135612,0.04753836,-0.15577359,0.19230675$, $-0.00088776,-0.00010853,-0.00098027,0.00012936,0.00049375,0.00041121,-$ $0.00025874,-0.00058549,-0.00069021,-0.00010111,0.00014736,0.00039168,-$ $0.00032184,0.00010977,0.00022334,0.00041700,0.00059169,-0.00011380,0.0$ $0018779,-0.00018660,0.00019742,-0.00002512,0.00001270,0.00007519,0.000$ $09143,0.00048938,0.00021692,0.00001703,-0.00004297,-0.00001654,-0.0003$ $9285,-0.00058436,-0.00012795,0.00002038,0.00001593,-0.00000613,-0.0028$ $7651,0.00201201,-0.00155927,0.00002393,0.00044980,-0.00059853,0.000156$ $98,0.00143119,-0.00116341,0.01011813,0.00295868,-0.00413665,-0.2019941$ $7,-0.08719056,0.11129089,-0.01610327,-0.01257592,0.01583086,-0.0013624$ $2,-0.00023608,0.00032735,-0.00001410,-0.00008035,0.00002109,0.00035059$ $, 0.00052831,-0.00042450,0.21179668,-0.00103172,-0.00168439,-0.00200176$ $,-0.00055103,0.00028126,0.00024179,0.00106878,0.00013893,-0.00046062,0$ $.00008395,0.00036326,0.00025989,0.00003383,0.00002135,-0.00024618,-0.0$ $0008619,0.00011941,-0.00006743,-0.00004741,0.00000847,-0.00010675,-0.0$ $0008229,0.00003741,-0.00018930,-0.00036519,-0.00036322,0.00006108,0.00$ $004823,-0.00001830,-0.00002791,-0.00003214,-0.00031177,-0.00002044,-0$. $00004684,-0.00000733,0.00000712,0.00186529,0.00398126,0.00319540,0.000$ $39722,-0.00074339,-0.00053330,0.00221518,0.00114094,0.00564337,-0.0157$ $4279,-0.00459642,0.01136344,-0.08724257,-0.10013275,0.07652720,0.00760$ $287,0.00679073,-0.00405752,0.00078100,0.00009310,-0.00002963,-0.000106$ $45,-0.00036275,-0.00035385,-0.00079360,-0.00043585,0.00045930,0.092476$ $99,0.09766118,0.00092076,-0.00193959,-0.00132448,0.00000936,-0.0007194$ $1,0.00107569,0.00057195,-0.00055447,-0.00120439,-0.00015956,0.00053236$ $, 0.00064406,-0.00046506,-0.00005091,0.00011281,0.00040755,0.00050415,0$ $.00004057,0.00009189,0.00000685,0.00009943,-0.00012783,0.00010389,-0.0$ $0019180,-0.00012158,0.00027181,0.00038847,0.00006682,-0.00009759,0.000$ $00108,-0.00036365,-0.00058166,-0.00008996,0.00001874,0.00001189,0.0000$ $0034,-0.00098283,0.00305976,0.00401913,-0.00090864,-0.00036230,-0.0006$ $6740,-0.00159148,0.00592041,0.00019669,0.01729780,0.00942329,-0.007630$ $32,0.11009128,0.07591133,-0.13004248,-0.00586716,-0.00167205,0.0061946$ $2,-0.00055950,0.00011722,0.00017689,0.00003710,-0.00037303,-0.00030489$ 
$, 0.00084425,0.00033001,-0.00069303,-0.11919409,-0.08716578,0.13073498$, $0.00044065,0.00098869,-0.00083494,0.00020522,-0.00004559,0.00002331,-0$ $.00006198,0.00013391,0.00019773,-0.00001012,-0.00012285,-0.00005647,0$. $00008171,0.00005270,0.00001705,0.00013128,-0.00016137,-0.00013270,-0.0$ $0045993,0.00019179,-0.00016364,-0.00001327,-0.00001803,-0.00002547,-0$. $00004258,-0.00017726,-0.00006756,-0.00002416,0.00001632,0.00001164,0.0$ $0001306,0.00010029,0.00005541,-0.00001446,-0.00000044,-0.00000256,0.00$ $638512,-0.01467737,0.01605014,-0.00289716,-0.00173606,0.00286641,-0.00$ $118427,-0.00009757,0.00012059,-0.00537745,0.00104344,0.00001811,-0.002$ $15445,0.01936918,-0.02111127,-0.07096388,0.04764406,-0.04944519,0.0012$ $8516,-0.00064844,0.00030051,0.00040471,-0.00070209,0.00083625,0.000063$ $94,0.00002222,-0.00011127,0.00102859,-0.00044511,-0.00001613,0.0731642$ $7,-0.00018961,0.00000223,0.00031494,-0.00024025,-0.00012803,-0.0000941$ $7,-0.00011031,0.00007378,0.00010262,0.00005496,0.00001047,-0.00008470$, $-0.00006862,-0.00014538,-0.00001675,0.00022736,0.00001554,-0.00006293$, $0.00011007,-0.00010138,-0.00007057,0.00000201,-0.00000720,-0.00001526$, $0.00002951,-0.00005880,-0.00004212,0.00001444,-0.00000376,-0.00000992$, $0.00001304,0.00001270,-0.00004286,0.00000648,0.00000539,-0.00000533,0$. $00634296,-0.00573336,0.00843546,-0.00229074,0.00272502,0.00279640,-0.0$ $0015332,-0.00038835,-0.00075631,0.00143875,0.00498333,0.00261782,0.000$ $16431,-0.00087700,0.00529088,0.04545271,-0.16619020,0.14430527,-0.0001$ $2089,-0.00078220,-0.00195831,0.00020430,0.00004416,0.00067213,-0.00003$ $004,-0.00042319,-0.00030898,-0.00012672,-0.00198112,-0.00267618,-0.050$ $73040,0.16894736,0.00057737,0.00038107,0.00002004,-0.00026501,-0.00016$ $852,-0.00005979,-0.00009230,0.00032406,0.00025533,0.00004325,-0.000141$ $97,-0.00018531,0.00008902,-0.00001546,-0.00004371,0.00008865,-0.000147$ $62,-0.00009162,-0.00016339,0.00001018,-0.00012920,0.00001434,-0.000030$ $23,-0.00002983,-0.00006881,-0.00033084,-0.00012801,0.00000031,0.000005$ $09,0.00001373,0.00007215,0.00015156,0.00002199,-0.00000712,-0.00000346$ $, 0.00000126,-0.00752834,0.01074380,-0.00888591,0.00353290,0.00270727,0$ $.00140015,0.00011914,-0.00098299,0.00004309,-0.00074031,0.00288631,0.0$ $0414024,0.00045014,0.00293919,0.00055208,-0.04685353,0.14392160,-0.190$ $36612,-0.00045569,-0.00181763,-0.00042372,-0.00022791,0.00077168,-0.00$ $016481,-0.00008032,-0.00031386,-0.00034298,0.00002541,-0.00249812,-0.0$ $0153601,0.05147005,-0.15839113,0.19593910 \backslash 0.00000608,-0.00001981,-0.0$ $0001835,-0.00000022,0.00000488,0.00000378,-0.00000127,0.00001831,0.000$ $00885,-0.00000146,-0.00002098,-0.00000825,0.00000025,-0.00000794,-0.00$ $000674,0.00000527,0.00002684,0.00003342,-0.00000412,-0.00000209,0.0000$ $0188,-0.00000438,0.00000051,-0.00000219,-0.00000203,-0.00000836,-0.000$ $00176,0.00000318,0.00000228,0.00000436,-0.00000062,-0.00000358,-0.0000$ $0524,0.00000139,0.00000605,0.00000700,-0.00000457,-0.00000648,0.000002$ $63,-0.00000377,0.00000468,-0.00000530,0.00000582,0.00000356,-0.0000001$ $4,-0.00000399,0.00000091,0.00000128,-0.00000353,-0.00000113,0.00000184$ $, 0.00000907,0.00000190,-0.00000514,0.00000105,-0.00000012,-0.00000490$, $-0.00000048,-0.00000012,-0.00000201,-0.00000191,0.00000063,0.00000007$, $-0.00000099,0.00000047,-0.00000326,0.00000121,-0.00000041,-0.00000183 \backslash$ II@

ortho-Addsition Product to 4-cyanoaniline

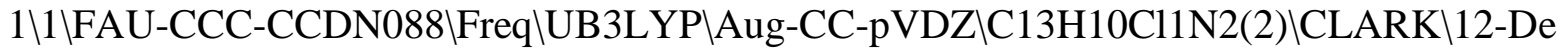
S466 
c-2014\0\\N Geom=AllCheck Guess=TCheck SCRF=Check GenChk UB3LYP/Aug-C C-pVDZ Freq \\Addition product p-Cl-Ph-radical + 4-cyano-aniline (ortho )\0,2\C,1.5459259907,0.1354380916,0.7684232677\C,2.2779915698,0.96381 $77611,-0.2756995305 \backslash \mathrm{C}, 3.3624039036,0.469719417,-0.9733999676 \backslash \mathrm{C}, 3.86658$ $36195,-0.8265808055,-0.7494619648 \backslash \mathrm{C}, 3.2438234815,-1.6625234676,0.22299$ $33429 \backslash \mathrm{C}, 2.1551291679,-1.2314434044,0.949077007 \backslash \mathrm{H}, 1.6272861242,0.684776$ $2502,1.728710814 \mathrm{IN}, 1.8375206689,2.2626394794,-0.4111684219 \backslash \mathrm{H}, 3.8489598$ 257,1.1063492662,-1.7147595764\C,3.7676692318,-2.9836639197,0.44608870 $05 \backslash \mathrm{H}, 1.695693205,-1.8856346247,1.688006673 \backslash \mathrm{H}, 4.7192808896,-1.194745323$ $5,-1.3144101329 \backslash \mathrm{C},-2.6995080726,-0.1236345466,-0.0784722636 \backslash \mathrm{C},-2.27221$ $47524,0.5634938831,1.0563046988 \backslash \mathrm{C},-0.9002391016,0.6415391451,1.3229583$ 233\C,0.0392710983,0.0449164415,0.4732572194\C,-0.4217495407,-0.644556 0486,-0.6589695902\C,-1.7851236875,-0.7326534543,-0.9424714792\Cl,-4.4 $222611623,-0.2313277344,-0.4287308597 \backslash \mathrm{H},-2.9977519445,1.0262561142,1.7$ $228350057 \backslash \mathrm{H},-0.5642843682,1.1712883746,2.2160163299 \mathrm{H}, 0.2931725547,-1$. $123643654,-1.3282733578 \mathrm{lH},-2.1386320598,-1.269536802,-1.8210221648 \mathrm{lH}, 2$ $.1967849869,2.8007634088,-1.1881561367 \backslash \mathrm{H}, 0.8698625337,2.4548182801,-0$. $1866812177 \mathrm{WN}, 4.197056644,-4.0510784977,0.6193624964 \mid \mathrm{Version}=E M 64 \mathrm{~L}-\mathrm{G} 09$ RevC.01 $\backslash S t a t e=2-A \backslash H F=-1071.1451328 \backslash S 2=0.782306 \backslash S 2-1=0 . \backslash S 2 A=0.750564 \backslash \mathrm{RM}$ $\mathrm{SD}=1.203 \mathrm{e}-09 \backslash \mathrm{RMSF}=1.360 \mathrm{e}-05 \backslash$ ZeroPoint $=0.1953827 \backslash \mathrm{Thermal}=0.2093839 \backslash \mathrm{ZPE}=$ 122.6045064\Dipole $=-0.6987371,2.4129933,-0.4233283 \backslash$ DipoleDeriv $=0.24581$ $92,-0.0914995,0.0570917,0.0836245,-0.0097663,0.1101183,0.0907388,0.178$ $863,0.1573887,0.2147196,-0.5525876,0.0068143,-0.5059591,1.3335758,-0.0$ $829572,-0.1909173,0.069986,0.0033023,-0.1925038,0.4127319,-0.1731872,-$ $0.0808688,-0.241761,0.0027794,0.0360668,-0.277386,-0.0342466,-0.098016$ $5,-0.1457412,0.0174198,-0.0227319,0.0143808,-0.0380884,-0.0416952,0.05$ $77898,-0.1151542,0.0177402,0.3846938,-0.208072,0.1644905,-0.0267088,-0$ $.0308678,-0.0623805,-0.3050076,0.2199822,-0.0973508,-0.3369768,0.05474$ $06,-0.1537112,0.020041,-0.096421,-0.1488837,0.2177363,-0.1448758,-0.00$ $24564,0.0255971,0.0468326,0.0536869,-0.0150368,-0.1229317,0.0358222,-0$ $.1057339,-0.0774649,-0.3836532,0.1912336,-0.093609,0.3984907,-1.615242$ $1,0.0820413,0.0188974,-0.2583696,-0.2510126,0.0600026,-0.0199653,0.063$ $9657,-0.0187605,-0.024739,0.1016403,0.1027162,0.0947442,0.0272921,0.19$ $0556,-0.1816668,0.0582494,-0.2447433,0.5430143,-0.0680947,0.1022057,-0$ $.0706436,0.1010845,0.0828395,-0.0040812,0.060803,-0.0050426,0.032737,0$ $.0521313,0.0639457,0.0451427,0.0531432,0.032484,0.0382004,0.0691371,0$. $0529947,0.0581661,-0.000391,0.0746108,0.0031627,0.0774886,1.26995,0.04$ $25722,0.2159099,0.1031105,-0.0177949,0.0480172,0.2903981,0.0383216,0.0$ $586857,-0.2765493,-0.0512839,-0.1113263,-0.0907626,-0.0636543,0.028044$ $3,-0.1450372,0.0358432,-0.0532131,-0.0320711,0.0210222,0.0516445,-0.07$ $51811,-0.0626429,0.0793831,-0.0697321,0.0951713,0.0451365,0.195947,-0$. $0130333,-0.0115813,0.048618,-0.063779,-0.0221005,0.0488219,-0.0186732$, $-0.0810808,-0.0987801,-0.0239341,-0.0277241,0.0084409,-0.0553875,0.039$ $7504,0.0117701,0.0318581,-0.0243995,-0.2694115,0.0324467,0.0257236,0.0$ $156155,-0.039654,0.062291,0.0070573,0.0597409,0.015309,-0.9140941,-0.0$ $21003,-0.1288143,-0.0510791,-0.0704246,-0.0171348,-0.1679841,-0.013322$ $9,-0.1046878,0.0048518,0.0369874,0.0483902,0.0322609,0.096722,-0.04286$ $05,0.0399782,-0.0436196,0.0616081,0.0505407,-0.0408144,-0.0725713,-0.0$ $160871,0.0697314,-0.0714956,-0.0533949,-0.0689203,-0.0056469,0.0308708$ $, 0.0351008,0.0528137,0.0420914,0.0693544,-0.0378693,0.0668724,-0.03727$ $31,0.0450234,0.0475232,-0.0163226,-0.0340919,-0.0171434,0.0894446,-0.0$ 
$581695,-0.043224,-0.0556688,0.0317782,0.139074,0.1189133,0.0396536,0.0$ $562284,0.2716878,0.0028555,0.0511647,0.0833387,0.1511715,0.1635623,0.0$ $636727,0.016013,0.0495056,0.263643,0.0388201,-0.0715045,0.1982978,0.16$ $28089,-0.3815942,0.0957378,-0.0242253,0.1729124,-0.5559069,0.0415097,-$ $0.0463128,0.0446222,-0.3194207 \backslash$ Polar $=245.6883763,-33.024799,192.330157$ $5,-8.489837,-0.1195566,145.1415323 \backslash \mathrm{PG}=\mathrm{C} 01[\mathrm{X}(\mathrm{C} 13 \mathrm{H} 10 \mathrm{Cl1N} 2)] \mathrm{NImag}=0 \backslash 0$. 41476914,-0.01496587,0.49596018,-0.01773852,0.02409058,0.49961578,-0.0 $9970139,-0.02144641,0.04070680,0.51847696,-0.01489811,-0.10912322,0.03$ $953337,-0.16793586,0.59322115,0.03834435,0.03669985,-0.12513083,-0.229$ $16939,-0.06334391,0.37775624,-0.00789929,0.00009520,0.01304147,-0.2466$ $4667,0.08133318,0.12484394,0.46219459,-0.01125642,0.01392400,0.0048857$ $2,0.04402264,-0.12544633,-0.00868072,-0.09759817,0.59169526,0.01900704$ ,-0.00289044,-0.00825992,0.14240925,-0.03565814,-0.16091892,-0.2614339 $3,-0.15318785,0.41489366,-0.00080255,-0.00405196,-0.00342732,-0.015907$ $51,0.03340761,0.00069355,-0.11066836,0.09785660,0.00722871,0.47417530$, $-0.00142402,-0.00733085,0.00066585,0.05436913,-0.02165001,-0.02914672$, $0.04791862,-0.25939964,0.06385065,-0.15878733,0.54427583,-0.00481648,0$ $.00417135,-0.00396151,-0.00986029,-0.01587735,0.01826579,0.03170547,0$. $02314812,-0.08563890,-0.24751871,-0.08754415,0.36340461,-0.00795043,0$. $01592193,0.00553282,-0.01363900,-0.00312681,0.00840527,0.03006144,-0.0$ $2717522,-0.00641280,-0.10525736,0.00396982,0.04234824,0.43033340,0.023$ $34353,-0.00687460,-0.01348478,-0.00541150,0.00144793,0.00260832,-0.004$ $47015,-0.03477742,0.02483705,-0.04709419,-0.15599068,0.09278732,-0.135$ $91533,0.57425168,0.00026686,-0.00707882,0.00710271,0.01354980,0.000809$ $89,-0.00996093,-0.01845692,0.04500579,-0.00137392,0.06849333,0.0486648$ $5,-0.13991719,-0.19628843,-0.11254789,0.35599255,-0.08856362,0.0453654$ $0,0.00502193,0.01754176,-0.01182469,-0.01070838,-0.01431609,0.02103448$ $, 0.00054520,-0.02926052,-0.03670487,0.05447028,-0.23597709,0.08970356$, $0.11742961,0.48470396,0.03874601,-0.18201216,0.02612838,-0.00211955,-0$ $.01979982,0.00891673,0.00542634,-0.00765071,-0.00100458,-0.00846660,0$. $02868122,-0.00157417,0.06702745,-0.12327662,-0.01986694,-0.08716984,0$. $50384037,0.00946888,0.02012837,-0.08026757,-0.01788046,0.02071025,0.00$ $088920,0.00932528,-0.01540549,-0.00085313,0.03915818,0.02325336,-0.034$ $77363,0.12891764,-0.03932756,-0.14115424,-0.27361719,-0.12396281,0.418$ $52554,-0.03785553,-0.00779018,-0.01198600,-0.00218952,0.00634926,0.018$ $57885,0.00145126,-0.00332377,0.00023090,-0.00087434,0.00182726,-0.0002$ $5269,-0.00020271,0.00090118,-0.00053585,-0.00107001,0.01071948,0.01137$ $853,0.05725999,-0.01302686,-0.09893757,-0.09729396,0.00377102,0.007865$ $44,0.01328930,-0.00225079,-0.00070563,0.00165035,0.00181794,-0.0000082$ $0,-0.00158147,-0.00363275,-0.00318677,0.00261575,0.00668758,-0.0189127$ $2,-0.02302072,0.00839651,0.11117728,-0.02112074,-0.09323426,-0.2163706$ $8,0.00687107,-0.00742801,-0.02480905,-0.00337115,0.00533660,-0.0011086$ $2,0.00083038,-0.00320610,0.00062870,-0.00068089,0.00104640,-0.00088596$ $, 0.00285298,-0.00342763,0.00800978,0.01593989,0.10712324,0.24025527,0$. $00811559,-0.01478696,0.00074134,-0.12093950,0.09326907,0.03407500,-0.0$ $1212863,0.04704927,0.00017665,-0.00032430,-0.00247155,0.00599711,-0.00$ $130524,0.00009990,0.00050402,-0.00180460,-0.00053852,0.00307556,0.0004$ $4994,-0.00133934,-0.00031551,0.63768700,0.00097529,-0.02981978,0.01011$ $431,0.07247653,-0.29621282,0.03546097,0.02335734,-0.02642936,-0.005747$ $76,-0.00159494,0.00128145,0.00467528,0.00801660,-0.00144370,-0.0063735$ $8,-0.00651232,0.00003366,0.00498459,0.00017982,0.00226091,-0.00077310$, 
$-0.12893345,0.55994320,-0.00769263,0.02822703,-0.00295196,0.02637667,0$ $.05971690,-0.07228566,0.01214259,-0.02272466,0.00844903,0.00353455,0.0$ $0263114,0.00016798,0.00087566,0.00007665,-0.00158100,0.00435289,0.0014$ $4164,-0.00209621,-0.00141373,0.00050694,0.00242283,-0.26276787,-0.2469$ $0208,0.38312197,0.00037778,0.00392503,0.00387233,-0.00781744,-0.015916$ $07,0.01796390,-0.10352929,-0.06601549,0.09428688,0.00752284,0.00679793$ ,-0.00817593,0.00181367,0.00358460,0.00110340,-0.00060938,0.00046214,-$0.00026196,-0.00062259,-0.00079409,0.00087542,-0.00021538,-0.00222848$, $-0.00184748,0.10358894,0.00321760,0.00051566,0.00055285,0.00621749,0.0$ $0695079,-0.00703225,-0.06632092,-0.14629565,0.11424579,-0.01435604,-0$. $01207343,0.01954048,0.00365025,-0.00307646,0.00085432,0.00033251,-0.00$ $010139,-0.00045799,-0.00015194,-0.00008433,0.00021965,-0.00059909,0.00$ $143090,-0.00206004,0.06945278,0.15248123,0.00373897,-0.00043370,0.0041$ $3763,0.00655552,0.01189547,-0.00778509,0.09518632,0.11309695,-0.172673$ $61,0.00234925,0.00203139,-0.00103326,0.00090535,0.00133783,0.00380942$, $-0.00012507,-0.00050311,-0.00019366,-0.00014984,-0.00035683,0.00028819$ ,-0.00297688,-0.00074061,-0.00078434,-0.10508842,-0.12542172,0.1768149 $3,0.00038574,0.00212768,0.00658576,-0.00174790,0.00084477,0.00105261,0$ $.00175329,-0.00274760,0.00427268,0.00961485,-0.00625503,-0.00491457,-0$ $.09591969,0.07140861,-0.00239113,-0.01624954,0.03377821,-0.00148302,-0$ $.00059067,0.00111500,-0.00033848,0.00038005,-0.00123778,-0.00064707,0$. $00067197,-0.00065325,0.00033903,0.29653718,0.00296435,0.00270095,0.001$ $06135,0.00232362,-0.00299238,-0.00133538,-0.00415591,0.00358940,0.0040$ $0605,0.00736592,-0.03536666,0.01200102,0.06329151,-0.24568045,0.042008$ $00,0.02713428,-0.02595962,-0.00912620,-0.00008525,0.00002676,-0.000366$ $45,-0.00103685,0.00406952,0.00053698,0.00039699,-0.00056186,0.00028477$ ,-0.48683362,1.30780838,0.00584620,0.00206713,0.00336369,0.00023213,0. $00055970,-0.00042110,0.00472253,0.00316194,-0.00073815,-0.01193987,0.0$ $2350844,-0.00133305,0.00244142,0.03505555,-0.07694185,0.00125029,-0.01$ $529126,0.00856377,-0.00015581,0.00057370,-0.00042802,-0.00008694,-0.00$ $119688,-0.00036058,-0.00021208,0.00122439,0.00024641,0.06433778,-0.203$ $70789,0.13257297,0.00533104,0.00792143,-0.00925781,-0.00081545,0.00293$ $562,0.00097852,-0.00038742,0.00037984,-0.00043775,-0.00072550,0.004044$ $22,0.00386434,-0.00562920,-0.01569535,0.01678363,-0.09600542,-0.065619$ $43,0.09456797,0.00034421,0.00013907,0.00042369,0.00050311,0.00021367,-$ $0.00094944,-0.00015201,-0.00018363,-0.00022420,-0.00151538,-0.00234895$ ,-0.00194687,0.09726995,-0.01141926,-0.01220300,0.01799643,0.00457071, $-0.00373448,-0.00149651,-0.00023167,-0.00040518,-0.00010538,0.00366015$ $, 0.00073323,-0.00025204,0.00624087,0.00848781,-0.00766781,-0.06775996$, $-0.15558013,0.12651620,-0.00043596,0.00075010,-0.00031160,-0.00135143$, $-0.00001093,0.00163961,-0.00018146,-0.00003227,-0.00006716,-0.00088455$ $, 0.00032534,-0.00207836,0.06805989,0.16077347,0.00096350,-0.00063394,0$ $.00085479,0.00069440,0.00013267,0.00173805,-0.00069919,-0.00026980,-0$. $00006502,0.00454335,-0.00107295,0.00296056,0.00549576,0.01178446,-0.00$ $668573,0.09575465,0.12348298,-0.17490425,-0.00032243,0.00091101,0.0003$ $1342,-0.00013641,-0.00012038,-0.00020936,-0.00023408,-0.00008144,-0.00$ $028920,-0.00279396,-0.00085092,-0.00233373,-0.10462179,-0.13372574,0.1$ $7803452,-0.00010787,-0.00043852,-0.00061407,0.00220961,0.00200862,0.00$ $075814,-0.00340580,0.00034785,0.00554549,-0.22392127,0.08430524,0.1329$ $4144,-0.00676187,0.00601327,0.00468851,0.00179951,-0.00146219,0.001893$ $82,0.00013753,-0.00001466,-0.00004973,-0.00032998,0.00001005,0.0003084$ 
$8,-0.00063962,-0.00070563,-0.00167687,-0.00071710,0.00004989,-0.001427$ $34,-0.00011709,0.00039463,0.00052645,0.23147386,-0.00025552,-0.0003314$ $9,-0.00014735,0.00175919,-0.00411018,0.00254004,0.02056888,-0.00364953$ ,-0.01498987,0.08563765,-0.08626331,-0.04359824,-0.01519150,0.00823992 $, 0.01017453,-0.00086543,-0.00218388,0.00346411,0.00009037,0.00003438,-$ $0.00007953,0.00135252,-0.00007225,-0.00068202,-0.00104079,0.00054826,-$ $0.00085224,-0.00145759,0.00108618,-0.00044311,-0.00067099,0.00046917,0$ $.00069973,-0.09035909,0.08674496,-0.00064345,-0.00011565,-0.00035121,0$ $.00082270,0.00242077,0.00220738,-0.00500866,0.00302353,0.00590660,0.13$ $269615,-0.04247087,-0.11974934,0.01514451,-0.00851055,-0.00705342,0.00$ $155818,0.00398114,-0.00049808,0.00009401,0.00005598,0.00001568,-0.0002$ $7451,0.00022874,0.00039913,-0.00148332,-0.00112563,-0.00114351,-0.0005$ $9330,-0.00178407,-0.00082765,0.00107957,-0.00023883,-0.00006151,-0.143$ $03060,0.04402660,0.12091524,-0.00117447,0.00017388,0.00027561,-0.00001$ $414,0.00011389,-0.00000809,-0.00004532,0.00020717,-0.00009130,0.000079$ $45,-0.00018295,0.00004144,0.00015509,-0.00021186,-0.00018813,-0.000155$ $51,0.00017579,0.00011459,0.00003964,0.00002683,0.00006490,0.00031135,0$ $.00008374,-0.00015295,0.00005529,0.00001826,-0.00006299,-0.00011049,0$. $00033038,-0.00010108,-0.00004800,0.00006382,-0.00005413,0.00002743,-0$. $00001814,-0.00002487,0.50531219,0.00063094,-0.00065493,-0.00038462,-0$. $00002074,-0.00019538,0.00037610,0.00006468,-0.00008890,0.00002433,0.00$ $007805,0.00020967,-0.00004593,-0.00005977,-0.00007137,0.00015596,0.000$ $28713,0.00020979,0.00023146,0.00018338,0.00014484,0.00011852,0.0000433$ $2,0.00023177,0.00025851,-0.00006727,0.00000080,-0.00005288,-0.00013649$ $, 0.00005668,-0.00007561,0.00002388,-0.00004082,-0.00003680,0.00000781$, $0.00001979,-0.00000261,-0.03231576,0.27872007,0.00086277,-0.00013826,-$ $0.00116996,0.00030629,-0.00054455,0.00065192,0.00003493,-0.00001814,0$. $00004025,0.00007537,0.00029538,-0.00006962,-0.00011488,-0.00005513,0.0$ $0028672,0.00052596,0.00028200,0.00028039,0.00023100,0.00000193,0.00033$ $921,-0.00012730,0.00049519,0.00047595,-0.00009647,-0.00002534,-0.00012$ $047,-0.00022543,0.00014792,-0.00012579,-0.00007664,-0.00000148,-0.0001$ $0988,0.00001912,-0.00000156,0.00000345,-0.01084767,0.23661819,0.499897$ $42,0.00049388,0.00148958,0.00232269,-0.00082182,0.00110267,-0.00074259$ $, 0.00007123,-0.00023814,-0.00006953,-0.00004411,-0.00023397,-0.0000017$ $3,0.00012548,0.00023952,-0.00023399,-0.00093866,-0.00056329,-0.0003260$ $5,-0.00013003,-0.00013747,-0.00041074,0.00018996,-0.00075743,-0.000506$ $14,0.00007663,0.00003297,0.00016296,0.00020902,-0.00015001,0.00016338$, $-0.00003606,0.00004332,0.00012897,-0.00000942,-0.00000232,0.00000376,-$ $0.12234338,-0.00818587,-0.01877869,0.69575310,0.00150888,0.00440512,-0$ $.00448376,0.00132668,-0.00052421,-0.00000327,-0.00002138,0.00058912,-0$ $.00009150,-0.00014391,-0.00032974,0.00008632,0.00018514,0.00034269,-0$. $00031914,-0.00135759,-0.00017447,0.00028150,0.00004852,-0.00002577,0.0$ $0001529,0.00012568,-0.00016251,-0.00000912,0.00004844,-0.00006157,-0.0$ $0006539,0.00008016,0.00002638,0.00015888,-0.00000519,-0.00005603,0.000$ 05256,-0.00000886,-0.00001339,0.00002509,-0.04208557,-0.13506993,-0.11 $488898,-0.05142193,0.29725196,0.00132181,-0.00467800,0.00008540,-0.000$ $49785,0.00020014,-0.00033003,-0.00021089,-0.00007116,0.00011925,0.0000$ $1126,-0.00026408,-0.00001324,0.00001714,-0.00014349,0.00005632,0.00090$ $520,0.00003686,-0.00029031,-0.00003257,0.00013778,0.00005083,-0.000194$ $76,-0.00001422,-0.00017700,0.00003746,0.00005959,0.00012274,0.00013450$ ,-0.00010386,0.00000268,0.00005862,-0.00006877,0.00004278,-0.00001288, 
$0.00001373,0.00001308,-0.07293214,-0.11212629,-0.25050081,-0.02404399$, $0.26002921,0.54287946,-0.02555200,0.01214057,0.01538541,-0.00222045,0$. $00223624,-0.00034133,0.00035996,-0.00113743,0.00063061,-0.00006832,0.0$ $0056900,-0.00013171,-0.00014529,0.00085864,-0.00027704,-0.00188118,-0$. $00131443,0.00144340,0.00086977,0.00176966,0.00324151,0.00070456,-0.000$ $11486,0.00053839,-0.00029632,0.00003020,0.00005721,-0.00037022,0.00005$ $808,0.00011459,0.00000180,0.00036004,-0.00035109,-0.00003618,0.0000035$ $5,-0.00003619,-0.00625877,-0.04733307,-0.07623150,-0.32611636,0.009704$ $70,-0.01182520,0.66872168,0.00106309,0.00468841,0.00073009,-0.00080414$ ,-0.00190067,0.00020035,-0.00011297,0.00003534,-0.00003580,-0.00004835 $, 0.00032321,0.00005190,-0.00034142,0.00001233,-0.00006485,-0.00027044$, $0.00003479,-0.00001821,-0.00140857,0.00014876,0.00030300,-0.00091380,0$ $.00002670,0.00033011,0.00012117,0.00003452,0.00013452,-0.00001514,-0.0$ $0018145,0.00003807,-0.00007183,0.00011144,0.00008771,-0.00000909,0.000$ $01404,-0.00002819,-0.01738478,0.01079158,0.00398783,-0.02490083,-0.080$ $30726,-0.03092370,-0.01318220,0.29715023,-0.00288400,0.00141928,0.0065$ $2624,-0.00200905,0.00102350,-0.00007703,0.00043493,-0.00093755,0.00006$ $626,-0.00022134,0.00047131,-0.00015840,-0.00032737,0.00043476,0.000361$ $16,-0.00181676,0.00001015,-0.00088110,-0.00204851,0.00067749,0.0008762$ $0,-0.00010797,-0.00084671,-0.00039765,0.00000381,0.00003597,0.00020747$ $, 0.00023991,-0.00014474,0.00026966,0.00013612,-0.00014961,0.00005469,-$ $0.00002967,-0.00001192,-0.00001454,-0.02911810,0.00118313,0.00618862,-$ $0.06788031,-0.02783209,-0.11367757,0.03569215,0.26046983,0.55685818,-0$ $.15064222,-0.01177412,-0.02550162,-0.02676472,-0.01147867,0.01216695,0$ $.00199507,-0.00268518,-0.00258581,-0.00115483,0.00135095,0.00036966,0$. $00069230,-0.00133906,-0.00219183,-0.02265243,0.01699344,-0.00374873,-0$ $.01068061,-0.00155835,-0.00275483,-0.00037870,0.00208979,-0.00165780,0$ $.00062330,0.00024624,0.00031809,0.00040081,0.00045604,0.00014515,0.001$ $76911,-0.00128500,0.00085528,0.00012964,0.00003523,-0.00005168,-0.0009$ $9809,0.00689715,0.01178468,-0.04360487,0.00851279,0.00866603,-0.209424$ $47,0.07627312,0.10773554,0.59670675,-0.00431886,-0.07014993,-0.0061043$ $6,-0.01360899,-0.00272015,-0.00417418,-0.00065834,-0.00150802,0.001462$ $69,0.00100667,0.00068946,-0.00140984,-0.00129410,-0.00332807,0.0034991$ $6,0.03107609,0.00040207,0.00277972,-0.01582023,-0.00197176,-0.00582306$ ,-0.00316359,0.00133856,0.00139044,0.00013206,0.00042042,0.00051280,-0 $.00017050,-0.00071745,-0.00129878,0.00003213,0.00049369,-0.00015083,0$. $00000363,0.00006876,0.00001579,0.00763888,-0.02619159,-0.02955629,0.03$ $379659,0.01791863,0.02009626,0.04234799,-0.11806129,-0.08145870,-0.028$ $02714,0.31628141,-0.01360802,-0.00610671,-0.07997589,0.02358386,-0.000$ $59437,0.00549403,-0.00034322,0.00399058,-0.00220416,0.00005126,-0.0008$ $9744,0.00087289,0.00048765,-0.00051823,-0.00077408,-0.00242603,0.00119$ $104,0.00084793,-0.02896641,-0.00489641,-0.01030019,0.00106658,-0.00021$ $089,0.00017457,0.00007998,-0.00055196,-0.00111716,0.00015461,0.0001047$ $7,-0.00015304,-0.00025658,-0.00028736,0.00045431,0.00011555,0.00001363$ $, 0.00013315,0.01250161,-0.02937561,-0.05226913,0.04796629,0.01763614,0$ $.03994765,0.05187218,-0.07790155,-0.18367538,-0.00546909,0.22136510,0$. $52259720,-0.01510493,-0.01561587,-0.02614505,0.00126776,0.00102094,-0$. $00310999,0.00005899,-0.00028406,0.00020413,-0.00019070,-0.00001254,0.0$ $0008483,0.00004423,0.00020270,-0.00029126,0.00078808,-0.00249196,-0.00$ $107776,-0.00605629,-0.00077360,-0.00205351,-0.00000282,-0.00071211,-0$. $00022562,0.00005864,0.00001575,0.00013643,0.00016559,-0.00014443,0.000$ 
$10943,-0.00012456,0.00019171,-0.00001074,-0.00002417,0.00000739,-0.000$ $00448,-0.04787688,0.03712198,0.05256889,-0.03580384,-0.01551144,-0.027$ $71176,0.05471515,-0.00192403,0.00257648,-0.13909119,-0.00890466,-0.022$ $54263,0.70381069,-0.00452312,0.00161609,-0.00081698,0.00048255,0.00061$ $305,-0.00045739,-0.00027628,0.00034236,-0.00042419,0.00013920,-0.00059$ $634,0.00003620,0.00055484,-0.00000962,-0.00009259,0.00046378,0.0000877$ $3,0.00048800,0.00035812,0.00109894,0.00000335,-0.00008789,-0.00023863$, $-0.00035869,0.00002059,0.00001243,-0.00010868,-0.00007737,0.00009488,-$ $0.00006696,-0.00004973,0.00033469,-0.00008159,0.00004421,0.00002527,-0$ $.00001609,0.00731201,0.01833046,0.01843645,-0.01599565,-0.00967166,-0$. $01166874,0.02175318,-0.01024773,-0.02641866,-0.04166238,-0.13085223,-0$ $.10498022,-0.04710161,0.29956450,-0.00875637,-0.00158436,-0.00171447,0$ $.00004281,0.00183346,-0.00081494,0.00026713,0.00019025,-0.00006336,0.0$ $0019344,-0.00040722,-0.00020240,0.00047773,-0.00037964,-0.00071291,-0$. $00037500,-0.00128892,0.00048637,-0.00013541,-0.00014913,0.00080922,0.0$ $0091008,-0.00033045,-0.00010093,-0.00000361,-0.00003899,-0.00002013,0$. $00002656,0.00005218,-0.00004968,-0.00004562,0.00012420,-0.00000861,-0$. $00000715,0.00000435,0.00006658,0.00576064,0.02169534,0.04204842,-0.028$ $70088,-0.01201816,-0.02494748,0.04006252,-0.02892541,-0.03358591,-0.07$ $379730,-0.10126668,-0.23339274,-0.01306736,0.25072728,0.52710422,0.003$ $74230,-0.00004990,-0.00090718,-0.00015061,-0.00008724,0.00063526,0.000$ $12338,-0.00014722,0.00020389,0.00008766,0.00030967,-0.00019801,-0.0002$ $1448,0.00020384,0.00049207,0.00040877,0.00000774,0.00035694,0.00032029$ $, 0.00021592,0.00046884,0.00000502,0.00030815,0.00074681,-0.00015181,-0$ $.00001584,-0.00004381,-0.00016102,-0.00002702,-0.00000049,-0.00006281$, $-0.00005478,-0.00016944,-0.00001967,0.00000488,0.00000096,-0.18613669$, $0.04355613,0.05617429,0.05476727,0.02159406,0.03858092,-0.05837722,0.0$ $1298925,0.01499124,-0.00632363,-0.04348799,-0.06958408,-0.33469683,0.0$ $0676481,-0.01804337,0.66863259,-0.00019403,0.00238591,-0.00484932,0.00$ $100164,0.00008661,0.00000243,0.00000473,0.00002055,-0.00007269,-0.0000$ $7927,0.00006337,0.00001664,-0.00009697,0.00017547,0.00008939,-0.000018$ $44,-0.00022564,0.00013836,-0.00088062,-0.00007672,-0.00042830,0.000027$ $68,0.00014455,-0.00008779,0.00002491,-0.00003156,-0.00002249,-0.000050$ $52,0.00002098,0.00001069,-0.00007637,0.00000439,-0.00007677,-0.0000034$ $0,-0.00002086,0.00000233,0.07834093,-0.12332292,-0.08301351,-0.0020519$ $2,-0.00862887,-0.02807901,0.01344853,-0.00632174,-0.00265376,-0.018162$ $79,0.01192387,0.00500819,-0.02923606,-0.08076616,-0.03323862,-0.006322$ $98,0.30494211,-0.00077288,-0.00492518,-0.00207747,0.00013567,0.0003600$ $1,-0.00043421,0.00006357,-0.00007903,0.00025997,-0.00015618,0.00009142$ $, 0.00002679,-0.00001848,-0.00001120,-0.00001974,0.00077948,-0.00018730$ $, 0.00020322,-0.00118951,-0.00018023,-0.00037290,0.00011097,-0.00013207$ $, 0.00016275,-0.00003203,-0.00000632,-0.00000002,-0.00004559,0.00003220$ ,-0.00005848,-0.00003928,0.00010029,0.00001145,0.00001777,-0.00000206, $-0.00002127,0.11048319,-0.08627220,-0.19000817,0.00138612,-0.02569199$, $-0.03132340,0.01547624,-0.00293449,-0.00629236,-0.02960314,0.00275212$, $0.00837947,-0.07452720,-0.02966729,-0.11626608,0.04620459,0.27212650,0$ $.56409144,0.00047539,0.00014737,0.00028174,-0.00024967,0.00034475,-0.0$ $0048024,0.00006131,-0.00036950,0.00012447,-0.00011441,0.00010980,-0.00$ $000036,-0.00007688,0.00027269,-0.00004994,-0.00030404,-0.00051988,-0.0$ $0007364,-0.00005589,0.00001855,0.00000499,-0.00028722,-0.00041443,0.00$ $014235,-0.00004338,0.00000505,0.00012014,0.00014544,-0.00031752,0.0001$ 
$5429,0.00006267,-0.00002554,0.00003682,-0.00002105,0.00001480,0.000019$ $32,-0.14855186,-0.00602223,-0.02266952,-0.03078611,-0.00227580,-0.0070$ $8958,0.00325455,-0.00168641,-0.00290886,-0.00166759,-0.00064695,-0.001$ $05682,0.00200958,0.00352031,0.00523006,-0.04122393,-0.00278782,-0.0090$ $3362,0.21777819,-0.00009377,-0.00046937,0.00042254,-0.00007951,0.00005$ $576,-0.00004405,-0.00001539,-0.00003574,0.00005718,-0.00001509,0.00001$ $305,0.00002001,-0.00001684,-0.00001177,-0.00003546,0.00003019,-0.00000$ $549,-0.00002950,0.00003934,0.00002669,0.00004418,-0.00002132,-0.000007$ $08,0.00002154,-0.00000850,0.00000236,0.00001354,0.00001588,-0.00002980$ $,-0.00000006,0.00000437,0.00000589,-0.00000213,-0.00000361,-0.00000170$ ,-0.00000073,-0.00627325,-0.02917745,-0.00839641,-0.01640678,0.0032496 $0,-0.00396663,0.00171348,0.00235603,-0.00393637,-0.00034142,0.00009719$ $, 0.00114913,-0.00007719,0.00221775,-0.00369920,0.01174815,0.00500992,0$ $.00167325,0.01031491,0.01963163,0.00004534,0.00047008,-0.00000680,-0.0$ $0003064,0.00002354,-0.00011436,0.00001233,-0.00007472,0.00003262,-0.00$ $003130,0.00001950,-0.00000183,-0.00002654,0.00010359,-0.00000033,-0.00$ $013076,-0.00012571,0.00003055,0.00006683,0.00003284,0.00004542,-0.0000$ $4879,-0.00005463,0.00004345,-0.00000662,-0.00000002,0.00002580,0.00002$ $909,-0.00005655,0.00004503,0.00001780,-0.00001112,-0.00000456,-0.00001$ $046,0.00000178,-0.00000074,-0.02259041,-0.00833910,-0.03992281,-0.0297$ $9067,-0.00257789,-0.00239752,0.00255719,-0.00428408,-0.00166771,-0.000$ $69909,0.00112020,0.00107223,-0.00034415,-0.00327815,-0.00069803,0.0136$ $0330,0.00017256,0.00647894,0.03779449,0.01328312,0.03698349,-0.0001975$ $2,0.00051618,0.00083242,-0.00026639,0.00024344,-0.00006879,0.00009440$, $-0.00018402,0.00000551,-0.00004432,0.00009964,-0.00000195,-0.00002759$, $0.00006672,-0.00004565,-0.00021229,-0.00009694,0.00000419,0.00004191,0$ $.00000037,0.00001403,0.00000187,-0.00017974,-0.00000833,-0.00001104,0$. $00000418,0.00003099,0.00000524,-0.00002493,0.00003894,-0.00000622,0.00$ $002443,-0.00000347,-0.00000646,-0.00000057,-0.00000319,0.00761204,-0.0$ $0535668,-0.00821413,-0.18200875,0.08331063,0.11837338,-0.01401966,0.01$ $316004,0.01914567,-0.00422099,-0.00092591,-0.00250752,-0.00042097,-0.0$ $0044351,-0.00063099,0.00027006,-0.00119396,-0.00239381,0.00117841,-0.0$ $0051869,-0.00044570,0.19069465,-0.00023892,0.00014415,-0.00003375,0.00$ $008573,0.00016847,0.00006823,-0.00000443,0.00000556,0.00000313,-0.0000$ $2744,-0.00004017,0.00003999,0.00008117,0.00001452,-0.00003219,-0.00014$ 436,-0.00002264,-0.00008095,0.00000048,0.00000824,-0.00000919,-0.00002 $264,-0.00010825,-0.00005194,-0.00001209,-0.00000222,-0.00000590,0.0000$ $1899,0.00001584,-0.00000286,0.00000694,-0.00000465,-0.00000557,-0.0000$ $0068,-0.00000467,-0.00000084,0.01127530,-0.00252505,-0.00853094,0.0817$ $9978,-0.09349180,-0.08460080,-0.00259284,0.00446969,0.00123021,-0.0005$ $2961,0.00531983,-0.00250406,-0.00050491,-0.00081035,0.00056814,-0.0015$ $0060,0.00293734,-0.00435606,0.00005308,-0.00161473,0.00179317,-0.08808$ $609,0.08881724,-0.00034149,0.00008384,0.00028459,-0.00008581,-0.000063$ $54,-0.00009365,0.00000064,-0.00000623,0.00002912,0.00001827,0.00002333$ $,-0.00003842,-0.00005865,0.00000520,0.00005310,0.00005202,-0.00002536$, $0.00002208,0.00000299,-0.00001061,-0.00000331,0.00002376,0.00005658,0$. $00000699,0.00000548,0.00000063,0.00000798,-0.00001283,-0.00000980,-0.0$ $0000152,-0.00000425,0.00000811,-0.00000170,0.00000005,0.00000252,-0.00$ $000046,0.01828076,-0.01006522,-0.01069597,0.11593955,-0.08462251,-0.15$ $903247,-0.00578090,0.00277652,0.00741087,-0.00210452,-0.00242181,0.002$ $70804,-0.00087636,0.00050128,-0.00047197,-0.00284497,-0.00432985,-0.00$ 
$161535,0.00049442,0.00174161,0.00011344,-0.12392718,0.09443654,0.16258$ $025,0.00106835,0.00080232,0.00184463,0.00009702,-0.00011790,-0.0001605$ $0,-0.00021819,0.00028827,0.00001040,0.00011615,-0.00024404,0.00001389$, $0.00002386,-0.00002466,-0.00001695,0.00002329,-0.00020373,-0.00003960$, $-0.00015369,-0.00025061,-0.00044182,-0.00004061,0.00018222,-0.00002803$ $, 0.00001159,0.00001855,0.00001196,-0.00001204,0.00005750,-0.00000045,0$ $.00001200,-0.00000723,0.00001400,-0.00000157,-0.00000497,0.00000470,-0$ $.00411590,0.00042818,-0.00046598,-0.00572558,-0.01526457,-0.02545219,-$ $0.08275664,-0.03987618,-0.06975805,0.00879630,0.00942759,0.01589836,-0$ $.00225297,0.00231721,0.00270152,-0.00101709,0.00022186,0.00038911,-0.0$ $0125191,-0.00054849,-0.00101668,0.00102777,-0.00016186,0.00028900,0.08$ $582084,-0.00157820,-0.00291140,0.00179349,-0.00059378,0.00076622,0.000$ $11280,-0.00002543,-0.00027698,0.00006228,0.00002162,0.00000636,0.00001$ $234,0.00002572,-0.00010334,0.00009561,0.00058361,0.00002684,-0.0001635$ $0,0.00050214,0.00038842,0.00039144,0.00008540,-0.00018355,-0.00002188$, $-0.00004884,0.00001662,0.00005198,-0.00001308,0.00000694,-0.00006105,0$ $.00005265,-0.00008794,-0.00004178,-0.00000745,0.00000025,0.00000365,0$. $00020430,0.00567421,-0.00222010,0.00069515,0.00238326,-0.00130808,-0.0$ $3964848,-0.10660012,-0.11913472,-0.00511658,-0.00278751,-0.01078560,0$. $00280485,0.00363093,-0.00387775,0.00029121,-0.00065056,0.00075506,0.00$ $004624,0.00006984,-0.00007688,-0.00030480,-0.00274408,0.00213420,0.042$ $57270,0.10404094,-0.00152802,0.00221155,-0.00084265,0.00063227,-0.0005$ $3508,0.00002820,-0.00017536,0.00029976,0.00008068,0.00000351,-0.000109$ $98,0.00004224,-0.00001843,0.00017319,-0.00006726,-0.00026411,-0.000061$ $77,0.00044250,0.00086803,0.00021838,0.00061902,0.00001861,0.00032688,0$ $.00024608,-0.00002004,-0.00002892,-0.00004943,-0.00003777,-0.00001992$, $0.00002739,-0.00003120,0.00000386,-0.00005039,-0.00000555,0.00000323,0$ $.00000084,-0.00072657,-0.00212455,0.00340924,0.00035852,-0.00293440,-0$ $.00118083,-0.06928134,-0.11958468,-0.23731233,-0.00851116,-0.00886530$, $-0.01158188,0.00352153,-0.00376832,0.00049200,0.00054509,0.00070200,0$. $00009329,-0.00006437,-0.00011975,-0.00009334,0.00002695,0.00212165,-0$. $00062870,0.07529774,0.13227994,0.24777482,-0.00016474,-0.00026935,-0.0$ $0011873,0.00029916,-0.00045158,-0.00035738,-0.00010676,0.00010389,0.00$ $017575,-0.00013016,0.00000105,0.00005276,-0.00013831,0.00007215,-0.000$ $08732,0.00002438,-0.00022530,-0.00025431,0.00015824,0.00008352,0.00016$ 476,-0.00015676,0.00032645,0.00005759,-0.00001087,0.00000655,-0.000012 $65,-0.00007744,0.00011423,-0.00003622,0.00001711,-0.00000339,0.0000723$ $9,0.00000761,-0.00000740,-0.00000323,-0.00349586,-0.00058805,-0.002137$ $91,-0.00037919,-0.00047207,-0.00065918,0.00016383,-0.00105031,-0.00237$ $442,0.01043720,-0.00455297,-0.00660278,-0.17642878,0.08154452,0.112272$ $61,-0.01539224,0.01355314,0.01918970,-0.00141493,0.00039030,0.00043660$ $, 0.00000534,0.00000546,0.00008896,0.00042974,-0.00036218,-0.00051832,0$ $.18533846,0.00049418,-0.00208722,0.00168630,-0.00034450,0.00021603,-0$. $00018319,0.00016994,-0.00006141,0.00040573,-0.00019679,0.00018420,-0.0$ $0003218,0.00005860,-0.00008186,-0.00029275,0.00004517,0.00025763,0.000$ $18157,0.00006258,-0.00006827,0.00008765,0.00013177,-0.00030252,0.00018$ $528,-0.00007464,-0.00001696,0.00000982,0.00006426,0.00011663,0.0000389$ $5,0.00006351,-0.00000601,0.00012320,0.00000822,-0.00000586,0.00000802$, $-0.00046574,0.00566213,-0.00266832,-0.00055254,-0.00084938,0.00054640$, $-0.00153288,0.00337824,-0.00485545,0.01101882,-0.00269273,-0.00867322$, $0.08157807,-0.09756772,-0.08580007,-0.00276407,0.00470742,0.00085466,-$ 
$0.00024932,0.00006248,0.00000998,0.00002916,-0.00051837,0.00033013,0.0$ $0055485,-0.00014800,-0.00047033,-0.08789974,0.09287022,0.00114940,0.00$ $159467,-0.00035332,0.00031018,-0.00059783,0.00013084,-0.00021946,0.000$ $06941,0.00001236,-0.00008002,-0.00009664,-0.00002458,-0.00024682,0.000$ $25443,0.00032132,0.00012943,0.00011351,-0.00003716,0.00010446,0.000079$ $95,0.00003247,-0.00018540,0.00038790,0.00000186,0.00005527,0.00006409$, $0.00002094,-0.00012238,0.00013970,0.00002722,-0.00008232,-0.00005023,-$ $0.00015117,0.00003822,0.00000474,-0.00001013,-0.00181962,-0.00276970,0$ $.00289352,-0.00071290,0.00054606,-0.00037234,-0.00302146,-0.00487022,-$ $0.00158267,0.01781464,-0.01035077,-0.01067993,0.11199326,-0.08557709$,$0.15802228,-0.00637791,0.00255398,0.00730056,-0.00052293,0.00006999,0$. $00010893,0.00010758,0.00034557,-0.00020065,0.00087911,-0.00053545,-0.0$ $0061124,-0.11960455,0.09633628,0.16198632,0.00006894,-0.00069117,-0.00$ $107445,0.00008418,0.00012278,-0.00009700,0.00004749,-0.00012039,0.0000$ $3046,-0.00002110,0.00009841,-0.00000040,-0.00004953,0.00005190,0.00004$ $087,0.00016551,-0.00012739,0.00000835,-0.00044245,-0.00008405,-0.00015$ $392,-0.00000108,-0.00004107,0.00006868,-0.00002376,-0.00000890,0.00000$ $785,0.00000140,-0.00004429,0.00000458,-0.00001142,0.00001464,-0.000013$ $40,-0.00001163,0.00000144,-0.00000355,0.00795190,0.01069513,0.01724608$ ,-0.00249743,0.00222129,0.00266499,-0.00105365,0.00018454,0.00031809,-$0.00517135,0.00056394,-0.00042458,-0.00464050,-0.01490347,-0.02435057$, $-0.08456447,-0.04548394,-0.07635334,0.00108933,0.00016021,0.00062166,0$ $.00052883,0.00050502,0.00084332,0.00005426,-0.00008242,-0.00005860,0.0$ $0095773,-0.00018001,0.00033855,0.08757020,0.00012822,0.00012043,-0.000$ $06952,-0.00016172,0.00003544,0.00002401,0.00004632,-0.00007961,-0.0000$ $2514,0.00000724,0.00004703,-0.00003773,-0.00003404,0.00000305,0.000066$ $61,0.00012837,-0.00008318,-0.00003563,-0.00003220,-0.00009467,0.000083$ $90,0.00002790,-0.00002749,0.00004512,-0.00001016,-0.00000556,0.0000025$ $1,0.00001810,0.00002870,0.00001945,0.00000384,-0.00000956,0.00000944,-$ $0.00000306,0.00000199,-0.00000283,-0.00609088,-0.00274181,-0.01032957$, $0.00246366,0.00318644,-0.00315085,0.00036928,-0.00059055,0.00068324,0$. $00033279,0.00583890,-0.00230134,0.00071249,0.00317224,-0.00114913,-0.0$ 4396666,-0.11221821,-0.12409304,-0.00042321,-0.00148992,0.00169475,-0. $00025152,-0.00003683,-0.00051659,-0.00006400,-0.00052242,0.00029460,-0$ $.00025417,-0.00282794,0.00233767,0.04708524,0.10832132,0.00029689,-0.0$ $0019136,-0.00009069,0.00005100,0.00004790,-0.00003652,0.00002302,-0.00$ $001049,0.00001513,-0.00002228,0.00000324,0.00002964,0.00001091,0.00001$ $082,-0.00006663,-0.00011708,0.00003971,0.00003040,-0.00008559,0.000052$ $63,-0.00007087,-0.00001273,-0.00003774,-0.00000976,0.00000306,0.000006$ $72,0.00000979,0.00001952,-0.00002893,0.00001153,0.00000364,0.00000337$, $-0.00001352,-0.00000286,-0.00000285,-0.00000459,-0.00909776,-0.0086637$ $1,-0.01101479,0.00306005,-0.00316957,0.00064707,0.00047630,0.00070771$, $0.00017046,-0.00091621,-0.00241900,0.00345024,0.00029625,-0.00276428$,$0.00025511,-0.07395835,-0.12406697,-0.23839104,-0.00027270,0.00174087$, $0.00000155,-0.00035093,-0.00042953,-0.00043713,-0.00002437,0.00030390$, $-0.00024011,0.00025006,0.00232623,-0.00070490,0.08036707,0.13649520,0$. $24696456,0.00093969,0.00273146,0.00068850,0.00733167,-0.00048009,-0.01$ $134133,-0.00049409,0.00152177,-0.00163192,-0.00063680,-0.00012249,-0.0$ $0039188,-0.00055837,0.00002984,0.00049207,0.00076900,0.00038162,-0.000$ $28941,-0.00025211,-0.00019008,0.00045316,-0.10195929,-0.04769215,0.117$ $53348,-0.00012309,-0.00016535,0.00102002,0.00006740,-0.00036772,0.0001$ 
$6655,-0.00000985,0.00008295,-0.00005486,0.00006423,0.00005911,0.000040$ $43,0.00003879,-0.00010351,-0.00015555,0.00021888,0.00002120,0.00008216$ ,-0.00000813,-0.00023253,0.00004180,-0.00004932,-0.00021106,-0.0000477 $7,0.00027567,0.00013359,0.00025811,-0.00023992,0.00004963,-0.00002887$, $-0.00000308,0.00000462,-0.00001026,0.00000884,-0.00000124,0.00000183,-$ $0.00002051,0.00003582,-0.00008557,0.00001483,-0.00005330,-0.00003518,-$ $0.00001117,-0.00001651,0.00000676,0.10044275,0.00468087,-0.00372614,-0$ $.00066854,-0.01419329,-0.01910495,0.02752520,-0.00357535,0.00265415,-0$ $.00025896,-0.00001215,-0.00093213,-0.00032457,-0.00022486,0.00031368,0$ $.00000801,-0.00115252,-0.00018158,0.00142109,0.00008087,0.00020529,0.0$ $0021142,-0.05430253,-0.15012661,0.16418760,0.00139291,0.00013465,-0.00$ $056243,0.00017502,-0.00021524,-0.00001714,0.00031854,-0.00048674,-0.00$ $001412,0.00002598,0.00011738,0.00005096,-0.00017029,-0.00001628,-0.000$ $00887,-0.00002781,0.00001890,0.00000916,-0.00004569,0.00010409,-0.0000$ $3163,0.00065923,0.00021736,-0.00011965,-0.00006432,0.00006123,-0.00006$ $520,0.00000237,-0.00002179,-0.00003370,0.00012094,0.00000316,0.0000183$ $3,0.00000798,0.00000397,-0.00000719,-0.00001210,-0.00000561,0.00005185$ $, 0.00000557,-0.00007745,-0.00000673,-0.00001347,-0.00000315,0.00000340$ $, 0.06413029,0.16605586,0.00028766,0.00052497,0.00229753,-0.00145686,-0$ $.00430212,-0.00168530,-0.00081081,-0.00230804,0.00084940,0.00007718,0$. $00051464,-0.00032188,-0.00024447,-0.00003967,0.00018526,0.00078683,-0$. $00008905,0.00025847,-0.00019680,-0.00034210,0.00055077,0.13747679,0.18$ $723338,-0.27098207,-0.00032193,0.00037578,-0.00021271,-0.00009190,-0.0$ $0002332,-0.00008983,-0.00005515,0.00003654,-0.00001077,0.00005426,0.00$ $000239,0.00005843,-0.00001092,-0.00001106,-0.00004963,0.00005490,-0.00$ $000774,0.00003397,0.00001709,-0.00013030,-0.00000586,-0.00006953,-0.00$ $000604,-0.00025523,0.00008378,0.00003035,0.00015171,-0.00005478,0.0000$ $3074,0.00000455,0.00000417,-0.00000143,-0.00000404,0.00000561,0.000007$ $26,-0.00000220,-0.00001589,0.00001694,0.00001633,0.00002753,-0.0000359$ $2,0.00000628,-0.00001202,-0.00001203,0.00000173,-0.13107217,-0.1822503$ $9,0.27398777,0.00016867,-0.00114966,0.00069413,-0.00817007,0.00179240$, $-0.00157989,0.00181632,-0.00056890,-0.00133125,0.00077971,0.00067364,0$ $.00077176,-0.00025018,-0.00001342,0.00015466,-0.00018828,-0.00018352,0$ $.00010396,0.00002950,0.00012262,-0.00057485,-0.40829302,0.08127736,0.1$ $1102298,-0.00027580,-0.00033353,-0.00047591,-0.00007082,0.00002040,-0$. $00006729,0.00011937,-0.00012733,0.00004240,0.00001899,0.00006024,-0.00$ $004978,-0.00039782,0.00012976,0.00023225,-0.00056857,-0.00007769,-0.00$ $014868,-0.00025710,0.00045939,0.00028814,0.00122317,-0.00033616,0.0000$ $5659,-0.00040946,-0.00031678,-0.00030065,0.00036488,-0.00006473,-0.000$ $05790,0.00013219,0.00000498,0.00002569,-0.00001647,0.00000028,-0.00000$ $816,0.00007122,-0.00013860,0.00005473,0.00002134,0.00004262,0.00005354$ ,-0.00001251,-0.00000204,-0.00000232,-0.00572997,0.00227727,-0.0044673 $7,0.41987806,0.00068934,0.00026505,-0.00049764,0.03575624,-0.00617372$, $-0.01256043,-0.00014400,-0.00437643,0.00393577,0.00139244,-0.00056190$, $-0.00081972,0.00023349,0.00032486,-0.00029044,-0.00009827,0.00003000,-$ $0.00024261,0.00005924,0.00011423,-0.00007631,0.06671161,-0.06302870,0$. $01208578,-0.00003123,0.00009655,-0.00014053,0.00000760,-0.00010532,0.0$ $0005041,-0.00005324,0.00006989,0.00002943,0.00004080,-0.00050796,0.000$ $01763,-0.00046392,0.00010325,0.00028145,-0.00020533,-0.00004845,-0.000$ $02907,-0.00055094,0.00035533,0.00054892,-0.00047521,0.00009667,0.00039$ $972,-0.00049558,-0.00037824,-0.00088741,0.00039307,-0.00013942,-0.0001$ 
$5618,0.00043899,0.00001376,0.00006570,0.00006584,0.00002760,-0.0000282$ $6,-0.00003388,0.00003179,-0.00010372,0.00002980,-0.00002990,0.00003787$ $, 0.00002786,0.00000060,0.00001521,-0.01974334,0.00484412,0.00077032,-0$ $.08351108,0.06890563,-0.00061723,0.00092838,-0.00036706,-0.00241518,-0$ $.00990157,-0.00230711,-0.00022455,0.00200996,0.00297346,0.00122821,0.0$ $0029482,0.00045082,-0.00122861,0.00002129,0.00116336,0.00054331,-0.000$ 05666,-0.00093364,-0.00000447,-0.00006814,-0.00097860,0.08315184,0.018 $94062,-0.04391049,-0.00048684,-0.00015833,-0.00011749,0.00025530,-0.00$ $067060,0.00021675,0.00000893,-0.00015398,-0.00000278,-0.00009206,-0.00$ $008256,-0.00014429,0.00024171,-0.00033532,-0.00050395,0.00071203,-0.00$ $007321,0.00024349,-0.00027099,-0.00009201,0.00043480,-0.00018763,0.000$ $04721,0.00018727,0.00042616,0.00031256,0.00016663,-0.00079910,0.000061$ $42,-0.00014573,-0.00010990,-0.00000553,-0.00005433,0.00003337,0.000024$ $48,0.00000859,0.00005833,0.00018974,-0.00060383,-0.00003230,-0.0001155$ $0,-0.00009459,-0.00002220,-0.00002859,0.00000805,0.02442130,-0.0090847$ $4,-0.00478706,-0.10444828,-0.00239272,0.04920105,-0.00021994,-0.000328$ $05,-0.00105684,0.00075413,-0.00080821,-0.00033146,-0.00024284,0.001039$ $76,-0.00109373,-0.00222951,-0.00000560,0.00360349,0.01085375,0.0131228$ $7,-0.00294439,0.00396440,-0.00261566,-0.00076518,0.00007413,-0.0001119$ 4,0.00006056,-0.00018132,0.00092175,0.00019597,-0.00027889,0.00024576, $-0.00010342,-0.19279568,0.39097331,-0.06375281,0.00021600,0.00006042,0$ $.00033701,0.00026233,0.00027906,-0.00025349,0.00014000,0.00004190,0.00$ $008005,-0.00009127,-0.00004218,-0.00004181,0.00005065,-0.00001759,-0.0$ $0011947,0.00005135,0.00010190,0.00000101,-0.00006948,0.00002889,0.0000$ $1107,0.00001022,0.00003077,0.00001543,-0.00013509,-0.00000659,-0.00002$ $824,-0.00001070,-0.00000453,0.00000407,0.00001428,-0.00000157,-0.00000$ $057,0.00002106,-0.00000820,0.00005733,-0.00000773,-0.00001328,-0.00000$ $135,-0.00007603,-0.00008344,0.00000477,0.00001666,-0.00004027,-0.00014$ $113,0.17990950,-0.00024932,-0.00009429,-0.00082668,-0.00144317,0.00226$ $141,0.00063367,0.00087596,-0.00166922,-0.00004867,0.00248298,0.0040350$ $1,-0.00348956,0.01785222,-0.01570120,0.00061461,-0.00899342,0.00256594$ $, 0.00729259,0.00013016,-0.00017787,0.00017892,0.00064485,-0.00258704,-$ $0.00038302,0.00006956,-0.00030194,-0.00003791,0.39087824,-1.00816332,0$ $.15777268,0.00057510,0.00009862,-0.00014844,-0.00024396,-0.00020525,0$. $00043665,-0.00030205,-0.00005863,-0.00014887,0.00016806,0.00004295,0.0$ $0006763,0.00008967,0.00017610,0.00022411,-0.00043008,-0.00012609,-0.00$ $015369,0.00019853,-0.00006387,-0.00008885,0.00003506,0.00000199,-0.000$ $01717,0.00033114,0.00002794,0.00006972,0.00003877,-0.00000863,0.000006$ $48,-0.00005700,-0.00002113,0.00003982,-0.00010015,-0.00011337,-0.00008$ $115,0.00004820,-0.00002523,0.00002751,0.00019746,0.00016382,0.00001592$ ,-0.00003752,0.00007072,0.00038898,-0.40275930,1.01987259,-0.00107987, $-0.00060638,-0.00037638,0.00012509,-0.00042109,0.00004309,-0.00085000$, $-0.00044471,0.00016992,0.00221109,-0.00110317,0.00038721,-0.00539374,0$ $.00448126,0.01786327,0.00262214,0.00184111,-0.00170721,-0.00001986,-0$. $00002769,0.00007119,-0.00009323,0.00057668,0.00017441,-0.00001617,-0.0$ $0019130,-0.00015122,-0.06374993,0.15785368,-0.06148464,0.00010610,0.00$ $023736,0.00043762,0.00005195,-0.00005807,0.00016118,0.00006229,0.00001$ $968,0.00004586,-0.00006101,-0.00004233,-0.00000171,-0.00001604,-0.0000$ $2415,-0.00009610,-0.00000605,0.00026417,0.00005983,-0.00001331,0.00001$ $047,0.00000189,-0.00002274,0.00000388,0.00001379,-0.00007478,0.0000002$ $8,-0.00001506,-0.00001698,0.00000623,-0.00000157,0.00000776,0.00002344$ 
,-0.00001064,0.00001057,-0.00000327,0.00000236,0.00000347,-0.00000450, $-0.00000450,-0.00006140,-0.00003315,0.00000441,0.00001151,-0.00001315$, $-0.00010334,0.06626314,-0.16234525,0.04451633 \backslash \backslash-0.00001981,-0.00002251$ $, 0.00000113,0.00000707,-0.00000137,-0.00000171,-0.00000220,-0.00000763$ ,-0.00000654,0.00001548,0.00003714,-0.00000743,-0.00000769,-0.00004826 $, 0.00002991,0.00000123,0.00003848,-0.00001158,0.00000080,0.00000463,-0$ $.00000102,0.00000704,0.00000942,0.00001187,-0.00000011,-0.00000085,-0$. $00000123,-0.00000426,0.00005619,-0.00001494,0.00000591,0.00000020,0.00$ $000622,-0.00000623,-0.00000397,-0.00000608,-0.00000151,-0.00000684,0.0$ $0000601,-0.00000217,0 .,-0.00000383,0.00000116,0.00000843,-0.00000995,0$ $.00001128,-0.00000539,0.00001054,-0.00000500,0.00000051,-0.00000552,0$. $00000555,-0.00000522,0.00000009,0.00000050,-0.00000648,0.00000145,-0.0$ $0000060,-0.00000198,-0.00000094,-0.00000189,-0.00000059,0.00000166,0.0$ $0000053,-0.00000338,0.00000325,0.00000021,-0.00000802,0.00000348,-0.00$ $001139,-0.00000111,-0.00001339,-0.00001082,-0.00000039,-0.00000992,0.0$ 0001694,-0.00003101,0.00001847III@

meta-Addition Product to 4-cyanoaniline

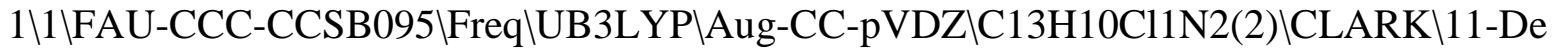
c-2014\0\\N Geom=AllCheck Guess=TCheck SCRF=Check GenChk UB3LYP/Aug-C C-pVDZ Freq \\Addition product p-Cl-Ph-radical + 4-cyano-aniline (meta) II0,2\C,-1.2636856618,0.8825446908,0.6177783412\C,-2.1042215753,-0.322 $5797156,0.943919159 \backslash \mathrm{C},-3.2070060113,-0.7035899958,0.2176523576 \backslash \mathrm{C},-3.63$ $35663311,0.0938926504,-0.8924136245 \backslash \mathrm{C},-2.950835539,1.2697003816,-1.232$ 9318521\C,-1.8287825115,1.6916064036,-0.5378851292\H,-1.2527073302,1.5 $442697774,1.5051089058 \backslash \mathrm{H},-1.7722803418,-0.928535662,1.7882946032 \mathrm{LN},-3$. $8997034471,-1.8896089742,0.4834825936 \backslash \mathrm{H},-3.312671063,1.8714219756,-2.0$ $665104567 \backslash \mathrm{C},-1.1852586965,2.9102758845,-0.8822476602 \backslash \mathrm{H},-4.5005419695,-$ $0.2152259079,-1.4758588247 \backslash \mathrm{C}, 2.8740412094,-0.2107431854,-0.0666896592 \backslash$ C,2.5700116318,0.7260260616,0.9190210222\C,1.2316885627,1.0727397987,1 $.1331528576 \backslash \mathrm{C}, 0.205787621,0.4946908191,0.378177649 \backslash \mathrm{C}, 0.5412609396,-0.4$ 442530991,-0.608160166\C,1.8703321653,-0.8016554059,-0.8380706949\Cl,4 $.5554064441,-0.6606697855,-0.3458549922 \backslash \mathrm{H}, 3.3644705821,1.1805733711,1$. $5078286699 \backslash \mathrm{H}, 0.9929483495,1.8118887779,1.8987574539 \backslash \mathrm{H},-0.2440234333,-0$ $.906977283,-1.2061042063 \backslash \mathrm{H}, 2.1278960851,-1.5298516637,-1.6051227022 \backslash \mathrm{H}$, $-3.7406317594,-2.2927052022,1.3978584972 \backslash \mathrm{H},-4.8801769604,-1.8882466244$ $, 0.2332758082 \mathrm{lN},-0.6523433586,3.9172748402,-1.1374197932 / \mathrm{Version}=\mathrm{EM} 64$ L-G09RevC.01\State=2-A $\backslash H F=-1071.1424691 \backslash S 2=0.788153 \backslash S 2-1=0 . \backslash S 2 A=0.7508$ 64\RMSD $=3.153 \mathrm{e}-09 \backslash \mathrm{RMSF}=8.069 \mathrm{e}-06$ ZeroPoint $=0.1950138 \backslash$ Thermal $=0.2091115$ $\backslash \mathrm{ZPE}=122.3730102 \backslash$ Dipole=-1.8895102,-1.6053409,0.8673162\DipoleDeriv=0. $1198858,0.0167403,-0.1197103,-0.0689525,0.1325533,0.0473747,-0.1366338$ $, 0.1272328,0.0827279,-0.3803891,-0.4719075,0.3276717,0.0880107,-0.3387$ $527,0.0167138,-0.0456628,-0.3153346,0.0549605,0.5498432,1.0417459,-0.4$ $199714,0.8487226,1.0121943,-0.1689206,-0.1508703,0.2077325,-0.2200815$, $-0.0083395,-0.0181322,0.160991,-0.121555,-0.1038223,-0.0843708,0.27759$ $15,-0.0611143,0.0957435,0.0966513,0.0153495,0.1083521,0.1995628,0.0556$ $699,-0.0343494,-0.0100857,-0.1047023,0.0722353,-0.1900083,-0.1418969,0$ $.0970692,-0.1430218,-0.1460233,0.053169,0.1655917,-0.0384478,0.0107498$ $, 0.0380542,-0.0192777,-0.0537342,-0.0237775,0.0180605,-0.0900438,-0.00$ $62608,-0.0877147,-0.0135,0.0802748,-0.0184621,-0.0485854,-0.0226451,0$. 
$0008398,0.0936372,-0.0404115,0.0821435,0.0050013,-0.4973708,-0.331926$, $0.173203,-0.8496651,-1.2338809,0.0670853,0.0888793,-0.118754,-0.197032$ $9,0.080195,0.004863,-0.0392025,-0.0100837,0.0484508,0.0745357,-0.07713$ $15,0.0729289,0.0282157,0.1928888,0.2178532,-0.1132229,0.1599898,0.4638$ $757,-0.0875678,-0.1019626,-0.057913,0.0648042,-0.0115323,-0.0615481,-0$ $.0691849,-0.0754561,0.0545518,0.0016244,-0.0800588,0.0011887,0.0605915$ ,1.1873891,-0.2985968,-0.1507004,-0.3521499,0.0745568,0.080448,-0.2322 $885,0.0890211,0.030523,-0.2522831,0.1239362,0.0923855,0.1509813,-0.080$ $6618,0.0179622,0.1233806,0.0124924,-0.0639513,-0.0157838,-0.0233982,-0$ $.0330206,0.0538504,-0.0026095,0.1089986,0.0682734,0.0623051,0.0057866$, $0.28319,-0.0776422,0.0470455,-0.1106544,-0.022401,-0.0363274,-0.007950$ $7,-0.0209113,-0.0570522,-0.1036025,0.0295126,0.0209361,-0.0034907,-0.0$ $426028,0.0343958,-0.0063122,0.0431905,-0.0338901,-0.2591325,0.0175044$, $-0.019361,0.0292264,-0.0268717,0.0680494,0.0092254,0.0676569,-0.006916$ $3,-0.873712,0.1971314,0.0869318,0.2152609,-0.1285406,-0.0348176,0.1272$ $112,-0.0427522,-0.0942918,-0.0029162,-0.0263333,-0.0525778,-0.0256151$, $0.0899723,-0.0409723,-0.0405901,-0.0395803,0.0756243,0.0533743,0.04466$ $29,0.0515589,0.0295671,0.0298155,-0.0911419,0.0381188,-0.0773728,0.028$ $099,0.0041819,-0.0442283,-0.0557362,-0.0438892,0.0722862,-0.0366492,-0$ $.0692094,-0.0258104,0.0473601,0.0534137,0.0285118,0.0189559,0.0285312$, $0.0549038,-0.0706484,0.0283522,-0.0725329,0.0507302,0.1081733,-0.08220$ $26,-0.0466414,0.0130905,0.294137,-0.0190497,-0.0137663,0.0703339,0.105$ $5426,0.1032664,-0.0317169,-0.007844,0.1034867,0.2616481,0.0636974,0.06$ $63304,0.1635846,0.1808654,-0.3557119,-0.0905424,0.0443925,-0.0693242,-$ $0.5373491,0.0671672,0.0262406,0.0631298,-0.312845 \backslash$ Polar $=248.1322888,19$ $.9180956,189.7174941,8.088908,-7.559065,145.664233 \backslash \mathrm{PG}=\mathrm{C} 01$ [X(C13H10Cl1 $\mathrm{N} 2)] \mathrm{NImag}=0 \backslash 10.41432735,0.03297346,0.48823812,0.01929221,0.03032857,0$ $.49326179,-0.10284078,-0.06419867,0.01166008,0.49894878,-0.05687999,-0$ $.16047920,0.03741175,0.14323424,0.44274787,0.00288983,0.02961981,-0.08$ $165249,0.23395277,-0.15644990,0.47474353,-0.01711002,-0.01067260,-0.00$ $924105,-0.24766343,-0.06274007,-0.12877922,0.49852561,-0.02149668,-0.0$ $0586462,-0.01336216,-0.09263611,-0.11558797,-0.03014747,0.19075247,0.5$ $1338204,0.00058167,-0.00400825,0.00345603,-0.10307470,-0.00466511,-0.1$ $4836139,0.15992288,-0.14419392,0.41977602,-0.00090524,0.00175737,0.004$ $68971,-0.00883633,0.01418771,-0.02787318,-0.10990011,0.02639411,-0.074$ $40446,0.49036027,0.00355903,-0.00382399,0.00064047,0.03511459,0.022281$ $53,0.02008002,-0.03277399,-0.13704347,0.03668026,0.19288469,0.47849840$ $, 0.00300622,-0.00146376,-0.00323658,-0.04695145,0.00073791,-0.03810953$ $,-0.02005824,0.09550898,-0.16074200,0.21243690,-0.13228403,0.42282460$, $-0.00849623,-0.00133661,-0.01678345,-0.02301064,-0.00835349,-0.0143953$ $9,0.04855220,-0.00422285,0.05042113,-0.12651468,-0.05374577,-0.0284253$ $6,0.44701130,0.00844262,0.01097651,0.00395825,-0.01726252,-0.00612421$, $-0.01096428,0.02436315,-0.03059252,0.05082166,-0.10991462,-0.22439927$, $0.04155290,0.11863706,0.50793760,-0.02247143,-0.00185096,-0.00991248$,$0.00776811,-0.00298845,-0.00447028,0.02515682,0.02422797,0.00525454,0$. $01985008,0.09438613,-0.11154477,0.20651791,-0.20745286,0.48592650,-0.0$ $8365112,0.01834195,-0.03817415,0.01977890,-0.00273110,0.02014702,-0.02$ $216144,0.01075150,-0.02865049,-0.03573278,-0.07053136,0.01818473,-0.22$ $271870,-0.03212544,-0.13277091,0.47232757,0.00363747,-0.10351714,0.041$ $96870,0.00978529,-0.01757689,0.02602767,-0.00500093,0.00557876,-0.0108$ $6332,-0.04190778,-0.03617581,-0.00248773,-0.05252439,-0.08667321,-0.02$ 
$128081,0.17542748,0.47362854,-0.02653144,0.04972591,-0.13330641,0.0093$ $2757,0.01256888,-0.00293329,-0.01557287,0.00288472,-0.01999109,-0.0069$ $4775,-0.03019787,0.02719874,-0.11351612,-0.00105029,-0.15687159,0.1734$ $9344,-0.10732789,0.37714212,-0.03372261,-0.00503931,-0.00482093,-0.004$ $68583,-0.01432610,-0.01360978,0.00031480,-0.00209283,0.00125106,-0.000$ $32944,-0.00017089,-0.00038440,0.00001041,0.00134541,-0.00051182,-0.001$ $86811,-0.00401994,-0.01299400,0.05454744,0.00072448,-0.12622990,-0.113$ $35985,-0.00719783,-0.01699682,-0.01722633,0.00121058,-0.00270981,0.000$ $06561,0.00007347,-0.00050298,0.00062347,0.00112025,-0.00126646,0.00143$ 032,0.00120154,0.01120674,0.01336096,0.00170704,0.14252313,0.00388744, $-0.11248473,-0.19510603,-0.00298062,0.00096201,0.00787839,0.00251765,-$ $0.00026857,0.00253660,-0.00064134,0.00018405,-0.00120247,0.00142731,0$. $00284718,-0.00242363,-0.00552785,-0.00825537,-0.02378765,0.00291690,0$. $12322135,0.21697868,0.00496052,-0.00886828,0.01421531,-0.07285961,0.03$ $804807,-0.07664520,-0.00159389,0.01466642,-0.02052151,-0.00148948,-0.0$ 0432056,-0.00370804,-0.00052113,-0.00019015,0.00012579,-0.00170060,-0. $00287588,-0.00012390,0.00044849,-0.00024551,-0.00045331,0.07196306,0.0$ 0842216,-0.01104303,0.01800146,0.03947933,-0.13179961,0.13089590,-0.00 $637123,0.00697615,-0.00628904,-0.00358566,0.00172365,0.00087134,0.0001$ $8735,-0.00039026,-0.00018244,-0.00394339,-0.00212434,-0.00031632,0.000$ $59438,0.00086489,-0.00034211,-0.03790370,0.13542689,-0.00189070,0.0012$ $4827,-0.00291312,-0.07681985,0.12753403,-0.21251307,-0.00399745,0.0127$ $0054,-0.00829443,-0.00469621,0.00009646,0.00208734,0.00005236,-0.00074$ $380,0.00005086,0.00069605,0.00051677,0.00190443,0.00037775,0.00080416$, $0.00029750,0.08489015,-0.14073002,0.22041936,0.00199005,-0.00064463,-0$ $.00458241,-0.02614546,-0.04259528,0.00059543,-0.13917179,-0.11569338,-$ $0.00493914,0.00832873,0.00880623,-0.00435443,-0.00391600,-0.00155901,-$ $0.00493142,0.00050817,0.00032302,0.00117171,-0.00094222,-0.00158556,0$. $00038717,-0.00021149,0.00171858,0.00293760,0.64423015,0.00168485,0.006$ $96494,0.00321184,-0.02724133,-0.01856497,-0.00418236,-0.07745718,-0.21$ $661571,0.04970414,-0.01500673,-0.03080680,0.01515121,0.00191701,0.0040$ $9514,0.00021461,-0.00370728,-0.00284819,-0.00062588,0.00026102,-0.0006$ $7716,0.00028291,0.00252702,-0.00012784,-0.00123288,0.13508106,0.376156$ $91,-0.00248863,0.00245074,0.00087896,-0.01903716,-0.01947664,0.0072094$ $9,0.00569560,0.07453366,-0.08618989,0.01682382,0.03997181,-0.01014494$, $-0.00907791,-0.00112661,-0.00395762,0.00269585,0.00069606,0.00158162,0$ $.00000604,0.00003842,-0.00048487,0.00100824,-0.00216071,-0.00016769,0$. $17570704,-0.26611540,0.51910175,-0.00011979,-0.00454855,-0.00368404,-0$ $.00049188,-0.00020913,0.00043866,0.00088348,-0.00453523,-0.00143898,0$. $00819510,-0.00933018,0.01199898,-0.08156828,0.04598954,-0.07843468,-0$. $00567763,0.01409669,-0.02033862,-0.00050981,0.00085978,-0.00094683,-0$. $00010132,0.00022826,0.00021270,0.00040583,-0.00024397,0.00073413,0.080$ $68267,-0.00364217,0.00159219,0.00184521,-0.00044302,-0.00030934,-0.000$ $71564,-0.00443703,-0.00129188,0.00190678,0.01000467,-0.00841286,0.0195$ $9068,0.04541359,-0.13953367,0.12537381,-0.00411439,0.00868531,-0.00759$ $746,0.00015018,-0.00029808,0.00019666,0.00022996,-0.00011028,-0.000122$ $03,0.00048275,-0.00049370,0.00107033,-0.04522348,0.14073498,-0.0037541$ $8,0.00044319,0.00386587,0.00021773,-0.00059431,0.00000150,-0.00101357$, $0.00214390,0.00385309,-0.00334339,0.00280700,-0.00195861,-0.08025760,0$ $.12441895,-0.21550090,-0.00373253,0.01047746,-0.00827424,0.00001422,-0$ $.00036827,0.00016249,0.00018817,-0.00013326,-0.00023754,-0.00018670,0$. 
$00016332,-0.00031969,0.08853310,-0.13724989,0.22118436,0.00971170,0.00$ $599657,0.00573469,-0.00241309,-0.00019022,-0.00320934,0.00197829,-0.00$ $207058,0.00382958,0.00403204,0.00825985,-0.01019843,-0.03311019,-0.044$ $13260,0.00232539,-0.10076184,-0.08076884,0.01515326,-0.00031479,-0.000$ $72604,-0.00198572,0.00075818,0.00044010,-0.00035697,0.00008856,0.00132$ $423,0.00000115,-0.00114358,0.00076159,0.00241820,0.38356764,-0.0031470$ $3,-0.02410192,0.01375302,-0.00016269,-0.00159479,0.00125111,0.00497053$ $,-0.00429573,0.00766328,0.00649108,0.02288497,-0.00693985,-0.04597698$, $-0.04049136,-0.00923077,-0.07026083,-0.21548425,0.06398714,-0.00133791$ ,-0.00048933,-0.00182091,-0.00089733,-0.00044218,0.00039055,-0.0000152 $2,0.00181769,-0.00090005,0.00258543,-0.00096752,-0.00116137,0.54076465$ $, 1.11349101,0.01290374,0.02338588,-0.00769123,-0.00307466,0.00211644,-$ $0.00313288,-0.00156285,0.00105421,-0.00275952,-0.00843310,-0.00436215$, $0.00352018,0.00383071,-0.00710010,0.01121572,0.00495115,0.05119600,-0$. $08486248,0.00063149,0.00123424,0.00084242,0.00077401,0.00148362,0.0000$ $1185,0.00041769,-0.00063800,0.00023575,0.00136745,-0.00244101,-0.00135$ $523,-0.12145521,-0.27400624,0.17189646,0.00010045,0.00057318,0.0005851$ $9,0.00167099,0.00040176,-0.00185499,-0.00413275,-0.00627140,-0.0024665$ $3,-0.22466214,-0.07126055,-0.13234681,-0.00816123,-0.00241251,-0.00915$ $543,0.00238703,-0.00292276,-0.00005303,0.00010537,-0.00004153,0.000004$ $76,-0.00039317,0.00005450,-0.00004089,0.00070161,0.00062144,0.00190225$ ,-0.00066543,0.00120786,0.00164307,-0.00041797,-0.00060498,-0.00056321 $, 0.23344950,0.00028684,-0.00041659,-0.00026098,0.00028598,-0.00125123$, $0.00410537,0.01244522,0.00795890,0.00882914,-0.07213673,-0.07150435,-0$ $.03206334,-0.02008459,-0.00270419,-0.01430394,-0.00323699,-0.00262666$, $0.00317001,-0.00009556,0.00002474,-0.00002791,0.00092187,0.00020121,0$. $00036943,0.00134375,0.00065158,-0.00125711,0.00136208,-0.00012277,-0.0$ $0130707,-0.00108453,0.00032521,-0.00063331,0.08009409,0.06969734,0.000$ $74328,-0.00010044,-0.00027435,-0.00155293,0.00431404,-0.00219891,-0.01$ $840069,-0.00895935,-0.00605239,-0.13255411,-0.03226124,-0.12886706,0.0$ $0692302,0.00327641,0.00718303,-0.00002539,0.00331752,0.00209231,-0.000$ $10436,0.00002437,-0.00003736,-0.00087319,-0.00049260,-0.00067833,0.000$ $16075,-0.00171276,0.00020140,0.00136366,-0.00144404,-0.00080632,0.0000$ $8820,0.00014241,0.00064378,0.14289895,0.03279472,0.12949418,-0.0009619$ $2,-0.00019618,-0.00030402,-0.00006257,-0.00017377,0.00000850,-0.000292$ $37,-0.00030964,0.00032462,0.00002530,0.00008924,-0.00008155,-0.0000060$ $9,-0.00002474,0.00014730,-0.00009504,0.00023935,-0.00010417,-0.0000666$ $5,-0.00002744,-0.00007283,-0.00007204,-0.00002041,0.00002490,0.0003140$ $1,0.00048602,-0.00002119,0.00005348,0.00001351,0.00004963,-0.00020008$, $0.00002013,0.00004587,0.00003527,0.00002403,0.00002601,0.50966346,-0.0$ $0065337,-0.00075540,-0.00029142,-0.00051210,0.00025759,0.00005206,0.00$ $013990,0.00072998,-0.00009354,0.00010038,-0.00019264,0.00030056,0.0001$ $4928,0.00029137,0.00012104,-0.00040215,0.00020081,0.00031498,-0.000189$ $29,0.00015870,0.00013417,-0.00000608,-0.00002537,-0.00005268,-0.000114$ $32,-0.00074591,-0.00003594,0.00008840,-0.00001857,-0.00005396,0.000018$ $92,-0.00013136,-0.00009748,-0.00001710,0.00000805,-0.00000036,0.003533$ $82,0.36076538,-0.00067001,-0.00041711,-0.00092169,-0.00065203,0.000276$ $21,0.00002182,0.00016919,0.00079777,-0.00008846,0.00010862,-0.00018489$ $, 0.00036518,0.00017553,0.00031298,0.00006884,-0.00058135,0.00028294,0$. $00038351,-0.00016298,0.00006730,0.00030007,0.00007675,-0.00000777,-0.0$ $0012153,-0.00009944,-0.00074002,-0.00003998,0.00010440,-0.00004263,-0$. 
00005799,-0.00011044,-0.00012937,-0.00001834,-0.00002196,-0.00000996,-$0.00001157,0.05131908,0.25679400,0.41357468,-0.00000584,-0.00162753,-0$ $.00240171,-0.00084257,0.00067691,0.00011456,0.00012667,0.00062968,-0.0$ $0017407,0.00015883,-0.00017549,0.00039670,0.00016176,0.00040904,-0.000$ $04843,-0.00105440,0.00018521,0.00056158,-0.00008498,0.00018165,0.00034$ $320,-0.00003526,-0.00006636,-0.00010644,-0.00012619,-0.00075284,-0.000$ $03128,0.00009056,-0.00007617,-0.00009645,-0.00024998,-0.00043195,-0.00$ $004431,-0.00001691,-0.00000617,0.00000619,-0.11407302,0.00376588,-0.00$ $185929,0.70360638,-0.00157401,0.00336221,-0.00428046,0.00115144,-0.000$ $24928,0.00032180,0.00008846,-0.00051927,-0.00007436,-0.00009316,0.0002$ $3600,-0.00029112,-0.00016156,-0.00005842,-0.00026817,-0.00073539,-0.00$ $027842,-0.00004067,-0.00001420,-0.00010538,-0.00010353,-0.00001758,-0$. $00003574,0.00009598,0.00011734,0.00068421,0.00009276,-0.00007384,-0.00$ $005516,-0.00002638,-0.00021340,-0.00009613,0.00007394,0.00000972,-0.00$ $000086,0.00001258,0.04699531,-0.18830439,-0.12882446,-0.00230004,0.389$ $62340,-0.00239840,-0.00463245,0.00161573,-0.00078478,0.00038874,-0.000$ $11951,-0.00024651,-0.00059603,0.00010433,-0.00002075,0.00010552,-0.000$ $16742,-0.00006327,-0.00055466,0.00011125,0.00140692,0.00000728,-0.0000$ $4556,0.00006803,0.00008195,0.00006551,-0.00006319,-0.00005013,0.000043$ $95,0.00001726,0.00032950,-0.00002979,-0.00007171,0.00010498,0.00011660$ $, 0.00000098,0.00002242,-0.00001375,0.00000934,0.00001483,0.00001083,0$. $04544757,-0.13379265,-0.20558319,0.06658882,0.28134110,0.44504363,-0.0$ $2821225,-0.00872997,-0.01332101,-0.00136103,0.00147818,-0.00145163,-0$. $00012704,-0.00135481,0.00058831,-0.00011559,0.00027225,-0.00028334,-0$. $00011836,0.00005596,-0.00065688,-0.00078280,-0.00174733,0.00047615,0.0$ $0088970,-0.00244252,-0.00289118,-0.00005917,-0.00026569,0.00045493,-0$. $00023973,0.00034902,-0.00018532,-0.00031317,-0.00003045,-0.00006037,-0$ $.00048632,-0.00002971,0.00000948,-0.00003609,0.00002222,0.00004160,0.0$ $0503118,0.06120720,0.06687258,-0.31777207,0.03029034,0.00193175,0.6648$ $7413,0.00586514,0.00640540,0.00292381,0.00138893,-0.00065886,0.0002089$ $0,-0.00023347,0.00000158,-0.00021033,0.00004780,-0.00008505,0.00002447$ $, 0.00026192,0.00006617,0.00035652,0.00126091,-0.00085078,-0.00028877,0$ $.00170909,0.00049583,0.00073312,0.00003366,0.00011057,-0.00001775,0.00$ $019911,0.00039299,0.00009144,-0.00011091,0.00015653,0.00019955,0.00026$ $175,0.00012868,0.00004741,0.00004425,-0.00001229,-0.00000459,0.0236418$ $5,-0.00150323,-0.00664800,0.07581264,-0.10379744,-0.03553747,-0.045099$ $15,0.41071766,0.00312580,0.00082708,0.00539548,0.00166868,-0.00038777$, $-0.00075897,0.00034287,-0.00033072,0.00070178,-0.00001099,0.00028800,-$ $0.00028212,-0.00015957,-0.00024532,0.00022009,0.00111598,-0.00023940$,$0.00017186,0.00170575,0.00061630,0.00074849,-0.00015116,-0.00014903,0$. $00004353,0.00008055,0.00069159,0.00004130,0.00006018,0.00002806,0.0000$ $7832,0.00007031,0.00074265,-0.00012563,0.00003841,-0.00000986,-0.00001$ $638,0.02500533,-0.00222505,0.00763625,0.05107928,-0.04067665,-0.100490$ $67,0.01639777,0.29296914,0.45431064,-0.14494995,0.02698160,0.02119319$, $-0.03008566,-0.01010991,0.00531960,0.00011080,0.00036876,0.00371269,-0$ $.00062199,-0.00042751,-0.00026918,0.00133964,0.00201770,0.00153340,-0$. $01945336,0.01370089,-0.01235089,-0.00831937,0.00270000,0.00120875,0.00$ $183416,0.00082693,-0.00126466,0.00053068,-0.00024002,-0.00044574,0.000$ $48772,-0.00016623,-0.00014161,0.00052205,-0.00216604,0.00072395,0.0000$ $9142,0.00001761,0.00001856,-0.00452856,-0.01336143,-0.01452522,-0.0490$ $0124,0.00194488,-0.00467089,-0.22723118,-0.08139091,-0.10869531,0.5981$ 
$4481,0.01819259,-0.07672559,-0.01116640,-0.02320382,0.00785599,0.00164$ $224,-0.00172448,-0.00150274,0.00053108,0.00043216,-0.00045546,0.000856$ $50,0.00026752,-0.00155226,0.00053944,0.01562731,-0.00417549,0.00044304$ $, 0.02003640,-0.00760113,-0.00646406,-0.00017642,0.00023087,-0.00005056$ ,-0.00058549,-0.00214114,-0.00079667,-0.00022016,0.00046785,0.00037821 $, 0.00002971,-0.00114085,0.00079135,-0.00009813,0.00007527,0.00002693,-$ $0.01450520,-0.03333903,-0.03043552,-0.02983492,0.03173816,0.02338441,-$ $0.03586880,-0.12785450,-0.07270815,-0.00882457,0.39606308,0.00892783$,$0.00946295,-0.07217974,0.00390522,-0.00028937,-0.00080099,0.00196242,-$ $0.00120581,0.00126278,-0.00069380,0.00052757,-0.00046363,0.00051088,0$. $00306437,-0.00288392,-0.02405015,0.00483197,0.00356010,0.02556998,-0.0$ $1030939,-0.00753359,0.00008691,-0.00022774,0.00067171,-0.00025035,0.00$ $049359,-0.00012014,-0.00002296,-0.00081291,-0.00100863,-0.00094712,0.0$ $0025747,0.00021401,-0.00010379,0.00005601,0.00010295,-0.01546662,-0.03$ $068995,-0.04237073,-0.03999233,0.02706984,0.03196992,-0.05871241,-0.07$ $864888,-0.15813924,0.04334458,0.24206972,0.44013739,-0.01214506,0.0214$ $7499,0.02180313,0.00043536,0.00243693,0.00068880,0.00009205,0.00145050$ ,-0.00010422,0.00010730,-0.00039524,0.00046506,0.00009995,0.00045539,$0.00010211,0.00059247,-0.00023122,0.00264551,-0.00549209,0.00221247,0$. $00177214,-0.00009367,-0.00009267,0.00001237,-0.00015851,-0.00131081,-0$ $.00010213,0.00008070,-0.00006424,-0.00011649,-0.00037699,-0.00005265,-$ $0.00011926,-0.00004457,-0.00000461,0.00000845,-0.05350887,-0.03310091$, $-0.04348302,-0.02991799,0.02312950,0.02201854,0.04998282,-0.00940116$, $0.00544062,-0.12909170,0.00998494,0.00280405,0.70814368,0.00742002,-0$. $00342735,-0.00485886,-0.00030252,-0.00024461,0.00053697,-0.00088707,0$. $00016217,-0.00059026,-0.00000043,-0.00043032,0.00001706,-0.00001637,-0$ $.00000120,-0.00030149,-0.00024051,0.00063805,-0.00117403,0.00072663,0$. $00066688,-0.00025506,0.00010797,0.00034499,-0.00015805,0.00014918,-0.0$ $0000379,0.00006194,-0.00005475,0.00002568,-0.00002399,0.00013717,-0.00$ $015000,-0.00003516,-0.00002861,0.00003778,0.00001874,0.00458017,0.0326$ $7795,0.02824600,0.02317628,-0.02122494,-0.01720401,-0.03955318,-0.0143$ $9320,-0.02872515,0.05091799,-0.18022823,-0.11785210,-0.01150068,0.3848$ $9165,0.00660569,-0.00227638,-0.00078346,0.00012373,-0.00139760,0.00031$ $701,0.00001419,-0.00015071,-0.00019816,-0.00019500,0.00006950,-0.00052$ $394,-0.00058125,0.00004116,-0.00012568,-0.00038974,0.00018033,0.000199$ $19,0.00012061,-0.00011405,0.00081466,0.00008087,0.00003443,0.00002475$, $-0.00006314,-0.00030606,-0.00011342,0.00001573,-0.00008215,-0.00005979$ $, 0.00055130,0.00034037,0.00006812,-0.00001600,-0.00000414,0.00003869,-$ $0.00231604,0.02416070,0.03319000,0.02257993,-0.01730548,-0.01955658,-0$ $.03891313,-0.02551615,-0.02445809,0.04850217,-0.12290272,-0.19488166,0$ $.05629607,0.27142621,0.43877744,0.00365153,-0.00000555,0.00007200,0.00$ $061158,-0.00006570,-0.00016559,-0.00010512,-0.00077585,-0.00013787,-0$. $00008233,0.00016218,-0.00023438,-0.00020154,-0.00035166,-0.00032936,0$. $00028545,-0.00048028,-0.00049142,0.00042356,-0.00029236,-0.00044999,-0$ $.00003741,0.00006176,0.00015524,-0.00006488,0.00051831,0.00001622,-0.0$ $0016256,0.00001093,-0.00000894,0.00016962,0.00006751,0.00011675,-0.000$ $00388,0.00000020,-0.00000063,-0.20535675,-0.04232487,-0.06354348,0.049$ $77252,-0.03831846,-0.03720104,-0.06191058,-0.00705178,-0.01404347,0.00$ $440331,0.05621061,0.06146811,-0.32374763,0.03570580,0.00675743,0.66109$ $794,0.00031411,0.00048330,-0.00531555,-0.00042957,-0.00010597,0.000331$ $10,0.00011463,0.00027702,0.00016247,-0.00003622,-0.00002822,0.00017819$ 
$, 0.00009218,0.00009213,-0.00011618,-0.00086734,0.00026478,-0.00023435$, $0.00097638,-0.00030138,-0.00030330,0.00011690,-0.00002738,-0.00012508$, $0.00002480,-0.00001353,-0.00003154,0.00002817,-0.00005454,-0.00005452$, $-0.00001019,0.00001997,0.00011151,0.00000338,-0.00001987,-0.00002018$, $0.08563542,-0.13079214,-0.08255988,-0.00835532,-0.01275325,-0.02471457$ ,-0.00704614,-0.00348096,-0.00096787,0.02394113,0.00007888,-0.00507469 $, 0.08051486,-0.10473171,-0.03800103,-0.05111712,0.41208888,0.00031068$, $-0.00508925,-0.00021312,-0.00065431,-0.00010786,0.00012057,0.00000123$, $-0.00013723,-0.00005854,0.00012181,0.00000545,0.00006547,0.00008203,0$. $00000257,0.00018109,-0.00002013,0.00020240,-0.00034956,0.00080647,-0.0$ $0041311,-0.00030725,0.00002007,0.00009162,0.00004105,0.00000573,0.0000$ $7314,0.00001956,0.00000885,0.00001277,0.00001801,0.00003326,-0.0002066$ $5,-0.00004550,-0.00001415,0.00000566,-0.00001644,-0.11106885,-0.077291$ $84,-0.16388496,-0.00433523,-0.02846660,-0.02248781,-0.01474185,-0.0005$ $8845,-0.00618670,0.02558750,-0.00157752,0.00936864,0.05636745,-0.04336$ $007,-0.10246769,0.00869199,0.30282562,0.46420438,0.00040121,-0.0002336$ $2,-0.00022107,-0.00044705,0.00054399,-0.00015958,0.00028597,0.00069007$ ,-0.00020475,0.00003273,-0.00021468,0.00031736,0.00013410,0.00034841,-$0.00015390,-0.00044529,-0.00022458,0.00041473,-0.00000408,-0.00000410$, $0.00004294,0.00007745,-0.00000931,-0.00001179,-0.00033815,-0.00091436$, $-0.00004586,-0.00001754,-0.00005803,-0.00010837,-0.00002007,-0.0002063$ $6,0.00000732,-0.00003892,-0.00002483,-0.00002535,-0.14221807,0.0284452$ $7,0.01612654,-0.02687458,0.00810923,0.00502608,0.00334436,0.00199707,0$ $.00192727,-0.00133161,0.00077471,0.00110239,0.00128280,-0.00421245,-0$. $00507856,-0.04128159,0.01208366,0.00747810,0.20779189,-0.00003695,-0.0$ $0027145,0.00050493,0.00013320,-0.00012790,0.00004060,-0.00009238,-0.00$ $026490,0.00002765,-0.00000377,0.00007208,-0.00010669,-0.00004037,-0.00$ $011849,0.00009768,0.00023021,0.00010089,-0.00011687,-0.00005495,0.0000$ $5151,0.00003819,-0.00001952,0.00000658,-0.00000425,0.00009491,0.000312$ $55,0.00002332,0.00000747,0.00001795,0.00003865,-0.00002014,0.00003825$, $0.00000134,0.00001227,0.00000455,0.00000730,0.02836926,-0.03860973,-0$. $01230391,0.02624220,-0.00297694,-0.00501859,-0.00240744,0.00074885,-0$. $00479527,0.00067193,0.00031302,0.00108688,0.00021584,0.00165527,-0.003$ $15175,-0.00606596,0.00557331,0.00027788,-0.04752556,0.03540183,0.00004$ $298,0.00050318,-0.00016060,0.00012782,-0.00010194,0.00010423,-0.000030$ $17,-0.00006436,0.00003487,-0.00001378,0.00003705,-0.00004432,-0.000025$ $35,-0.00007359,0.00001279,0.00005562,0.00001071,-0.00008997,-0.0000674$ $1,0.00002682,0.00001831,-0.00001505,-0.00000624,-0.00000437,0.00005865$ $, 0.00016247,0.00001676,0.00000448,0.00000368,0.00001231,-0.00000611,0$. $00001620,0.00000040,0.00001125,0.00000270,0.00000395,0.01626902,-0.012$ $35648,-0.03553840,0.02474135,-0.00729230,-0.00025654,-0.00288958,-0.00$ $428400,-0.00008852,0.00073155,0.00116776,0.00065759,-0.00021216,-0.003$ $67679,0.00046369,-0.01239438,0.00259319,0.00591759,-0.02731850,0.01967$ $941,0.03003406,-0.00041123,-0.00057143,-0.00066551,-0.00019366,0.00014$ $529,-0.00006126,0.00001813,0.00004842,0.00001904,-0.00000948,-0.000021$ $49,0.00001748,0.00001363,0.00004531,-0.00003711,-0.00014997,-0.0000244$ $9,0.00009952,0.00005890,-0.00002222,-0.00001510,-0.00000532,-0.0000226$ $7,0.00001696,-0.00004713,-0.00011674,-0.00001839,-0.00000906,-0.000010$ $77,-0.00002457,-0.00005134,-0.00009085,0.00001152,-0.00000749,-0.00000$ $045,0.00000025,0.00622841,0.00506053,0.00577560,-0.20772054,-0.0880034$ $8,-0.11618424,-0.01535038,-0.01309602,-0.01650516,-0.00359858,0.002645$ 
$82,0.00157114,-0.00030383,0.00051777,0.00064439,0.00080171,0.00172961$, $0.00137885,0.00121244,0.00018882,0.00068954,0.21801618,0.00039092,0.00$ $015789,0.00014607,0.00004260,-0.00008326,-0.00009357,0.00008756,-0.000$ $01413,0.00011751,-0.00000876,0.00004882,-0.00005859,-0.00003866,-0.000$ $02386,0.00000036,0.00003574,0.00004631,0.00001191,-0.00000946,0.000004$ $80,0.00001168,-0.00000280,-0.00000553,-0.00000504,-0.00001452,0.000037$ $87,-0.00000529,0.00001654,-0.00000548,-0.00000271,0.00005315,0.0000635$ $8,-0.00001175,0.00000054,-0.00000202,-0.00000254,-0.01603044,-0.004483$ $78,-0.01057916,-0.08621609,-0.09565086,-0.07585727,0.00685367,0.006907$ $25,0.00430802,0.00233743,0.00383090,-0.00299777,0.00062740,-0.00087752$ $, 0.00046088,0.00211435,0.00065785,-0.00508985,-0.00052607,-0.00092341$, $0.00189563,0.09165641,0.09268160,0.00033705,0.00006114,0.00017800,0.00$ $001966,-0.00000694,0.00007118,-0.00007907,0.00003822,-0.00007281,0.000$ $01533,-0.00005549,0.00003986,0.00003003,0.00004253,0.00004327,0.000005$ $37,-0.00003779,-0.00009696,-0.00000553,0.00000263,-0.00001845,0.000010$ $29,0.00000902,-0.00000338,0.00001723,-0.00001541,0.00000940,-0.0000099$ $1,0.00000533,0.00001079,-0.00002208,-0.00004344,0.00001732,0.00000092$, $0.00000102,-0.00000003,-0.01722713,-0.00823935,-0.00749095,-0.11410344$ ,-0.07576376,-0.13149592,0.00518129,0.00181645,0.00640573,0.00124230,$0.00300341,0.00376584,0.00080746,0.00048861,-0.00056852,0.00179514,-0$. $00512604,0.00013532,-0.00010099,0.00199011,-0.00057883,0.12269629,0.08$ $554022,0.13178755,0.00126598,-0.00157377,-0.00116639,0.00000354,0.0001$ $3643,0.00007109,-0.00005626,-0.00009913,0.00001341,0.00000372,0.000046$ $71,-0.00000095,-0.00002128,-0.00006874,0.00004336,-0.00001340,0.000005$ $77,0.00005273,-0.00025792,0.00038245,0.00047297,-0.00000359,0.00000129$ ,-0.00002881,0.00005693,0.00014362,-0.00000154,0.00001528,-0.00000227, $-0.00000411,0.00003606,0.00006991,-0.00004107,0.00000650,0.00000047,-0$ $.00000137,-0.00392803,0.00116201,-0.00008699,-0.00306846,0.02072648,0$. $02142229,-0.06986334,0.04213942,0.04154927,0.00742902,-0.01454042,-0.0$ $1532447,-0.00290640,-0.00174470,-0.00302682,-0.00108790,-0.00020180,-0$ $.00022475,-0.00115974,0.00091766,0.00086074,0.00099829,-0.00046664,0.0$ $0015667,0.07216639,0.00112460,-0.00197862,0.00208525,-0.00051444,0.000$ $05908,-0.00006526,-0.00002892,0.00000026,0.00010656,-0.00000179,0.0000$ $0267,0.00000987,0.00003270,-0.00019425,0.00012177,0.00050582,0.0004060$ $7,0.00014382,-0.00054164,0.00066057,0.00042271,-0.00006734,-0.00006990$ ,-0.00003296,0.00001411,-0.00006504,-0.00002533,0.00004348,0.00001312, $0.00004502,0.00006725,0.00002166,0.00001127,0.00000328,0.00000170,0.00$ $000687,0.00132776,0.00452310,-0.00239804,-0.00006183,-0.00142780,-0.00$ $533882,0.04181037,-0.17394893,-0.14354829,0.00488415,-0.00498271,-0.00$ $888742,-0.00230207,0.00295975,-0.00373888,-0.00029948,-0.00026855,0.00$ $085056,0.00013594,-0.00009451,-0.00022117,-0.00024143,-0.00185211,0.00$ $237268,-0.04615888,0.17695617,0.00152993,0.00232829,-0.00145351,0.0004$ $1828,0.00002395,0.00052618,-0.00003909,0.00004644,-0.00016660,0.000029$ $70,0.00001713,0.00002635,-0.00001901,0.00012618,-0.00006028,-0.0005949$ $5,-0.00004975,-0.00006940,-0.00075287,0.00020663,0.00049220,0.00003541$ $, 0.00001330,-0.00003326,0.00007901,0.00012451,0.00004247,0.00001576,-0$ $.00004402,-0.00005496,-0.00021399,-0.00038998,0.00003617,0.00000595,0$. $00000648,0.00000533,0.00017869,-0.00254632,0.00431059,-0.00082261,-0.0$ $0311195,0.00021367,0.04083601,-0.14269174,-0.18488893,0.00617931,-0.01$ $178027,-0.00796782,-0.00372906,-0.00359812,0.00183965,-0.00029439,0.00$ $088424,-0.00017218,0.00003707,-0.00014420,-0.00004512,0.00036266,0.002$ 
$41502,-0.00152799,-0.04393570,0.15825247,0.18962622,-0.00000921,-0.000$ $16285,0.00039968,-0.00002977,0.00032725,0.00020976,-0.00035775,-0.0001$ $9380,0.00030188,-0.00009135,0.00006092,-0.00014694,-0.00004397,0.00003$ $227,-0.00006987,0.00002469,0.00010960,0.00013666,0.00011162,-0.0001498$ $2,-0.00010383,-0.00000300,0.00001220,-0.00002930,0.00002087,0.00020281$ $, 0.00001335,0.00000103,-0.00000888,0.00001061,0.00009026,0.00008504,-0$ $.00007308,0.00000060,0.00000890,-0.00000069,-0.00303985,0.00230773,0.0$ $0136164,-0.00024866,0.00050983,0.00065452,0.00074144,0.00171550,0.0012$ $4765,0.00856311,0.00343501,0.00448631,-0.20111979,-0.08445831,-0.11203$ $348,-0.01671695,-0.01305544,-0.01691662,-0.00145248,-0.00021114,-0.000$ $39690,-0.00000731,-0.00009623,-0.00002036,0.00038461,0.00032486,0.0003$ $2719,0.21232882,-0.00089271,-0.00125218,0.00188700,-0.00040760,0.00021$ $603,-0.00004942,0.00060666,0.00018359,0.00010831,0.00016091,0.00024274$ ,-0.00010695,-0.00004146,-0.00008471,0.00028378,0.00027279,-0.00009153 ,-0.00004086,-0.00018499,0.00001325,0.00010376,-0.00004552,-0.00001243 $, 0.00012470,-0.00012362,-0.00029229,-0.00006084,0.00004391,-0.00000521$ $, 0.00003040,-0.00009337,-0.00017821,-0.00000895,-0.00001408,-0.0000054$ $7,-0.00000486,0.00209117,0.00411415,-0.00322868,0.00050356,-0.00082292$ $, 0.00054276,0.00233924,0.00098240,-0.00568358,-0.01629270,-0.00452469$, $-0.01050639,-0.08427669,-0.09760562,-0.07655376,0.00740533,0.00677973$, $0.00406312,0.00057267,0.00006165,0.00007445,-0.00010942,-0.00035931,0$. $00036156,-0.00077532,-0.00028186,-0.00057979,0.08969784,0.09472276,-0$. $00059227,0.00189935,-0.00099482,0.00008774,0.00023603,0.00025795,-0.00$ $012086,0.00058609,-0.00035636,0.00010431,-0.00035768,0.00048368,0.0003$ $0088,0.00005024,-0.00005220,-0.00012563,-0.00007185,-0.00005546,-0.000$ $08020,0.00008938,0.00001526,0.00008256,-0.00007862,-0.00018642,0.00003$ $318,-0.00016071,0.00007769,-0.00004521,0.00005917,0.00001493,-0.000200$ $54,-0.00012585,0.00006460,-0.00002575,-0.00000138,-0.00001181,0.001032$ $54,-0.00313340,0.00401795,0.00078319,0.00046736,-0.00059195,0.00181910$ ,-0.00574325,0.00030279,-0.01693397,-0.00817159,-0.00753452,-0.1117586 $9,-0.07628293,-0.13298737,0.00546531,0.00150160,0.00628030,0.00040477$, $-0.00001917,0.00009302,-0.00003444,0.00037181,-0.00032256,-0.00086940$, $-0.00044412,-0.00045475,0.12040426,0.08657743,0.13351060,0.00002195,0$. $00086366,0.00086571,0.00014168,0.00008234,-0.00006323,0.00001999,0.000$ $10006,-0.00004238,0.00000732,-0.00004075,0.00000889,-0.00000739,0.0000$ $0944,-0.00004774,0.00009535,-0.00010117,0.00017084,-0.00037275,0.00015$ $359,0.00014137,-0.00001445,-0.00001368,0.00002941,-0.00003329,-0.00014$ $668,-0.00002044,-0.00001868,0.00000719,-0.00000572,-0.00001026,-0.0000$ $2512,-0.00001520,-0.00001210,0.00000165,0.00000056,0.00688489,-0.01524$ $640,-0.01636390,-0.00311884,-0.00172011,-0.00285547,-0.00112778,-0.000$ $10773,-0.00017144,-0.00489060,0.00118371,-0.00016127,-0.00206517,0.019$ $65413,0.02076031,-0.07036524,0.04676971,0.04708428,0.00106511,-0.00064$ $114,-0.00023331,0.00046537,-0.00074391,-0.00079323,0.00006297,0.000005$ $04,0.00009831,0.00091344,-0.00046483,0.00011595,0.07236579,-0.00013540$ $, 0.00001170,-0.00029494,-0.00011797,-0.00009740,-0.00001685,-0.0000068$ $4,0.00004494,0.00004623,0.00002147,-0.00000965,-0.00001982,-0.00002250$ ,-0.00002654,0.00001028,0.00005189,-0.00001779,0.00003174,0.00011205,-$0.00009154,0.00003662,-0.00000634,-0.00000323,0.00000865,0.00001079,-0$ $.00001260,0.00000291,0.00001289,-0.00000406,-0.00000081,0.00005055,0.0$ $0002397,-0.00002530,0.00000111,0.00000384,0.00000378,0.00577572,-0.005$ $01532,-0.00848757,-0.00203241,0.00278705,-0.00301360,-0.00027499,-0.00$ 
$026326,0.00082809,0.00150323,0.00471669,-0.00259802,-0.00008441,-0.000$ $83052,-0.00510208,0.04494342,-0.17374580,-0.14613435,0.00004973,-0.000$ $86433,0.00195987,0.00019720,-0.00012218,-0.00045021,-0.00002209,-0.000$ $39829,0.00030843,-0.00034152,-0.00195861,0.00260423,-0.04963881,0.1758$ $9198,-0.00030713,-0.00020163,-0.00000467,0.00014166,0.00004439,0.00005$ $333,-0.00006370,-0.00010753,-0.00000540,-0.00000084,0.00001941,-0.0000$ $4931,-0.00002766,-0.00000816,0.00003810,0.00000443,0.00002659,-0.00009$ $263,0.00006126,0.00003358,-0.00006597,0.00000260,0.00000230,-0.0000226$ $6,0.00002517,0.00012450,0.00000497,-0.00000556,0.00000831,0.00000205,-$ $0.00003164,0.00001231,0.00002686,0.00000050,0.00000259,0.00000581,0.00$ $674337,-0.01103527,-0.00782288,-0.00329735,-0.00293453,0.00161900,-0.0$ $0033513,0.00083801,-0.00012790,0.00028898,-0.00261606,0.00434236,-0.00$ $071965,-0.00266569,0.00092818,0.04491130,-0.14594067,-0.19021853,0.000$ $55562,0.00185073,-0.00057380,0.00025203,-0.00056064,-0.00027849,0.0000$ $8182,0.00030491,-0.00034012,0.00020962,0.00255991,-0.00154466,-0.04852$ $743,0.16026639,0.19416093,-0.00046689,0.00069161,-0.00005574,0.0001597$ $9,-0.00144178,0.00131599,0.00574604,-0.00043700,0.01791144,-0.00101990$ $,-0.00374239,-0.00038672,0.00137743,-0.00016991,0.00015549,-0.00029705$ $,-0.00010736,-0.00015002,0.00004462,-0.00008492,0.00008884,-0.00036690$ $, 0.00001872,-0.00114903,-0.06613071,-0.00144607,-0.05824554,-0.0002060$ $7,-0.00011269,0.00013297,0.00009124,0.00025622,-0.00014549,0.00004384$, $0.00005504,0.00036514,-0.00003509,-0.00005506,-0.00005757,-0.00005966$, $0.00006245,0.00002674,0.00003709,0.00001583,0.00003014,-0.00005532,-0$. $00021683,0.00007083,-0.00012488,0.00002833,-0.00001296,0.00005576,0.00$ $002022,0.00000687,-0.00002925,0.00001665,0.00000642,-0.00000692,0.0000$ $0379,-0.00000054,0.00000822,-0.00001587,0.00001775,0.00002661,-0.00003$ $937,0.00001789,-0.00001523,-0.00000914,0.00001695,0.06293794,0.0002981$ $9,-0.00075993,-0.00077349,0.00360435,0.00187494,0.00002437,0.00561464$, $-0.01745446,0.03066089,-0.00514153,-0.00273417,-0.00000822,0.00042153$, $-0.00040037,0.00182343,0.00050762,0.00040284,-0.00001535,-0.00011361,-$ $0.00005823,0.00007420,-0.00106298,0.00056009,-0.00032539,0.00357626,-0$ $.08296454,0.13618790,-0.00016135,-0.00042749,-0.00005137,-0.00021144,-$ $0.00022184,-0.00000627,0.00016273,-0.00002636,-0.00009170,-0.00006883$, $0.00007006,0.00006847,0.00006410,-0.00007784,-0.00001643,0.00003233,-0$ $.00003393,-0.00006225,-0.00003750,0.00031900,-0.00004160,0.00013733,-0$ $.00002050,0.00005447,-0.00002541,-0.00001274,0.00000044,0.00012237,-0$. $00004214,-0.00002360,0.00001752,0.00000031,-0.00000168,-0.00001847,-0$. $00000095,-0.00001977,-0.00003304,0.00004767,-0.00002139,0.00002315,0.0$ $0000289,-0.00002288,-0.00850190,0.10286552,-0.00011339,0.00028189,0.00$ $011634,-0.00064735,-0.00198461,0.00148760,0.00009948,-0.00458882,-0.00$ $375505,0.00050326,0.00088028,0.00090727,-0.00127449,-0.00001258,0.0004$ $1376,0.00029813,0.00006639,0.00016217,-0.00005090,-0.00006982,-0.00001$ $642,0.00064456,0.00011530,-0.00031321,-0.07751893,0.17123616,-0.368258$ $30,0.00013570,-0.00001696,-0.00005985,0.00008132,-0.00012547,-0.000070$ 44,0.00019566,-0.00033790,-0.00000036,0.00000534,-0.00000112,-0.000001 $50,-0.00000642,-0.00000025,-0.00000162,0.00000092,0.00000692,-0.000026$ $31,-0.00006983,0.00000833,0.00000074,0.00004385,0.00001206,0.00005947$, $0.00000405,-0.00001651,-0.00001398,0.00000092,-0.00000388,-0.00000414$, $-0.00000038,-0.00000298,0.00000084,-0.00000393,-0.00001053,-0.00000289$ $,-0.00001648,0.00002855,-0.00003457,0.00001333,0.00000201,-0.00000614$, $0.07243861,-0.16458491,0.37520291,0.00106351,-0.00049451,-0.00143949$,- 
$0.00078953,-0.00194198,0.00147959,-0.01336337,0.00495761,-0.00104103,0$ $.00162937,0.00244984,-0.00150532,-0.00067982,-0.00110049,-0.00048355,0$ $.00065902,0.00036698,0.00032556,-0.00025762,-0.00034051,0.00013013,-0$. $00026738,0.00046452,0.00048592,-0.41955909,-0.01569885,-0.11498163,0.0$ $0000172,0.00006430,-0.00001086,-0.00017078,-0.00017851,0.00022456,0.00$ $007022,-0.00026973,0.00075701,-0.00000393,0.00002565,0.00002525,0.0000$ $2107,-0.00002641,-0.00000230,-0.00002442,0.00000671,-0.00002035,0.0001$ $4403,0.00015735,-0.00009049,0.00001285,-0.00001474,0.00000273,-0.00001$ $873,0.00001747,-0.00000347,0.00001415,-0.00000374,0.00000071,0.0000018$ $2,-0.00000306,0.00000312,0.00000607,0.00001107,0.00000649,-0.00001140$, $-0.00000403,-0.00000380,-0.00000616,-0.00000300,0.00000393,-0.00164390$ $, 0.00068042,0.00524204,0.43310292,-0.00127134,0.00004320,-0.00061853,-$ $0.00077633,-0.00397626,0.00388517,-0.03619171,-0.00431163,-0.01270933$, $0.00025251,0.00069346,-0.00224740,0.00022828,-0.00049157,0.00001366,0$. $00026898,0.00055228,-0.00019944,-0.00010043,-0.00007019,0.00003857,0.0$ $0006949,-0.00000835,-0.00022684,0.01118899,-0.03330384,0.03638915,-0.0$ $0001890,0.00006734,0.00002699,-0.00009714,-0.00024603,0.00016790,0.000$ $37945,0.00021708,0.00103892,-0.00005578,0.00005418,0.00005582,0.000050$ $34,-0.00001878,-0.00005636,-0.00011149,-0.00000694,-0.00000830,-0.0000$ $6642,-0.00021100,-0.00002044,0.00006310,0.00000176,0.00002215,-0.00004$ $559,0.00001345,-0.00001844,0.00006495,-0.00002360,-0.00000863,0.000001$ $82,0.00000010,0.00000132,-0.00001263,-0.00000409,0.00000372,-0.0000201$ $4,0.00001287,0.00004169,0.00000955,-0.00000041,-0.00000560,0.01532555$, $-0.00107833,-0.00093029,0.01075765,0.04191195,-0.00130025,0.00046023,0$ $.00087942,0.00218610,0.00213984,0.00181239,0.00269015,-0.00961900,-0.0$ $0119875,0.00265793,0.00045888,0.00083303,0.00018007,-0.00039845,-0.000$ $22334,0.00029675,0.00008791,0.00003950,0.00016992,0.00045047,-0.000164$ $90,0.00042778,-0.00018852,-0.00006520,-0.08477863,0.03327744,-0.059515$ $59,-0.00002567,0.00007753,0.00004616,-0.00019780,-0.00015749,0.0000351$ $2,-0.00082307,0.00070420,-0.00051963,-0.00005479,0.00006771,0.00008659$ $, 0.00007411,-0.00004771,-0.00007004,-0.00007832,-0.00002871,-0.0000082$ $3,-0.00017095,-0.00005315,0.00000871,0.00012979,-0.00001930,-0.0000098$ $4,-0.00005167,0.00001184,-0.00001749,0.00009025,-0.00003288,-0.0000160$ $3,0.00000544,-0.00000032,-0.00000015,-0.00002079,-0.00001381,-0.000009$ $89,-0.00001788,0.00004617,0.00004140,0.00001846,-0.00000103,-0.0000116$ $9,-0.03245038,-0.00278456,-0.00578901,0.11095407,-0.02455787,0.0639010$ $1,-0.00348609,-0.00075032,-0.00364989,0.00100825,0.00005940,0.00103678$ $,-0.00061867,0.00137970,-0.00167758,-0.00256986,-0.00455468,0.00250324$ $, 0.01039510,0.00716109,0.00211722,-0.00009512,-0.02494081,0.00514500,0$ $.00027335,0.00063305,0.00072702,-0.00021297,-0.00021532,0.00004576,-0$. $00009095,-0.00083357,0.00009905,0.00010723,0.00004328,-0.00034225,-0.2$ $6131838,-0.42972632,0.10951176,-0.00007014,0.00007421,0.00013710,0.000$ $21291,-0.00002189,0.00010249,0.00033340,0.00011666,-0.00005629,0.00023$ $057,0.00008957,-0.00024718,-0.00044162,0.00082140,0.00000511,0.0002671$ $7,-0.00010716,-0.00042904,-0.00012993,0.00000735,-0.00000583,0.0000162$ $1,0.00002264,0.00000256,0.00005737,-0.00006347,0.00004411,-0.00007726$, $-0.00005671,0.00024415,-0.00007563,0.00007163,0.00013909,0.00001409,-0$ $.00003463,0.00001684,-0.00007080,0.00011350,-0.00000506,0.00006938,0.0$ $0010726,0.00008937,0.25627240,-0.00360685,0.00241146,-0.00408369,0.001$ $18655,0.00053552,0.00088798,-0.00177589,0.00277345,-0.00373410,-0.0049$ $7117,-0.01007929,0.00265439,0.01783694,0.01166790,0.00897527,-0.031587$ 
$58,-0.02927087,0.00258292,0.00067250,0.00072737,0.00103838,-0.00001536$ $,-0.00022044,-0.00006222,-0.00019984,-0.00143682,0.00038168,-0.0006321$ $8,0.00034965,-0.00014473,-0.42854698,-0.84878349,0.20769062,0.00010207$ ,-0.00051460,0.00011007,-0.00013947,0.00006556,0.00009550,0.00023963,0 $.00002277,0.00005954,0.00025089,-0.00022455,-0.00053626,0.00026900,0.0$ $0074509,0.00016803,0.00011410,0.00010759,-0.00018726,0.00001967,0.0000$ $0675,0.00006999,0.00021588,-0.00004091,-0.00002404,0.00007692,-0.00003$ $384,0.00001268,-0.00004736,-0.00003655,0.00019910,-0.00009858,0.000098$ $20,0.00012755,0.00002708,-0.00002151,0.00000472,-0.00011413,0.00017049$ $, 0.00004876,0.00012000,0.00018335,0.00012058,0.45060417,0.87079773,-0$. $00166653,-0.00157559,0.00115670,0.00016091,-0.00030373,0.00044612,0.00$ $063289,-0.00079083,0.00131080,0.00248723,0.00244835,-0.00101500,-0.005$ $84544,-0.00078467,-0.00269316,0.01129184,0.01071722,0.01673446,-0.0001$ $5431,-0.00051712,-0.00000199,-0.00011497,-0.00016375,-0.00004211,0.000$ $01041,0.00048854,-0.00007919,0.00003041,0.00028546,0.00032728,0.108647$ $74,0.20665848,-0.08875375,0.00018936,0.00015472,-0.00029130,0.00008941$ $,-0.00000842,-0.00000332,0.00005046,0.00003304,-0.00000405,-0.00008974$ ,-0.00000762,-0.00011485,-0.00004831,-0.00011292,-0.00017813,0.0000247 $3,-0.00002653,0.00001335,-0.00010693,0.00000220,0.00008777,-0.00010264$ $, 0.00003279,0.00003105,0.00000546,-0.00001162,-0.00001006,0.00001274,-$ $0.00000913,0.00012888,0.00006231,0.00003217,-0.00003273,-0.00000665,0$. $00003534,-0.00001675,0.00005193,-0.00004331,0.00000270,-0.00005882,-0$. $00007758,-0.00006356,-0.11555351,-0.21645549,0.07306083 \backslash \backslash-0.00001834,0$ $.00001874,-0.00000075,0.00000500,-0.00000867,0.00000015,-0.00000265,-0$ $.00002207,0.00000555,-0.00000656,0.00000737,0.00000004,0.00000638,0.00$ $000469,-0.00000145,-0.00000115,-0.00001751,0.00000338,0.00000562,-0.00$ $000069,-0.00000280,0.00000292,0.00000250,0.00000216,0.00000096,0.00002$ $145,0.00002821,-0.00000251,-0.00000228,0.00000186,-0.00001319,-0.00002$ $192,-0.00000126,-0.00000219,-0.00000584,0.00000650,0.00000246,0.000004$ $49,-0.00000169,0.00000258,-0.00000076,-0.00000421,-0.00000233,-0.00000$ $142,-0.00000353,0.00000708,0.00000676,-0.00000702,0.00000287,-0.000004$ $33,0.00000094,-0.00000596,-0.00000072,-0.00000178,-0.00000064,0.000000$ $32,-0.00000407,0.00000104,0.00000355,-0.00000549,0.00000146,0.00000329$ ,-0.00000309,-0.00000130,-0.00000240,0.00000150,-0.00000134,-0.0000017 $2,-0.00000023,-0.00000129,0.00000109,0.00000176,0.00001152,-0.00000518$ ,-0.00000468,0.00000958,0.00002126,-0.00001000III@ 